Prepared for the Missouri River Recovery-Integrated Science Program U.S. Army Corps of Engineers, Yankton, South Dakota

\title{
Hydraulic and Substrate Maps of Reaches Used by Sturgeon (Genus Scaphirhynchus) in the Lower Missouri River, 2005-07
}

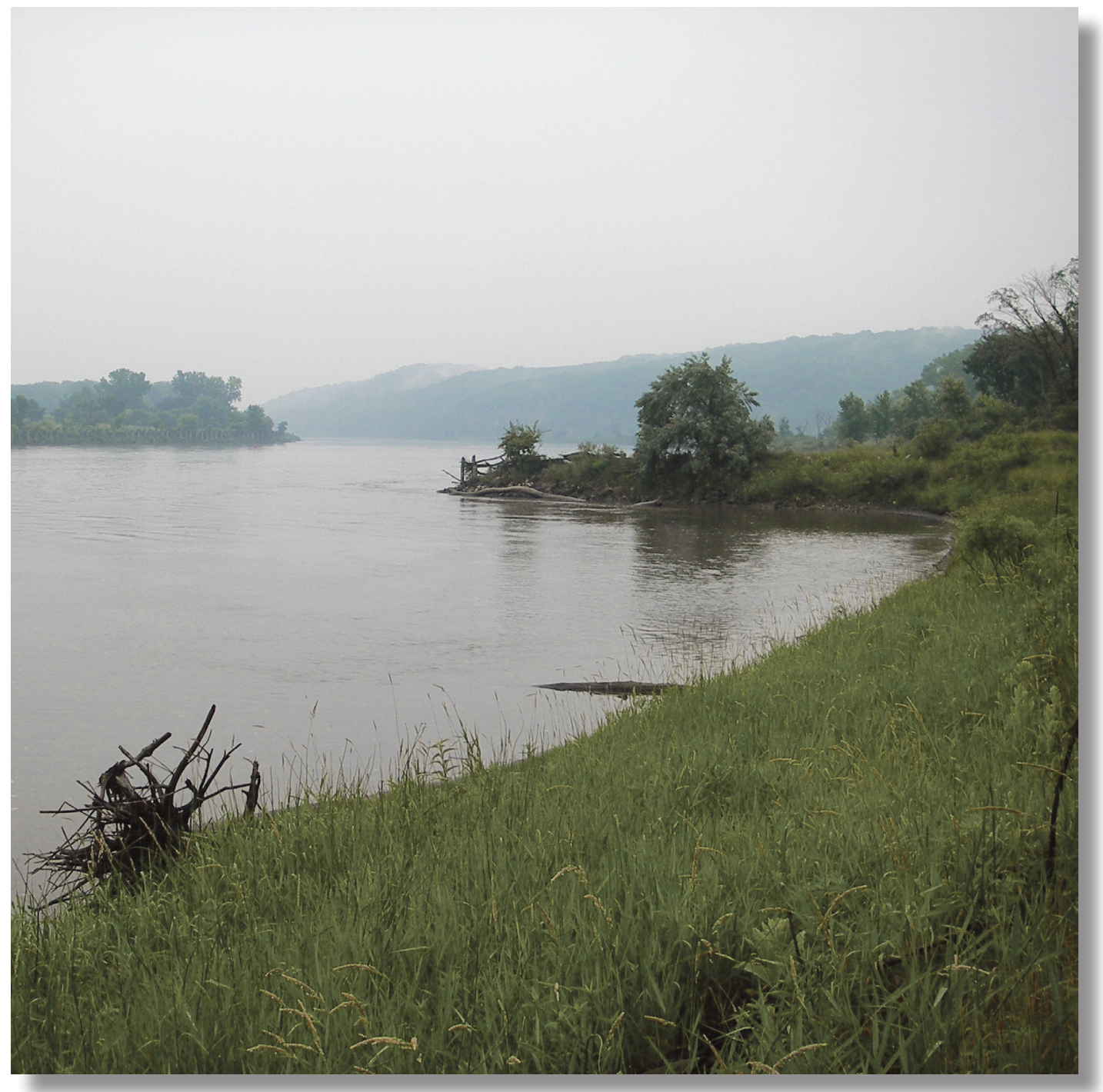

Data Series 386 
Cover: Photograph of the channelized Missouri River near river mile 707.5.

Photograph taken by Joanna M. Reuter, U.S. Geological Survey, June 13, 2007. 


\section{Hydraulic and Substrate Maps of Reaches Used by Sturgeon (Genus Scaphirhynchus) in the Lower Missouri River, 2005-07}

By Joanna M. Reuter, Robert B. Jacobson, Caroline M. Elliott, Harold E. Johnson III, and Aaron J. DeLonay

Prepared for the Missouri River Recovery-Integrated Science Program U.S. Army Corps of Engineers, Yankton, South Dakota

Data Series 386 


\section{U.S. Department of the Interior DIRK KEMPTHORNE, Secretary}

\section{U.S. Geological Survey \\ Mark D. Myers, Director}

\section{U.S. Geological Survey, Reston, Virginia: 2008}

For product and ordering information:

World Wide Web: http://www.usgs.gov/pubprod

Telephone: 1-888-ASK-USGS

For more information on the USGS - the Federal source for science about the Earth, its natural and living resources, natural hazards, and the environment:

World Wide Web: http://www.usgs.gov

Telephone: 1-888-ASK-USGS

Any use of trade, product, or firm names is for descriptive purposes only and does not imply endorsement by the U.S. Government.

Although this report is in the public domain, permission must be secured from the individual copyright owners to reproduce any copyrighted materials contained within this report.

Suggested citation:

Reuter, J.M., Jacobson, R.B., Elliott, C.M., Johnson, H.E., III, and DeLonay, A.J., 2008, Hydraulic and substrate maps of reaches used by sturgeon (genus Scaphirhynchus) in the Lower Missouri River, 2005-07: U.S. Geological Survey Data Series 386, 442 p. 


\section{Contents}

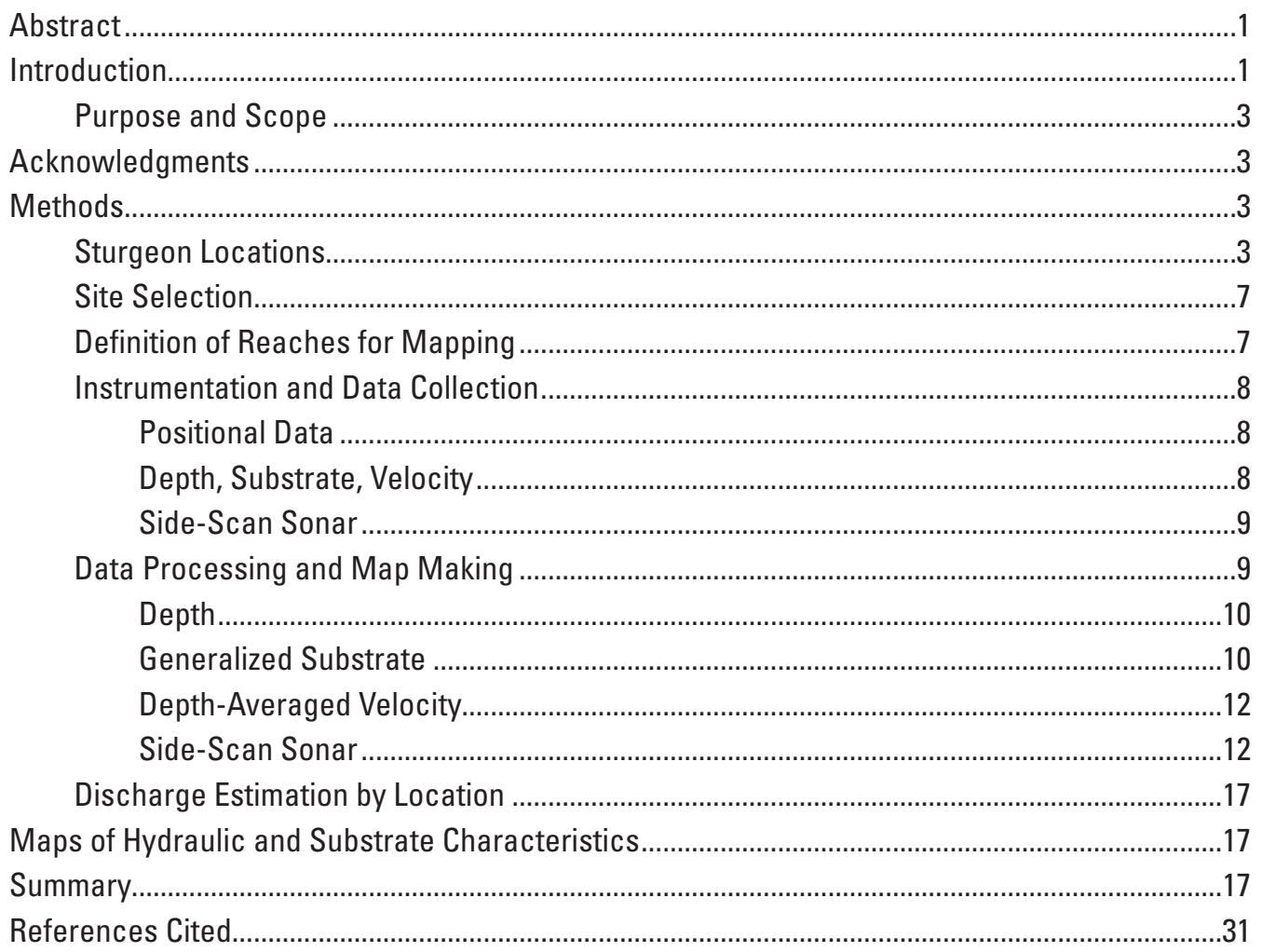




\section{Figures}

1. Map of the Lower Missouri River including U.S. Geological Survey streamflow-gaging stations and river segments.

2. Hydrographs showing Missouri River daily mean discharge for April 1 through September 1 in each of the study years.

3. Map of example reach with $(A)$ line file showing all planned transects, $(B)$ locations of data points, and $(C)$ extent of reach for data interpolation. .6

4. Photograph of the research vessel (R/V) Slim Funk actively collecting data.

5. Illustrations of the schematic framework for interpretation of RoxAnn data ..................11

6. Images of nongeoreferenced side-scan sonar data ......................................................13

7. Maps of an example reach, including primary maps, variance of prediction

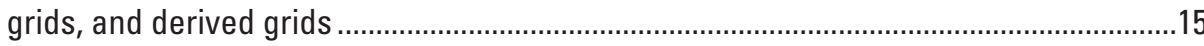

8-416. Maps of depth, substrate, velocity, and side-scan sonar.......................................33-441

\section{Tables}

1. Instrumentation used during each survey year, by boat.................................................

2. Gages used for discharge estimation by river subsegment.............................................18

3. Locations, dates, and available data sets for habitat surveys.......................................20

4. Sturgeon relocations and nets targeted for mapping .................................................25 


\section{Conversion Factors}

\section{Inch/Pound to SI}

\begin{tabular}{lcl}
\hline Multiply & By & To obtain \\
\hline mile $(\mathrm{mi})$ & Length & \\
\hline & 1.609 & kilometer $(\mathrm{km})$ \\
\hline cubic foot per second $\left(\mathrm{ft}^{3} / \mathrm{s}\right)$ & Flow rate & \\
\hline
\end{tabular}

\section{SI to Inch/Pound}

\begin{tabular}{lll}
\hline Multiply & By & To obtain \\
\hline centimeter $(\mathrm{cm})$ & Length & \\
meter $(\mathrm{m})$ & 0.3937 & inch (in.) \\
kilometer $(\mathrm{km})$ & 3.281 & foot $(\mathrm{ft})$ \\
meter $(\mathrm{m})$ & 0.6214 & mile $(\mathrm{mi})$ \\
\hline & 1.094 & yard $(\mathrm{yd})$ \\
\hline cubic meter per second $\left(\mathrm{m}^{3} / \mathrm{s}\right)$ & Flow rate & \\
\hline
\end{tabular}

Distances along the Missouri River are given in river miles upstream from the junction with the Mississippi River at St. Louis, Missouri.

Horizontal coordinate information is referenced to the World Geodetic System of 1984 (WGS 84). 



\title{
Hydraulic and Substrate Maps of Reaches Used by Sturgeon (Genus Scaphirhynchus) in the Lower Missouri River, 2005-07
}

\author{
By Joanna M. Reuter, Robert B. Jacobson, Caroline M. Elliott, Harold E. Johnson III, and Aaron J. DeLonay
}

\begin{abstract}
This report is a repository of reach-scale maps of hydraulic and substrate characteristics generated for the habitat-use portion of an interdisciplinary sturgeon research project on the Lower Missouri River (from Gavins Point Dam to the junction with the Mississippi River). The maps were derived from hydroacoustic data sets that were collected for the purpose of assessing physical aquatic habitat in the vicinity of locations of adult shovelnose sturgeon (Scaphirhynchus platorynchus) and pallid sturgeon (S. albus). Hydroacoustic data sets were collected at the reach scale (mean reach length, 2.4 kilometers) in order to include the immediate vicinity of a targeted sturgeon location as well as the full range of habitat available at the bend and crossover scale. Reaches typically were surveyed on the day following the relocation of a telemetered sturgeon and at a discharge within 10 percent of the discharge on the sturgeon relocation date in order to characterize as closely as possible the channel morphology and flow-field conditions at the time that the sturgeon was present. One hundred fiftythree reaches were mapped during April-September in the years 2005 through 2007, with the majority of data collection occurring in the months of May and June (coinciding with the period of sturgeon migration and spawning in the Lower Missouri River). Interpolated maps (grid cell size, 5 meters) depict depth, generalized substrate, and depth-averaged velocity. Side-scan sonar imagery is also available for a subset of reaches. Collectively, the maps represent more than 20 percent of the length of the Lower Missouri River.
\end{abstract}

\section{Introduction}

The physical character and the aquatic habitat of the Missouri River have changed substantially relative to the historical condition due to human alteration, including dam construction and operation, bank stabilization, and channelization (National Research Council, 2002; Galat and others, 2005). Altered habitat conditions are common among the large rivers that are inhabited by sturgeon throughout North America and the Northern Hemisphere. Many different species in the sturgeon family (Acipenseridae) are on the decline (Birstein and others, 1997). Habitat degradation is one of the factors often hypothesized to be a factor in the decline of sturgeon populations in general and pallid sturgeon (Scaphirhynchus albus) on the Missouri River in particular (U.S. Fish and Wildlife Service, $2000,2003)$. Because of the assumed importance of habitat to sturgeon survival and reproduction, habitat assessments were identified as a research priority for pallid sturgeon in the Missouri River (Quist and others, 2004). One step in this process is to determine what habitats sturgeon utilize in the context of available habitat. To address the question of habitat use, mapping efforts were coordinated with a telemetry-based, sturgeon-tracking project to map depth, substrate, and water velocity at sites individual sturgeon were known to have occupied in the Lower Missouri River.

The Lower Missouri River is an approximately 1,305-kilometer (811-mile) section of river from Gavins Point Dam (the lowest main-stem dam on the Missouri River) to the junction with the Mississippi River (fig. 1). The degree of hydrologic and geomorphic alteration varies in different segments of the Lower Missouri River. Deviation from the natural flow regime is greatest immediately downstream from the dam but is progressively moderated by inputs from unregulated tributaries in the downstream direction (Galat and Lipkin, 2000; Jacobson and Heuser, 2002; Pegg and others, 2003). In contrast, the channel morphology most resembles the historical state in the unchannelized segment just downstream from Gavins Point Dam (Elliott and Jacobson, 2006). Downstream from Sioux City, Iowa, the river has been narrowed and stabilized for navigation, although the type and quantity of engineered structures changes longitudinally, as does the size of the river.

Riverine physical aquatic habitat is substantially dependent on interaction between flow regime and channel morphology (Jacobson and Galat, 2006). Ongoing, related studies are characterizing the sensitivity of physical aquatic habitat to changes in discharge and geomorphic change on the Missouri River (Jacobson and others, 2007). Preliminary analyses indicate that channel morphology within the stabilized banks can be very dynamic on the time scale of weeks to months. Because of the dynamic nature of both discharge and channel morphology on the Lower Missouri 


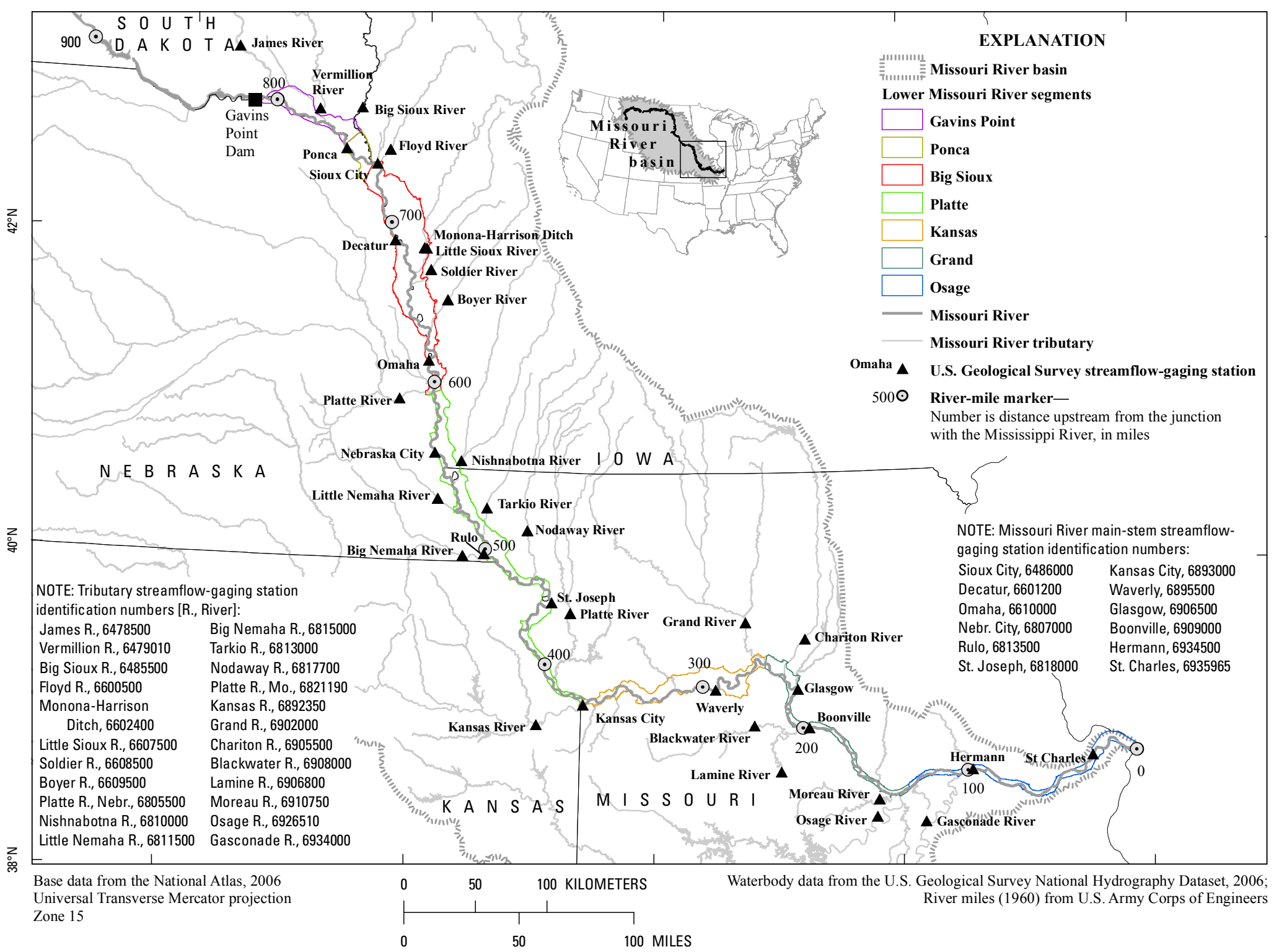

Figure 1. Map of the Lower Missouri River including U.S. Geological Survey streamflow-gaging stations and river segments. Segments are lengths of river bounded by major tributaries or defined by changes in channel morphology. Segment names are based on the tributary or feature bounding the segment on the upstream end. 
River, physical aquatic habitat assessments are best completed near in time and at discharges similar to the discharge when sturgeon were relocated (fig. 2). Obtaining such map data sets requires a high degree of coordination with fisheries crews tracking telemetered sturgeon.

Two sturgeon species of the genus Scaphirhynchus are native inhabitants of the Missouri River. These species are the pallid sturgeon (S. albus), listed as endangered in 1990, and the more common shovelnose sturgeon (S. platorynchus). Both species exhibit periodical migratory behavior associated with spawning (DeLonay and others, 2007). Gravid female sturgeon typically migrate upstream, reach an upstream-most location (apex), and descend downstream (DeLonay and others, 2007). Spawning is assumed to occur at or near the apex location. Although spawning of both shovelnose and pallid sturgeon has been confirmed on the Lower Missouri River (U.S. Geological Survey, 2007), the exact spawning locations have not been identified. Lower Missouri River sturgeon migrate during spring and summer; the peak of spawning in the Lower Missouri River appears to occur during May and June (DeLonay and others, 2007; Simpkins and LaBay, 2007).

Habitat mapping coincided with the spring through summer sturgeon migration, including the time period when spawning occurs. The sturgeon targeted for mapping were predominantly gravid female shovelnose sturgeon (as determined at the time they were implanted with transmitters); some of the mapped sturgeon were later confirmed to have spawned (DeLonay and others, 2007; Aaron J. DeLonay, unpub. data, 2008). Some locations of male shovelnose sturgeon as well as male and female pallid sturgeon also were mapped.

\section{Purpose and Scope}

This report serves as a repository of maps of hydraulic and substrate characteristics that were generated during 2005 through 2007 as part of the Comprehensive Sturgeon Research Project at the Columbia Environmental Research Center (CERC). The report documents methods used for reach selection, data collection, data processing, and map production. Maps are presented for 153 reaches that collectively represent more than 20 percent of the length of the Lower Missouri River. Analysis of the data set is not within the scope of this report.

\section{Acknowledgments}

Funding for this project was provided by the U.S. Army Corps of Engineers (USACE), Missouri River RecoveryIntegrated Science Program and the U.S. Geological Survey (USGS). This work is part of the Comprehensive Sturgeon Research Project, a large, interdisciplinary, multiyear research project to which numerous individuals have contributed. The following individuals in particular contributed to the habitat-use component of this project: Matt Smith and Chad
Vishy (Arctic Slope Regional Corporation) worked as field technicians and assisted with data processing. Mark Laustrup (USGS) and David Gaeuman (National Research Council Post-Doctoral Fellow) also participated in data collection. Aaron DeLonay and his tracking crews (USGS) tagged and relocated sturgeon during 2005 to 2007. In addition, tracking crews with the Nebraska Game and Parks Commission provided some of the telemetry locations in 2007. Kim Chojnacki, Emily Tracy-Smith, and Sandy Clark-Kolaks (USGS) provided support through management and quality control of the sturgeon telemetry data, making possible the near-real-time analysis that allowed for selection of target mapping locations. Reviewers David Heimann and Jason Alexander (USGS) provided input that helped to improve this report.

\section{Methods}

The "Methods" section provides details relevant to map production, including information about the sturgeon telemetry project, target sturgeon selection, reach definition, data collection, and data processing. Each mapping crew used recent sturgeon relocations to select a target location for a mapping day; one or two mapping crews were deployed each day. After selecting a target location, mapping crews defined a reach to include the habitat in the immediate vicinity of the sturgeon and in the surrounding bend and crossover macrohabitats (fig. 3). The target location and surroundings were mapped by driving the hydroacoustic mapping boat (fig. 4) along predefined transects throughout the reach. We used the data to generate interpolated maps for each reach.

\section{Sturgeon Locations}

In the late winter/early spring of 2005, 2006, and 2007, CERC collaborators caught sturgeon and implanted selected individuals with telemetry transmitters (DeLonay and others, 2007); several hundred sturgeon were tagged and implanted with transmitters. Tagged, telemetered sturgeon were subsequently released and tracked to determine their movement patterns. Crews tracked sturgeon by navigating through sections of river, using boats outfitted with acoustic and radio receivers to determine the locations of telemetered sturgeon. These relocation points, accurate to approximately 2 meters, were obtained using a differential global positioning system (DGPS) receiver and were recorded in Universal Transverse Mercator (UTM) coordinates, Zone 15 North, using the World Geodetic System of 1984 (WGS 84) datum. Coordinates were typically made available to habitat-mapping crews on the evening that the relocation data were collected.

The spatial extent of habitat mapping was determined by the sampling design used in the telemetry studies and by the movement of tagged sturgeon. During 2005 and 2006 the sample design called for comparison between upstream and downstream locations. Downstream sturgeon were released in 
A

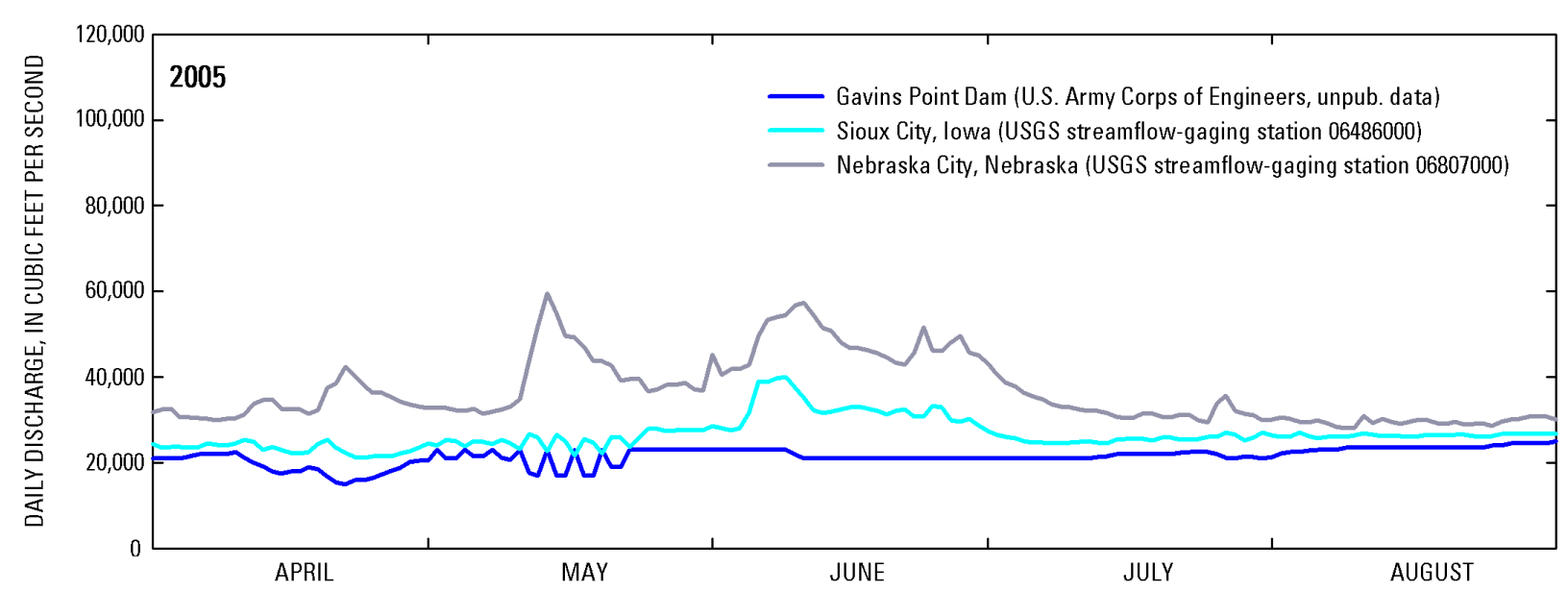

B

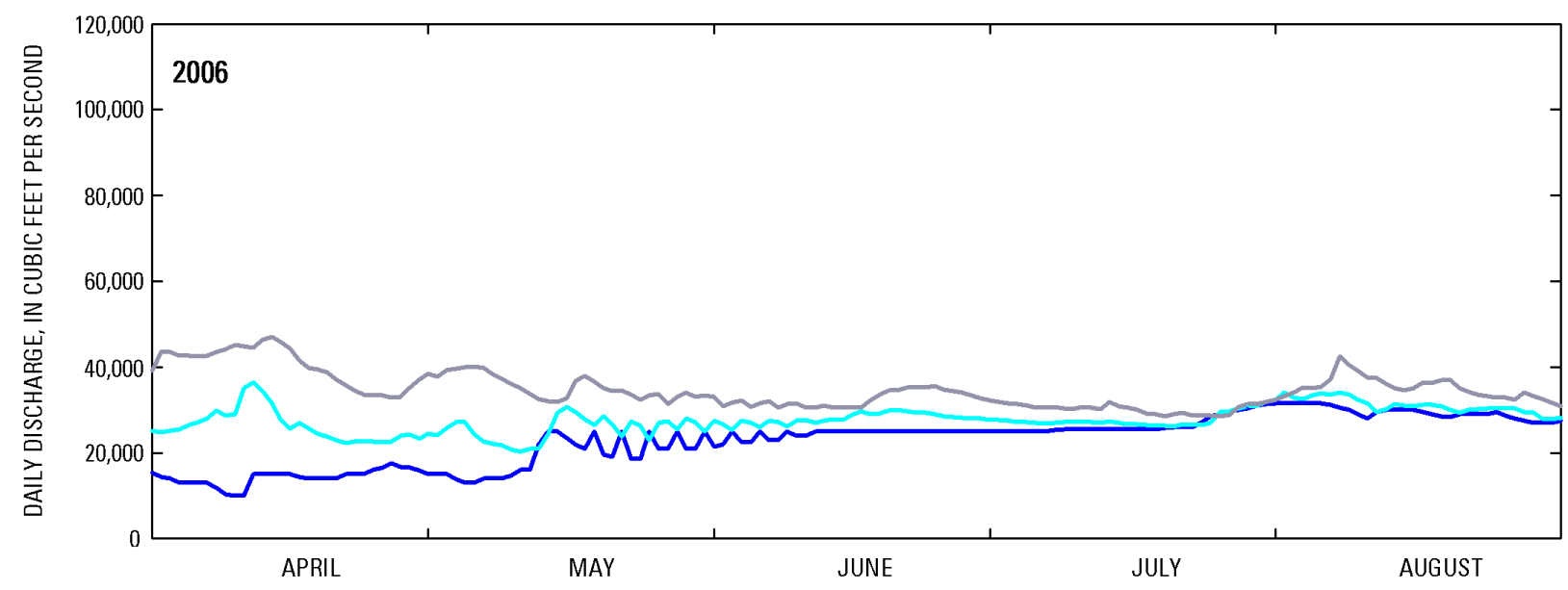

C

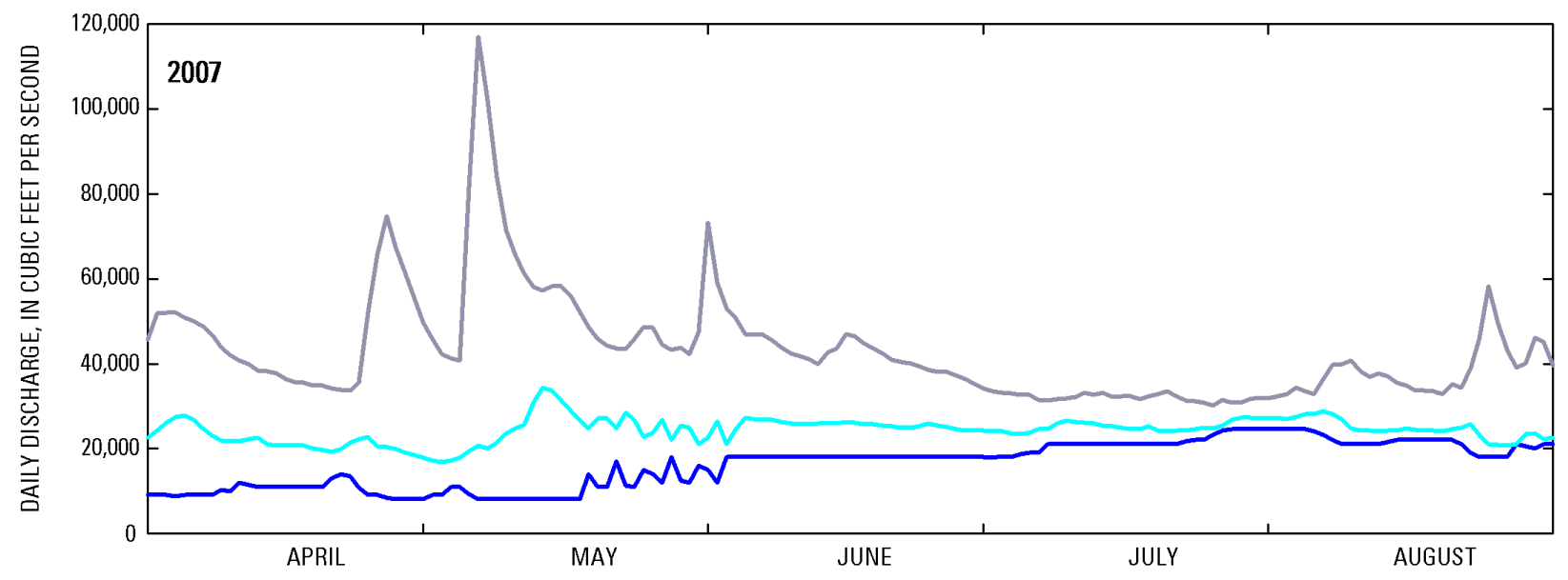

Figure 2. Hydrographs showing Missouri River daily mean discharge for April 1 through September 1 in each of the study years. Gages representing the Gavins Point segment (Gavins Point Dam), the Big Sioux segment (Sioux City), and the Platte segment (Nebraska City) are shown for (A) 2005, (B) 2006, and (C) 2007. Gages representing the Kansas segment (Waverly), the Grand segment (Boonville), and the Osage segment (Hermann) are shown for (D) 2005, (E) 2006, and (F) 2007. 


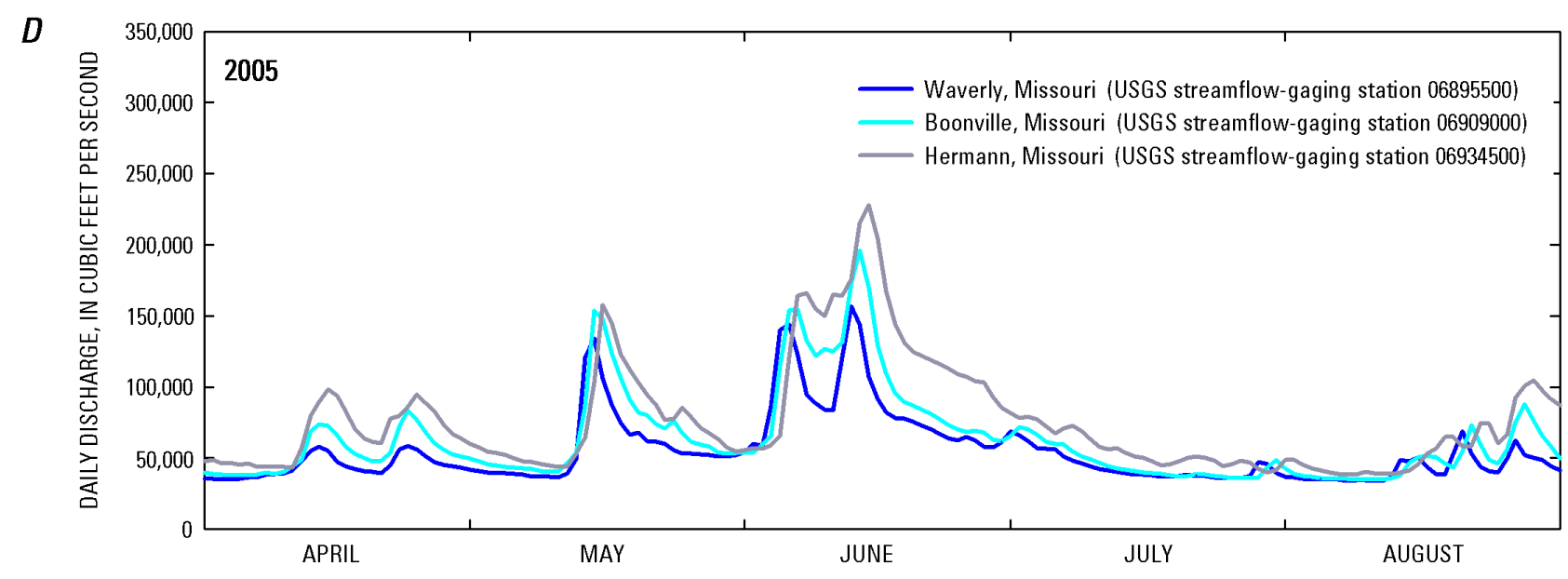

$E$

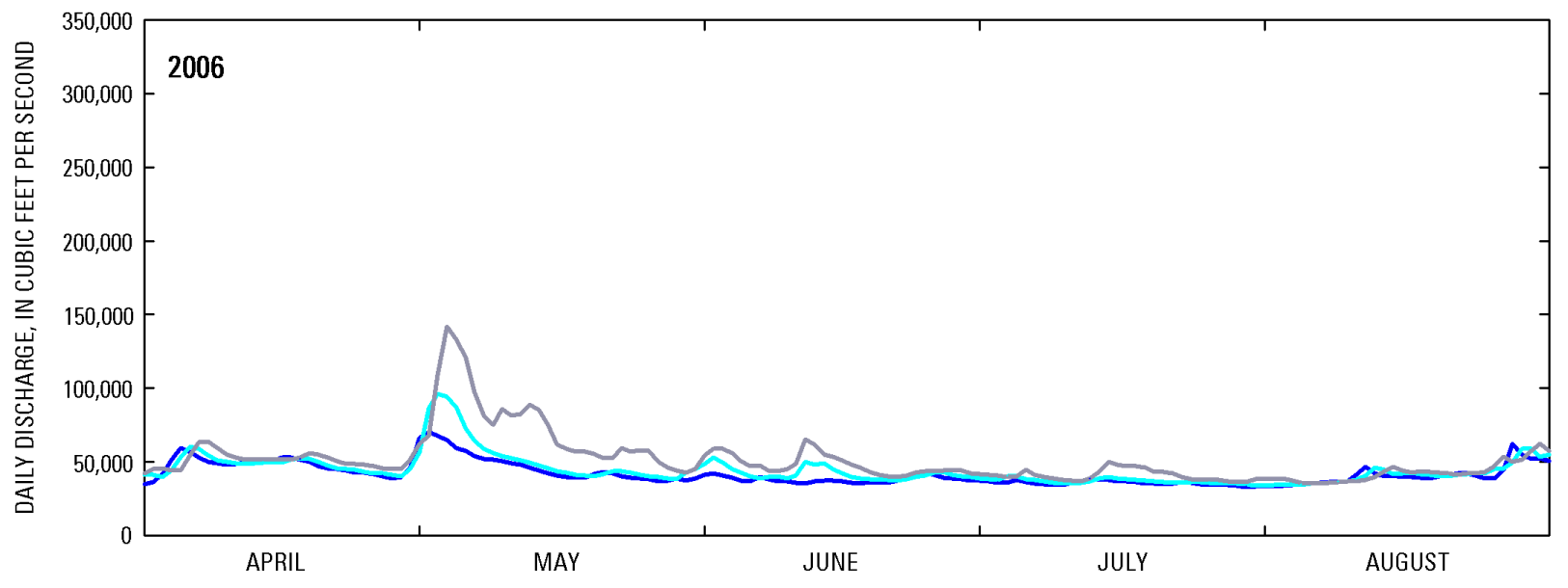

$F$

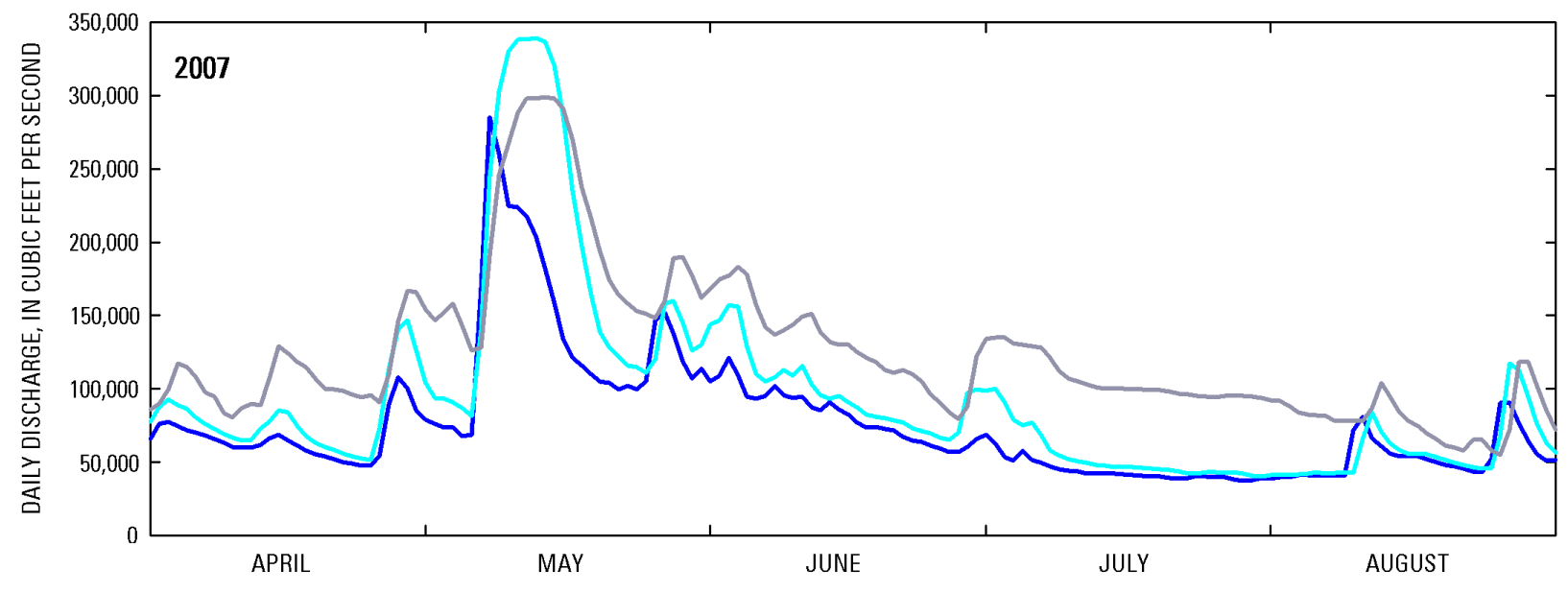

Figure 2. Hydrographs showing Missouri River daily mean discharge for April 1 through September 1 in each of the study years. Gages representing the Gavins Point segment (Gavins Point Dam), the Big Sioux segment (Sioux City), and the Platte segment (Nebraska City) are shown for (A) 2005, (B) 2006, and (C) 2007. Gages representing the Kansas segment (Waverly), the Grand segment (Boonville), and the 0sage segment (Hermann) are shown for (D) 2005, (E) 2006, and (F) 2007.-Continued 

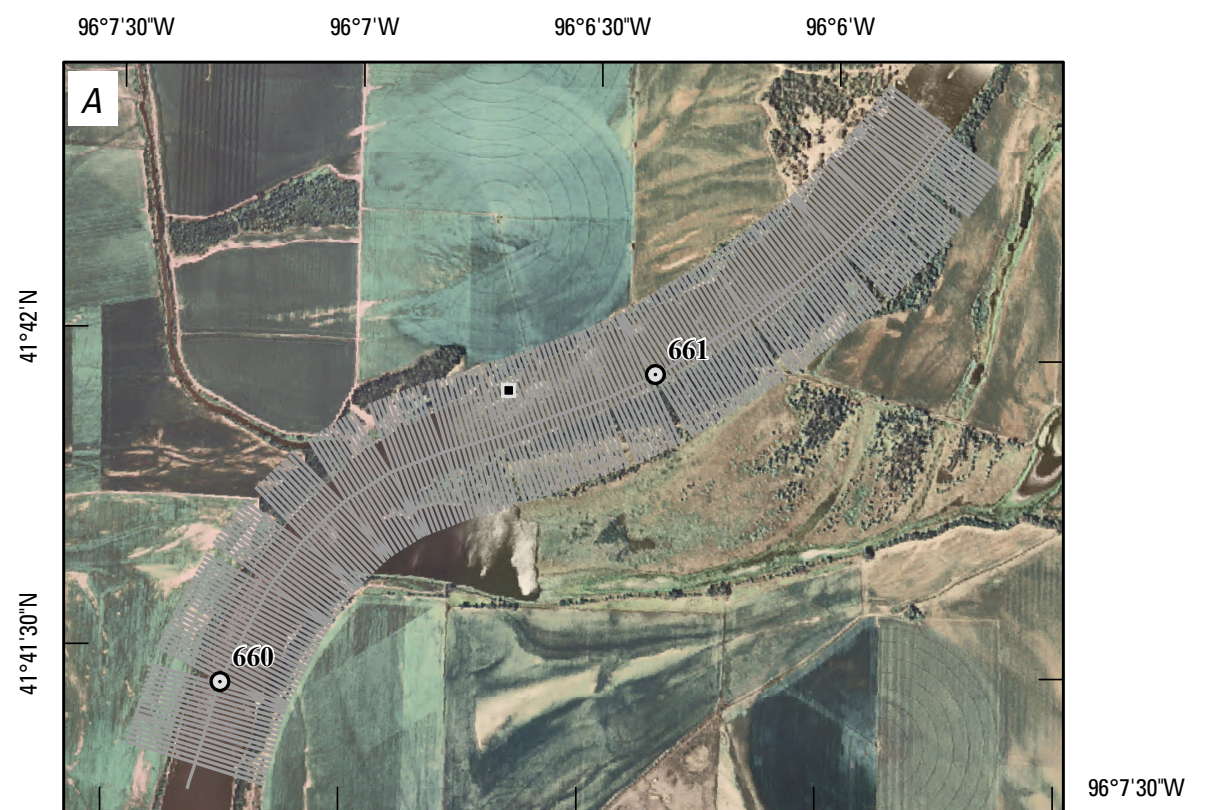

\section{EXPLANATION}

Planned survey line (A)

- Data points along survey line (B)

\section{Extent of reach for data interpolation $(C)$}

- Sturgeon relocation targeted for mapping

${ }^{661} \odot$ River-mile marker-Number is distance upstream from the junction with the Mississippi River, in miles

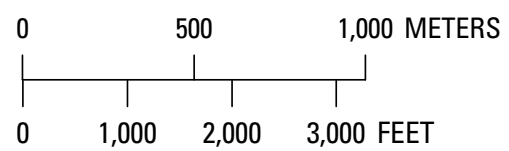

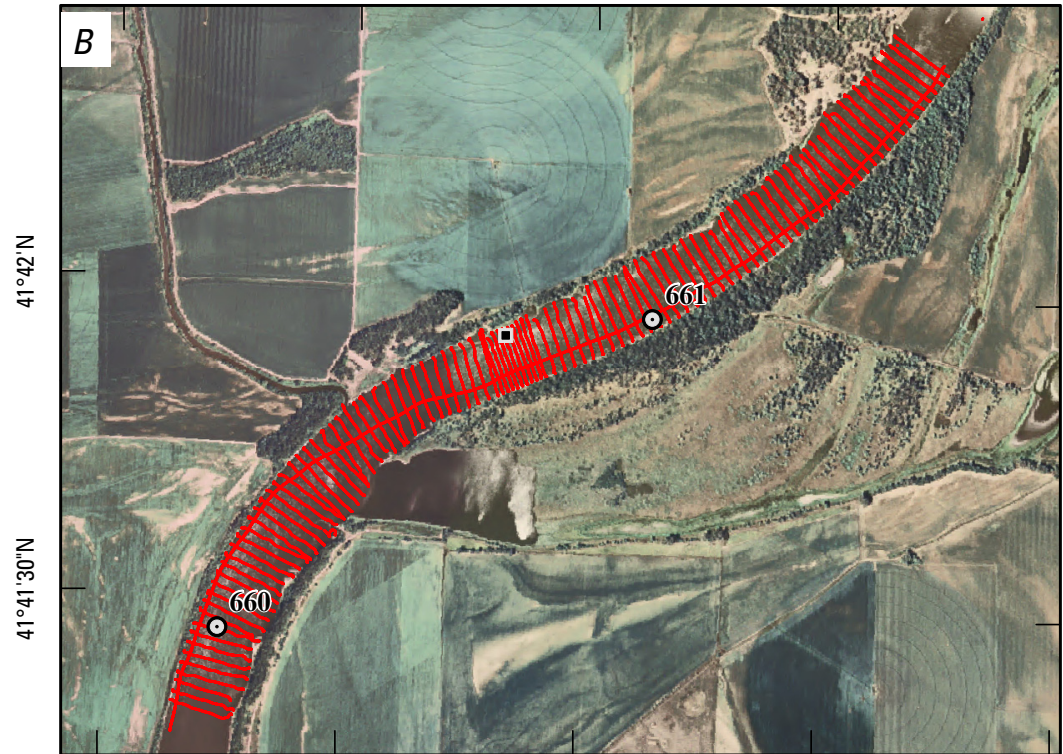

Base from U.S. Department of Agriculture National Agriculture Imagery Program, digital data, 2006 Base from U.S. Department of Agriculture
Universal Transverse Mercator projection Universa
Zone 15

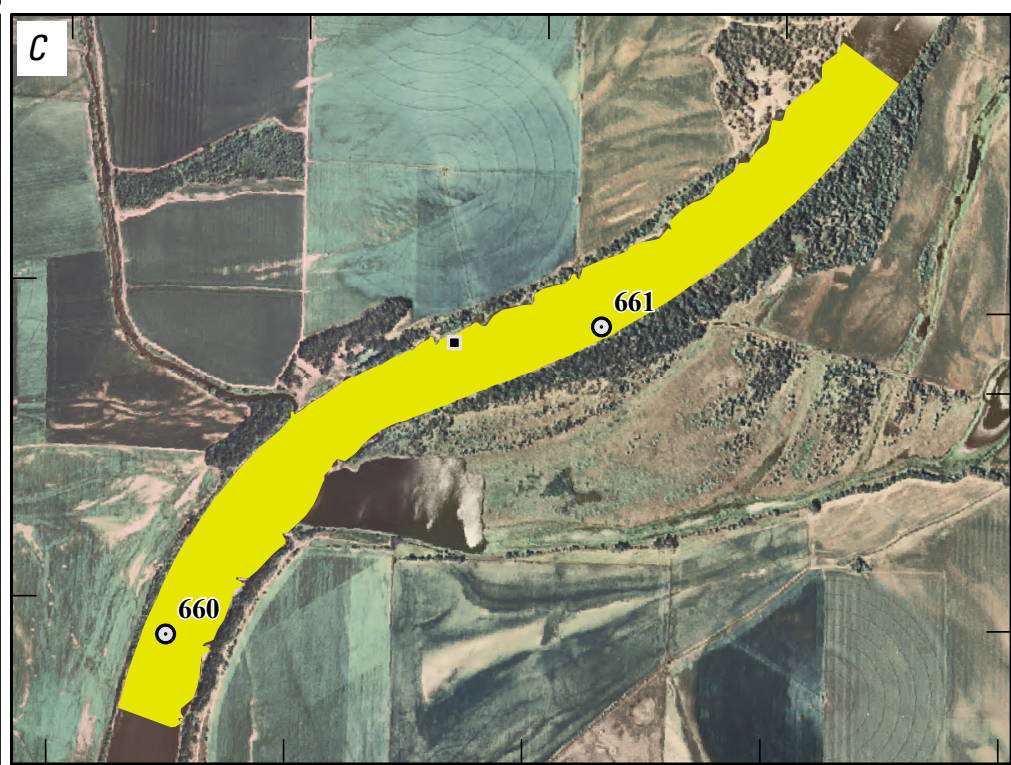

River miles (1960) from U.S. Army Corps of Engineers; Sturgeon relocations from U.S. Geological Survey, DeLonay and others, 2007; DeLonay, unpub, data, 2007

Figure 3. Map of example reach with (A) line file showing all planned transects, (B) locations of data points, and (C) extent of reach for data interpolation. 


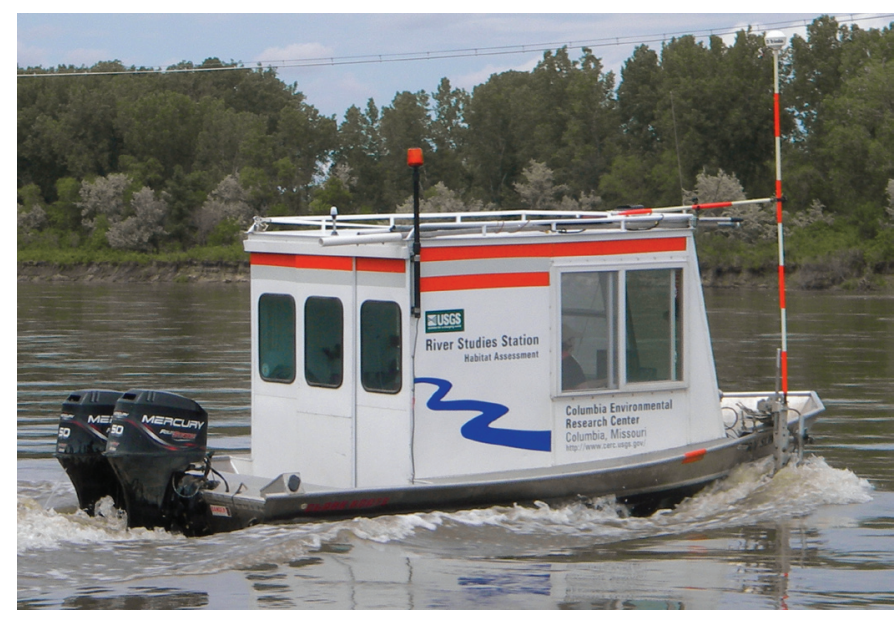

Figure 4. Photograph of the research vessel (R/V) Slim Funk actively collecting data.

the Missouri River between the Osage River and the Kansas River junctions, and the upstream sturgeon were released between the junctions of the Platte River and the Vermillion River in South Dakota (fig. 1). During 2007, releases were upstream from the Platte River.

\section{Site Selection}

Selection of a mapping site typically began with selection of a recent sturgeon relocation point, usually from the previous day of tracking. Locations for mapping were selected through either random or nonrandom methods. The randomized approach was used because randomly selected locations are best suited for unbiased assessments of habitat use and availability (Millspaugh and Marzluff, 2001). Sites were selected through nonrandom procedures for a variety of reasons, including the desire to target locations where spawning was suspected. Both random and nonrandom sites were mapped each year.

One prerequisite for site selection was to check that discharge on the day of mapping was anticipated to be within 10 percent of the discharge on the sturgeon-relocation date. We established this discharge goal because physical aquatic habitat conditions change with discharge, and it is important for the conditions at the time of mapping to be as similar as possible to conditions when the sturgeon was relocated. However, in some cases discharge changed more than the mapping crew anticipated, so not all maps meet the 10-percent criterion. In addition, the rapidly fluctuating flows from Gavins Point Dam in parts of May and June of 2006 and 2007 made strict adherence to this guideline difficult (fig. 2).

Randomization for the selection of reaches was implemented by assigning a random ranking to each qualifying sturgeon relocation point from the previous tracking day (typically the previous calendar day). The rankings were stratified by location either upstream or downstream from
Rulo, Nebraska, to accommodate the location of staged boats. In 2005 and 2006, most of the tracked sturgeon were gravid female shovelnose, and all sturgeon relocations were considered for randomization. In 2007, only gravid female shovelnose sturgeon were targeted for random mapping. Occasionally, the relocation point with the highest randomized ranking had to be rejected for one of the following reasons: (1) the discharge criterion was not met at the site; (2) river or weather conditions threatened the safety of the boat or crew; (3) the site had already been mapped in the same year and at a similar discharge. In these cases, either the sturgeon with the second random ranking was mapped or a nonrandom site was selected.

Maps based on nonrandom selection of sturgeon relocations were made for a variety of specific reasons including the following: (1) to target suspected spawning sites; (2) to map sites where pallid sturgeon had been relocated (due to the endangered status of this species); (3) to map locations of nets where crews fished for gravid sturgeon; (4) to target relocations of sturgeon considered to be post-spawn; (5) to re-map sites with real-time kinematic GPS equipment; or (6) because fluctuations in discharge, threat of severe weather, or other logistical constraints precluded the use of randomly selected locations.

Suspected spawning sites were selected on the basis of judgment of collaborators, timing that coincided with the expected spawning period (late May/early June), and ongoing analysis of preliminary sturgeon movement data. Working under the assumption that spawning would occur at or near the migration apex (DeLonay and others, 2007; Papoulias and others, 2007), we looked at the movement histories of individual sturgeon to judge when upstream movement slowed or stopped, suggesting that the sturgeon might be near its apex. The best opportunity to use this approach was in 2007, when the large number and high frequency of relocations for many individual sturgeon allowed us to explore detailed movement histories of sturgeon individuals. Other approaches to suspected spawning site identification included looking for male and female sturgeon in close proximity to each other as well as looking for general aggregations of sturgeon. Some of our maps do include the upstream-most relocation for female shovelnose sturgeon that were later confirmed to have spawned. However, exact sturgeon spawning locations were not confirmed in the Lower Missouri River during the study period because of difficulties in documenting actual release of eggs, egg deposition, and egg hatch.

\section{Definition of Reaches for Mapping}

In the channelized river (downstream from river mile 753 near Ponca, Nebraska, fig. 1), the standard protocol was to define mapping reaches as a bend-crossover-bend sequence or a crossover-bend-crossover sequence, up to a maximum length of 3 kilometers (the most that can reasonably be mapped in a single day). In the Gavins Point segment (fig. 1), which 
is characterized by multithread channels, reach length was determined by the area that could be mapped in one day, and reaches were considerably shorter because of the difficulties of maneuvering boats in shallow water. Maps for some dates differ from the ideal of this protocol. Occasionally, mapping crews stopped collecting data short of the intended full reach length due to adverse weather conditions or equipment problems. At the beginning of the study in 2005, a few sites were mapped as a series of very short reaches in order to obtain multiple sturgeon positions (or, in some cases, net positions where sturgeon had been caught) in a single day. In 2007, a number of the suspected spawning reaches were shorter in length than called for by the standard protocol because only a single boat was available for both side-scan sonar and depth/ substrate/velocity surveys, and both types of surveys were carried out in the same day.

Data collection for depth, substrate, and velocity took place along cross-sectional transects and a longitudinal profile (fig. 3). Planned transects were defined with Hypack software (Hypack, Inc., Middletown, Connecticut) and saved as a line file. Cross-sectional transects were spaced 15 meters apart upstream from Rulo, Nebraska; downstream from Rulo, transect spacing was 20 meters. Transect spacing increased downstream to scale with channel size; spacing was selected to produce accurate and realistic interpolated maps at the reach scale. To ensure high-resolution data near the sturgeon relocation, mapping crews collected data along every transect for a set of 10 transects centered on the target sturgeon relocation. In the rest of the reach, mapping crews collected data on every other transect (resulting in a 30-meter transect spacing upstream from Rulo and 40-meter transect spacing downstream from Rulo). In each reach, mapping crews also collected data along a longitudinal profile that was driven from upstream to downstream approximately following the thalweg. The collection of side-scan sonar data involved driving a series of longitudinal passes down the reach.

\section{Instrumentation and Data Collection}

Mapping was accomplished using boats outfitted with hydroacoustic instrumentation. The standard instrument configuration allowed for concurrent collection of depth, substrate, and velocity data through use of a single-beam echo sounder, a substrate sensor, and an acoustic Doppler current profiler (ADCP). In addition, at some reaches, we used sidescan sonar as an imaging tool. Side-scan sonar data sets were collected separately (either in time or by a second boat) from depth, substrate, and velocity data sets.

\section{Positional Data}

Global positioning system (GPS) data provided submeter or better positional accuracy for hydroacoustic data. Most sites were mapped with differential global positioning system (DGPS) instrumentation. Boats were equipped with Trimble
(Trimble Navigation Ltd., Sunnyvale, California) 12-channel AgGPS 132 receivers that used OmniSTAR (OmniSTAR, Inc., Houston, Texas) satellites for differential correction. GPS positions were collected at 0.2 -second intervals. For a few sites, we used survey-grade, 12-channel, real-time kinematic GPS equipment to georeference data to subdecimeter horizontal and vertical accuracy.

\section{Depth, Substrate, Velocity}

Hydroacoustic instrumentation included single-beam echo sounders, RoxAnn instruments (Marine Microsystems and Sonavision, Ltd., Aberdeen, United Kingdom), and acoustic Doppler current profilers (ADCP; Teledyne RD Instruments, Poway, California). Computers logged hydroacoustic data while the boat was driven along the planned transects from bank to bank or as depth allowed; transects ended where the water was too shallow for the boat to navigate (at depths less than approximately 0.6 meter). Boat speeds typically averaged around 2 meters per second for a survey, generally with lower speeds in shallow or slow water. In general, data collection followed standard procedures for hydroacoustic data as described in Elliott and others (2004).

Two boats were used for the collection of depth, substrate, and velocity data: the R/V (research vessel) Lucien M. Brush and the R/V Slim Funk (fig. 4). Both boats had mounts that suspended the echo sounder and ADCP transducers in the water on the starboard side of the boat near the bow. The GPS antenna was mounted above the transducers. The R/V Funk worked primarily in the upstream segments of the river (Gavins Point, Ponca, Big Sioux, and Platte; fig. 1). The R/V Brush worked in the downstream segments (Kansas, Grand, and Osage; fig. 1). The instrument configuration varied slightly between the boats and from year to year (table 1).

Depth data sets were generally collected with highresolution, single-beam Hydrotrac echo sounders (Odom Hydrographic Systems, Inc., Baton Rouge, Louisiana). (The exception is that the R/V Brush did not have a Hydrotrac echo sounder installed in 2005, so depths were determined from ADCP data for that year.) Each echo sounder was calibrated regularly by using standard bar-check procedures: A metal plate was suspended below the echo-sounder transducer at alternating depths of one and two meters, and the speed of sound and draft were adjusted as needed to read the correct depth. Bar checks were performed approximately one time per week or as needed due to changes in water temperature. Data-collection rates of 5 hertz $(\mathrm{Hz})$ were used along crosssectional transects; collection rates were either 5 or $10 \mathrm{~Hz}$ for longitudinal profiles. A laptop computer logged depth data using Hypack software (version 4.3 or 4.3 a Gold). Depth data were logged simultaneously with substrate and GPS data. Depth data values were visually inspected in cross-sectional view during collection, and gain was adjusted as needed to maximize data quality.

Substrate data sets were collected with RoxAnn instrumentation. RoxAnn instruments analyze the return 
Table 1. Instrumentation used during each survey year, by boat.

[R/V, research vessel; $\mathrm{x}$, standard equipment used; select, equipment used for selected surveys only; --, no data; kHz, kilohertz]

\begin{tabular}{|c|c|c|c|c|c|c|c|}
\hline \multirow[b]{2}{*}{ Instrumentation } & \multicolumn{3}{|c|}{$\mathrm{R} / \mathrm{V}$ Funk } & \multicolumn{2}{|c|}{ R/V Brush } & \multicolumn{2}{|c|}{ R/V Schmudde } \\
\hline & 2005 & 2006 & 2007 & 2005 & 2006 & 2006 & 2007 \\
\hline Differential global positioning system & $\mathrm{x}$ & $\mathrm{x}$ & $\mathrm{x}$ & $\mathrm{x}$ & $\mathrm{x}$ & $\mathrm{x}$ & $\mathrm{x}$ \\
\hline Real-time kinematic global positioning system & select & -- & -- & select & -- & -- & -- \\
\hline $\begin{array}{l}1200 \mathrm{kHz} \text { Workhorse Rio Grande acoustic } \\
\text { Doppler current profiler }\end{array}$ & $\mathrm{x}$ & $\mathrm{x}$ & $\mathrm{x}$ & $\mathrm{x}$ & $\mathrm{x}$ & -- & -- \\
\hline Odom Hydrotrac echo sounder & $\mathrm{x}$ & $\mathrm{x}$ & $\mathrm{x}$ & -- & $\mathrm{x}$ & -- & -- \\
\hline RoxAnn GD, digital, with function generator & -- & -- & -- & -- & $\mathrm{x}$ & -- & -- \\
\hline RoxAnn, analog & $\mathrm{x}$ & $\mathrm{x}$ & $\mathrm{x}$ & -- & -- & -- & -- \\
\hline $900 \mathrm{kHz}$ side-scan sonar & -- & select & select & -- & select & select & select \\
\hline
\end{tabular}

signals from the echo sounder and generate two parameters, E1 and E2, based on the first and second returns, respectively (Hamilton, 2001). Two different RoxAnn instruments were used; an analog RoxAnn was used on the R/V Funk, and a digital model (RoxAnn GD) was used on the R/V Brush. Each RoxAnn instrument was kept on the same boat, and the units were not recalibrated through the study period. The computer operator on the boat monitored collection of RoxAnn data; however, few options exist to adjust quality characteristics during data collection.

Velocity data sets were collected with $1,200 \mathrm{kHz}$ Workhorse Rio Grande acoustic Doppler current profiler (ADCP) units. ADCP data were logged simultaneously with GPS data on a second laptop computer running WinRiver (version 10.06, Teledyne RD Instruments, Poway, California). Magnetic variation was set for each reach mapped by using either GeoMagix software (Interpex, Ltd., Golden, Colorado) or data from the National Geophysical Data Center of the National Oceanic and Atmospheric Administration (National Geophysical Data Center, 2008). Mapping crews performed the "Method 3" compass calibration procedure at each site by rotating the boat in a tight circle (RD Instruments, 2003). This procedure corrects for one-cycle errors. The procedure was repeated until the total error reading was less than 1 degree. Configuration settings for ADCP data collection included a bin (vertical cell) size of 25 centimeters and a blanking distance of 50 centimeters below the transducer. ADCP data were collected using water mode 1 with 6 water pings and bottom mode 5 with 1 bottom ping.

\section{Side-Scan Sonar}

In 2006 and 2007, mapping crews collected side-scan sonar data in a subset of reaches that had potential to contain spawning areas. Side-scan sonar provides an image of the riverbed based on hydroacoustic returns from a towfish that is deployed from the boat. The images are useful for visualizing channel substrate and detecting the presence of relatively large fish, including adult sturgeon. Data sets were collected with a 900-kHz towfish (Marine Sonic Technology, Ltd, White Marsh, Virginia) using an image width of 20 meters on each side, providing a 40-meter total swath width (Marine Sonic Technology Ltd., 2001). Side-scan sonar data were collected while making multiple longitudinal passes downstream through the reach. The highest quality images result from keeping the towfish a uniform distance off of the bottom, a task that can be challenging in a river environment. A powered winch was used to raise and lower the towfish by its cable while collecting data. Sea Scan PC software (Marine Sonic Technology, Ltd., White Marsh, Virginia) logged the data to a computer. Differential GPS was used to record the position of the boat. The GPS position provides only approximate georeferencing for the side-scan imagery, because the towfish position changes based on the amount of cable that is released to compensate for depth. Thus, the position of the towfish is not known precisely. Each side-scan data set was collected from one of three boats: the R/V Funk, the R/V Brush, or the R/V Schmudde (table 1).

\section{Data Processing and Map Making}

Converting raw data sets into finalized maps is a multistep process that includes editing to remove spurious data points, digitizing a blanking polygon to define the extent of the map, and interpolating the point data to a grid (raster) format. Additional details of these steps are described for each data type in the sections that follow. Processing steps that did not require human judgment were automated with Python scripts (Python Software Foundation, Hampton, New Hampshire), utilizing ArcGIS processing tools (version 9.x, Environmental Systems Research Institute, Inc., Redlands, California). 
All depth, substrate, and velocity maps were generated with 5-meter grid cells and are stored in the Environmental Systems Research Institute (ESRI) grid format. All maps were projected to UTM Zone 15 North with the WGS 84 datum.

\section{Depth}

Depth maps were derived from echo-sounder data for most surveys (table 1). Echo-sounder data sets were edited in Hypack software by viewing each transect in cross-sectional view and removing data points with spurious depth values. Spurious depth data were associated with fish or rapidly varying bed conditions that prevented acquisition of accurate depth values. Depth data sets were exported to text files, and a Python script was used to convert the text files containing point locations and attributes to shapefiles. Blanking polygons were digitized on screen to define the spatial extent of the reach to which data could reasonably be interpolated based on the extent of the echo-sounder points. In a few reaches, depth values recorded by either the RoxAnn or the ADCP were used to supplement transects with missing echo-sounder data. A Python script automated the remaining map-production steps; these steps accomplish the interpolation of point data values to a 5-meter grid. Point data sets were prepared for interpolation by using filters to reduce the point density because point spacing of raw data along transects is very dense relative to the grid-cell size. The interpolation technique utilized was ordinary kriging, executed with the ArcGIS kriging tool from ArcToolbox. Variance of prediction grids were generated as an assessment of uncertainty of kriged values. Grids were finalized by cropping to the extent of the associated blanking polygons.

For surveys lacking echo-sounder data, depth grids were generated from ADCP data sets. This applies to most of the reaches from 2005 in the Osage, Grand, and Kansas segments because the R/V Brush did not have an echo sounder in that year (table 1). The ADCP data sets contain depth data at each of four beam locations for each data ensemble. Depth data from the ADCP were obtained by computing the position and depth for each of the four beams and exporting these values to a shapefile. Kriging and blanking procedures were similar to those used for the echo sounder data, although different kriging parameters were used because of the different spatial distribution of data points.

\section{Generalized Substrate}

We developed an interpretational framework for mapping substrate classes using the RoxAnn substrate classification systems, validated through sediment sampling, side-scan sonar interpretation, and repeat substrate mapping. These methods generally follow those established by other seabed classification studies (Cholwek and others, 2000; Brown and others, 2005), although methods had to be optimized for the turbid and high-velocity conditions of the Lower Missouri River. In particular, high turbidity prevented us from using videography to establish correlations between acoustic signatures and substrate types. To generate substrate maps, RoxAnn data were first classified into three classes based on signatures of the E1 and E2 parameters, as described below. The RoxAnn-based classifications were supplemented with other geospatial data to produce the generalized substrate maps in this report.

Three distinct signatures of E1 and E2 values are common in RoxAnn data on the Lower Missouri River (fig. 5). The first signature, defined by very high E1 or E2 values, corresponds to rock, gravel, and coarse, hard sand. The second signature is characterized by low and spatially uniform E1 values in combination with spatially fluctuating E2 values; this signature corresponds to sand dunes. Spatial variability in E2 was interpreted to be the result of differences in the hydroacoustic return across dune forms. The third signature, defined by low and spatially uniform E1 and E2, was interpreted to represent two distinct but hydroacoustically similar substrate types: fine sediment and transporting sand. This signature is associated with fine sediment (mud, silt) in areas of low velocity, such as behind wing dikes. Low, uniform E1 and E2 values were also recorded in the main channel, typically at relatively high discharges, and we interpret these patches to be transporting sand. The transporting sand is distinguished from sand dunes by consistently low values of E2, suggesting a more uniformly soft riverbed.

For shallow and relatively slow moving water, we established "ground-truth" correlations between hydroacoustic signatures and sediment types through sampling with a 5 -cm coring device or with a "mini ponar" sediment sampler. Retrieved sediments were classified qualitatively into dominant particle-size classes. In deep and swift parts of the Missouri River, direct sampling was not possible. For these areas, correlations between acoustic signatures and sediment type were established through comparisons with side-scan imagery from selected locations. Side-scan imagery provided reliable identification of gravel and cobble based on bright acoustic returns and variable texture. Mud was identified as uniform areas of low acoustic returns. Sand dunes were readily apparent based on morphology, and transported sand was apparent as blurry uniform areas within sand-dune fields.

We developed an automated procedure to categorize data points into one of these three classes. To describe spatial variability, summary statistics were computed for E1 and E2 in the vicinity of each data point. For each data point, all other points within a 5-meter radius were identified, and quartile, mean, and standard deviation values were calculated. Following some exploration of data sets using multivariate statistics (discriminant analysis and K-means classification), as well as visual examination of the data sets, the third quartile for E1 and the third quartile for E2 emerged as reliable values for the basis of the classification.

Distributions of the spatial third-quartile values for E1 and E2 were used to determine cutoff values between substrate classes. Populations of these values varied between the two RoxAnn instruments used, and values also varied by water 
$A$
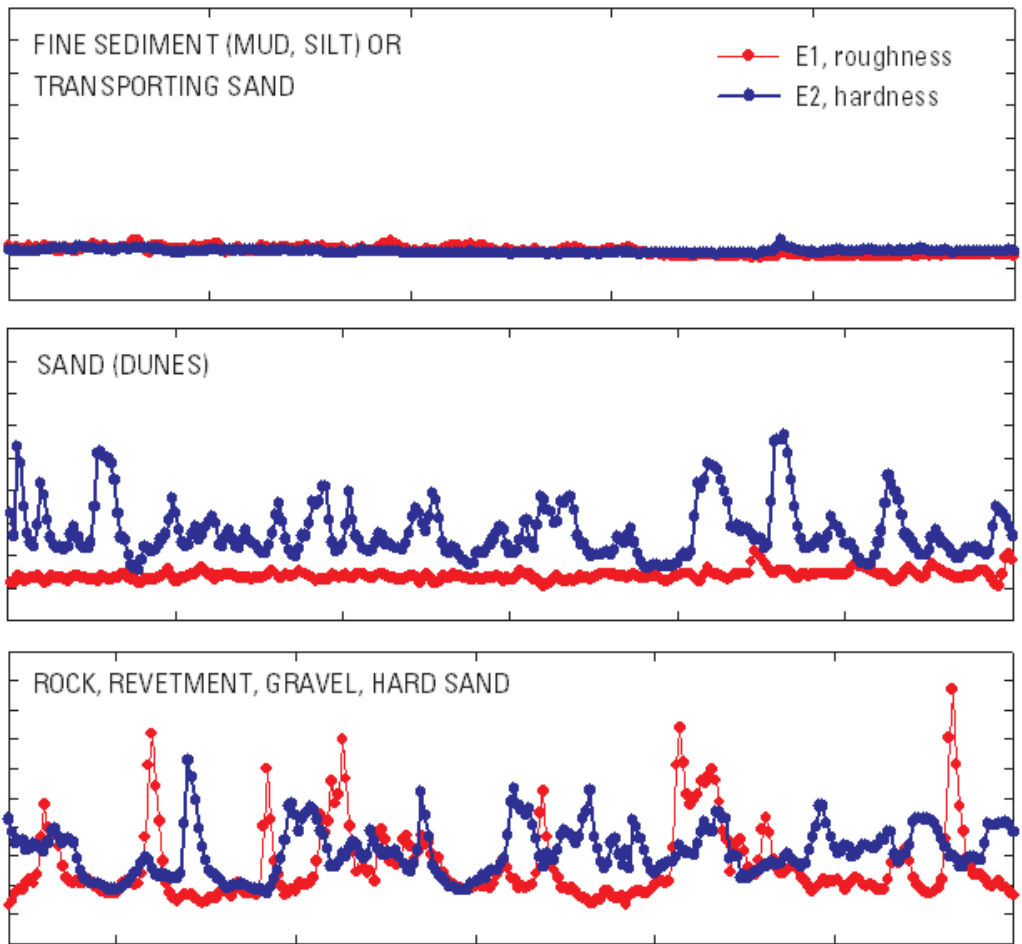

$B$

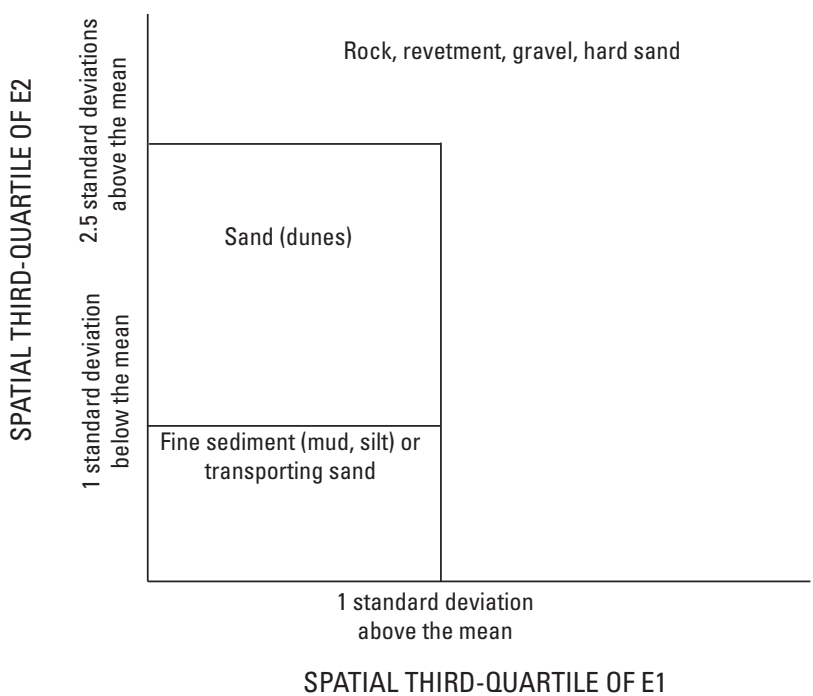

Figure 5. Illustrations of the schematic framework for interpretation of RoxAnn data. (A) Characteristic signatures of RoxAnn data along transect lines. (B) Automated classification of the three substrate signatures was based on cutoff values determined from the distribution of the spatial third-quartile values of E1 and E2. 
temperature. To adjust for differences in environmental conditions and between instruments, we used relative cutoff values to define substrate classes based on the distribution of the spatial third-quartile E1 and E2 values in each reach (fig. 5B).

RoxAnn data were brought into Hypack software with the RoxAnn selected as the echo sounder, and points with spurious depth values were deleted because E1 and E2 values associated with spurious depths were unreliable. Data were then exported from Hypack software to a text file and converted to a shapefile. For each data point, quartile values were calculated based on the data points falling within a 5-meter radius of the point. Cutoff values between classes were determined by computing the standard deviations of the spatial thirdquartile values for E1 and E2, as described previously. Each point was then classified into one of three general substrate classes. After classifying each data point, grids of substrate were made based on a discrete-value gridding approach: First, each grid cell that contained substrate data points was assigned the substrate class with the majority representation in the cell. Next, cells without points were assigned the value based on the nearest neighbor grid cell with an assigned substrate class. The resulting grids were smoothed with a majority operation in a moving window of 3 by 3 cells; larger windows were used to break ties. The result was a substrate grid based on RoxAnn values only with three preliminary classes: fine sediment/ transporting sand, sand dunes, and rock/gravel.

Mud and transporting sand have similar RoxAnn signatures, but these substrate classes can be differentiated based on spatial context. We used the velocity maps to differentiate between these two classes, using a threshold value for transporting sand of 0.6 meter per second $(\mathrm{m} / \mathrm{s})$ based on Shield's diagram for sediment transport (Knighton, 1998). Grid cells that were classified as fine sediment/transporting sand were thus reclassified to fine sediment if the velocity was less than $0.6 \mathrm{~m} / \mathrm{s}$ or transporting sand if the velocity was greater than $0.6 \mathrm{~m} / \mathrm{s}$. The new substrate grids were blanked to the extent of the velocity grids.

Bedrock and engineered rock structures at the margins of the channel were poorly represented in the initial substrate maps, largely because RoxAnn data were not viable for depths less than approximately 1.5 meters, the RoxAnn blanking depth. Therefore, we supplemented the substrate maps with two aerial-photograph-derived polygon data sets representing (1) distribution of navigation structures and bank revetments (U.S. Army Corps of Engineers, unpub. data, 2008) and (2) locations of bedrock (Laustrup and others, 2007). These supplemental data sets were superimposed onto the substrate grids, overriding existing classifications.

Substrate data sets are not available for some mapped reaches for two main reasons. The R/V Brush was not instrumented with a RoxAnn instrument in 2005 (table 1), so no substrate grids are available for most of the reaches downstream from Rulo, Nebraska, for that year. Other reaches lack substrate grids due to instrument malfunction or poor data quality. The RoxAnn's relatively large blanking distance, combined with generally poor performance in shallow water, resulted in rejection of data for the majority of the mapped reaches in the Gavins Point segment (fig. 1), which is characterized by relatively shallow depths.

\section{Depth-Averaged Velocity}

Acoustic Doppler current profiler (ADCP) data sets contain velocity values in ensembles (vertical sets of bins through the water column). Depth-averaged values of velocity for each ensemble, as computed from the bin values, were used for the purpose of generating the two-dimensional velocity grids contained in this report. The raw velocity data sets contain additional information that may be used for future analyses.

ADCP data processing consisted of several steps. The first step was to export the data from WinRiver, using the GPS-based frame of reference. The WinRiver export files were then parsed by a Python script that also calculated depthaveraged velocity values for each vertical data ensemble, converted the data into a shapefile format, projected the shapefile to UTM Zone 15 North, and performed some basic filtering to flag spurious data points. The depth-averaged velocities were computed by averaging the easting and northing components for each bin, then calculating the magnitude and direction of the depth-averaged velocity from the component vectors. We examined the shapefiles manually to identify and flag other spurious data points. For example, spurious velocity magnitudes tend to be associated with poor GPS values, often as a result of bridge interference with GPS signals. The point shapefiles, with good values selected, were used to guide digitization of blanking polygons that define the extent of the area for which values can be reasonably interpolated to a 5-meter grid. For each data set, the point velocity values were interpolated to a grid with a Python script that executes kriging in ArcGIS and clips the grid to the extent of the blanking polygon. In addition to the velocity grid, the procedure computed a variance of prediction grid that estimates the uncertainty of the kriged values.

Directional bias of recorded velocities is common in association with the standard method of driving the boat in alternating directions across the river (left bank to right bank for one transect, then right bank to left bank for the next). This bias can appear as slight striping in velocity grids. The magnitude of error associated with striping is small (less than 0.05 meter per second) and the effect is primarily visual. This error apparently relates to slight spatial variations in magnetic field that are not compensated by the daily compass calibration (Gaeuman and Jacobson, 2005). In postprocessing, we adjusted magnetic variation as needed to minimize the directional bias.

\section{Side-Scan Sonar}

Side-scan sonar data sets were used to generate images of the channel bottom in a high-resolution, minimally processed form (fig. 6) and also as georeferenced mosaics (fig. 7). 

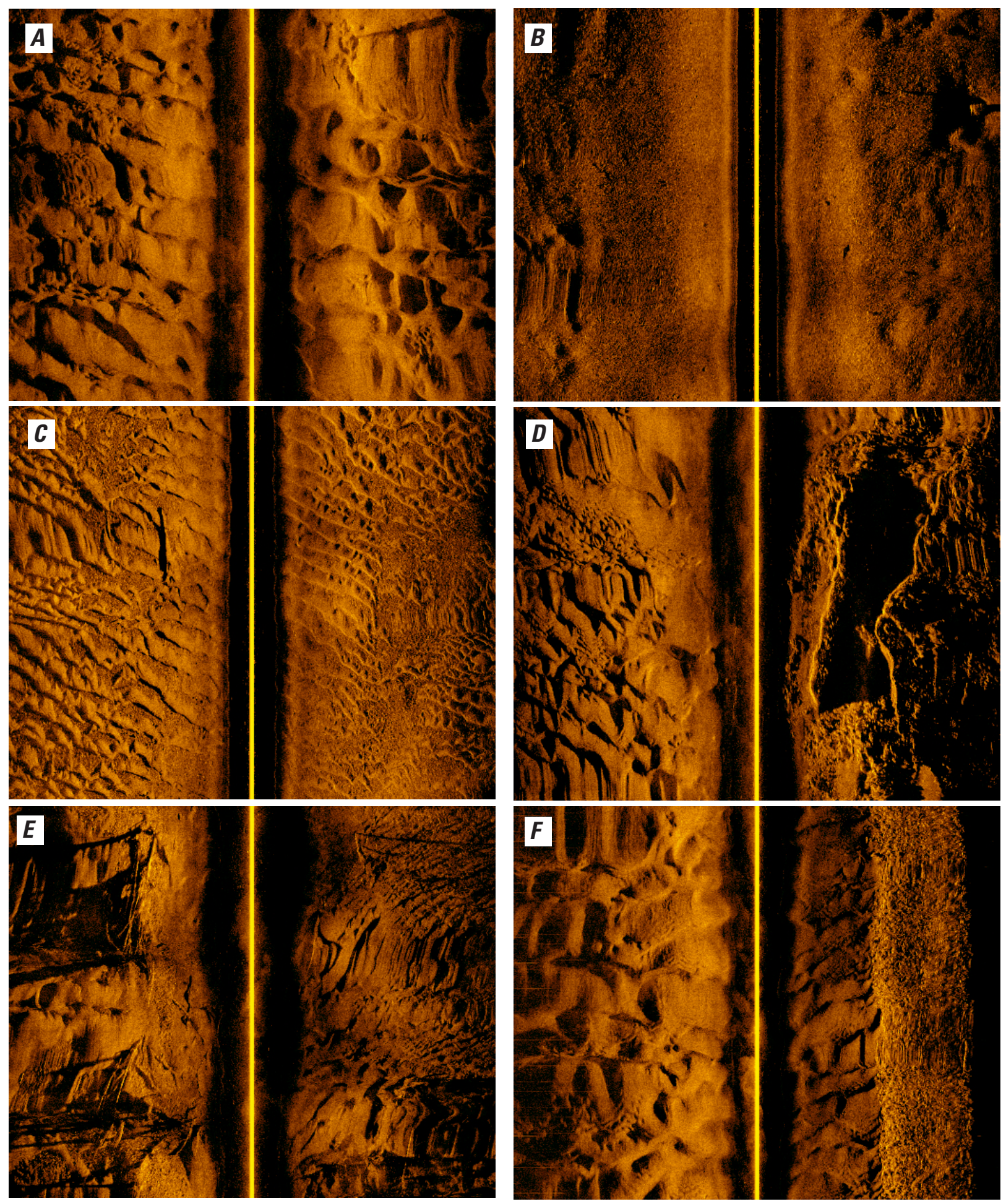

Figure 6. Images of nongeoreferenced side-scan sonar data. Examples illustrate substrate diversity and fish-detection capabilities. All images are oriented such that the bottom of the image is in the downstream direction. (A) Mid-channel dunes (June 2, 2007, Big Sioux segment), (B) gravel (May 22, 2007, Gavins Point segment), (C) sand dunes over gravel (May 22, 2007, Gavins Point segment), (D) bedrock, right side of image (May 23, 2007, Ponca segment), (E) large woody debris, left side of image (May 23, 2007, Ponca segment), (F) revetment, right side of image (June 2, 2007, Big Sioux segment), (G) aggregation of fish (species undetermined) on the lee side of a bar (May 23, 2007, Ponca segment), (H) fish and soft substrate in a dike field, left side of image (June 2, 2007, Big Sioux segment), (I) some fish visible; car bodies on left side demonstrate scale (May 24, 2007, Gavins Point segment). 

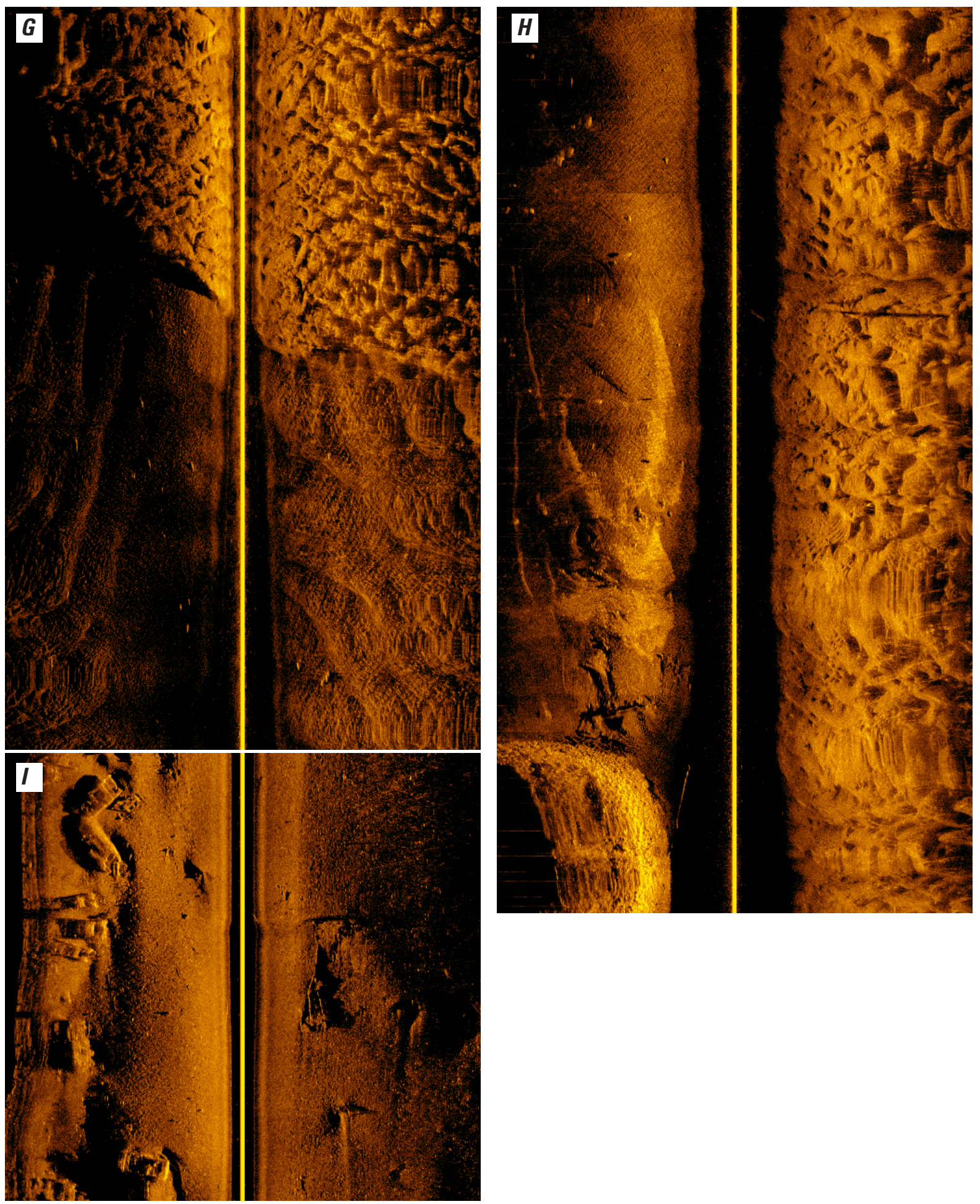

Figure 6. Images of nongeoreferenced side-scan sonar data. Examples illustrate substrate diversity and fish-detection capabilities. All images are oriented such that the bottom of the image is in the downstream direction. (A) Mid-channel dunes (June 2, 2007, Big Sioux segment), (B) gravel (May 22, 2007, Gavins Point segment), (C) sand dunes over gravel (May 22, 2007, Gavins Point segment), (D) bedrock, right side of image (May 23, 2007, Ponca segment), (E) large woody debris, left side of image (May 23, 2007, Ponca segment), (F) revetment, right side of image (June 2, 2007, Big Sioux segment), (G) aggregation of fish (species undetermined) on the lee side of a bar (May 23, 2007, Ponca segment), (H) fish and soft substrate in a dike field, left side of image (June 2, 2007, Big Sioux segment), (I) some fish visible; car bodies on left side demonstrate scale (May 24, 2007, Gavins Point segment).-Continued 

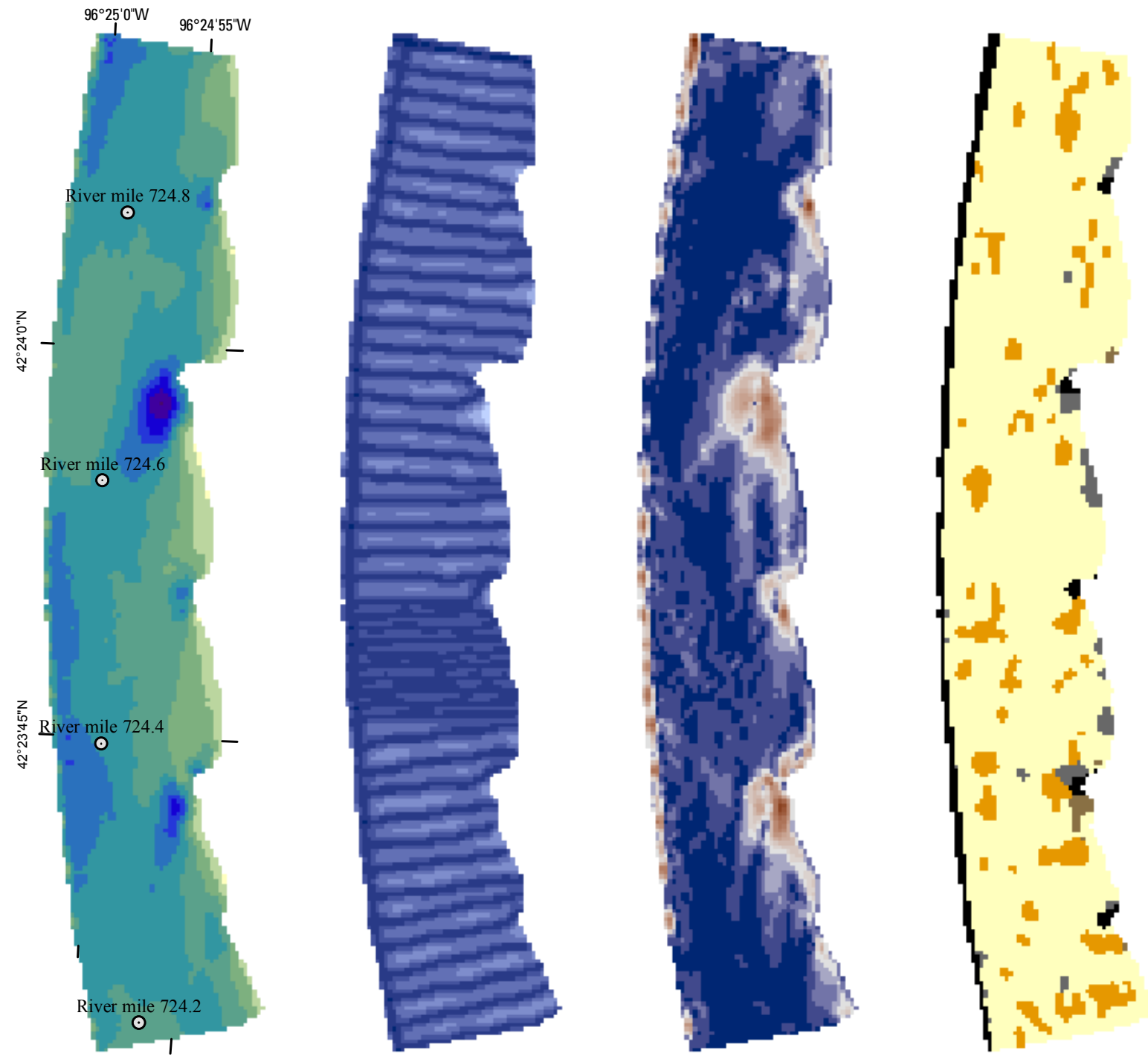

\section{EXPLANATION}
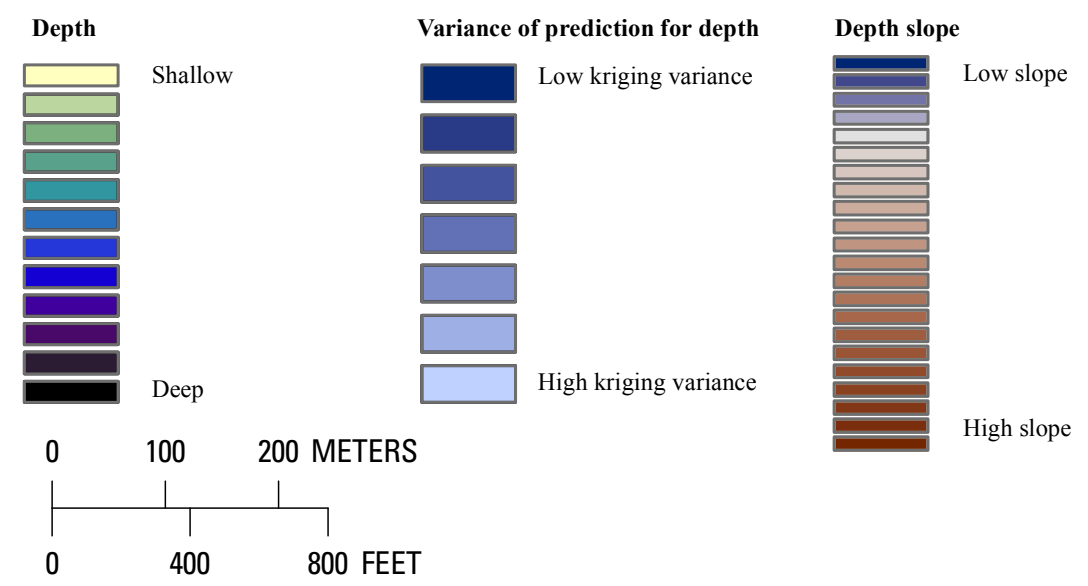

\section{Generalized substrate}

RoxAnn-derived classes

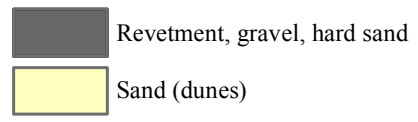

RoxAnn- and velocity-derived classes

Transporting sand

Fine sediment (mud, silt)

Class derived from aerial photographs

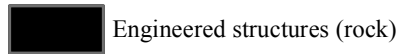

Figure 7. Maps of an example reach. Maps shown include the primary data types (depth, substrate, velocity, side-scan sonar), variance of prediction grids (estimates of the uncertainty of kriged values), and examples of derived grids (depth slope and velocity gradient). 


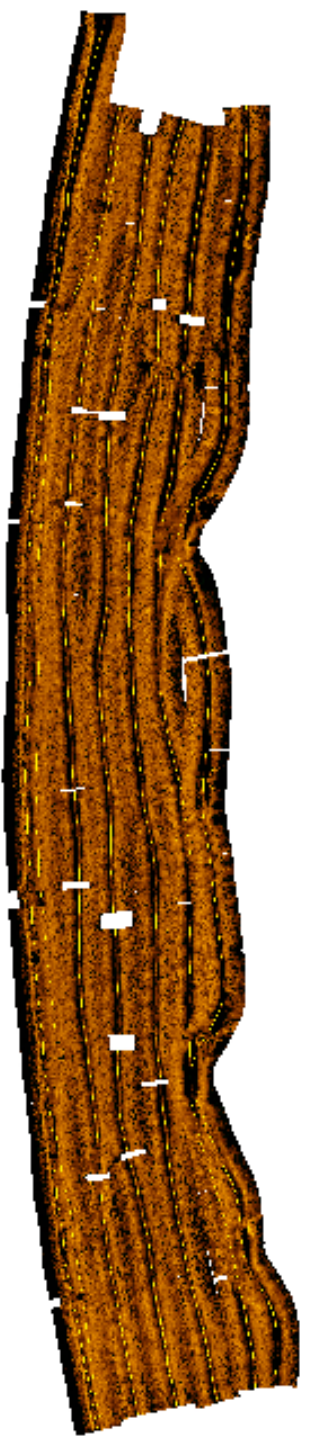

Side-scan sonar

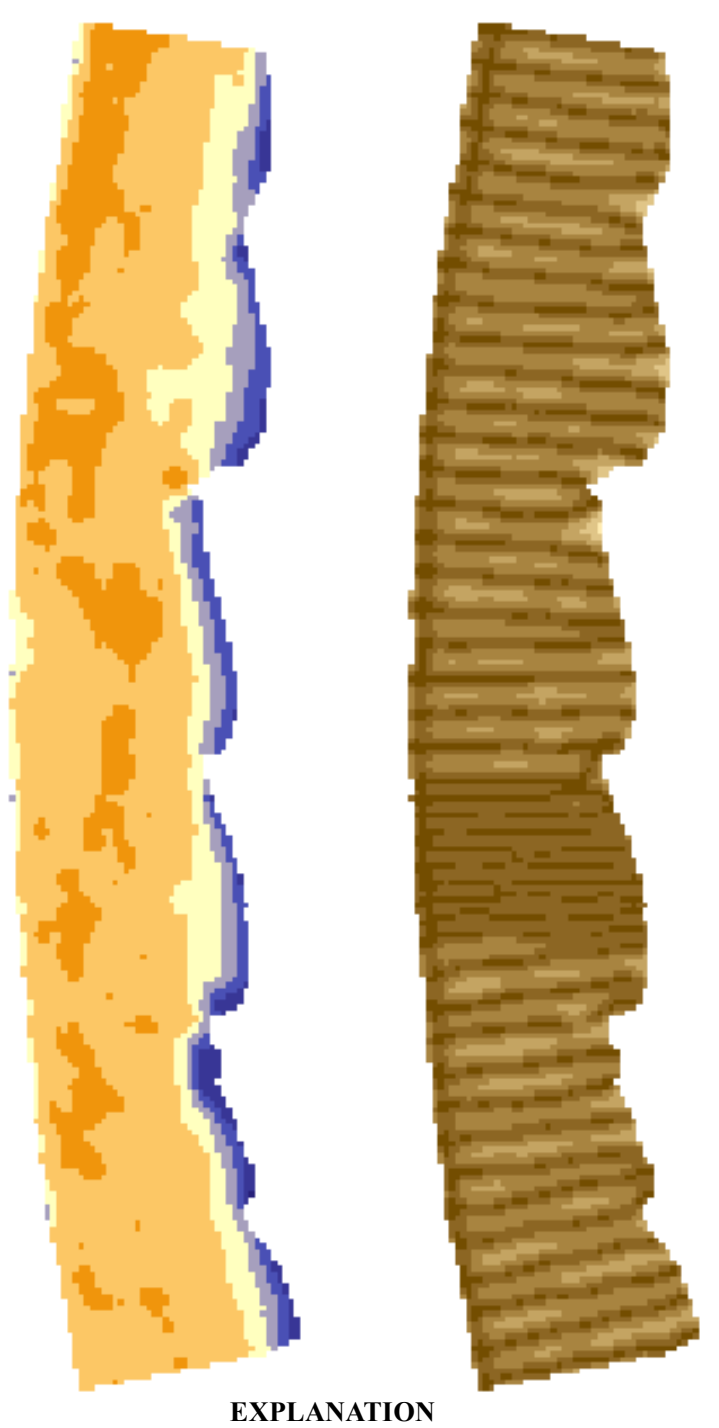

\section{EXPLANATION}

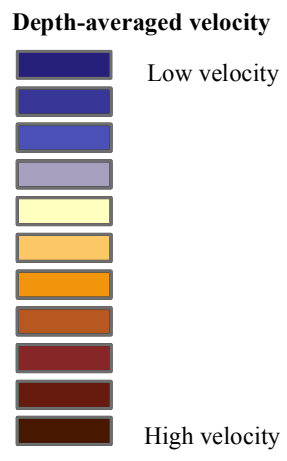

Variance of prediction for depth-averaged velocity

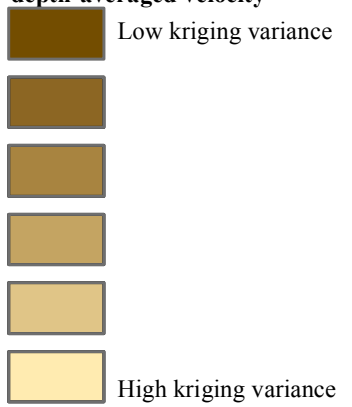

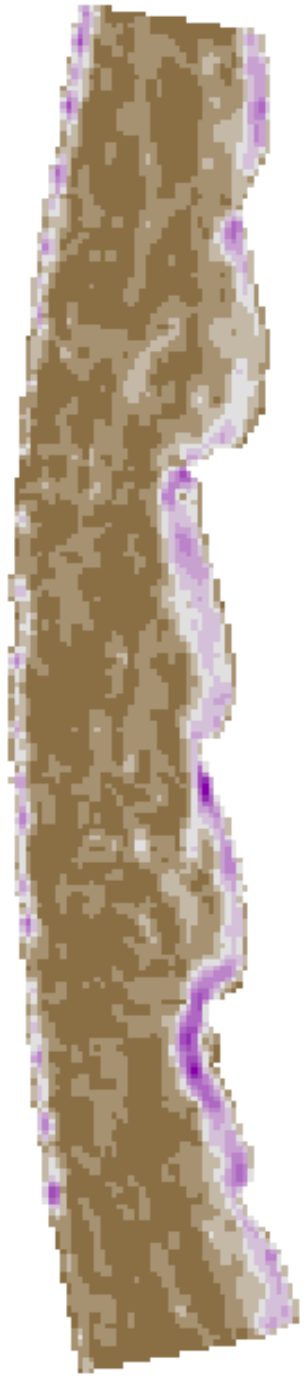

Gradient of depth-averaged velocity

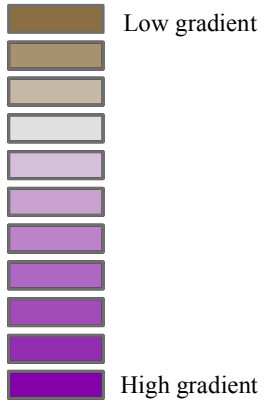

Figure 7. Maps of an example reach. Maps shown include the primary data types (depth, substrate, velocity, side-scan sonar), variance of prediction grids (estimates of the uncertainty of kriged values), and examples of derived grids (depth slope and velocity gradient).-Continued 
We used SonarWeb software (Chesapeake Technology, Inc., Mountain View, California) for all side-scan sonar processing. Georeferenced images are based on DGPS navigation data. We did not compensate for the length of cable utilized during data collection (typically $3-10 \mathrm{~m}$ ), so georeferencing is approximate. Because of stretching that occurs to the image during georeferencing, the quality and resolution of georeferenced images is degraded relative to the unprocessed images. However, both versions of the image can be used in conjunction for interpretation. For example, locations of fish or other features often can be discerned from the original images, and the georeferenced images can be used to determine the approximate locations of such features. The georeferenced images tend to be of sufficient quality to assist with identification of substrate. Where side-scan sonar data were collected, they were used to verify substrate classification produced by RoxAnn processing.

\section{Discharge Estimation by Location}

Estimates of discharge values were needed at the site of each mapped sturgeon for both the time period when the sturgeon was present and the time period when the reach was mapped. Discharge values are available at USGS streamflow-gaging stations on the Missouri River main stem and on tributaries (fig. 1). However, many mapped reaches are located some distance from gages, and in some locations substantial tributaries enter the Missouri River between a mapped reach and the nearest main-stem gage. We developed a systematic procedure to specify which gage or combination of gages to use to estimate discharge at any location along the Lower Missouri River, using daily mean discharge values served through the National Water Information System (U.S. Geological Survey, 2008). In addition, we used discharge values for Gavins Point Dam from the U.S. Army Corps of Engineers (Michael A. Swenson, unpub. data, 2007). The gages we used include Missouri River main-stem streamflowgaging stations and streamflow-gaging stations for tributaries with drainage-basin areas greater than 1,000 square kilometers at the gage site.

The procedure uses daily values from the nearest Missouri River main-stem gage or combination of main-stem and tributary gages. For the length of the Lower Missouri River, subsegments of river were defined such that each end of a subsegment is bounded by either a gage or a tributary (table 2). For each of these subsegments, the gage or gage combination that would typically provide the best (primary) discharge estimate was identified; gages or gage combinations that can provide an alternate (secondary) estimate for verification were also identified (table 2). Differences between the primary and secondary estimates can be used as an indicator of uncertainty of the discharge estimate; the comparison often serves as an alert to rapidly changing conditions, as differences between discharge estimates tend to be highest during time periods of rapidly fluctuating flow.
With the use of daily data, travel time was only considered if travel time between gages exceeded approximately one-half of a day. This was the case for some of the gages used in the secondary estimates, in which case the previous-day or nextday data were used, as appropriate.

The procedure involved accessing NWIS data by script and storing the data by river subsegment in a table that could be queried by date and location. Through this automated process, the discharge values that we used were regularly synchronized with the ones available from NWIS, so the best available values were accessible in the local database.

\section{Maps of Hydraulic and Substrate Characteristics}

Reach maps (figs. 8-416) are organized in order from upstream to downstream (in descending order by river mile). All data types available for a reach are on successive pages, ordered by depth, substrate, velocity, and side-scan sonar. Maps of side-scan sonar are included primarily to document the extent of mapping. Maps are in UTM Zone 15 North downstream from river mile 640; upstream from river mile 640, maps have been projected to UTM Zone 14 North. Two tables provide supplemental information about the maps. Table 3 contains the estimated daily discharge at the mapped reach on the day of mapping. Table 4 contains information about sturgeon relocation points and nets that were specifically targeted for mapping; these targeted sturgeon relocations also are shown on the maps. The maps depict physical conditions dependent on the discharge and channel morphology at the time of mapping.

\section{Summary}

The reach-scale maps presented in this report contain information about physical aquatic habitat available to and used by adult sturgeon of the genus Scaphirhynchus in the Lower Missouri River in the years 2005 to 2007. Close coordination with sturgeon tracking crews allowed us to rapidly obtain information about the sturgeon locations that we targeted for mapping. Reaches were typically mapped on the day after sturgeon were relocated, and usually within 10 percent of the discharge at the time of relocation.

Hydroacoustic data sets were collected from a boat mounted with a single-beam echo sounder for depth data, a RoxAnn instrument for substrate data, and an acoustic Doppler current profiler for velocity data. These data sets were collected along a longitudinal profile of the reach and in crosssectional transects (at 15- to 40-meter spacing). Side-scan sonar data sets also were collected at reaches that were considered to be potential spawning areas based on the information available at the time. Data sets were georeferenced at the 
Table 2. Gages used for discharge estimation by river subsegment.

[R., River; Gavins, Gavins Point Dam (U.S. Army Corps of Engineers); Missouri River main-stem streamflow-gaging stations and identification numbers: Sioux City, 6486000; Decatur, 6601200; Omaha, 6610000; Nebr. City, 6807000; Rulo, 6813500; St. Joseph, 6818000; Kansas City, 6893000; Waverly, 6895500; Glasgow, 6906500; Boonville, 69 09000; Hermann,

6934500; St. Charles, 6935965; Tributary streamflow-gaging stations and identification numbers: James R., 6478500; Vermillion R., 6479010; Big Sioux R., 6485500; Floyd R., 6600500; Mon.-H. (Monona-Harrison Ditch), 6602400; Little Sioux R., 6607500; Soldier R., 6608500; Boyer R., 6609500; Platte R., Nebr., 6805500; Nishnabotna R., 6810000; Little Nemaha R., 6811500; Big Nemaha R., 6815000; Tarkio R., 6813000; Nodaway R., 6817700; Platte R., Mo., 6821190; Kansas R., 6892350; Grand R., 6902000; Chariton R., 6905500; Blackwater R., 6908000; Lamine R., 6906800; Moreau R., 6910750; Osage R., 6926510; Gasconade R., 6934000; (+1), data from next day used; (-1), data from previous day used]

\begin{tabular}{|c|c|c|c|c|}
\hline Subsegment & $\begin{array}{l}\text { Upper } \\
\text { river mile }\end{array}$ & $\begin{array}{l}\text { Lower } \\
\text { river mile }\end{array}$ & $\begin{array}{l}\text { Primary } \\
\text { estimate }\end{array}$ & $\begin{array}{l}\text { Secondary } \\
\text { estimate }\end{array}$ \\
\hline Gavins Point Dam to James R. & 811.1 & 800.5 & Gavins & Sioux City $(+1)$ - Big Sioux (+1) - Vermillion $(+1)$ - James \\
\hline James R. to Vermillion R. & 800.5 & 772.0 & Gavins + James & Sioux City (+1) - Big Sioux (+1) - Vermillion \\
\hline Vermillion R. to Big Sioux R. & 772.0 & 733.9 & Sioux City - Big Sioux & Gavins $(-1)+$ James $(-1)+$ Vermillion \\
\hline Big Sioux R. to Sioux City gage & 733.9 & 732.4 & Sioux City & Gavins (-1) + James (-1) + Vermillion (-1) + Big Sioux \\
\hline Sioux City gage to Floyd R. & 732.4 & 731.2 & Sioux City & Decatur (+1) - Floyd \\
\hline Floyd R. to Decatur gage & 731.2 & 691.1 & Decatur & Sioux City $(-1)+$ Floyd $(-1)$ \\
\hline Decatur gage to Monona-Harrison Ditch & 691.1 & 670.1 & Decatur & Omaha (-1) - Boyer (-1) - Soldier - Little Sioux - Mon.-H. \\
\hline Monona-H. Ditch to Little Sioux R. & 670.1 & 669.2 & Decatur + Mon.-H. & Omaha (+1) - Boyer - Soldier - Little Sioux \\
\hline Little Sioux R. to Soldier R. & 669.2 & 664.0 & $\begin{array}{l}\text { Decatur + Mon.-H. + Little } \\
\quad \text { Sioux }\end{array}$ & Omaha $(+1)$ - Boyer - Soldier \\
\hline Soldier R. to Boyer R. & 664.0 & 635.2 & Omaha - Boyer & Decatur (-1) + Mon.-H. + Little Sioux + Soldier \\
\hline Boyer R. to Omaha gage & 635.2 & 616.0 & Omaha & $\begin{array}{l}\text { Decatur }(-1)+\text { Mon.-H. }(-1)+\text { Little Sioux }(-1)+\text { Soldier }(-1) \\
\quad+\text { Boyer }\end{array}$ \\
\hline Omaha gage to Platte R., Nebr. & 616.0 & 594.7 & Omaha & Nebr. City (+1) - Platte, Nebr. \\
\hline Platte R., Nebr. to Nebraska City gage & 594.7 & 562.6 & Nebr. City & Omaha $(-1)+$ Platte, Nebr. \\
\hline Nebr. City gage to Nishnabotna R. & 562.6 & 542.0 & Nebr. City & Rulo (+1) - Tarkio (+1) - Little Nemaha - Nishnabotna \\
\hline Nishnabotna R. to Little Nemaha R. & 542.0 & 527.8 & Nebr. City + Nishnabotna & Rulo (+1) - Tarkio - Little Nemaha \\
\hline Little Nemaha R. to Tarkio R. & 527.8 & 507.6 & Rulo - Tarkio & Nebr. City $(-1)+$ Nishnabotna + Little Nemaha \\
\hline Tarkio R. to Rulo gage & 507.6 & 498.0 & Rulo & Nebr. City $(-1)+$ Nishnabotna (-1) + Little Nemaha + Tarkio \\
\hline Rulo gage to Big Nemaha R. & 498.0 & 494.9 & Rulo & St. Joseph (+1) - Nodaway - Big Nemaha \\
\hline Big Nemaha R. to Nodaway R. & 494.9 & 463.0 & Rulo + Big Nemaha & St. Joseph (+1) - Nodaway \\
\hline Nodaway R. to St. Joseph gage & 463.0 & 448.2 & St. Joseph & Rulo (-1) + Big Nemaha (-1) + Nodaway \\
\hline St. Joseph gage to Platte R., Mo. & 448.2 & 391.2 & St. Joseph & Kansas City (+1) - Kansas (+1) - Platte, Mo. \\
\hline Platte R., Mo. to Kansas R. & 391.2 & 367.4 & Kansas City - Kansas & St. Joseph (-1) + Platte, Mo. \\
\hline Kansas R. to Kansas City gage & 367.4 & 366.1 & Kansas City & St. Joseph $(-1)+$ Platte, Mo. + Kansas \\
\hline Kansas City gage to Crooked R. & 366.1 & 313.7 & Kansas City & Waverly $(+1)$ \\
\hline Crooked R. to Waverly gage & 313.7 & 293.4 & Waverly & Kansas City $(-1)$ \\
\hline
\end{tabular}


Table 2. Gages used for discharge estimation by river subsegment.—Continued

[R., River; Gavins, Gavins Point Dam (U.S. Army Corps of Engineers); Missouri River main-stem streamflow-gaging stations and identification numbers: Sioux City, 6486000; Decatur, 6601200; Omaha, 6610000; Nebr. City, 6807000; Rulo, 6813500; St. Joseph, 6818000; Kansas City, 6893000; Waverly, 6895500; Glasgow, 6906500; Boonville, 69 09000; Hermann, 6934500; St. Charles, 6935965; Tributary streamflow-gaging stations and identification numbers: James R., 6478500; Vermillion R., 6479010; Big Sioux R., 6485500; Floyd R., 6600500; Mon.-H. (Monona-Harrison Ditch), 6602400; Little Sioux R., 6607500; Soldier R., 6608500; Boyer R., 6609500; Platte R., Nebr., 6805500; Nishnabotna R., 6810000; Little Nemaha R., 6811500; Big Nemaha R., 6815000; Tarkio R., 6813000; Nodaway R., 6817700; Platte R., Mo., 6821190; Kansas R., 6892350; Grand R., 6902000; Chariton R., 6905500; Blackwater R., 6908000; Lamine R., 6906800; Moreau R., 6910750; Osage R., 6926510; Gasconade R., 6934000; (+1), data from next day used; (-1), data from previous day used]

\begin{tabular}{lccll}
\hline \multicolumn{1}{c}{ Subsegment } & $\begin{array}{c}\text { Upper } \\
\text { river mile }\end{array}$ & $\begin{array}{c}\text { Lower } \\
\text { river mile }\end{array}$ & $\begin{array}{c}\text { Primary } \\
\text { estimate }\end{array}$ & $\begin{array}{c}\text { Secondary } \\
\text { estimate }\end{array}$ \\
\hline Waverly gage to Grand R. & 293.4 & 249.9 & Waverly & Glasgow (+1) - Chariton (+1) - Grand (+1) \\
Grand R. to Chariton R. & 249.9 & 238.8 & Glasgow - Chariton & Waverly $(-1)+$ Grand \\
Chariton R. to Glasgow gage & 238.8 & 226.3 & Glasgow & Waverly $(-1)+$ Grand + Chariton \\
Glasgow gage to Lamine R. & 226.3 & 202.5 & Glasgow & Boonville - Lamine - Blackwater \\
Lamine R. to Boonville gage & 202.5 & 197.1 & Boonville & Glasgow + Lamine + Blackwater \\
Boonville gage to Moreau R. & 197.1 & 138.3 & Boonville & Hermann $(+1)-$ Gasconade $(+1)-$ Osage $(+1)-$ Moreau (+1) \\
Moreau R. to Osage R. & 138.3 & 130.0 & Boonville $(-1)+$ Moreau & Hermann - Gasconade - Osage \\
Osage R. to Gasconade R. & 130.0 & 104.4 & Hermann - Gasconade & Boonville $(-1)+$ Moreau + Osage \\
Gasconade R. to Hermann gage & 104.4 & 97.9 & Hermann & Boonville $(-1)+$ Moreau + Osage + Gasconade \\
Hermann gage to Charrette Creek & 97.9 & 67.7 & Hermann & St. Charles $(+1)$ \\
Charrette Creek to St. Charles gage & 67.7 & 27.7 & St. Charles & Hermann $(-1)$ \\
St. Charles gage to mouth & 27.7 & 0.0 & St. Charles & St. Charles \\
\hline
\end{tabular}


Table 3. Locations, dates, and available data sets for habitat surveys.

[ft³/s, cubic feet per second; GPS, global positioning system; R/V, research vessel; x, data available; --, data not available; DGPS, differential GPS; RTK GPS, real-time kinematic GPS]

\begin{tabular}{|c|c|c|c|c|c|c|c|c|}
\hline \multirow{2}{*}{$\begin{array}{l}\text { River } \\
\text { mile }\end{array}$} & \multirow{2}{*}{$\begin{array}{l}\text { Map } \\
\text { date }\end{array}$} & \multirow[b]{2}{*}{ Boat } & \multirow{2}{*}{$\begin{array}{c}\text { Estimated } \\
\text { discharge } \\
\left(\mathrm{ft}^{3} / \mathrm{s}\right)\end{array}$} & \multicolumn{5}{|c|}{ Data types available } \\
\hline & & & & Depth & Substrate & Velocity & $\begin{array}{l}\text { Side-scan } \\
\text { sonar }\end{array}$ & GPS type \\
\hline \multicolumn{9}{|c|}{ Gavins Point Dam to Ponca } \\
\hline 807.9 & July 11, 2006 & R/V Funk & 25,500 & $\mathrm{x}$ & -- & $\mathrm{x}$ & -- & DGPS \\
\hline 806.9 & May 16, 2007 & R/V Funk & 8,000 & $\mathrm{x}$ & -- & $\mathrm{x}$ & -- & DGPS \\
\hline 806.6 & May 24, 2007 & R/V Funk & 11,000 & $\mathrm{x}$ & -- & $\mathrm{x}$ & $\mathrm{x}$ & DGPS \\
\hline 805.4 & June 13, 2006 & R/V Funk & 25,000 & $\mathrm{x}$ & -- & $\mathrm{x}$ & -- & DGPS \\
\hline 805.4 & June 14, 2006 & R/V Funk & 25,000 & -- & -- & -- & $\mathrm{x}$ & DGPS \\
\hline 805.4 & June 21, 2006 & R/V Funk & 25,000 & -- & -- & -- & $\mathrm{x}$ & DGPS \\
\hline 801.9 & May 10, 2007 & R/V Funk & 8,000 & $\mathrm{x}$ & -- & $\mathrm{x}$ & -- & DGPS \\
\hline 799.8 & May 22, 2007 & R/V Funk & 24,700 & $\mathrm{x}$ & -- & $\mathrm{x}$ & $\mathrm{x}$ & DGPS \\
\hline 797.1 & June 01, 2007 & R/V Funk & 19,600 & $\mathrm{x}$ & -- & $\mathrm{x}$ & $\mathrm{x}$ & DGPS \\
\hline 791.3 & July 17, 2007 & R/V Funk & 23,000 & $\mathrm{x}$ & -- & $\mathrm{x}$ & $\mathrm{x}$ & DGPS \\
\hline 782.5 & May 18, 2007 & R/V Funk & 18,900 & $\mathrm{x}$ & -- & $\mathrm{x}$ & -- & DGPS \\
\hline 775.2 & $\begin{array}{l}\text { May 30-31, } \\
2006\end{array}$ & R/V Funk & 21,700 & $\mathrm{x}$ & -- & $\mathrm{x}$ & $\mathrm{x}$ & DGPS \\
\hline 775.1 & May 30, 2007 & R/V Funk & 17,000 & $\mathrm{x}$ & -- & $\mathrm{x}$ & $\mathrm{x}$ & DGPS \\
\hline 774.2 & June 08, 2006 & R/V Funk & 23,700 & $\mathrm{x}$ & $\mathrm{x}$ & $\mathrm{x}$ & -- & DGPS \\
\hline 759.8 & June 15, 2005 & R/V Funk & 27,500 & $\mathrm{x}$ & -- & $\mathrm{x}$ & -- & DGPS \\
\hline 759.6 & May 21, 2007 & R/V Funk & 23,700 & $\mathrm{x}$ & -- & $\mathrm{x}$ & $\mathrm{x}$ & DGPS \\
\hline \multicolumn{9}{|c|}{ Ponca to Big Sioux River } \\
\hline 752.7 & May 23, 2007 & R/V Funk & 25,300 & $\mathrm{x}$ & -- & $\mathrm{x}$ & $\mathrm{x}$ & DGPS \\
\hline 751.8 & June 22, 2006 & R/V Funk & 26,800 & $\mathrm{x}$ & $\mathrm{x}$ & $\mathrm{x}$ & -- & DGPS \\
\hline 749.3 & June 20, 2006 & R/V Funk & 26,400 & $\mathrm{x}$ & -- & $\mathrm{x}$ & -- & DGPS \\
\hline 746.3 & June 10, 2005 & R/V Funk & 32,400 & $\mathrm{x}$ & $\mathrm{x}$ & $\mathrm{x}$ & -- & DGPS \\
\hline 746.3 & July 11, 2005 & R/V Funk & 22,800 & $\mathrm{x}$ & $\mathrm{x}$ & $\mathrm{x}$ & -- & RTK GPS \\
\hline 745.0 & May 25, 2007 & R/V Funk & 19,600 & $\mathrm{x}$ & $\mathrm{x}$ & $\mathrm{x}$ & $\mathrm{x}$ & DGPS \\
\hline 743.7 & July 12, 2006 & R/V Funk & 26,200 & $\mathrm{x}$ & $\mathrm{x}$ & $\mathrm{x}$ & -- & DGPS \\
\hline 739.9 & May 31, 2007 & R/V Funk & 18,200 & $\mathrm{x}$ & -- & $\mathrm{x}$ & $\mathrm{x}$ & DGPS \\
\hline 738.2 & May 02, 2007 & R/V Funk & 12,100 & $\mathrm{x}$ & $\mathrm{x}$ & $\mathrm{x}$ & -- & DGPS \\
\hline 736.6 & May 09, 2007 & R/V Funk & 15,000 & $\mathrm{x}$ & $\mathrm{x}$ & $\mathrm{x}$ & -- & DGPS \\
\hline \multicolumn{9}{|c|}{ Big Sioux River to Platte River } \\
\hline 733.8 & May 03, 2007 & R/V Funk & 16,900 & $\mathrm{x}$ & $\mathrm{x}$ & $\mathrm{x}$ & -- & DGPS \\
\hline 733.8 & June 12, 2007 & R/V Funk & 25,700 & $\mathrm{x}$ & $\mathrm{x}$ & $\mathrm{x}$ & -- & DGPS \\
\hline 732.4 & June 16, 2005 & R/V Funk & 32,900 & $\mathrm{x}$ & $\mathrm{x}$ & $\mathrm{x}$ & -- & DGPS \\
\hline 729.5 & June 15, 2007 & R/V Funk & 26,900 & $\mathrm{x}$ & $\mathrm{x}$ & $\mathrm{x}$ & -- & DGPS \\
\hline 726.5 & May 04, 2007 & R/V Funk & 19,200 & $\mathrm{x}$ & $\mathrm{x}$ & $\mathrm{x}$ & -- & DGPS \\
\hline 725.0 & May 23, 2006 & R/V Funk & 25,200 & $\mathrm{x}$ & $\mathrm{x}$ & $\mathrm{x}$ & -- & DGPS \\
\hline 724.6 & June 02, 2007 & R/V Funk & 26,500 & $\mathrm{x}$ & $\mathrm{x}$ & $\mathrm{x}$ & $\mathrm{x}$ & DGPS \\
\hline 717.8 & June 09, 2007 & R/V Funk & 27,300 & $\mathrm{x}$ & $\mathrm{x}$ & $\mathrm{x}$ & -- & DGPS \\
\hline 716.9 & Aug. 09, 2005 & R/V Funk & 27,300 & $\mathrm{x}$ & $\mathrm{x}$ & $\mathrm{x}$ & -- & DGPS \\
\hline
\end{tabular}


Table 3. Locations, dates, and available data sets for habitat surveys.-Continued

$\left[\mathrm{ft}^{3} / \mathrm{s}\right.$, cubic feet per second; GPS, global positioning system; R/V, research vessel; $\mathrm{x}$, data available; --, data not available; DGPS, differential GPS; RTK GPS, real-time kinematic GPS

\begin{tabular}{|c|c|c|c|c|c|c|c|c|}
\hline \multirow{2}{*}{$\begin{array}{l}\text { River } \\
\text { mile }\end{array}$} & \multirow{2}{*}{$\begin{array}{l}\text { Map } \\
\text { date }\end{array}$} & \multirow[b]{2}{*}{ Boat } & \multirow{2}{*}{$\begin{array}{c}\text { Estimated } \\
\text { discharge } \\
\left(\mathrm{ft}^{3} / \mathrm{s}\right)\end{array}$} & \multicolumn{5}{|c|}{ Data types available } \\
\hline & & & & Depth & Substrate & Velocity & $\begin{array}{l}\text { Side-scan } \\
\text { sonar }\end{array}$ & GPS type \\
\hline \multicolumn{9}{|c|}{ Big Sioux River to Platte River-Continued } \\
\hline 709.3 & June 07, 2006 & R/V Funk & 26,800 & $\mathrm{x}$ & $\mathrm{x}$ & $\mathrm{x}$ & -- & DGPS \\
\hline 707.5 & June 13, 2007 & R/V Funk & 26,900 & $\mathrm{x}$ & $\mathrm{x}$ & $\mathrm{x}$ & -- & DGPS \\
\hline 706.2 & May 15, 2007 & R/V Funk & 35,200 & $\mathrm{x}$ & -- & $\mathrm{x}$ & -- & DGPS \\
\hline 702.2 & June 07, 2007 & R/V Funk & 27,600 & $\mathrm{x}$ & $\mathrm{x}$ & $\mathrm{x}$ & -- & DGPS \\
\hline 701.1 & Aug. 10, 2005 & R/V Funk & 27,500 & $\mathrm{x}$ & -- & $\mathrm{x}$ & -- & DGPS \\
\hline 697.8 & June 03, 2005 & R/V Funk & 28,700 & $\mathrm{x}$ & $\mathrm{x}$ & $\mathrm{x}$ & -- & DGPS \\
\hline 693.4 & June 09, 2005 & R/V Funk & 42,900 & $\mathrm{x}$ & $\mathrm{x}$ & $\mathrm{x}$ & -- & DGPS \\
\hline 693.0 & June 17, 2005 & R/V Funk & 34,200 & $\mathrm{x}$ & $\mathrm{x}$ & $\mathrm{x}$ & -- & DGPS \\
\hline 692.7 & July 12, 2005 & R/V Funk & 26,200 & $\mathrm{x}$ & $\mathrm{x}$ & $\mathrm{x}$ & -- & RTK GPS \\
\hline 691.9 & May 19, 2007 & R/V Funk & 26,800 & $\mathrm{x}$ & $\mathrm{x}$ & $\mathrm{x}$ & -- & DGPS \\
\hline 689.2 & May 17, 2007 & R/V Funk & 30,900 & $\mathrm{x}$ & -- & $\mathrm{x}$ & -- & DGPS \\
\hline 687.7 & May 26, 2007 & R/V Funk & 24,000 & $\mathrm{x}$ & $\mathrm{x}$ & $\mathrm{x}$ & $\mathrm{x}$ & DGPS \\
\hline 686.6 & May 18, 2005 & R/V Funk & 23,900 & $\mathrm{x}$ & $\mathrm{x}$ & $\mathrm{x}$ & -- & DGPS \\
\hline 683.9 & June 24, 2005 & R/V Funk & 31,900 & $\mathrm{x}$ & $\mathrm{x}$ & $\mathrm{x}$ & -- & DGPS \\
\hline 673.8 & May 10, 2005 & R/V Funk & 26,400 & $\mathrm{x}$ & $\mathrm{x}$ & $\mathrm{x}$ & -- & DGPS \\
\hline 672.4 & June 02, 2005 & R/V Funk & 29,300 & $\mathrm{x}$ & $\mathrm{x}$ & $\mathrm{x}$ & -- & DGPS \\
\hline 670.4 & Aug. 11, 2005 & R/V Funk & 28,000 & $\mathrm{x}$ & -- & $\mathrm{x}$ & -- & DGPS \\
\hline 669.2 & June 01, 2006 & R/V Funk & 26,600 & $\mathrm{x}$ & $\mathrm{x}$ & $\mathrm{x}$ & -- & DGPS \\
\hline 668.9 & Apr. 22, 2005 & R/V Funk & 27,200 & $\mathrm{x}$ & $\mathrm{x}$ & $\mathrm{x}$ & -- & DGPS \\
\hline 665.6 & June 06, 2006 & R/V Funk & 28,700 & $\mathrm{x}$ & $\mathrm{x}$ & $\mathrm{x}$ & -- & DGPS \\
\hline 663.0 & June 08, 2005 & R/V Funk & 44,700 & $\mathrm{x}$ & $\mathrm{x}$ & $\mathrm{x}$ & -- & DGPS \\
\hline 660.8 & May 12, 2007 & R/V Funk & 36,500 & $\mathrm{x}$ & -- & $\mathrm{x}$ & -- & DGPS \\
\hline 657.4 & May 19, 2005 & R/V Funk & 30,900 & $\mathrm{x}$ & -- & -- & -- & DGPS \\
\hline 654.7 & May 07, 2005 & R/V Funk & 26,500 & $\mathrm{x}$ & $\mathrm{x}$ & $\mathrm{x}$ & -- & DGPS \\
\hline 652.0 & May 25, 2005 & R/V Funk & 29,400 & $\mathrm{x}$ & $\mathrm{x}$ & $\mathrm{x}$ & -- & DGPS \\
\hline 651.8 & July 13, 2005 & R/V Funk & 28,400 & $\mathrm{x}$ & $\mathrm{x}$ & $\mathrm{x}$ & -- & RTK GPS \\
\hline 650.4 & June 23, 2005 & R/V Funk & 33,800 & $\mathrm{x}$ & $\mathrm{x}$ & $\mathrm{x}$ & -- & DGPS \\
\hline 650.3 & June 08, 2007 & R/V Funk & 31,200 & $\mathrm{x}$ & $\mathrm{x}$ & $\mathrm{x}$ & -- & DGPS \\
\hline 647.6 & May 01, 2007 & R/V Funk & 28,000 & $\mathrm{x}$ & $\mathrm{x}$ & $\mathrm{x}$ & -- & DGPS \\
\hline 647.5 & May 11, 2005 & R/V Funk & 31,200 & $\mathrm{x}$ & $\mathrm{x}$ & $\mathrm{x}$ & -- & DGPS \\
\hline 644.8 & June 14, 2005 & R/V Funk & 36,200 & $\mathrm{x}$ & $\mathrm{x}$ & $\mathrm{x}$ & -- & DGPS \\
\hline 643.6 & June 14, 2007 & R/V Funk & 30,100 & $\mathrm{x}$ & $\mathrm{x}$ & $\mathrm{x}$ & -- & DGPS \\
\hline 642.7 & Aug. 12, 2005 & R/V Funk & 28,100 & $\mathrm{x}$ & -- & $\mathrm{x}$ & -- & DGPS \\
\hline 641.5 & May 18, 2006 & R/V Funk & 34,400 & $\mathrm{x}$ & $\mathrm{x}$ & $\mathrm{x}$ & -- & DGPS \\
\hline 641.3 & June 16, 2007 & R/V Funk & 29,600 & $\mathrm{x}$ & $\mathrm{x}$ & $\mathrm{x}$ & -- & DGPS \\
\hline 640.5 & May 05, 2005 & R/V Funk & 27,400 & $\mathrm{x}$ & $\mathrm{x}$ & $\mathrm{x}$ & -- & DGPS \\
\hline 638.9 & June 01, 2005 & R/V Funk & 32,600 & $\mathrm{x}$ & $\mathrm{x}$ & $\mathrm{x}$ & -- & DGPS \\
\hline 638.3 & Apr. 20, 2005 & R/V Funk & 27,400 & $\mathrm{x}$ & $\mathrm{x}$ & $\mathrm{x}$ & -- & DGPS \\
\hline
\end{tabular}




\section{Hydraulic and Substrate Maps of Reaches Used by Sturgeon in the Lower Missouri River, 2005-07}

Table 3. Locations, dates, and available data sets for habitat surveys._- Continued

[ft³/s, cubic feet per second; GPS, global positioning system; R/V, research vessel; x, data available; --, data not available; DGPS, differential GPS; RTK GPS, real-time kinematic GPS]

\begin{tabular}{|c|c|c|c|c|c|c|c|c|}
\hline \multirow{2}{*}{$\begin{array}{l}\text { River } \\
\text { mile }\end{array}$} & \multirow{2}{*}{$\begin{array}{l}\text { Map } \\
\text { date }\end{array}$} & \multirow[b]{2}{*}{ Boat } & \multirow{2}{*}{$\begin{array}{c}\text { Estimated } \\
\text { discharge } \\
\left(\mathrm{ft}^{3} / \mathrm{s}\right)\end{array}$} & \multicolumn{5}{|c|}{ Data types available } \\
\hline & & & & Depth & Substrate & Velocity & $\begin{array}{l}\text { Side-scan } \\
\text { sonar }\end{array}$ & GPS type \\
\hline \multicolumn{9}{|c|}{ Big Sioux River to Platte River-Continued } \\
\hline 627.8 & July 14,2005 & R/V Funk & 28,600 & $\mathrm{x}$ & $\mathrm{x}$ & $\mathrm{x}$ & -- & RTK GPS \\
\hline 627.5 & May 20, 2005 & R/V Funk & 35,000 & $\mathrm{x}$ & $\mathrm{x}$ & $\mathrm{x}$ & -- & DGPS \\
\hline 626.1 & Sept. 01, 2005 & R/V Funk & 27,800 & $\mathrm{x}$ & $\mathrm{x}$ & $\mathrm{x}$ & -- & DGPS \\
\hline 622.8 & June 22, 2005 & R/V Funk & 33,800 & $\mathrm{x}$ & $\mathrm{x}$ & $\mathrm{x}$ & -- & DGPS \\
\hline 620.1 & May 12,2005 & R/V Funk & 34,000 & $\mathrm{x}$ & $\mathrm{x}$ & $\mathrm{x}$ & -- & DGPS \\
\hline 618.6 & June 21, 2005 & R/V Funk & 34,600 & $\mathrm{x}$ & $\mathrm{x}$ & $\mathrm{x}$ & -- & DGPS \\
\hline 616.5 & Aug. 31, 2005 & R/V Funk & 27,800 & $\mathrm{x}$ & $\mathrm{x}$ & $\mathrm{x}$ & -- & DGPS \\
\hline 614.5 & Apr. 30, 2005 & R/V Funk & 25,200 & $\mathrm{x}$ & $\mathrm{x}$ & $\mathrm{x}$ & -- & DGPS \\
\hline 611.6 & Apr. 28, 2005 & R/V Funk & 25,100 & $\mathrm{x}$ & $\mathrm{x}$ & $\mathrm{x}$ & -- & DGPS \\
\hline 609.9 & May 06, 2005 & R/V Funk & 26,800 & $\mathrm{x}$ & $\mathrm{x}$ & $\mathrm{x}$ & -- & DGPS \\
\hline 609.7 & June 05, 2005 & R/V Funk & 33,100 & $\mathrm{x}$ & $\mathrm{x}$ & $\mathrm{x}$ & -- & DGPS \\
\hline 609.1 & May 08, 2005 & R/V Funk & 28,000 & $\mathrm{x}$ & $\mathrm{x}$ & $\mathrm{x}$ & -- & DGPS \\
\hline 608.2 & Apr. 29, 2005 & R/V Funk & 25,100 & $\mathrm{x}$ & $\mathrm{x}$ & $\mathrm{x}$ & -- & DGPS \\
\hline 606.5 & May 17, 2005 & R/V Funk & 36,100 & $\mathrm{x}$ & $\mathrm{x}$ & $\mathrm{x}$ & -- & DGPS \\
\hline 606.4 & Apr. 27, 2005 & R/V Funk & 25,300 & $\mathrm{x}$ & $\mathrm{x}$ & $\mathrm{x}$ & -- & DGPS \\
\hline 604.0 & June 04, 2005 & R/V Funk & 32,900 & $\mathrm{x}$ & $\mathrm{x}$ & $\mathrm{x}$ & -- & DGPS \\
\hline 602.4 & May 09, 2005 & R/V Funk & 28,500 & $\mathrm{x}$ & $\mathrm{x}$ & $\mathrm{x}$ & -- & DGPS \\
\hline 601.2 & May 22, 2005 & R/V Funk & 31,100 & $\mathrm{x}$ & $\mathrm{x}$ & $\mathrm{x}$ & -- & DGPS \\
\hline \multicolumn{9}{|c|}{ Platte River to Kansas River } \\
\hline 593.7 & June 11, 2005 & R/V Funk & 57,300 & $\mathrm{x}$ & $\mathrm{x}$ & $\mathrm{x}$ & -- & DGPS \\
\hline 588.6 & May 23, 2005 & R/V Funk & 39,400 & $\mathrm{x}$ & $\mathrm{x}$ & $\mathrm{x}$ & -- & DGPS \\
\hline 583.8 & July 13, 2006 & R/V Funk & 30,200 & $\mathrm{x}$ & $\mathrm{x}$ & $\mathrm{x}$ & -- & DGPS \\
\hline 565.5 & May 24, 2005 & R/V Funk & 39,600 & $\mathrm{x}$ & $\mathrm{x}$ & $\mathrm{x}$ & -- & DGPS \\
\hline 554.2 & June 25, 2005 & R/V Funk & 46,300 & $\mathrm{x}$ & $\mathrm{x}$ & $\mathrm{x}$ & -- & DGPS \\
\hline \multicolumn{9}{|c|}{ Kansas River to Grand River } \\
\hline 362.7 & May 04, 2005 & R/V Brush & 39,200 & $\mathrm{x}$ & -- & $\mathrm{x}$ & -- & DGPS \\
\hline 354.4 & May 25, 2005 & R/V Brush & 52,900 & $\mathrm{x}$ & -- & $\mathrm{x}$ & -- & DGPS \\
\hline 331.9 & June 20, 2006 & R/V Brush & 35,600 & $\mathrm{x}$ & $\mathrm{x}$ & $\mathrm{x}$ & -- & DGPS \\
\hline 330.1 & Apr. 29, 2005 & R/V Brush & 43,300 & $\mathrm{x}$ & -- & $\mathrm{x}$ & -- & DGPS \\
\hline 326.8 & May 24, 2006 & R/V Brush & 38,600 & $\mathrm{x}$ & $\mathrm{x}$ & $\mathrm{x}$ & -- & DGPS \\
\hline 325.2 & May 11,2005 & R/V Brush & 37,600 & $\mathrm{x}$ & -- & $\mathrm{x}$ & -- & DGPS \\
\hline 315.1 & Apr. 27, 2005 & R/V Brush & 45,000 & $\mathrm{x}$ & -- & $\mathrm{x}$ & -- & DGPS \\
\hline 311.6 & May 05, 2005 & R/V Brush & 39,200 & $\mathrm{x}$ & -- & $\mathrm{x}$ & -- & DGPS \\
\hline 291.8 & May 26, 2005 & R/V Brush & 53,000 & $\mathrm{x}$ & -- & $\mathrm{x}$ & -- & DGPS \\
\hline 289.4 & July 06, 2006 & R/V Brush & 36,200 & $\mathrm{x}$ & $\mathrm{x}$ & $\mathrm{x}$ & -- & DGPS \\
\hline 282.3 & June 13, 2006 & R/V Brush & 36,700 & $\mathrm{x}$ & $\mathrm{x}$ & $\mathrm{x}$ & -- & DGPS \\
\hline 282.0 & May 04, 2006 & R/V Brush & 65,100 & $\mathrm{x}$ & $\mathrm{x}$ & $\mathrm{x}$ & -- & DGPS \\
\hline 281.5 & June 13, 2006 & $\mathrm{R} / \mathrm{V}$ Brush & 36,700 & $\mathrm{x}$ & $\mathrm{x}$ & $\mathrm{x}$ & -- & DGPS \\
\hline
\end{tabular}


Table 3. Locations, dates, and available data sets for habitat surveys.-Continued

[ft³/s, cubic feet per second; GPS, global positioning system; R/V, research vessel; x, data available; --, data not available; DGPS, differential GPS; RTK GPS, real-time kinematic GPS]

\begin{tabular}{|c|c|c|c|c|c|c|c|c|}
\hline \multirow{2}{*}{$\begin{array}{l}\text { River } \\
\text { mile }\end{array}$} & \multirow{2}{*}{$\begin{array}{l}\text { Map } \\
\text { date }\end{array}$} & \multirow[b]{2}{*}{ Boat } & \multirow{2}{*}{$\begin{array}{c}\text { Estimated } \\
\text { discharge } \\
\left(\mathrm{ft}^{3} / \mathrm{s}\right)\end{array}$} & \multicolumn{5}{|c|}{ Data types available } \\
\hline & & & & Depth & Substrate & Velocity & $\begin{array}{l}\text { Side-scan } \\
\text { sonar }\end{array}$ & GPS type \\
\hline \multicolumn{9}{|c|}{ Kansas River to Grand River-Continued } \\
\hline 280.0 & June 27, 2006 & R/V Brush & 39,400 & -- & -- & -- & $\mathrm{x}$ & DGPS \\
\hline 280.0 & June 28, 2006 & R/V Brush & 38,800 & -- & -- & -- & $\mathrm{x}$ & DGPS \\
\hline 280.0 & July 25, 2006 & R/V Funk & 35,000 & -- & -- & -- & $\mathrm{x}$ & DGPS \\
\hline 279.7 & June 08, 2006 & R/V Brush & 38,200 & $\mathrm{x}$ & $\mathrm{x}$ & $\mathrm{x}$ & -- & DGPS \\
\hline 278.1 & June 14, 2006 & $\mathrm{R} / \mathrm{V}$ Brush & 37,400 & $\mathrm{x}$ & $\mathrm{x}$ & $\mathrm{x}$ & -- & DGPS \\
\hline 275.8 & June 10, 2005 & R/V Brush & 84,400 & $\mathrm{x}$ & -- & $\mathrm{x}$ & -- & DGPS \\
\hline \multicolumn{9}{|c|}{ Grand River to Osage River } \\
\hline 247.6 & June 02, 2006 & R/V Brush & 45,700 & $\mathrm{x}$ & $\mathrm{x}$ & $\mathrm{x}$ & -- & DGPS \\
\hline 247.5 & May 12, 2005 & R/V Brush & 44,400 & $\mathrm{x}$ & -- & $\mathrm{x}$ & -- & DGPS \\
\hline 230.5 & May 06, 2005 & $\mathrm{R} / \mathrm{V}$ Brush & 42,700 & $\mathrm{x}$ & -- & $\mathrm{x}$ & -- & DGPS \\
\hline 219.2 & Apr. 15, 2005 & $\mathrm{R} / \mathrm{V}$ Brush & 69,500 & $\mathrm{x}$ & -- & $\mathrm{x}$ & -- & DGPS \\
\hline 218.8 & May 19, 2006 & $\mathrm{R} / \mathrm{V}$ Brush & 39,700 & $\mathrm{x}$ & $\mathrm{x}$ & $\mathrm{x}$ & -- & DGPS \\
\hline 217.4 & June 27, 2005 & R/V Brush & 68,900 & $\mathrm{x}$ & -- & $\mathrm{x}$ & -- & DGPS \\
\hline 208.7 & July 05, 2005 & R/V Brush & 59,300 & $\mathrm{x}$ & -- & $\mathrm{x}$ & -- & DGPS \\
\hline 206.1 & June 07, 2006 & R/V Brush & 38,000 & $\mathrm{x}$ & $\mathrm{x}$ & $\mathrm{x}$ & -- & DGPS \\
\hline 206.1 & June 09, 2006 & R/V Brush & 38,800 & -- & -- & -- & $\mathrm{x}$ & DGPS \\
\hline 203.3 & Apr. 28, 2005 & R/V Brush & 53,300 & $\mathrm{x}$ & -- & $\mathrm{x}$ & -- & DGPS \\
\hline 202.3 & June 06, 2006 & R/V Brush & 40,100 & $\mathrm{x}$ & $\mathrm{x}$ & $\mathrm{x}$ & -- & DGPS \\
\hline 201.3 & Apr. 07, 2005 & R/V Brush & 38,700 & $\mathrm{x}$ & -- & $\mathrm{x}$ & -- & DGPS \\
\hline 201.3 & Apr. 07, 2005 & R/V Funk & 38,700 & $\mathrm{x}$ & -- & -- & -- & DGPS \\
\hline 199.7 & Apr. 08, 2005 & R/V Funk & 40,000 & $\mathrm{x}$ & $\mathrm{x}$ & $\mathrm{x}$ & -- & DGPS \\
\hline 197.5 & May 18, 2005 & R/V Brush & 106,000 & $\mathrm{x}$ & -- & $\mathrm{x}$ & -- & DGPS \\
\hline 197.0 & July 13, 2005 & R/V Brush & 43,000 & $\mathrm{x}$ & -- & $\mathrm{x}$ & -- & RTK GPS \\
\hline 196.0 & July 07, 2006 & R/V Brush & 38,000 & $\mathrm{x}$ & $\mathrm{x}$ & $\mathrm{x}$ & -- & DGPS \\
\hline 186.8 & May 27, 2005 & R/V Brush & 59,500 & $\mathrm{x}$ & -- & $\mathrm{x}$ & -- & DGPS \\
\hline 178.0 & Apr. 12, 2005 & R/V Brush & 48,500 & $\mathrm{x}$ & -- & $\mathrm{x}$ & -- & DGPS \\
\hline 177.4 & May 10, 2006 & R/V Brush & 54,000 & $\mathrm{x}$ & $\mathrm{x}$ & $\mathrm{x}$ & -- & DGPS \\
\hline 173.5 & June 01, 2005 & R/V Brush & 53,600 & $\mathrm{x}$ & -- & $\mathrm{x}$ & -- & DGPS \\
\hline 173.3 & Apr. 22, 2005 & R/V Brush & 53,800 & $\mathrm{x}$ & -- & $\mathrm{x}$ & -- & DGPS \\
\hline 168.0 & July 01, 2005 & R/V Brush & 65,400 & $\mathrm{x}$ & -- & $\mathrm{x}$ & -- & DGPS \\
\hline 166.8 & May 19, 2005 & R/V Brush & 90,900 & $\mathrm{x}$ & -- & $\mathrm{x}$ & -- & DGPS \\
\hline 165.5 & June 21, 2006 & R/V Brush & 37,600 & $\mathrm{x}$ & $\mathrm{x}$ & $\mathrm{x}$ & -- & DGPS \\
\hline 160.5 & May 10, 2005 & R/V Brush & 40,500 & $\mathrm{x}$ & -- & $\mathrm{x}$ & -- & DGPS \\
\hline 146.5 & June 03, 2005 & R/V Brush & 59,600 & $\mathrm{x}$ & -- & $\mathrm{x}$ & -- & DGPS \\
\hline 146.5 & Aug. 10, 2005 & R/V Brush & 35,000 & $\mathrm{x}$ & -- & $\mathrm{x}$ & -- & DGPS \\
\hline 142.7 & May 23, 2005 & R/V Brush & 71,000 & $\mathrm{x}$ & -- & $\mathrm{x}$ & -- & DGPS \\
\hline 142.0 & Aug. 09, 2005 & R/V Brush & 35,100 & $\mathrm{x}$ & -- & $\mathrm{x}$ & -- & DGPS \\
\hline 140.9 & June 02, 2005 & R/V Brush & 54,000 & $\mathrm{x}$ & -- & $\mathrm{x}$ & -- & DGPS \\
\hline
\end{tabular}




\section{Hydraulic and Substrate Maps of Reaches Used by Sturgeon in the Lower Missouri River, 2005-07}

Table 3. Locations, dates, and available data sets for habitat surveys._Continued

[ft³/s, cubic feet per second; GPS, global positioning system; R/V, research vessel; x, data available; --, data not available; DGPS, differential GPS; RTK GPS, real-time kinematic GPS]

\begin{tabular}{|c|c|c|c|c|c|c|c|c|}
\hline \multirow{2}{*}{$\begin{array}{l}\text { River } \\
\text { mile }\end{array}$} & \multirow{2}{*}{$\begin{array}{l}\text { Map } \\
\text { date }\end{array}$} & \multirow[b]{2}{*}{ Boat } & \multirow{2}{*}{$\begin{array}{c}\text { Estimated } \\
\text { discharge } \\
\left(\mathrm{ft}^{3} / \mathrm{s}\right)\end{array}$} & \multicolumn{5}{|c|}{ Data types available } \\
\hline & & & & Depth & Substrate & Velocity & $\begin{array}{l}\text { Side-scan } \\
\text { sonar }\end{array}$ & GPS type \\
\hline 136.3 & Apr. 14, 2005 & R/V Funk & 69,300 & $\mathrm{x}$ & $\mathrm{x}$ & $\mathrm{x}$ & -- & DGPS \\
\hline 130.6 & Apr. 19, 2005 & R/V Brush & 53,600 & $\mathrm{x}$ & -- & $\mathrm{x}$ & -- & DGPS \\
\hline \multicolumn{9}{|c|}{ Osage River to mouth } \\
\hline 128.5 & May 31, 2005 & R/V Brush & 53,600 & $\mathrm{x}$ & -- & $\mathrm{x}$ & -- & DGPS \\
\hline 127.6 & May 09, 2006 & R/V Brush & 69,700 & $\mathrm{x}$ & $\mathrm{x}$ & $\mathrm{x}$ & -- & DGPS \\
\hline 127.3 & Apr. 26, 2005 & R/V Brush & 86,500 & $\mathrm{x}$ & -- & $\mathrm{x}$ & -- & DGPS \\
\hline 120.6 & Apr. 05, 2005 & $\mathrm{R} / \mathrm{V}$ Brush & 43,500 & $\mathrm{x}$ & -- & $\mathrm{x}$ & -- & DGPS \\
\hline 119.6 & June 30, 2006 & R/V Brush & 41,700 & $\mathrm{x}$ & $\mathrm{x}$ & $\mathrm{x}$ & -- & DGPS \\
\hline 118.5 & May 03, 2005 & R/V Brush & 52,700 & $\mathrm{x}$ & -- & $\mathrm{x}$ & -- & DGPS \\
\hline 118.2 & July 06, 2005 & R/V Brush & 66,800 & $\mathrm{x}$ & -- & $\mathrm{x}$ & -- & DGPS \\
\hline 109.3 & Aug. 31, 2005 & R/V Brush & 90,600 & $\mathrm{x}$ & -- & $\mathrm{x}$ & -- & DGPS \\
\hline 81.9 & Sept. 01, 2005 & R/V Brush & 87,200 & $\mathrm{x}$ & -- & $\mathrm{x}$ & -- & DGPS \\
\hline 75.8 & July 07, 2005 & $\mathrm{R} / \mathrm{V}$ Brush & 70,600 & $\mathrm{x}$ & -- & $\mathrm{x}$ & -- & DGPS \\
\hline 27.3 & July 08, 2005 & R/V Brush & 71,800 & $\mathrm{x}$ & -- & $\mathrm{x}$ & -- & DGPS \\
\hline
\end{tabular}


Table 4. Sturgeon relocations and nets targeted for mapping.

[ft $\mathrm{ft}^{3} / \mathrm{s}$, cubic feet per second; SNS, shovelnose sturgeon; PLS pallid sturgeon; --, no data]

\begin{tabular}{|c|c|c|c|c|c|c|c|c|c|}
\hline $\begin{array}{l}\text { Sturgeon } \\
\text { relocation } \\
\text { date }\end{array}$ & $\begin{array}{c}\text { River } \\
\text { mile } \\
\text { of } \\
\text { sturgeon } \\
\text { re- } \\
\text { location }\end{array}$ & $\begin{array}{c}\text { Estimated } \\
\text { discharge, } \\
\text { sturgeon } \\
\text { relocation } \\
\text { date }\left(\mathrm{ft}^{3} / \mathrm{s}\right)\end{array}$ & $\begin{array}{c}\text { Sturgeon } \\
\text { identification } \\
\text { code }\end{array}$ & Species & Sex & $\begin{array}{c}\text { Selection } \\
\text { method }\end{array}$ & $\begin{array}{l}\text { Map } \\
\text { date }\end{array}$ & Boat & $\begin{array}{c}\text { Estimated } \\
\text { discharge, } \\
\text { map } \\
\text { date } \\
\left(\mathrm{ft}^{3} / \mathrm{s}\right)\end{array}$ \\
\hline July 10, 2006 & 808.2 & 25,500 & PLS05-006 & PLS & unknown & random & July 11,2006 & R/V Funk & 25,500 \\
\hline May 15, 2007 & 807.0 & 8,000 & SNS07-093 & SNS & female & random & May 16, 2007 & R/V Funk & 8,000 \\
\hline May 23, 2007 & 806.6 & 11,300 & SNS07-093 & SNS & female & random & May 24, 2007 & R/V Funk & 11,000 \\
\hline June 5, 2006 & 805.8 & 22,500 & Net & -- & -- & nonrandom & June 13, 2006 & R/V Funk & 25,000 \\
\hline May 9, 2007 & 801.9 & 8,000 & SNS07-081 & SNS & female & random & May 10, 2007 & R/V Funk & 8,000 \\
\hline May 21, 2007 & 799.8 & 19,400 & SNS07-062 & SNS & female & random & May 22, 2007 & R/V Funk & 24,700 \\
\hline May 21, 2007 & 799.7 & 19,400 & SNS07-088 & SNS & female & random & May 22, 2007 & R/V Funk & 24,700 \\
\hline May 21, 2007 & 799.1 & 19,400 & SNS07-169 & SNS & male & random & May 22, 2007 & R/V Funk & 24,700 \\
\hline May 31, 2007 & 797.0 & 20,800 & SNS07-085 & SNS & female & random & June 01, 2007 & R/V Funk & 19,600 \\
\hline May 23, 2007 & 791.2 & 18,500 & SNS07-152 & SNS & female & random & July 17, 2007 & R/V Funk & 23,000 \\
\hline June 2, 2007 & 791.1 & 16,500 & SNS07-152 & SNS & female & random & July 17, 2007 & R/V Funk & 23,000 \\
\hline June 4, 2007 & 791.1 & 22,300 & SNS07-152 & SNS & female & random & July 17, 2007 & R/V Funk & 23,000 \\
\hline June 8, 2007 & 791.1 & 21,900 & SNS07-152 & SNS & female & random & July 17, 2007 & R/V Funk & 23,000 \\
\hline June 10, 2007 & 791.1 & 21,900 & SNS07-152 & SNS & female & random & July 17, 2007 & R/V Funk & 23,000 \\
\hline May 27, 2007 & 791.0 & 17,700 & SNS07-152 & SNS & female & random & July 17, 2007 & R/V Funk & 23,000 \\
\hline May 17, 2007 & 782.5 & 20,100 & SNS07-122 & SNS & female & random & May 18, 2007 & R/V Funk & 18,900 \\
\hline May 20, 2006 & 775.1 & 20,400 & SNS06-059 & SNS & female & random & May 30, 2006 & R/V Funk & 21,700 \\
\hline May 29, 2007 & 775.1 & 17,700 & SNS07-139 & SNS & female & random & May 30, 2007 & R/V Funk & 17,000 \\
\hline May 29, 2007 & 775.1 & 17,700 & SNS07-127 & SNS & female & random & May 30, 2007 & R/V Funk & 17,000 \\
\hline May 29, 2007 & 775.1 & 17,700 & SNS07-164 & SNS & female & random & May 30, 2007 & R/V Funk & 17,000 \\
\hline May 25, 2006 & 774.2 & 25,800 & SNS06-061 & SNS & female & random & June 08, 2006 & R/V Funk & 23,700 \\
\hline June 14, 2005 & 759.7 & 27,300 & SNS05-066 & SNS & female & random & June 15,2005 & R/V Funk & 27,500 \\
\hline May 20, 2007 & 759.5 & 23,400 & PLS07-004 & PLS & female & random & May 21, 2007 & R/V Funk & 23,700 \\
\hline May 20, 2007 & 759.5 & 23,400 & PLS07-004 & PLS & female & random & May 21, 2007 & R/V Funk & 23,700 \\
\hline May 22, 2007 & 752.9 & 21,500 & SNS07-141 & SNS & male & random & May 23, 2007 & R/V Funk & 25,300 \\
\hline May 22, 2007 & 752.9 & 21,500 & SNS07-104 & SNS & female & random & May 23, 2007 & R/V Funk & 25,300 \\
\hline June 14, 2006 & 752.2 & 26,400 & SNS06-060 & SNS & female & random & June 22, 2006 & R/V Funk & 26,800 \\
\hline June 16, 2006 & 752.1 & 27,400 & SNS06-060 & SNS & female & random & June 22, 2006 & R/V Funk & 26,800 \\
\hline May 22, 2007 & 752.0 & 21,500 & SNS06-060 & SNS & female & random & May 23, 2007 & R/V Funk & 25,300 \\
\hline June 1, 2006 & 751.8 & 25,200 & PLS06-007 & PLS & male & random & June 22, 2006 & R/V Funk & 26,800 \\
\hline June 16, 2006 & 749.5 & 27,400 & SNS06-085 & SNS & female & random & June 20, 2006 & R/V Funk & 26,400 \\
\hline June 16, 2006 & 749.4 & 27,400 & SNS06-083 & SNS & female & random & June 20, 2006 & R/V Funk & 26,400 \\
\hline June 9, 2005 & 746.3 & 34,000 & SNS05-063 & SNS & female & random & June 10, 2005 & R/V Funk & 32,400 \\
\hline June 15, 2005 & 746.3 & 27,500 & SNS05-063 & SNS & female & random & July 11, 2005 & R/V Funk & 22,800 \\
\hline May 24, 2007 & 745.1 & 23,500 & SNS07-052 & SNS & male & random & May 25, 2007 & R/V Funk & 19,600 \\
\hline July 11, 2006 & 743.8 & 26,200 & SNS06-083 & SNS & female & random & July 12, 2006 & R/V Funk & 26,200 \\
\hline May 30, 2007 & 740.0 & 22,300 & SNS07-057 & SNS & female & random & May 31, 2007 & R/V Funk & 18,200 \\
\hline May 1, 2007 & 738.2 & 12,400 & SNS07-162 & SNS & female & random & May 02, 2007 & R/V Funk & 12,100 \\
\hline May 8, 2007 & 736.4 & 13,700 & SNS07-040 & SNS & female & random & May 09, 2007 & R/V Funk & 15,000 \\
\hline
\end{tabular}


Table 4. Sturgeon relocations and nets targeted for mapping.-Continued

$\left[\mathrm{ft}^{3} / \mathrm{s}\right.$, cubic feet per second; SNS, shovelnose sturgeon; PLS pallid sturgeon; --, no data]

\begin{tabular}{|c|c|c|c|c|c|c|c|c|c|}
\hline $\begin{array}{l}\text { Sturgeon } \\
\text { relocation } \\
\text { date }\end{array}$ & $\begin{array}{c}\text { River } \\
\text { mile } \\
\text { of } \\
\text { sturgeon } \\
\text { re- } \\
\text { location }\end{array}$ & $\begin{array}{c}\text { Estimated } \\
\text { discharge, } \\
\text { sturgeon } \\
\text { relocation } \\
\text { date }\left(\mathrm{ft}^{3} / \mathrm{s}\right)\end{array}$ & $\begin{array}{c}\text { Sturgeon } \\
\text { identification } \\
\text { code }\end{array}$ & Species & Sex & $\begin{array}{l}\text { Selection } \\
\text { method }\end{array}$ & $\begin{array}{l}\text { Map } \\
\text { date }\end{array}$ & Boat & $\begin{array}{c}\text { Estimated } \\
\text { discharge, } \\
\text { map } \\
\text { date } \\
\left(\mathrm{ft}^{3} / \mathrm{s}\right)\end{array}$ \\
\hline May 2, 2007 & 733.8 & 17,100 & SNS07-092 & SNS & female & random & May 03, 2007 & R/V Funk & 16,900 \\
\hline June 11, 2007 & 733.8 & 25,700 & SNS07-154 & SNS & female & random & June 12, 2007 & R/V Funk & 25,700 \\
\hline June 15, 2005 & 732.4 & 32,400 & SNS05-084 & SNS & female & random & June 16, 2005 & R/V Funk & 32,900 \\
\hline June 14, 2007 & 730.2 & 27,500 & SNS07-079 & SNS & female & random & June 15, 2007 & R/V Funk & 26,900 \\
\hline May 3, 2007 & 726.6 & 18,600 & SNS07-155 & SNS & female & random & May 04, 2007 & R/V Funk & 19,200 \\
\hline May 22, 2006 & 724.8 & 25,400 & SNS06-058 & SNS & female & random & May 23, 2006 & R/V Funk & 25,200 \\
\hline May 31,2007 & 724.5 & 24,200 & SNS07-059 & SNS & female & random & June 02, 2007 & R/V Funk & 26,500 \\
\hline June 8, 2007 & 717.8 & 27,600 & SNS07-072 & SNS & female & random & June 09, 2007 & R/V Funk & 27,300 \\
\hline July 7, 2005 & 716.7 & 26,100 & SNS05-084 & SNS & female & random & Aug. 09, 2005 & R/V Funk & 27,300 \\
\hline July 18, 2005 & 716.5 & 26,300 & SNS05-084 & SNS & female & random & Aug. 09, 2005 & R/V Funk & 27,300 \\
\hline May 31, 2006 & 709.8 & 26,400 & SNS06-051 & SNS & female & random & June 07, 2006 & R/V Funk & 26,800 \\
\hline May 22, 2006 & 709.7 & 25,400 & SNS06-051 & SNS & female & random & June 07, 2006 & R/V Funk & 26,800 \\
\hline June 12, 2007 & 707.2 & 26,700 & SNS07-175 & SNS & female & random & June 13, 2007 & R/V Funk & 26,900 \\
\hline May 14, 2007 & 706.2 & 33,900 & SNS07-057 & SNS & female & random & May 15, 2007 & R/V Funk & 35,200 \\
\hline June 6, 2007 & 702.1 & 27,800 & SNS07-055 & SNS & female & random & June 07, 2007 & R/V Funk & 27,600 \\
\hline July 7,2005 & 700.8 & 26,100 & SNS05-064 & SNS & female & random & Aug. 10, 2005 & R/V Funk & 27,500 \\
\hline July 19, 2005 & 700.8 & 26,100 & SNS05-064 & SNS & female & random & Aug. 10, 2005 & R/V Funk & 27,500 \\
\hline June 2, 2005 & 697.7 & 29,300 & SNS05-084 & SNS & female & random & June 03, 2005 & R/V Funk & 28,700 \\
\hline June 8, 2005 & 693.4 & 41,900 & SNS05-071 & SNS & female & random & June 09, 2005 & R/V Funk & 42,900 \\
\hline June 16, 2005 & 693.0 & 34,100 & SNS05-071 & SNS & female & random & June 17, 2005 & R/V Funk & 34,200 \\
\hline May 27, 2005 & 692.6 & 27,900 & SNS05-077 & SNS & female & random & July 12,2005 & R/V Funk & 26,200 \\
\hline May 18,2007 & 692.0 & 28,500 & SNS07-021 & SNS & female & random & May 19, 2007 & R/V Funk & 26,800 \\
\hline May 16, 2007 & 689.1 & 33,400 & SNS07-046 & SNS & female & random & May 17, 2007 & R/V Funk & 30,900 \\
\hline May 24, 2007 & 687.7 & 29,700 & SNS07-047 & SNS & female & random & May 26, 2007 & R/V Funk & 24,000 \\
\hline May 17, 2005 & 686.4 & 24,100 & SNS05-064 & SNS & female & random & May 18, 2005 & R/V Funk & 23,900 \\
\hline May 17,2005 & 686.4 & 24,100 & SNS05-062 & SNS & female & random & May 18, 2005 & R/V Funk & 23,900 \\
\hline June 23, 2005 & 683.9 & 32,500 & SNS05-057 & SNS & female & random & June 24, 2005 & R/V Funk & 31,900 \\
\hline June 23, 2005 & 683.4 & 32,500 & SNS05-063 & SNS & female & random & June 24, 2005 & R/V Funk & 31,900 \\
\hline May 9, 2005 & 674.2 & 25,700 & SNS05-064 & SNS & female & random & May 10, 2005 & R/V Funk & 26,400 \\
\hline May 9, 2005 & 673.3 & 25,700 & SNS05-062 & SNS & female & random & May 10, 2005 & R/V Funk & 26,400 \\
\hline June 1, 2005 & 672.5 & 28,800 & SNS05-058 & SNS & female & random & June 02, 2005 & R/V Funk & 29,300 \\
\hline June 1, 2005 & 671.8 & 28,800 & SNS05-052 & SNS & female & random & June 02, 2005 & R/V Funk & 29,300 \\
\hline July 6, 2005 & 670.1 & 26,300 & SNS05-063 & SNS & female & random & Aug. 11, 2005 & R/V Funk & 28,000 \\
\hline June 30, 2005 & 670.1 & 30,900 & SNS05-063 & SNS & female & random & Aug. 11, 2005 & R/V Funk & 28,000 \\
\hline May 31, 2006 & 669.4 & 26,600 & SNS06-067 & SNS & female & random & June 01, 2006 & R/V Funk & 26,600 \\
\hline May 31, 2006 & 669.1 & 28,900 & SNS06-072 & SNS & female & random & June 01, 2006 & R/V Funk & 26,600 \\
\hline Apr. 21, 2005 & 668.5 & 30,000 & Net & -- & -- & nonrandom & Apr. 22, 2005 & R/V Funk & 27,200 \\
\hline May 31, 2006 & 665.8 & 28,900 & SNS06-089 & SNS & female & random & June 06, 2006 & R/V Funk & 28,700 \\
\hline June 7, 2005 & 662.8 & 40,900 & SNS05-074 & SNS & female & random & June 08, 2005 & R/V Funk & 44,700 \\
\hline
\end{tabular}


Table 4. Sturgeon relocations and nets targeted for mapping.-Continued

[ft $\mathrm{ft}^{3} / \mathrm{s}$, cubic feet per second; SNS, shovelnose sturgeon; PLS pallid sturgeon; --, no data]

\begin{tabular}{|c|c|c|c|c|c|c|c|c|c|}
\hline $\begin{array}{l}\text { Sturgeon } \\
\text { relocation } \\
\text { date }\end{array}$ & $\begin{array}{c}\text { River } \\
\text { mile } \\
\text { of } \\
\text { sturgeon } \\
\text { re- } \\
\text { location }\end{array}$ & $\begin{array}{c}\text { Estimated } \\
\text { discharge, } \\
\text { sturgeon } \\
\text { relocation } \\
\text { date }\left(\mathrm{ft}^{3} / \mathrm{s}\right)\end{array}$ & $\begin{array}{c}\text { Sturgeon } \\
\text { identification } \\
\text { code }\end{array}$ & Species & Sex & $\begin{array}{l}\text { Selection } \\
\text { method }\end{array}$ & $\begin{array}{l}\text { Map } \\
\text { date }\end{array}$ & Boat & $\begin{array}{c}\text { Estimated } \\
\text { discharge, } \\
\text { map } \\
\text { date } \\
\left(\mathrm{ft}^{3} / \mathrm{s}\right)\end{array}$ \\
\hline May 11, 2007 & 660.7 & 36,300 & SNS07-033 & SNS & female & random & May 12, 2007 & R/V Funk & 36,500 \\
\hline May 18, 2005 & 657.9 & 31,400 & SNS05-071 & SNS & female & random & May 19, 2005 & R/V Funk & 30,900 \\
\hline May 18, 2005 & 657.3 & 31,400 & SNS05-097 & SNS & female & random & May 19, 2005 & R/V Funk & 30,900 \\
\hline May 6, 2005 & 654.6 & 26,500 & SNS05-058 & SNS & female & random & May 07, 2005 & R/V Funk & 26,500 \\
\hline May 24,2005 & 651.9 & 30,400 & SNS05-084 & SNS & female & random & May 25, 2005 & R/V Funk & 29,400 \\
\hline May 24,2005 & 651.8 & 30,400 & SNS05-061 & SNS & female & random & May 25, 2005 & R/V Funk & 29,400 \\
\hline May 18, 2005 & 651.8 & 31,400 & SNS05-061 & SNS & female & random & July 13, 2005 & R/V Funk & 28,400 \\
\hline June 22, 2005 & 650.5 & 33,300 & SNS05-094 & SNS & female & random & June 23, 2005 & R/V Funk & 33,800 \\
\hline June 7, 2007 & 650.4 & 31,600 & SNS07-025 & SNS & female & random & June 08, 2007 & R/V Funk & 31,200 \\
\hline May 10,2005 & 648.0 & 28,300 & SNS05-060 & SNS & female & random & May 11, 2005 & R/V Funk & 31,200 \\
\hline Apr. 30, 2007 & 647.8 & 29,900 & SNS07-045 & SNS & female & random & May 01, 2007 & R/V Funk & 28,000 \\
\hline May 10,2005 & 647.2 & 28,300 & SNS05-052 & SNS & female & random & May 11, 2005 & R/V Funk & 31,200 \\
\hline May 10, 2005 & 647.2 & 28,300 & SNS05-053 & SNS & female & random & May 11,2005 & R/V Funk & 31,200 \\
\hline June 13, 2005 & 644.8 & 38,000 & SNS05-054 & SNS & female & random & June 14, 2005 & R/V Funk & 36,200 \\
\hline July 20, 2005 & 643.4 & 28,200 & SNS05-099 & SNS & female & random & Aug. 12, 2005 & R/V Funk & 28,100 \\
\hline Aug. 2, 2005 & 643.4 & 28,400 & SNS05-099 & SNS & female & random & Aug. 12, 2005 & R/V Funk & 28,100 \\
\hline Aug. 9, 2005 & 643.4 & 27,100 & SNS05-099 & SNS & female & random & Aug. 12, 2005 & R/V Funk & 28,100 \\
\hline June 13, 2007 & 643.4 & 29,800 & SNS07-077 & SNS & female & random & June 14, 2007 & R/V Funk & 30,100 \\
\hline May 17, 2006 & 641.5 & 35,400 & SNS06-077 & SNS & female & random & May 18, 2006 & R/V Funk & 34,400 \\
\hline June 14, 2007 & 640.9 & 30,100 & SNS07-176 & SNS & female & random & June 16, 2007 & R/V Funk & 29,600 \\
\hline May 5, 2005 & 640.6 & 27,400 & SNS05-053 & SNS & female & random & May 05, 2005 & R/V Funk & 27,400 \\
\hline May 31, 2005 & 639.6 & 31,600 & SNS05-061 & SNS & female & random & June 01, 2005 & R/V Funk & 32,600 \\
\hline Apr. 19, 2005 & 638.4 & 26,900 & Net & -- & -- & nonrandom & Apr. 20, 2005 & R/V Funk & 27,400 \\
\hline May 31, 2005 & 638.2 & 31,600 & SNS05-101 & SNS & female & random & June 01, 2005 & R/V Funk & 32,600 \\
\hline May 19, 2005 & 628.2 & 31,600 & SNS05-076 & SNS & female & random & May 20, 2005 & R/V Funk & 35,000 \\
\hline May 19, 2005 & 627.8 & 31,600 & SNS05-098 & SNS & female & random & May 20, 2005 & R/V Funk & 35,000 \\
\hline May 19, 2005 & 627.8 & 31,600 & SNS05-098 & SNS & female & random & July 14, 2005 & R/V Funk & 28,600 \\
\hline May 25, 2005 & 627.7 & 29,900 & SNS05-100 & SNS & female & random & July 14, 2005 & R/V Funk & 28,600 \\
\hline May 19, 2005 & 627.6 & 31,600 & SNS05-100 & SNS & female & random & May 20, 2005 & R/V Funk & 35,000 \\
\hline May 19, 2005 & 626.7 & 31,600 & SNS05-095 & SNS & female & random & May 20, 2005 & R/V Funk & 35,000 \\
\hline July 21, 2005 & 626.3 & 28,400 & SNS05-098 & SNS & female & random & Sept. 01, 2005 & R/V Funk & 27,800 \\
\hline July 26, 2005 & 626.3 & 28,700 & SNS05-098 & SNS & female & random & Sept. 01, 2005 & R/V Funk & 27,800 \\
\hline June 20, 2005 & 622.7 & 35,500 & SNS05-076 & SNS & female & random & June 22, 2005 & R/V Funk & 33,800 \\
\hline May 11,2005 & 620.0 & 32,200 & SNS05-095 & SNS & female & random & May 12, 2005 & R/V Funk & 34,000 \\
\hline June 20, 2005 & 618.8 & 35,500 & SNS05-100 & SNS & female & random & June 21, 2005 & R/V Funk & 34,600 \\
\hline July 21, 2005 & 616.4 & 28,400 & SNS05-101 & SNS & female & random & Aug. 31, 2005 & R/V Funk & 27,800 \\
\hline July 26, 2005 & 616.4 & 28,700 & SNS05-101 & SNS & female & random & Aug. 31, 2005 & R/V Funk & 27,800 \\
\hline-- & 614.5 & -- & Net & -- & -- & nonrandom & Apr. 30, 2005 & R/V Funk & 25,200 \\
\hline-- & 611.6 & -- & Net & -- & -- & nonrandom & Apr. 28, 2005 & R/V Funk & 25,100 \\
\hline
\end{tabular}


Table 4. Sturgeon relocations and nets targeted for mapping.-Continued

$\left[\mathrm{ft}^{3} / \mathrm{s}\right.$, cubic feet per second; SNS, shovelnose sturgeon; PLS pallid sturgeon; --, no data]

\begin{tabular}{|c|c|c|c|c|c|c|c|c|c|}
\hline $\begin{array}{l}\text { Sturgeon } \\
\text { relocation } \\
\text { date }\end{array}$ & $\begin{array}{c}\text { River } \\
\text { mile } \\
\text { of } \\
\text { sturgeon } \\
\text { re- } \\
\text { location }\end{array}$ & $\begin{array}{l}\text { Estimated } \\
\text { discharge, } \\
\text { sturgeon } \\
\text { relocation } \\
\text { date }\left(\mathrm{ft}^{3} / \mathrm{s}\right)\end{array}$ & $\begin{array}{c}\text { Sturgeon } \\
\text { identification } \\
\text { code }\end{array}$ & Species & Sex & $\begin{array}{c}\text { Selection } \\
\text { method }\end{array}$ & $\begin{array}{l}\text { Map } \\
\text { date }\end{array}$ & Boat & $\begin{array}{c}\text { Estimated } \\
\text { discharge, } \\
\text { map } \\
\text { date } \\
\left(\mathrm{ft}^{3} / \mathrm{s}\right)\end{array}$ \\
\hline May 5, 2005 & 610.7 & 27,700 & SNS05-098 & SNS & female & random & May 06, 2005 & R/V Funk & 26,800 \\
\hline May 5, 2005 & 609.9 & 27,700 & SNS05-096 & SNS & female & random & May 06, 2005 & R/V Funk & 26,800 \\
\hline June 3, 2005 & 609.9 & 33,600 & SNS05-072 & SNS & female & random & June 05,2005 & R/V Funk & 33,100 \\
\hline June 3, 2005 & 609.7 & 33,600 & SNS05-068 & SNS & female & random & June 05,2005 & R/V Funk & 33,100 \\
\hline May 5, 2005 & 609.4 & 27,700 & SNS05-071 & SNS & female & random & May 06, 2005 & R/V Funk & 26,800 \\
\hline May 7, 2005 & 609.1 & 26,800 & SNS05-076 & SNS & female & random & May 08, 2005 & R/V Funk & 28,000 \\
\hline May 7, 2005 & 608.8 & 26,800 & SNS05-084 & SNS & female & random & May 08, 2005 & R/V Funk & 28,000 \\
\hline May 7, 2005 & 608.8 & 26,800 & SNS05-072 & SNS & female & random & May 08, 2005 & R/V Funk & 28,000 \\
\hline May 7, 2005 & 608.7 & 26,800 & SNS05-095 & SNS & female & random & May 08, 2005 & R/V Funk & 28,000 \\
\hline May 7, 2005 & 608.6 & 26,800 & SNS05-082 & SNS & female & random & May 08, 2005 & R/V Funk & 28,000 \\
\hline-- & 608.2 & -- & Net & -- & -- & nonrandom & Apr. 29, 2005 & R/V Funk & 25,100 \\
\hline May 7, 2005 & 608.1 & 26,800 & SNS05-077 & SNS & female & random & May 08, 2005 & R/V Funk & 28,000 \\
\hline May 7, 2005 & 607.6 & 26,800 & SNS05-101 & SNS & female & random & May 08, 2005 & R/V Funk & 28,000 \\
\hline-- & 606.4 & -- & Net & -- & -- & nonrandom & Apr. 27, 2005 & R/V Funk & 25,300 \\
\hline May 16, 2005 & 606.4 & 33,300 & SNS05-082 & SNS & female & random & May 17, 2005 & R/V Funk & 36,100 \\
\hline June 3,2005 & 604.6 & 33,600 & SNS05-082 & SNS & female & random & June 04, 2005 & R/V Funk & 32,900 \\
\hline May 16, 2005 & 604.0 & 33,300 & SNS05-089 & SNS & female & random & May 17, 2005 & R/V Funk & 36,100 \\
\hline June 3,2005 & 603.3 & 33,600 & SNS05-056 & SNS & female & random & June 04,2005 & R/V Funk & 32,900 \\
\hline May 8, 2005 & 602.9 & 28,000 & SNS05-073 & SNS & female & random & May 09, 2005 & R/V Funk & 28,500 \\
\hline May 8, 2005 & 601.9 & 28,000 & SNS05-069 & SNS & female & random & May 09, 2005 & R/V Funk & 28,500 \\
\hline May 20, 2005 & 601.2 & 35,000 & SNS05-087 & SNS & female & random & May 22, 2005 & R/V Funk & 31,100 \\
\hline June 10, 2005 & 593.4 & 56,700 & SNS05-073 & SNS & female & random & June 11, 2005 & R/V Funk & 57,300 \\
\hline May 20, 2005 & 588.6 & 43,700 & SNS05-085 & SNS & female & random & May 23, 2005 & R/V Funk & 39,400 \\
\hline July 12, 2006 & 583.8 & 30,500 & PLS06-006 & PLS & male & random & July 13, 2006 & R/V Funk & 30,200 \\
\hline May 20, 2005 & 565.3 & 43,700 & SNS05-070 & SNS & female & random & May 24, 2005 & R/V Funk & 39,600 \\
\hline June 24, 2005 & 554.1 & 51,600 & SNS05-056 & SNS & female & random & June 25, 2005 & R/V Funk & 46,300 \\
\hline May 3, 2005 & 362.1 & 39,300 & SNS05-040 & SNS & female & random & May 04, 2005 & R/V Brush & 39,200 \\
\hline May 24, 2005 & 354.6 & 52,900 & SNS05-022 & SNS & female & random & May 25, 2005 & R/V Brush & 52,900 \\
\hline June 19, 2006 & 331.6 & 35,600 & PLS06-003 & PLS & female & random & June 20, 2006 & R/V Brush & 35,600 \\
\hline Apr. 28, 2005 & 330.1 & 44,400 & SNS05-006 & SNS & female & random & Apr. 29, 2005 & R/V Brush & 43,300 \\
\hline May 23, 2006 & 326.6 & 38,700 & PLS06-004 & PLS & female & random & May 24, 2006 & R/V Brush & 38,600 \\
\hline May 10, 2005 & 324.9 & 36,600 & SNS05-041 & SNS & female & random & May 11,2005 & R/V Brush & 37,600 \\
\hline Apr. 26, 2005 & 315.3 & 47,500 & SNS05-037 & SNS & female & random & Apr. 27, 2005 & R/V Brush & 45,000 \\
\hline May 4, 2005 & 311.5 & 39,400 & SNS05-007 & SNS & female & random & May 05,2005 & R/V Brush & 39,200 \\
\hline May 25, 2005 & 291.7 & 53,000 & SNS05-003 & SNS & female & random & May 26, 2005 & R/V Brush & 53,000 \\
\hline July 5, 2006 & 289.4 & 38,000 & SNS06-038 & SNS & female & random & July 06, 2006 & R/V Brush & 36,200 \\
\hline May 3, 2006 & 280.4 & 67,700 & SNS06-044 & SNS & female & random & May 04, 2006 & R/V Brush & 65,100 \\
\hline May 31, 2006 & 279.8 & 38,600 & SNS06-044 & SNS & female & random & June 08, 2006 & R/V Brush & 38,200 \\
\hline June 7, 2006 & 279.8 & 39,600 & SNS06-044 & SNS & female & random & June 13, 2006 & R/V Brush & 36,700 \\
\hline
\end{tabular}


Table 4. Sturgeon relocations and nets targeted for mapping.-Continued

[ft $\mathrm{ft}^{3} / \mathrm{s}$, cubic feet per second; SNS, shovelnose sturgeon; PLS pallid sturgeon; --, no data]

\begin{tabular}{|c|c|c|c|c|c|c|c|c|c|}
\hline $\begin{array}{c}\text { Sturgeon } \\
\text { relocation } \\
\text { date }\end{array}$ & $\begin{array}{c}\text { River } \\
\text { mile } \\
\text { of } \\
\text { sturgeon } \\
\text { re- } \\
\text { location }\end{array}$ & $\begin{array}{l}\text { Estimated } \\
\text { discharge, } \\
\text { sturgeon } \\
\text { relocation } \\
\text { date }\left(\mathrm{ft}^{3} / \mathrm{s}\right)\end{array}$ & $\begin{array}{c}\text { Sturgeon } \\
\text { identification } \\
\text { code }\end{array}$ & Species & Sex & $\begin{array}{l}\text { Selection } \\
\text { method }\end{array}$ & $\begin{array}{l}\text { Map } \\
\text { date }\end{array}$ & Boat & $\begin{array}{c}\text { Estimated } \\
\text { discharge, } \\
\text { map } \\
\text { date } \\
\left(\mathrm{ft}^{3} / \mathrm{s}\right)\end{array}$ \\
\hline June 9, 2005 & 275.6 & 88,700 & SNS05-015 & SNS & female & random & June 10, 2005 & R/V Brush & 84,400 \\
\hline June 1, 2006 & 248.1 & 45,400 & SNS06-034 & SNS & female & random & June 02, 2006 & R/V Brush & 45,700 \\
\hline May 11, 2005 & 247.4 & 39,200 & SNS05-016 & SNS & female & random & May 12, 2005 & R/V Brush & 44,400 \\
\hline May 5, 2005 & 230.6 & 43,100 & SNS05-019 & SNS & female & random & May 06, 2005 & R/V Brush & 42,700 \\
\hline Apr. 14, 2005 & 219.2 & 72,100 & PLS04-001 & PLS & unknown & random & Apr. 15, 2005 & R/V Brush & 69,500 \\
\hline May 18, 2006 & 219.2 & 40,200 & SNS06-028 & SNS & female & random & May 19, 2006 & R/V Brush & 39,700 \\
\hline June 24,2005 & 216.7 & 70,700 & SNS05-089 & SNS & female & random & June 27, 2005 & R/V Brush & 68,900 \\
\hline June 24, 2005 & 216.5 & 70,700 & SNS05-028 & SNS & female & random & June 27, 2005 & R/V Brush & 68,900 \\
\hline July 1,2005 & 208.2 & 66,000 & SNS05-011 & SNS & female & random & July 05, 2005 & R/V Brush & 59,300 \\
\hline July 1,2005 & 208.1 & 66,000 & SNS05-089 & SNS & female & random & July 05, 2005 & R/V Brush & 59,300 \\
\hline June 1, 2006 & 206.5 & 46,500 & SNS06-011 & SNS & female & random & June 07, 2006 & R/V Brush & 38,000 \\
\hline May 22, 2006 & 206.4 & 43,300 & SNS06-011 & SNS & female & random & June 07, 2006 & R/V Brush & 38,000 \\
\hline May 25, 2006 & 206.4 & 39,700 & SNS06-011 & SNS & female & random & June 07, 2006 & R/V Brush & 38,000 \\
\hline Apr. 27, 2005 & 202.8 & 57,900 & SNS05-017 & SNS & female & random & Apr. 28, 2005 & R/V Brush & 53,300 \\
\hline May 20, 2006 & 202.8 & 39,800 & SNS06-041 & SNS & female & random & June 06, 2006 & R/V Brush & 40,100 \\
\hline Apr. 6, 2005 & 201.3 & 38,100 & SNS05-034 & SNS & female & random & Apr. 07, 2005 & R/V Brush & 38,700 \\
\hline Apr. 6, 2005 & 201.3 & 38,100 & SNS05-034 & SNS & female & random & Apr. 07, 2005 & R/V Funk & 38,700 \\
\hline Apr. 7, 2005 & 199.6 & 38,700 & PLS04-002 & PLS & unknown & random & Apr. 08, 2005 & R/V Funk & 40,000 \\
\hline May 17, 2005 & 197.7 & 124,000 & SNS05-012 & SNS & female & random & May 18, 2005 & R/V Brush & 106,000 \\
\hline May 6, 2005 & 197.0 & 43,600 & SNS05-009 & SNS & female & random & July 13, 2005 & R/V Brush & 43,000 \\
\hline July 6, 2006 & 195.9 & 38,500 & SNS06-011 & SNS & female & random & July 07, 2006 & R/V Brush & 38,000 \\
\hline May 26, 2005 & 186.5 & 61,300 & SNS05-026 & SNS & female & random & May 27, 2005 & R/V Brush & 59,500 \\
\hline Apr. 11, 2005 & 177.9 & 44,100 & SNS05-027 & SNS & female & random & Apr. 12, 2005 & R/V Brush & 48,500 \\
\hline May 9, 2006 & 177.2 & 55,700 & PLS06-001 & PLS & male & random & May 10, 2006 & R/V Brush & 54,000 \\
\hline Apr. 21, 2005 & 173.6 & 48,200 & SNS05-027 & SNS & female & random & Apr. 22, 2005 & R/V Brush & 53,800 \\
\hline May 27, 2005 & 173.5 & 59,500 & SNS05-031 & SNS & female & random & June 01, 2005 & R/V Brush & 53,600 \\
\hline June 30,2005 & 168.0 & 62,100 & PLS04-001 & PLS & unknown & random & July 01, 2005 & R/V Brush & 65,400 \\
\hline May 18, 2005 & 166.8 & 106,000 & SNS05-043 & SNS & female & random & May 19, 2005 & R/V Brush & 90,900 \\
\hline June 20, 2006 & 165.5 & 37,700 & SNS06-010 & SNS & female & random & June 21, 2006 & R/V Brush & 37,600 \\
\hline May 6, 2005 & 160.7 & 43,600 & SNS05-043 & SNS & female & random & May 10, 2005 & R/V Brush & 40,500 \\
\hline June 2, 2005 & 146.6 & 54,000 & SNS05-031 & SNS & female & random & June 03, 2005 & R/V Brush & 59,600 \\
\hline Aug. 3, 2005 & 146.4 & 37,600 & SNS05-031 & SNS & female & random & Aug. 10, 2005 & R/V Brush & 35,000 \\
\hline May 19, 2005 & 142.3 & 90,900 & SNS05-024 & SNS & female & random & May 23, 2005 & R/V Brush & 71,000 \\
\hline July 5, 2005 & 142.3 & 61,400 & SNS05-024 & SNS & female & random & Aug. 09, 2005 & R/V Brush & 35,100 \\
\hline Aug. 3, 2005 & 142.1 & 37,600 & SNS05-021 & SNS & female & random & Aug. 09, 2005 & R/V Brush & 35,100 \\
\hline May 27, 2005 & 140.9 & 59,500 & PLS05-003 & PLS & female & random & June 02, 2005 & R/V Brush & 54,000 \\
\hline Apr. 13, 2005 & 136.4 & 49,700 & SNS05-011 & SNS & female & random & Apr. 14, 2005 & R/V Funk & 69,300 \\
\hline Apr. 18, 2005 & 130.8 & 58,300 & PLS05-001 & PLS & unknown & random & Apr. 19, 2005 & R/V Brush & 53,600 \\
\hline May 27, 2005 & 128.7 & 70,400 & PLS05-001 & PLS & unknown & random & May 31, 2005 & R/V Brush & 53,600 \\
\hline
\end{tabular}


Table 4. Sturgeon relocations and nets targeted for mapping.-Continued

[ft $3 / \mathrm{s}$, cubic feet per second; SNS, shovelnose sturgeon; PLS pallid sturgeon; --, no data]

\begin{tabular}{|c|c|c|c|c|c|c|c|c|c|}
\hline $\begin{array}{l}\text { Sturgeon } \\
\text { relocation } \\
\text { date }\end{array}$ & $\begin{array}{c}\text { River } \\
\text { mile } \\
\text { of } \\
\text { sturgeon } \\
\text { re- } \\
\text { location }\end{array}$ & $\begin{array}{l}\text { Estimated } \\
\text { discharge, } \\
\text { sturgeon } \\
\text { relocation } \\
\text { date }\left(\mathrm{ft}^{3} / \mathrm{s}\right)\end{array}$ & $\begin{array}{c}\text { Sturgeon } \\
\text { identification } \\
\text { code }\end{array}$ & Species & Sex & $\begin{array}{l}\text { Selection } \\
\text { method }\end{array}$ & $\begin{array}{l}\text { Map } \\
\text { date }\end{array}$ & Boat & $\begin{array}{c}\text { Estimated } \\
\text { discharge, } \\
\text { map } \\
\text { date } \\
\left(\mathrm{ft}^{3} / \mathrm{s}\right)\end{array}$ \\
\hline May 8, 2006 & 127.5 & 74,400 & SNS06-012 & SNS & female & random & May 09, 2006 & R/V Brush & 69,700 \\
\hline Apr. 25, 2005 & 127.1 & 92,700 & PLS05-002 & PLS & unknown & random & Apr. 26, 2005 & R/V Brush & 86,500 \\
\hline Apr. 4, 2005 & 120.5 & 45,100 & PLS04-003 & PLS & male & random & Apr. 5, 2005 & R/V Brush & 43,500 \\
\hline June 29, 2006 & 119.6 & 43,300 & PLS06-001 & PLS & male & random & June 30, 2006 & R/V Brush & 41,700 \\
\hline May 2, 2005 & 118.4 & 55,500 & PLS04-003 & PLS & unknown & random & May 03, 2005 & R/V Brush & 52,700 \\
\hline July 5, 2005 & 117.8 & 71,800 & PLS04-003 & PLS & unknown & random & July 06, 2005 & R/V Brush & 66,800 \\
\hline June 21, 2005 & 109.1 & 121,000 & PLS05-001 & PLS & unknown & random & Aug. 31, 2005 & R/V Brush & 90,600 \\
\hline July 5, 2005 & 109.1 & 71,800 & PLS05-001 & PLS & unknown & random & Aug. 31, 2005 & R/V Brush & 90,600 \\
\hline Aug. 22, 2005 & 109.0 & 58,000 & PLS05-001 & PLS & unknown & random & Aug. 31, 2005 & R/V Brush & 90,600 \\
\hline July 28,2005 & 108.9 & 46,400 & PLS05-001 & PLS & unknown & random & Aug. 31, 2005 & R/V Brush & 90,600 \\
\hline July 6, 2005 & 75.7 & 67,400 & PLS05-004 & PLS & unknown & random & July 07, 2005 & R/V Brush & 70,600 \\
\hline July 7, 2005 & 27.3 & 68,500 & SNS05-017 & SNS & unknown & random & July 08, 2005 & R/V Brush & 71,800 \\
\hline
\end{tabular}


submeter scale, utilizing differential global positioning system (DGPS) data for most survey dates and real-time kinematic global positioning system for a few surveys. Data processing included removal of spurious data points, digitization of data extent, and interpolation of data to grids with 5-meter cell resolution.

The maps depict depth, velocity, and substrate conditions dependent on the discharge and channel morphology at the time of mapping. Collectively, the maps represent more than 20 percent of the length of the Lower Missouri River. This extensive set of maps is intended to provide better understanding of physical aquatic habitat use by sturgeon in the Lower Missouri River.

\section{References Cited}

Birstein, V.J., Bemin, W.E., and Waldman, J.R., 1997, The threatened status of acipenseriform species-A summary: Environmental Biology of Fishes, v. 48, no. 1-4.

Brown, C.J., Mitchell, A., Limpenny, D.S., Robertson, M.R., Service, M., and Golding, N., 2005, Mapping seabed habitats in the Firth of Lorn off the west coast of ScotlandEvaluation and comparison of habitat maps produced using the acoustic ground-discrimination system, RoxAnn, and sidescan sonar: Journal of Marine Science, v. 62, p. 790-802.

Cholwek, Gary, Bonde, J., Li, X., Richards, C., and Yin, K., 2000, Processing RoxAnn sonar data to improve its categorization of lake bed surficial substrates: Marine Geophysical Researches, v. 21, p. 409-421.

DeLonay, A.J., Papoulias, D.M., Wildhaber, M.L., Mestl, G.E., Everitt, D.W., and Chojnacki, K.A., 2007, Movement, habitat use, and reproductive behavior of shovelnose sturgeon and pallid sturgeon in the Lower Missouri River, in Korschgen, C.E., ed., Factors affecting the reproduction, recruitment, habitat, and population dynamics of pallid sturgeon and shovelnose sturgeon in the Missouri River: U.S. Geological Survey Open-File Report 2007-1262, p. 23-102.

Elliott, C.M., and Jacobson, R.B., 2006, Geomorphic classification and assessment of channel dynamics in the Missouri National Recreational River, South Dakota and Nebraska: U.S. Geological Survey Scientific Investigations Report 2006-5313, 66 p.

Elliott, C.M., Jacobson, R.B., and DeLonay, A.J., 2004, Physical aquatic habitat assessment, Fort Randall segment of the Missouri River, Nebraska and South Dakota: U.S. Geological Survey Open-File Report 2004-1060, 34 p.
Gaeuman, David, and Jacobson, R.B., 2005, Aquatic habitat mapping with an acoustic Doppler current profilerConsiderations for data quality: U.S. Geological Survey Open-File Report 2005-1163, 20 p.

Galat, D.L., Berry, C.R., Jr., Peters, E.J., and White, R.G., 2005, Missouri River basin, in Benke, A.C., and Cushing, C.E., eds., Rivers of North America: Oxford, Elsevier Academic Press, p. 427-480.

Galat, D.L., and Lipkin, R., 2000, Restoring ecological integrity of great rivers-Historical hydrographs aid in defining reference conditions for the Missouri River: Hydrobiologia, v. 422/423, p. 29-48.

Hamilton, L.J., 2001, Acoustic seabed classification systems: Defence Science \& Technology Organisation [Australia] DTSO-TN-0401, 66 p.

Jacobson, R.B., and Galat, D.L., 2006, Flow and form in rehabilitation of large-river ecosystems-An example from the Lower Missouri River: Geomorphology, v. 77, p. 249-269.

Jacobson, R.B., and Heuser, J., 2002, Visualization of flow alternatives, Lower Missouri River: U.S. Geological Survey Open-File Report 2002-122.

Jacobson, R.B., Johnson, H.E., Reuter, J.M., and Elliott, C.M., 2007, The roles of physical habitat in reproduction and survival of pallid sturgeon and shovelnose sturgeon in the Lower Missouri River, progress 2005-06, in Korschgen, C.E., ed., Factors affecting the reproduction, recruitment, habitat, and population dynamics of pallid sturgeon and shovelnose sturgeon in the Missouri River: U.S. Geological Survey Open-File Report 2007-1262, p. 143-212.

Knighton, David, 1998, Fluvial forms and processes-A new perspective: New York, Oxford University Press Inc., 383 p.

Laustrup, M.S., Jacobson, R.B., and Simpkins, D.G., 2007, Distribution of potential spawning habitat for sturgeon in the Lower Missouri River, 2003-06: U.S. Geological Survey Open-File Report 2007-1192, 26 p.

Marine Sonic Technology Ltd., 2001, Sea Scan PC Operator's Manual-Version 1.6: Gloucester, Va., 198 p.

Millspaugh, J.J., and Marzluff, J.M., eds., 2001, Radio tracking and animal populations: San Diego, Calif., Academic Press, 474 p.

National Geophysical Data Center, 2008, Magnetic declination (variation): last accessed on June 9, 2008, at http://www. ngdc.noaa.gov/geomag/declination.shtml

National Research Council, 2002, The Missouri River ecosystem-Exploring the prospects for recovery: Washington, D.C., National Academy Press, 176 p. 
Papoulias, D.M., Annis, M.L., DeLonay, A.J., and Tillitt, D.E., 2007, Reproductive physiology of Missouri River gravid pallid sturgeon and shovelnose sturgeon during the 2005 and 2006 spawning seasons, in Korschgen, C.E., ed., Factors affecting the reproduction, recruitment, habitat, and population dynamics of pallid sturgeon and shovelnose sturgeon in the Missouri River: U.S. Geological Survey Open-File Report 2007-1262, p. 107-136.

Pegg, M.A., Pierce, C.L., and Roy, A., 2003, Hydrological alteration along the Missouri River Basin-A time series approach: Aquatic Sciences, v. 65, p. 63-72.

Quist, M.C., Boelter, A.M., Lovato, J.M., Korfanta, N.M., Bergman, H.L., Latka, D.C., Korschgen, C., Galat, D.L., Krentz, S., Oetker, M., Olson, M., Scott, C.M., and Berkley, J., 2004, Research and assessment needs for pallid sturgeon recovery in the Missouri River-Final report to the U.S. Geological Survey, U.S. Army Corps of Engineers, U.S. Fish and Wildlife Service, and U.S. Environmental Protection Agency: Laramie, Wyo., William D. Ruckelshaus Institute of Environment and Natural Resources, University of Wyoming, $82 \mathrm{p}$.

RD Instruments, 2003, WinRiver user's guide-USGS Version, 144 p., accessed October 8, 2008, at http:// hydroacoustics.usgs.gov/downloads/RDI/WinRiver-UserGuide-USGS-Oct03.pdf

Simpkins, D.G., and LaBay, S.R., 2007, Site-specific assessment of spawning behavior and habitat use, in Korschgen, C.E., ed., Factors affecting the reproduction, recruitment, habitat, and population dynamics of pallid sturgeon and shovelnose sturgeon in the Missouri River: U.S. Geological Survey Open-File Report 2007-1262, p. 217-254.
U.S. Fish and Wildlife Service, 2000, Biological opinion on the operation of the Missouri River main stem reservoir system, operation and maintenance of the Missouri River Bank Stabilization and Navigation Project, and operation of the Kansas River Reservoir System: U.S. Fish and Wildlife Service., 296 p., accessed October 8, 2008, at http://www. nwd-mr.usace.army.mil/mmanual/opinion.html

U.S. Fish and Wildlife Service, 2003, Amendment to the 2000 biological opinion on the operation of the Missouri River main stem reservoir system, operation and maintenance of the Missouri River Bank Stabilization and Navigation Project, and operation of the Kansas River Reservoir System: U.S. Fish and Wildlife Service, 308 p., accessed October 8, 2008, at http://www.nwd-mr.usace.army.mil/ mmanual/FinalBO2003.pdf

U.S. Geological Survey, 2007, Sturgeon research updateConfirmed pallid sturgeon spawning in the Missouri River in 2007: U.S. Geological Survey Fact Sheet 2007-3053, 4 p.

U.S. Geological Survey, 2008, National Water Information System-Web interface: accessed June 9, 2008, at http:// waterdata.usgs.gov/nwis 


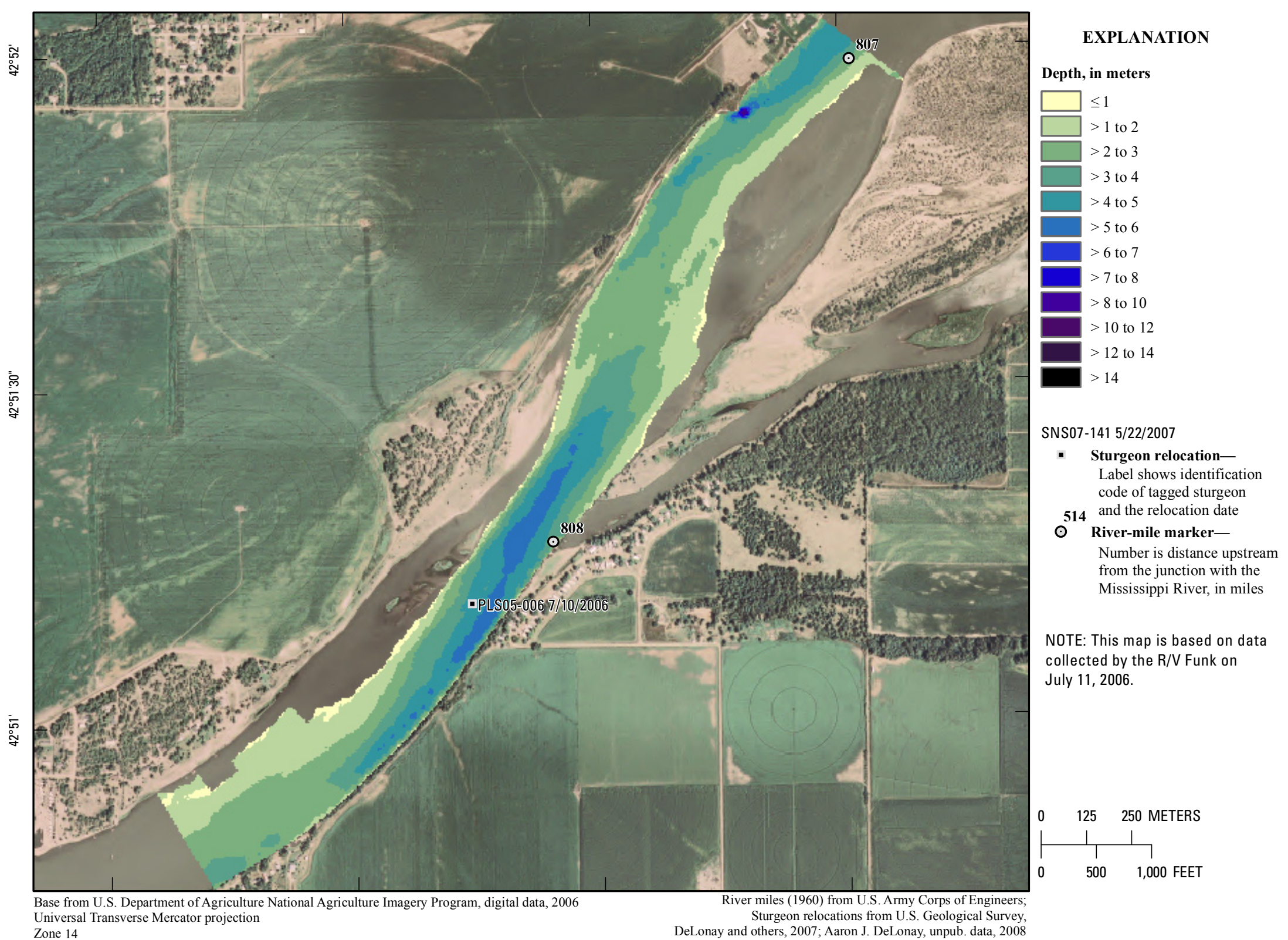
Universal Transverse Mercator projection Dturgeon relocations from U.S. Geonogical Survey,
DeLonay and others, 2007; Aaron J. DeLonay, unpub. data, 2008 


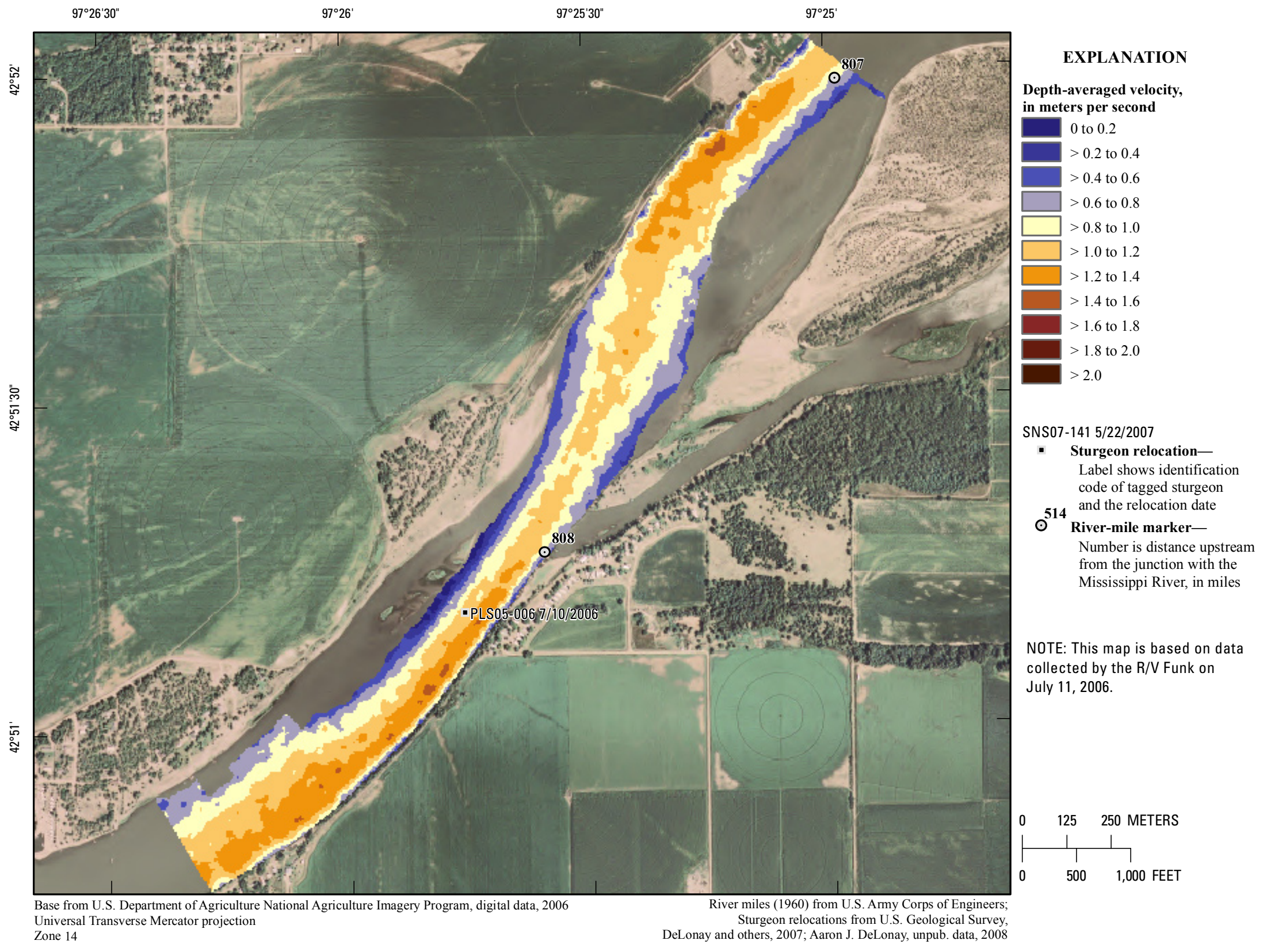

Figure 9. Map of depth-averaged velocity based on data collected on July 11, 2006, in the vicinity of river mile 808 . 


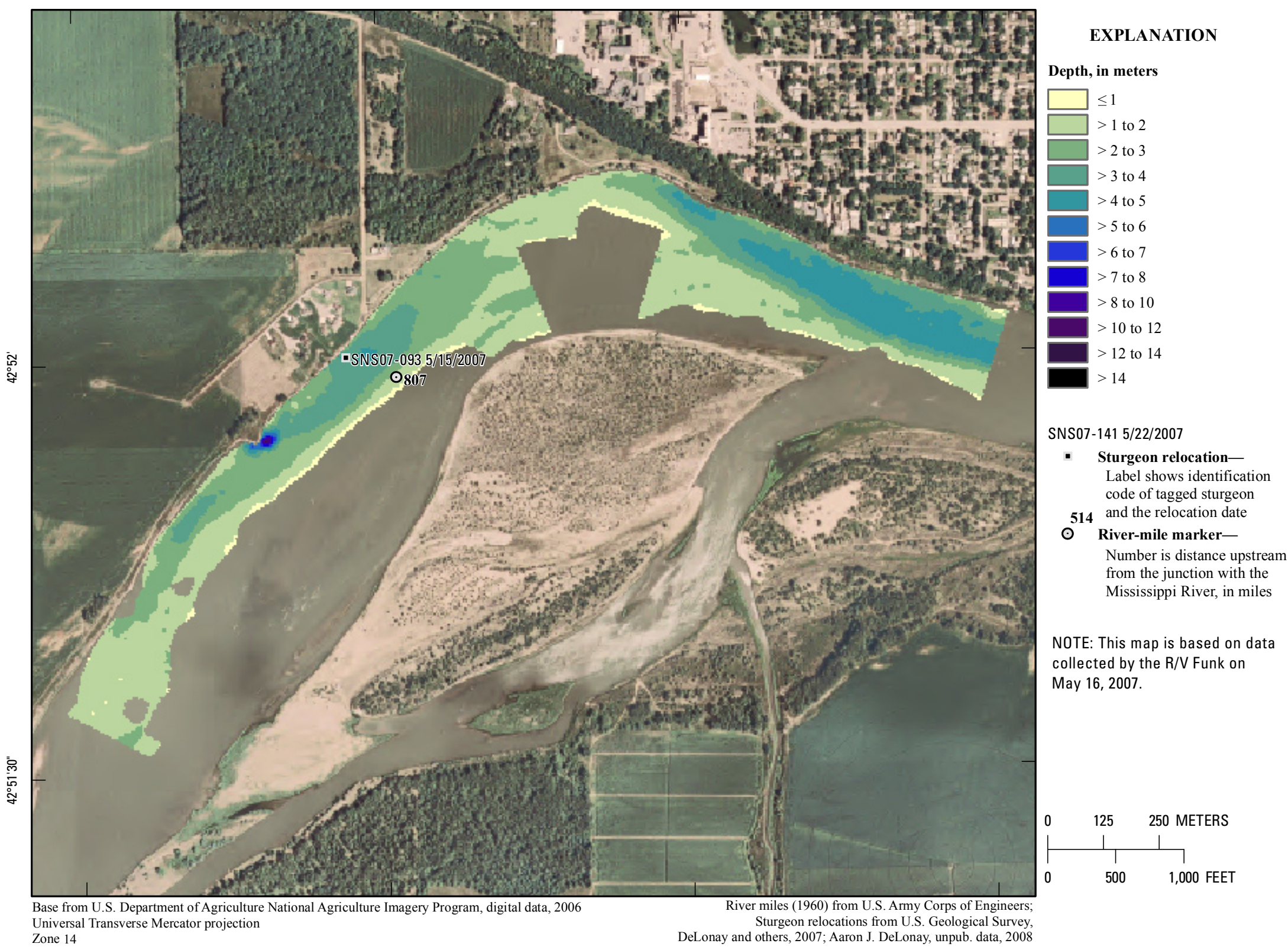




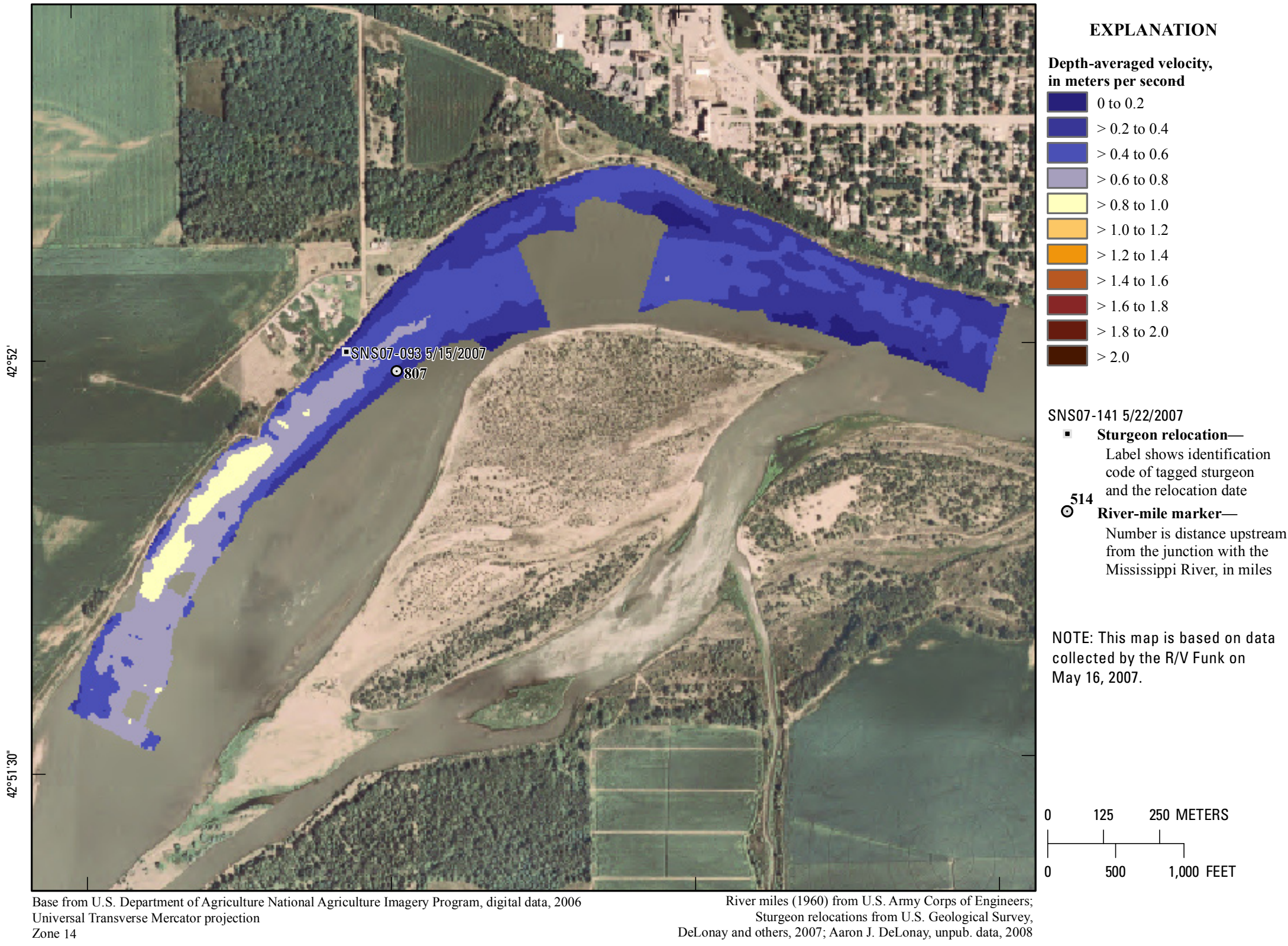

Figure 11. Map of depth-averaged velocity based on data collected on May 16, 2007, in the vicinity of river mile 807. 


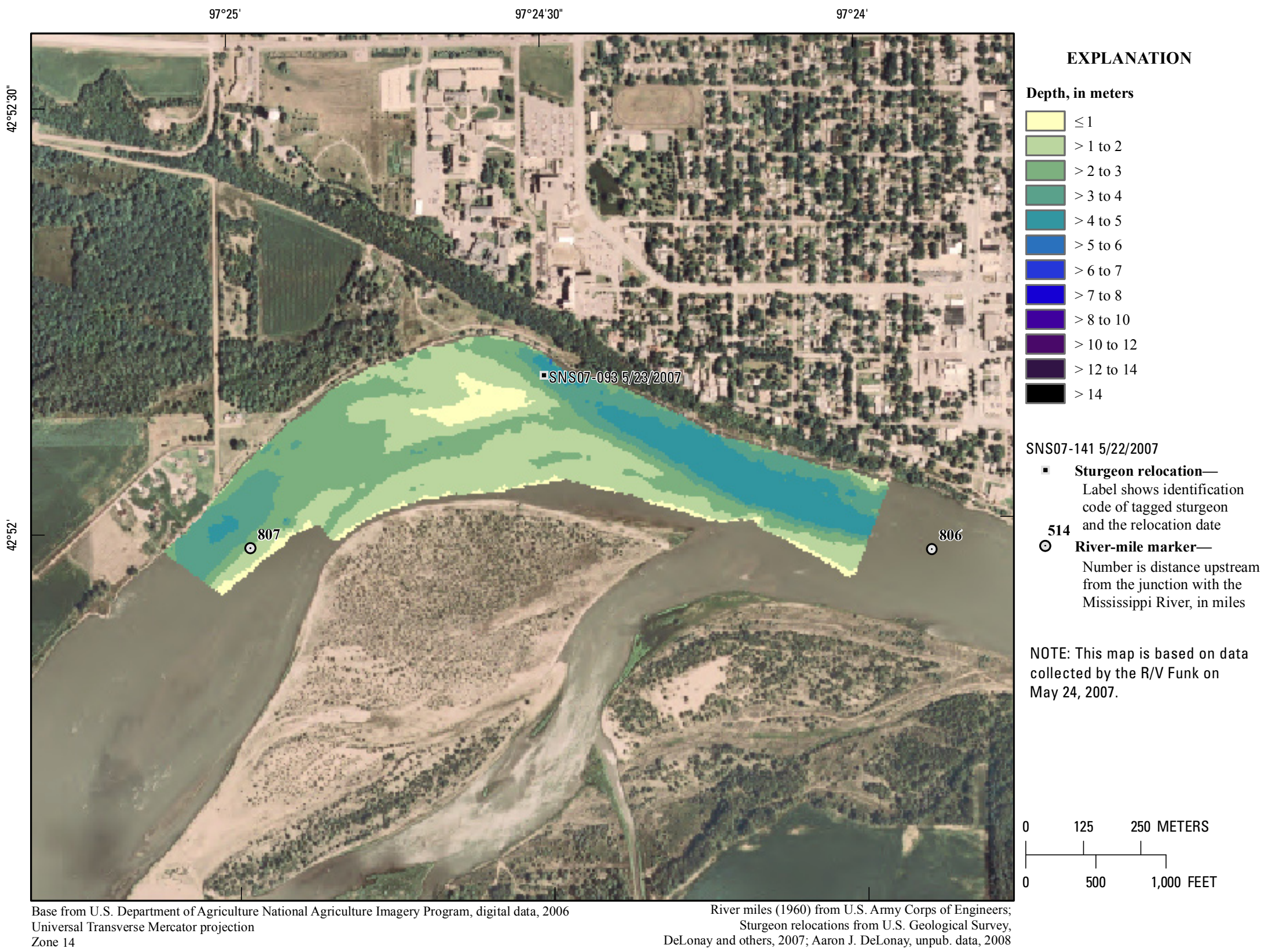

Figure 12. Map of depth based on data collected on May 24, 2007, in the vicinity of river mile 807 . 


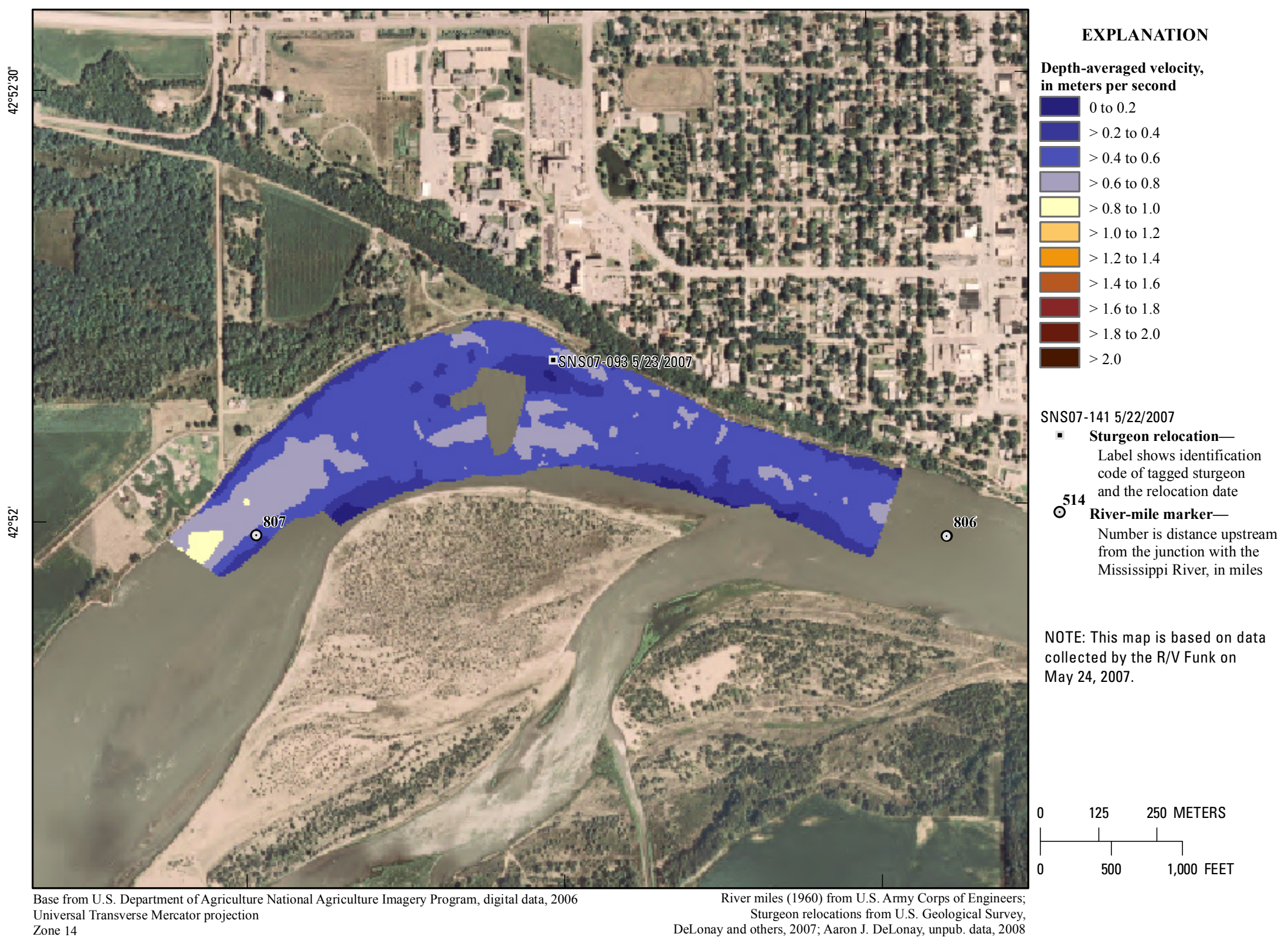

Figure 13. Map of depth-averaged velocity based on data collected on May 24, 2007, in the vicinity of river mile 807 . 


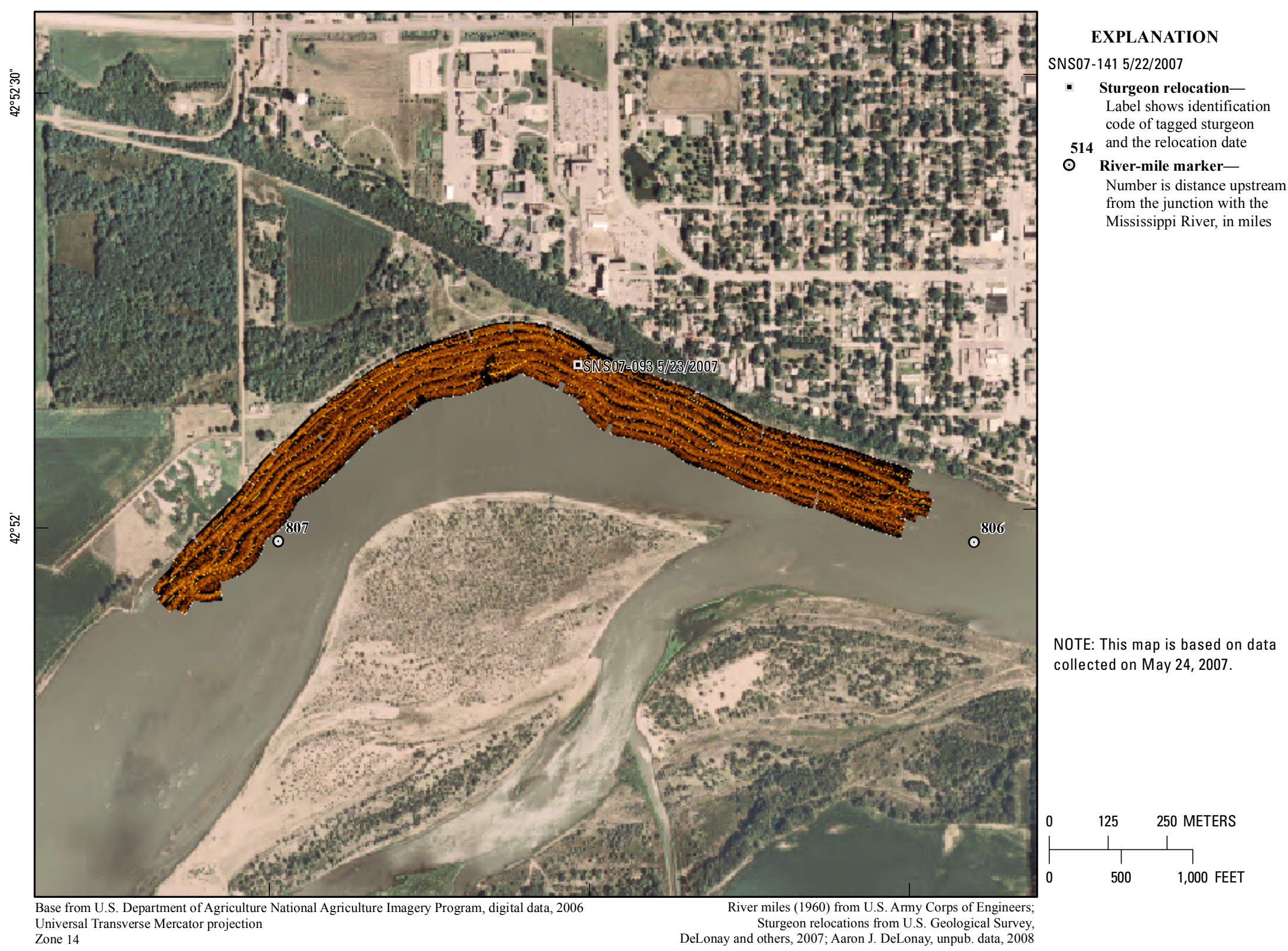




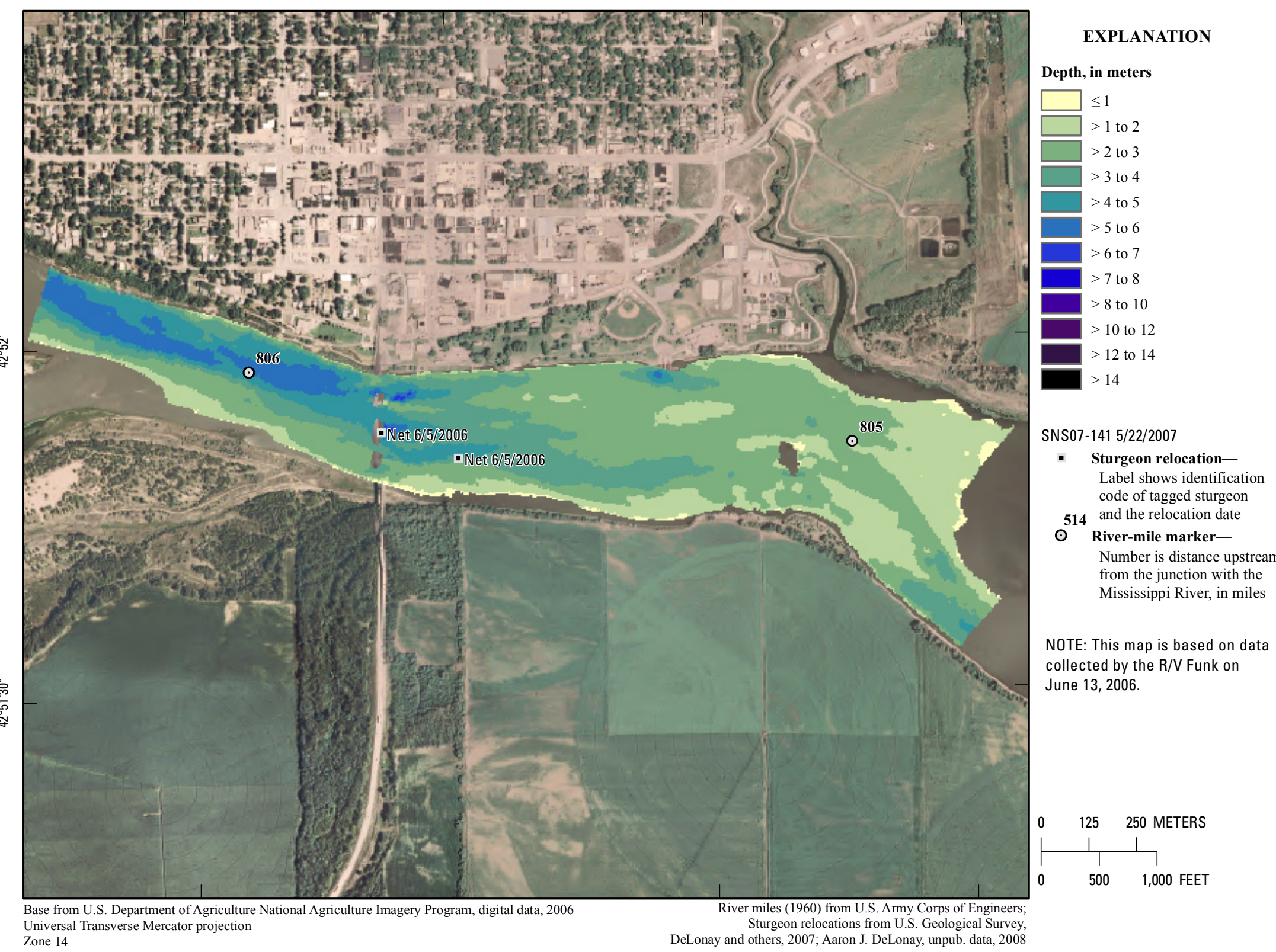

Figure 15. Map of depth based on data collected on June 13, 2006, in the vicinity of river mile 805 . The target location for this survey was a drifted trammel net. The two points indicate starting (upstream) and stopping (downstream) positions. 
$97^{\circ} 24$

$97^{\circ} 23^{\prime} 30^{\prime \prime}$

$97^{\circ} 23^{\prime}$

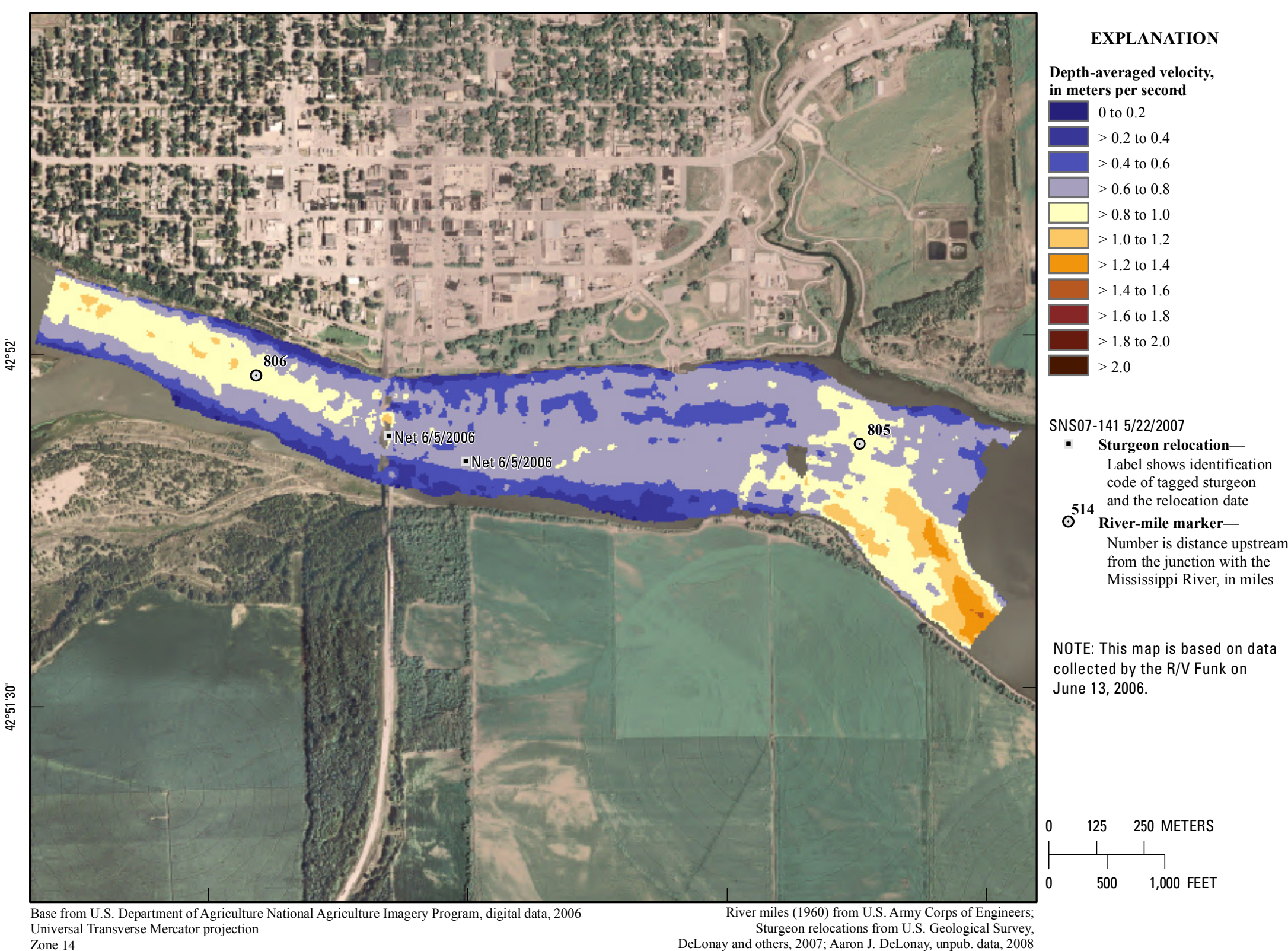

DeLonay and others, 2007; Aaron J. DeLonay, unpub. data, 2008

Figure 16. Map of depth-averaged velocity based on data collected on June 13,2006 , in the vicinity of river mile 805 . The target location for this survey was a drifted trammel net. The two points indicate starting (upstream) and stopping (downstream) positions. 


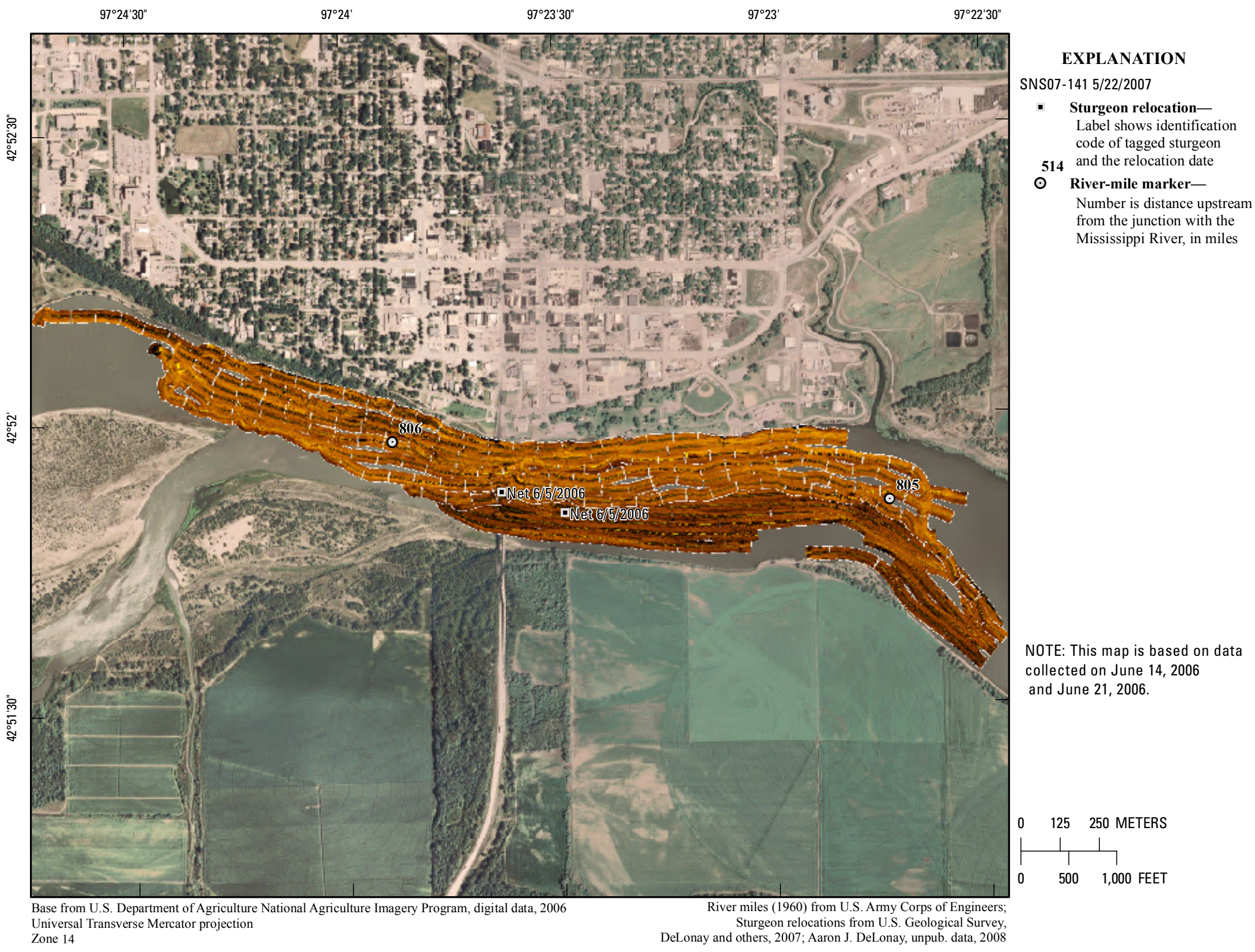

Figure 17. Map of side-scan sonar imagery based on data collected on June 14, 2006, and June 21,2006, in the vicinity of river mile 805 . 


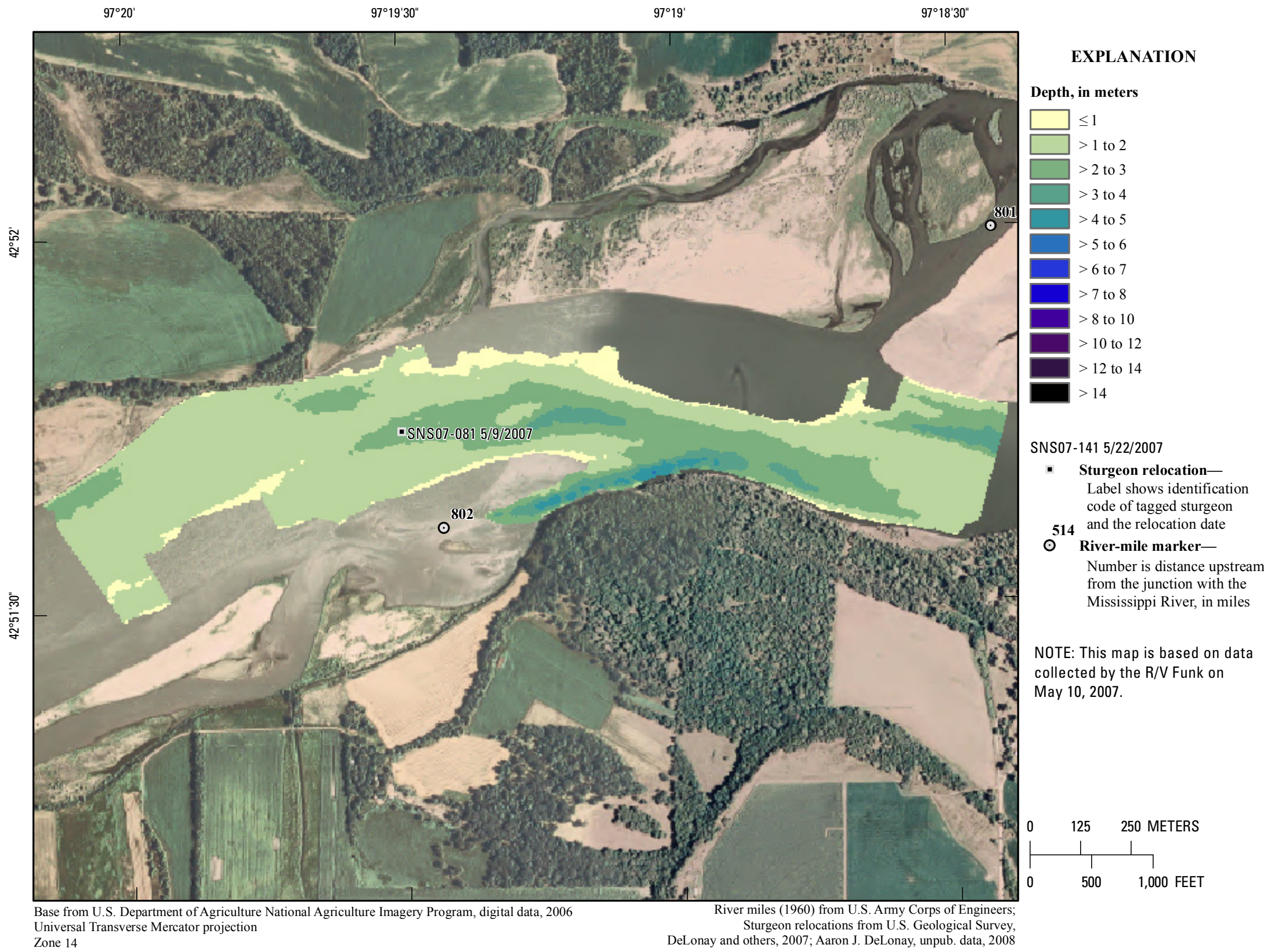

Figure 18. Map of depth based on data collected on May 10, 2007, in the vicinity of river mile 802 . 


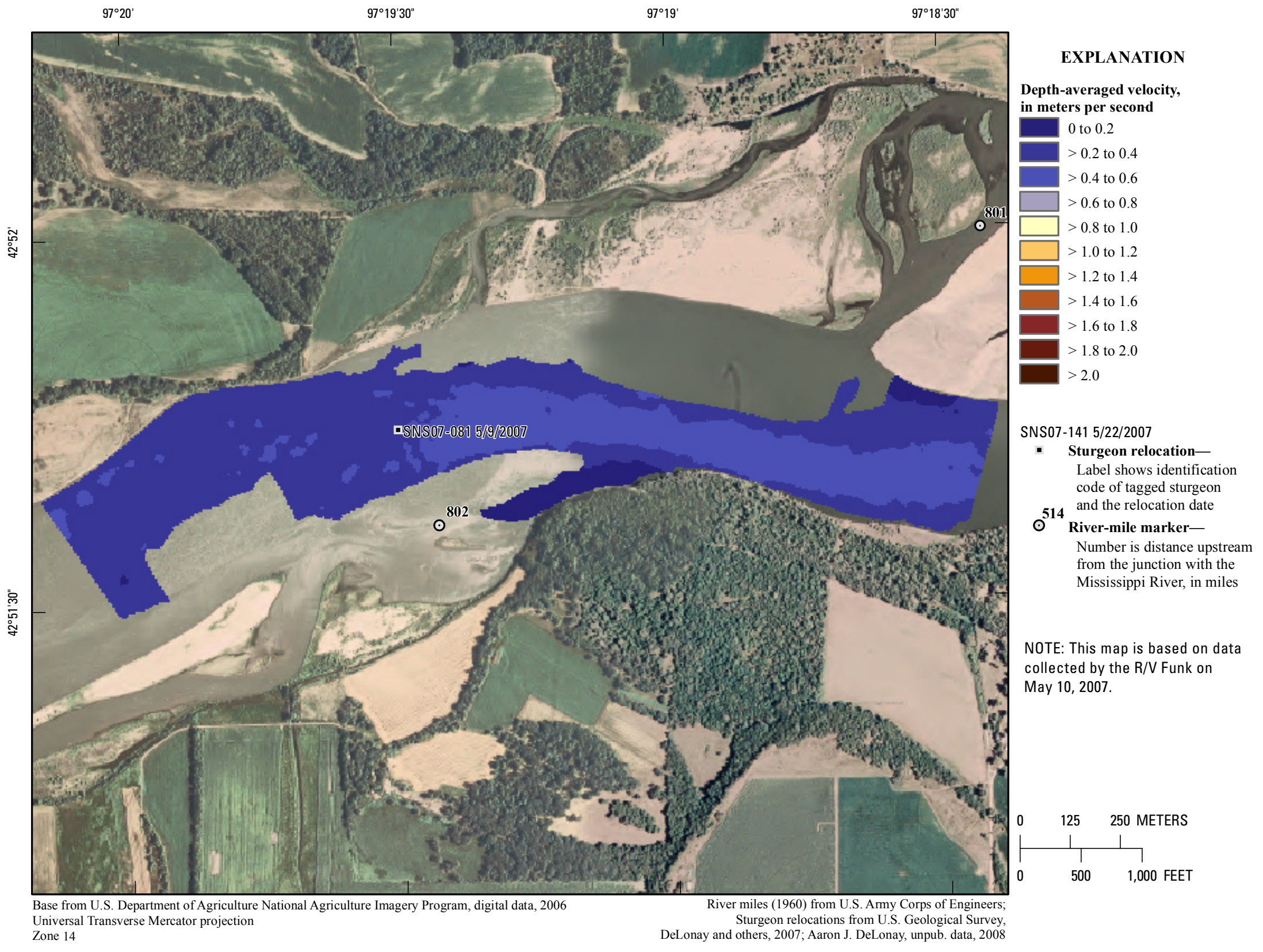

Figure 19. Map of depth-averaged velocity based on data collected on May 10, 2007, in the vicinity of river mile 802 . 
$97^{\circ} 18^{\prime}$

$97^{\circ} 17^{\prime} 30^{\prime \prime}$

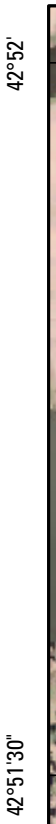

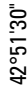

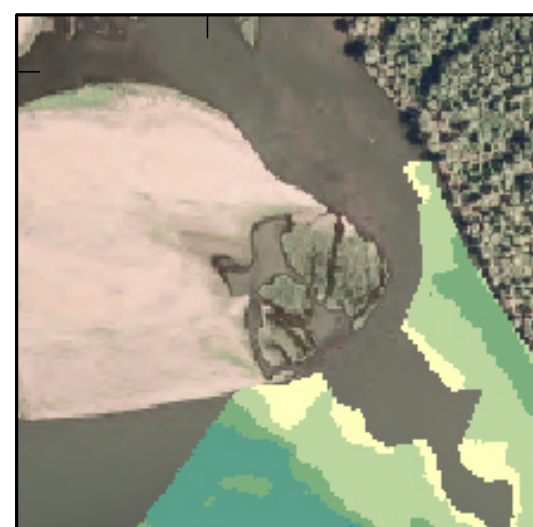

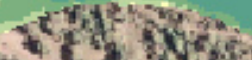
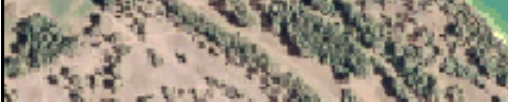

a

$\stackrel{⿱ 亠 乂}{\check{\alpha}}$

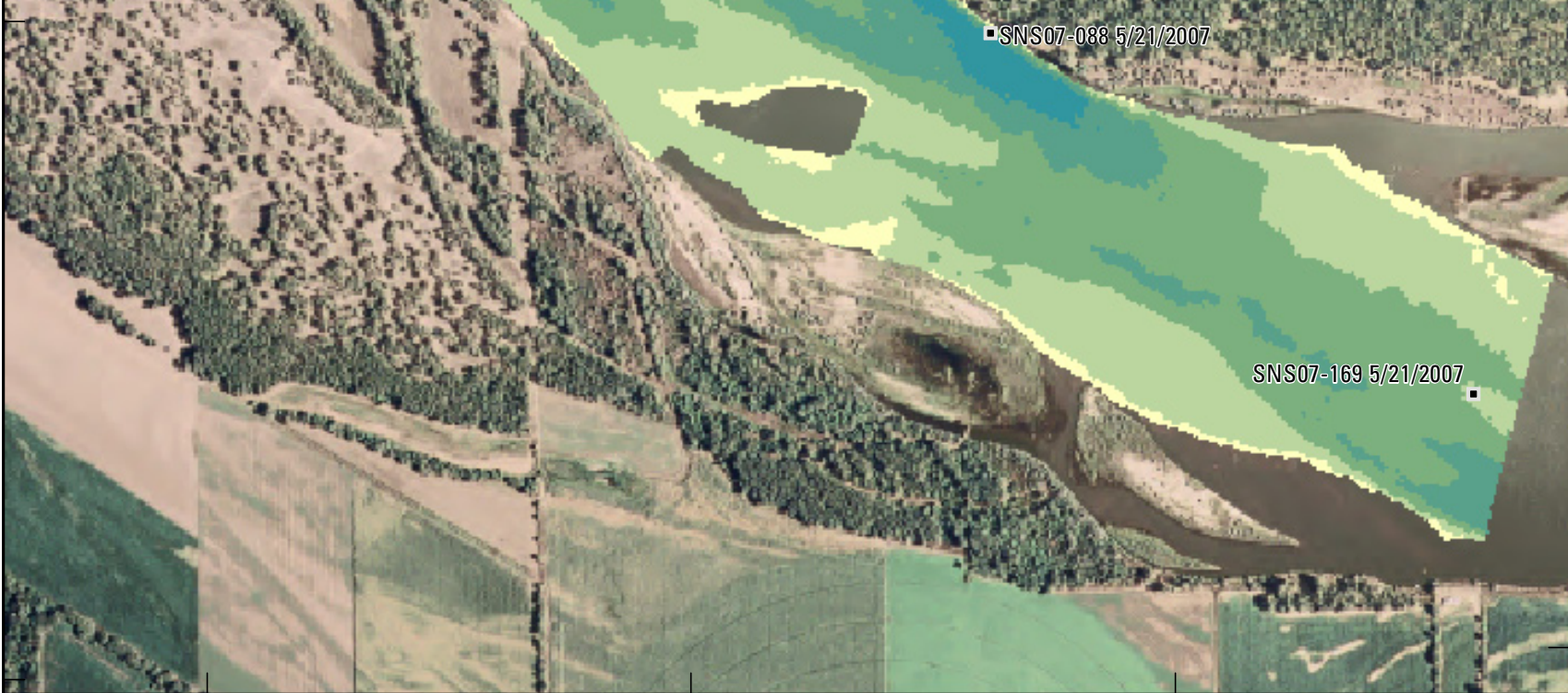

Base from U.S. Department of Agriculture National Agriculture Imagery Program, digital data, 2006 Universal Transverse Mercator projection $97^{\circ} 17^{\prime}$

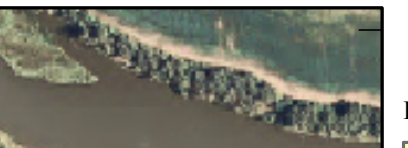

Depth, in meters

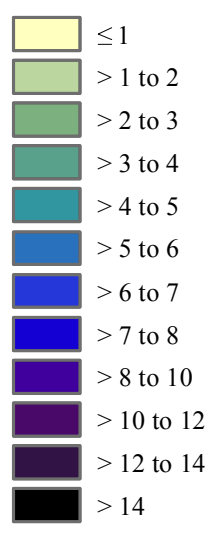

SNS07-141 5/22/2007

- Sturgeon relocationLabel shows identification code of tagged sturgeo 514 and the relocation date

$\odot \quad$ River-mile marker-

Number is distance upstream from the junction with the Mississippi River, in miles

NOTE: This map is based on data collected by the R/V Funk on May 22, 2007.

\begin{tabular}{|c|c|c|}
\hline 0 & 125 & 250 METERS \\
\hline & $\downarrow$ & \\
\hline & 500 & 1,000 FEET \\
\hline
\end{tabular}
Sturgeon relocations from U.S. Geological Survey,
DeLonay and others, 2007; Aaron J. DeLonay, unpub. data, 2008 


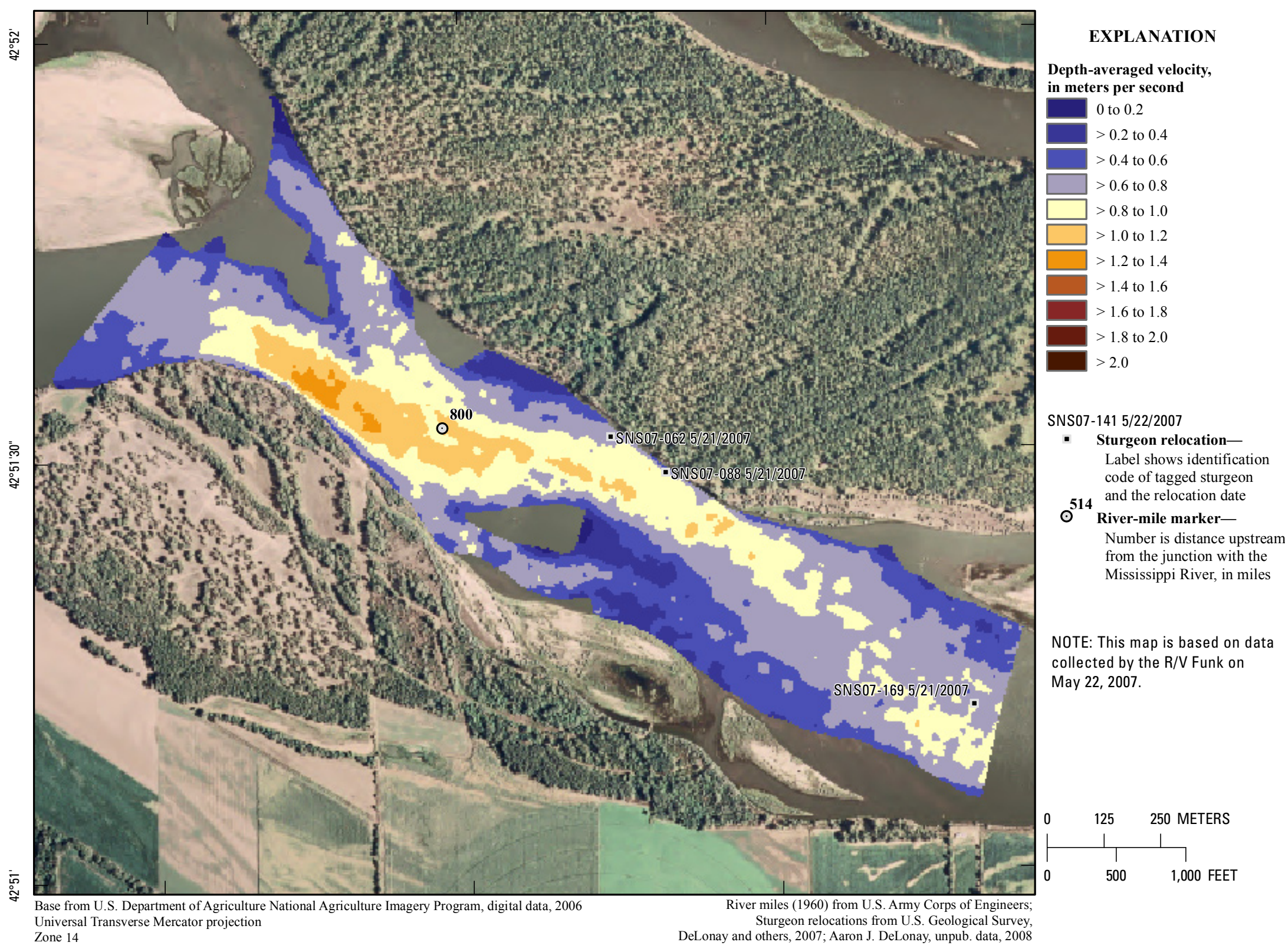

Figure 21. Map of depth-averaged velocity based on data collected on May 22, 2007, in the vicinity of river mile 800 . 


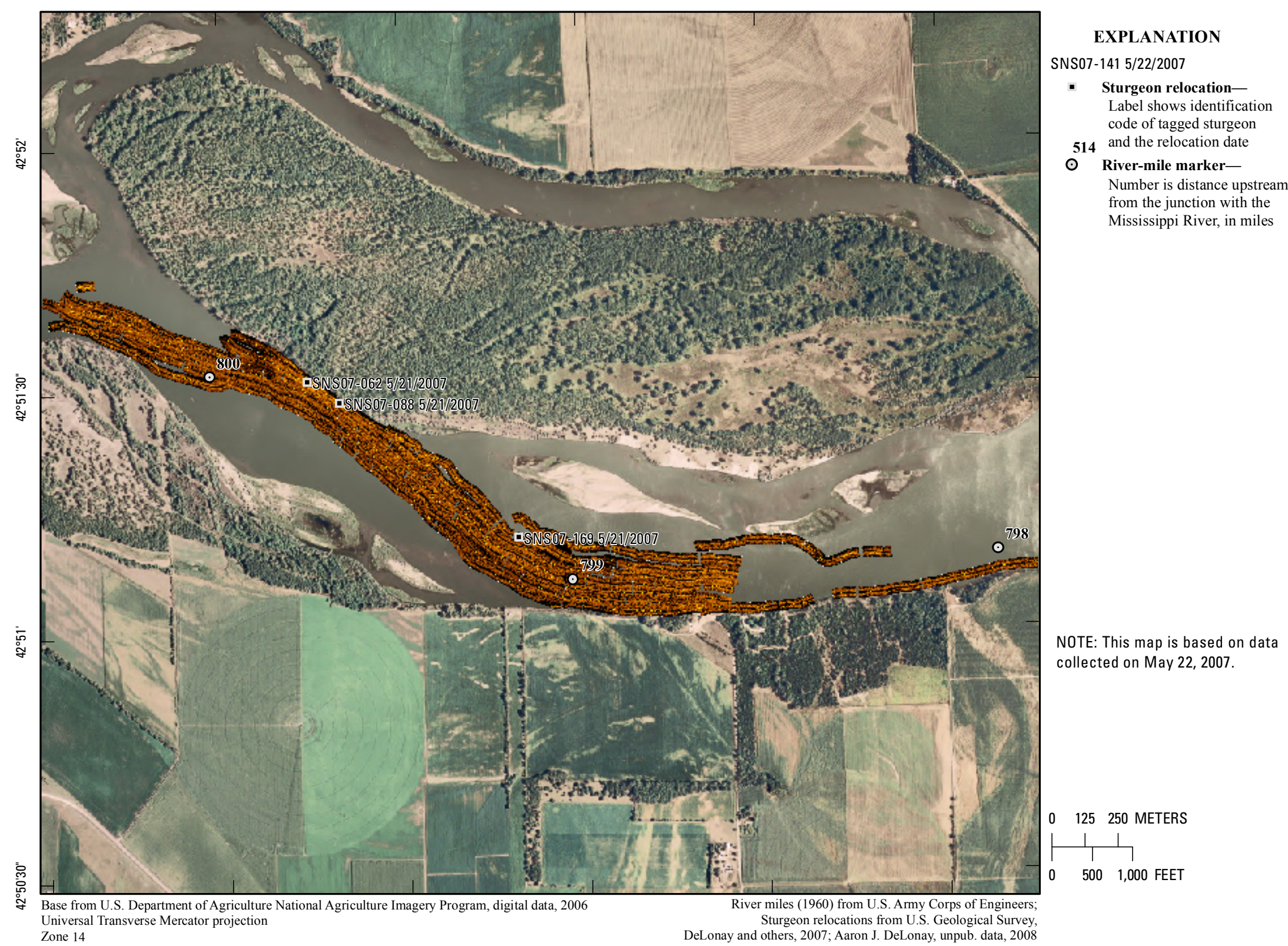
Zniversal 14 DeLonay and others, 2007; Aaron J. DeLonay, unpub. data, 2008 


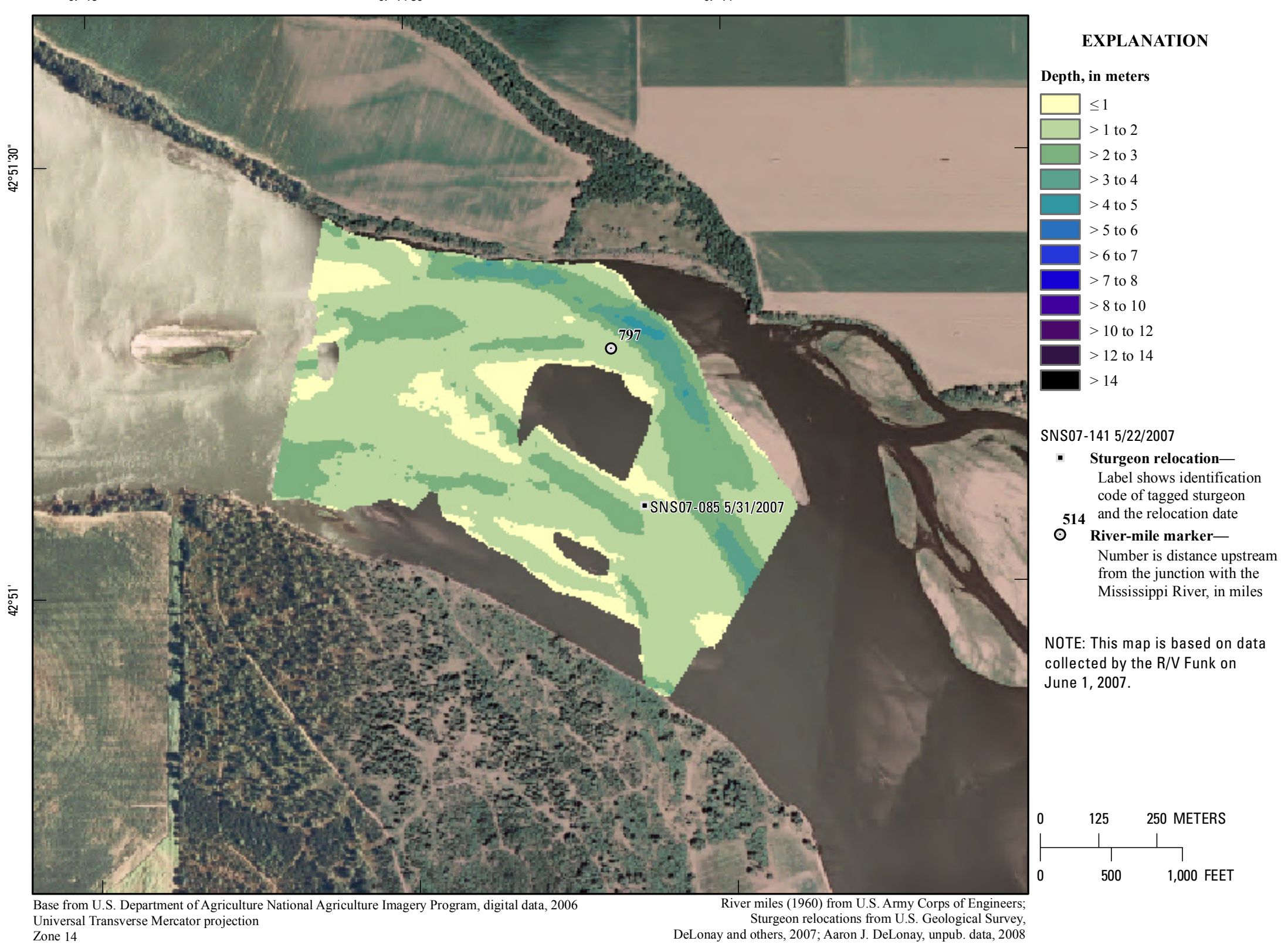

Figure 23. Map of depth based on data collected on June 1, 2007, in the vicinity of river mile 797 . 


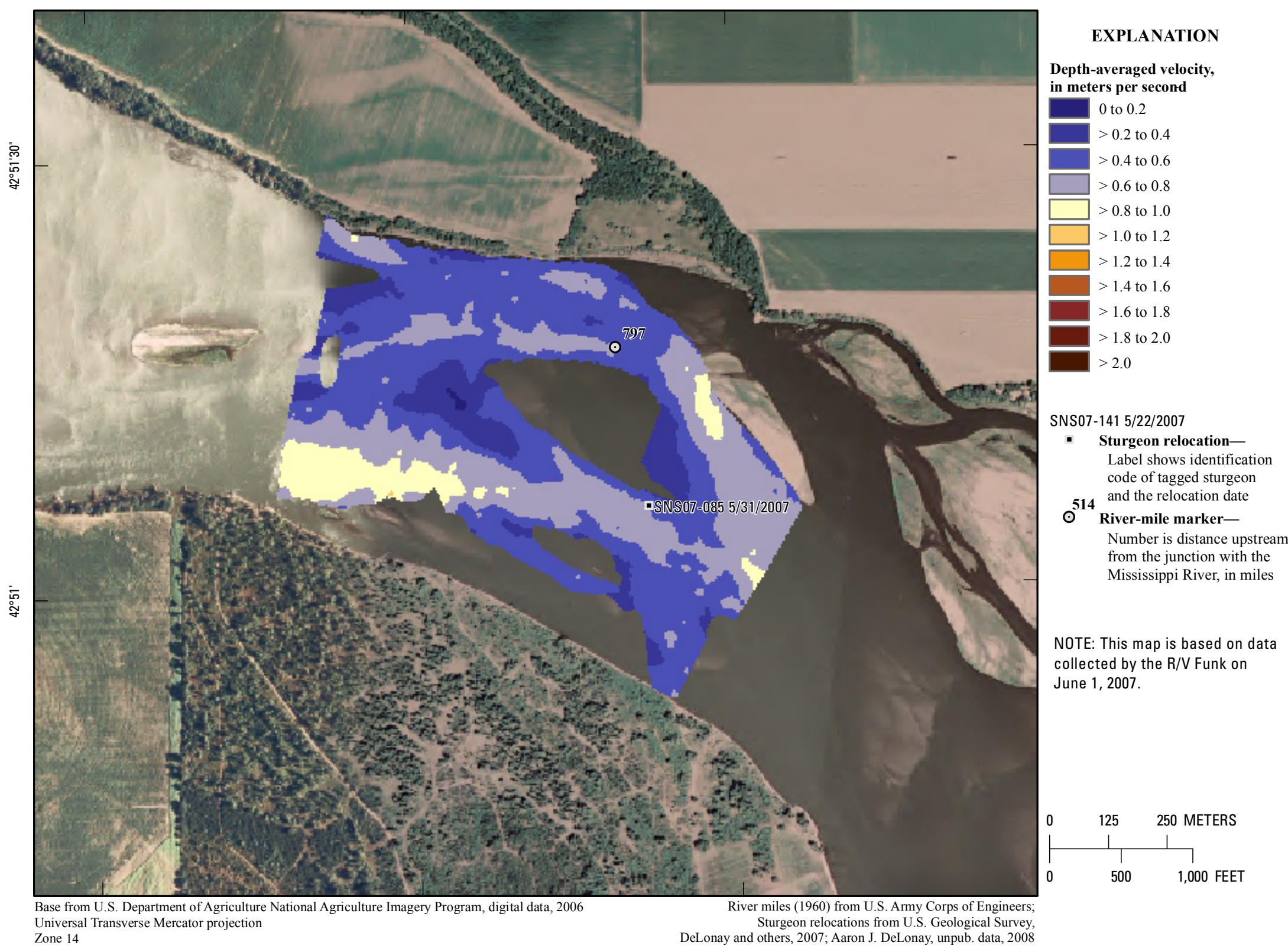




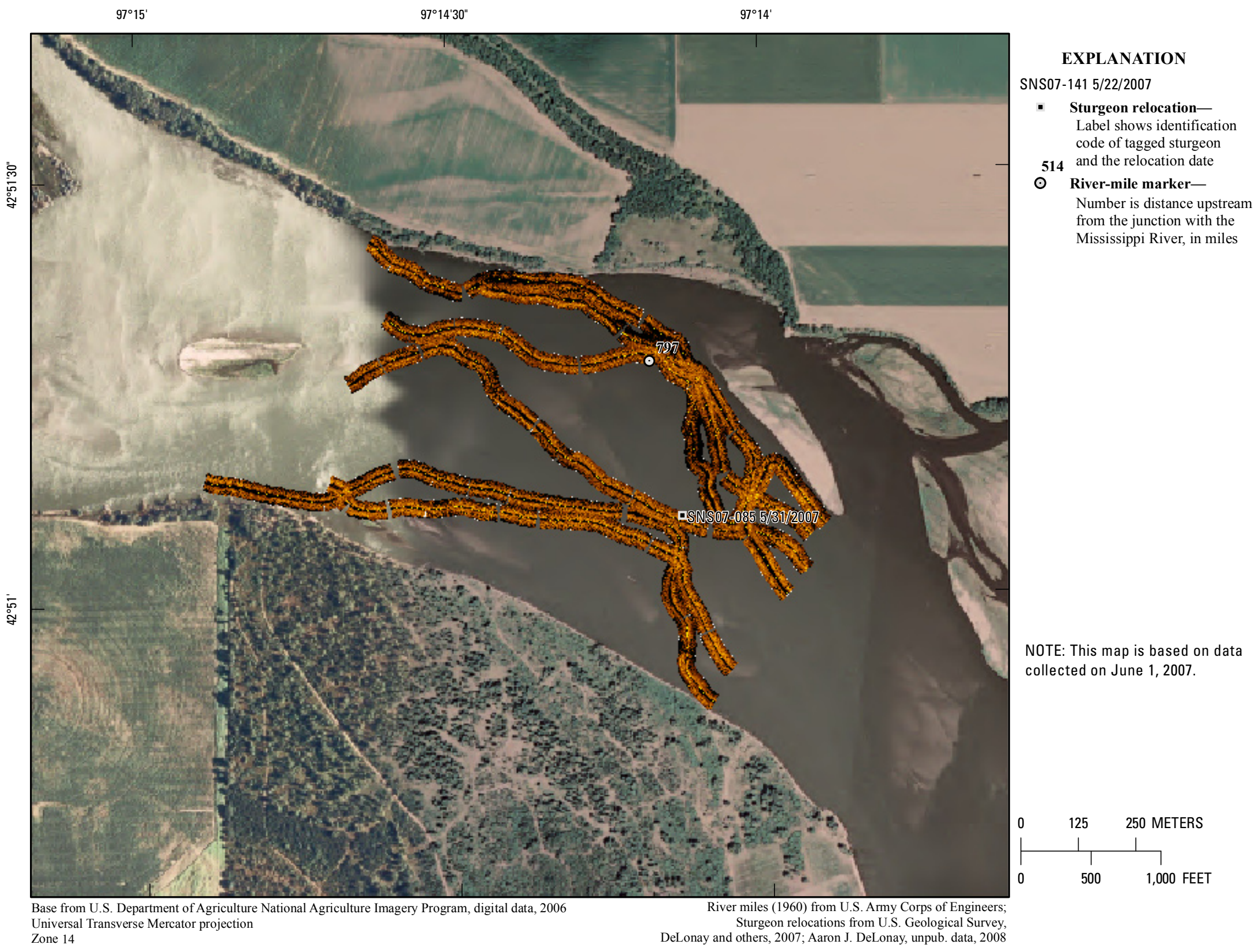

Figure 25. Map of side-scan sonar imagery based on data collected on June 1, 2007, in the vicinity of river mile 797. 


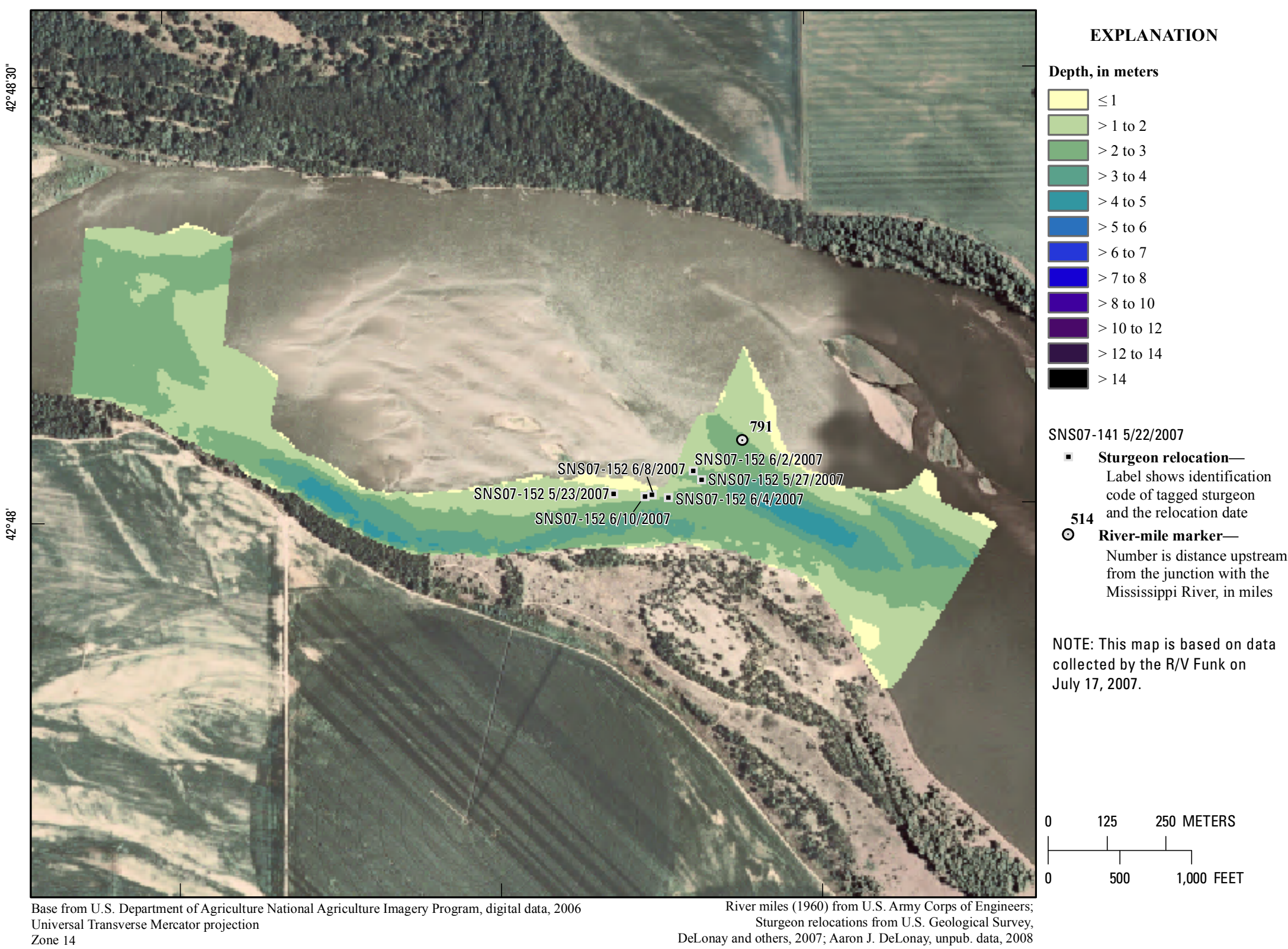

Figure 26. Map of depth based on data collected on July 17, 2007, in the vicinity of river mile 791 
$97^{\circ} 11$

$97^{\circ} 10^{\prime} 30^{\circ}$

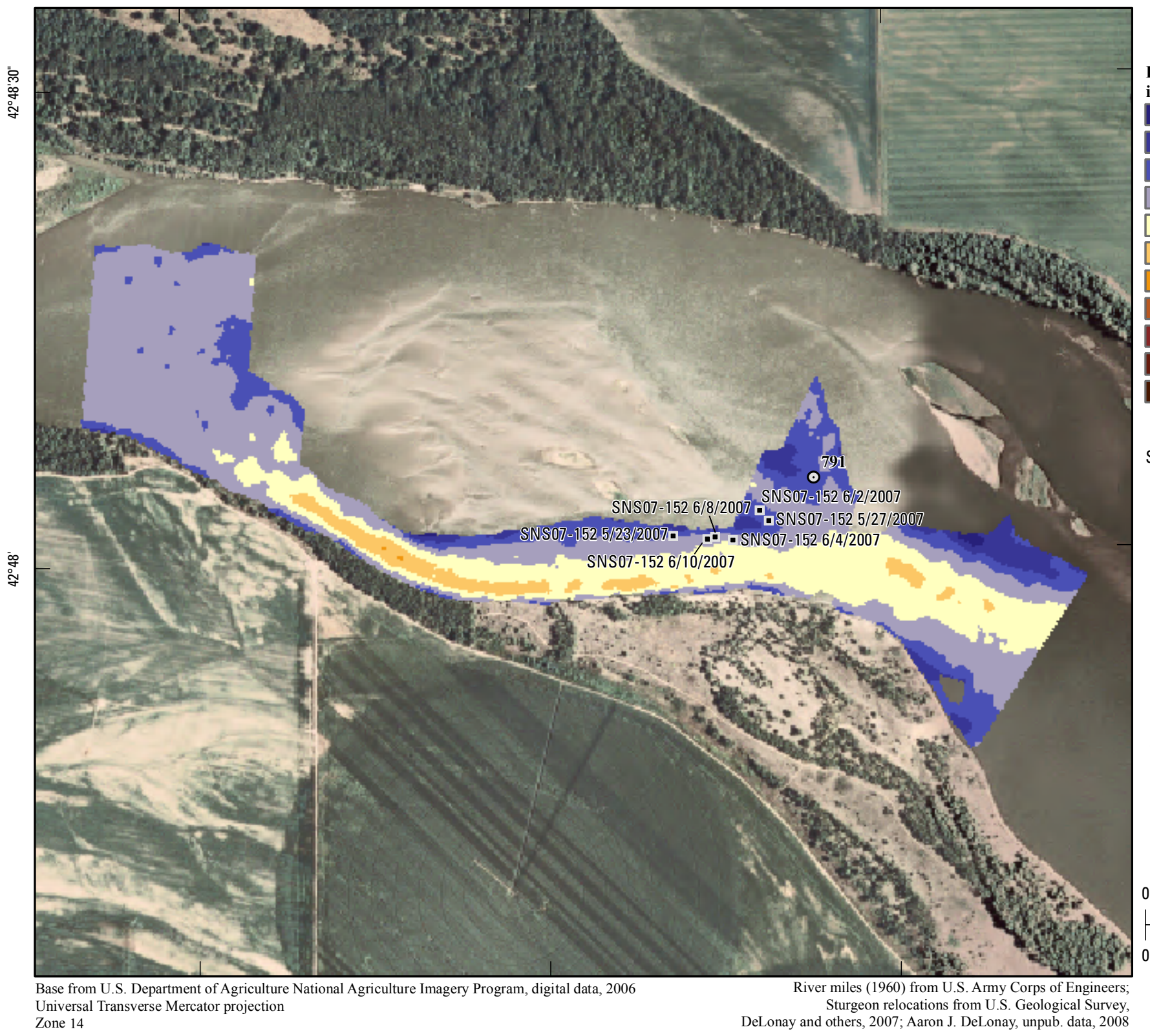

EXPLANATION

Depth-averaged velocity,

in meters per second

0 to 0.2

$>0.2$ to 0.4

0.4 to 0.6

$>0.6$ to 0.8

$>0.8$ to 1.0

$>1.0$ to 1.2

$>1.2$ to 1.4

$>1.4$ to 1.6

$>1.6$ to 1.8

$>1.8$ to 2.0

$>2.0$

\section{NSS07-141 5/22/2007}

Sturgeon relocation-

Label shows identification code of tagged sturgeo

$\mathbf{5 1 4}$ and the relocation date

$\odot^{514}$ River-mile marker-

Number is distance upstream from the junction with the Mississippi River, in miles

NOTE: This map is based on data collected by the R/V Funk on July 17,2007

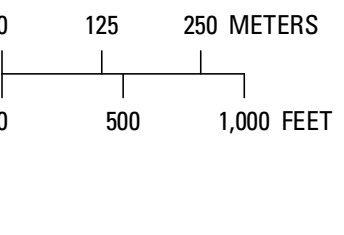

Figure 27. Map of depth-averaged velocity based on data collected on July 17, 2007, in the vicinity of river mile 791. 


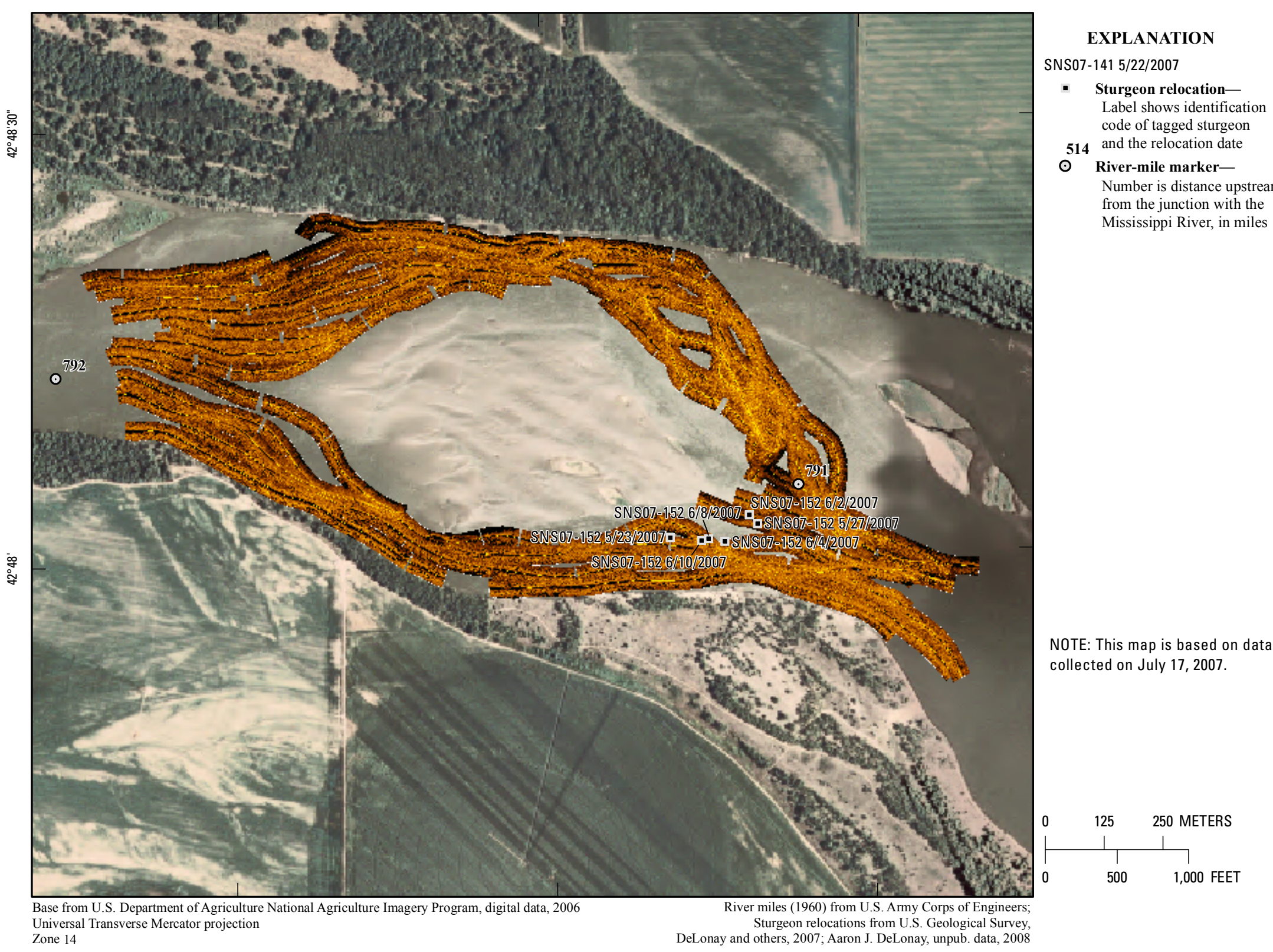

Figure 28. Map of side-scan sonar imagery based on data collected on July 17, 2007, in the vicinity of river mile 791 . 


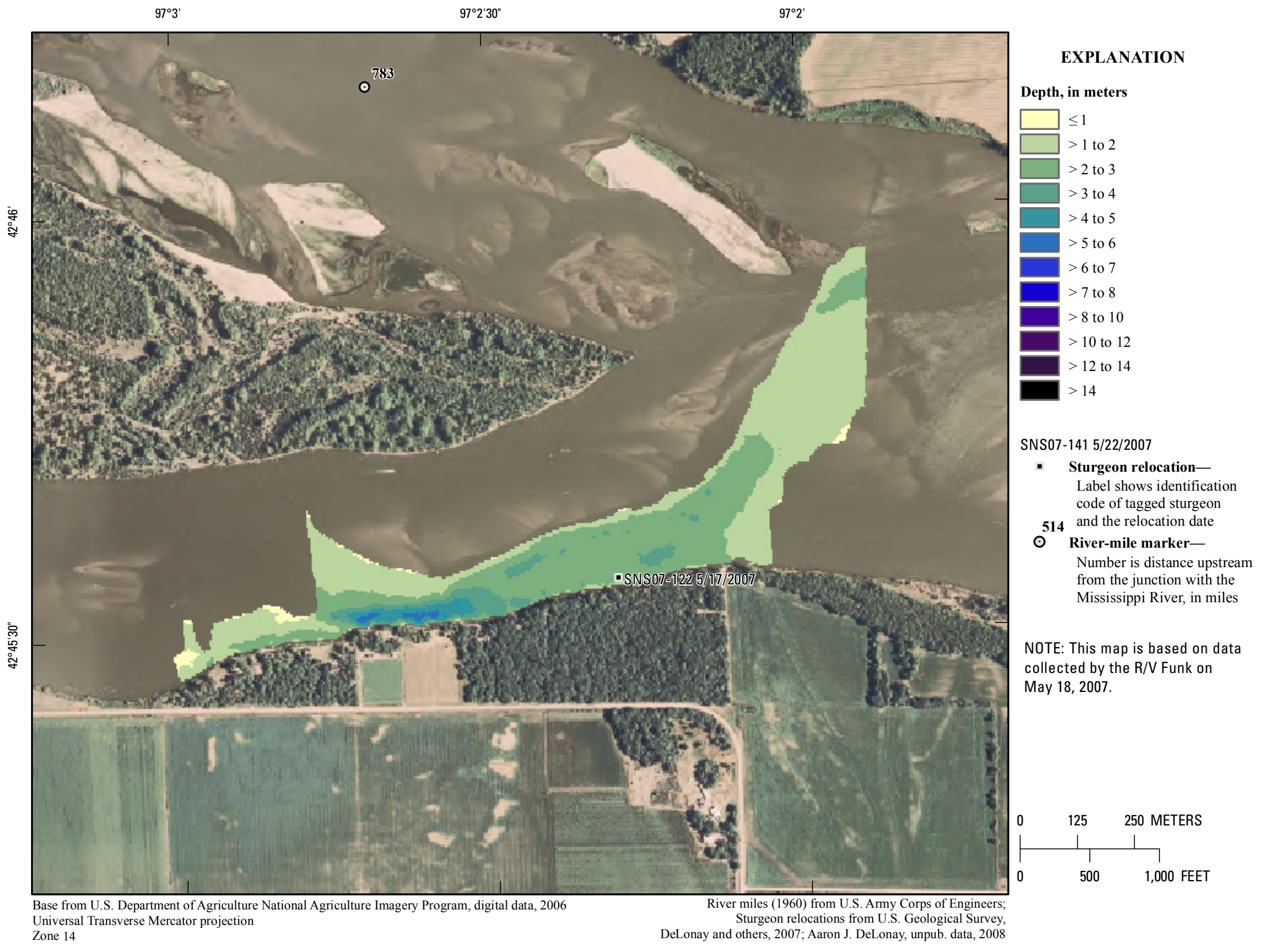

Figure 29. Map of depth based on data collected on May 18, 2007, in the vicinity of river mile 783 . 


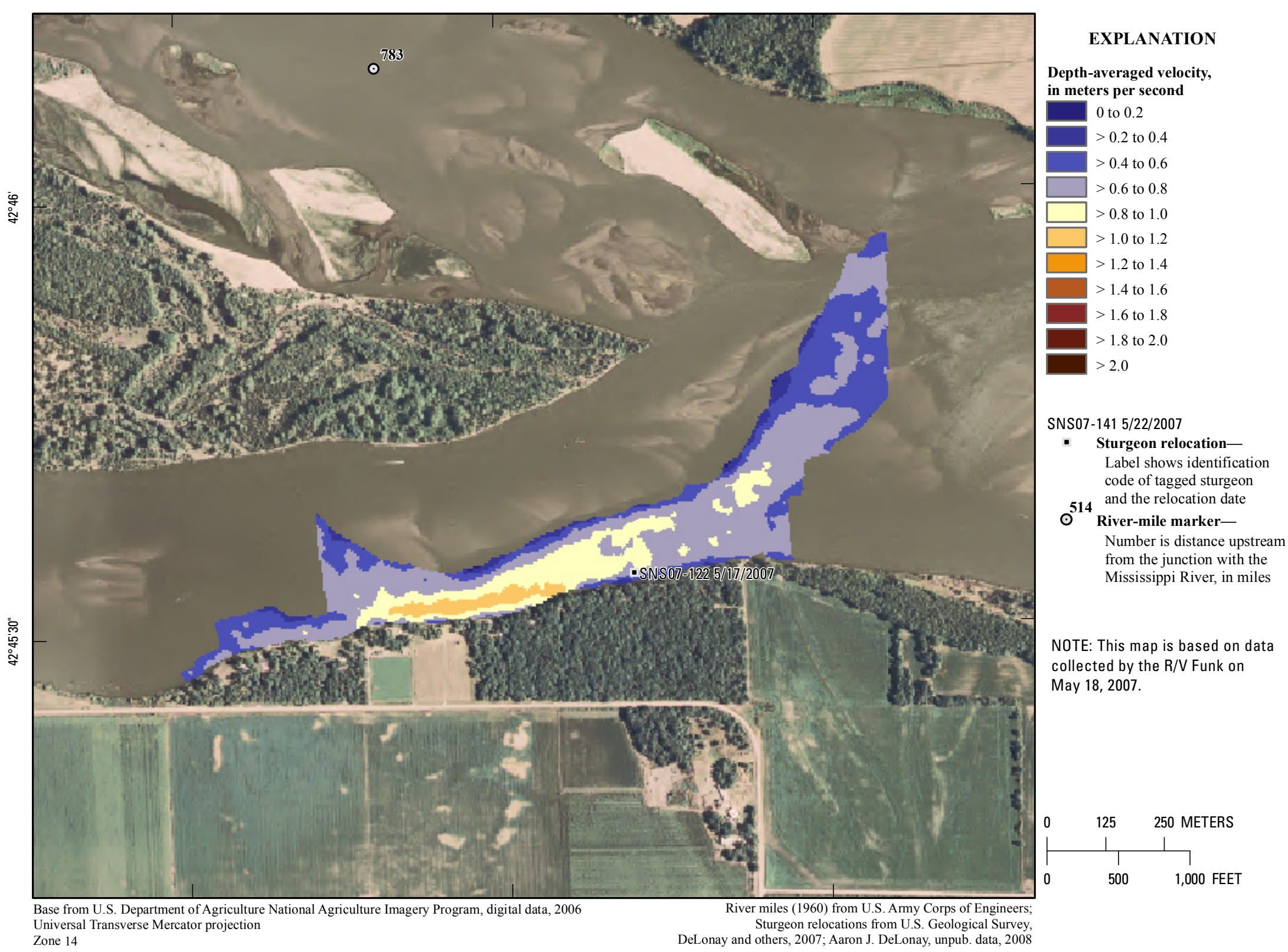




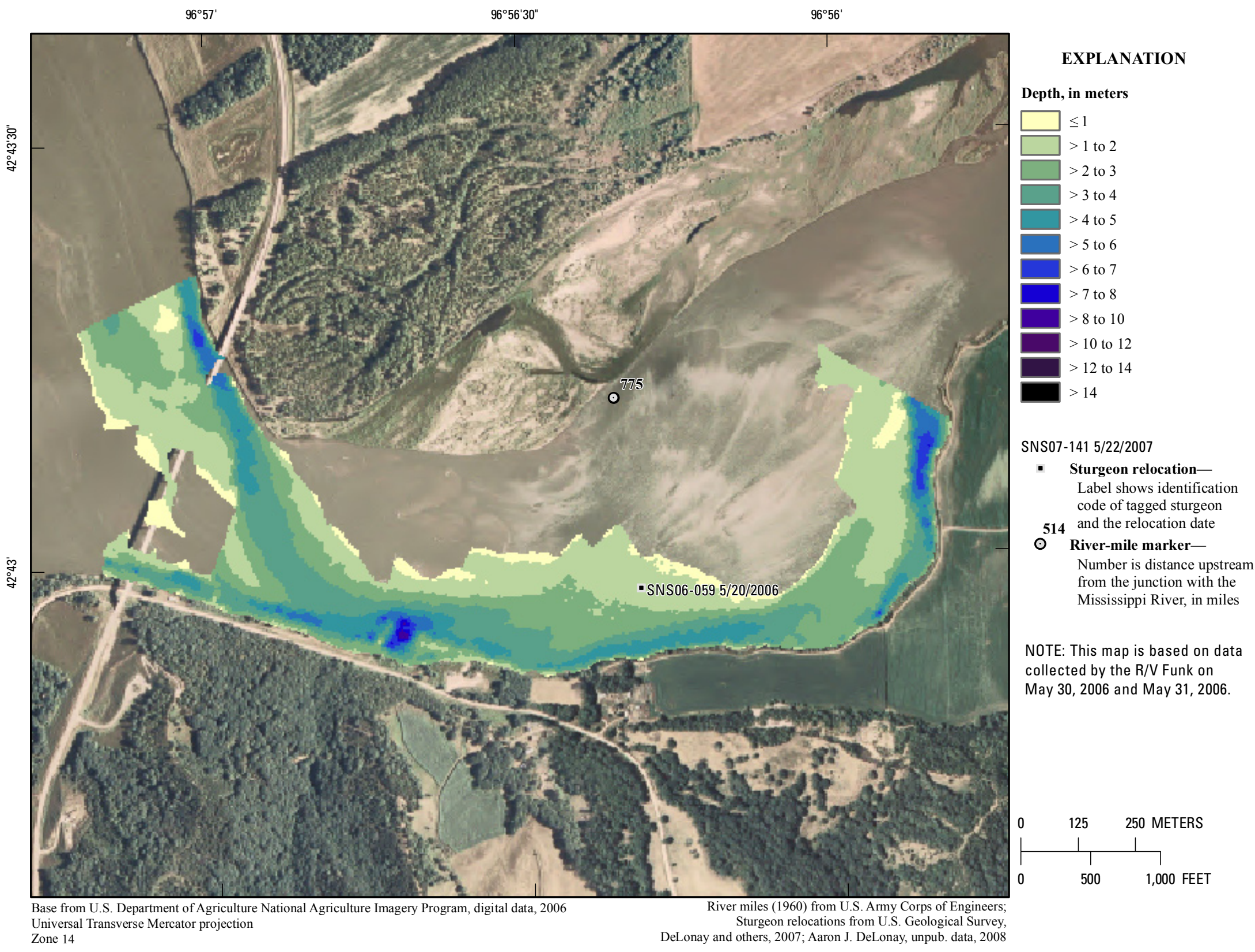

Figure 31. Map of depth based on data collected on May 30, 2006, and May 31, 2006, in the vicinity of river mile 775 . 


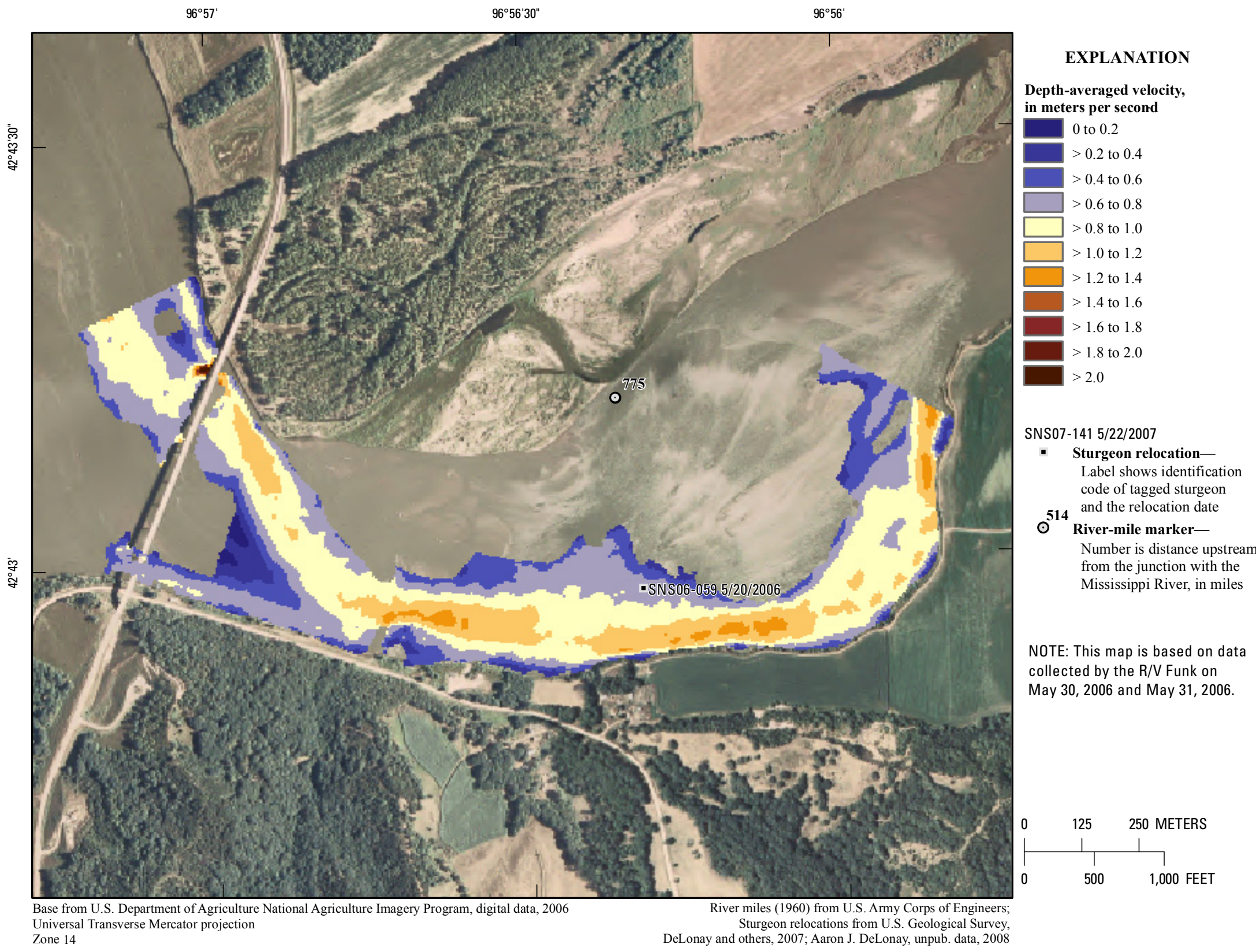

Figure 32. Map of depth-averaged velocity based on data collected on May 30, 2006, and May 31, 2006, in the vicinity of river mile 775 . 


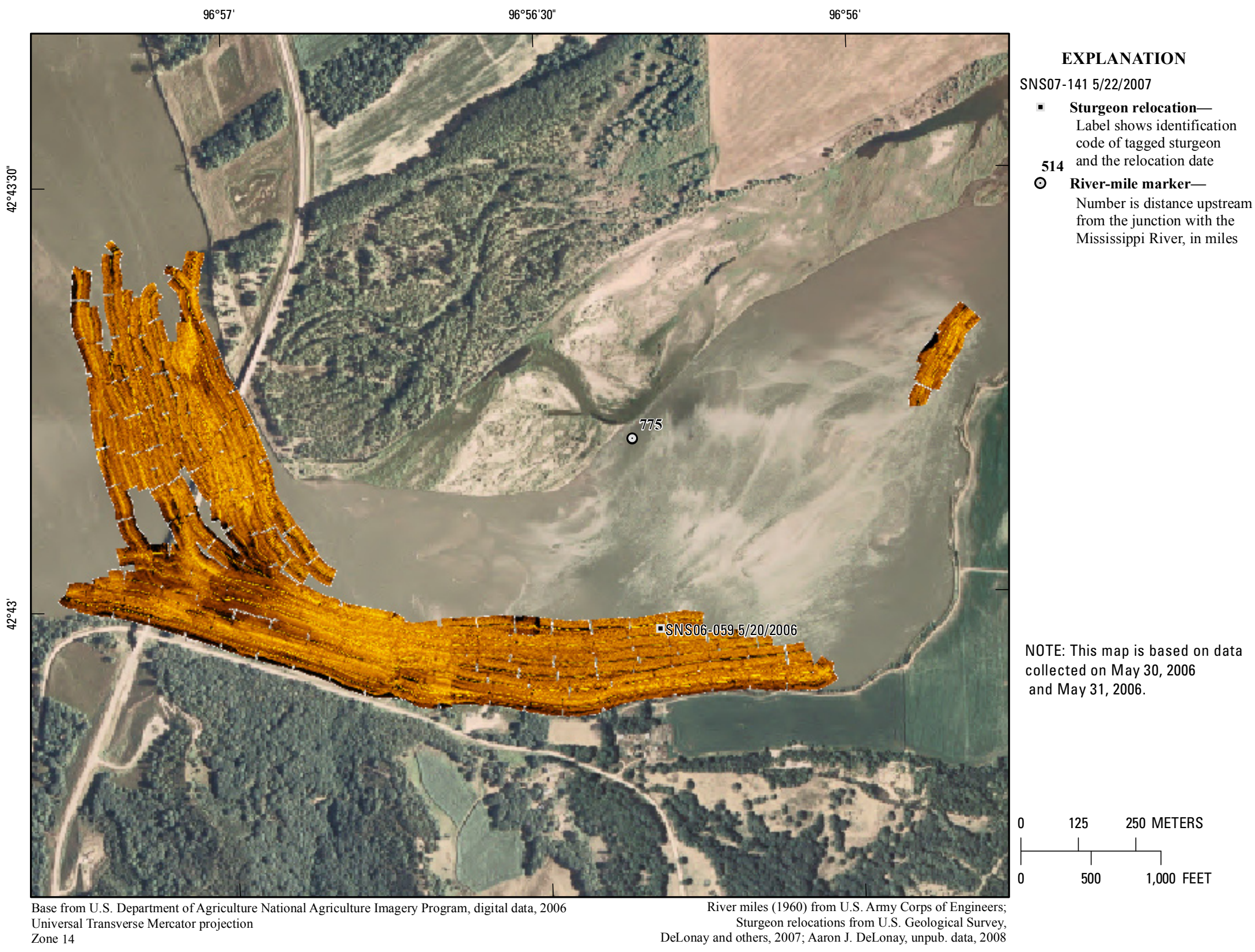

Figure 33. Map of side-scan sonar imagery based on data collected on May 30, 2006, and May 31, 2006, in the vicinity of river mile 775. 


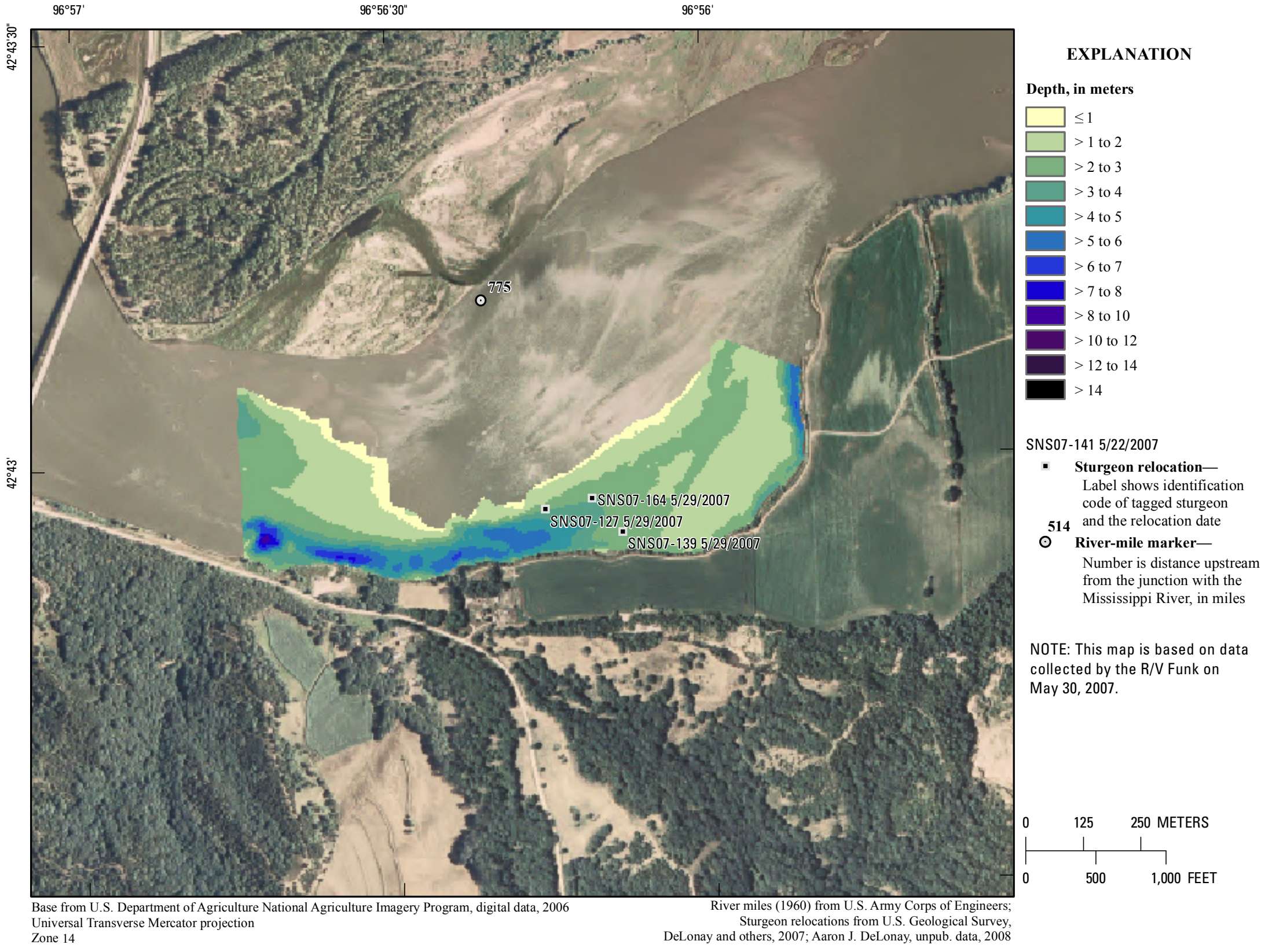

Figure 34. Map of depth based on data collected on May 30, 2007, in the vicinity of river mile 775 . 


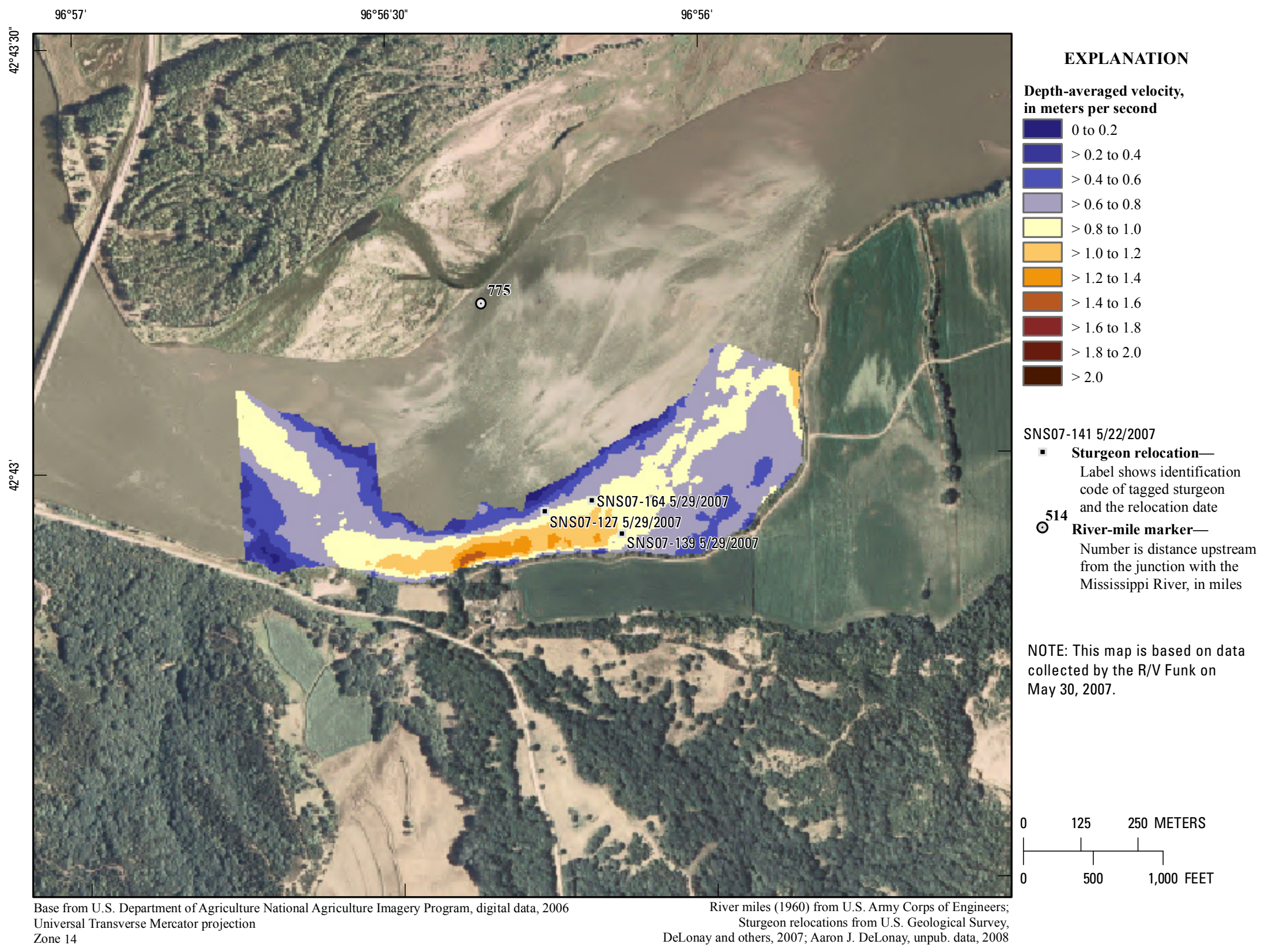

Figure 35. Map of depth-averaged velocity based on data collected on May 30, 2007, in the vicinity of river mile 775 . 


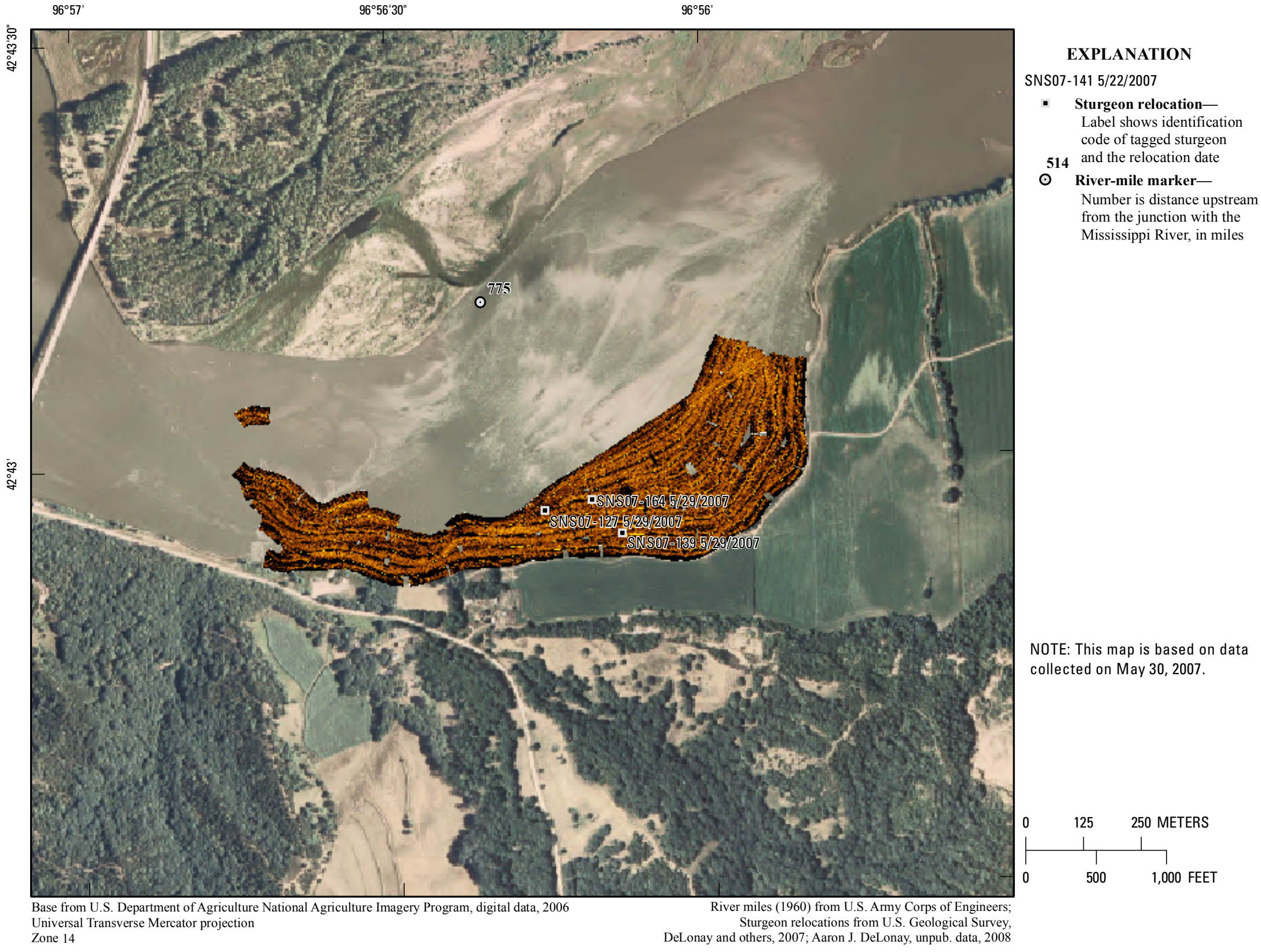

Figure 36. Map of side-scan sonar imagery based on data collected on May 30, 2007, in the vicinity of river mile 775 . 


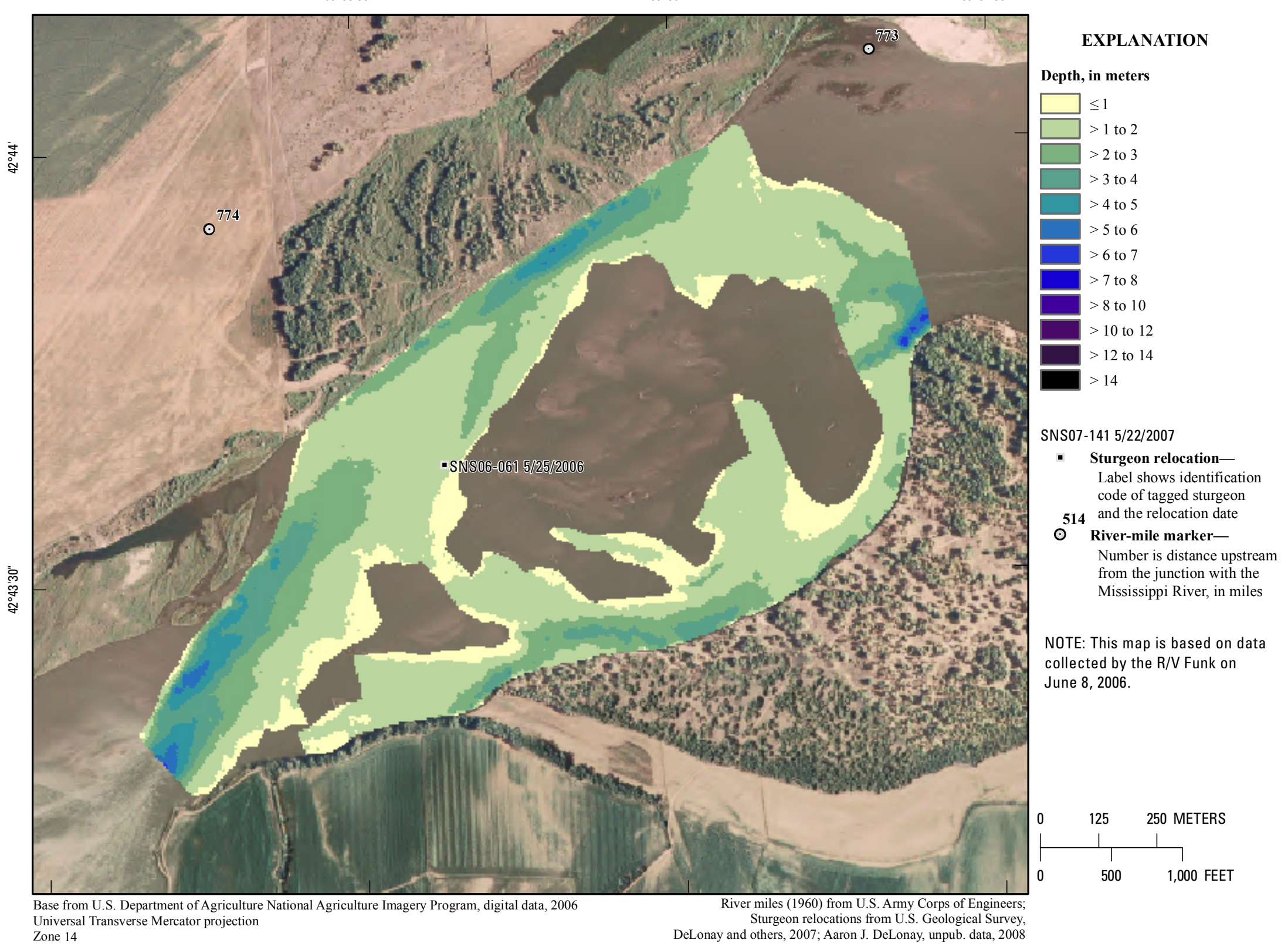

Figure 37. Map of depth based on data collected on June 8, 2006, in the vicinity of river mile 774 . 


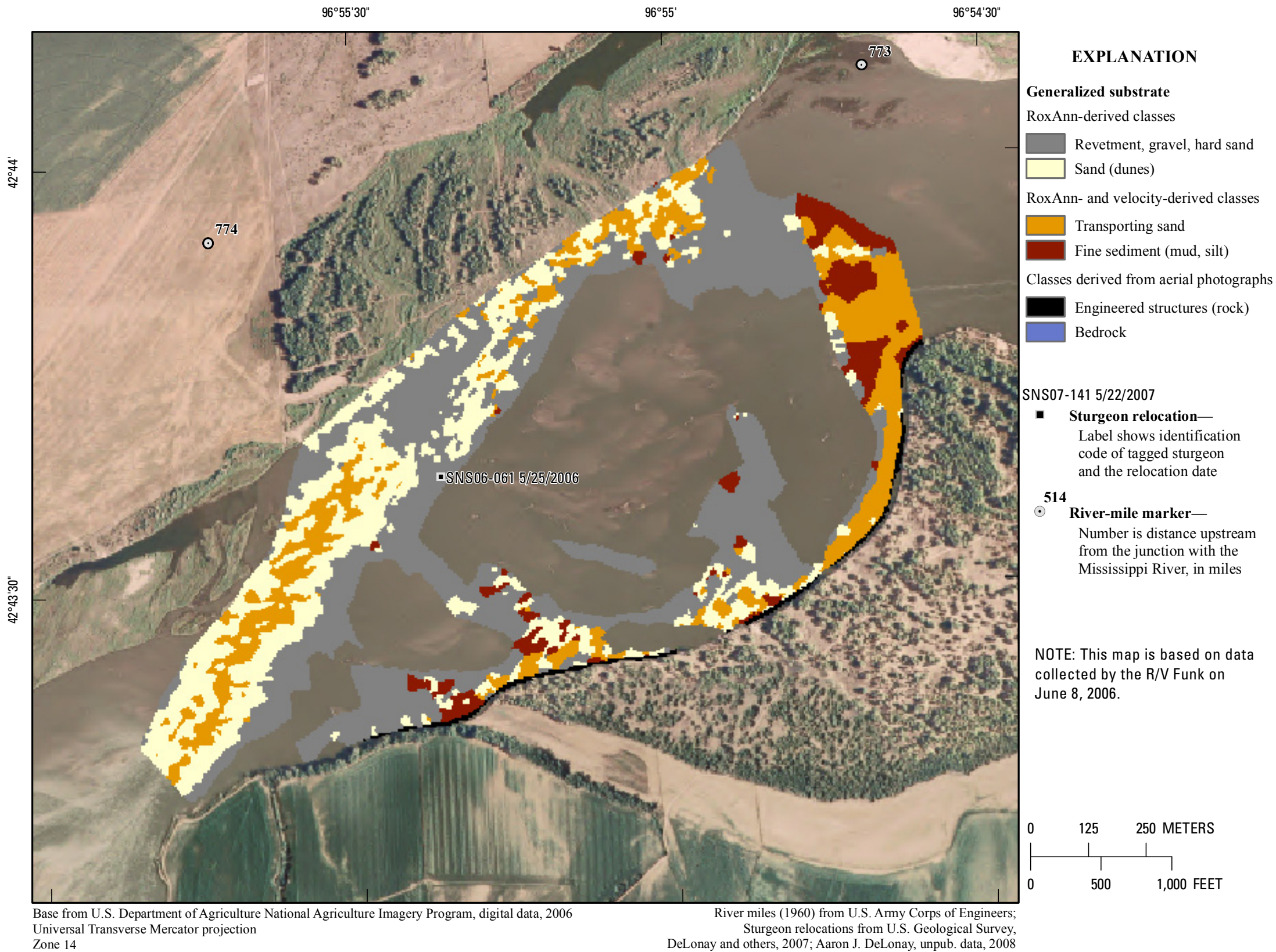

Figure 38. Map of generalized substrate based on data collected on June 8, 2006, in the vicinity of river mile 774 . 


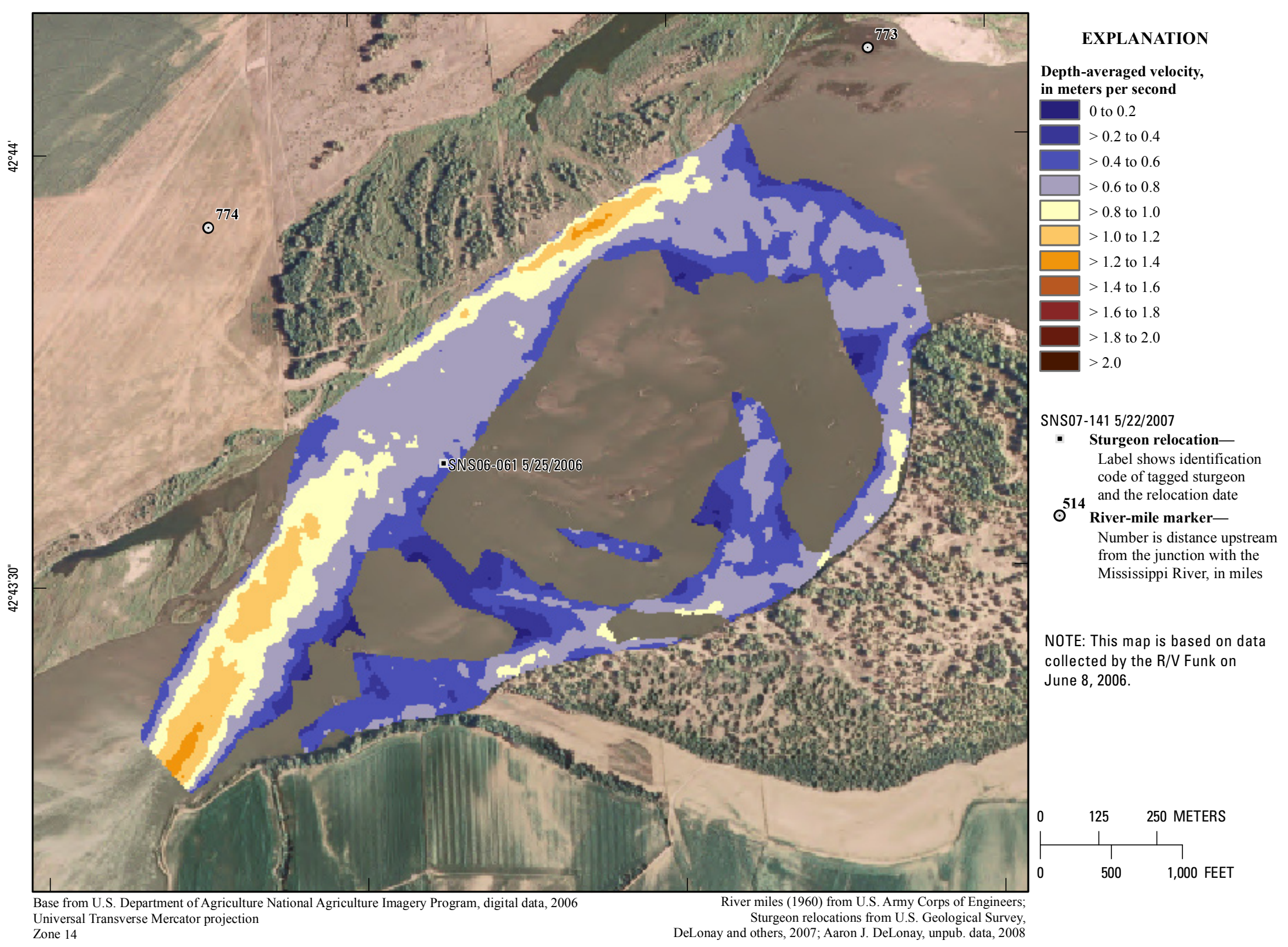

Figure 39. Map of depth-averaged velocity based on data collected on June 8, 2006, in the vicinity of river mile 774 . 
$96^{\circ} 44^{\prime}$

$96^{\circ} 43^{\prime} 30^{\prime \prime}$

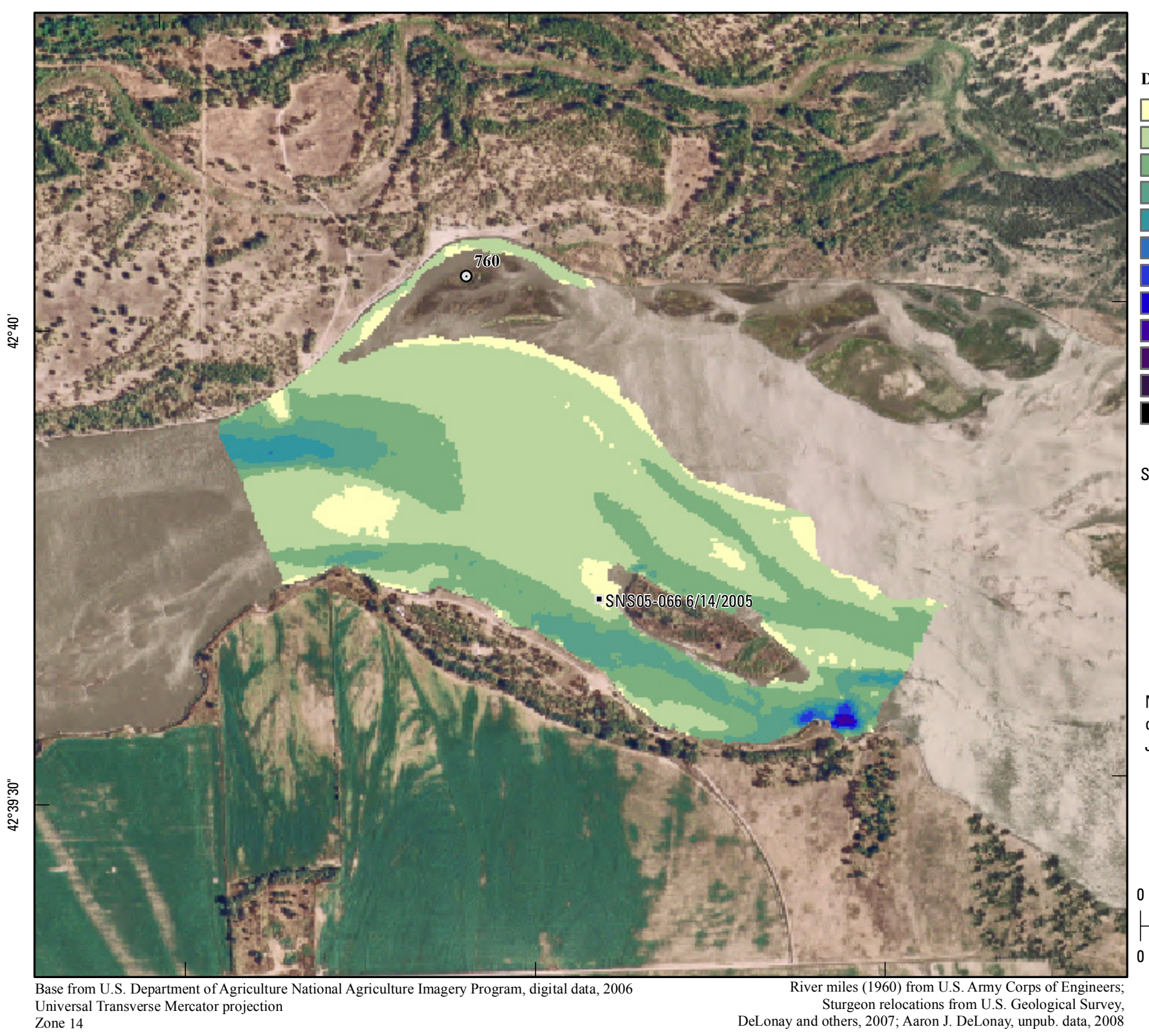

EXPLANATION

Depth, in meters

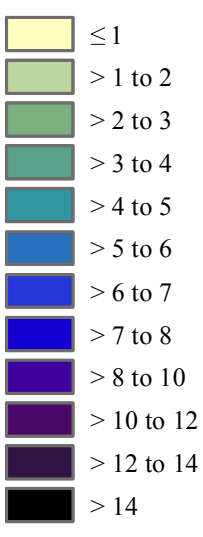

SNS07-141 5/22/2007

Sturgeon relocationLabel shows identification code of tagged sturgeo 514 and the relocation date

$\odot \quad$ River-mile marker-

Number is distance upstream from the junction with the Mississippi River, in miles

NOTE: This map is based on data collected by the R/V Funk on June 15, 2005.

$125 \quad 250$ METERS $\left.\right|_{500} ^{1} 1,000$ FEET

Figure 40. Map of depth based on data collected on June 15, 2005, in the vicinity of river mile 760 . 
$96^{\circ} 44^{\prime}$

$96^{\circ} 43^{\prime} 30^{\prime \prime}$

$96^{\circ} 43^{\prime}$

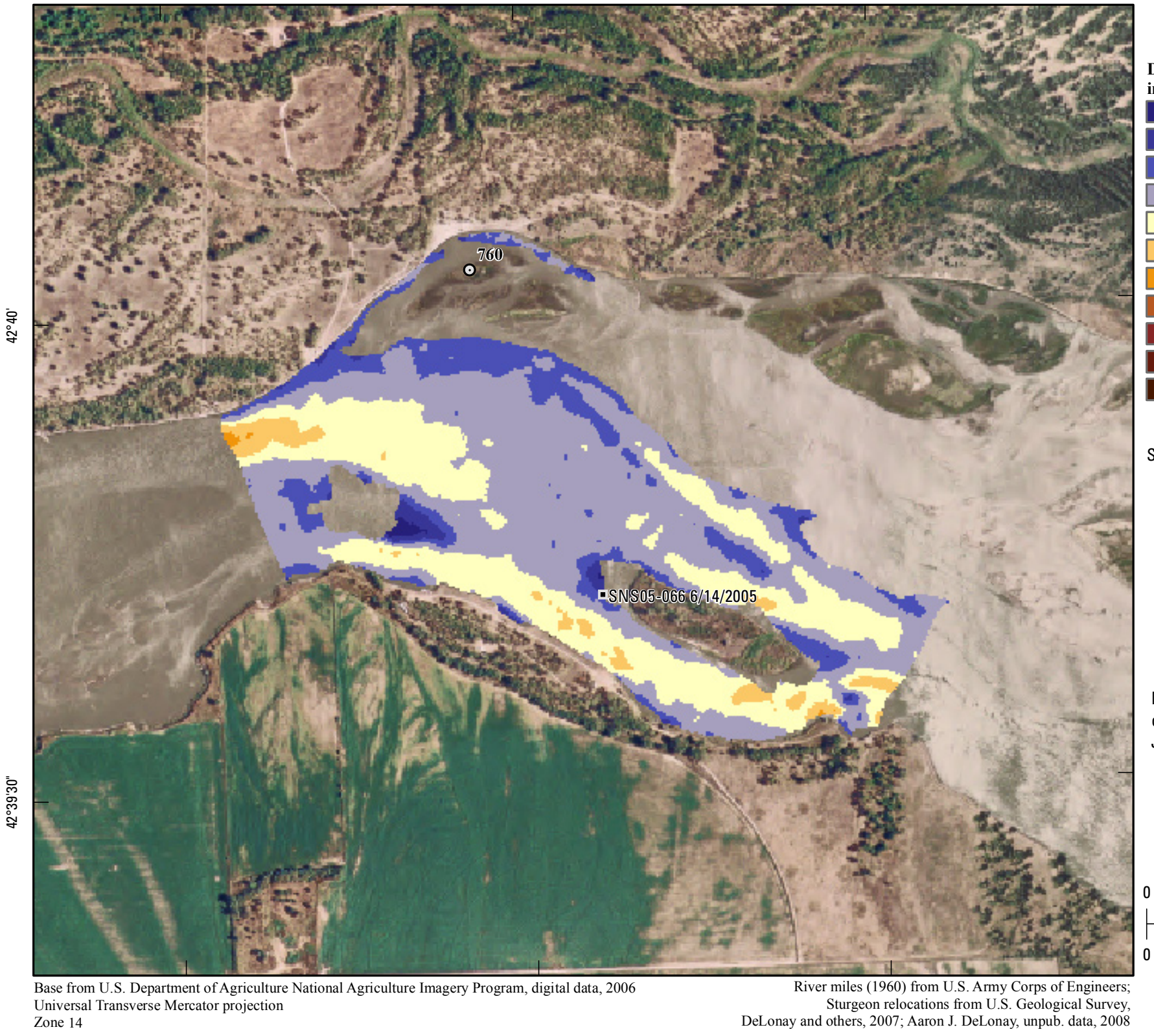

요

EXPLANATION

Depth-averaged velocity,

in meters per second

0 to 0.2

$>0.2$ to 0.4

$>0.4$ to 0.6

$>0.6$ to 0.8

$>0.8$ to 1.0

$>1.0$ to 1.2

$>1.2$ to 1.4

$>1.4$ to 1.6

$>1.6$ to 1.8

$>1.8$ to 2.0

$>2.0$

\section{NS07-141 5/22/2007}

Sturgeon relocation-

Label shows identification code of tagged sturgeo

514 and the relocation date

River-mile marker-

Number is distance upstream from the junction with the Mississippi River, in miles

NOTE: This map is based on data

collected by the R/V Funk on

June 15, 2005

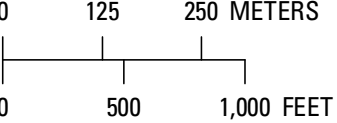

Figure 41. Map of depth-averaged velocity based on data collected on June 15, 2005, in the vicinity of river mile 760 . 


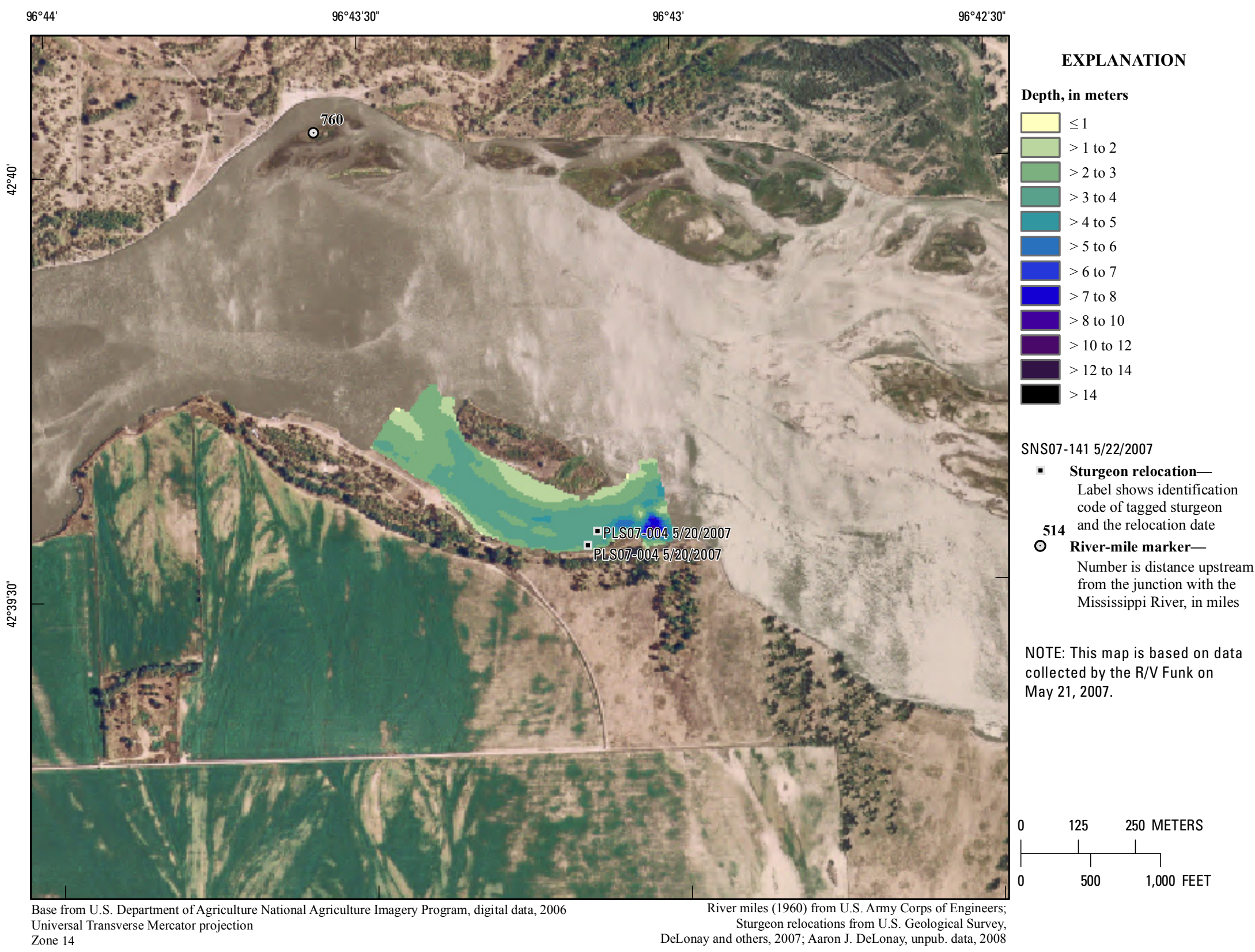

Figure 42. Map of depth based on data collected on May 21, 2007, in the vicinity of river mile 760 . 


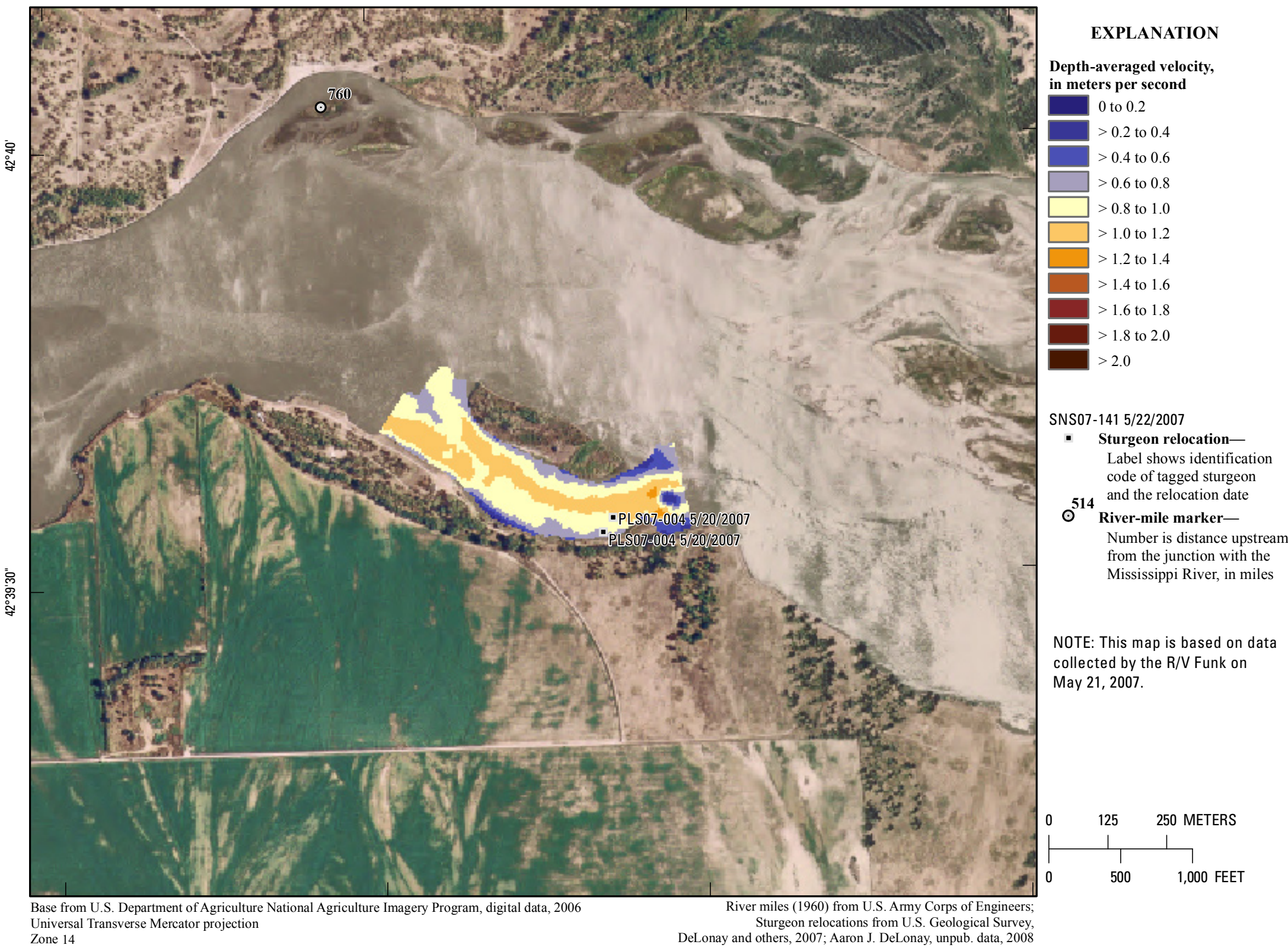

Figure 43. Map of depth-averaged velocity based on data collected on May 21, 2007, in the vicinity of river mile 760. 


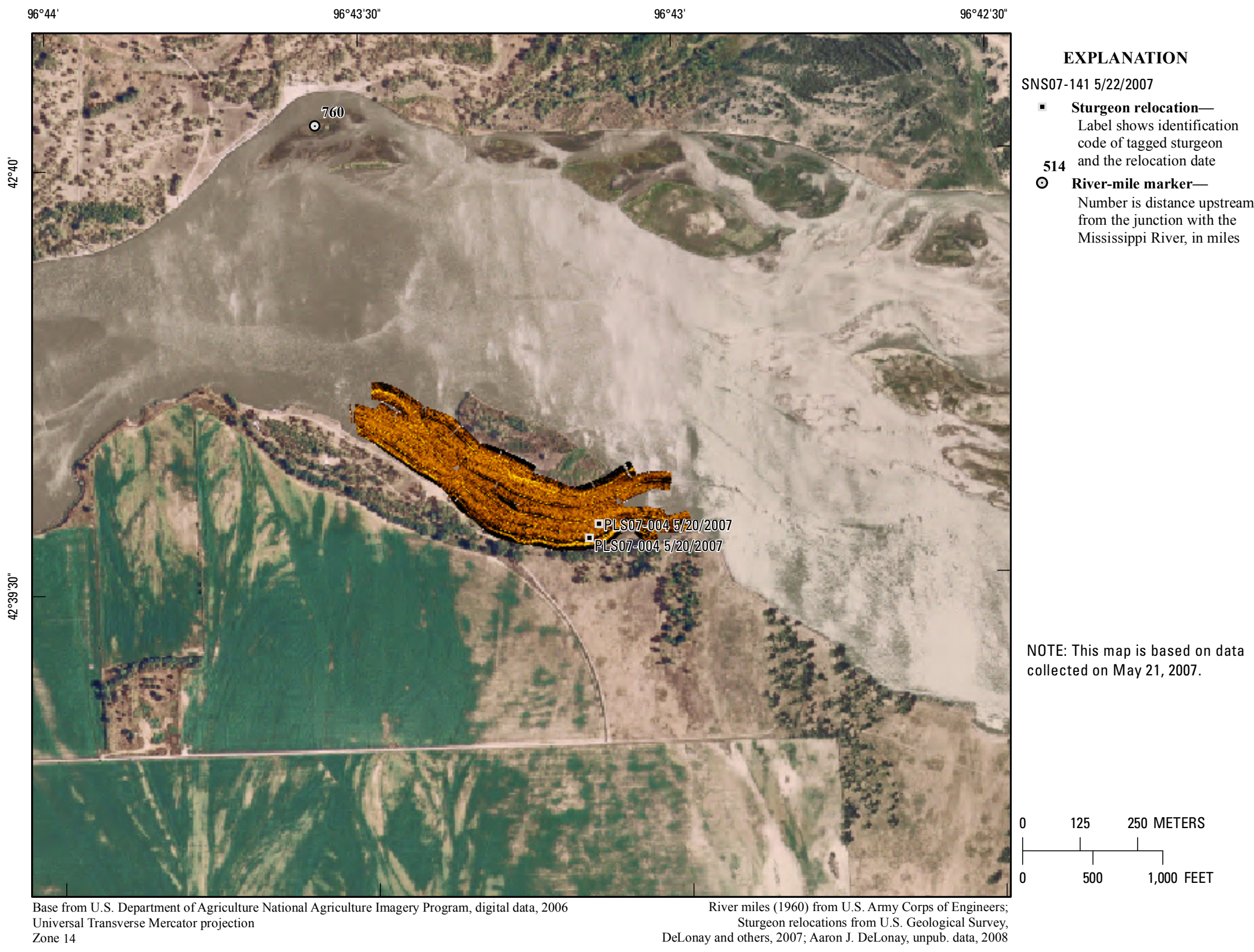

Figure 44. Map of side-scan sonar imagery based on data collected on May 21, 2007, in the vicinity of river mile 760. 


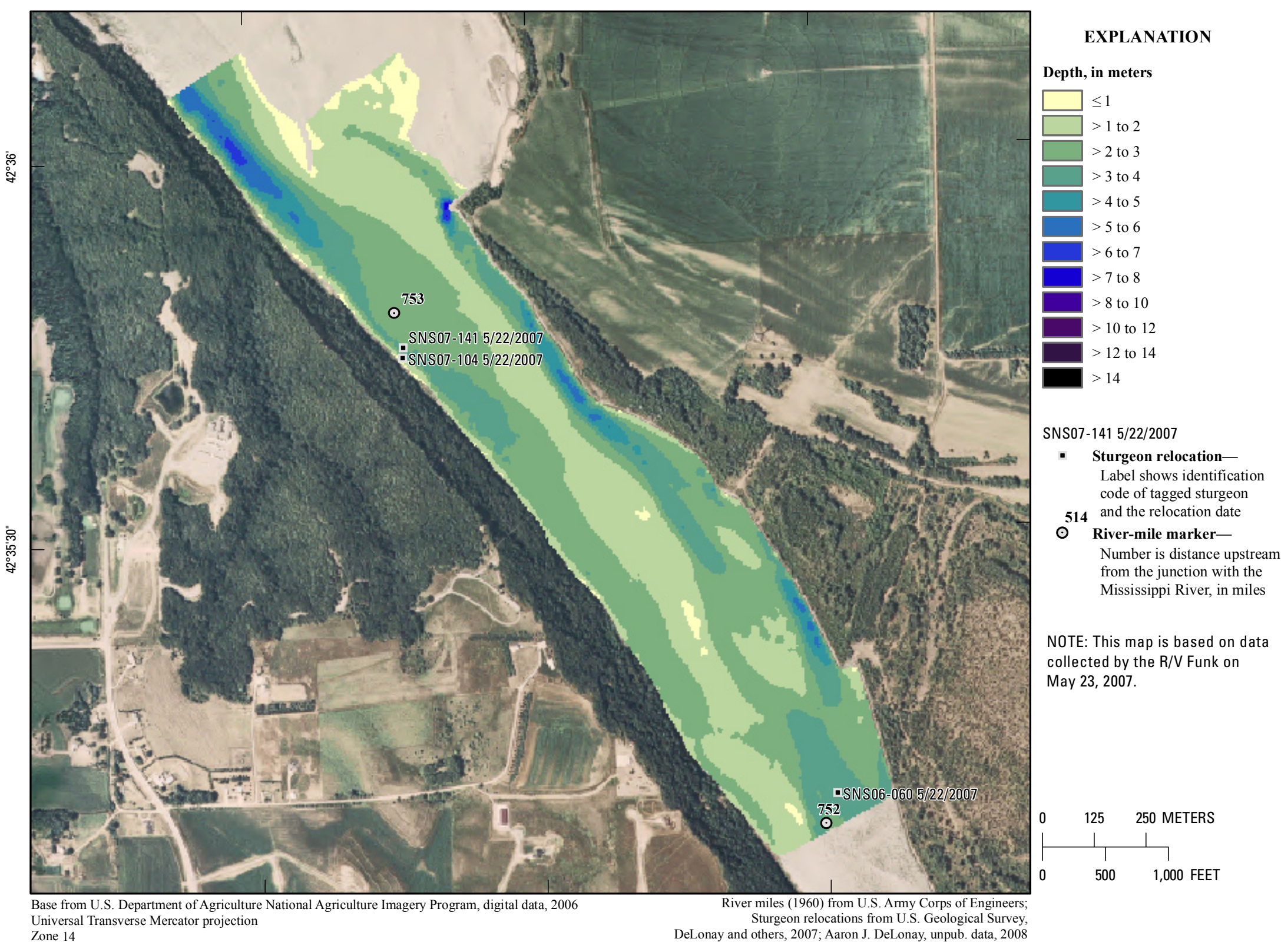

Figure 45. Map of depth based on data collected on May 23, 2007, in the vicinity of river mile 753 


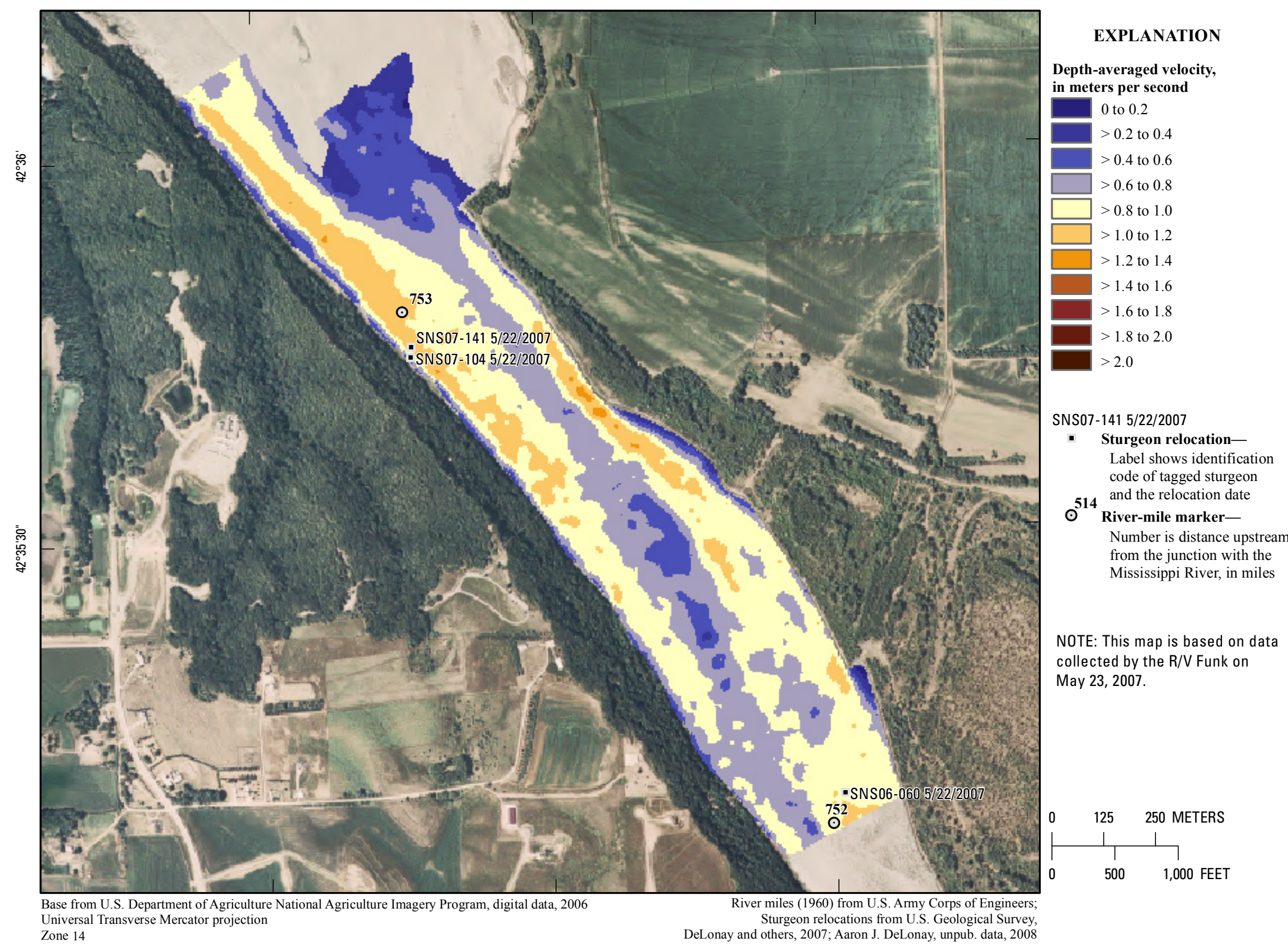




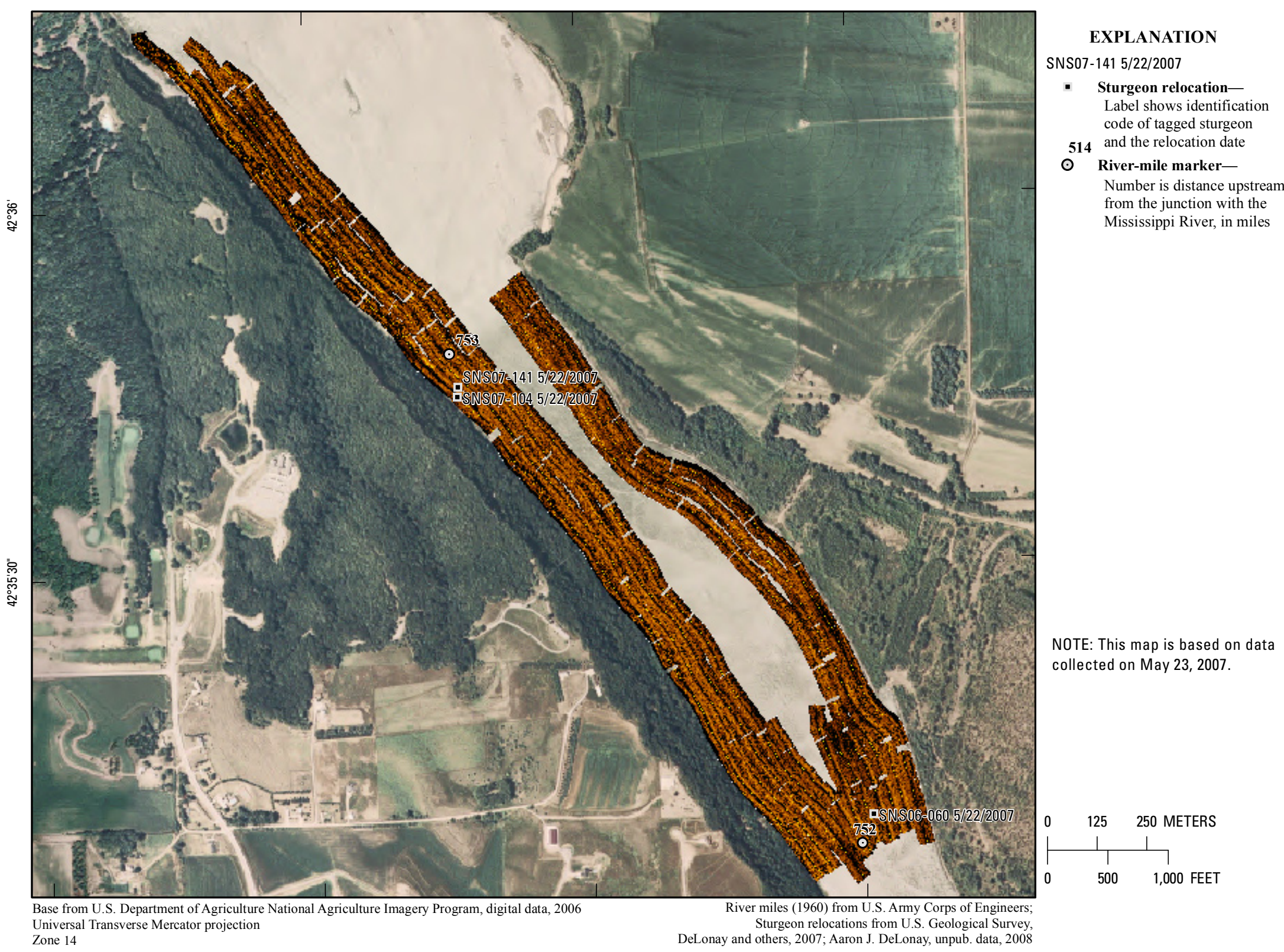

Figure 47. Map of side-scan sonar imagery based on data collected on May 23, 2007, in the vicinity of river mile 753. 


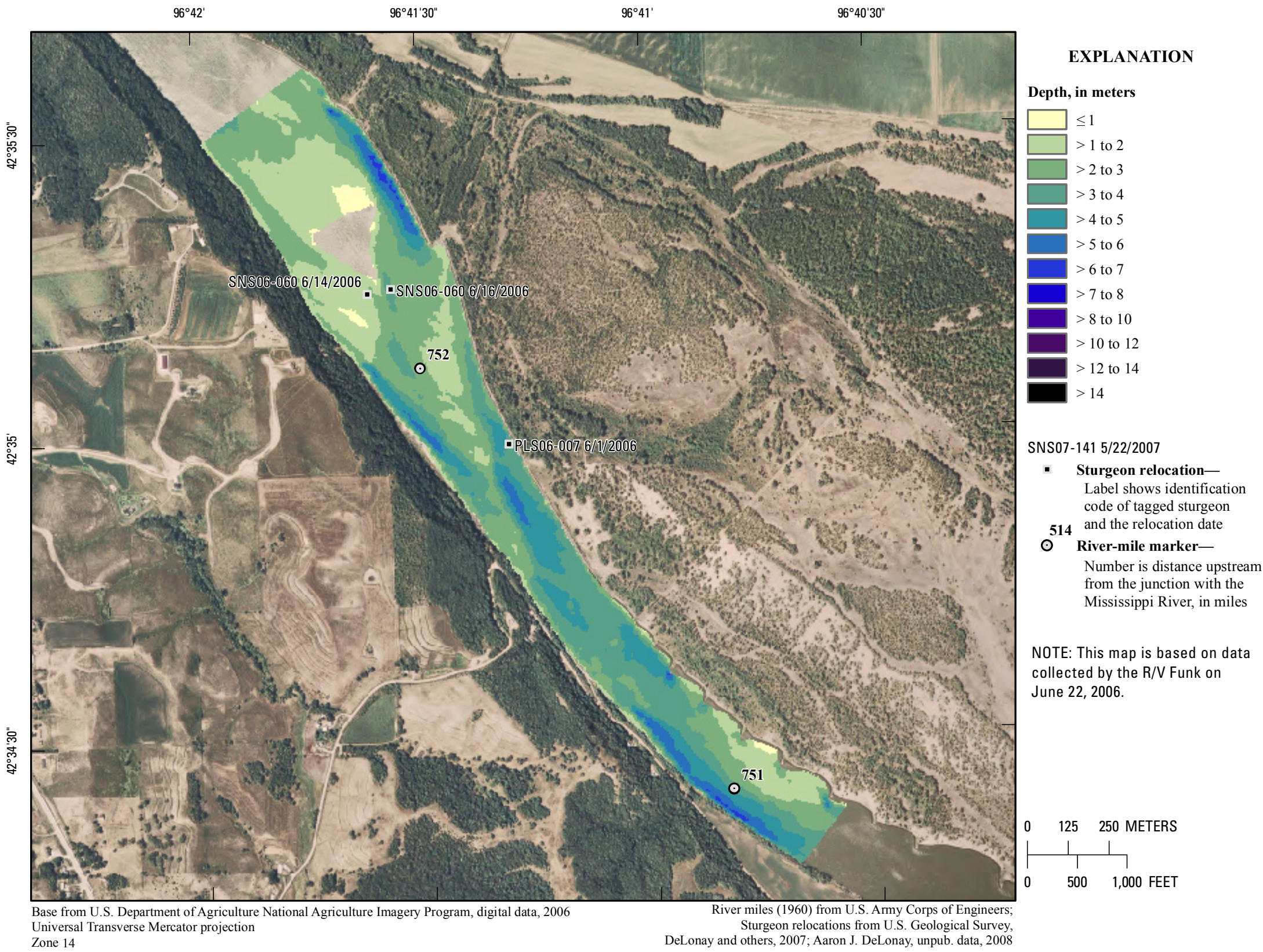

Figure 48. Map of depth based on data collected on June 22, 2006, in the vicinity of river mile 752. 


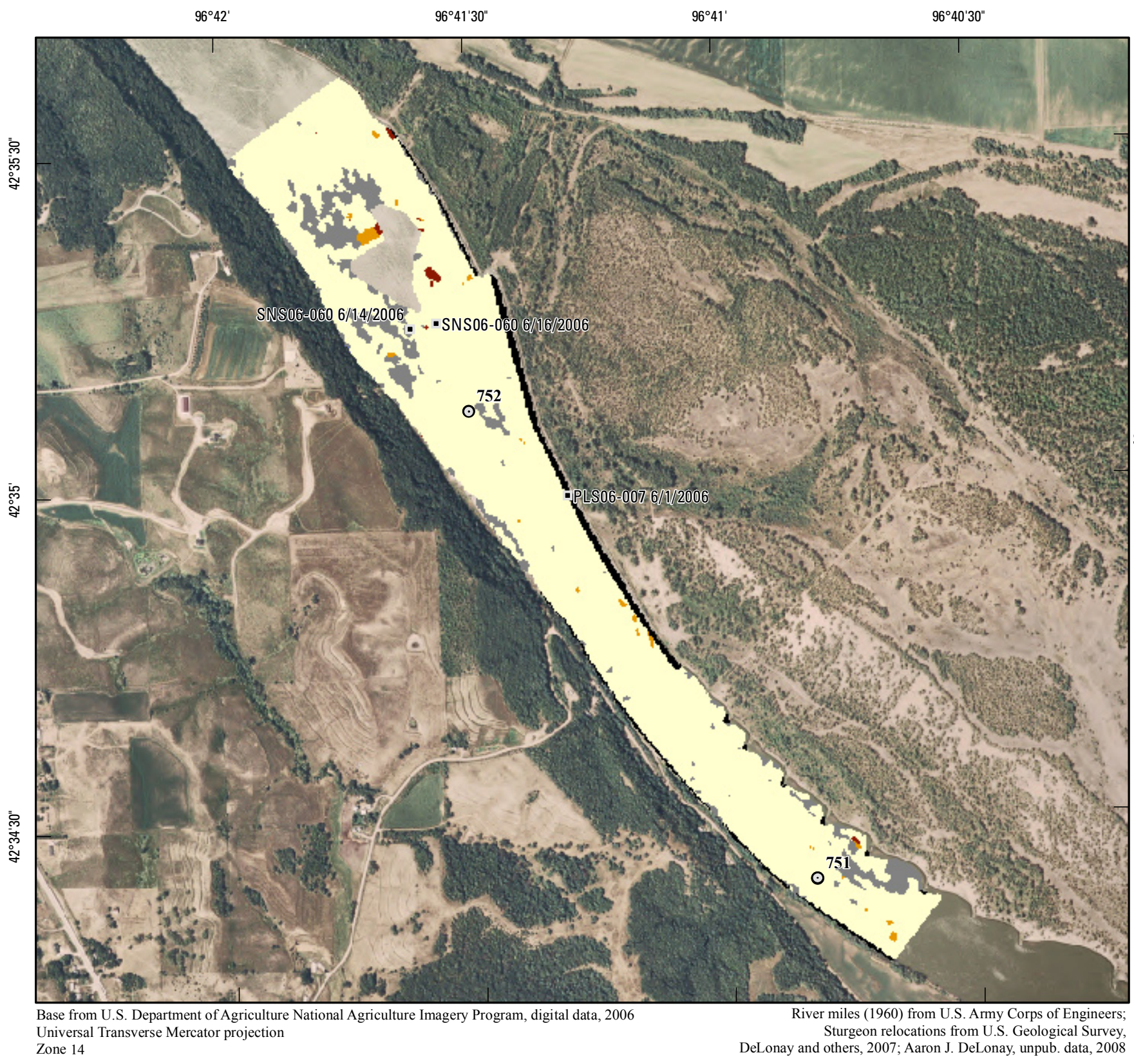

EXPLANATION

\section{Generalized substrate}

RoxAnn-derived classes

Revetment, gravel, hard sand Sand (dunes)

RoxAnn- and velocity-derived classes

Transporting san

Fine sediment (mud, silt)

Classes derived from aerial photographs

Engineered structures (rock) Bedrock

\section{SNS07-141 5/22/2007}

- Sturgeon relocationLabel shows identification code of tagged sturgeon and the relocation date

514

Number is distance upstream

from the junction with the

Mississippi River, in miles

NOTE: This map is based on data

collected by the R/V Funk on

June 22, 2006

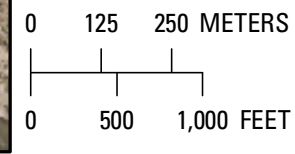

5001,000 FEET

Figure 49. Map of generalized substrate based on data collected on June 22, 2006, in the vicinity of river mile 752 . 


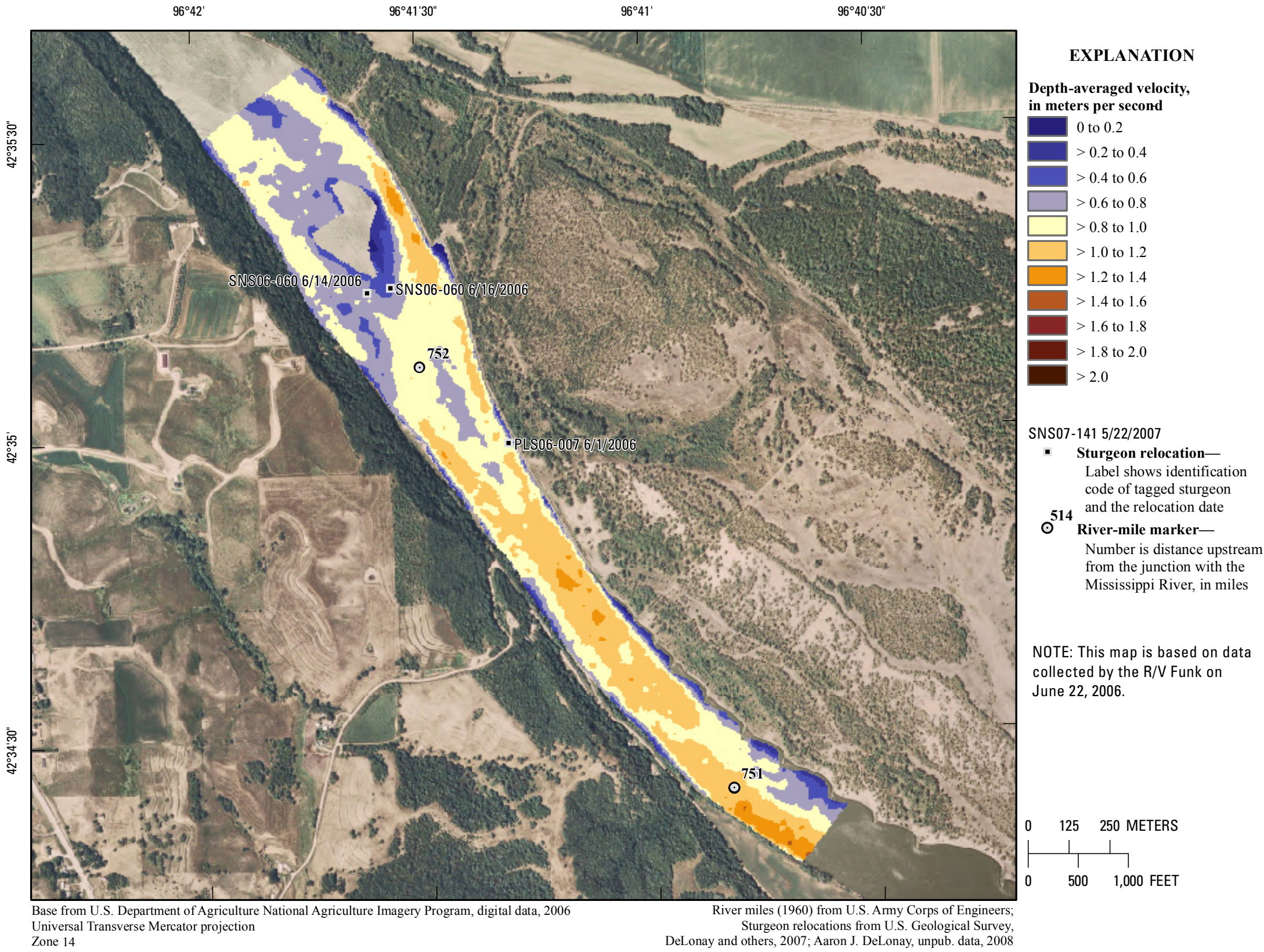

Figure 50. Map of depth-averaged velocity based on data collected on June 22, 2006, in the vicinity of river mile 752 . 


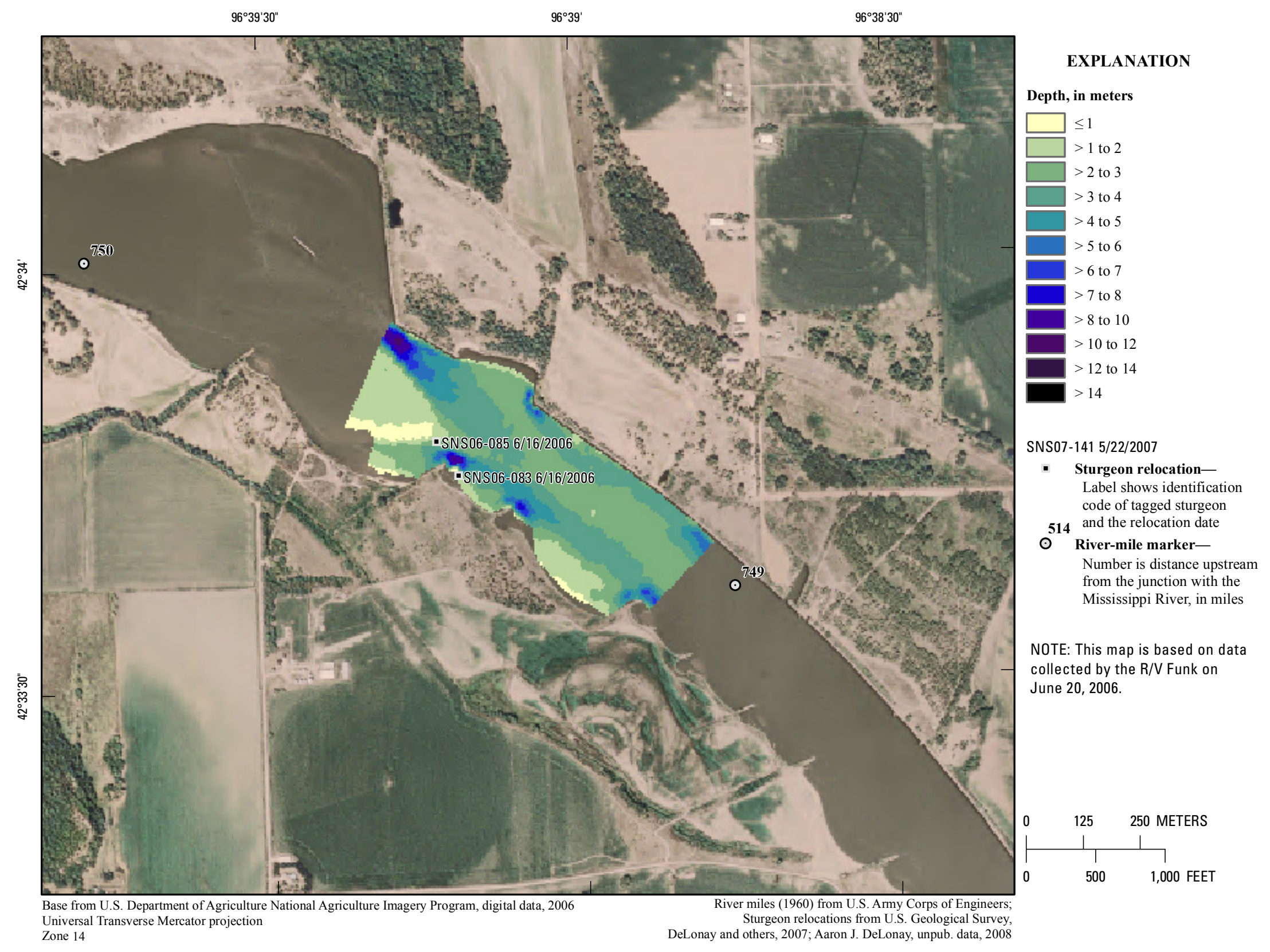

Figure 51. Map of depth based on data collected on June 20, 2006, in the vicinity of river mile 749 . 


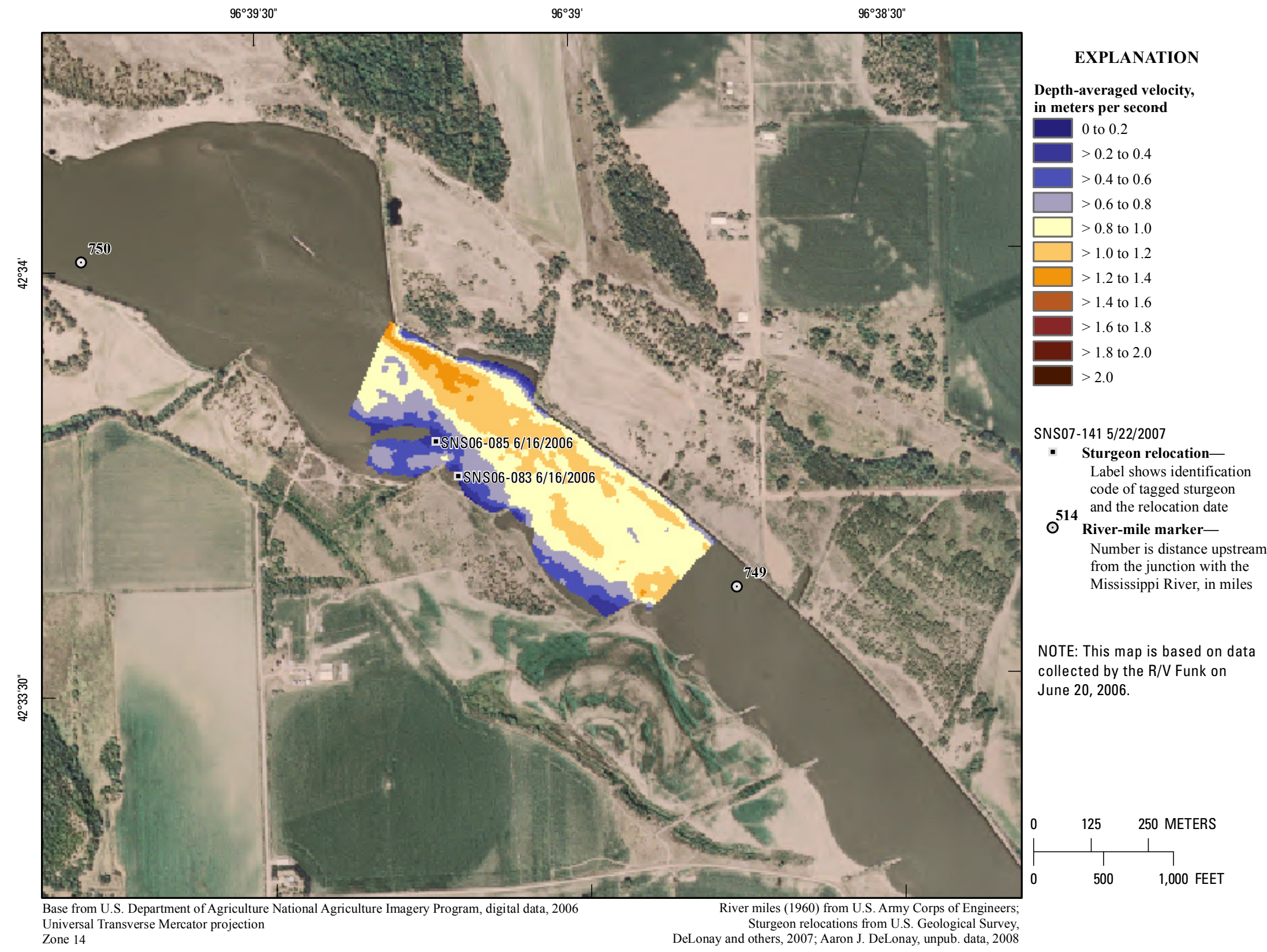

Figure 52. Map of depth-averaged velocity based on data collected on June 20, 2006, in the vicinity of river mile 749 . 


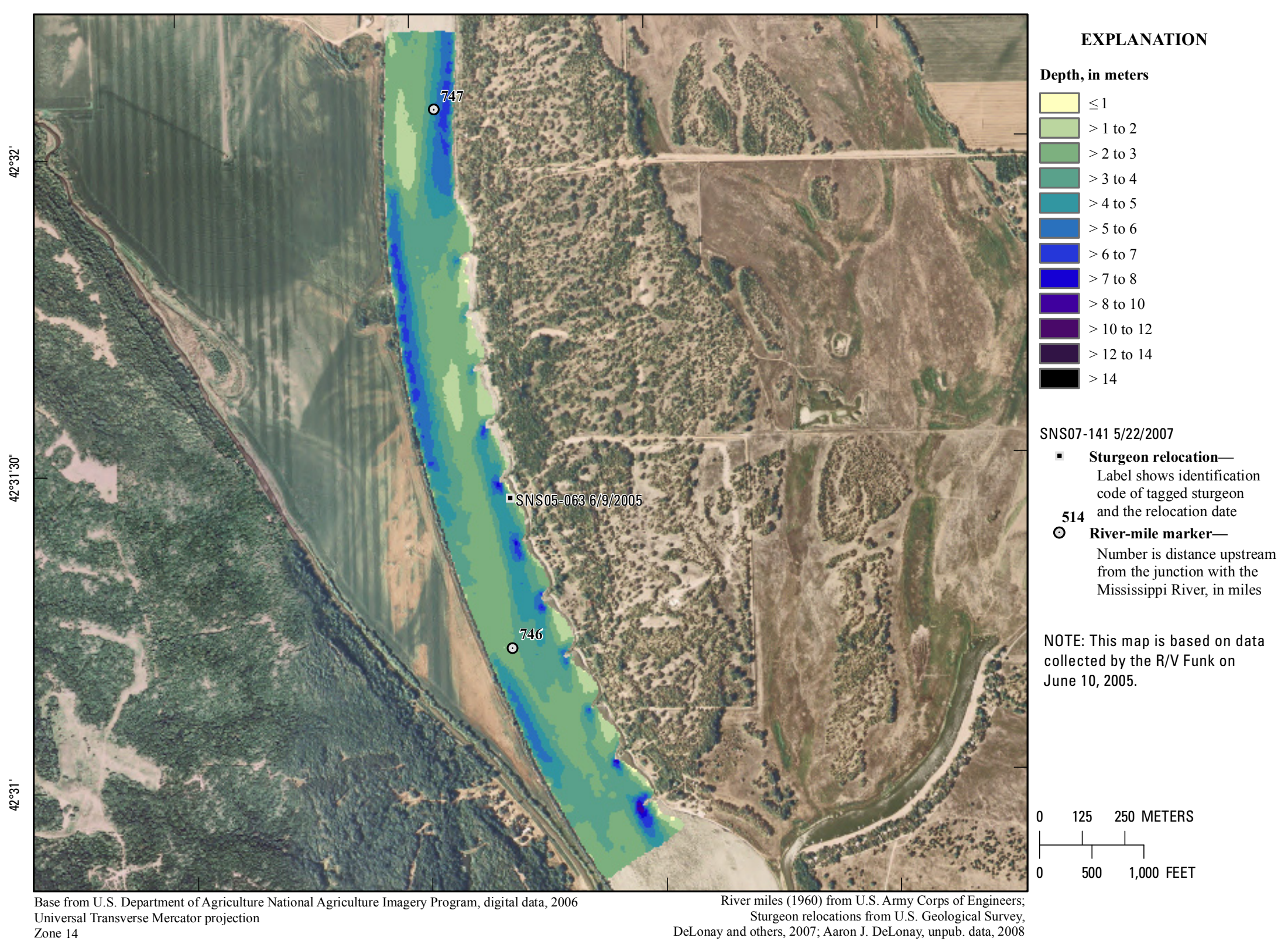

Figure 53. Map of depth based on data collected on June 10, 2005, in the vicinity of river mile 746 . 


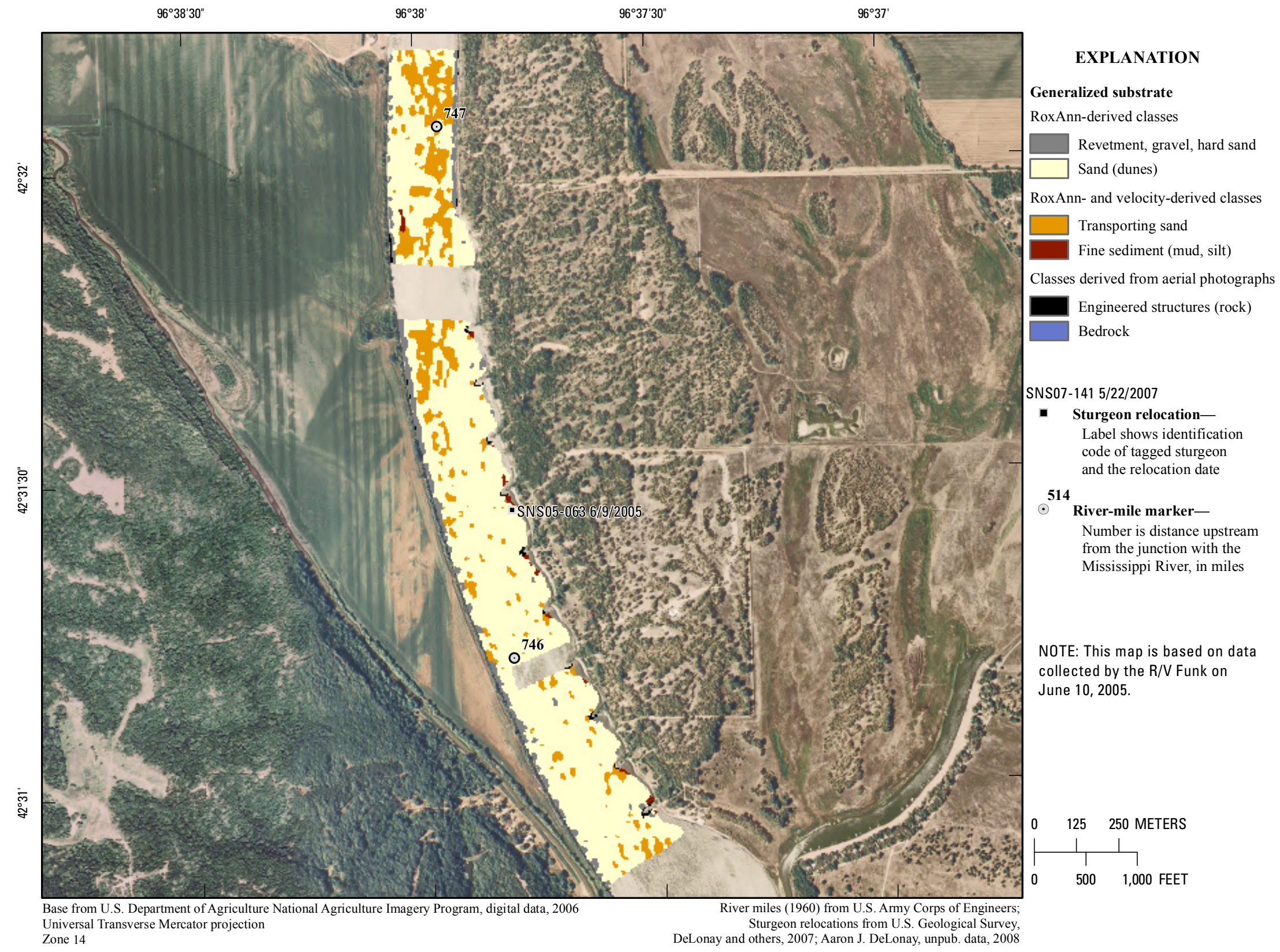

Figure 54. Map of generalized substrate based on data collected on June 10, 2005, in the vicinity of river mile 746 . 


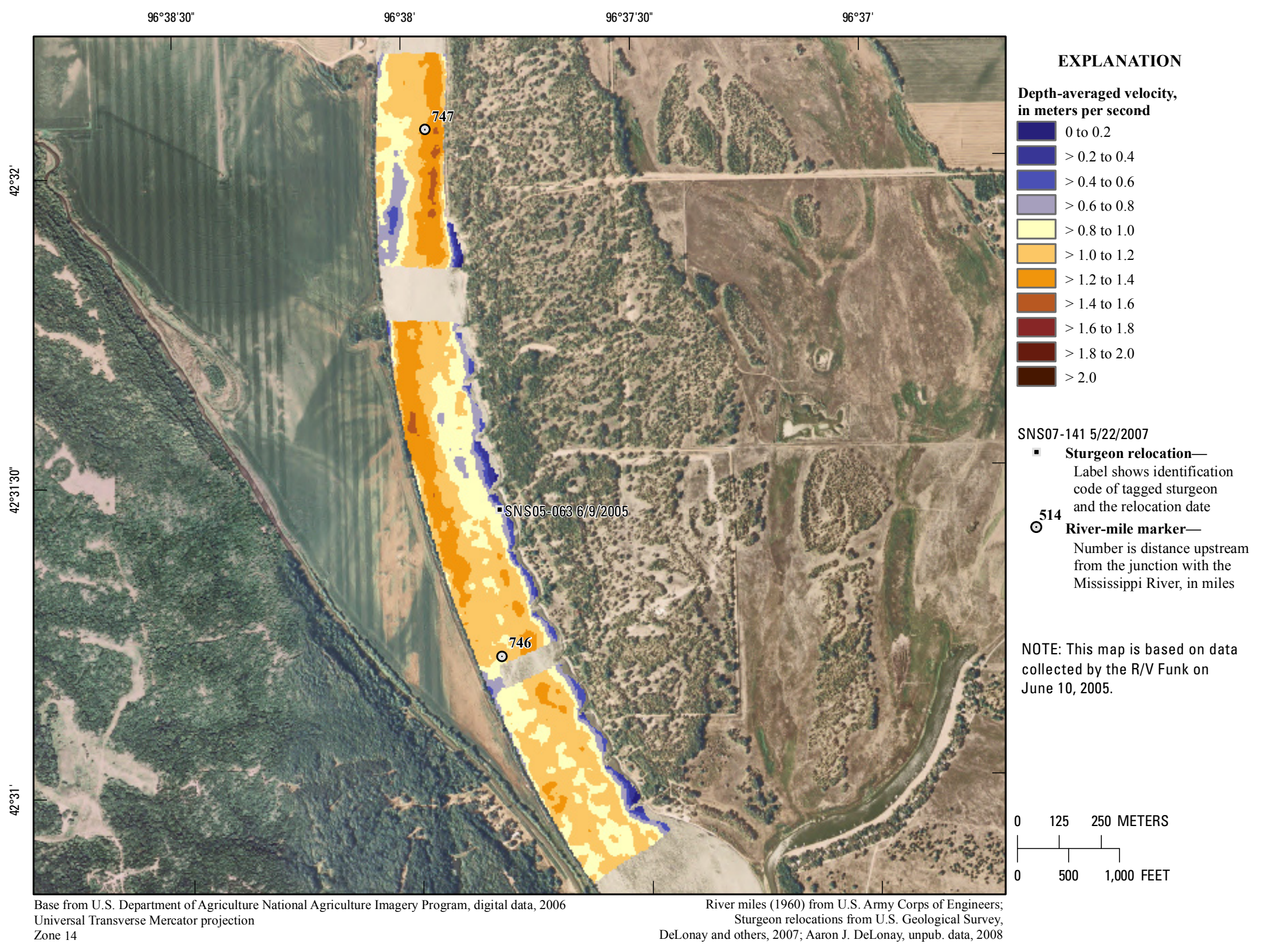

Figure 55. Map of depth-averaged velocity based on data collected on June 10, 2005, in the vicinity of river mile 746 . 


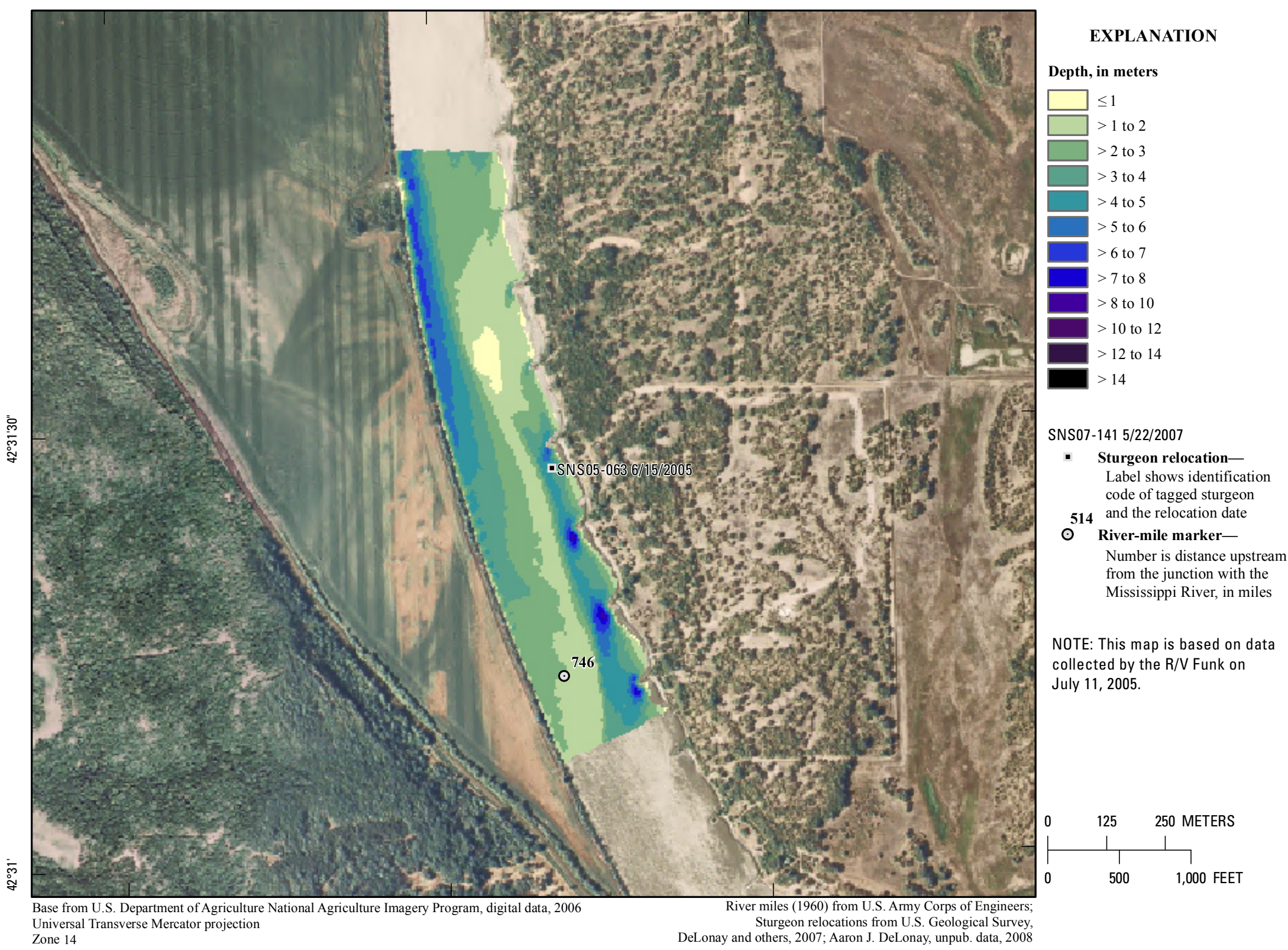
Universal Transverse Mercator projection Sturgeon relocations from U.S. Geological Survey,
DeLonay and others, 2007; Aaron J. DeLonay, unpub. data, 2008 


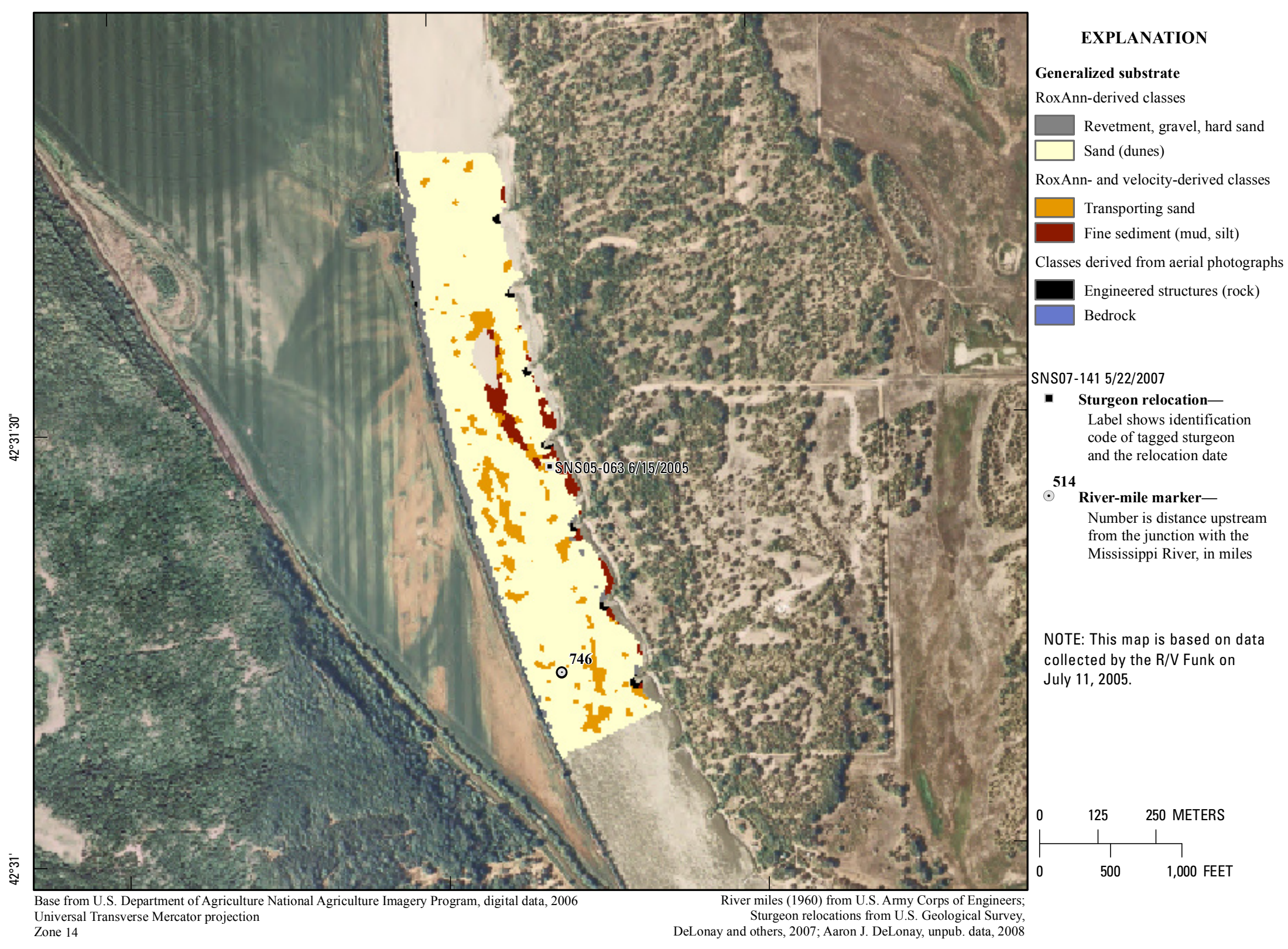

Figure 57. Map of generalized substrate based on data collected on July 11, 2005, in the vicinity of river mile 746 . 


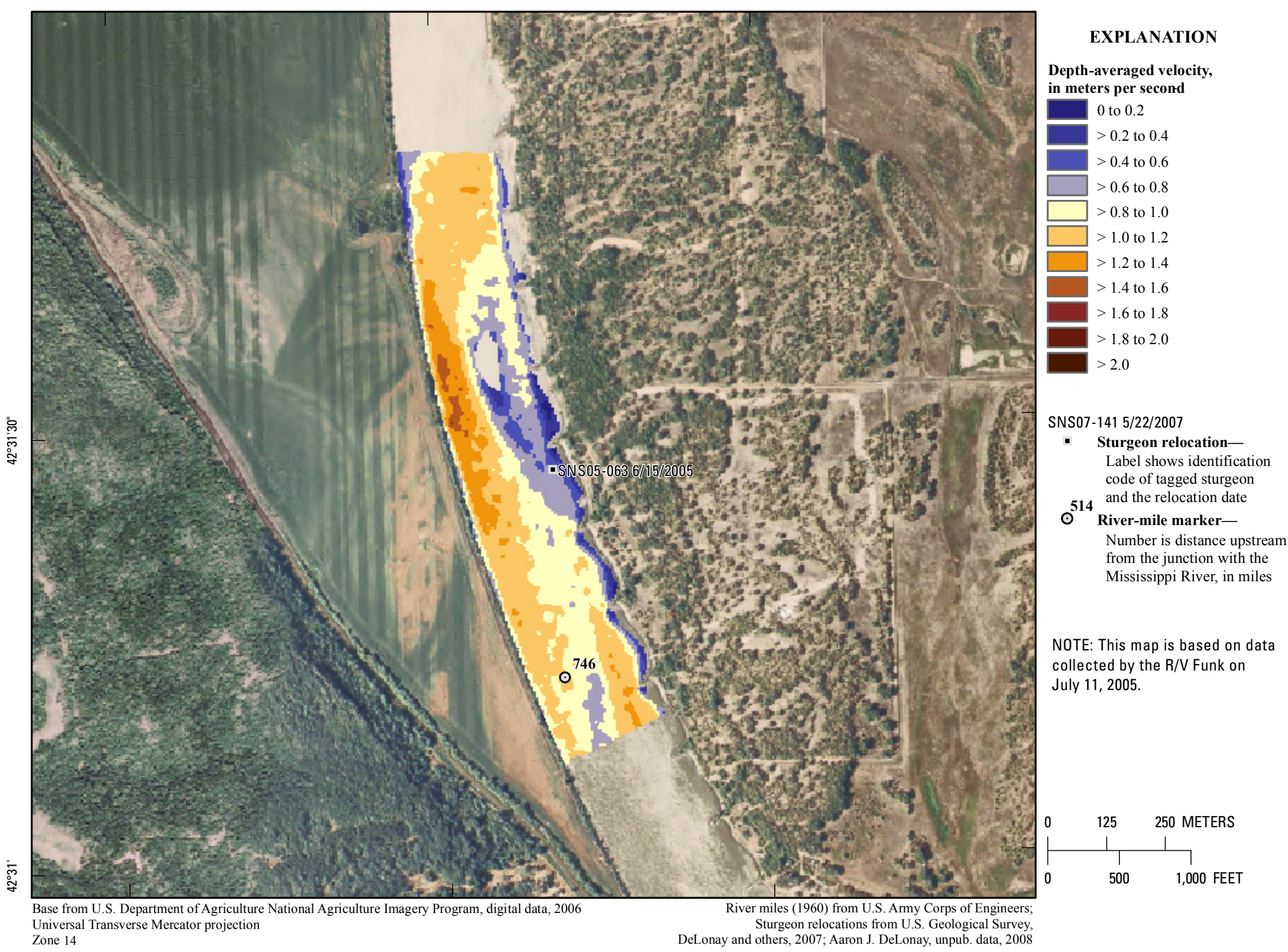
Universal Transverse Mercator projection Sturgeon relocations from U.S. Geological Survey,
DeLonay and others, 2007; Aaron J. DeLonay, unpub. data, 2008 


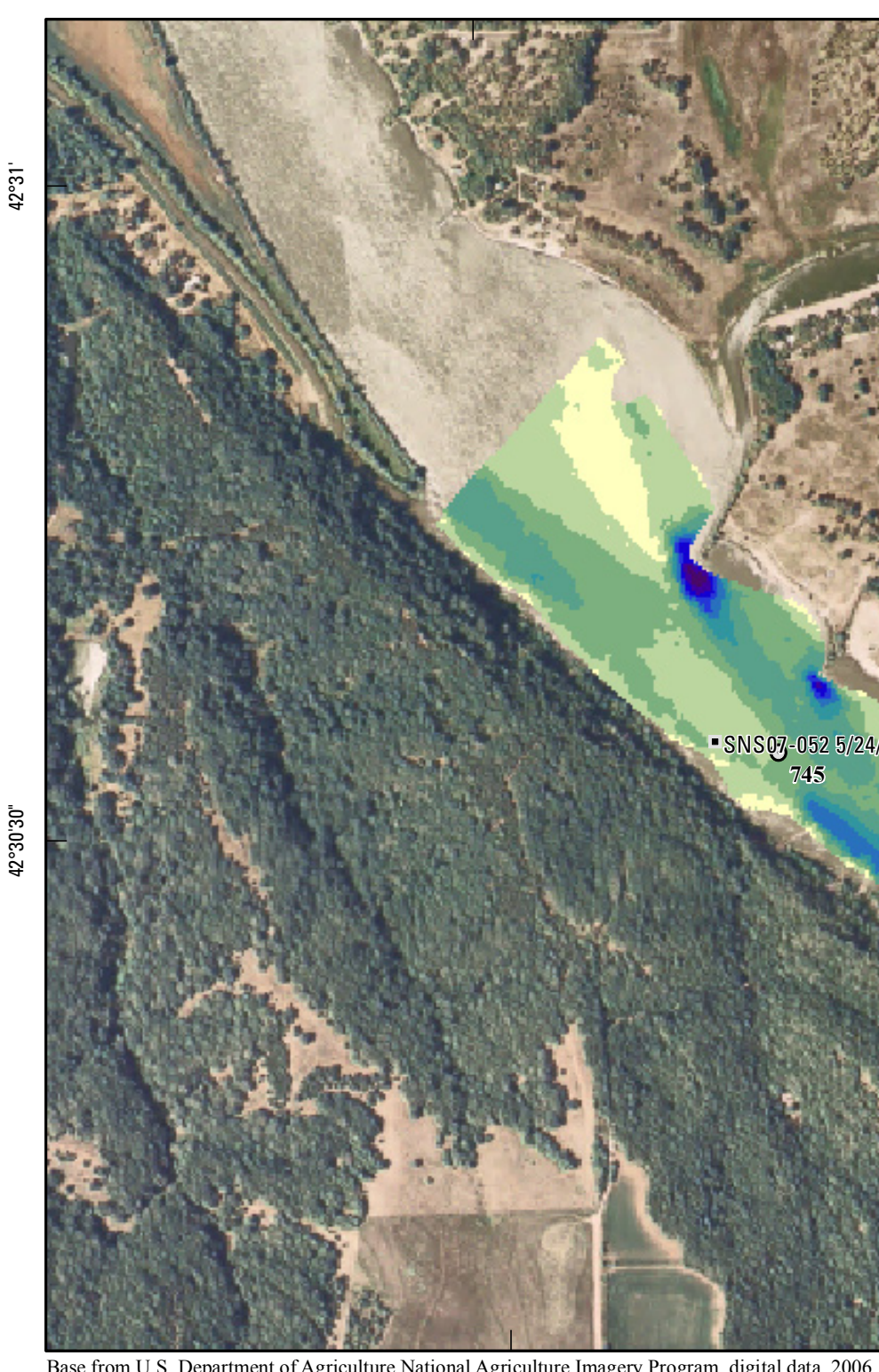

Base from U.S. Department of Agriculture National Agriculture Imagery Program, digital data, 2006 Universal Transverse Mercator projection Zone 14

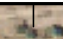

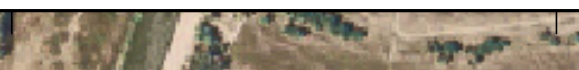
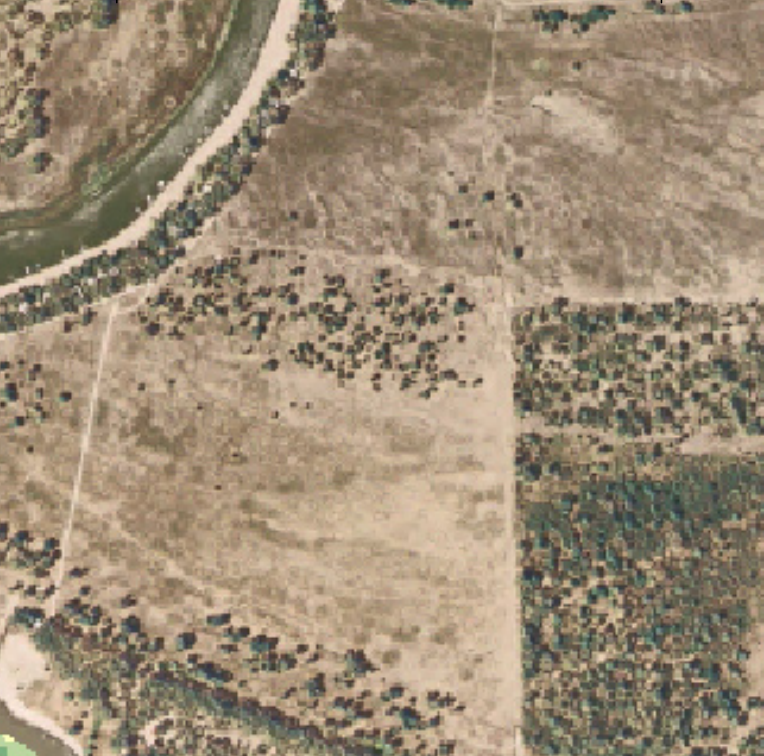

$(2 x+5=$

EXPLANATION

Depth, in meters

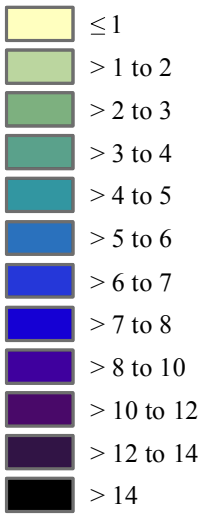

SNS07-141 5/22/2007

- Sturgeon relocationLabel shows identification code of tagged sturgeon 514 and the relocation date

$\odot$ River-mile markerNumber is distance upstream from the junction with the Mississippi River, in miles

NOTE: This map is based on data collected by the R/V Funk on May 25, 2007.

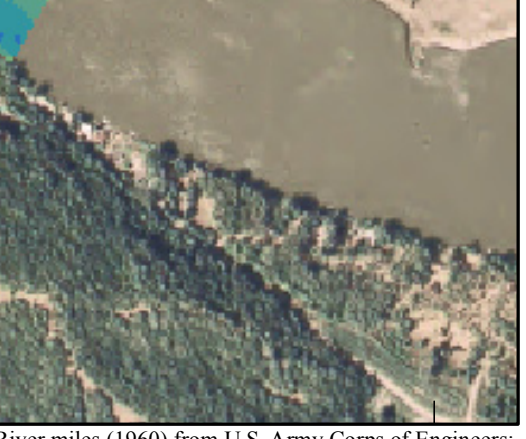

River miles (1960) from U.S. Army Corps of Engineer

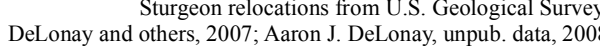

Figure 59. Map of depth based on data collected on May 25, 2007, in the vicinity of river mile 745. 


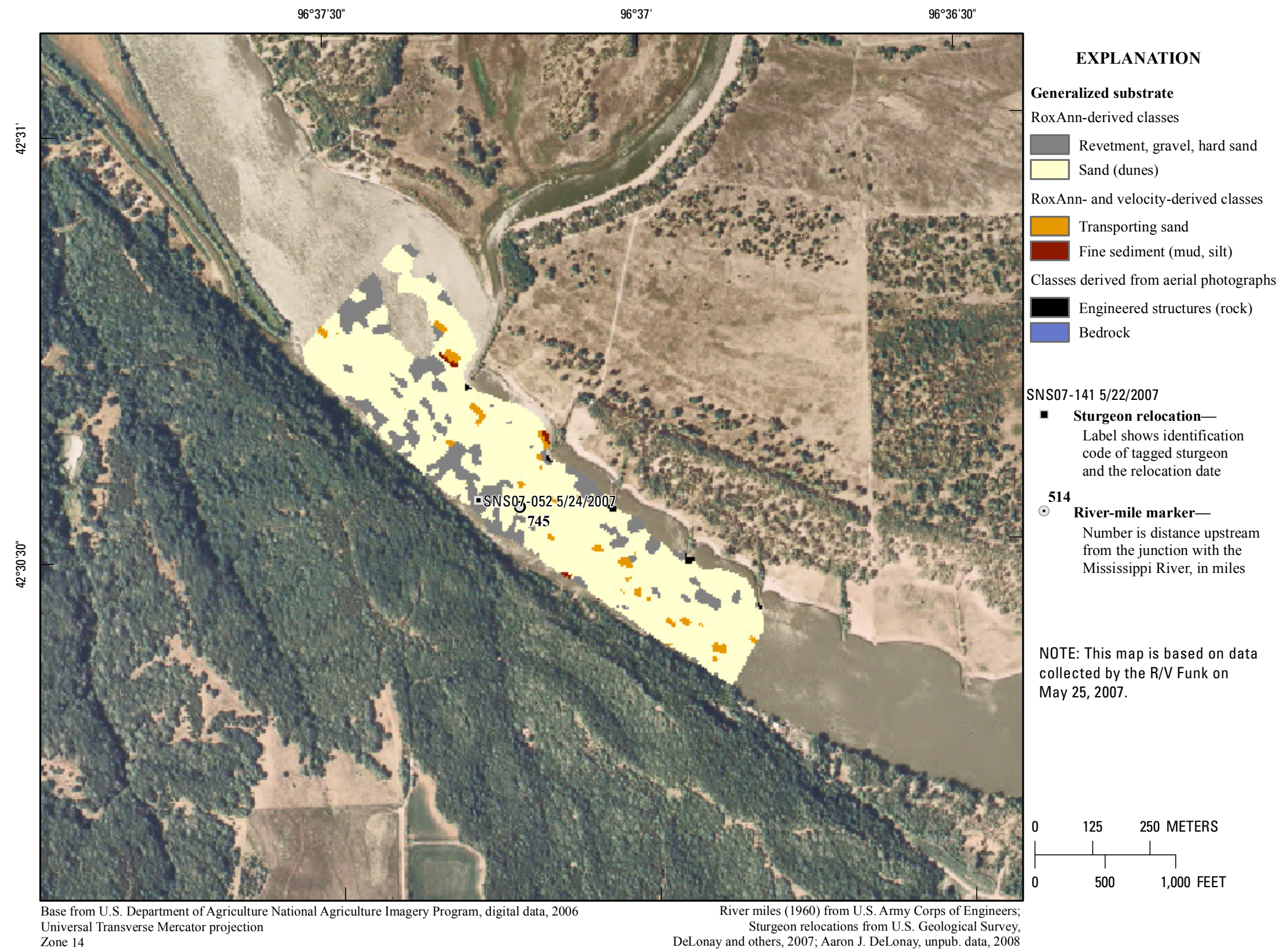

Figure 60. Map of generalized substrate based on data collected on May 25, 2007, in the vicinity of river mile 745. 


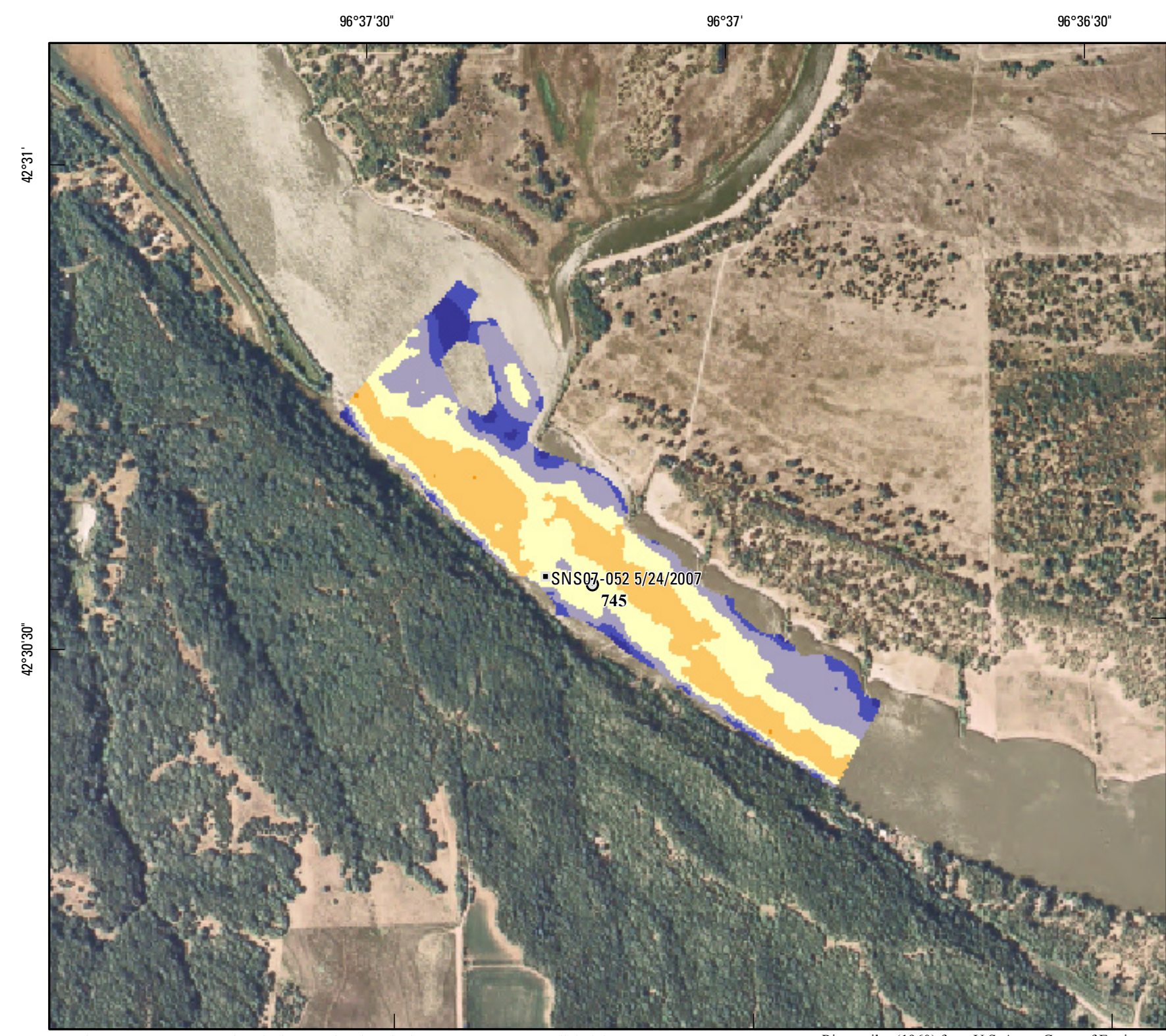

EXPLANATION

Depth-averaged velocity,

in meters per second

0 to 0.2

$>0.2$ to 0.4

$>0.4$ to 0.6

$>0.6$ to 0.8

$>0.8$ to 1.0

$>1.0$ to 1.2

$>1.2$ to 1.4

$>1.4$ to 1.6

$>1.6$ to 1.8

$>1.8$ to 2.0

$>2.0$

SNS07-141 5/22/2007

- Sturgeon relocationLabel shows identification code of tagged sturgeon

514 and the relocation date

River-mile marker-

Number is distance upstrean from the junction with the

Mississippi River, in miles

NOTE: This map is based on data

collected by the R/V Funk on

May 25, 2007.

Base from U.S. Department of Agriculture National Agriculture Imagery Program, digital data, 2006

Universal Transverse Mercator projection

River miles (1960) from U.S. Army Corps of Engineer Sturgeon relocations from U.S. Geological Survey,
DeLonay and others, 2007; Aaron J. DeLonay, unpub. data, 2008

\section{$\begin{array}{ll}125 & 250 \text { METERS }\end{array}$ \\ $\prod_{500}^{1}$ \\ 1,000 FEET}

Figure 61. Map of depth-averaged velocity based on data collected on May 25, 2007, in the vicinity of river mile 745. 
$96^{\circ} 37^{\prime} 30^{\prime \prime}$

$96^{\circ} 37^{\prime}$

$96^{\circ} 36^{\prime} 30^{\prime \prime}$

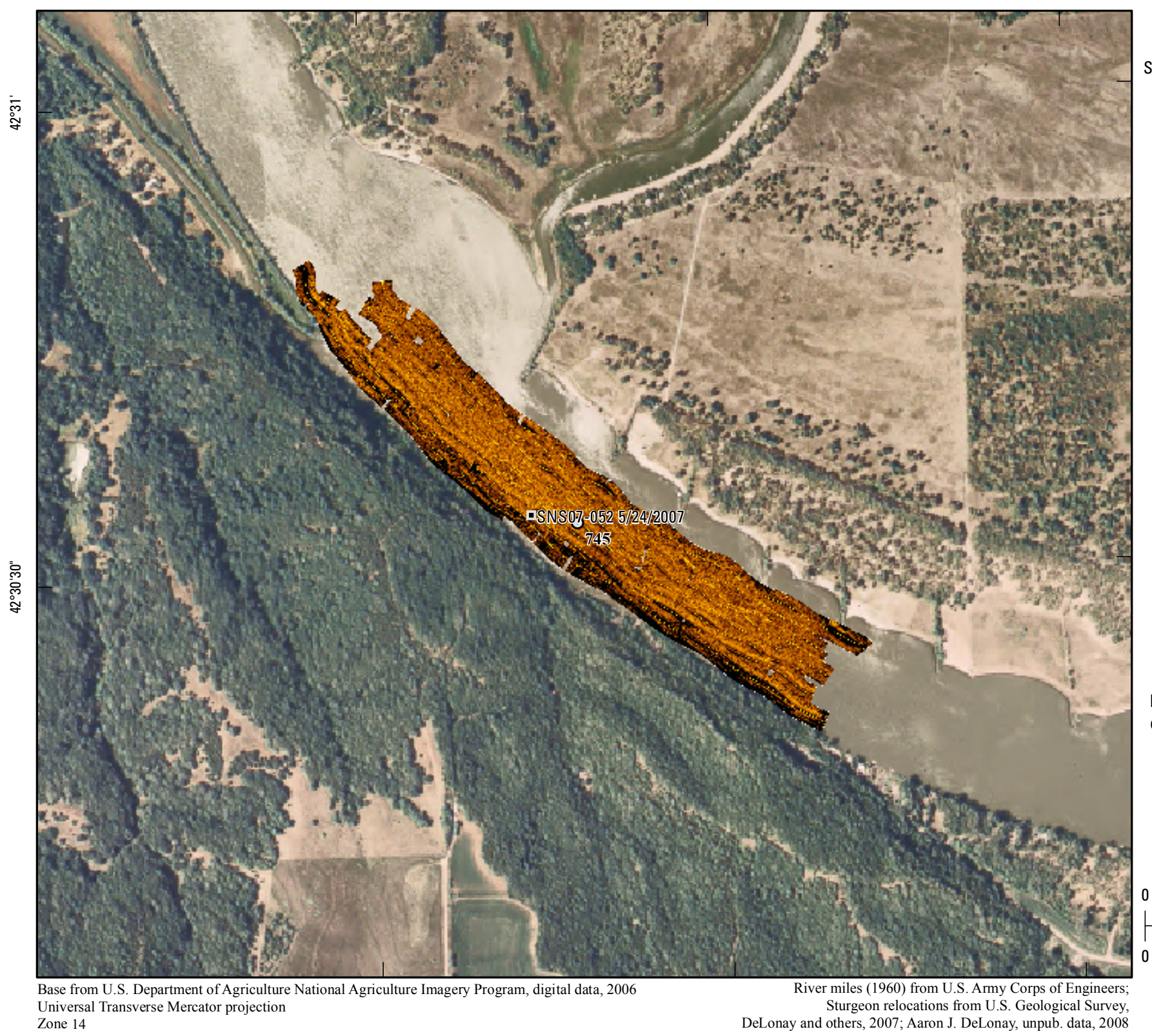

EXPLANATION

SNS07-141 5/22/2007

- Sturgeon relocation-

Label shows identification code of tagged sturgeon 514 and the relocation date

$\odot$ River-mile markerNumber is distance upstream from the junction with the Mississippi River, in miles

NOTE: This map is based on data collected on May 25, 2007.

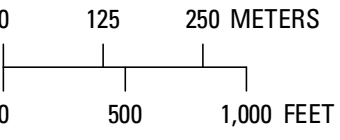




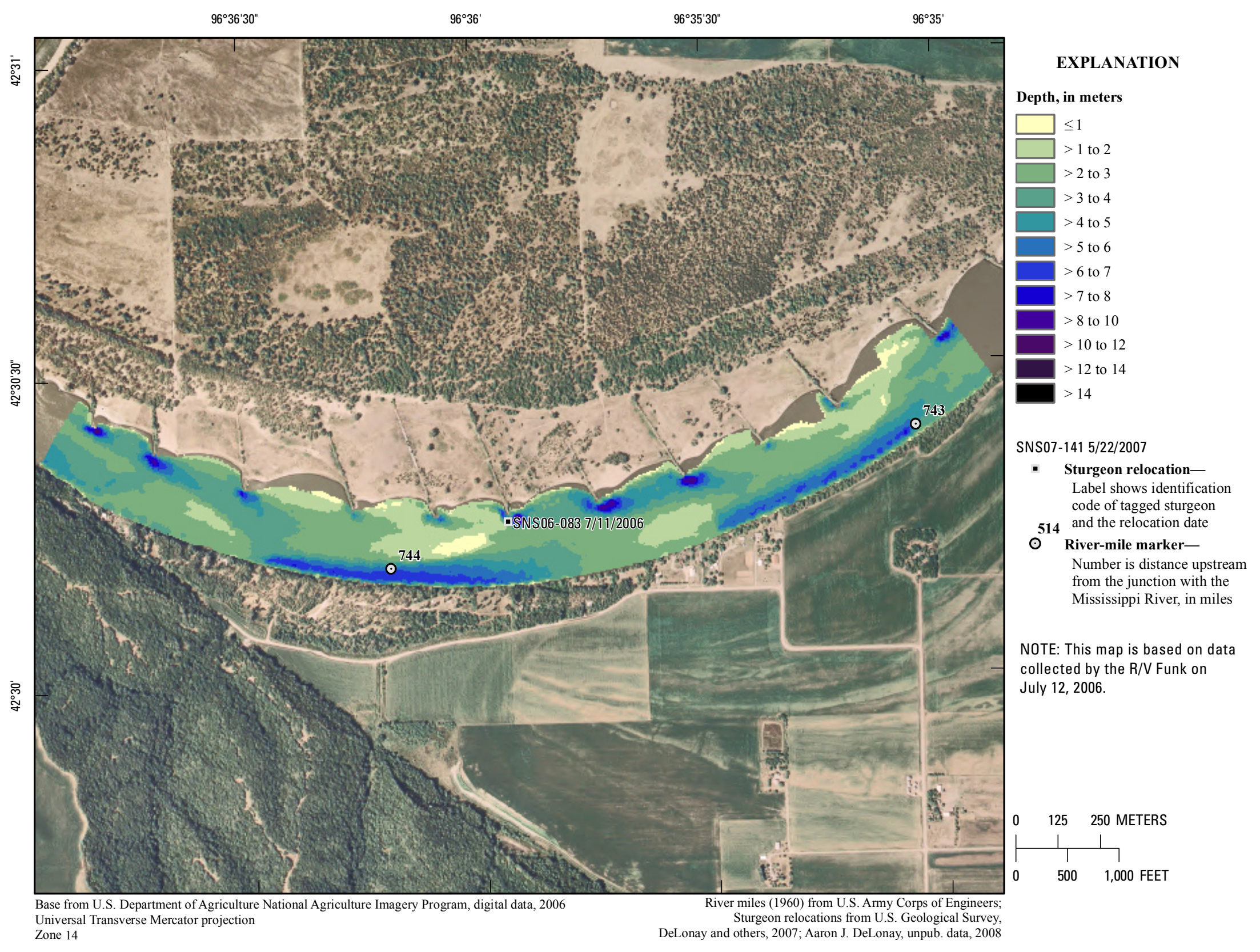

Figure 63. Map of depth based on data collected on July 12, 2006, in the vicinity of river mile 744 . 


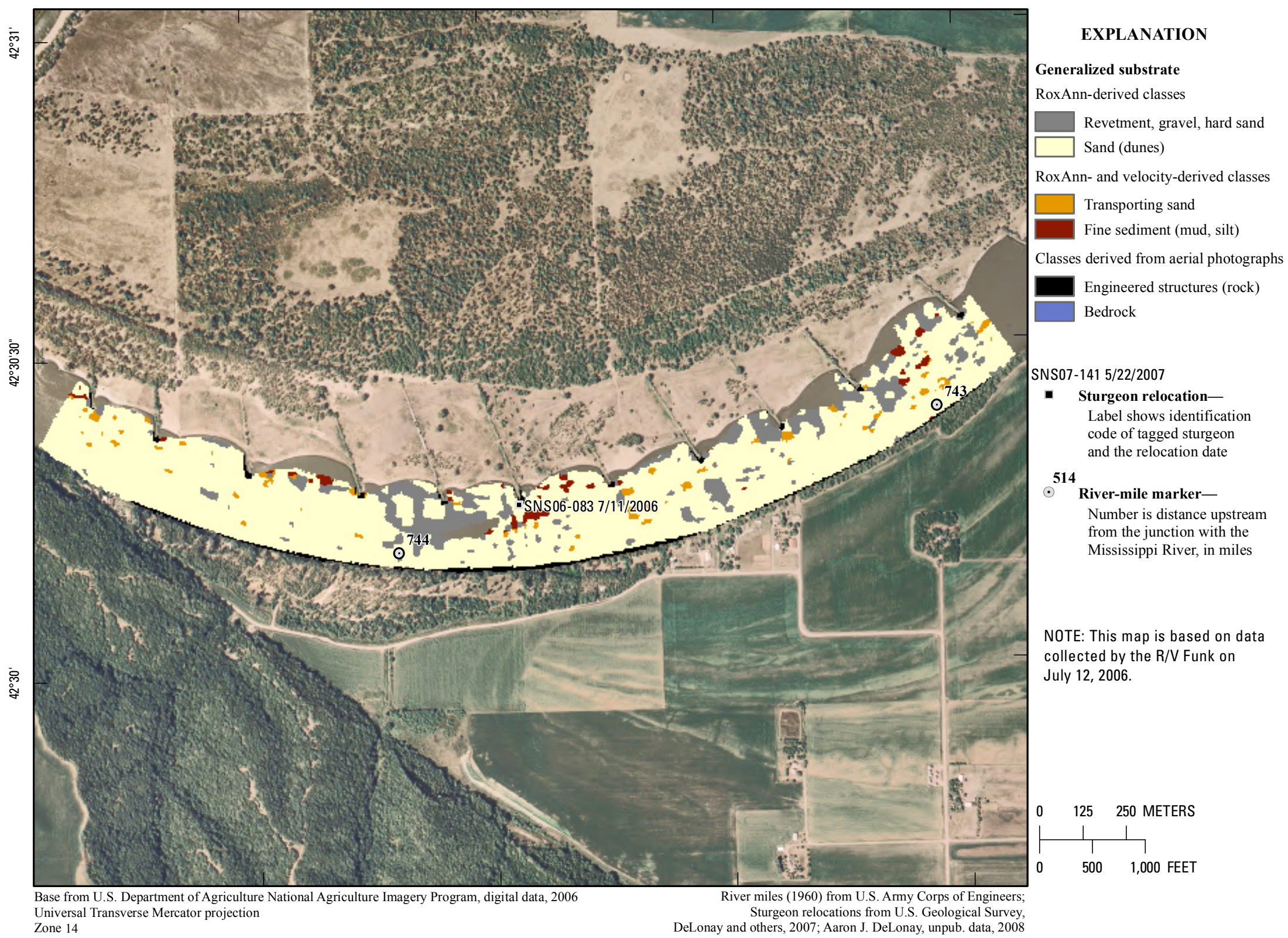

Figure 64. Map of generalized substrate based on data collected on July 12, 2006, in the vicinity of river mile 744 . 


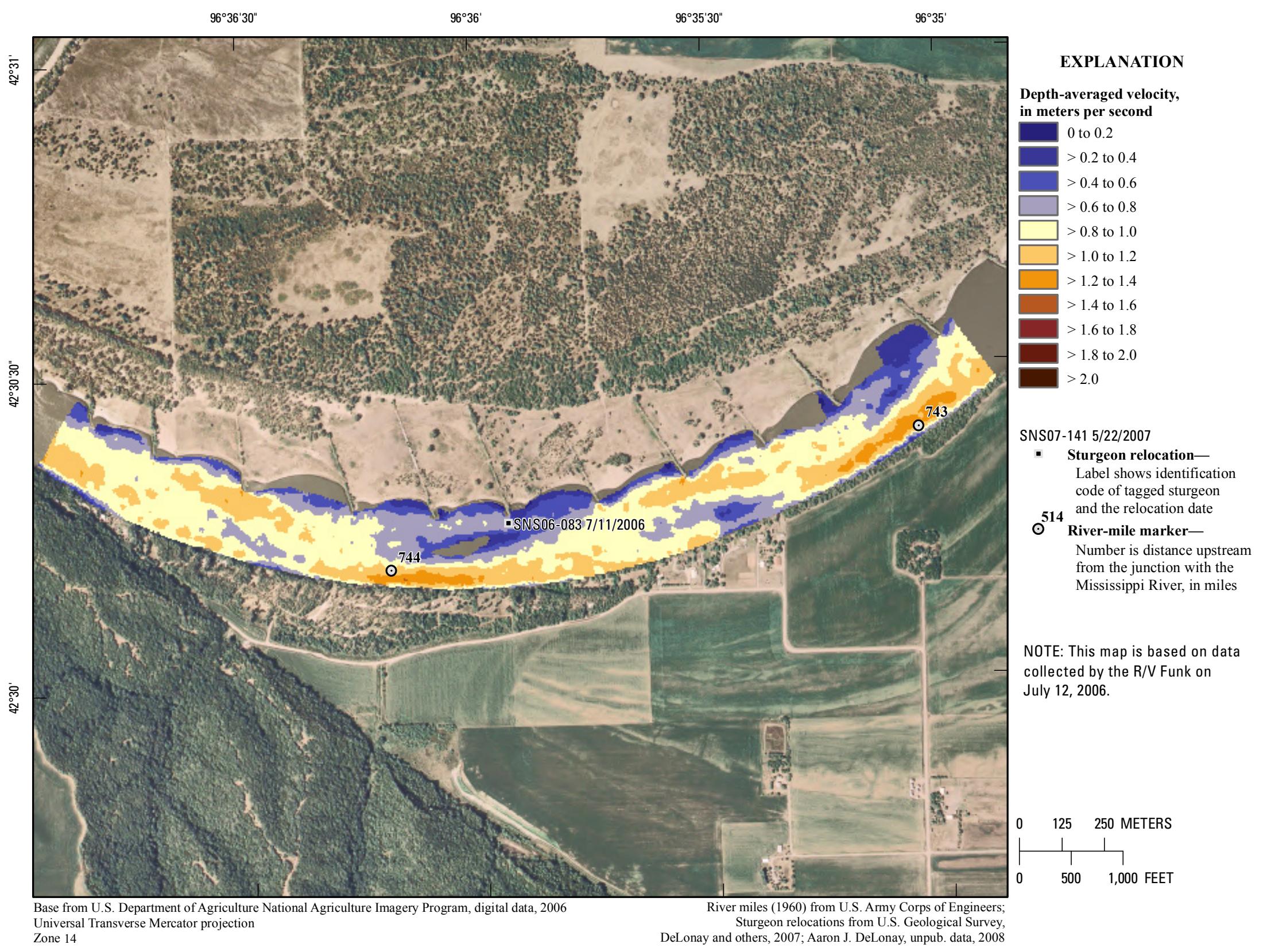

Figure 65. Map of depth-averaged velocity based on data collected on July 12, 2006, in the vicinity of river mile 744 . 


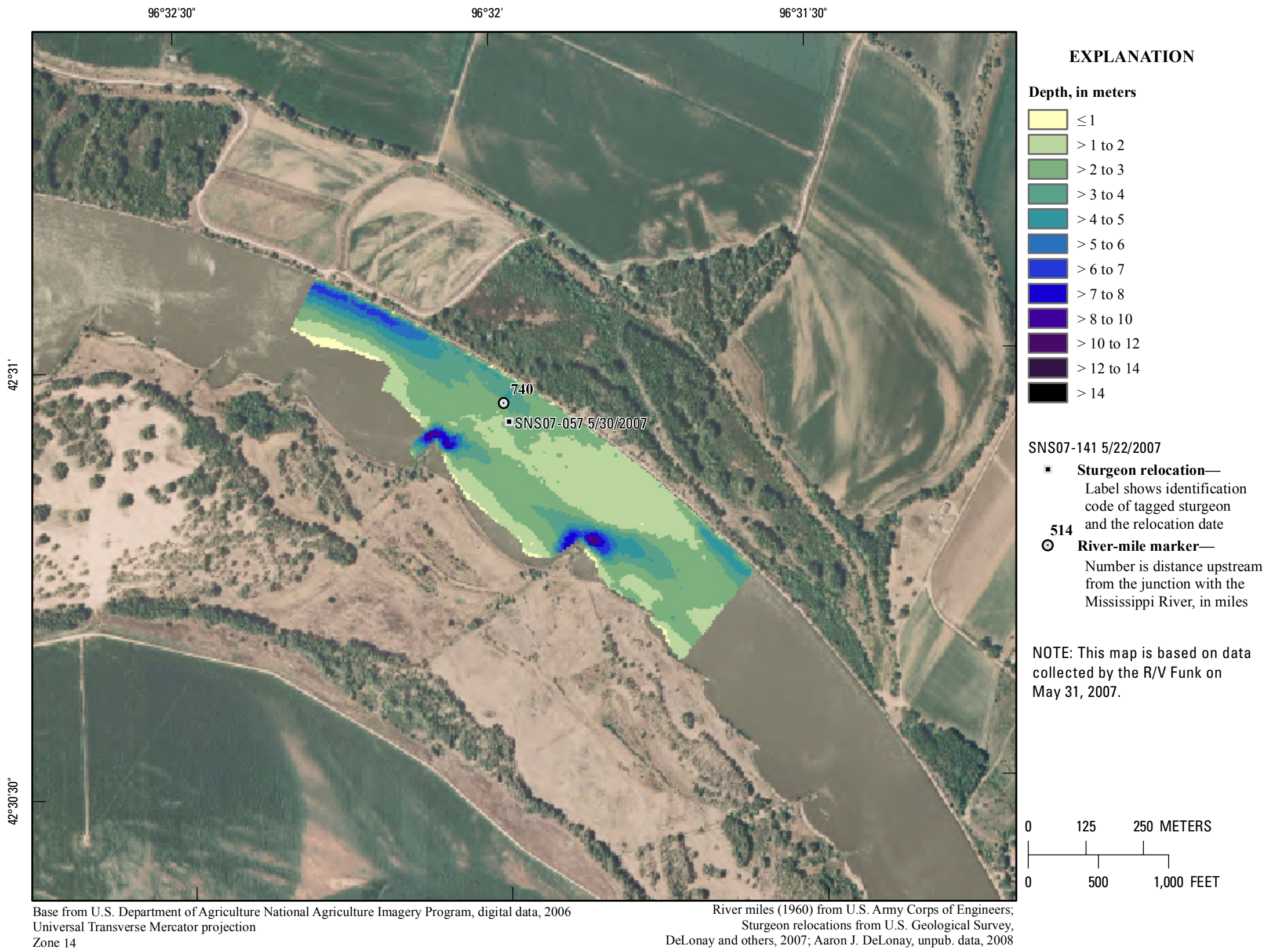

Figure 66. Map of depth based on data collected on May 31, 2007, in the vicinity of river mile 740 . 


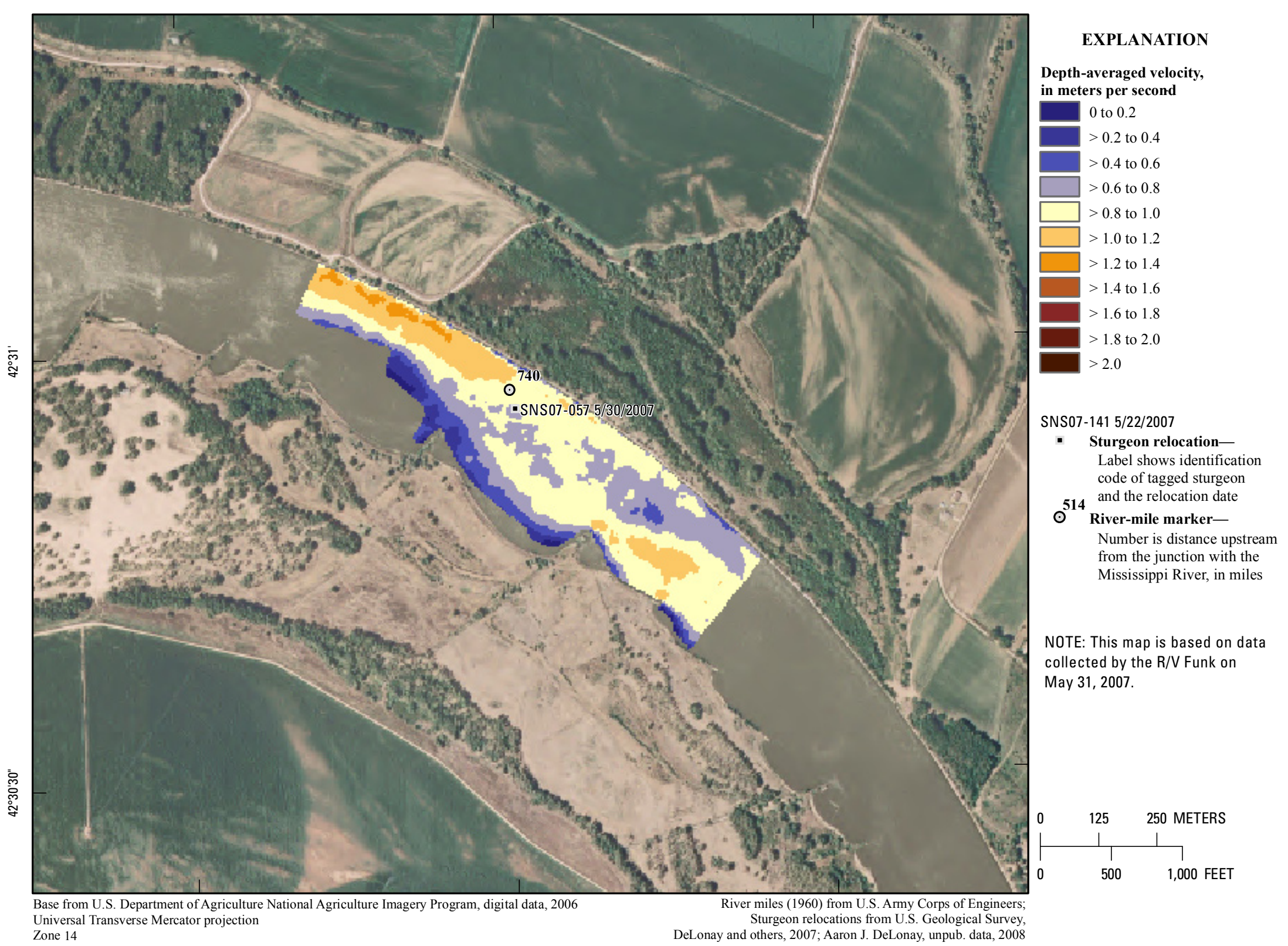

Figure 67. Map of depth-averaged velocity based on data collected on May 31, 2007, in the vicinity of river mile 740. 


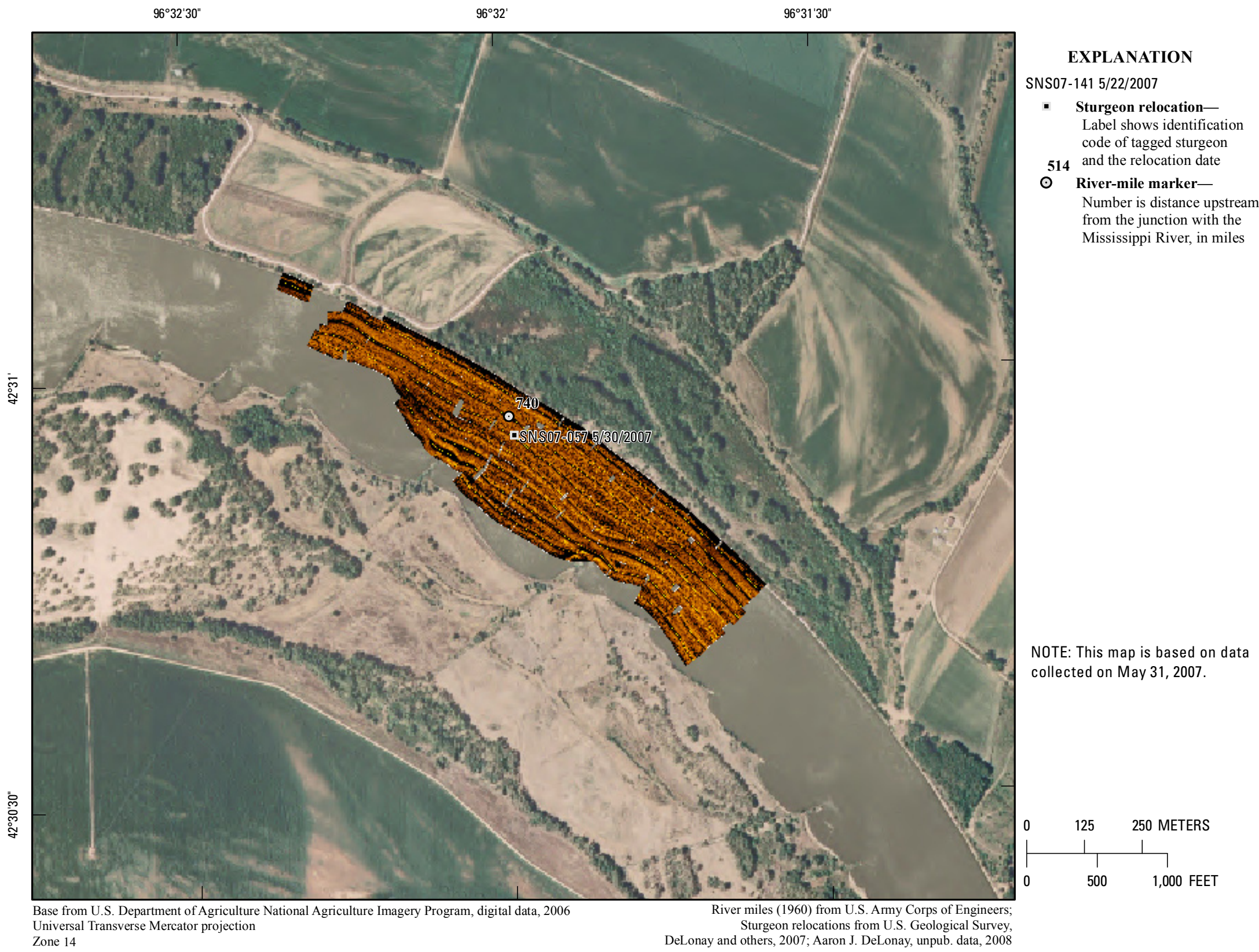

Figure 68. Map of side-scan sonar imagery based on data collected on May 31, 2007, in the vicinity of river mile 740. 


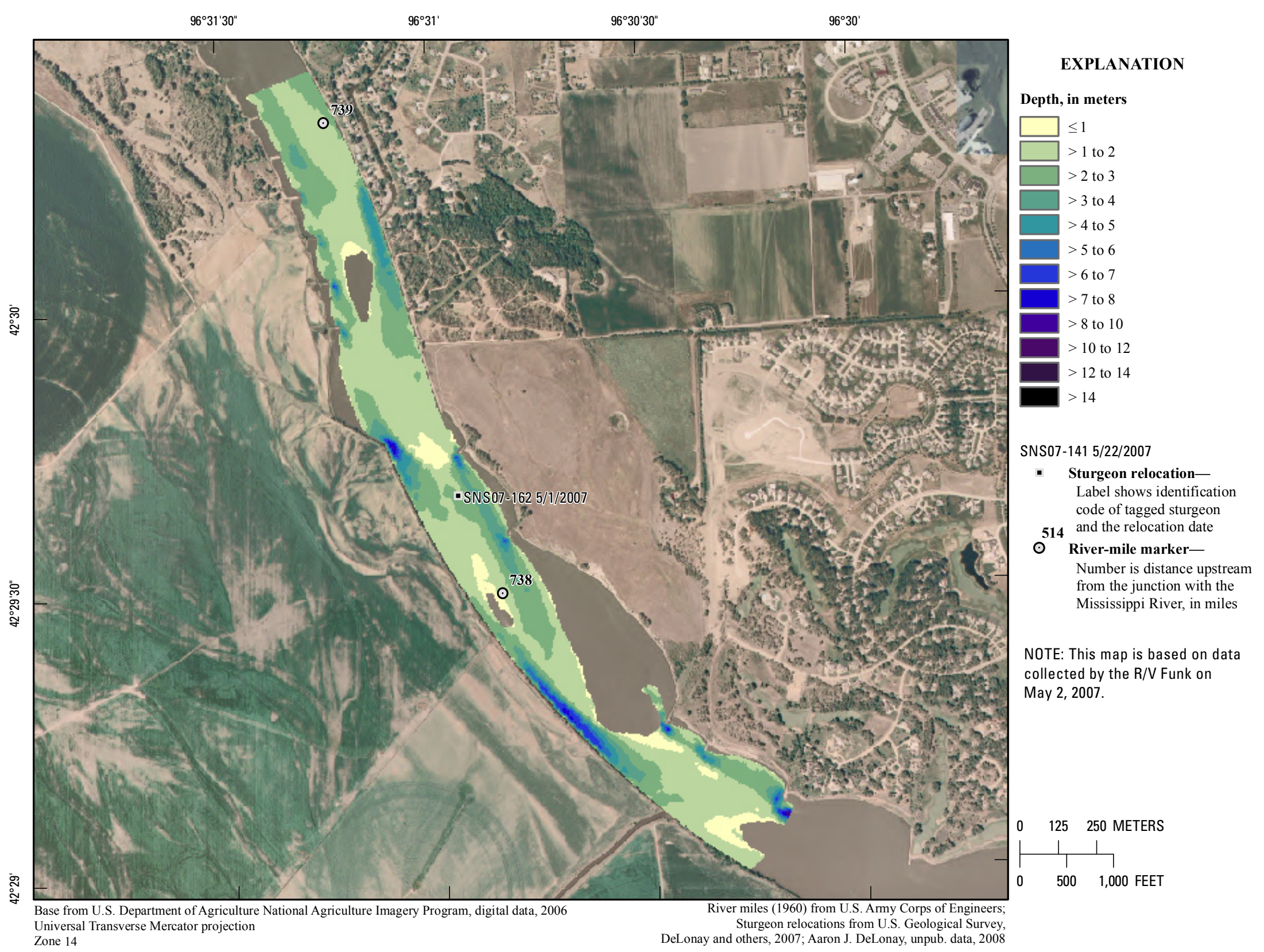

Figure 69. Map of depth based on data collected on May 2, 2007, in the vicinity of river mile 738. 


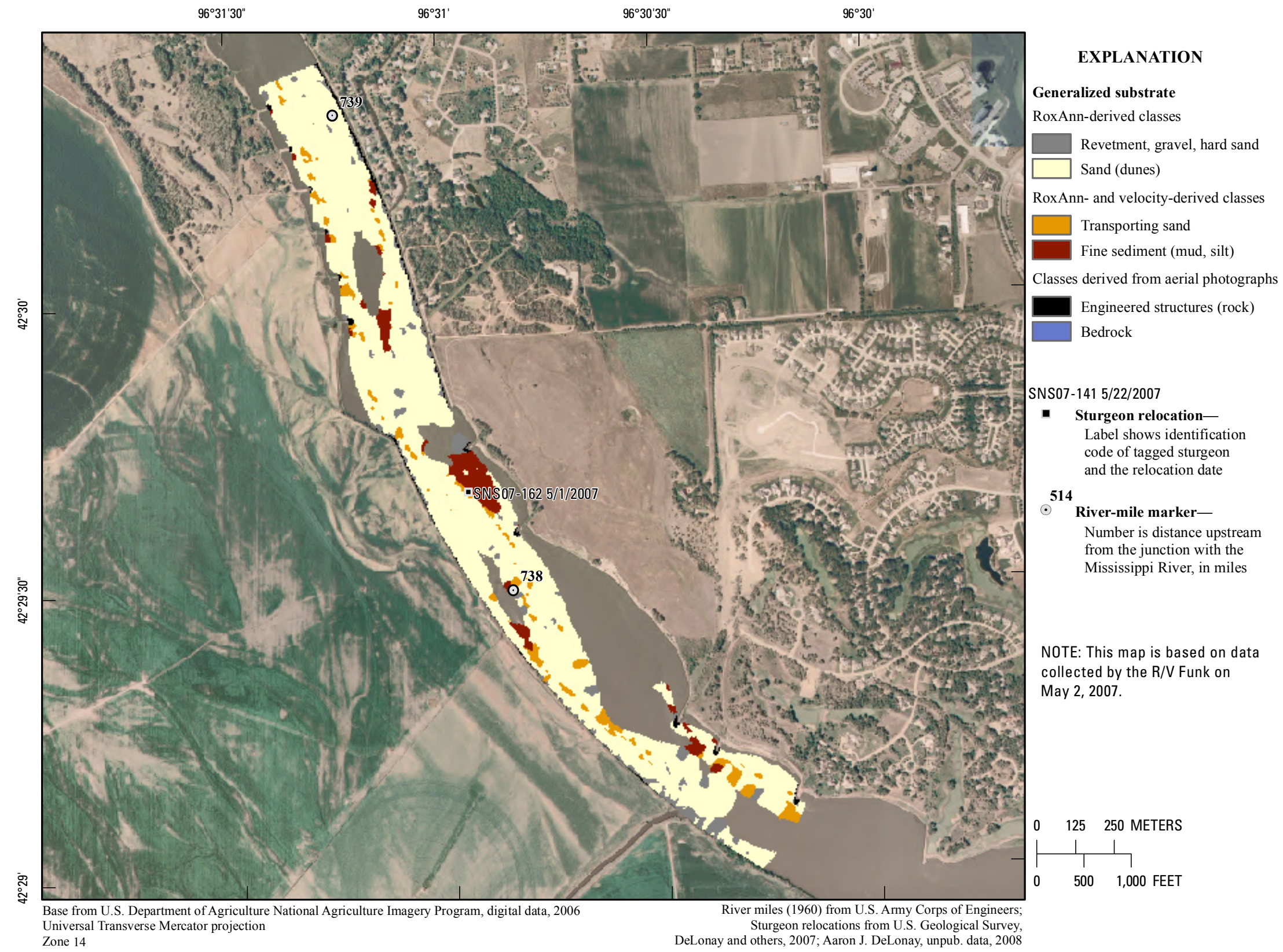

Figure 70. Map of generalized substrate based on data collected on May 2, 2007, in the vicinity of river mile 738. 


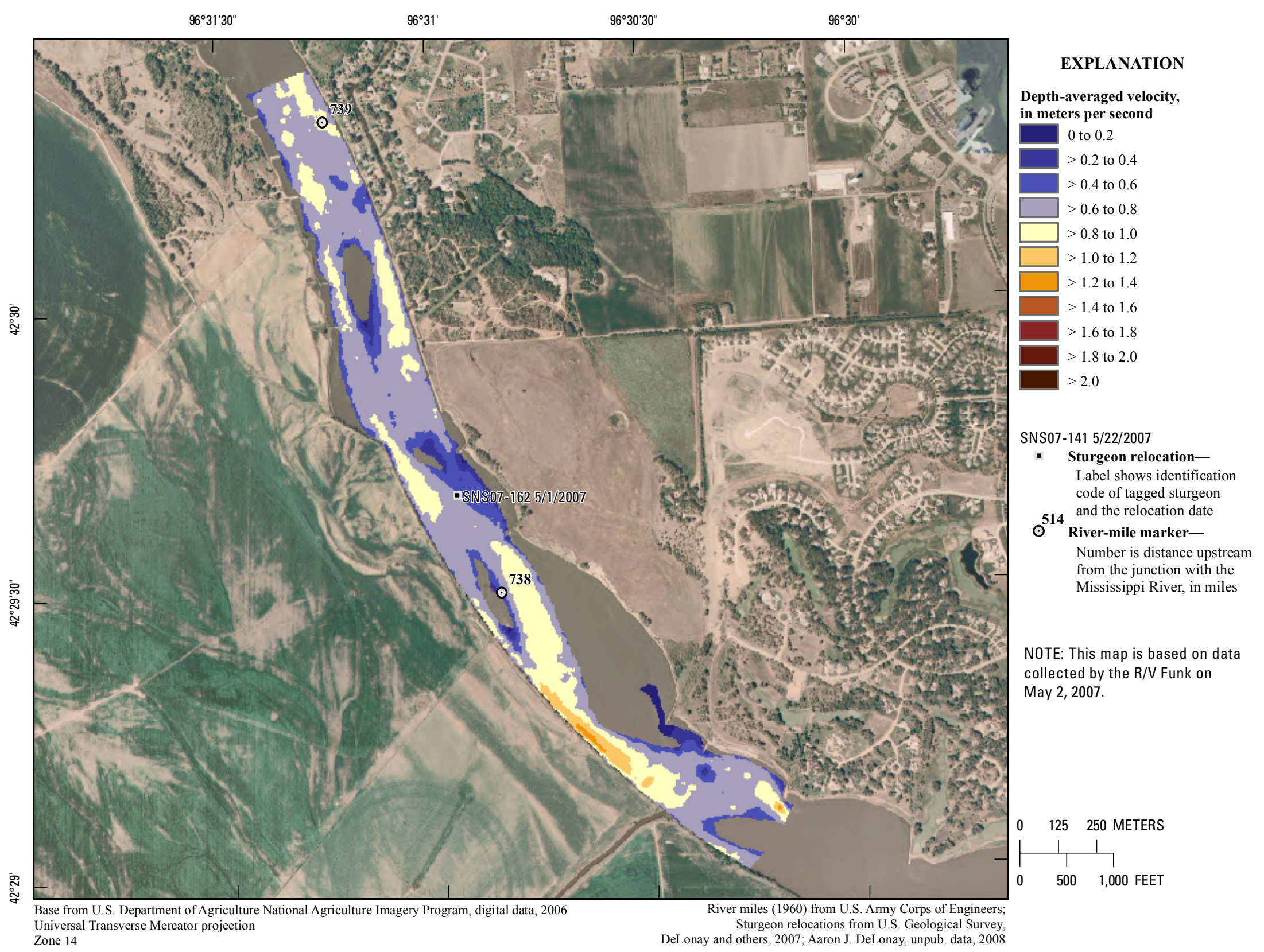

Figure 71. Map of depth-averaged velocity based on data collected on May 2, 2007, in the vicinity of river mile 738. 


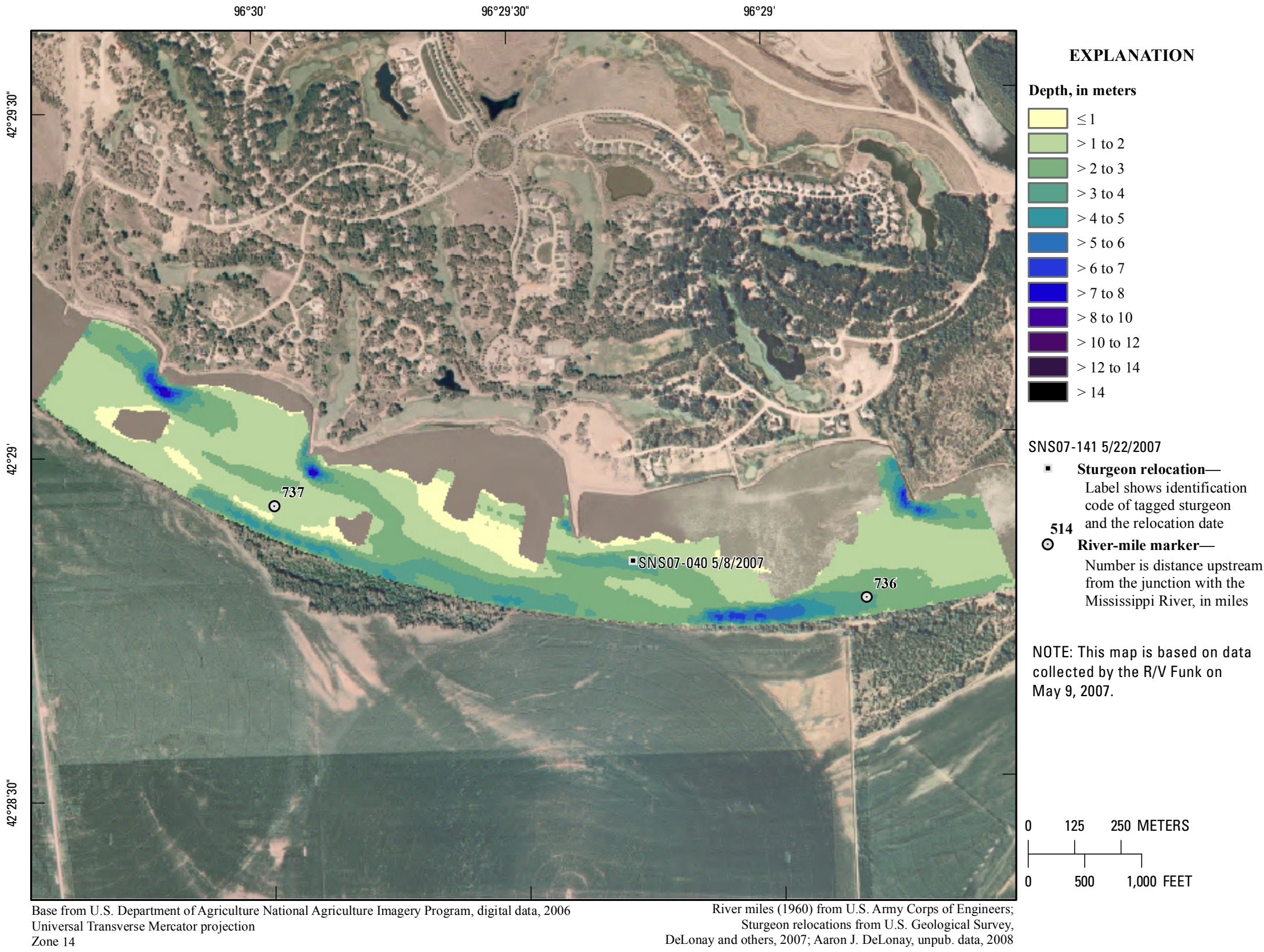

Figure 72. Map of depth based on data collected on May 9, 2007, in the vicinity of river mile 737. 


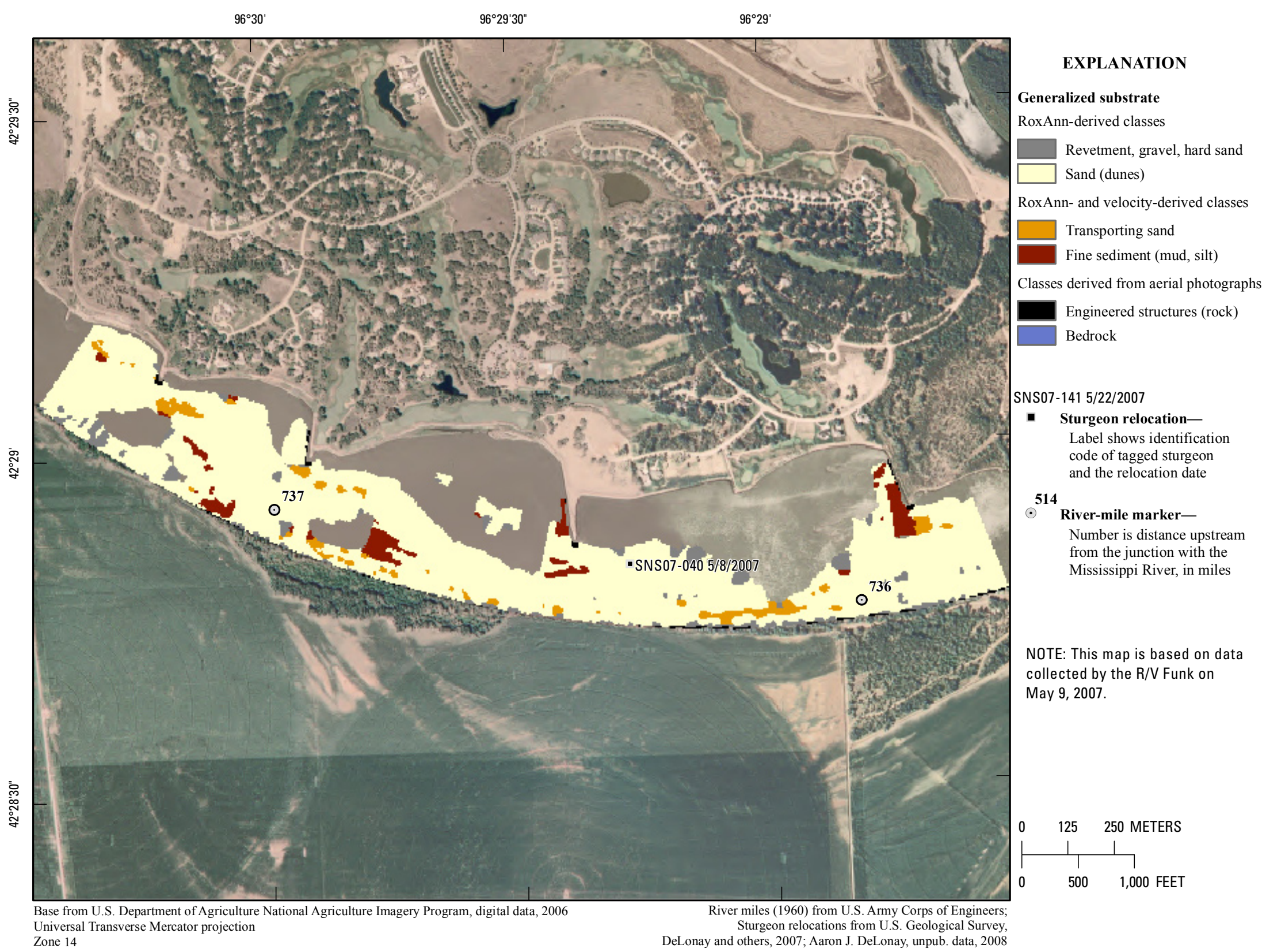

Figure 73. Map of generalized substrate based on data collected on May 9, 2007, in the vicinity of river mile 737 . 


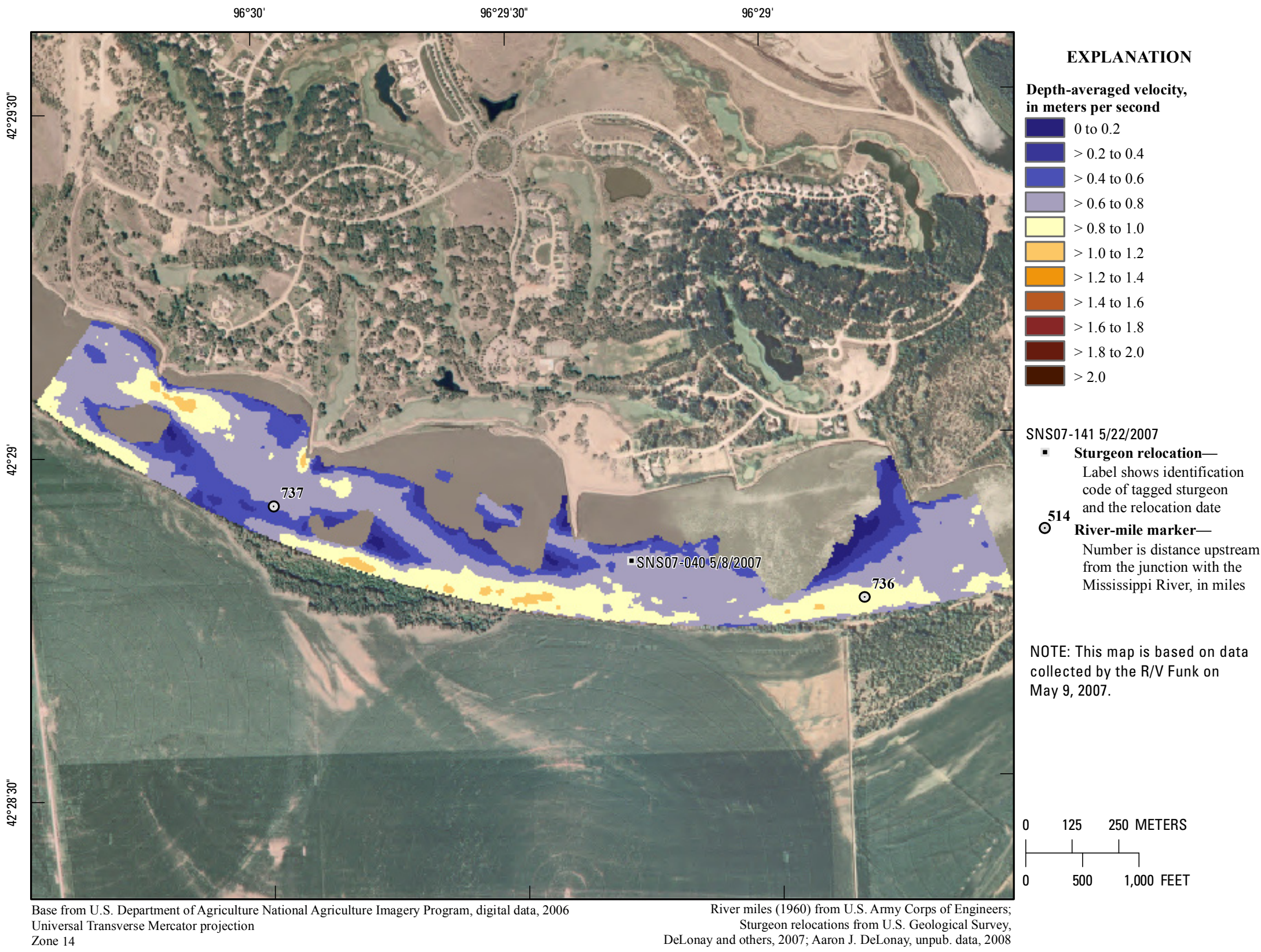

Figure 74. Map of depth-averaged velocity based on data collected on May 9, 2007, in the vicinity of river mile 737. 


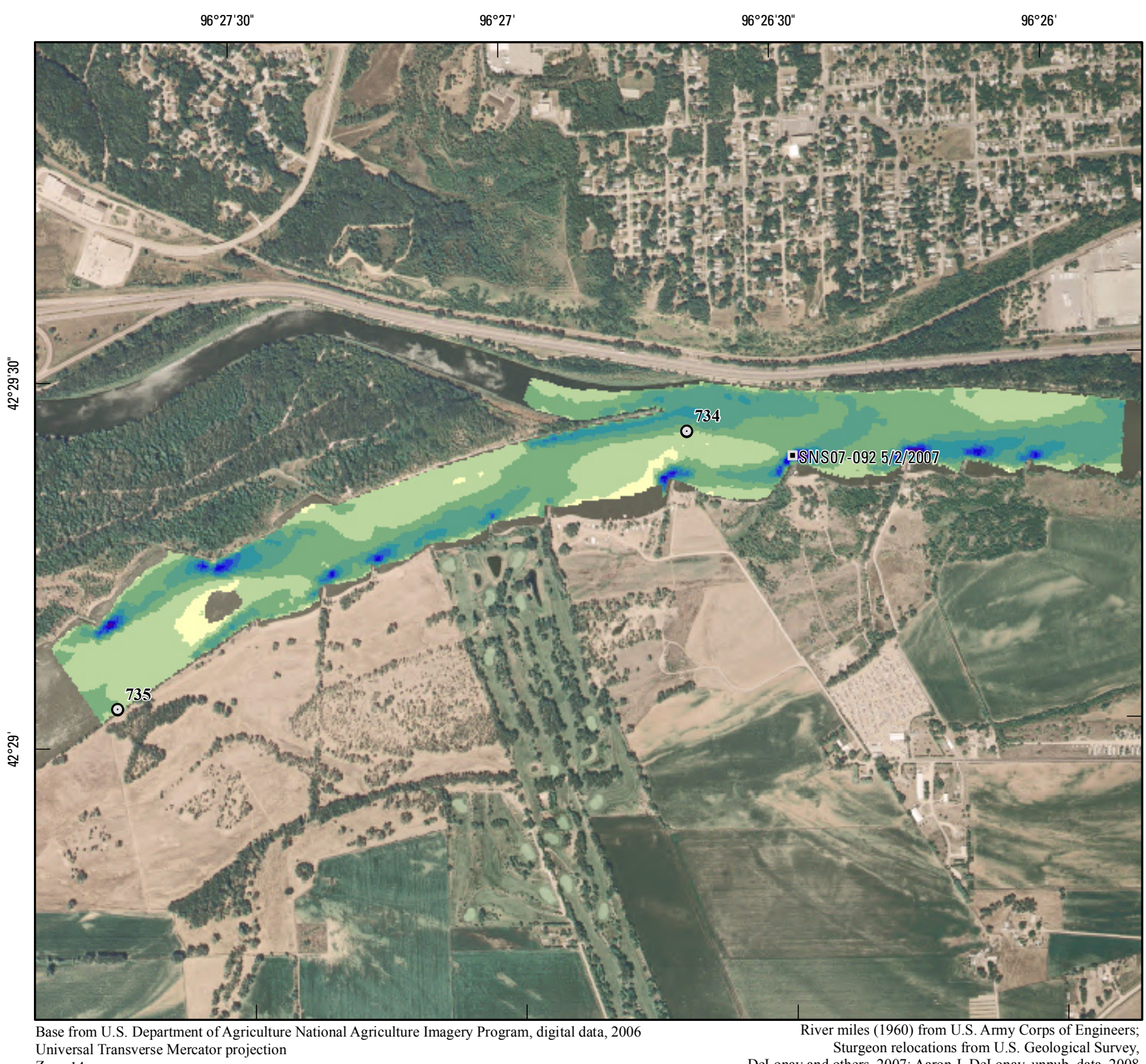

EXPLANATION

Depth, in meters

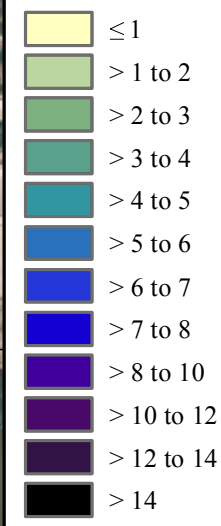

SNS07-141 5/22/2007

- Sturgeon relocationLabel shows identification code of tagged sturgeon 514 and the relocation date

$\odot$ River-mile markerNumber is distance upstrean from the junction with the Mississippi River, in miles

NOTE: This map is based on data collected by the R/V Funk on May 3, 2007. Zone 14

Figure 75. Map of depth based on data collected on May 3, 2007, in the vicinity of river mile 734 


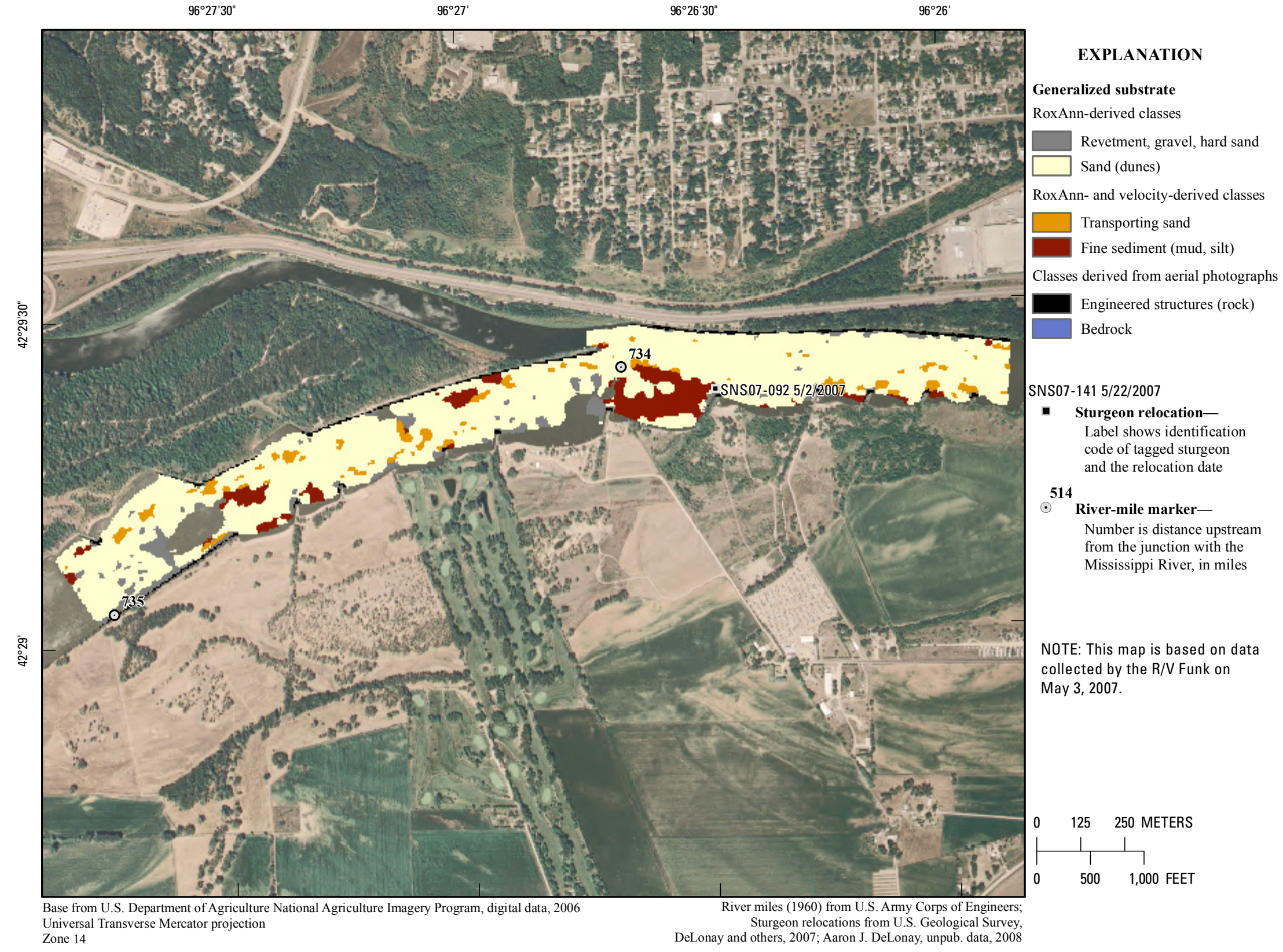

Figure 76. Map of generalized substrate based on data collected on May 3, 2007, in the vicinity of river mile 734. 


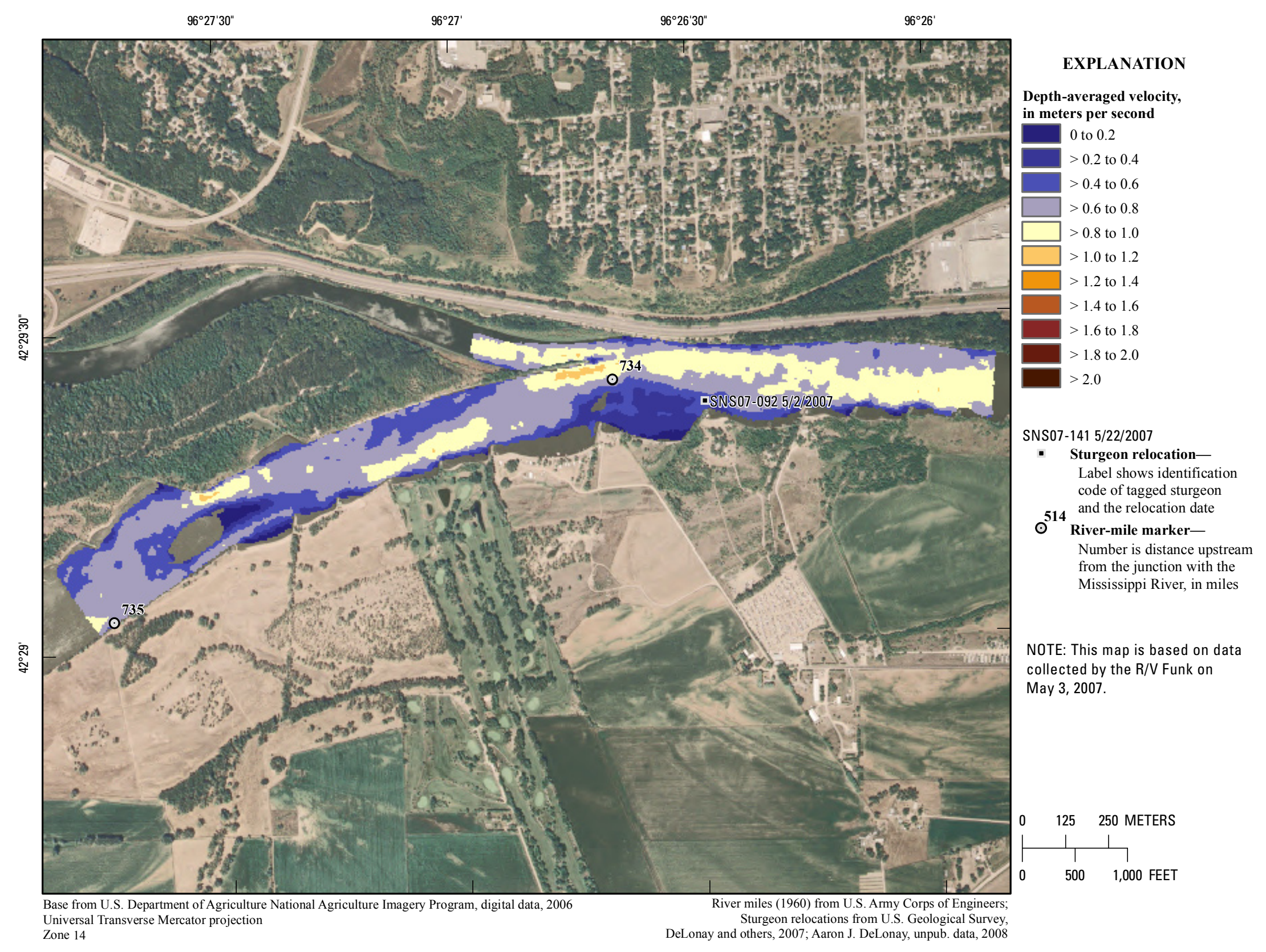

Figure 77. Map of depth-averaged velocity based on data collected on May 3, 2007, in the vicinity of river mile 734. 


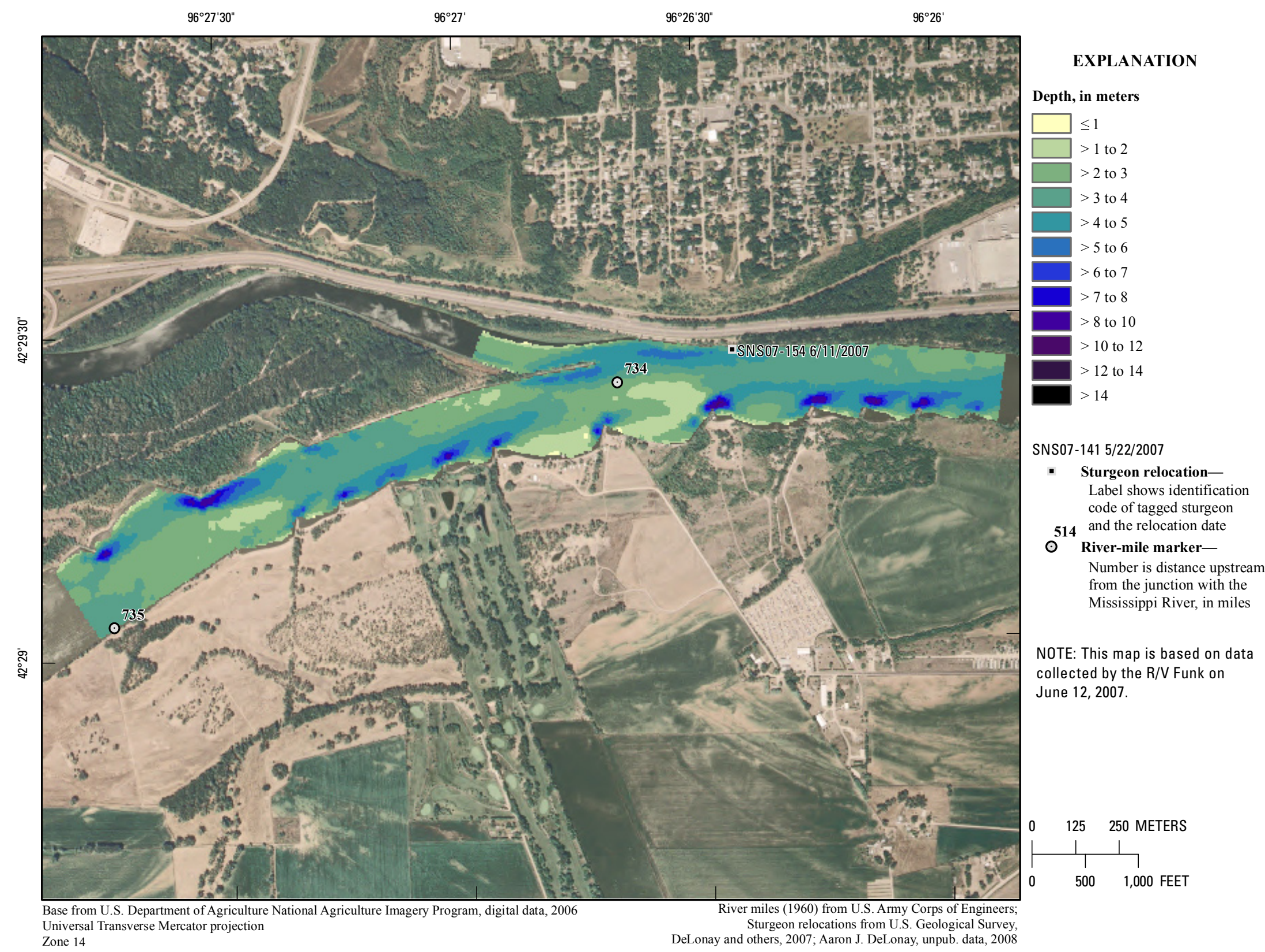

Figure 78. Map of depth based on data collected on June 12, 2007, in the vicinity of river mile 734 . 


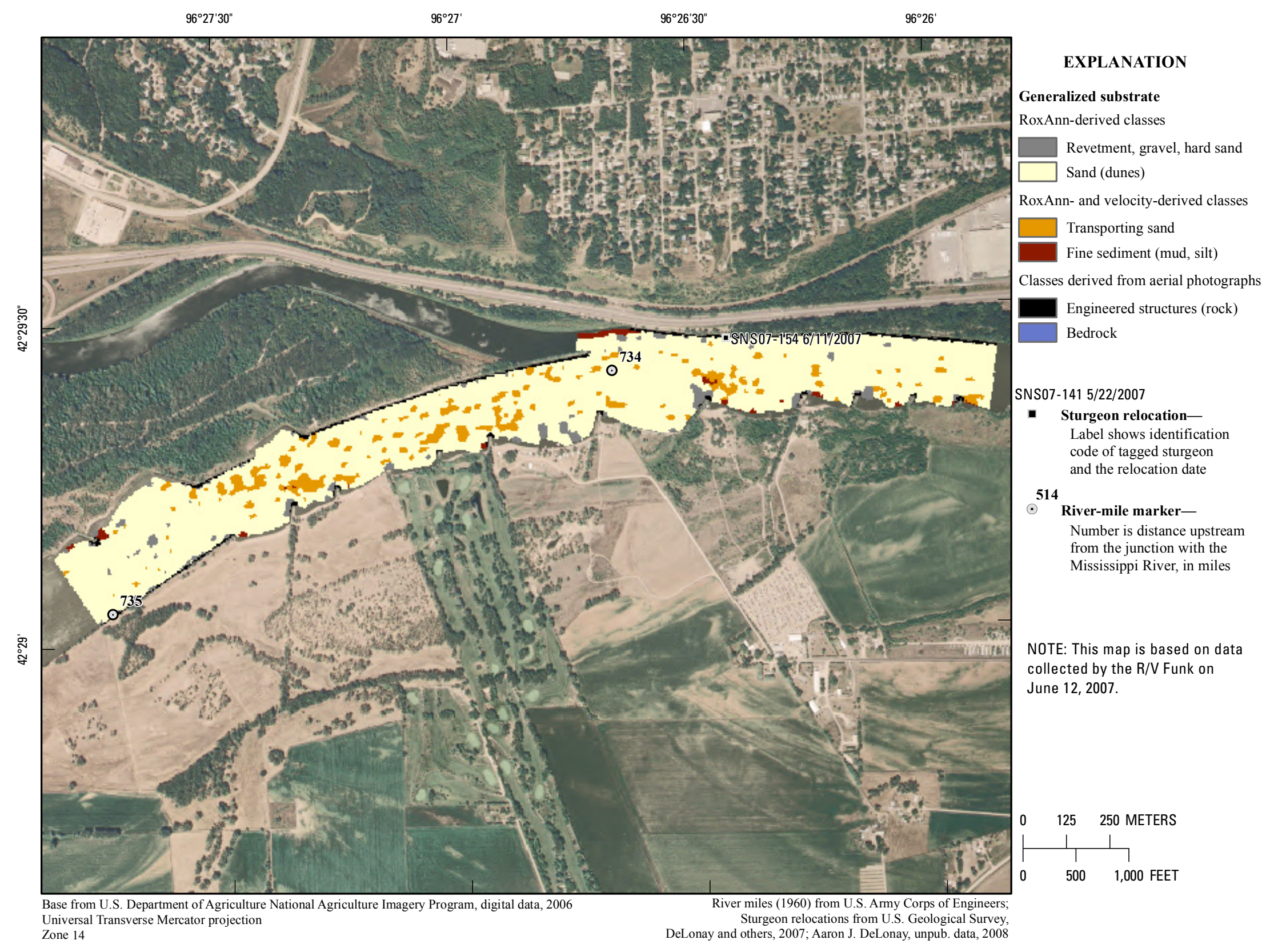

Figure 79. Map of generalized substrate based on data collected on June 12, 2007, in the vicinity of river mile 734 . 


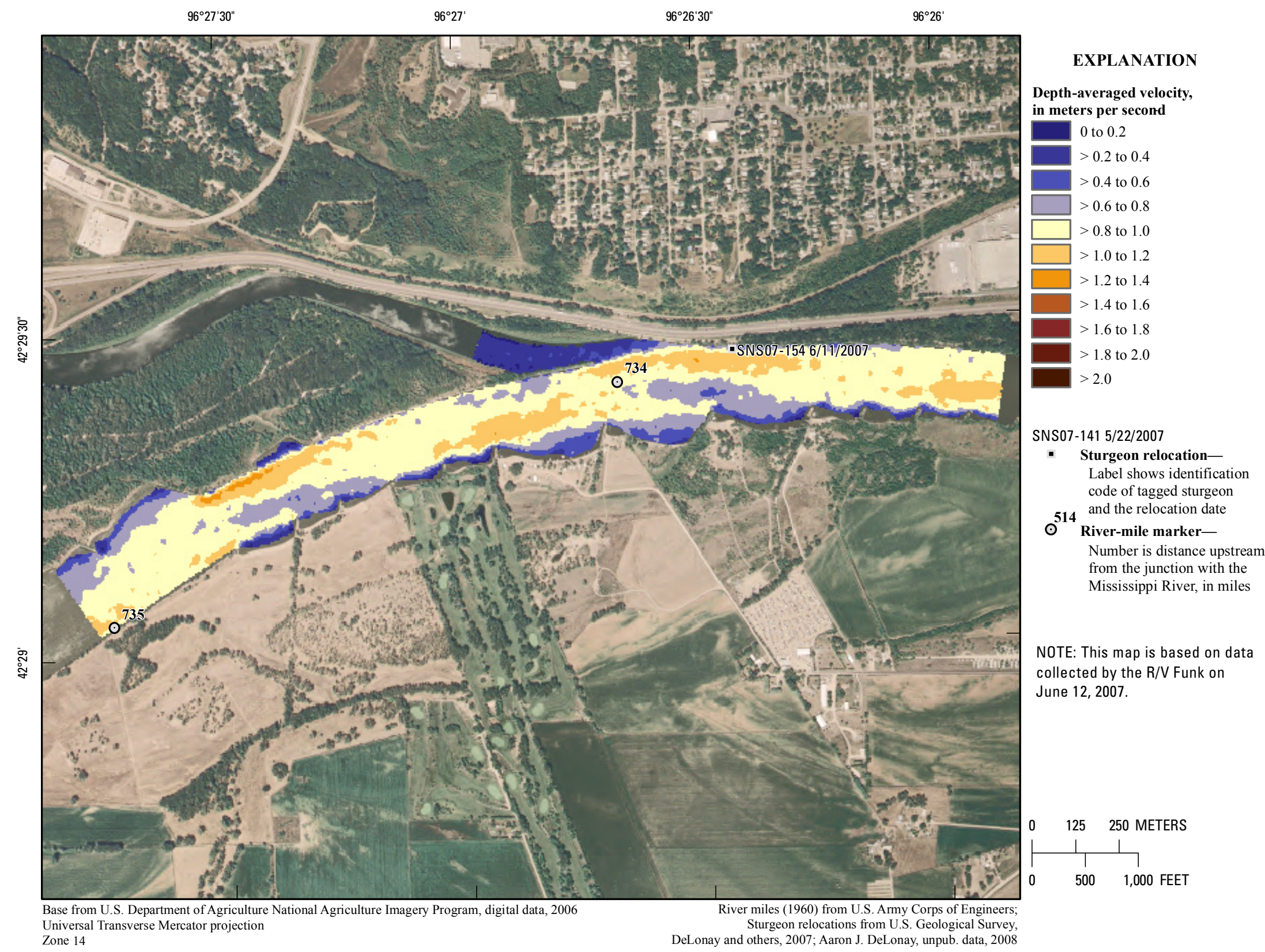

Figure 80. Map of depth-averaged velocity based on data collected on June 12, 2007, in the vicinity of river mile 734 . 


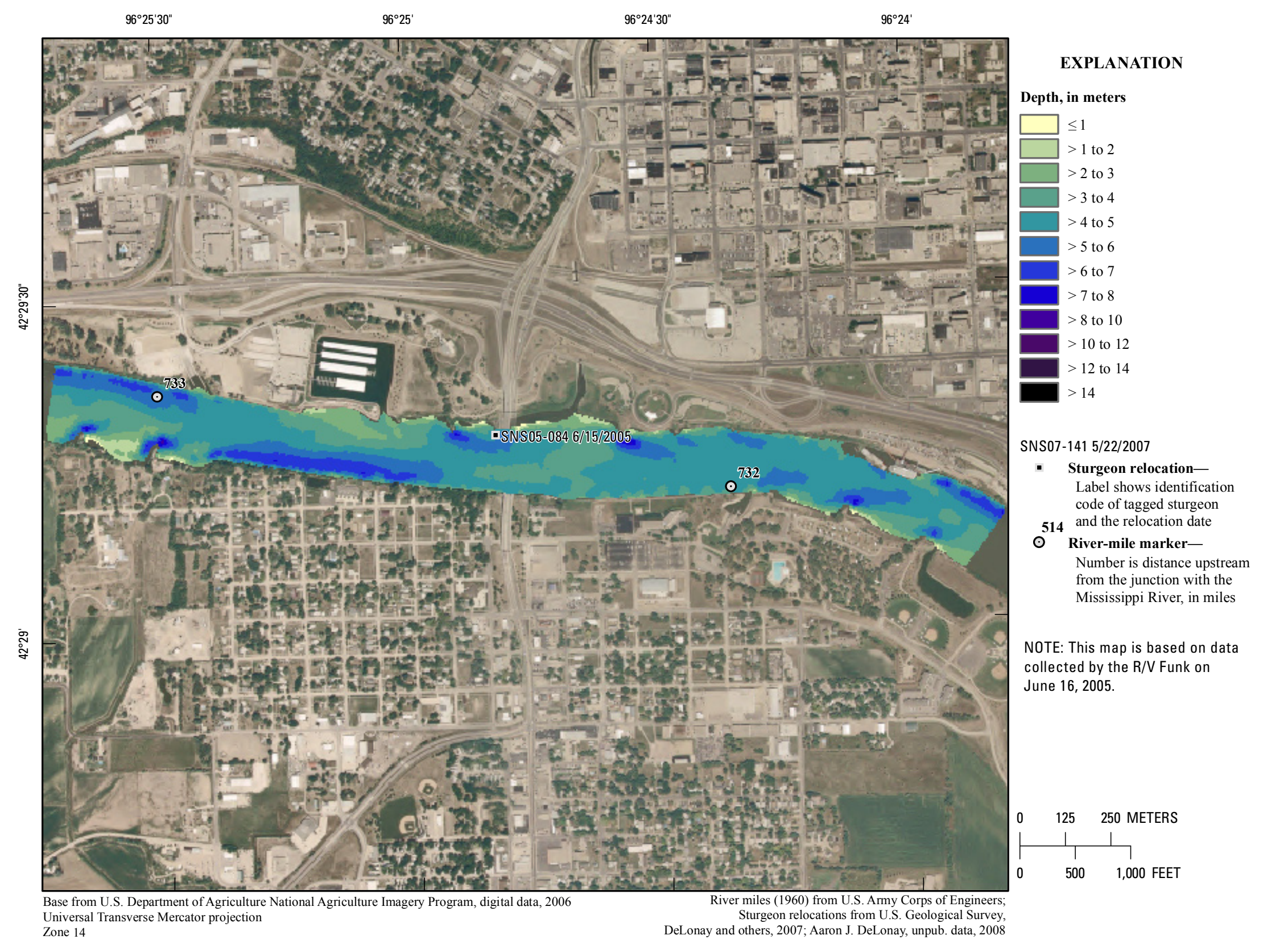

Figure 81. Map of depth based on data collected on June 16, 2005, in the vicinity of river mile 732 . 


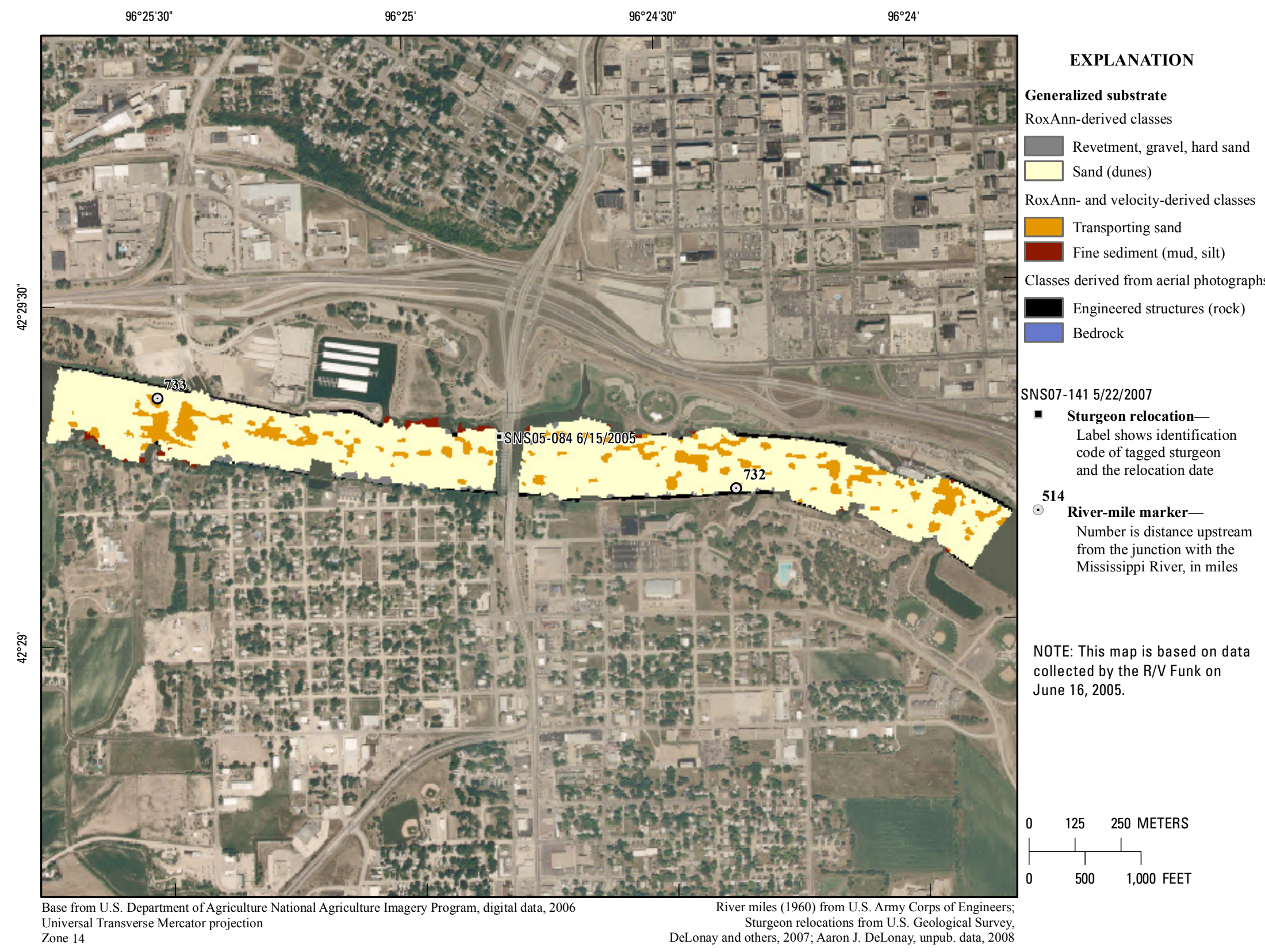

Figure 82. Map of generalized substrate based on data collected on June 16, 2005, in the vicinity of river mile 732 . 


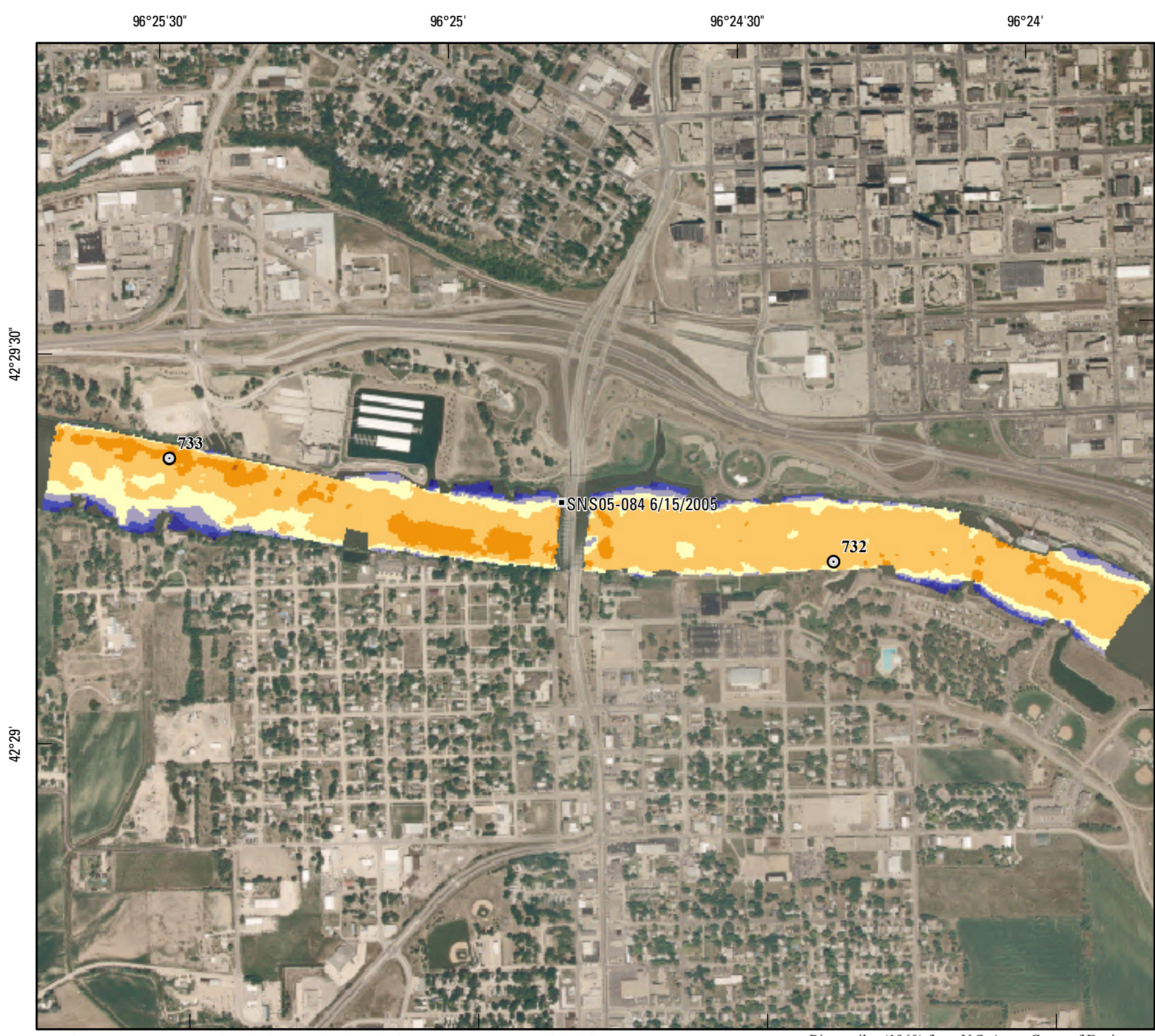

Depth-averaged velocity,

in meters per second

0 to 0.2

$>0.2$ to 0.4

$>0.4$ to 0.6

$>0.6$ to 0.8

$>0.8$ to 1.0

$>1.0$ to 1.2

$>1.2$ to 1.4

$>1.4$ to 1.6

1.6 to 1.8

$>1.8$ to 2.0

$>2.0$

\section{SNS07-141 5/22/2007}

- Sturgeon relocation-

Label shows identification code of tagged sturgeon

514 and the relocation date

River-mile marker-

Number is distance upstrean

from the junction with the

Mississippi River, in miles

NOTE: This map is based on data

collected by the R/V Funk on

June 16, 2005.

\section{METERS \\ $500 \quad 1,000$ FEET}

ver miles (1960) from U.S. Army Corps of Engineer

Sturgeon relocations from U.S. Geological Survey,
DeLonay and others, 2007; Aaron J. DeLonay, unpub. data, 2008

$\vec{\varnothing}$

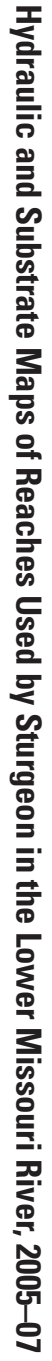

Figure 83. Map of depth-averaged velocity based on data collected on June 16,2005 , in the vicinity of river mile 732 


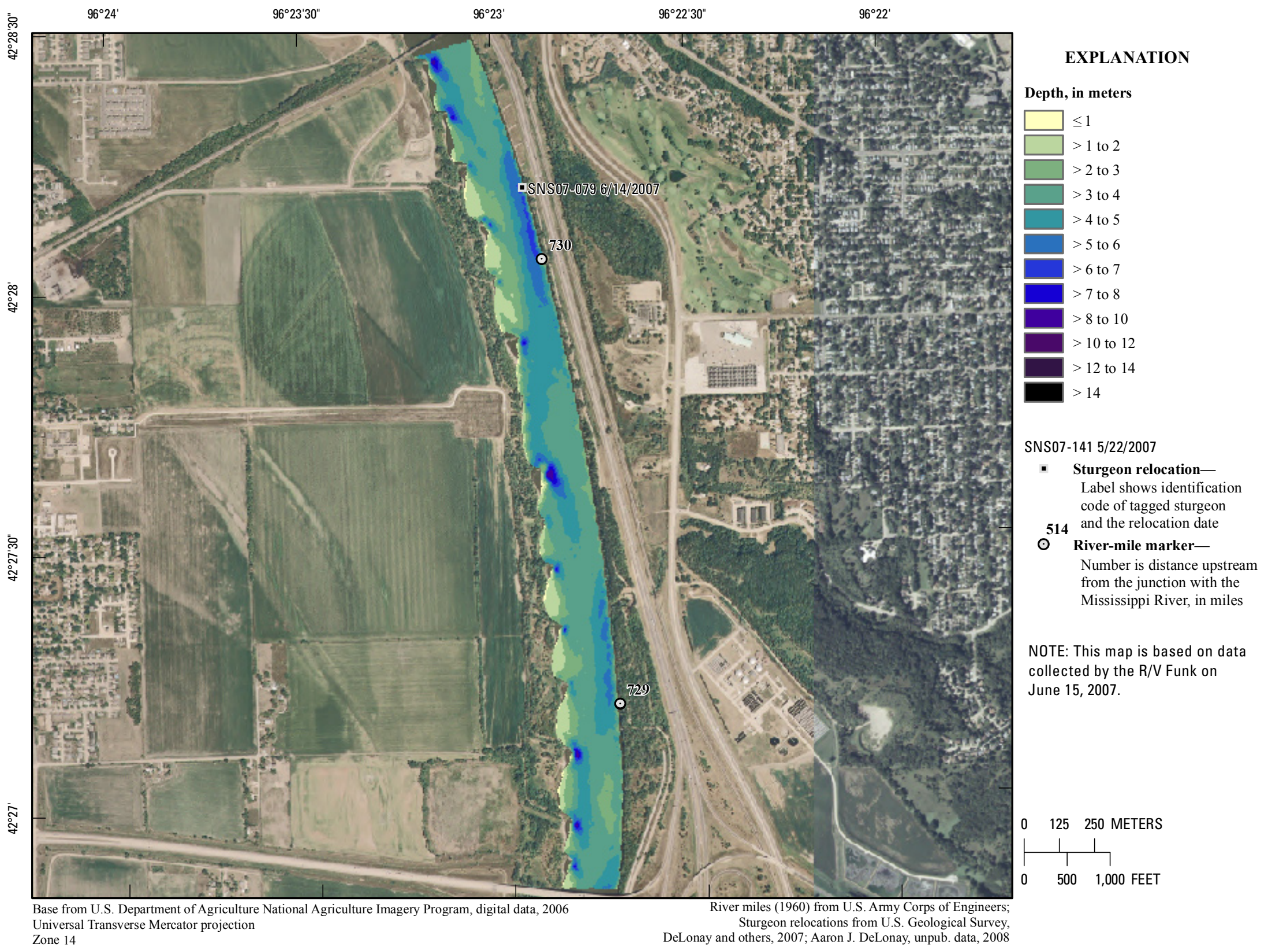

Figure 84. Map of depth based on data collected on June 15, 2007, in the vicinity of river mile 730 . 


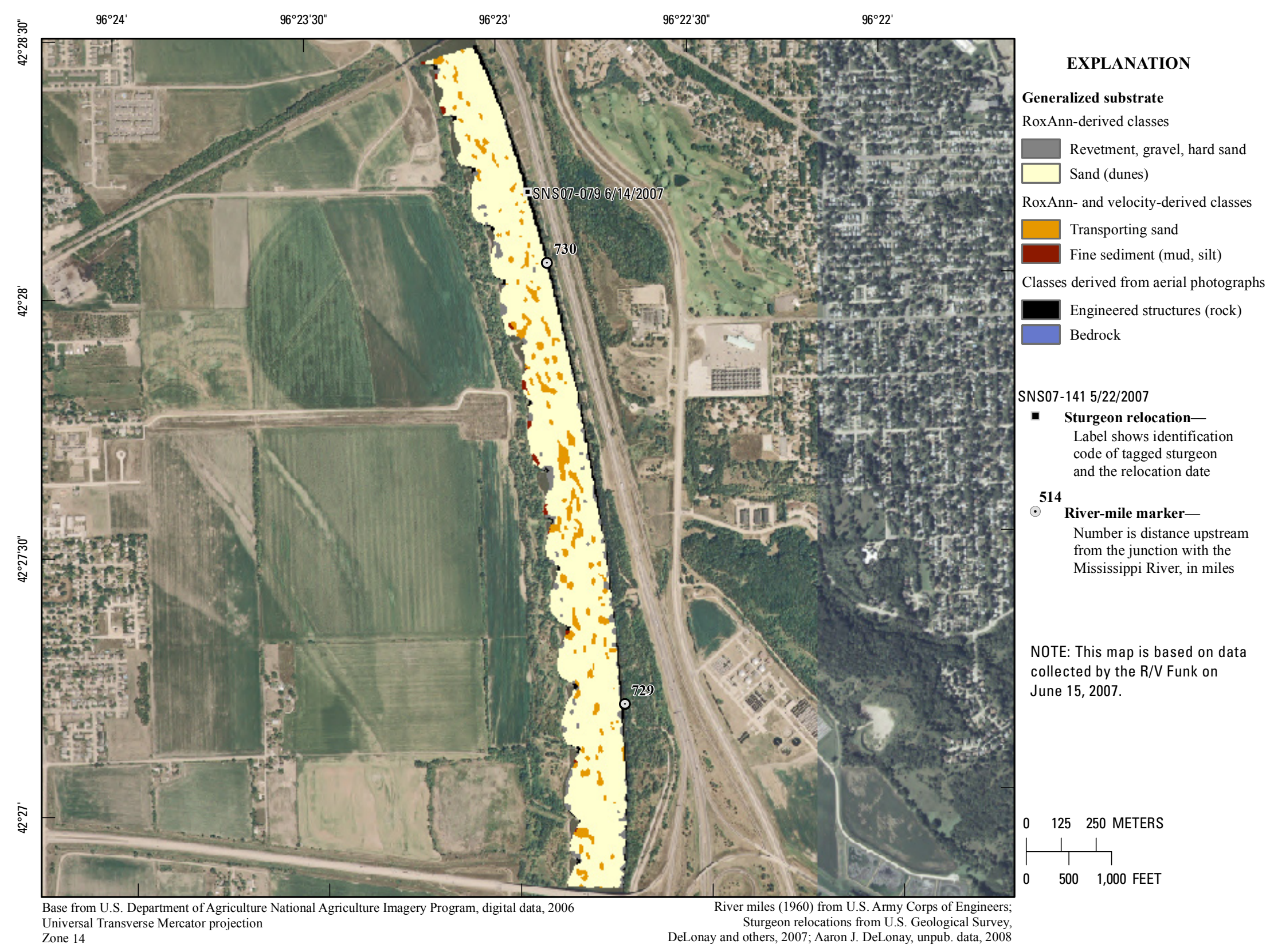

Figure 85. Map of generalized substrate based on data collected on June 15, 2007, in the vicinity of river mile 730 . 


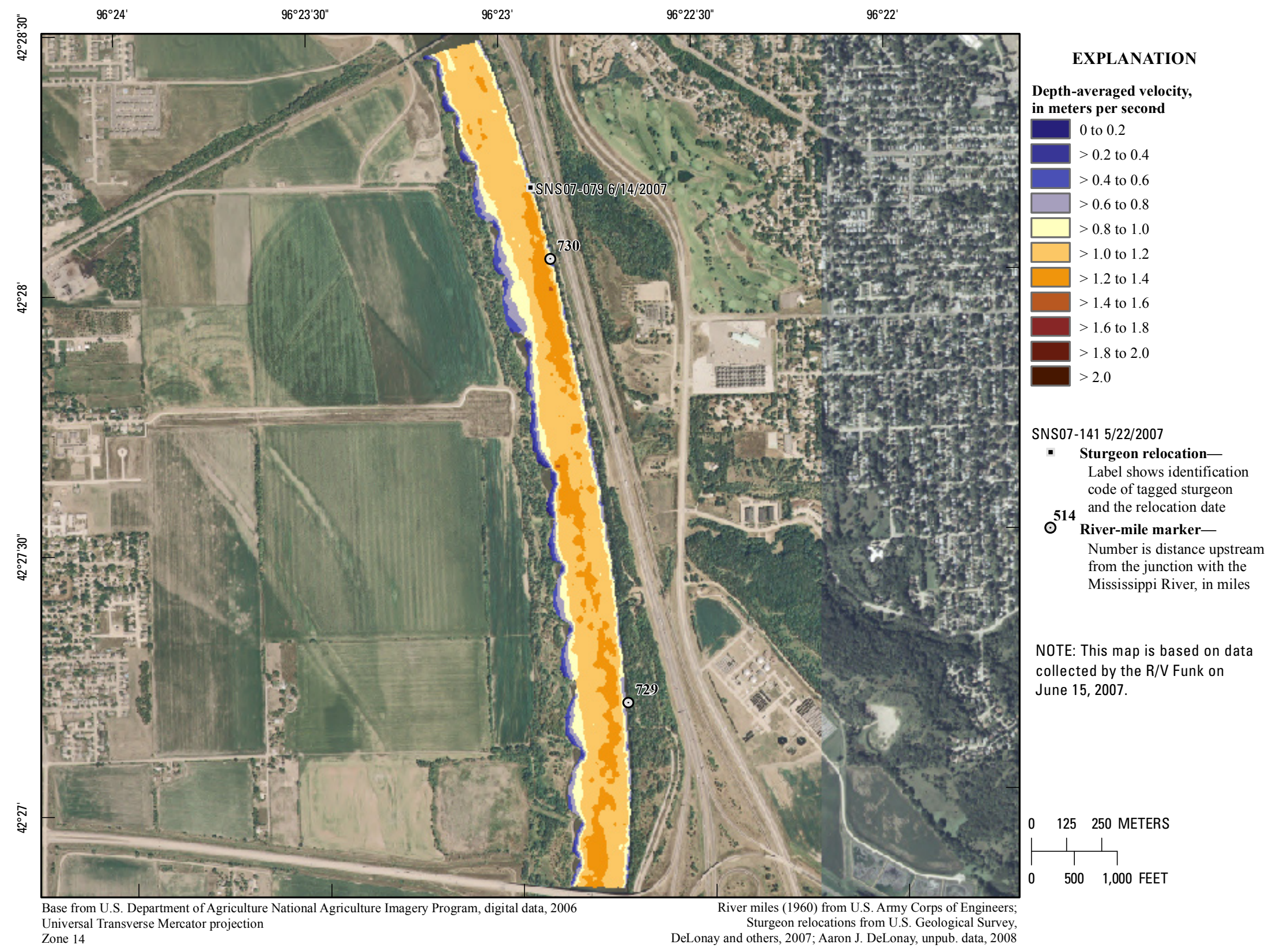

Figure 86. Map of depth-averaged velocity based on data collected on June 15, 2007, in the vicinity of river mile 730 . 


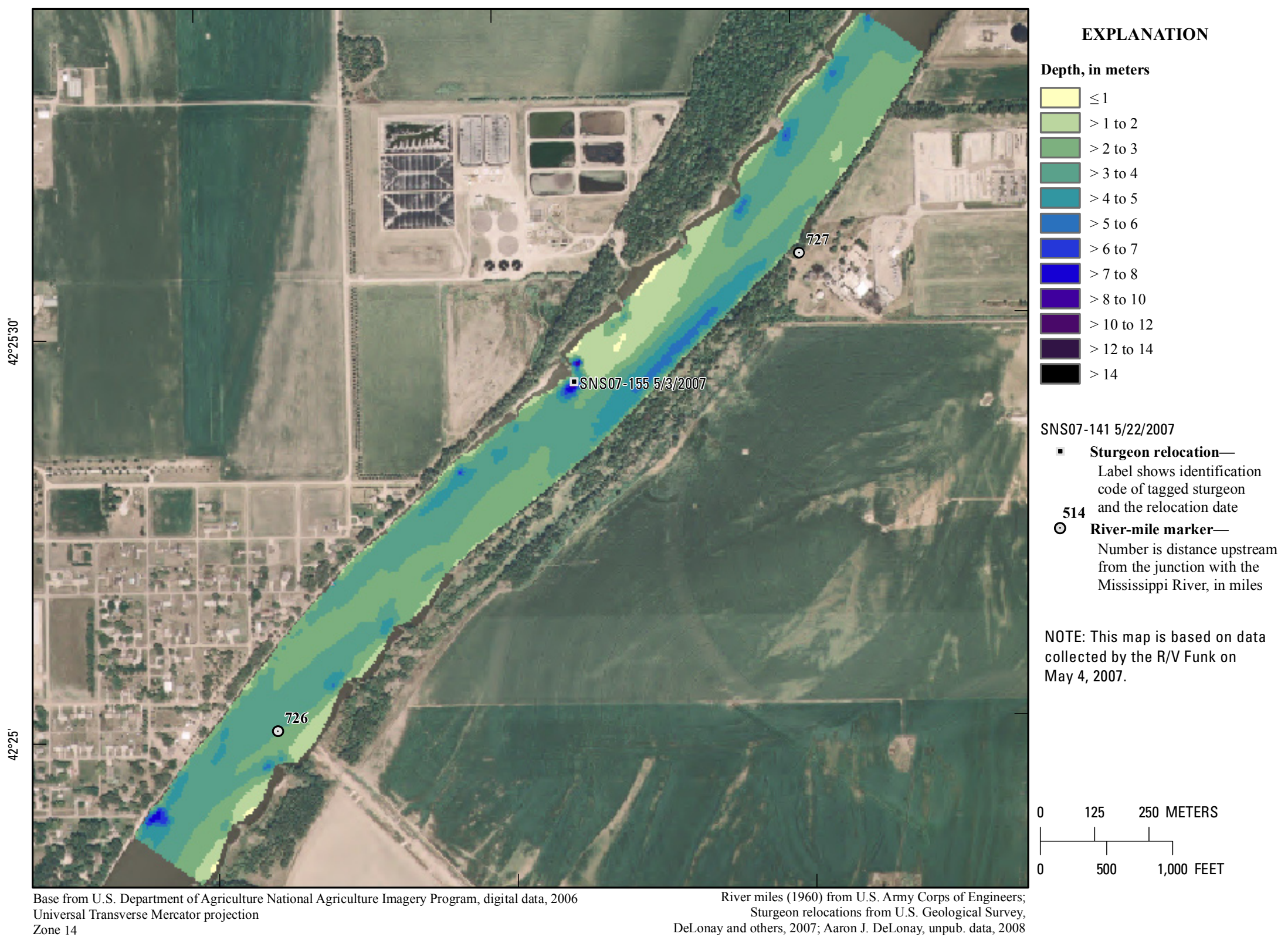

Figure 87. Map of depth based on data collected on May 4, 2007, in the vicinity of river mile 727. 


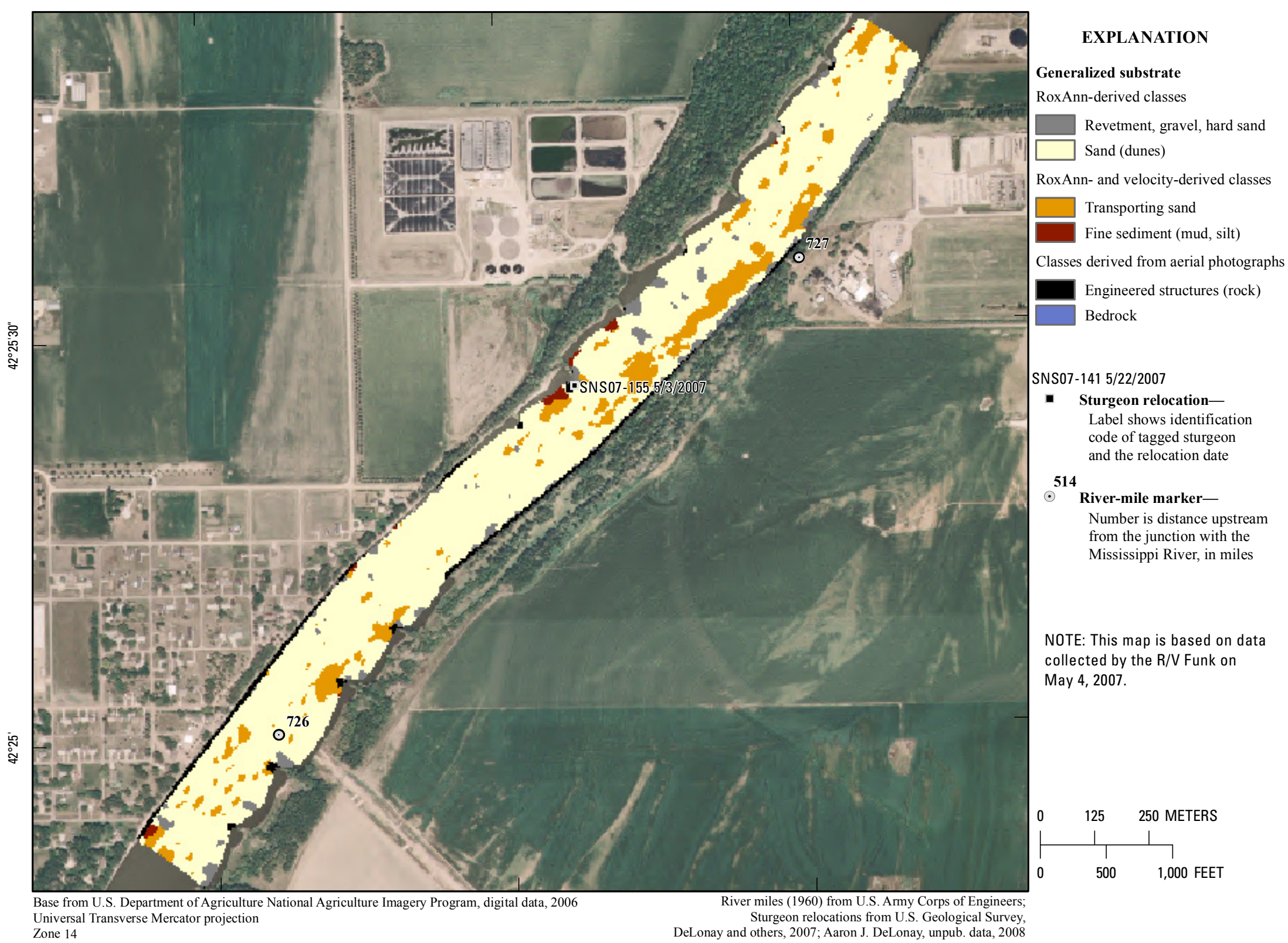




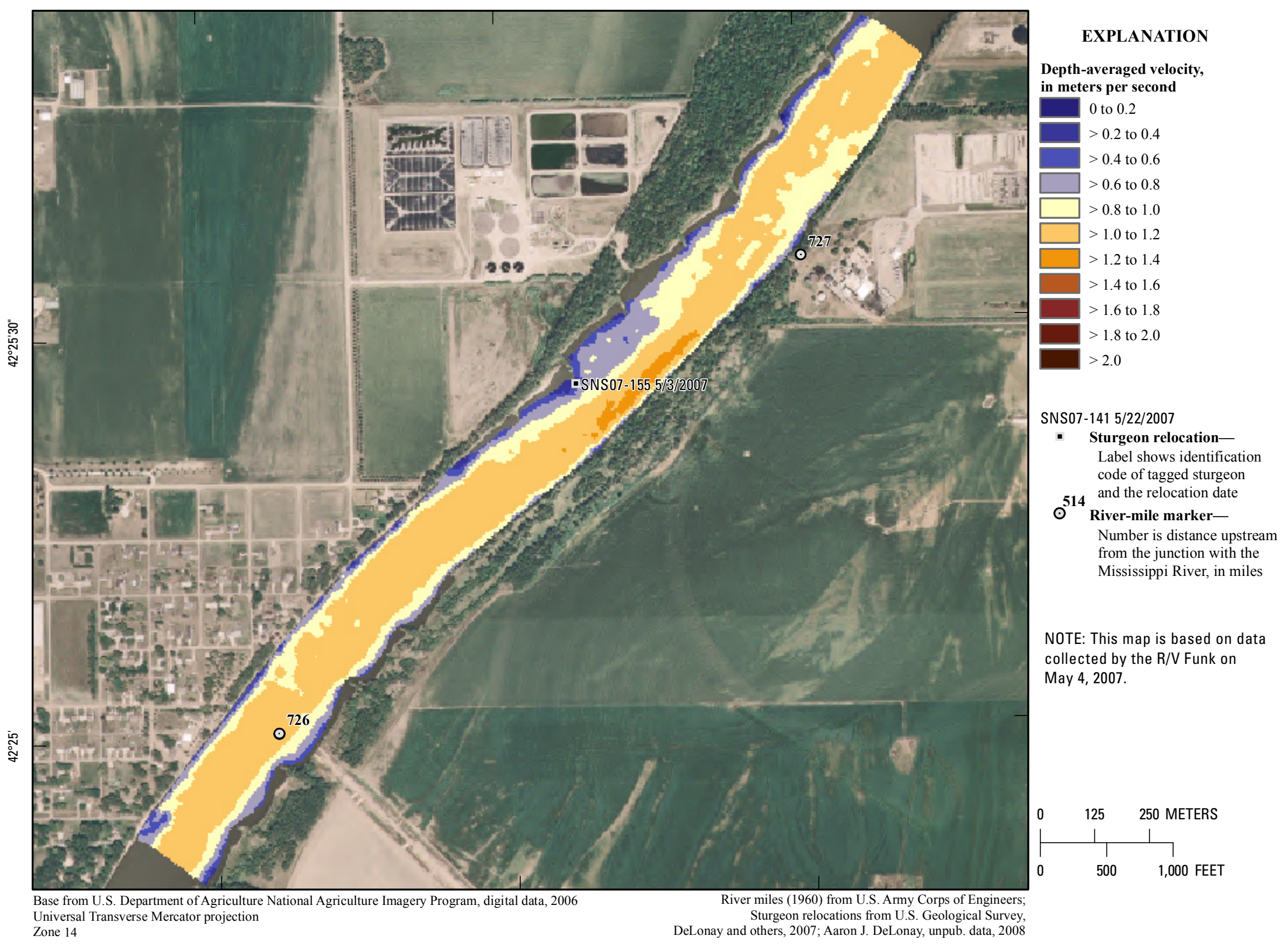

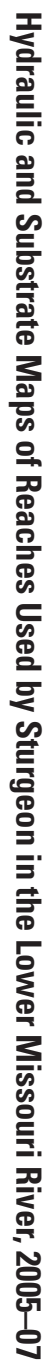

Figure 89. Map of depth-averaged velocity based on data collected on May 4, 2007, in the vicinity of river mile 727 . 


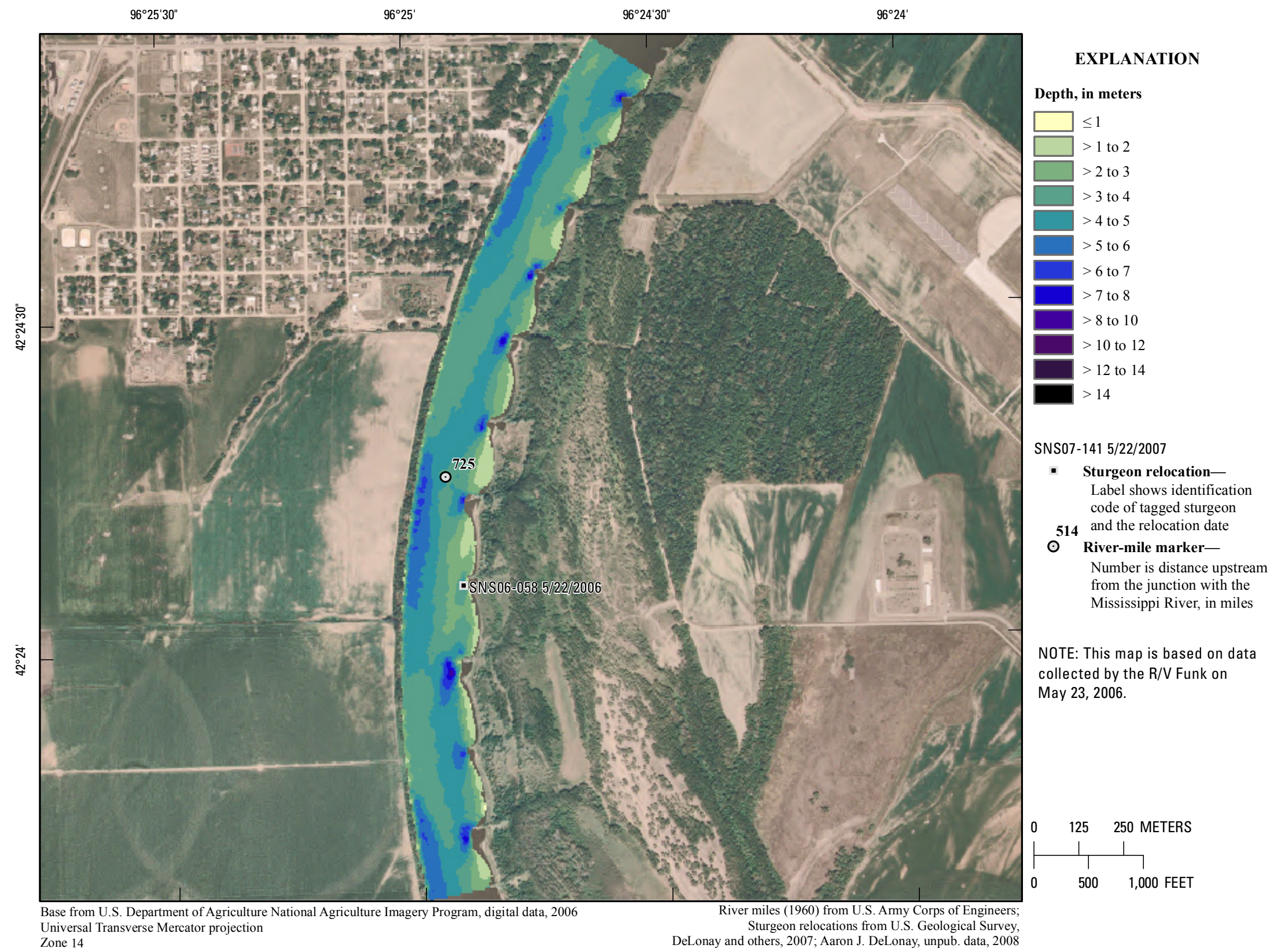

Figure 90. Map of depth based on data collected on May 23, 2006, in the vicinity of river mile 725 . 


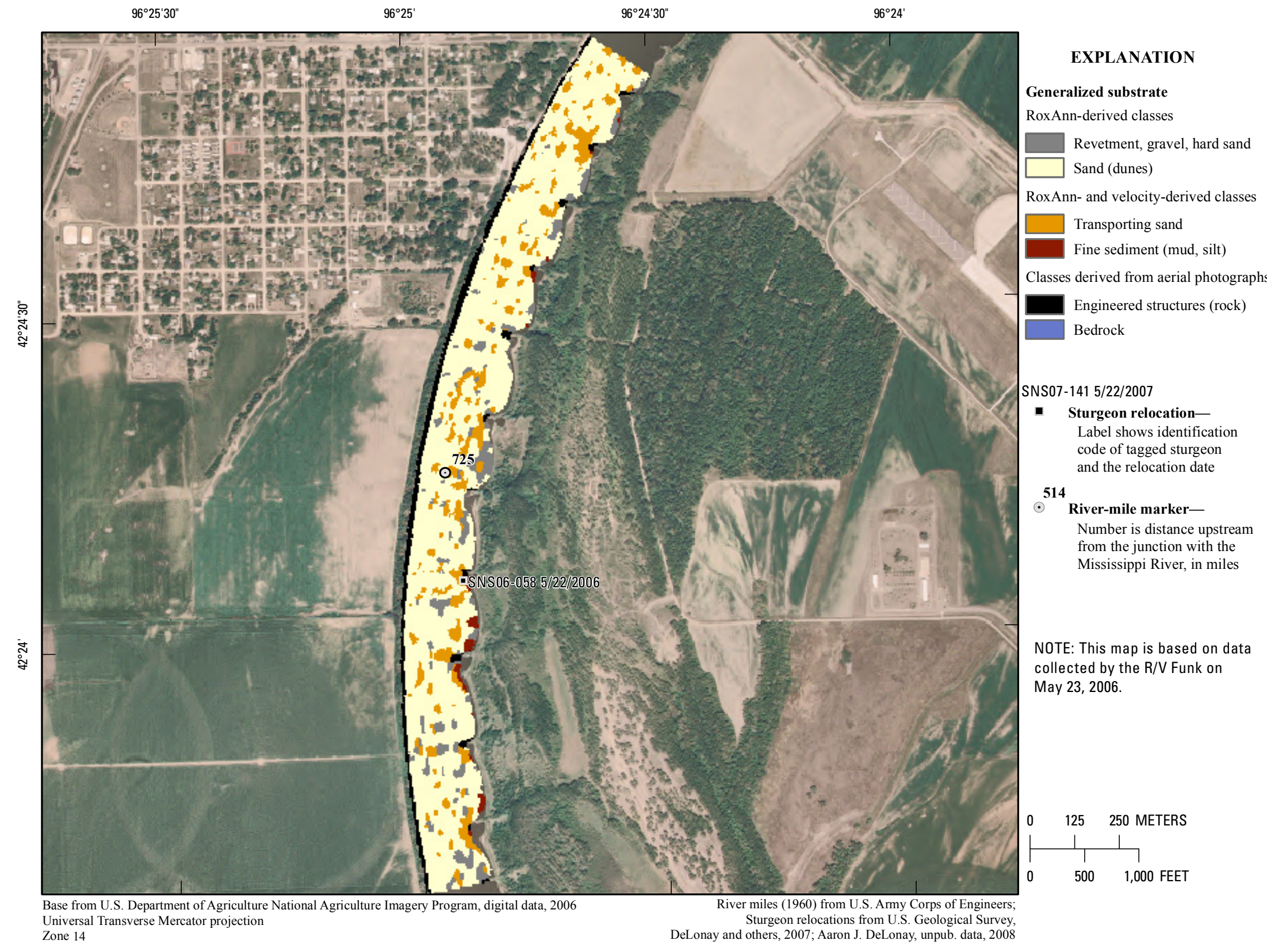

Figure 91. Map of generalized substrate based on data collected on May 23, 2006, in the vicinity of river mile 725 . 


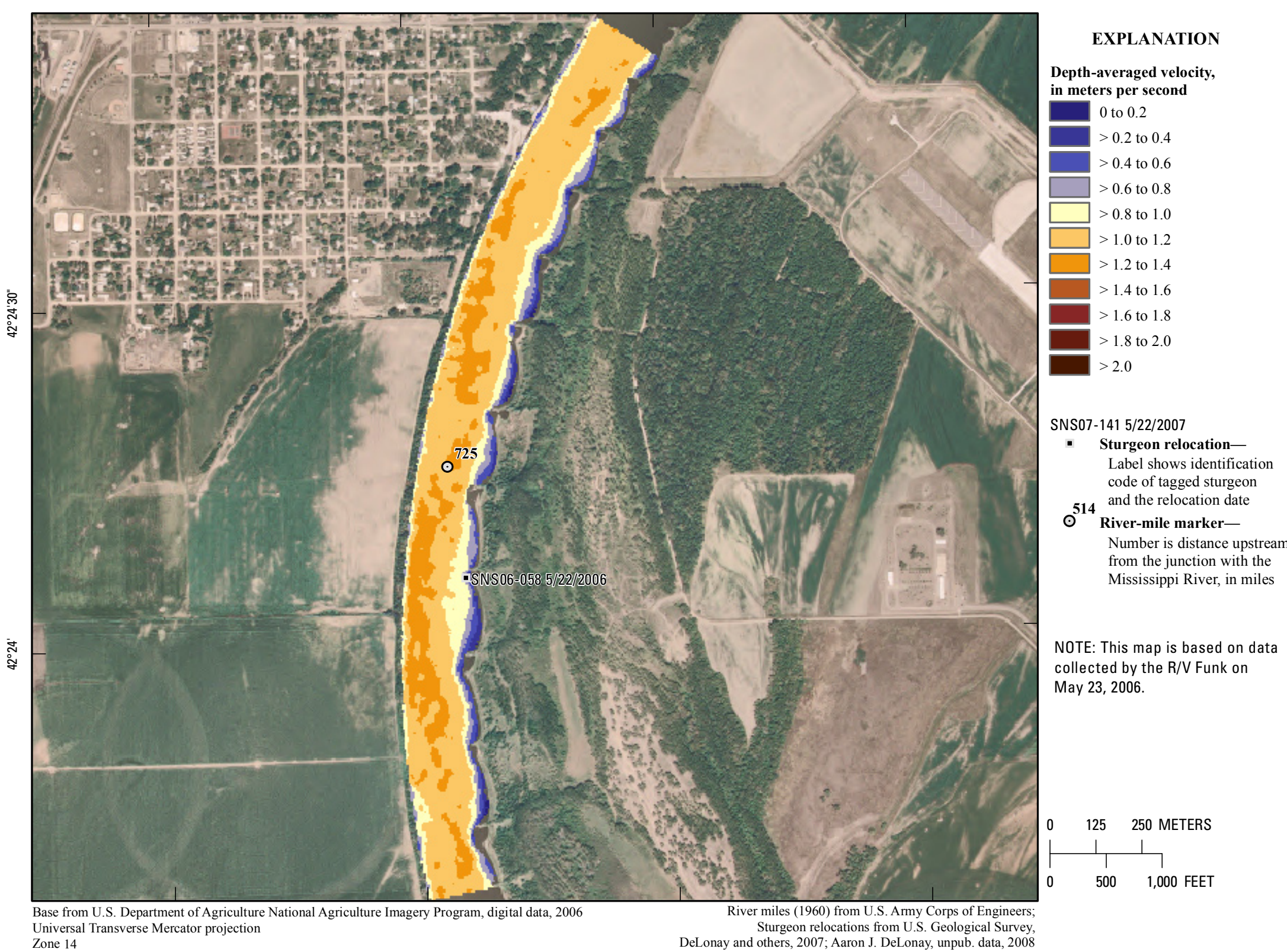




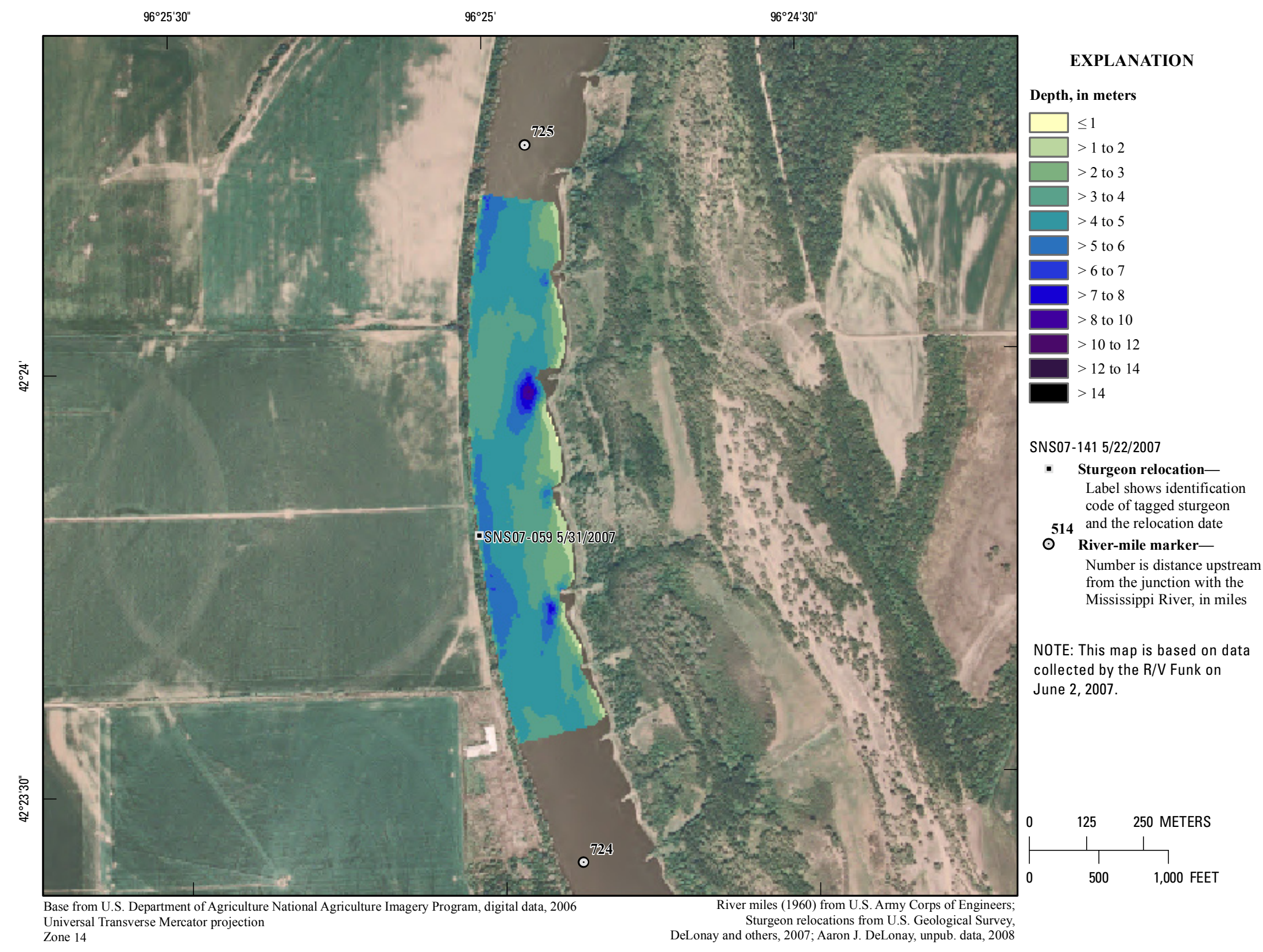

Figure 93. Map of depth based on data collected on June 2, 2007, in the vicinity of river mile 725 . 


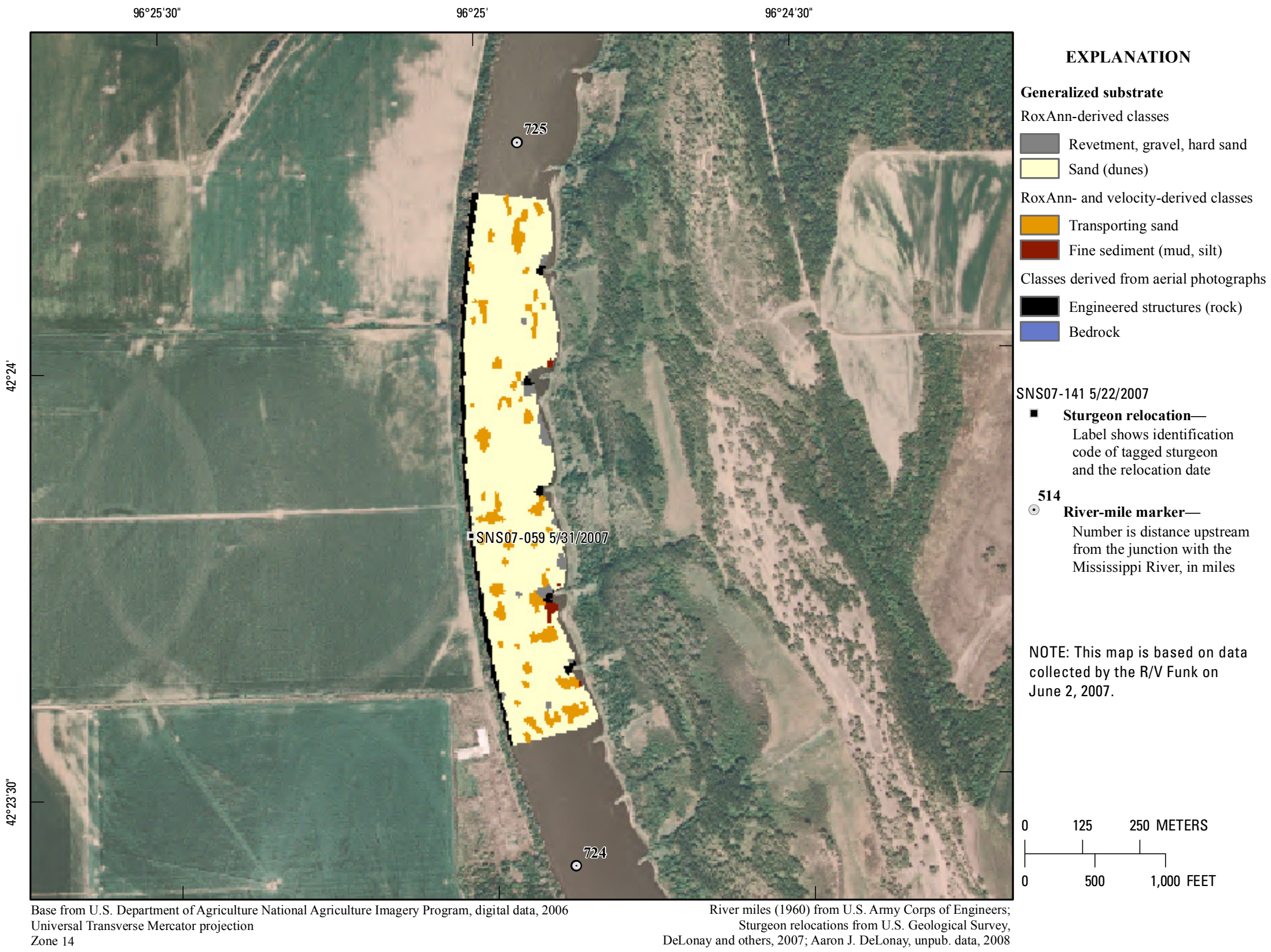

Figure 94. Map of generalized substrate based on data collected on June 2, 2007, in the vicinity of river mile 725 . 


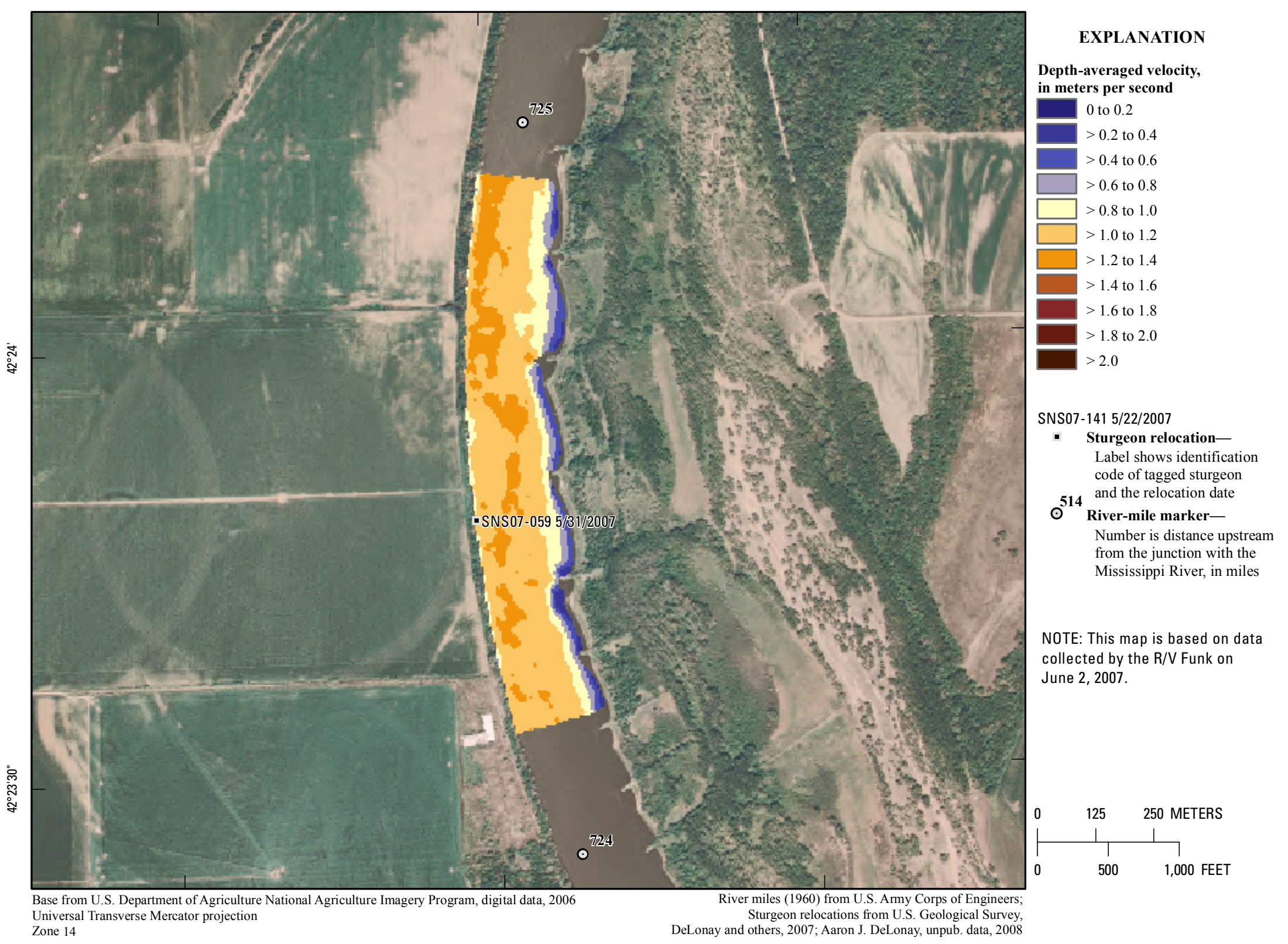

Figure 95. Map of depth-averaged velocity based on data collected on June 2, 2007, in the vicinity of river mile 725. 


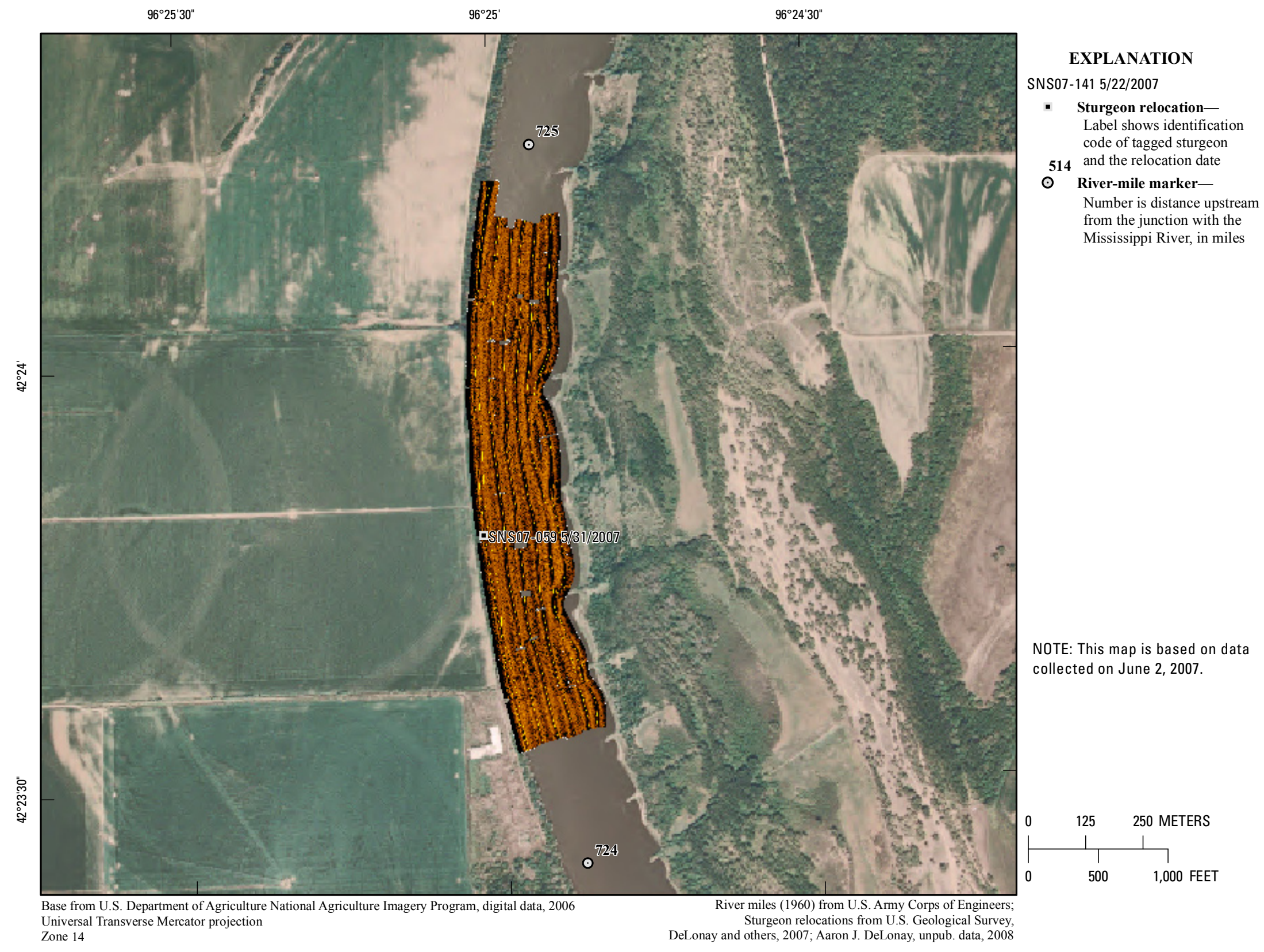

Figure 96. Map of side-scan sonar imagery based on data collected on June 2, 2007, in the vicinity of river mile 725 . 


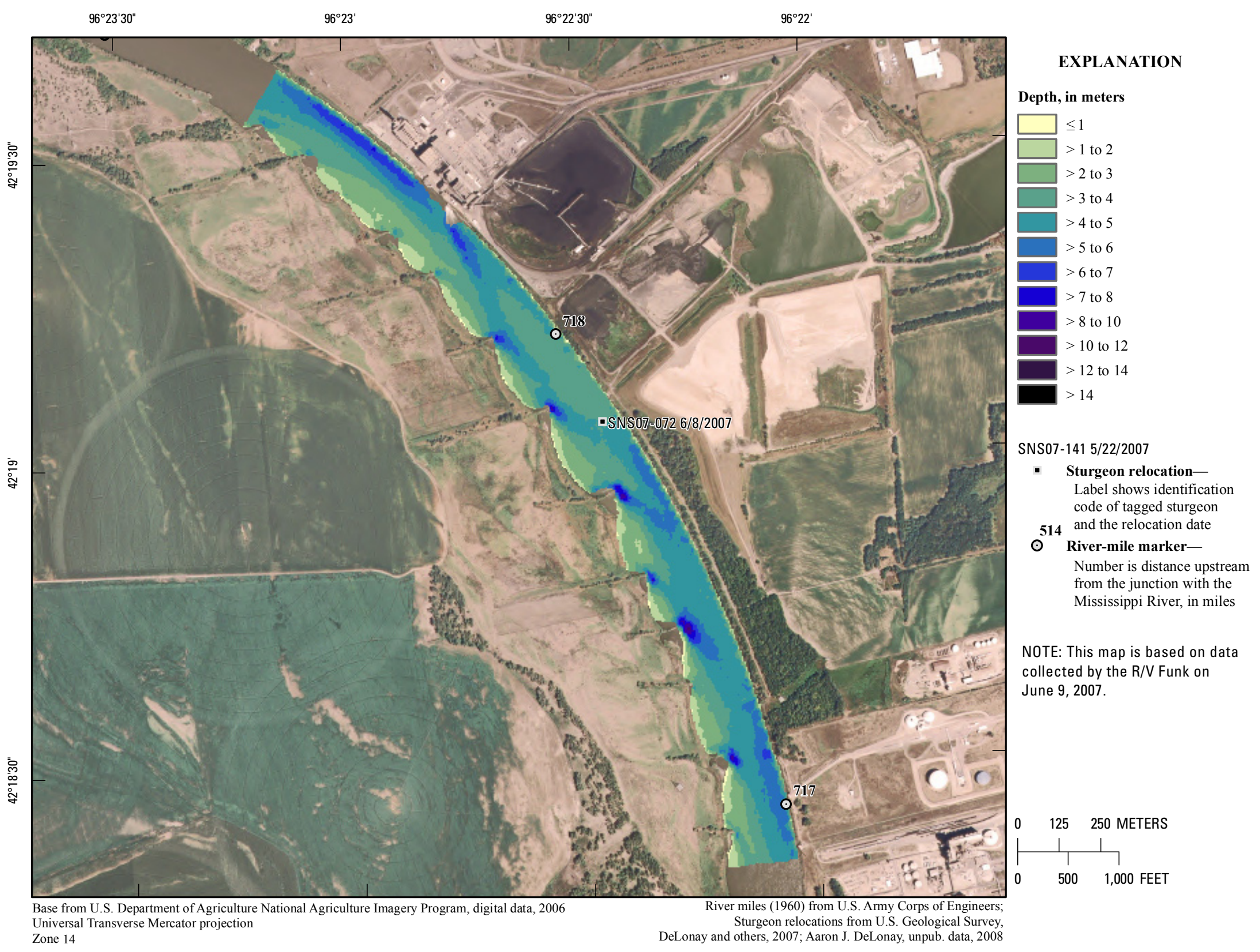

Figure 97. Map of depth based on data collected on June 9, 2007, in the vicinity of river mile 718 . 


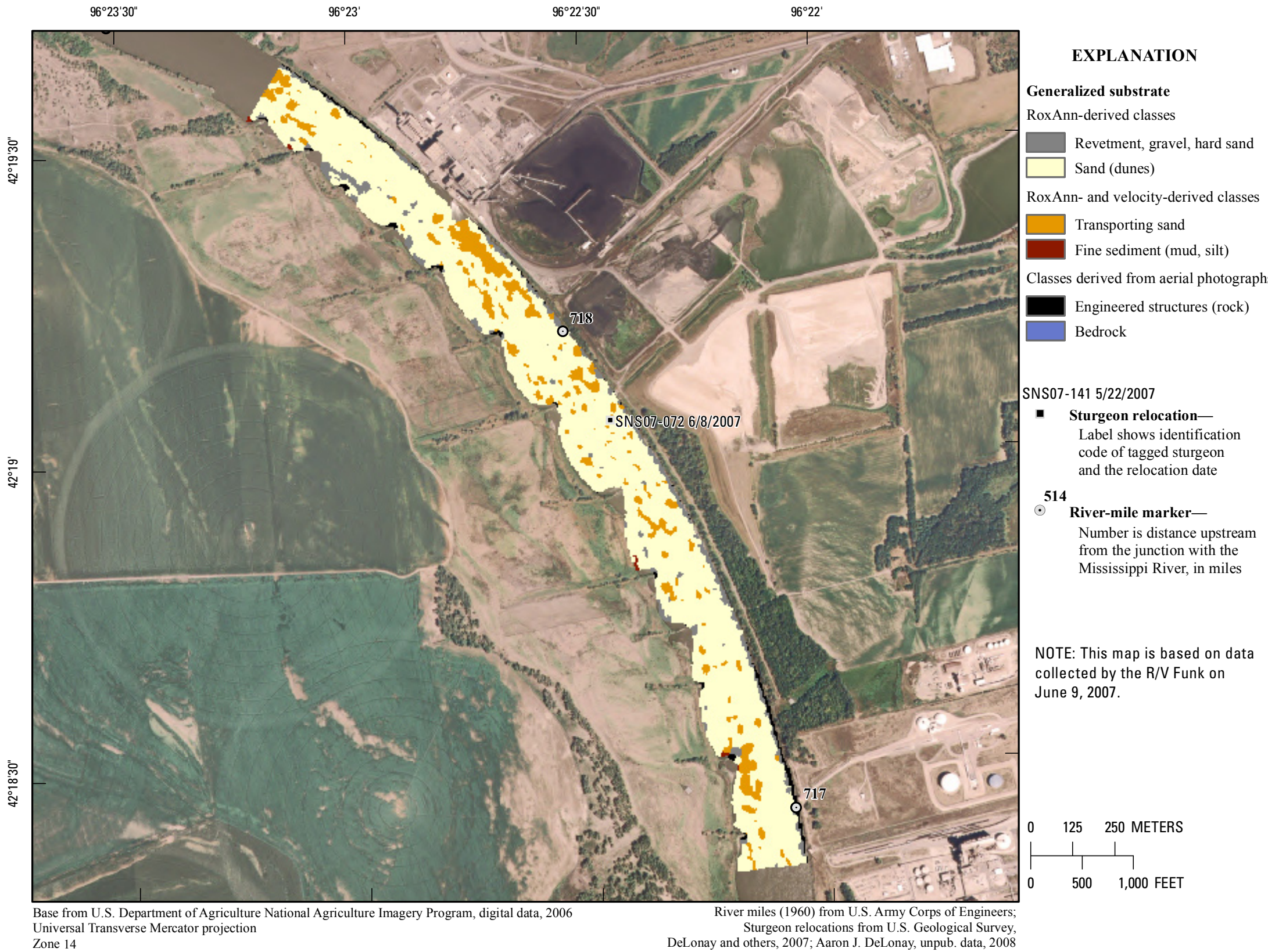

Figure 98. Map of generalized substrate based on data collected on June 9, 2007, in the vicinity of river mile 718 . 


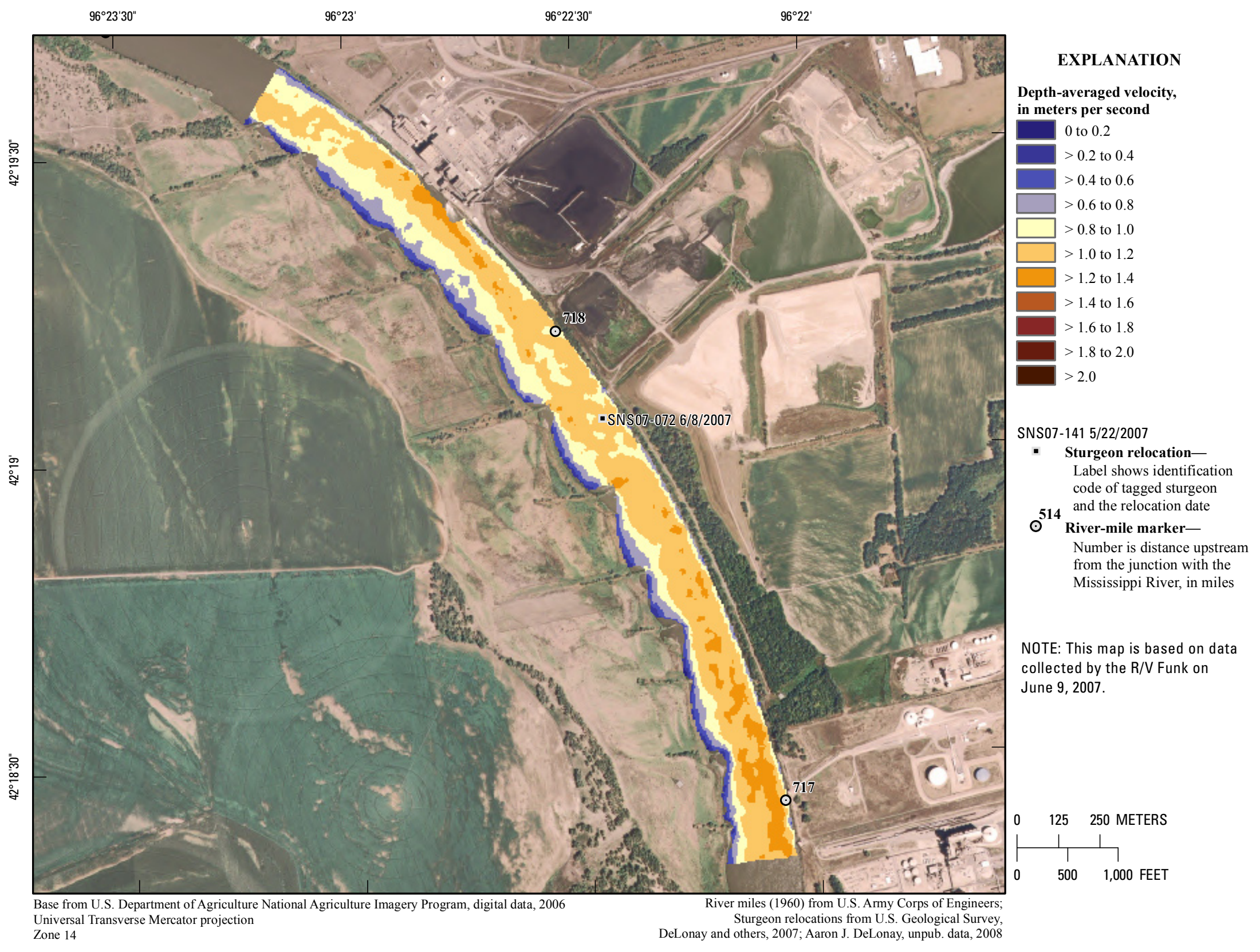

Figure 99. Map of depth-averaged velocity based on data collected on June 9, 2007, in the vicinity of river mile 718. 


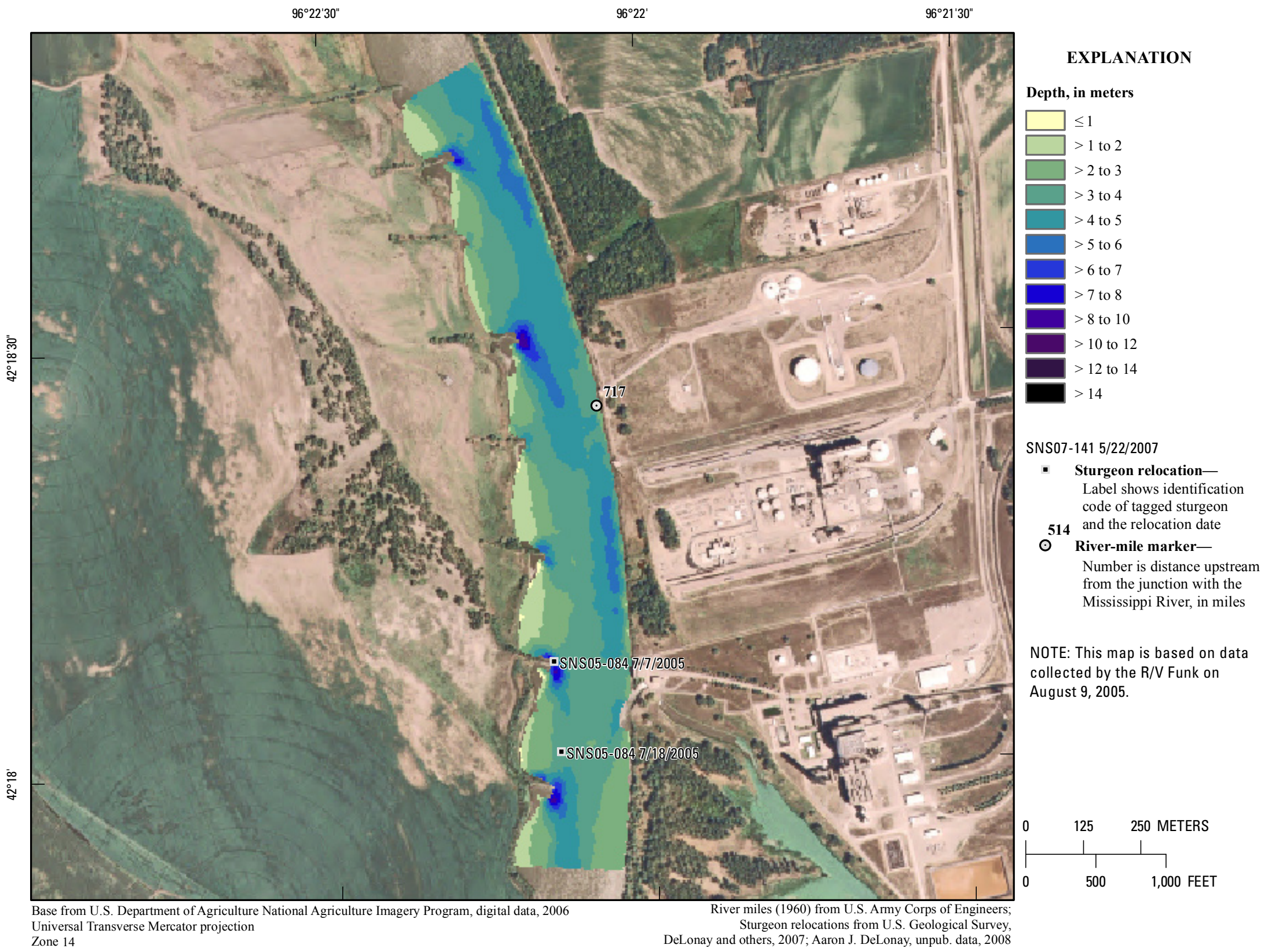

Figure 100. Map of depth based on data collected on August 9, 2005, in the vicinity of river mile 717 . 


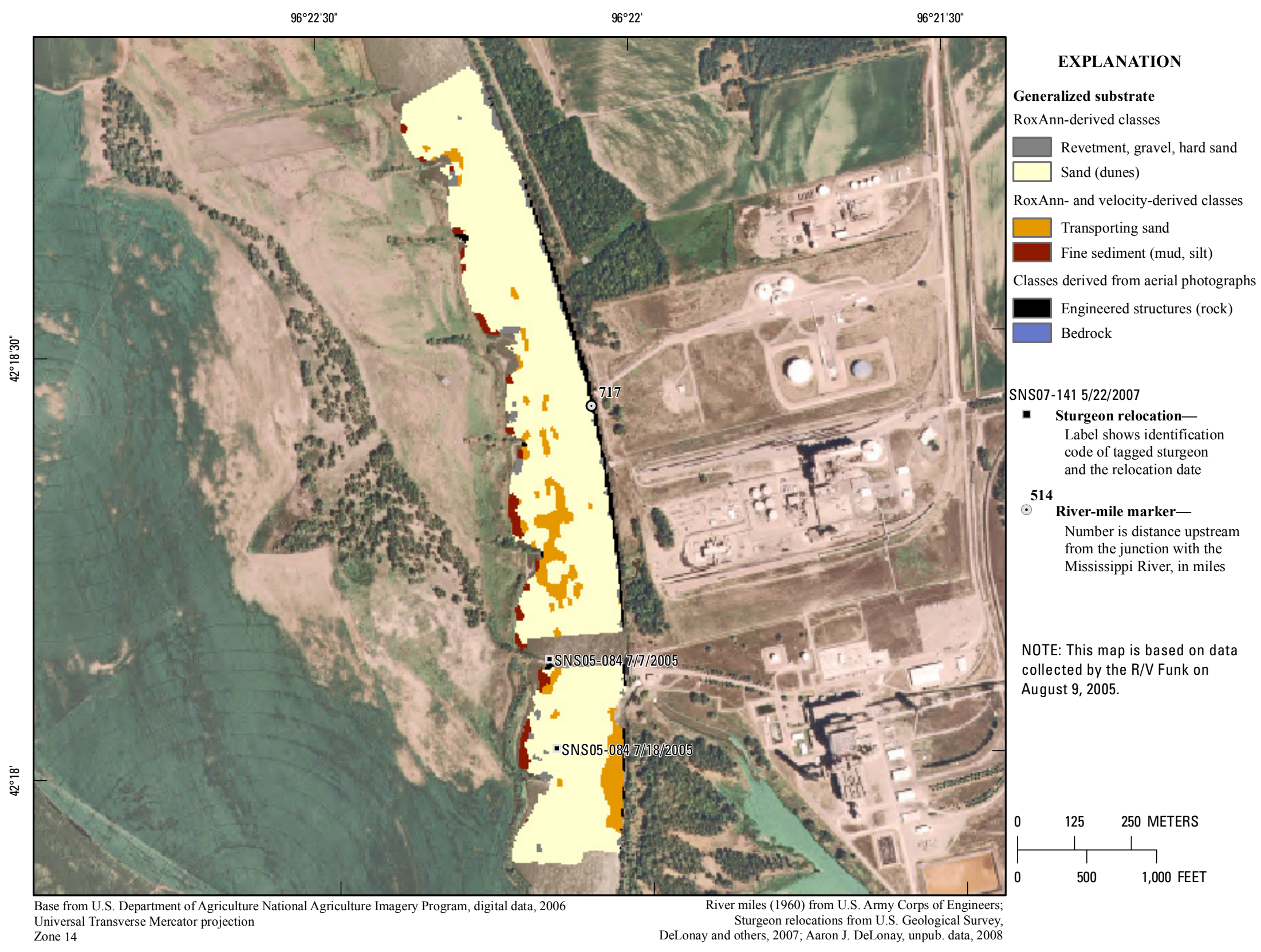

Figure 101. Map of generalized substrate based on data collected on August 9, 2005, in the vicinity of river mile 717 . 
$96^{\circ} 22^{\prime} 30^{\prime \prime}$

$96^{\circ} 22^{\prime}$

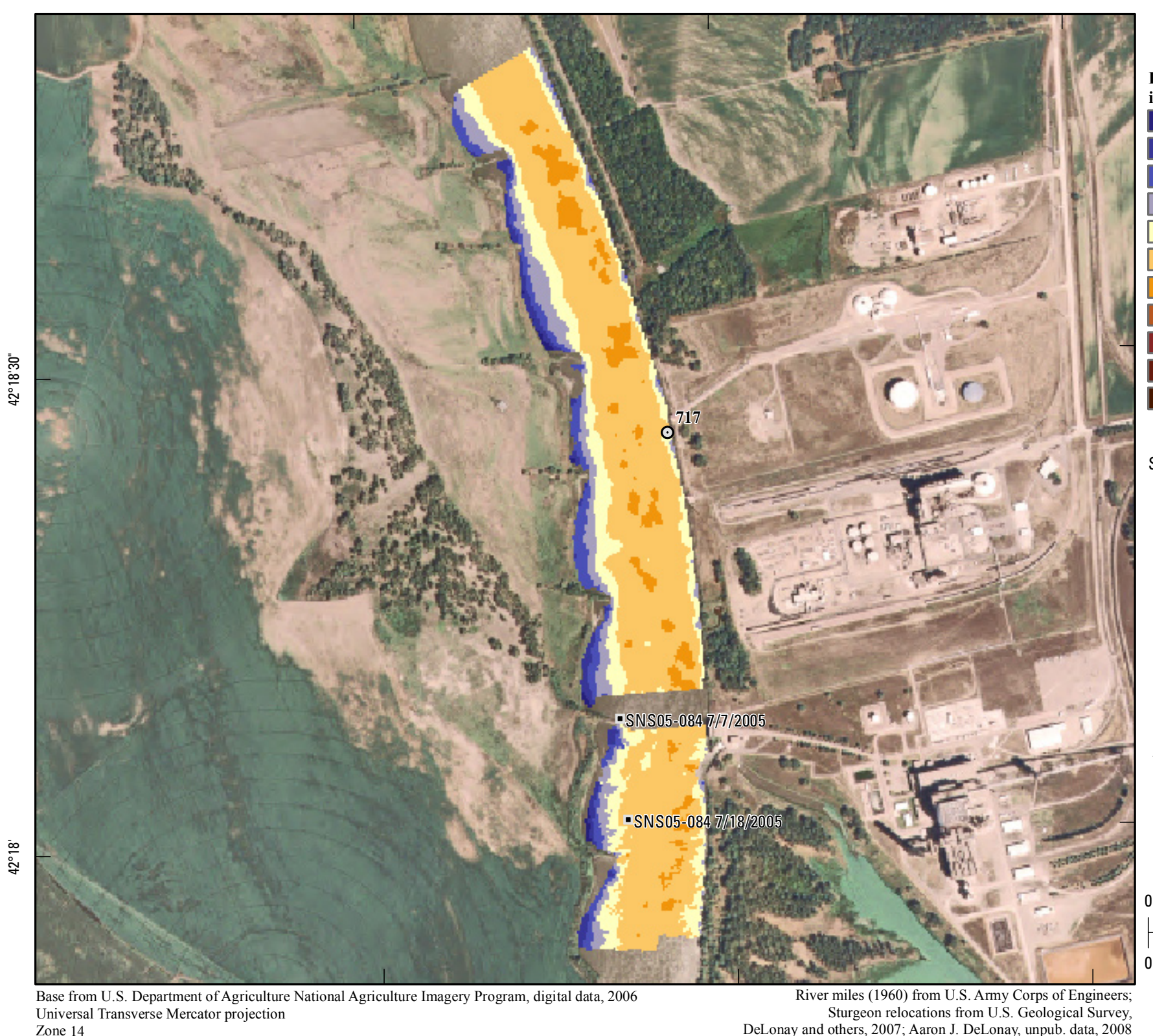

DeLonay and others, 2007; Aaron J. DeLonay, unpub. data, 2008
EXPLANATION

Depth-averaged velocity,

in meters per second

0 to 0.2

$>0.2$ to 0.4

$>0.4$ to 0.6

$>0.6$ to 0.8

$>0.8$ to 1.0

$>1.0$ to 1.2

$>1.2$ to 1.4

$>1.4$ to 1.6

$>1.6$ to 1.8

$>1.8$ to 2.0

$>2.0$

\section{SNS07-141 5/22/2007}

- Sturgeon relocation-

Label shows identification code of tagged sturgeo

$\mathbf{5 1 4}$ and the relocation date

$\odot^{514}$ River-mile marker-

Number is distance upstream

from the junction with the

Mississippi River, in miles

NOTE: This map is based on data

collected by the R/V Funk on

August 9, 2005

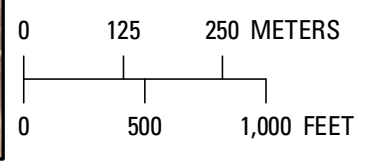

Figure 102. Map of depth-averaged velocity based on data collected on August 9, 2005, in the vicinity of river mile 717 . 


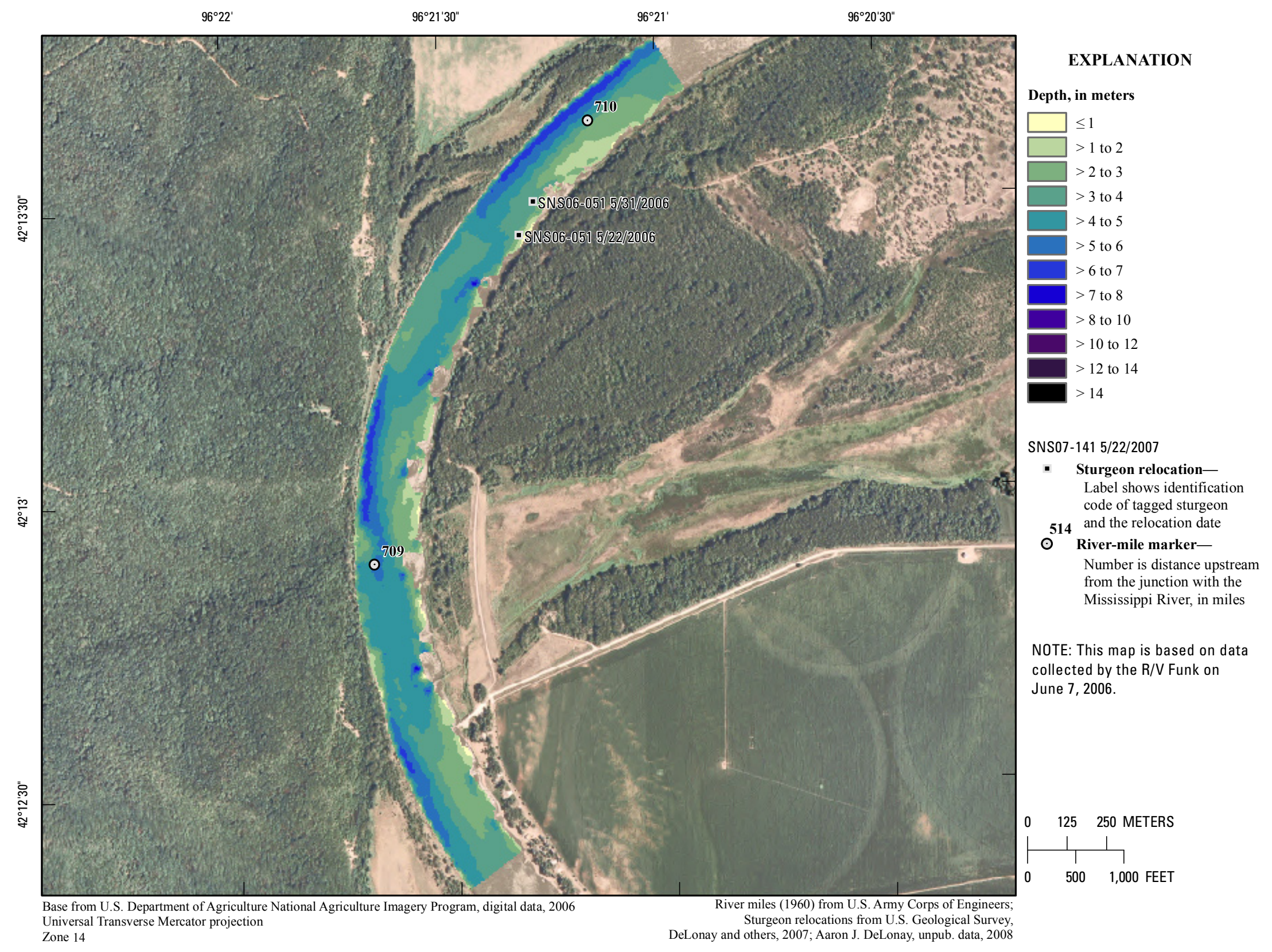

Figure 103. Map of depth based on data collected on June 7, 2006, in the vicinity of river mile 709 


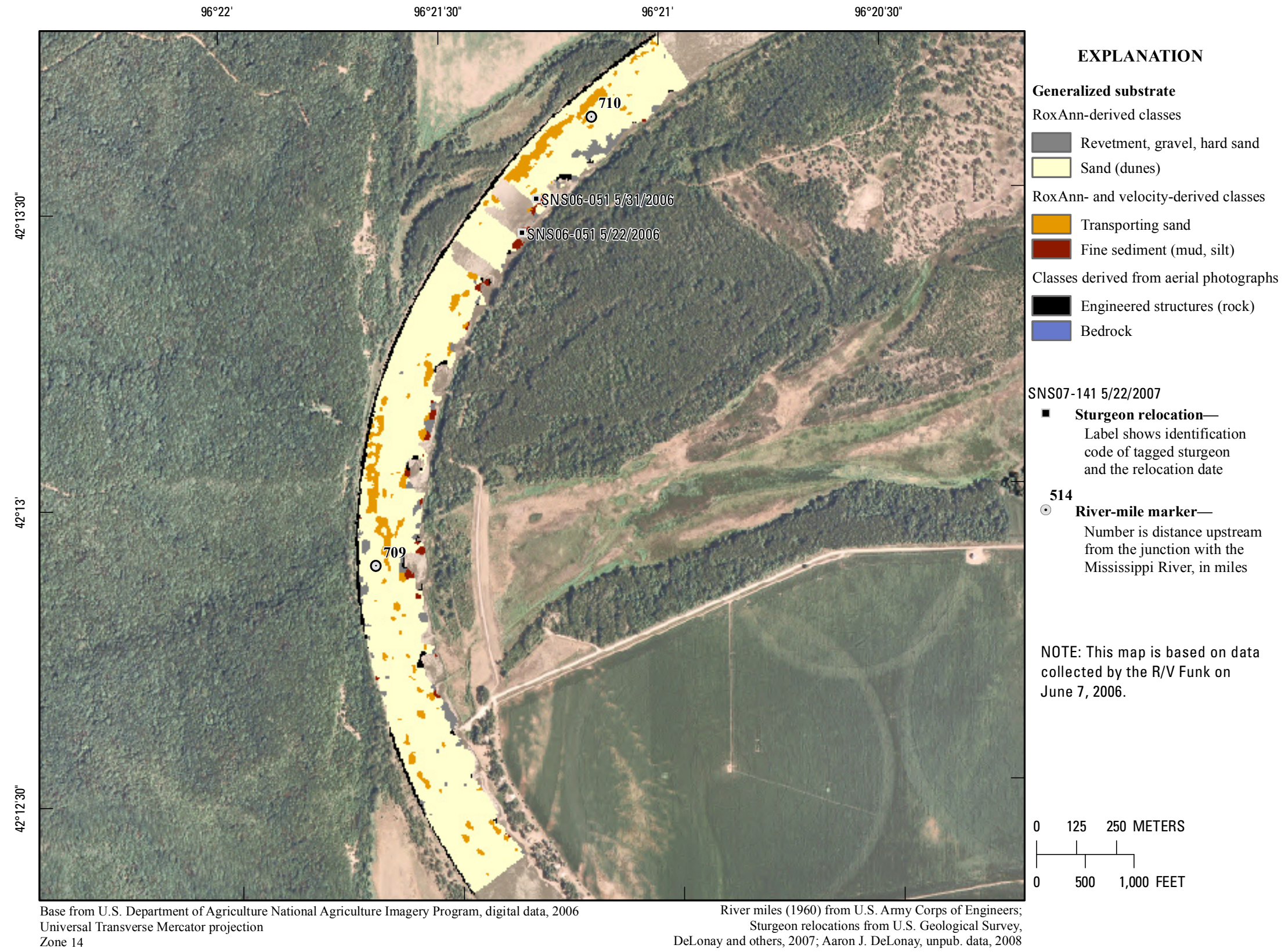

Figure 104. Map of generalized substrate based on data collected on June 7, 2006, in the vicinity of river mile 709. 


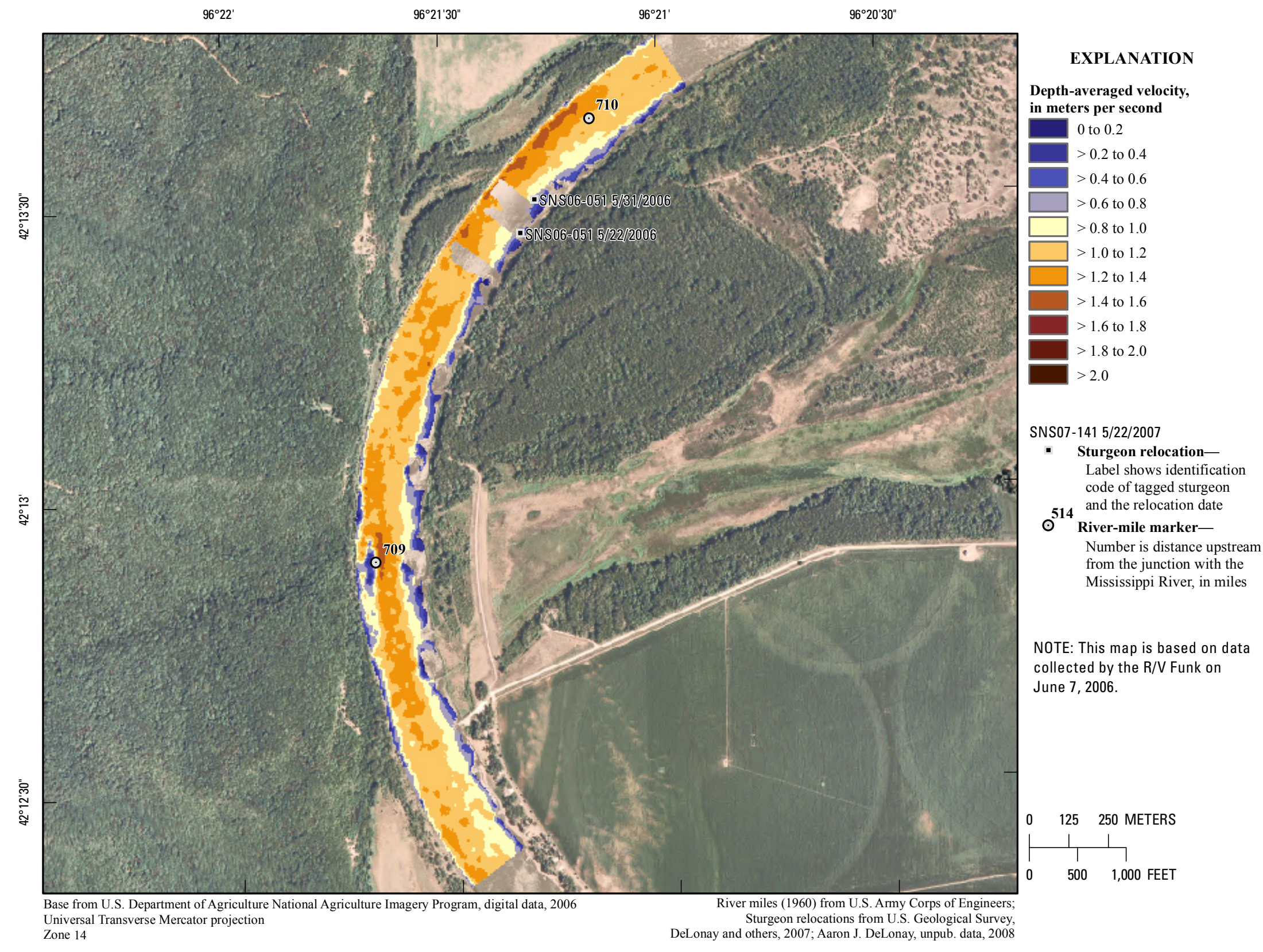

Figure 105. Map of depth-averaged velocity based on data collected on June 7, 2006, in the vicinity of river mile 709 . 


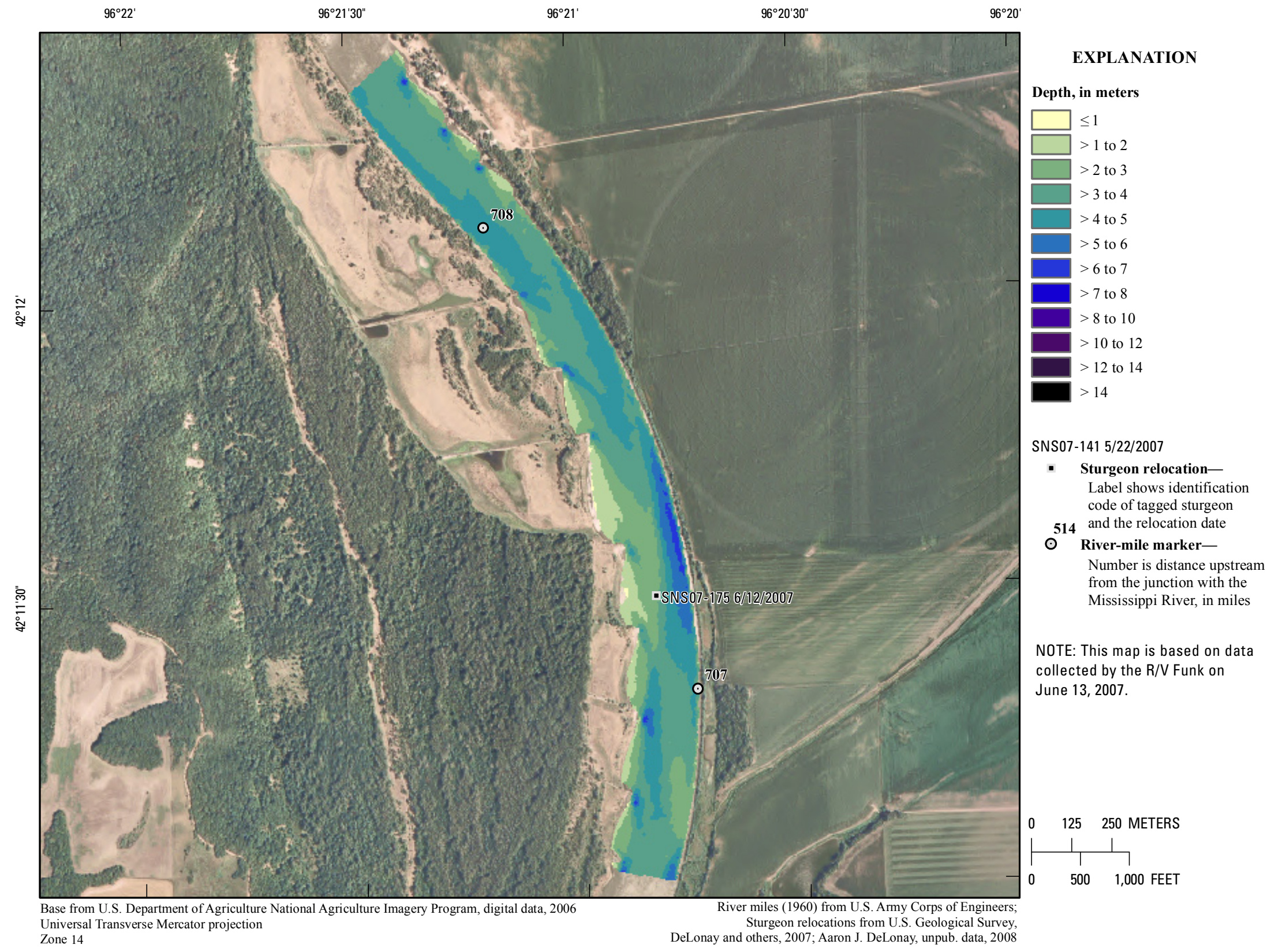

Figure 106. Map of depth based on data collected on June 13, 2007, in the vicinity of river mile 708 . 


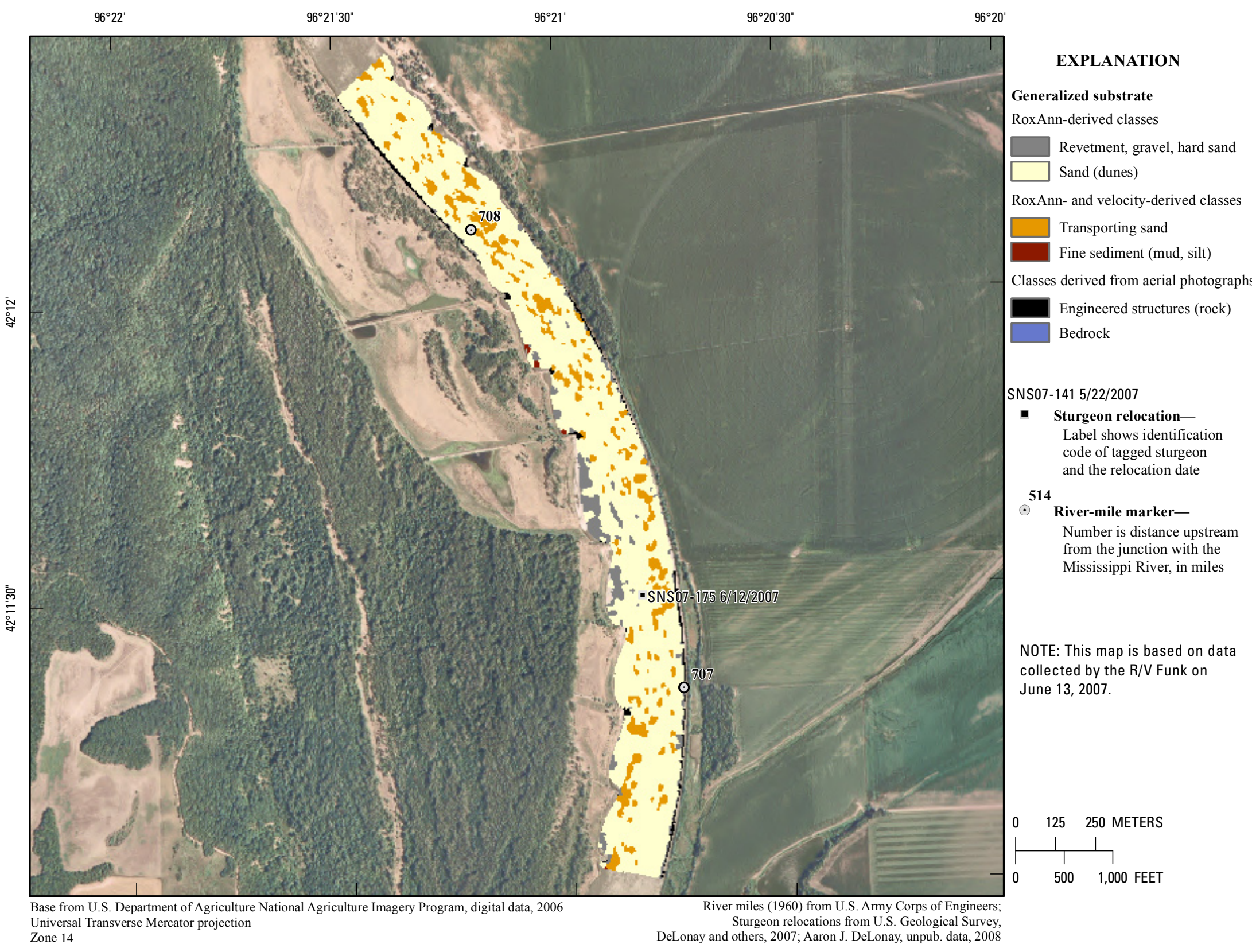

Figure 107. Map of generalized substrate based on data collected on June 13,2007 , in the vicinity of river mile 708 


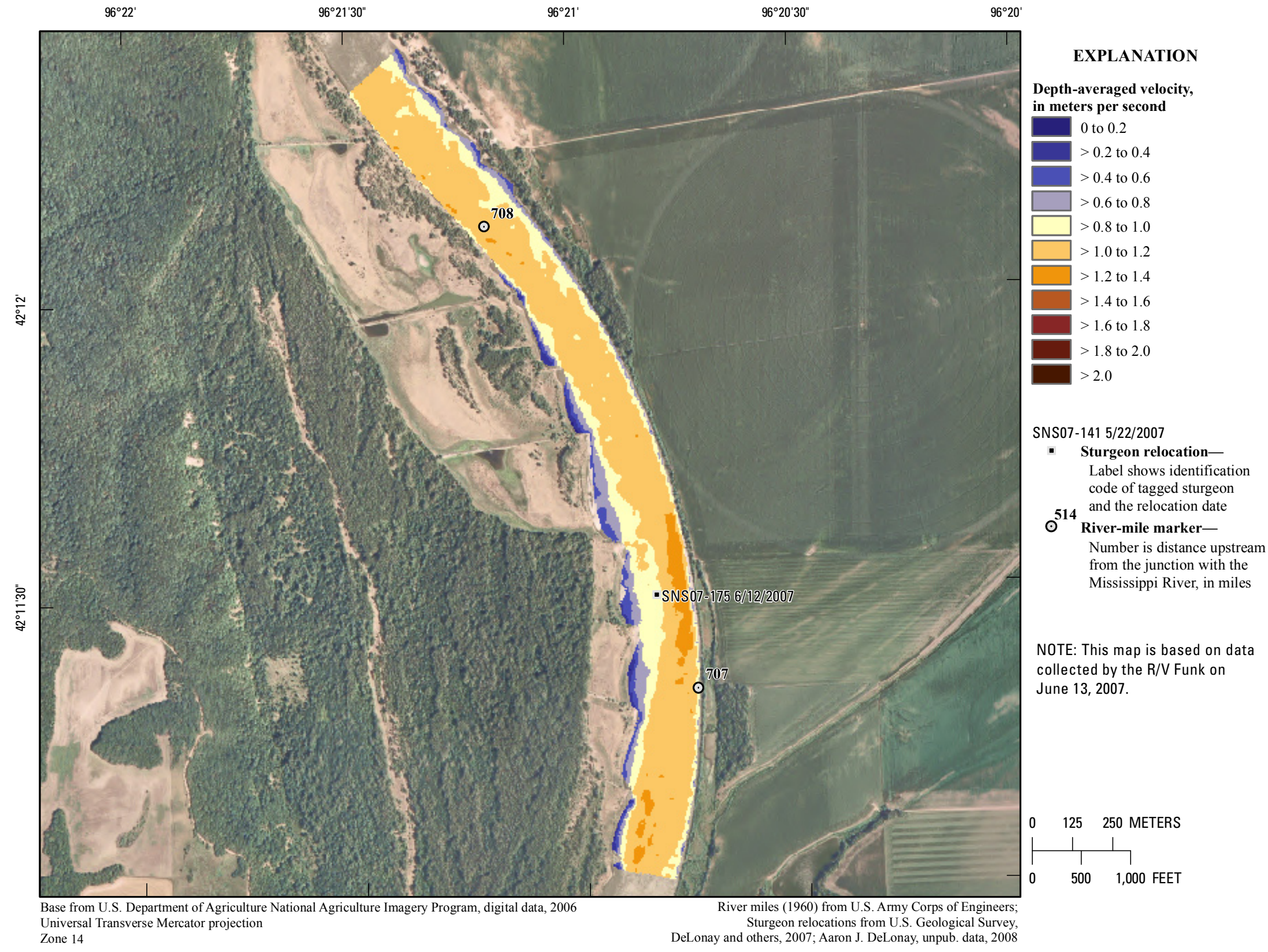

Figure 108. Map of depth-averaged velocity based on data collected on June 13, 2007, in the vicinity of river mile 708. 


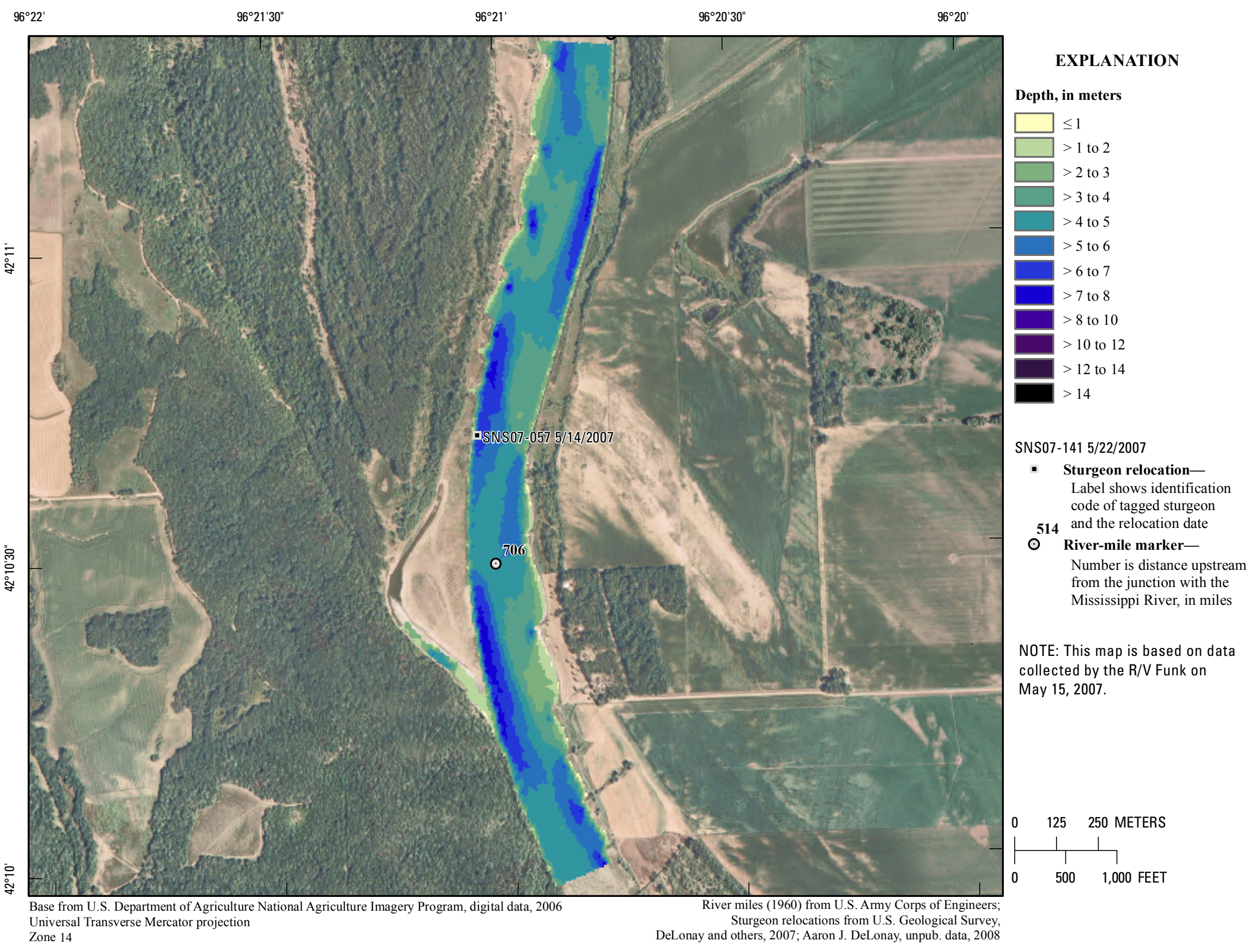

Figure 109. Map of depth based on data collected on May 15, 2007, in the vicinity of river mile 706. 


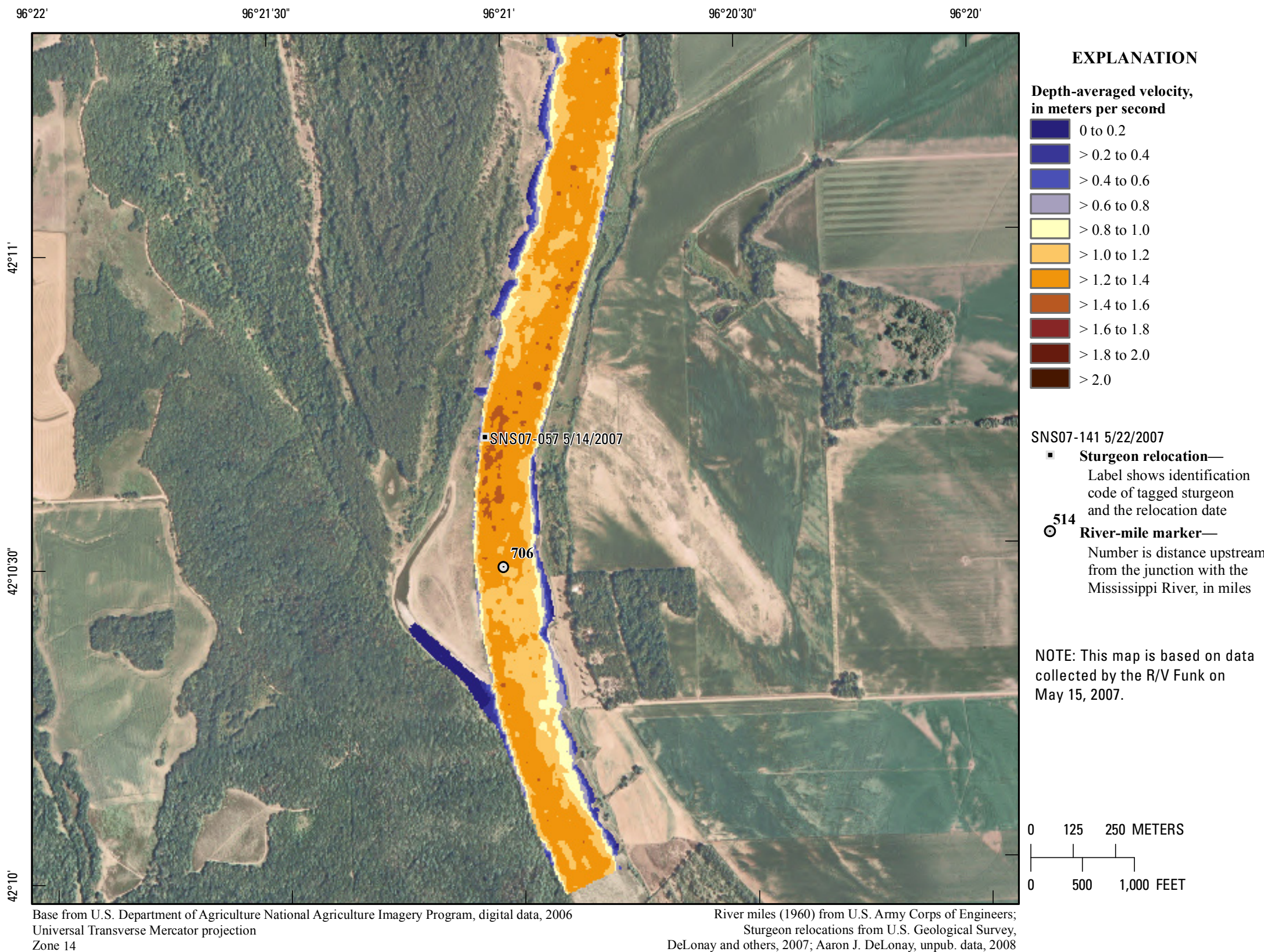

Figure 110. Map of depth-averaged velocity based on data collected on May 15, 2007, in the vicinity of river mile 706 . 


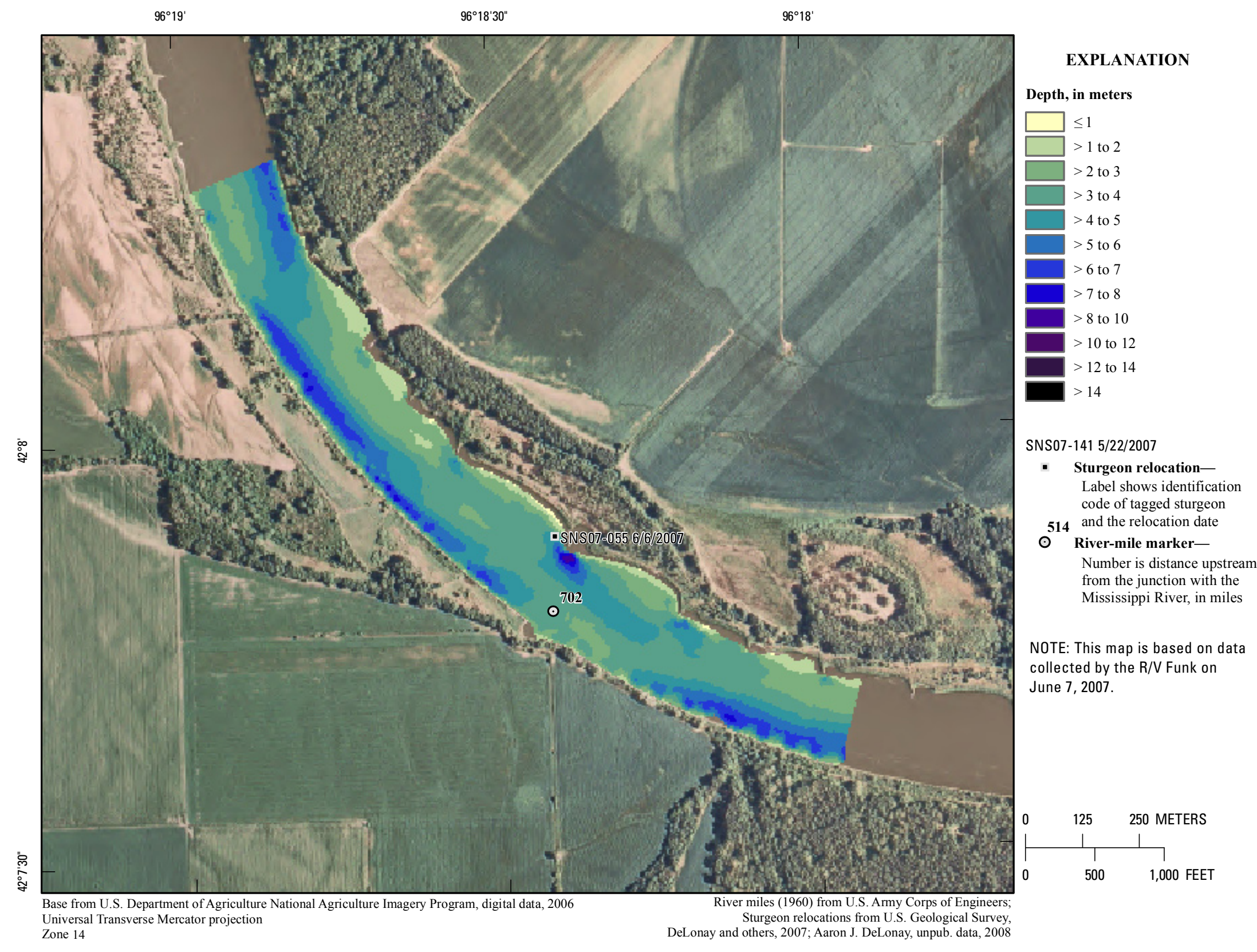

Figure 111. Map of depth based on data collected on June 7, 2007, in the vicinity of river mile 702. 


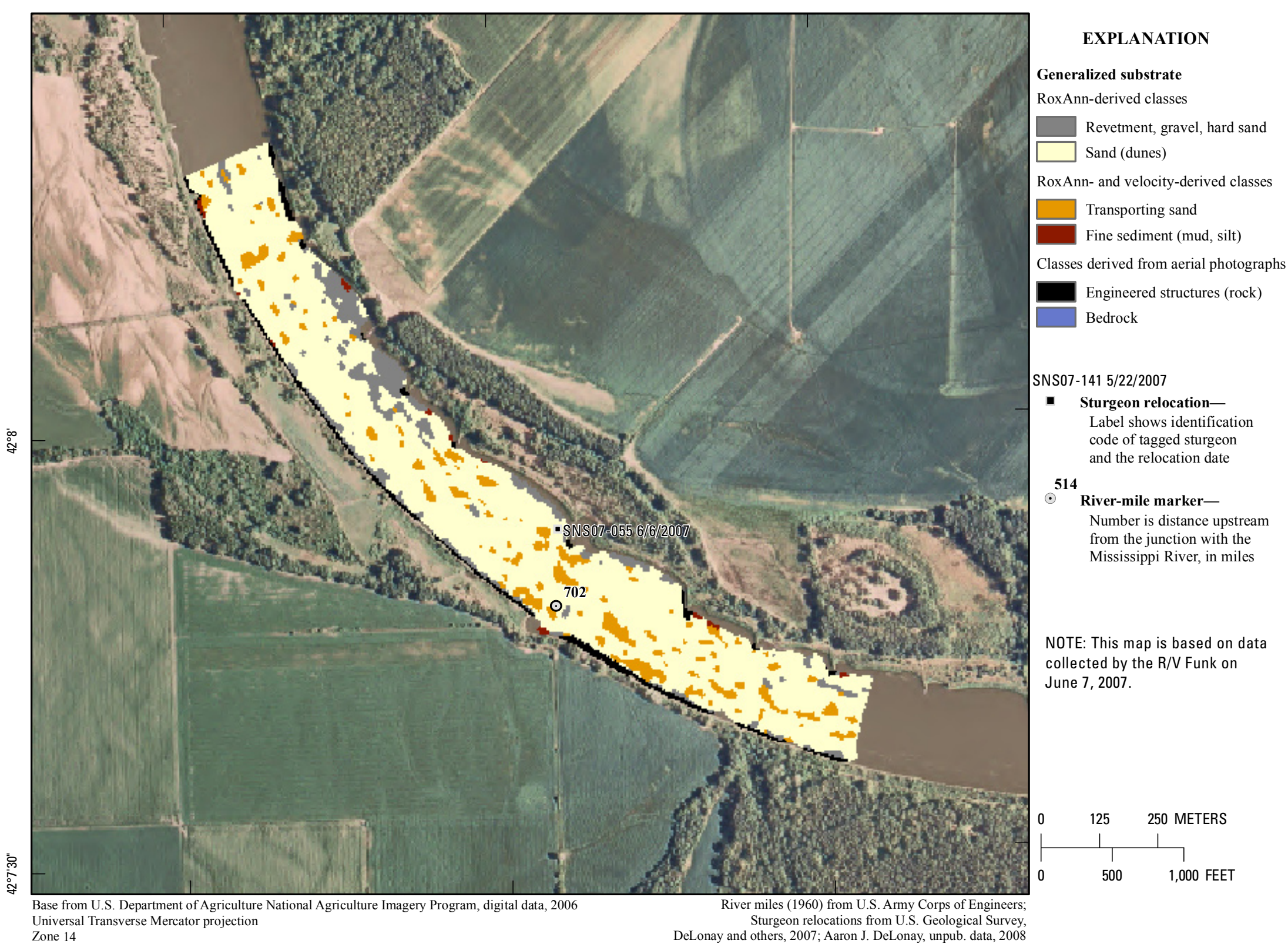
Universal Transverse Mercator projection Sturgeon relocations from U.S. Geological Survey,
DeLonay and others, 2007; Aaron J. DeLonay, unpub. data, 2008 


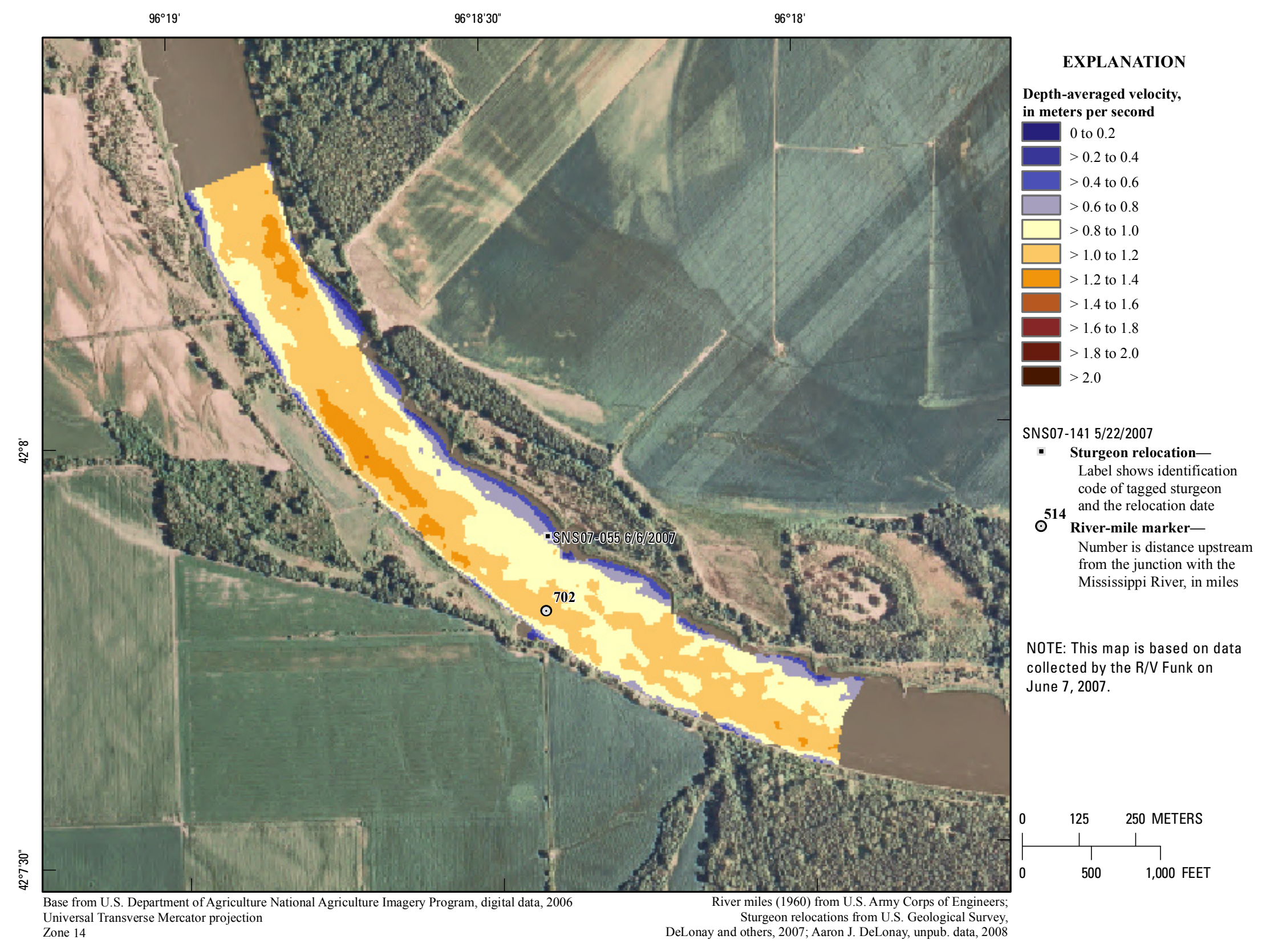

Figure 113. Map of depth-averaged velocity based on data collected on June 7, 2007, in the vicinity of river mile 702 . 


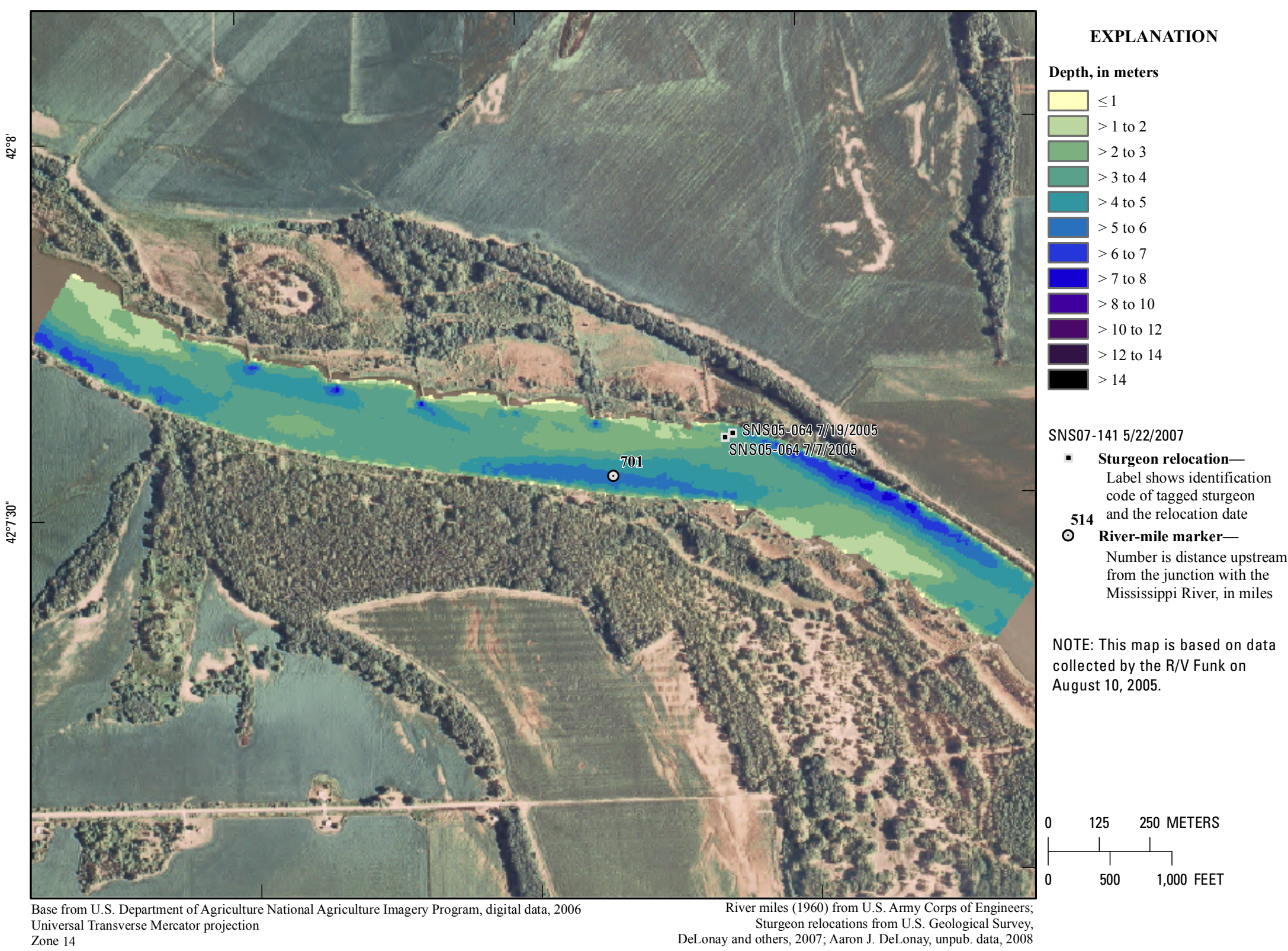
Universal Transverse Mercator projection 


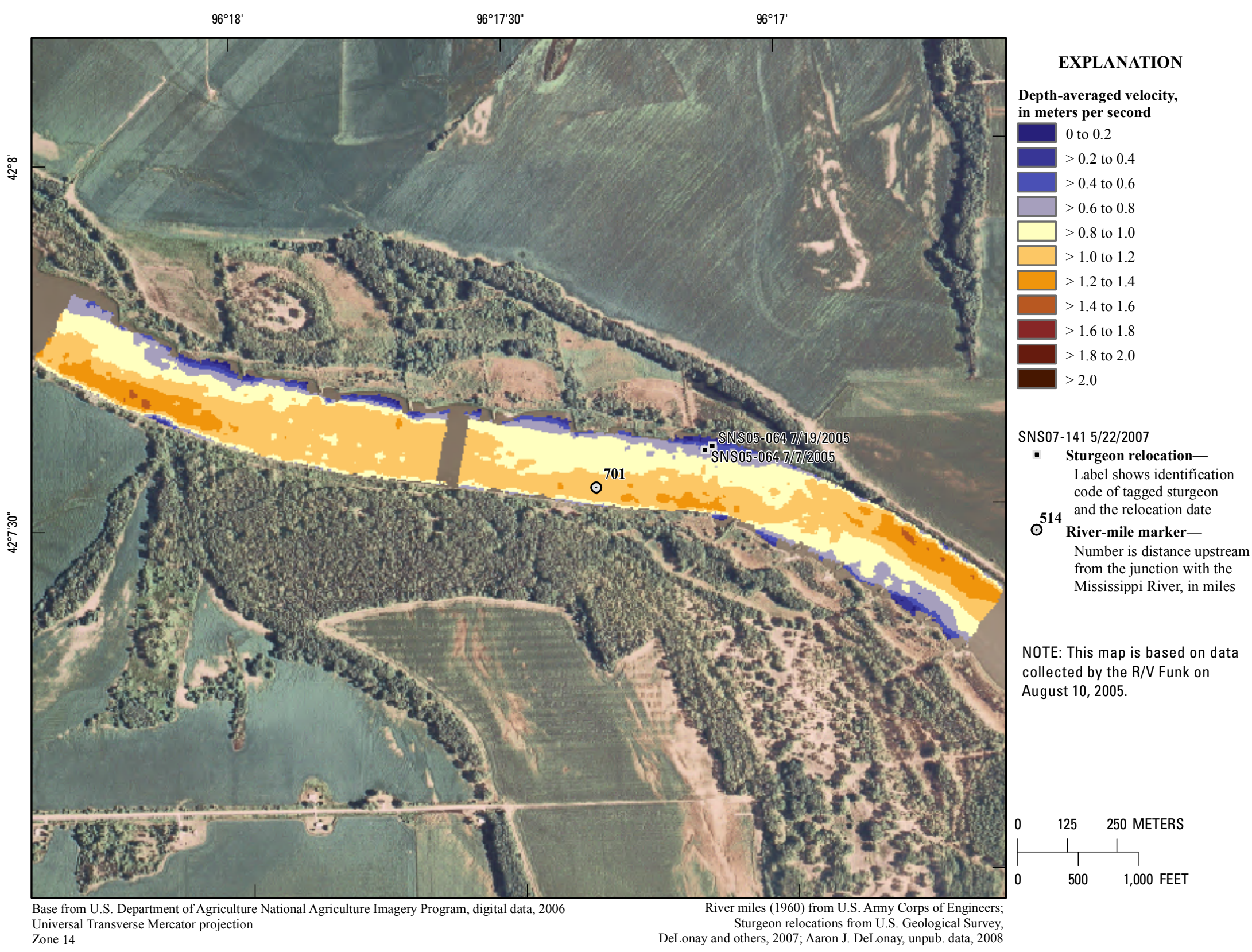

Figure 115. Map of depth-averaged velocity based on data collected on August 10, 2005, in the vicinity of river mile 701. 
$96^{\circ} 17$

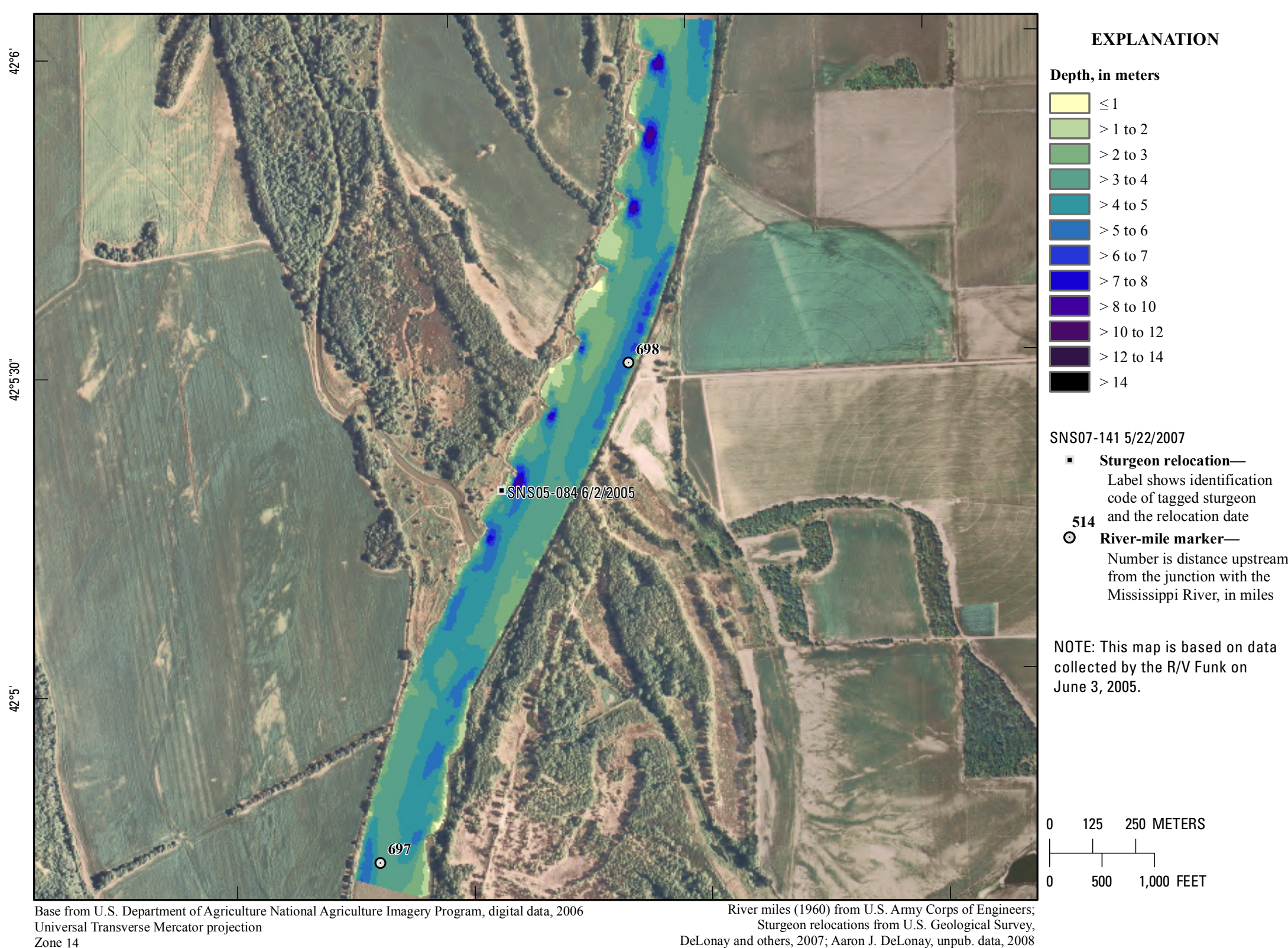




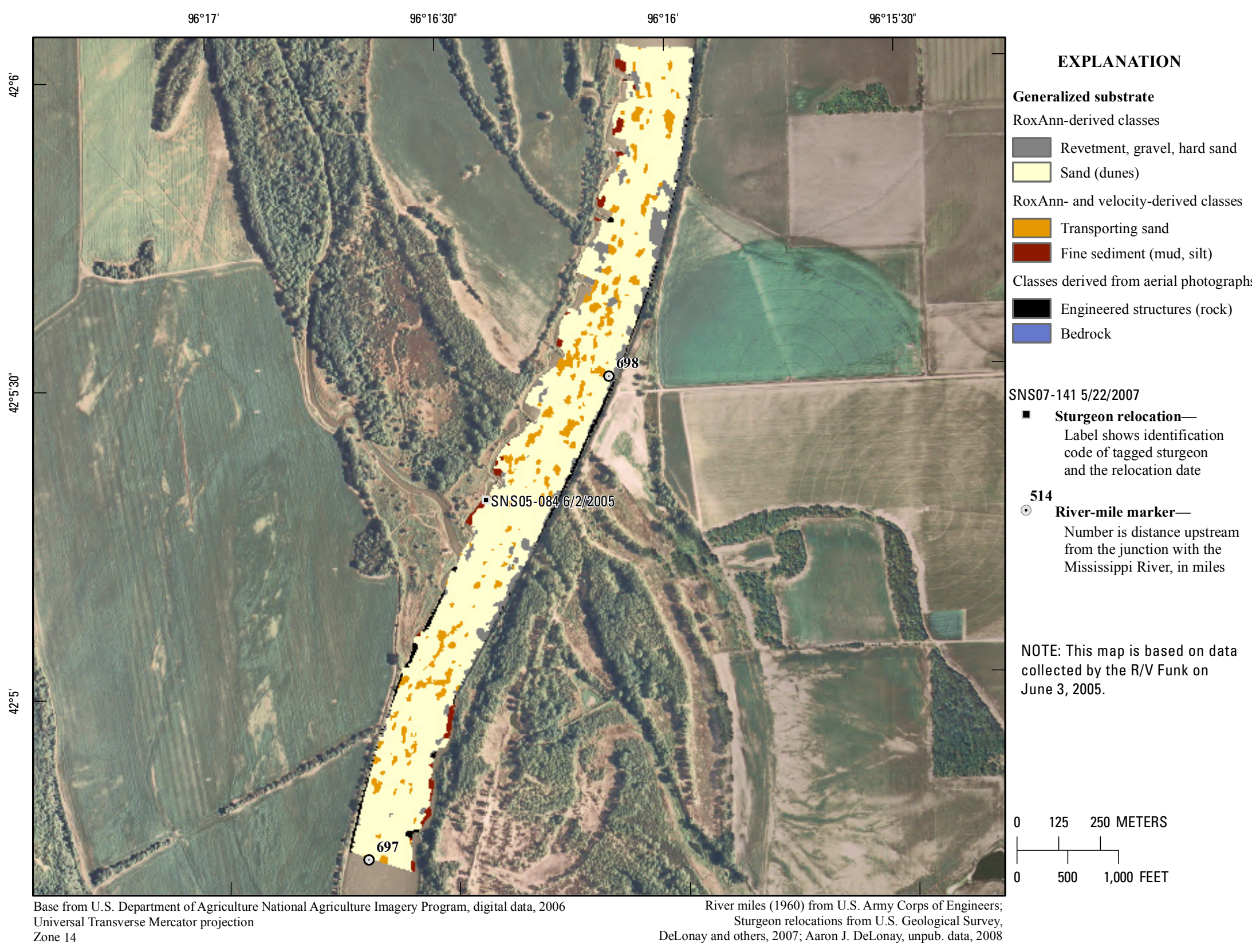

Figure 117. Map of generalized substrate based on data collected on June 3,2005 , in the vicinity of river mile 698 . 


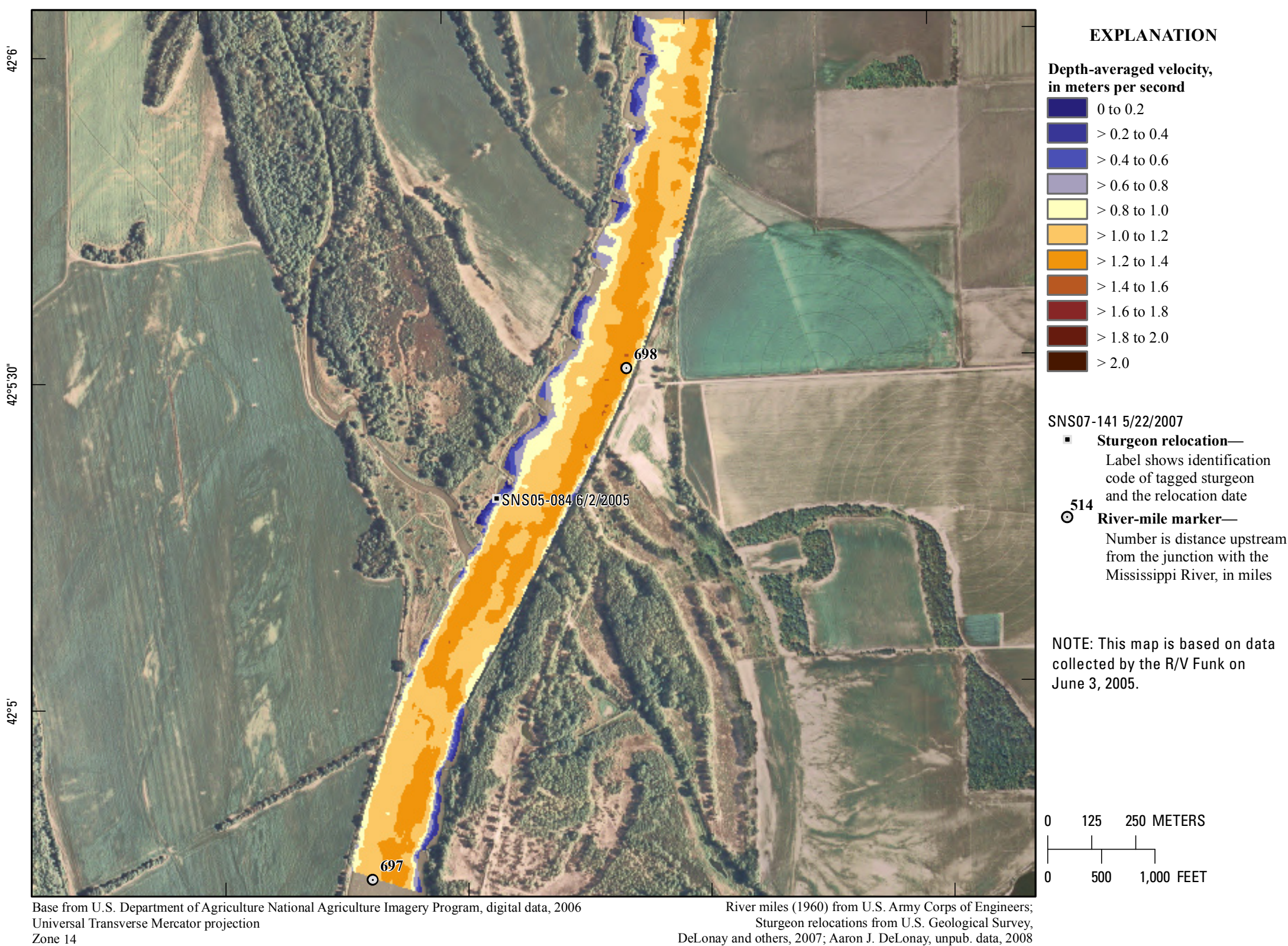




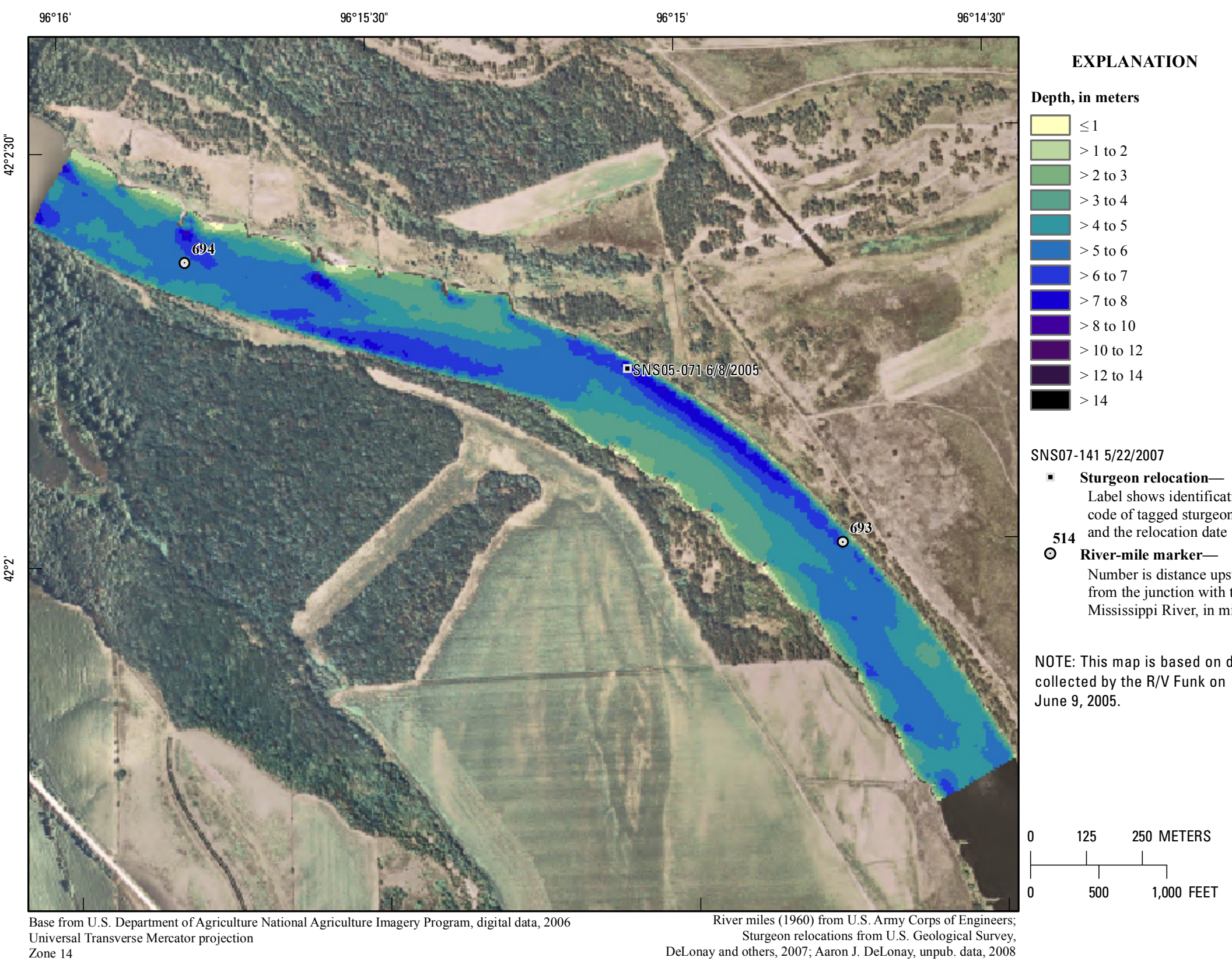

Figure 119. Map of depth based on data collected on June 9, 2005, in the vicinity of river mile 693 . 


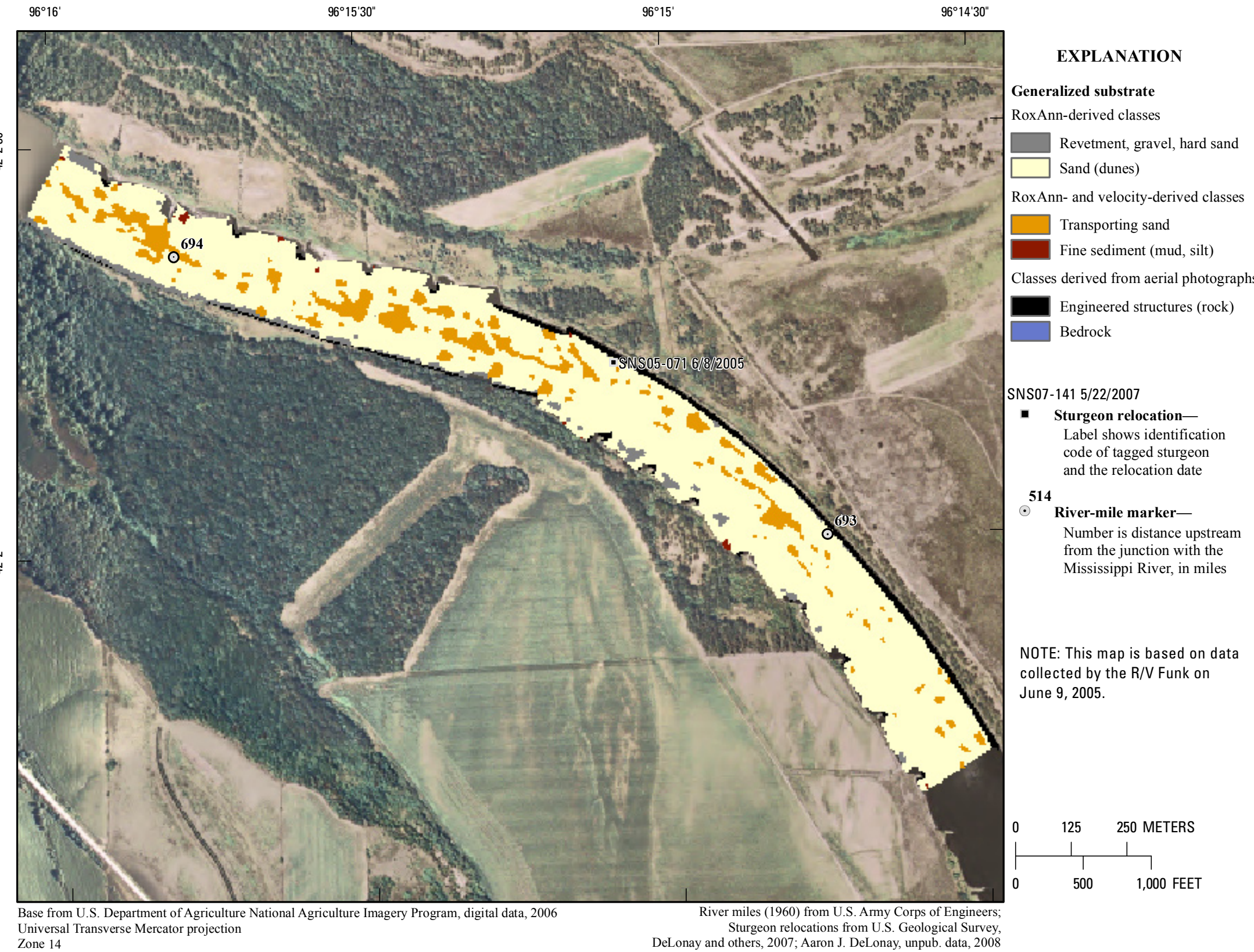

Figure 120. Map of generalized substrate based on data collected on June 9, 2005, in the vicinity of river mile 693. 


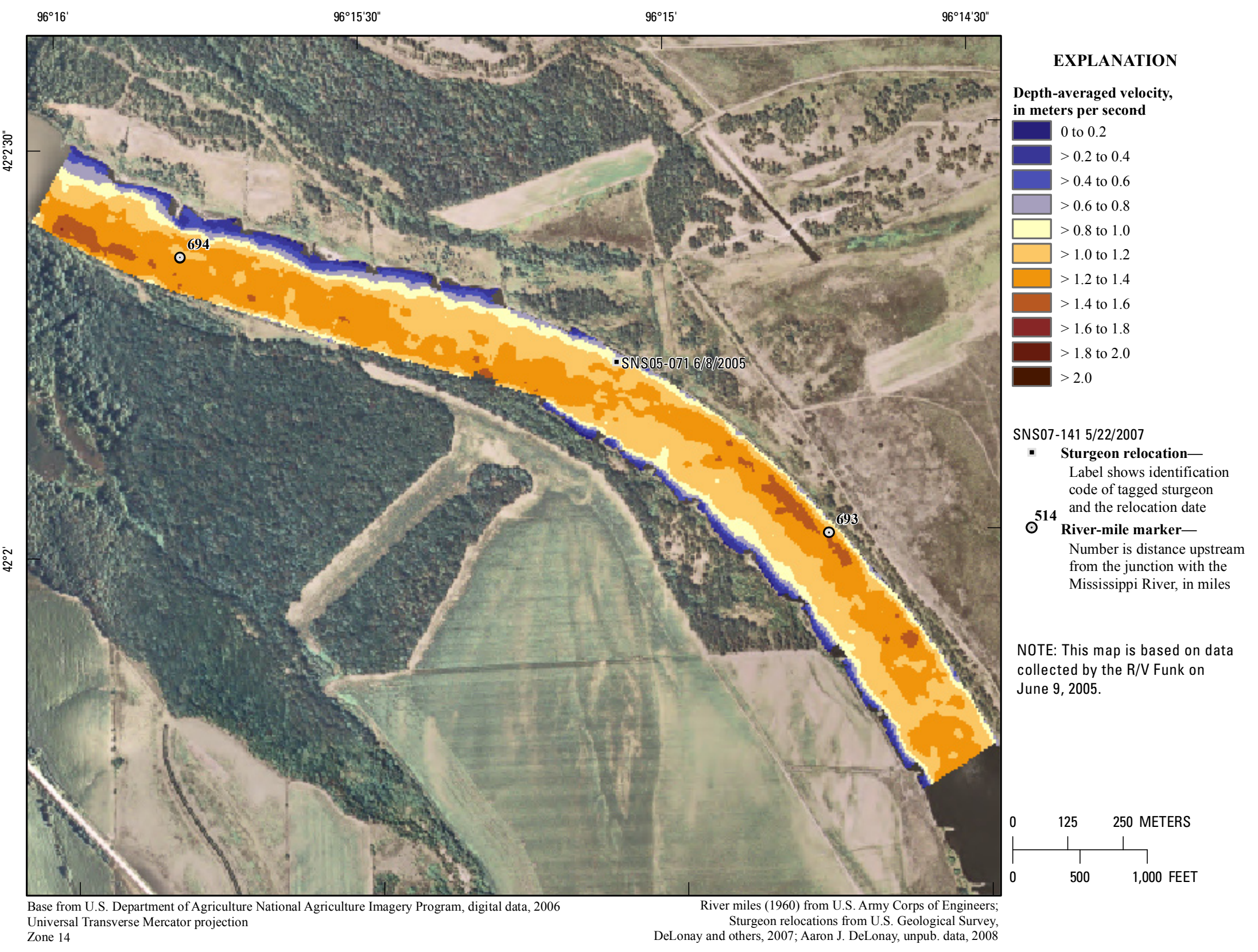

Figure 121. Map of depth-averaged velocity based on data collected on June 9, 2005, in the vicinity of river mile 693 . 


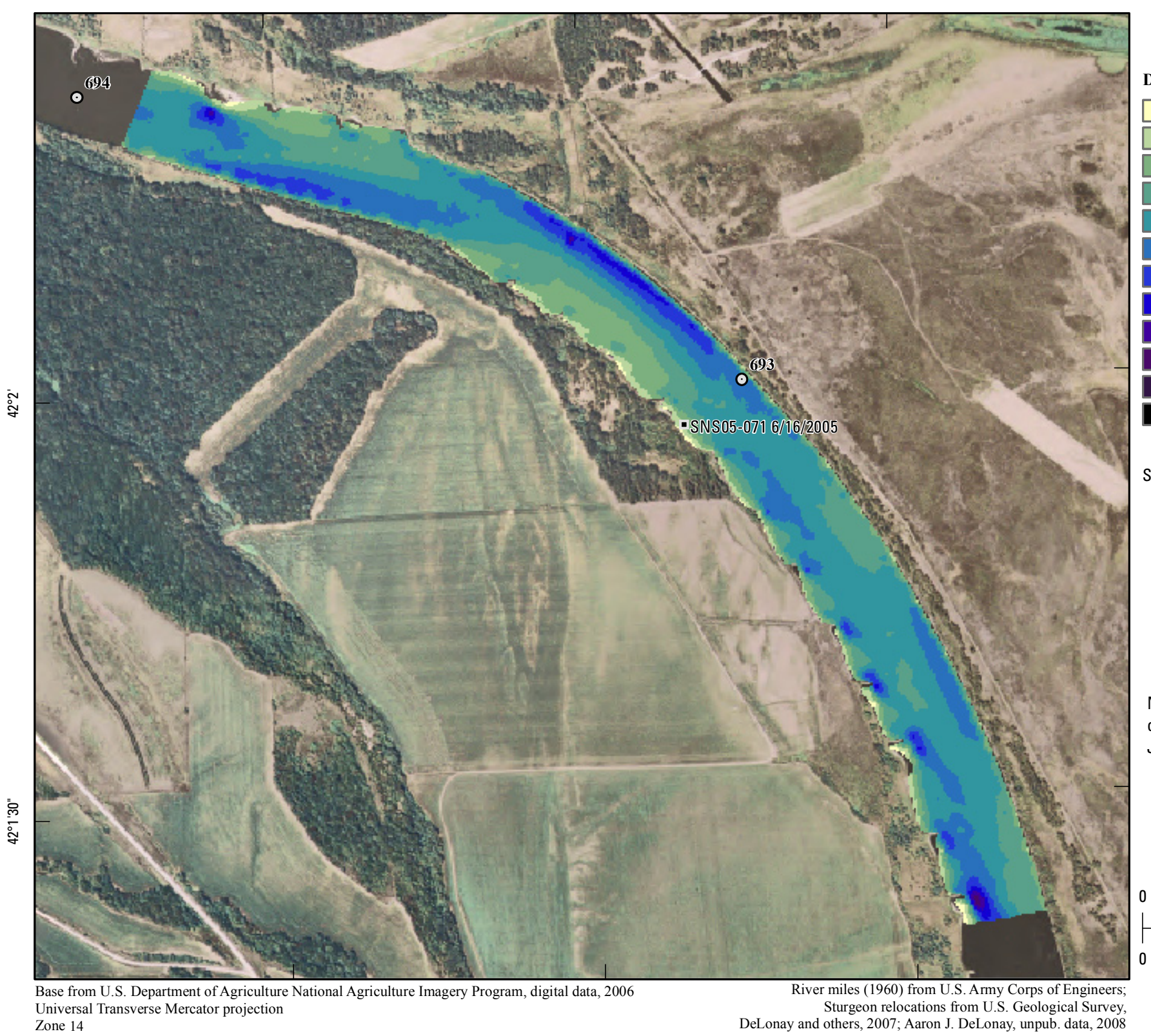

EXPLANATION

Depth, in meters

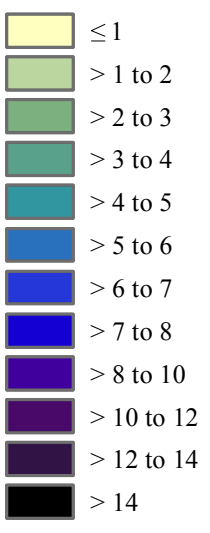

SNS07-141 5/22/2007

- Sturgeon relocationLabel shows identification code of tagged sturgeon ${ }_{0}^{514}$ and the relocation date Number is distance upstream from the junction with the Mississippi River, in miles

NOTE: This map is based on data collected by the R/V Funk on June 17, 2005.

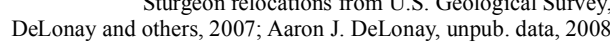

\begin{tabular}{|c|c|}
\hline 125 & 250 METERS \\
\hline 1 & $\perp$ \\
\hline 500 & 1,000 FEET \\
\hline
\end{tabular}




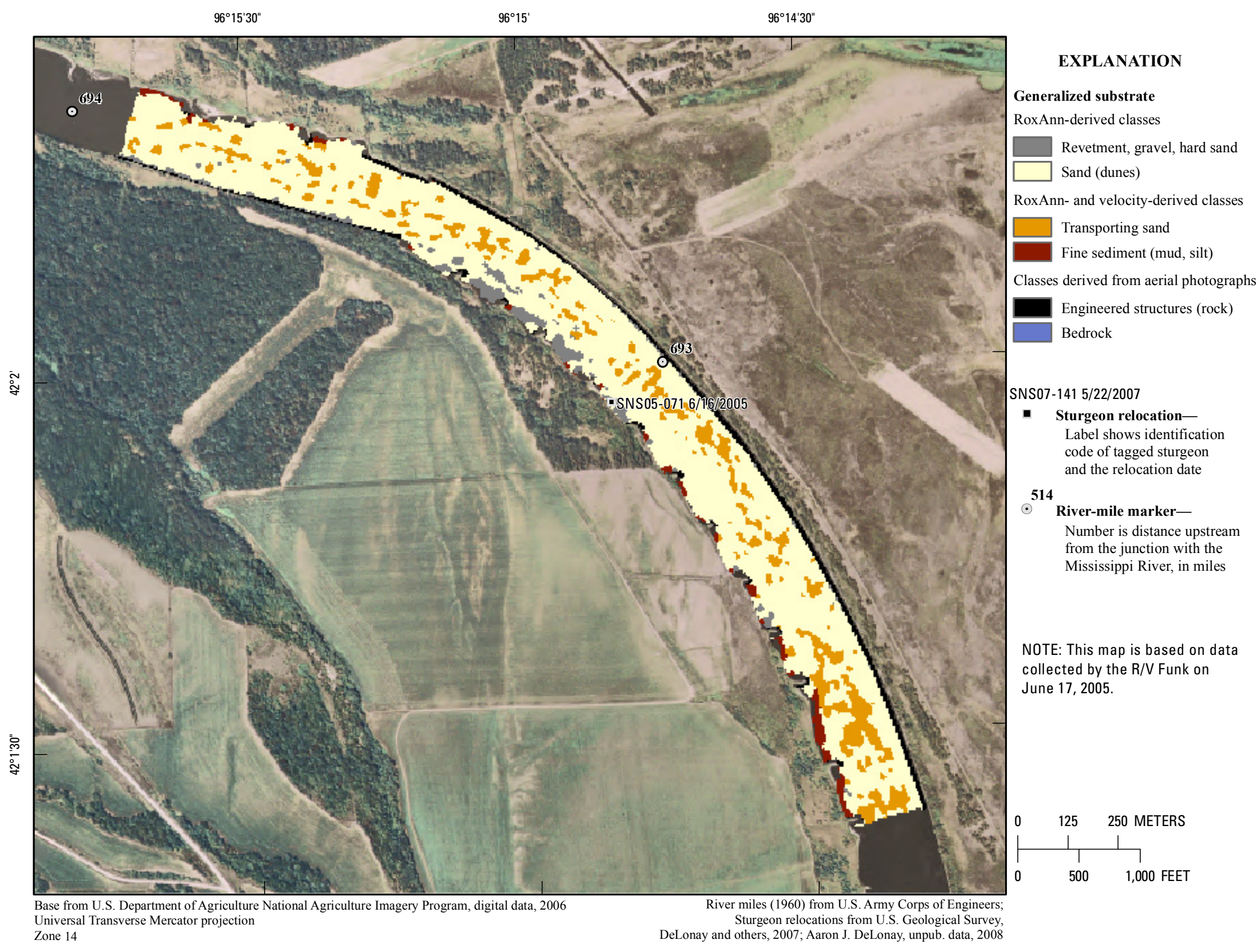

Figure 123. Map of generalized substrate based on data collected on June 17, 2005, in the vicinity of river mile 693 . 


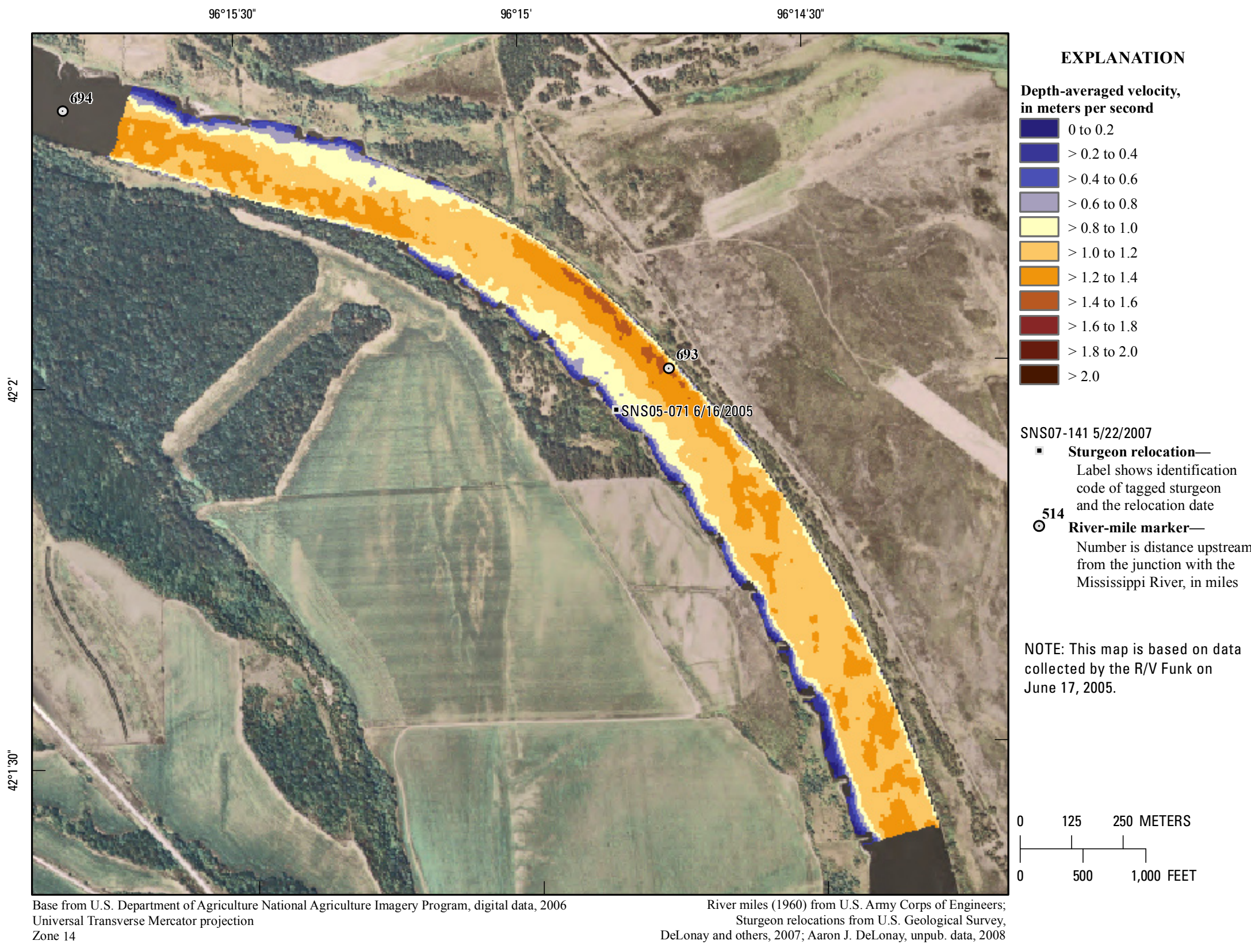

Figure 124. Map of depth-averaged velocity based on data collected on June 17, 2005, in the vicinity of river mile 693. 


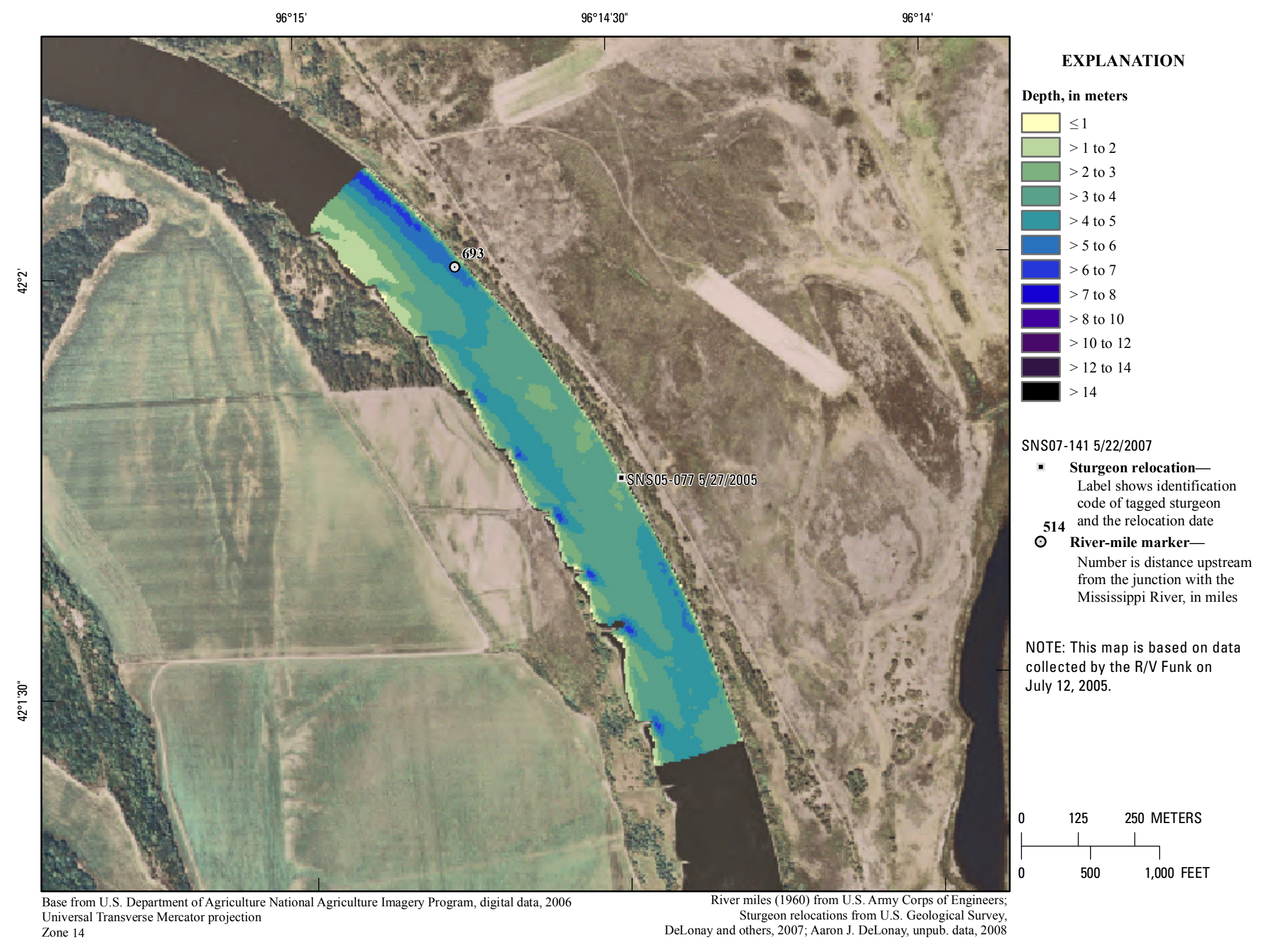

Figure 125. Map of depth based on data collected on July 12, 2005, in the vicinity of river mile 693 . 


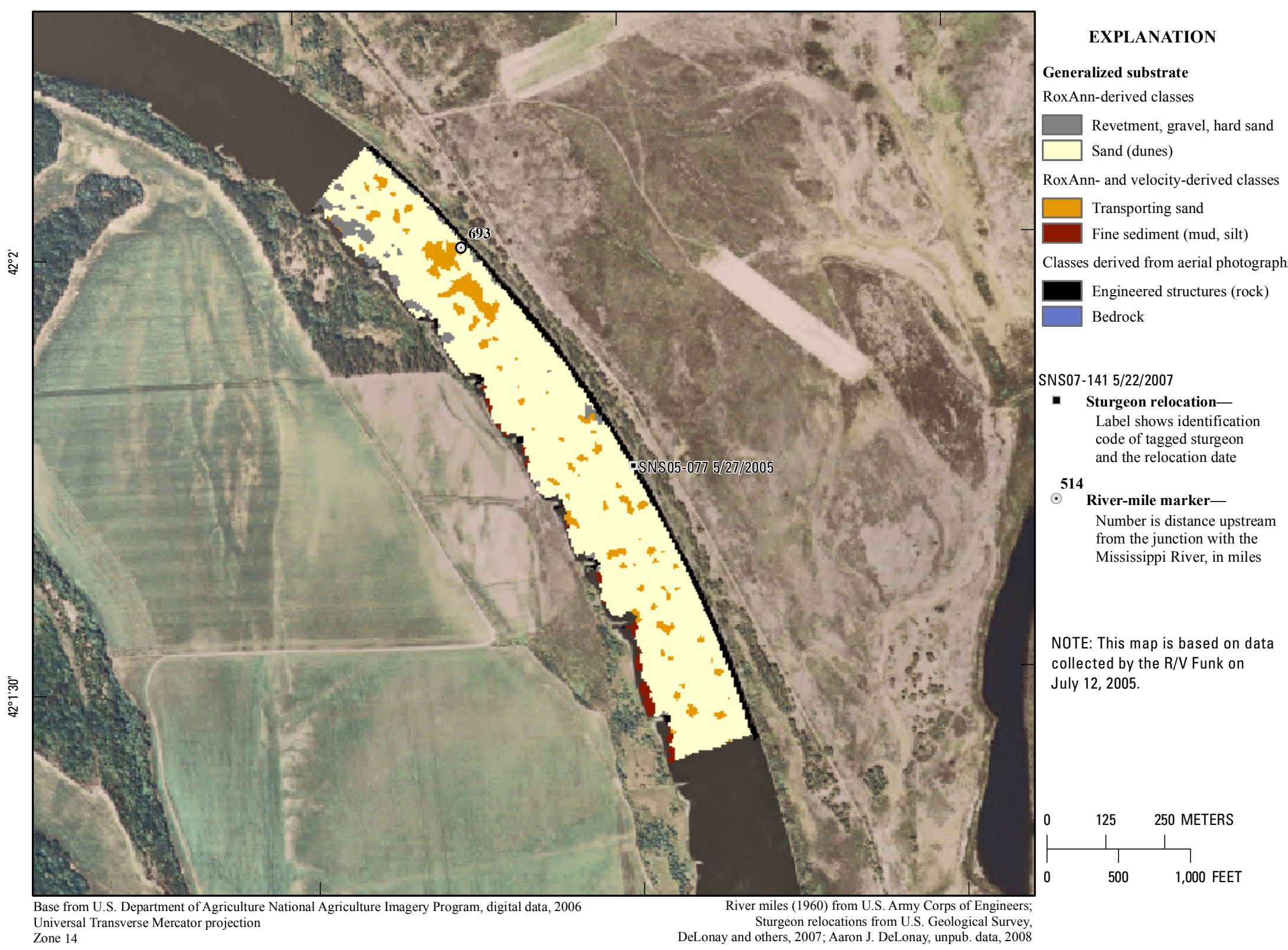

Figure 126. Map of generalized substrate based on data collected on July 12, 2005, in the vicinity of river mile 693. 


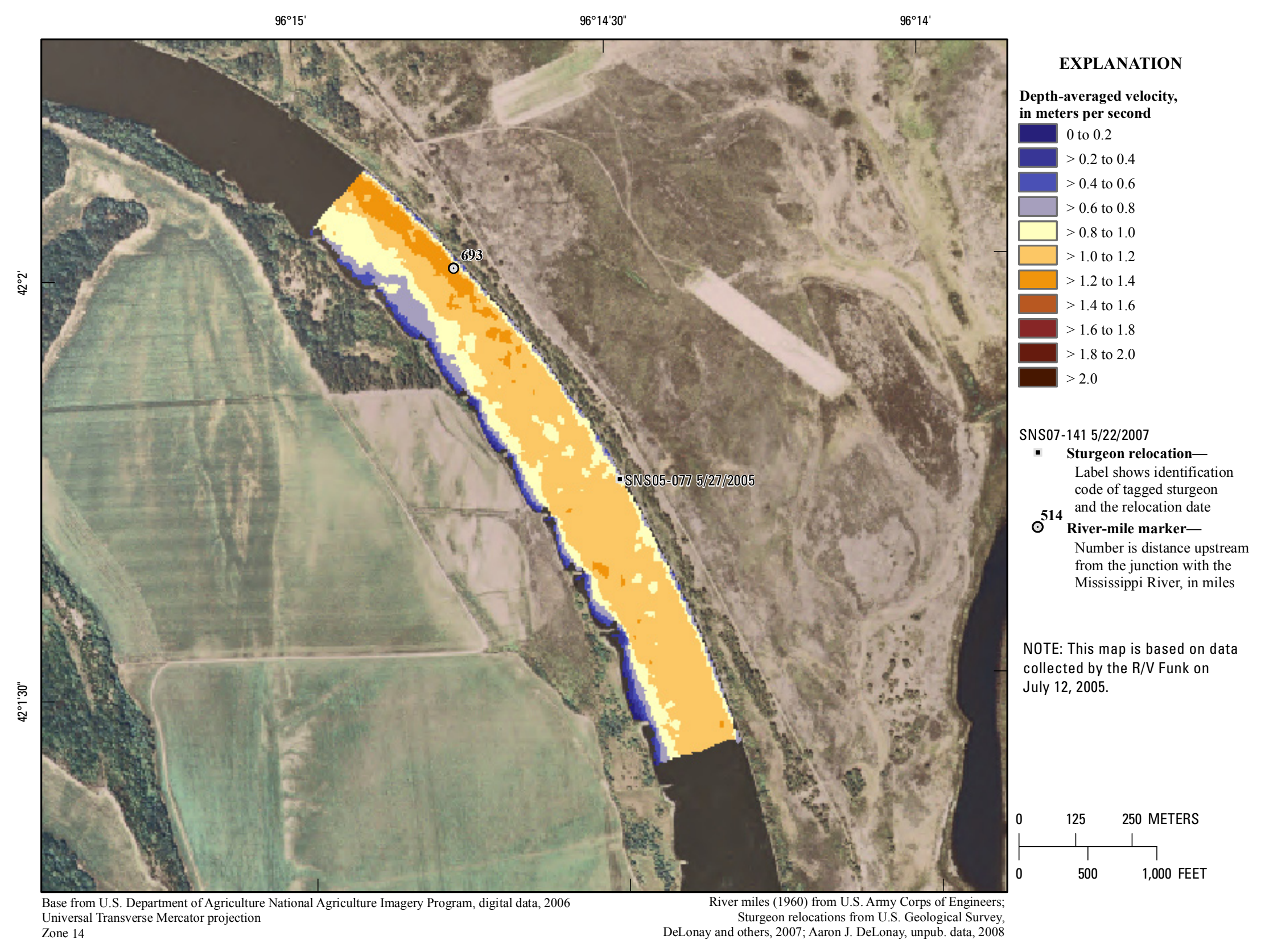

Figure 127. Map of depth-averaged velocity based on data collected on July 12, 2005, in the vicinity of river mile 693 . 


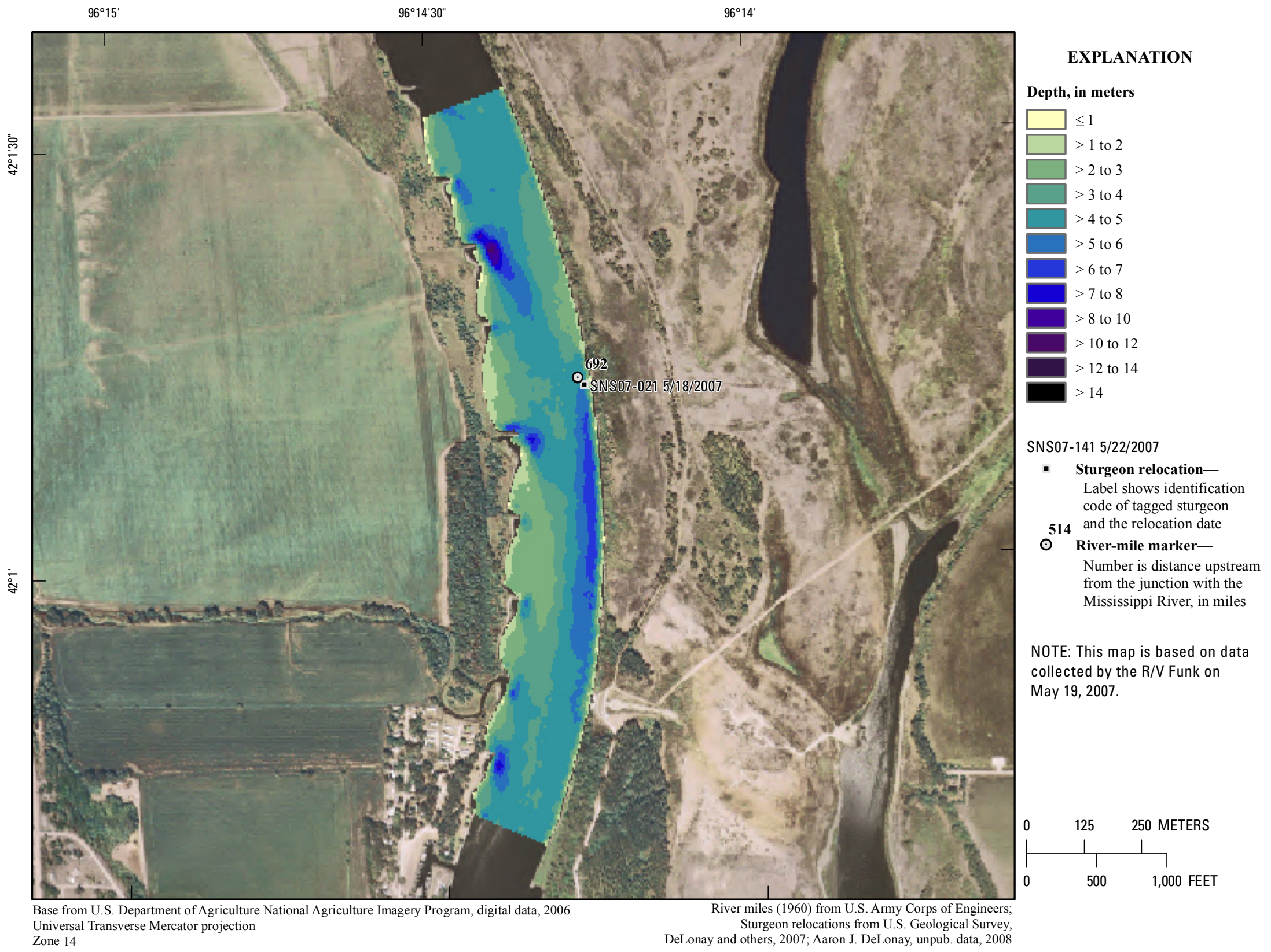

Figure 128. Map of depth based on data collected on May 19, 2007, in the vicinity of river mile 692. 


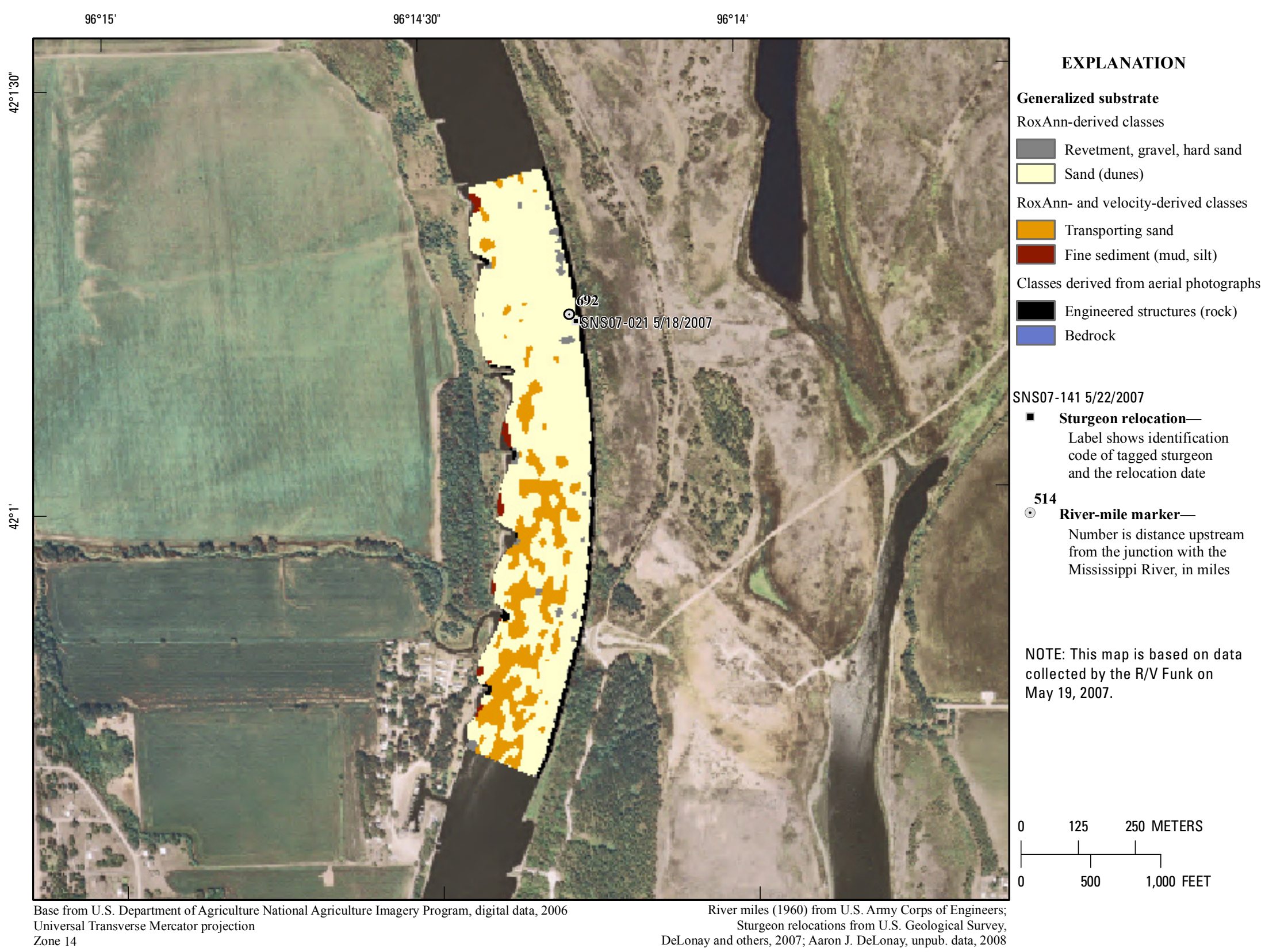

Figure 129. Map of generalized substrate based on data collected on May 19, 2007, in the vicinity of river mile 692 . 


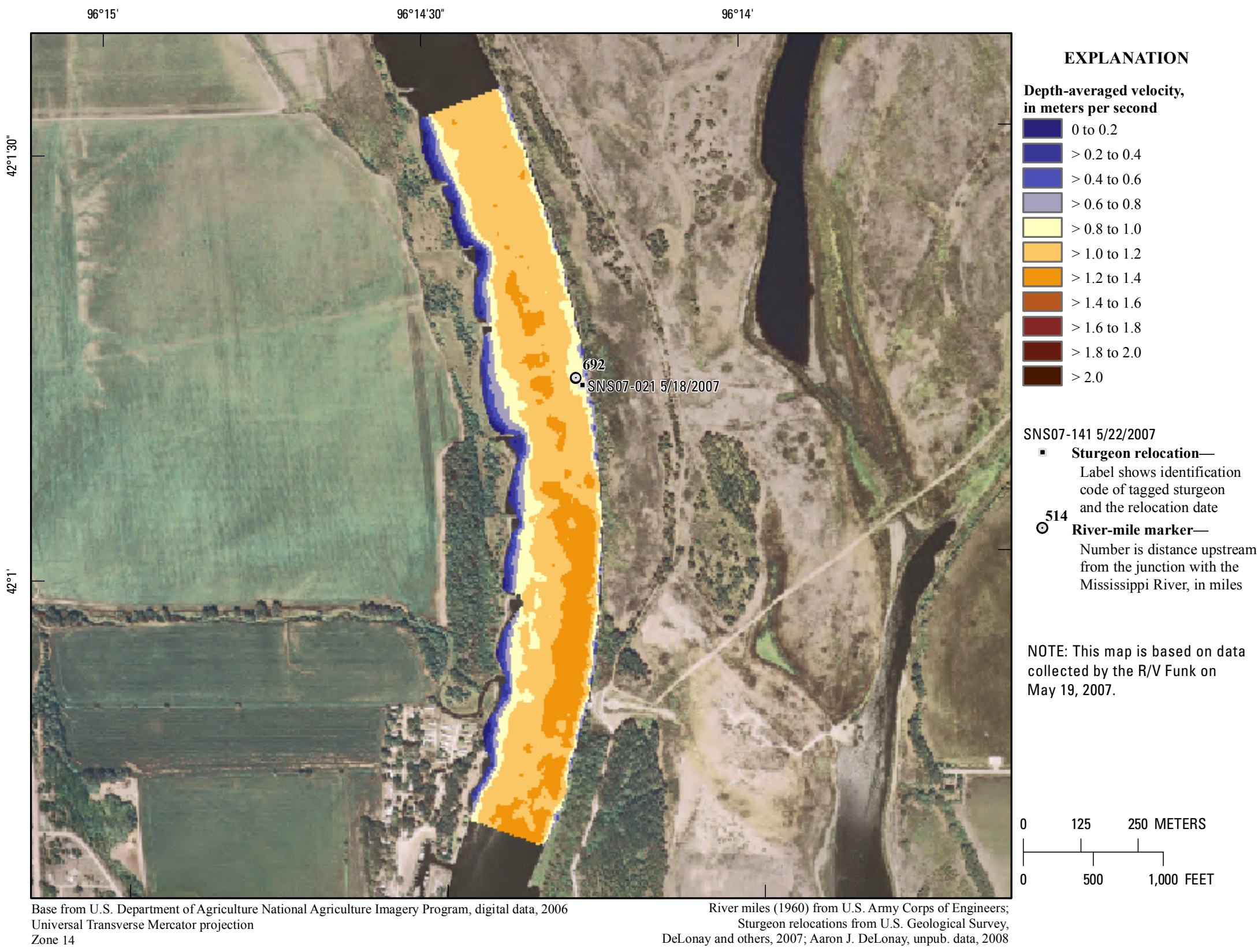

Figure 130. Map of depth-averaged velocity based on data collected on May 19, 2007, in the vicinity of river mile 692 . 


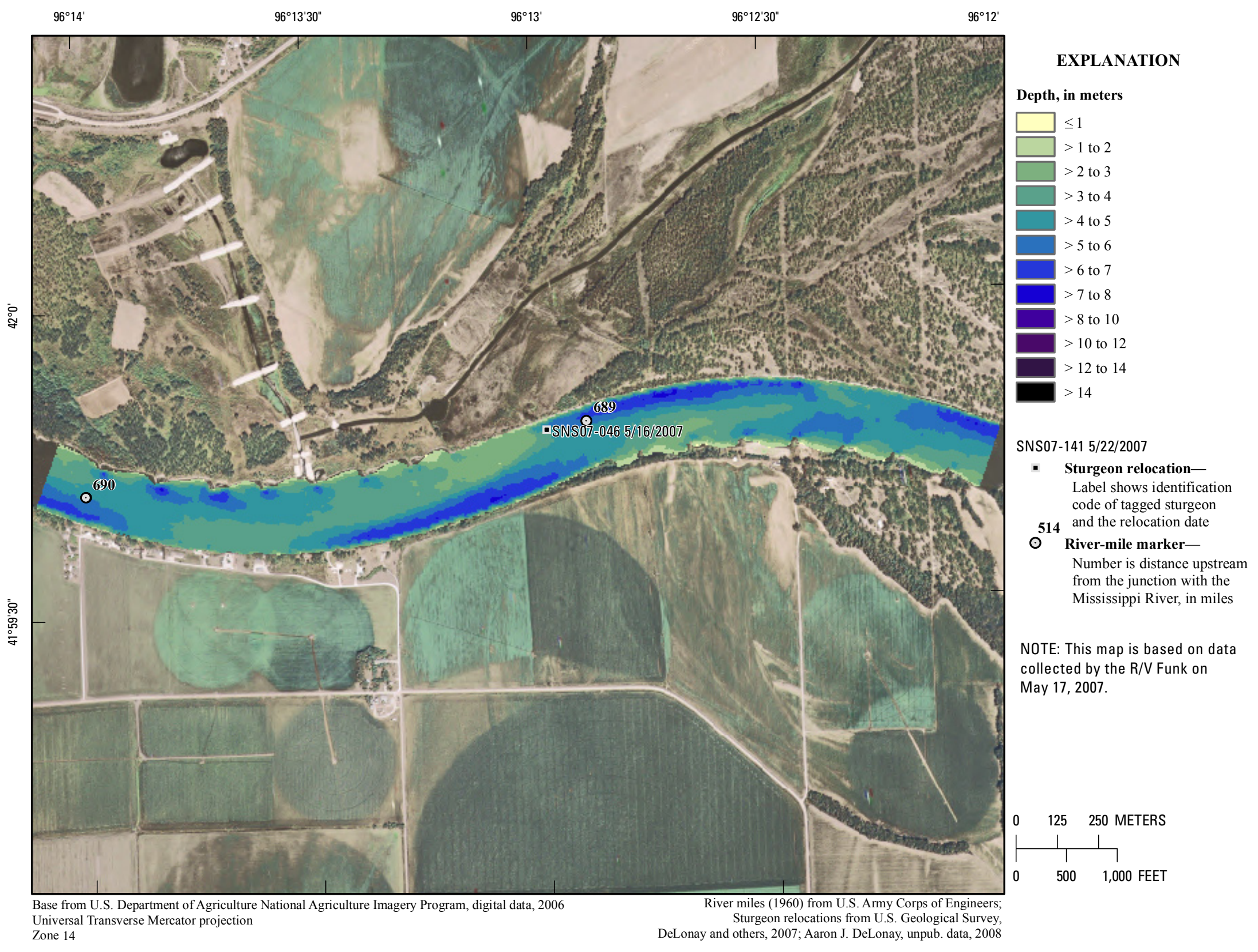

Figure 131. Map of depth based on data collected on May 17, 2007, in the vicinity of river mile 689. 


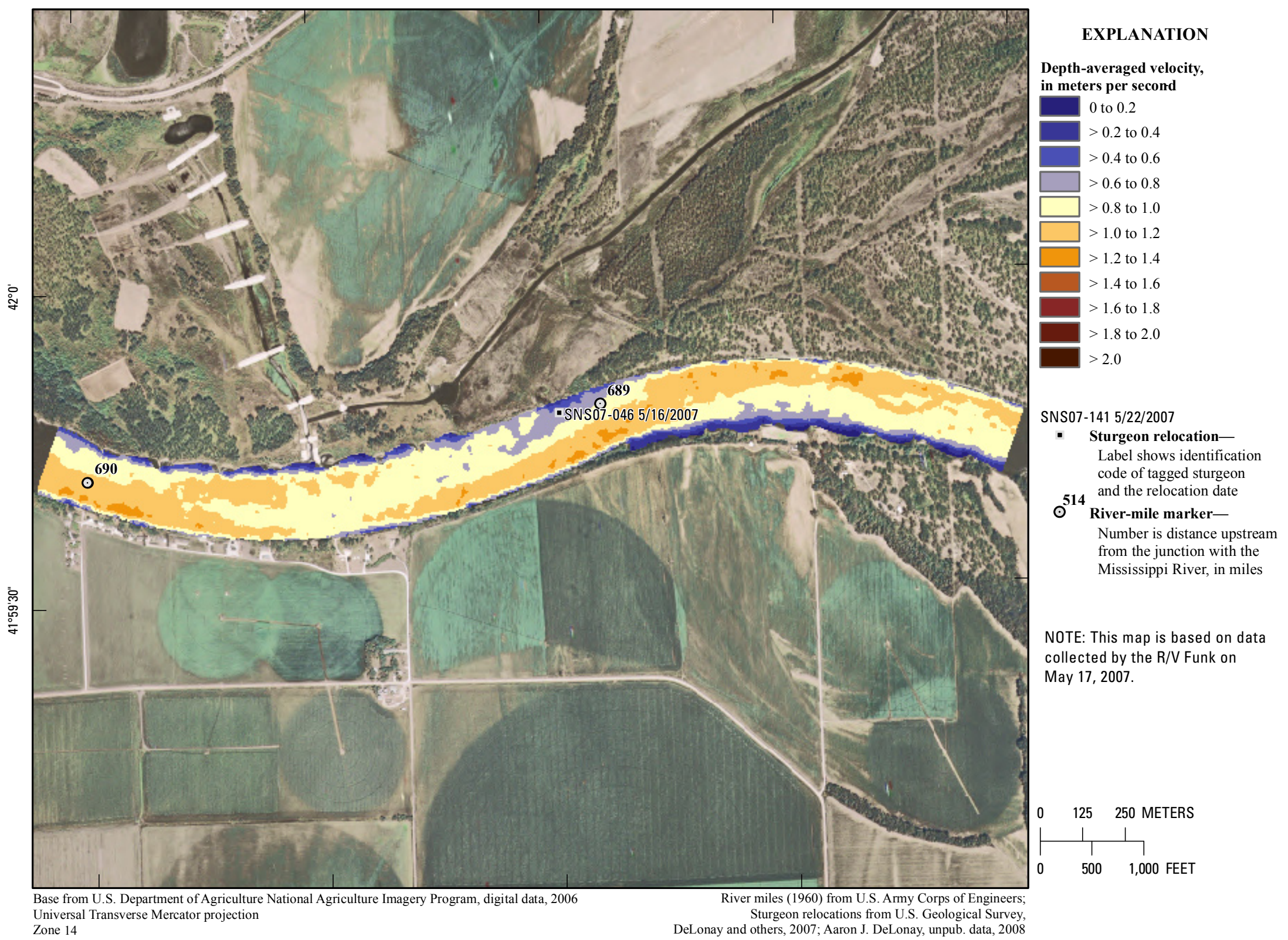

Figure 132. Map of depth-averaged velocity based on data collected on May 17, 2007, in the vicinity of river mile 689. 


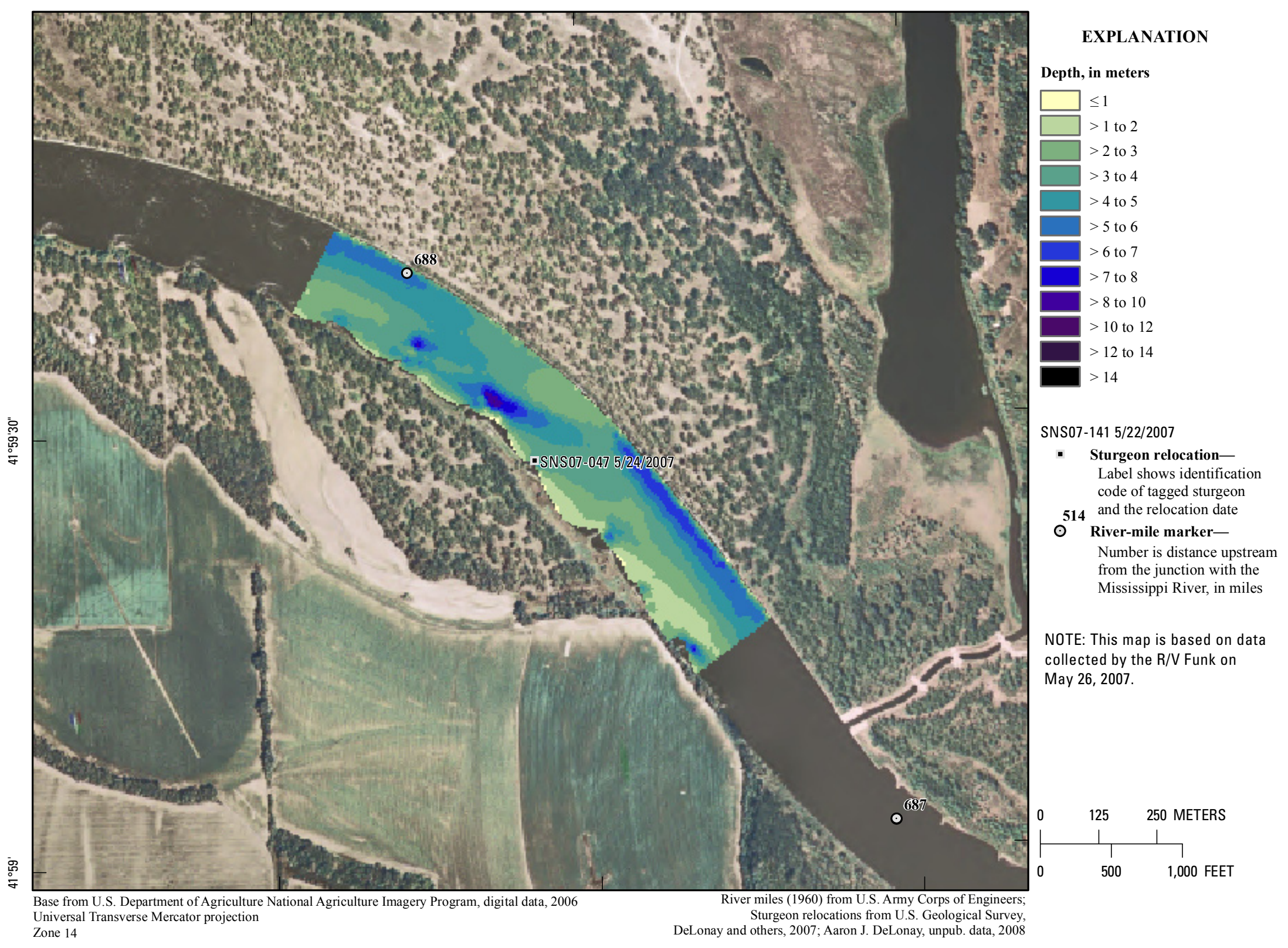

Figure 133. Map of depth based on data collected on May 26, 2007, in the vicinity of river mile 688 . 


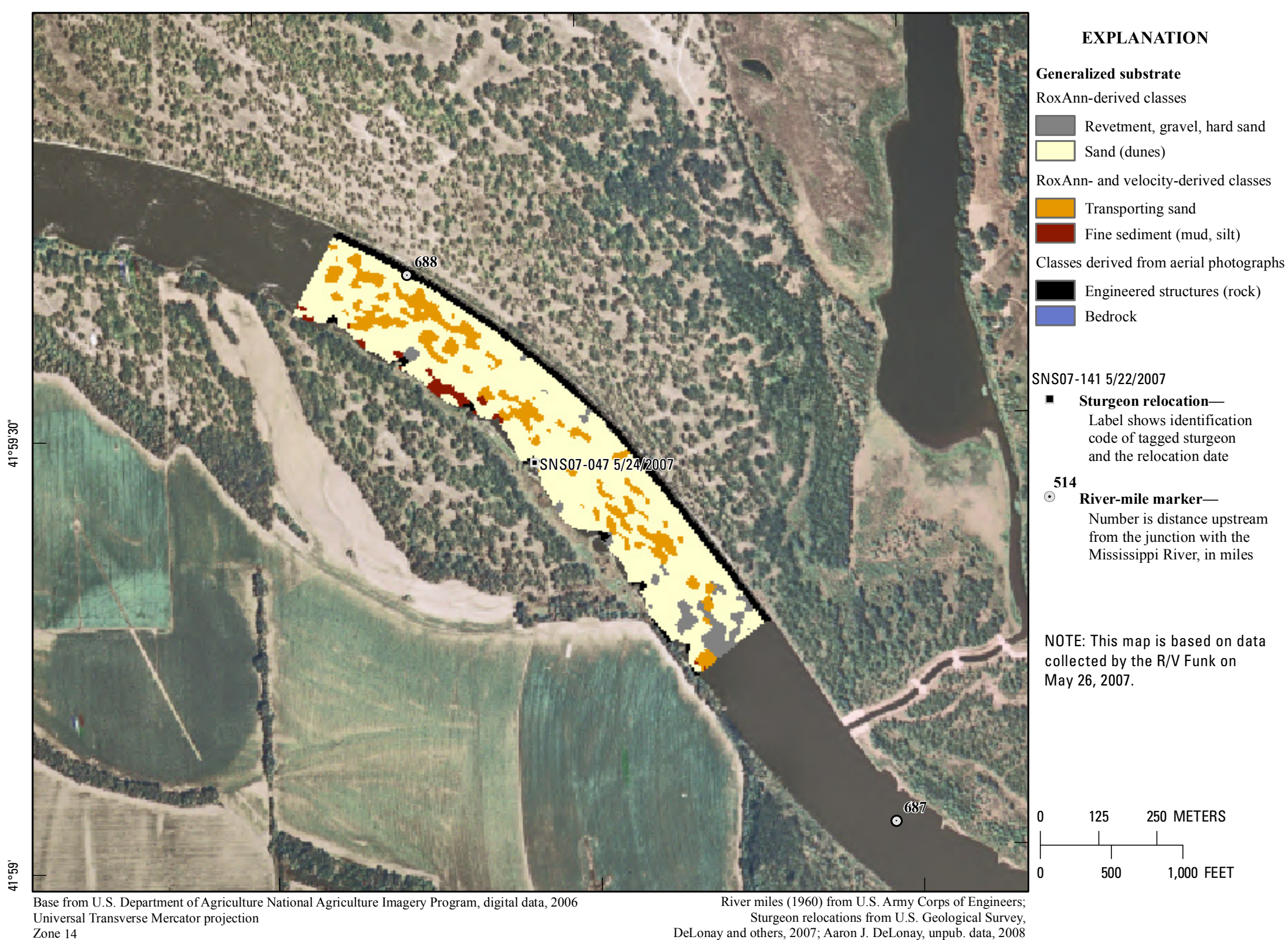




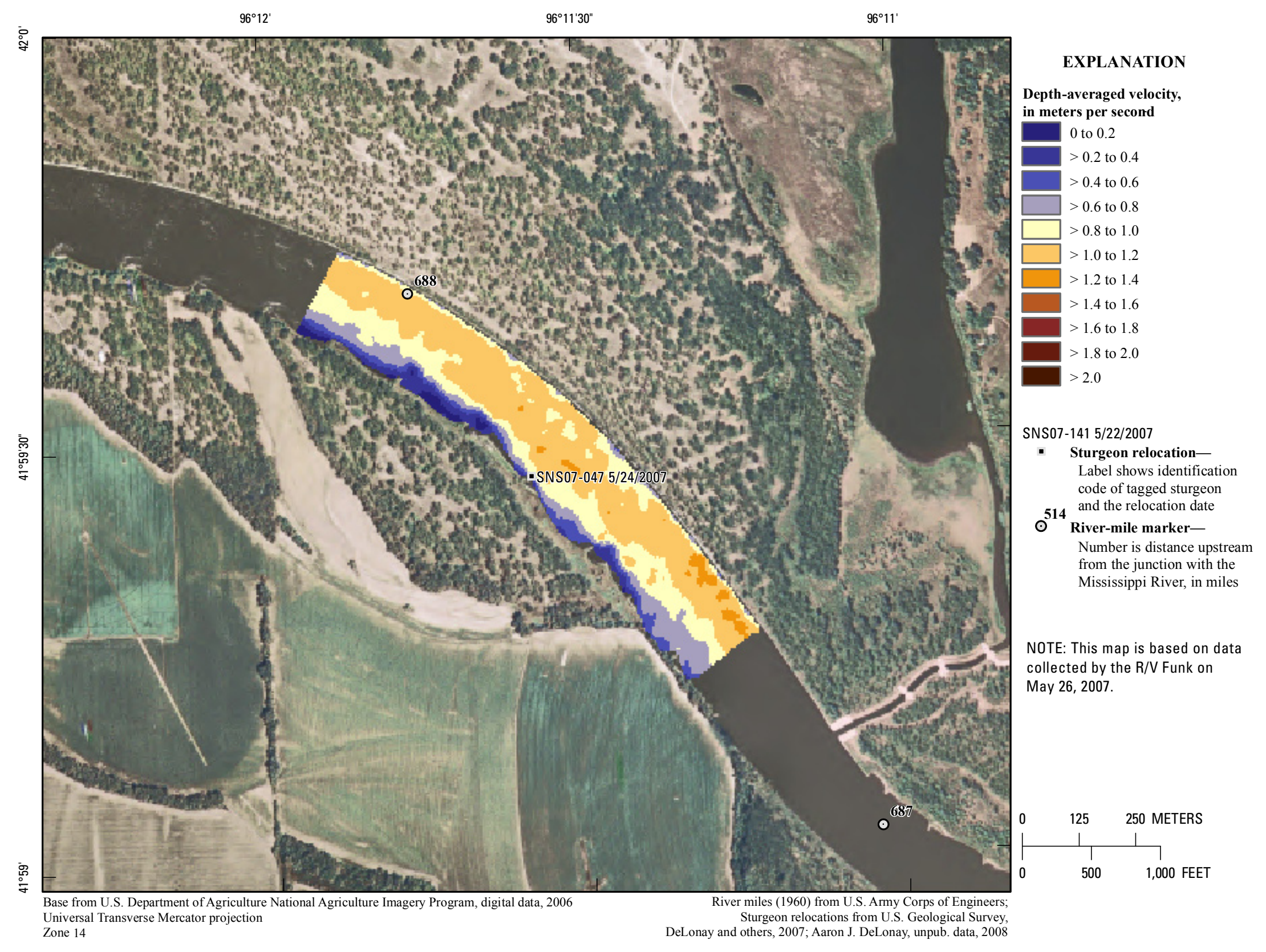

Figure 135. Map of depth-averaged velocity based on data collected on May 26, 2007, in the vicinity of river mile 688 . 


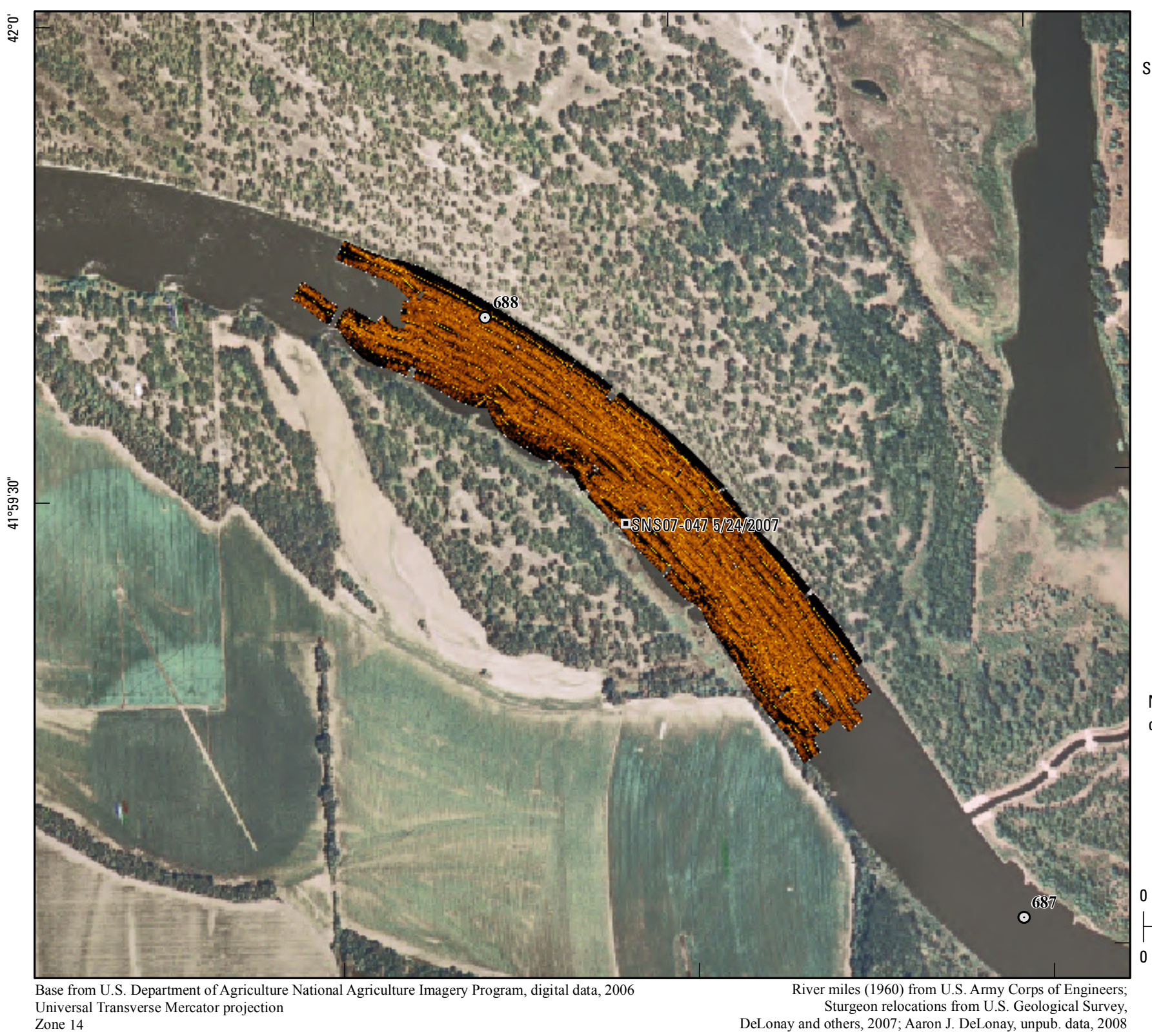

EXPLANATION

SNS07-141 5/22/2007

- Sturgeon relocation-

Label shows identification

code of tagged sturgeon

514 and the relocation date

$\odot$ River-mile marker-

Number is distance upstream

from the junction with the

Mississippi River, in miles

NOTE: This map is based on data collected on May 26, 2007. 


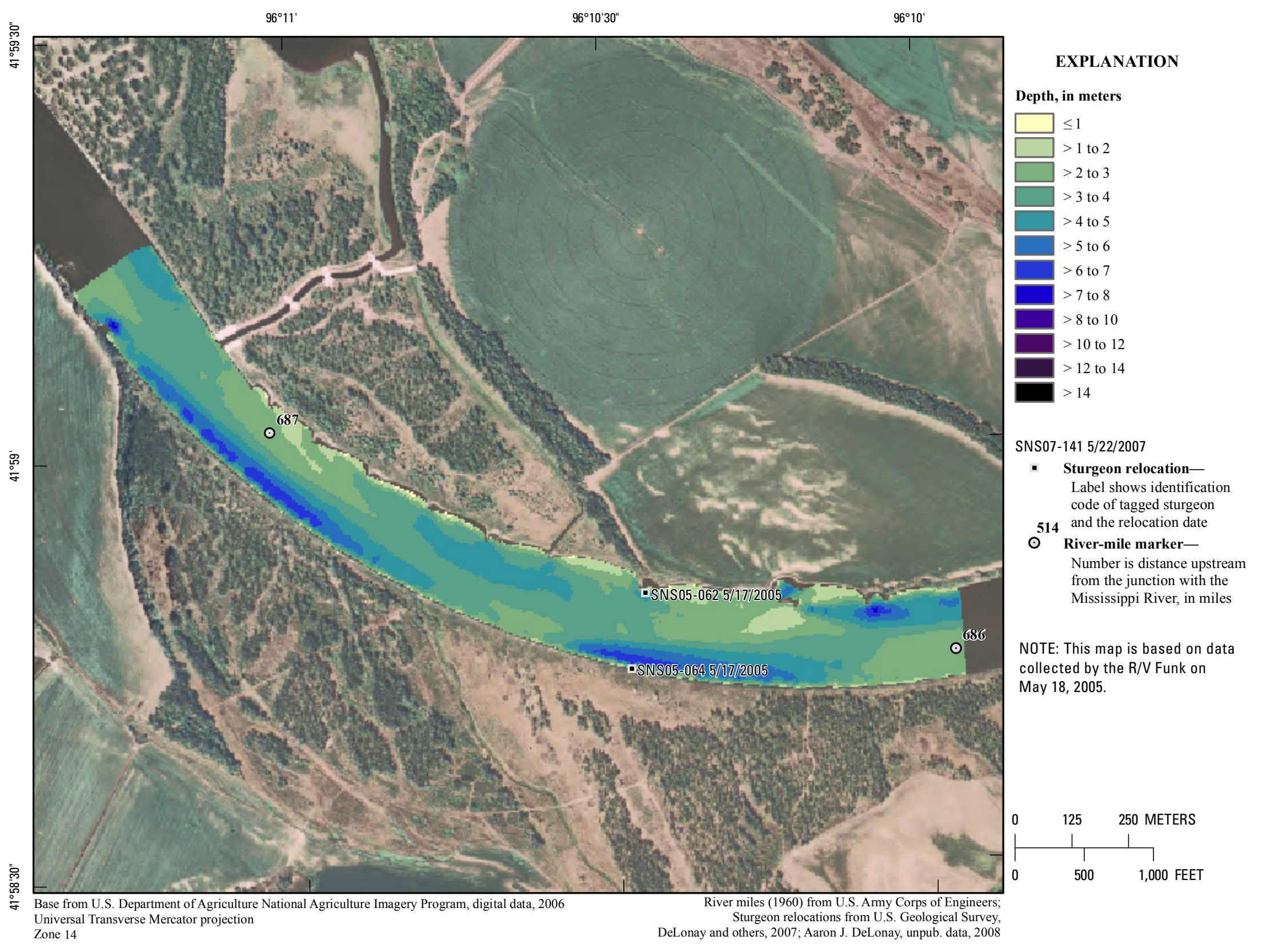

Figure 137. Map of depth based on data collected on May 18, 2005, in the vicinity of river mile 687 . 


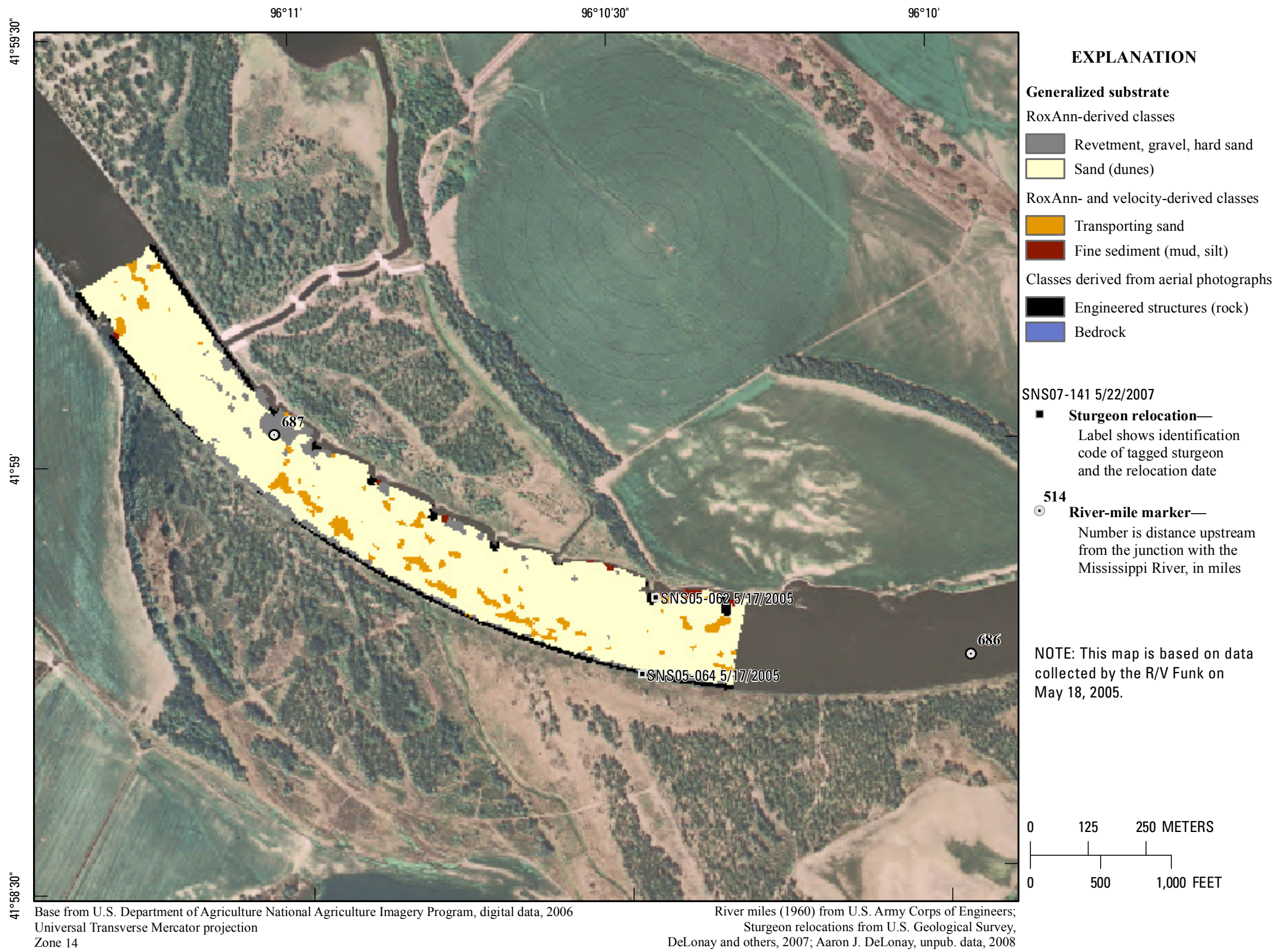

Figure 138. Map of generalized substrate based on data collected on May 18, 2005, in the vicinity of river mile 687 . 


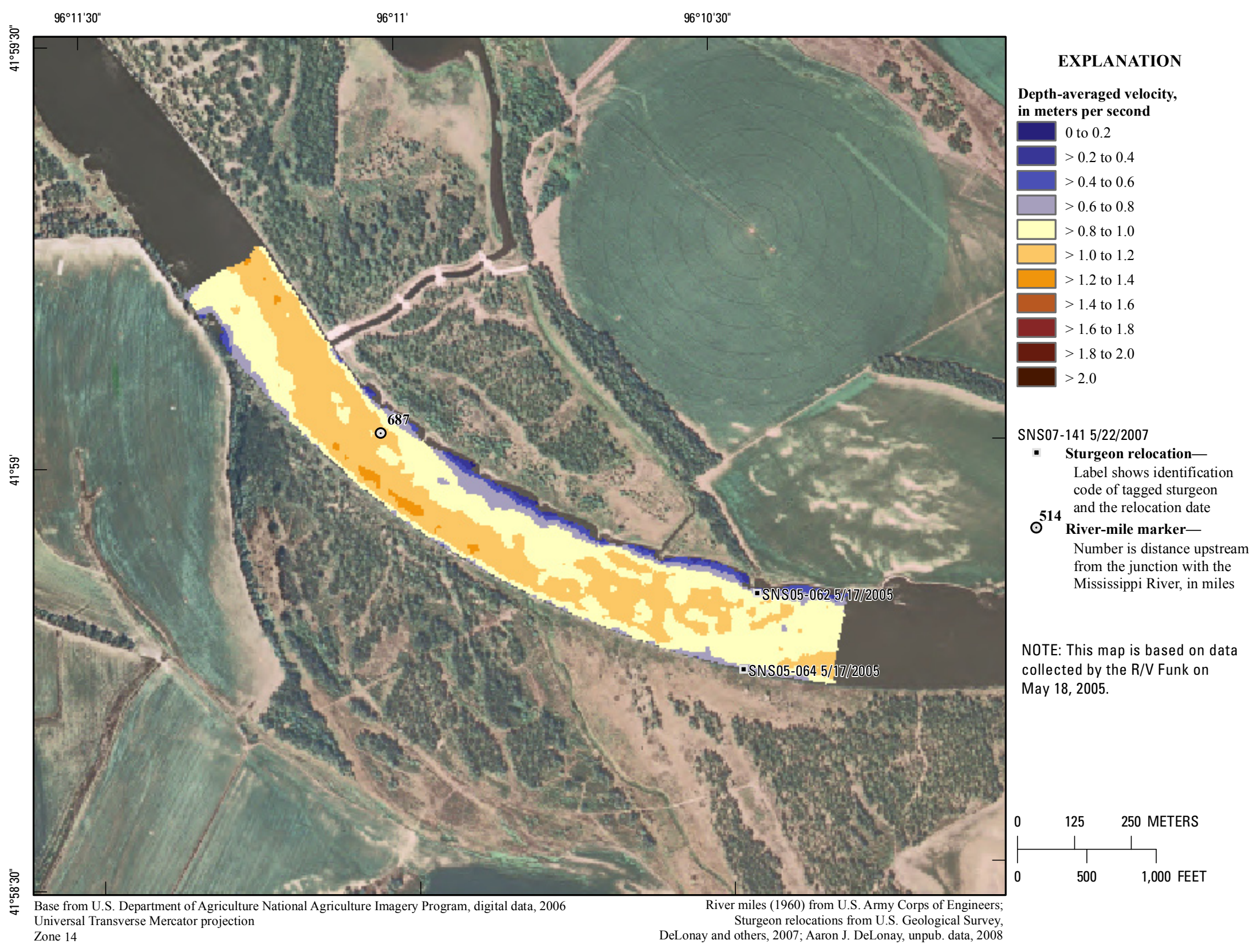

Figure 139. Map of depth-averaged velocity based on data collected on May 18, 2005, in the vicinity of river mile 687 . 


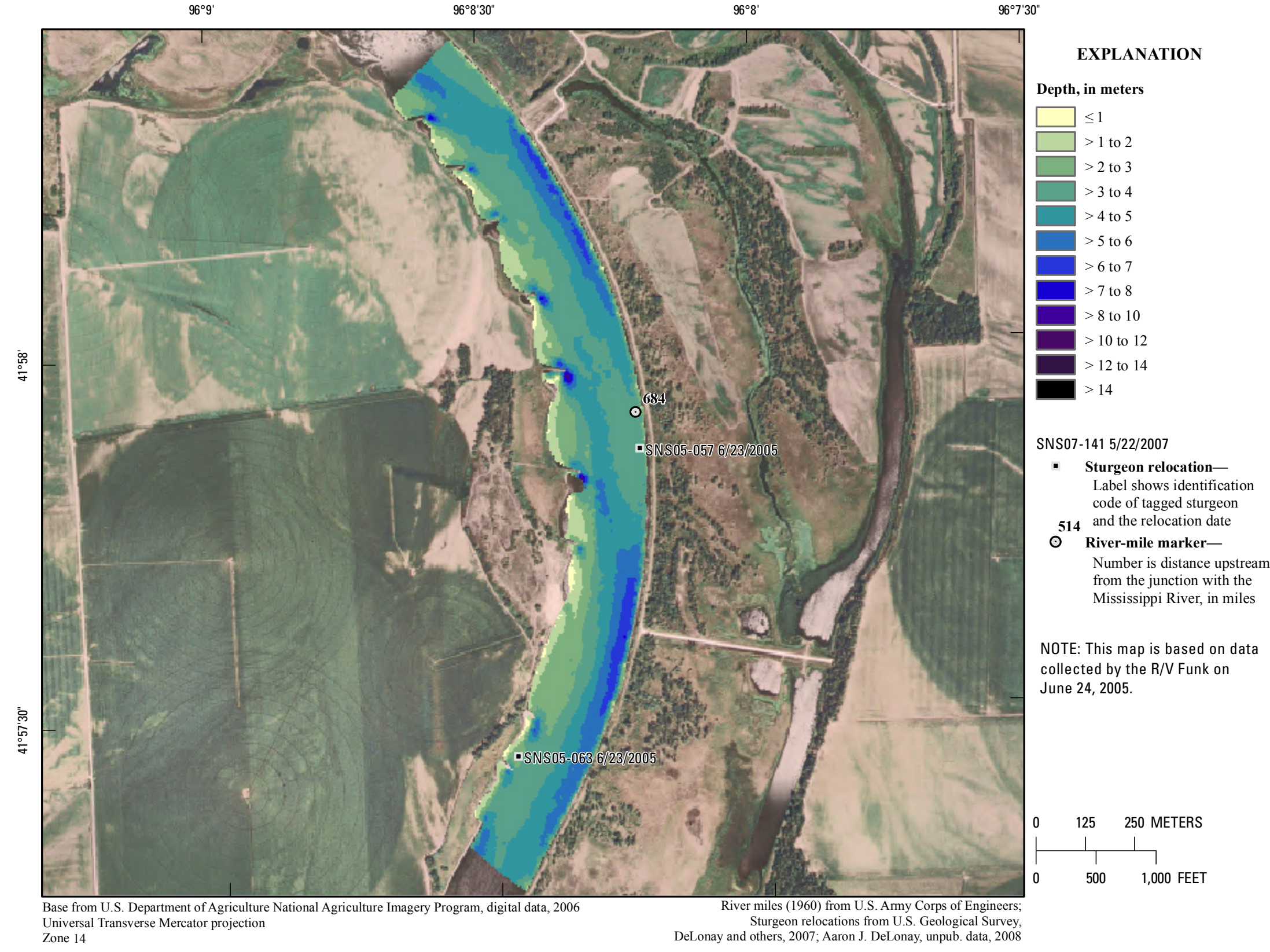

Figure 140. Map of depth based on data collected on June 24, 2005, in the vicinity of river mile 684 . 


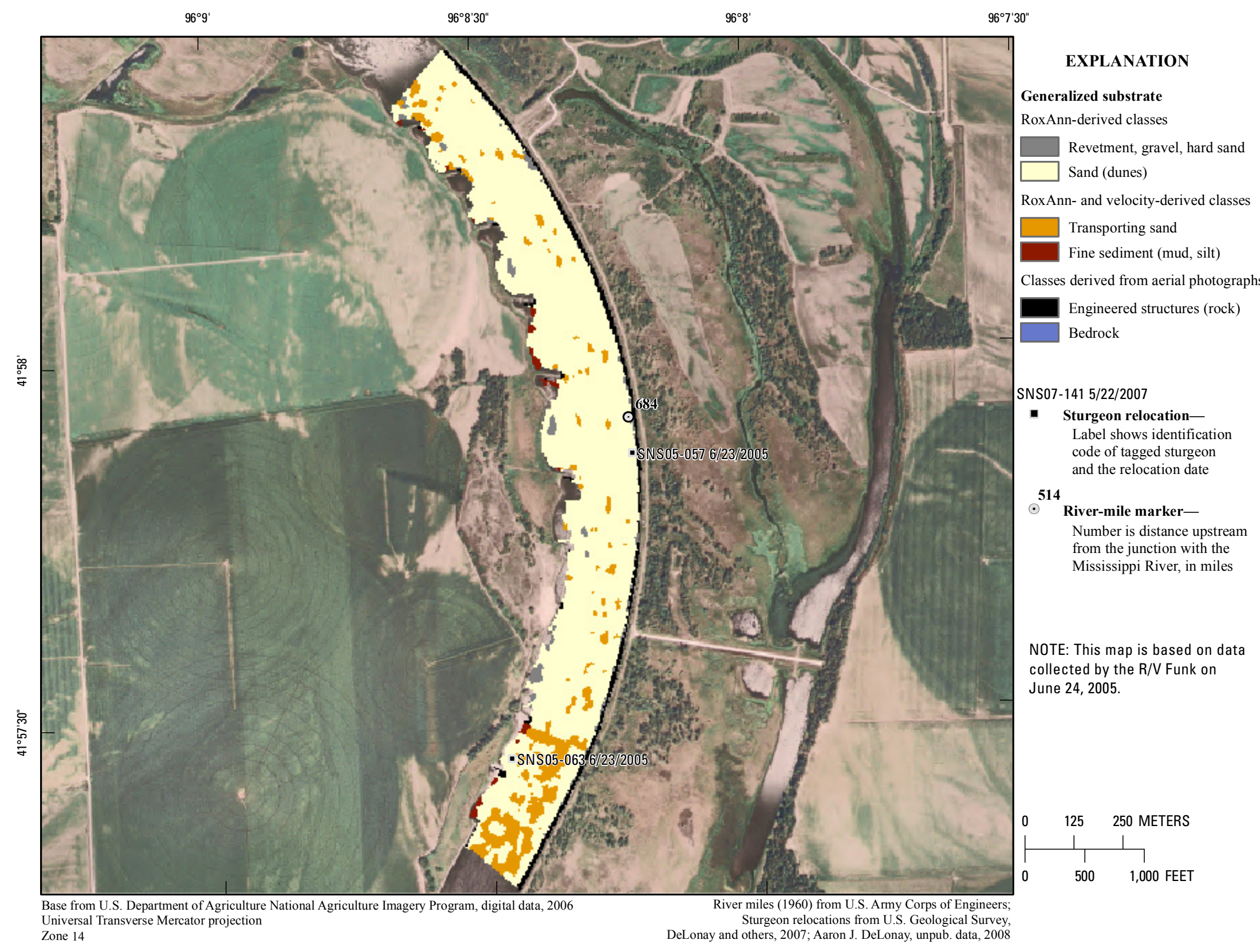

Figure 141. Map of generalized substrate based on data collected on June 24,2005 , in the vicinity of river mile 684 . 


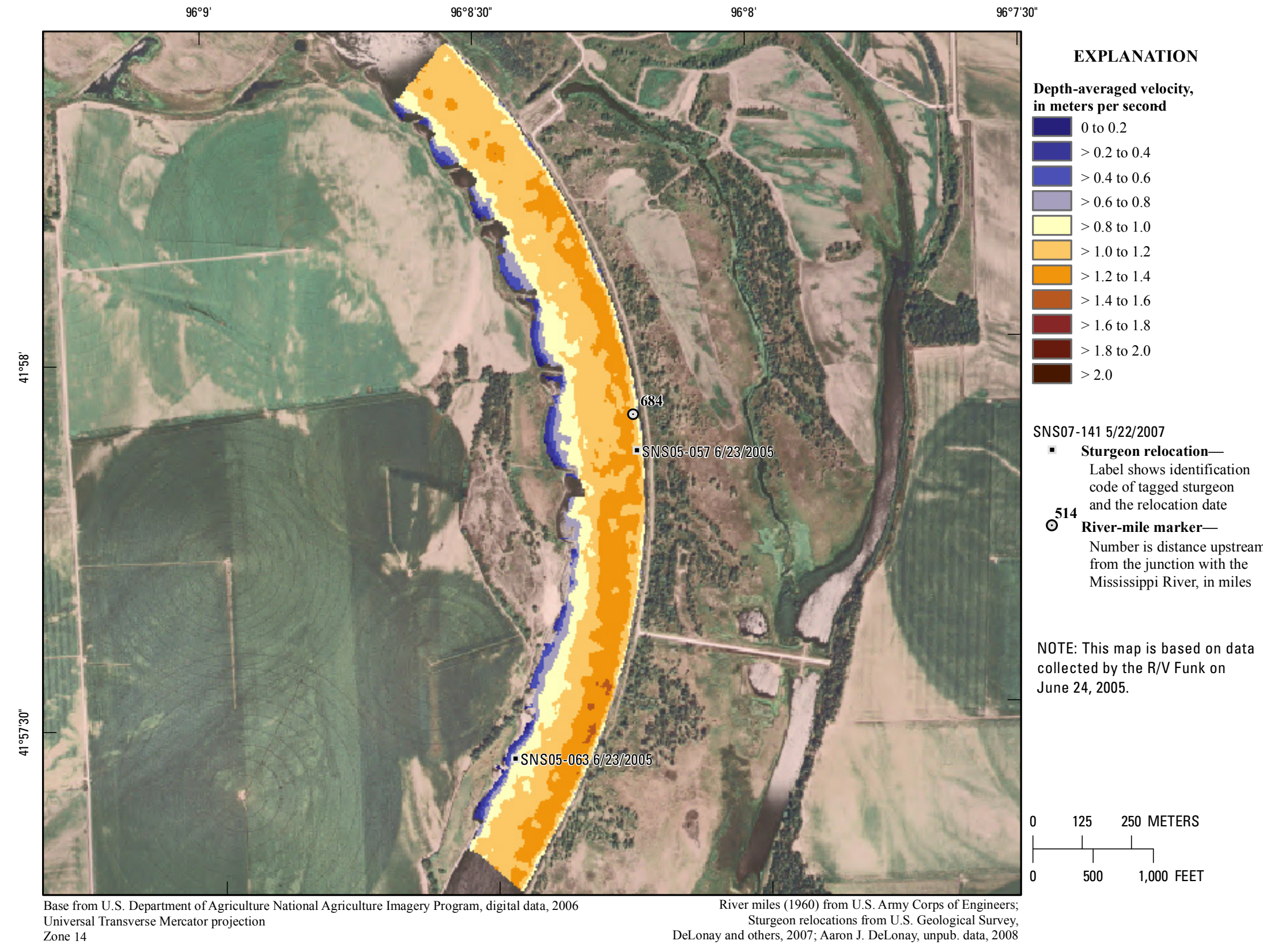

Figure 142. Map of depth-averaged velocity based on data collected on June 24, 2005, in the vicinity of river mile 684 . 


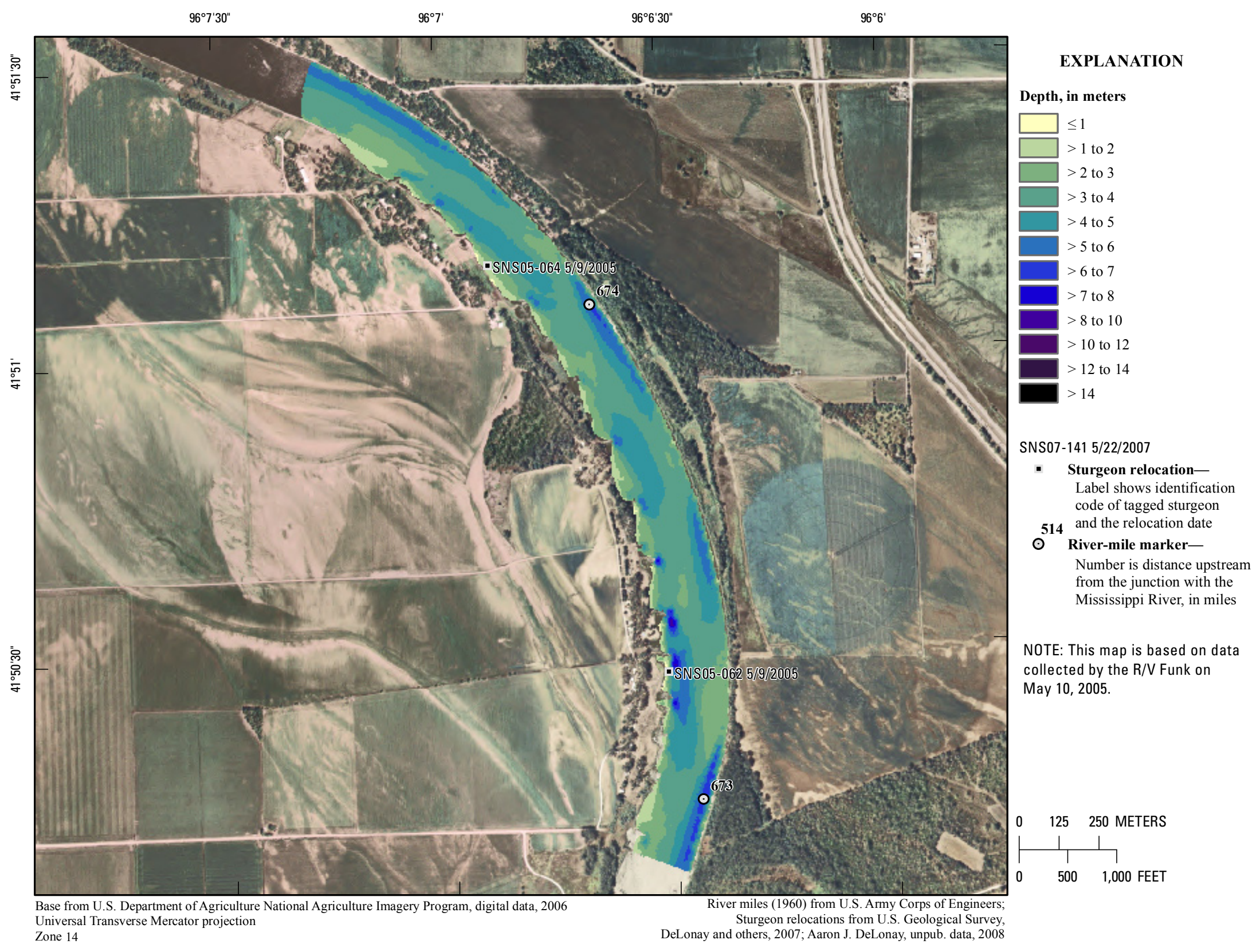

Figure 143. Map of depth based on data collected on May 10, 2005, in the vicinity of river mile 674 . 


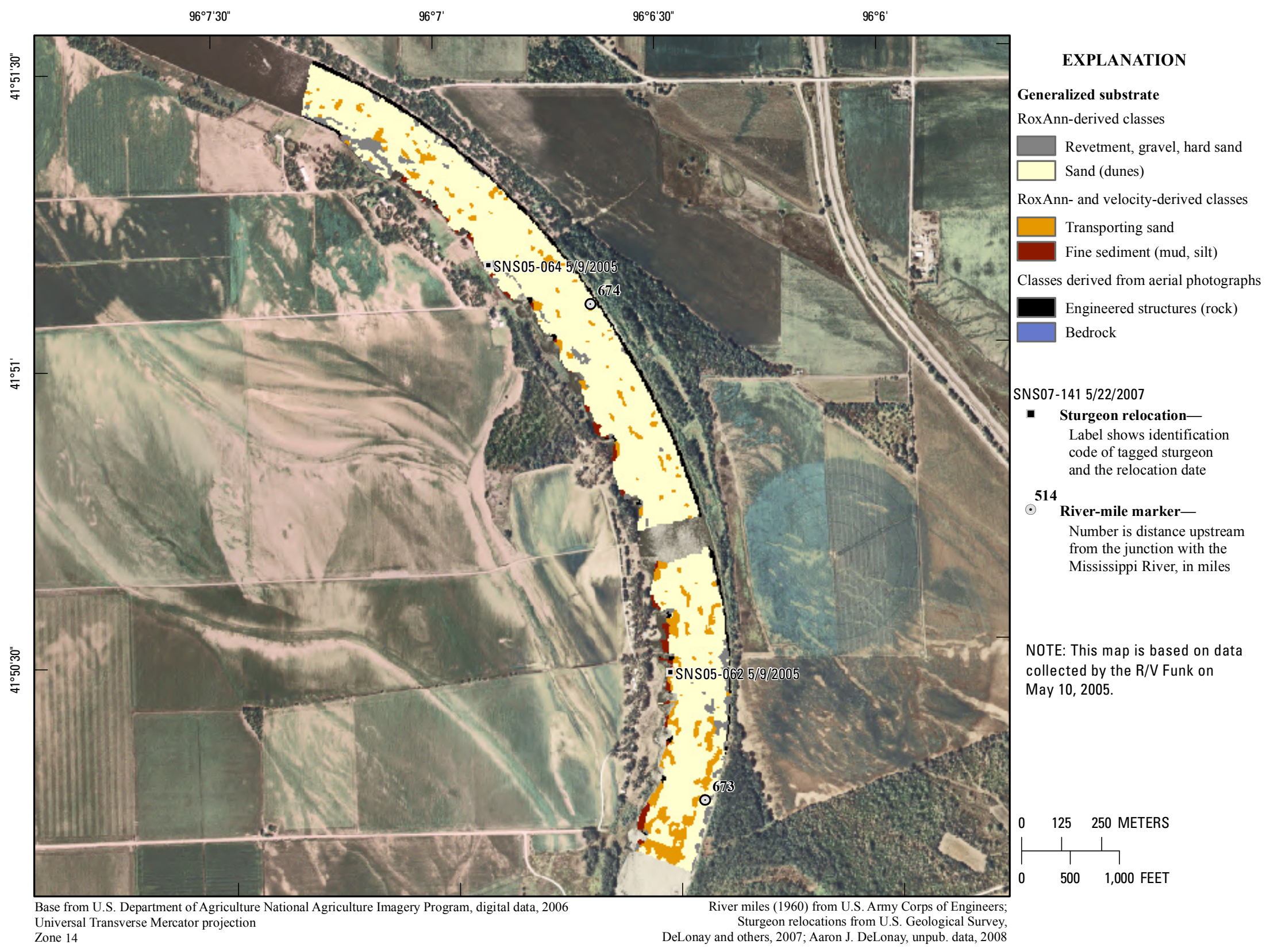

Figure 144. Map of generalized substrate based on data collected on May 10, 2005, in the vicinity of river mile 674 . 


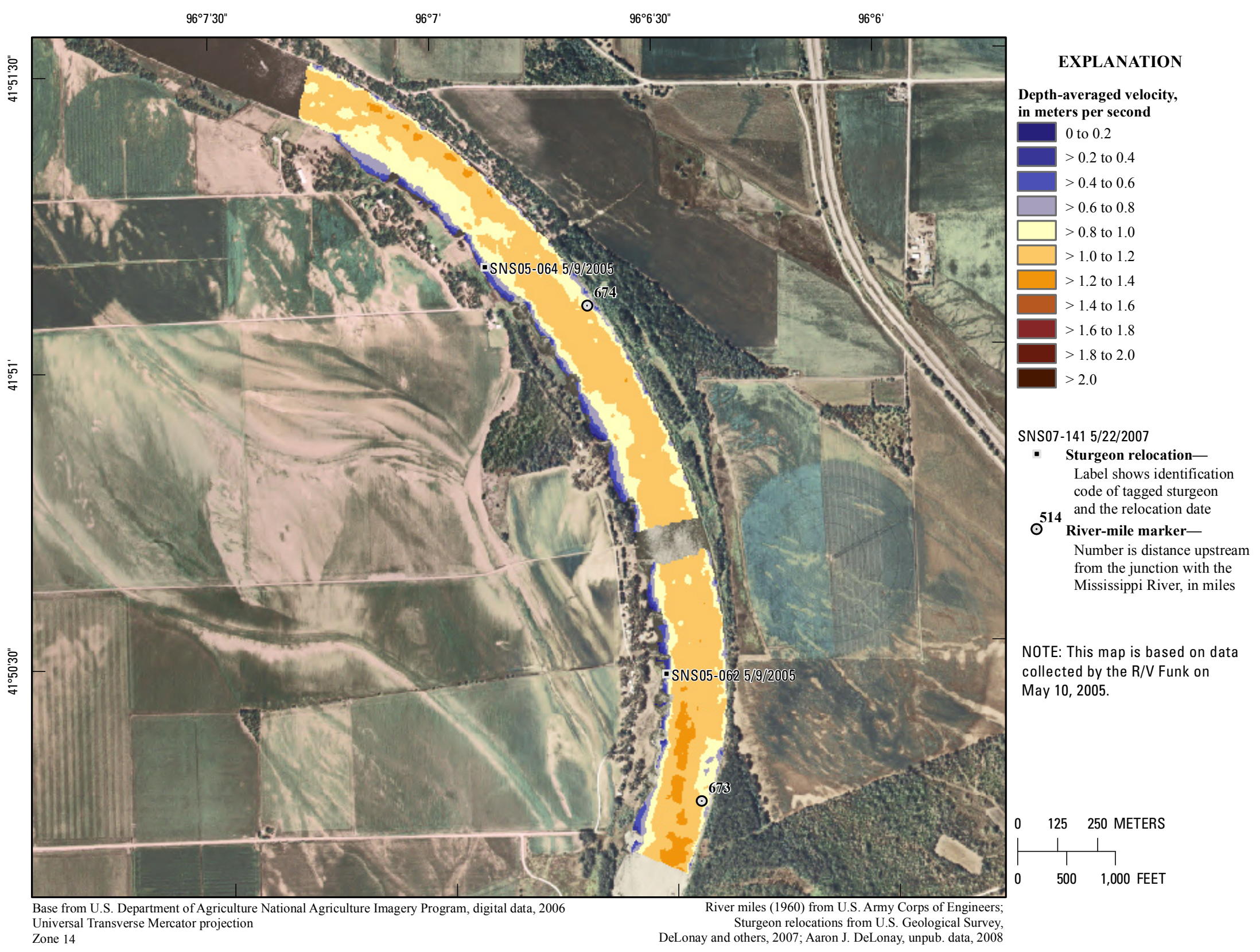

Figure 145. Map of depth-averaged velocity based on data collected on May 10, 2005, in the vicinity of river mile 674 


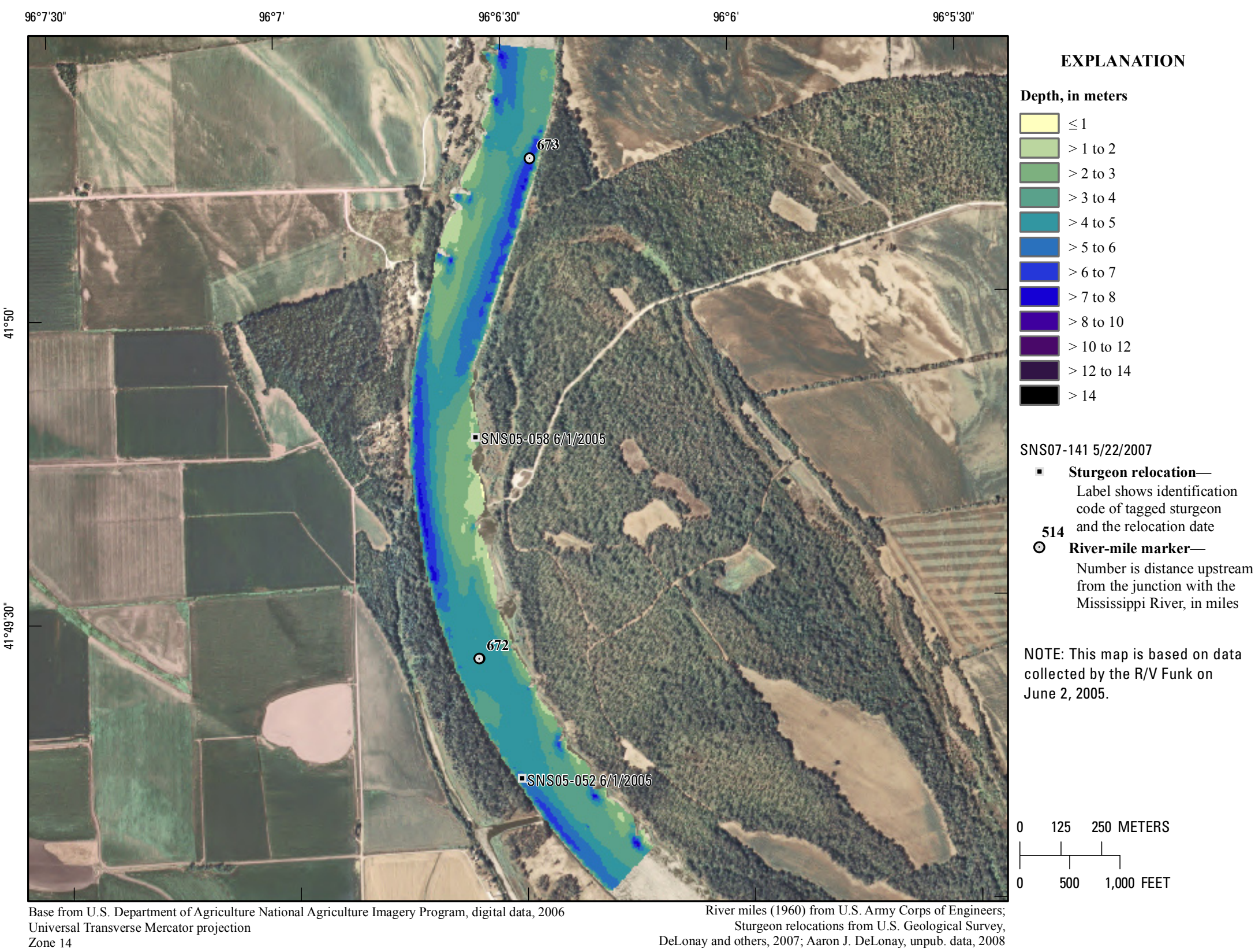

Figure 146. Map of depth based on data collected on June 2, 2005, in the vicinity of river mile 672 . 


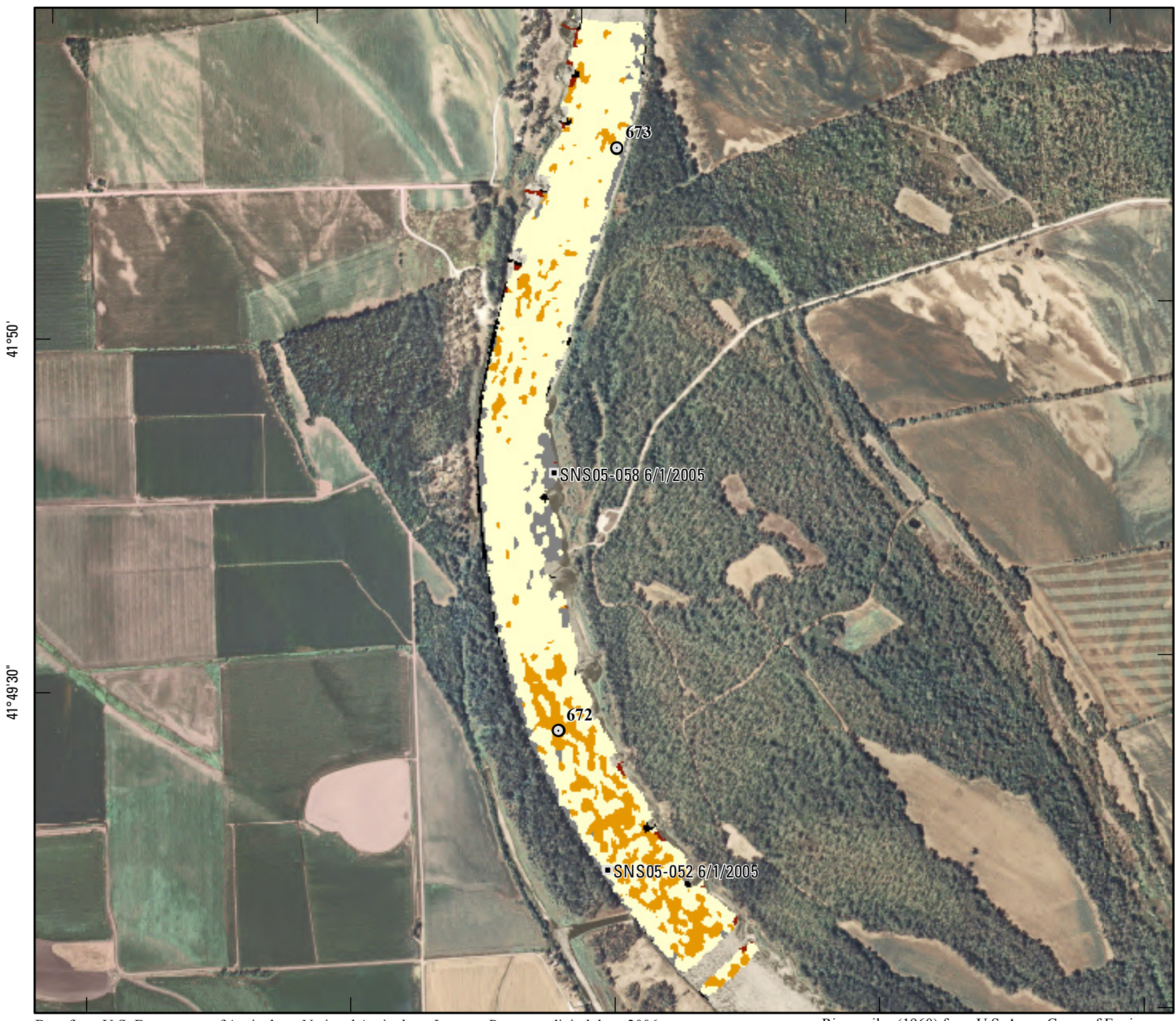

EXPLANATION

\section{Generalized substrate}

RoxAnn-derived classes

Revetment, gravel, hard sand Sand (dunes)

RoxAnn- and velocity-derived classes

$\square$ Transporting sand

Fine sediment (mud, silt)

Classes derived from aerial photographs

Engineered structures (rock) Bedrock

\section{SNS07-141 5/22/2007}

- Sturgeon relocationLabel shows identification code of tagged sturgeon and the relocation date

514

River-mile marker-

Number is distance upstream from the junction with the Mississippi River, in miles

NOTE: This map is based on data collected by the R/V Funk on June 2, 2005.

\section{$0 \quad 125 \quad 250$ METERS $\begin{array}{ll}1 & 1 \\ 1 & \end{array}$ \\ $500 \quad 1,000$ FEET \\ 1,000}

Figure 147. Map of generalized substrate based on data collected on June 2, 2005, in the vicinity of river mile 672. 


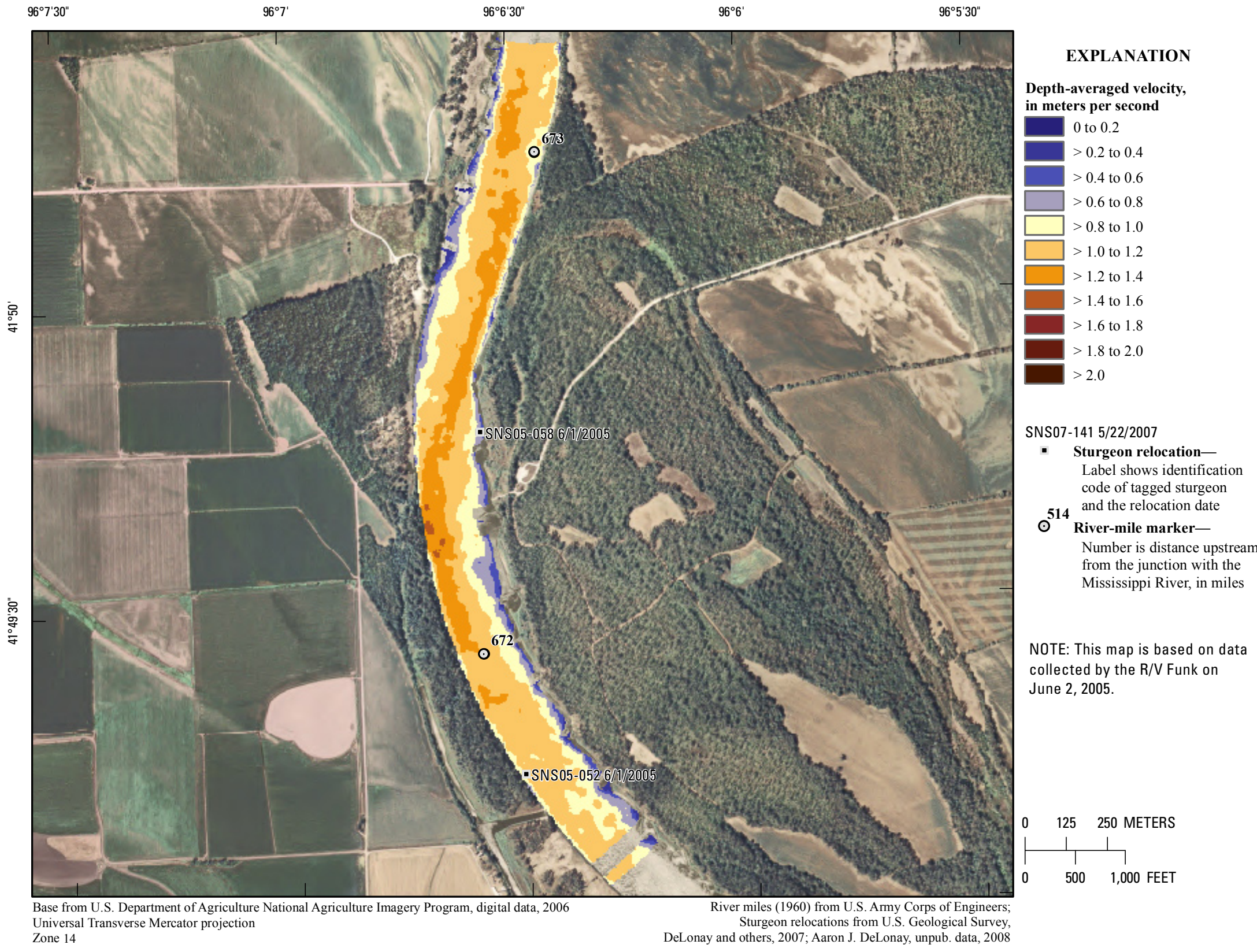

Figure 148. Map of depth-averaged velocity based on data collected on June 2, 2005, in the vicinity of river mile 672 . 


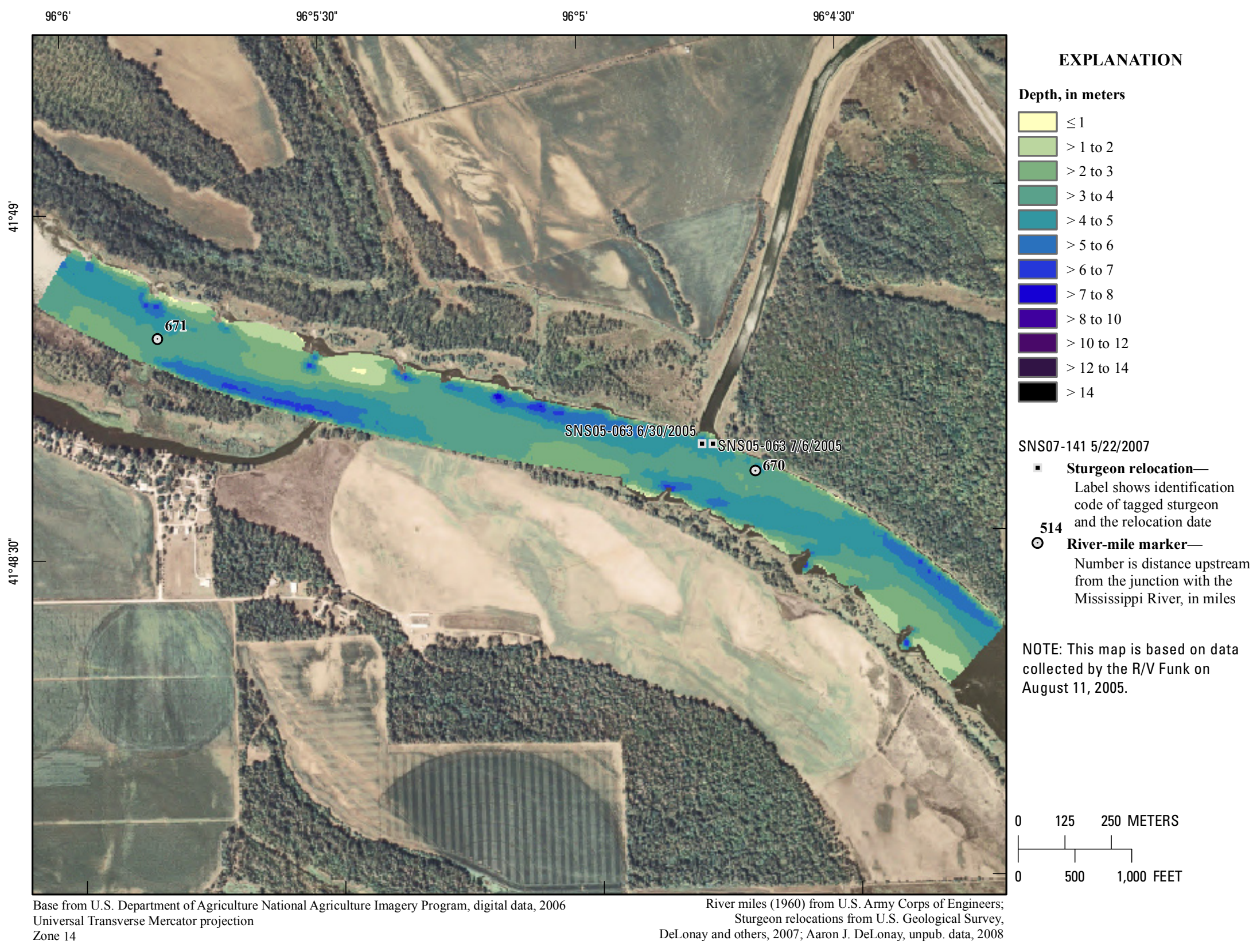

Figure 149. Map of depth based on data collected on August 11, 2005, in the vicinity of river mile 670 . 


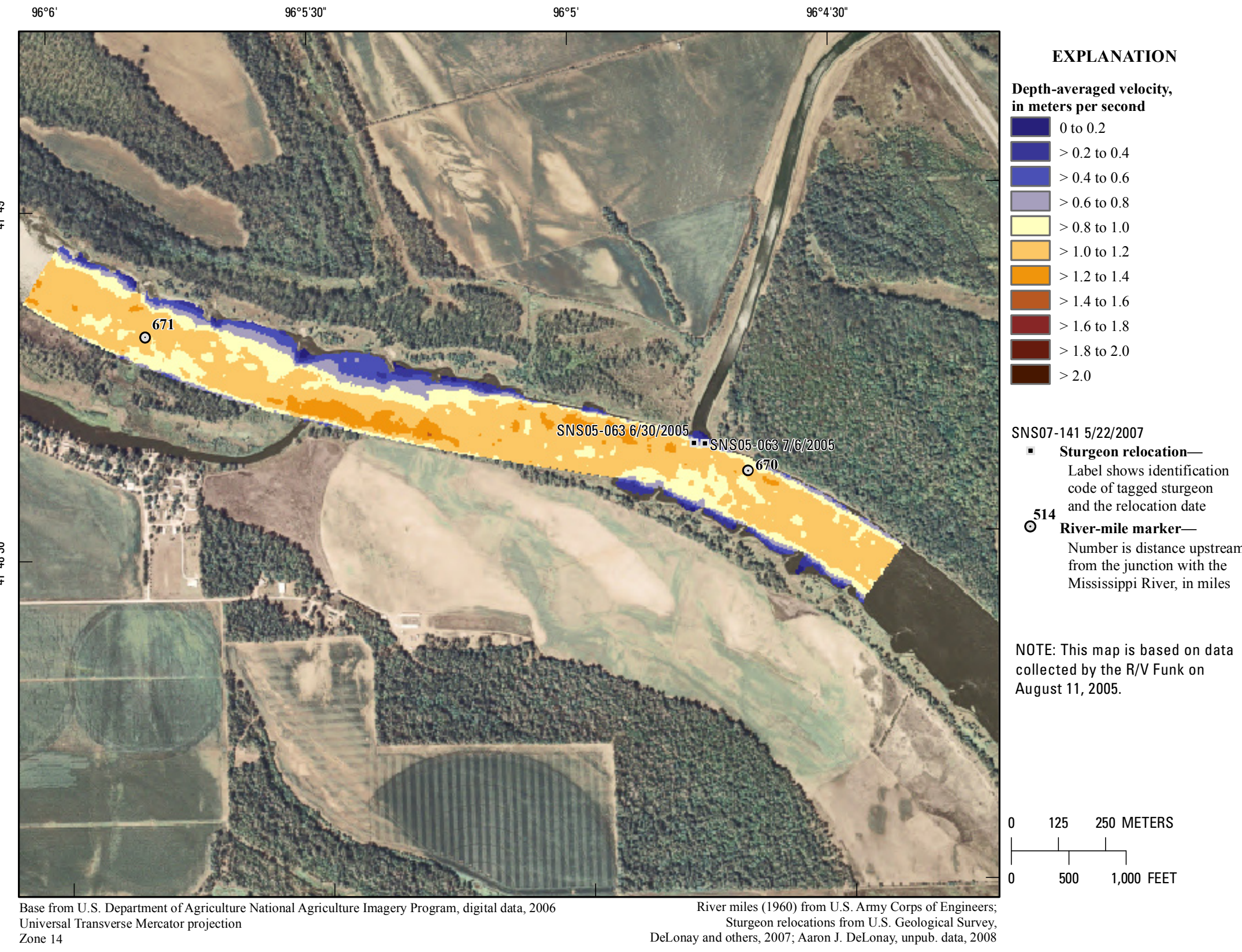

Figure 150. Map of depth-averaged velocity based on data collected on August 11, 2005, in the vicinity of river mile 670. 


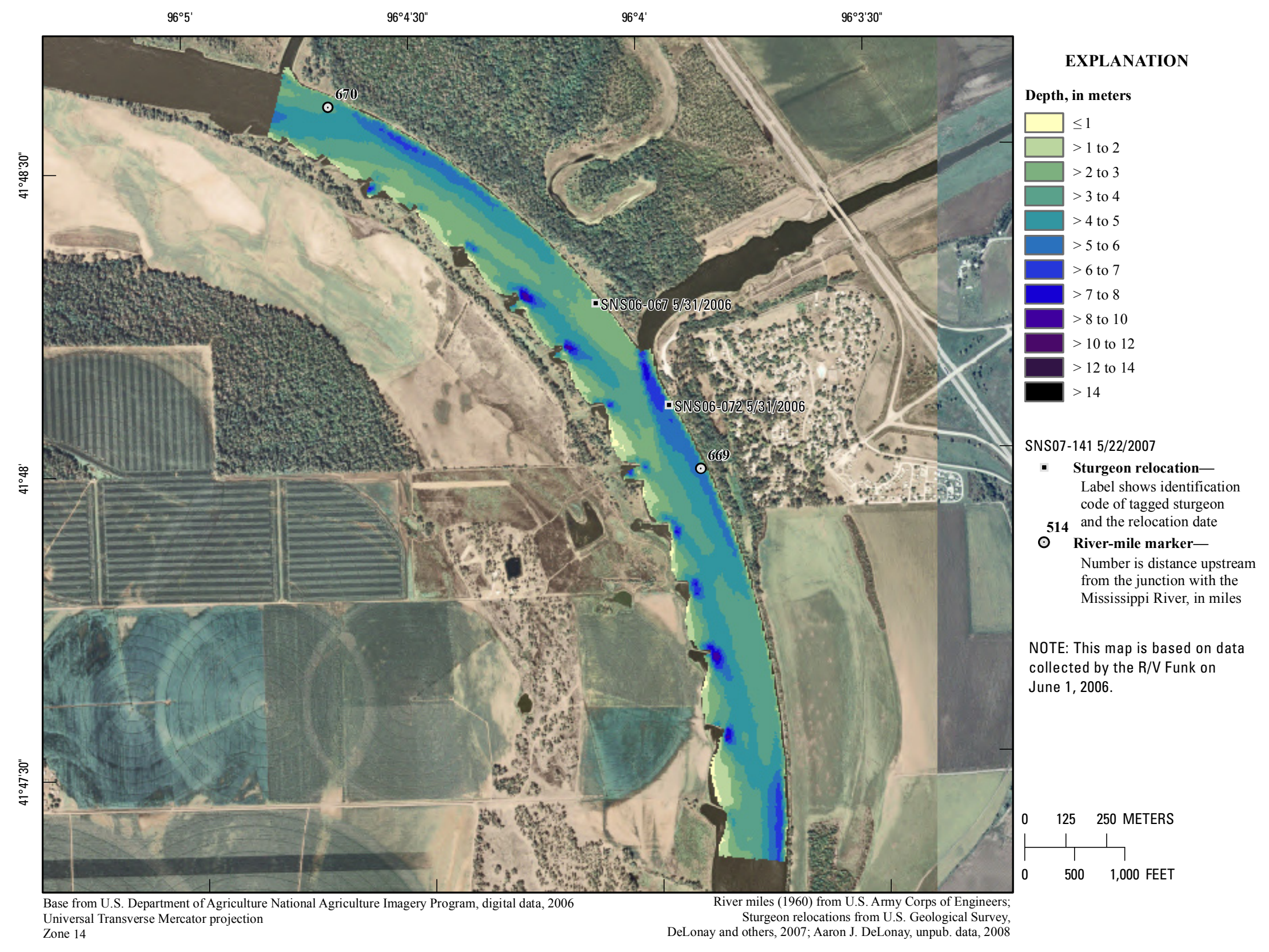

Figure 151. Map of depth based on data collected on June 1, 2006, in the vicinity of river mile 669 . 
$96^{\circ} 5^{\prime}$

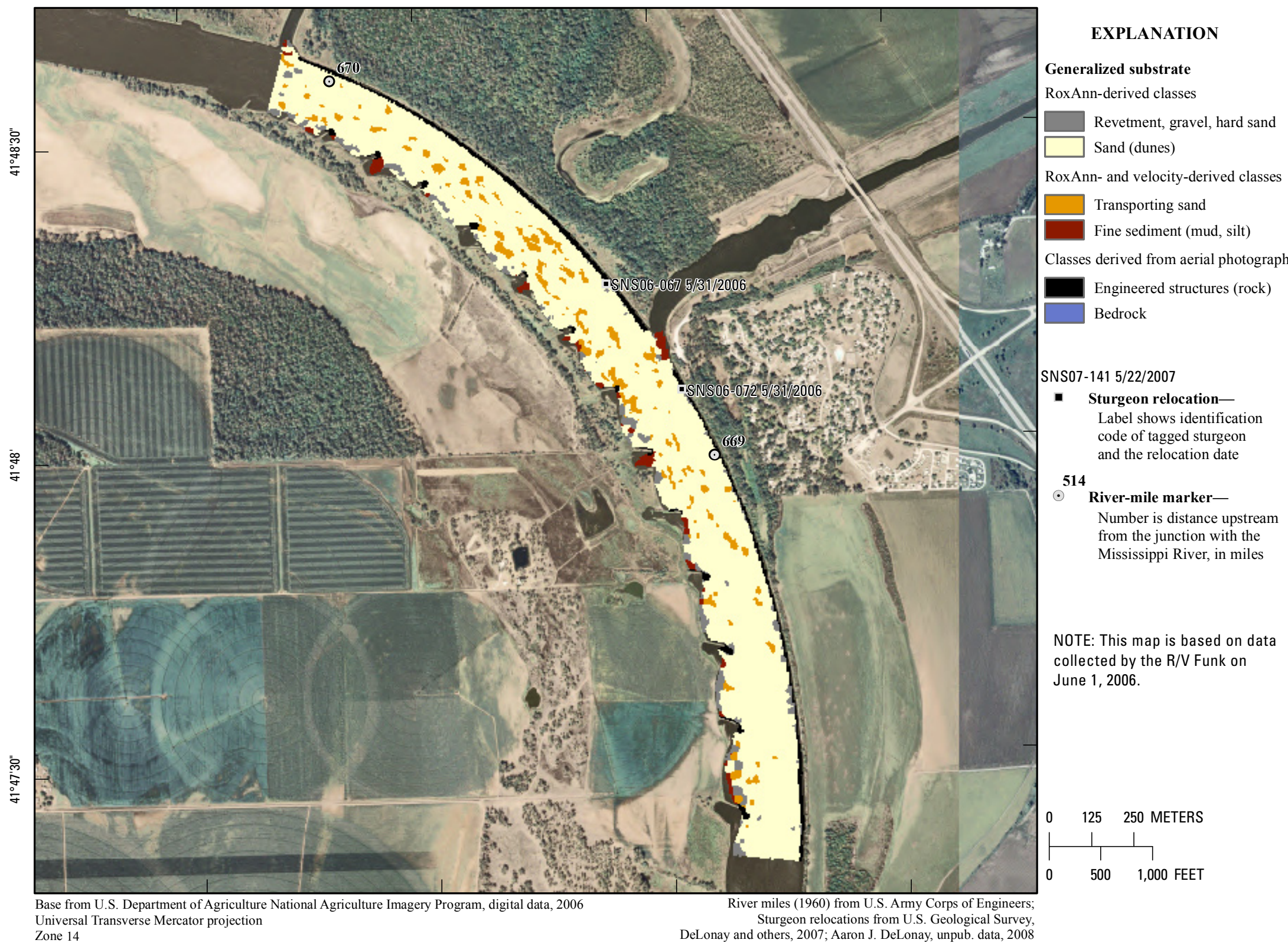




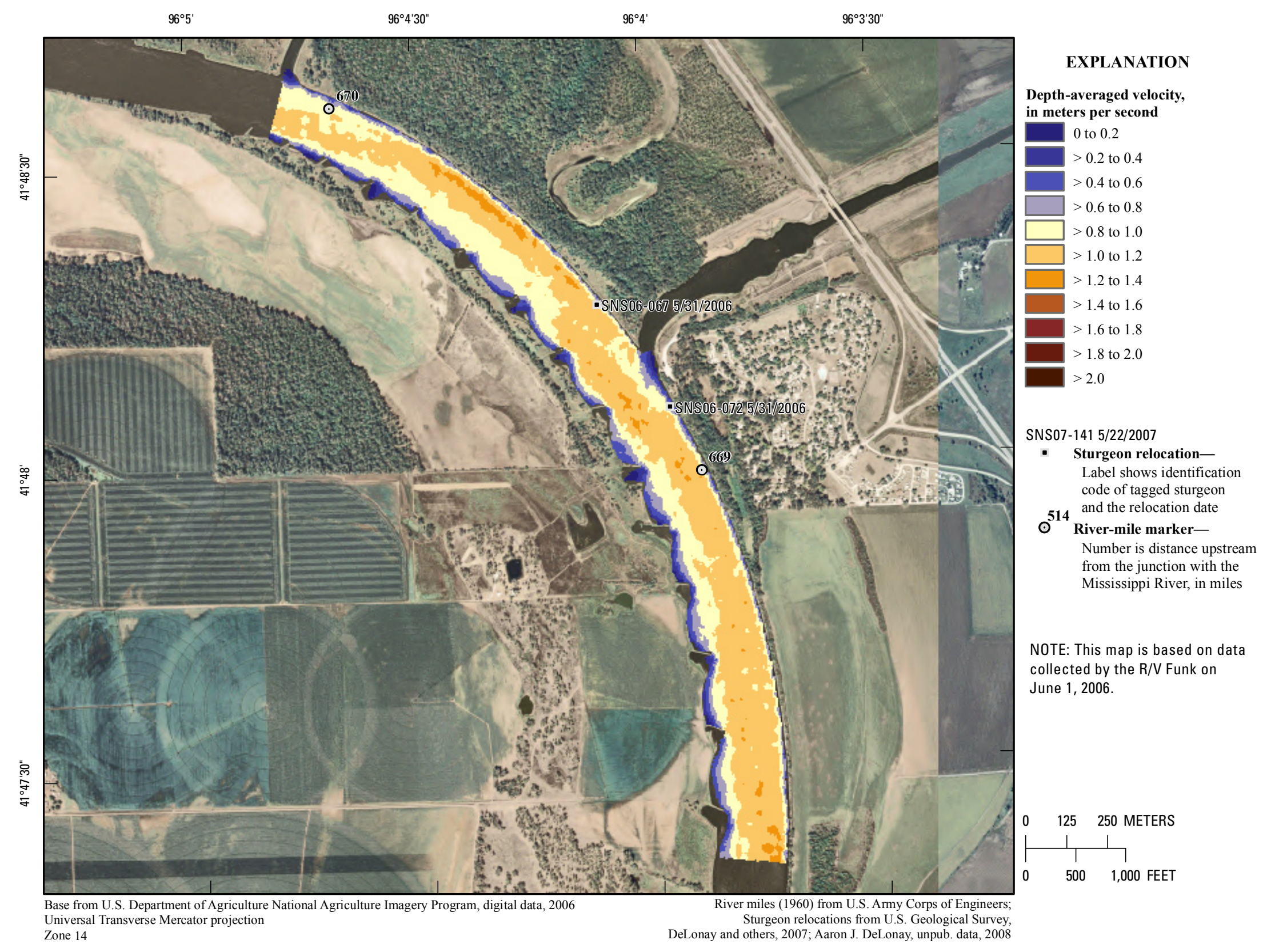

Figure 153. Map of depth-averaged velocity based on data collected on June 1, 2006, in the vicinity of river mile 669 . 


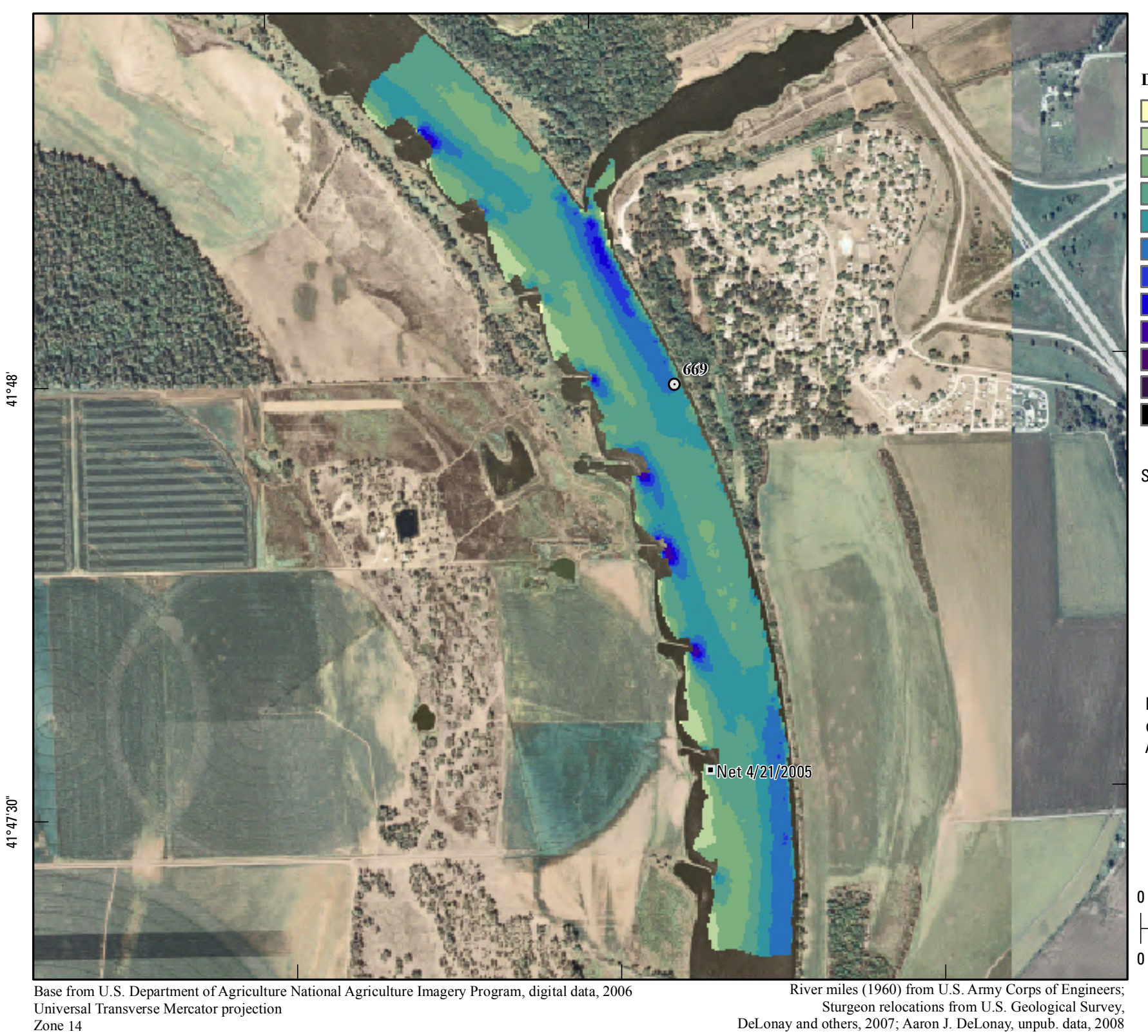

EXPLANATION

Depth, in meters

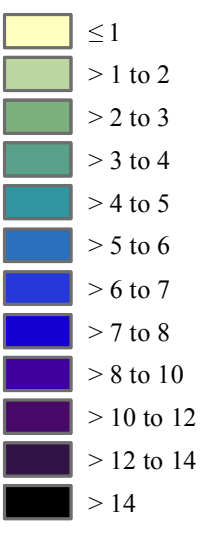

SNS07-141 5/22/2007

- Sturgeon relocationLabel shows identification code of tagged sturgeo 514 and the relocation date

Number is distance upstream from the junction with the Mississippi River, in miles

NOTE: This map is based on data collected by the R/V Funk on April 22, 2005. Zone 14

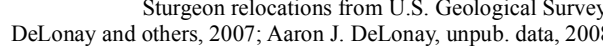

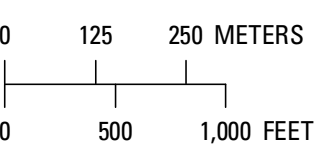




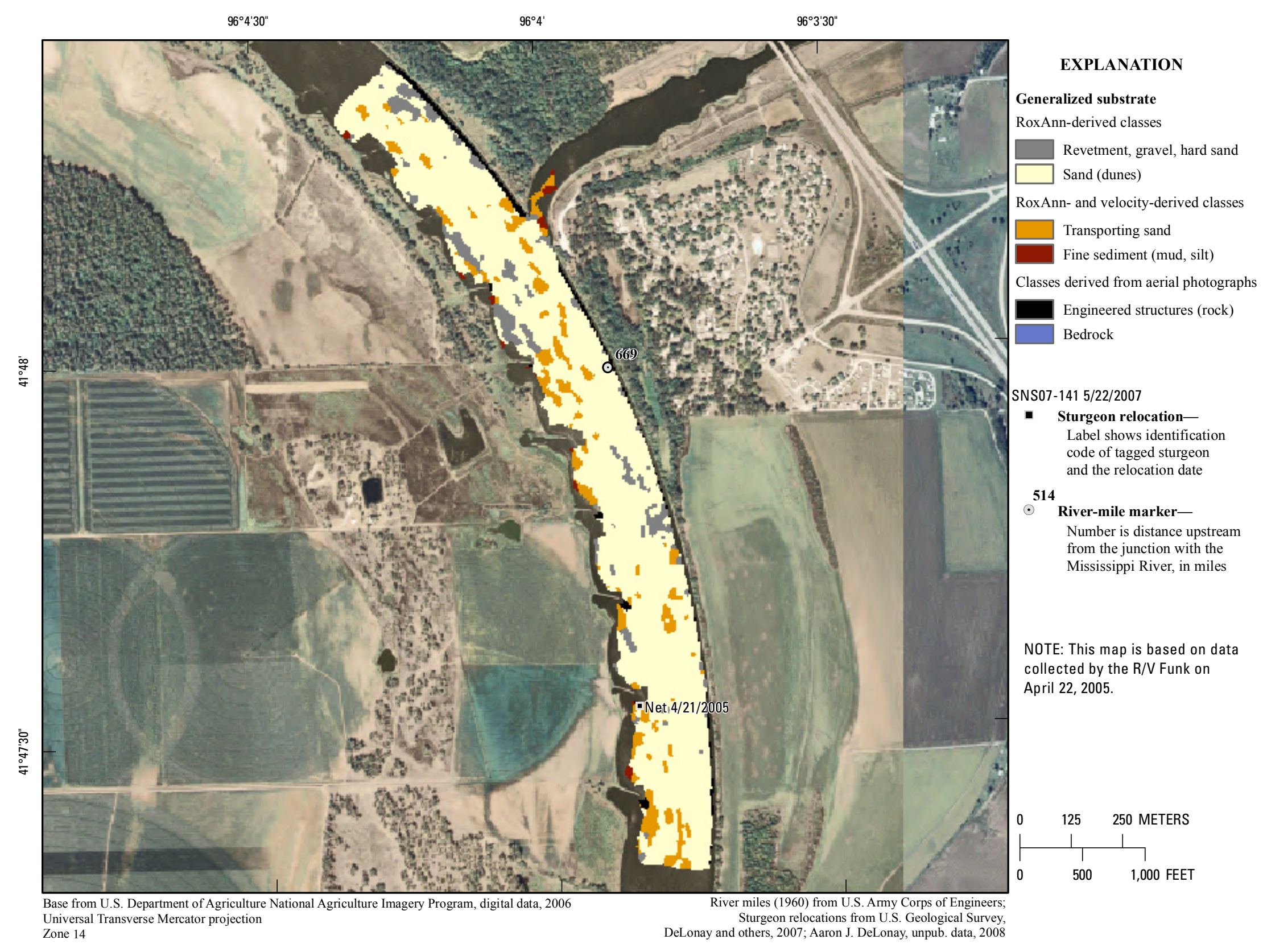

Figure 155. Map of generalized substrate based on data collected on April 22, 2005, in the vicinity of river mile 669 . 


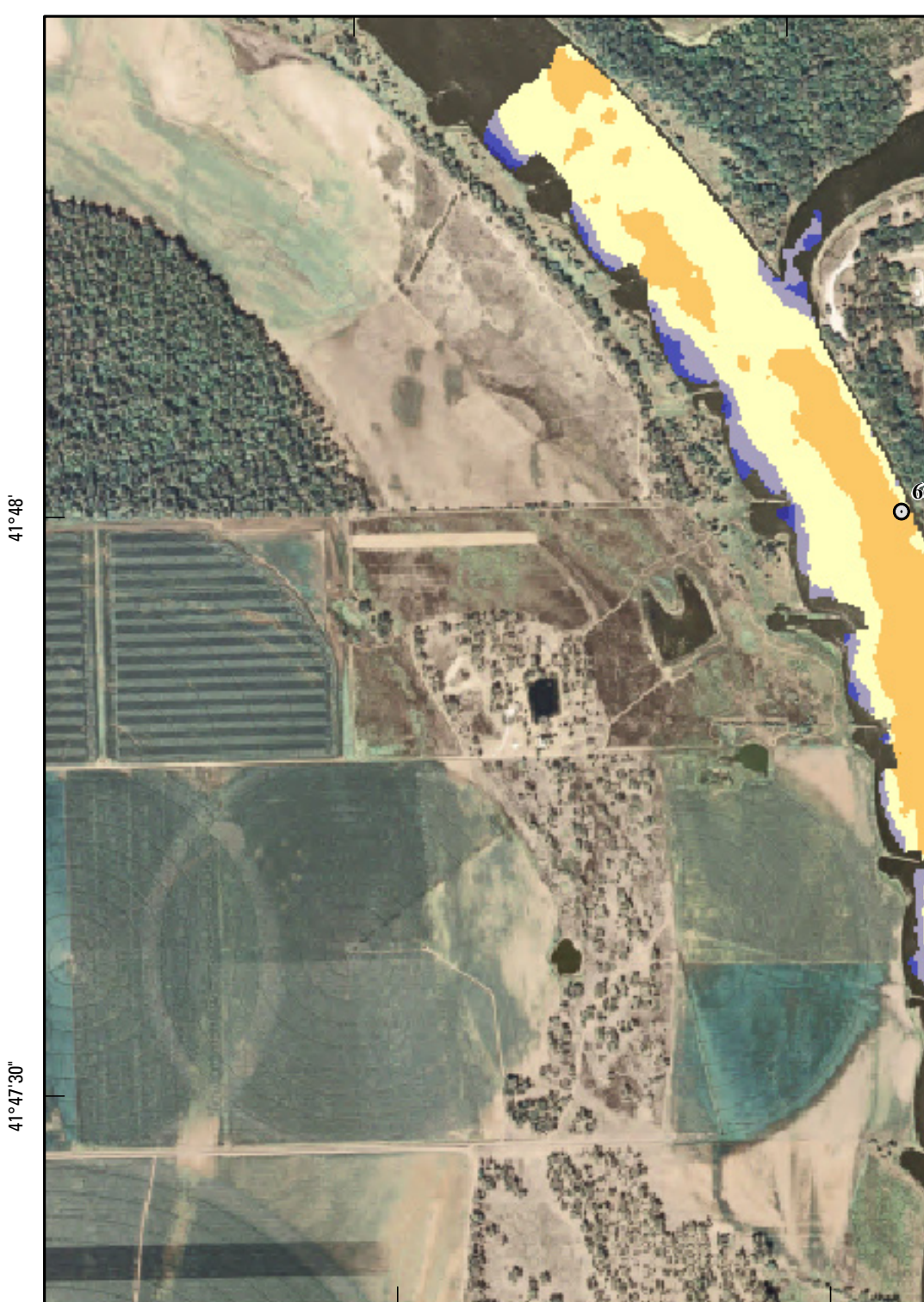

Base from U.S. Department of Agriculture National Agriculture Imagery Program, digital data, 2006 Universal Transverse Mercator projection
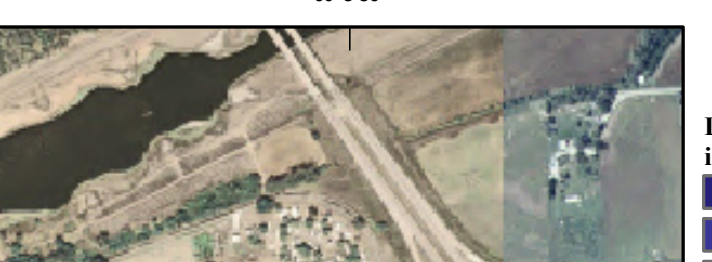

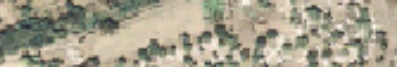

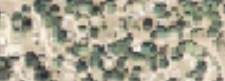

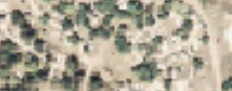

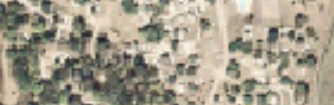
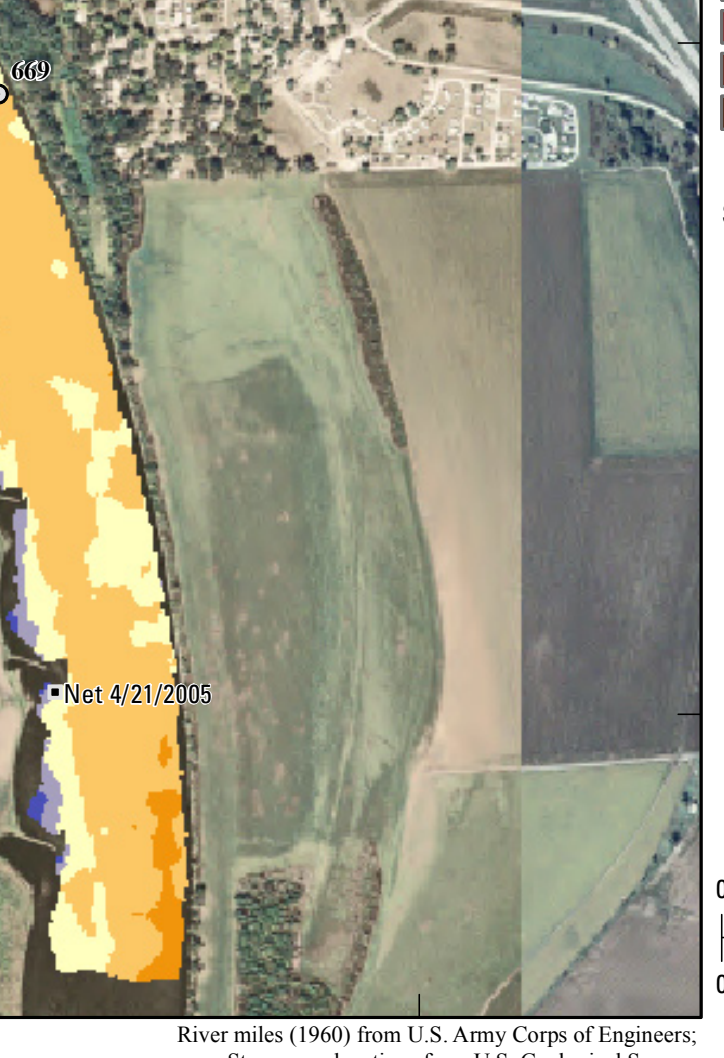

Stheren relocations from U.S. Geological Survey,
EXPLANATION

Depth-averaged velocity, in meters per second

0 to 0.2

$>0.2$ to 0.4

$>0.4$ to 0.6

$>0.6$ to 0.8

$>0.8$ to 1.0

$>1.0$ to 1.2

$>1.2$ to 1.4

$>1.4$ to 1.6

$>1.6$ to 1.8

$>1.8$ to 2.0

$>2.0$

SNS07-141 5/22/2007

- Sturgeon relocationLabel shows identification code of tagged sturgeo

$\mathbf{5 1 4}$ and the relocation date Number is distance upstream
from the junction with the Mississippi River, in miles

NOTE: This map is based on data collected by the R/V Funk on Ap ril 22, 2005.

\section{METERS $\left.\right|_{500} 1$}




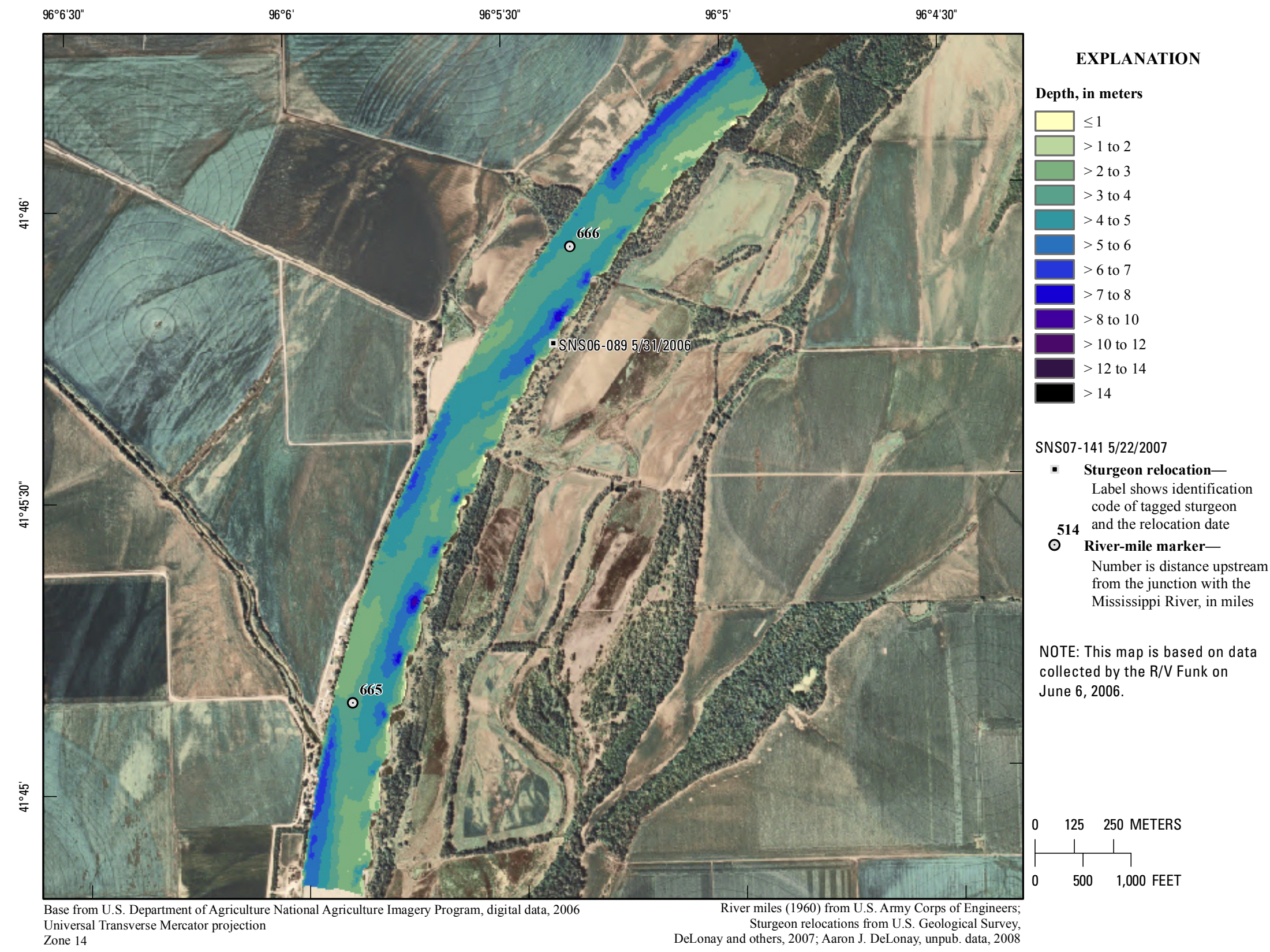

Figure 157. Map of depth based on data collected on June 6, 2006, in the vicinity of river mile 666 


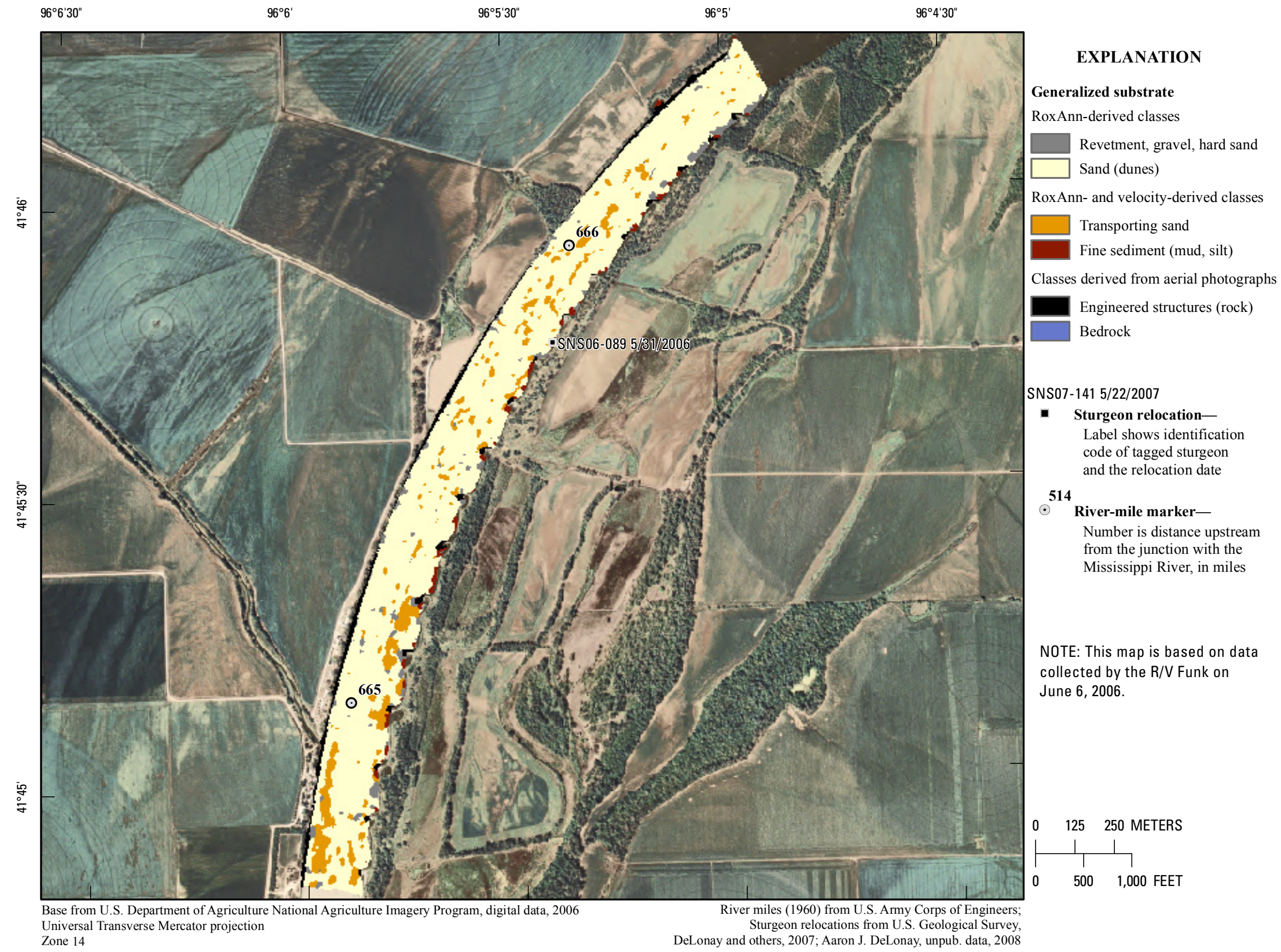

Figure 158. Map of generalized substrate based on data collected on June 6, 2006, in the vicinity of river mile 666 . 


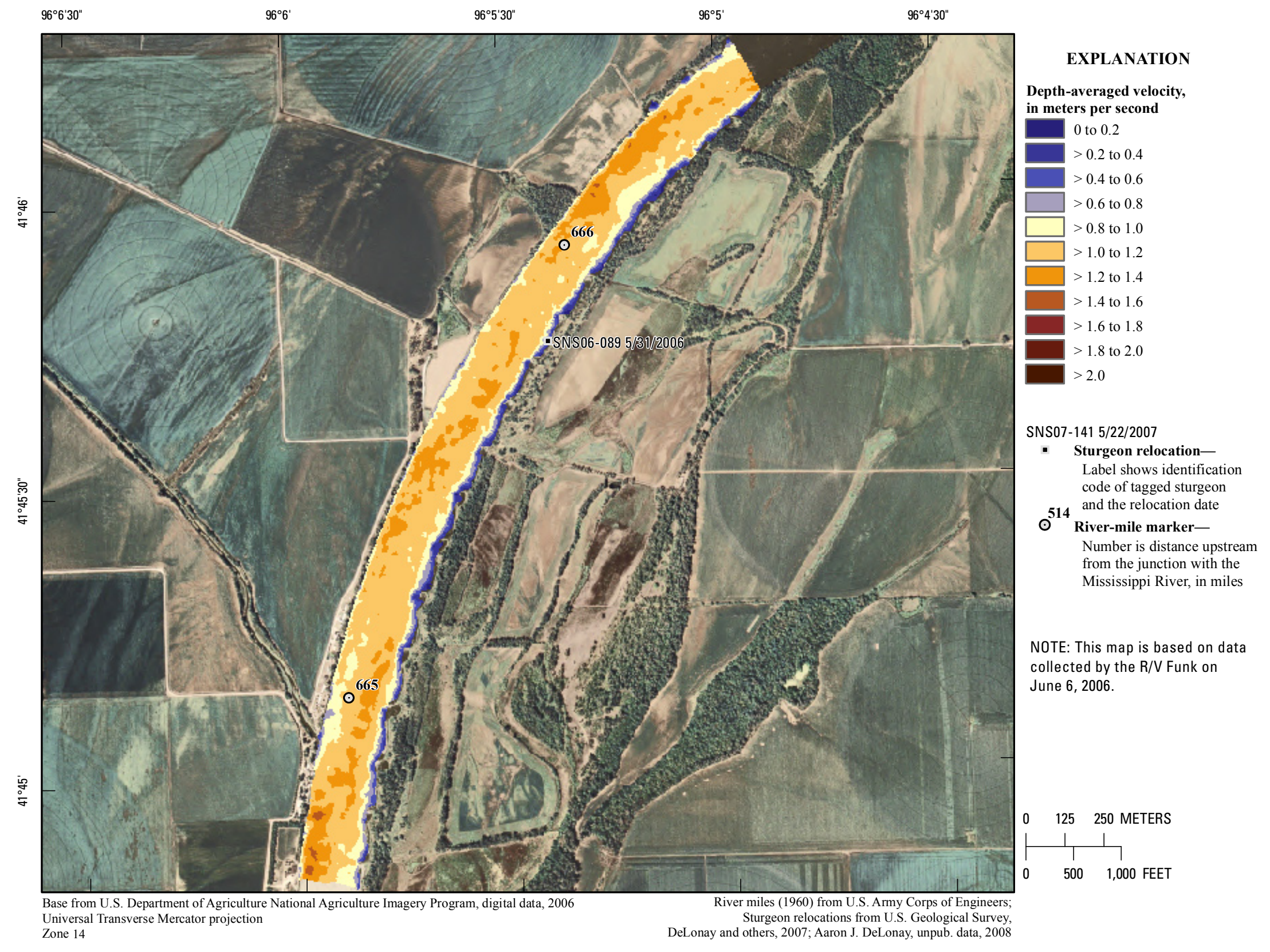

Figure 159. Map of depth-averaged velocity based on data collected on June 6, 2006, in the vicinity of river mile 666 . 


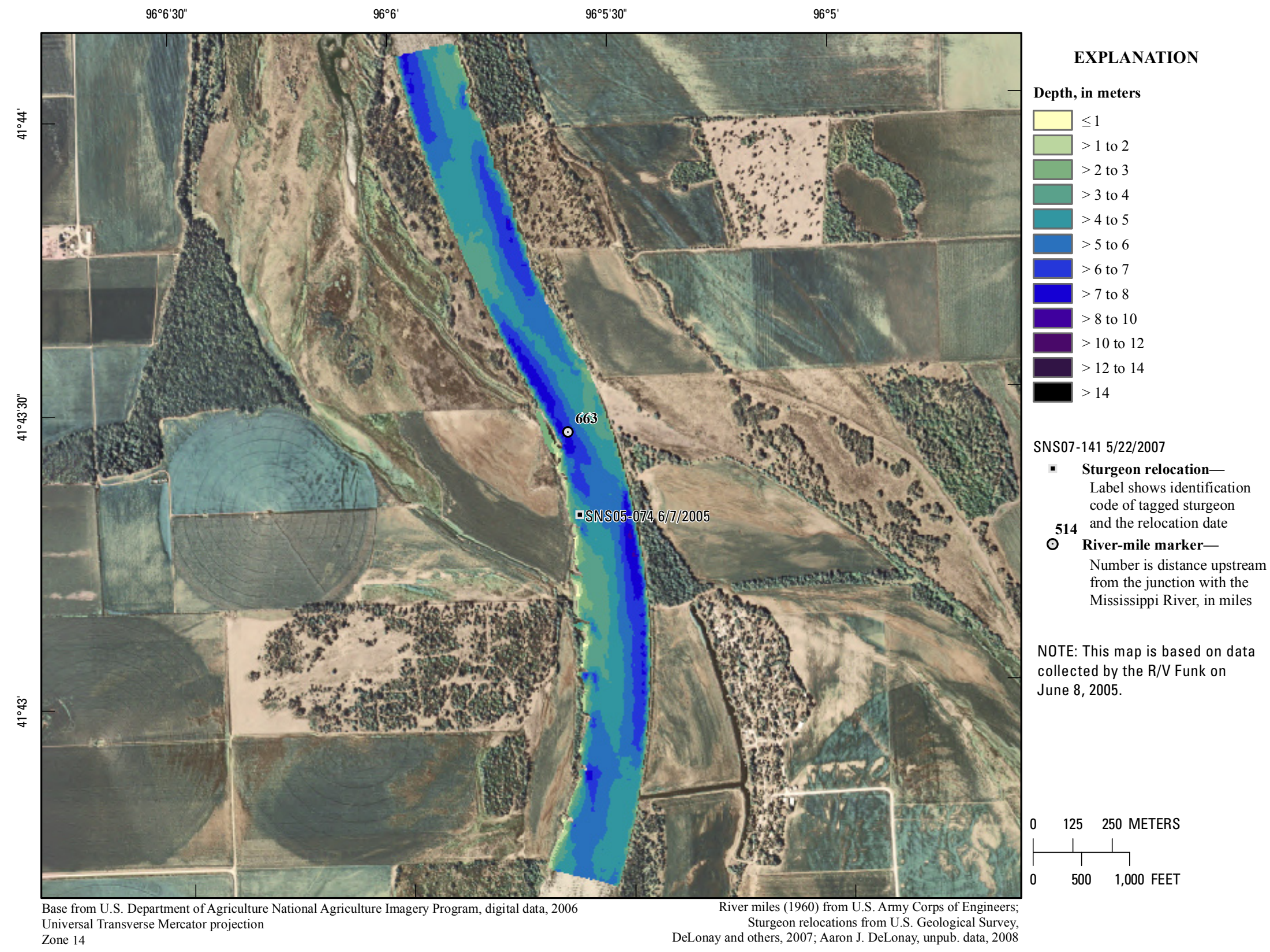

Figure 160. Map of depth based on data collected on June 8, 2005, in the vicinity of river mile 663 . 


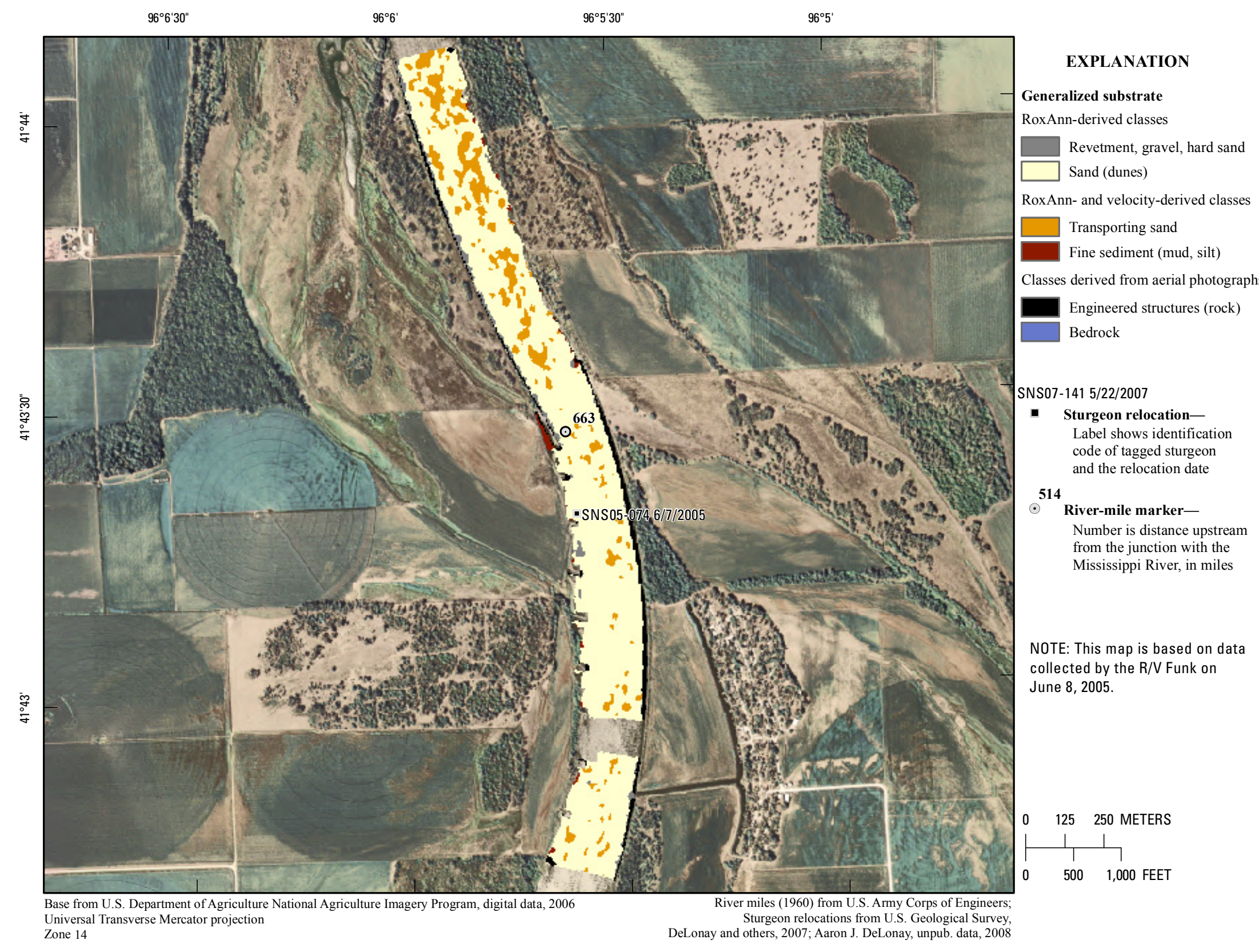

Figure 161. Map of generalized substrate based on data collected on June 8, 2005, in the vicinity of river mile 663 . 


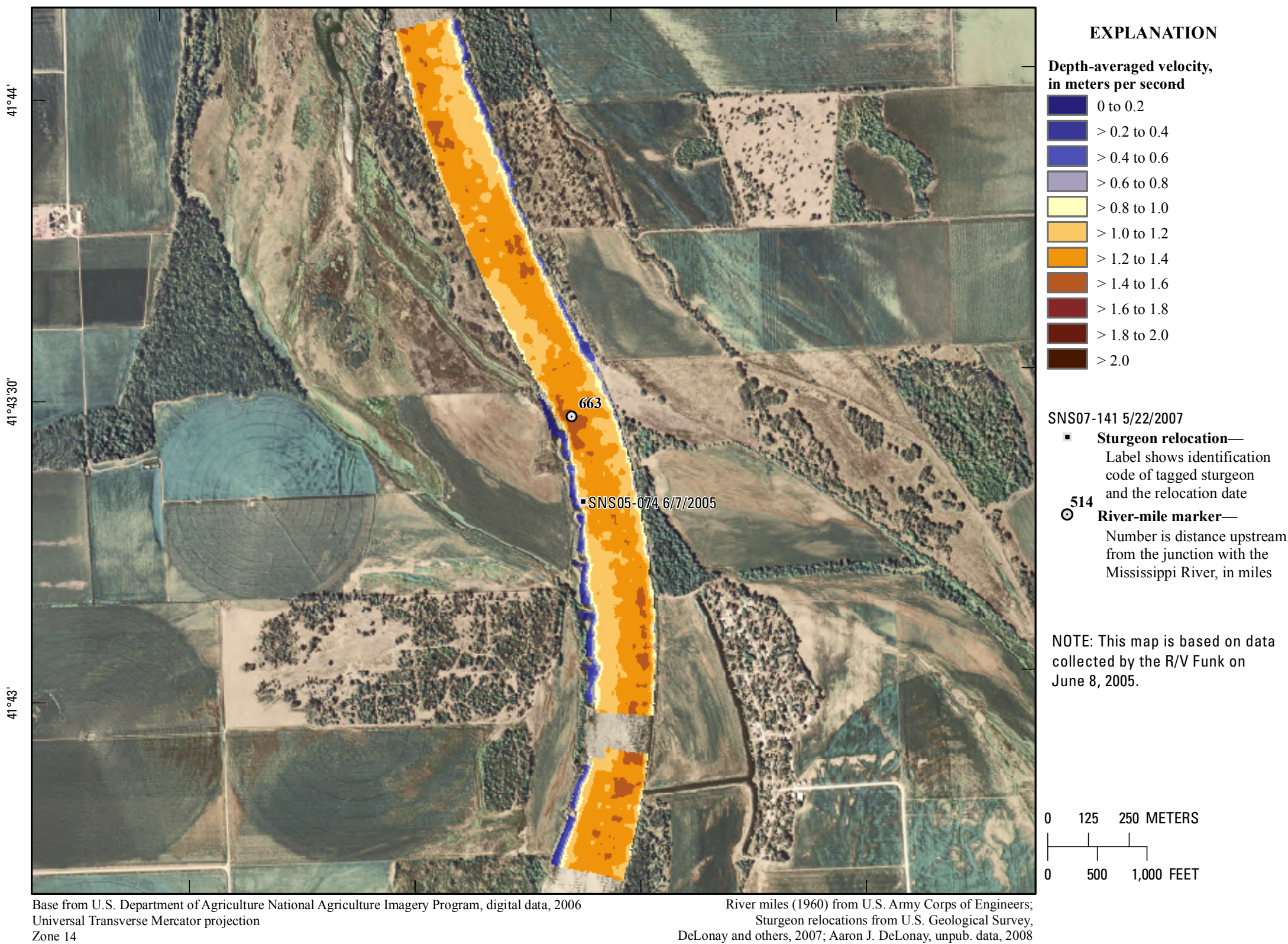




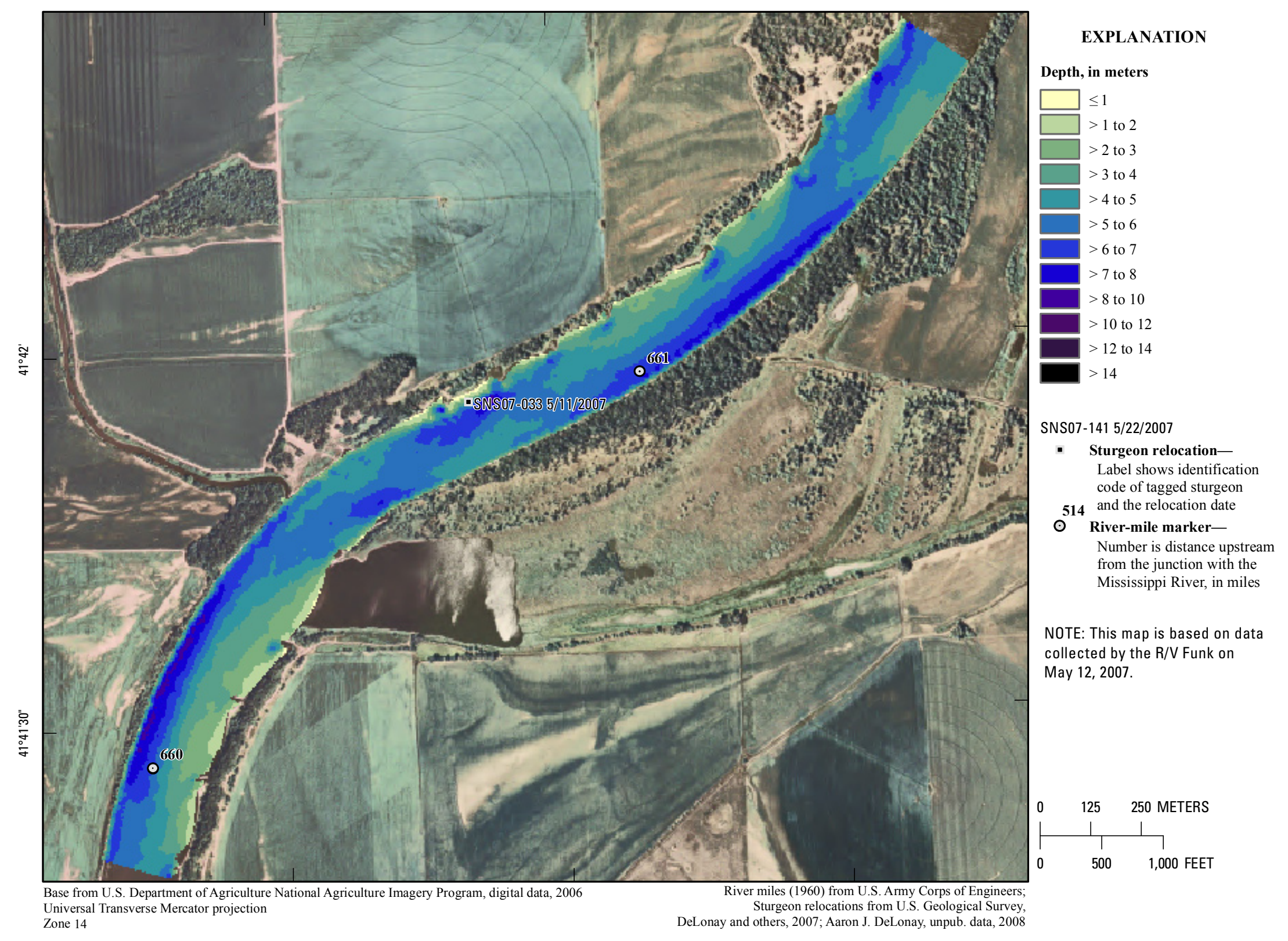

Figure 163. Map of depth based on data collected on May 12, 2007, in the vicinity of river mile 661. 


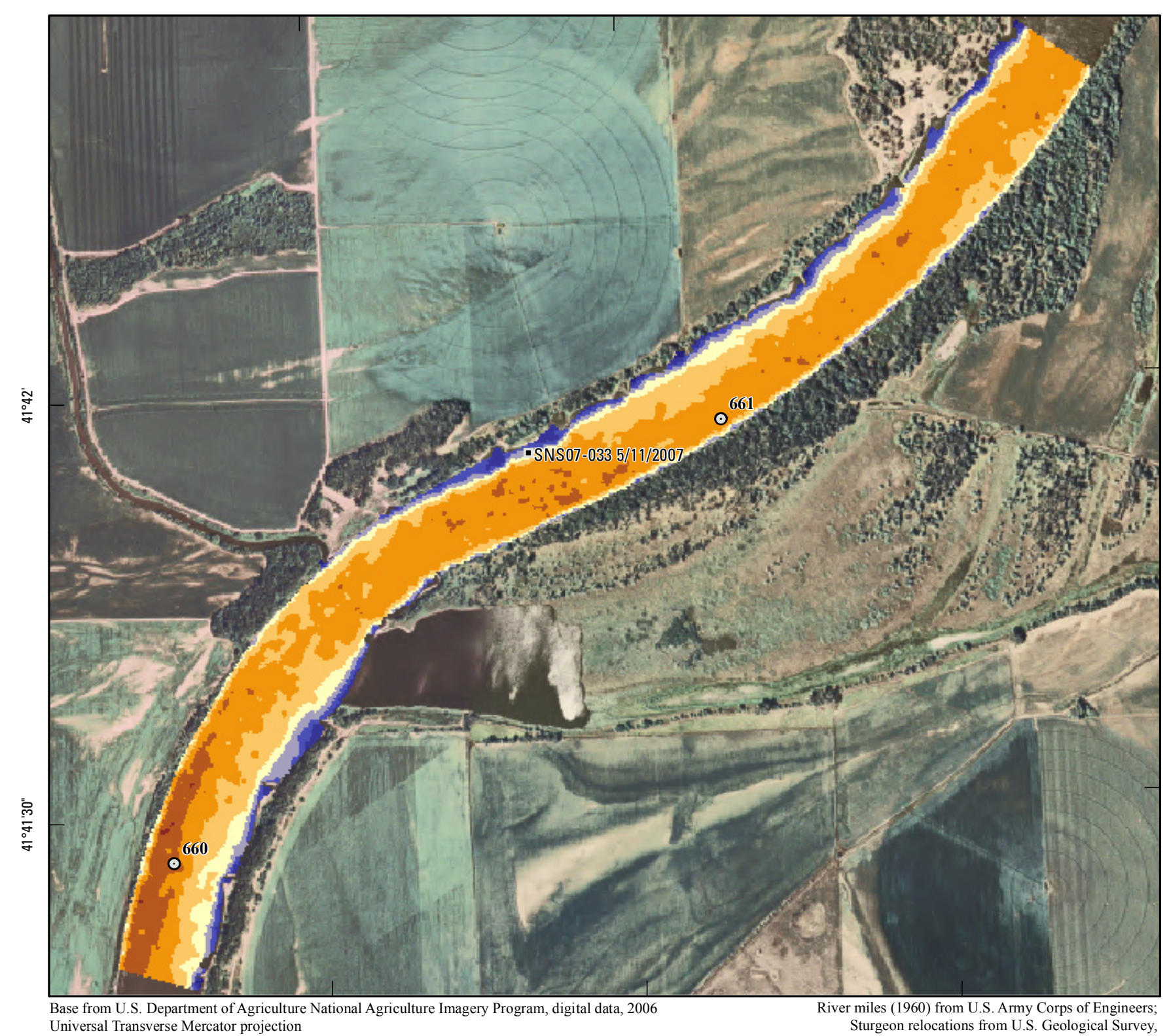

EXPLANATION

Depth-averaged velocity,

in meters per second

0 to 0.2

$>0.2$ to 0.4

$>0.4$ to 0.6

$>0.6$ to 0.8

$>0.8$ to 1.0

$>1.0$ to 1.2

$>1.2$ to 1.4

$>1.4$ to 1.6

$>1.6$ to 1.8

$>1.8$ to 2.0

$\stackrel{d}{\frac{d}{2}}$ Universal Transverse Mercator projection

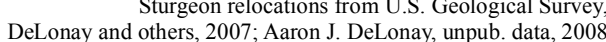

\section{METERS

$\left.\right|_{500} ^{\mid} \mid$

Figure 164. Map of depth-averaged velocity based on data collected on May 12, 2007, in the vicinity of river mile 661 . 


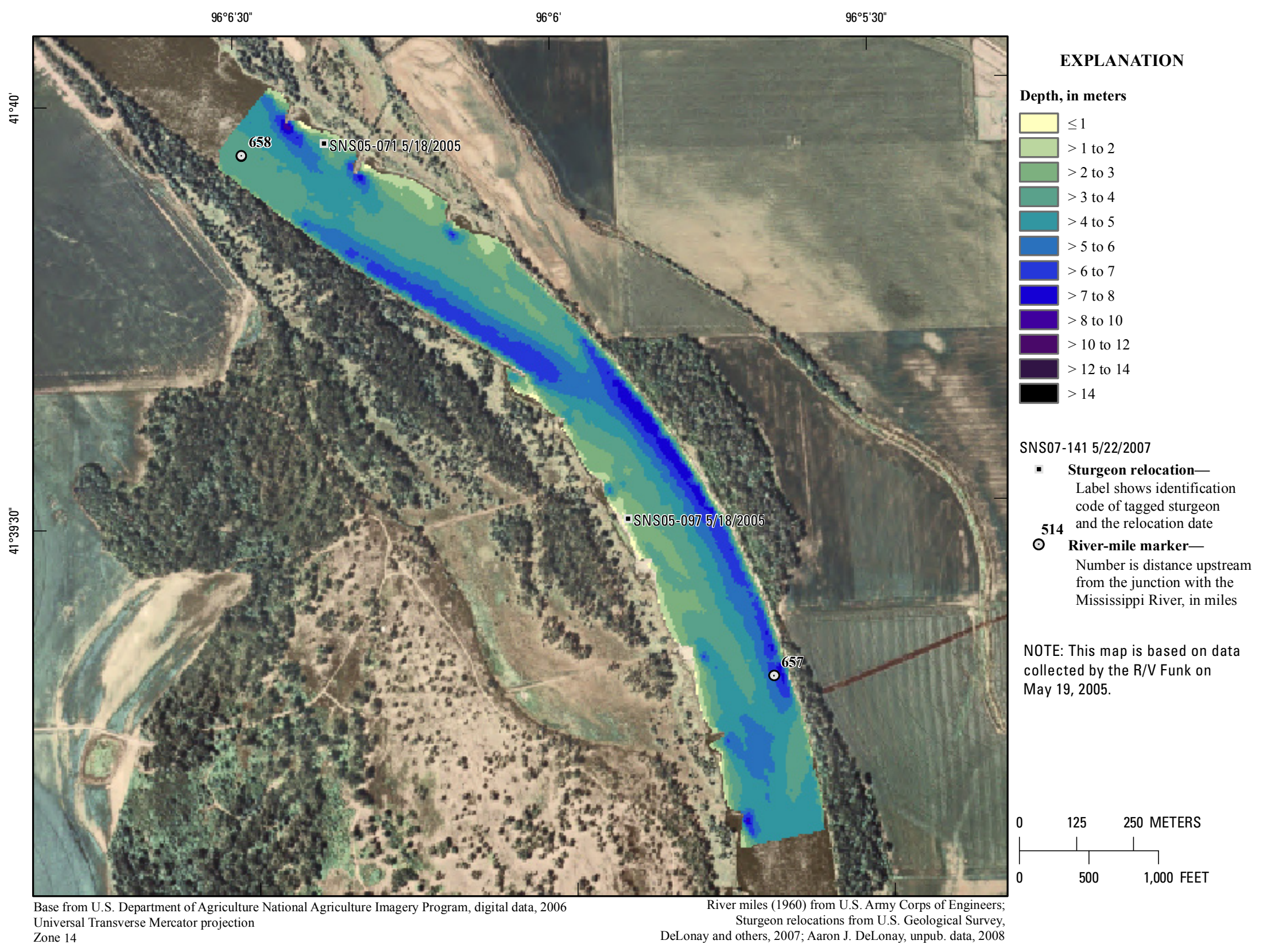

Figure 165. Map of depth based on data collected on May 19, 2005, in the vicinity of river mile 657 . 
$96^{\circ} 7$

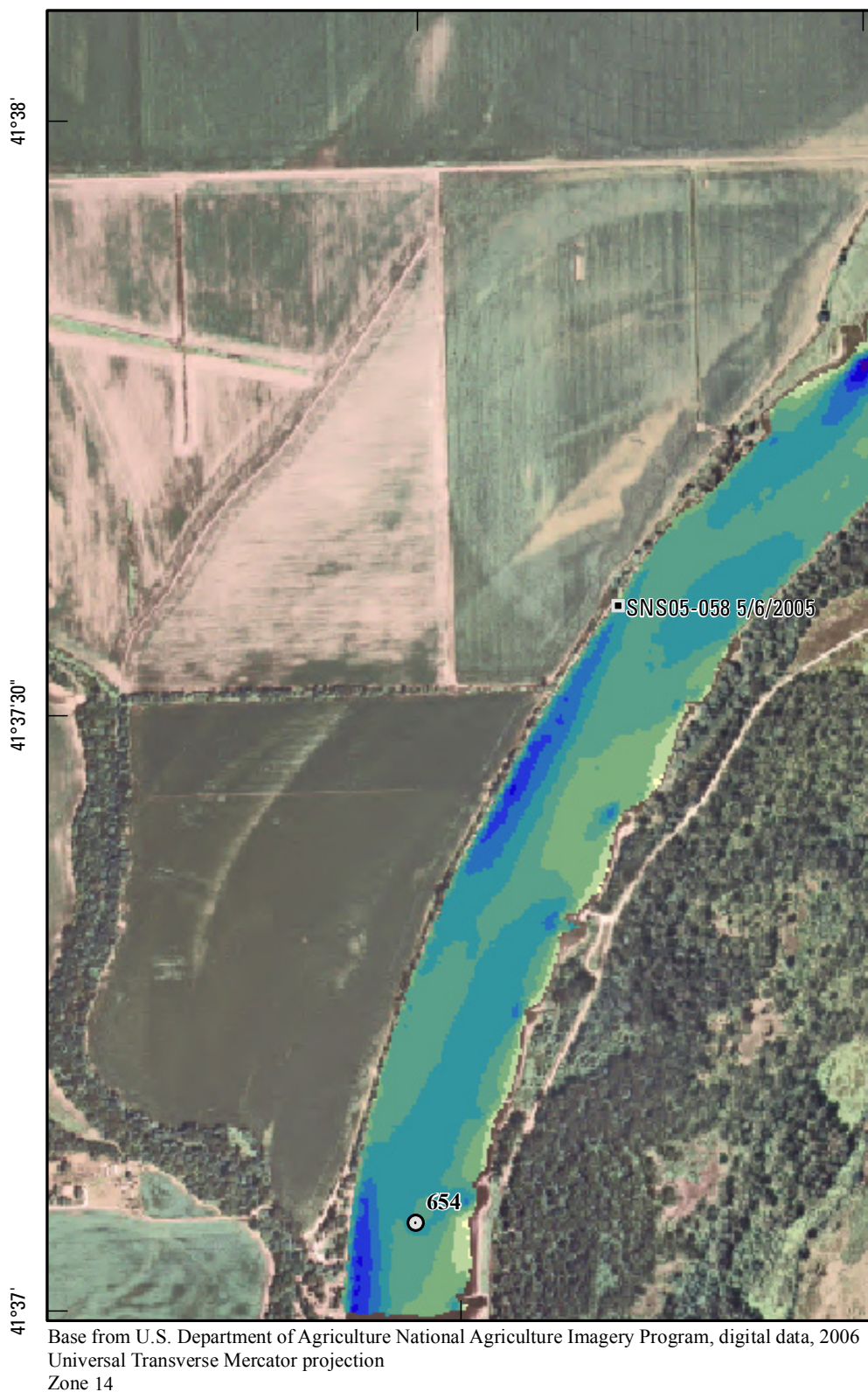

$96^{\circ} 6^{\prime}$

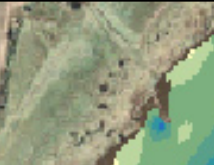

(1)
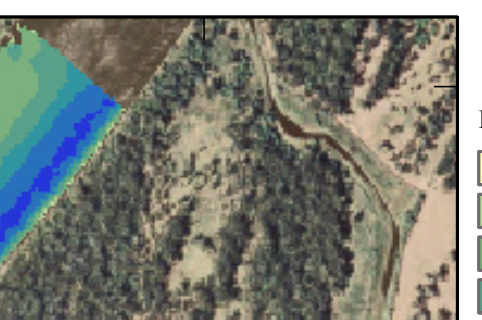

ming
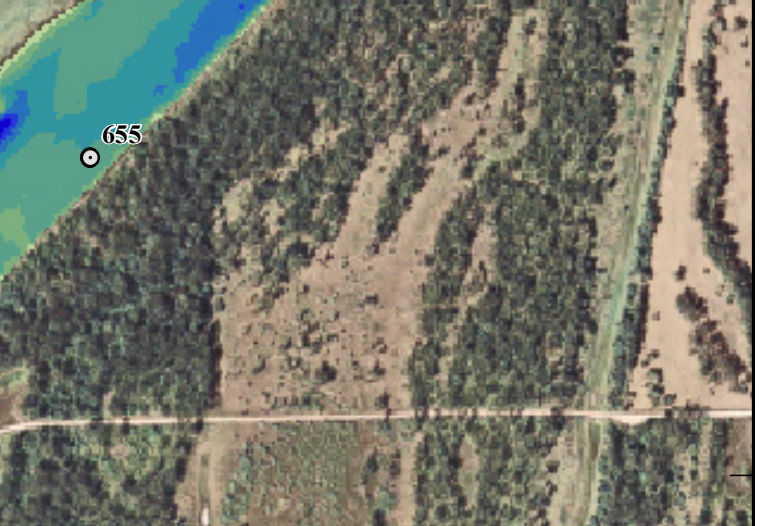

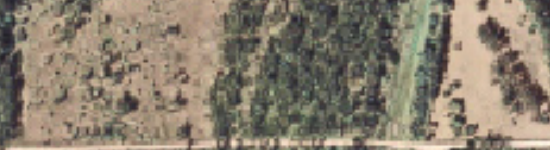
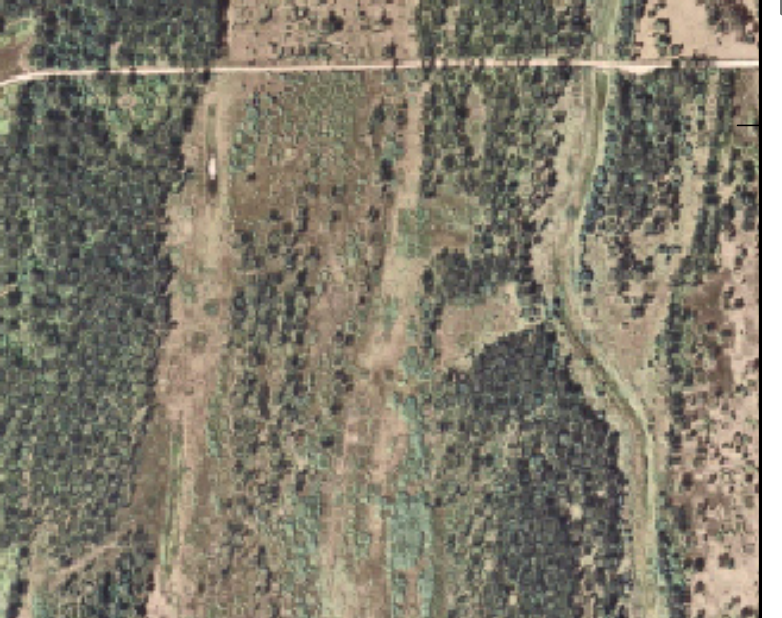

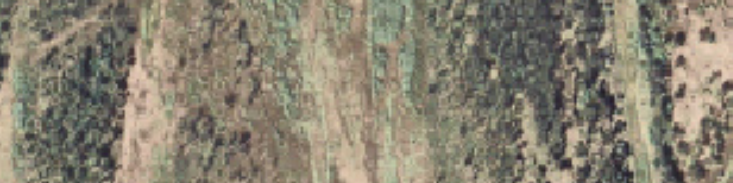

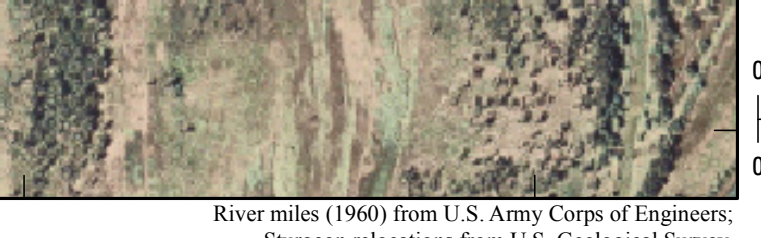

Sturgeon relocations from U.S. Geological Survey,
DeLonay and others, 2007; Aaron J. DeLonay, unpub. data, 2008

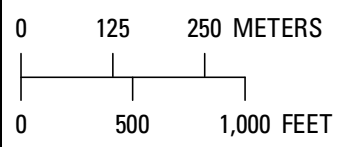

NOTE: This map is based on data collected by the R/V Funk on

May 7, 2005.

NS07-141 5/22/2007

- Sturgeon relocationLabel shows identification code of tagged sturgeon 514 and the relocation date

Number is distance upstream from the junction with the Mississippi River, in miles 
$96^{\circ} 7$

$96^{\circ} 6^{\prime} 30^{\prime \prime}$

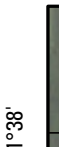

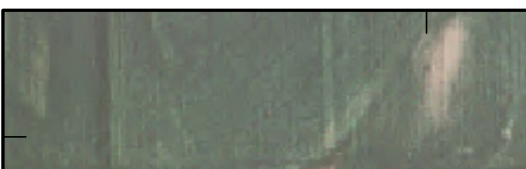

$\mid$

Ey
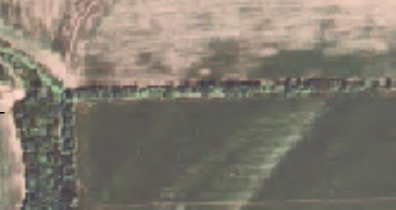

के

Base from U.S. Department of Agriculture National Agriculture Imagery Program, digital data, 2006 Universal Transverse Mercator projection
Zone 14

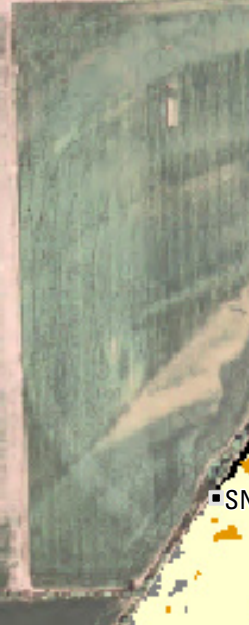

…

if $1:$ 19ucer

$96^{\circ} 6$

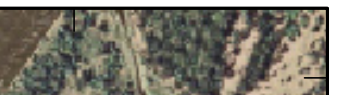

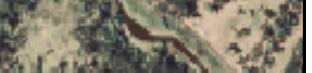

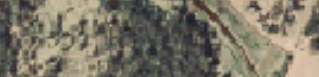

t?

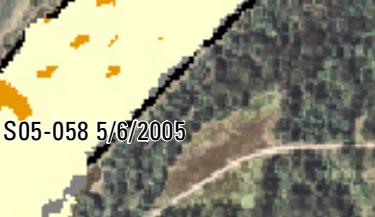

4 $655^{7}$

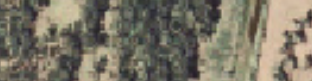

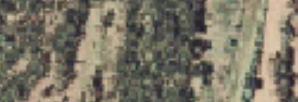

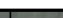

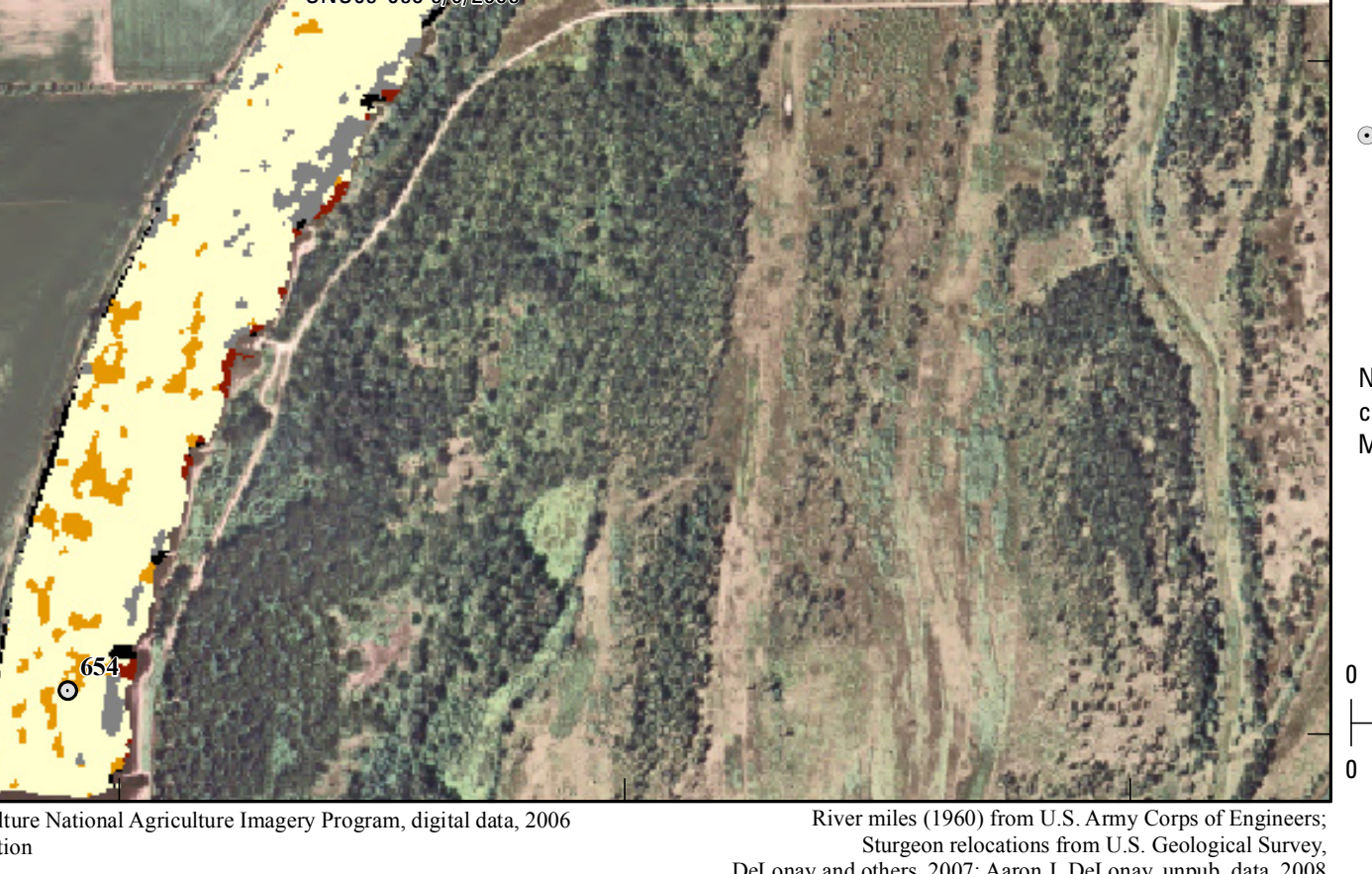

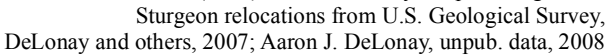

EXPLANATION

Generalized substrate

RoxAnn-derived classes

Revetment, gravel, hard sand Sand (dunes)

RoxAnn- and velocity-derived classes

Transporting sand

Fine sediment (mud, silt)

Classes derived from aerial photographs

Engineered structures (rock) Bedrock

\section{SNS07-141 5/22/2007}

- Sturgeon relocationLabel shows identification

514 and the relocation date

River-mile markerNumber is distance upstream from the junction with the Mississippi River, in miles

NOTE: This map is based on data collected by the R/V Funk on May 7, 2005.

\section{METERS

Figure 167. Map of generalized substrate based on data collected on May 7, 2005, in the vicinity of river mile 655. 
$96^{\circ} 7^{\prime}$

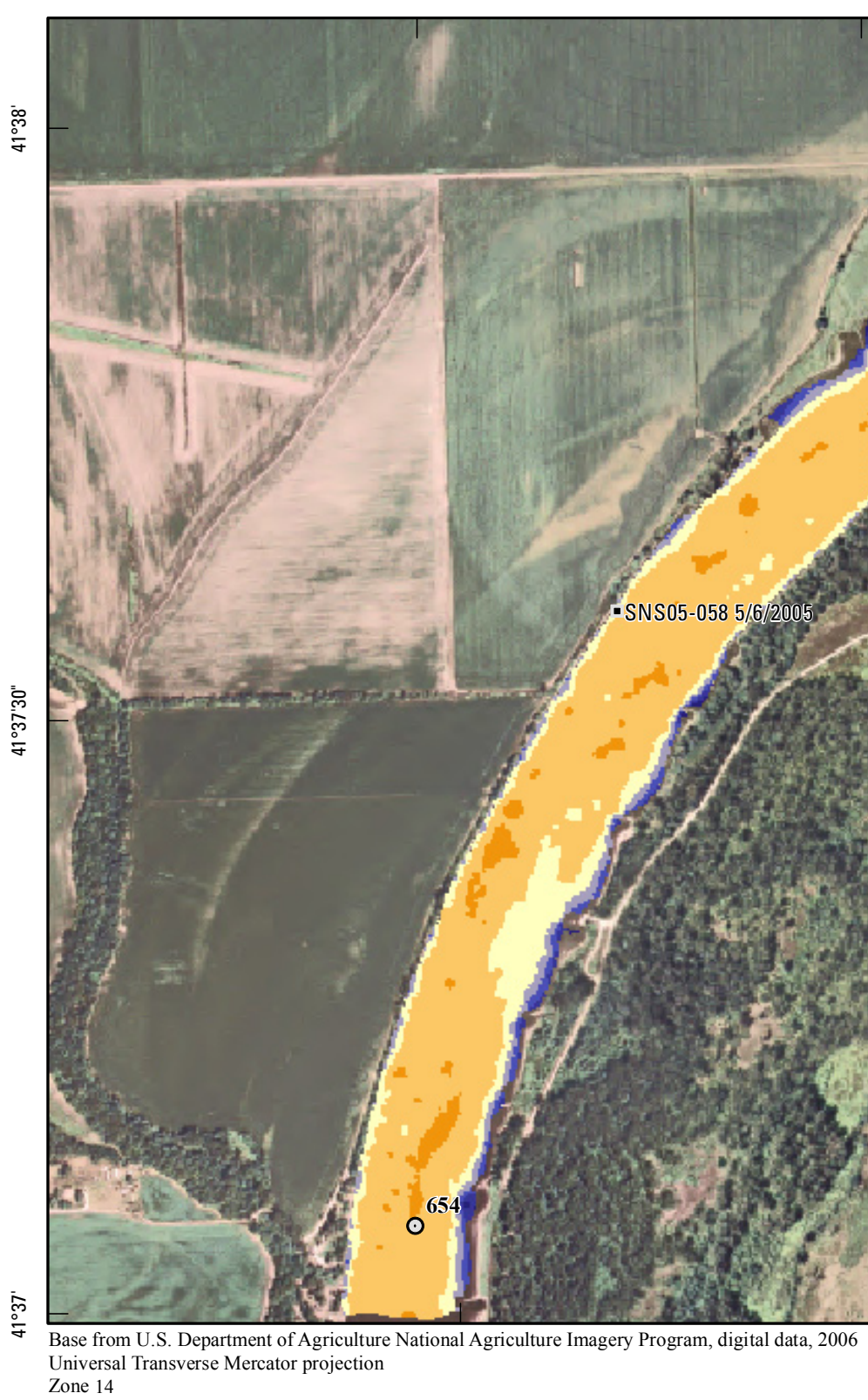

$96^{\circ} 6^{\prime} 30^{\prime \prime}$

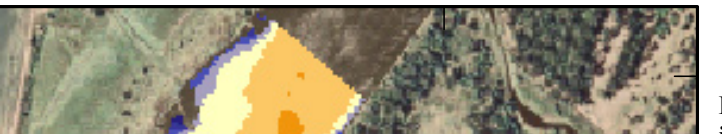

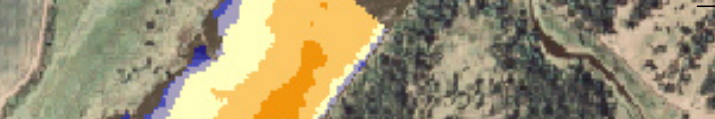
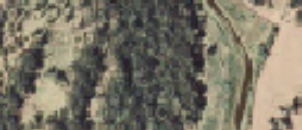

3.t.
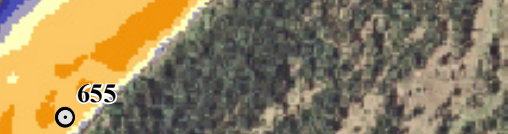

$0^{655}$
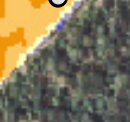

with
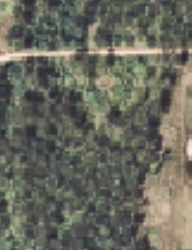

3.
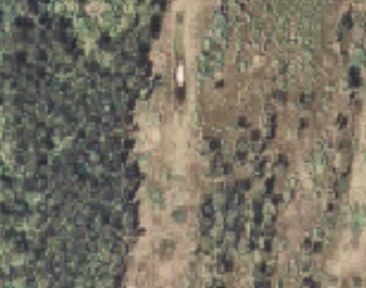

4. 798
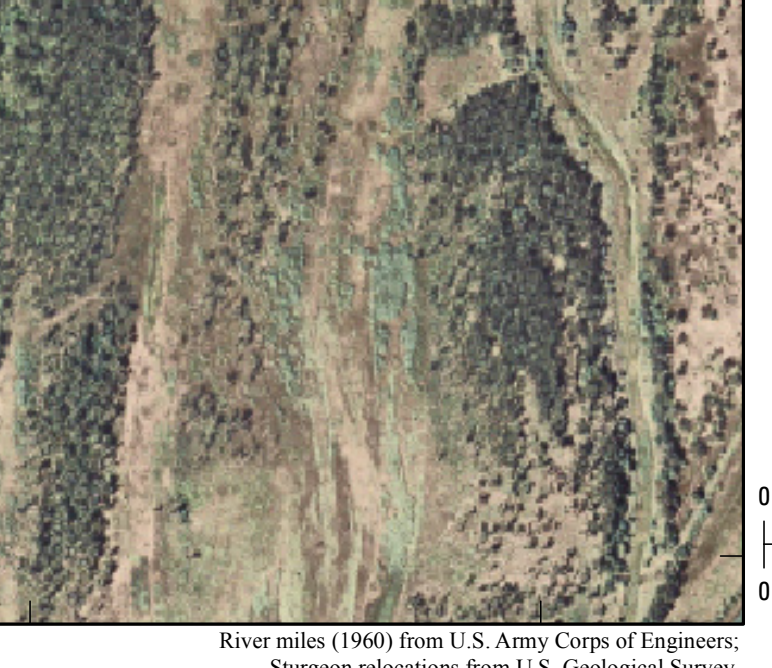
Sturgeon relocations from U.S. Geological Survey,
DeLonay and others, 2007; Aaron J. DeLonay, unpub. data, 2008
EXPLANATION

Depth-averaged velocity,

in meters per second

0 to 0.2

$>0.2$ to 0.4

$>0.4$ to 0.6

$>0.6$ to 0.8

$>0.8$ to 1.0

$>1.0$ to 1.2

$>1.2$ to 1.4

$>1.4$ to 1.6

$>1.6$ to 1.8

$>1.8$ to 2.0

$>2.0$

\section{SNS07-141 5/22/2007}

- Sturgeon relocationLabel shows identification code of tagged sturgeo

$0^{514}$ and the relocation date

Number is distance upstream

from the junction with the

Mississippi River, in miles

NOTE: This map is based on data

collected by the R/V Funk on

May 7, 2005.

\section{METERS \\ $500 \quad 1,000$ FEET}

Figure 168. Map of depth-averaged velocity based on data collected on May 7, 2005, in the vicinity of river mile 655 . 


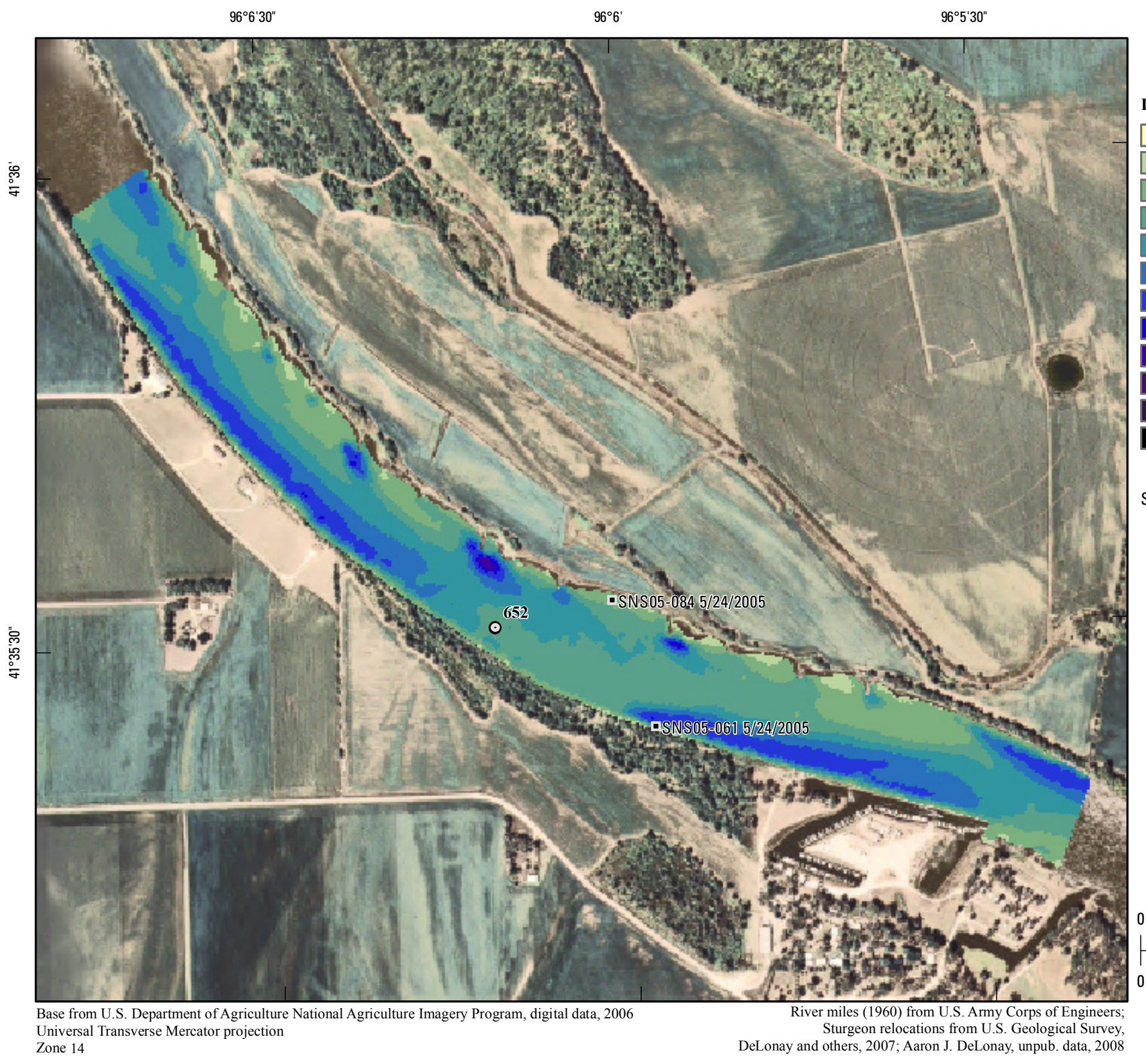

EXPLANATION

Depth, in meters

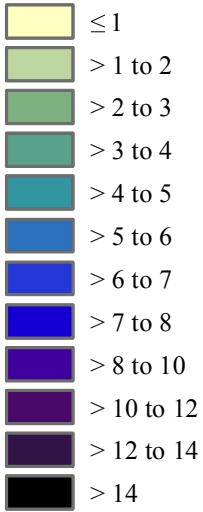

SNS07-141 5/22/2007

- Sturgeon relocationLabel shows identification code of tagged sturgeon 514 and the relocation date

$\odot$ River-mile markerNumber is distance upstream from the junction with the Mississippi River, in miles

NOTE: This map is based on data collected by the R/V Funk on May 25, 2005.

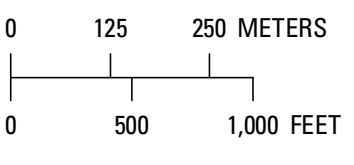

Figure 169. Map of depth based on data collected on May 25, 2005, in the vicinity of river mile 652. 


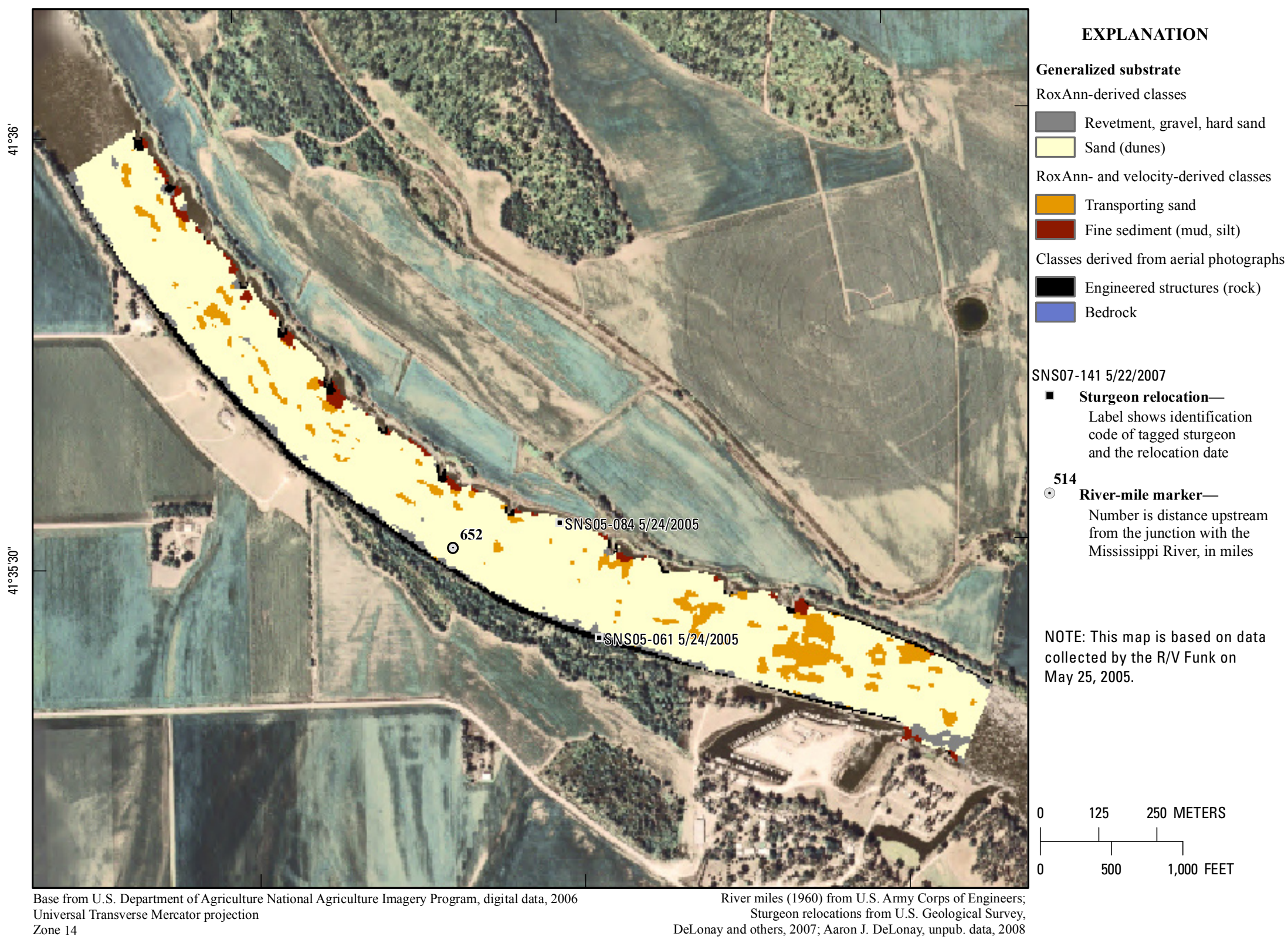




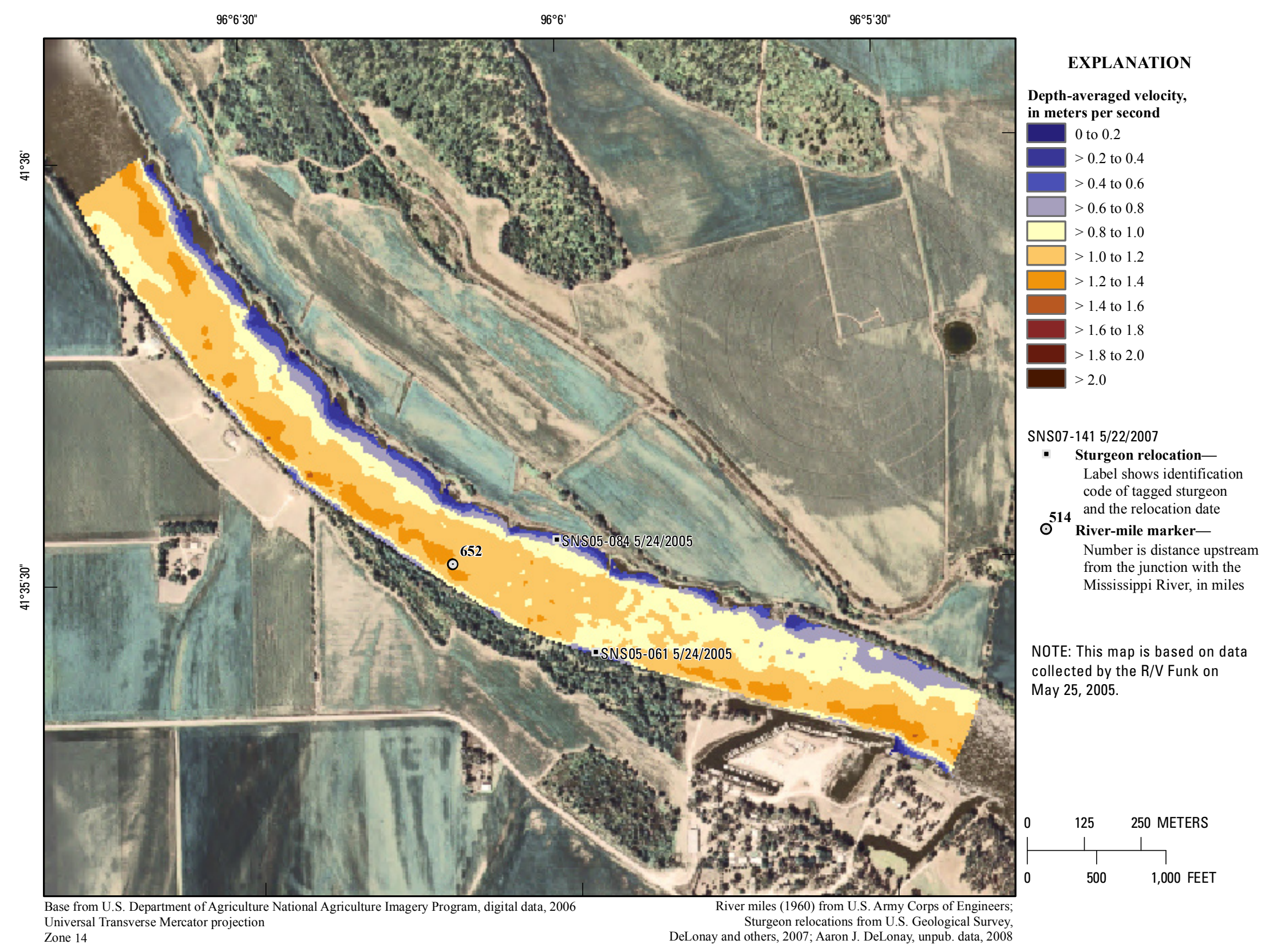

Figure 171. Map of depth-averaged velocity based on data collected on May 25, 2005, in the vicinity of river mile 652 . 


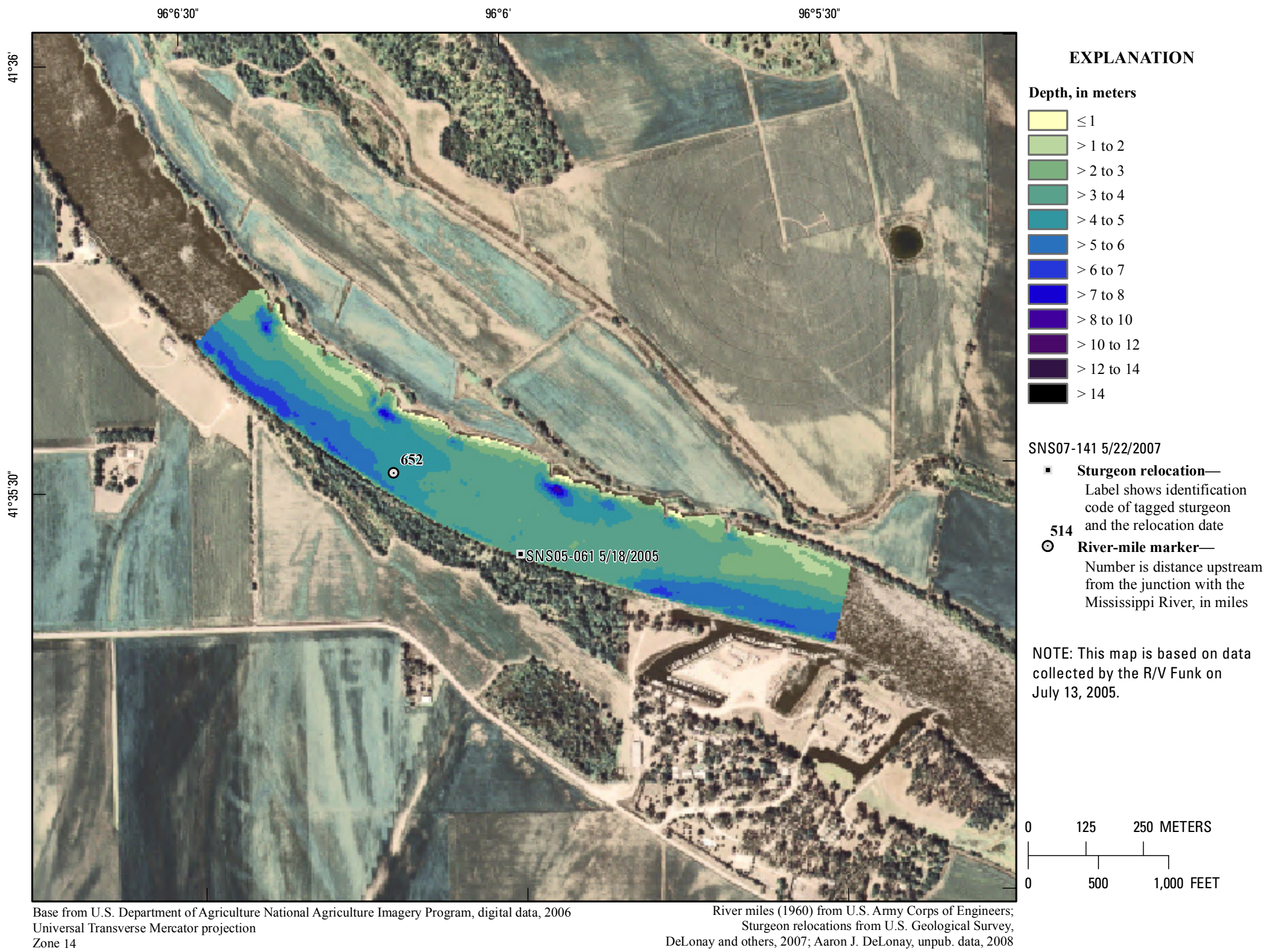

Figure 172. Map of depth based on data collected on July 13, 2005, in the vicinity of river mile 652 . 


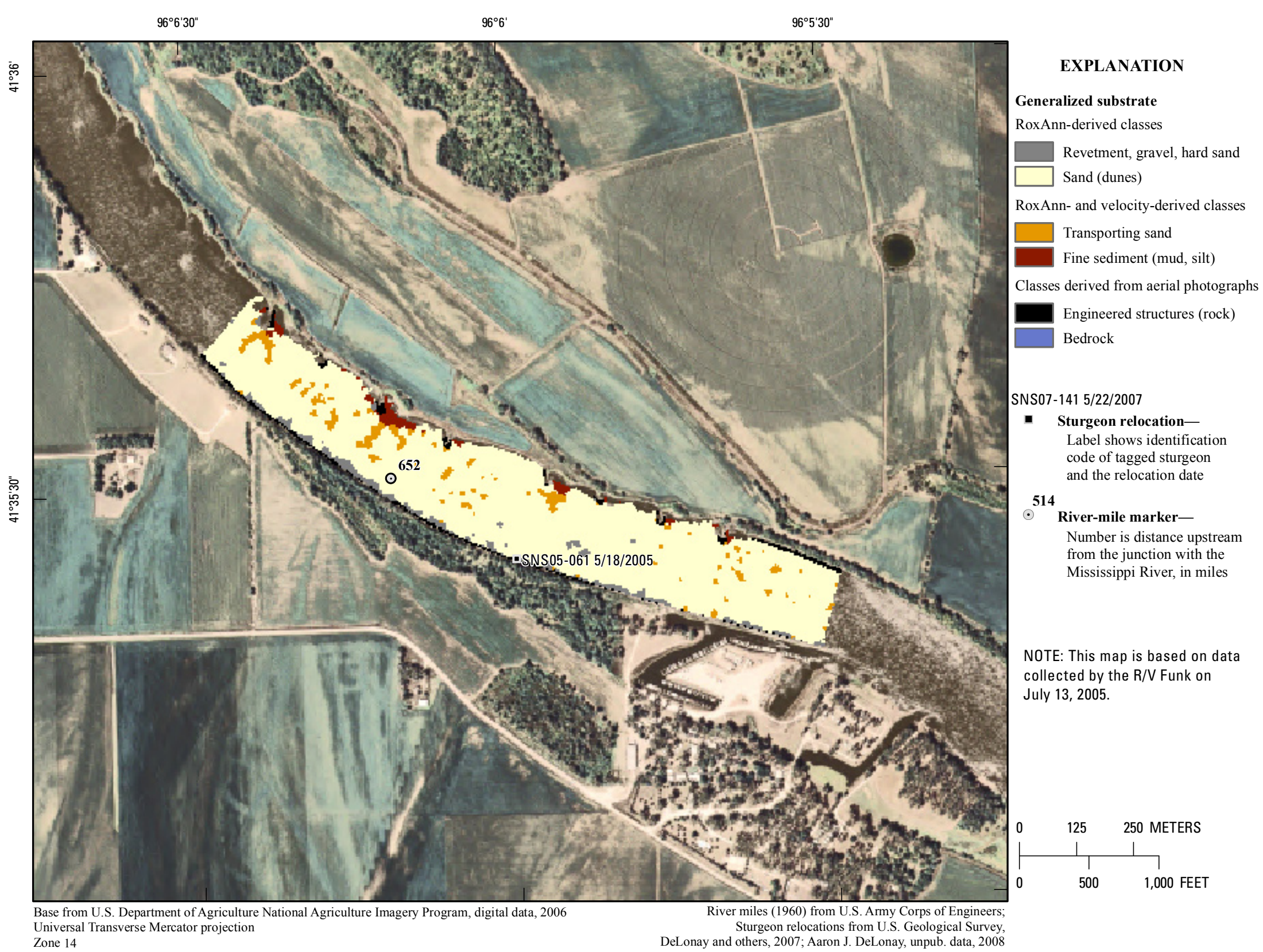

Figure 173. Map of generalized substrate based on data collected on July 13,2005 , in the vicinity of river mile 652 . 


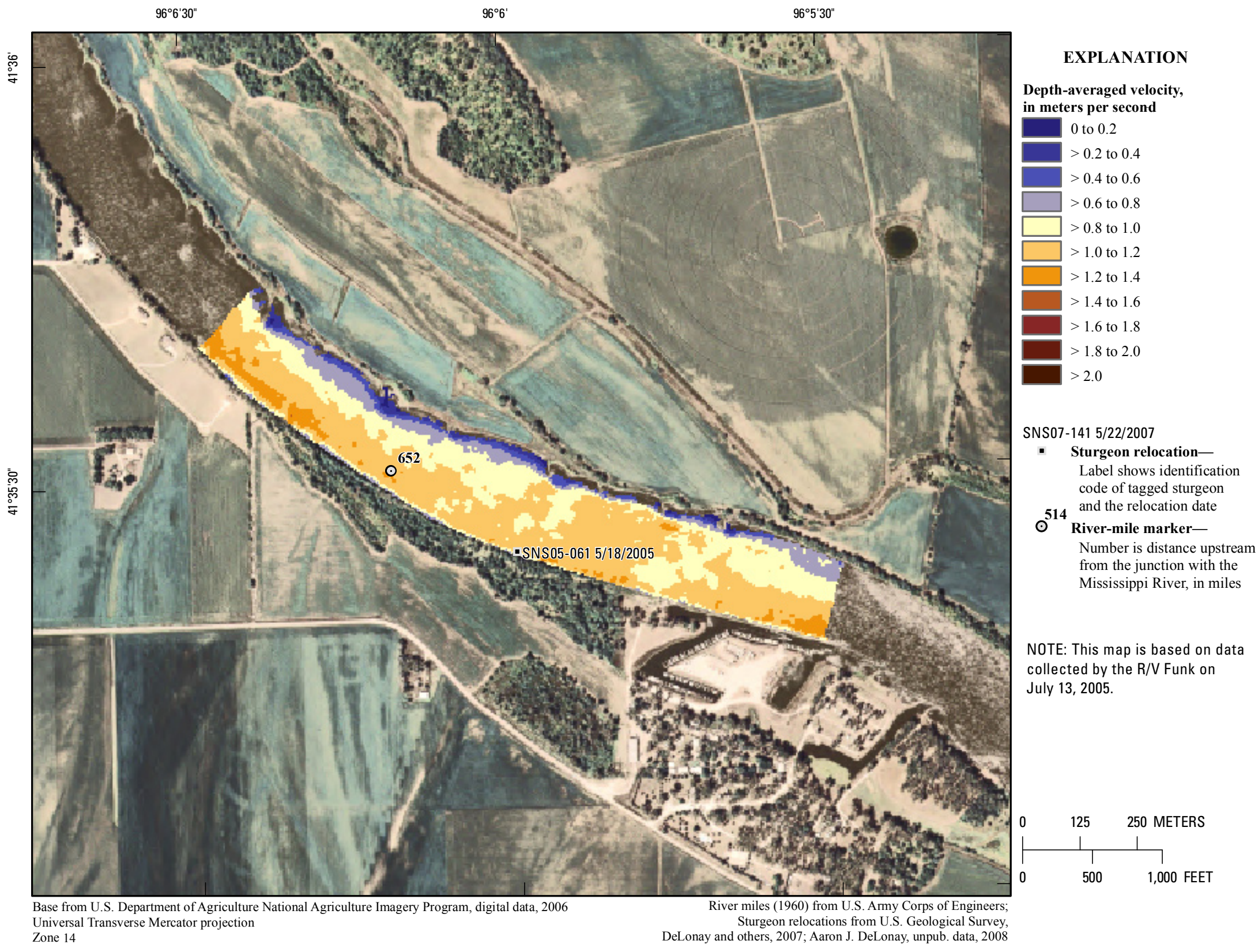

Figure 174. Map of depth-averaged velocity based on data collected on July 13, 2005, in the vicinity of river mile 652 . 


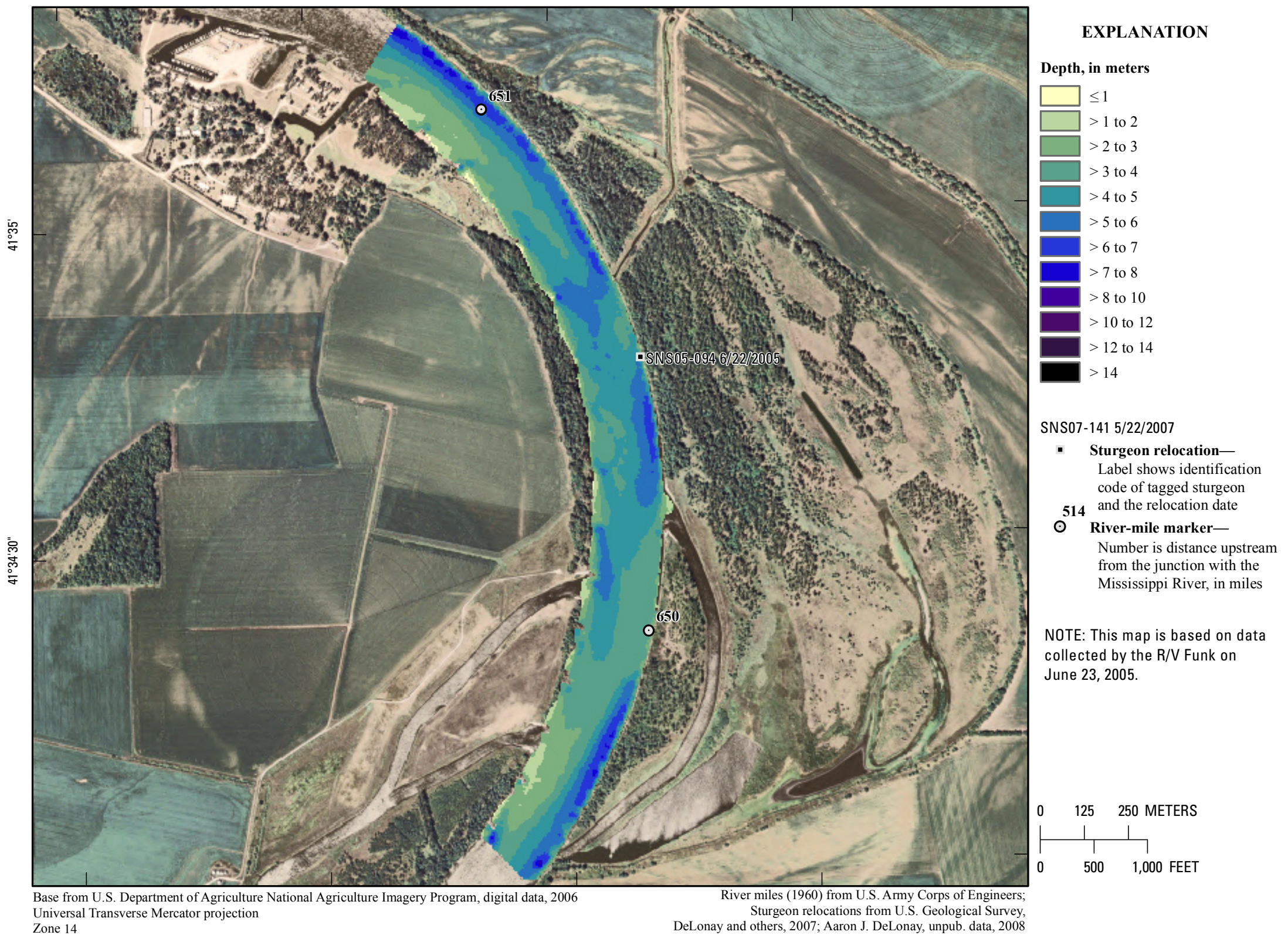

Figure 175. Map of depth based on data collected on June 23, 2005, in the vicinity of river mile 650 . 


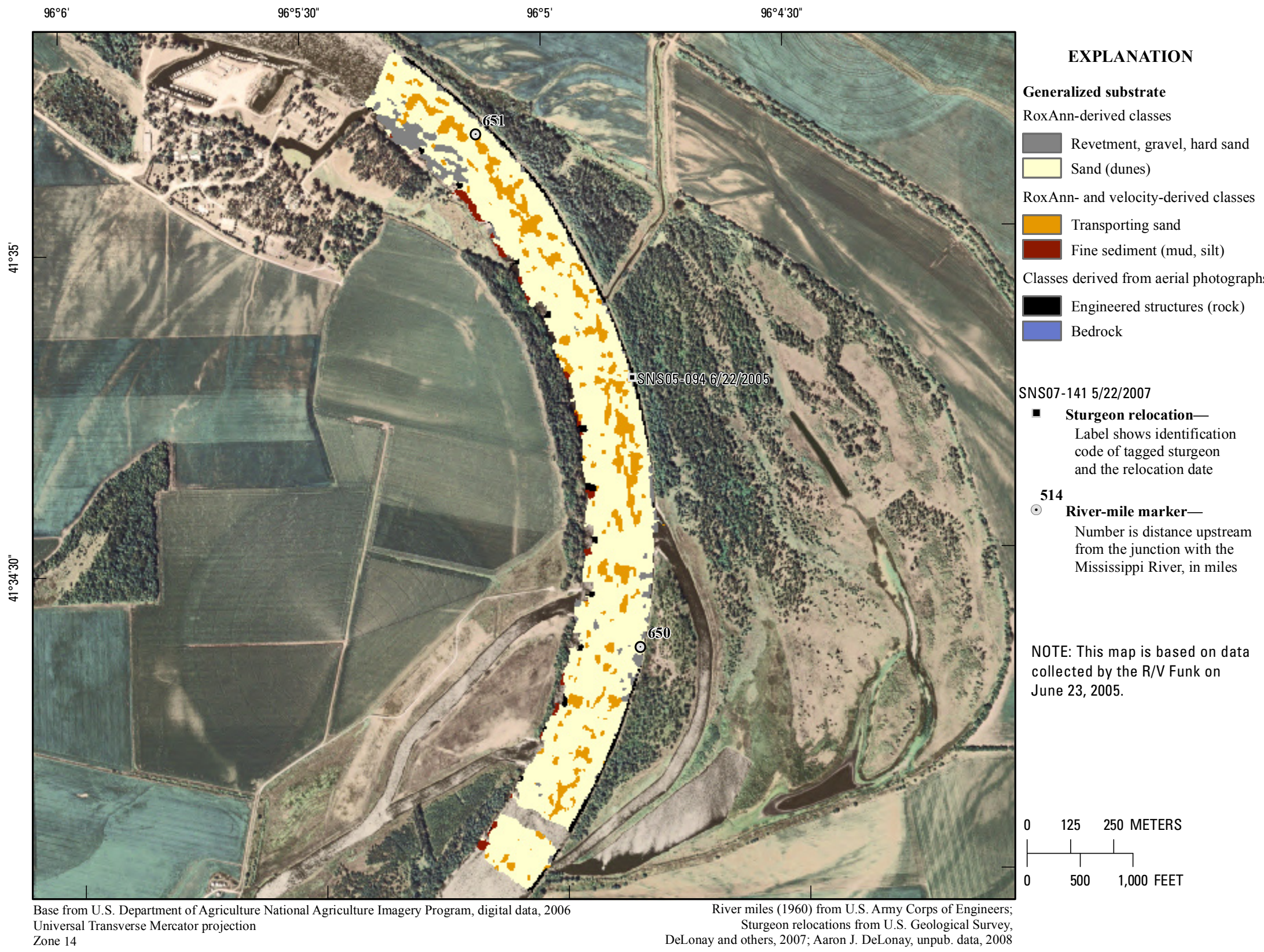

Figure 176. Map of generalized substrate based on data collected on June 23,2005 , in the vicinity of river mile 650 . 


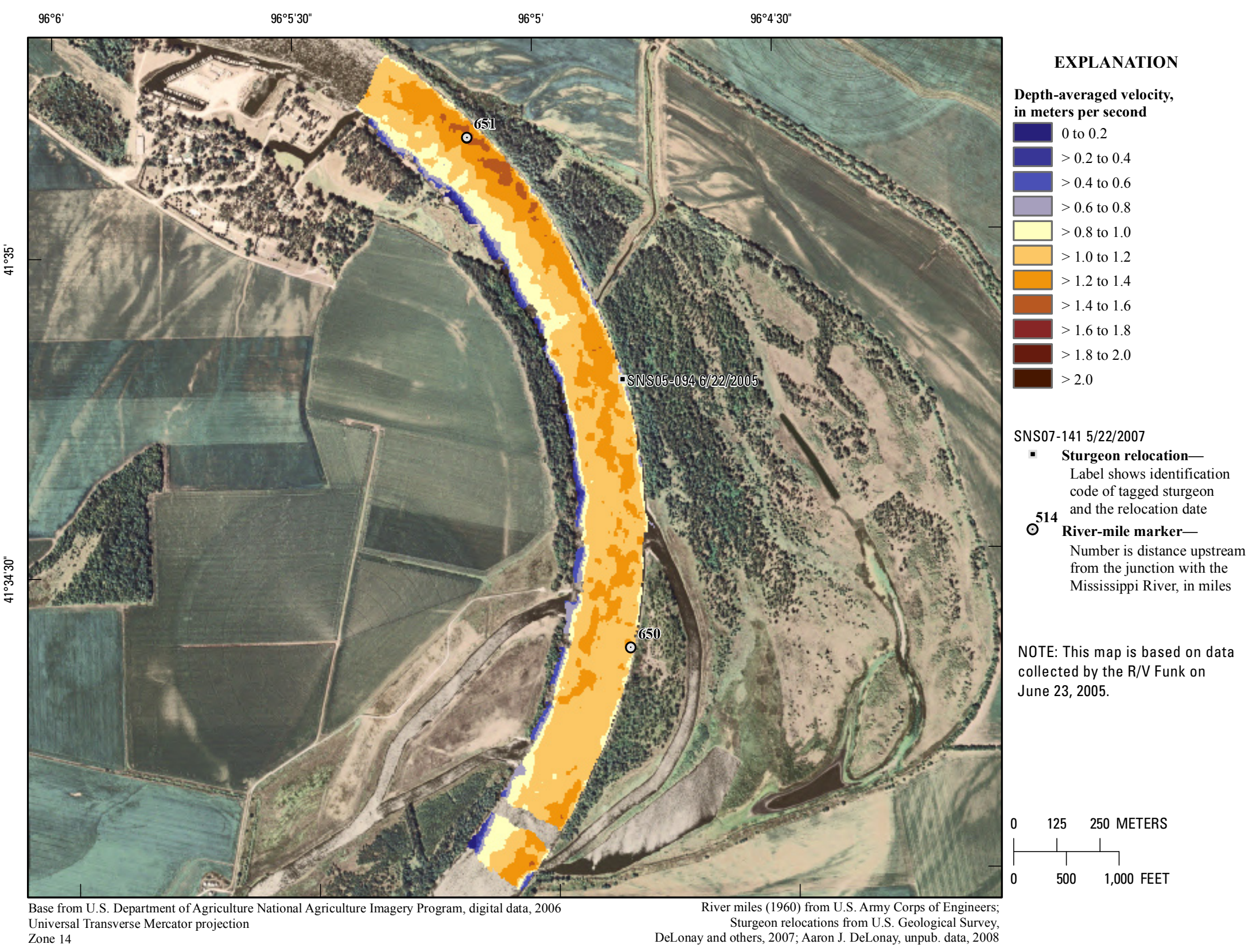

Figure 177. Map of depth-averaged velocity based on data collected on June 23, 2005, in the vicinity of river mile 650 . 


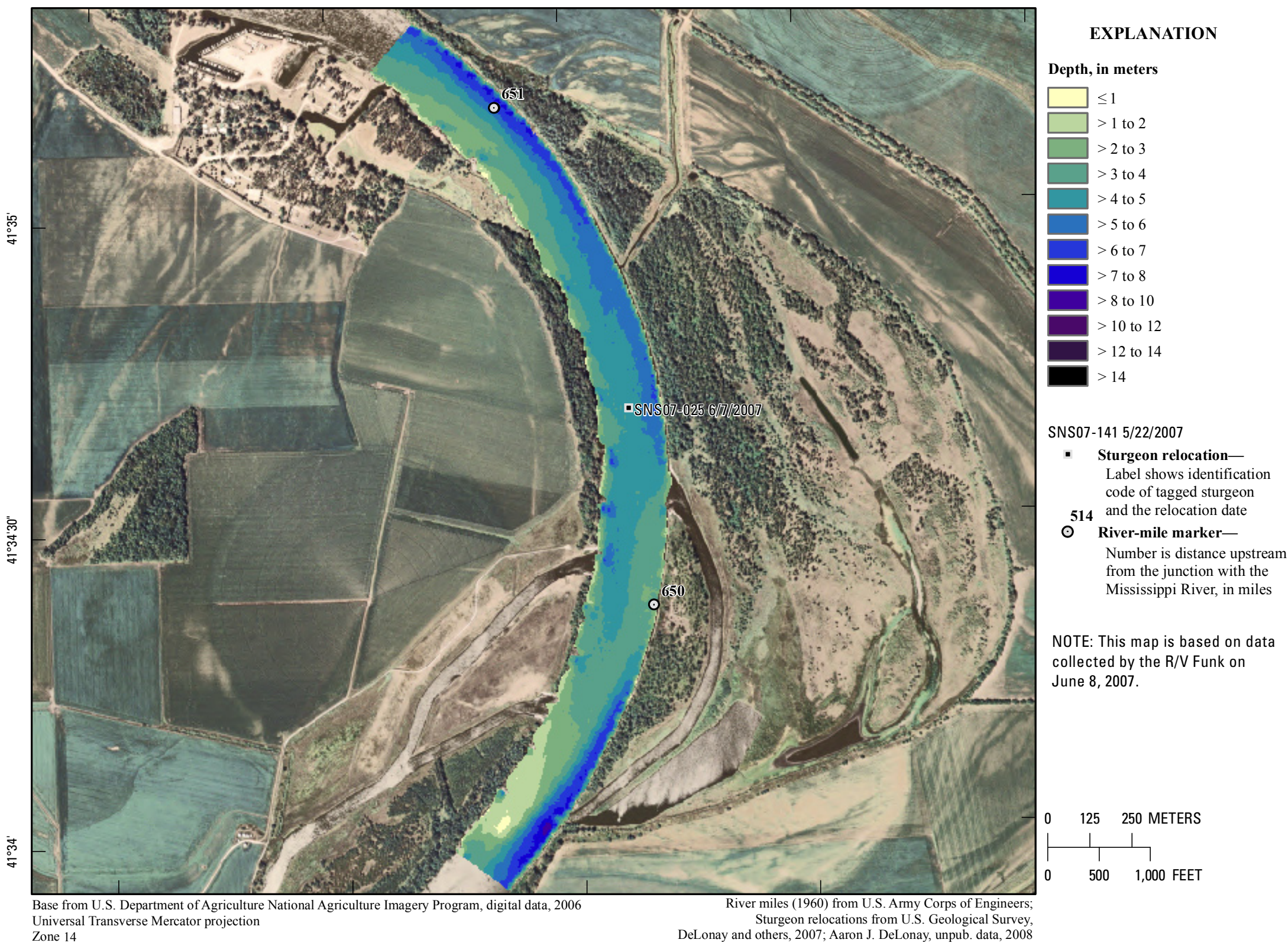
Universal Transverse Mercator projection DeLonay and others, 2007; Aaron J. DeLonay, unpub. data, 2008 


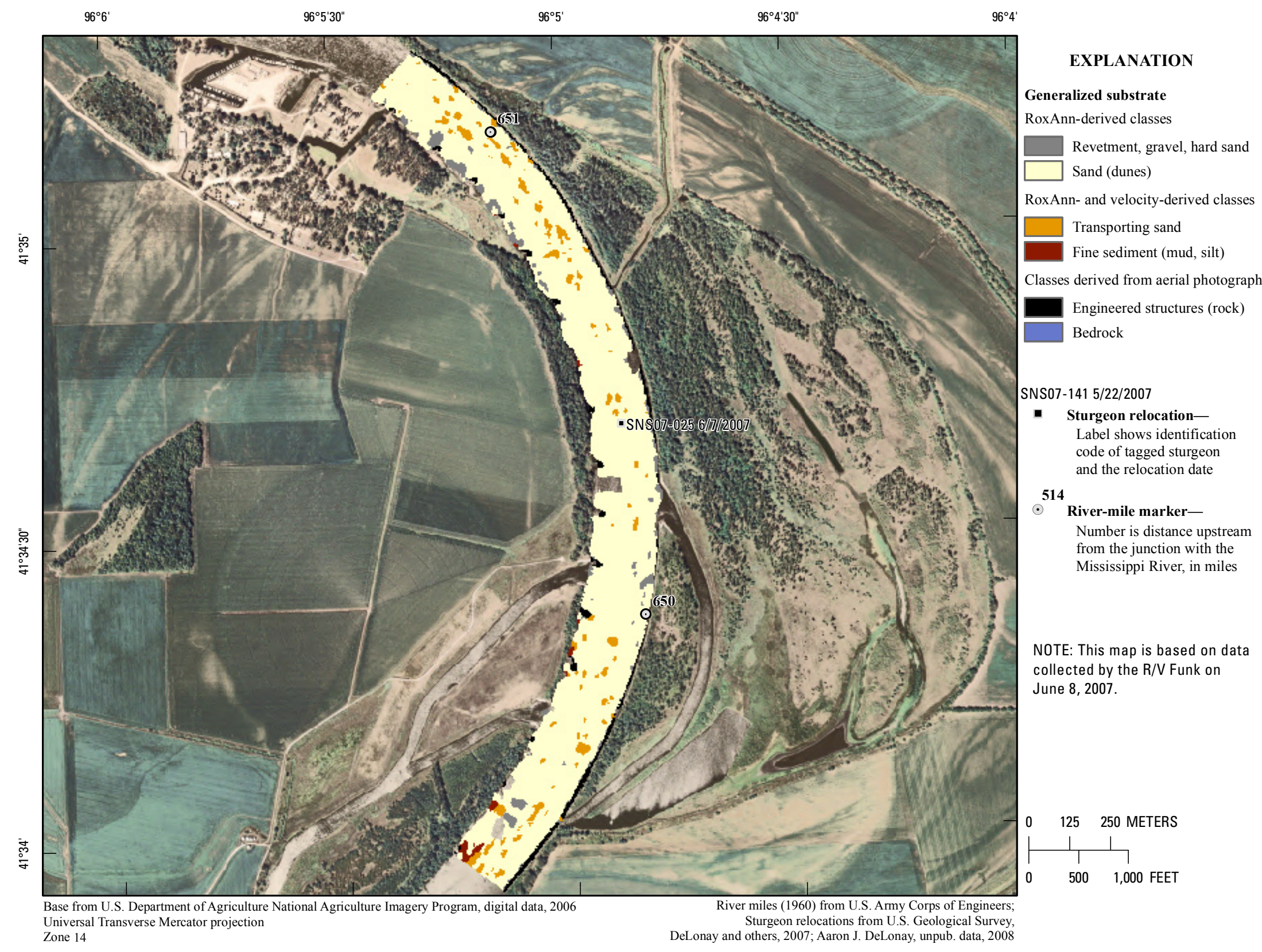

Figure 179. Map of generalized substrate based on data collected on June 8, 2007, in the vicinity of river mile 650 . 


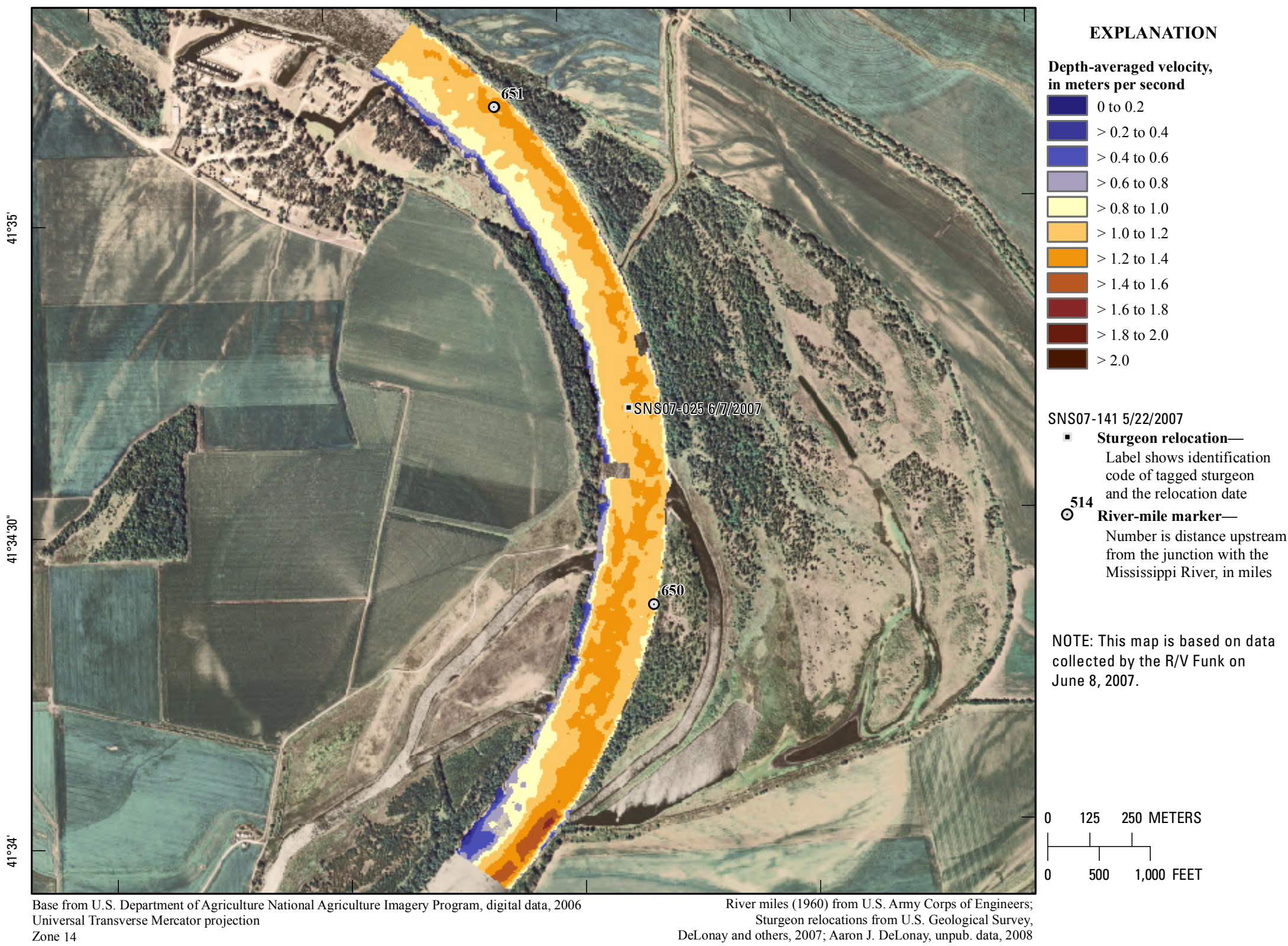




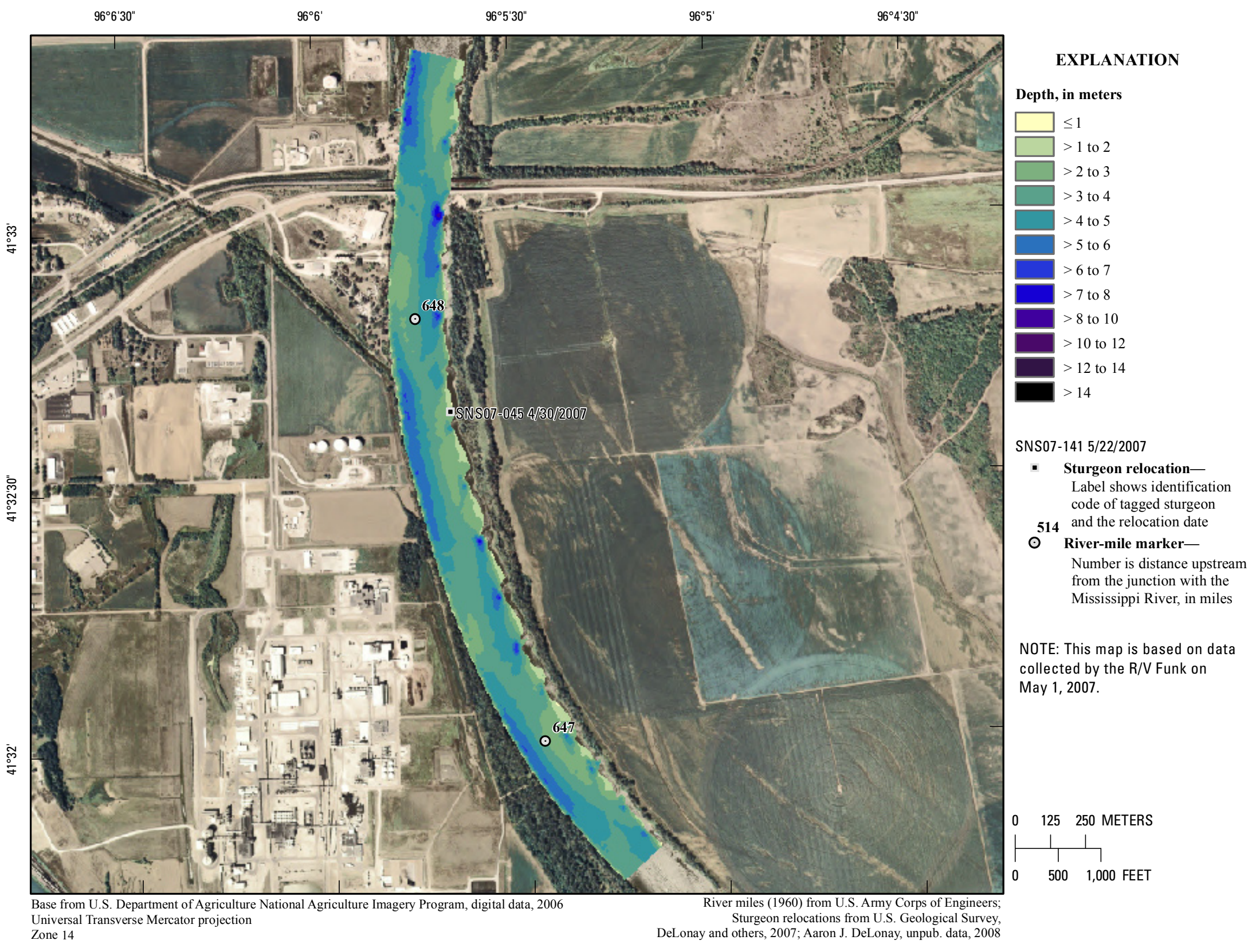

Figure 181. Map of depth based on data collected on May 1, 2007, in the vicinity of river mile 648 . 


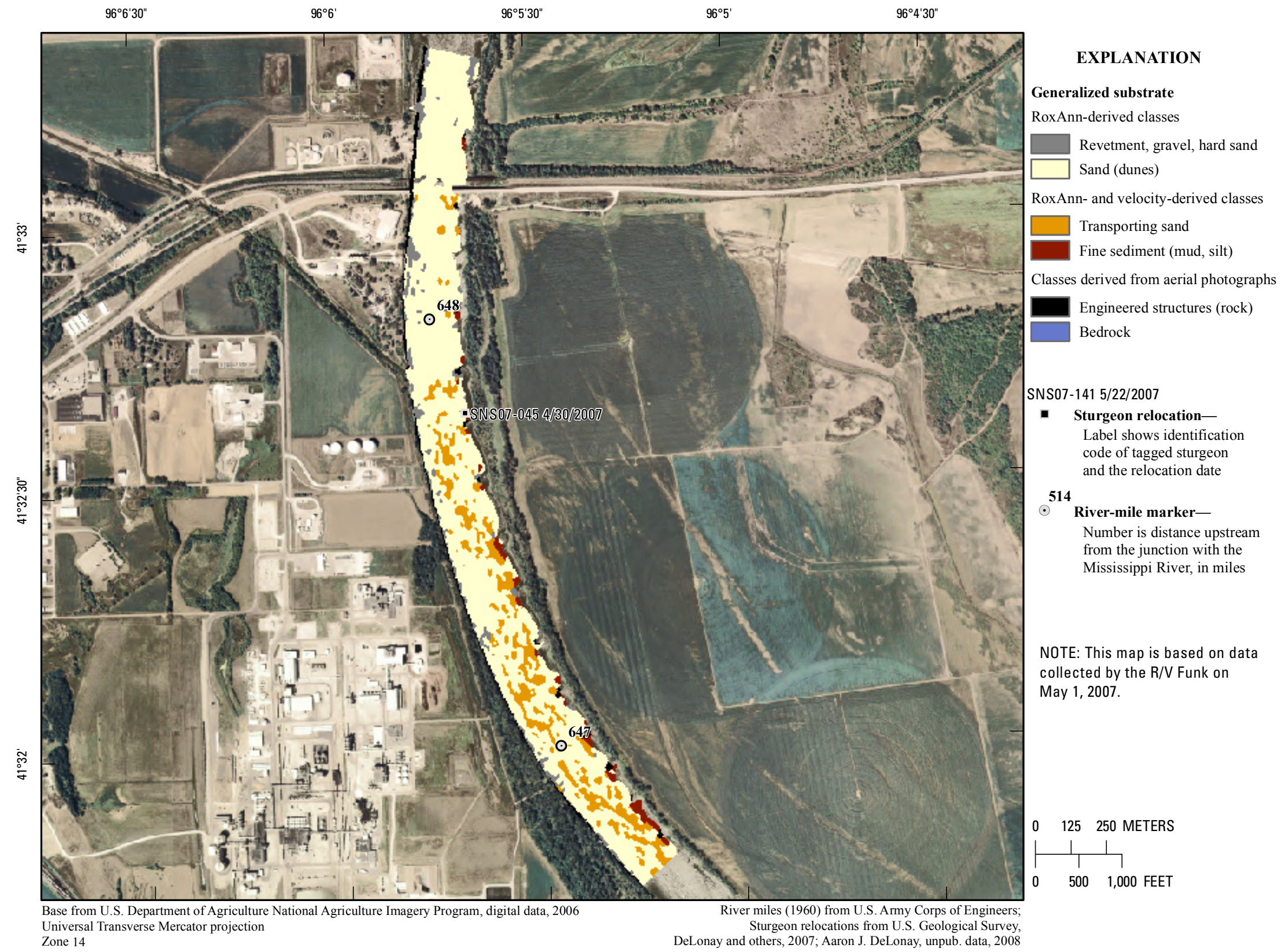

Figure 182. Map of generalized substrate based on data collected on May 1, 2007, in the vicinity of river mile 648 . 


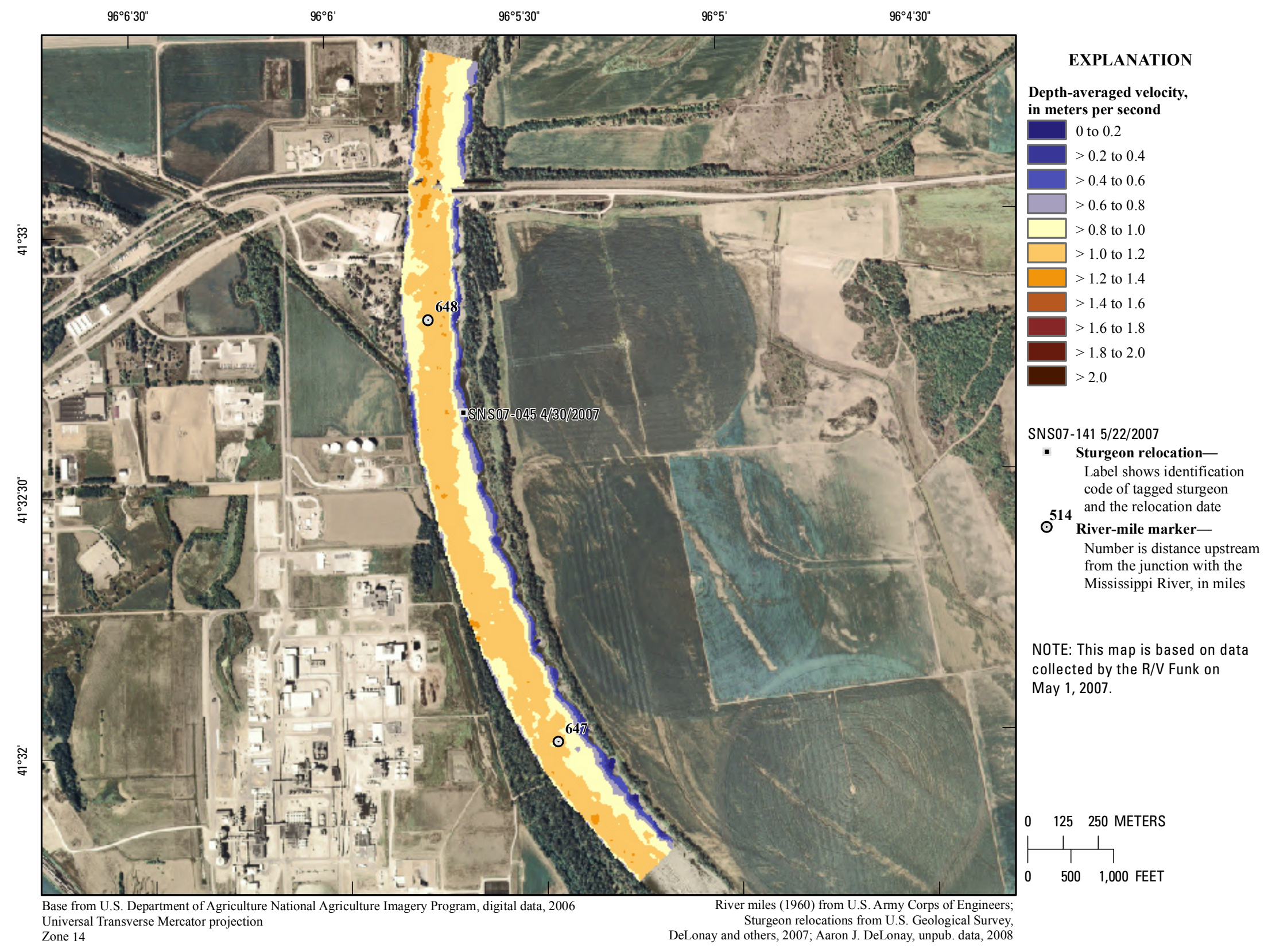

Figure 183. Map of depth-averaged velocity based on data collected on May 1, 2007, in the vicinity of river mile 648 . 


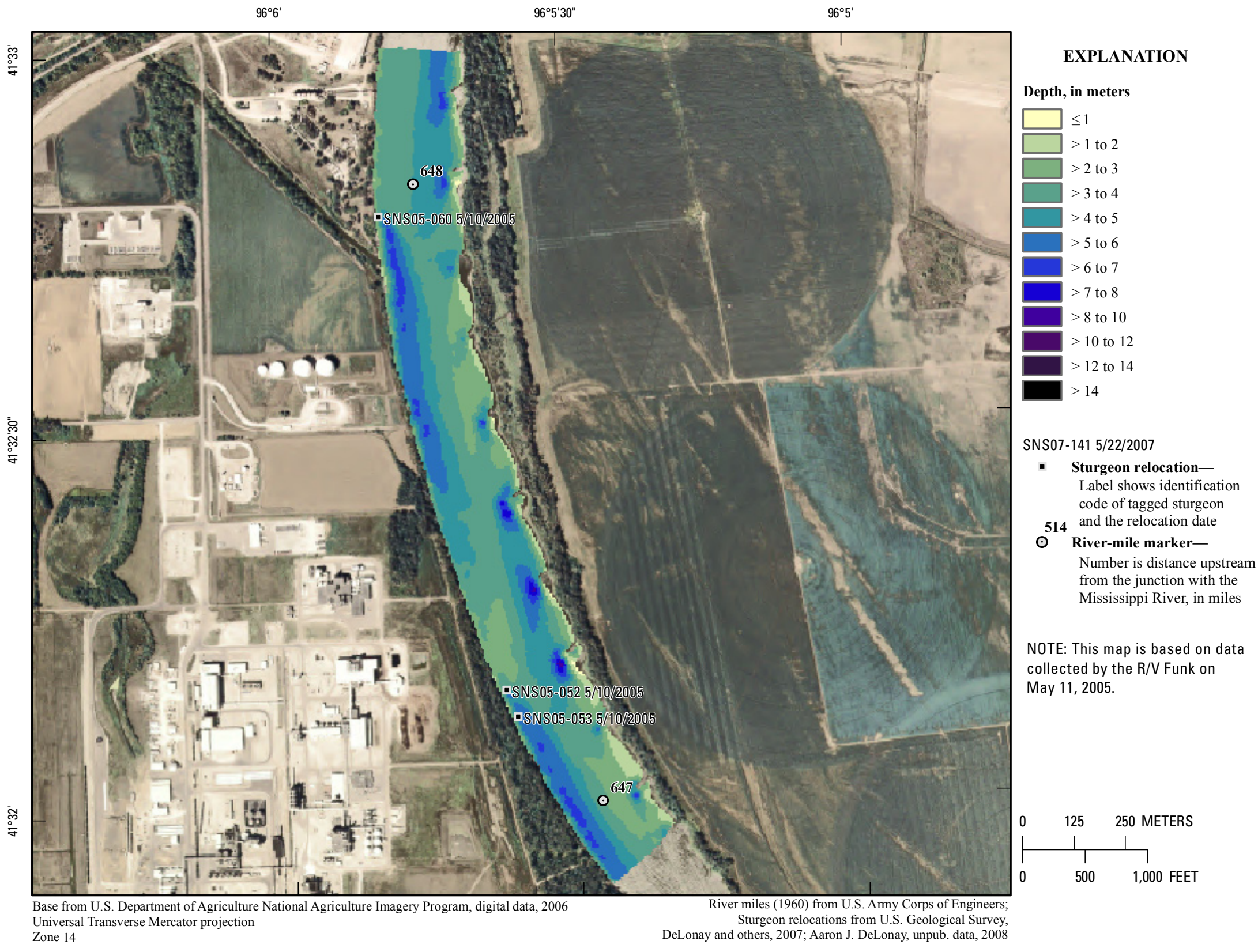

Figure 184. Map of depth based on data collected on May 11, 2005, in the vicinity of river mile 648. 


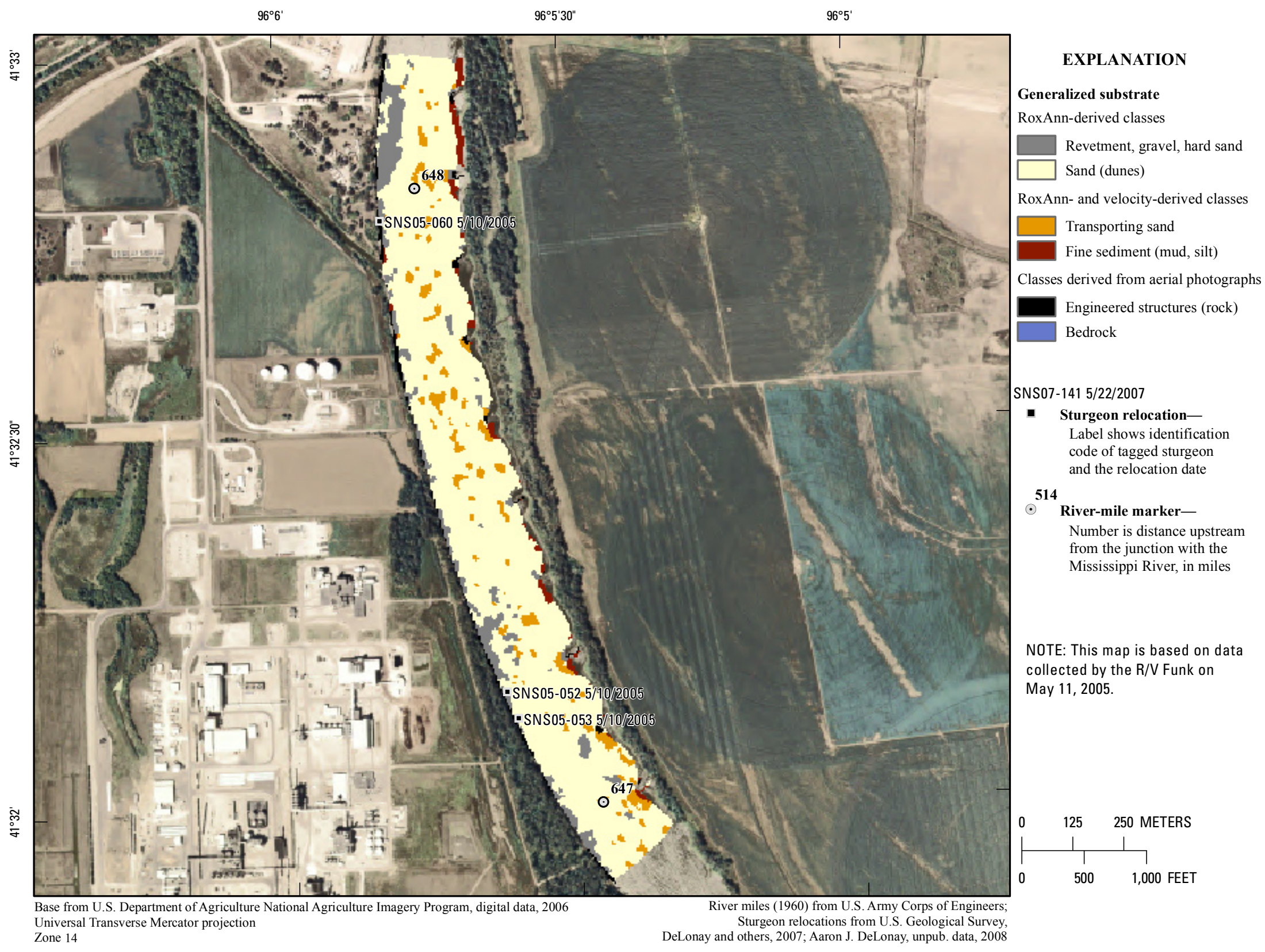

Figure 185. Map of generalized substrate based on data collected on May 11, 2005, in the vicinity of river mile 648 . 


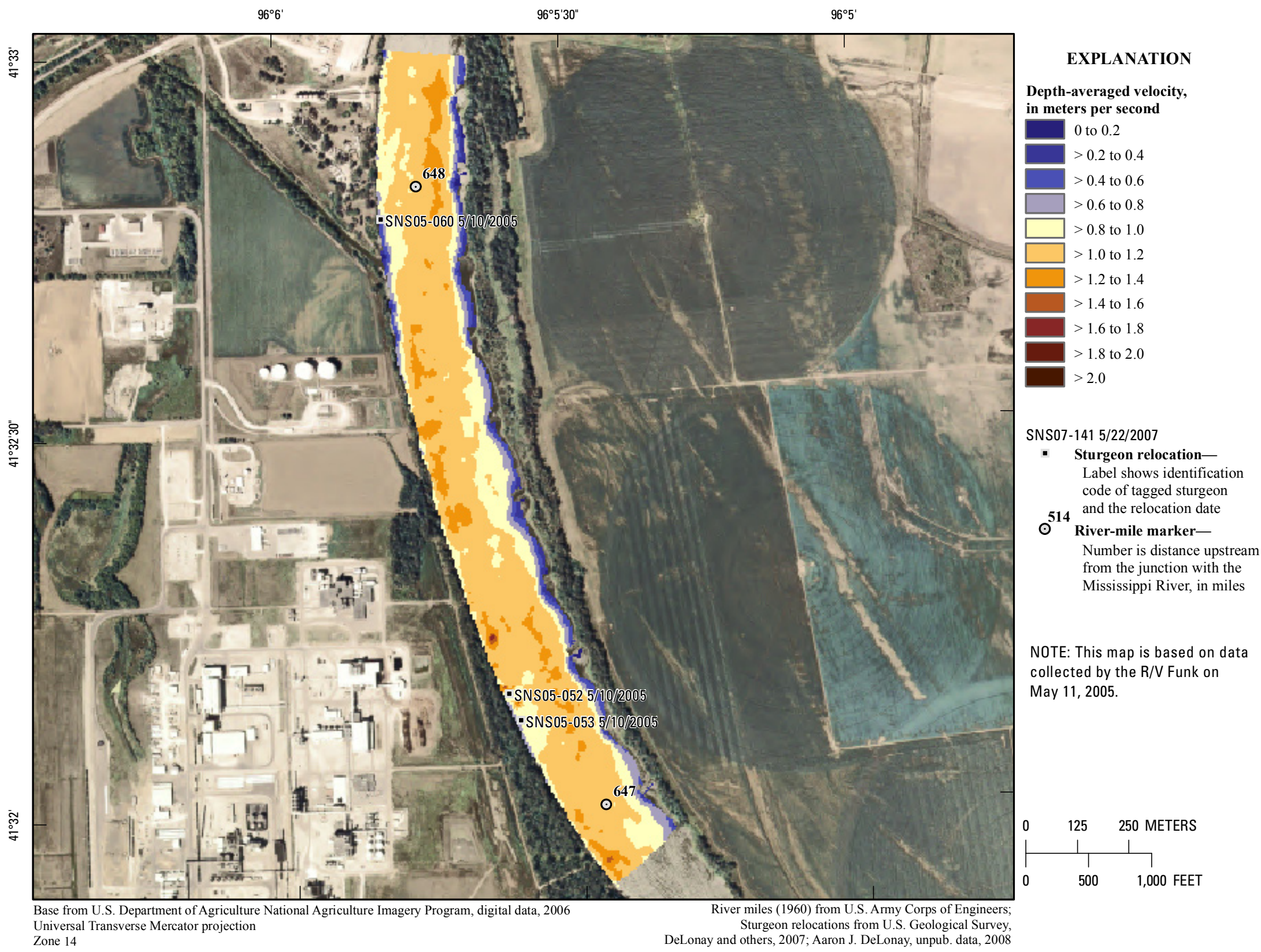

Figure 186. Map of depth-averaged velocity based on data collected on May 11, 2005, in the vicinity of river mile 648 . 


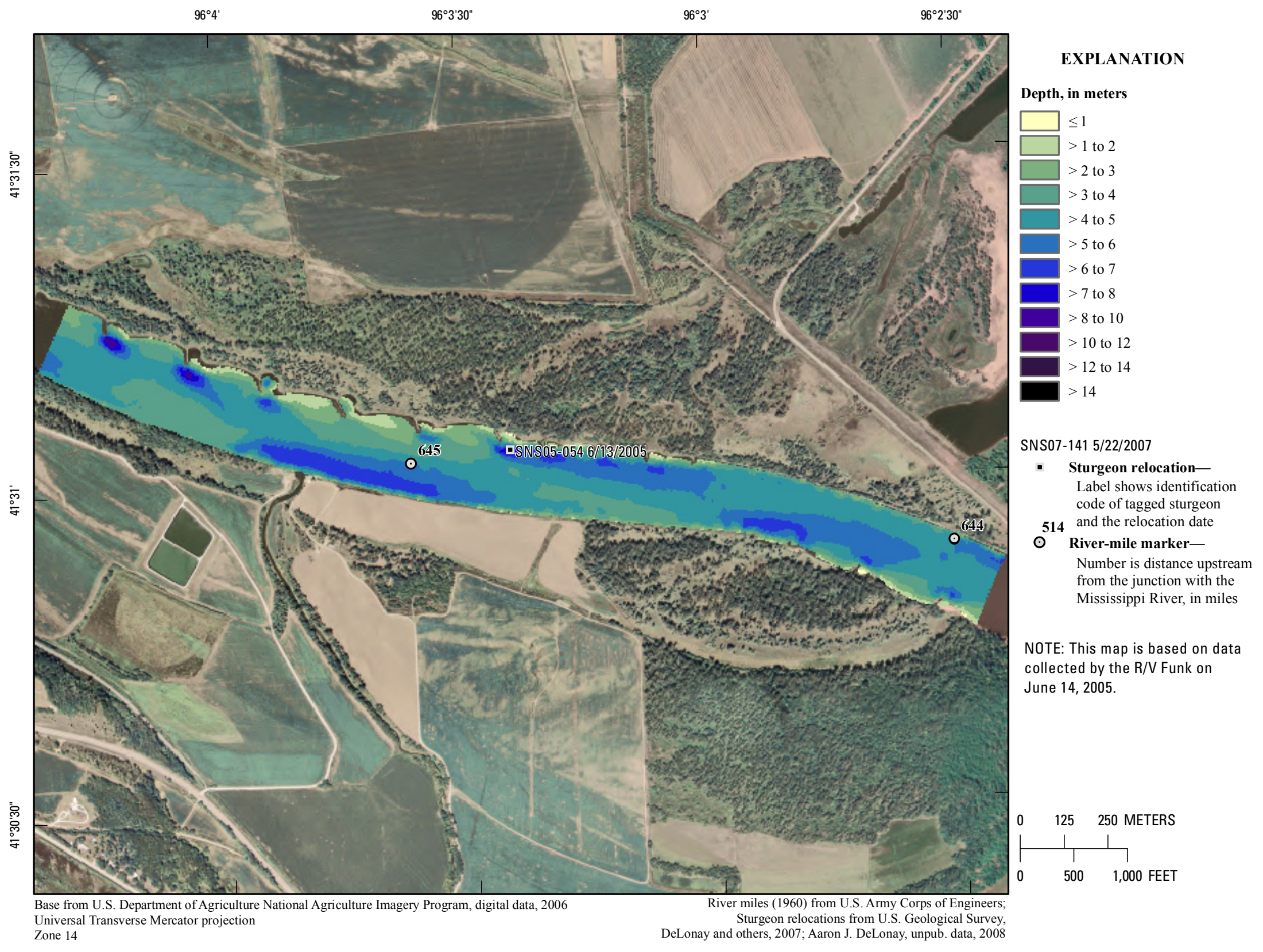

Figure 187. Map of depth based on data collected on June 14, 2005, in the vicinity of river mile 645 . 


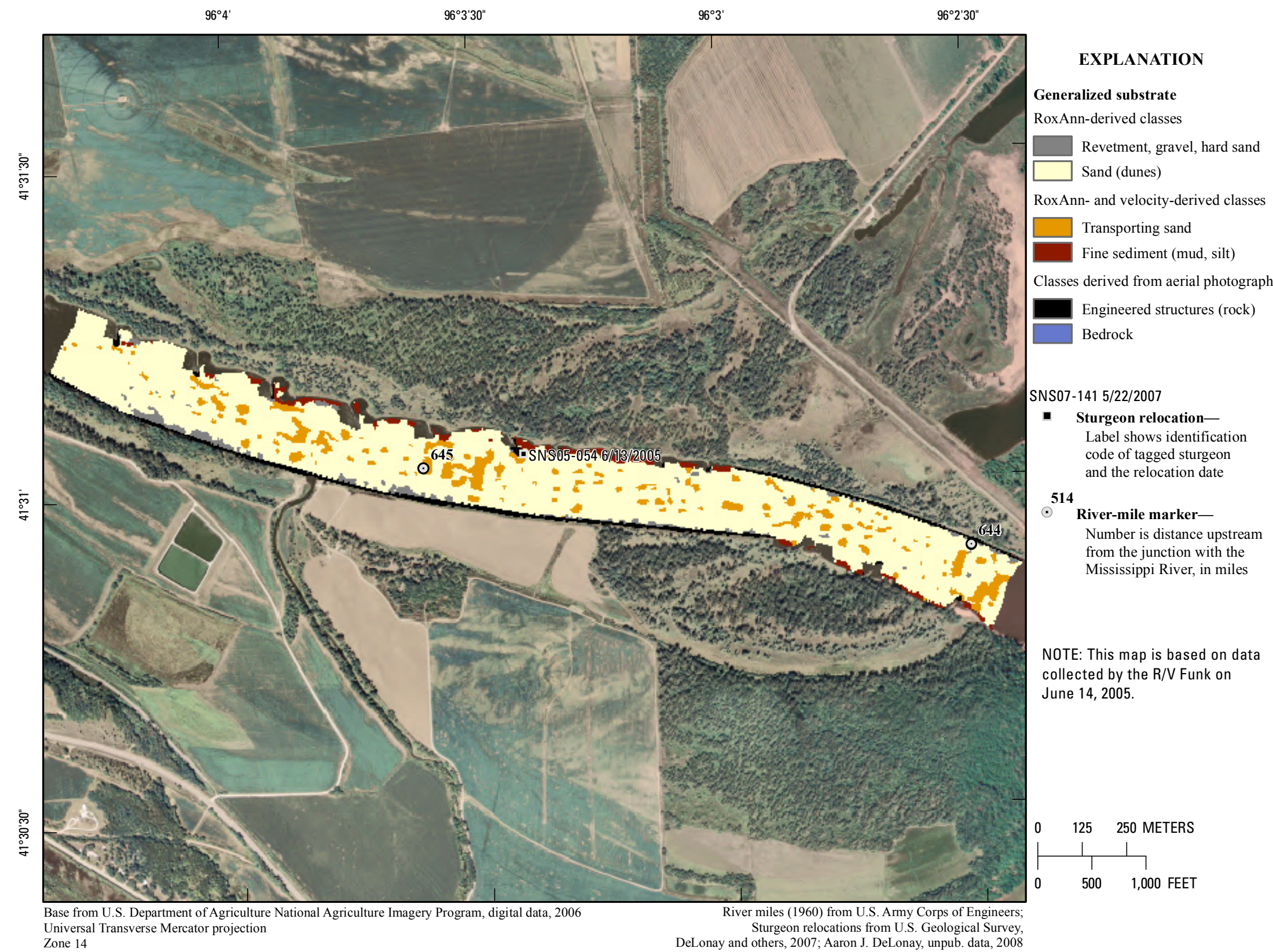

Figure 188. Map of generalized substrate based on data collected on June 14, 2005, in the vicinity of river mile 645 . 


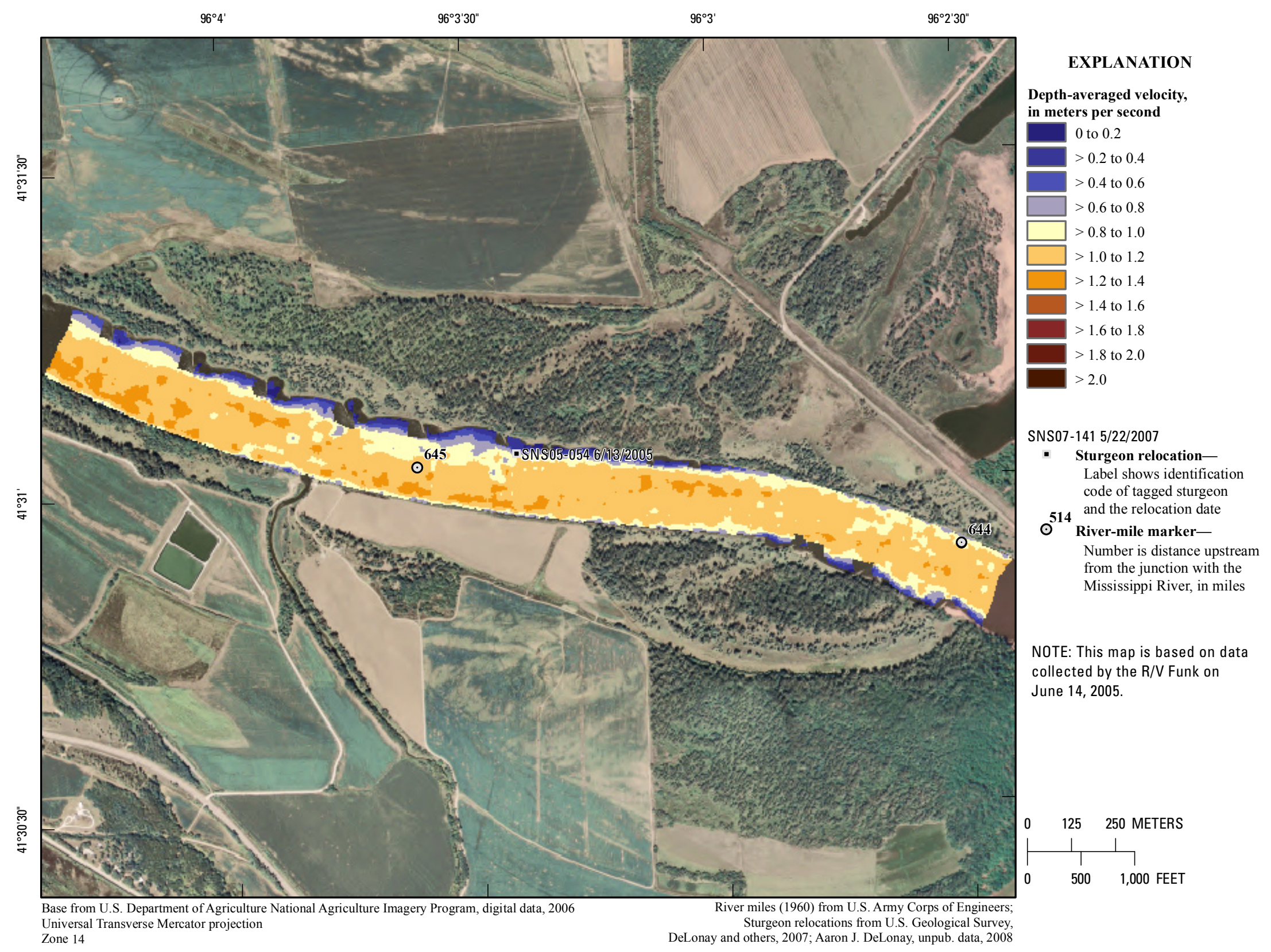

Figure 189. Map of depth-averaged velocity based on data collected on June 14, 2005, in the vicinity of river mile 645 . 


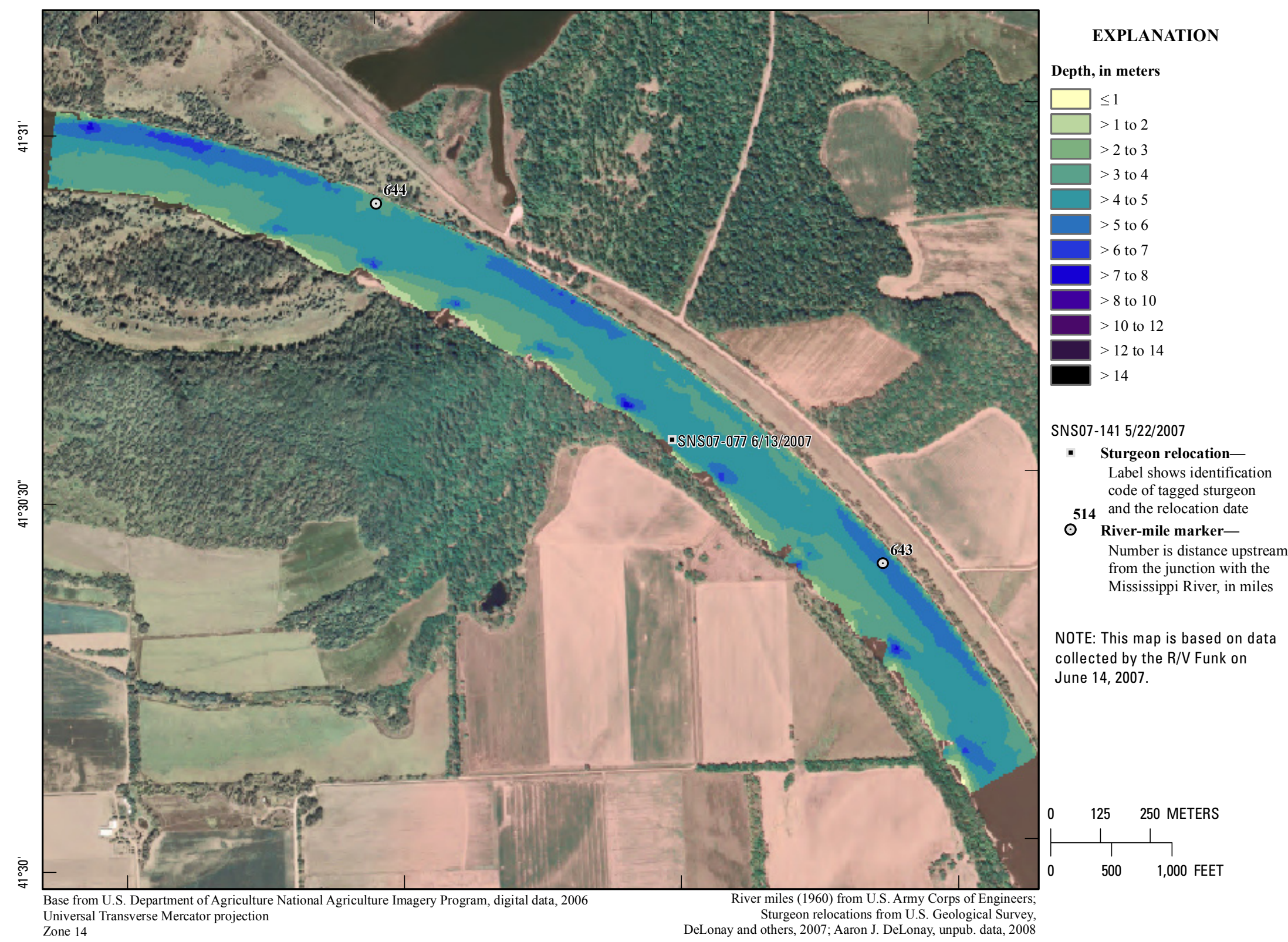
Universal Transverse Mercator projection
Zone 14 Sturgeon relocations from U.S. Geological Survey,
DeLonay and others, 2007; Aaron J. DeLonay, unpub. data, 2008 


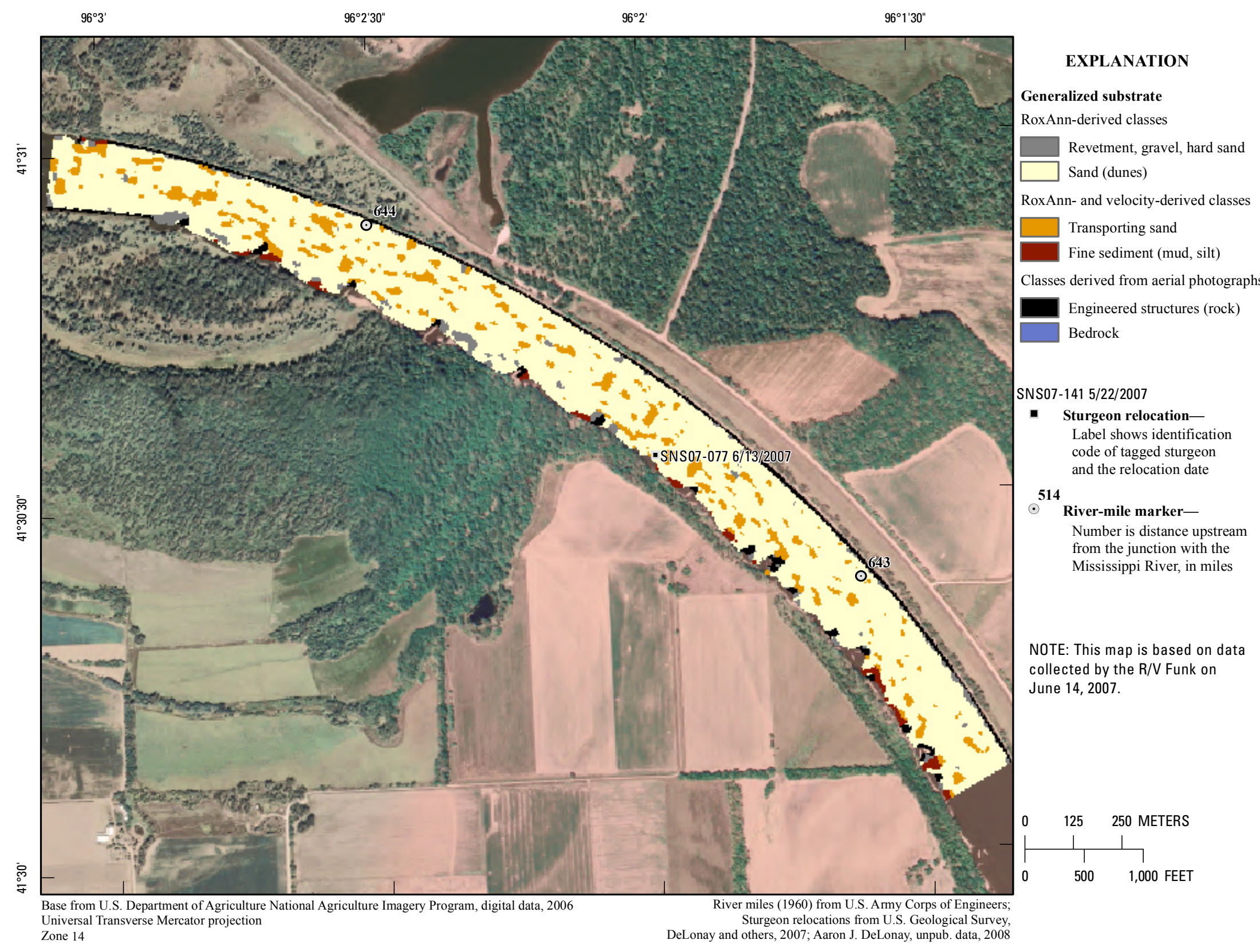

Figure 191. Map of generalized substrate based on data collected on June 14, 2007, in the vicinity of river mile 644 . 


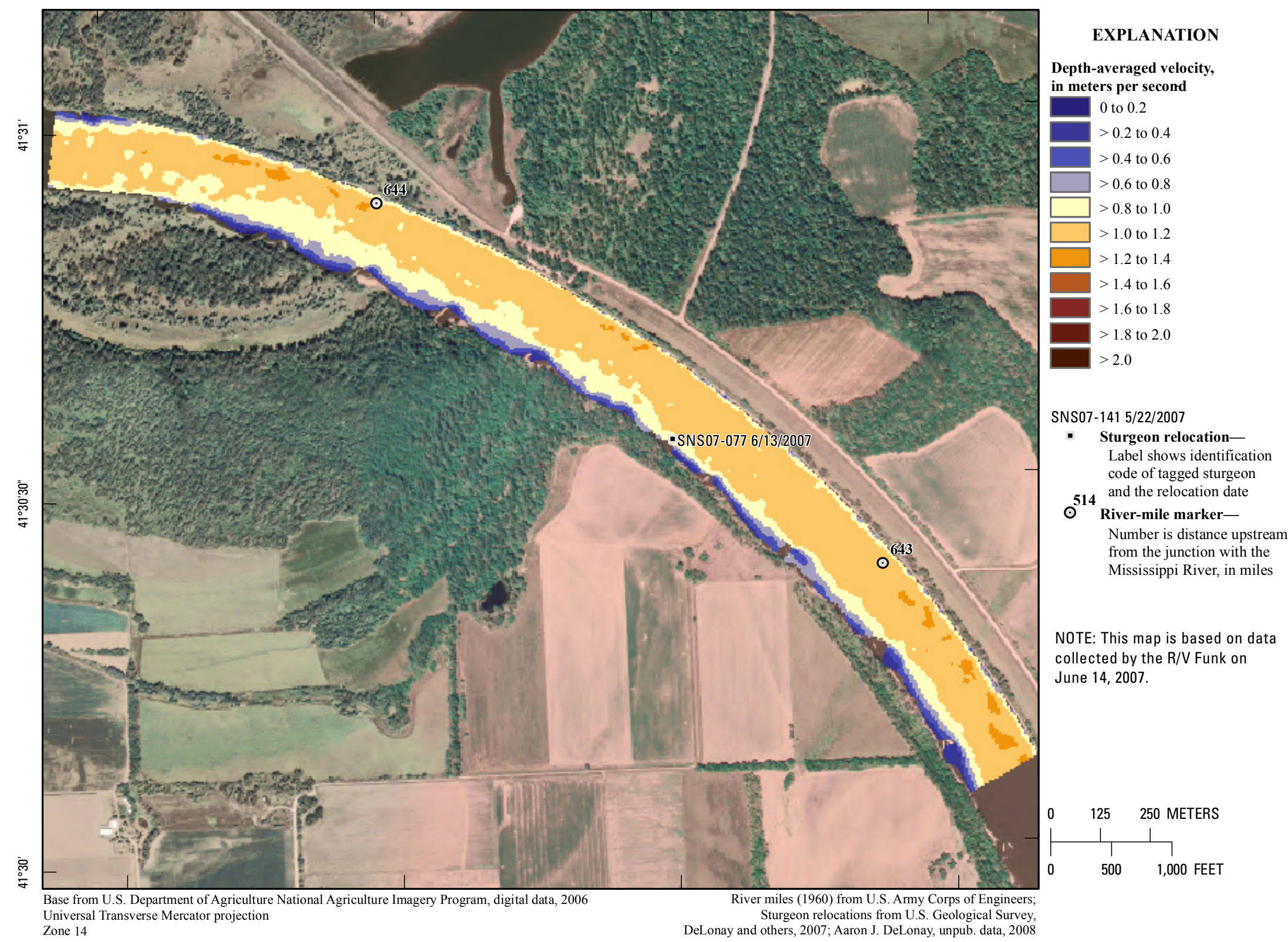
Universal Transverse Mercator projection Sturgeon relocations from U.S. Geological Survey,
DeLonay and others, 2007; Aaron J. DeLonay, unpub. data, 2008 


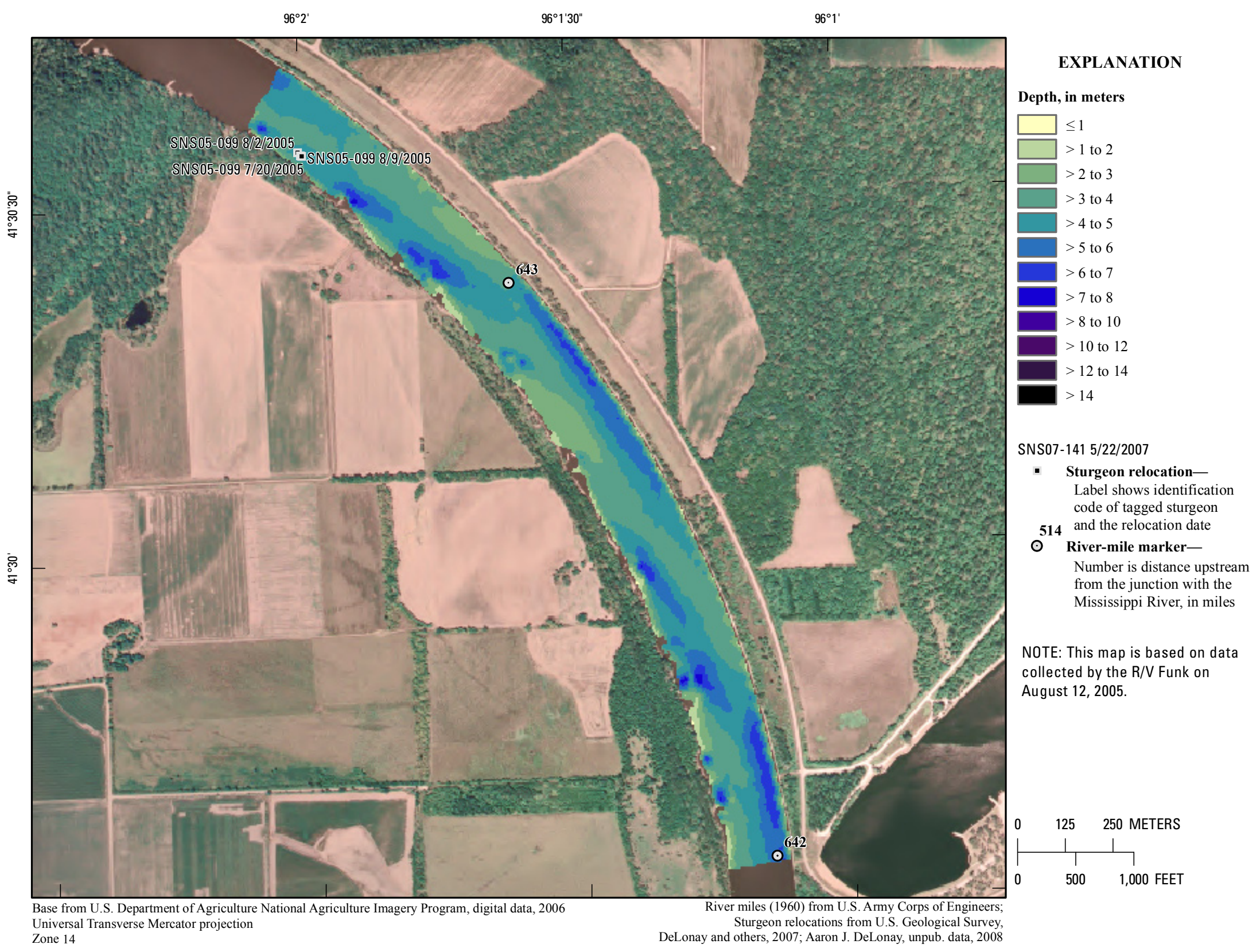

Figure 193. Map of depth based on data collected on August 12, 2005, in the vicinity of river mile 643 . 
$96^{\circ} 2$

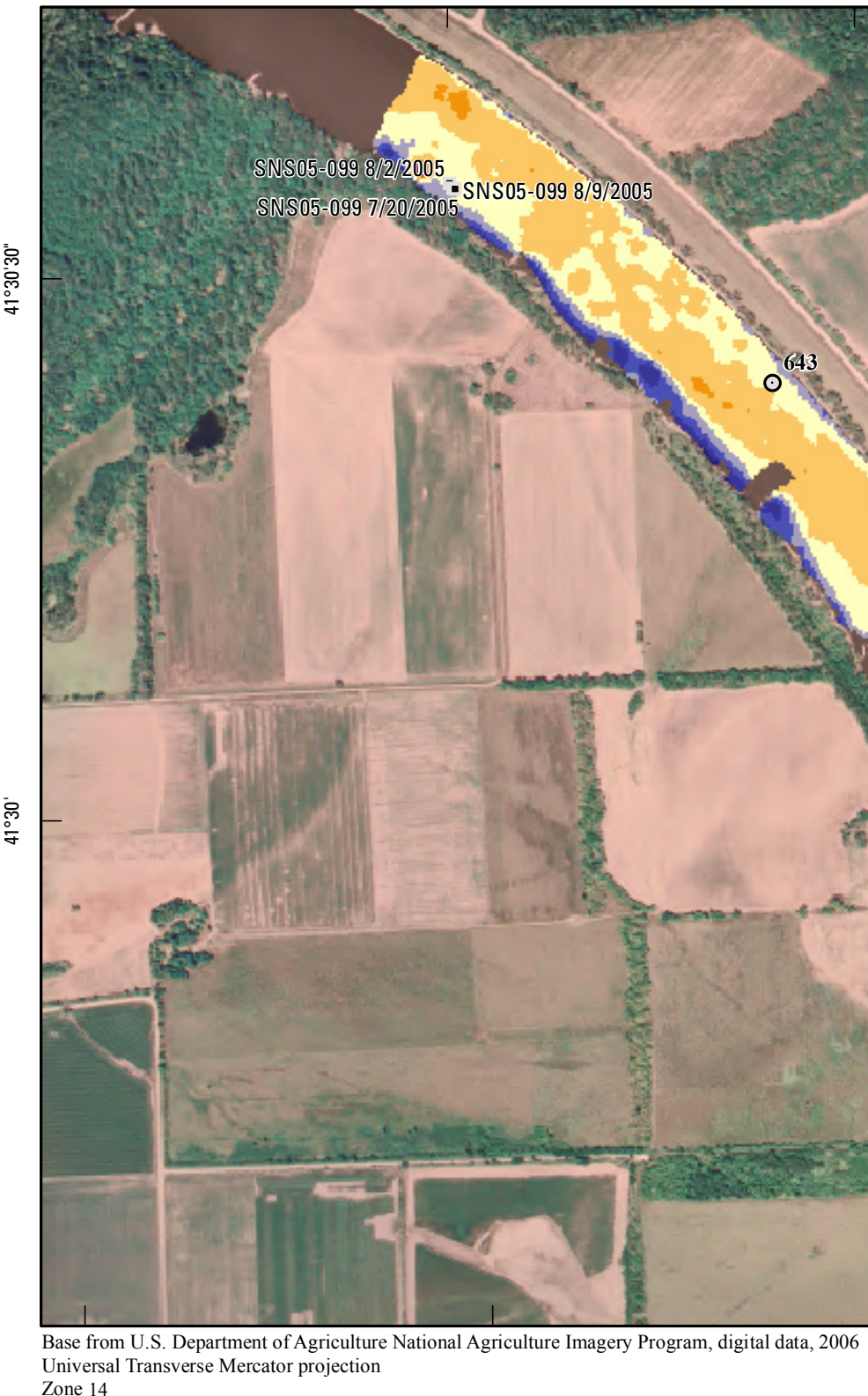

$96^{\circ} 1^{\prime} 30^{\prime \prime}$ $96^{\circ} 1$
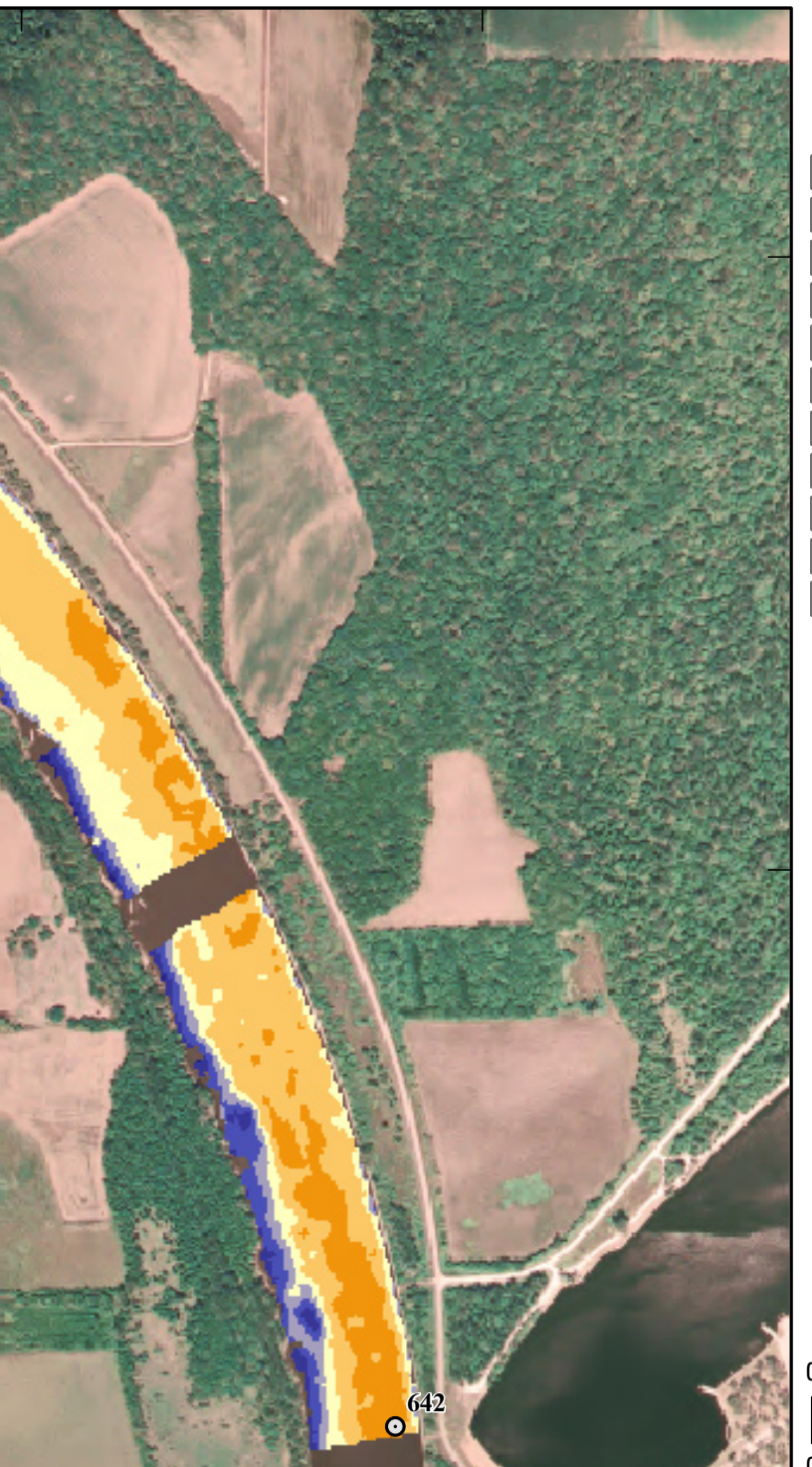
miles (1960) from U.S. Army Corps of Engineers DeLonay and others, 2007; Aaron J. DeLonay, unpub. data, 2008
EXPLANATION

Depth-averaged velocity,

in meters per second

0 to 0.2

$>0.2$ to 0.4

$>0.4$ to 0.6

$>0.6$ to 0.8

$>0.8$ to 1.0

$>1.0$ to 1.2

$>1.2$ to 1.4

$>1.4$ to 1.6

$>1.6$ to 1.8

$>1.8$ to 2.0

$>2.0$

\section{SNS07-141 5/22/2007}

- Sturgeon relocationLabel shows identification code of tagged sturgeo

$\mathbf{5 1 4}$ and the relocation date

Number is distance upstream
from the junction with the Mississippi River, in miles

NOTE: This map is based on data collected by the R/V Funk on August 12, 2005.

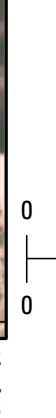

\section{METERS

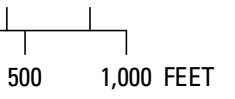

Figure 194. Map of depth-averaged velocity based on data collected on August 12, 2005, in the vicinity of river mile 643. 


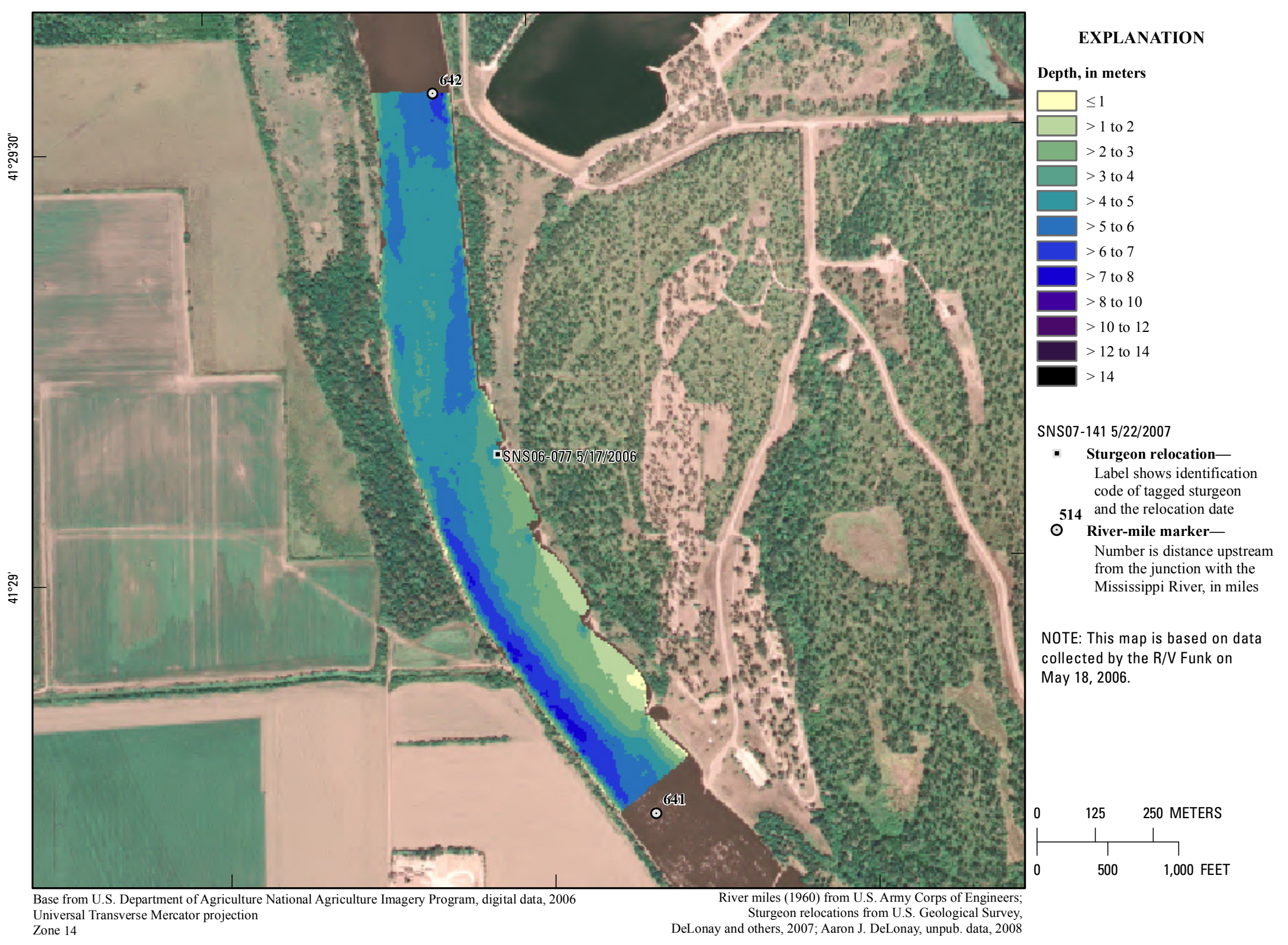

Figure 195. Map of depth based on data collected on May 18, 2006, in the vicinity of river mile 642. 


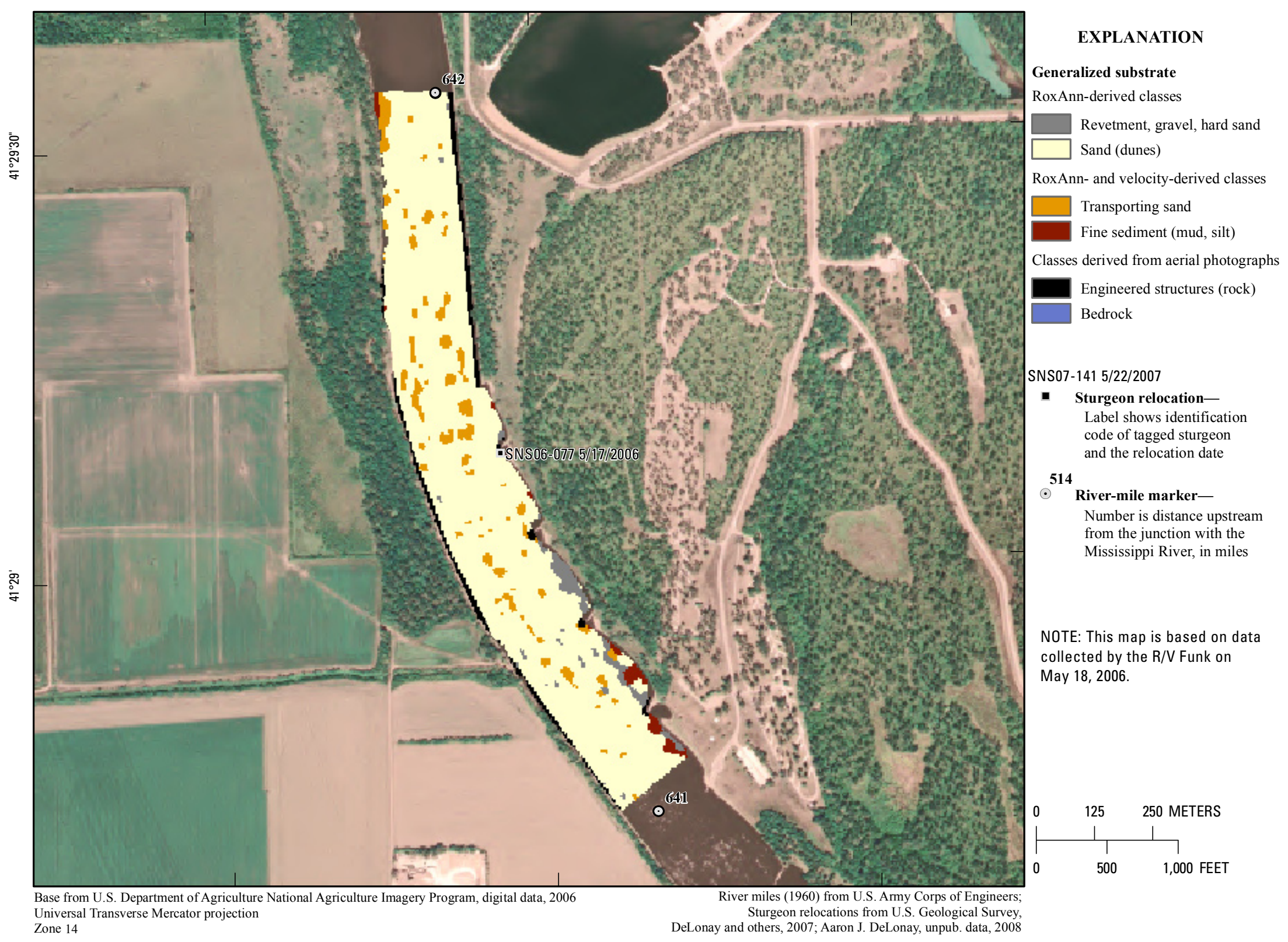




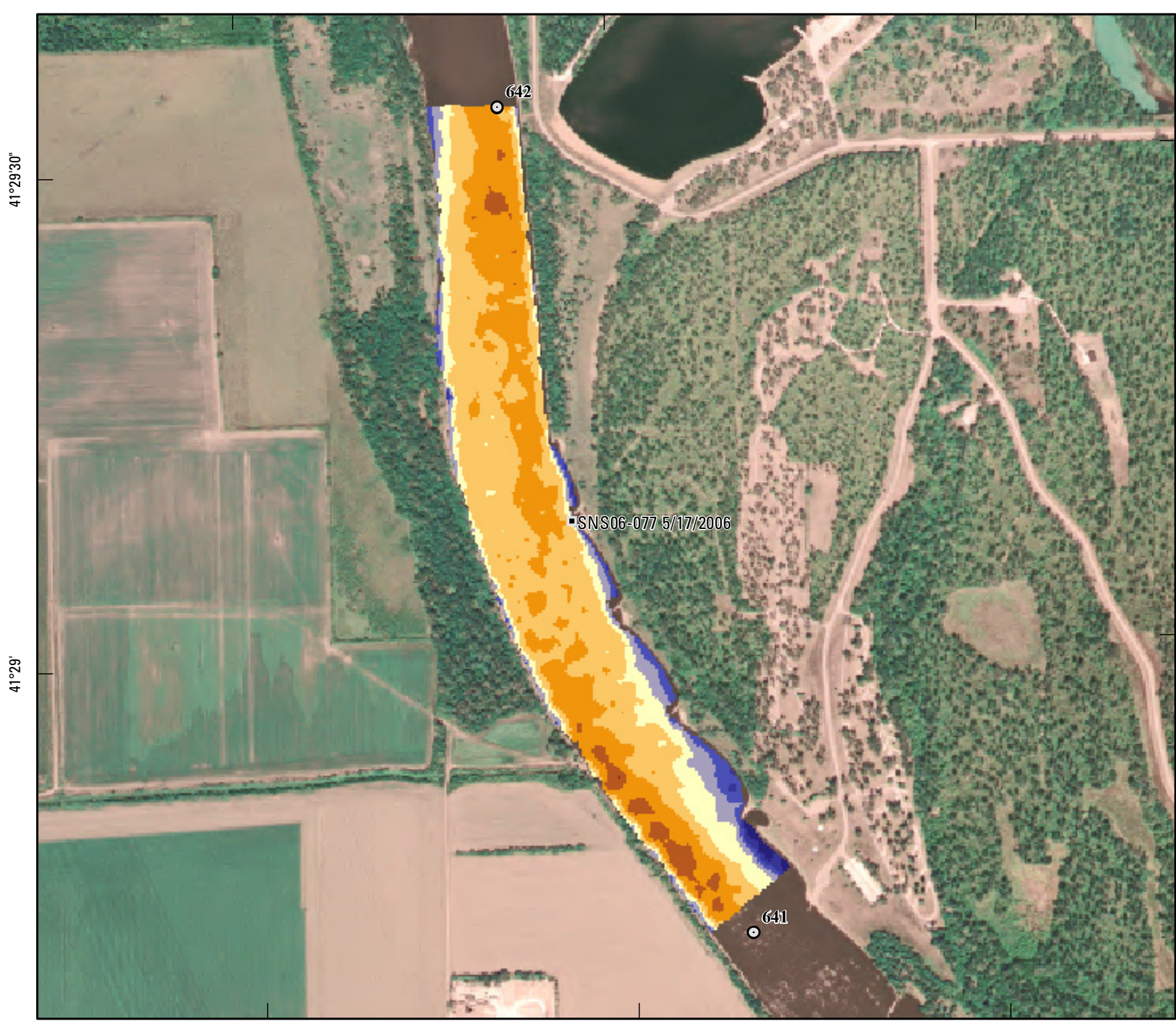

EXPLANATION

Depth-averaged velocity,

in meters per second

0 to 0.2

$>0.2$ to 0.4

$>0.4$ to 0.6

$>0.6$ to 0.8

$>0.8$ to 1.0

$>1.0$ to 1.2

$>1.2$ to 1.4

$>1.4$ to 1.6

$>1.6$ to 1.8

$>1.8$ to 2.0

$>2.0$

\section{SNS07-141 5/22/2007}

- Sturgeon relocation-

Label shows identification

code of tagged sturgeon

514 and the relocation date

${ }^{514}$ River-mile marker-

Number is distance upstream from the junction with the Mississippi River, in mile

NOTE: This map is based on data collected by the R/V Funk on May 18, 2006.

Base from U.S. Department of Agriculture National Agriculture Imagery Program, digital data, 2006 Universal Transverse Mercator projection

River miles (1960) from U.S. Army Corps of Engineers Sturgeon relocations from U.S. Geological Survey,
DeLonay and others, 2007; Aaron J. DeLonay, unpub. data, 2008

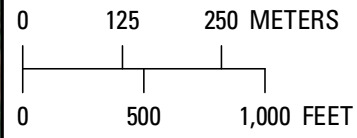

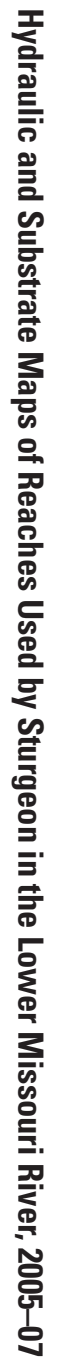

Figure 197. Map of depth-averaged velocity based on data collected on May 18, 2006, in the vicinity of river mile 642 . 


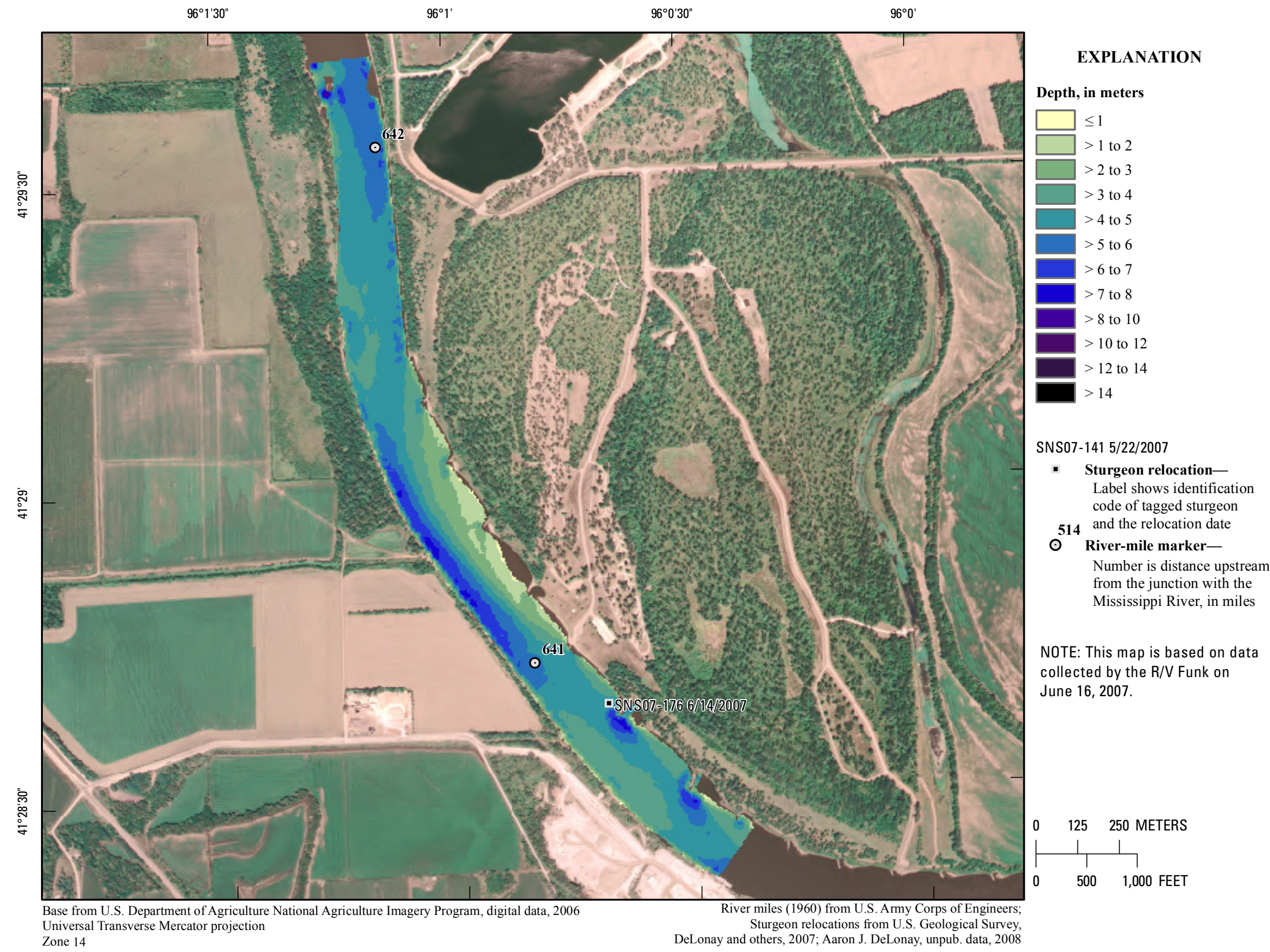

Figure 198. Map of depth based on data collected on June 16, 2007, in the vicinity of river mile 641 . 


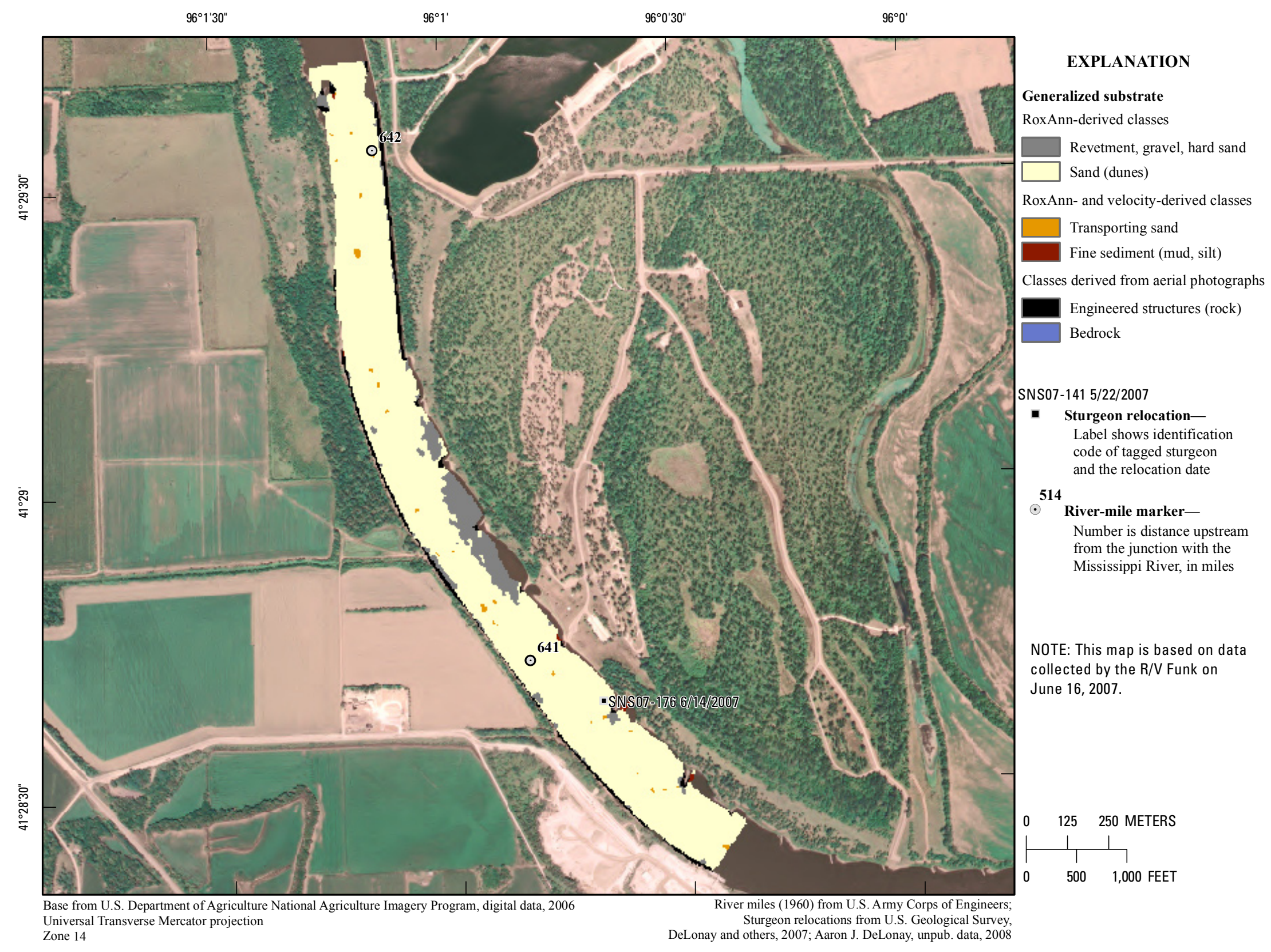

Figure 199. Map of generalized substrate based on data collected on June 16, 2007, in the vicinity of river mile 641 . 


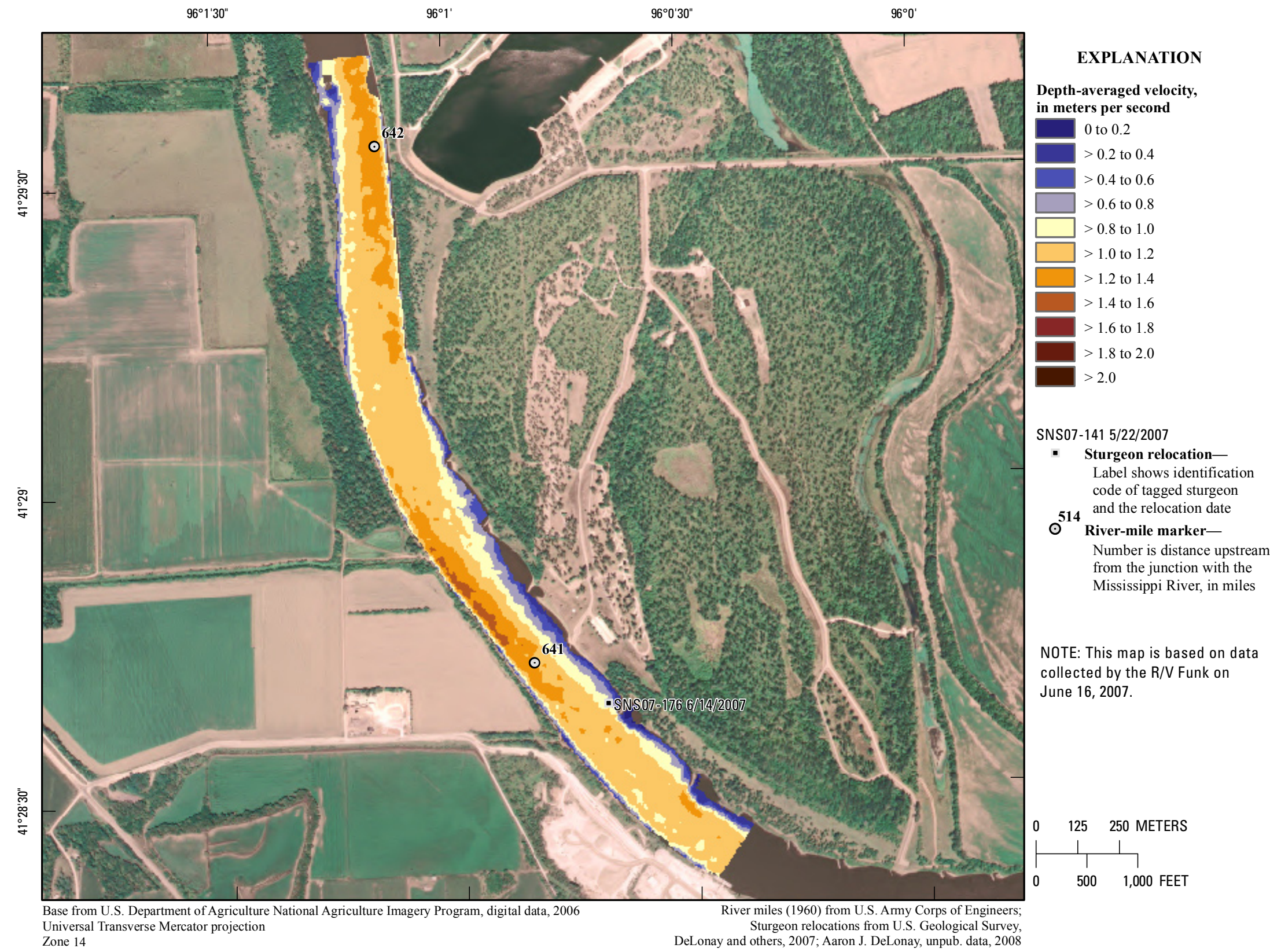

Figure 200. Map of depth-averaged velocity based on data collected on June 16, 2007, in the vicinity of river mile 641 . 


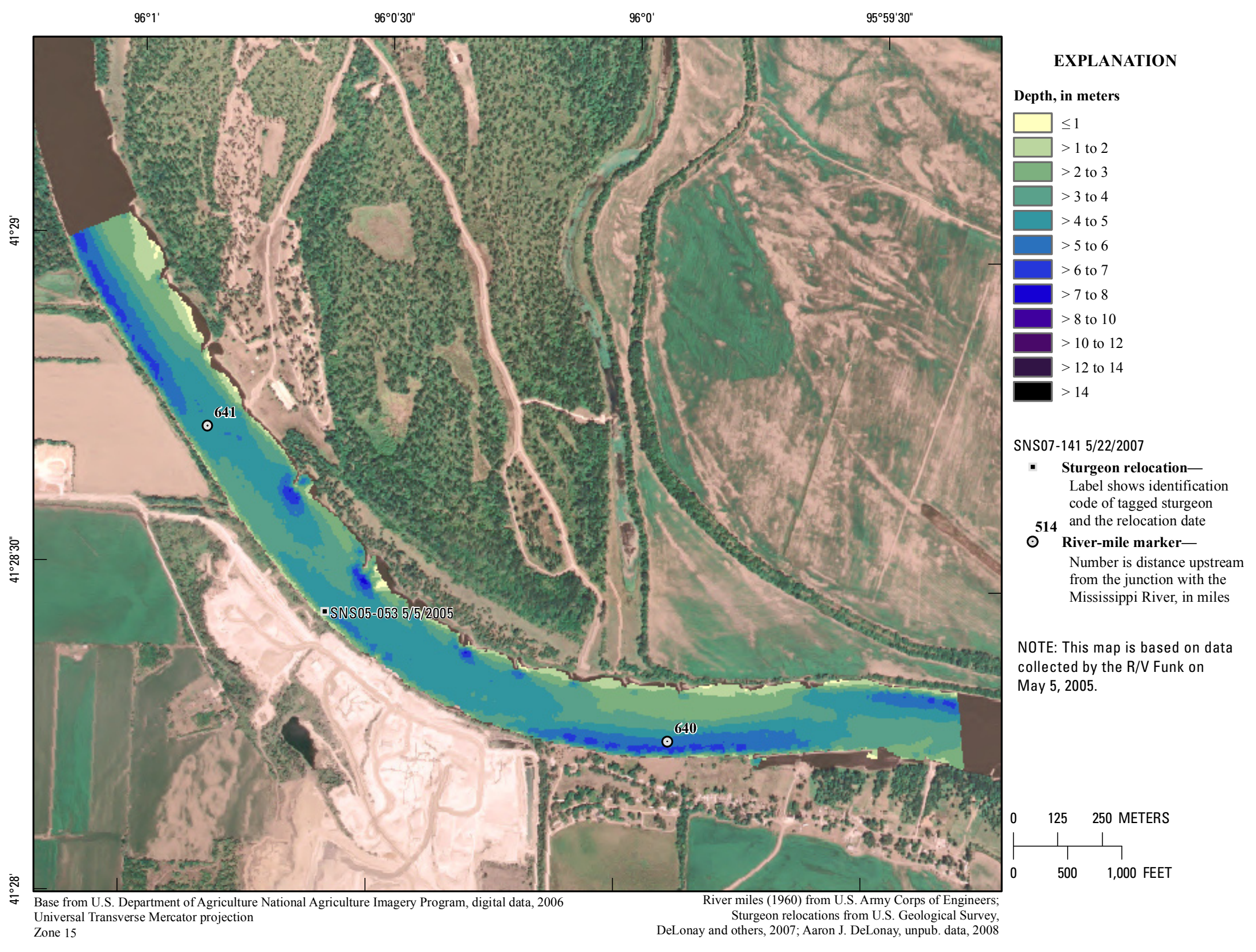

Figure 201. Map of depth based on data collected on May 5, 2005, in the vicinity of river mile 641 . 


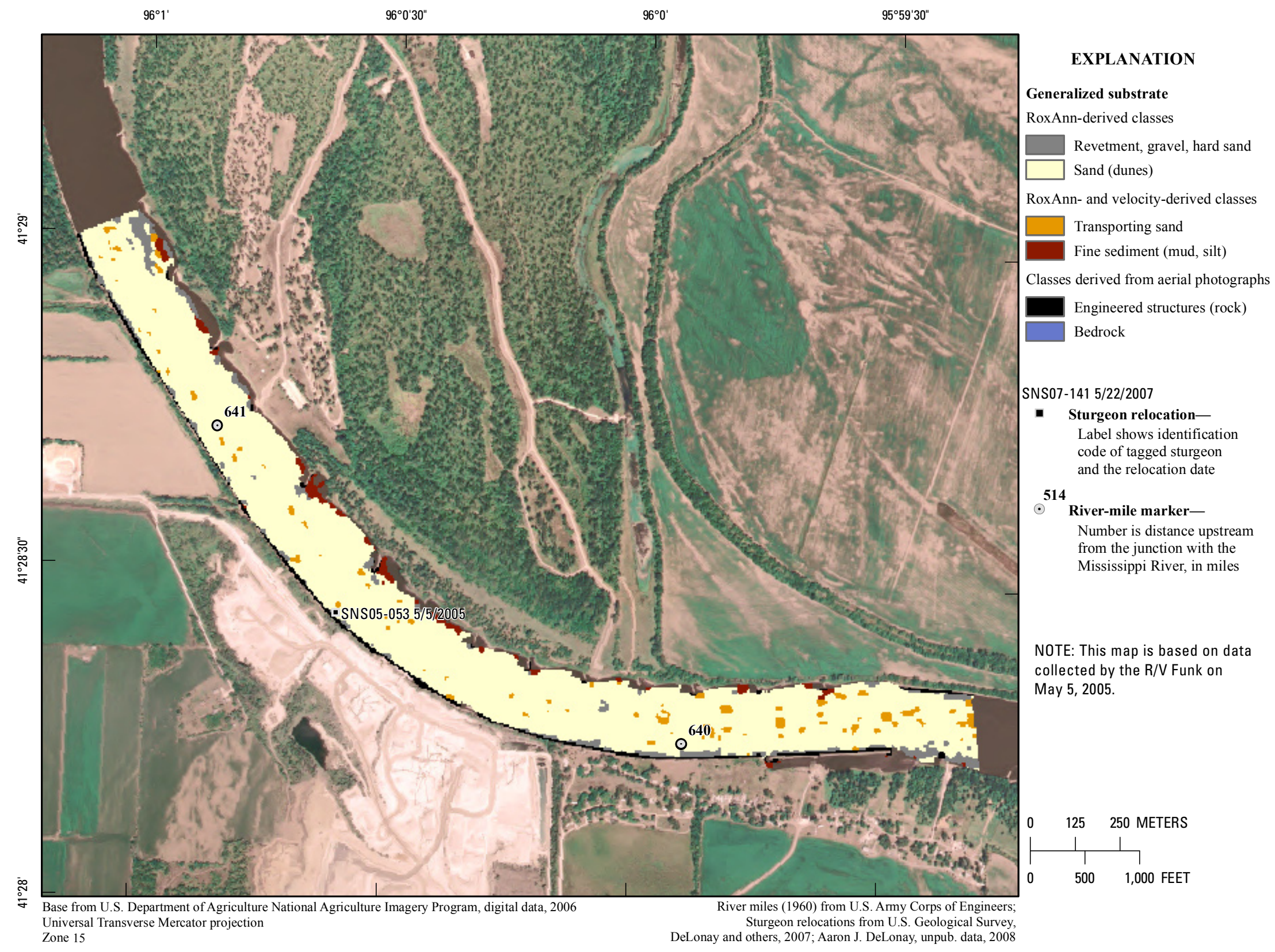

Figure 202. Map of generalized substrate based on data collected on May 5, 2005, in the vicinity of river mile 641 . 


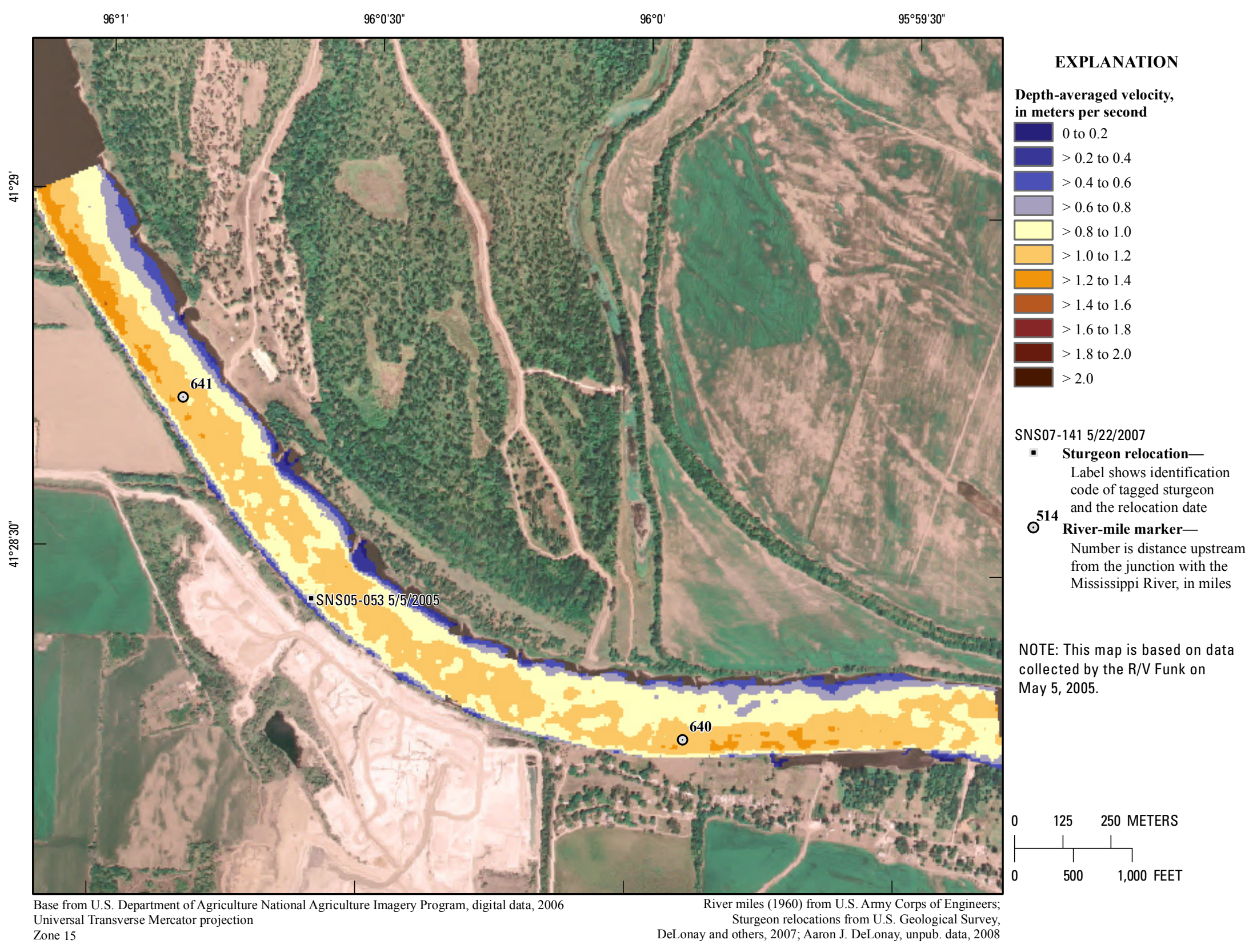

Figure 203. Map of depth-averaged velocity based on data collected on May 5, 2005, in the vicinity of river mile 641 . 


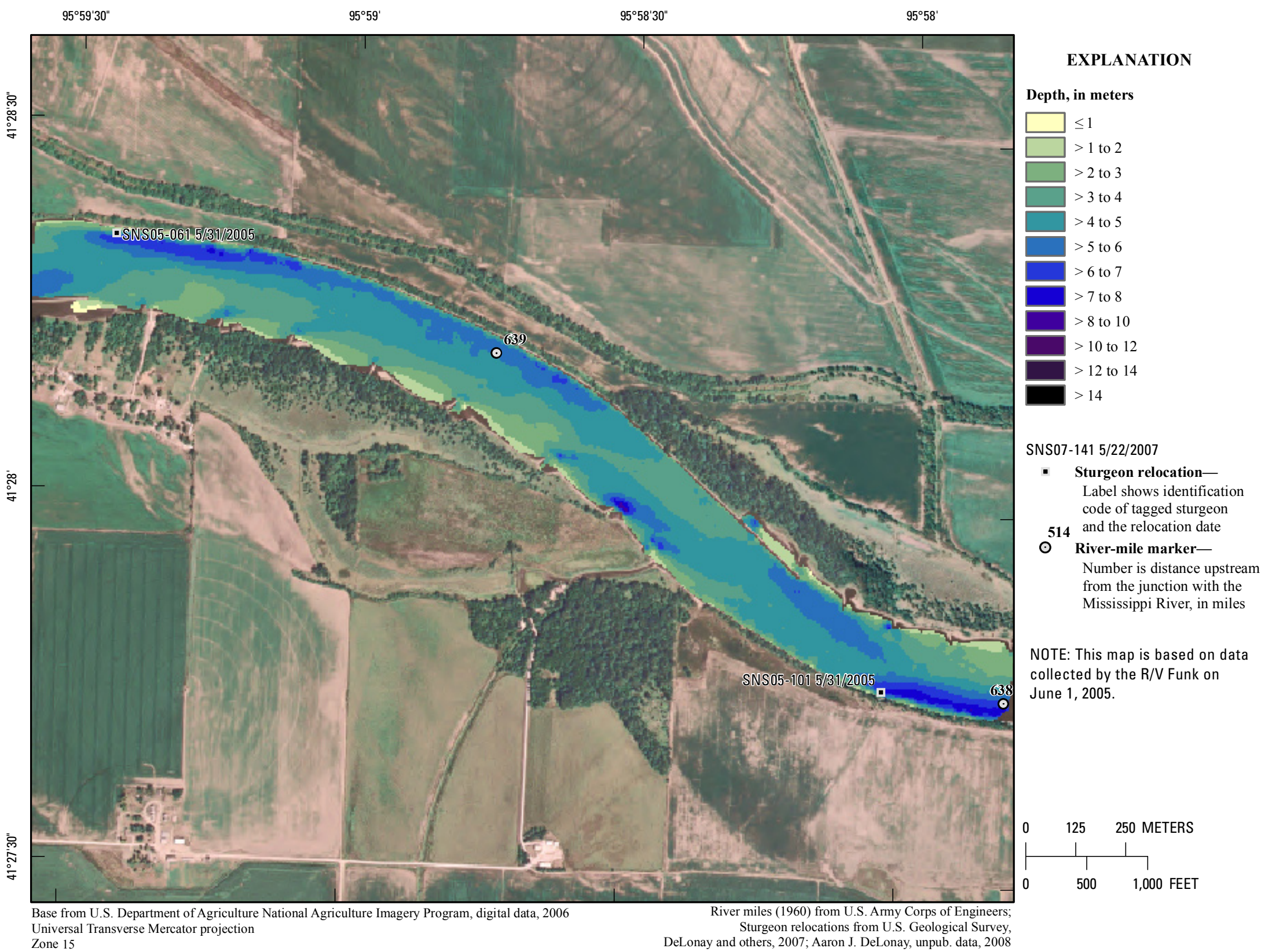

Figure 204. Map of depth based on data collected on June 1, 2005, in the vicinity of river mile 639 . 


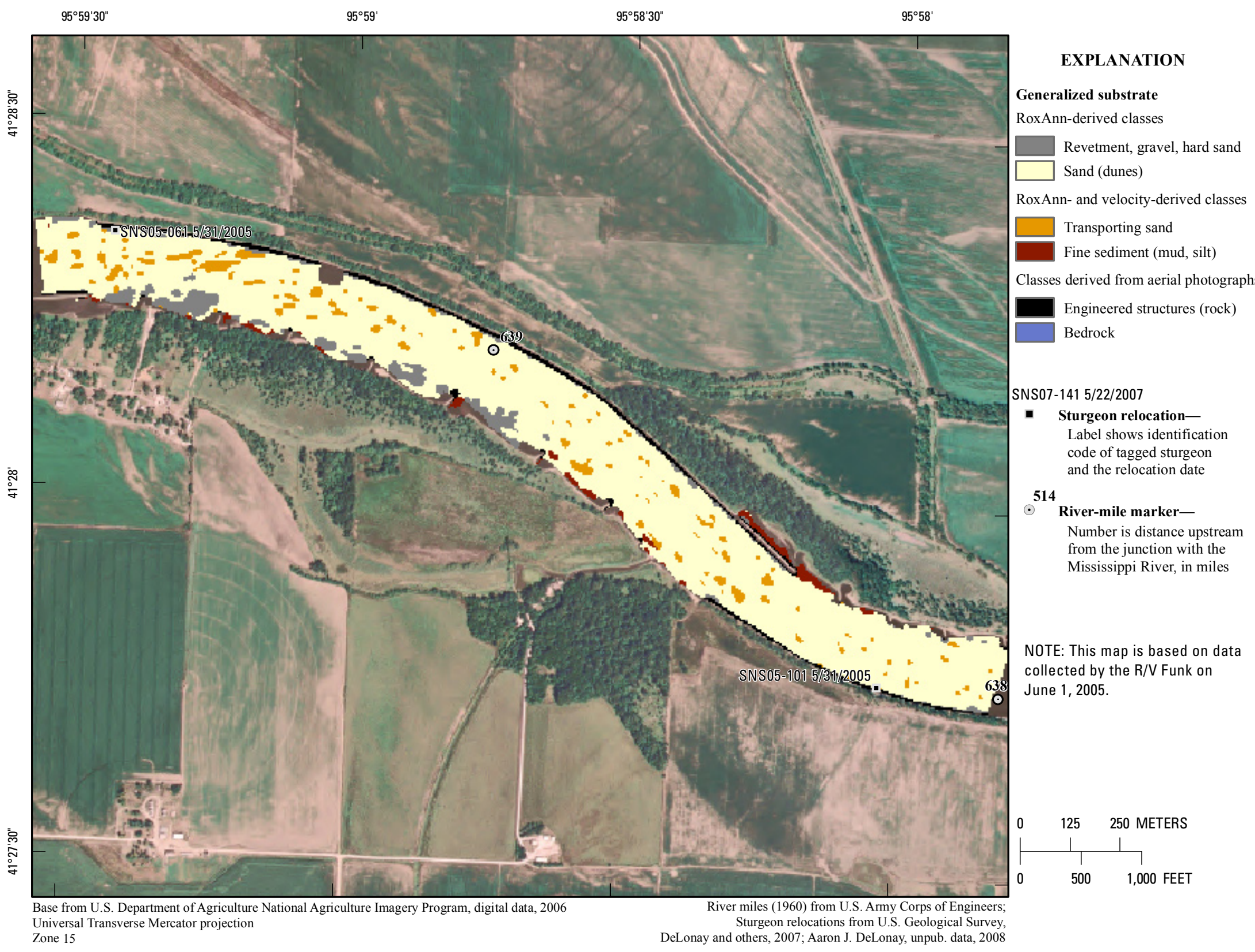

Figure 205. Map of generalized substrate based on data collected on June 1, 2005, in the vicinity of river mile 639 . 


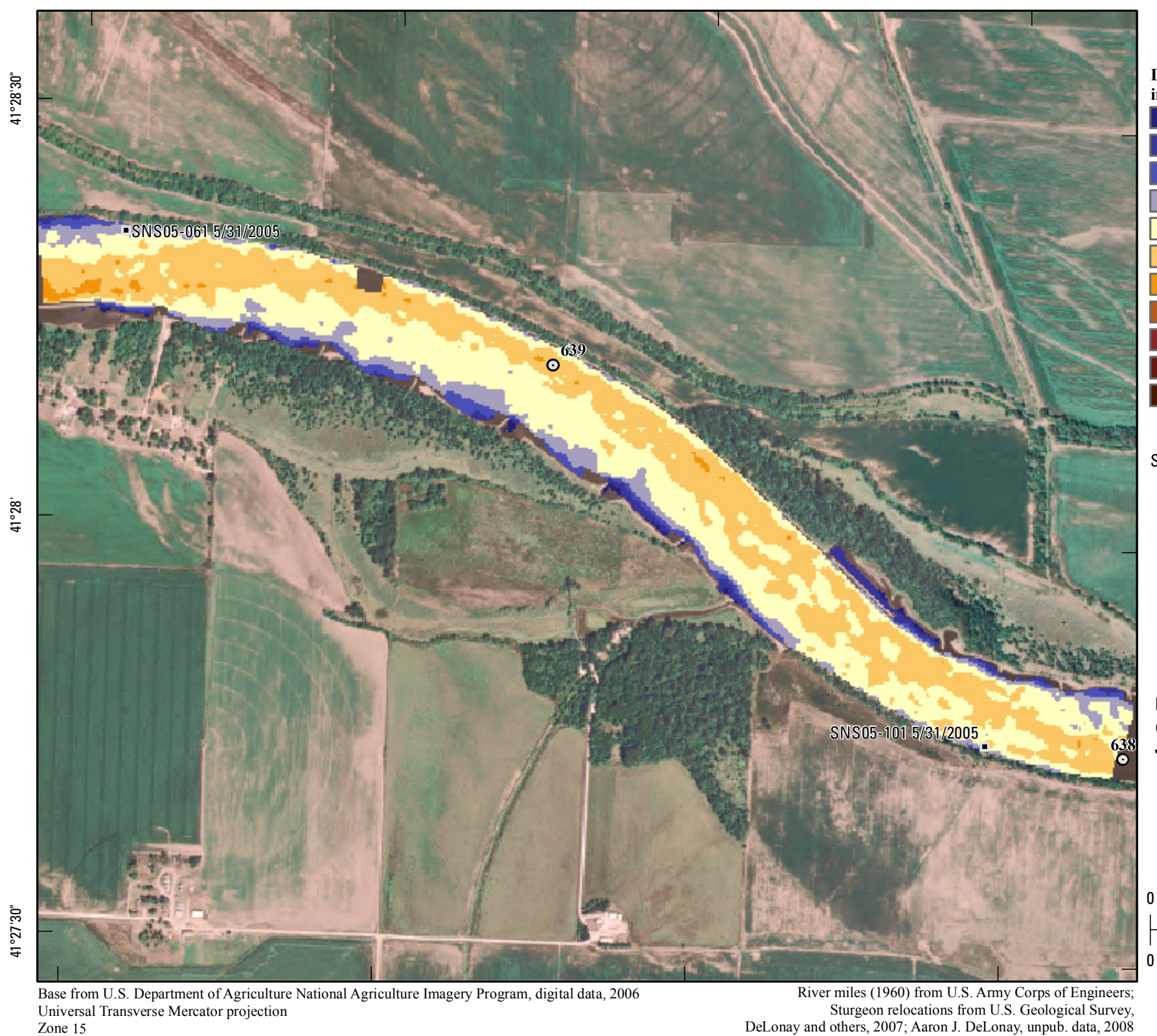

EXPLANATION

Depth-averaged velocity, in meters per second

0 to 0.2

$>0.2$ to 0.4

$>0.4$ to 0.6

$>0.6$ to 0.8

$>0.8$ to 1.0

$>1.0$ to 1.2

$>1.2$ to 1.4

$>1.4$ to 1.6

$>1.6$ to 1.8

$>1.8$ to 2.0

$>2.0$

\section{SNS07-141 5/22/2007}

- Sturgeon relocationLabel shows identification code of tagged sturgeo $\odot^{514}$ and the relocation date

Number is distance upstream from the junction with the Mississippi River, in miles

NOTE: This map is based on data collected by the R/V Funk on June 1, 2005

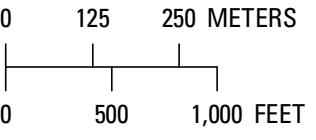
. DeLonay and others, 2007; Aaron J. DeLonay Geological Survey, 


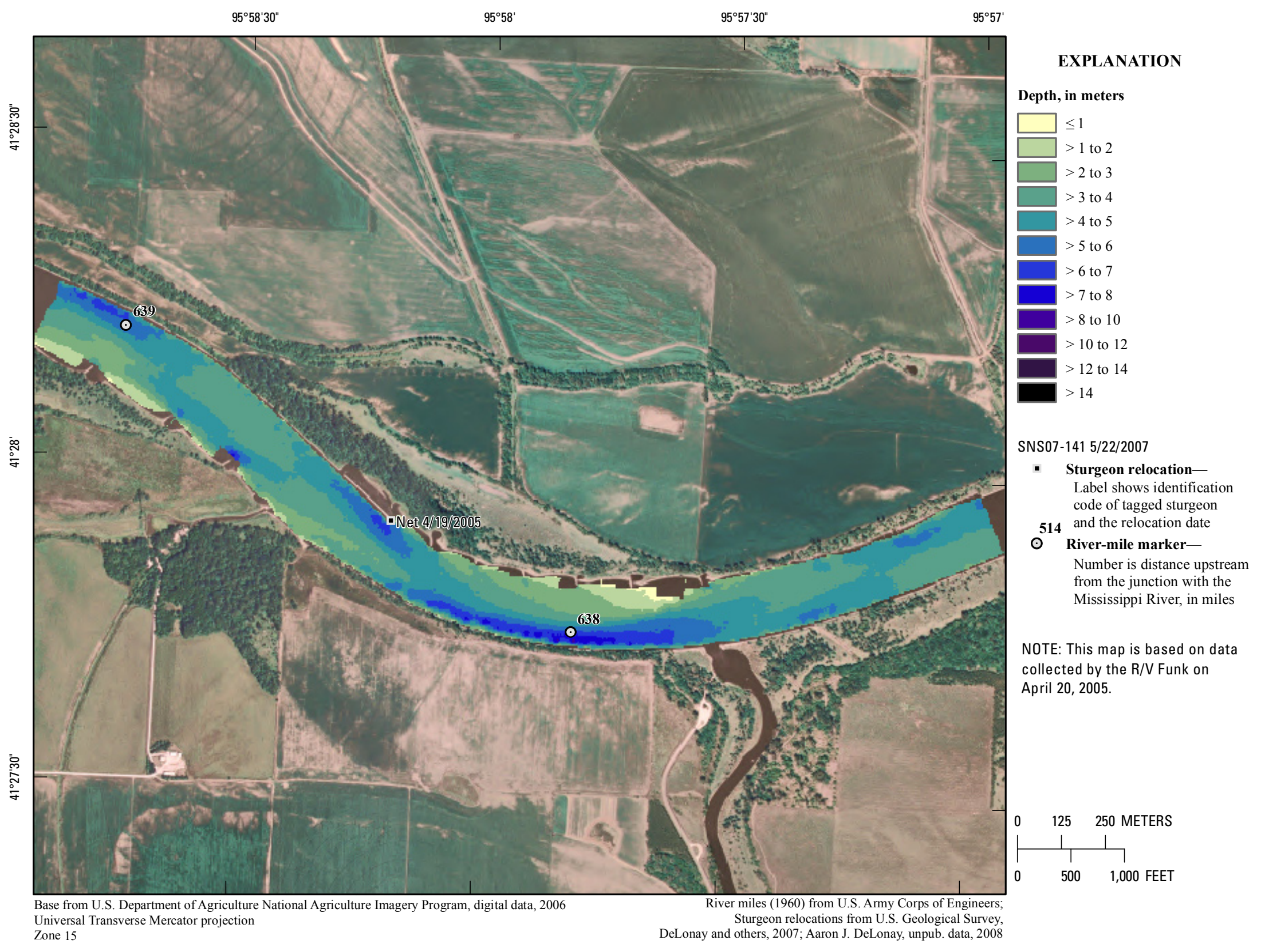

Figure 207. Map of depth based on data collected on April 20, 2005, in the vicinity of river mile 638 . 


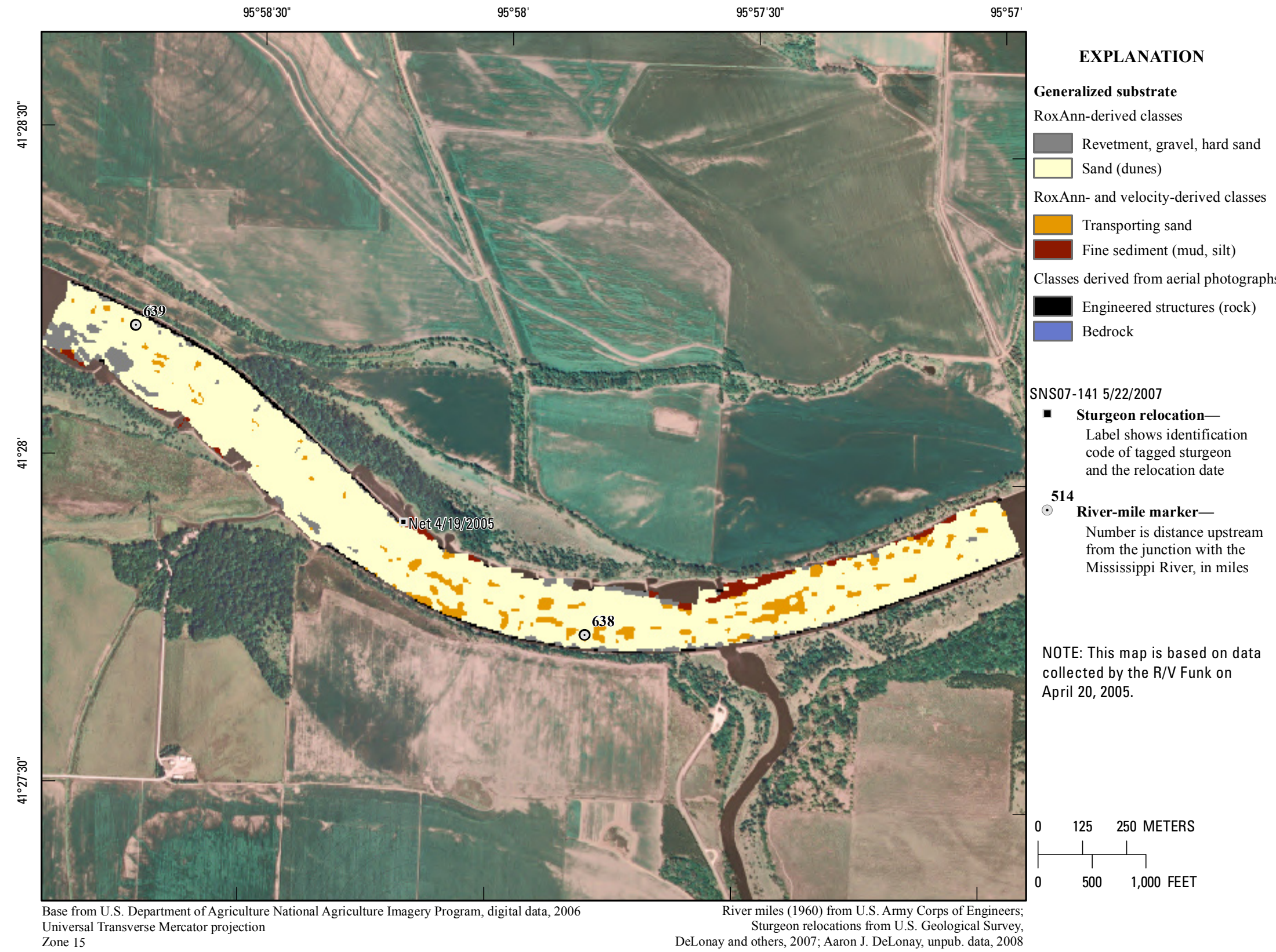

Figure 208. Map of generalized substrate based on data collected on April 20, 2005, in the vicinity of river mile 638 . 


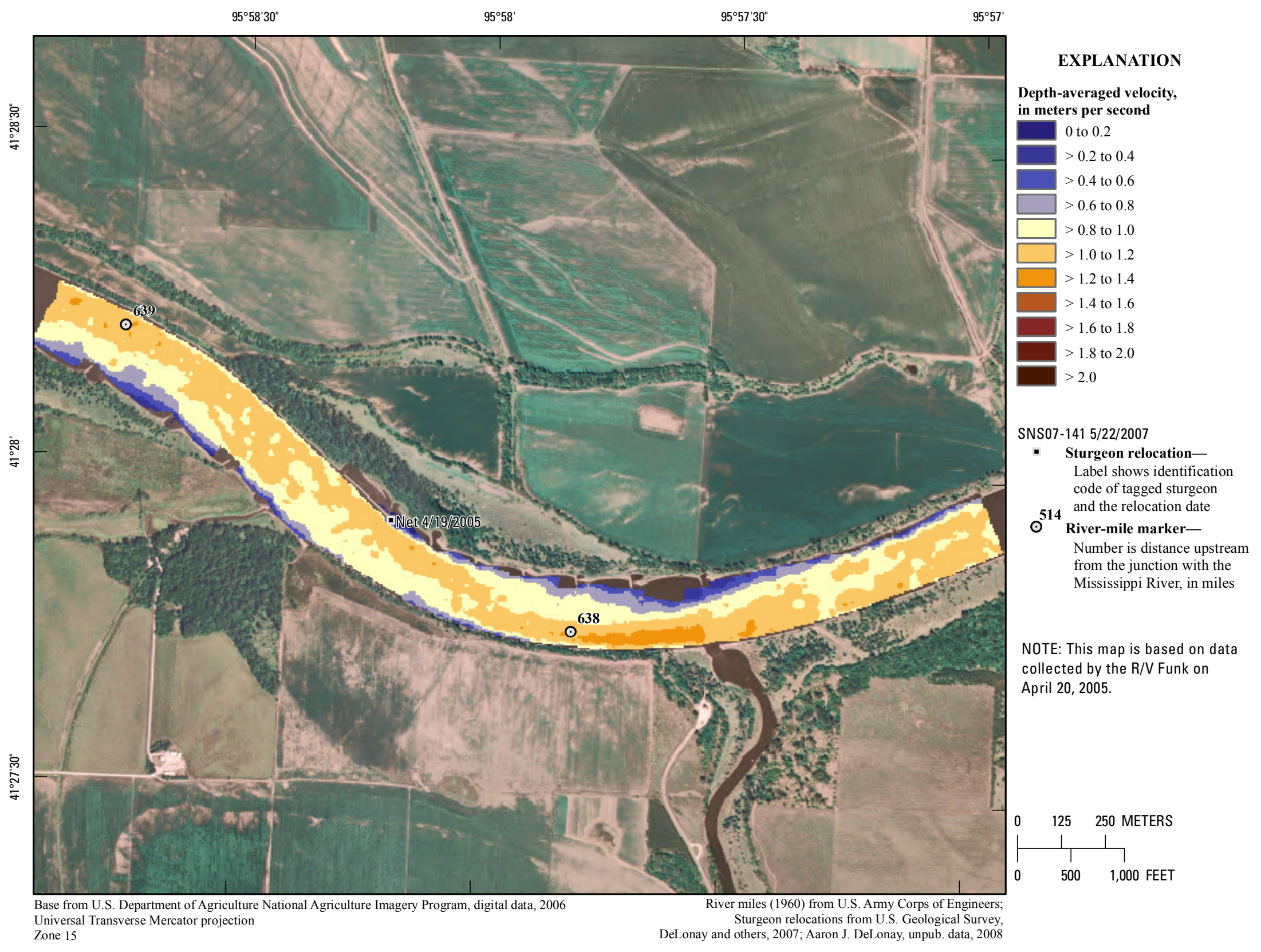

Figure 209. Map of depth-averaged velocity based on data collected on April 20, 2005, in the vicinity of river mile 638 . 


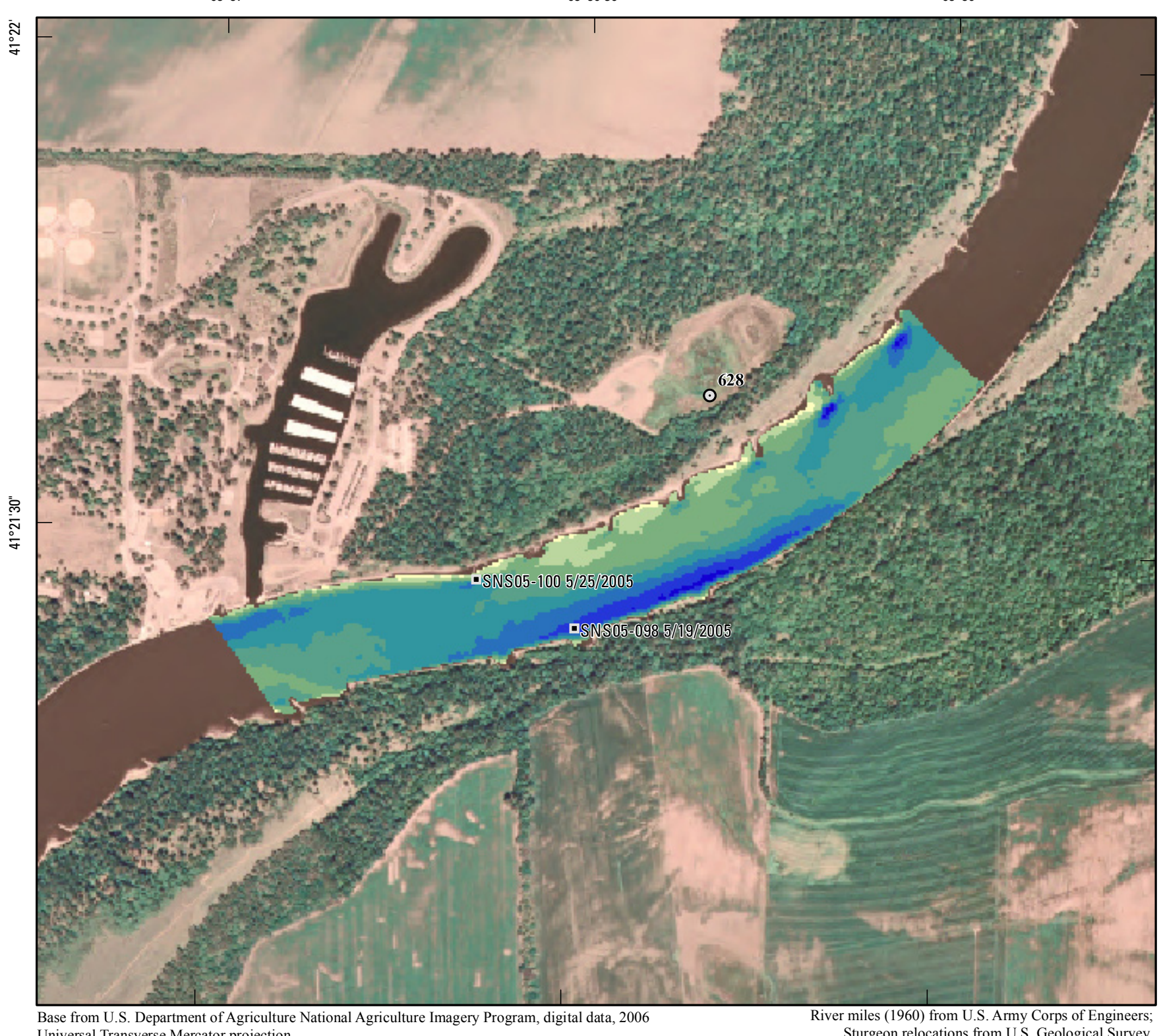

EXPLANATION

Depth, in meters

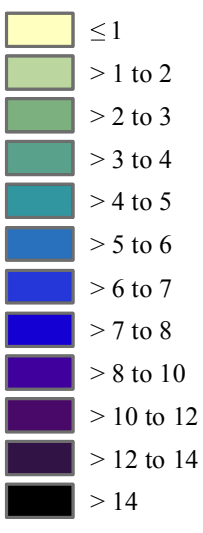

SNS07-141 5/22/2007

- Sturgeon relocationLabel shows identification code of tagged sturgeon $\odot^{514}$ and the relocation date Number is distance upstream from the junction with the Mississippi River, in miles

NOTE: This map is based on data collected by the R/V Funk on July 14, 2005. 


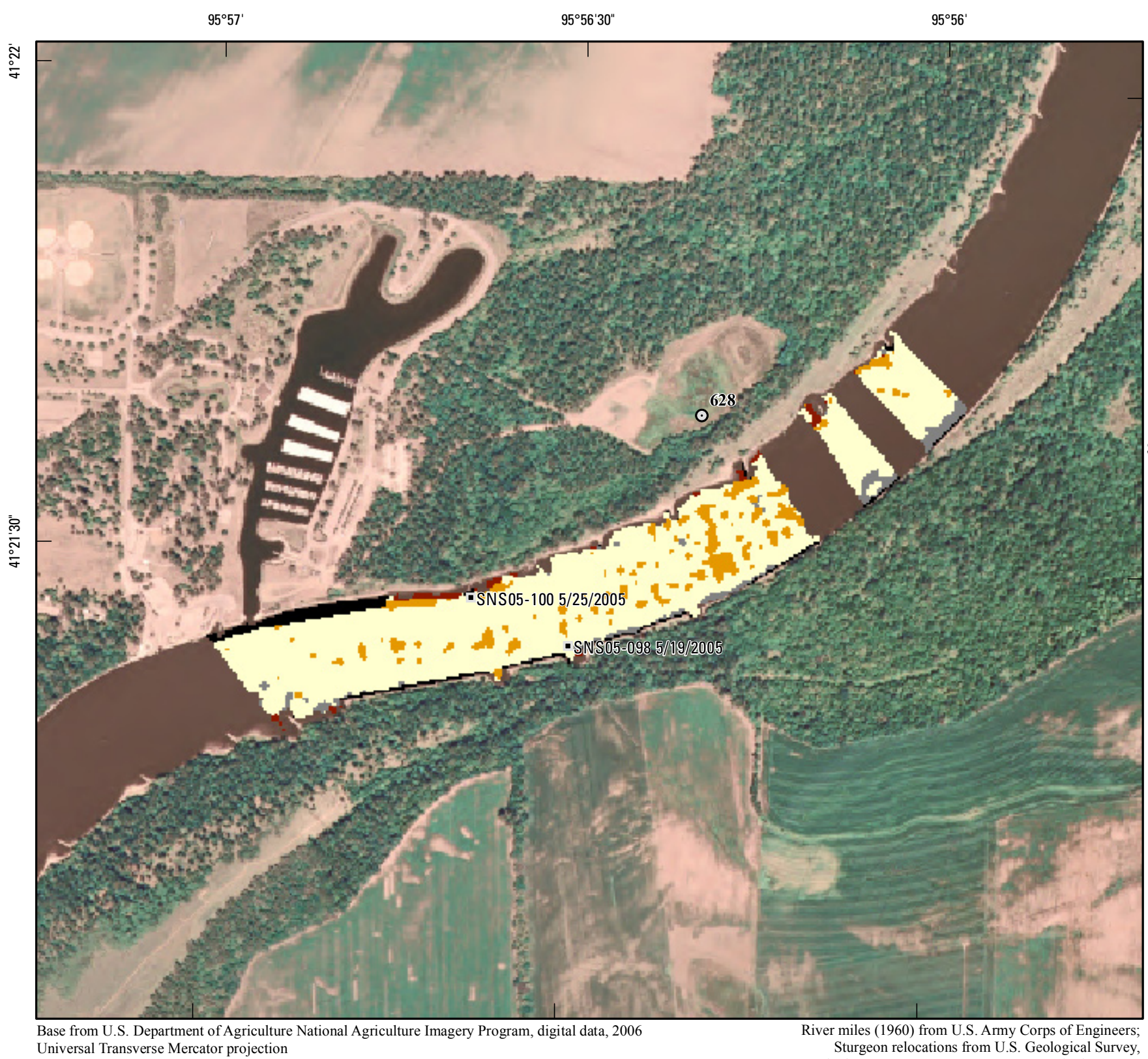

EXPLANATION

\section{Generalized substrate}

RoxAnn-derived classes

Revetment, gravel, hard sand Sand (dunes)

RoxAnn- and velocity-derived classes

$\square$ Transporting sand

Fine sediment (mud, silt)

Classes derived from aerial photographs

Engineered structures (rock)

Bedrock

\section{SNS07-141 5/22/2007}

- Sturgeon relocation-

Label shows identification code of tagged sturgeon and the relocation date

514

River-mile marker-

Number is distance upstream from the junction with the Mississippi River, in miles

NOTE: This map is based on data collected by the R/V Funk on July 14, 2005.

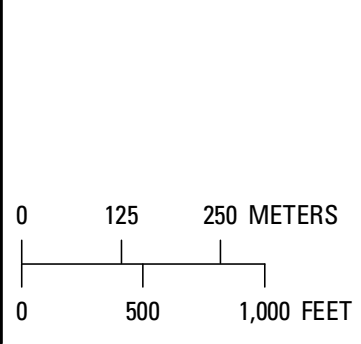
DeLonay and others, 2007; Aaron J. DeLonay, unpub. data, 2008

Figure 211. Map of generalized substrate based on data collected on July 14, 2005, in the vicinity of river mile 628 . 


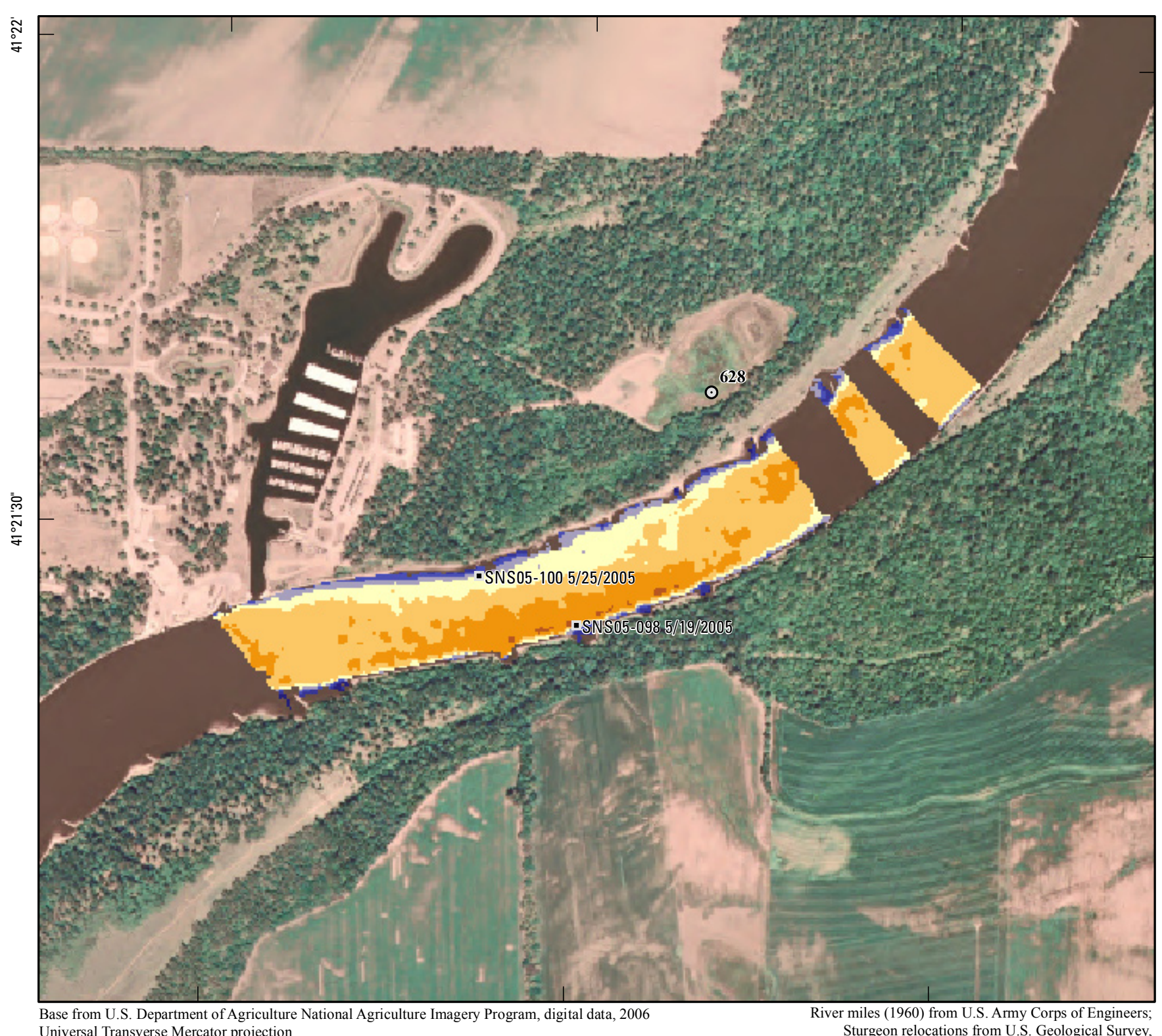

EXPLANATION

Depth-averaged velocity, in meters per second

0 to 0.2

$>0.2$ to 0.4

$>0.4$ to 0.6

$>0.6$ to 0.8

$>0.8$ to 1.0

$>1.0$ to 1.2

$>1.2$ to 1.4

$>1.4$ to 1.6

$>1.6$ to 1.8

$>1.8$ to 2.0

$>2.0$

\section{SNS07-141 5/22/2007}

- Sturgeon relocationLabel shows identification code of tagged sturgeo

$\odot^{514}$ and the relocation date

Number is distance upstream

from the junction with the

Mississippi River, in miles

NOTE: This map is based on data

collected by the R/V Funk on

July 14, 2005.

Universal Transverse Mercator projection

Sturgeon relocations from U.S. Geological Survey,
DeLonay and others, 2007; Aaron J. DeLonay, unpub. data, 2008

\section{$0 \quad 125 \quad 250$ METERS

$\left.\right|_{500} ^{1}$ \\ $500 \quad 1,000$ FEET}




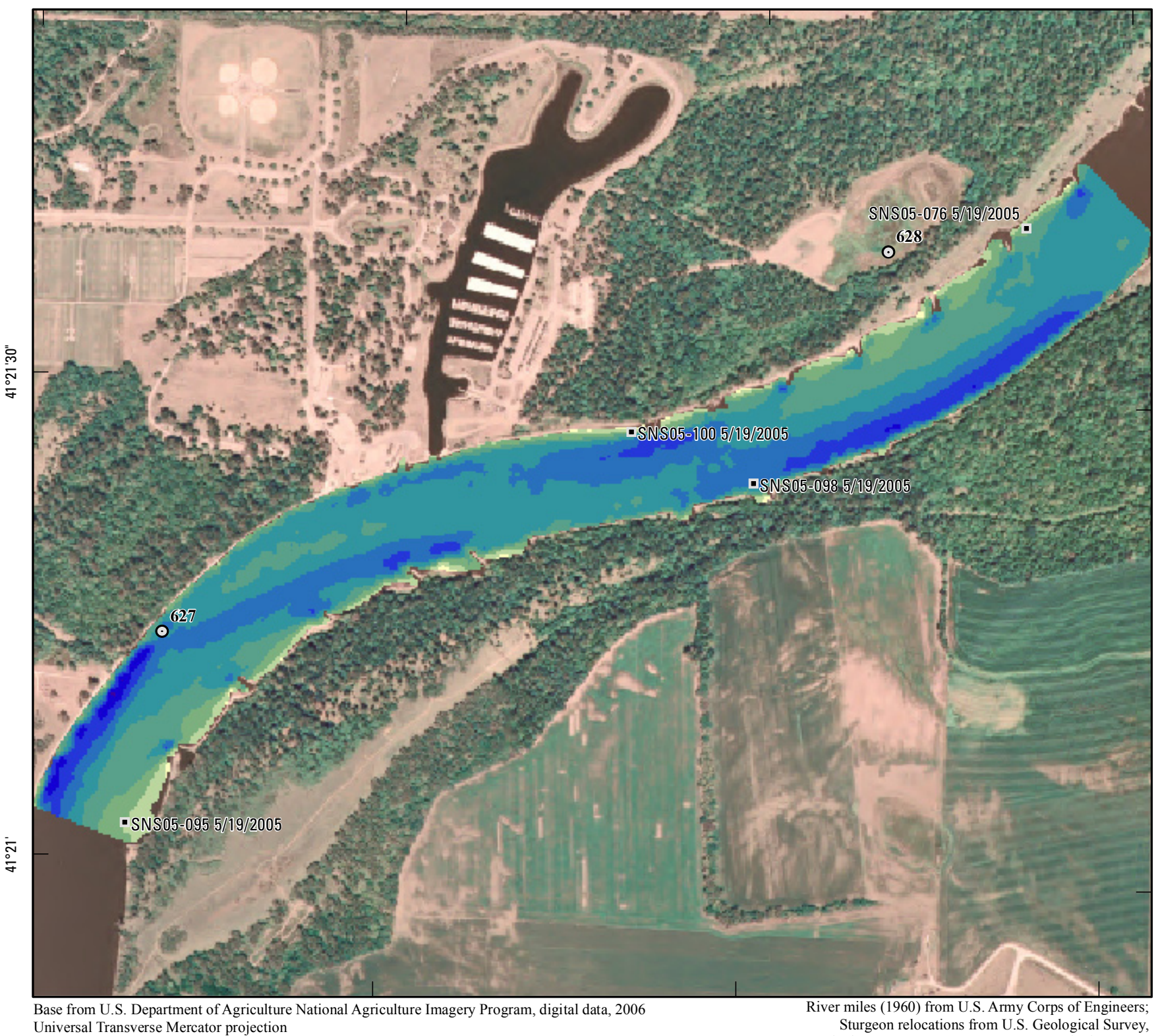

EXPLANATION

\section{Depth, in meters}

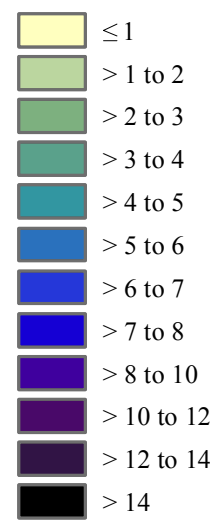

\section{SNS07-141 5/22/2007}

- Sturgeon relocationLabel shows identification code of tagged sturgeon 514 and the relocation date

$\odot$ River-mile markerNumber is distance upstream from the junction with the Mississippi River, in miles

NOTE: This map is based on data collected by the R/V Funk on May 20, 2005.

Figure 213. Map of depth based on data collected on May 20, 2005, in the vicinity of river mile 628. 


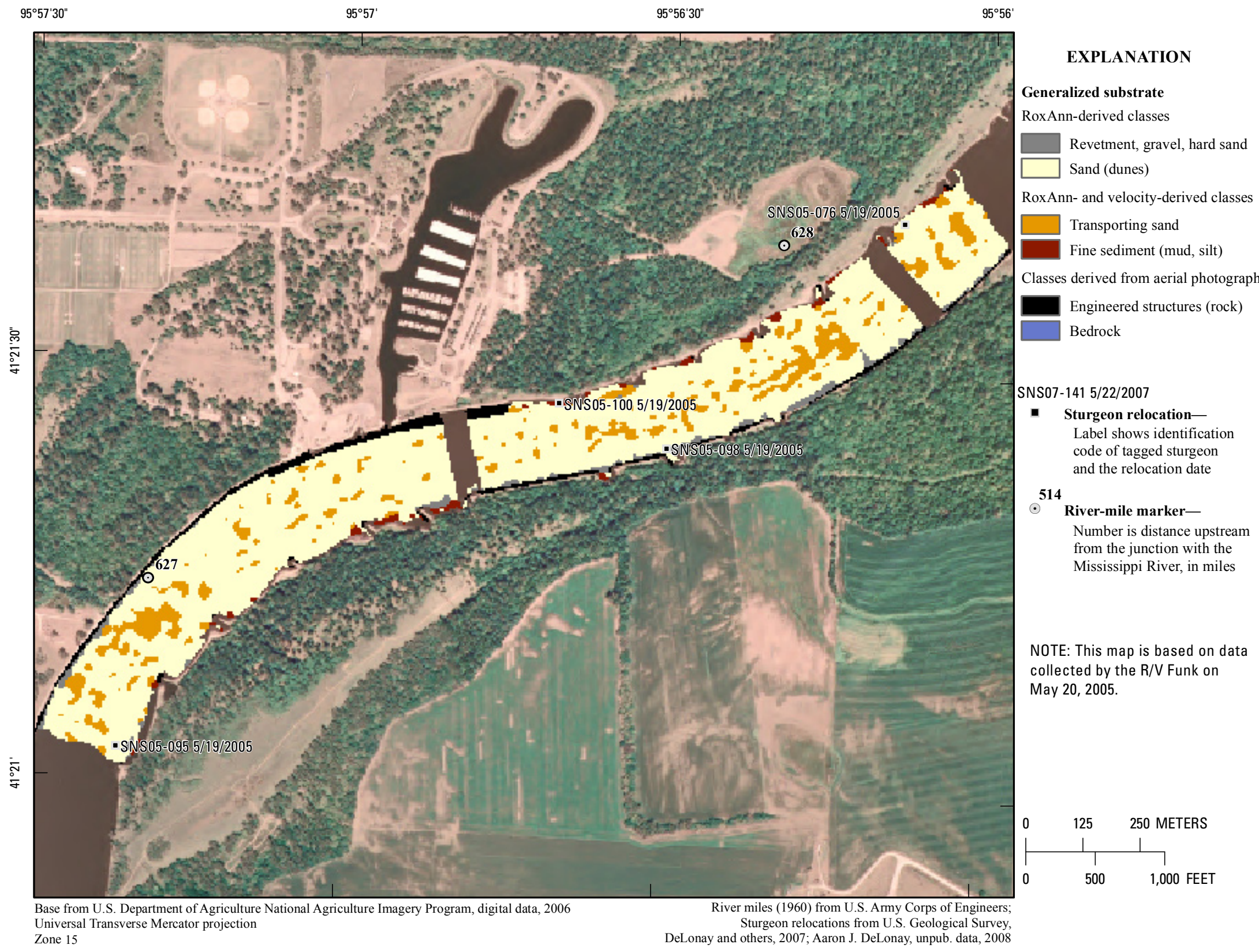

Figure 214. Map of generalized substrate based on data collected on May 20, 2005, in the vicinity of river mile 628 . 


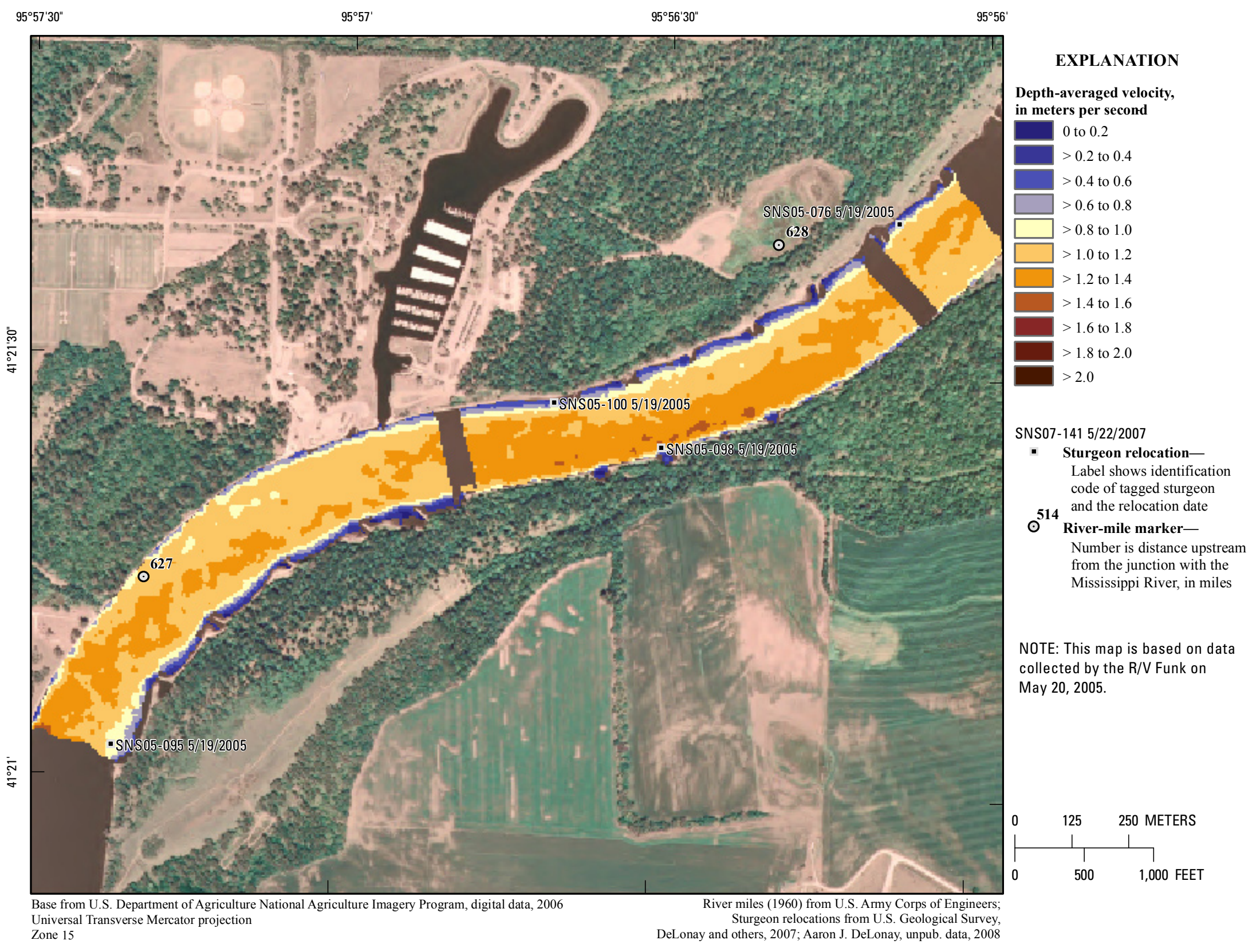

Figure 215. Map of depth-averaged velocity based on data collected on May 20, 2005, in the vicinity of river mile 628 . 
$95^{\circ} 57^{\prime}$

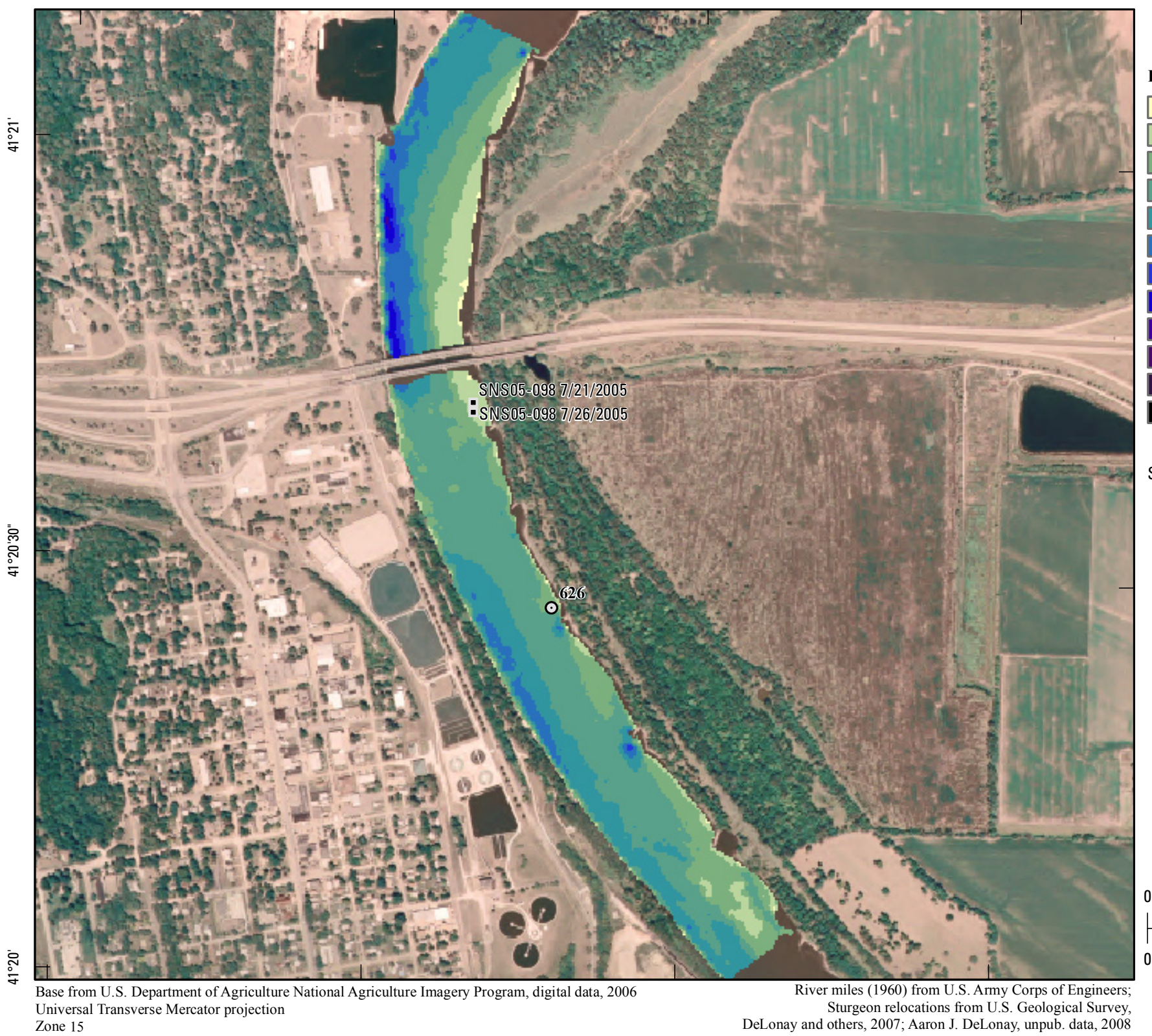

EXPLANATION

Depth, in meters

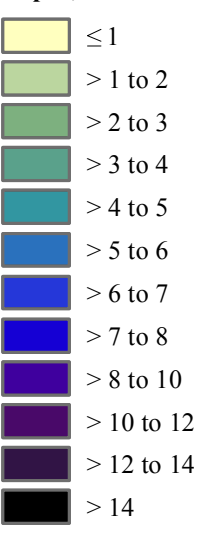

SNS07-141 5/22/2007

- Sturgeon relocationLabel shows identification code of tagged sturgeon 514 and the relocation date - River-mile markerNumber is distance upstream
from the junction with the Mississippi River, in miles

NOTE: This map is based on data collected by the R/V Funk on September 1, 2005.

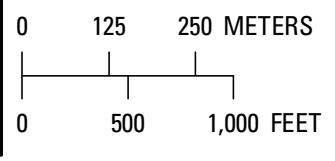
Sturgeon relocations from L.S. Geological Survey,
DeLonay and others, 2007; Aaron J. DeLonay, unpub. data, 2008 


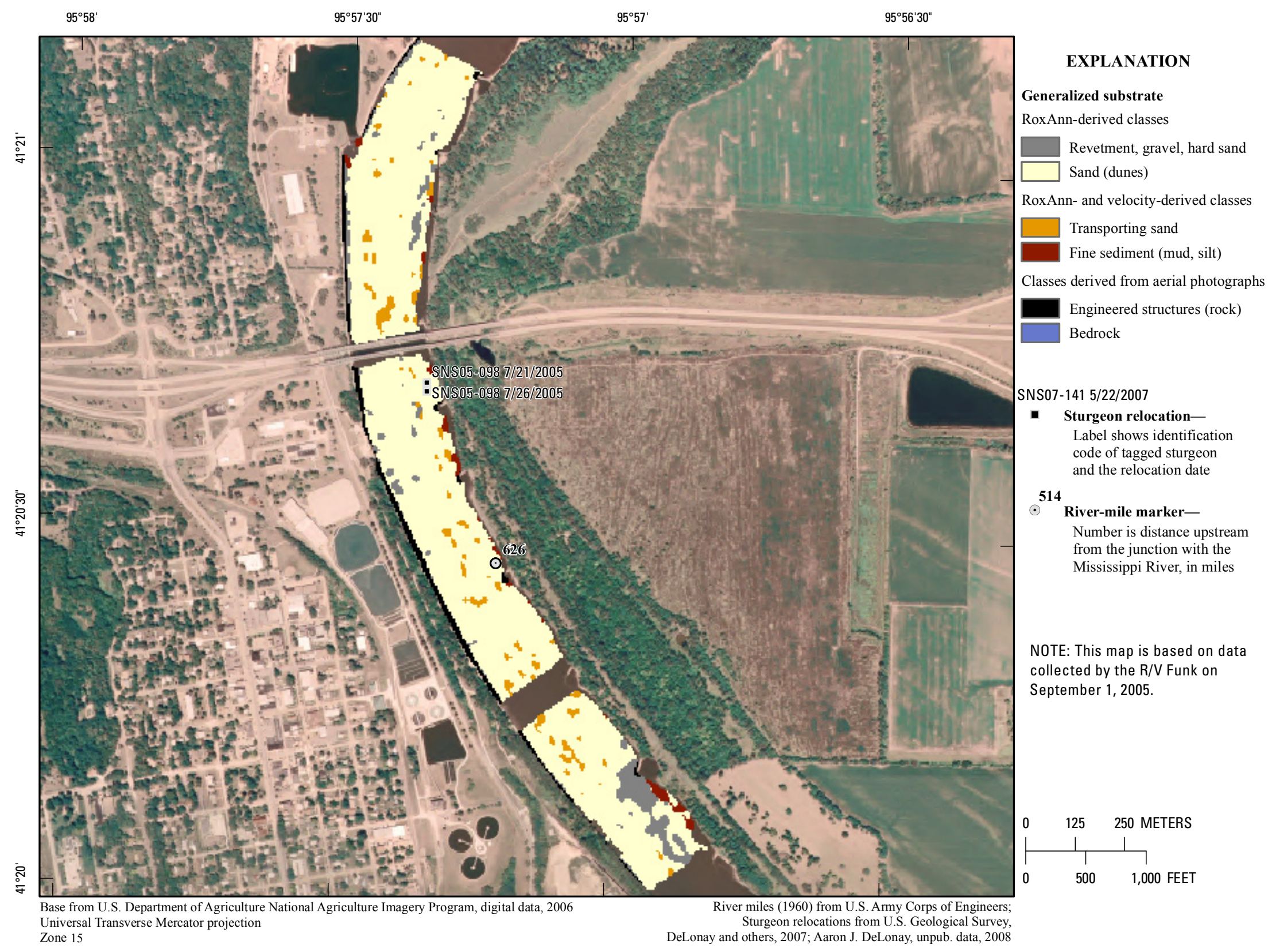

Figure 217. Map of generalized substrate based on data collected on September 1, 2005, in the vicinity of river mile 626 . 

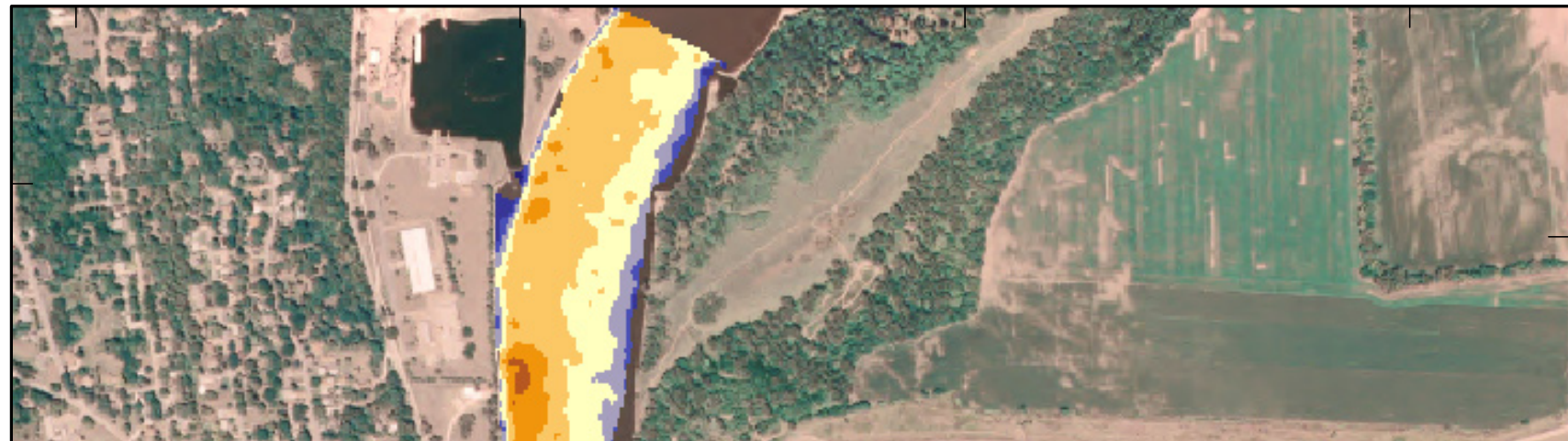

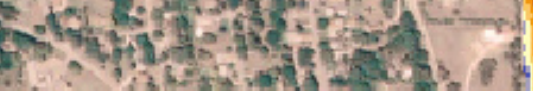

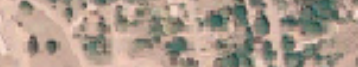

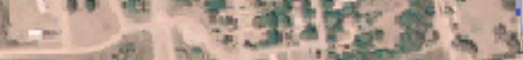
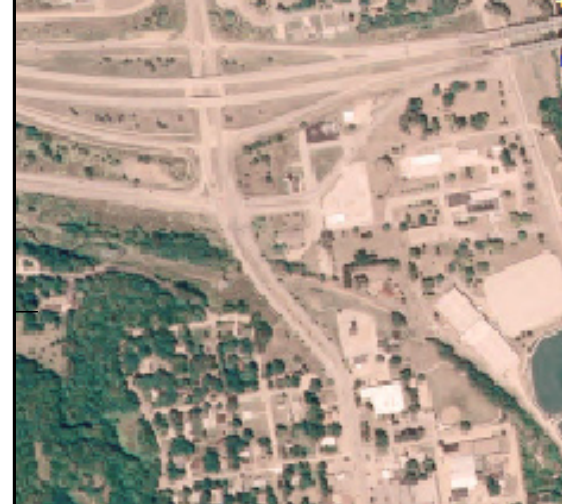

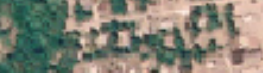

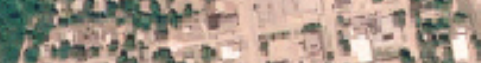

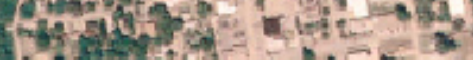

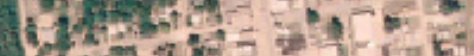

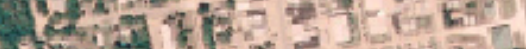

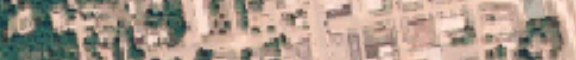

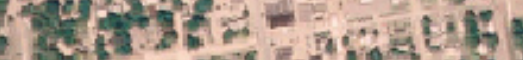

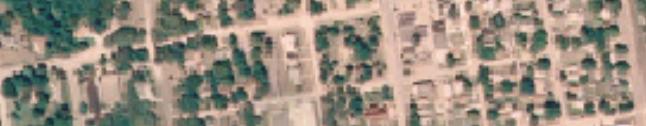

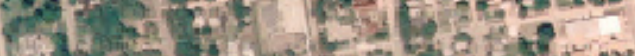

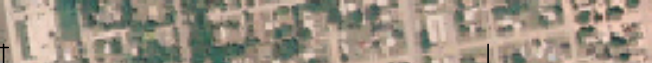

Base from U.S. Department of Agriculture National Agriculture Imagery Program, digital data, 2006 Universal Transverse Mercator projectio
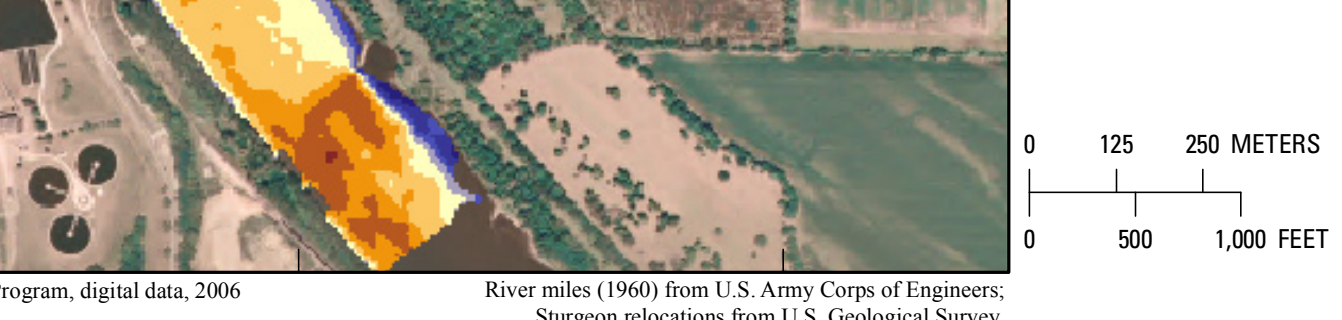
Sturgeon relocations from U.S. Geological Survey,
DeLonay and others, 2007; Aaron J. DeLonay, unpub. data, 2008

NOTE: This map is based on data collected by the R/V Funk on

September 1, 2005.

in meters per second

0 to 0.2

0.2 to 0.4

$>0.4$ to 0.6

$>0.6$ to 0.8

$>0.8$ to 1.0

$>1.0$ to 1.2

$>1.2$ to 1.4

1.4 to 1.6

$>1.6$ to 1.8

$>1.8$ to 2.0

$>2.0$

SNS07-141 5/22/2007

Sturgeon relocation-

Label shows identification

code of tagged sturgeo

$\odot^{514}$ River-mile marker-

Number is distance upstream

from the junction with the

Mississippi River, in miles 


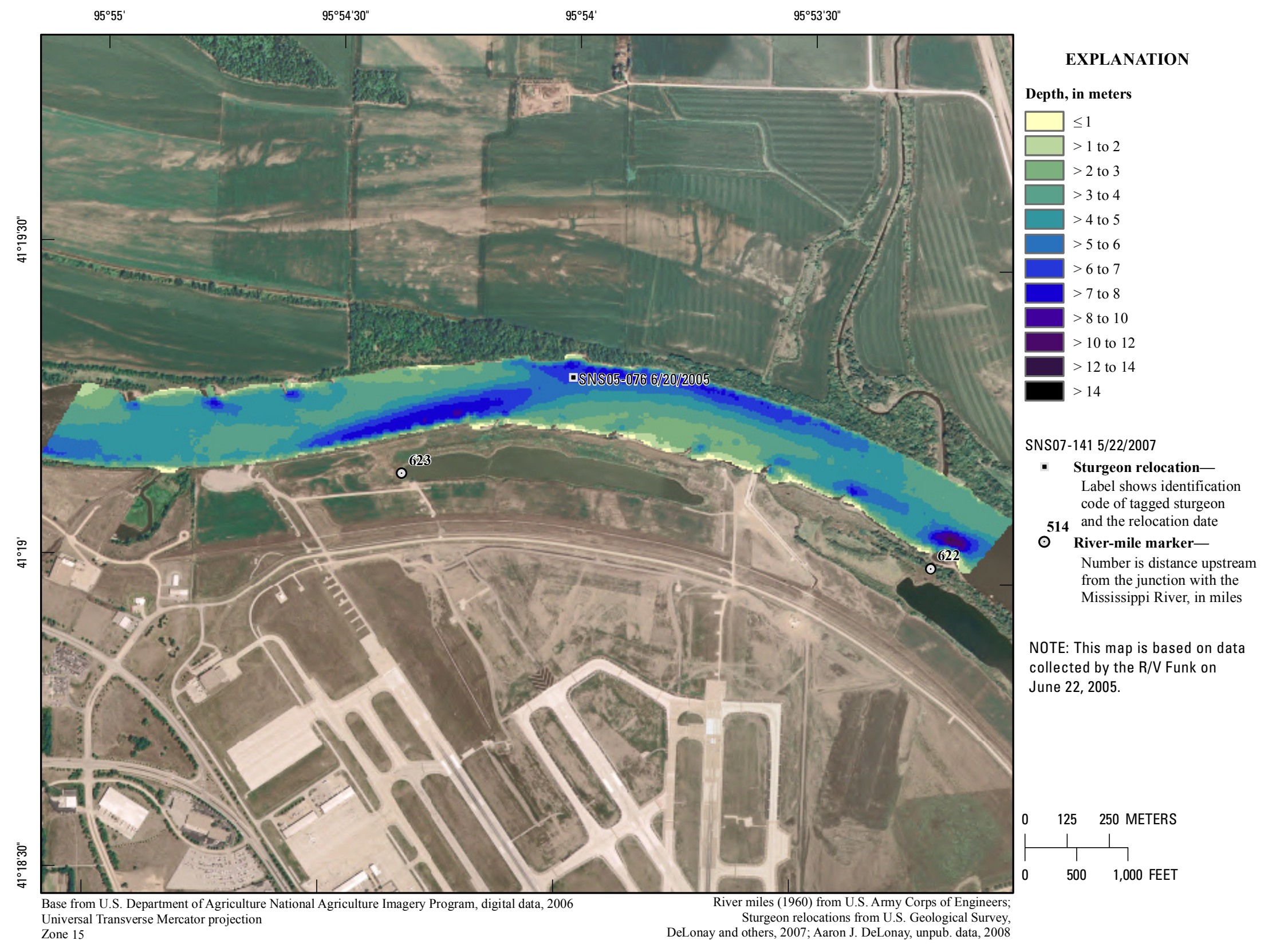

Figure 219. Map of depth based on data collected on June 22, 2005, in the vicinity of river mile 623 . 


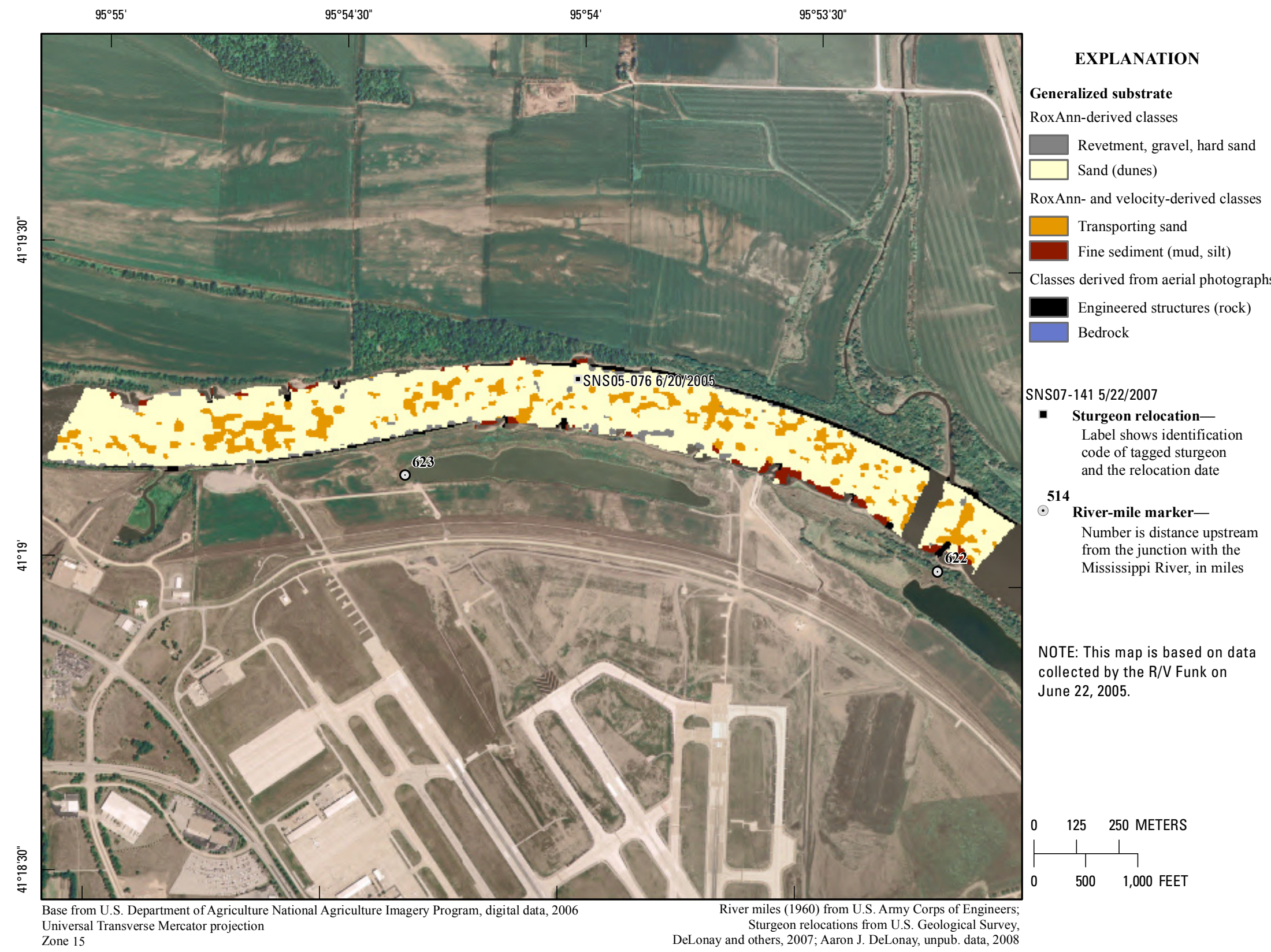

Figure 220. Map of generalized substrate based on data collected on June 22, 2005, in the vicinity of river mile 623 . 


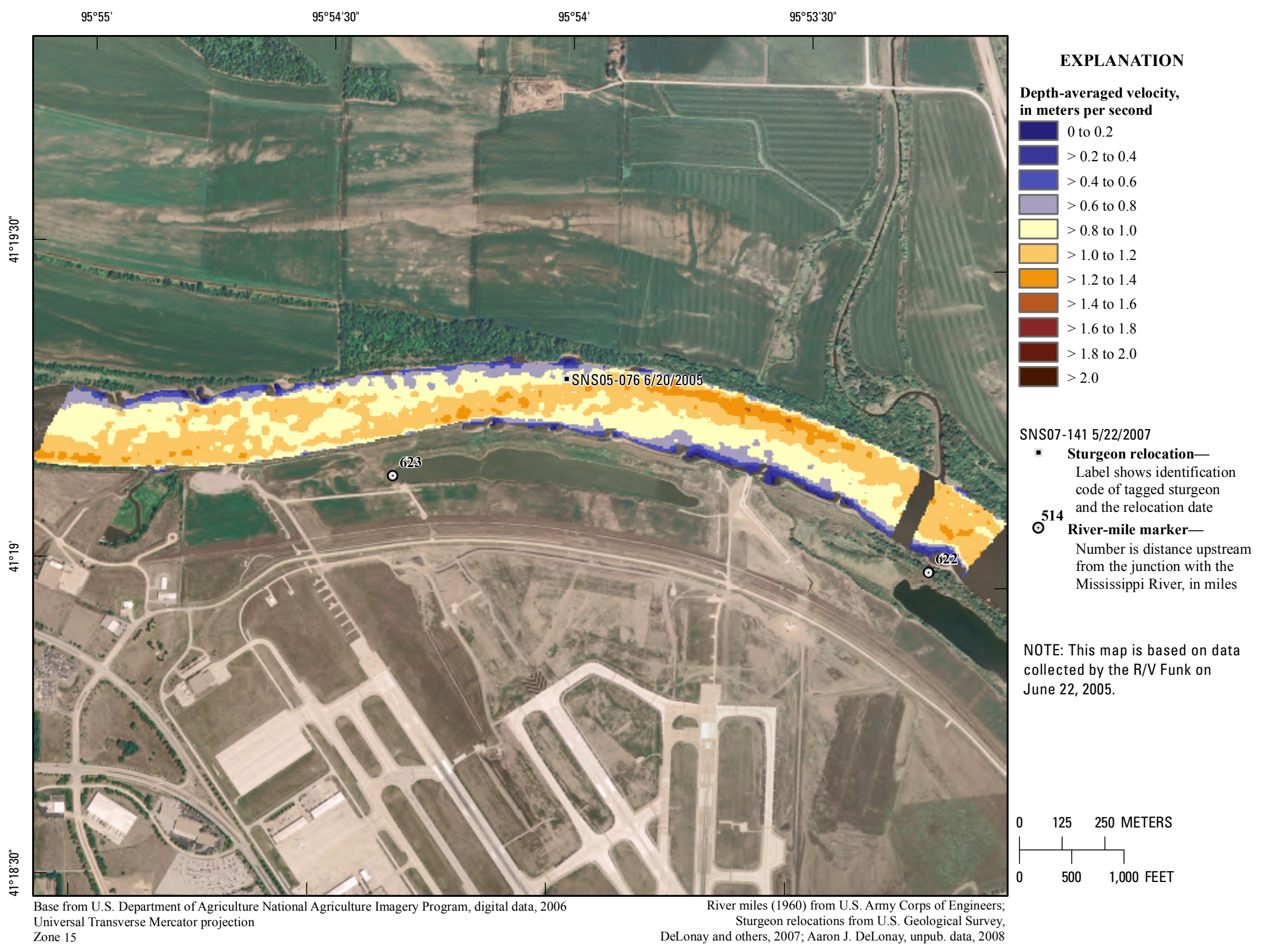

Figure 221. Map of depth-averaged velocity based on data collected on June 22, 2005, in the vicinity of river mile 623. 


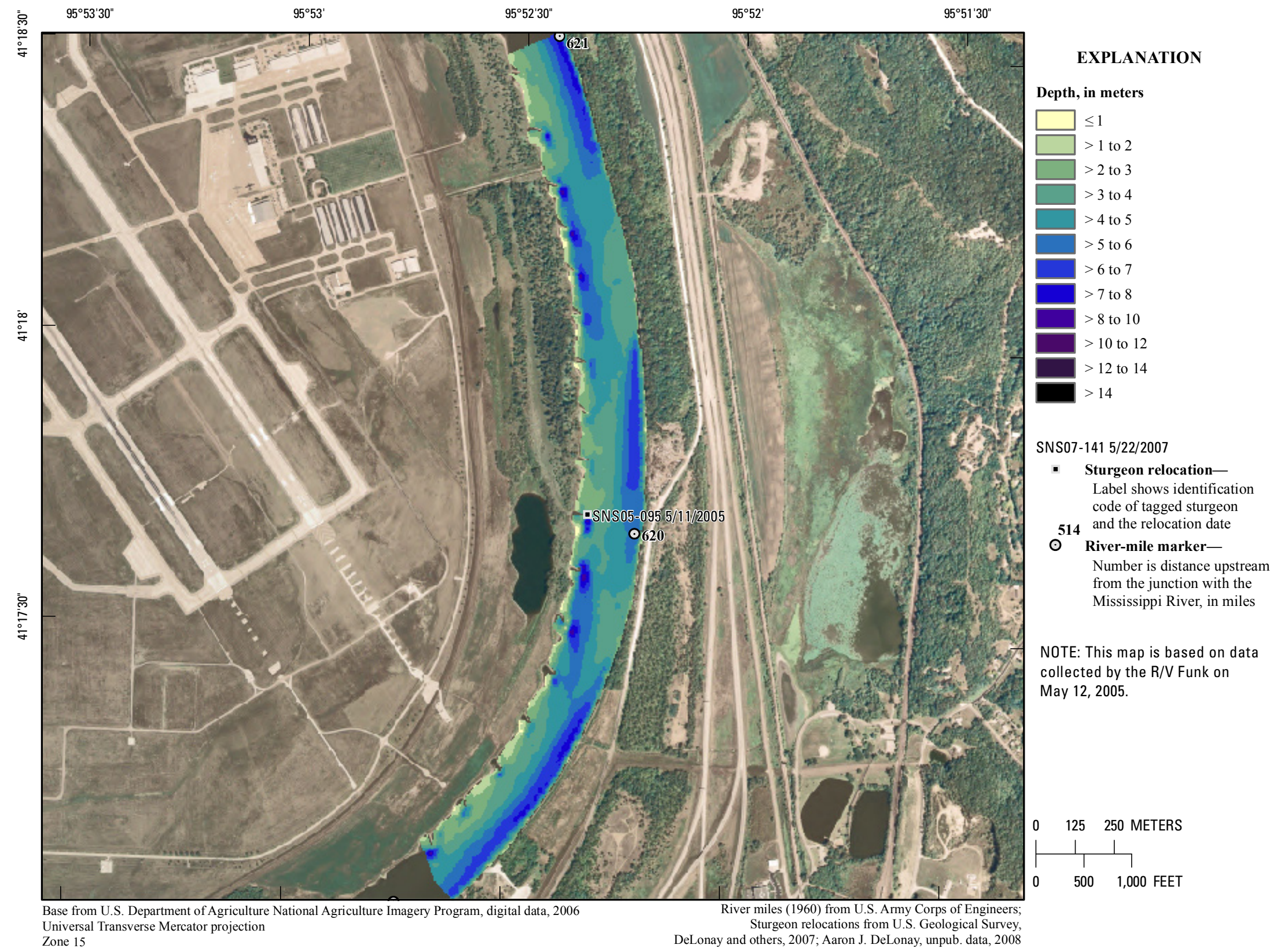

Figure 222. Map of depth based on data collected on May 12, 2005, in the vicinity of river mile 620 . 


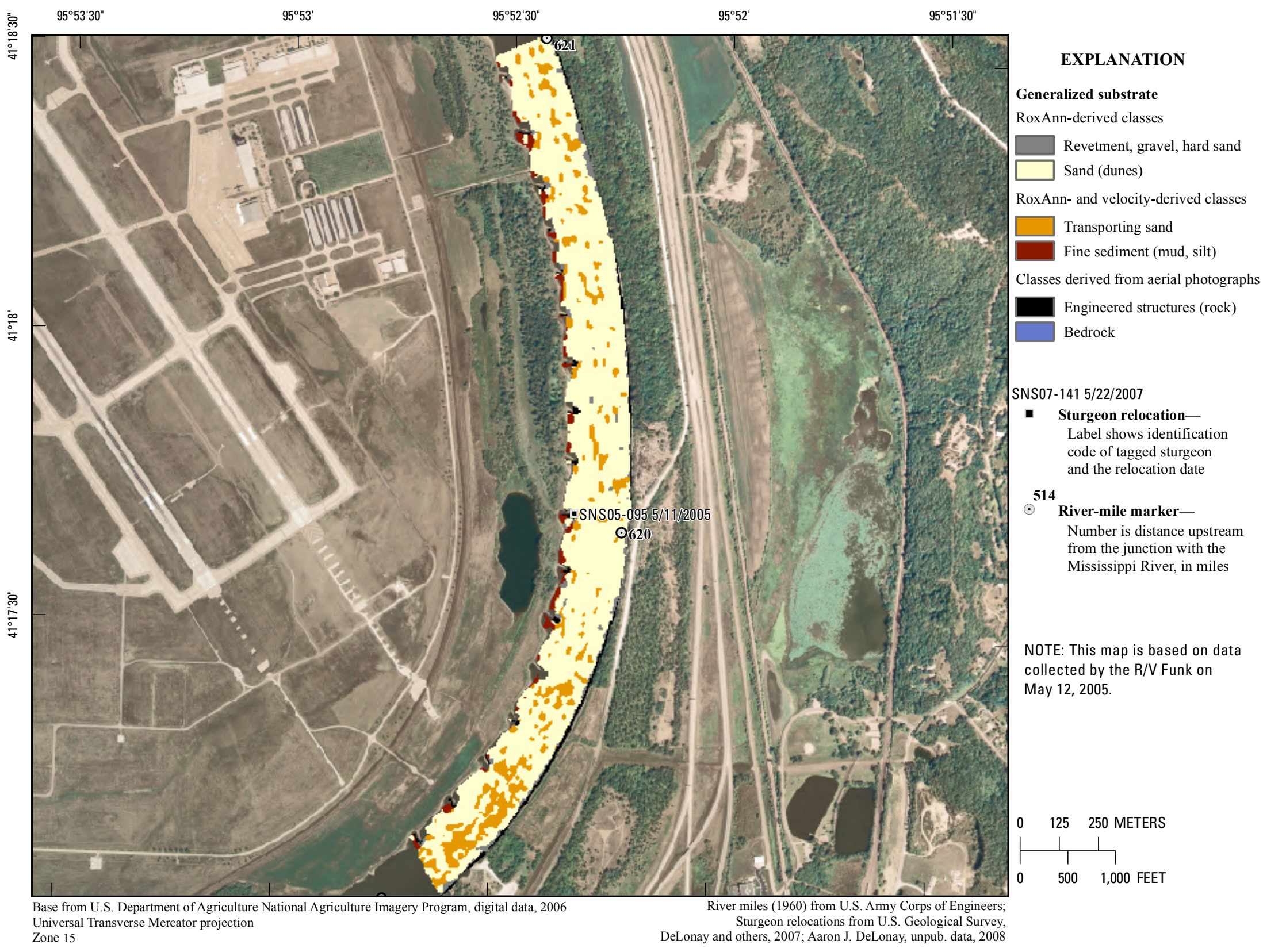

Figure 223. Map of generalized substrate based on data collected on May 12, 2005, in the vicinity of river mile 620 . 


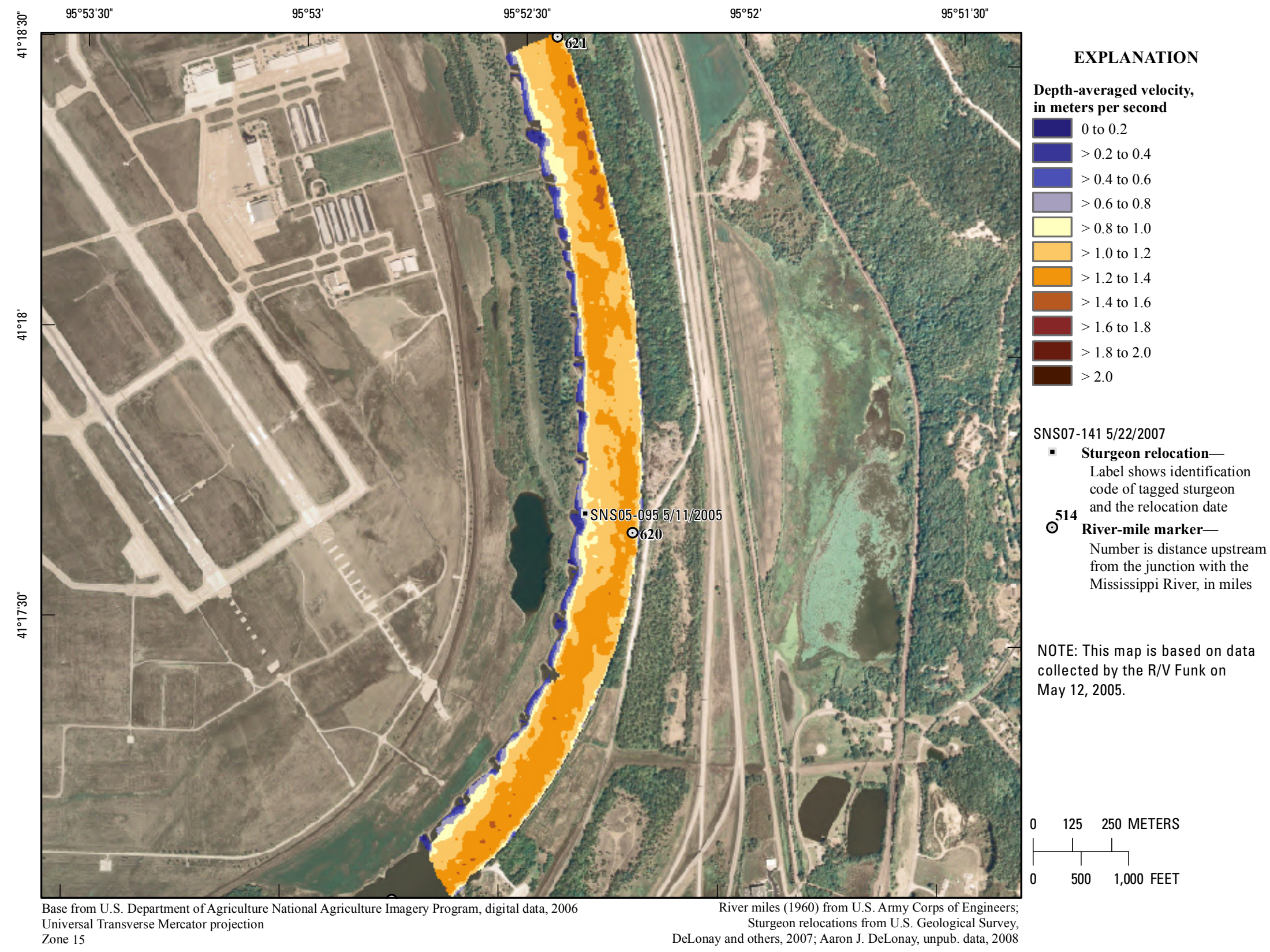

Figure 224. Map of depth-averaged velocity based on data collected on May 12, 2005, in the vicinity of river mile 620 . 


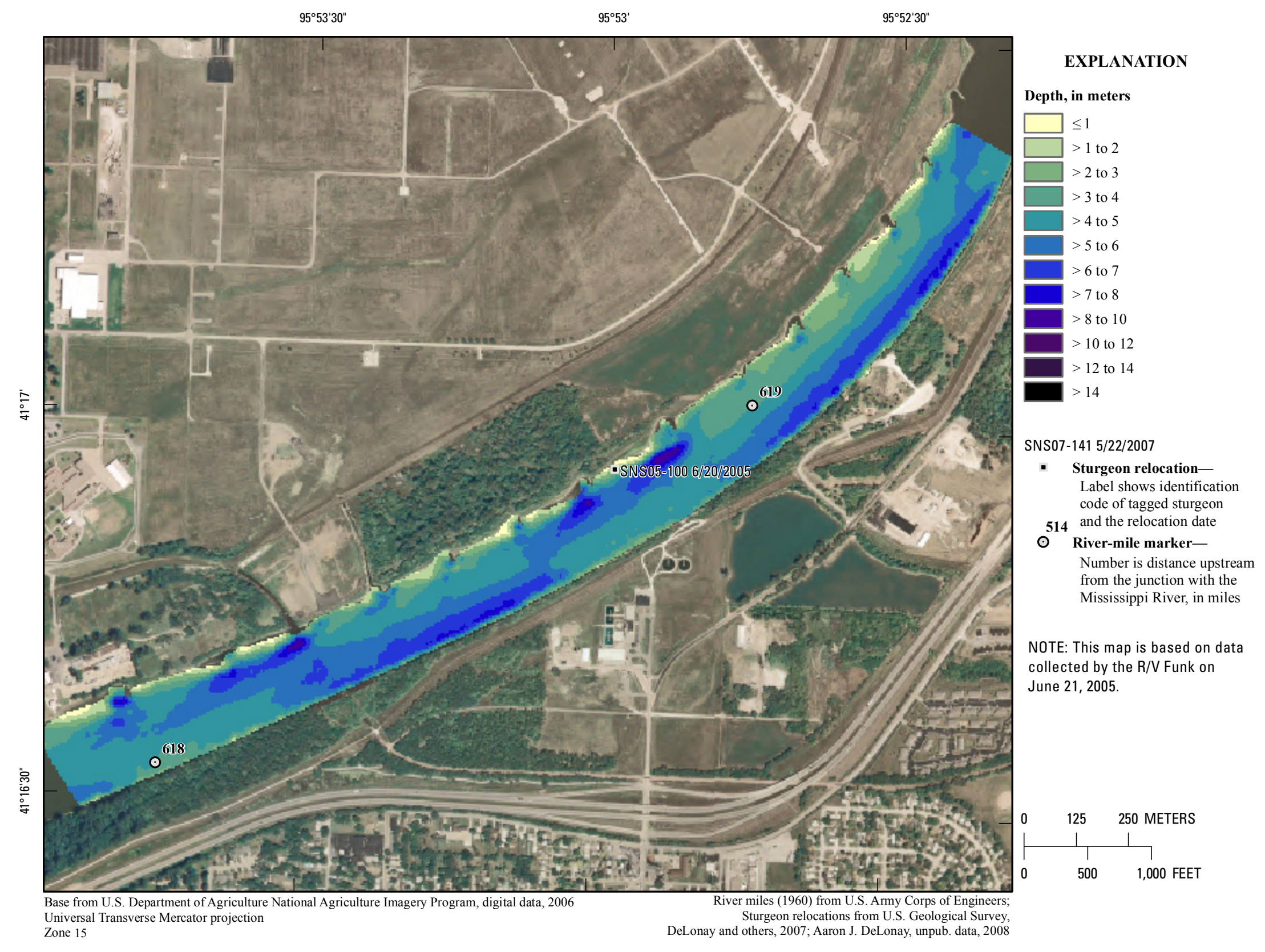

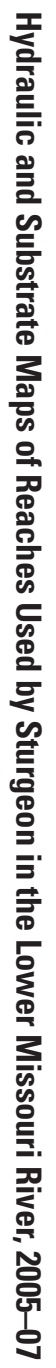

Figure 225. Map of depth based on data collected on June 21, 2005, in the vicinity of river mile 619 . 


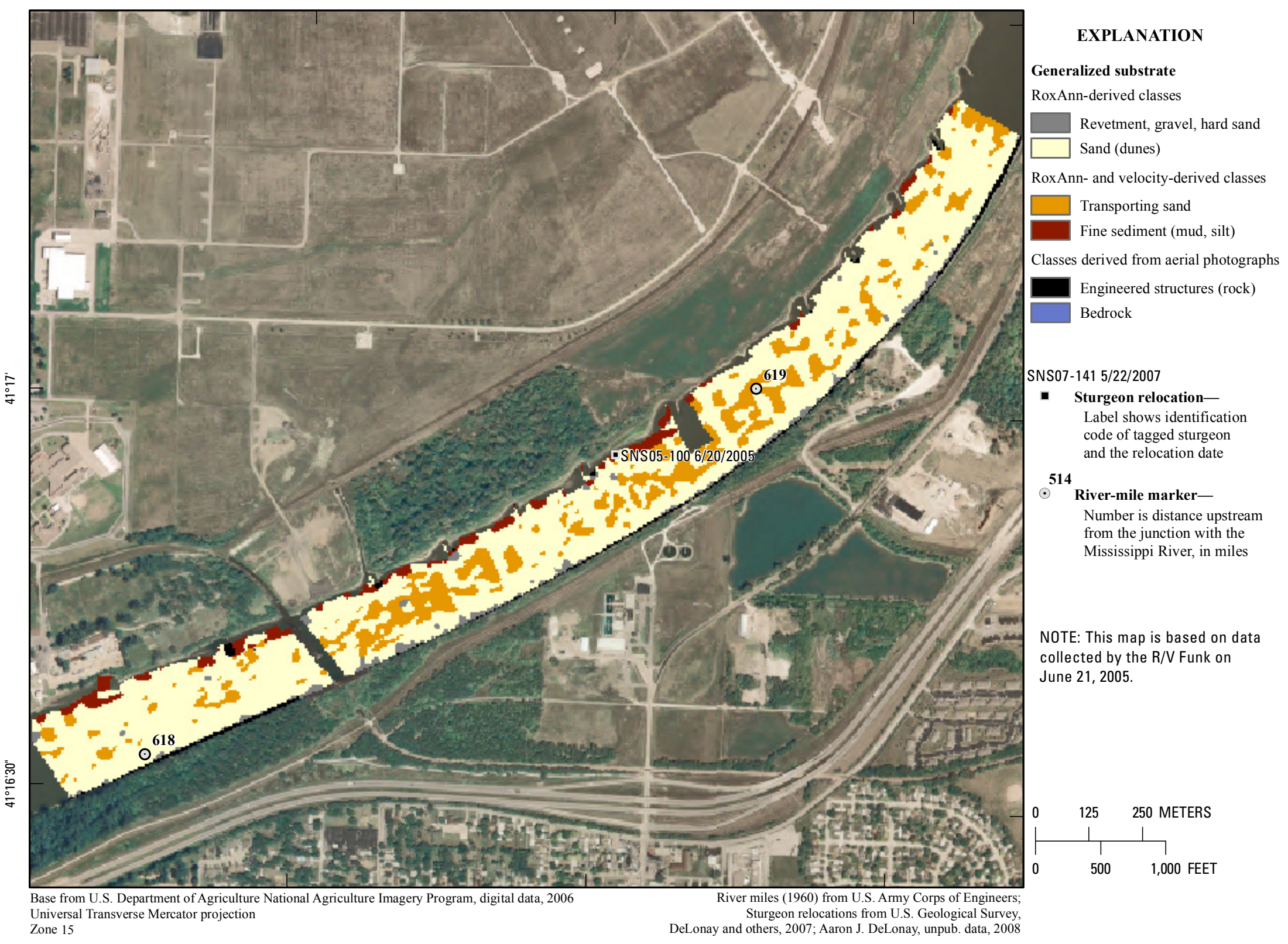




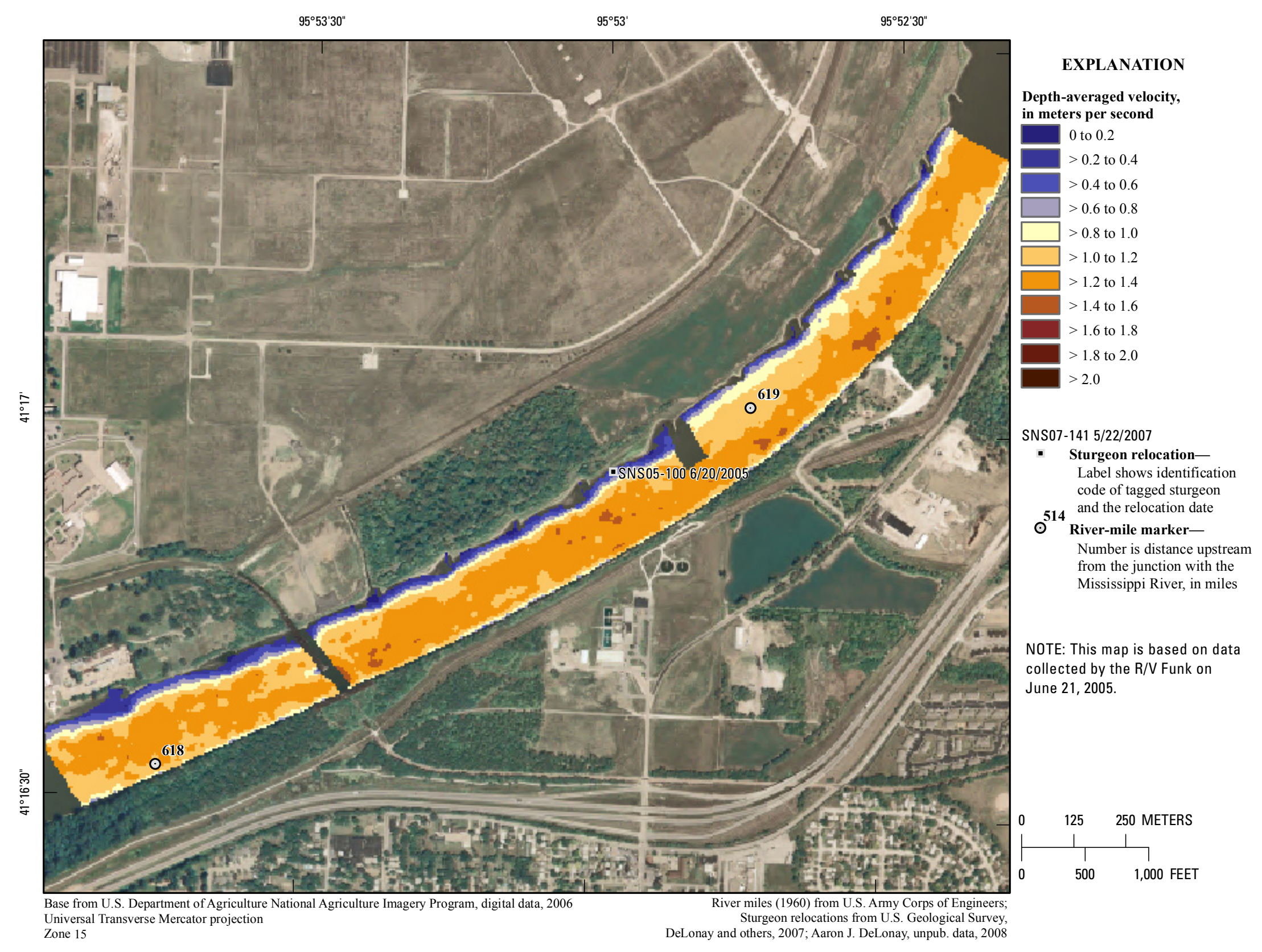

Figure 227. Map of depth-averaged velocity based on data collected on June 21, 2005, in the vicinity of river mile 619 . 


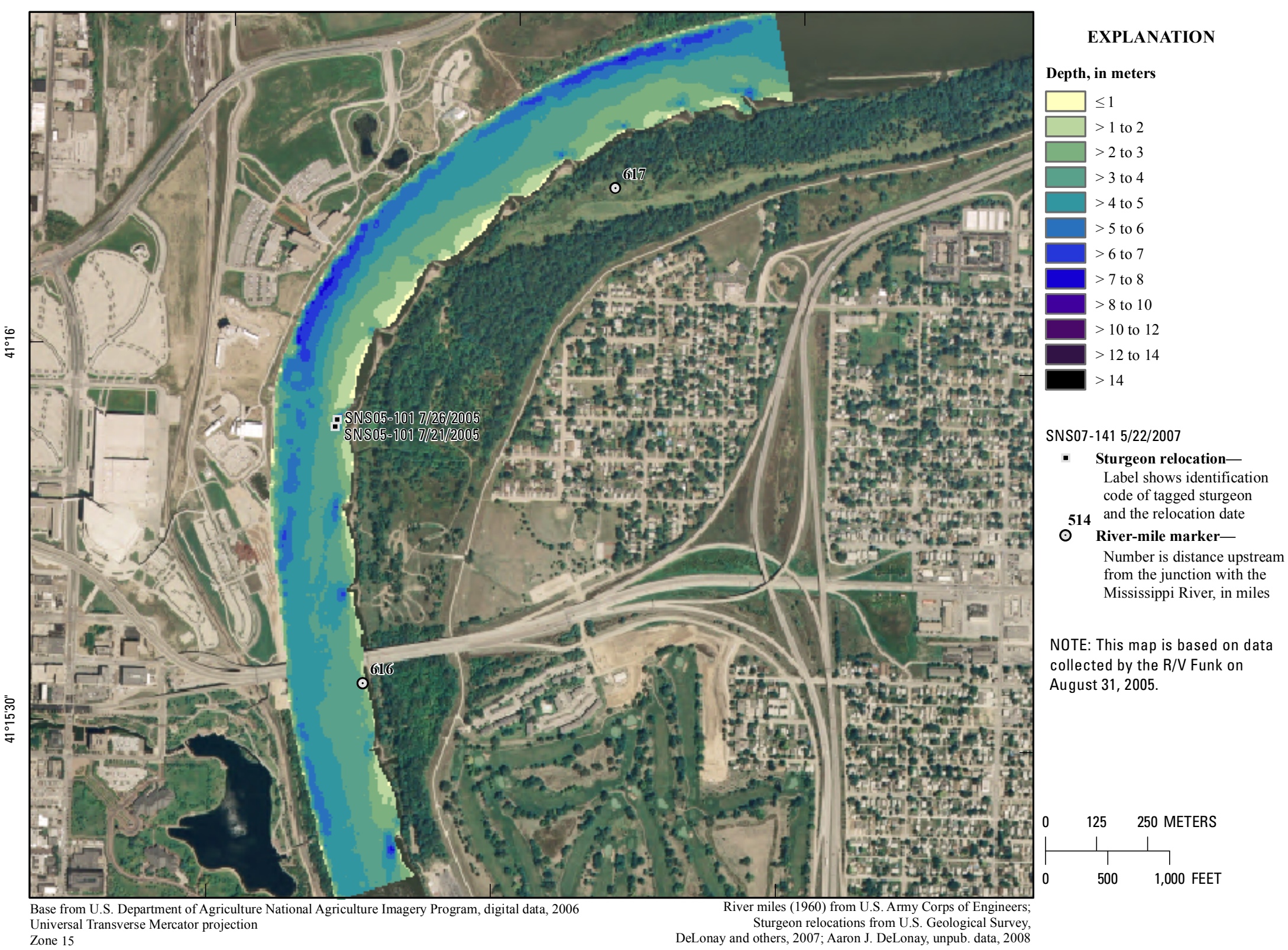




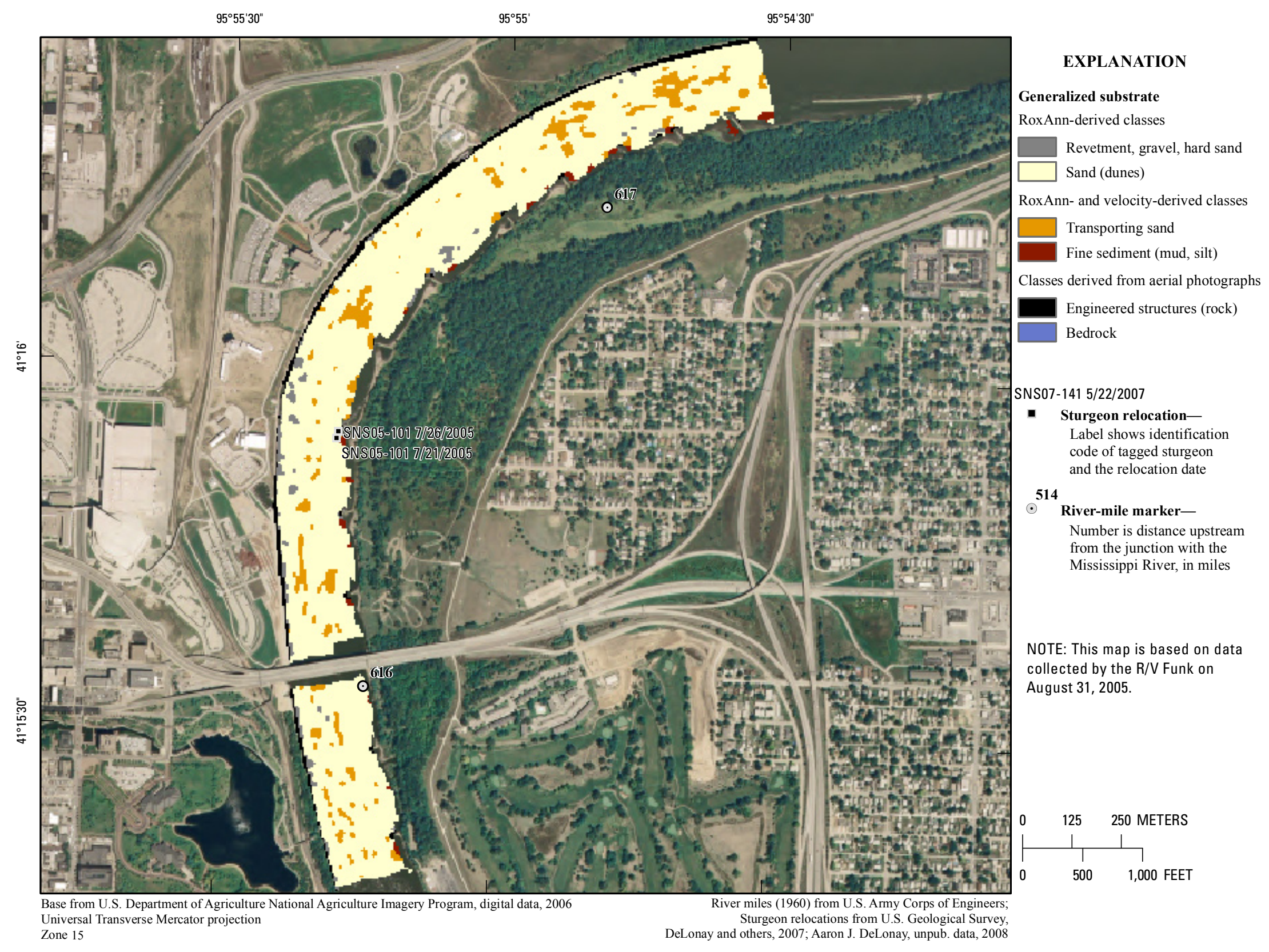

Figure 229. Map of generalized substrate based on data collected on August 31, 2005, in the vicinity of river mile 617 . 


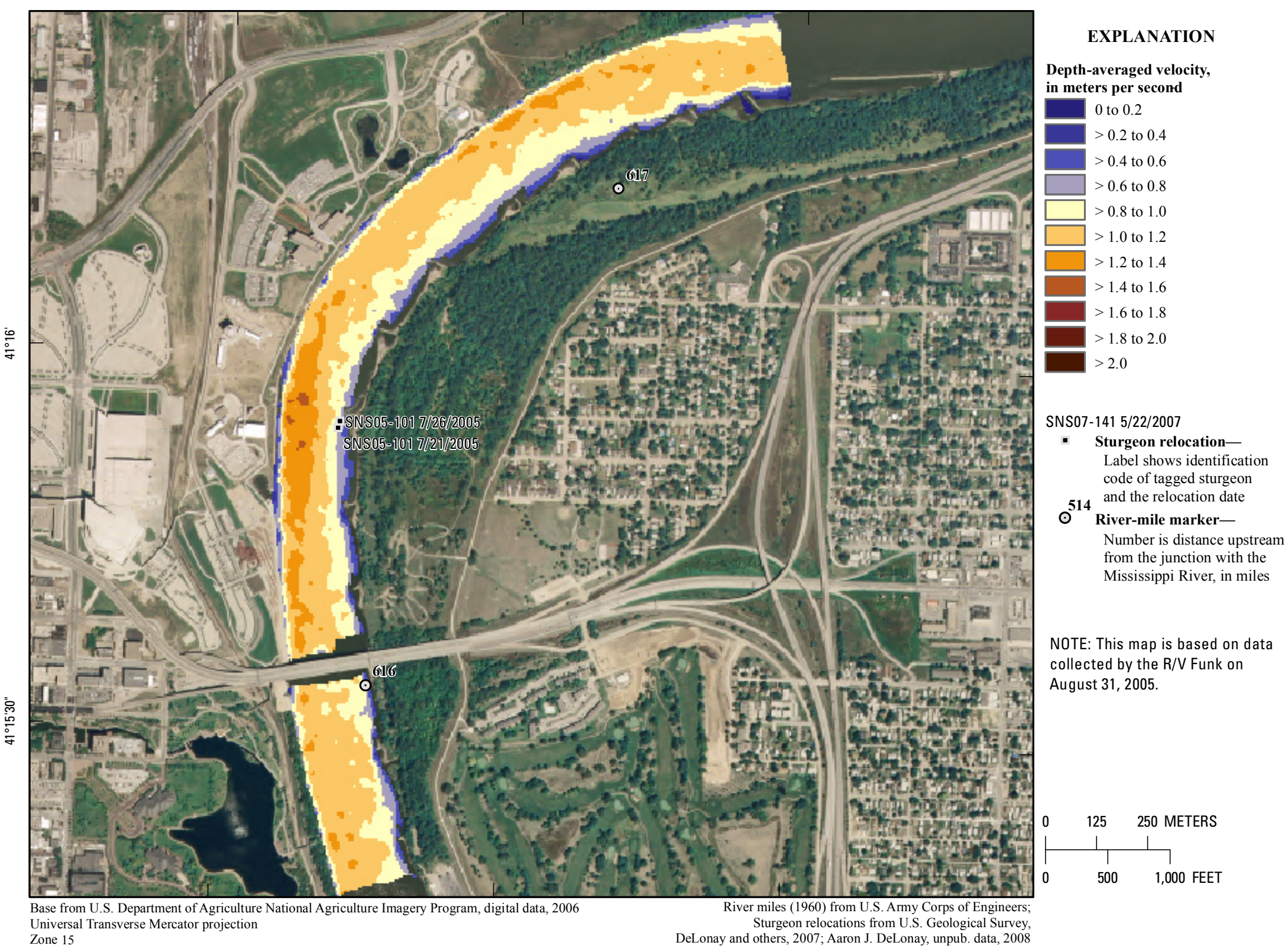




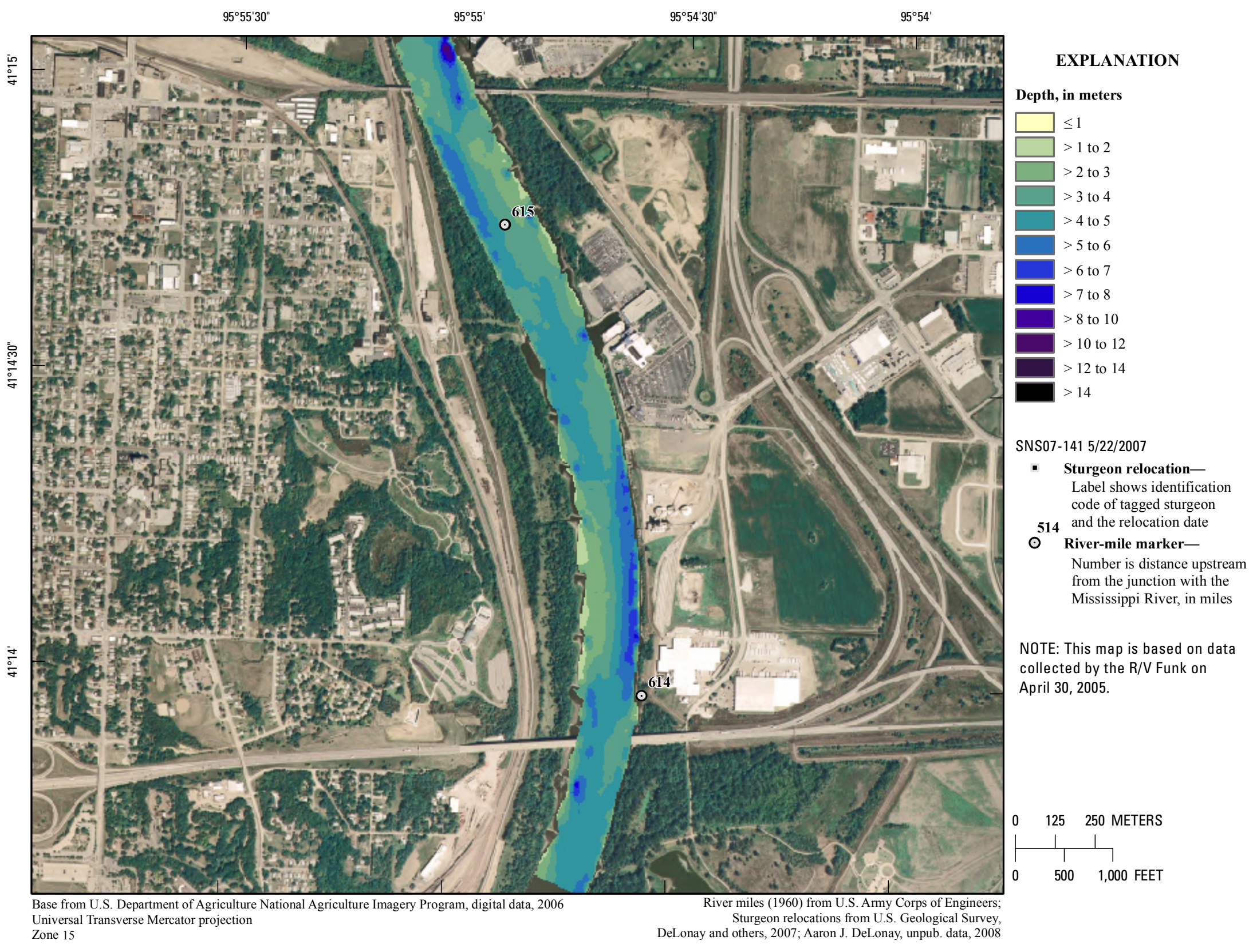

Figure 231. Map of depth based on data collected on April 30, 2005, in the vicinity of river mile 615 . 


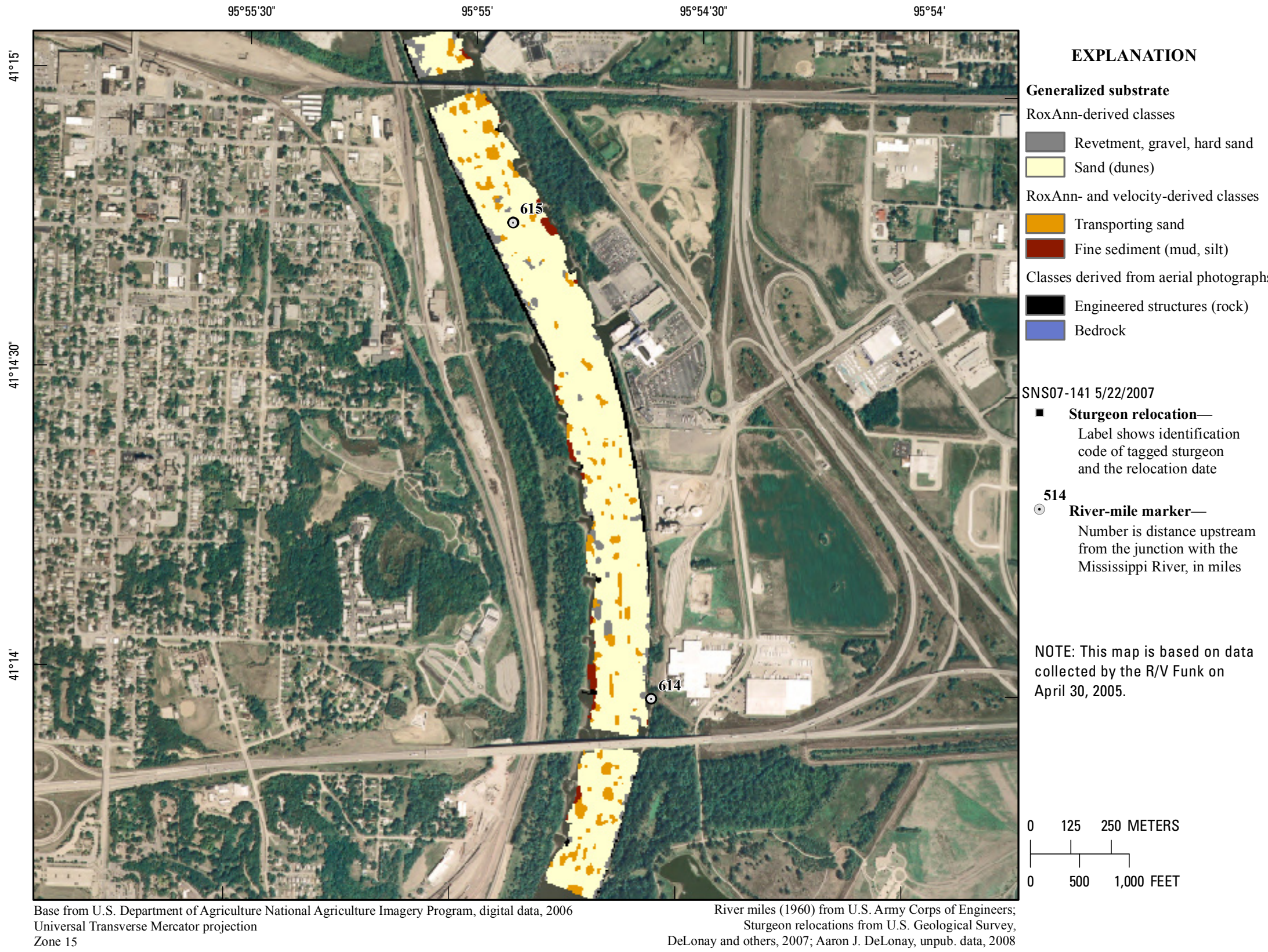

Figure 232. Map of generalized substrate based on data collected on April 30, 2005, in the vicinity of river mile 615 . 


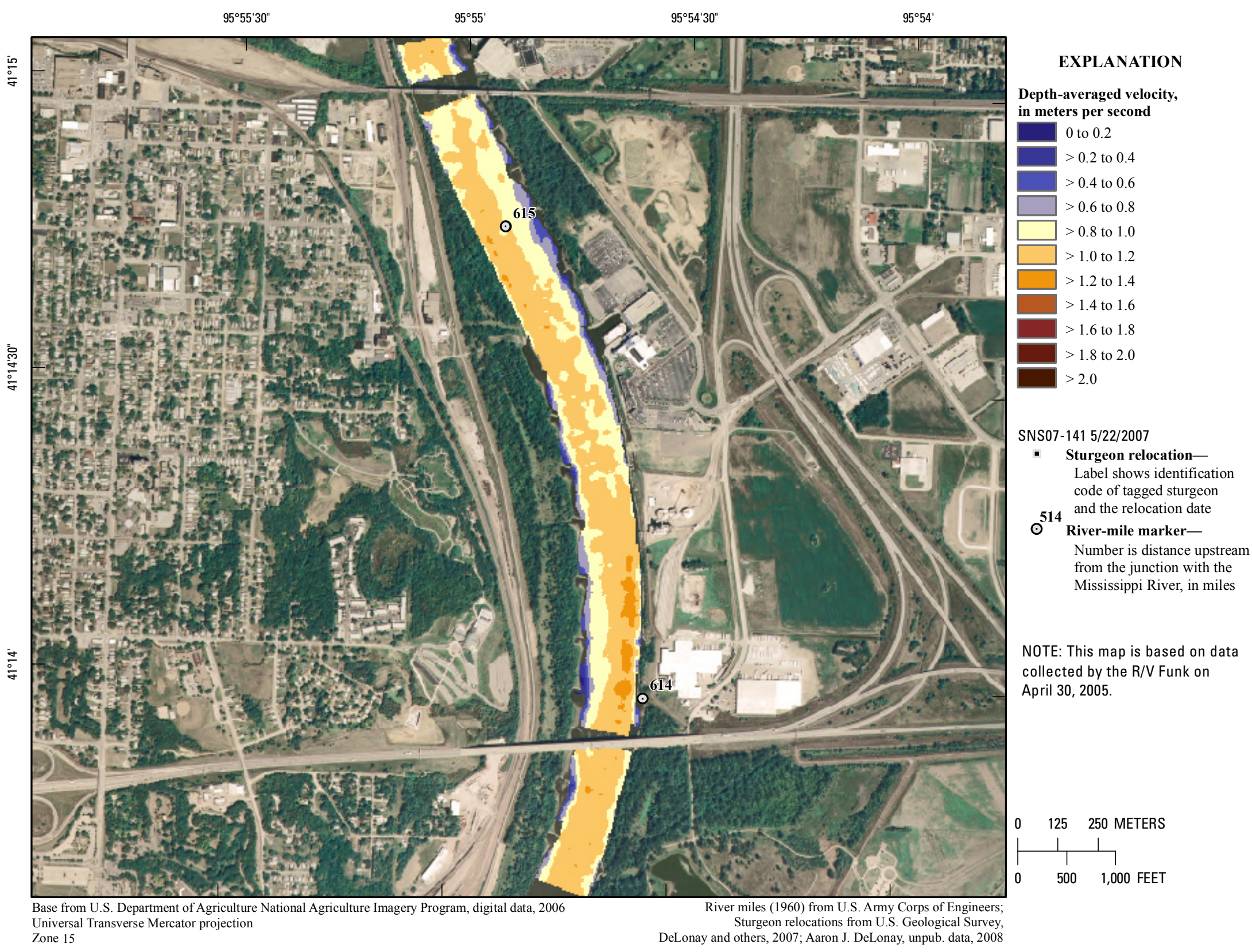

Figure 233. Map of depth-averaged velocity based on data collected on April 30, 2005, in the vicinity of river mile 615 . 


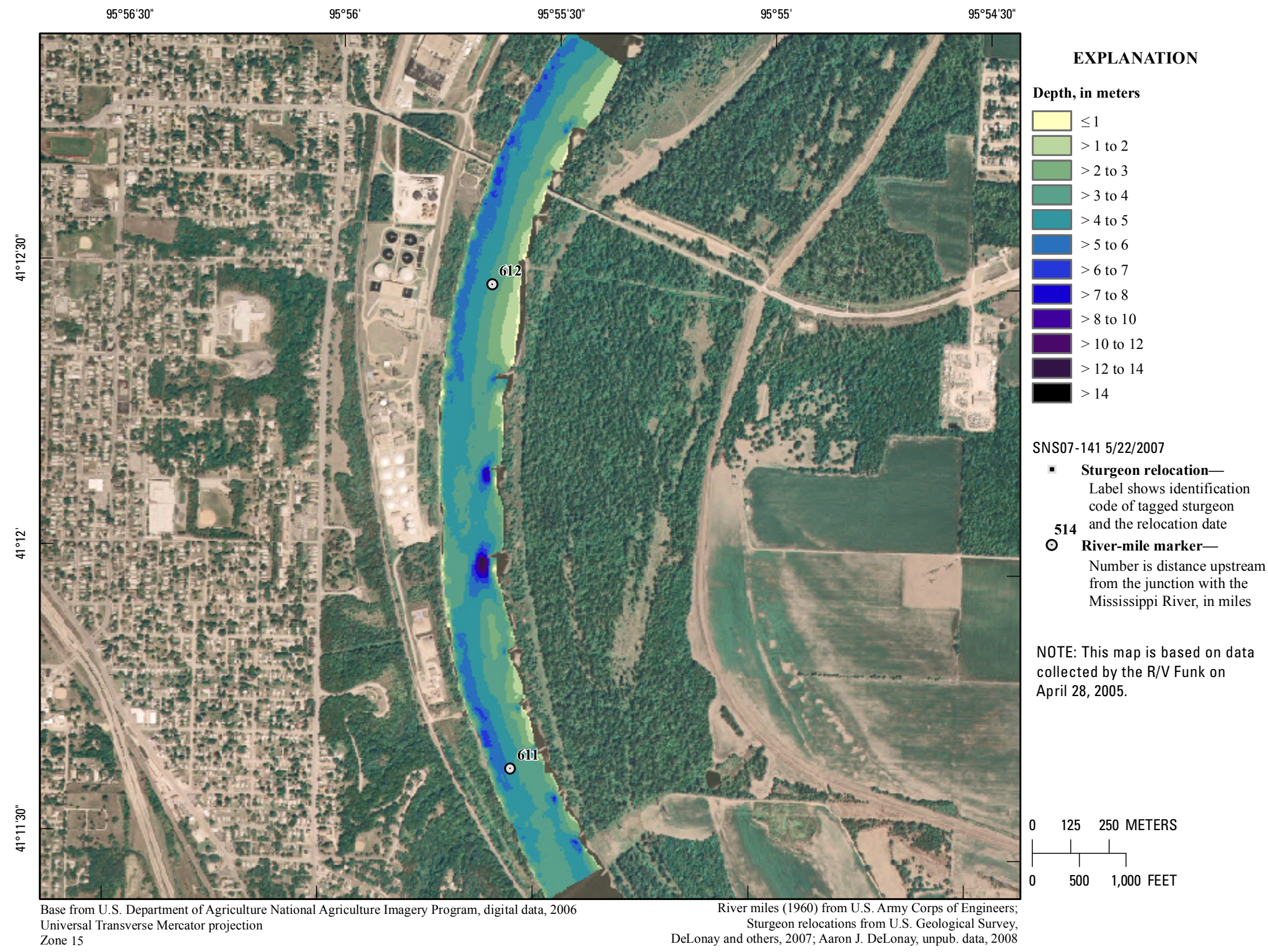

Figure 234. Map of depth based on data collected on April 28, 2005, in the vicinity of river mile 612 . 


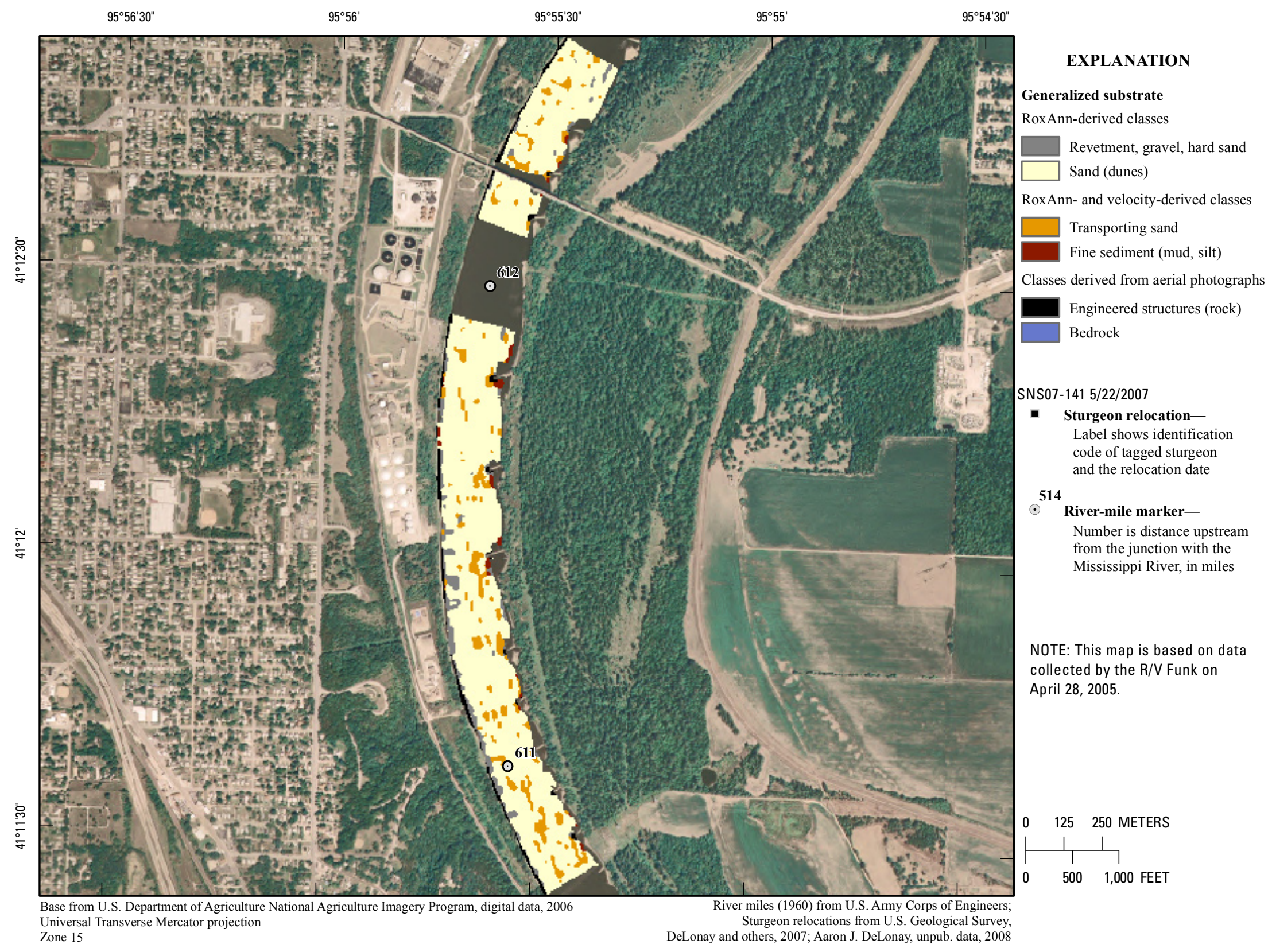

Figure 235. Map of generalized substrate based on data collected on April 28, 2005, in the vicinity of river mile 612 . 


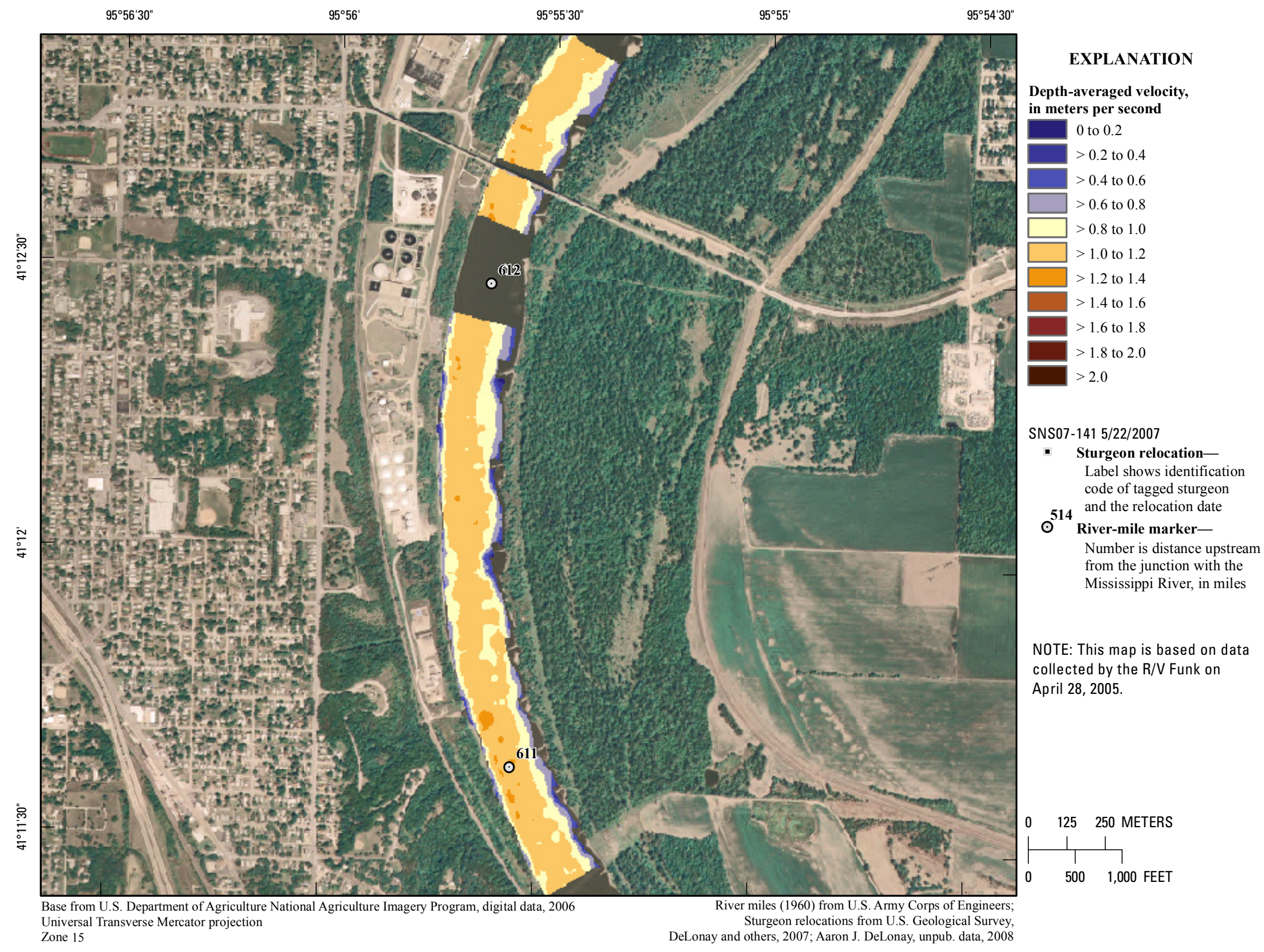

Figure 236. Map of depth-averaged velocity based on data collected on April 28, 2005, in the vicinity of river mile 612 . 


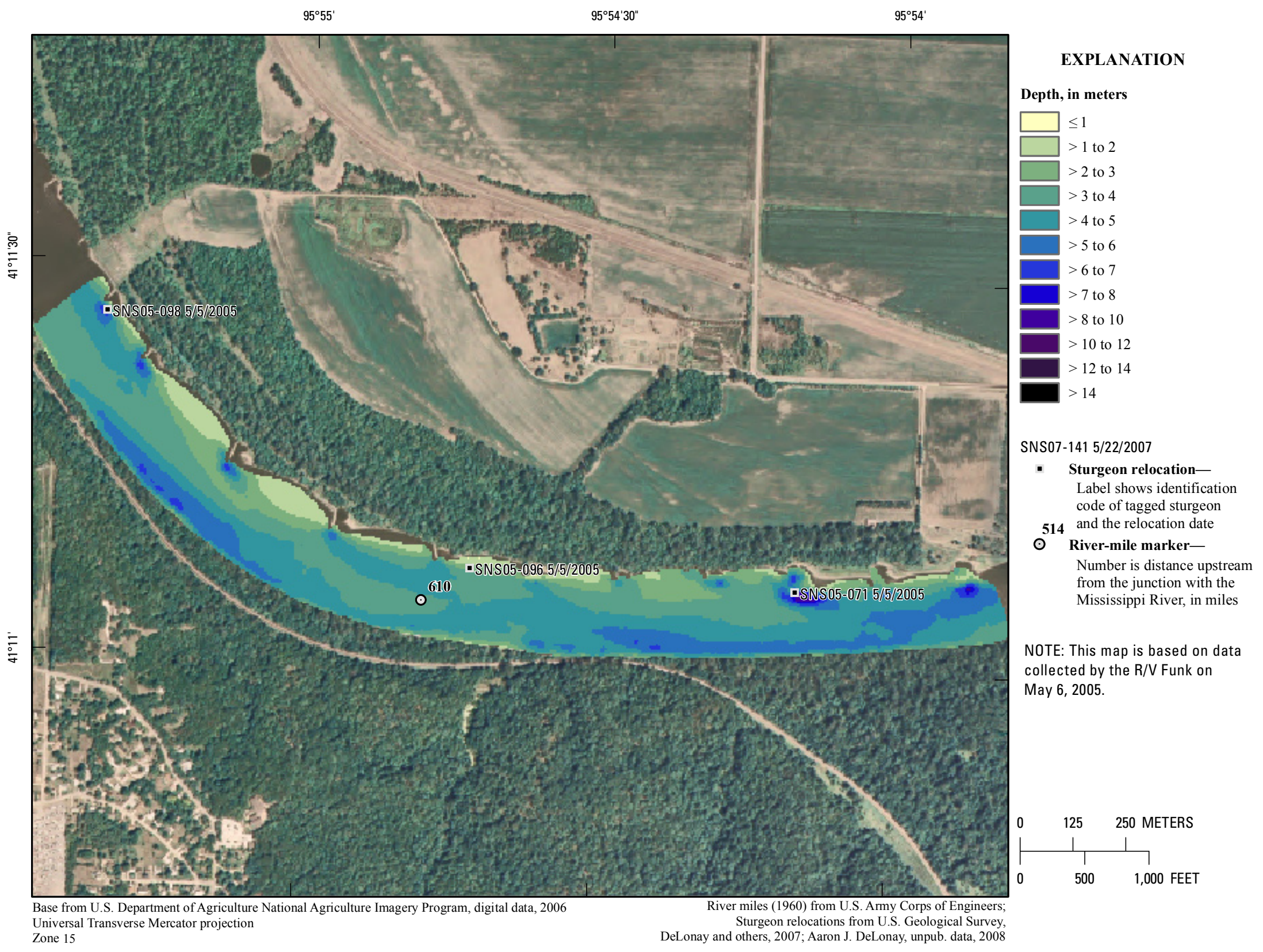

Figure 237. Map of depth based on data collected on May 6, 2005, in the vicinity of river mile 610. 


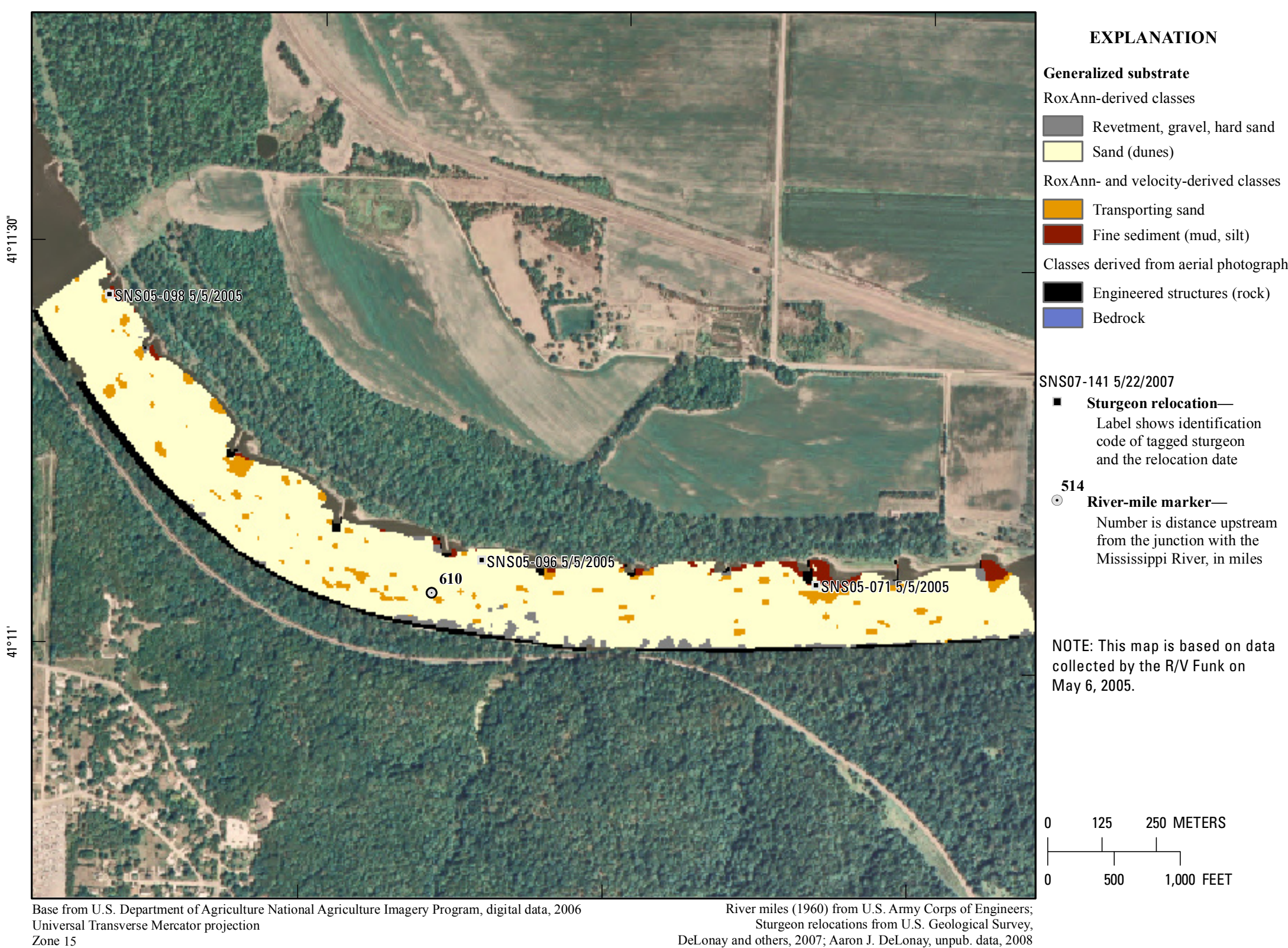




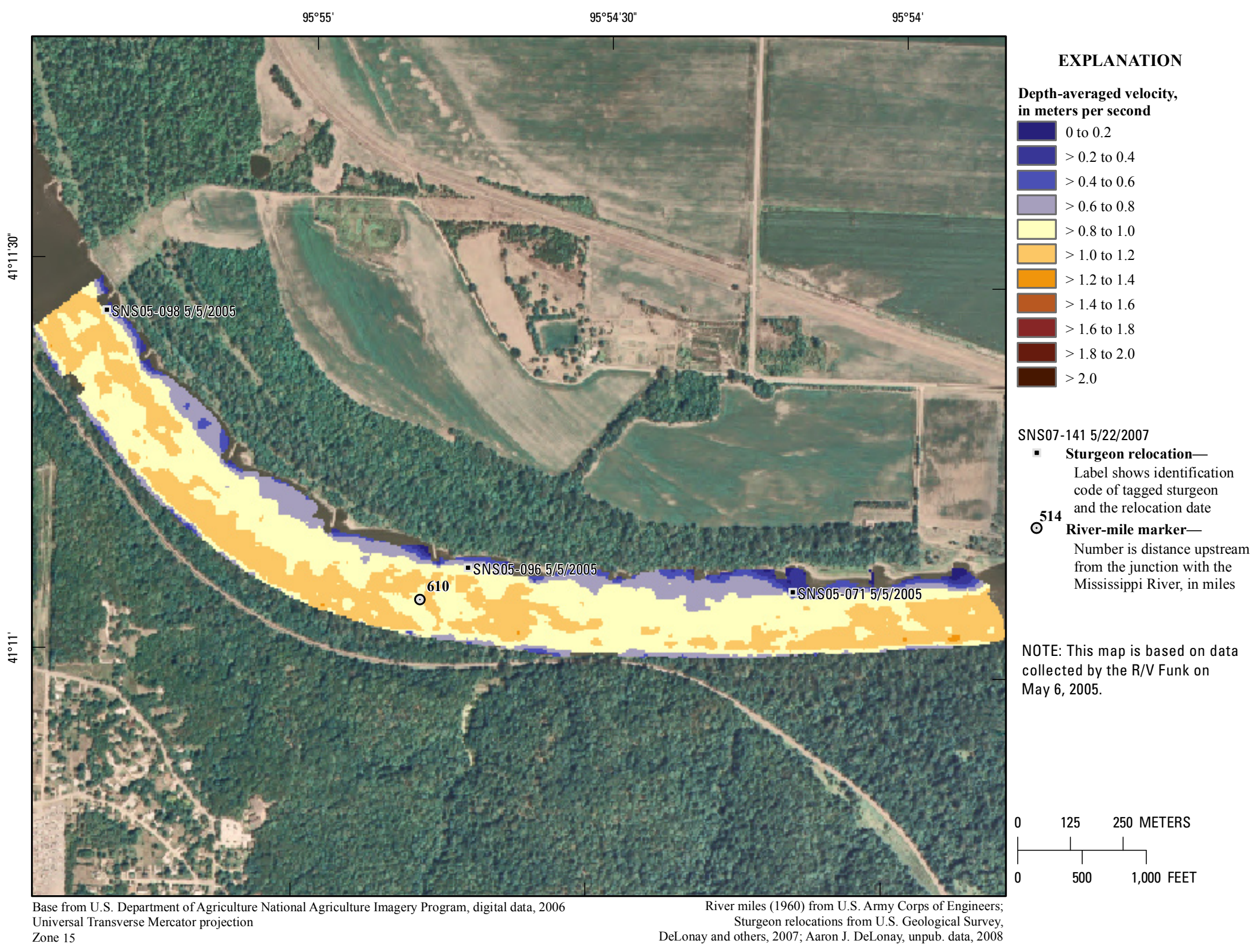

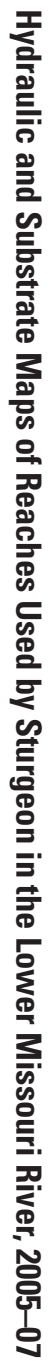

Figure 239. Map of depth-averaged velocity based on data collected on May 6, 2005, in the vicinity of river mile 610 . 


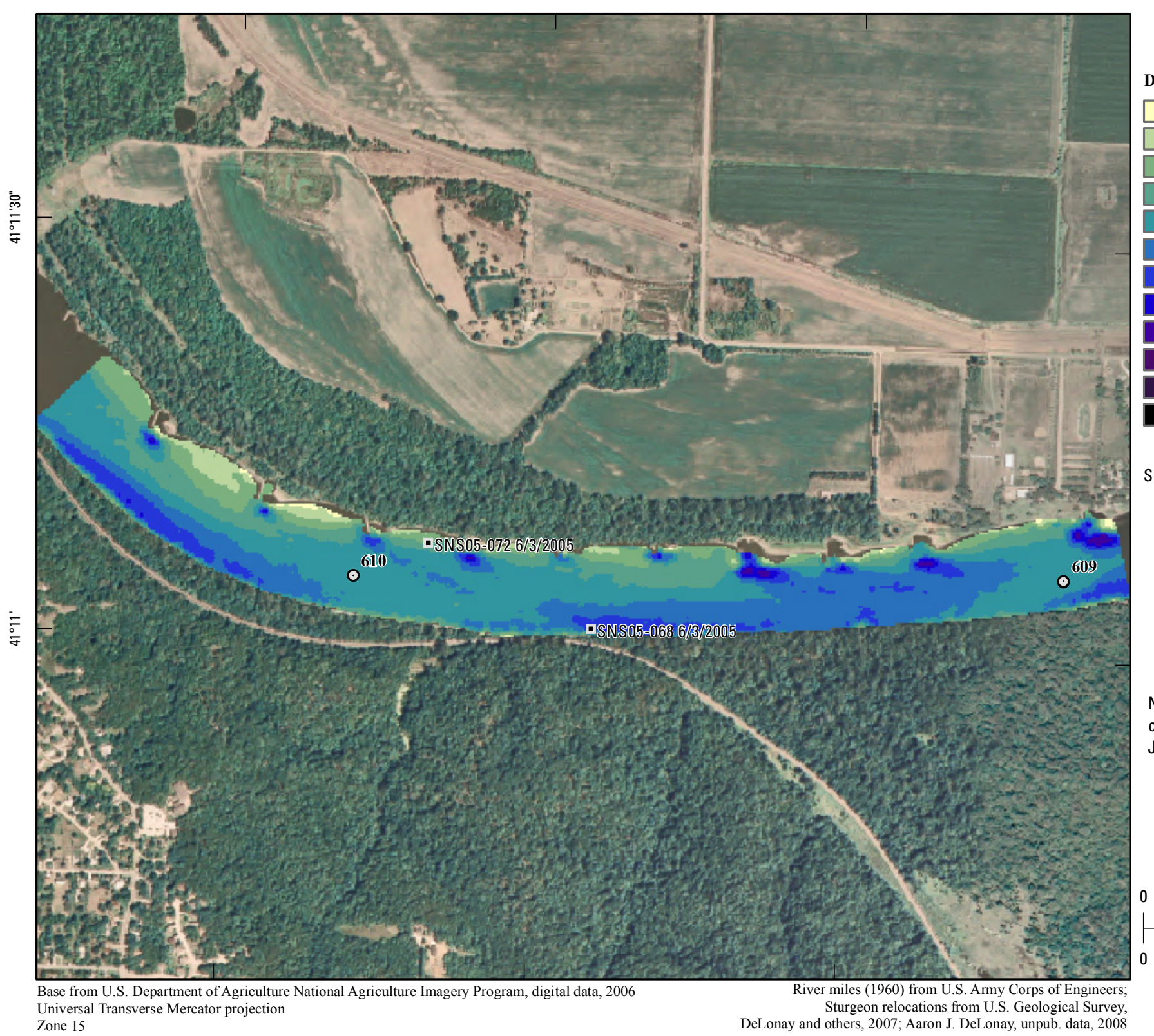

EXPLANATION

Depth, in meters

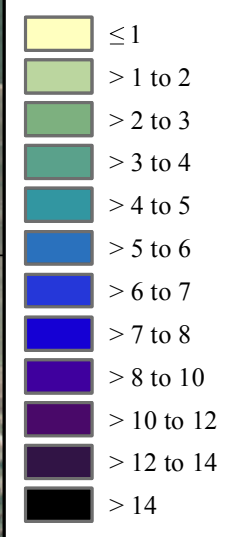

SNS07-141 5/22/2007

- Sturgeon relocationLabel shows identification code of tagged sturgeo 514 and the relocation date

Number is distance upstream from the junction with the Mississippi River, in miles

NOTE: This map is based on data

collected by the R/V Funk on

June 5, 2005.

\begin{tabular}{|c|c|c|}
\hline 0 & 125 & 250 METERS \\
\hline & $\perp$ & $\perp$ \\
\hline & 500 & 1,000 FEET \\
\hline
\end{tabular}
Universal Transverse Mercator projection Sturgeon relocations from U.S. Geological Survey,
DeLonay and others, 2007; Aaron J. DeLonay, unpub. data, 2008

$500 \quad 1,000$ FEET 


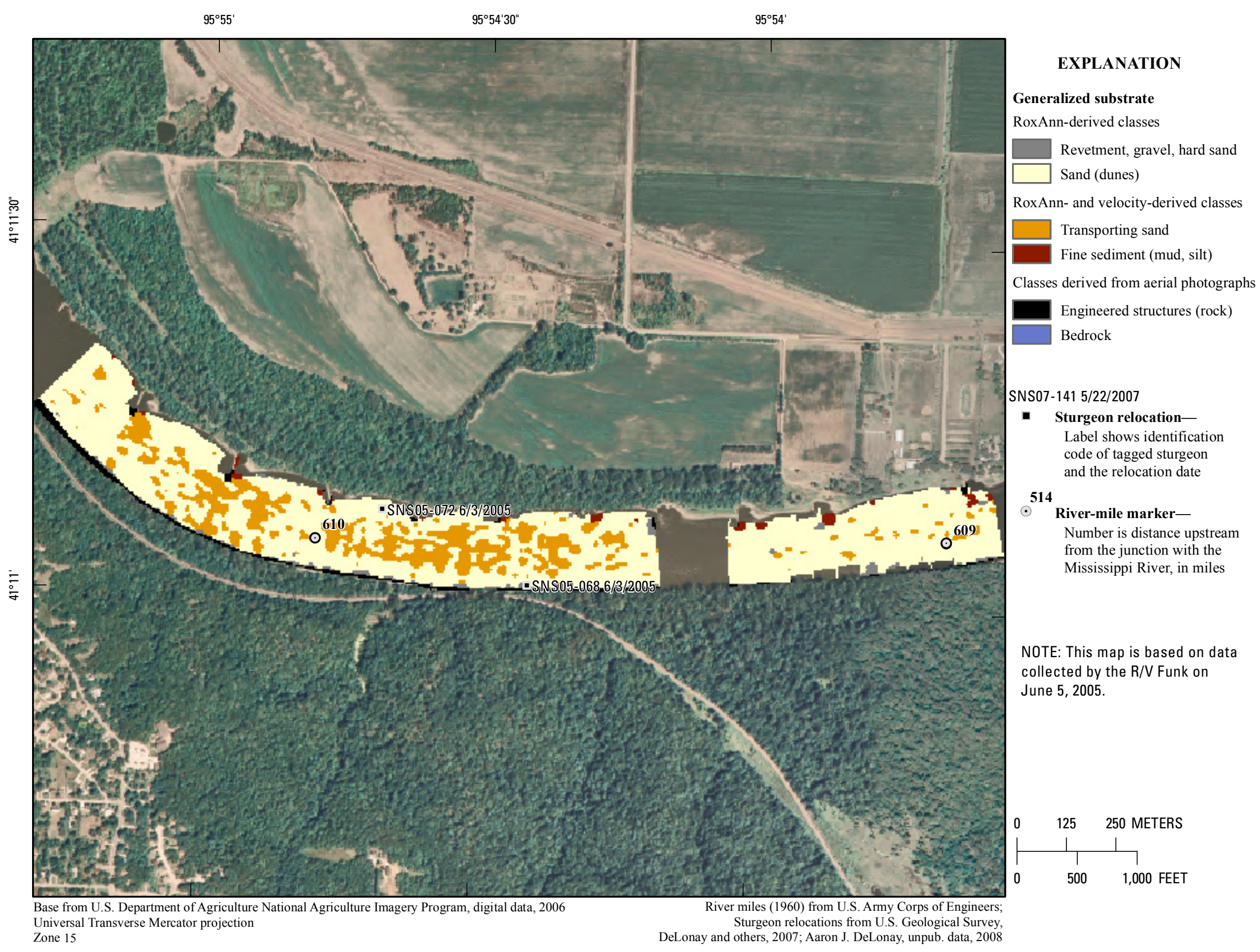

Figure 241. Map of generalized substrate based on data collected on June 5, 2005, in the vicinity of river mile 610 . 


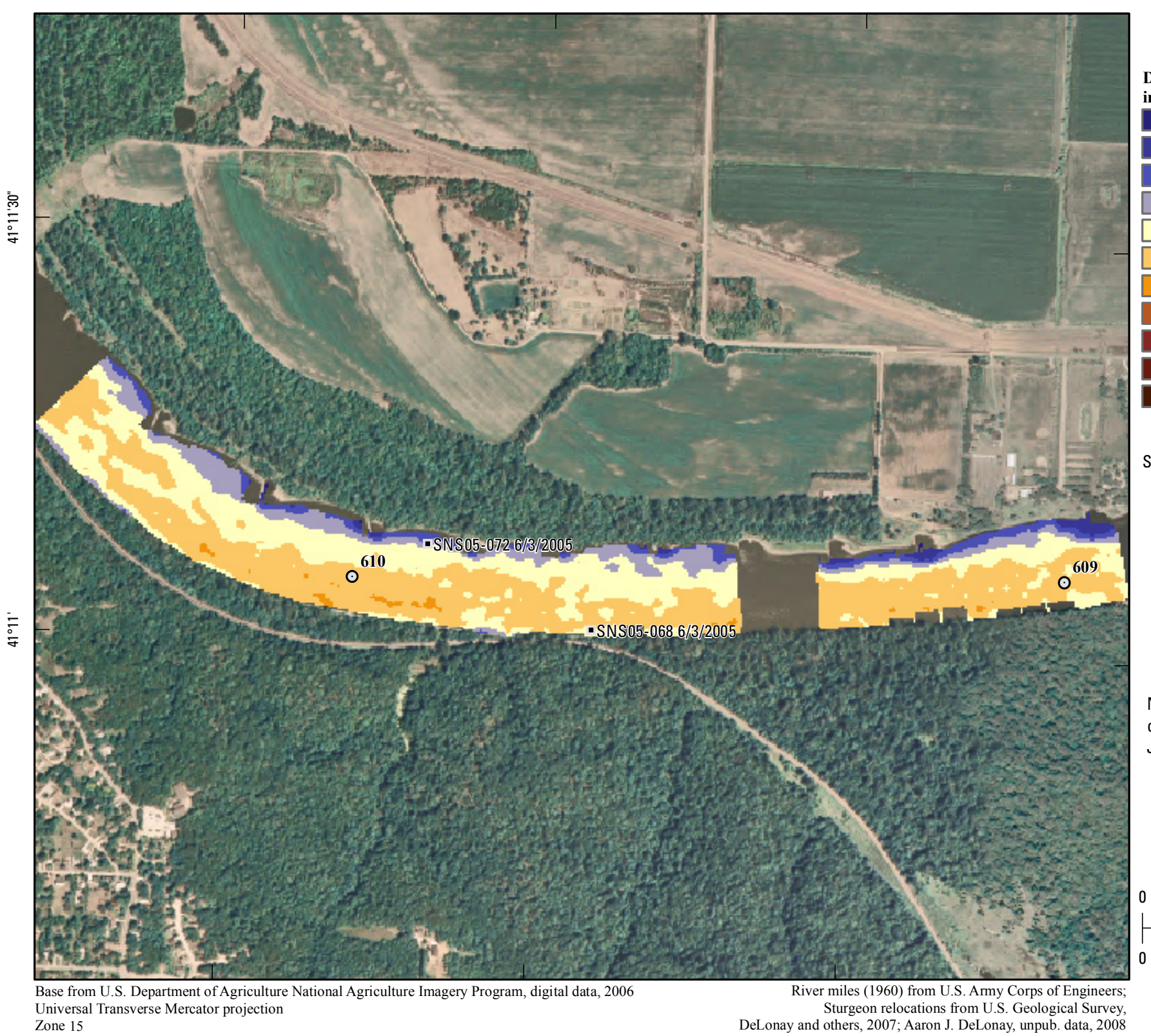

EXPLANATION

Depth-averaged velocity,

in meters per second

0 to 0.2

$>0.2$ to 0.4

$>0.4$ to 0.6

$>0.6$ to 0.8

$>0.8$ to 1.0

$>1.0$ to 1.2

$>1.2$ to 1.4

$>1.4$ to 1.6

$>1.6$ to 1.8

$>1.8$ to 2.0

$>2.0$

SNS07-141 5/22/2007

- Sturgeon relocationLabel shows identification code of tagged sturgeo

514 and the relocation date

Number is distance upstream
from the junction with the

Mississippi River, in miles

NOTE: This map is based on data

collected by the R/V Funk on

June 5, 2005

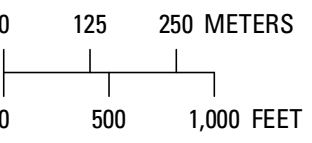

DeLonay and

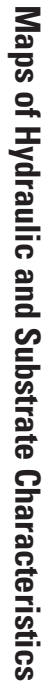

Figure 242. Map of depth-averaged velocity based on data collected on June 5, 2005, in the vicinity of river mile 610 . 


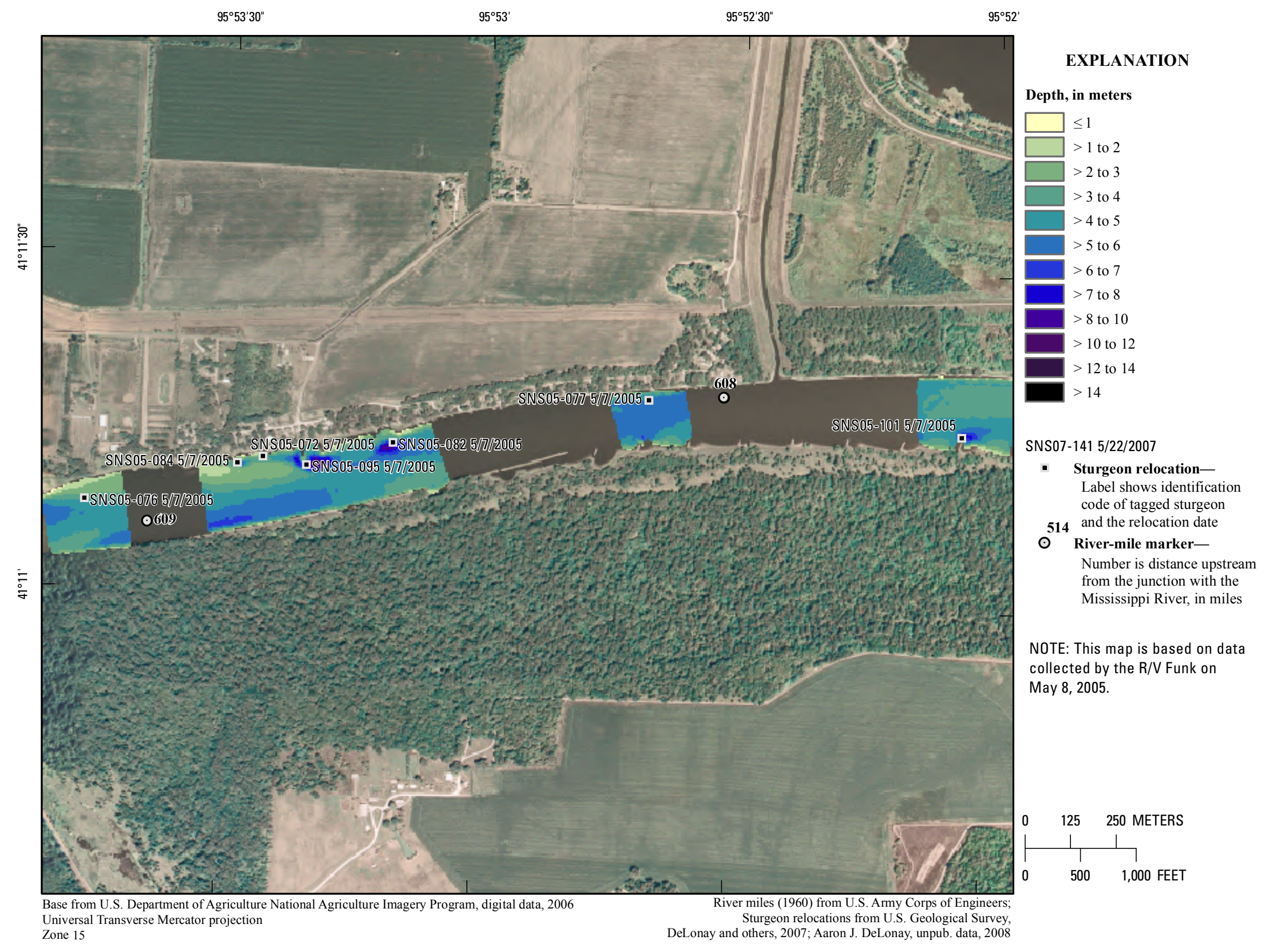

Figure 243. Map of depth based on data collected on May 8, 2005, in the vicinity of river mile 609 . 


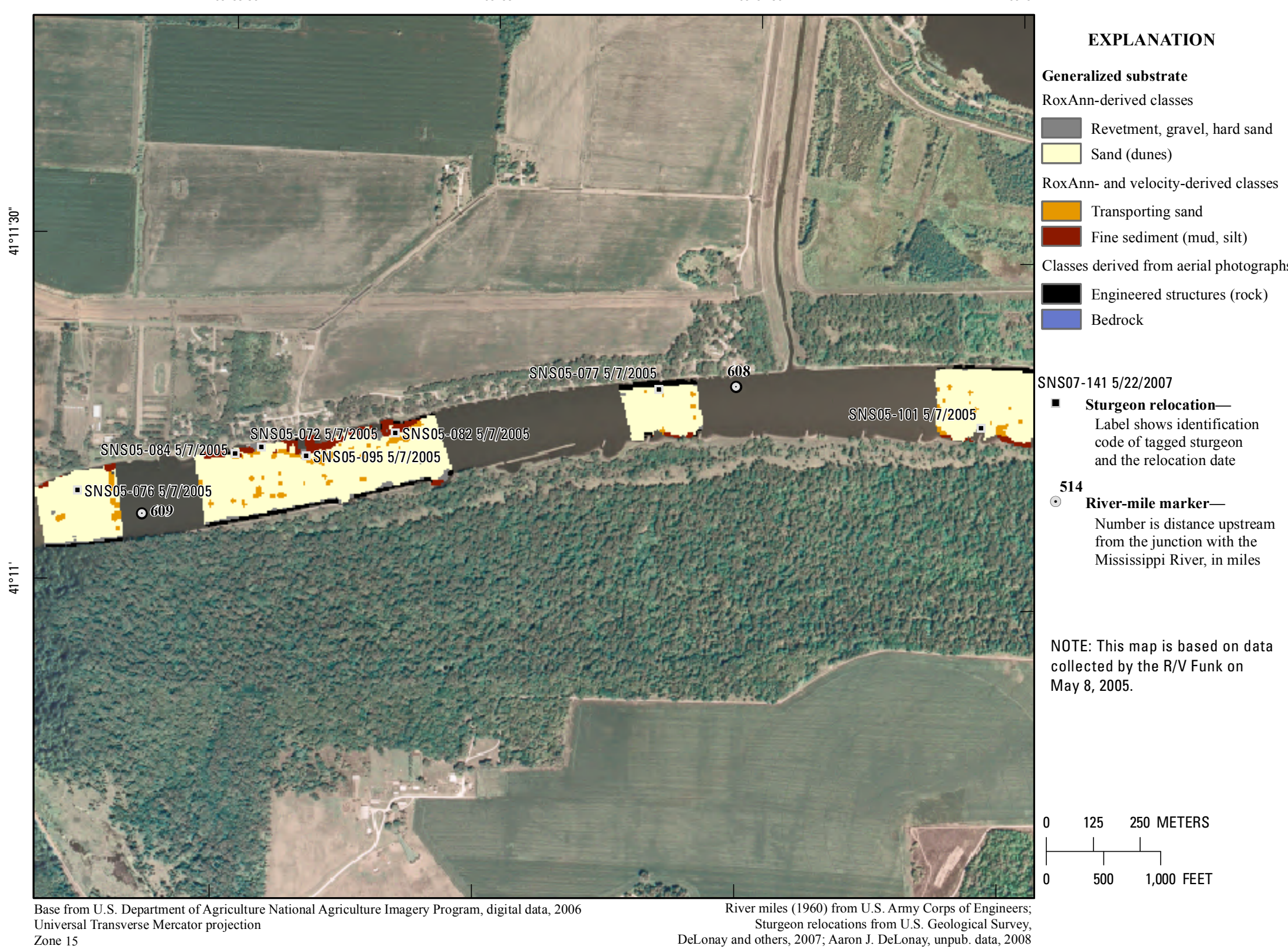




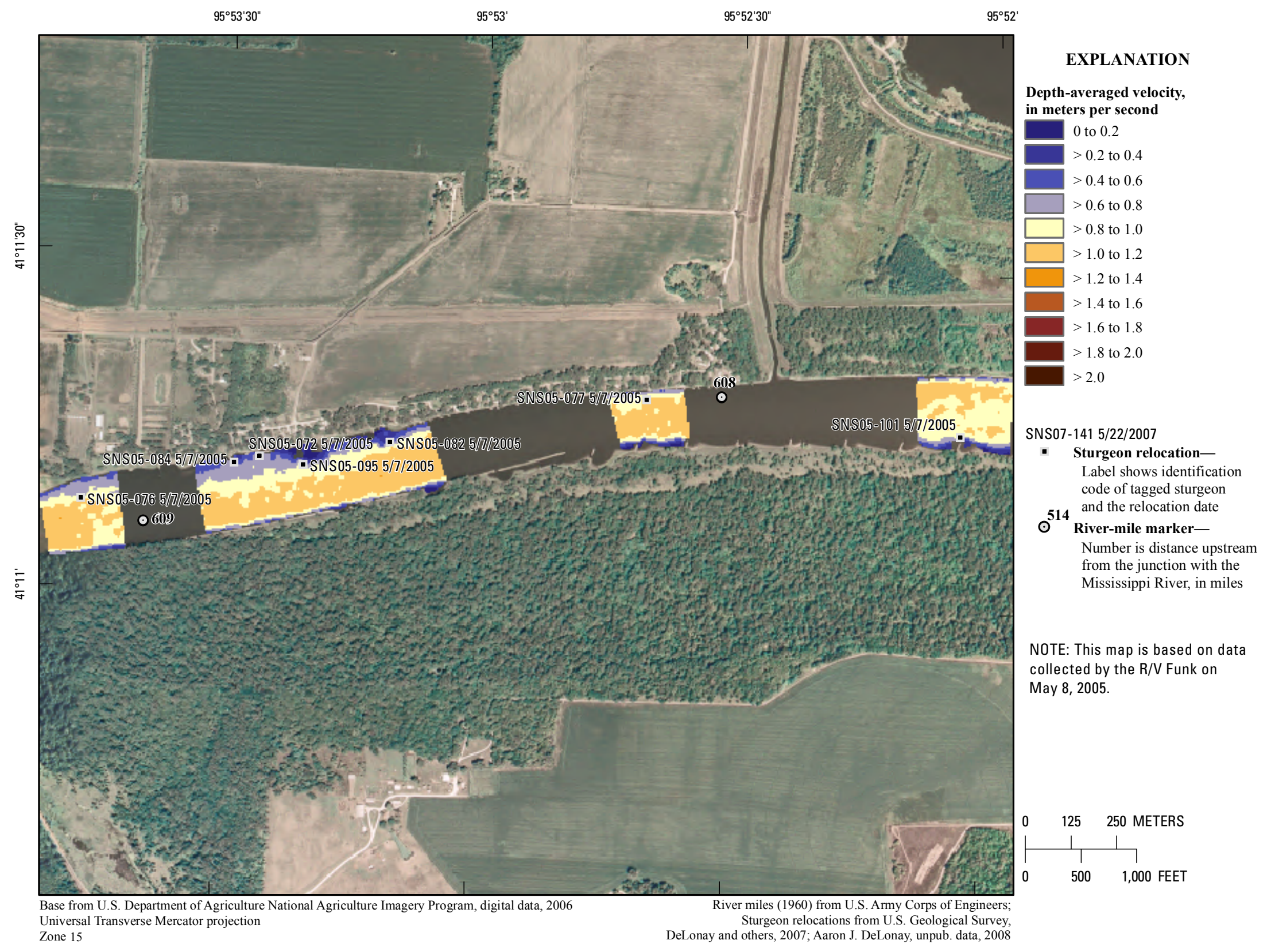

Figure 245. Map of depth-averaged velocity based on data collected on May 8, 2005, in the vicinity of river mile 609 . 


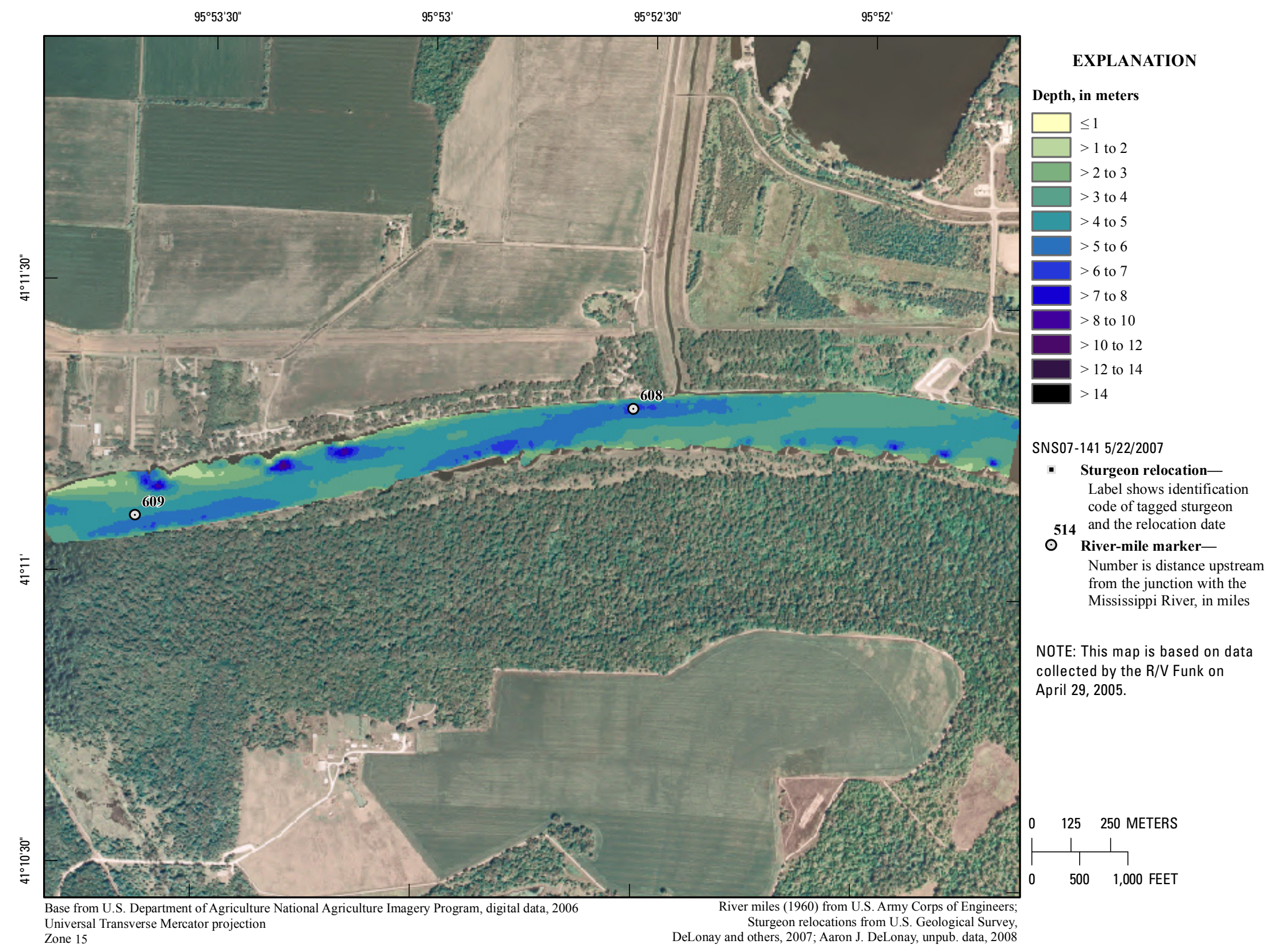

Figure 246. Map of depth based on data collected on April 29, 2005, in the vicinity of river mile 608 . 


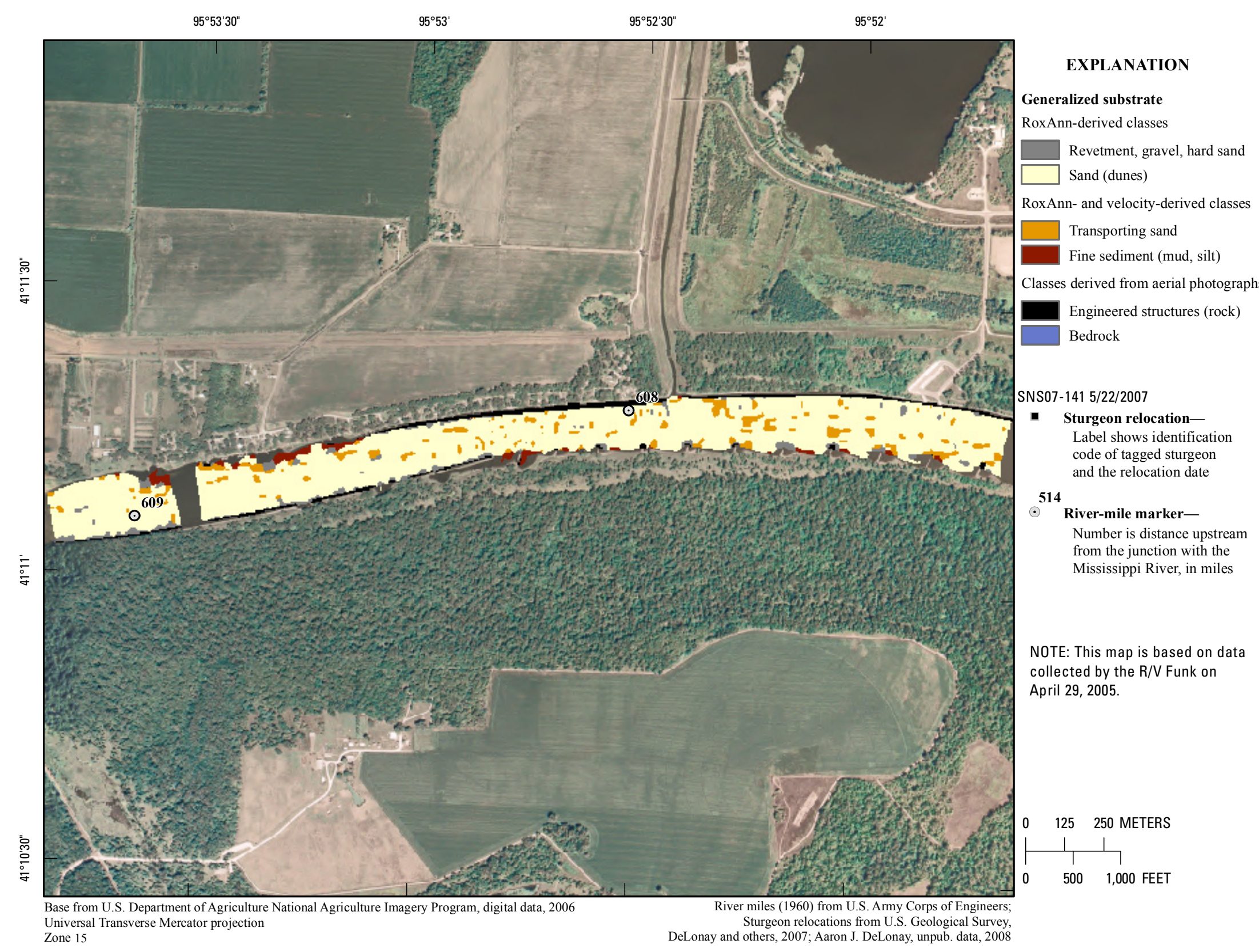

Figure 247. Map of generalized substrate based on data collected on April 29, 2005, in the vicinity of river mile 608 . 


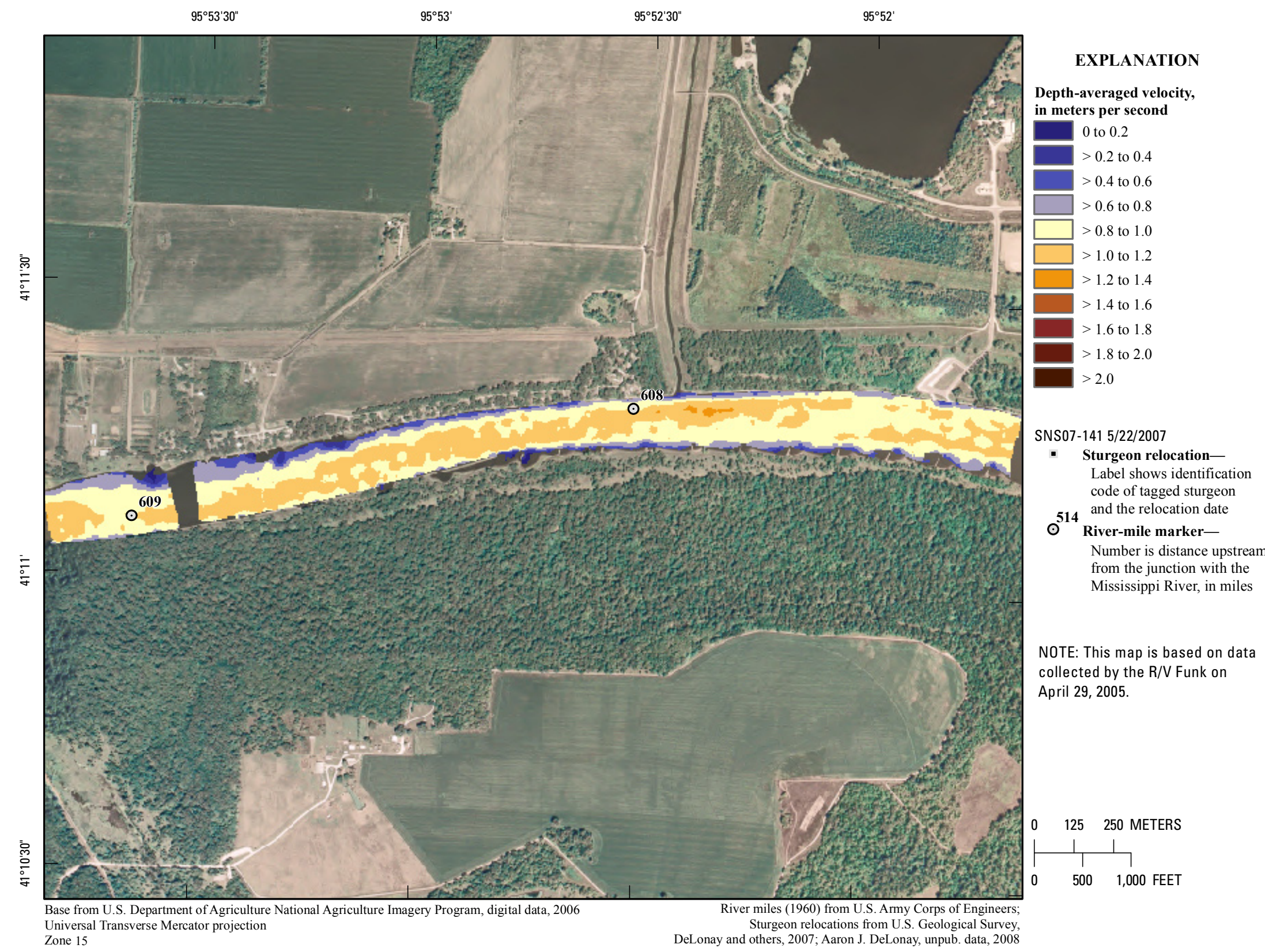

Figure 248. Map of depth-averaged velocity based on data collected on April 29, 2005, in the vicinity of river mile 608 . 


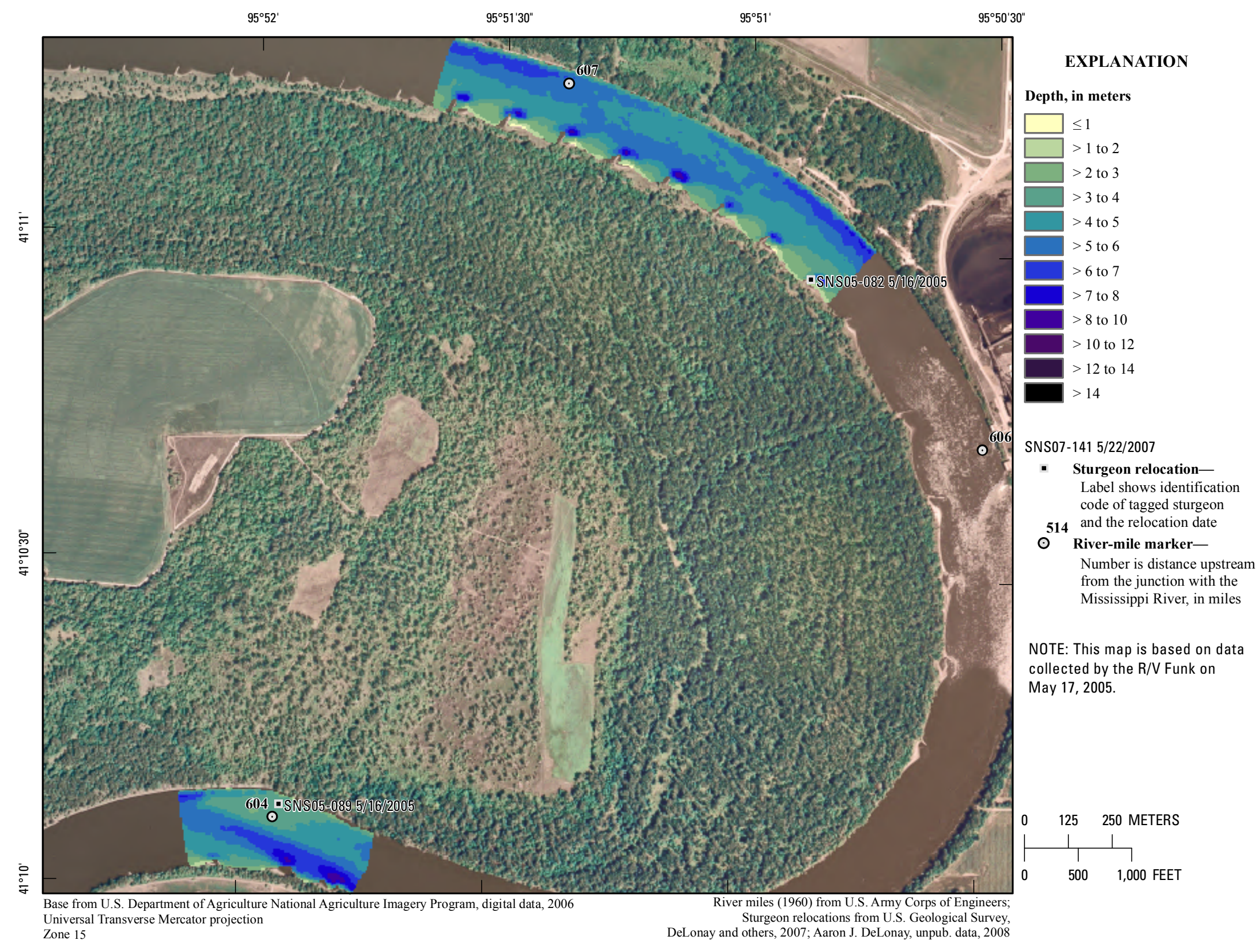

Figure 249. Map of depth based on data collected on May 17, 2005, in the vicinity of river mile 607 . 


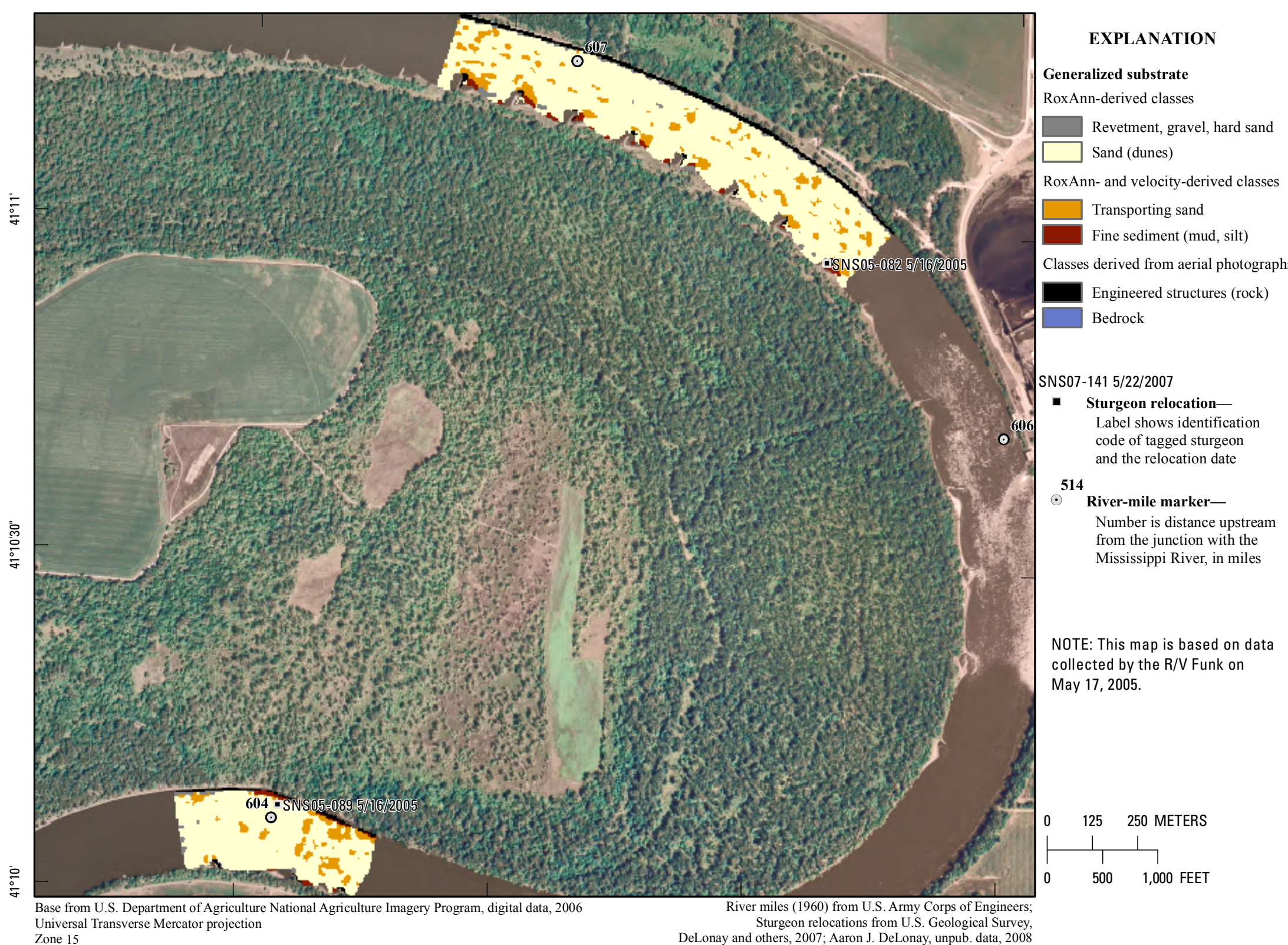




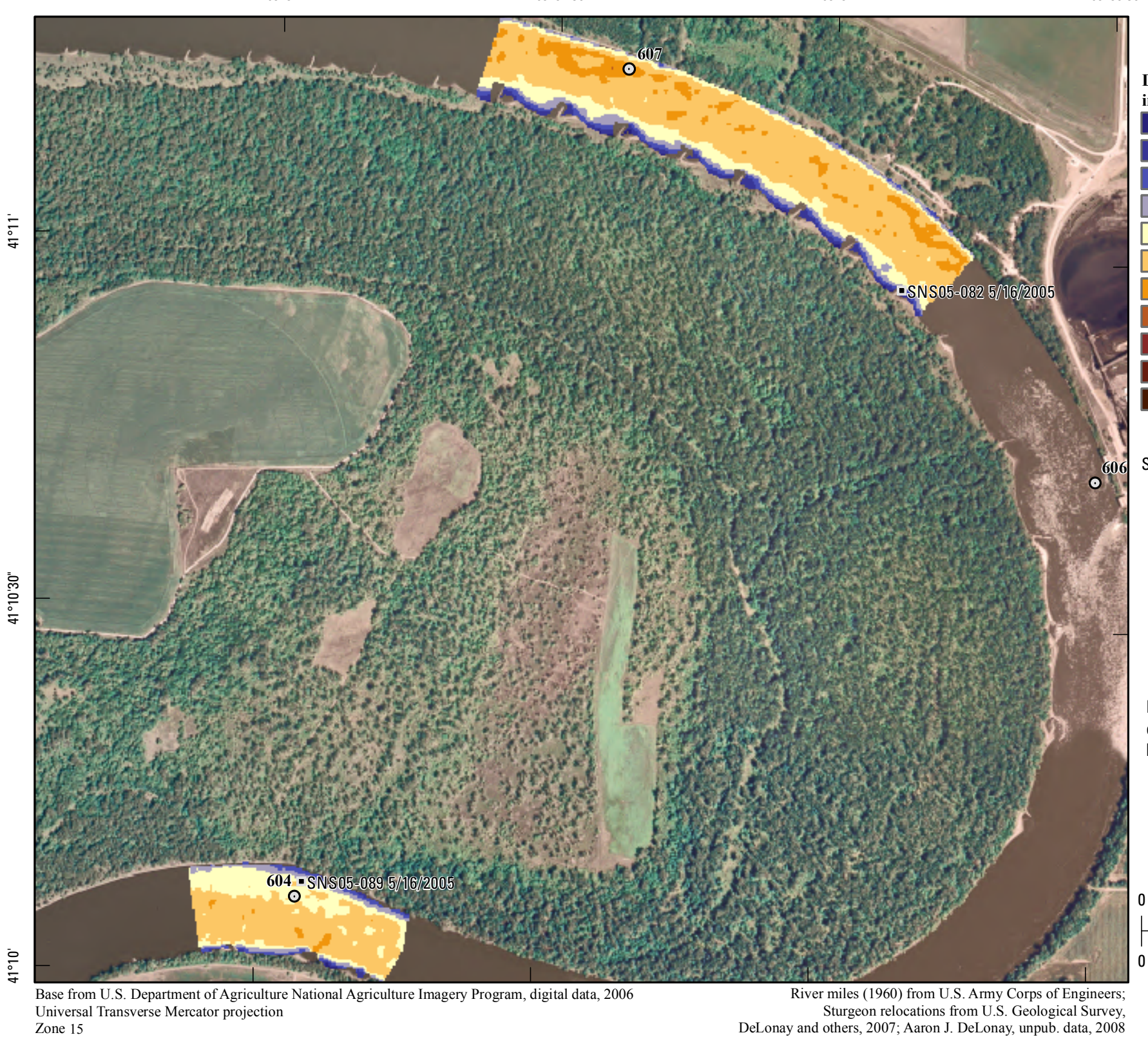

\section{EXPLANATION}

Depth-averaged velocity, in meters per second

0 to 0.2

$>0.2$ to 0.4

$>0.4$ to 0.6

$>0.6$ to 0.8

$>0.8$ to 1.0

$>1.0$ to 1.2

$>1.2$ to 1.4

$>1.4$ to 1.6

$>1.6$ to 1.8

$>1.8$ to 2.0

$>2.0$

\section{SNS07-141 5/22/2007}

Sturgeon relocationLabel shows identification code of tagged sturgeon

514 and the relocation date

514 River-mile marker-

Number is distance upstream

from the junction with the

Mississippi River, in miles

NOTE: This map is based on data

collected by the R/V Funk on

May 17, 2005.

\section{$125 \quad 250$ METERS

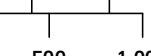 \\ $500 \quad 1,000$ FEET}

Figure 251. Map of depth-averaged velocity based on data collected on May 17, 2005, in the vicinity of river mile 607 


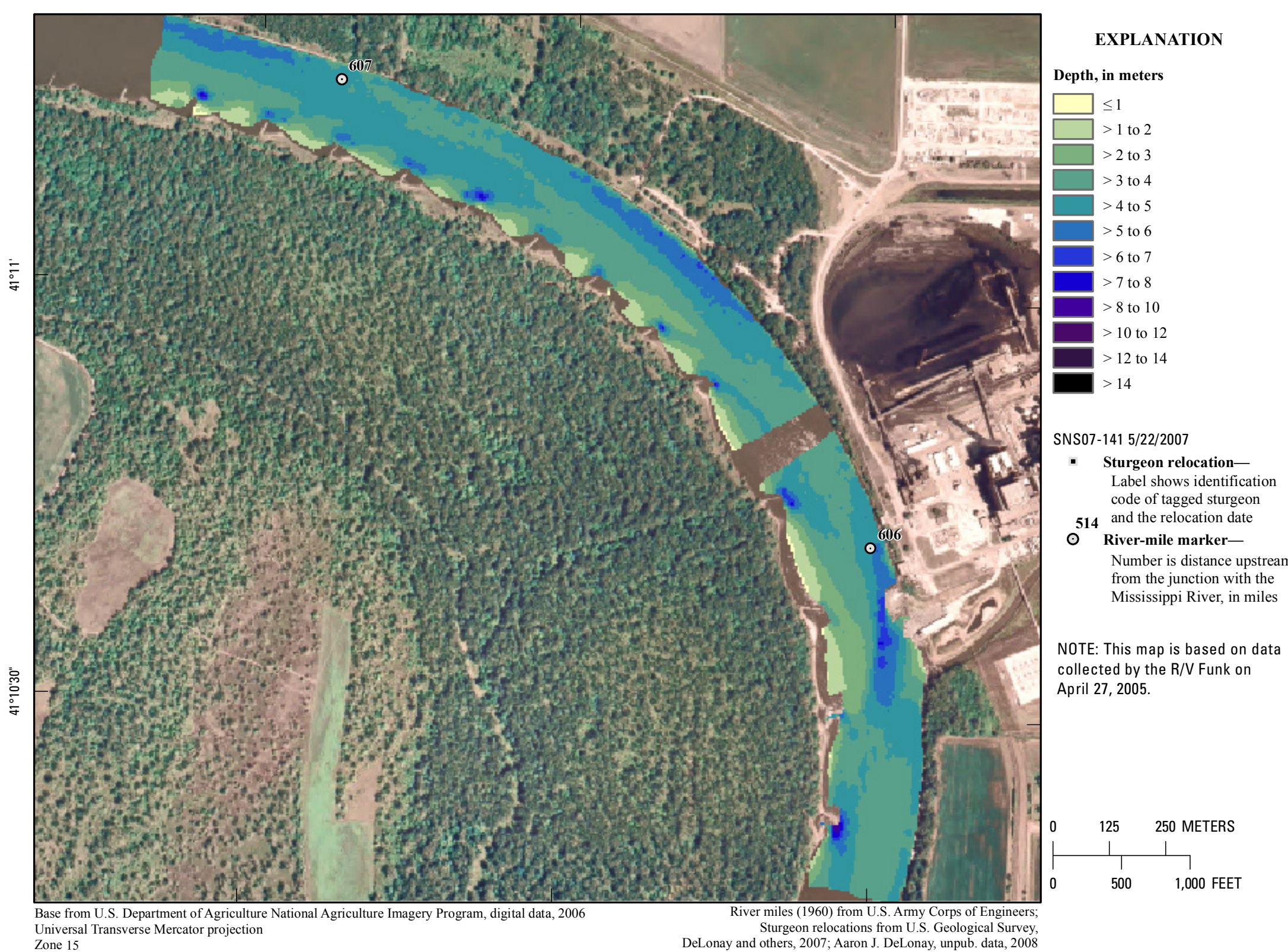




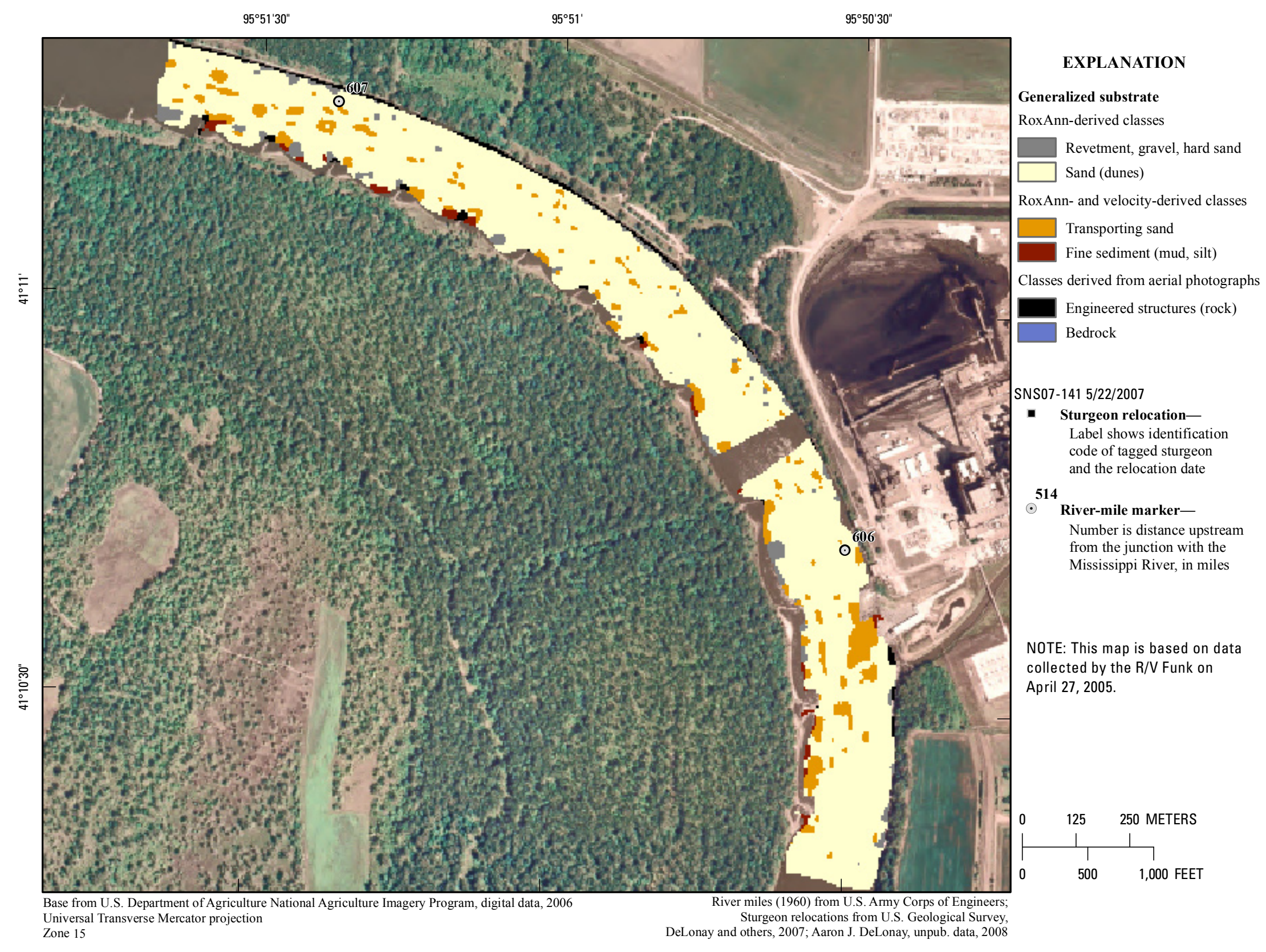

Figure 253. Map of generalized substrate based on data collected on April 27, 2005, in the vicinity of river mile 606 . 


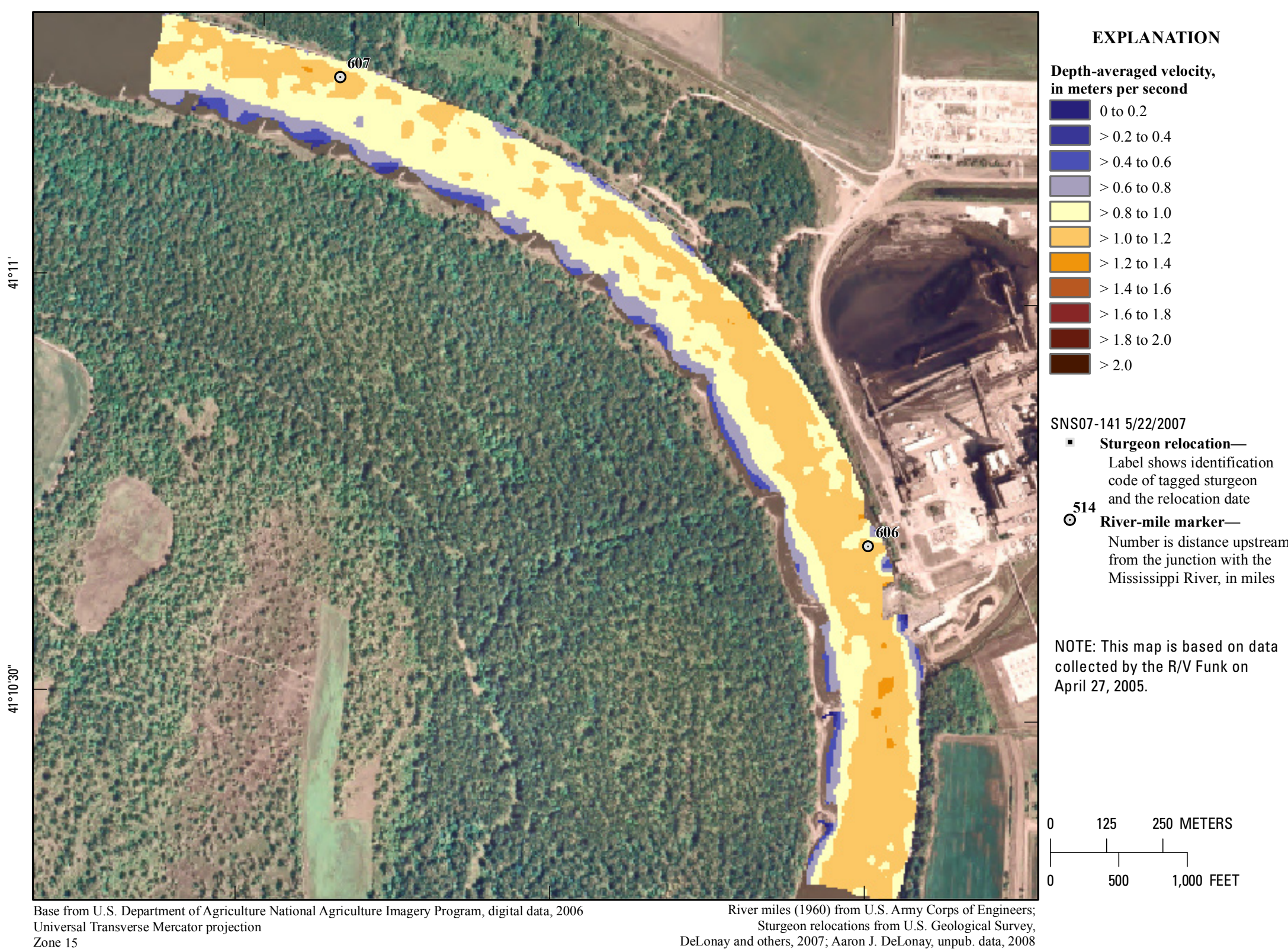




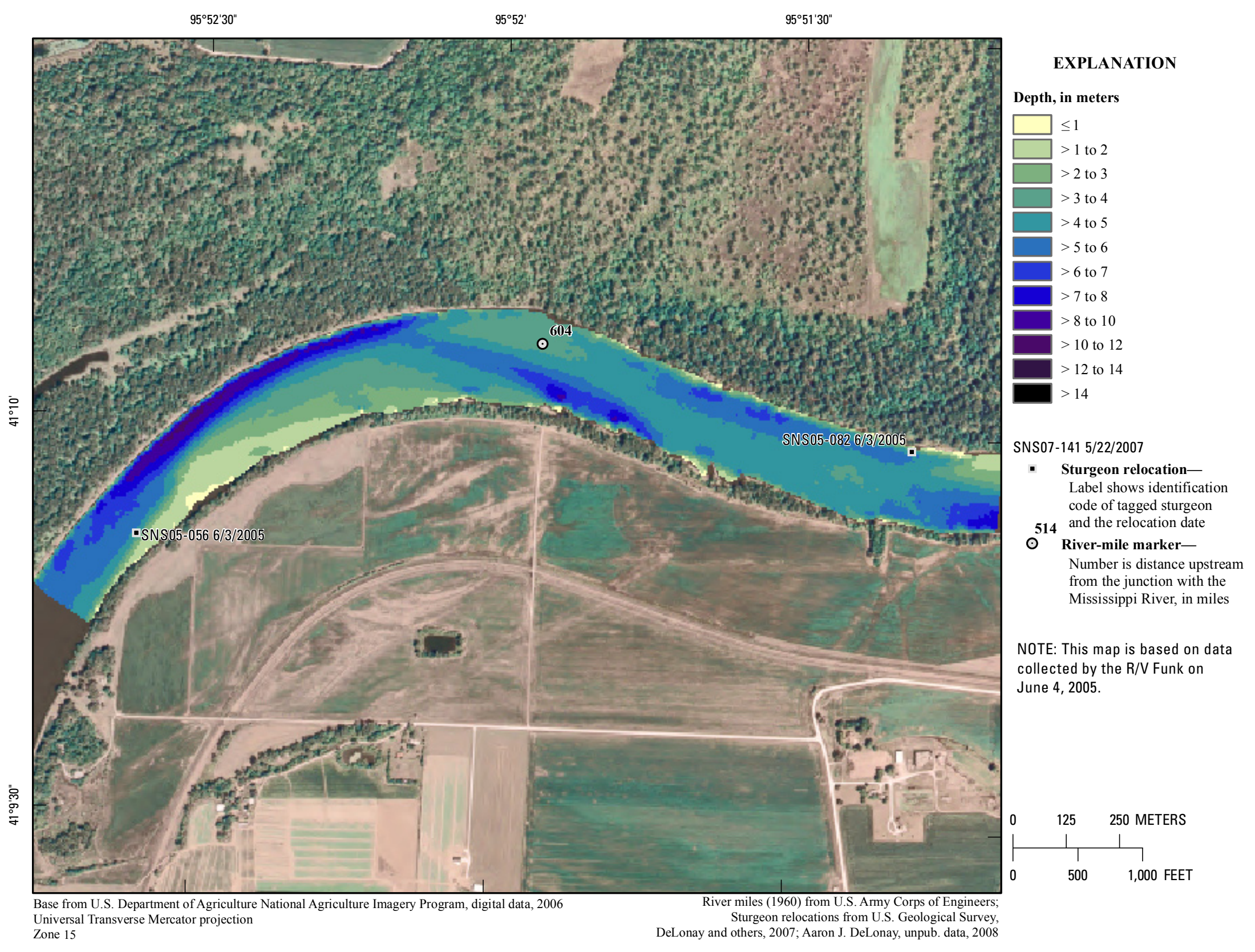

Figure 255. Map of depth based on data collected on June 4, 2005, in the vicinity of river mile 604 . 


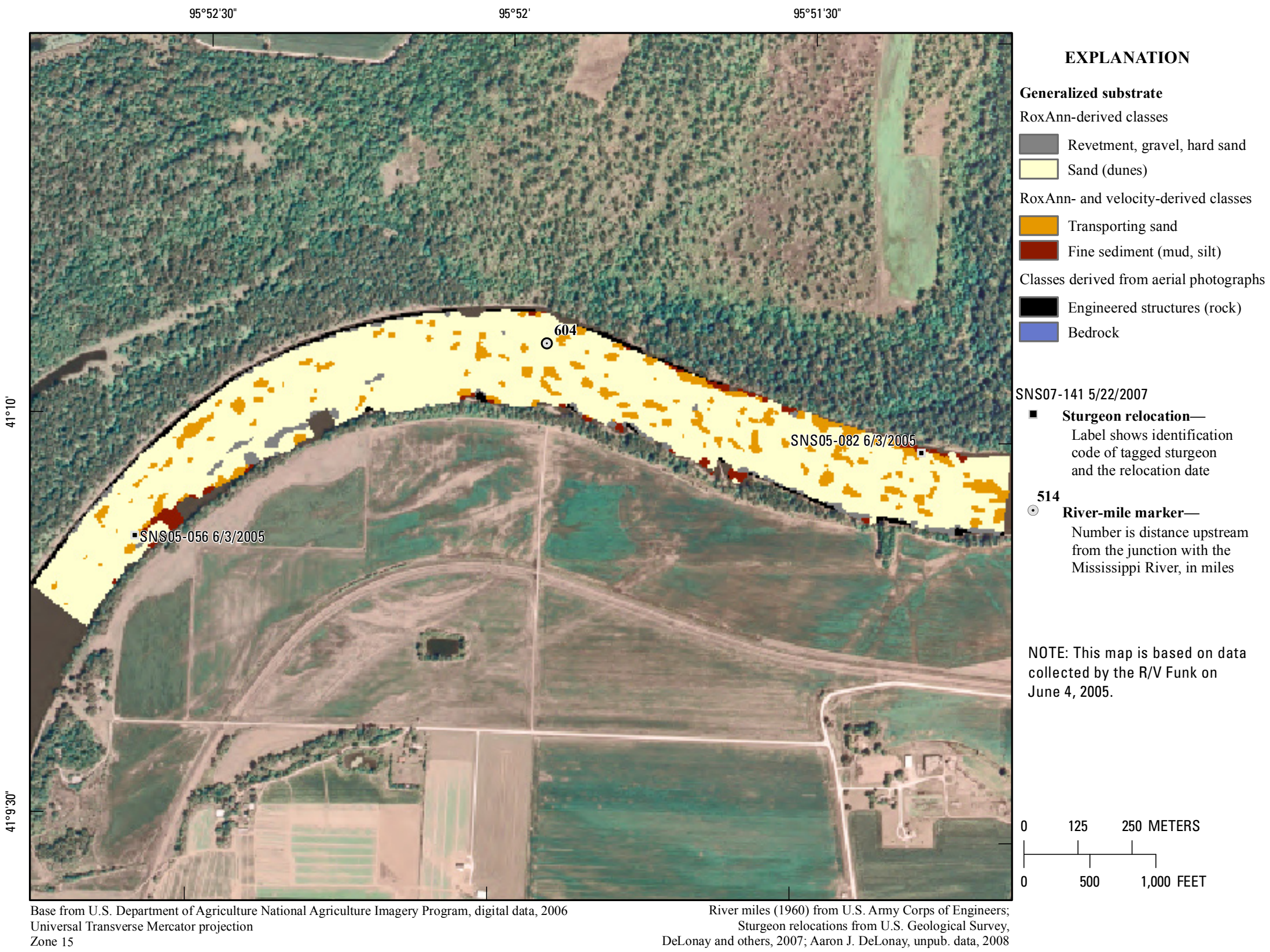

Figure 256. Map of generalized substrate based on data collected on June 4, 2005, in the vicinity of river mile 604 . 


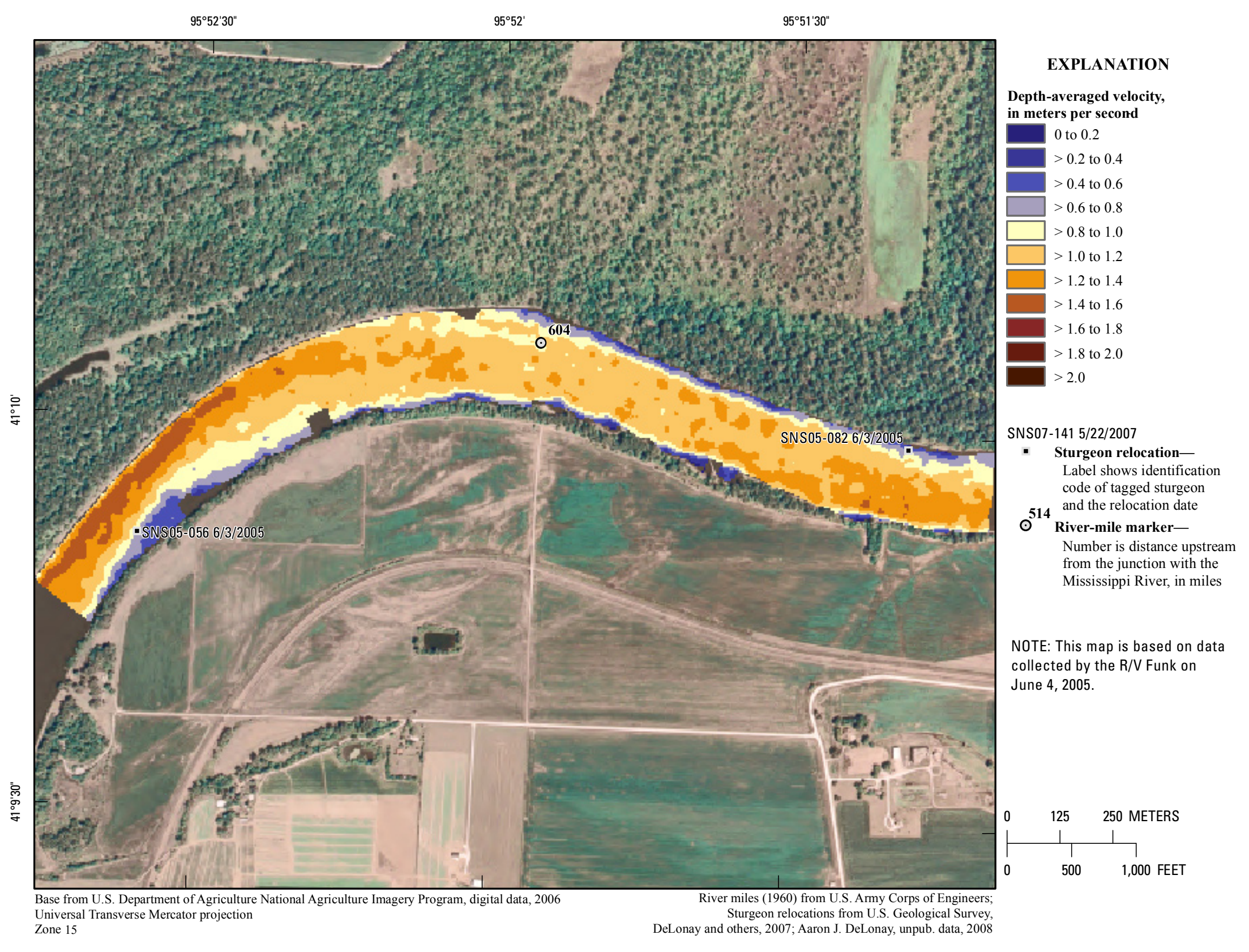

Figure 257. Map of depth-averaged velocity based on data collected on June 4, 2005, in the vicinity of river mile 604 . 


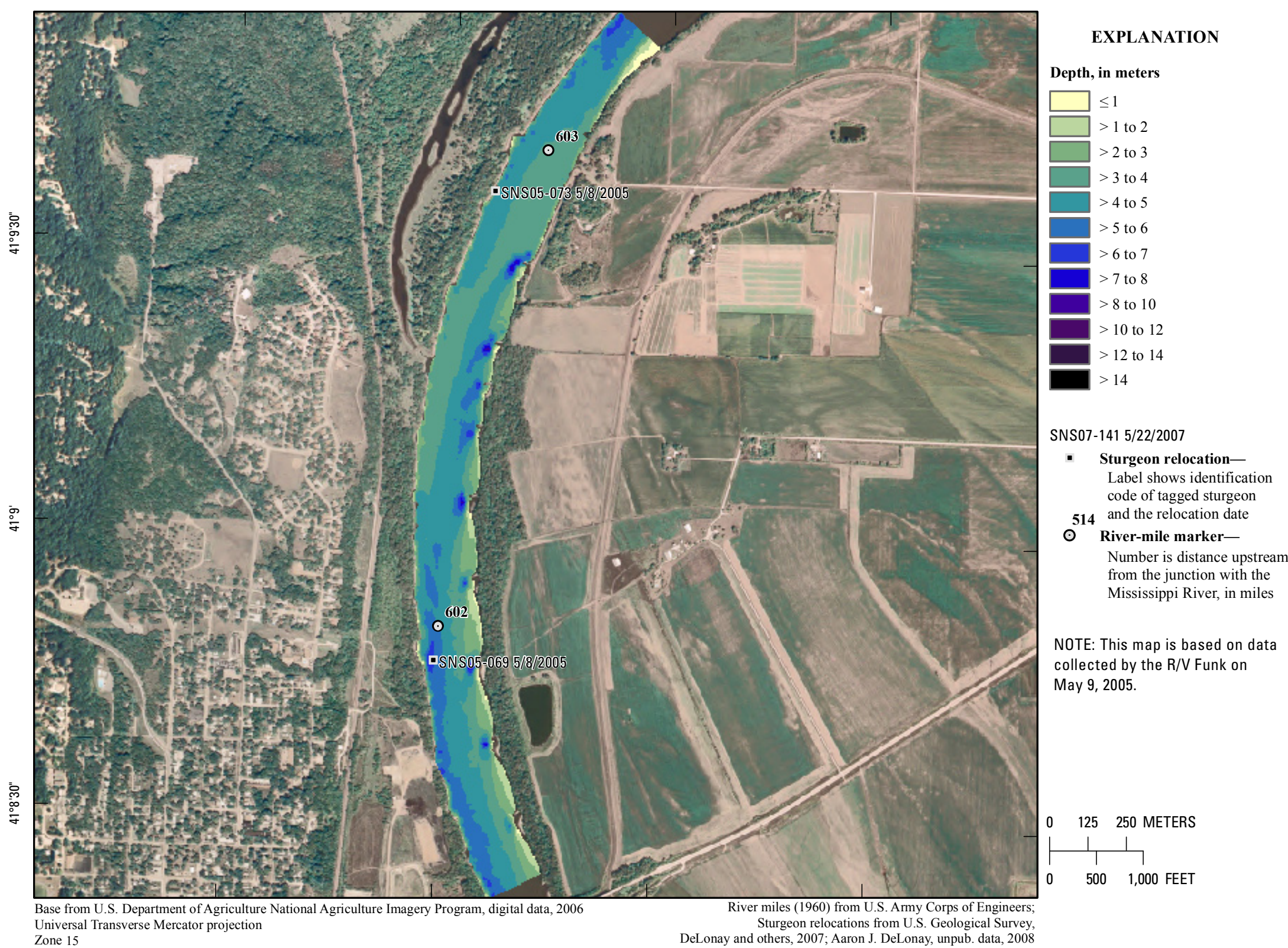




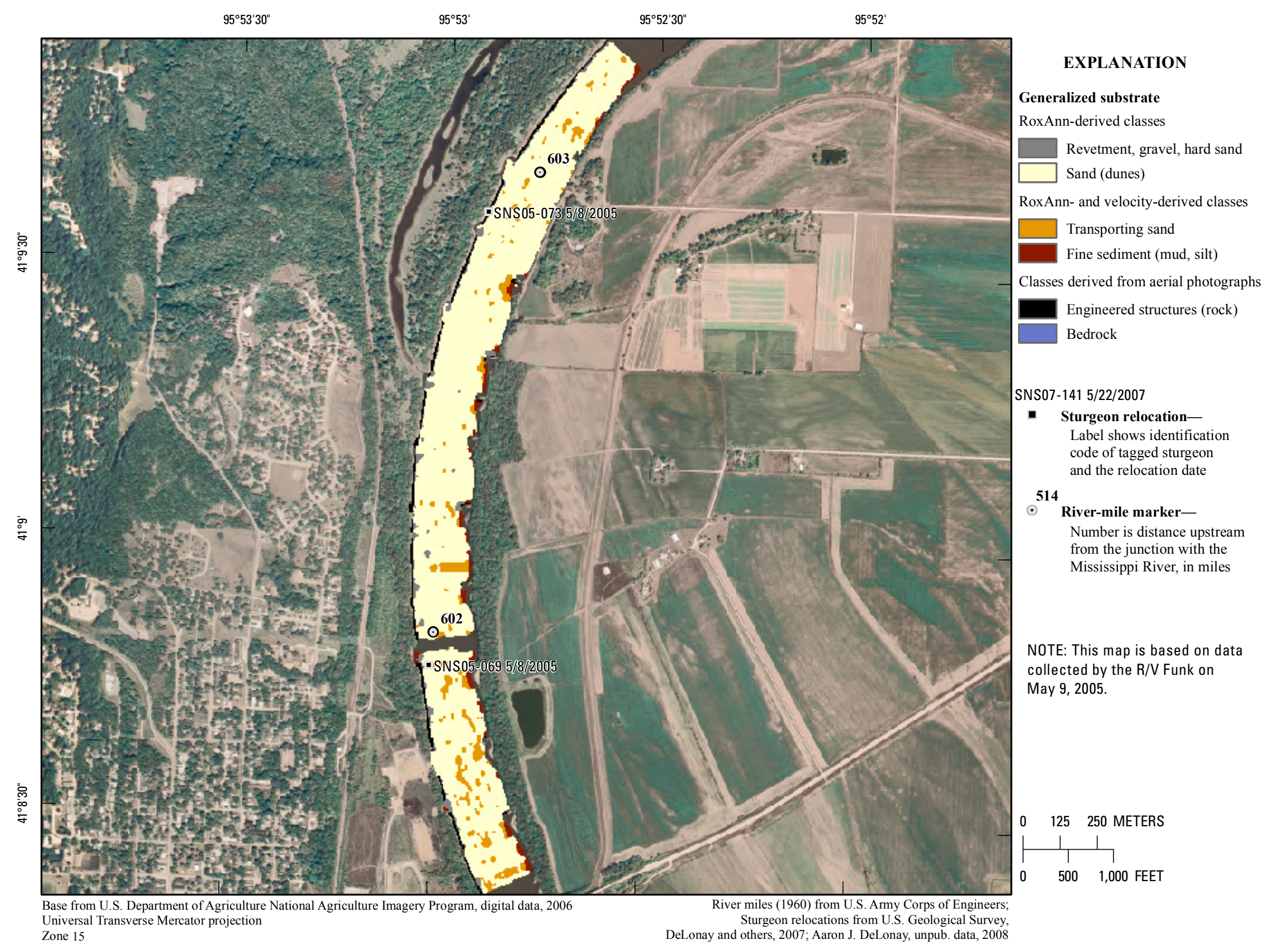

Figure 259. Map of generalized substrate based on data collected on May 9, 2005, in the vicinity of river mile 602 . 


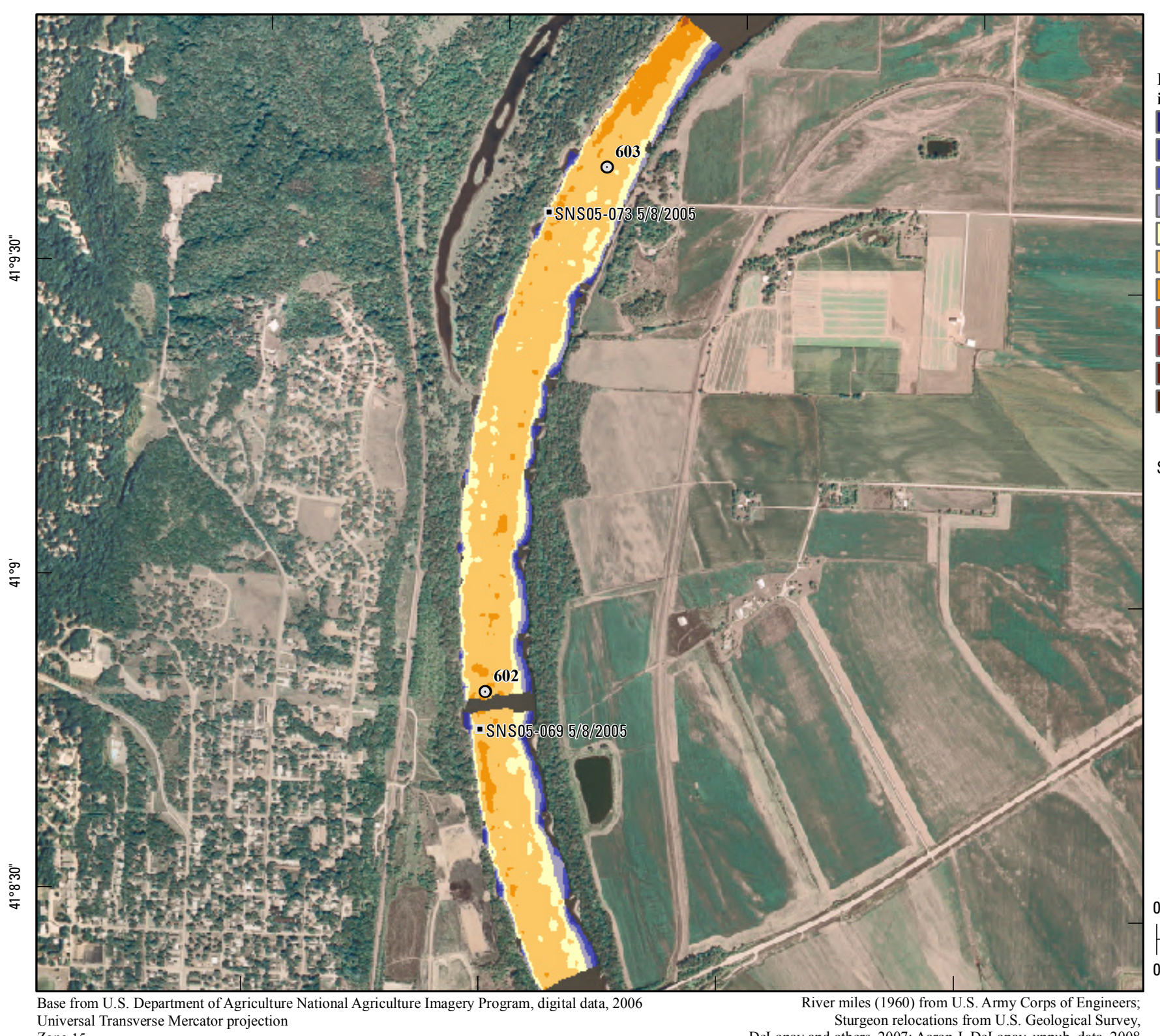

EXPLANATION

Depth-averaged velocity, in meters per second

0 to 0.2

$>0.2$ to 0.4

$>0.4$ to 0.6

$>0.6$ to 0.8

$>0.8$ to 1.0

$>1.0$ to 1.2

$>1.2$ to 1.4

$>1.4$ to 1.6

$>1.6$ to 1.8

$>1.8$ to 2.0

$>2.0$

\section{SNS07-141 5/22/2007}

- Sturgeon relocationLabel shows identification code of tagged sturgeo

$\odot^{514}$ and the relocation date

Number is distance upstream

Number is distance upstrean
from the junction with the

Mississippi River, in miles

NOTE: This map is based on data collected by the R/V Funk on

May 9, 2005.

\section{METERS $\left.\right|_{500} ^{1,000}$ \\ $500 \quad 1,000$ FEET} Sturgeon relocations from U.S. Geological Survey,
DeLonay and others, 2007; Aaron J. DeLonay, unpub. data, 2008 


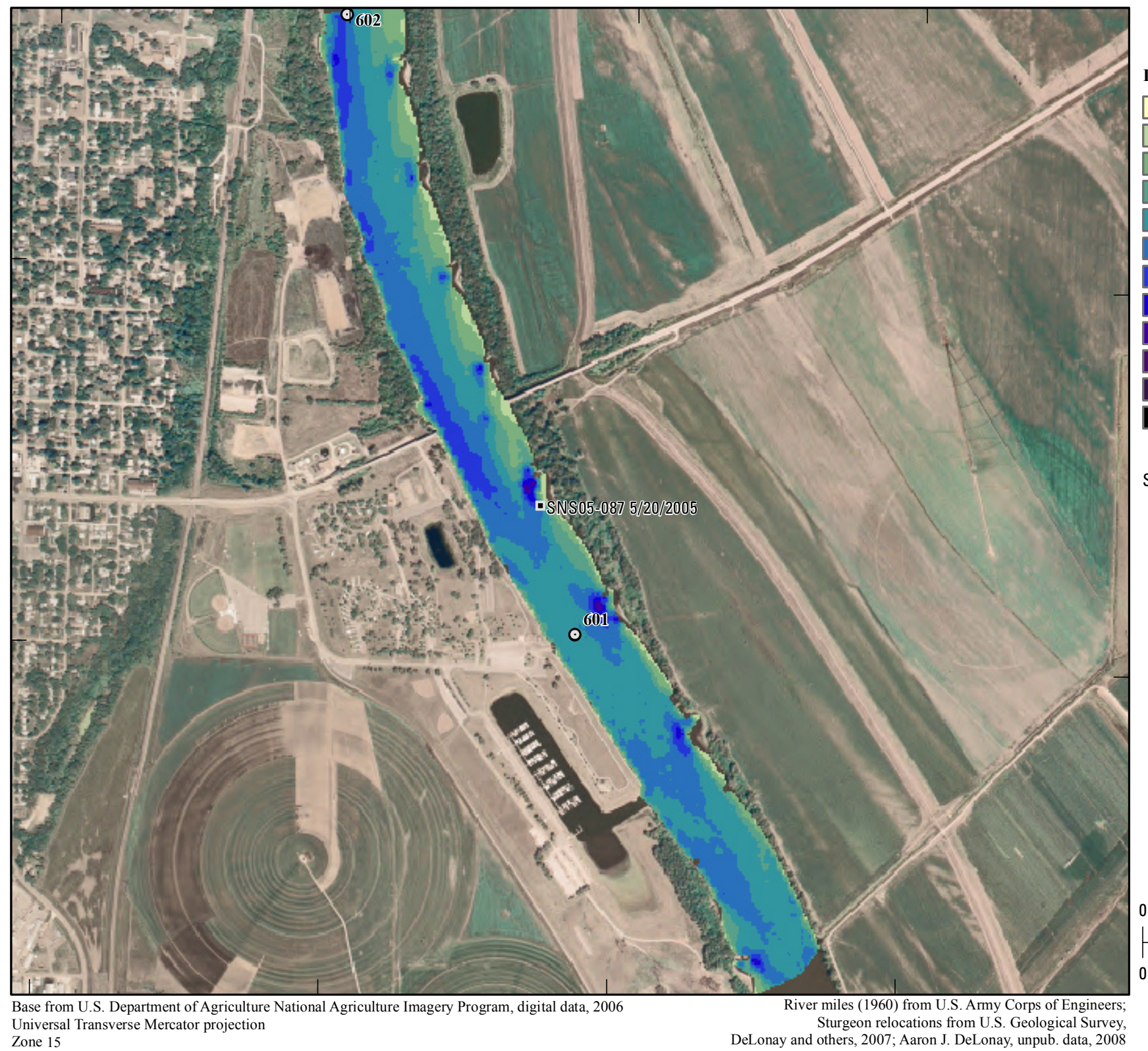

\section{EXPLANATION}

Depth, in meters

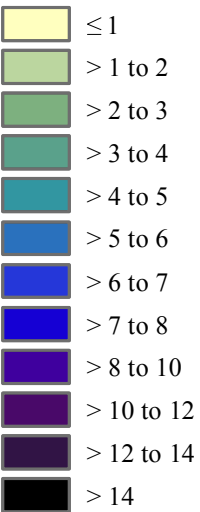

\section{SNS07-141 5/22/2007}

- Sturgeon relocationLabel shows identificatio code of tagged sturgeo

514 and the relocation date

$\circ$ River-mile markerNumber is distance upstrean from the junction with the Mississippi River, in miles

NOTE: This map is based on data collected by the R/V Funk on May 22, 2005.

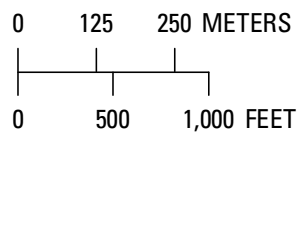

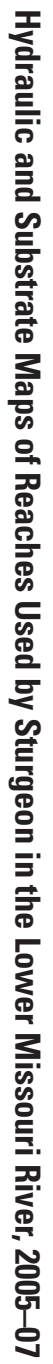

Figure 261. Map of depth based on data collected on May 22, 2005, in the vicinity of river mile 601 . 


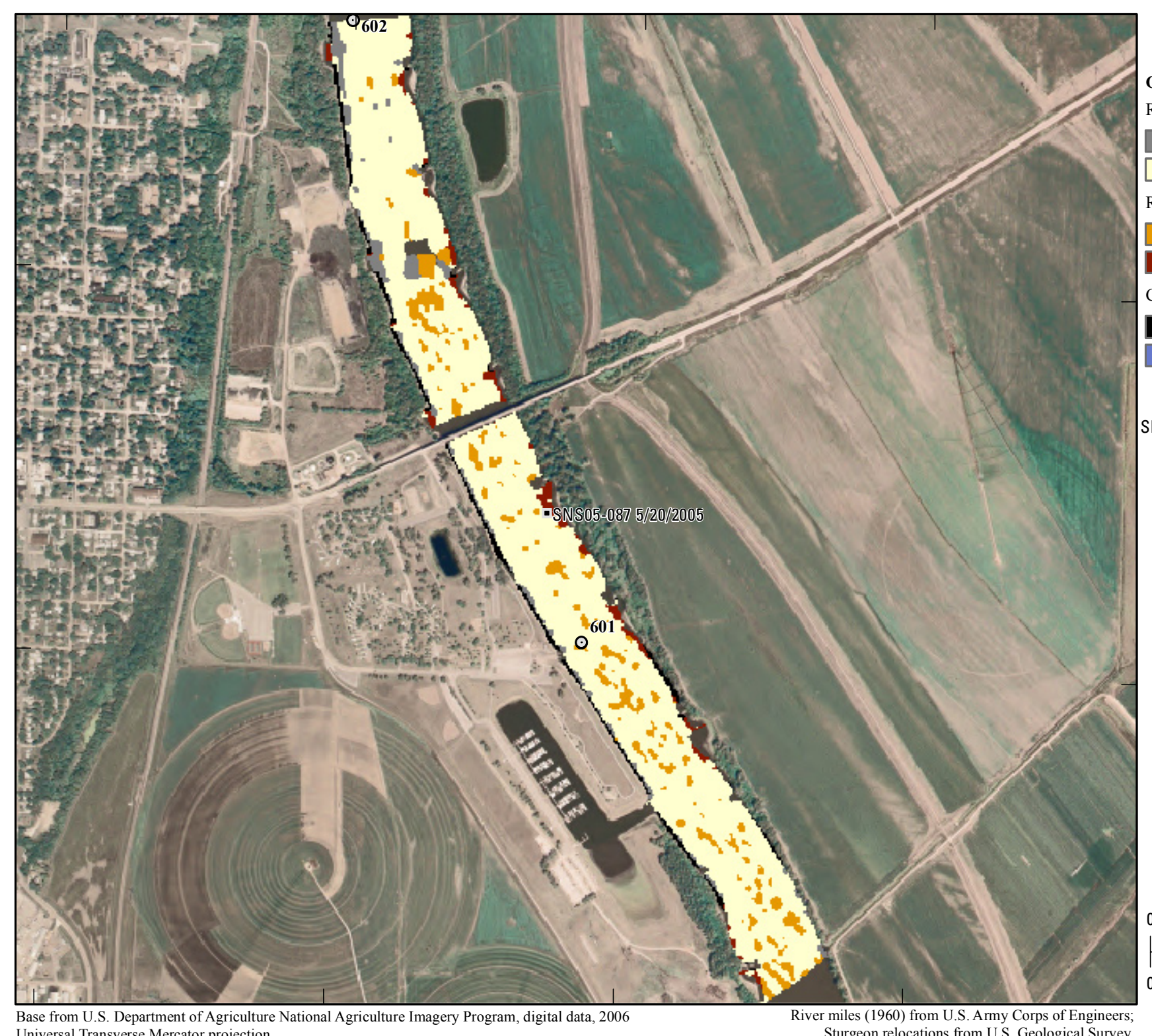

EXPLANATION

\section{Generalized substrate}

RoxAnn-derived classes

Revetment, gravel, hard sand Sand (dunes)

RoxAnn- and velocity-derived classes

Transporting sand

Fine sediment (mud, silt)

Classes derived from aerial photographs

Engineered structures (rock) Bedrock

\section{SNS07-141 5/22/2007}

- Sturgeon relocationLabel shows identification code of tagged sturgeon and the relocation date

514

River-mile marker-

Number is distance upstream from the junction with the Mississippi River, in miles

NOTE: This map is based on data collected by the R/V Funk on May 22, 2005.

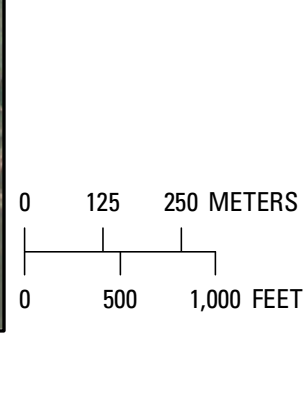
Sturgeon relocations from U.S. Geological Survey,
DeLonay and others, 2007; Aaron J. DeLonay, unpub. data, 2008 Universal Transverse Mercator projection 


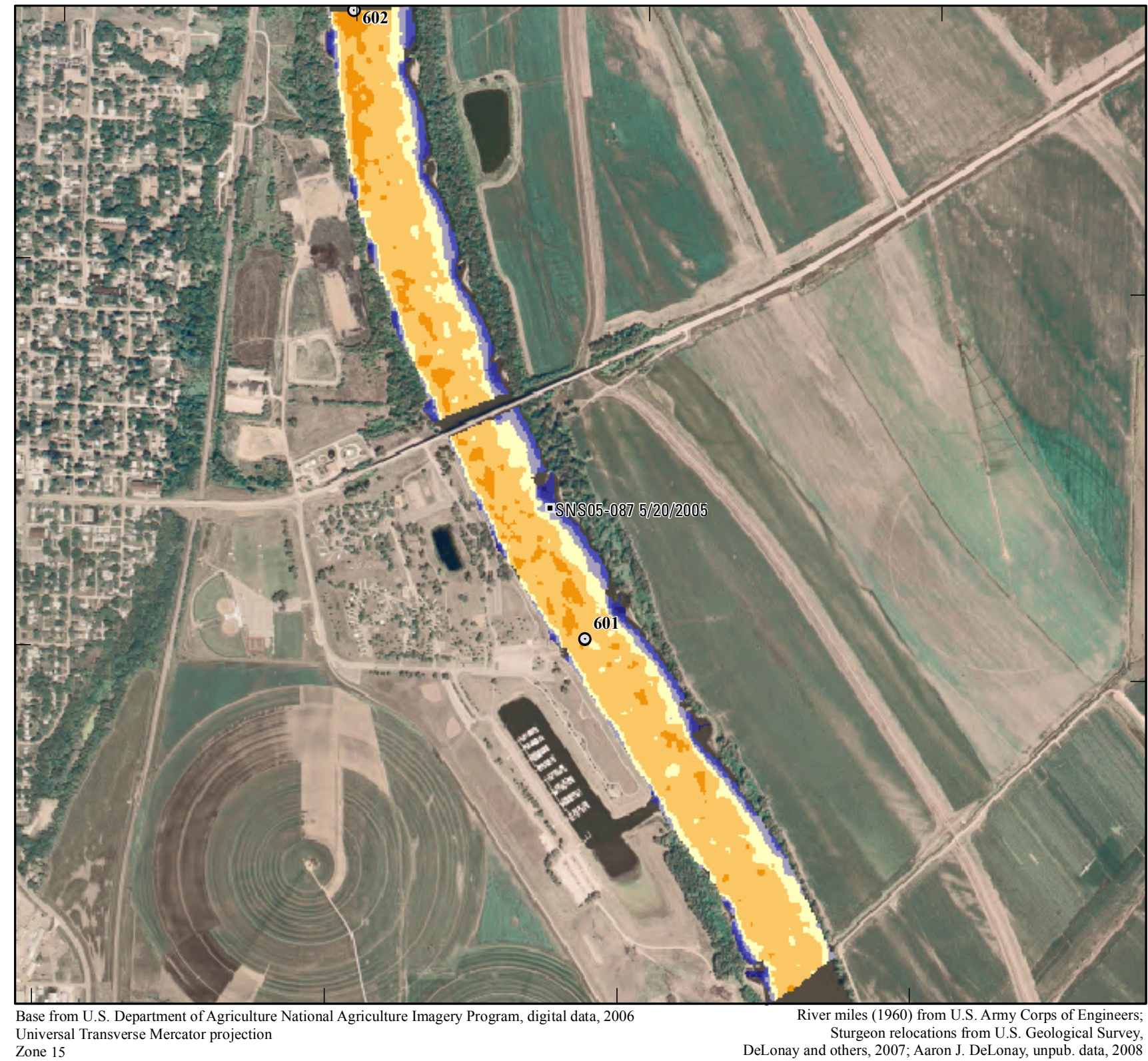

\section{EXPLANATION}

Depth-averaged velocity,

in meters per second

0 to 0.2

$>0.2$ to 0.4

$>0.4$ to 0.6

$>0.6$ to 0.8

$>0.8$ to 1.0

$>1.0$ to 1.2

$>1.2$ to 1.4

$>1.4$ to 1.6

$>1.6$ to 1.8

$>1.8$ to 2.0

$>2.0$

SNS07-141 5/22/2007

- Sturgeon relocationLabel shows identification code of tagged sturgeon

514 and the relocation date

River-mile marker-

Number is distance upstream from the junction with the Mississippi River, in miles

NOTE: This map is based on data

collected by the R/V Funk on

May 22, 2005.

Figure 263. Map of depth-averaged velocity based on data collected on May 22, 2005, in the vicinity of river mile 601. 
$95^{\circ} 52^{\prime} 30^{\prime \prime}$

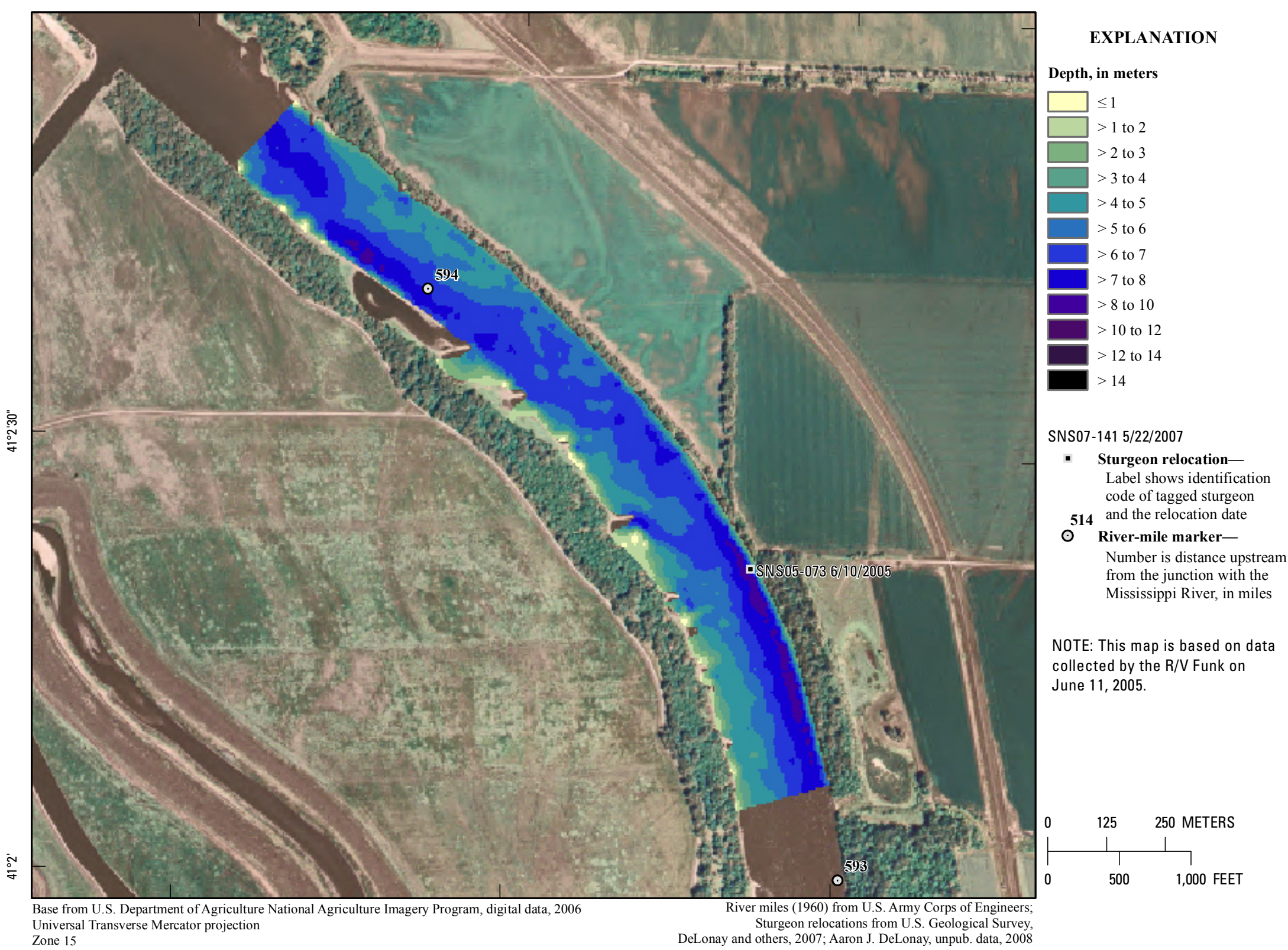
Universal Transverse Mercator projection Sturgeon relocations from U.S. Geological Survey,
DeLonay and others, 2007; Aaron J. DeLonay, unpub. data, 2008 


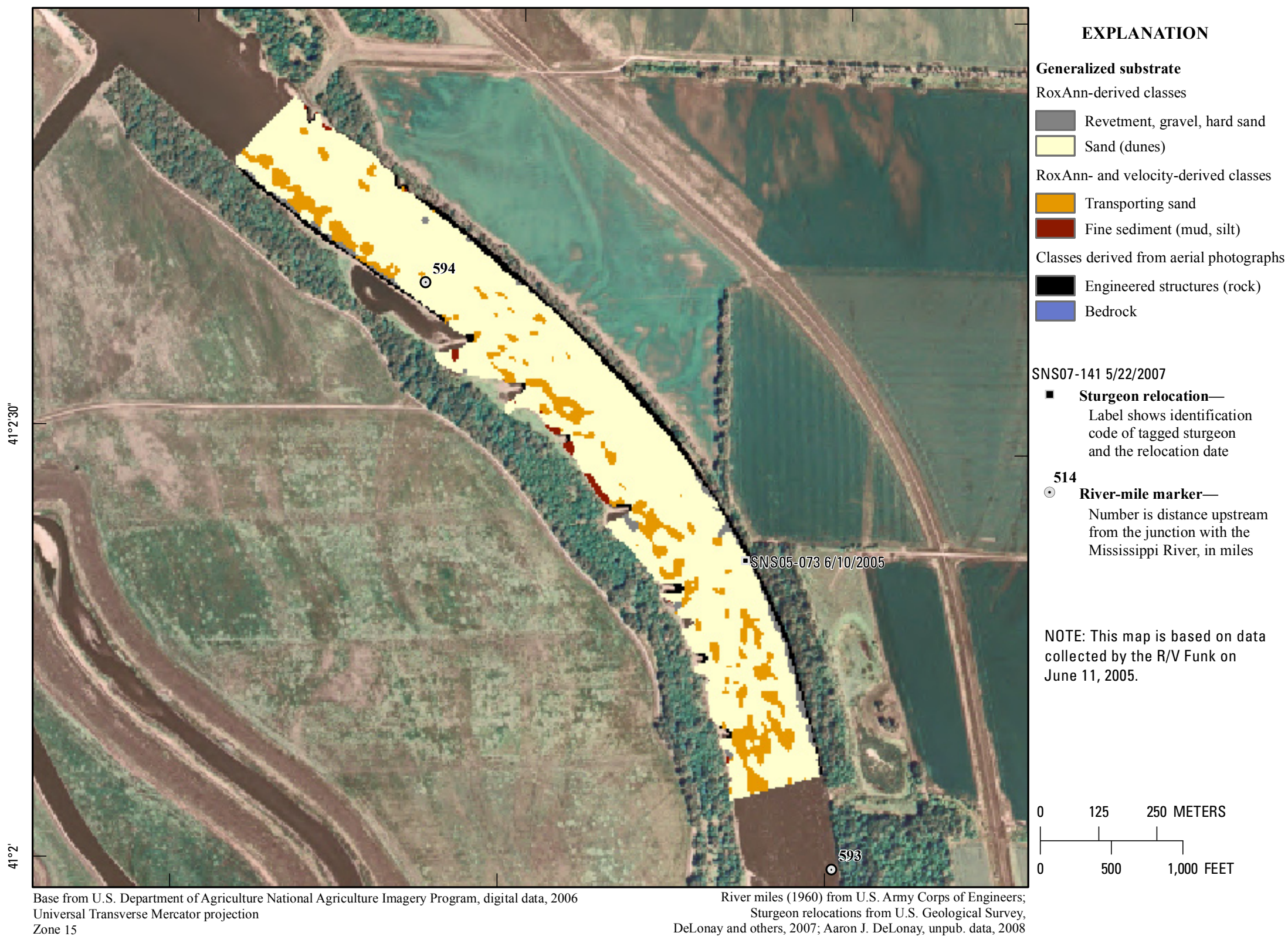

Figure 265. Map of generalized substrate based on data collected on June 11, 2005, in the vicinity of river mile 594 . 


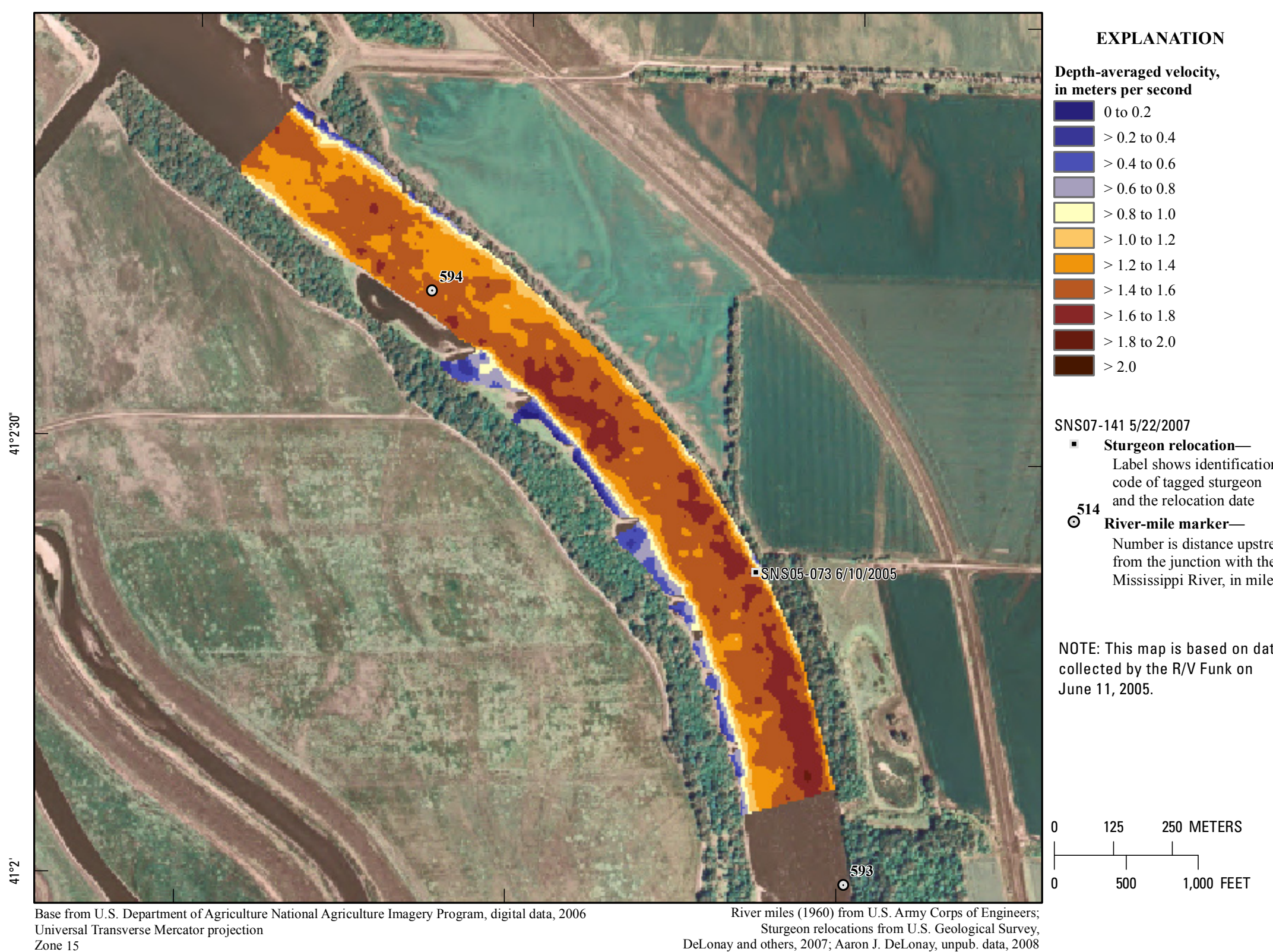
Universal Transverse Mercator projection Sturgeon relocations from U.S. Geological Survey,
DeLonay and others, 2007; Aaron J. DeLonay, unpub. data, 2008 


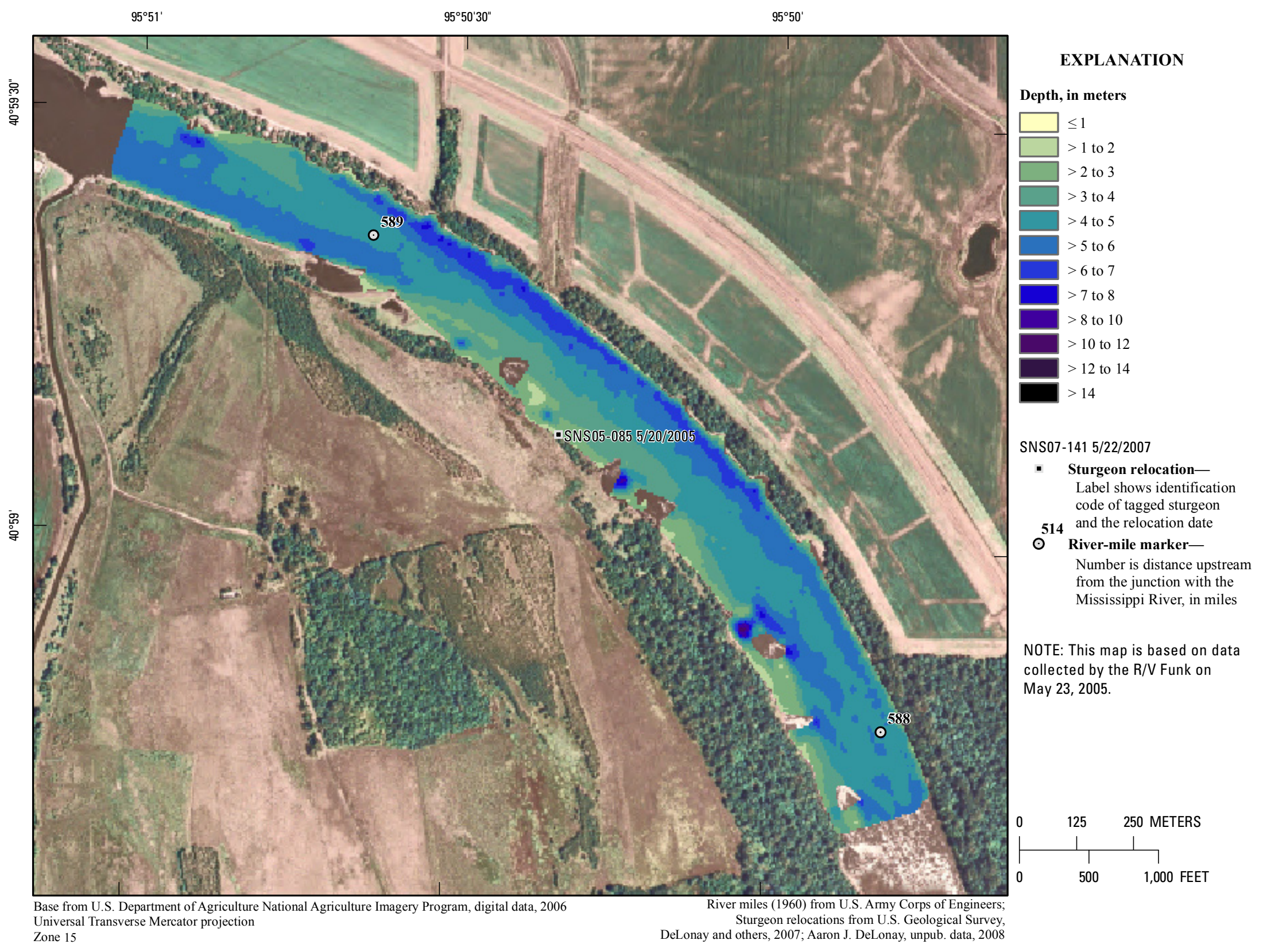

Figure 267. Map of depth based on data collected on May 23, 2005, in the vicinity of river mile 589. 


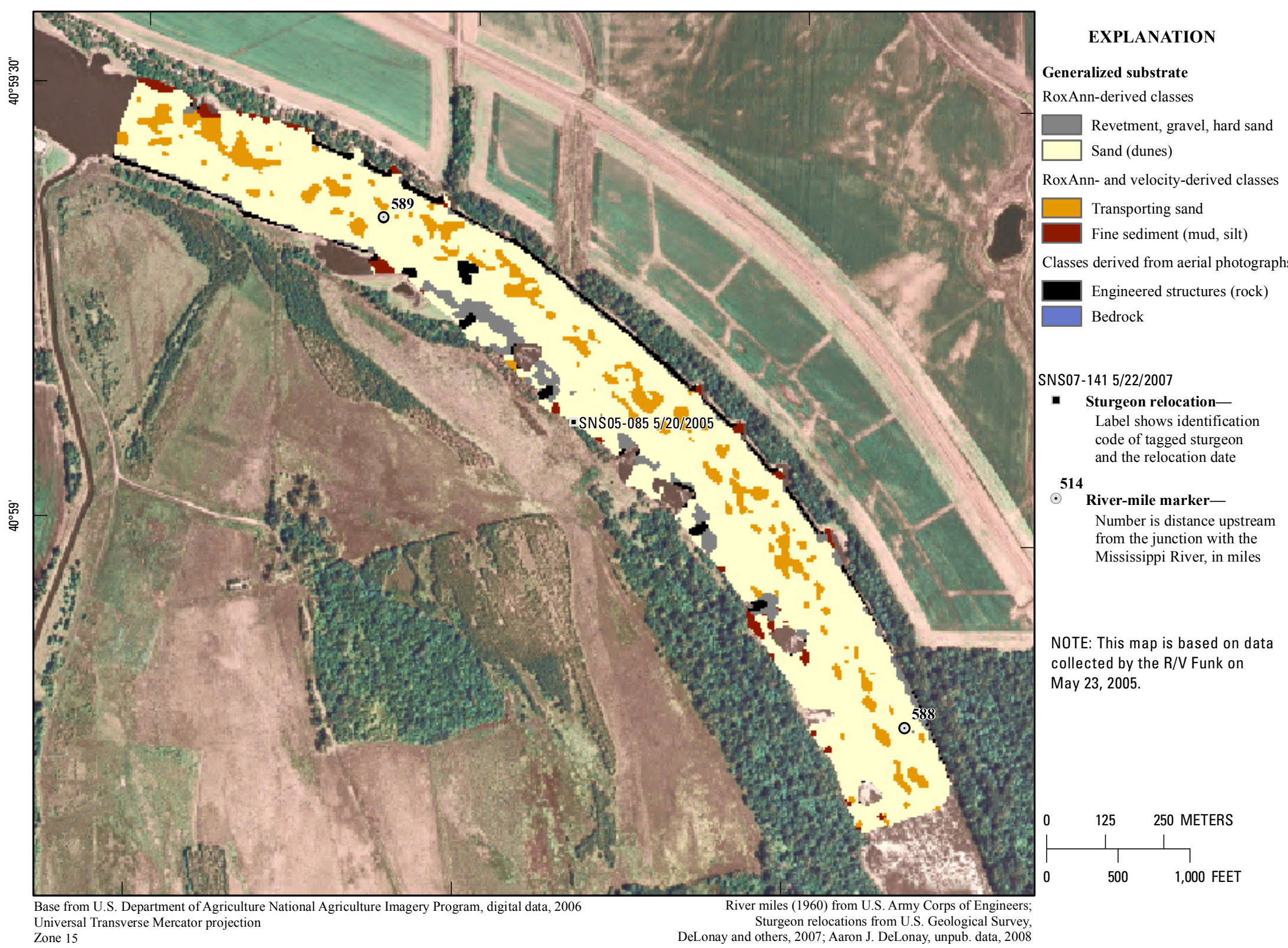




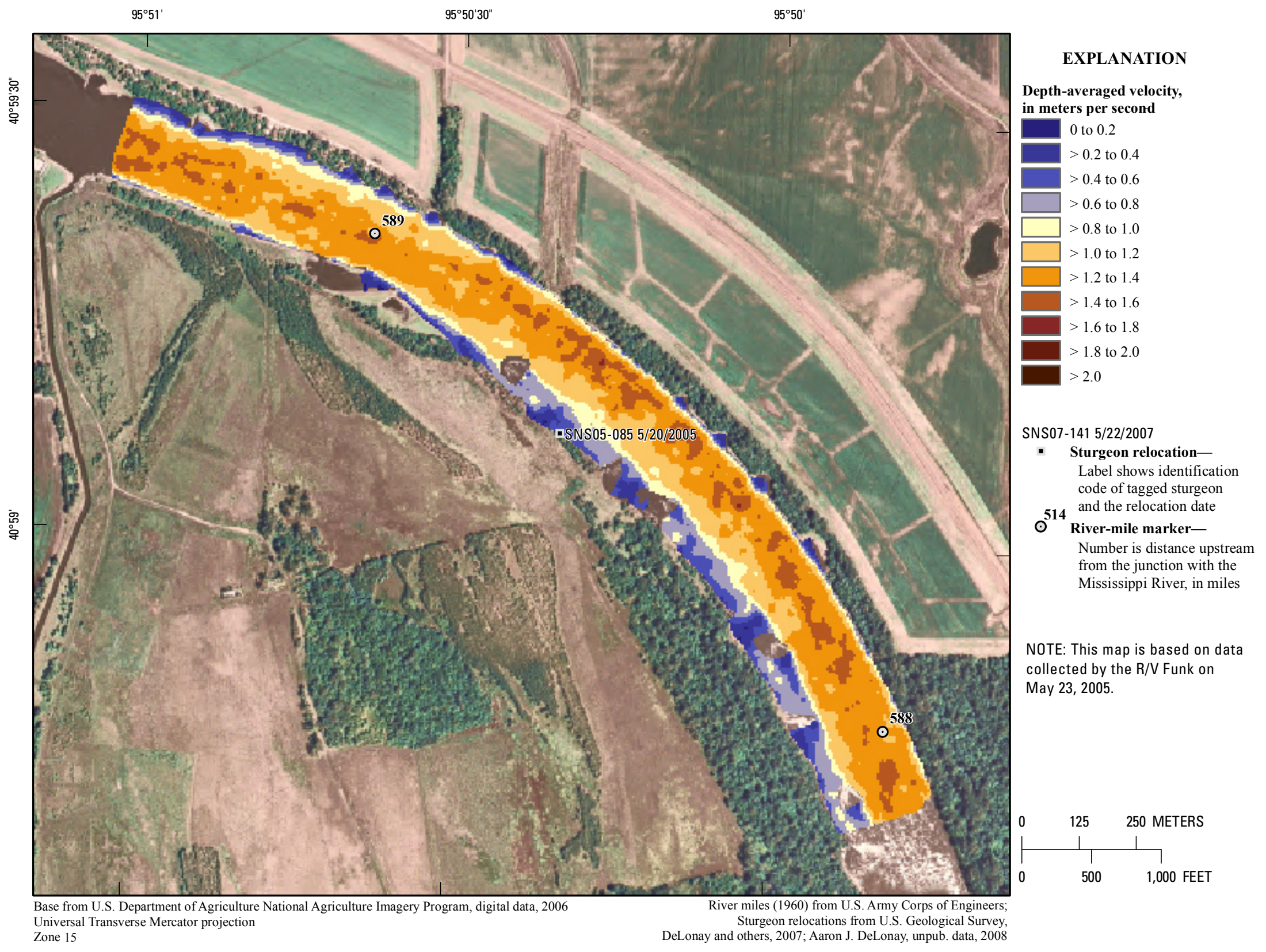

Figure 269. Map of depth-averaged velocity based on data collected on May 23, 2005, in the vicinity of river mile 589 . 


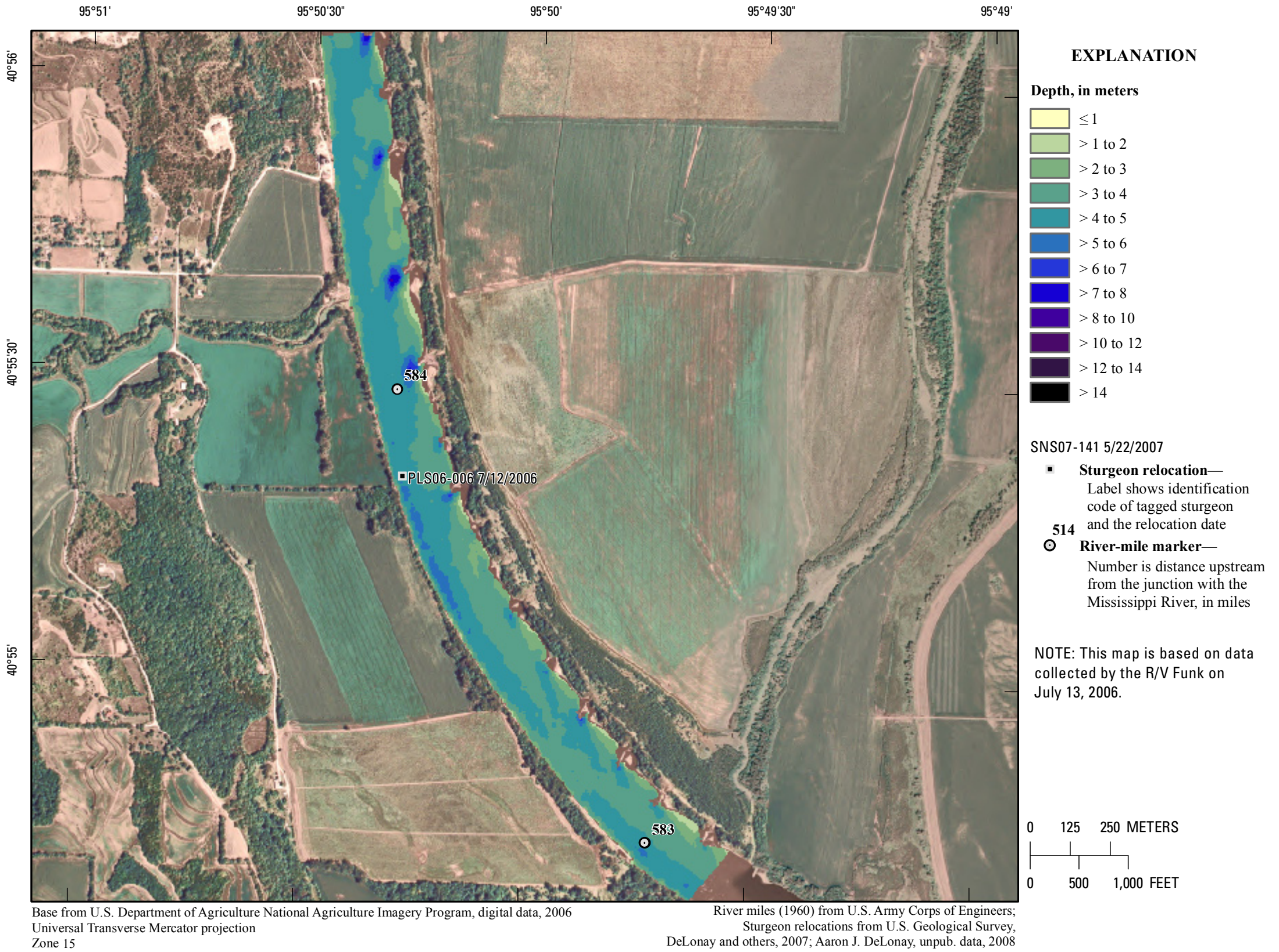

Figure 270. Map of depth based on data collected on July 13, 2006, in the vicinity of river mile 584 . 


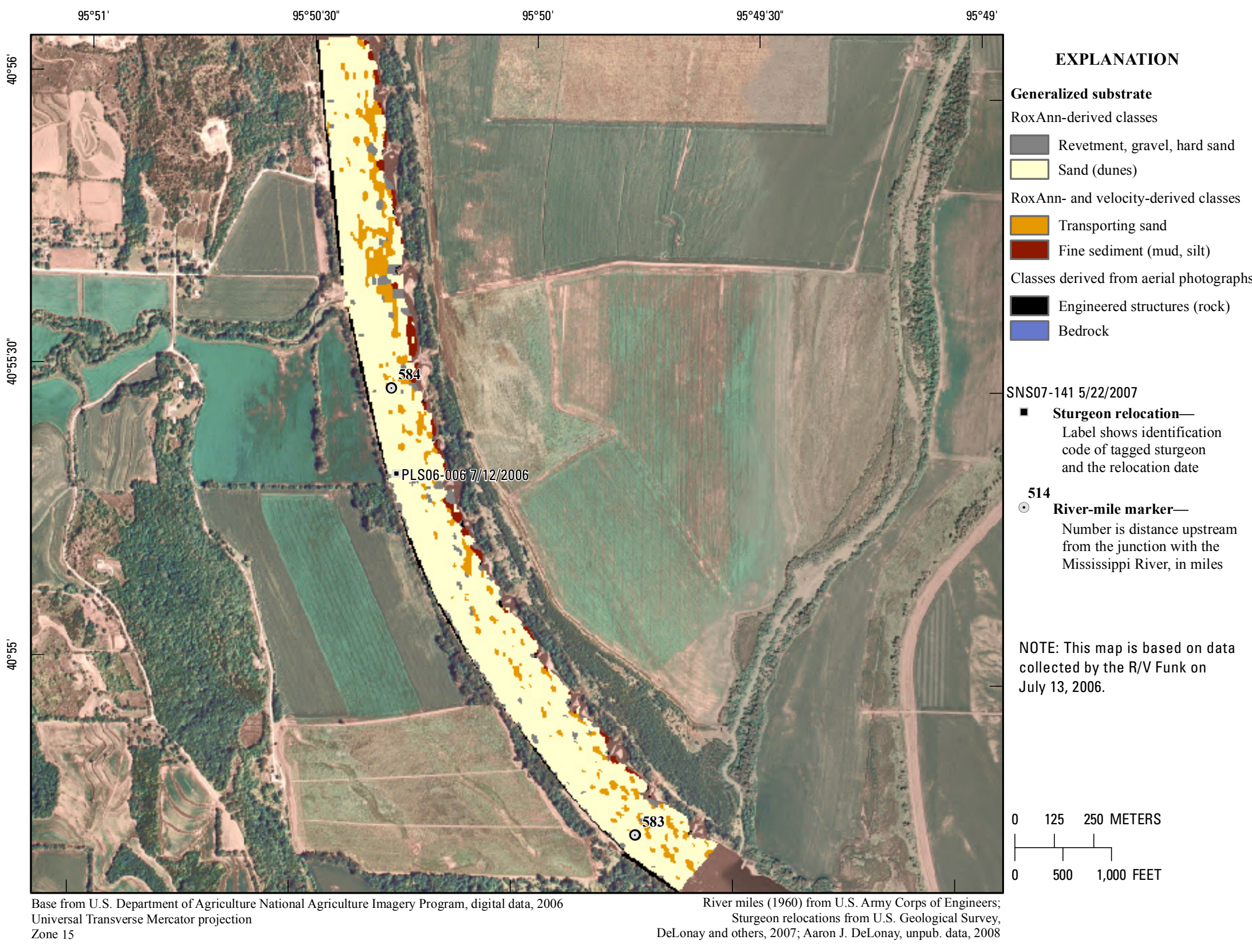

Figure 271. Map of generalized substrate based on data collected on July 13,2006 , in the vicinity of river mile 584 . 


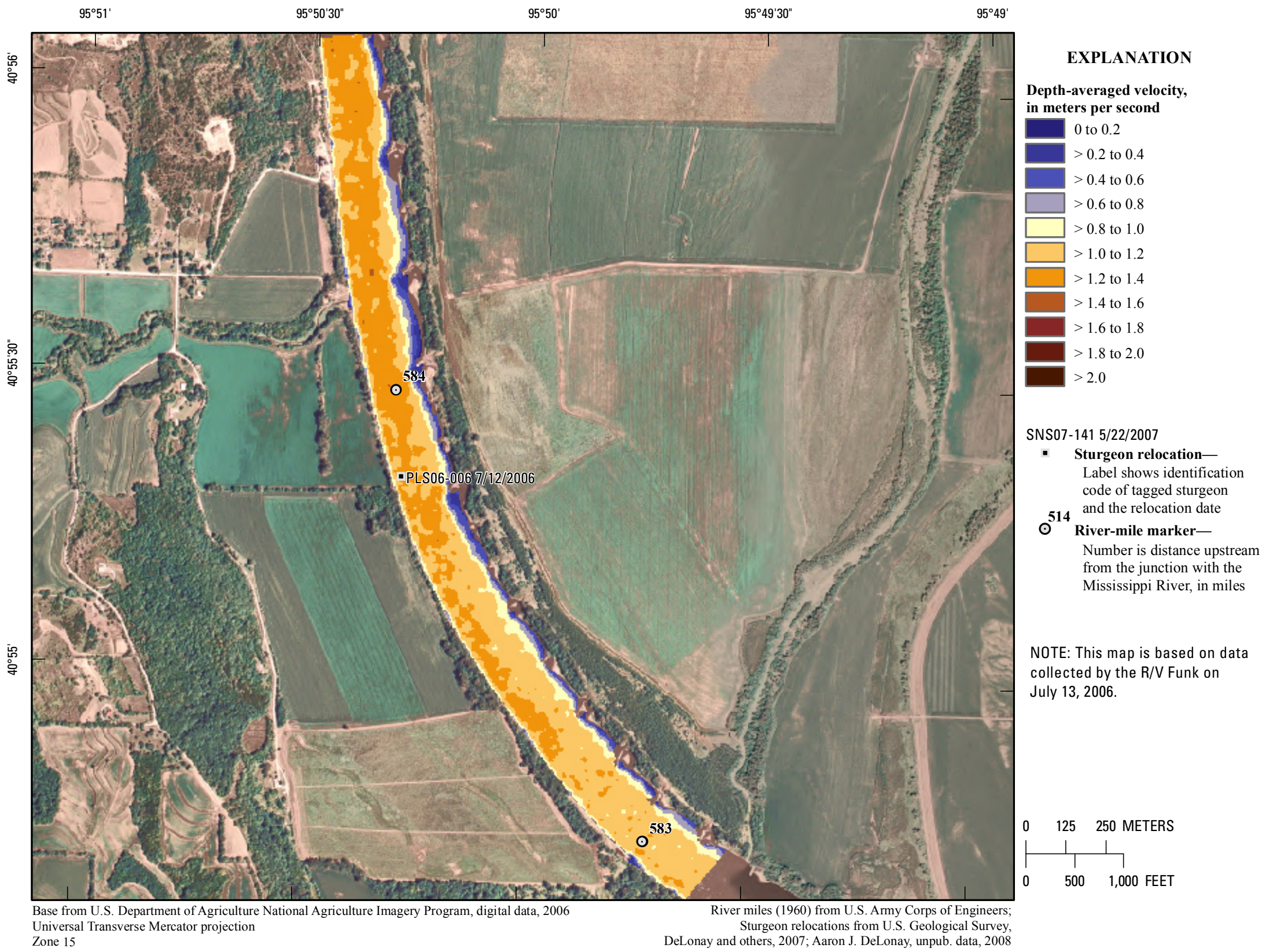

Figure 272. Map of depth-averaged velocity based on data collected on July 13, 2006, in the vicinity of river mile 584 . 


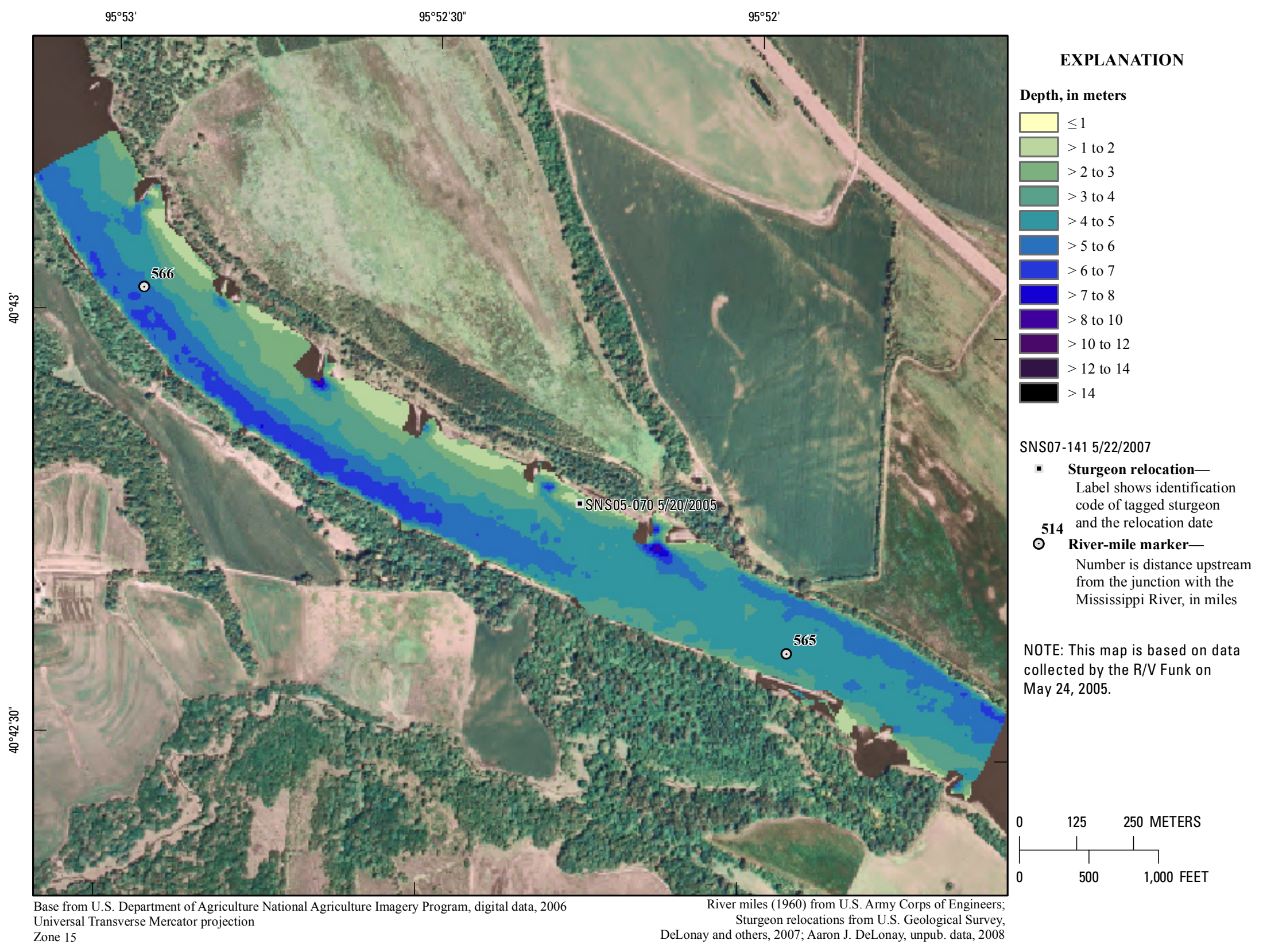

Figure 273. Map of depth based on data collected on May 24, 2005, in the vicinity of river mile 566. 


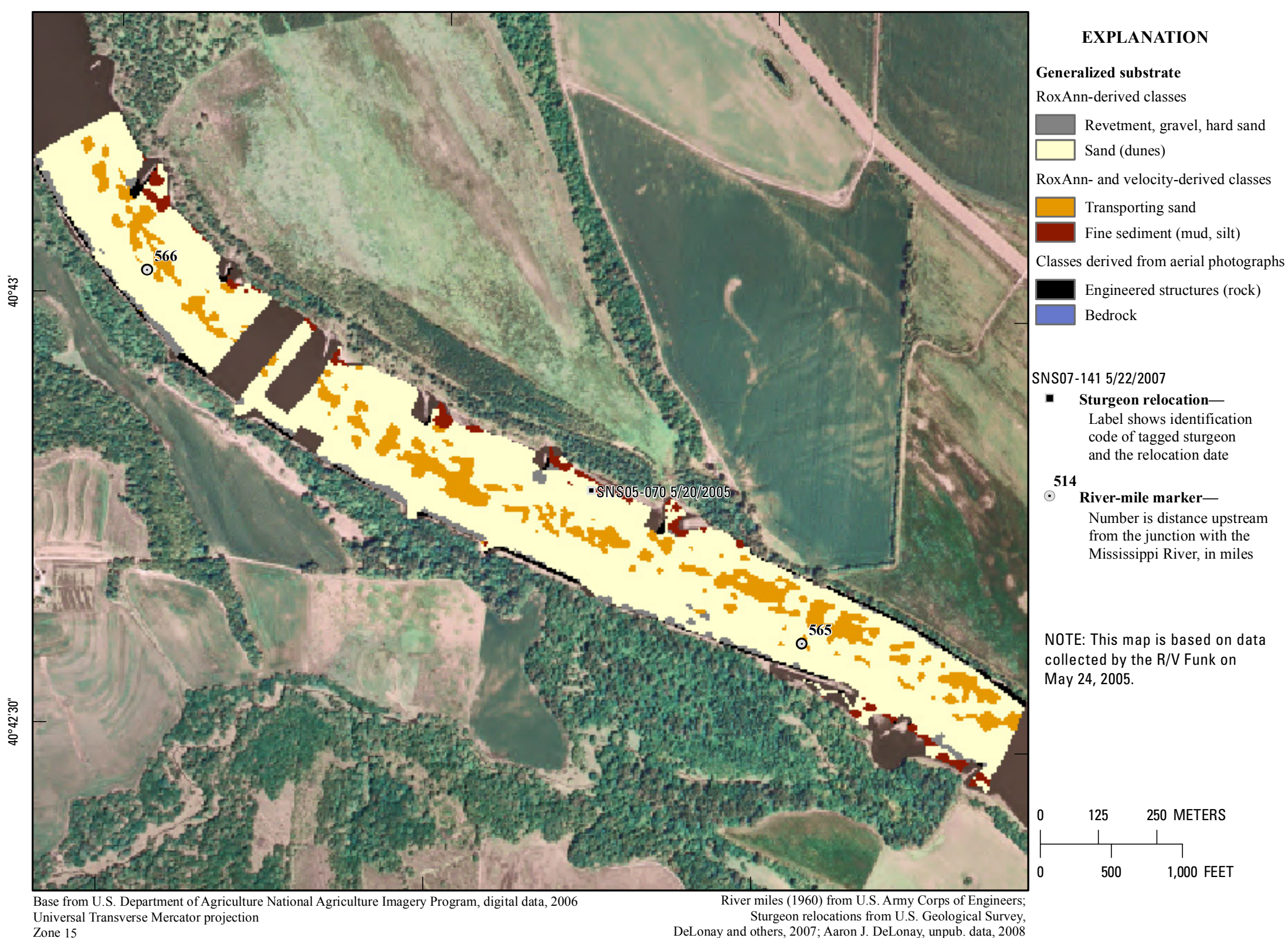




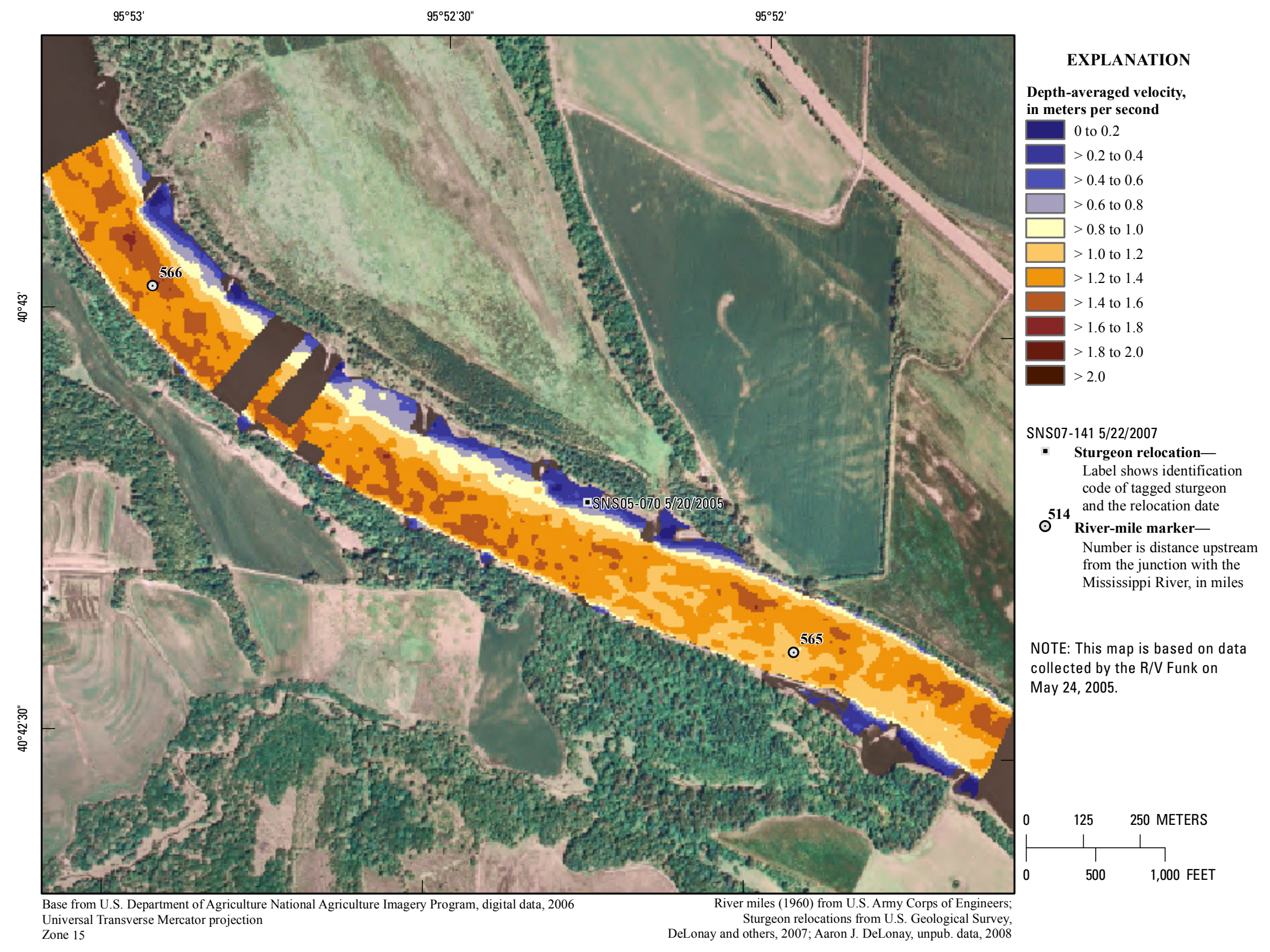

Figure 275. Map of depth-averaged velocity based on data collected on May 24, 2005, in the vicinity of river mile 566 . 


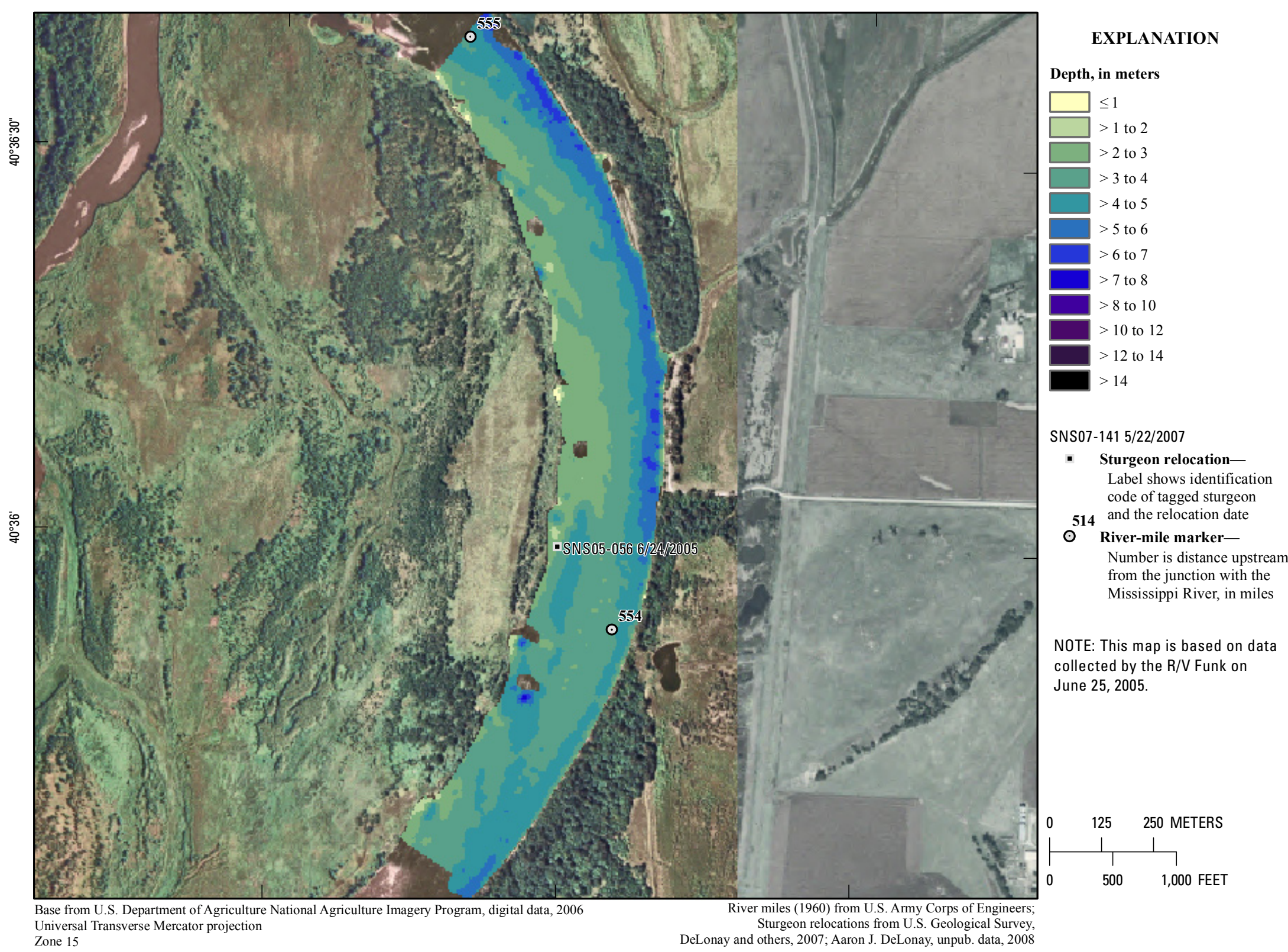




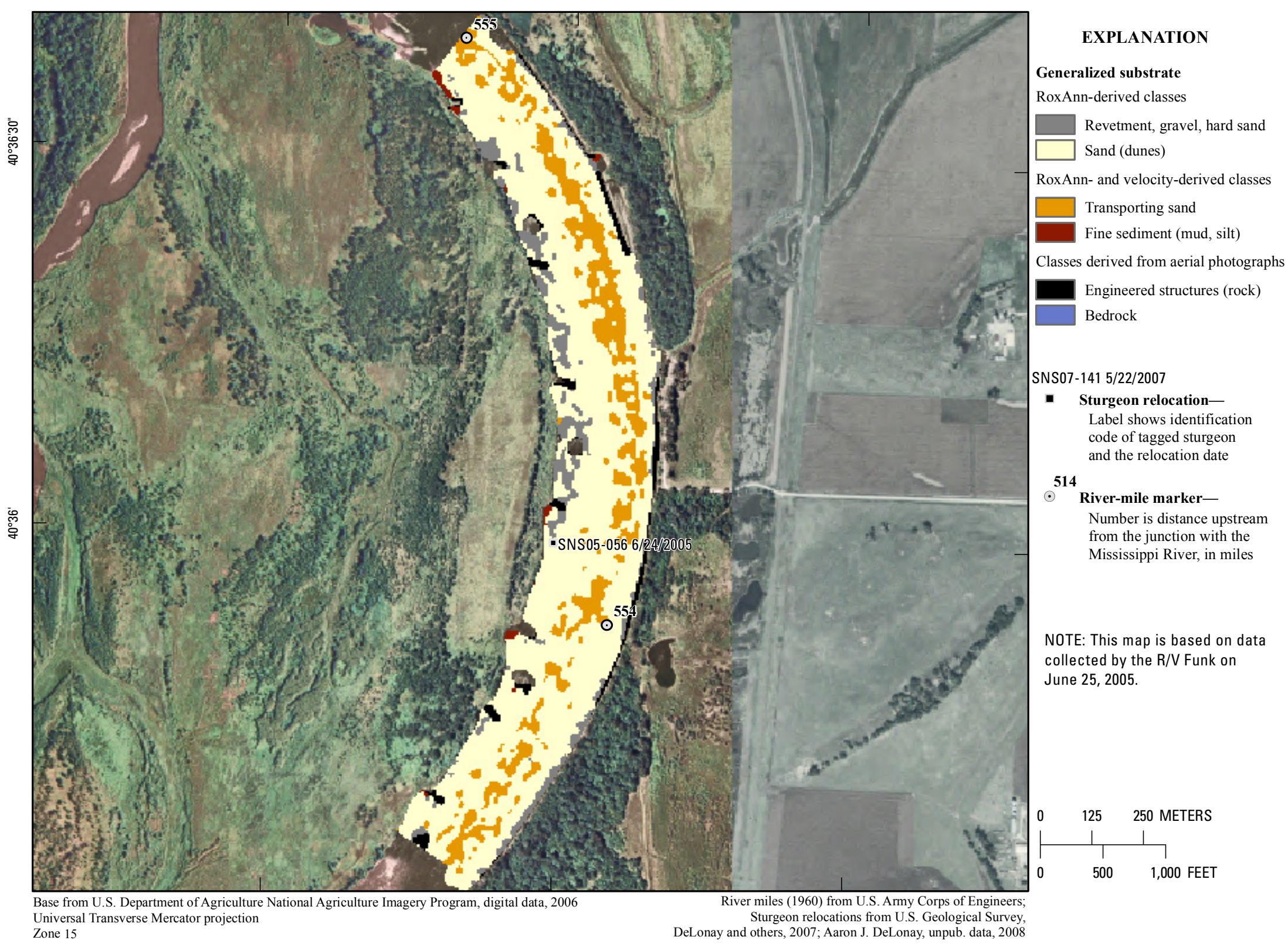

Figure 277. Map of generalized substrate based on data collected on June 25, 2005, in the vicinity of river mile 554 


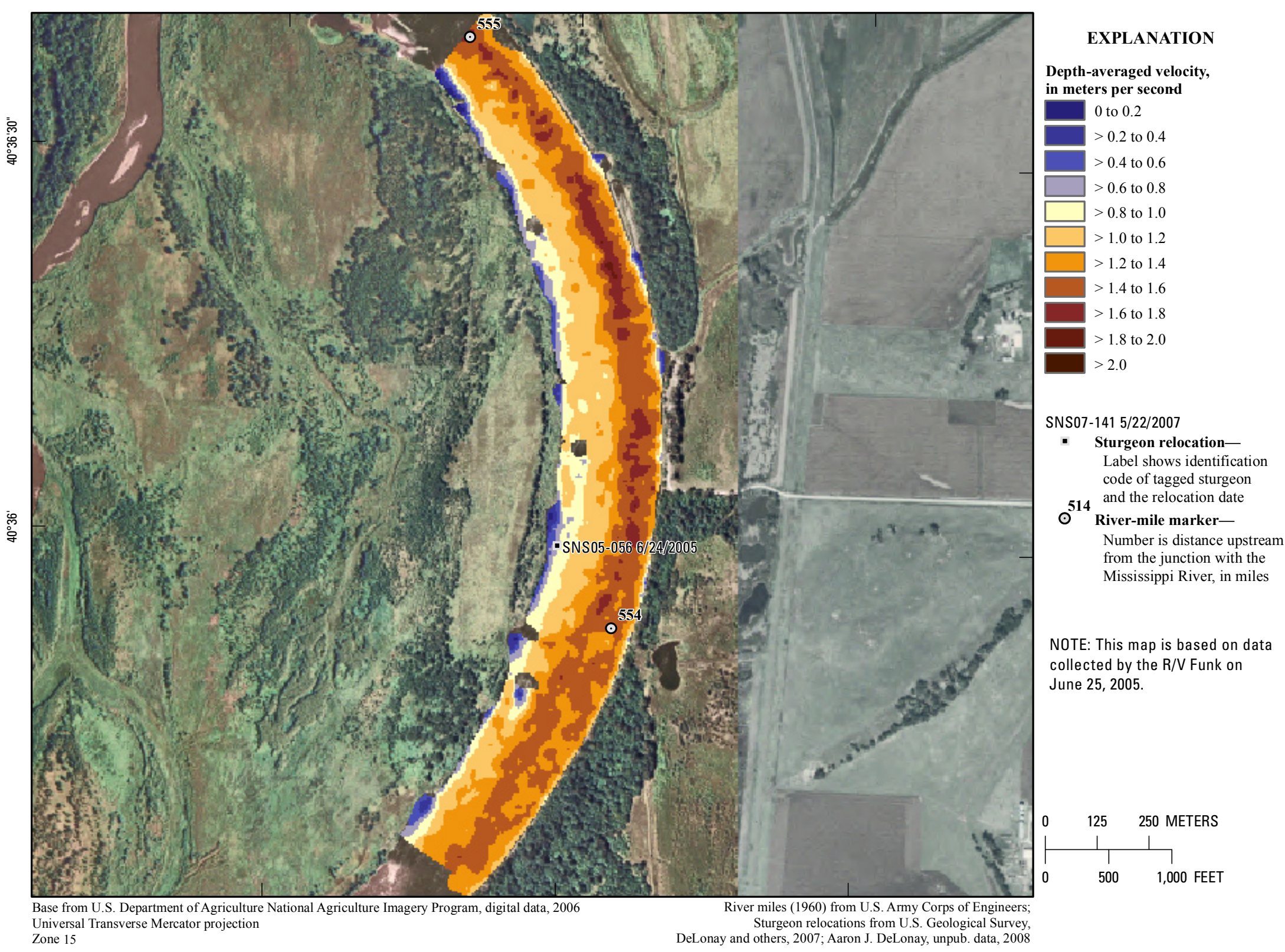




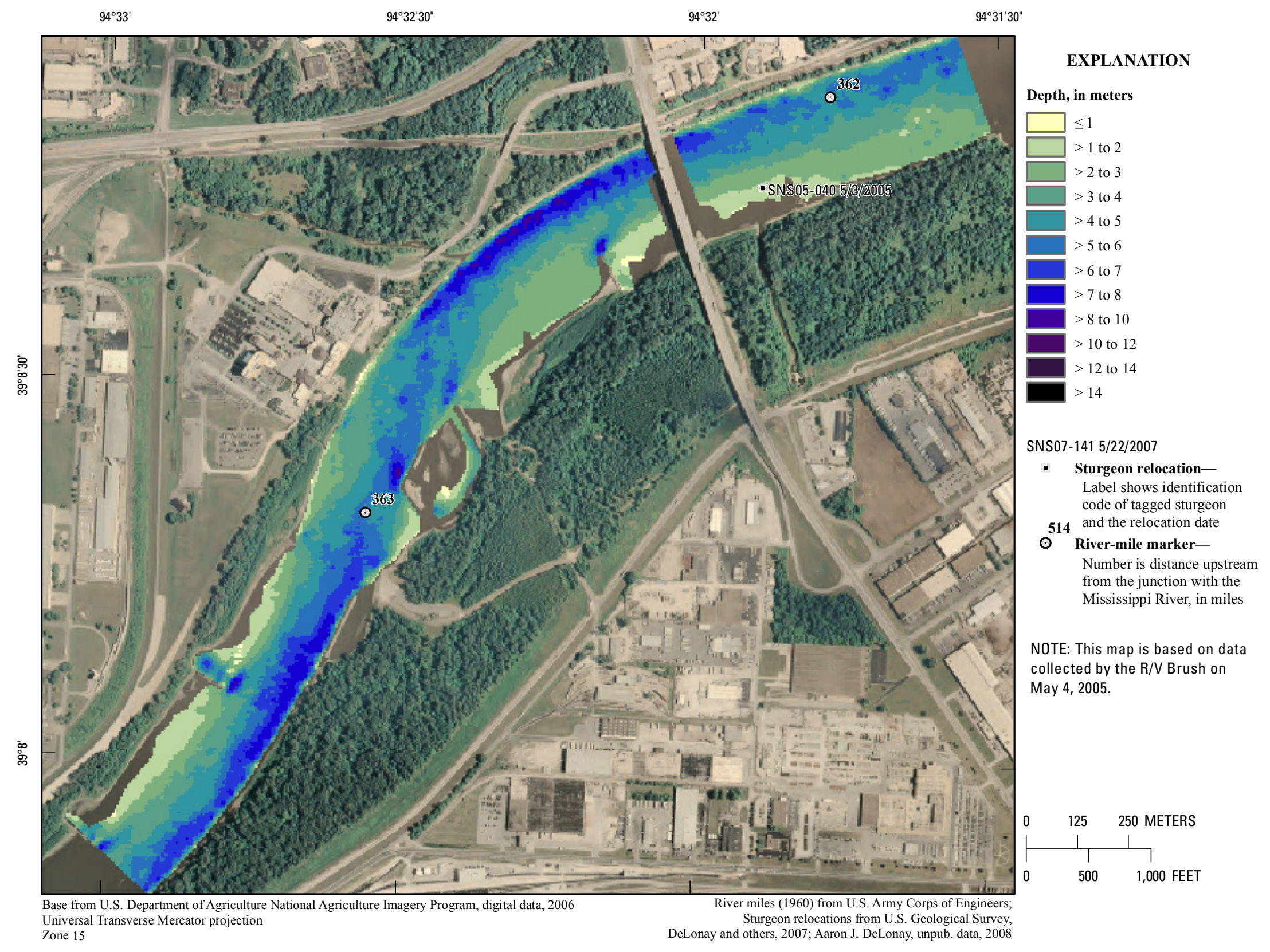

Figure 279. Map of depth based on data collected on May 4, 2005, in the vicinity of river mile 363 . 


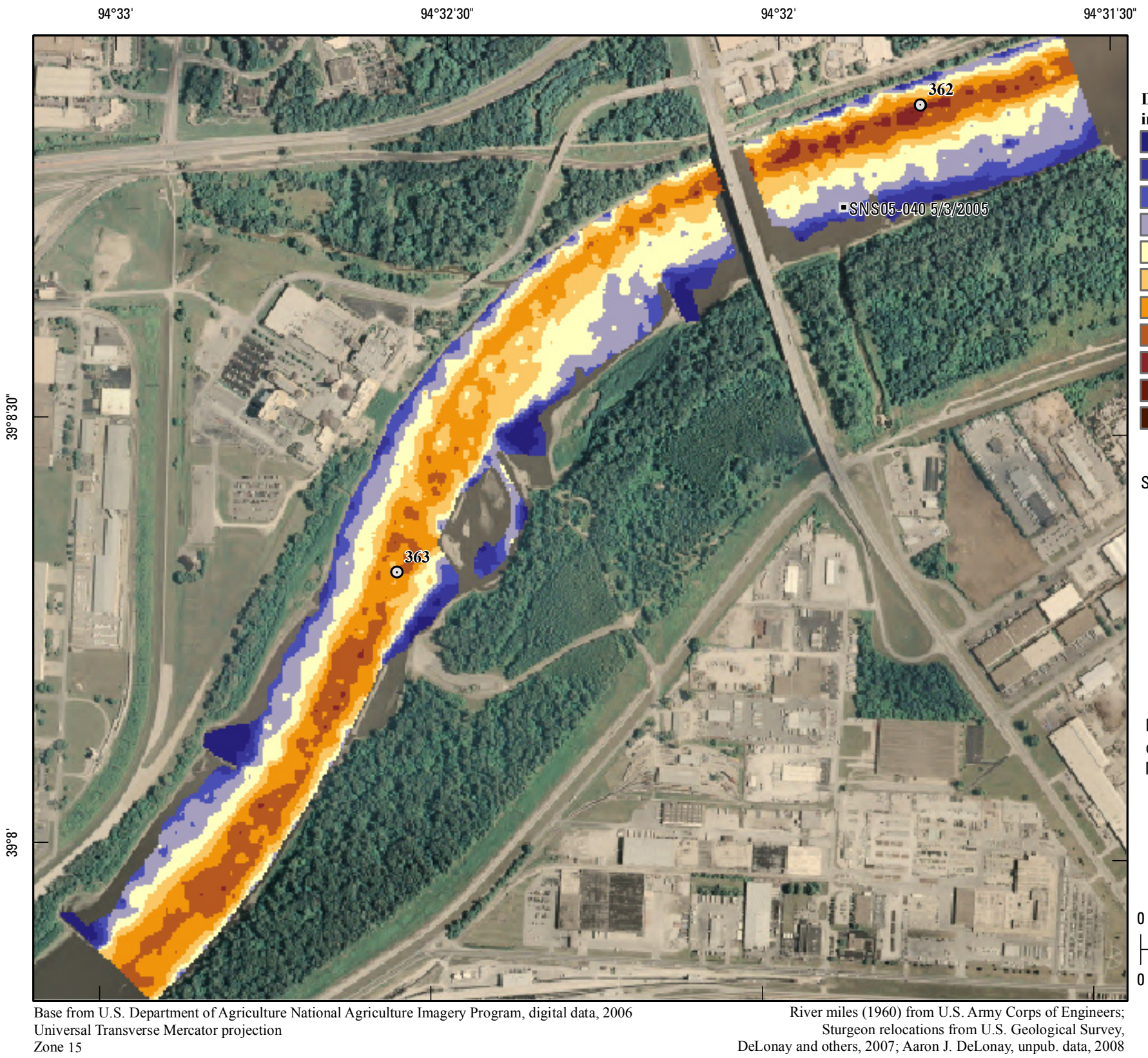

EXPLANATION

Depth-averaged velocity,

in meters per second

0 to 0.2

$>0.2$ to 0.4

$>0.4$ to 0.6

$>0.6$ to 0.8

$>0.8$ to 1.0

$>1.0$ to 1.2

$>1.2$ to 1.4

$>1.4$ to 1.6

$>1.6$ to 1.8

$>1.8$ to 2.0

$>2.0$

SNS07-141 5/22/2007

- Sturgeon relocationLabel shows identification code of tagged sturgeo

514 and the relocation date

Number is distance upstream
from the junction with the

Mississippi River, in miles

NOTE: This map is based on data

collected by the R/V Brush on

May 4, 2005.

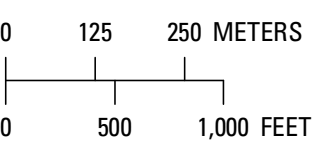
Sturgeon relocations from U.S. Geological Survey, 


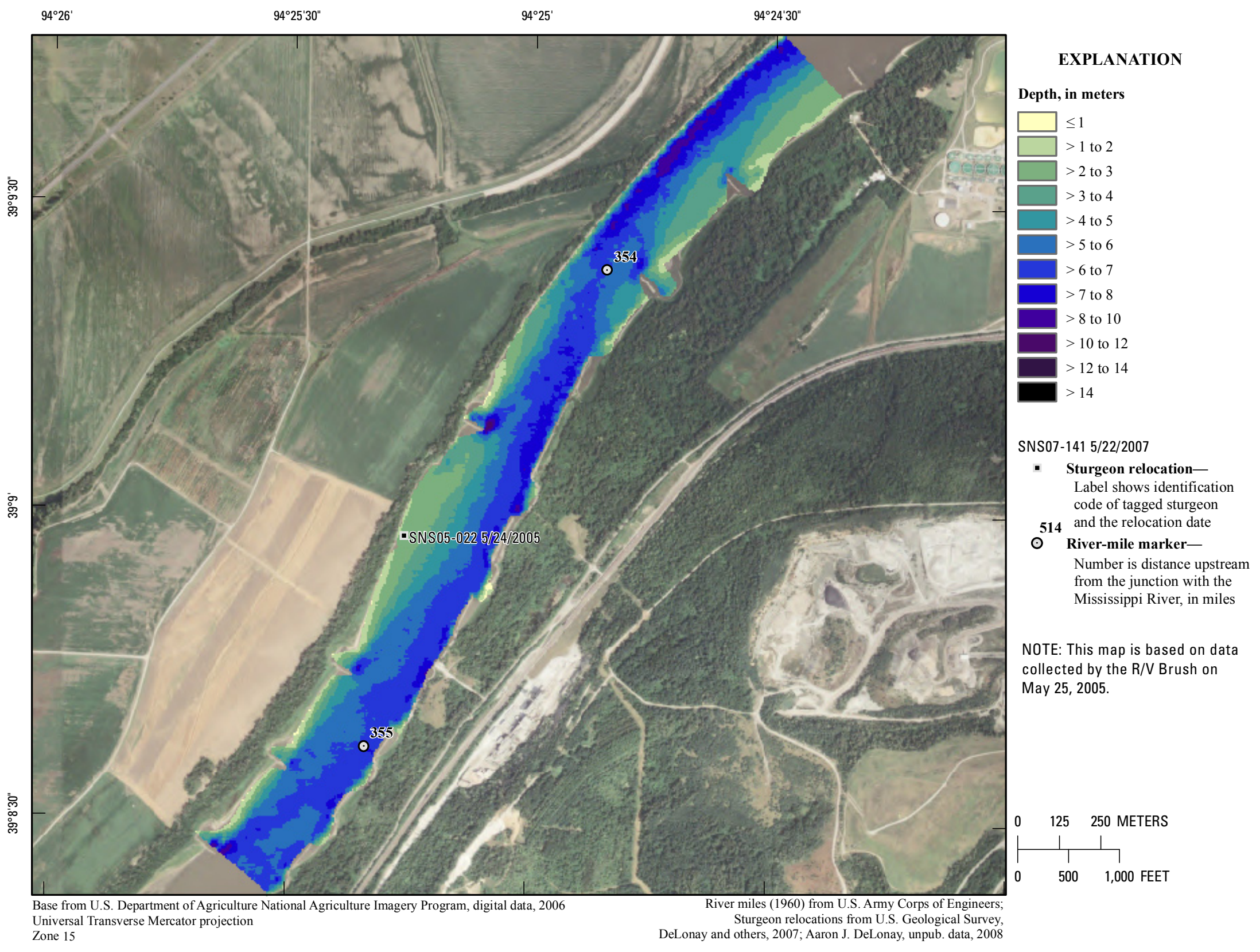

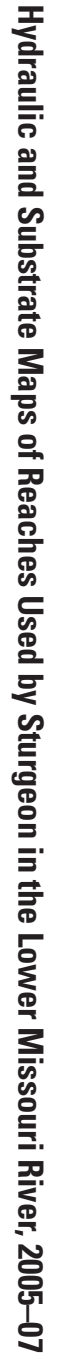

Figure 281. Map of depth based on data collected on May 25, 2005, in the vicinity of river mile 354 . 


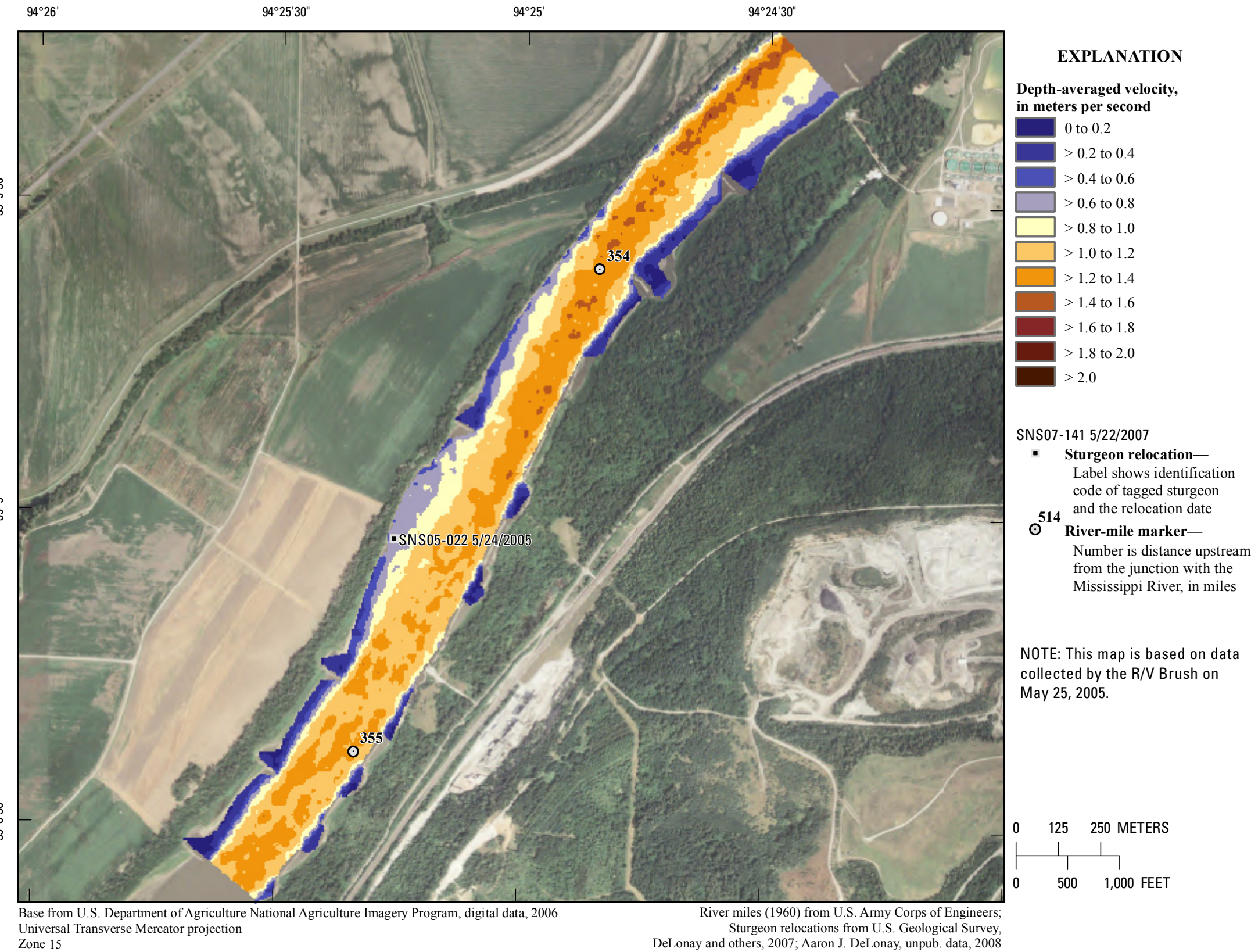

Figure 282. Map of depth-averaged velocity based on data collected on May 25, 2005, in the vicinity of river mile 354 . 


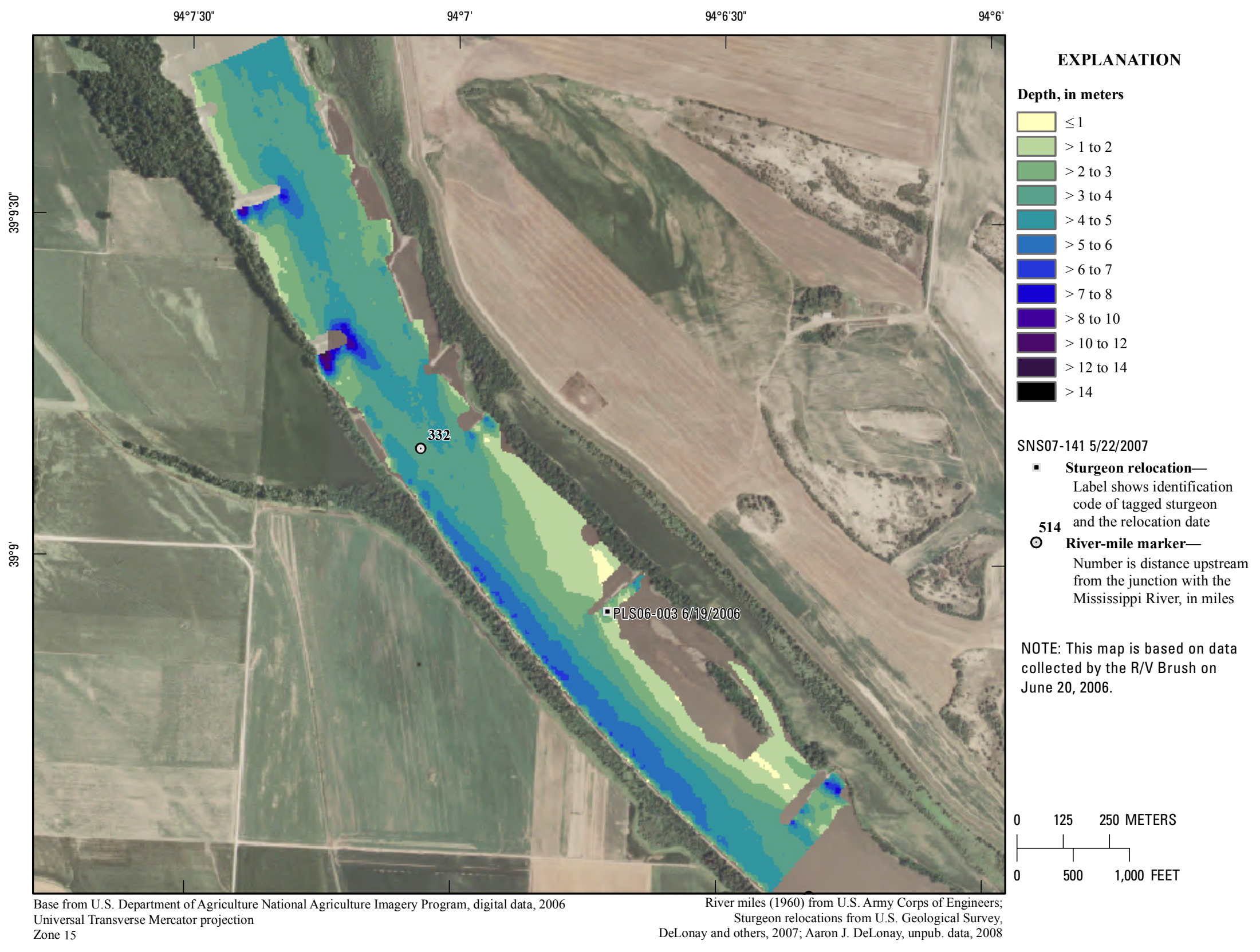

Figure 283. Map of depth based on data collected on June 20, 2006, in the vicinity of river mile 332 . 


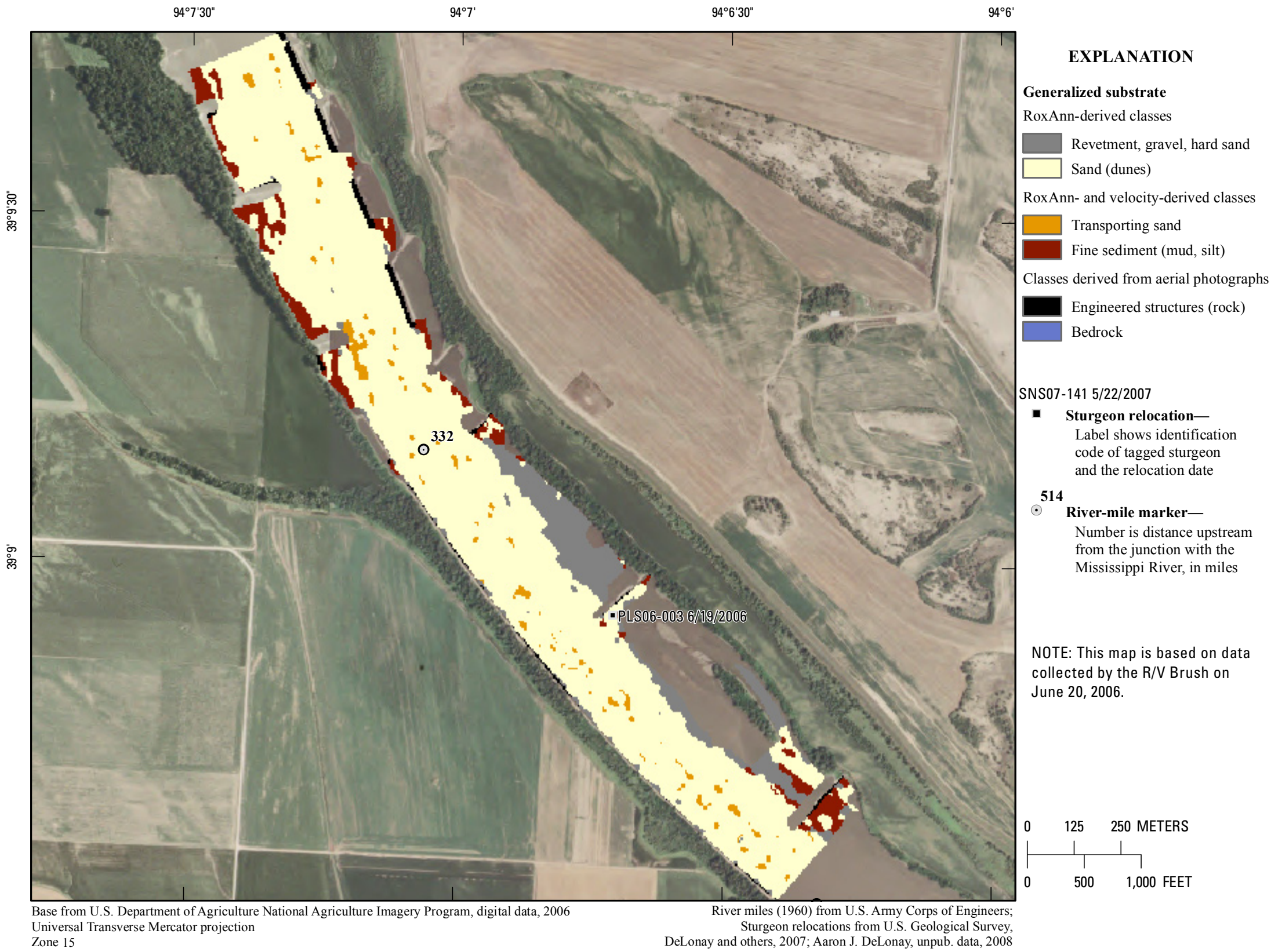

Figure 284. Map of generalized substrate based on data collected on June 20, 2006, in the vicinity of river mile 332 . 


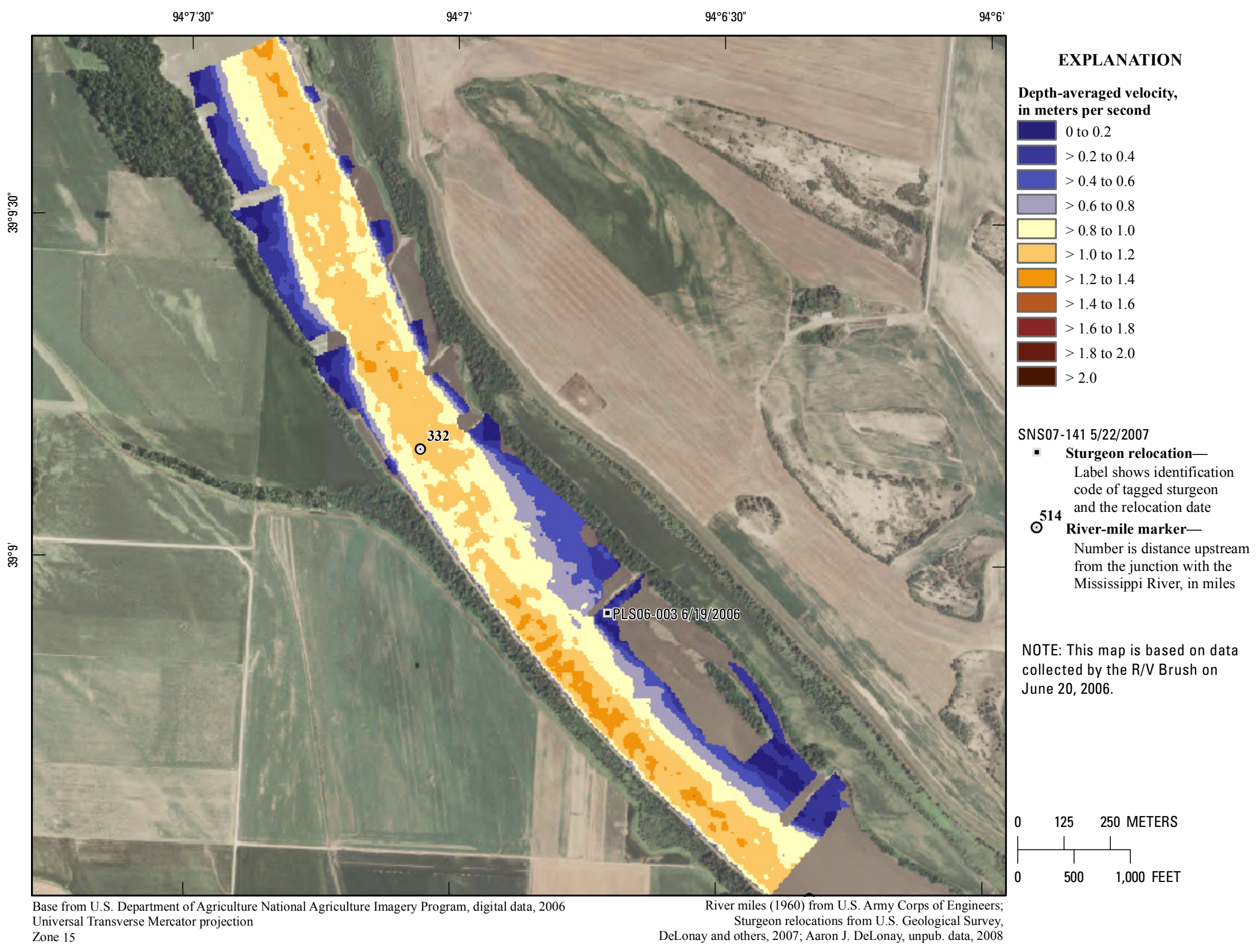

Figure 285. Map of depth-averaged velocity based on data collected on June 20, 2006, in the vicinity of river mile 332 . 


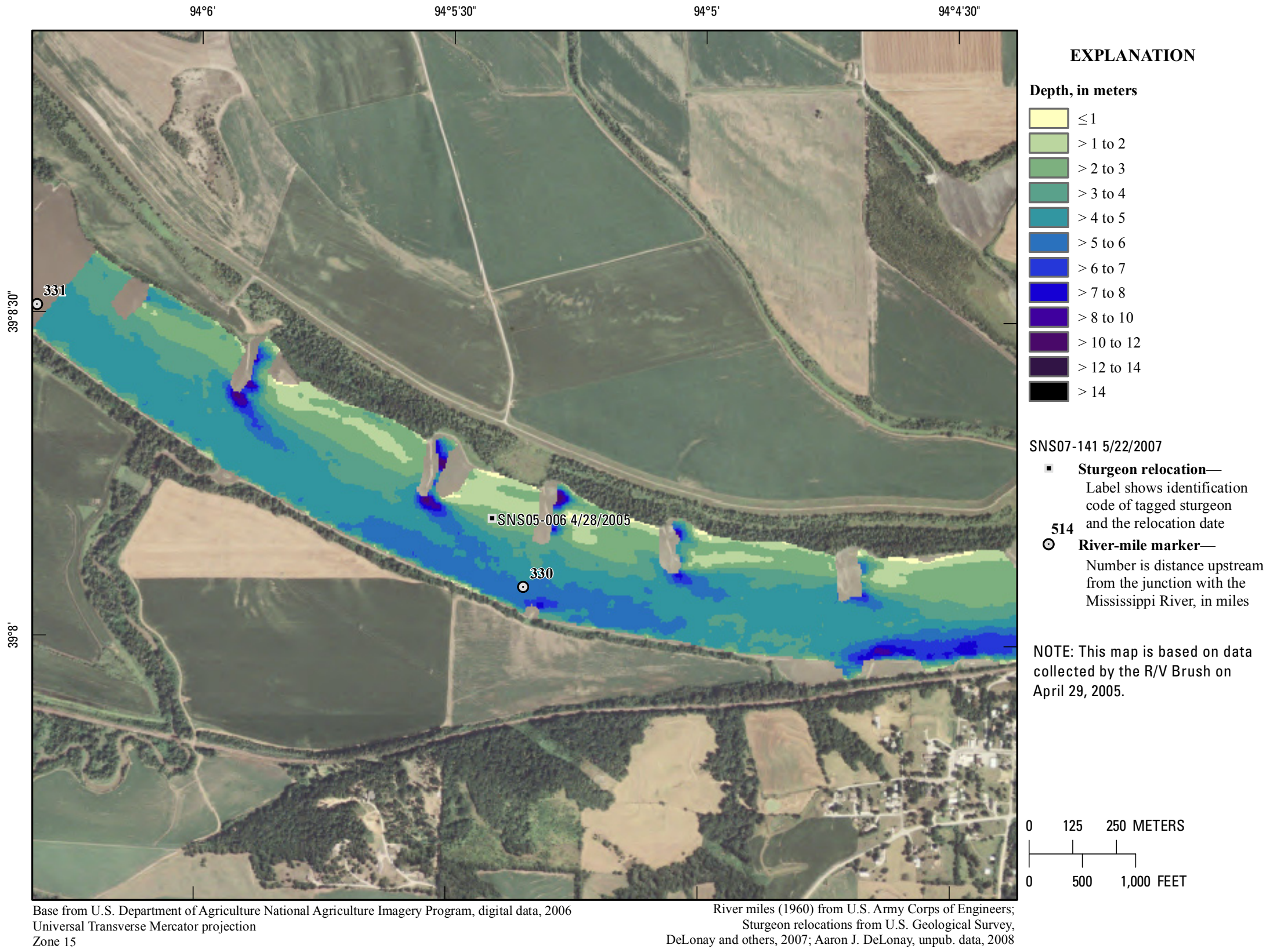

Figure 286. Map of depth based on data collected on April 29, 2005, in the vicinity of river mile 330 . 


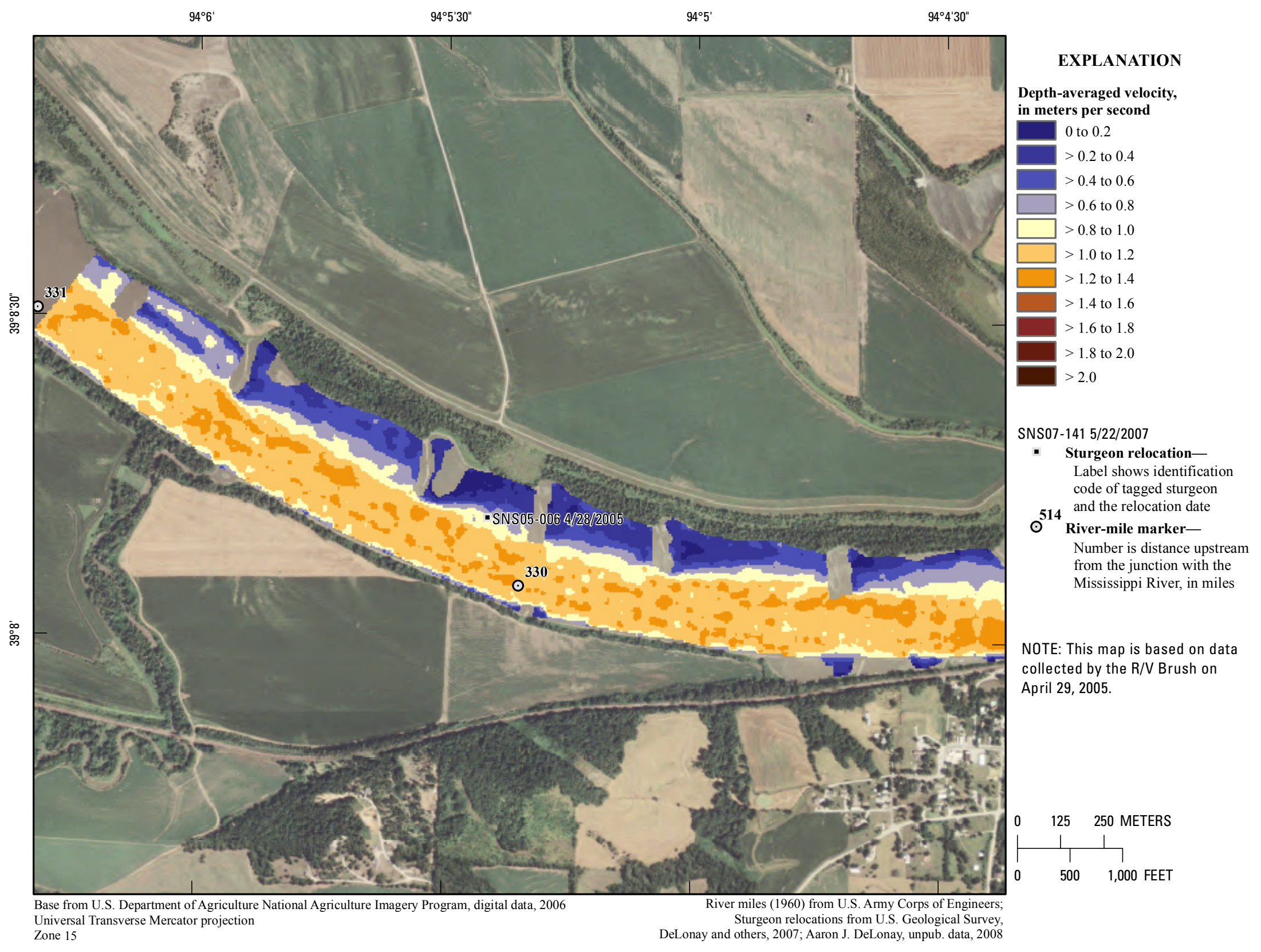

Figure 287. Map of depth-averaged velocity based on data collected on April 29, 2005, in the vicinity of river mile 330 


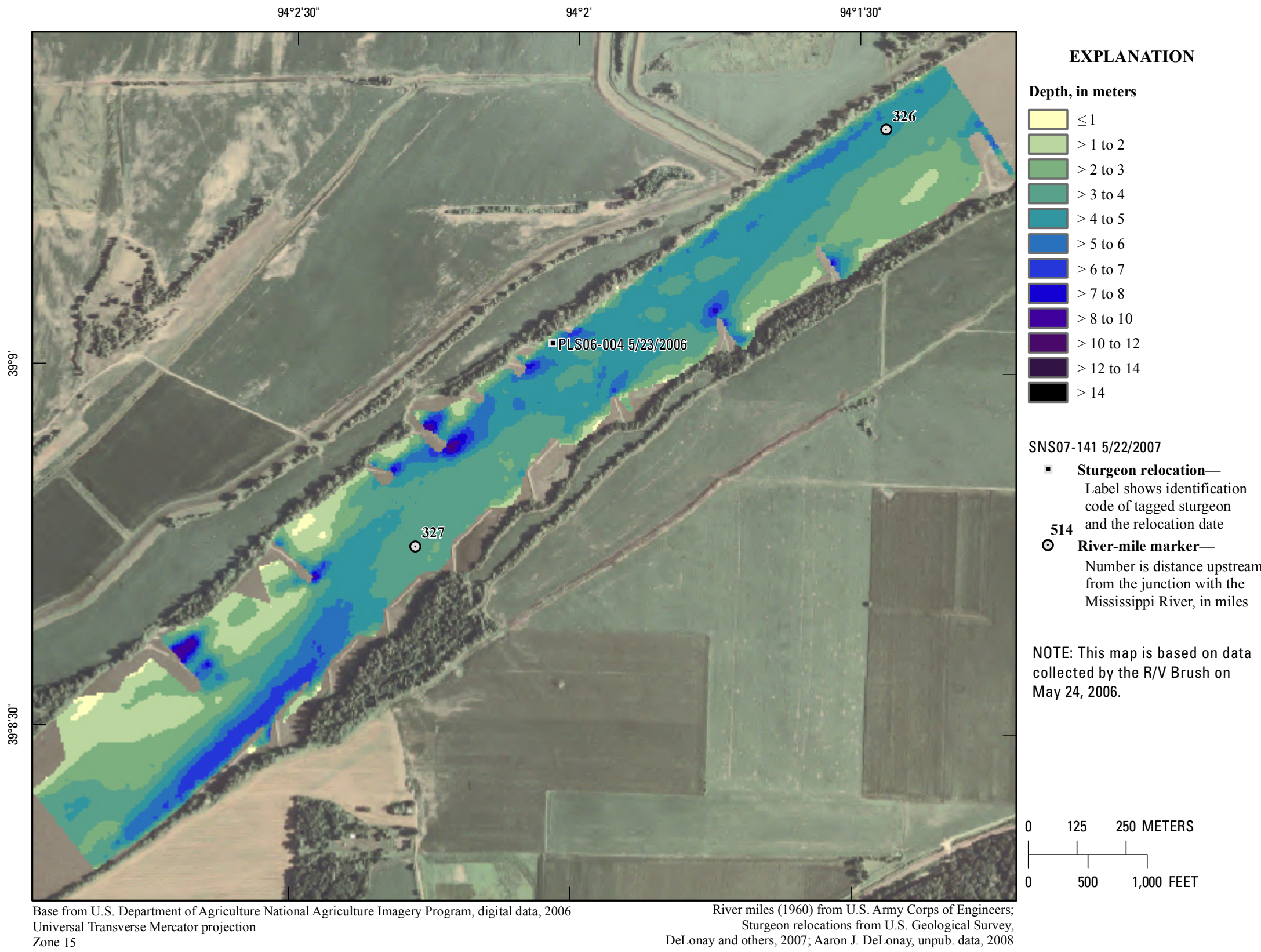

Figure 288. Map of depth based on data collected on May 24, 2006, in the vicinity of river mile 327. 


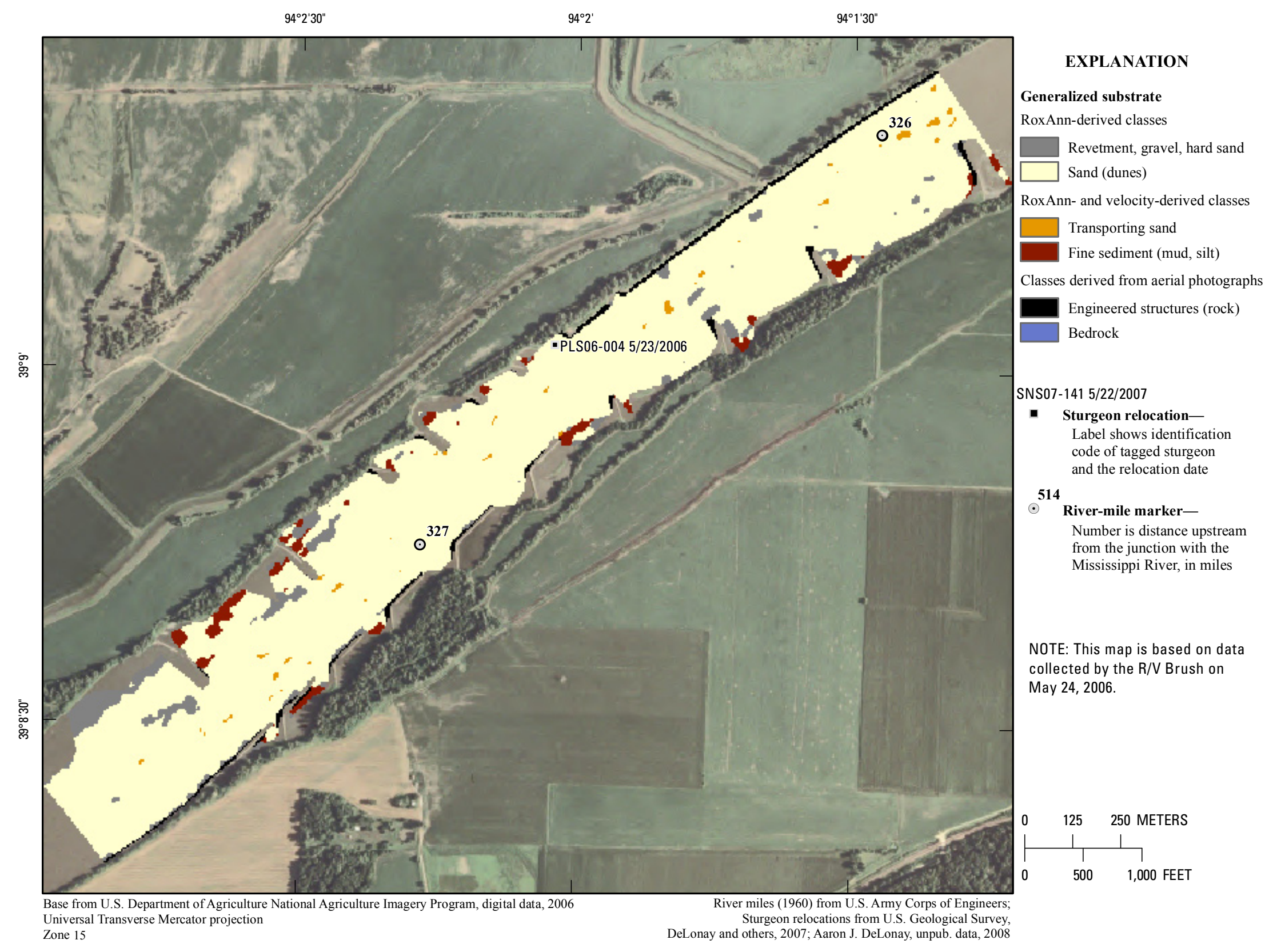

Figure 289. Map of generalized substrate based on data collected on May 24, 2006, in the vicinity of river mile 327 . 


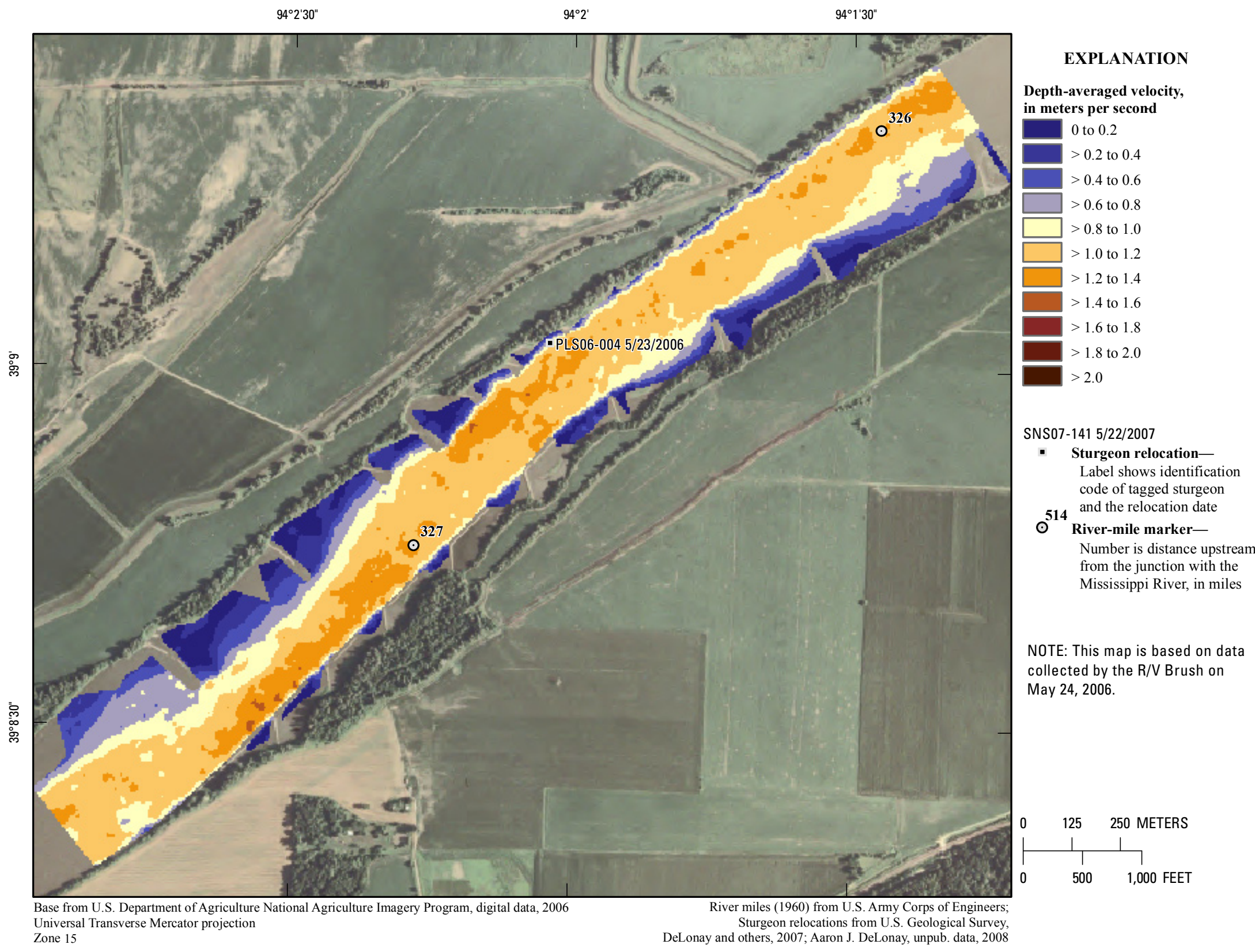

Figure 290. Map of depth-averaged velocity based on data collected on May 24, 2006, in the vicinity of river mile 327 . 


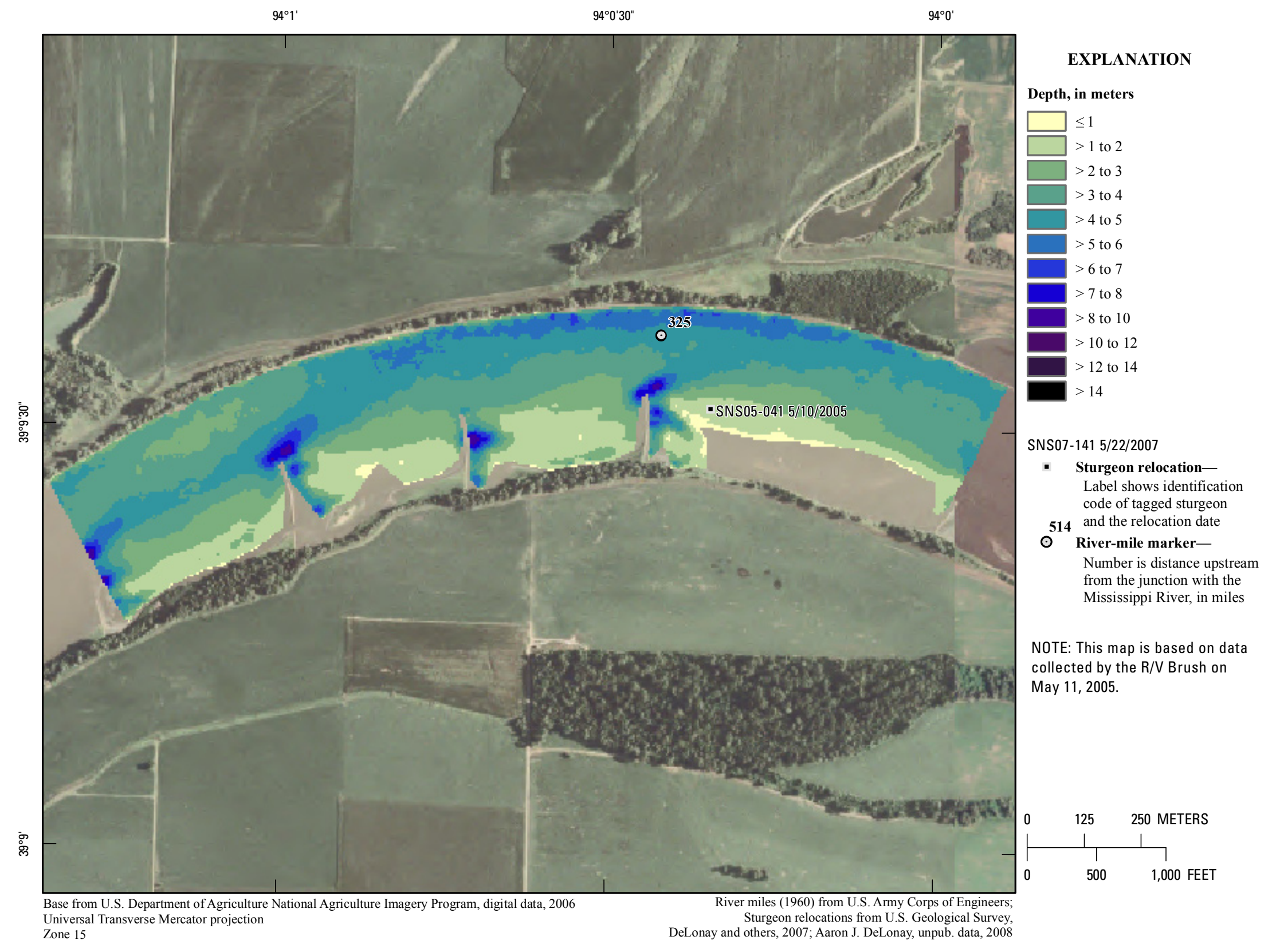

Figure 291. Map of depth based on data collected on May 11, 2005, in the vicinity of river mile 325. 


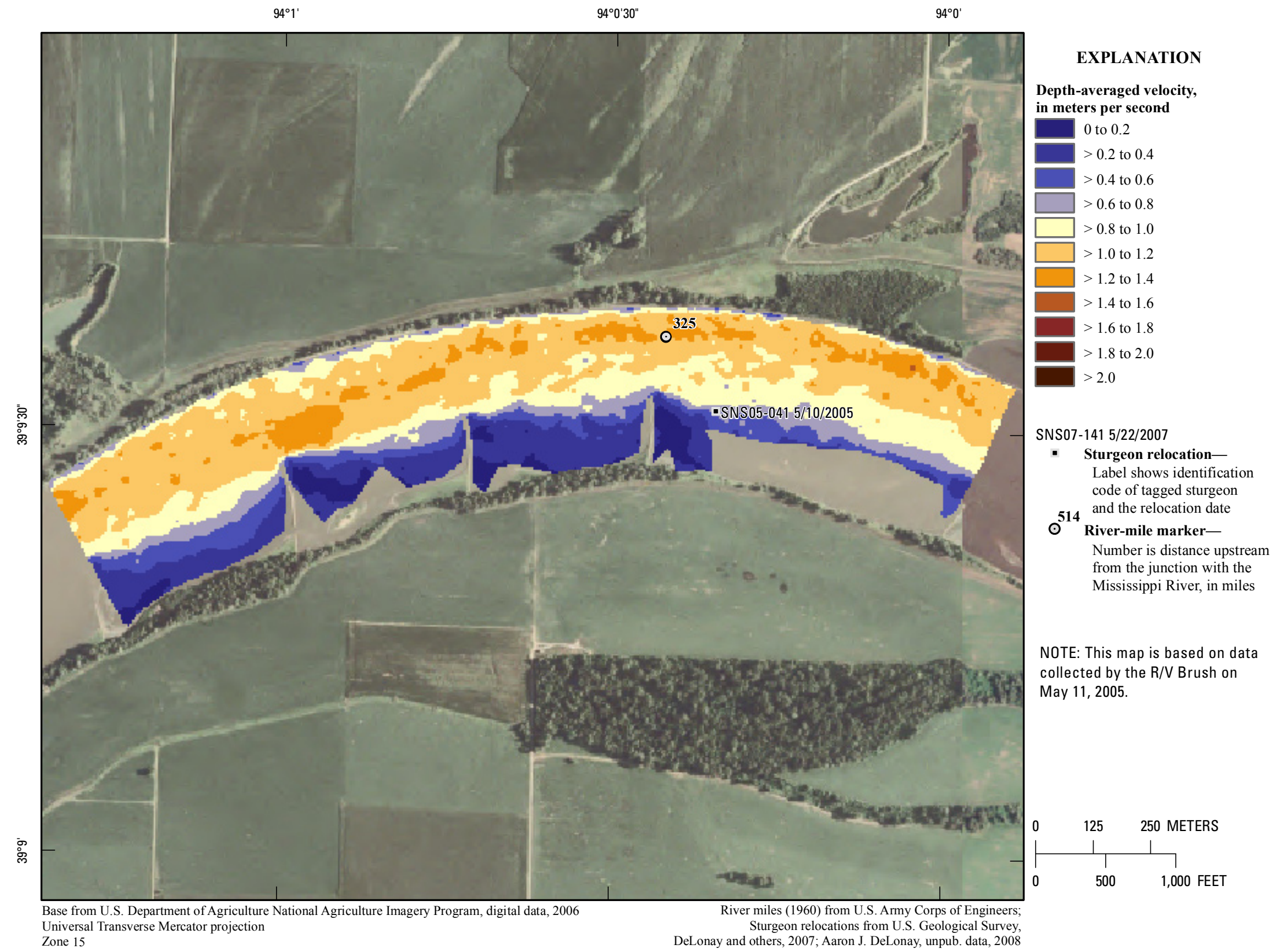

Figure 292. Map of depth-averaged velocity based on data collected on May 11, 2005, in the vicinity of river mile 325 . 


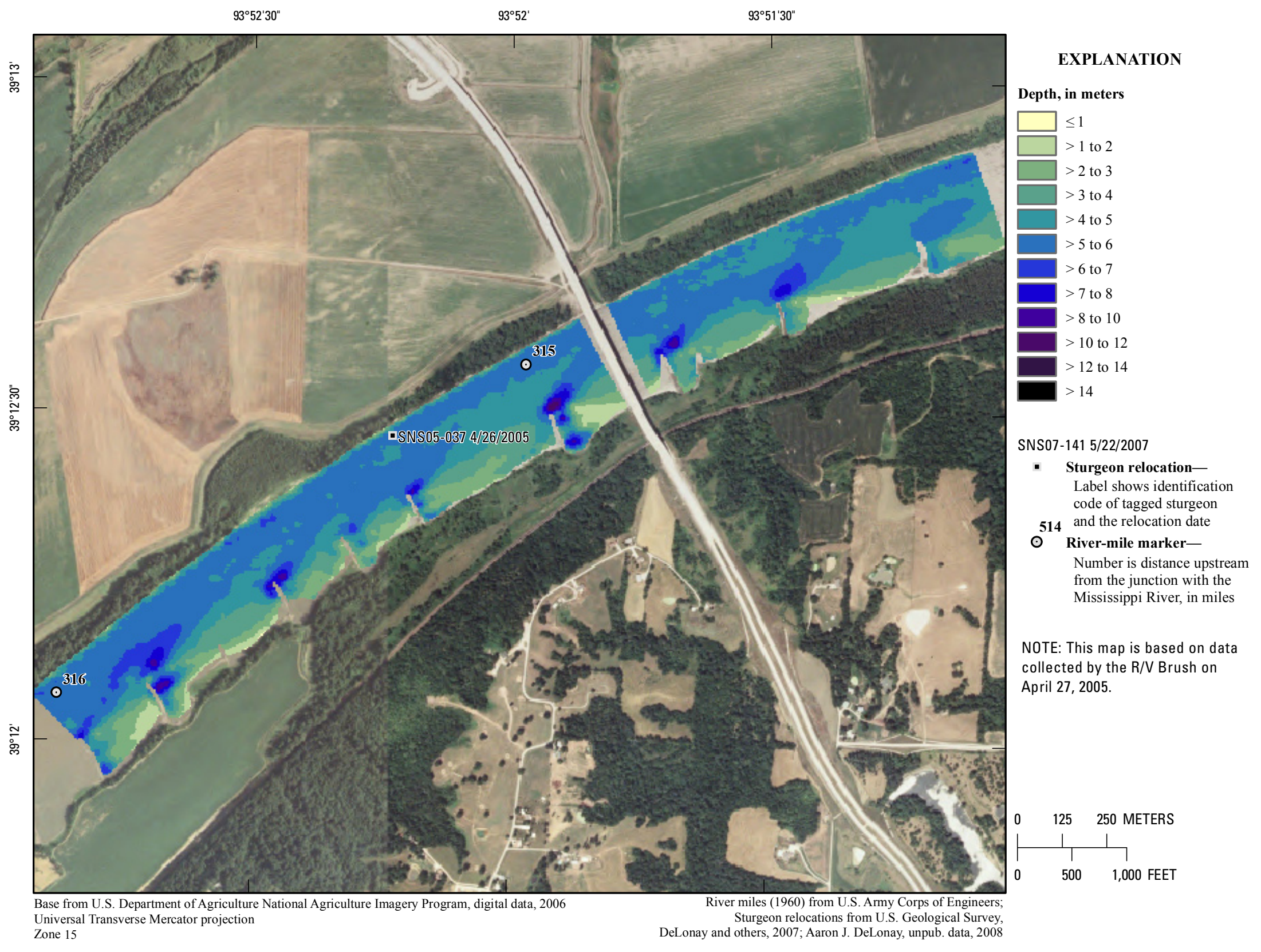

Figure 293. Map of depth based on data collected on April 27, 2005, in the vicinity of river mile 315. 


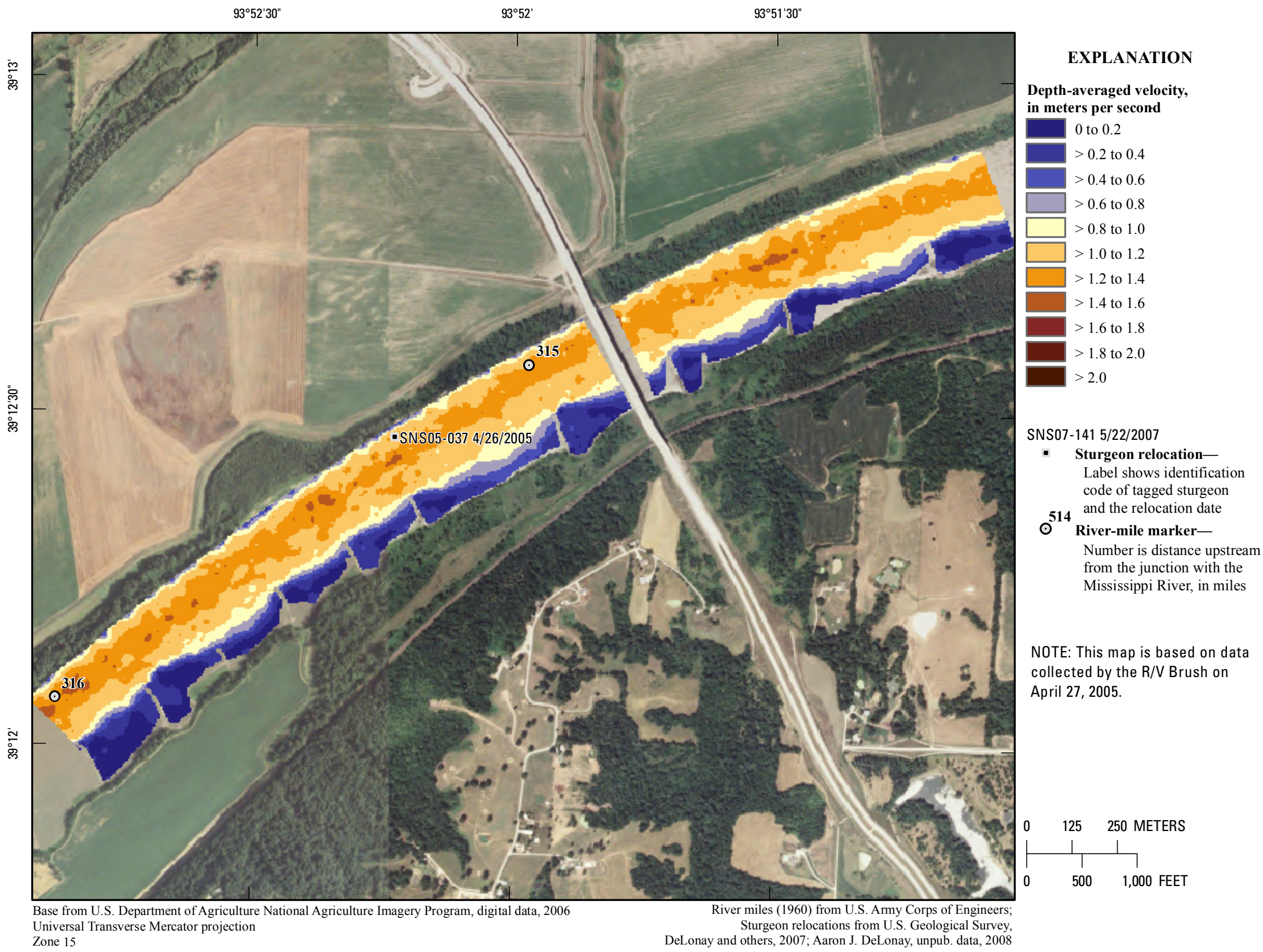

Figure 294. Map of depth-averaged velocity based on data collected on April 27, 2005, in the vicinity of river mile 315 . 


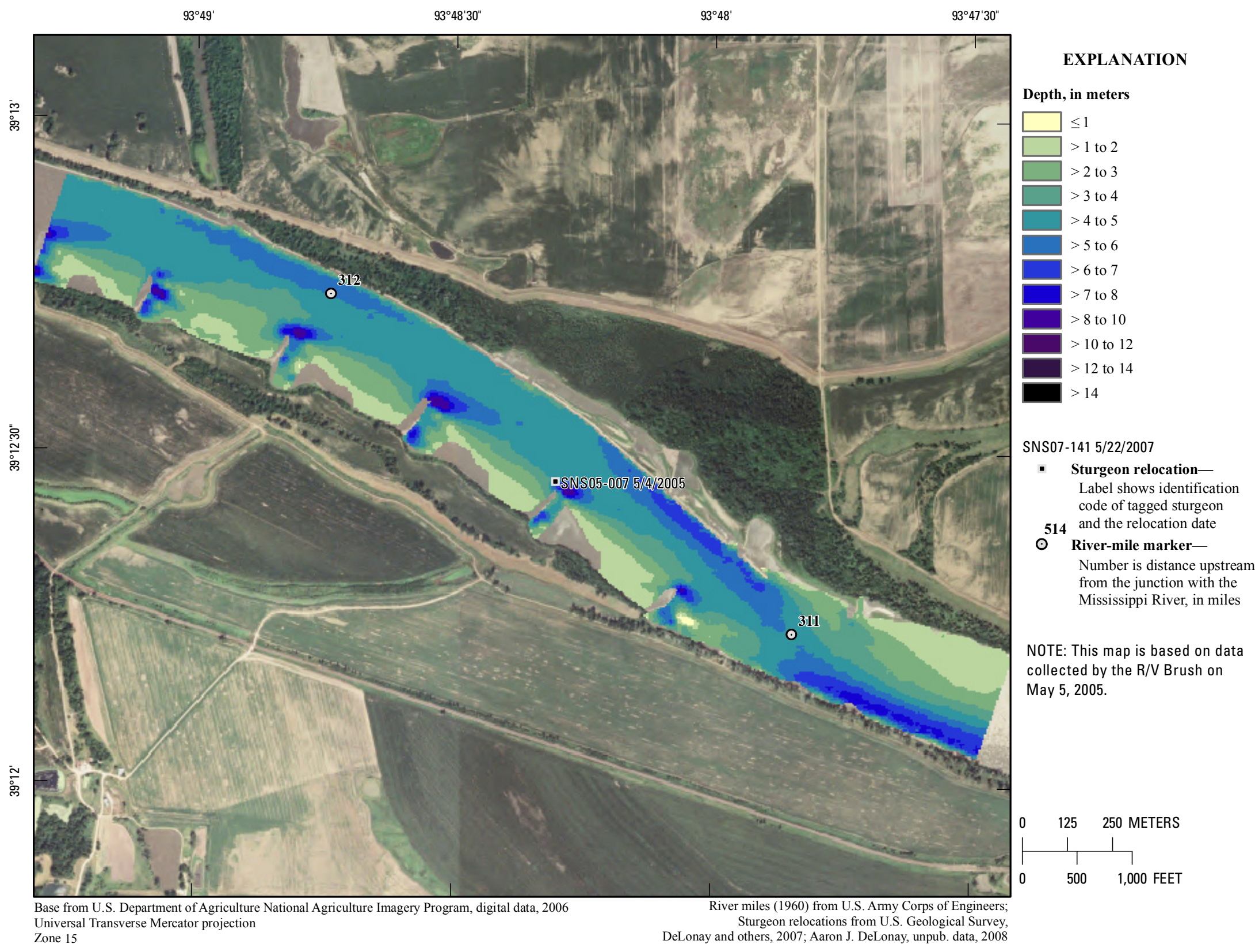

Figure 295. Map of depth based on data collected on May 5, 2005, in the vicinity of river mile 312 . 


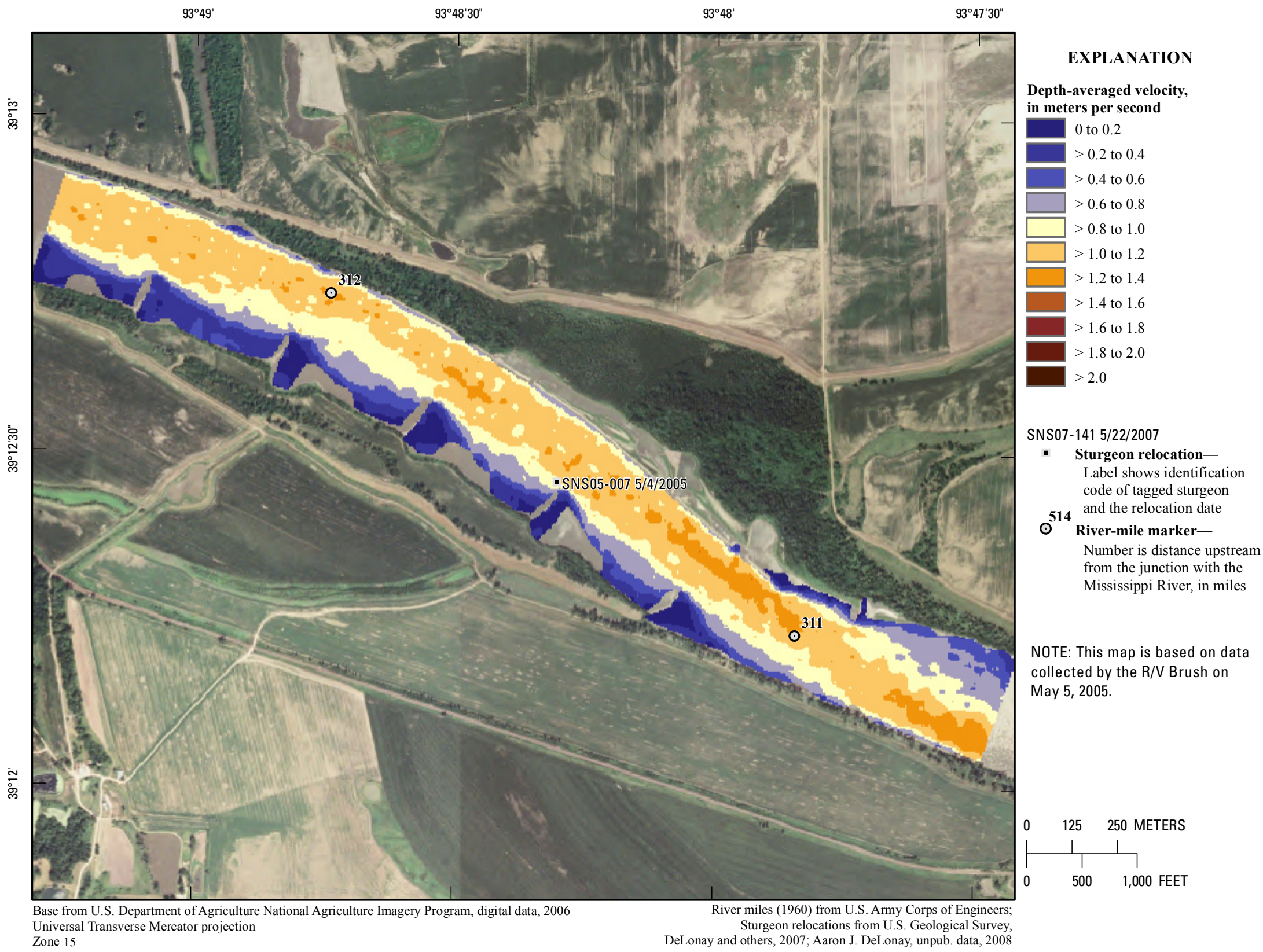

Figure 296. Map of depth-averaged velocity based on data collected on May 5, 2005, in the vicinity of river mile 312 . 


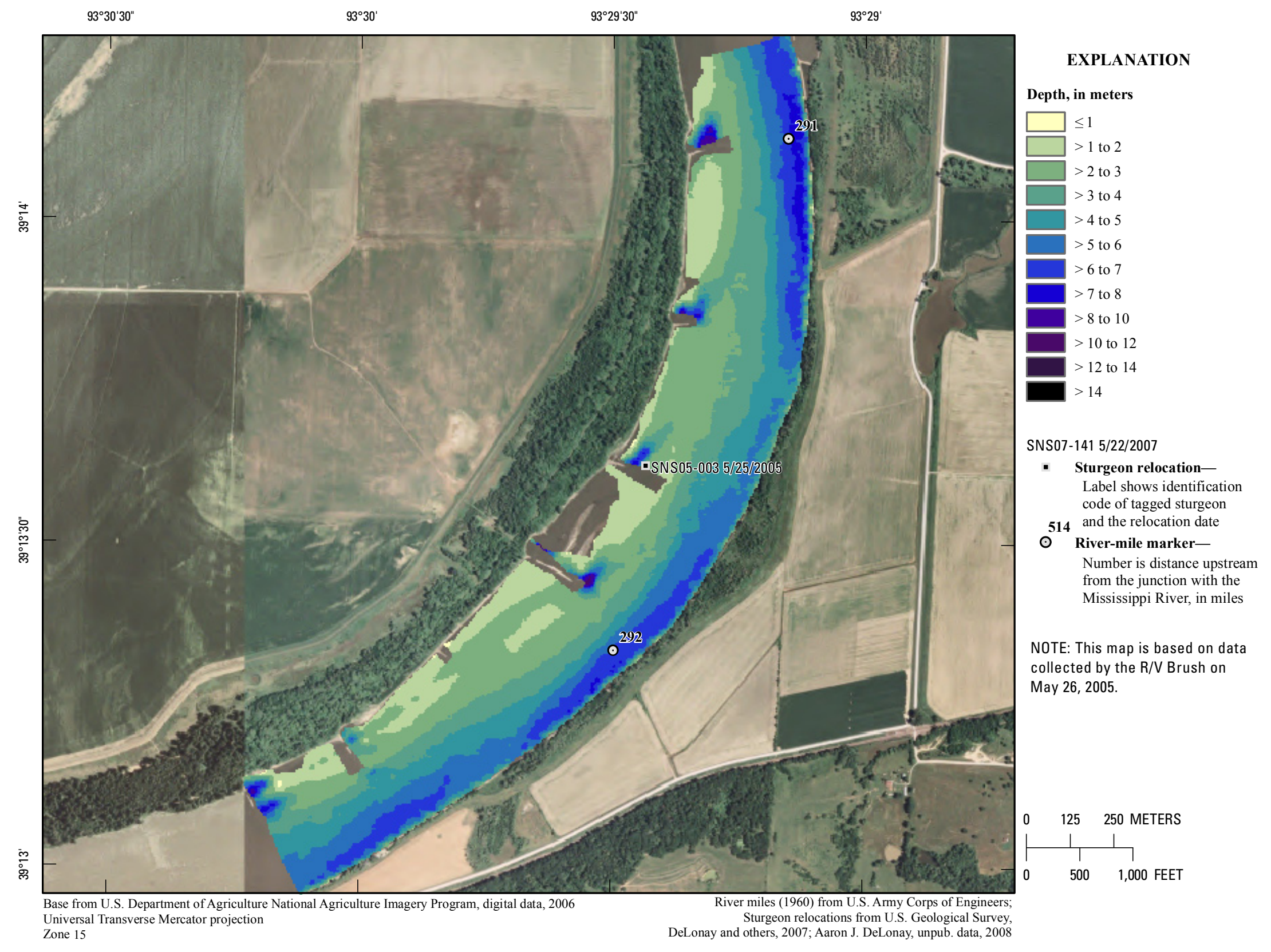

Figure 297. Map of depth based on data collected on May 26, 2005, in the vicinity of river mile 292. 


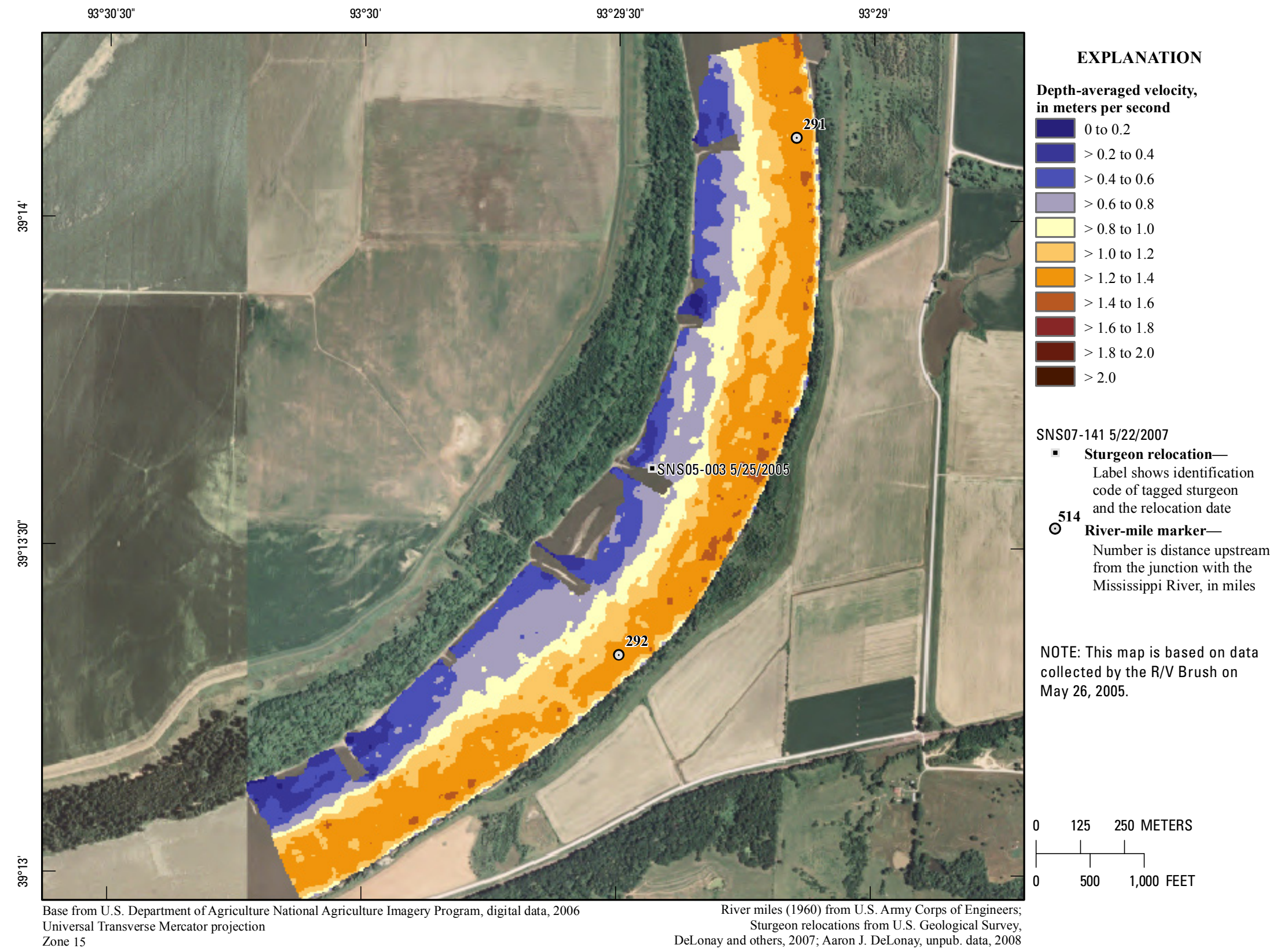

Figure 298. Map of depth-averaged velocity based on data collected on May 26, 2005, in the vicinity of river mile 292. 


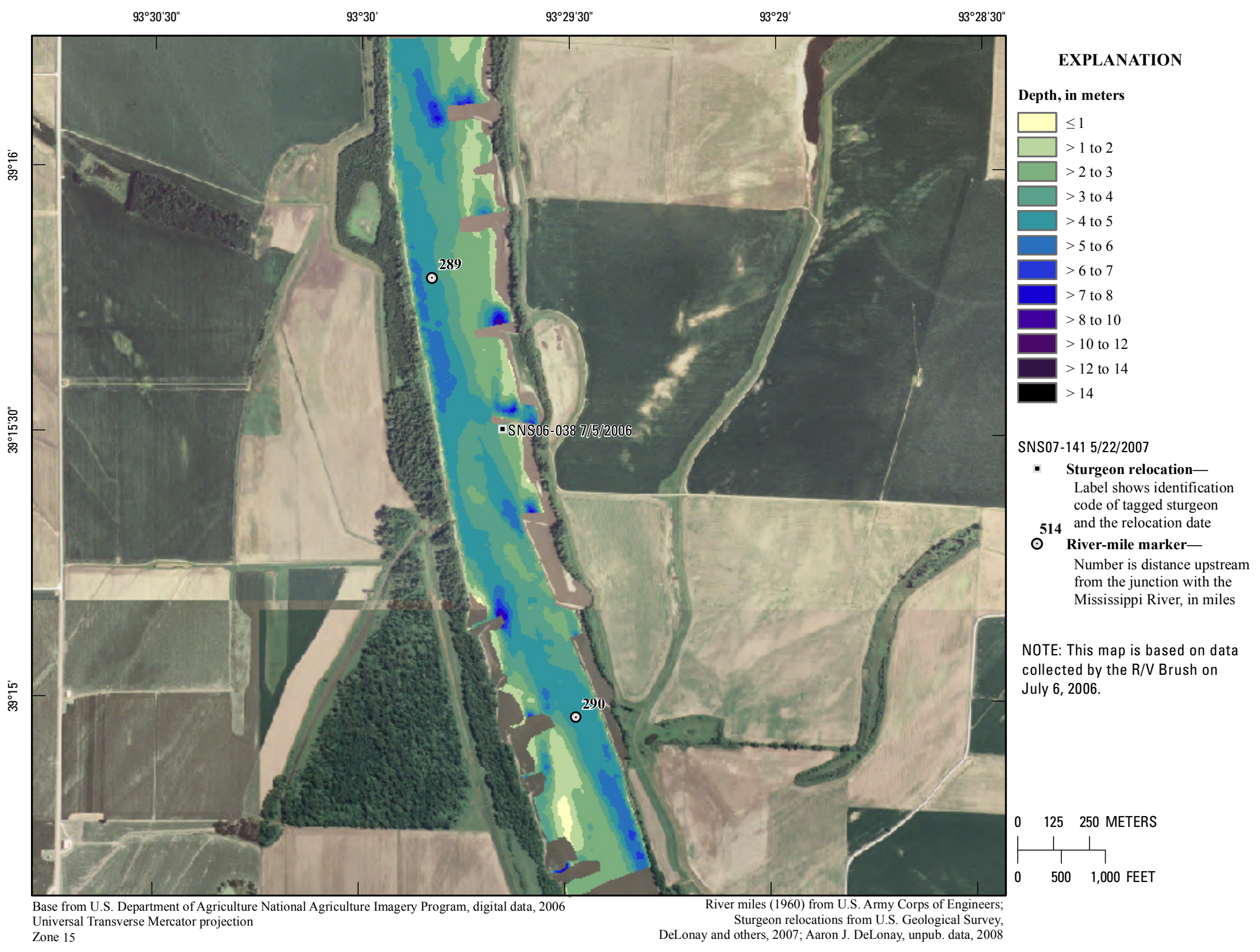

Figure 299. Map of depth based on data collected on July 6, 2006, in the vicinity of river mile 289. 


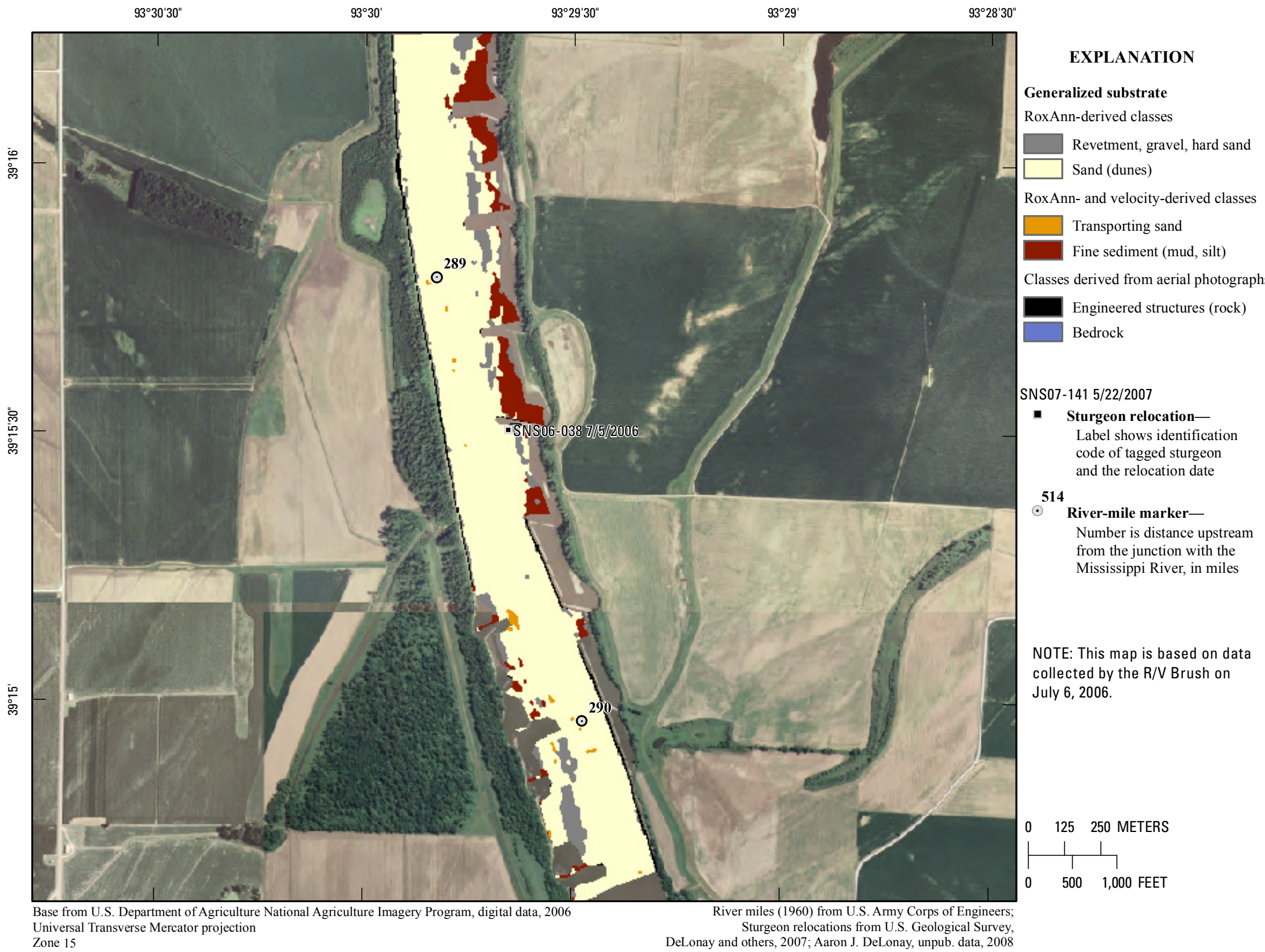

Figure 300. Map of generalized substrate based on data collected on July 6, 2006, in the vicinity of river mile 289. 


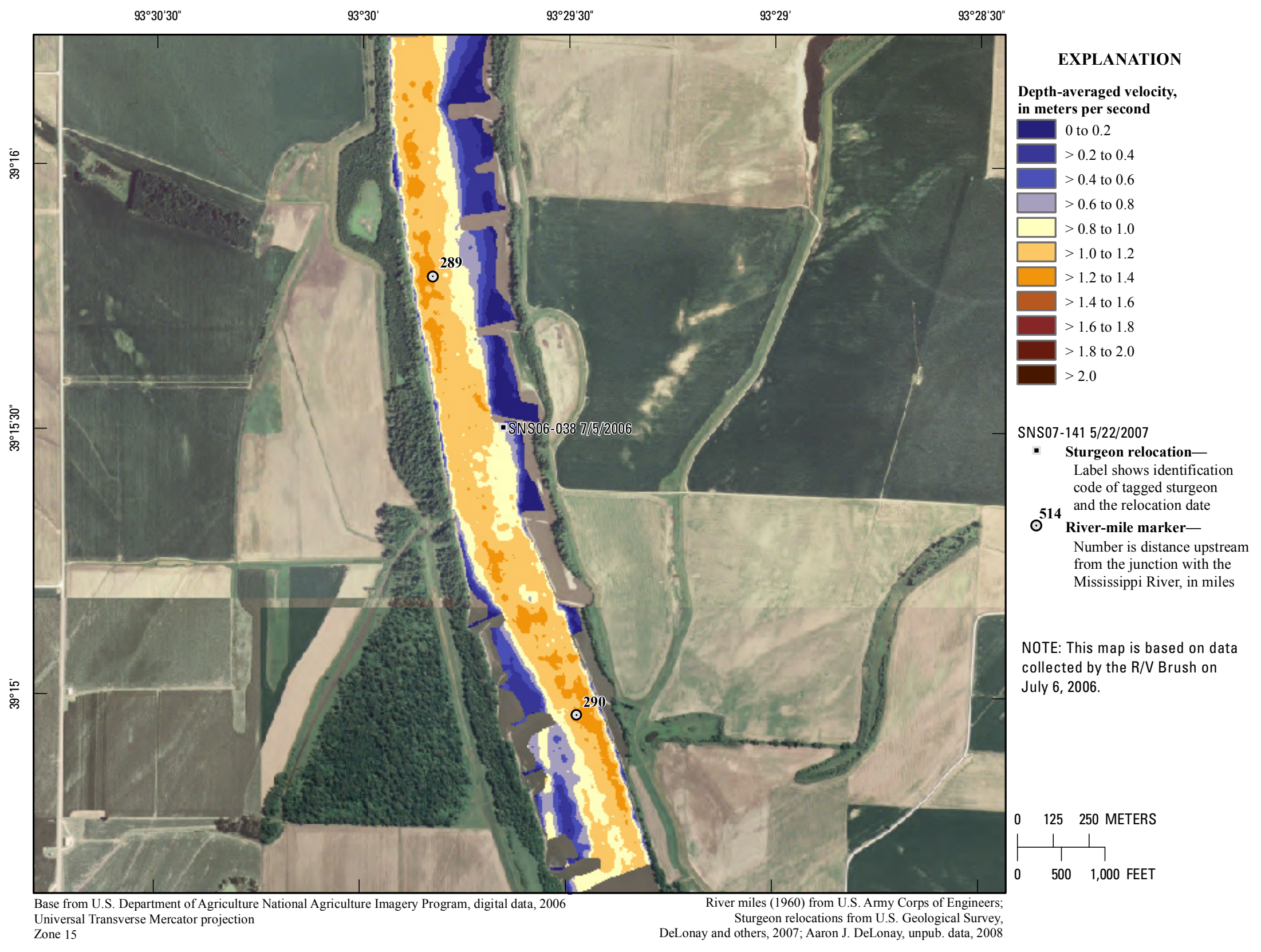

Figure 301. Map of depth-averaged velocity based on data collected on July 6, 2006, in the vicinity of river mile 289. 


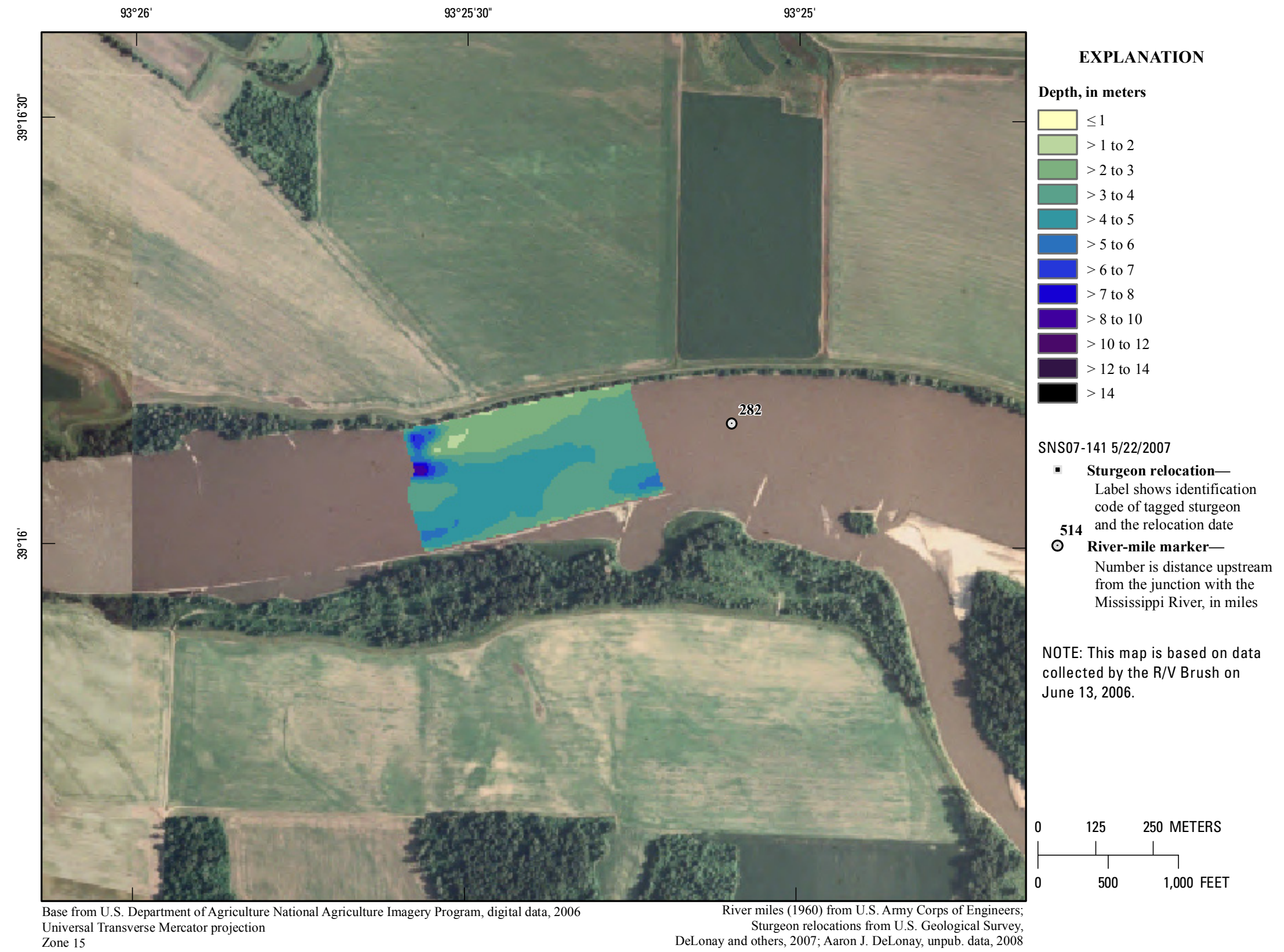

Figure 302. Map of depth based on data collected on June 13, 2006, in the vicinity of river mile 282. 


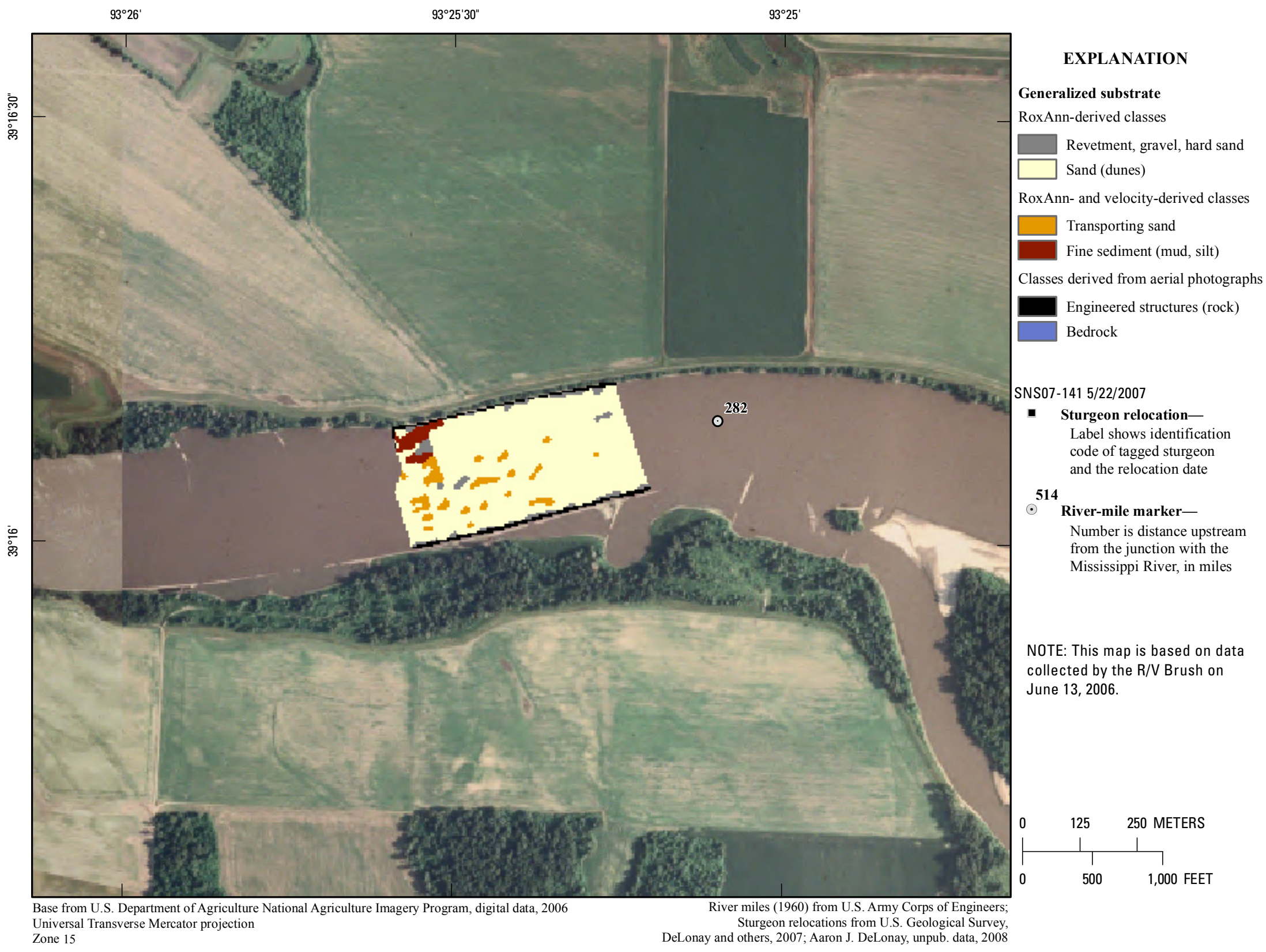

Figure 303. Map of generalized substrate based on data collected on June 13, 2006, in the vicinity of river mile 282 . 


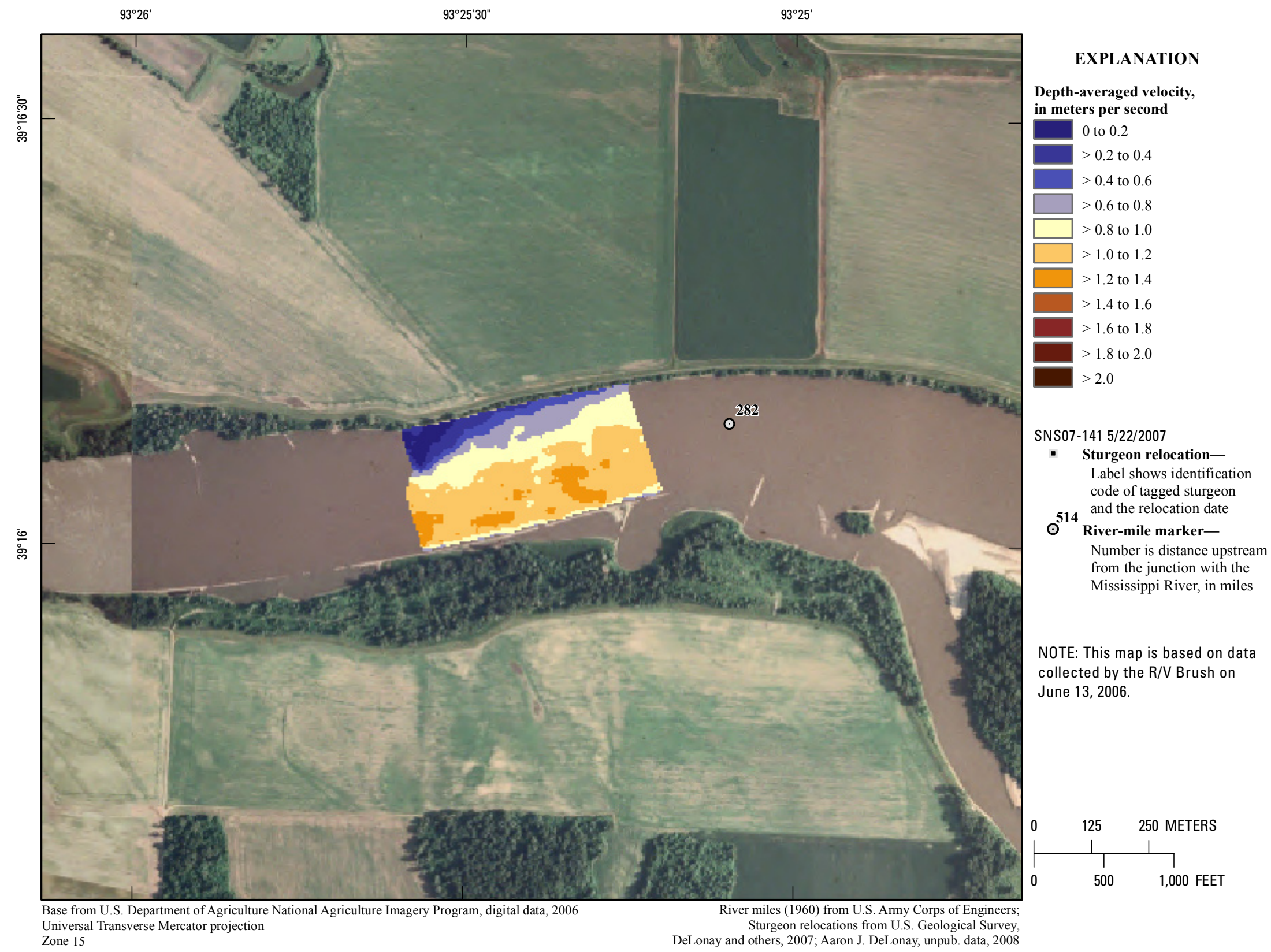

Figure 304. Map of depth-averaged velocity based on data collected on June 13, 2006, in the vicinity of river mile 282. 


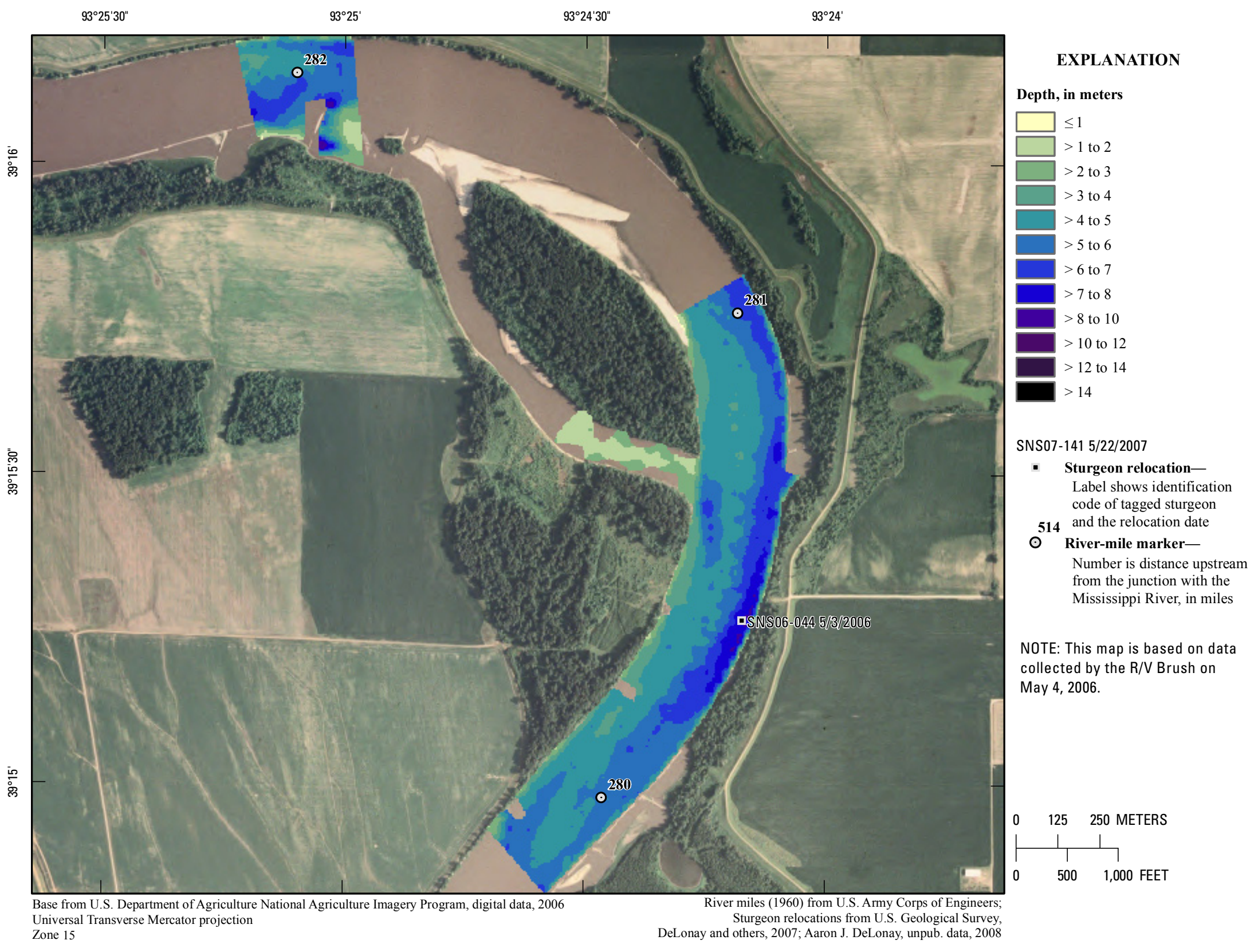

Figure 305. Map of depth based on data collected on May 4, 2006, in the vicinity of river mile 282. 


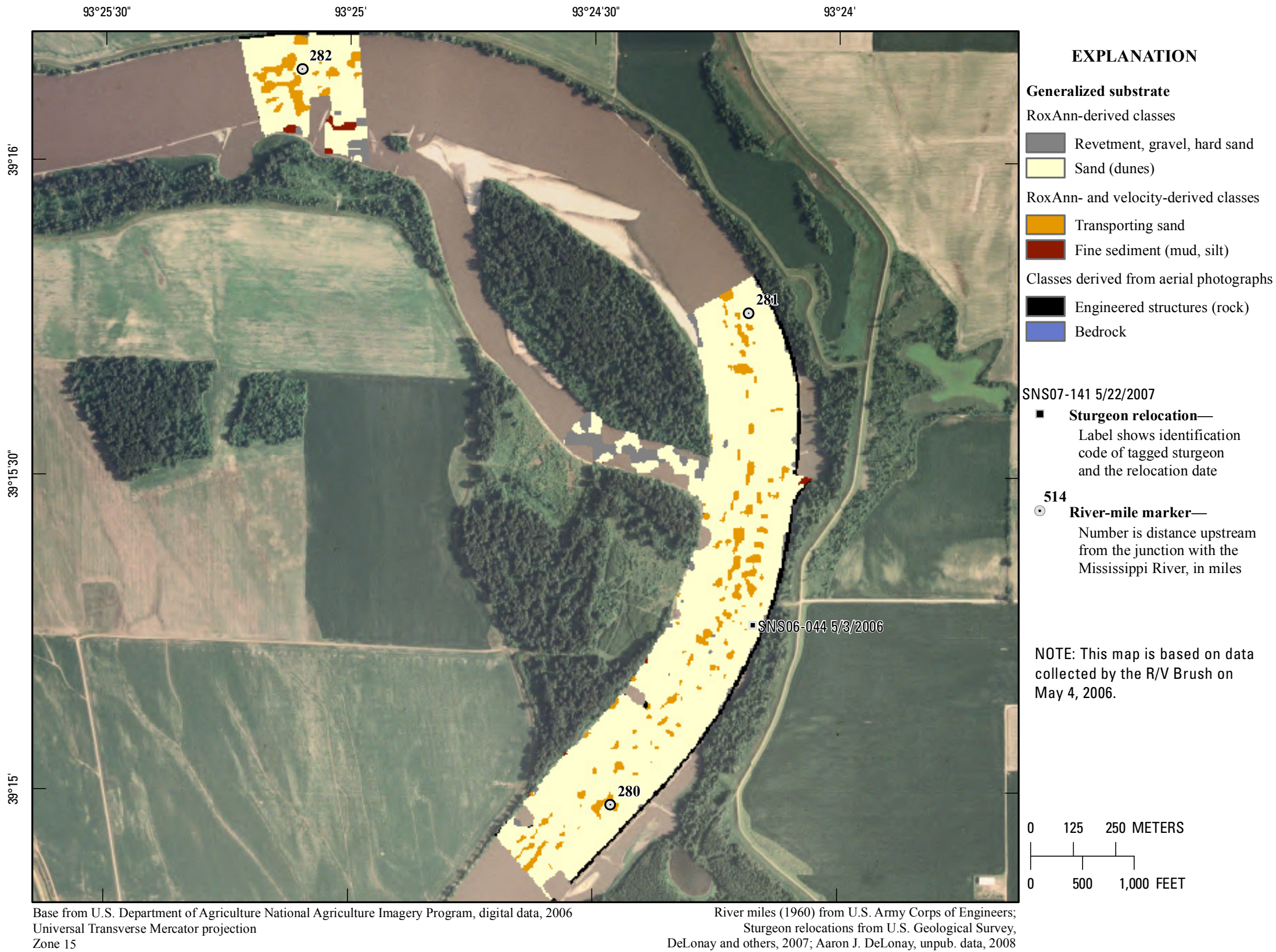

Figure 306. Map of generalized substrate based on data collected on May 4, 2006, in the vicinity of river mile 282. 


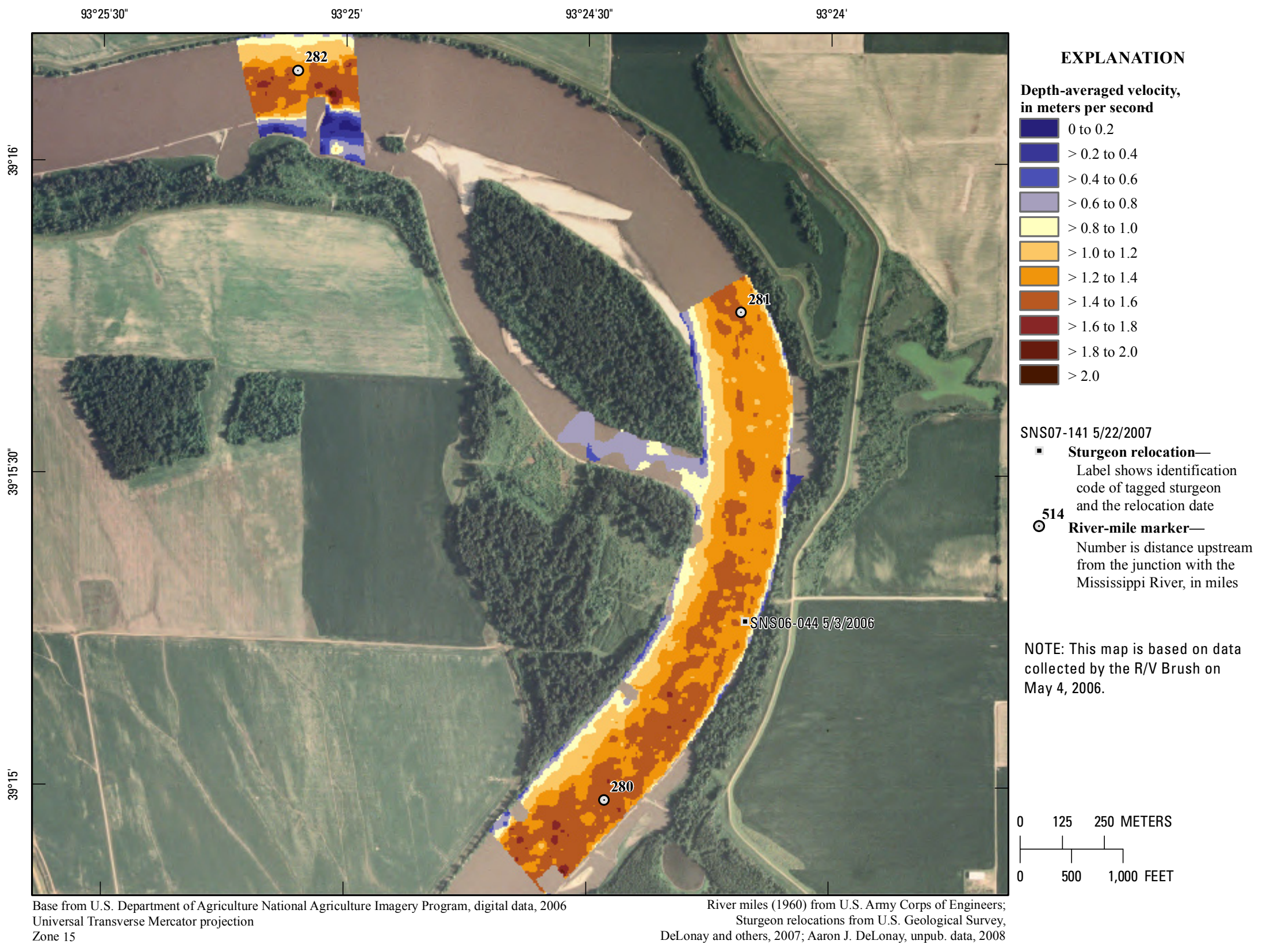

Figure 307. Map of depth-averaged velocity based on data collected on May 4, 2006, in the vicinity of river mile 282. 


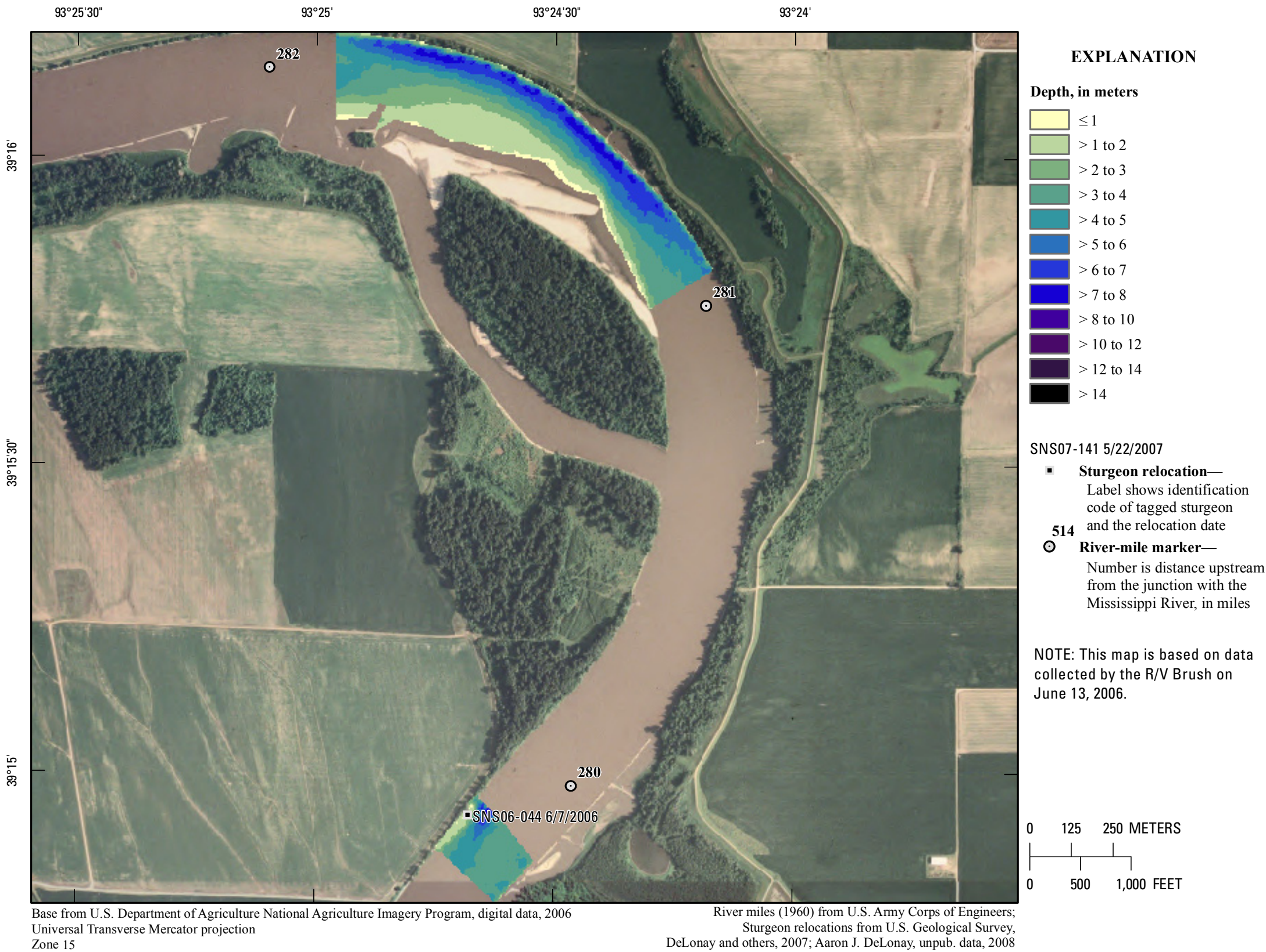

Figure 308. Map of depth based on data collected on June 13, 2006, in the vicinity of river mile 282. 


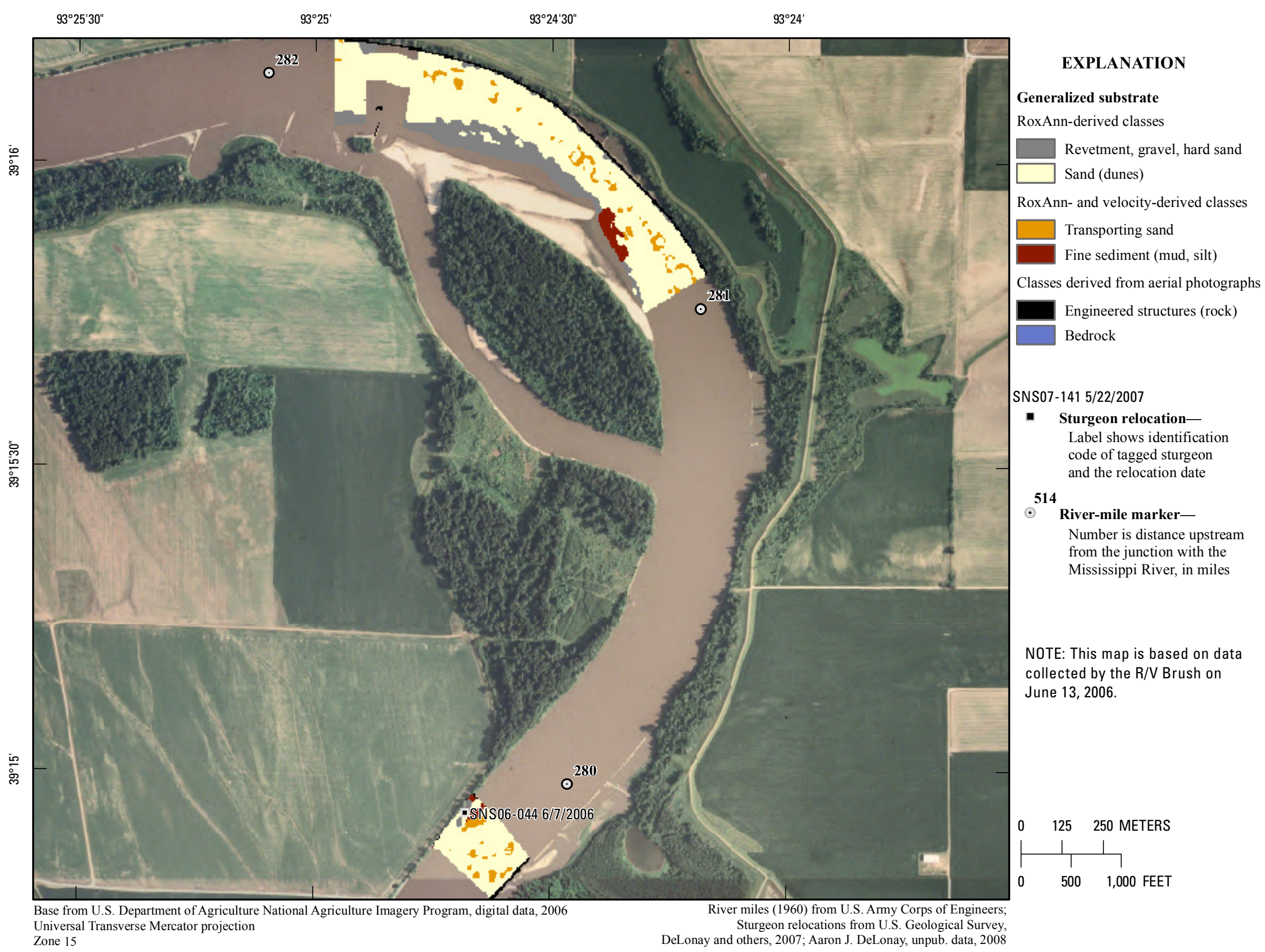

Figure 309. Map of generalized substrate based on data collected on June 13, 2006, in the vicinity of river mile 282 . 


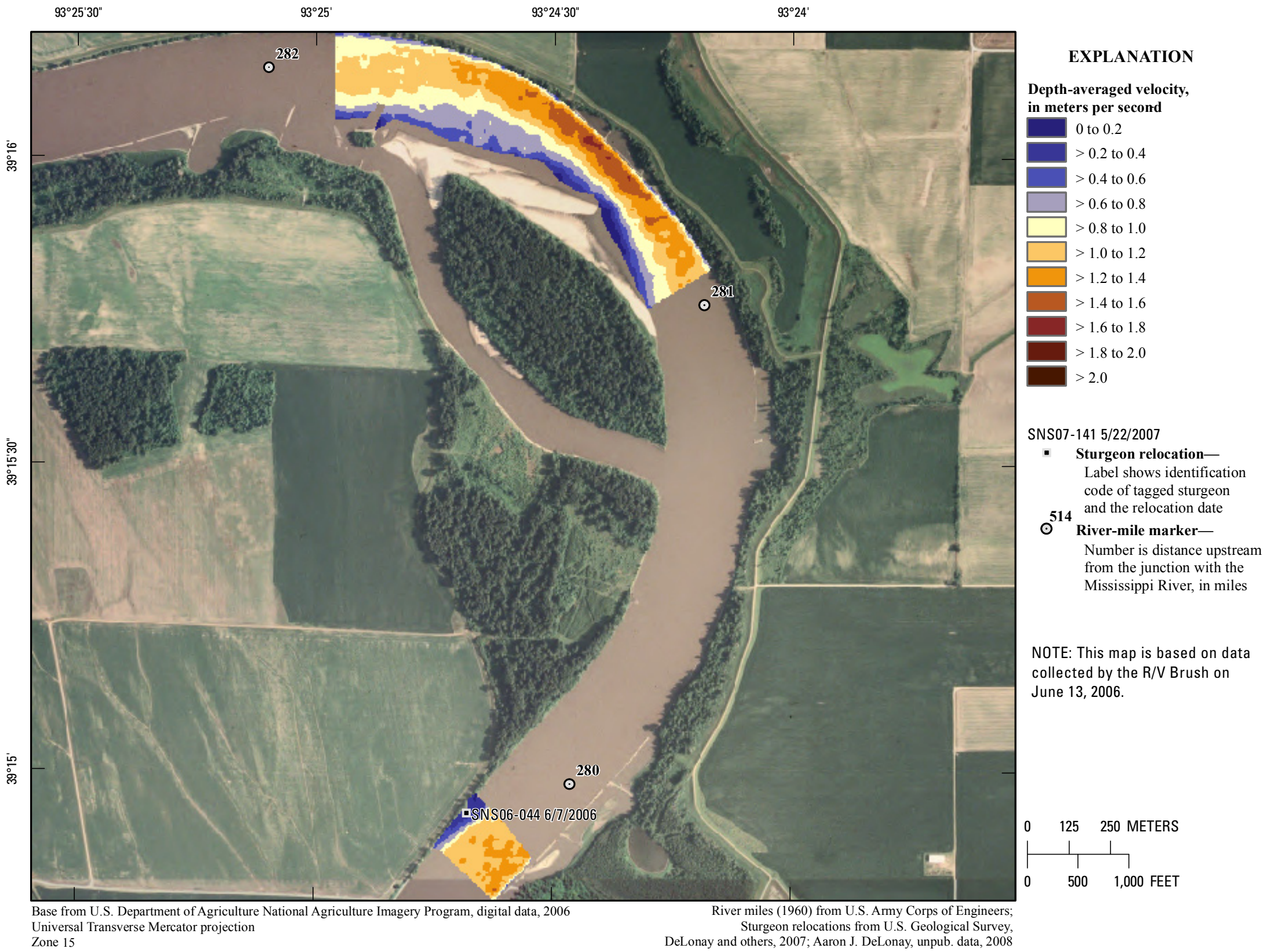

Figure 310. Map of depth-averaged velocity based on data collected on June 13, 2006, in the vicinity of river mile 282. 


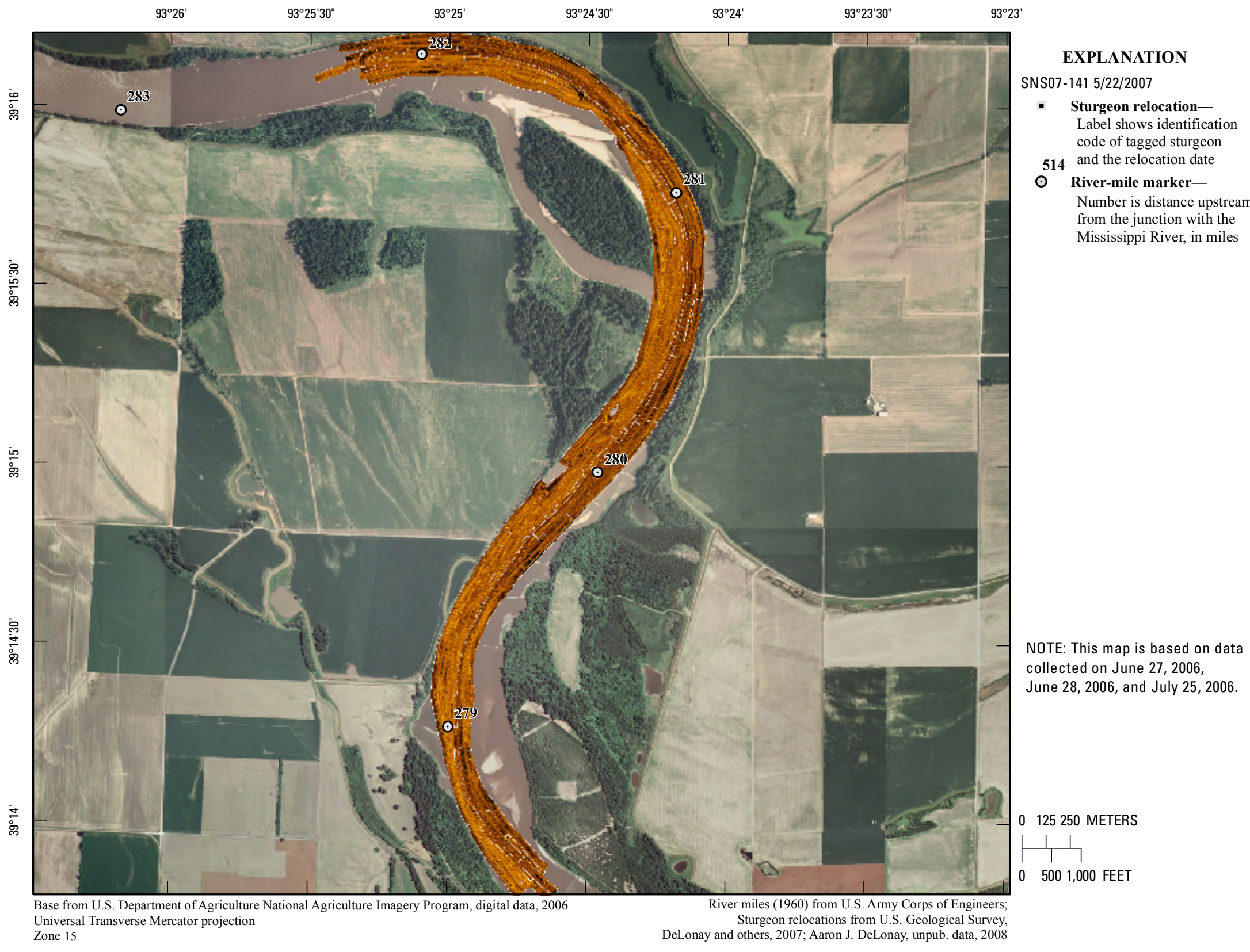

Figure 311. Map of side-scan sonar imagery based on data collected on June 27, 2006, June 28,2006, and July 25, 2006, in the vicinity of river mile 280. 


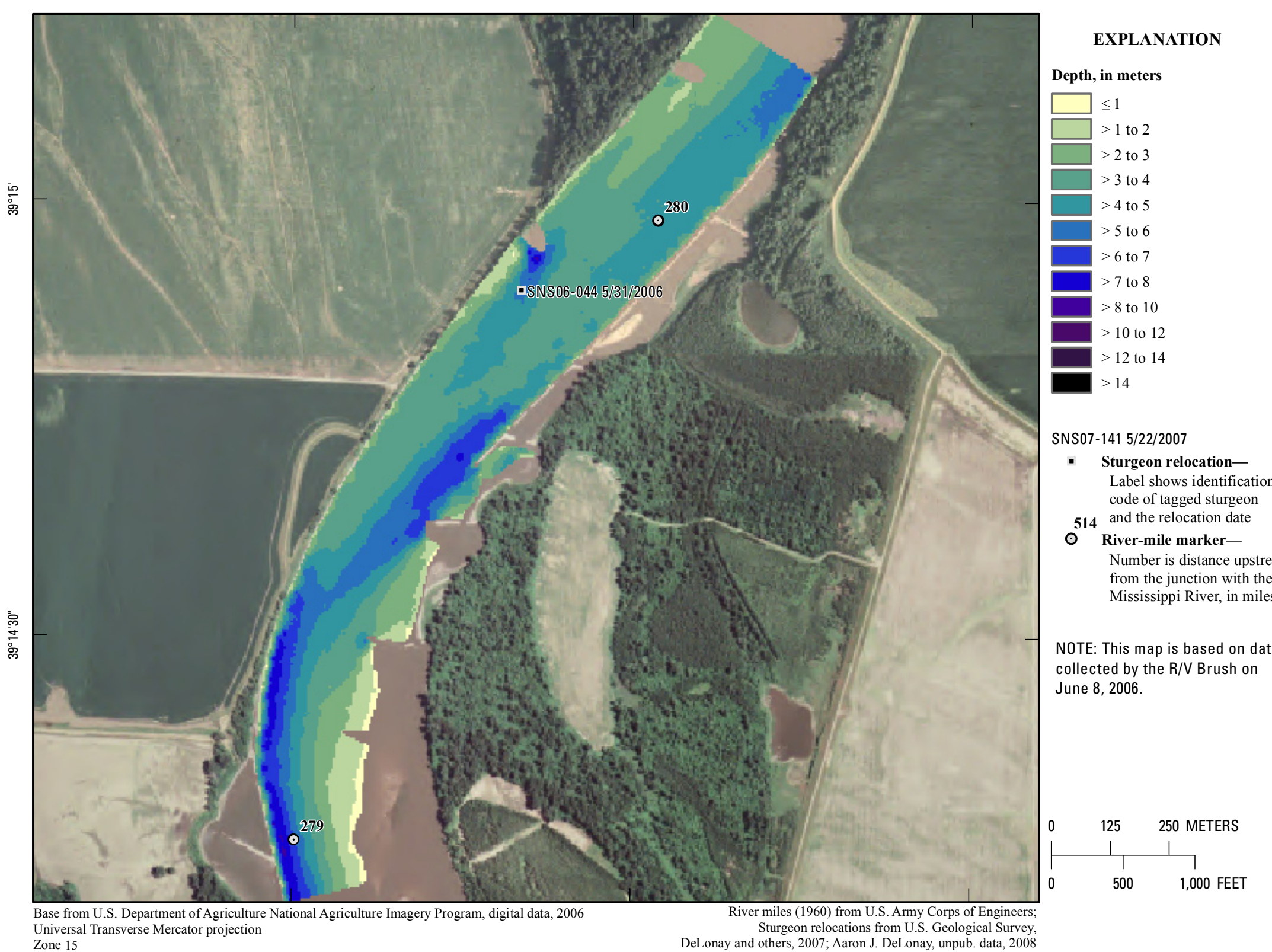

Depth, in meters

NS07-141 5/22/2007

abel shows identification and the relocation date

mer is distance upstream from the junction with the

NOTE: This map is based on data collected by the R/V Brush on June 8, 2006. DeLonay and others, 2007; Aaron J. DeLonay, unpub data, 2008 


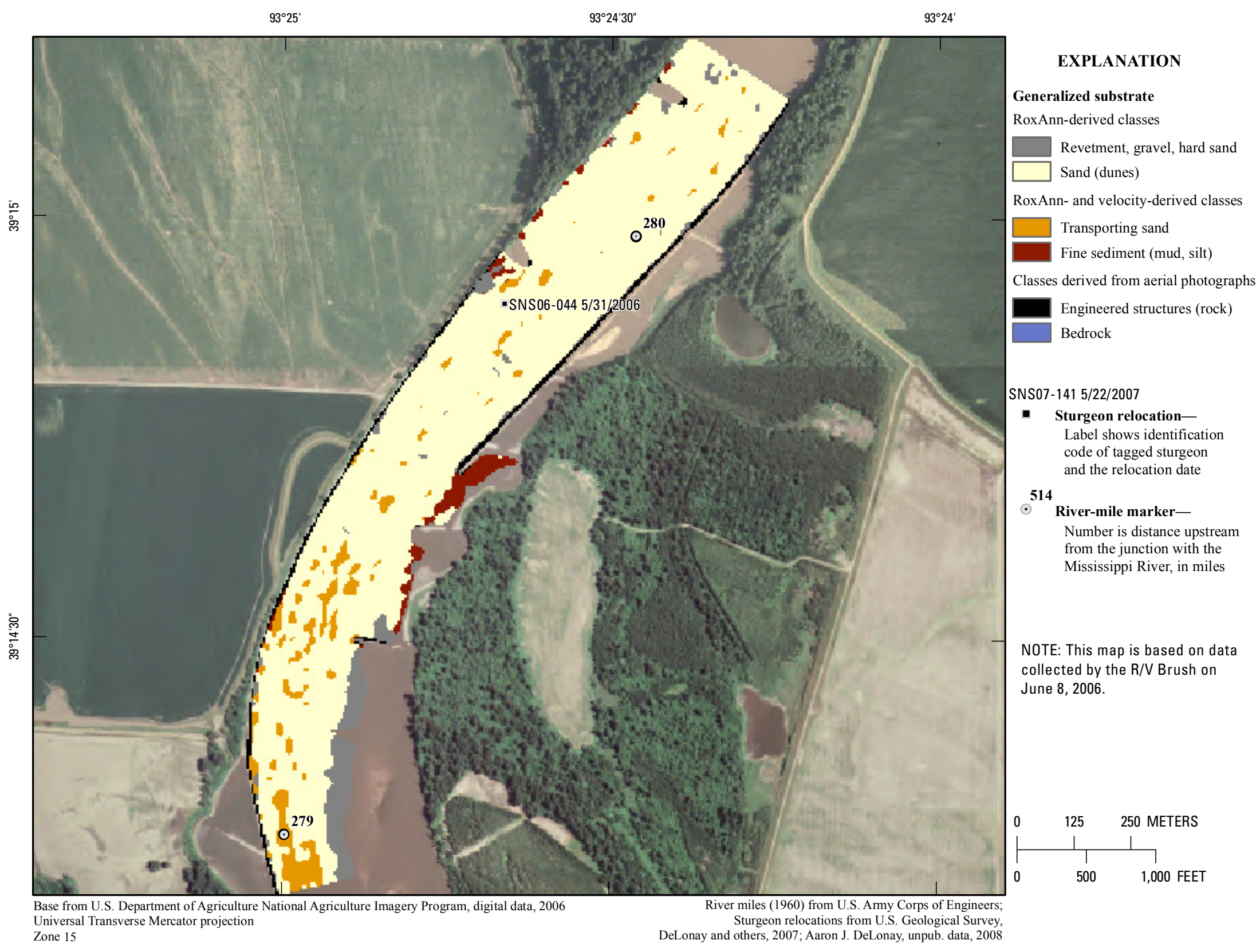

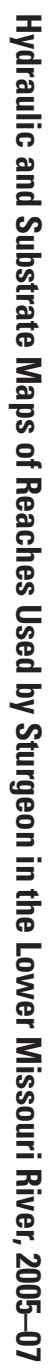

Figure 313. Map of generalized substrate based on data collected on June 8, 2006, in the vicinity of river mile 280 . 


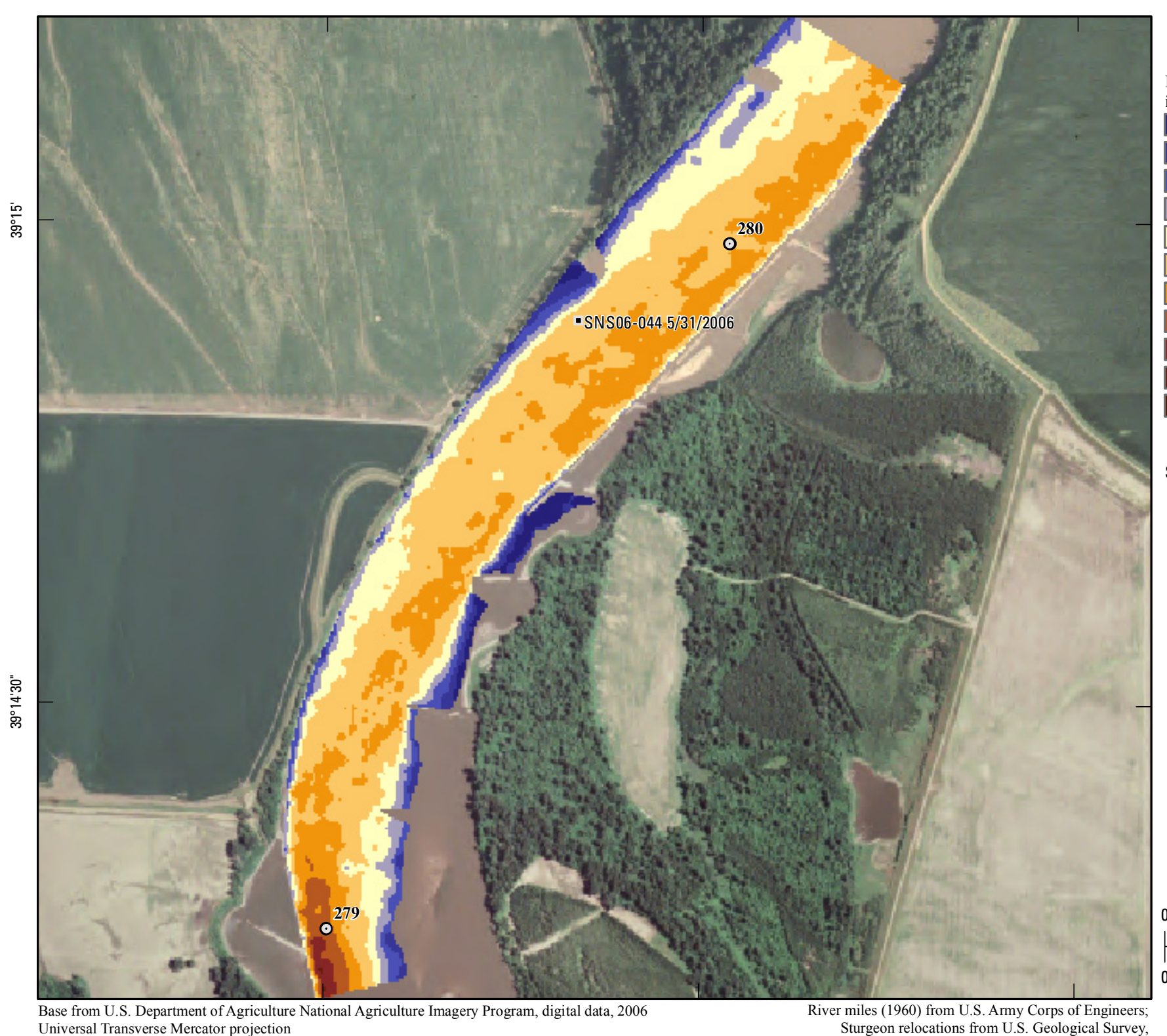

EXPLANATION

Depth-averaged velocity,

in meters per second

0 to 0.2

$>0.2$ to 0.4

$>0.4$ to 0.6

$>0.6$ to 0.8

$>0.8$ to 1.0

$>1.0$ to 1.2

$>1.2$ to 1.4

$>1.4$ to 1.6

$>1.6$ to 1.8

$>1.8$ to 2.0

$>2.0$

\section{SNS07-141 5/22/2007}

- Sturgeon relocationLabel shows identification code of tagged sturgeo

$\mathbf{5 1 4}$ and the relocation date

Number is distance upstream

Number is distance upstrea
from the junction with the

Mississippi River, in miles

NOTE: This map is based on data

collected by the R/V Brush on

June 8, 2006

Universal Transverse Mercator projection

Sturgeon relocations from U.S. Geological Survey,
DeLonay and others, 2007; Aaron J. DeLonay, unpub. data, 2008

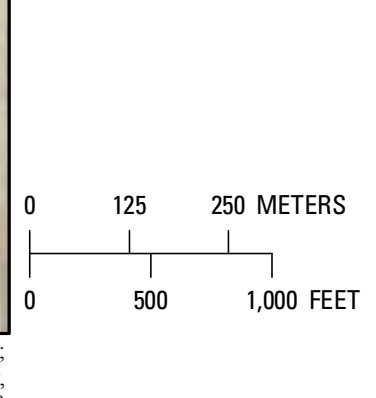




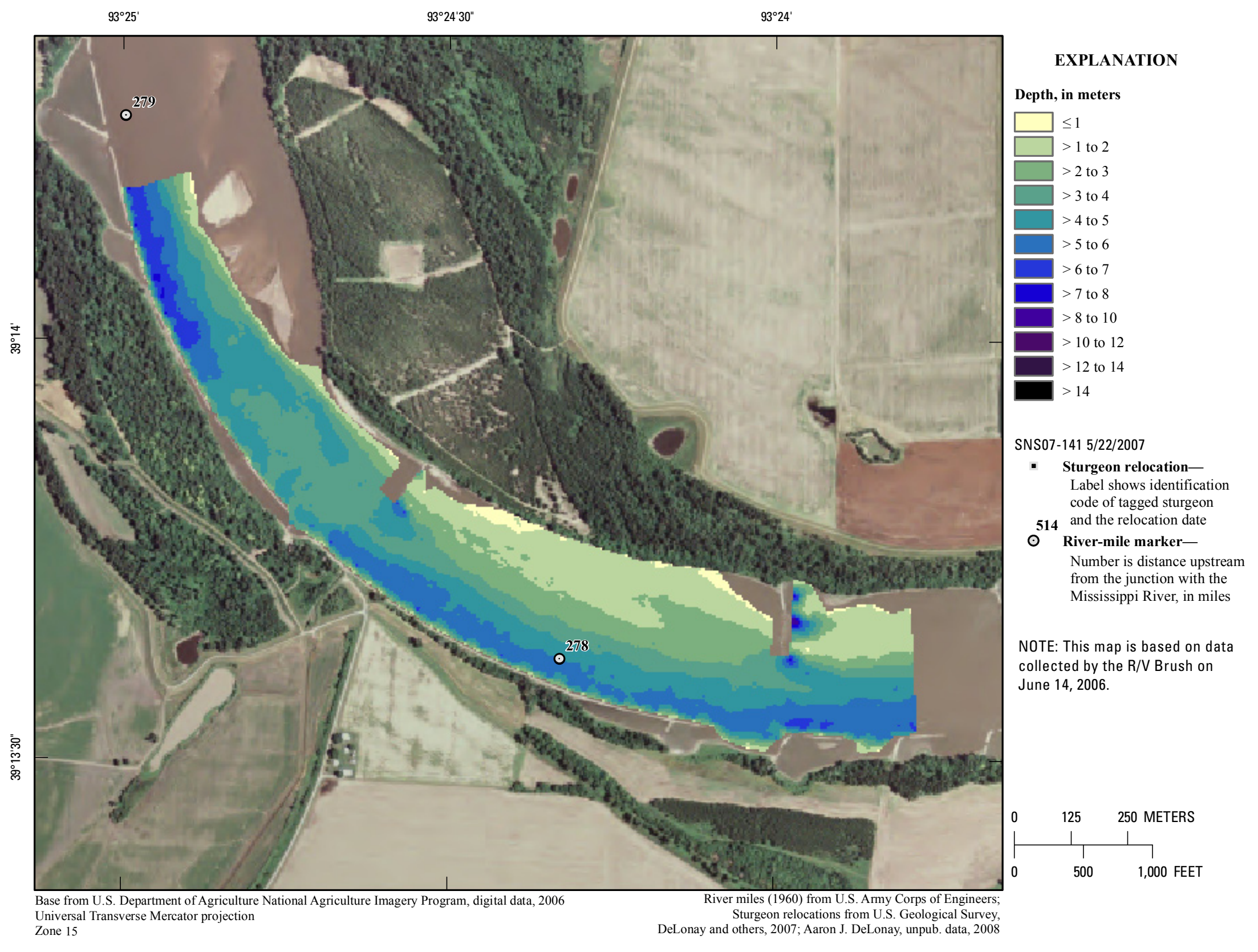

Figure 315. Map of depth based on data collected on June 14, 2006, in the vicinity of river mile 278 . 


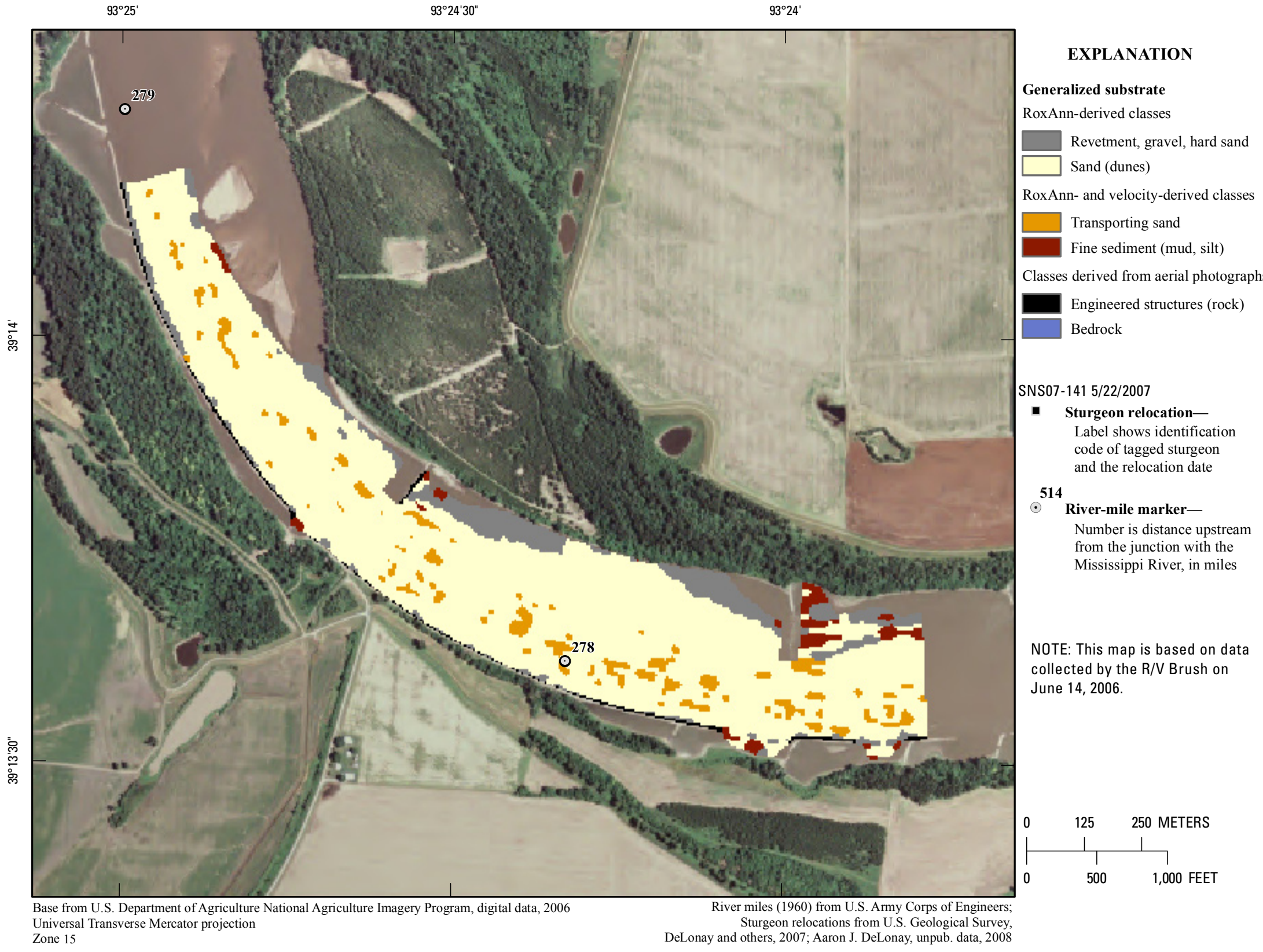

Figure 316. Map of generalized substrate based on data collected on June 14, 2006, in the vicinity of river mile 278 . 


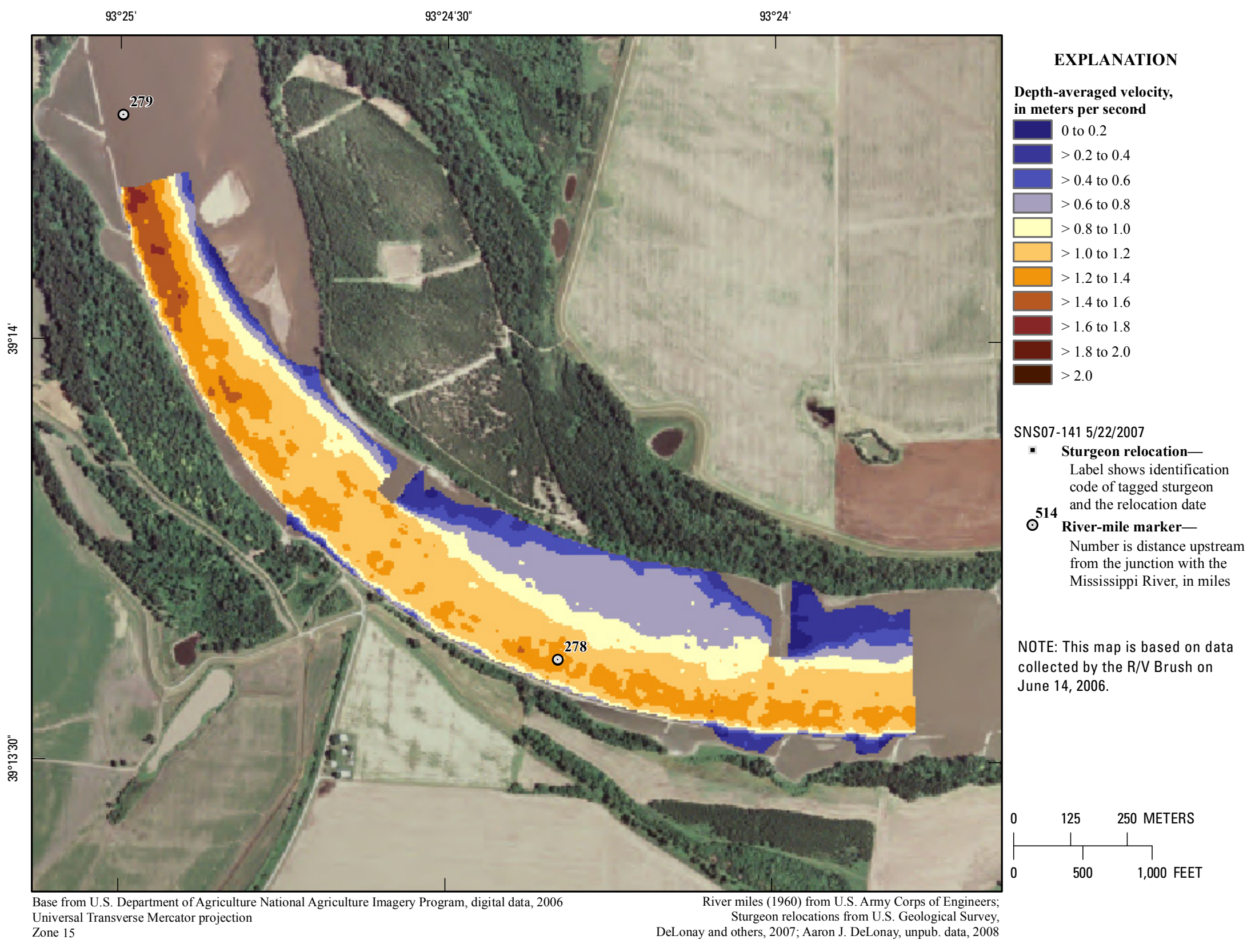

Figure 317. Map of depth-averaged velocity based on data collected on June 14, 2006, in the vicinity of river mile 278 . 


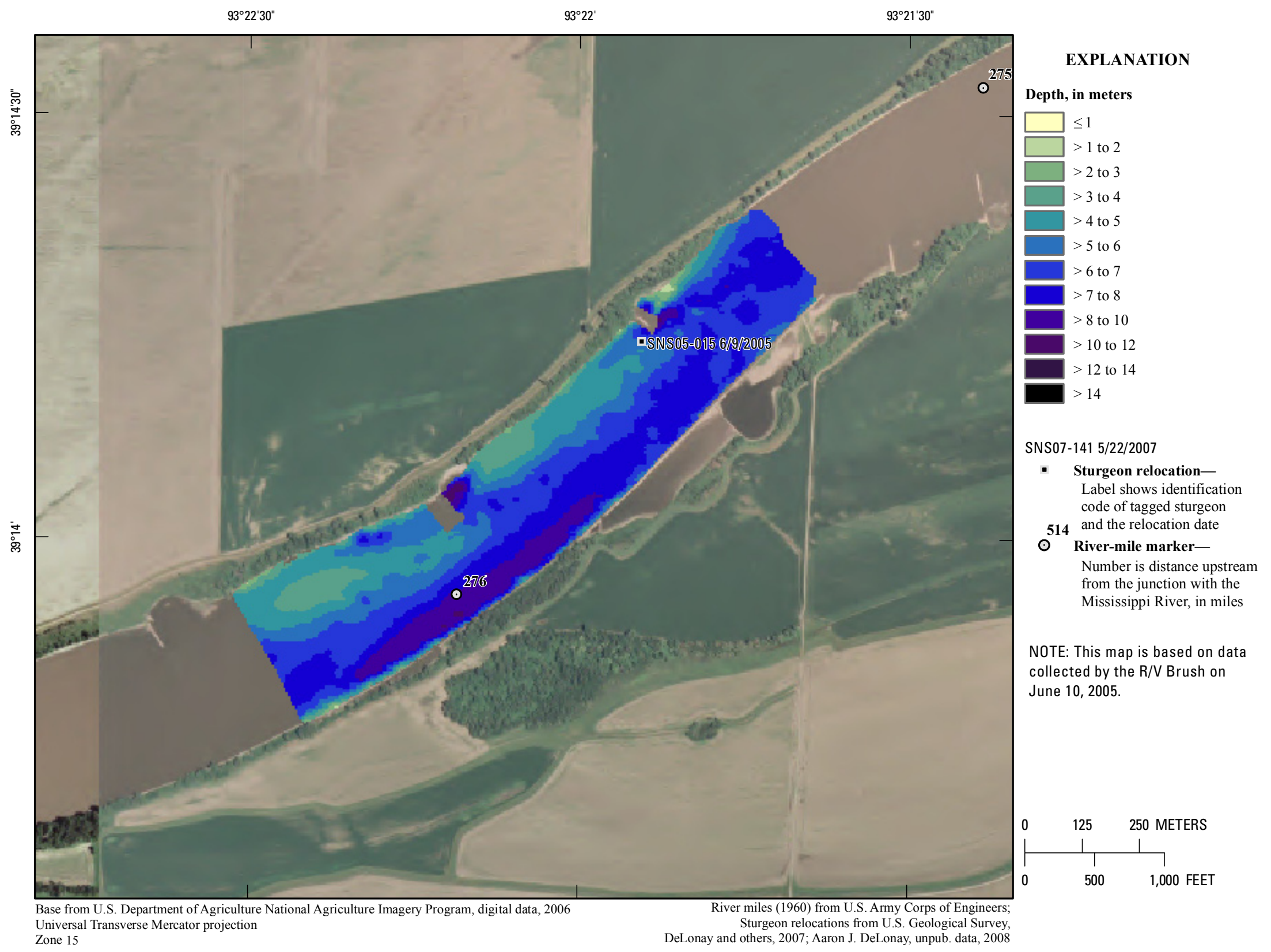

Figure 318. Map of depth based on data collected on June 10, 2005, in the vicinity of river mile 276. 


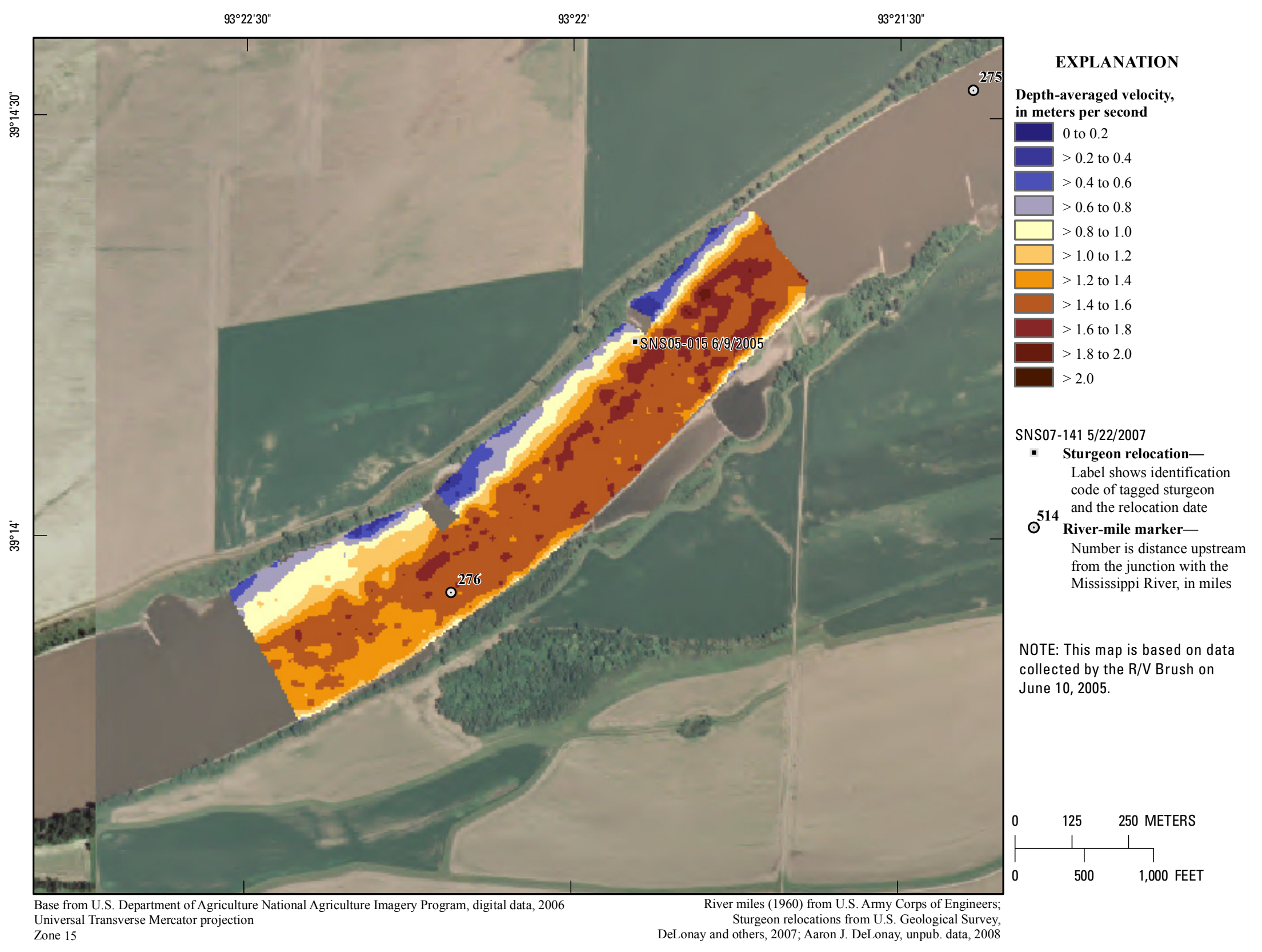

Figure 319. Map of depth-averaged velocity based on data collected on June 10, 2005, in the vicinity of river mile 276. 


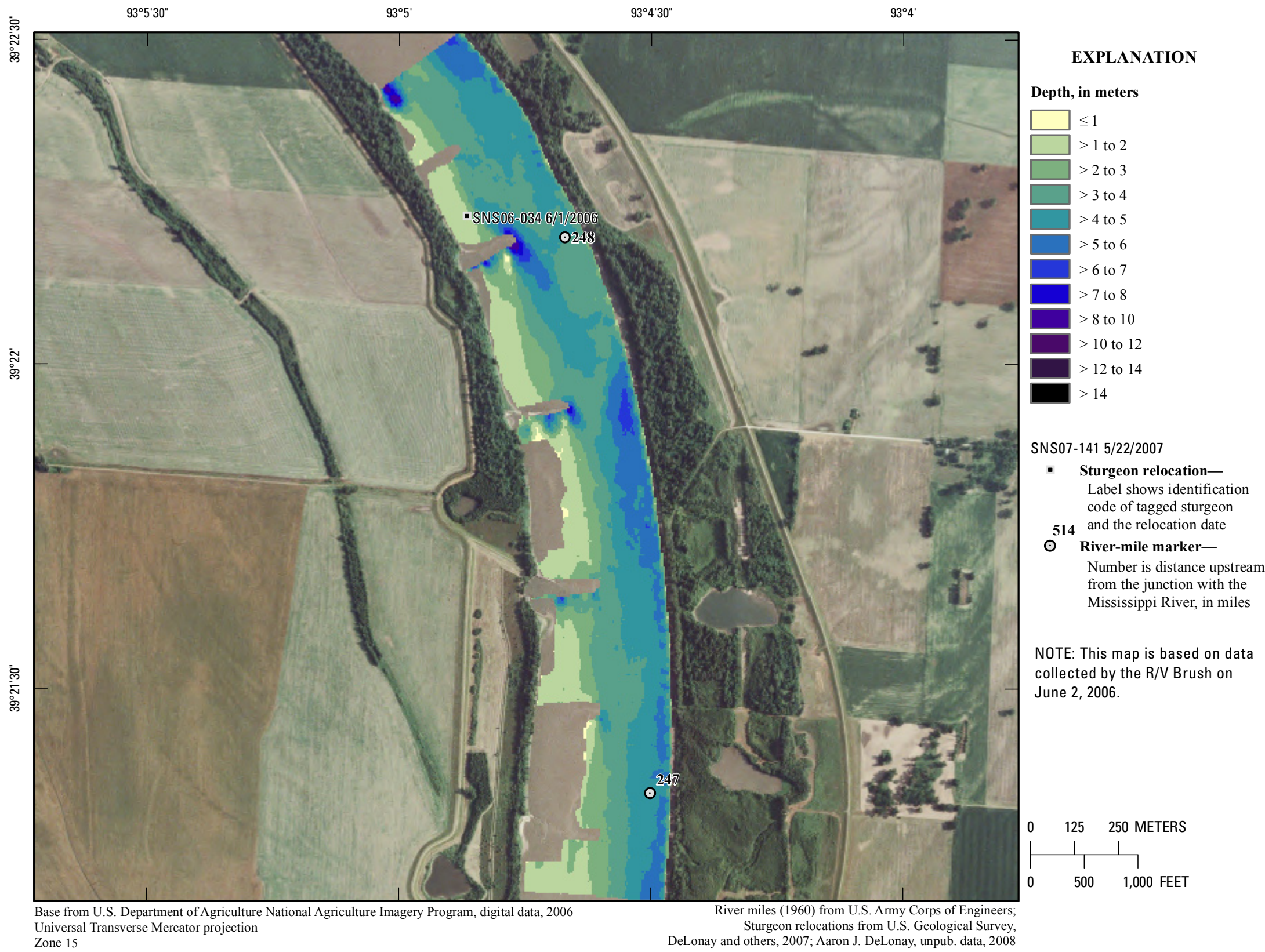

Figure 320. Map of depth based on data collected on June 2, 2006, in the vicinity of river mile 248. 


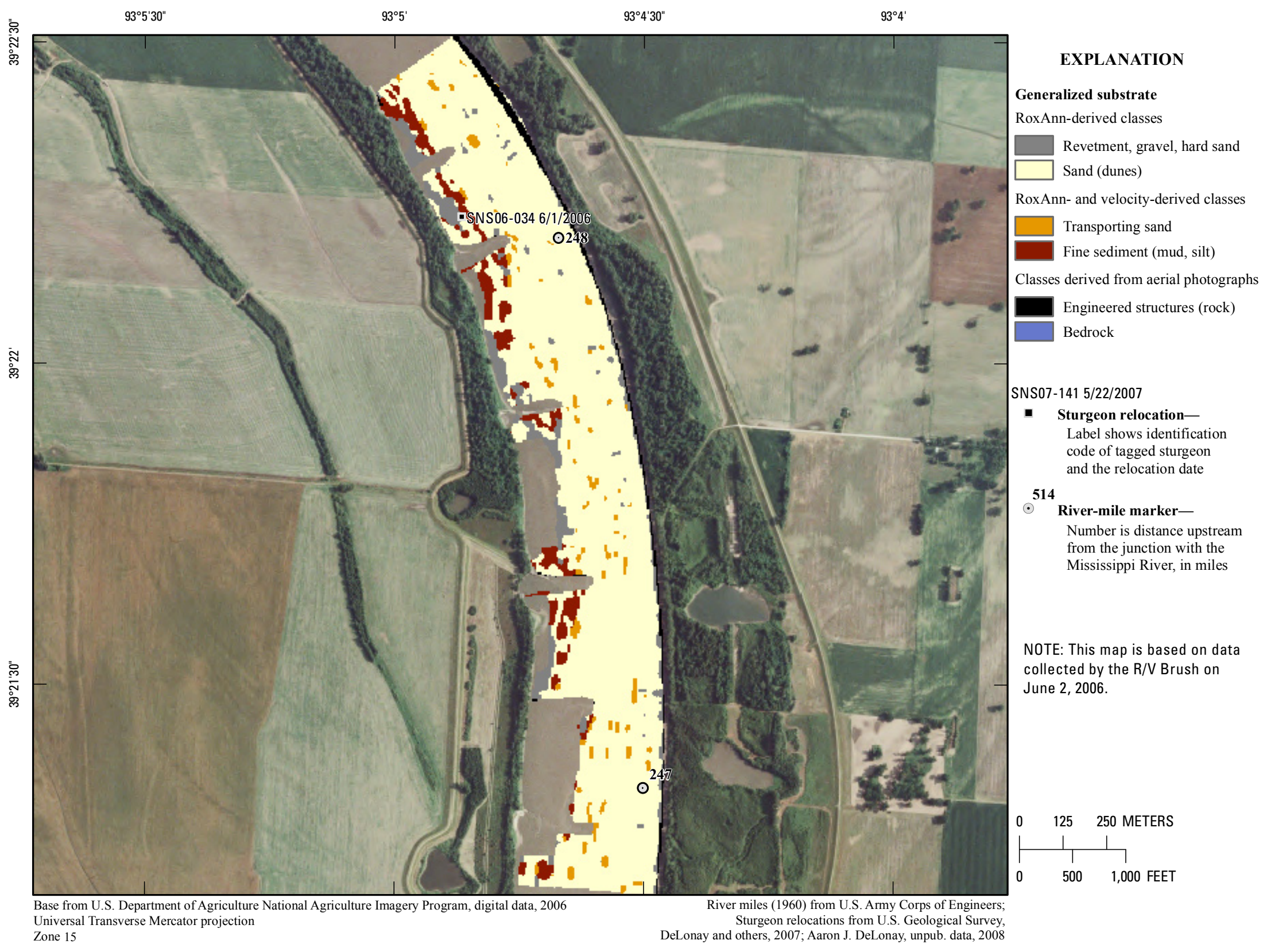

Figure 321. Map of generalized substrate based on data collected on June 2, 2006, in the vicinity of river mile 248. 


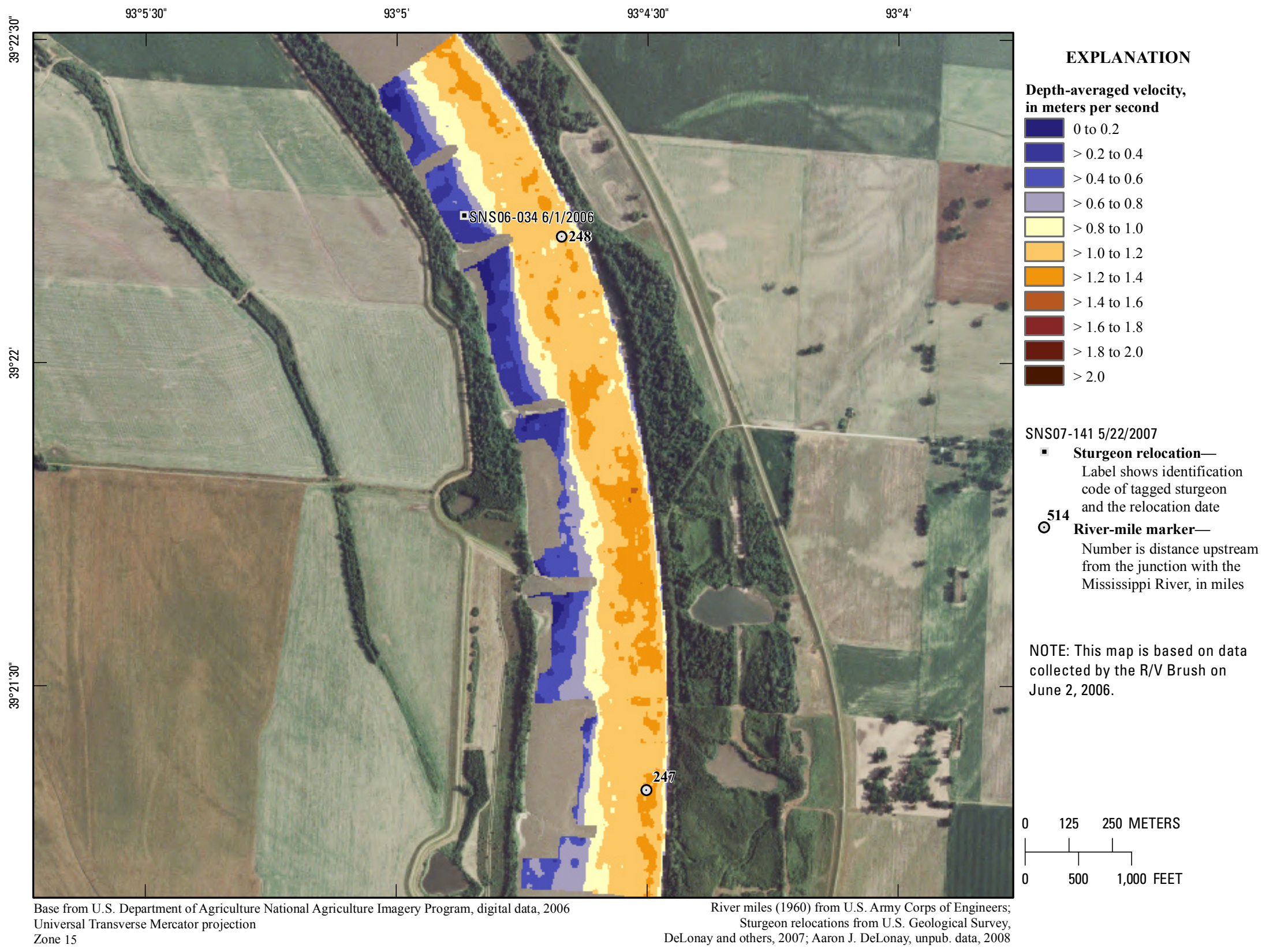

Figure 322. Map of depth-averaged velocity based on data collected on June 2, 2006, in the vicinity of river mile 248 . 


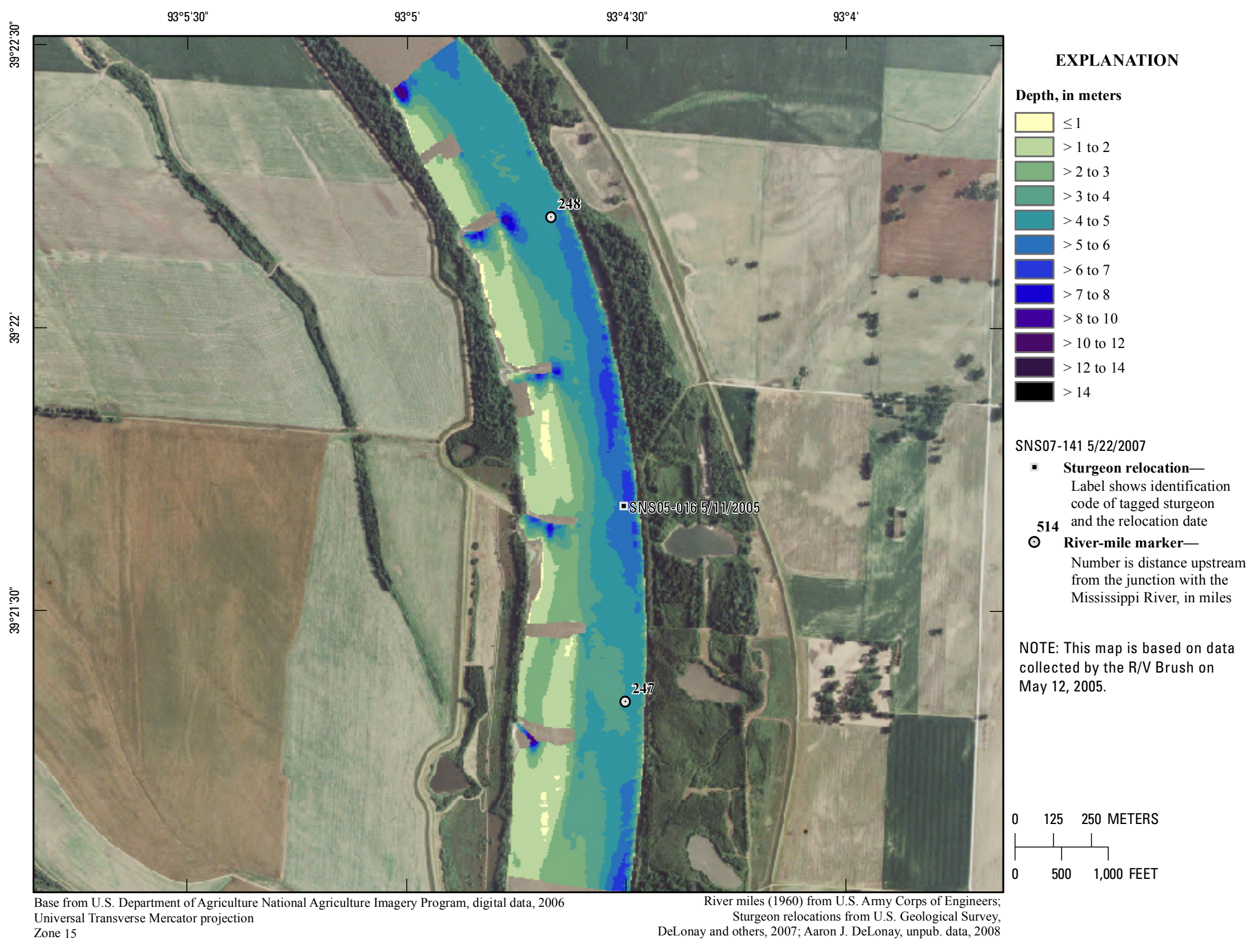

Figure 323. Map of depth based on data collected on May 12, 2005, in the vicinity of river mile 248. 


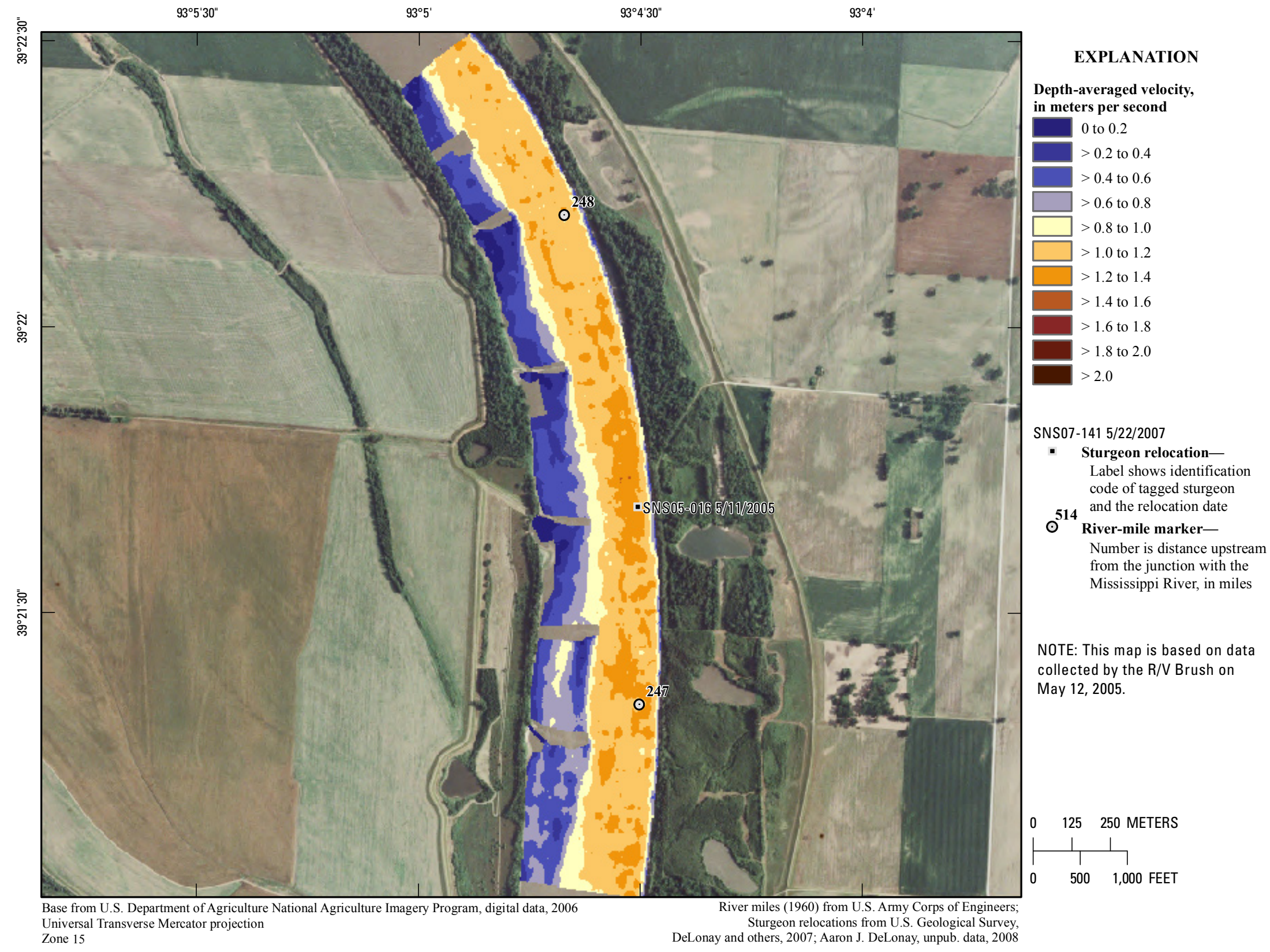

Figure 324. Map of depth-averaged velocity based on data collected on May 12, 2005, in the vicinity of river mile 248 . 


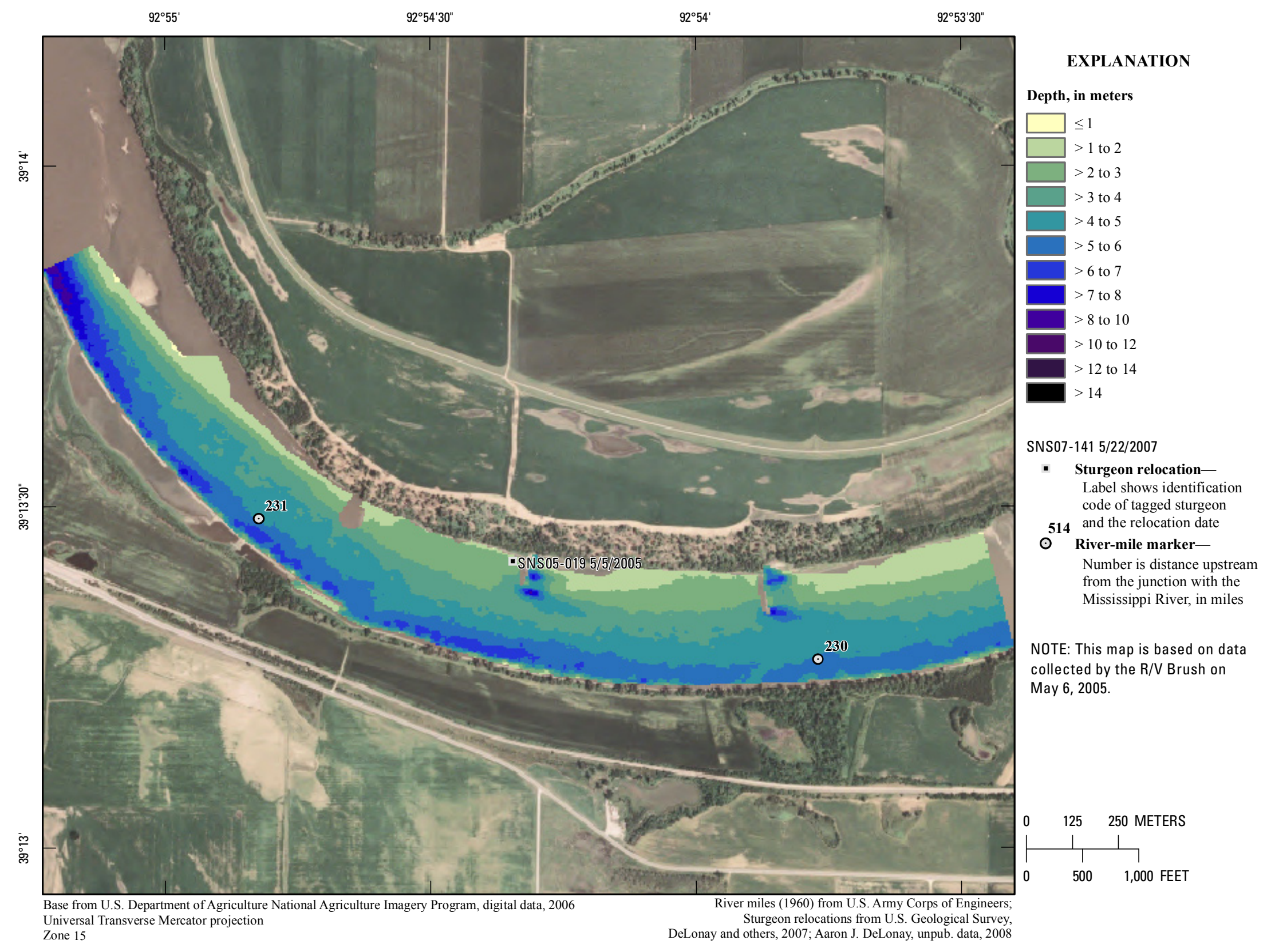

Figure 325. Map of depth based on data collected on May 6, 2005, in the vicinity of river mile 231 . 


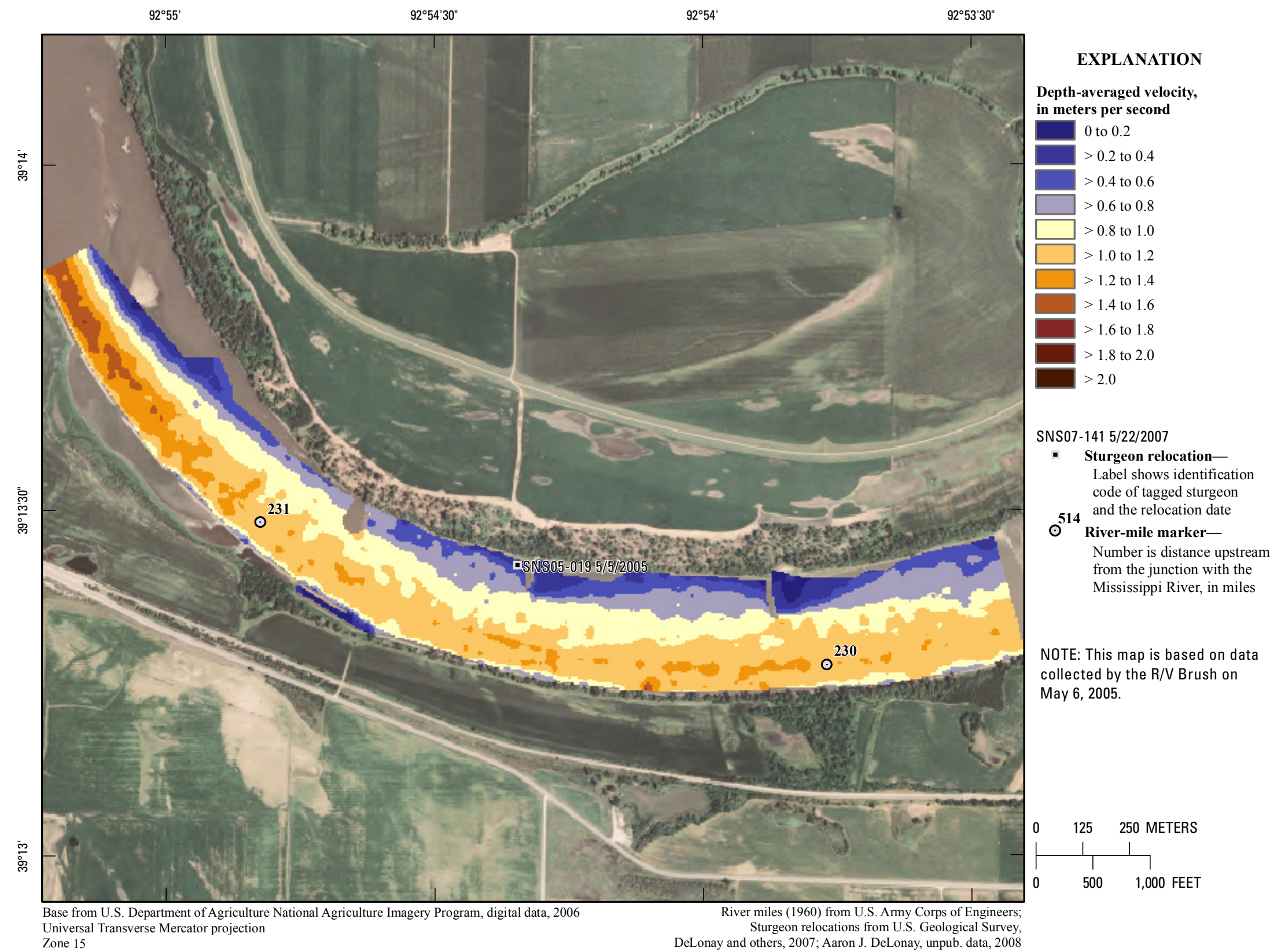

Figure 326. Map of depth-averaged velocity based on data collected on May 6, 2005, in the vicinity of river mile 231 . 


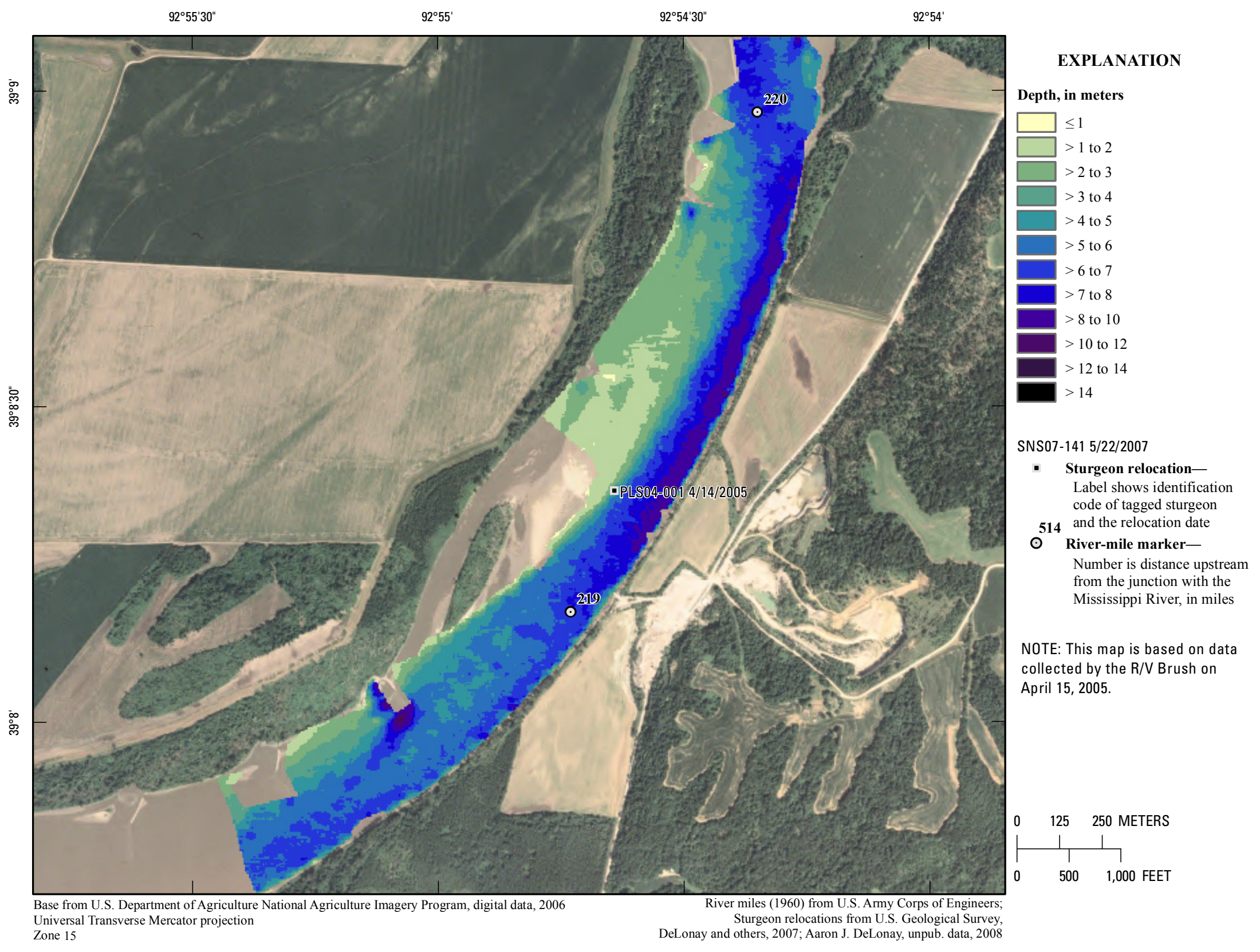

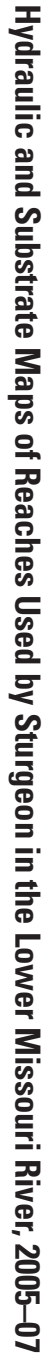

Figure 327. Map of depth based on data collected on April 15, 2005, in the vicinity of river mile 219. 


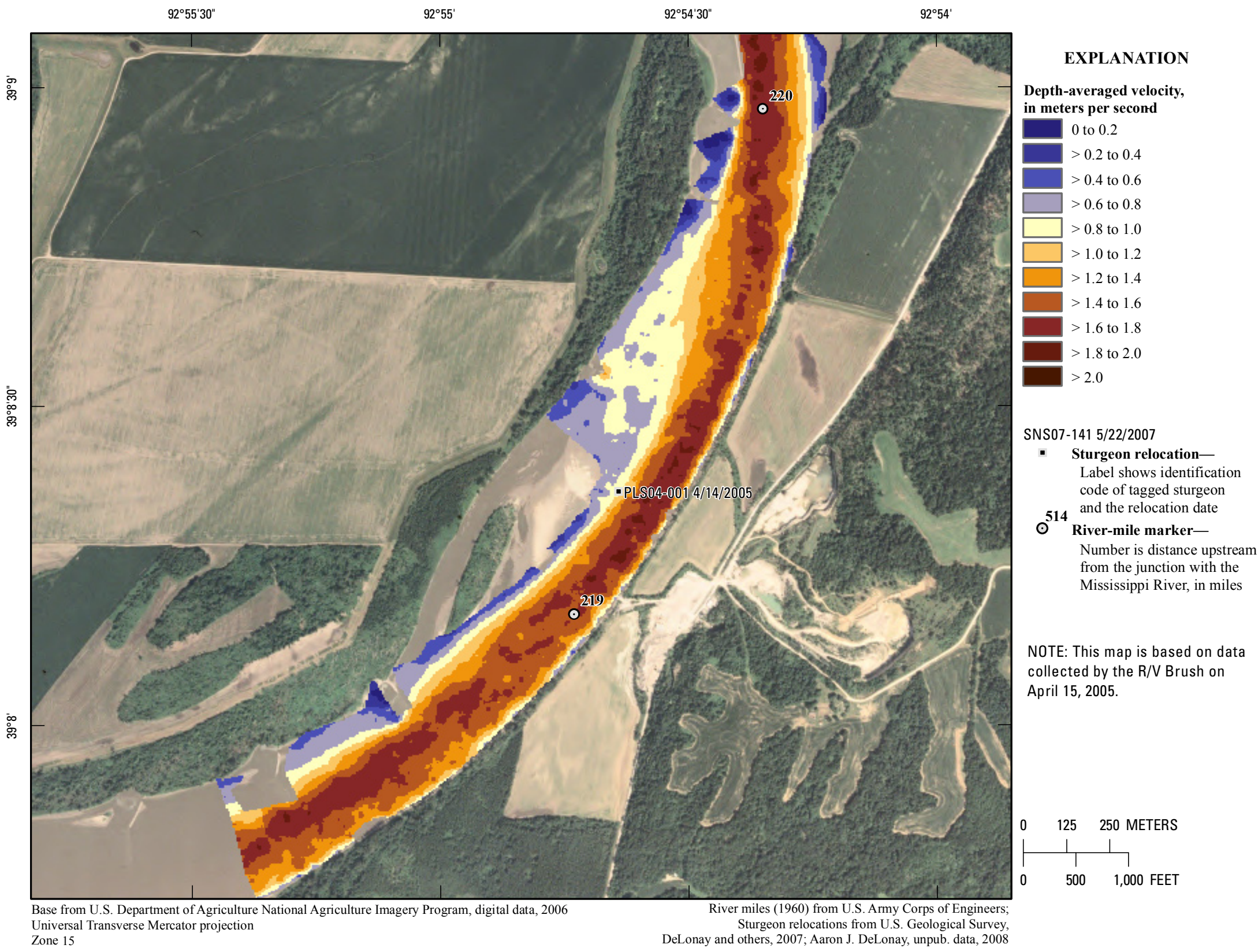

Figure 328. Map of depth-averaged velocity based on data collected on April 15, 2005, in the vicinity of river mile 219 . 


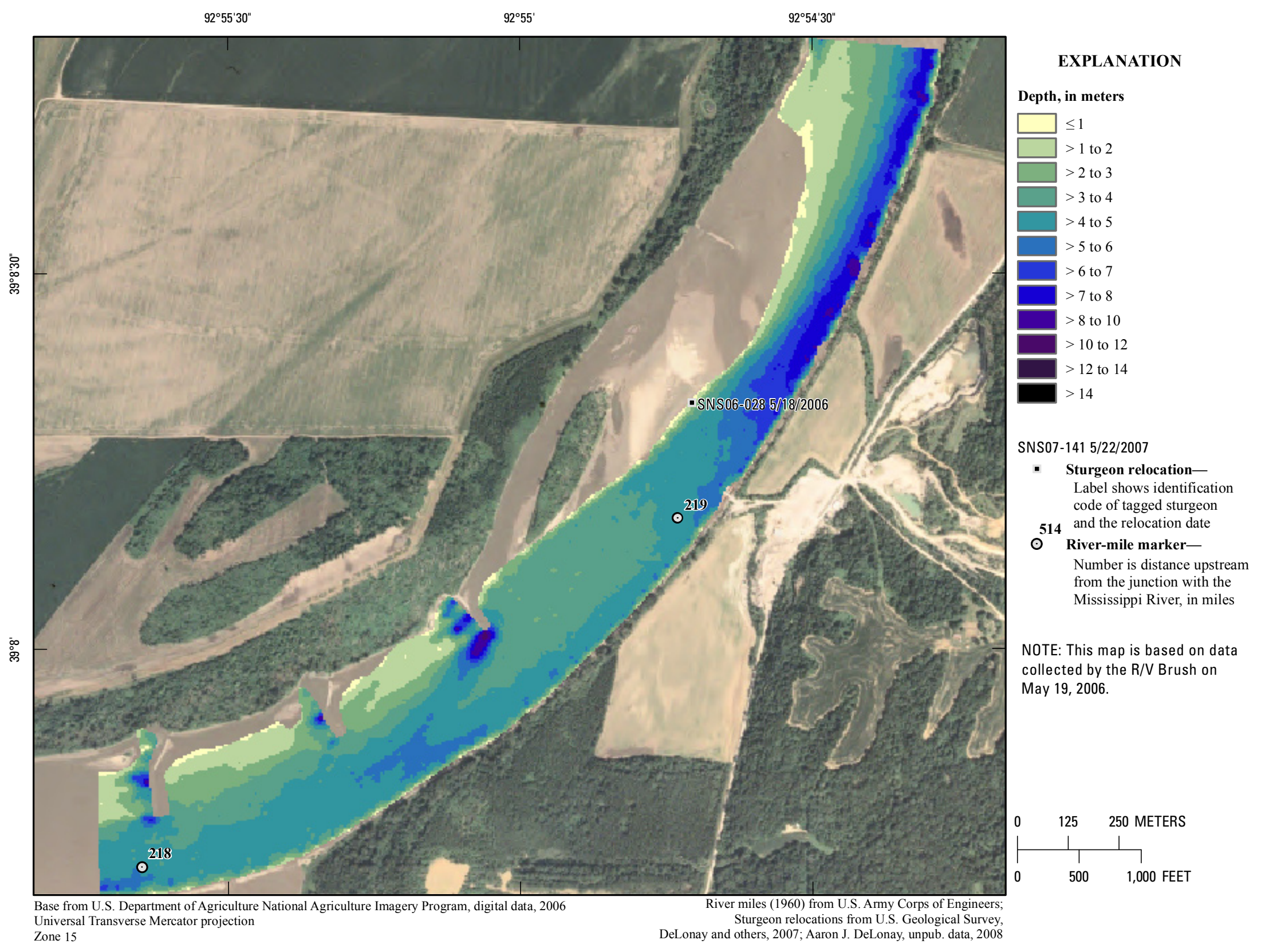

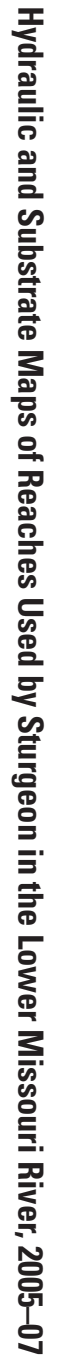

Figure 329. Map of depth based on data collected on May 19, 2006, in the vicinity of river mile 219. 


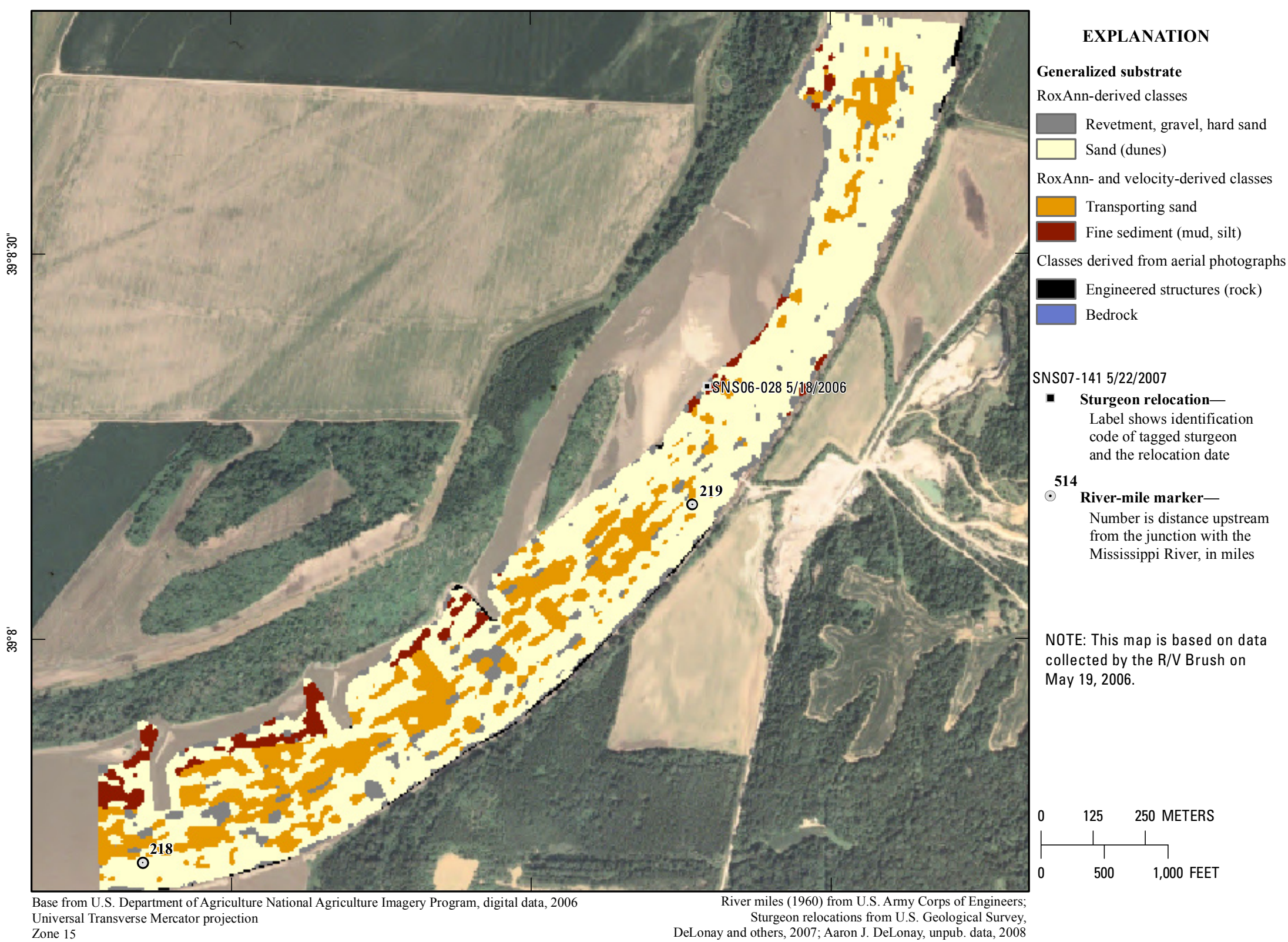




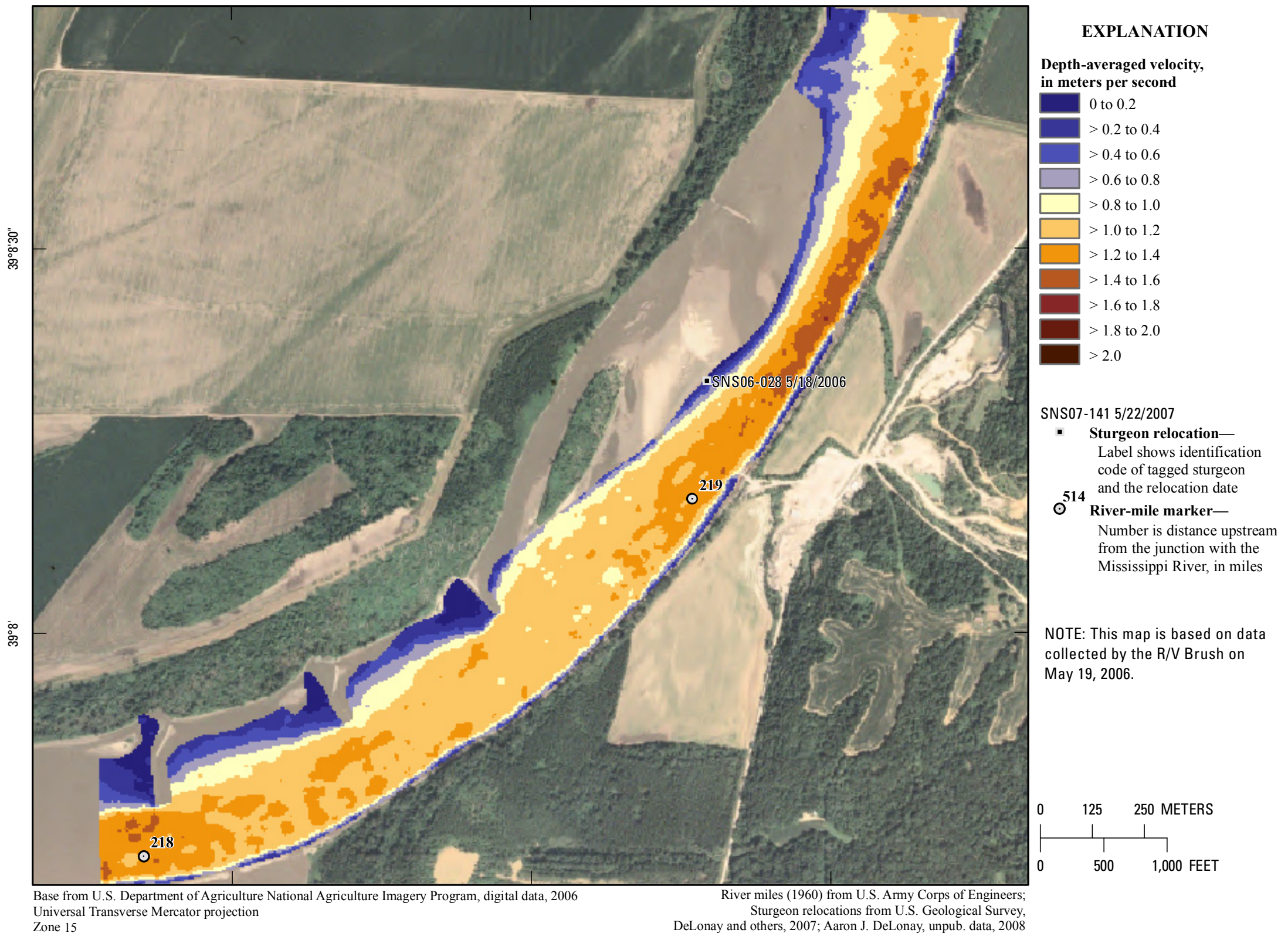

Figure 331. Map of depth-averaged velocity based on data collected on May 19, 2006, in the vicinity of river mile 219. 


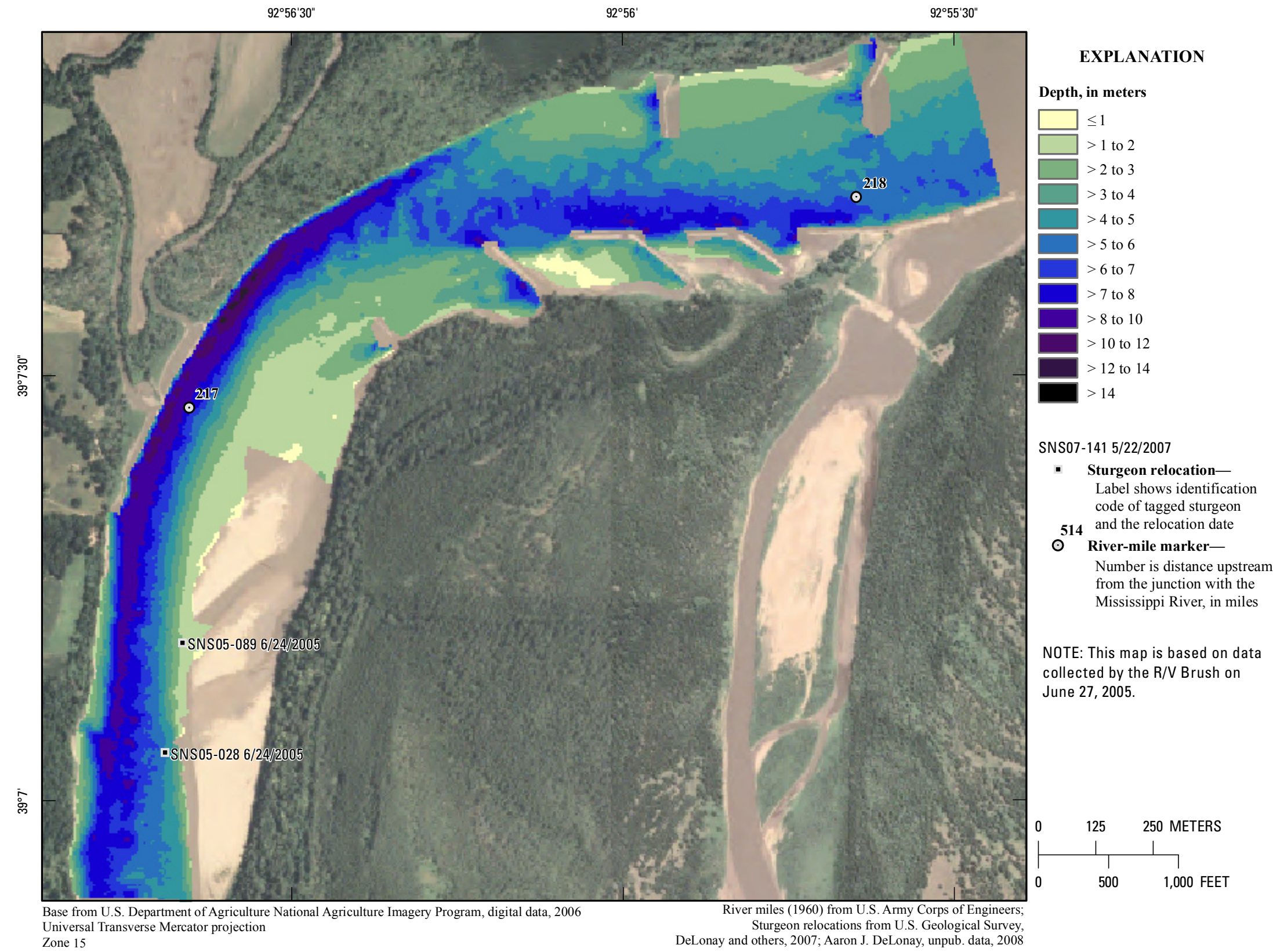
Universal Transverse Mercator projection
Zone 15 Sturgeon relocations from U.S. Geological Survey,
DeLonay and others, 2007; Aaron J. DeLonay, unpub. data, 2008 


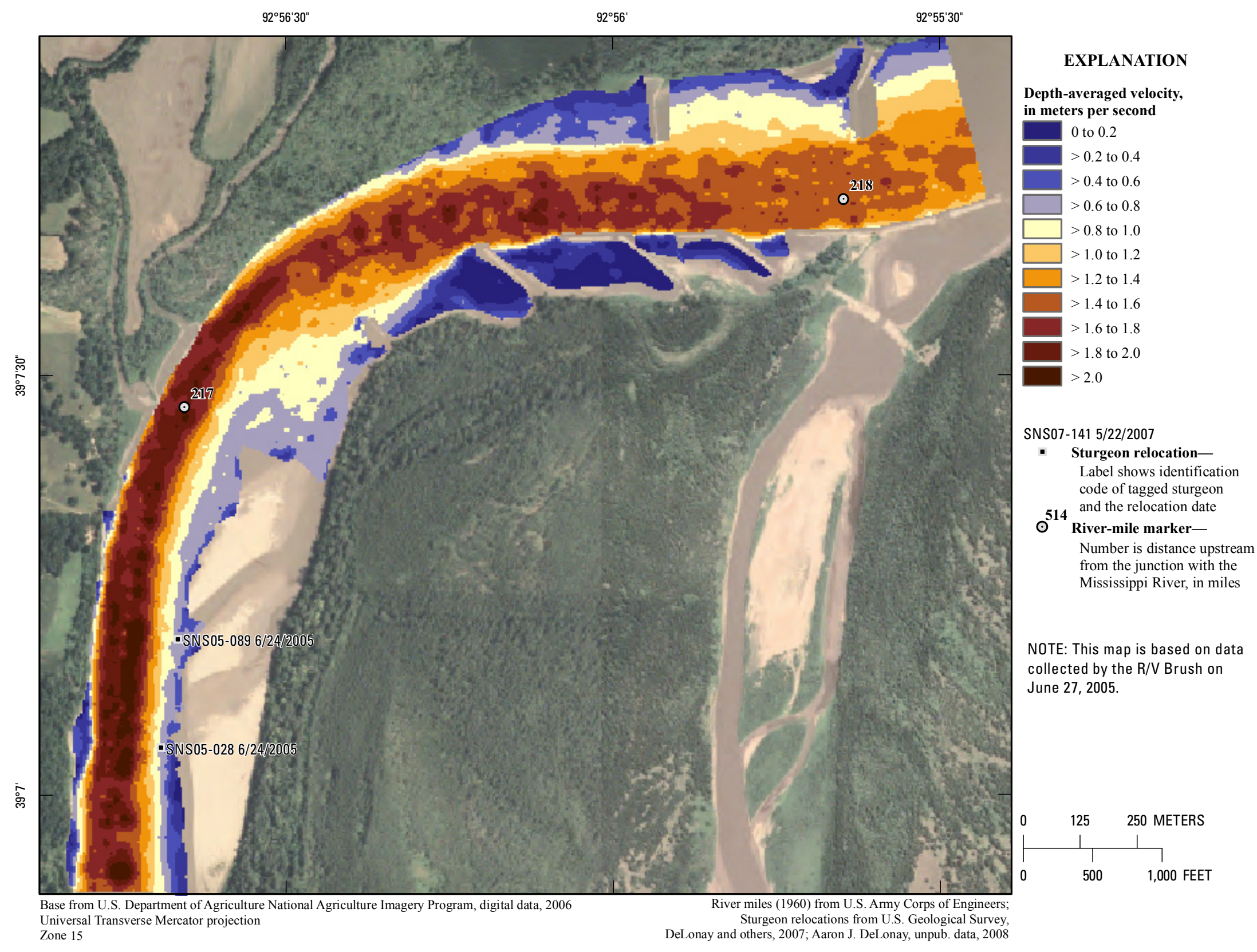

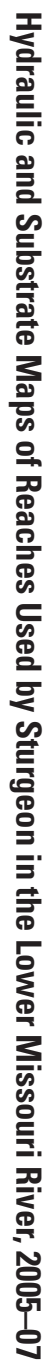

Figure 333. Map of depth-averaged velocity based on data collected on June 27, 2005, in the vicinity of river mile 217. 


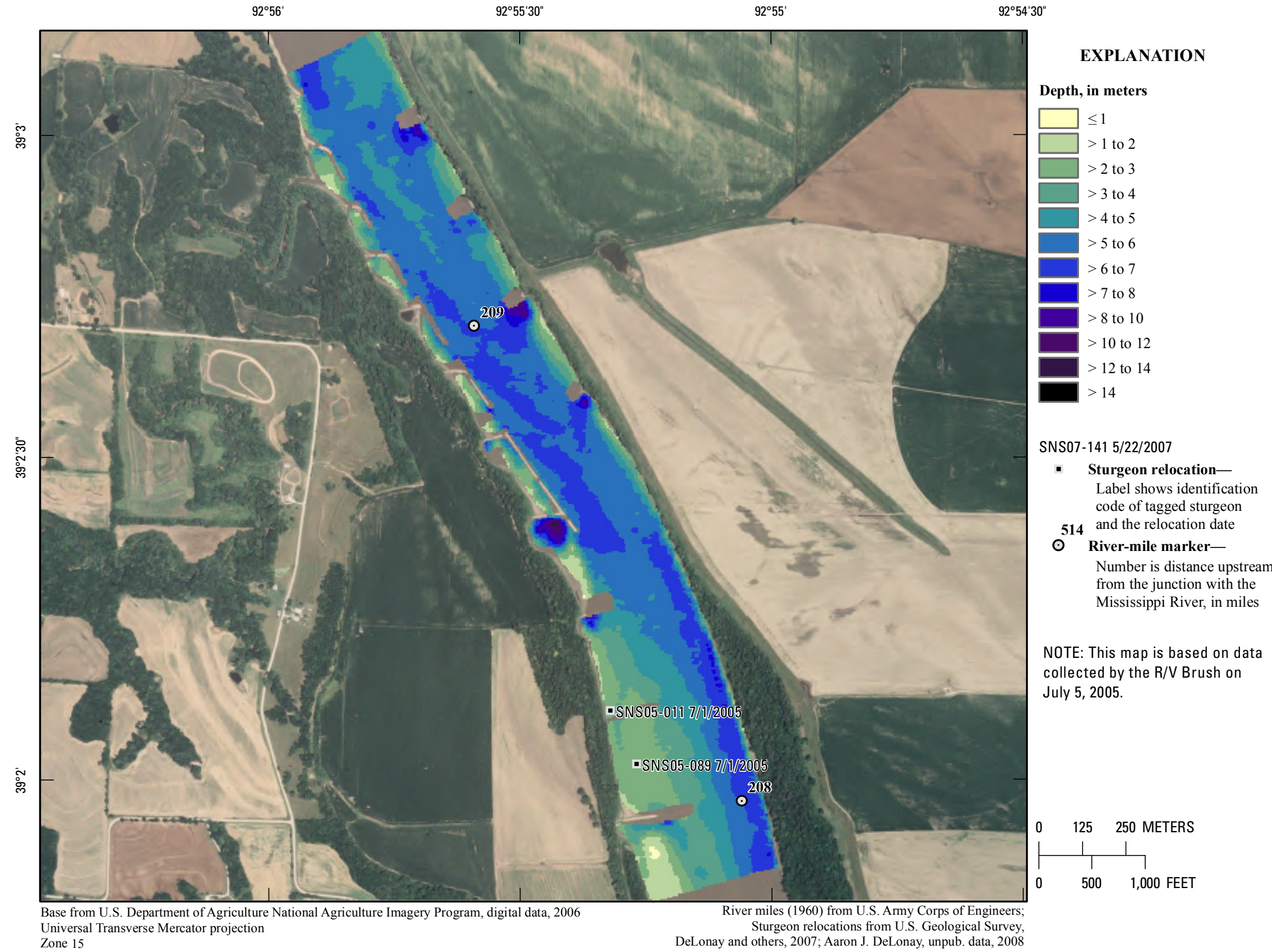

Figure 334. Map of depth based on data collected on July 5, 2005, in the vicinity of river mile 209. 


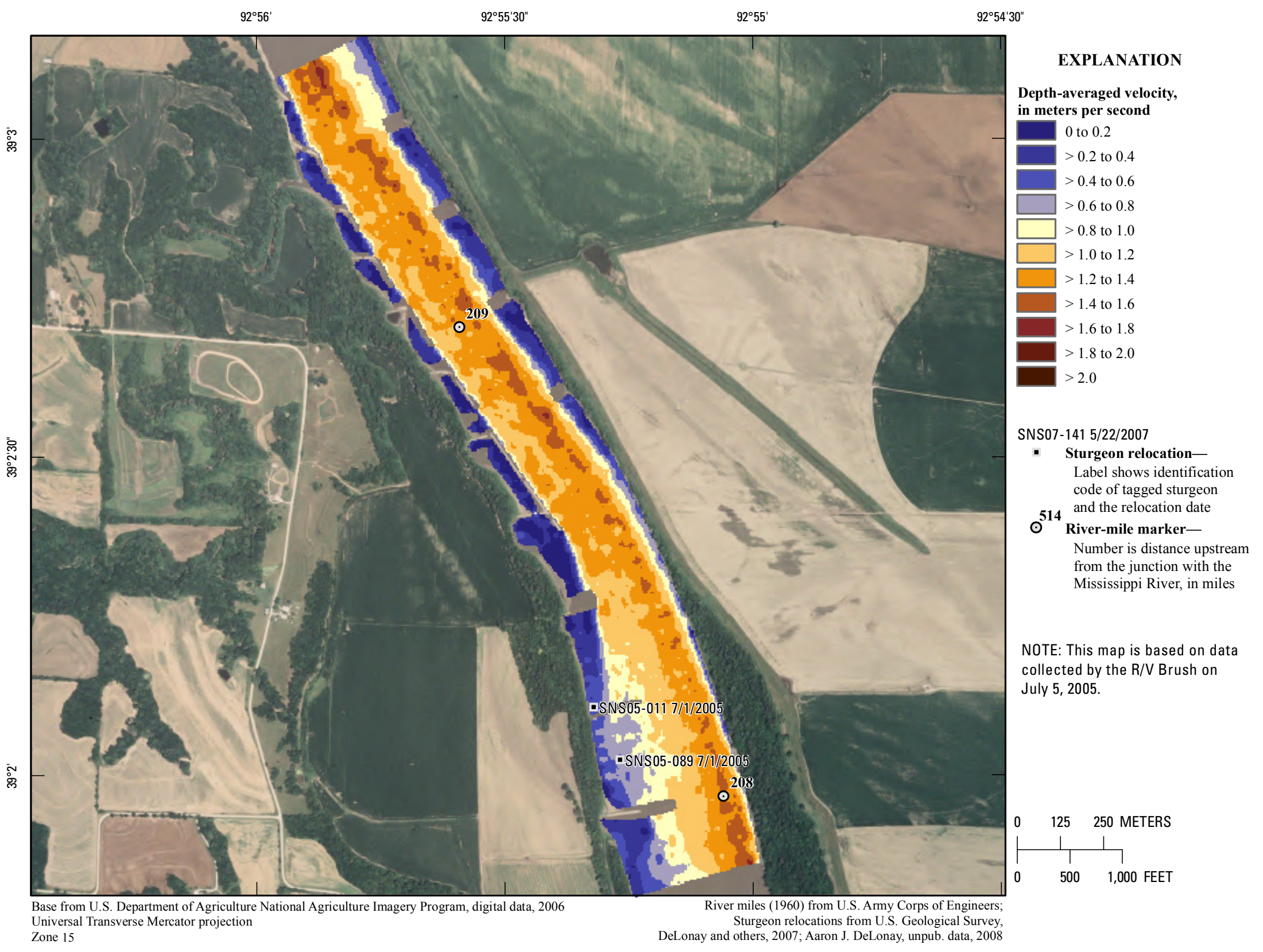

Figure 335. Map of depth-averaged velocity based on data collected on July 5, 2005, in the vicinity of river mile 209. 


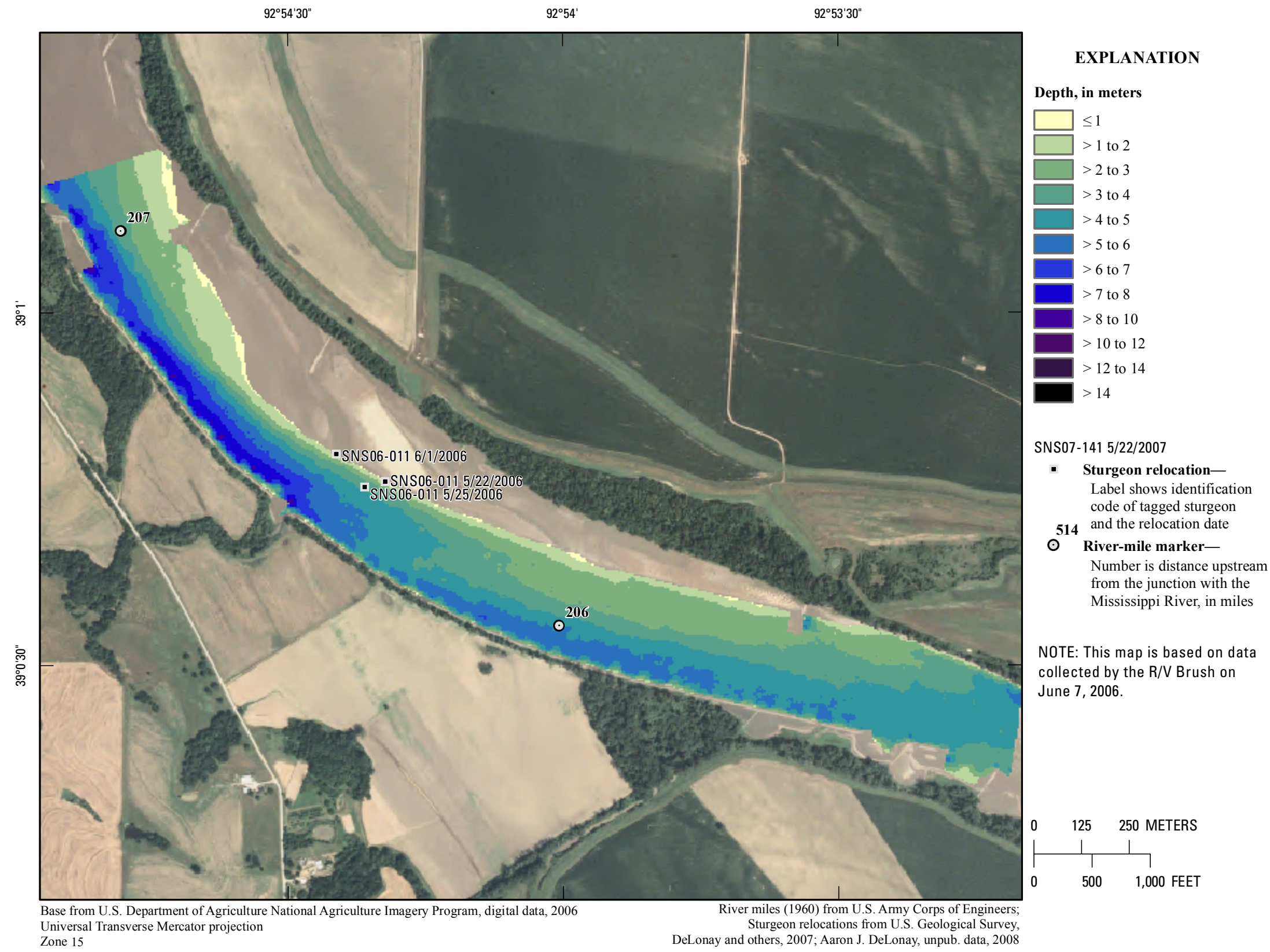

Figure 336. Map of depth based on data collected on June 7, 2006, in the vicinity of river mile 206. 


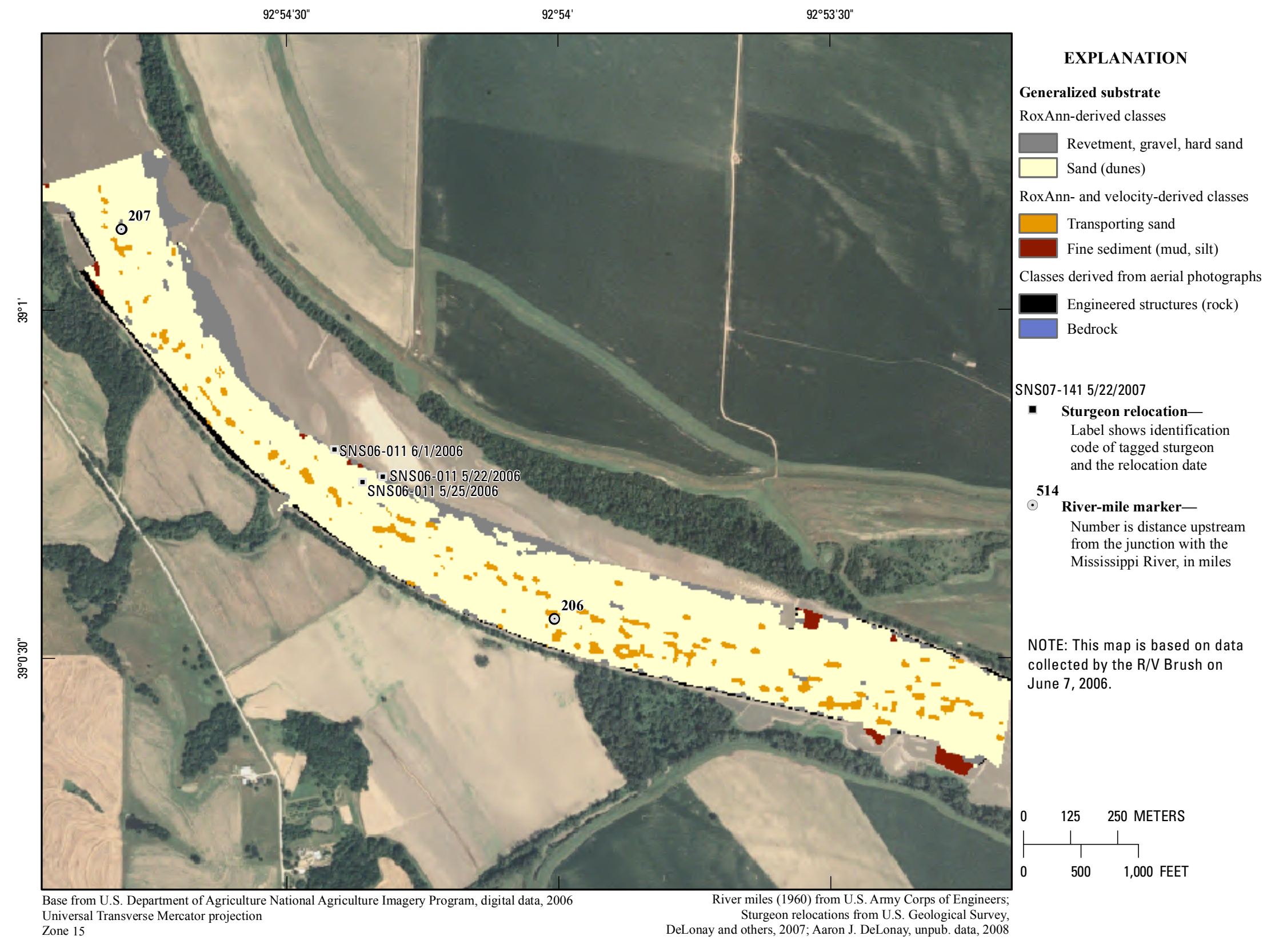

Figure 337. Map of generalized substrate based on data collected on June 7, 2006, in the vicinity of river mile 206. 


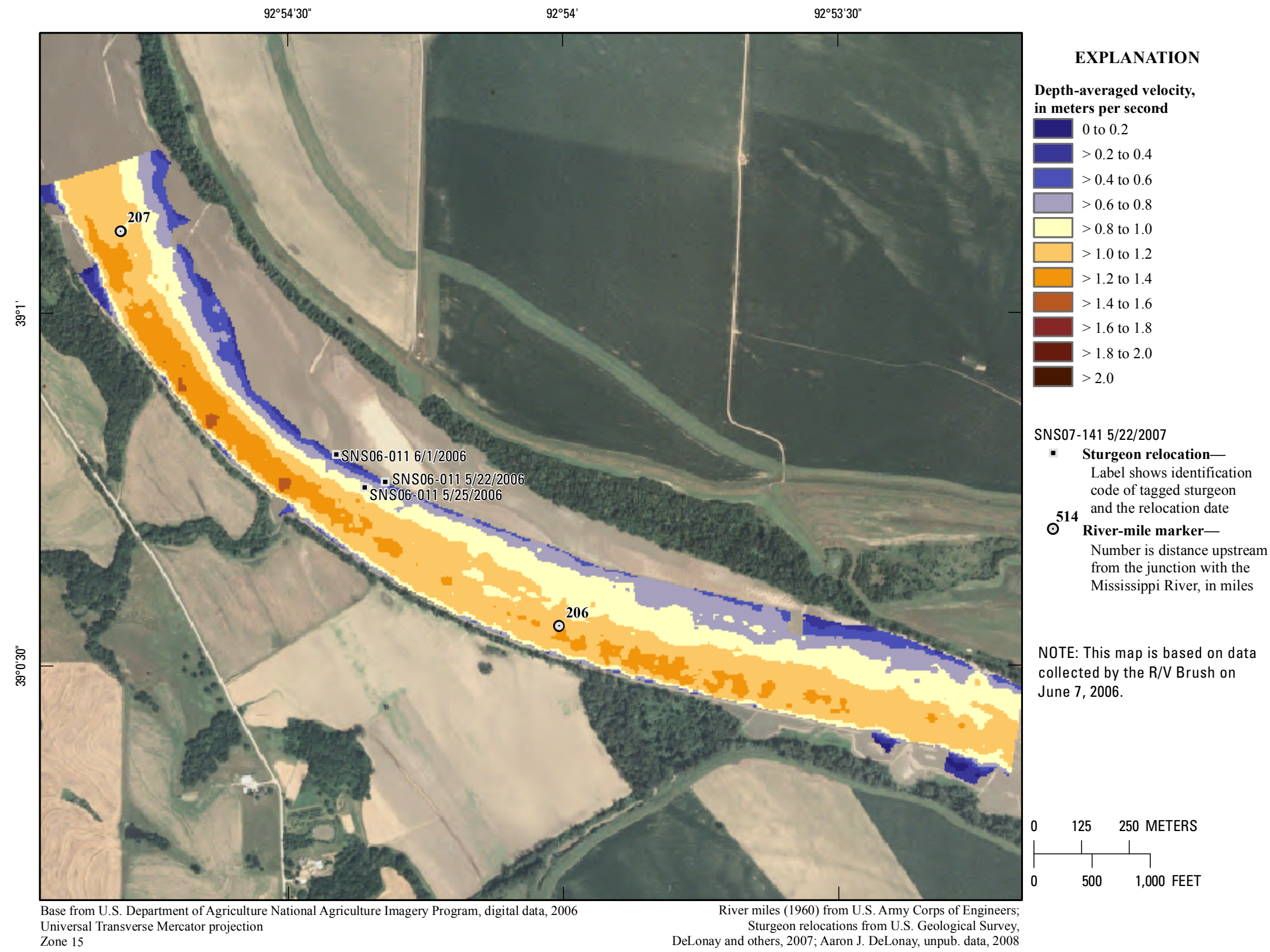

Figure 338. Map of depth-averaged velocity based on data collected on June 7, 2006, in the vicinity of river mile 206 . 


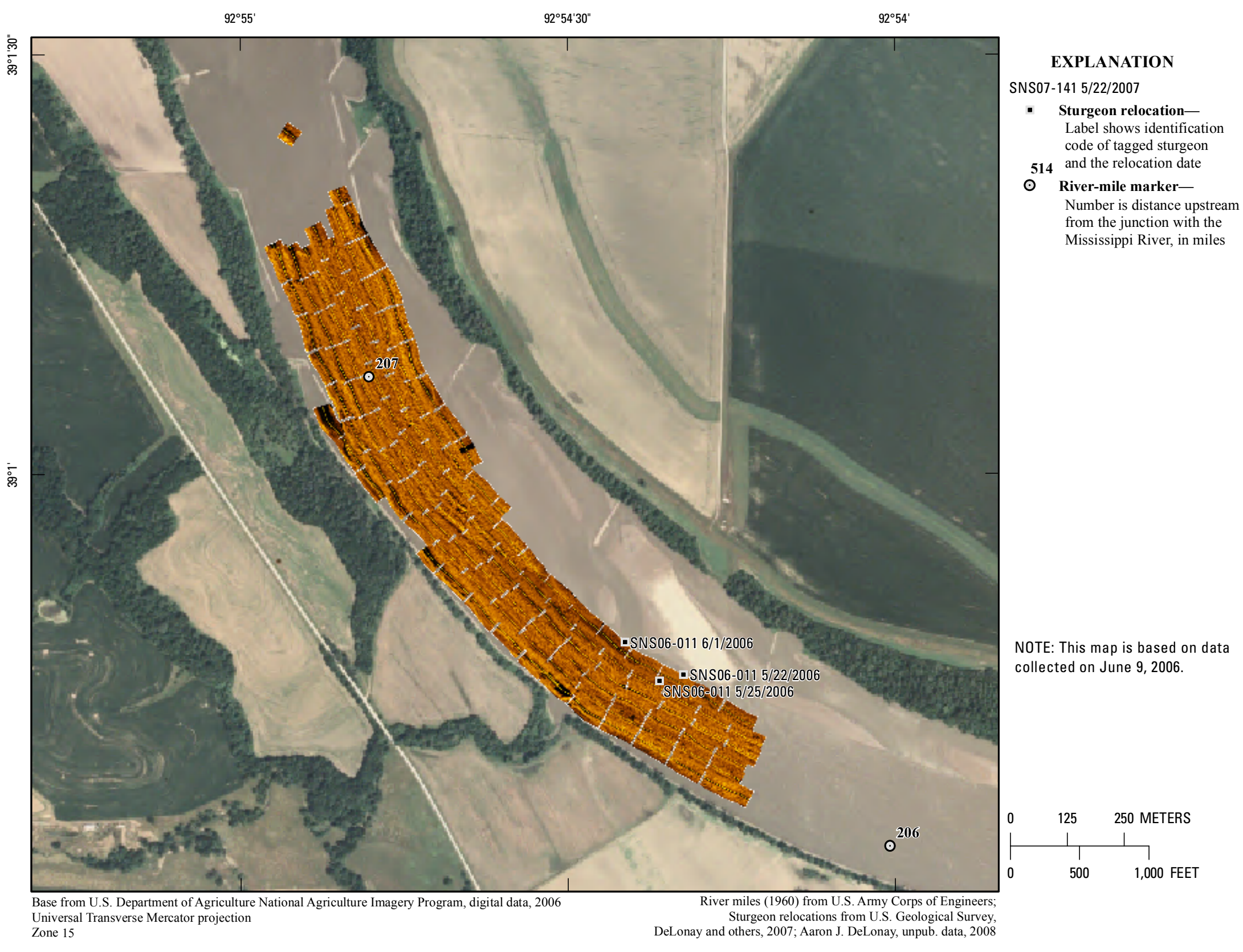

Figure 339. Map of side-scan sonar imagery based on data collected on June 9, 2006, in the vicinity of river mile 206. 


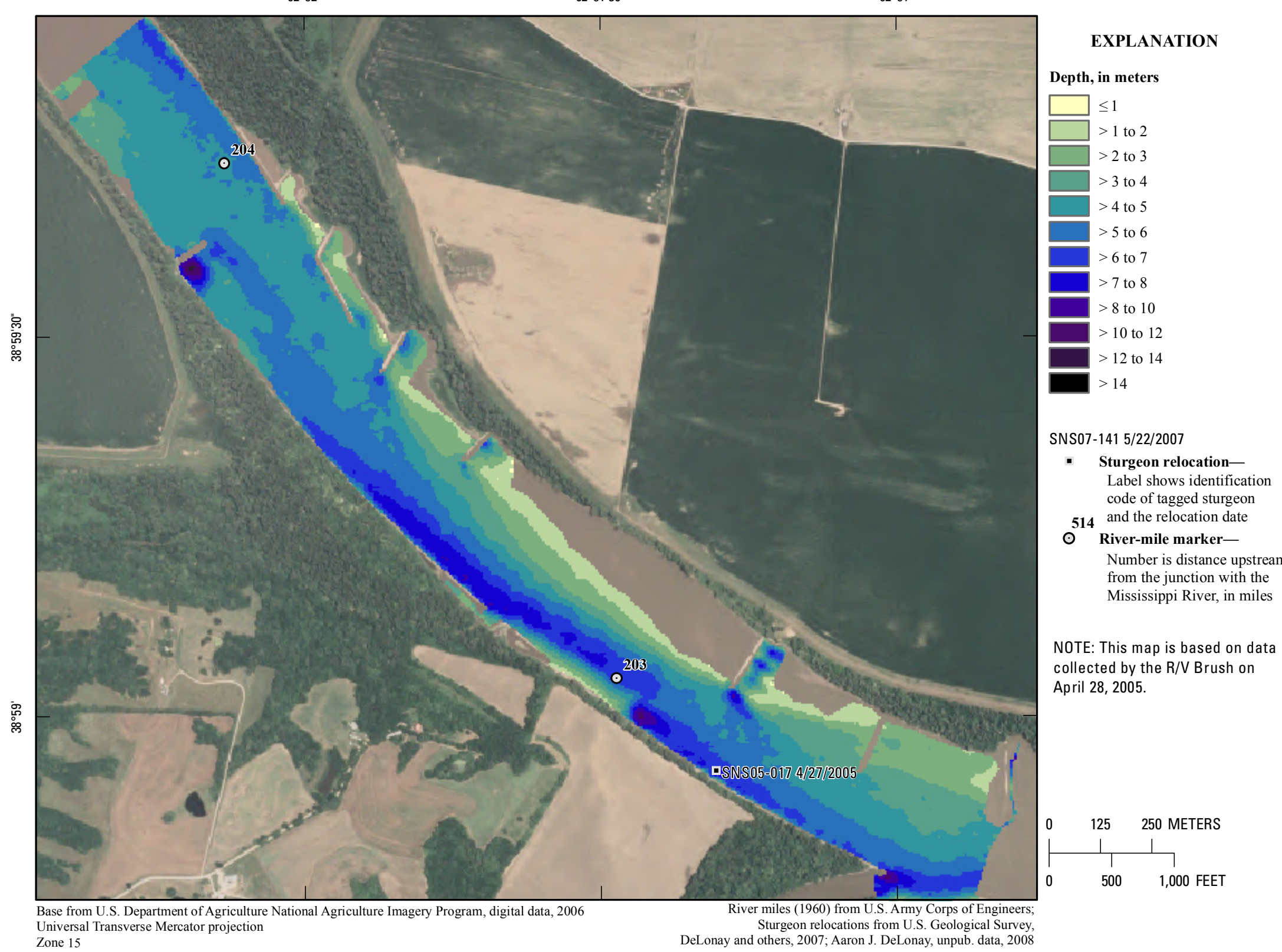
Universal Transverse Mercator projection
Zone 15 Sturgeon relocations from U.S. Geological Survey,
DeLonay and others, 2007; Aaron J. DeLonay, unpub. data, 2008 


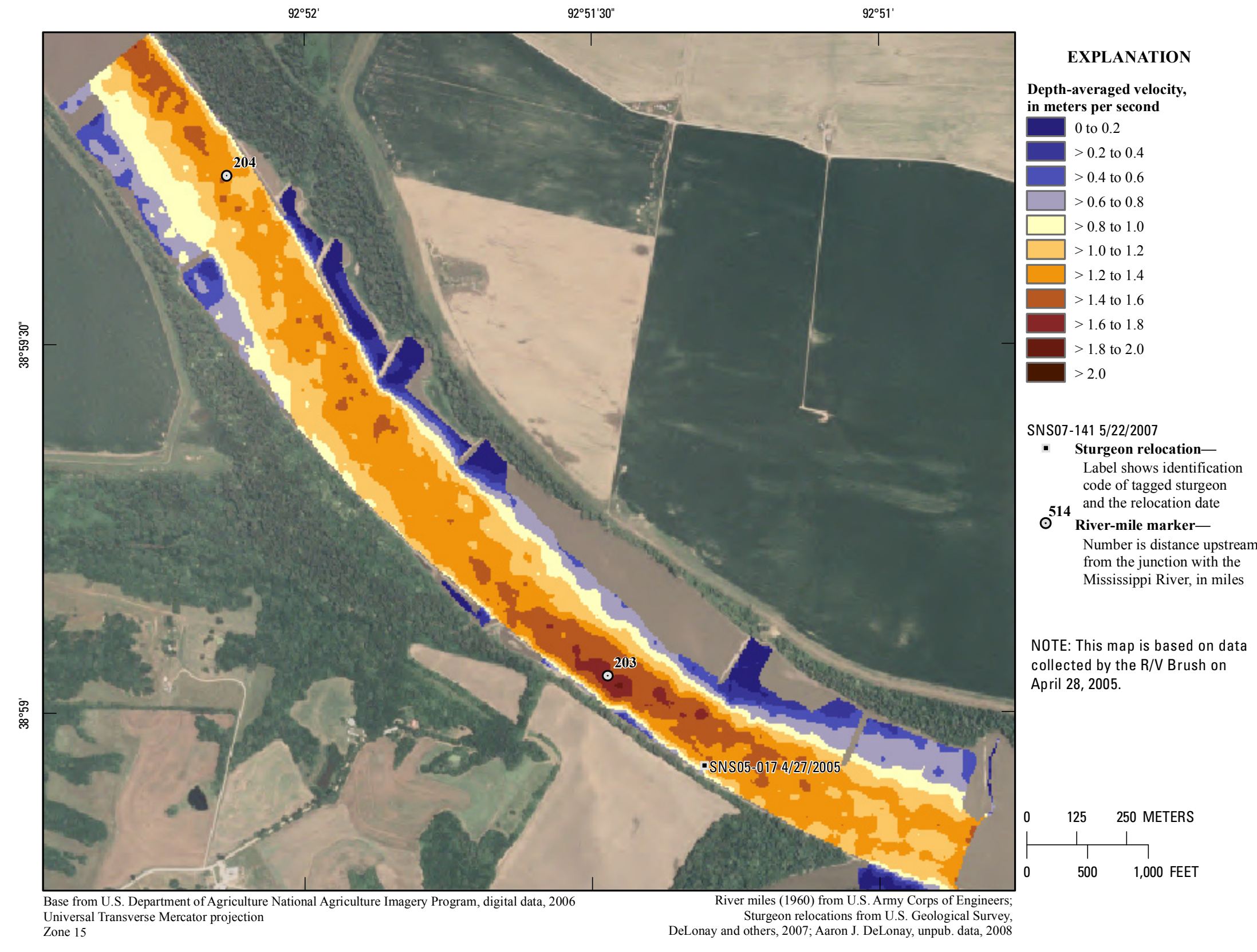

Figure 341. Map of depth-averaged velocity based on data collected on April 28, 2005, in the vicinity of river mile 203. 


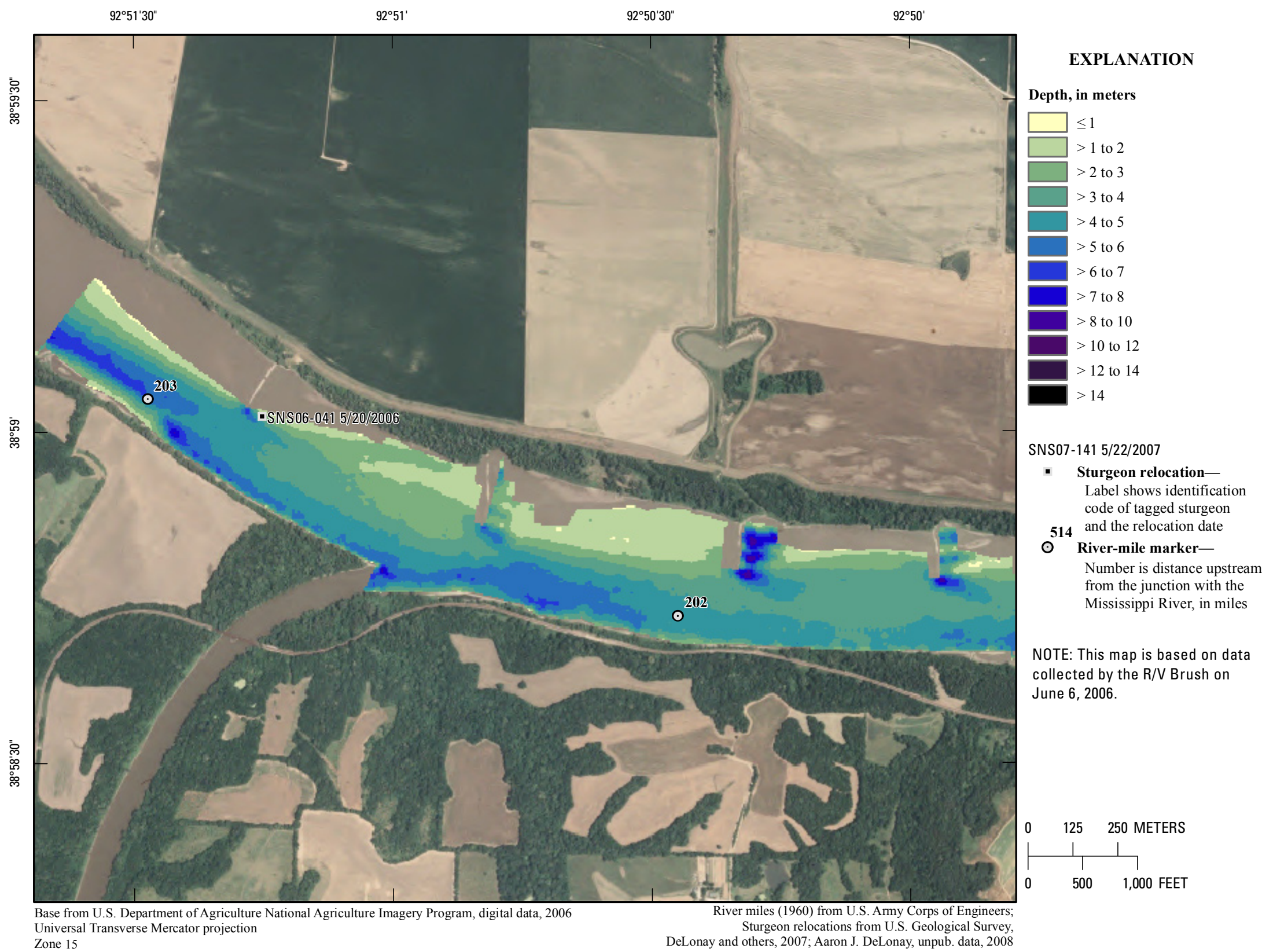

Figure 342. Map of depth based on data collected on June 6, 2006, in the vicinity of river mile 202. 


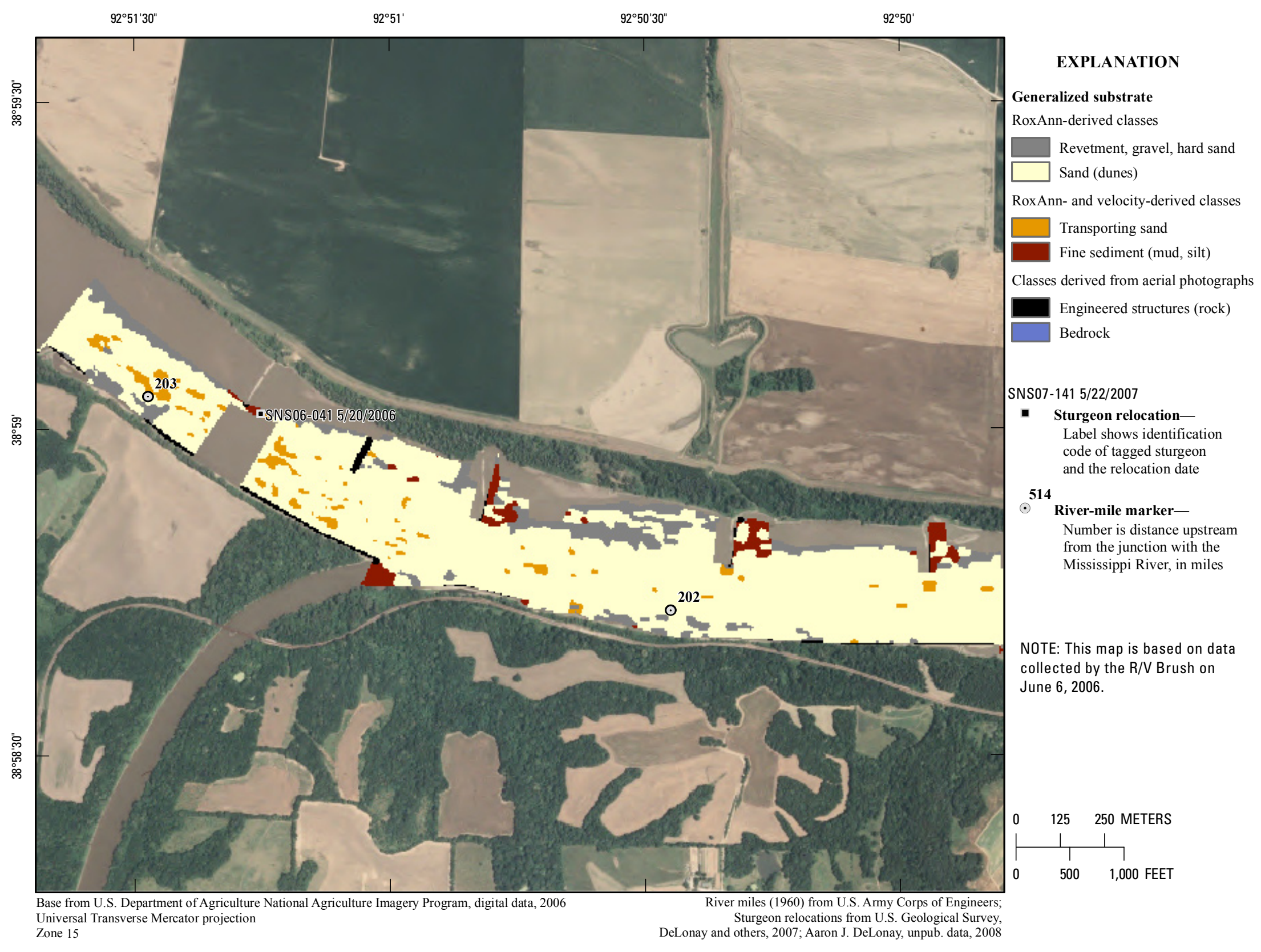

Figure 343. Map of generalized substrate based on data collected on June 6, 2006, in the vicinity of river mile 202. 


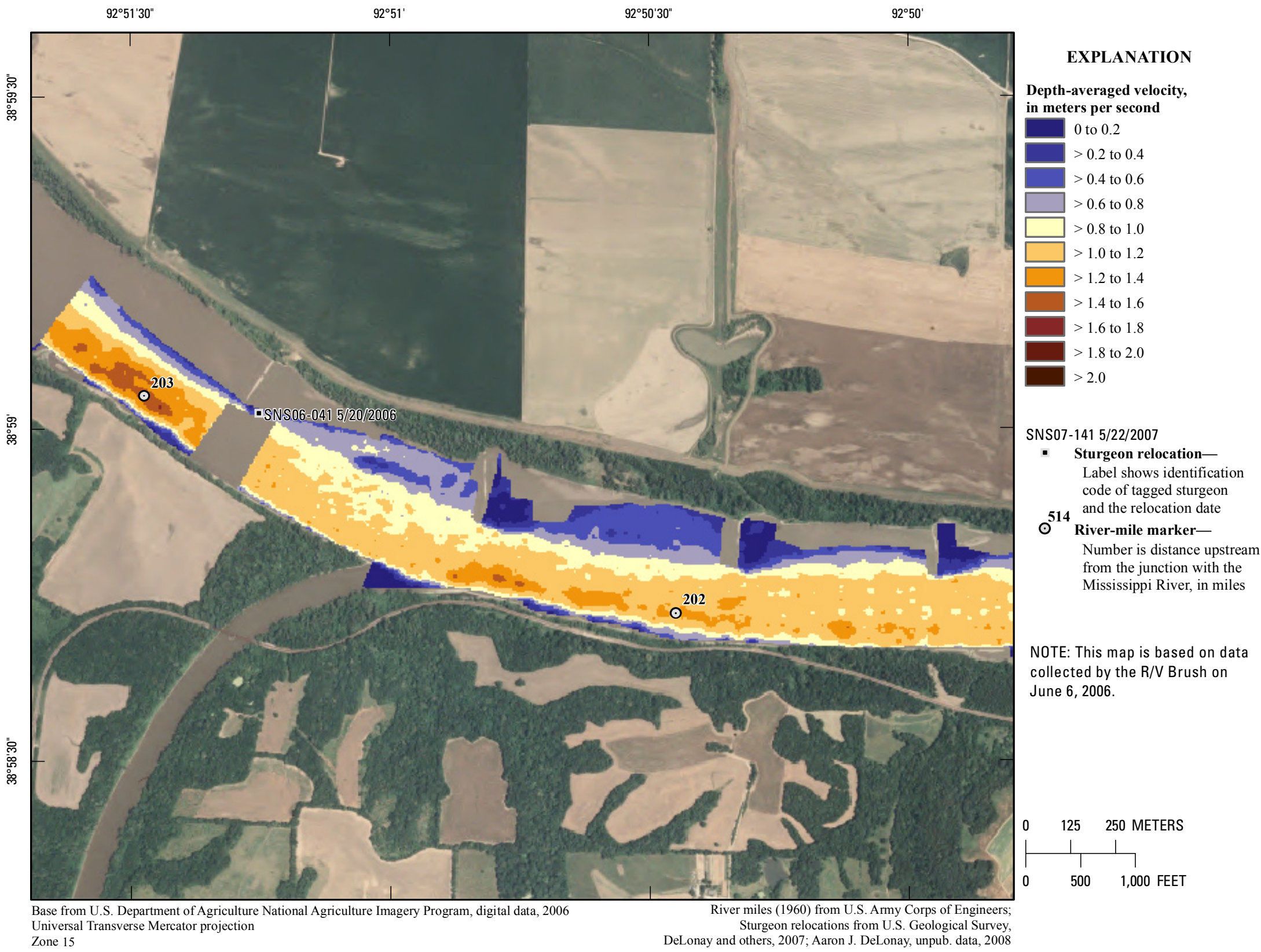

Figure 344. Map of depth-averaged velocity based on data collected on June 6, 2006, in the vicinity of river mile 202. 


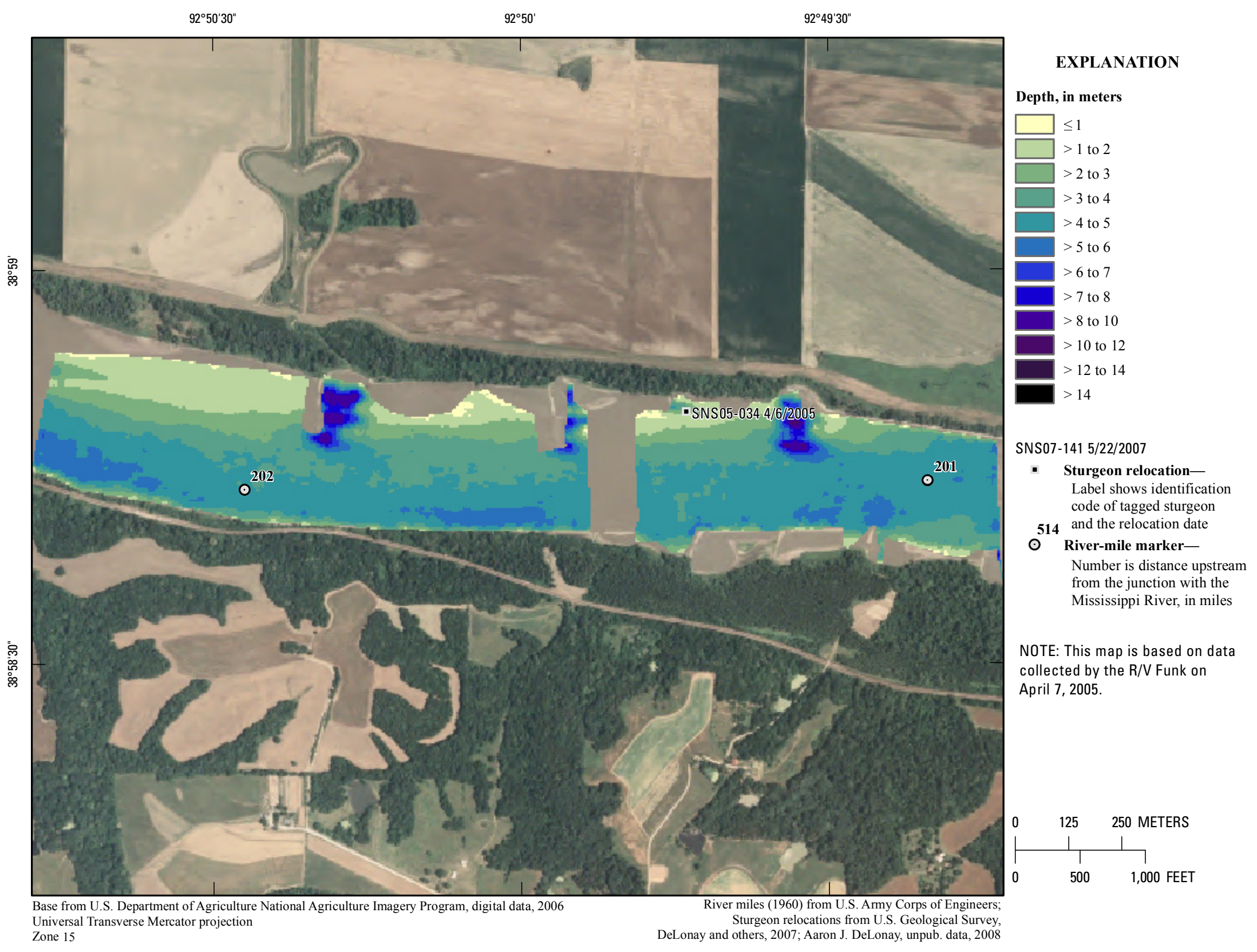

Figure 345. Map of depth based on data collected on April 7, 2005, in the vicinity of river mile 201. 


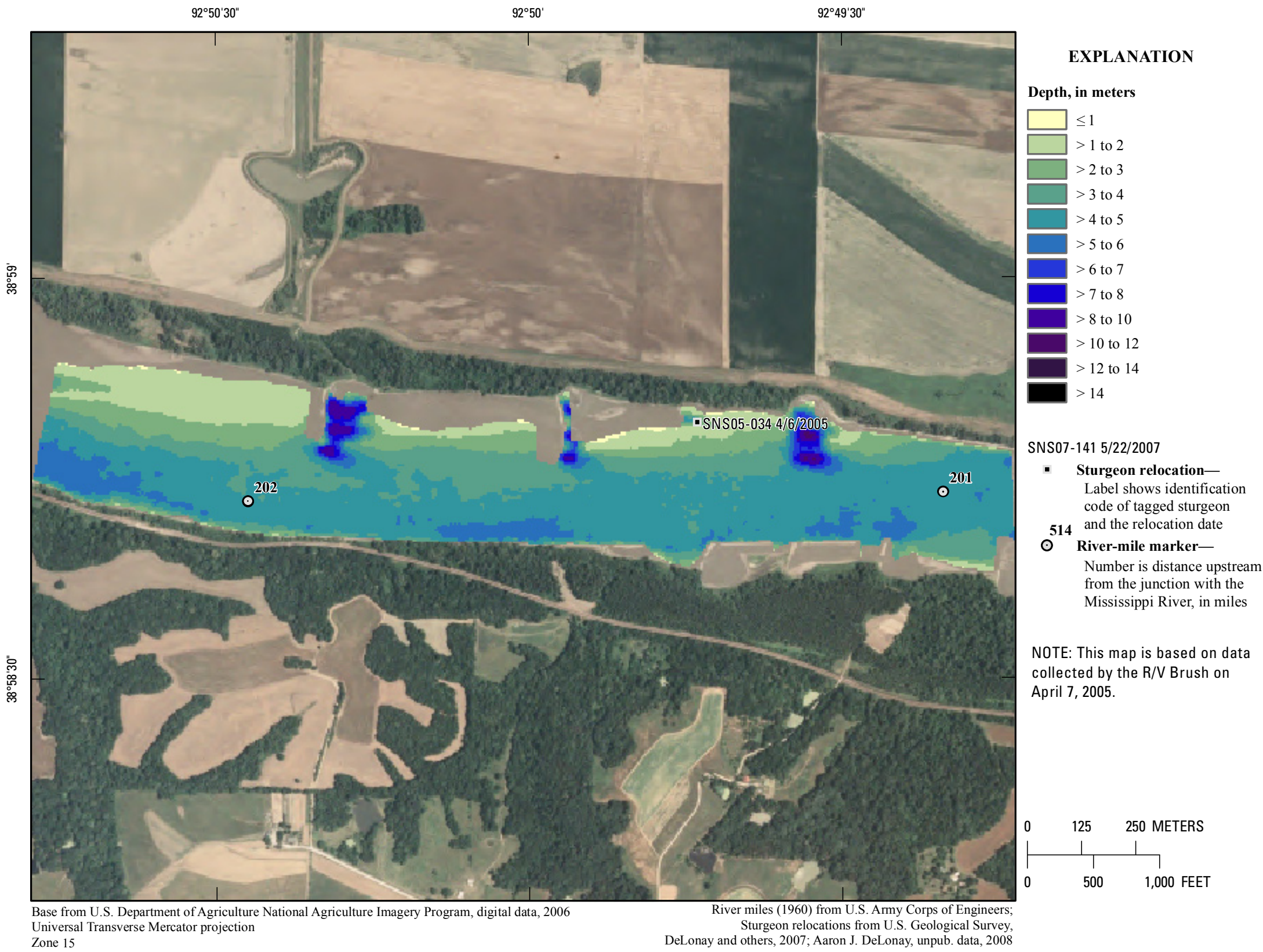

Figure 346. Map of depth based on data collected on April 7, 2005, in the vicinity of river mile 201. 


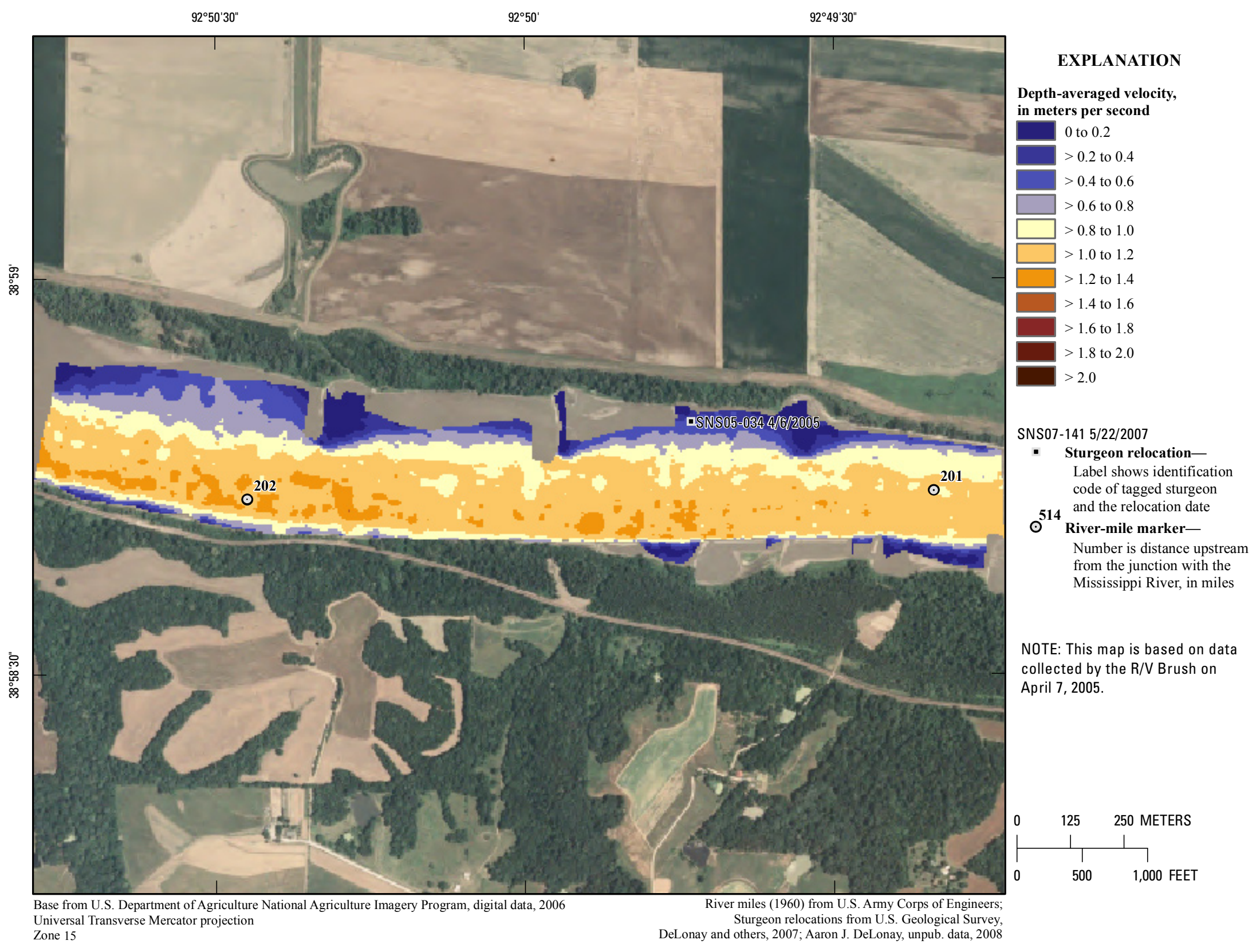

Figure 347. Map of depth-averaged velocity based on data collected on April 7, 2005, in the vicinity of river mile 201. 


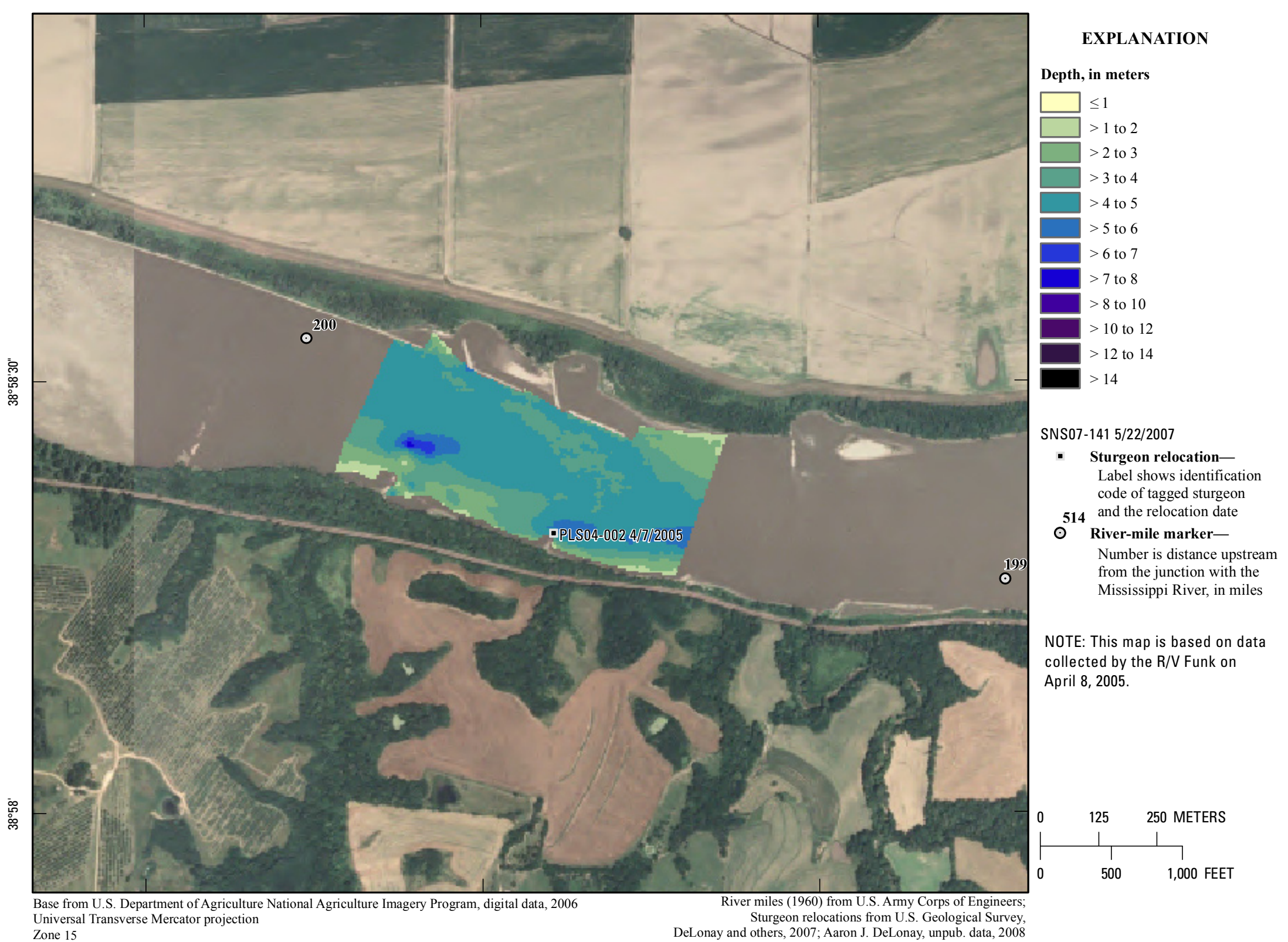




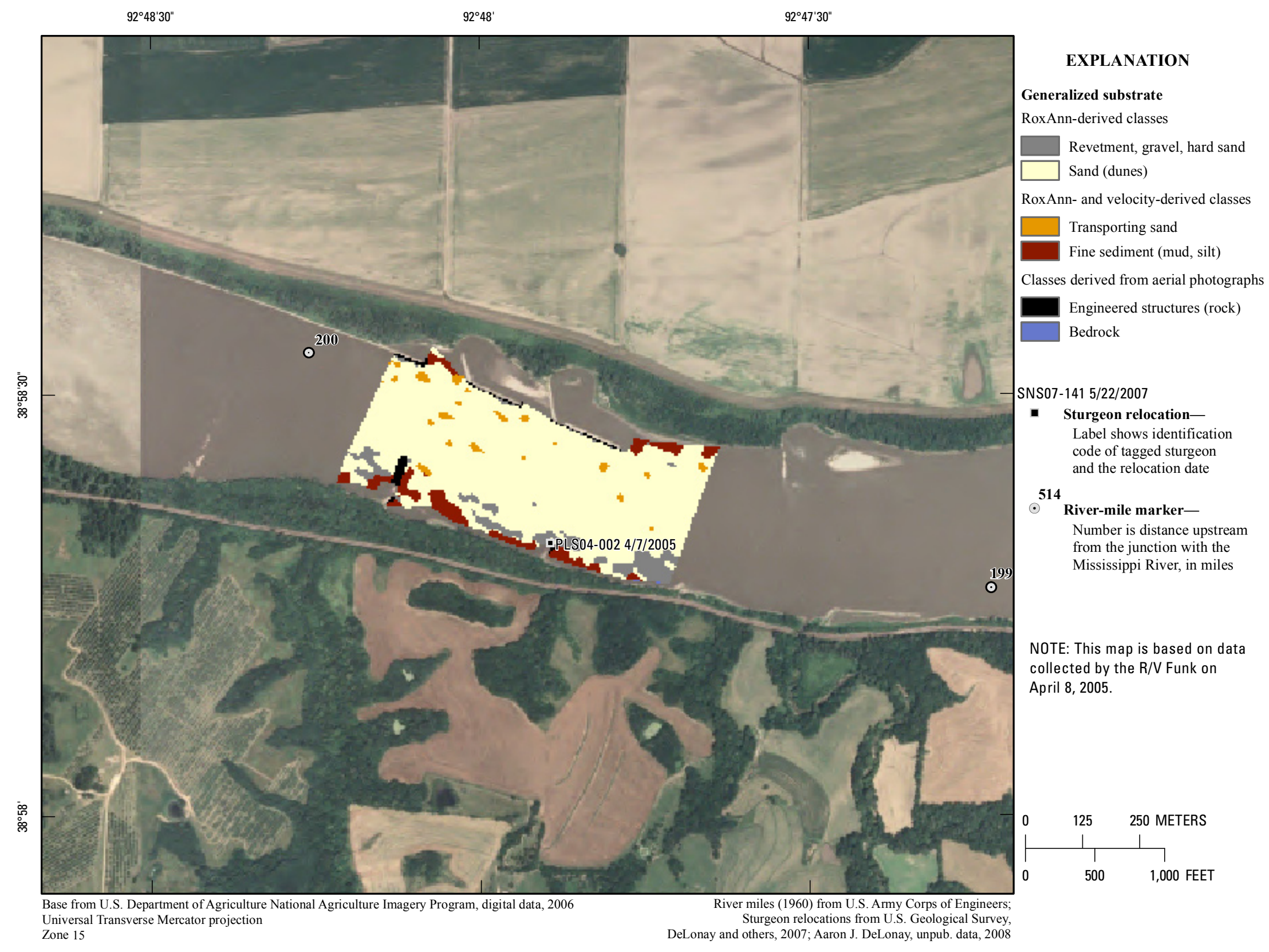

Figure 349. Map of generalized substrate based on data collected on April 8, 2005, in the vicinity of river mile 200. 


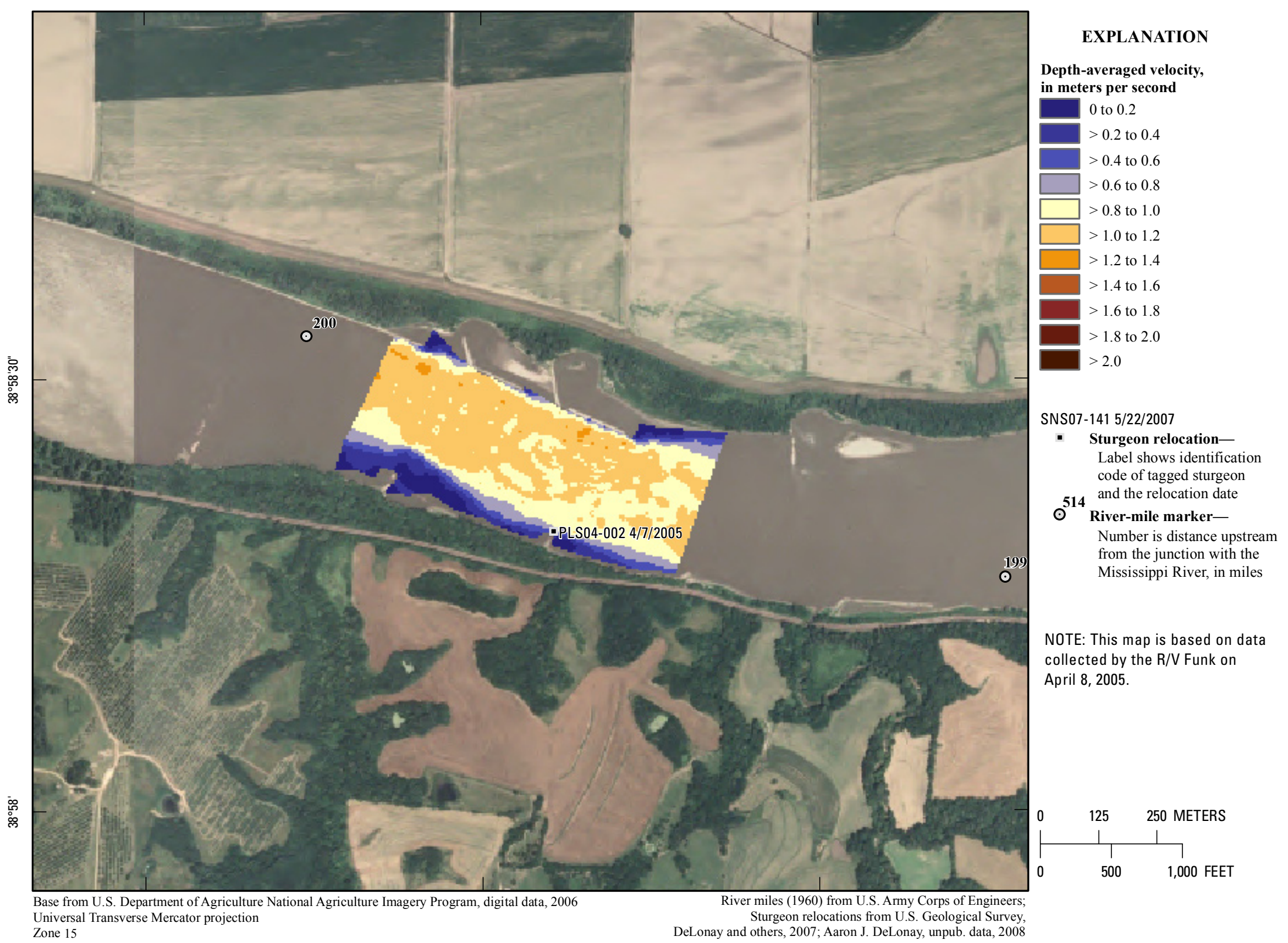




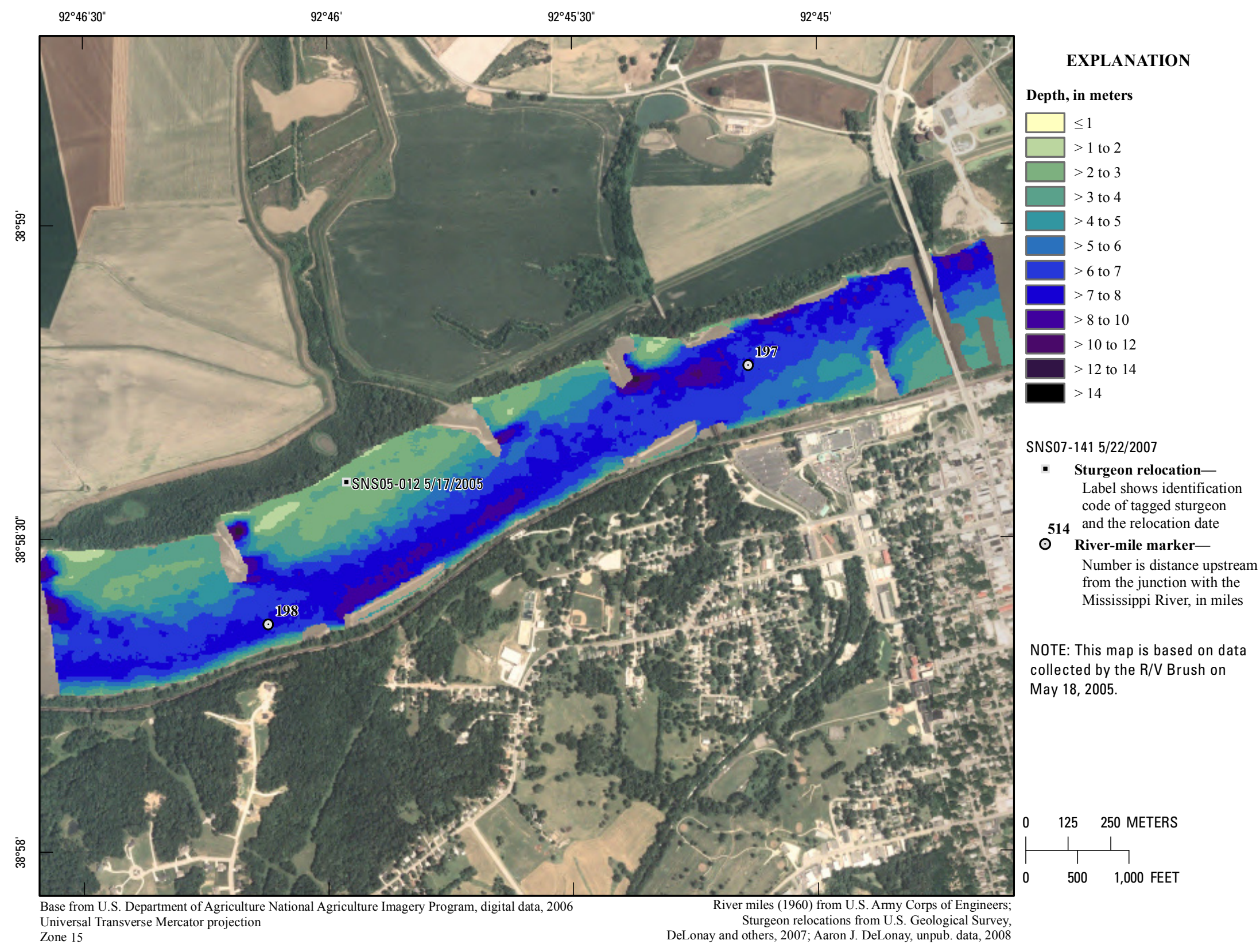

Figure 351. Map of depth based on data collected on May 18, 2005, in the vicinity of river mile 198. 


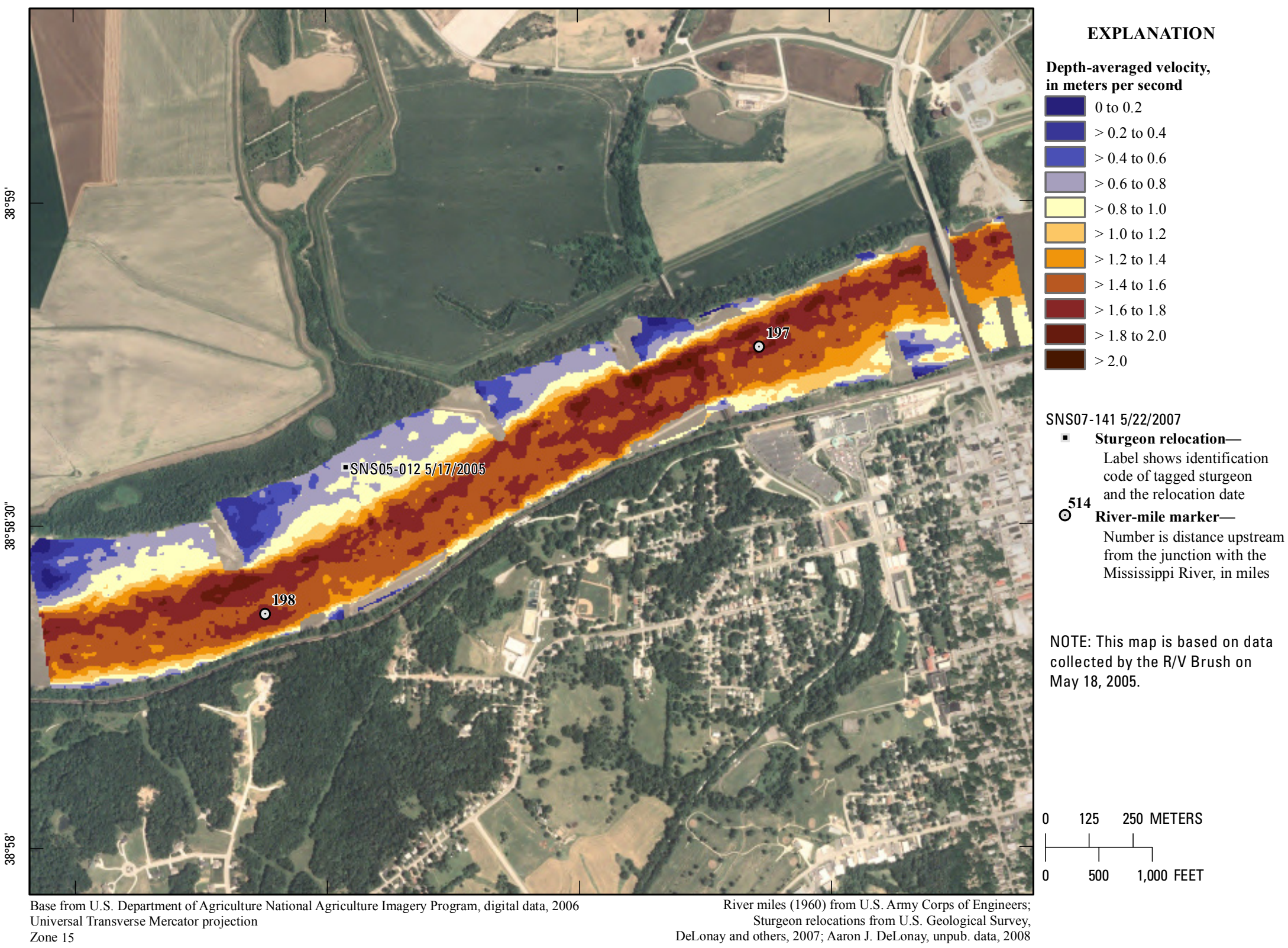
Universal Transverse Mercator projectio DeLonay and others, 2007; Aaron J. DeLonay, unpub. data, 2008 


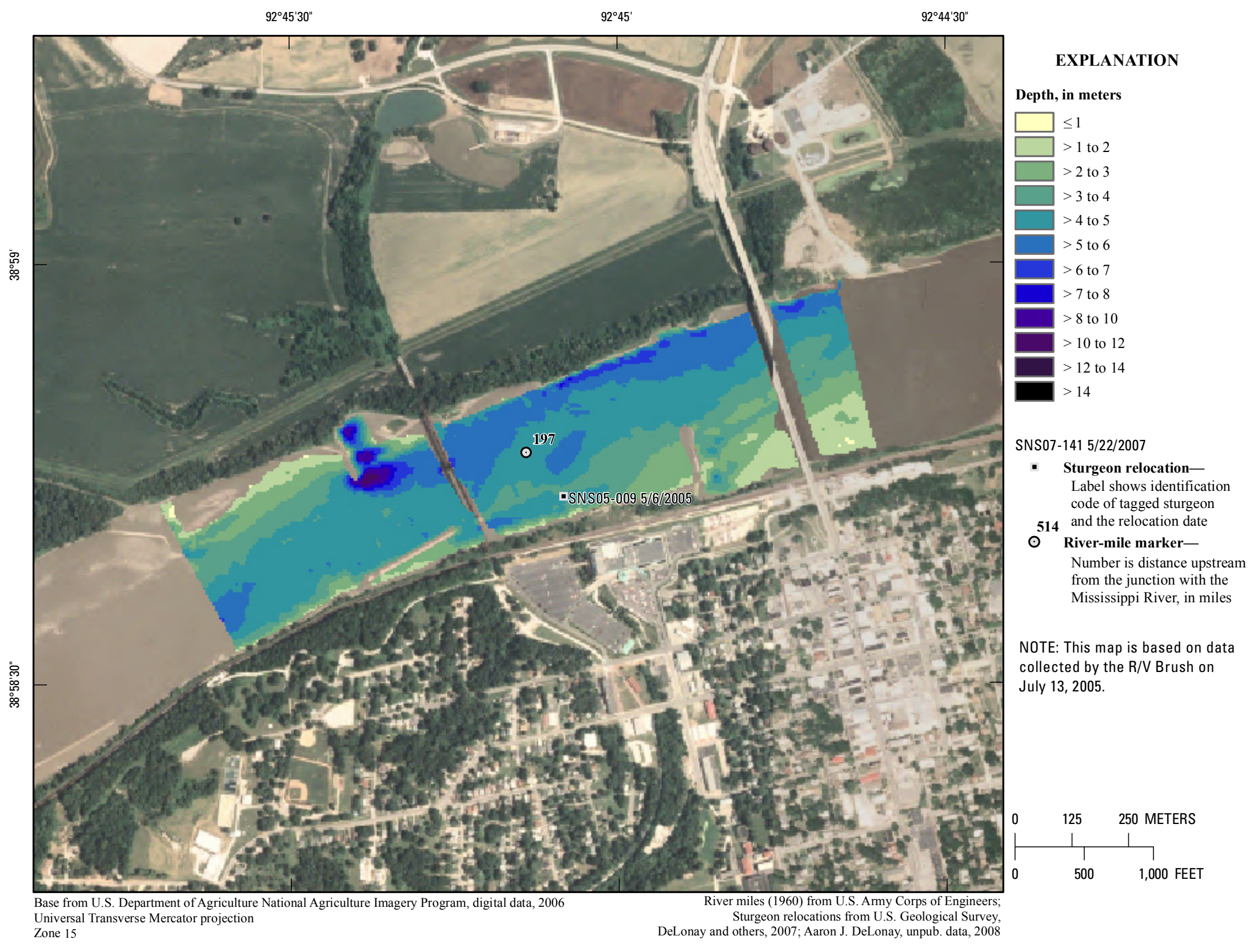

Figure 353. Map of depth based on data collected on July 13, 2005, in the vicinity of river mile 197. 


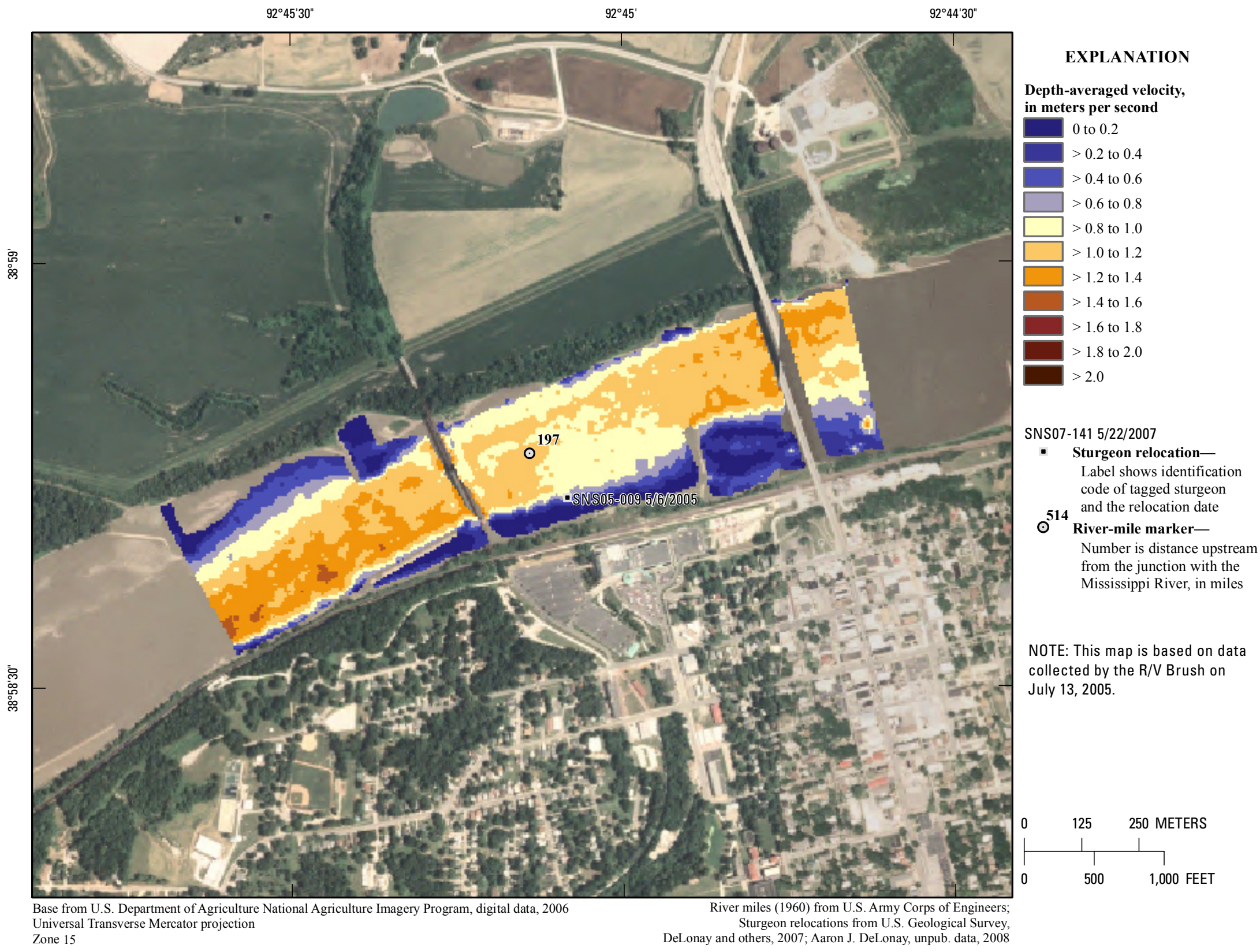

Figure 354. Map of depth-averaged velocity based on data collected on July 13, 2005, in the vicinity of river mile 197. 


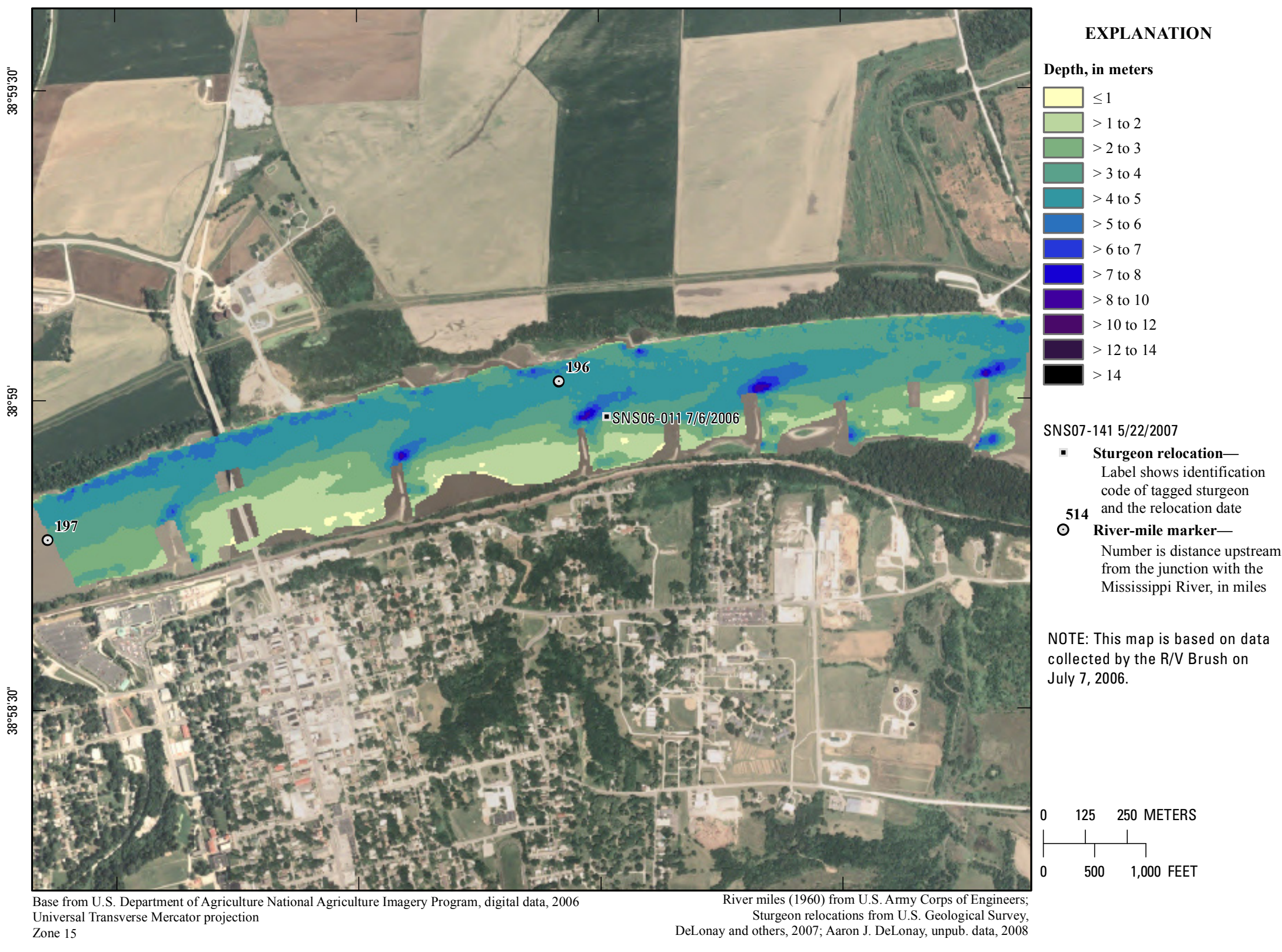

Figure 355. Map of depth based on data collected on July 7, 2006, in the vicinity of river mile 196. 


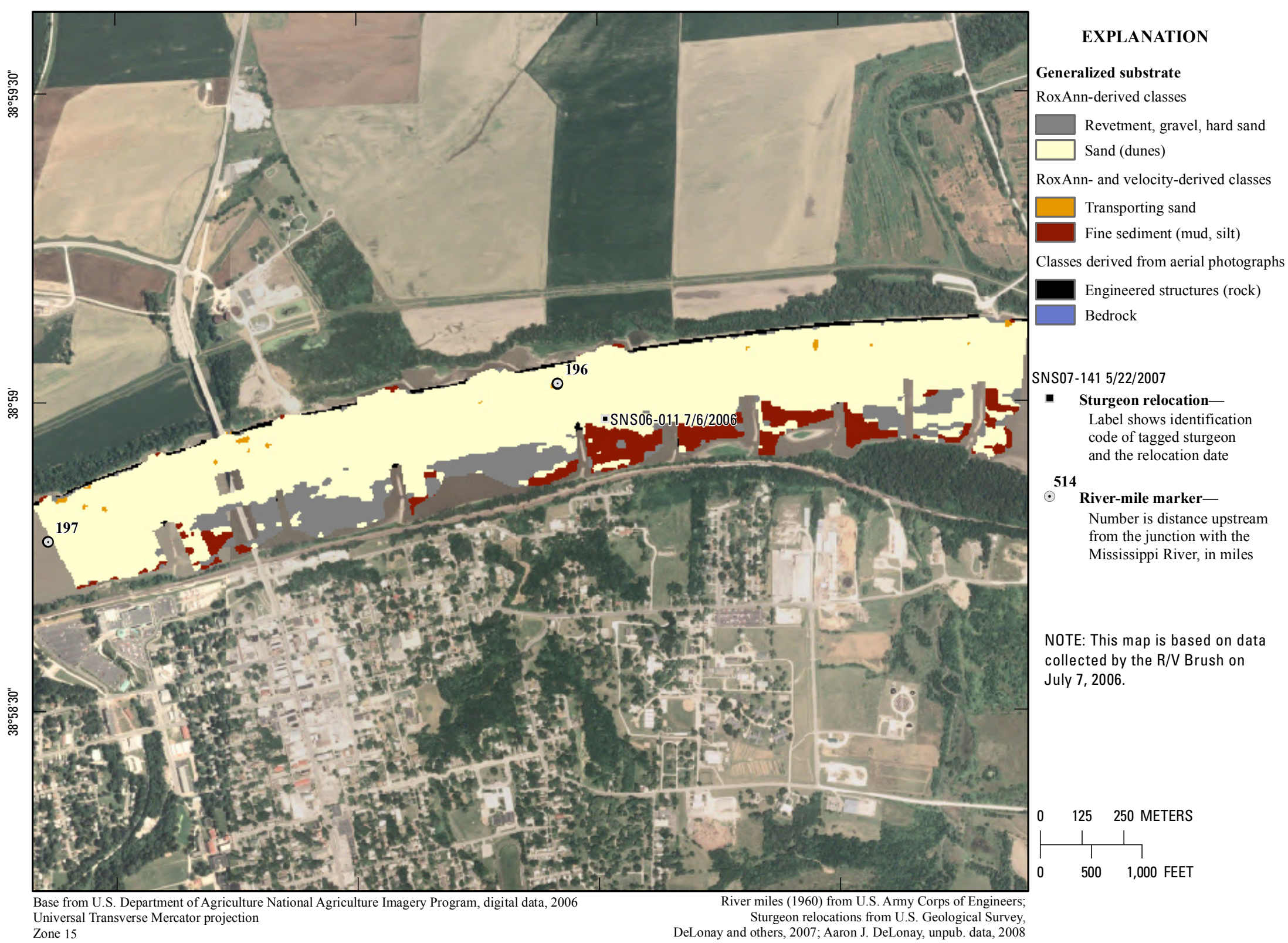

Figure 356. Map of generalized substrate based on data collected on July 7, 2006, in the vicinity of river mile 196. 


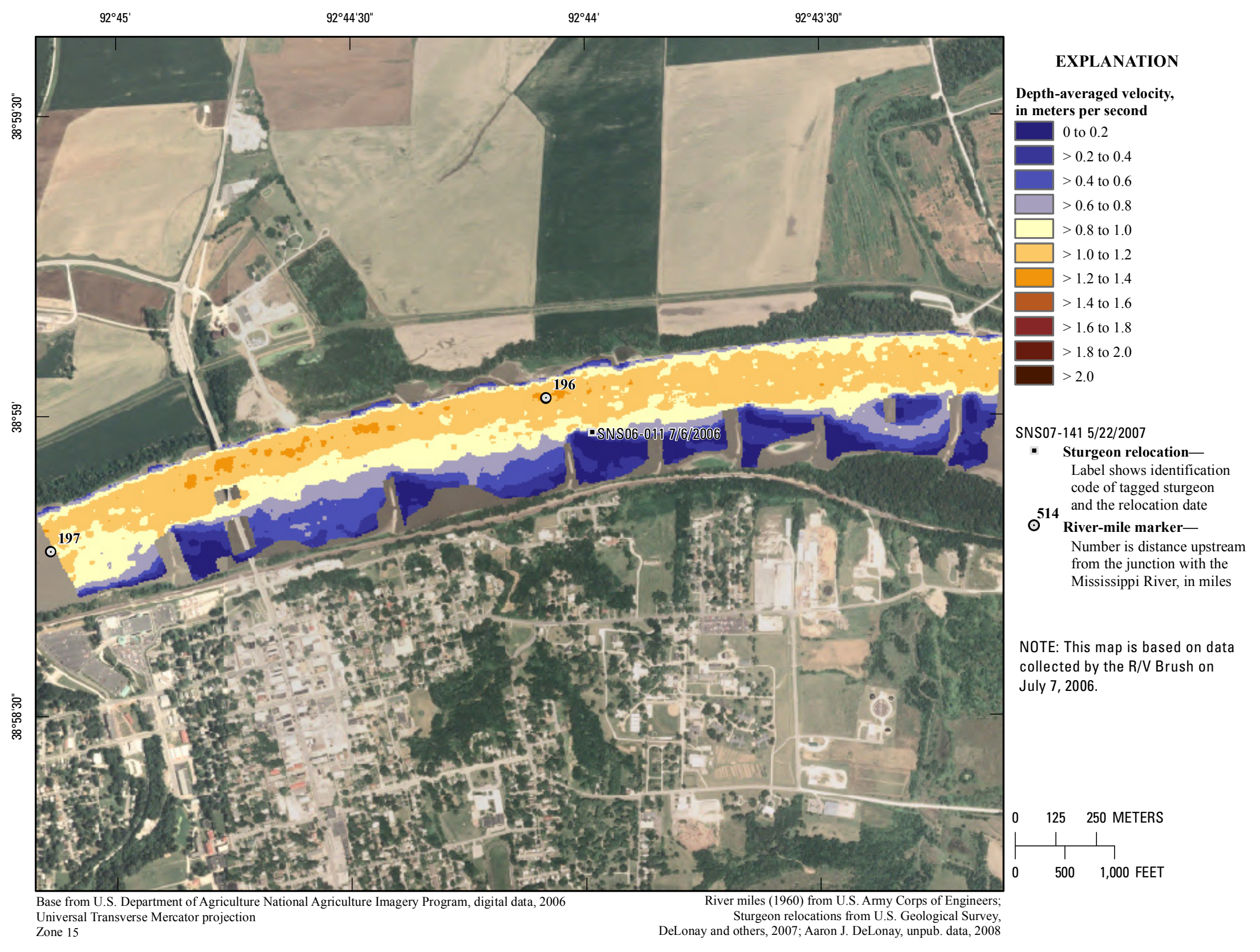

Figure 357. Map of depth-averaged velocity based on data collected on July 7, 2006, in the vicinity of river mile 196. 


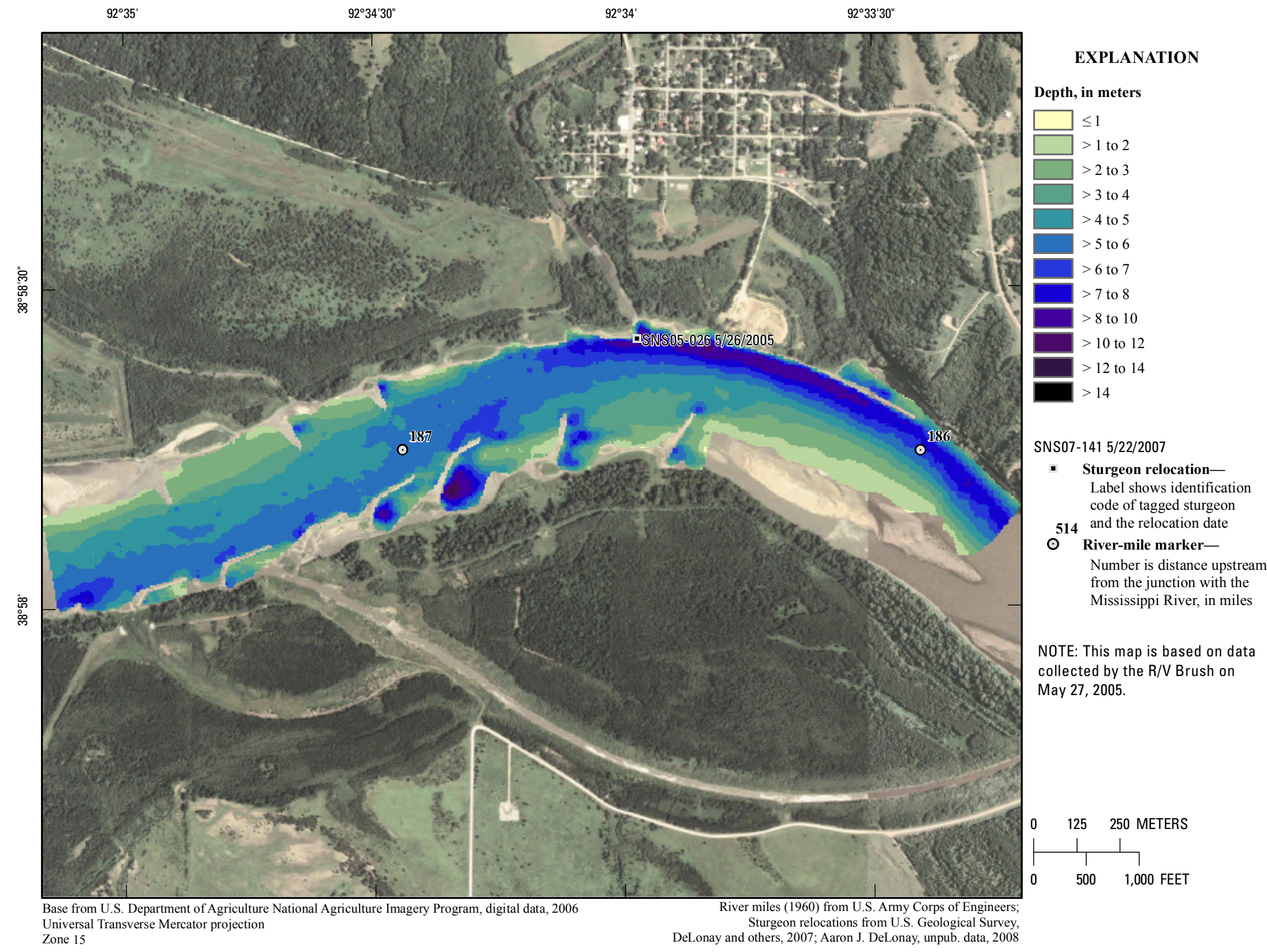

Figure 358. Map of depth based on data collected on May 27, 2005, in the vicinity of river mile 187. 


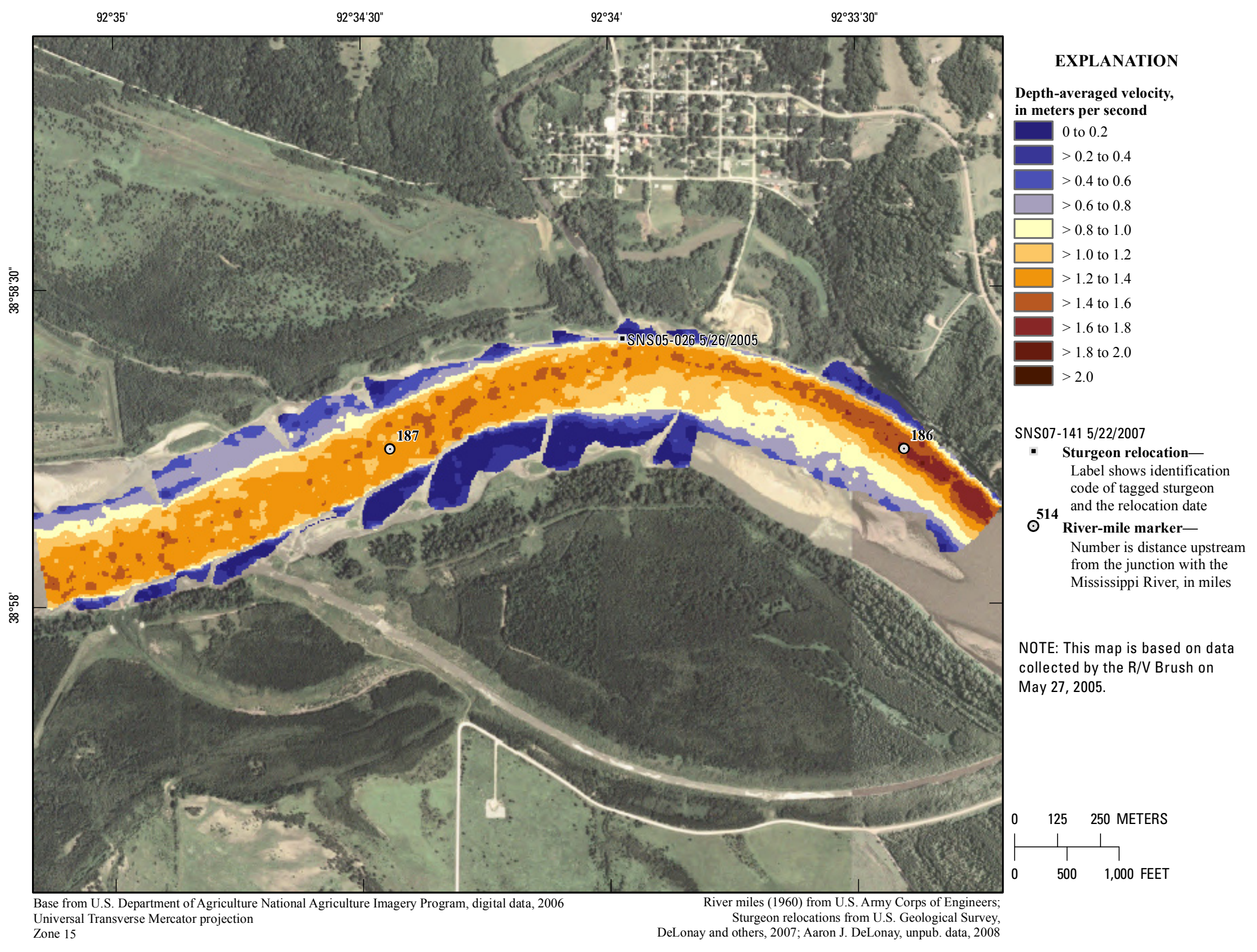

Figure 359. Map of depth-averaged velocity based on data collected on May 27, 2005, in the vicinity of river mile 187 . 


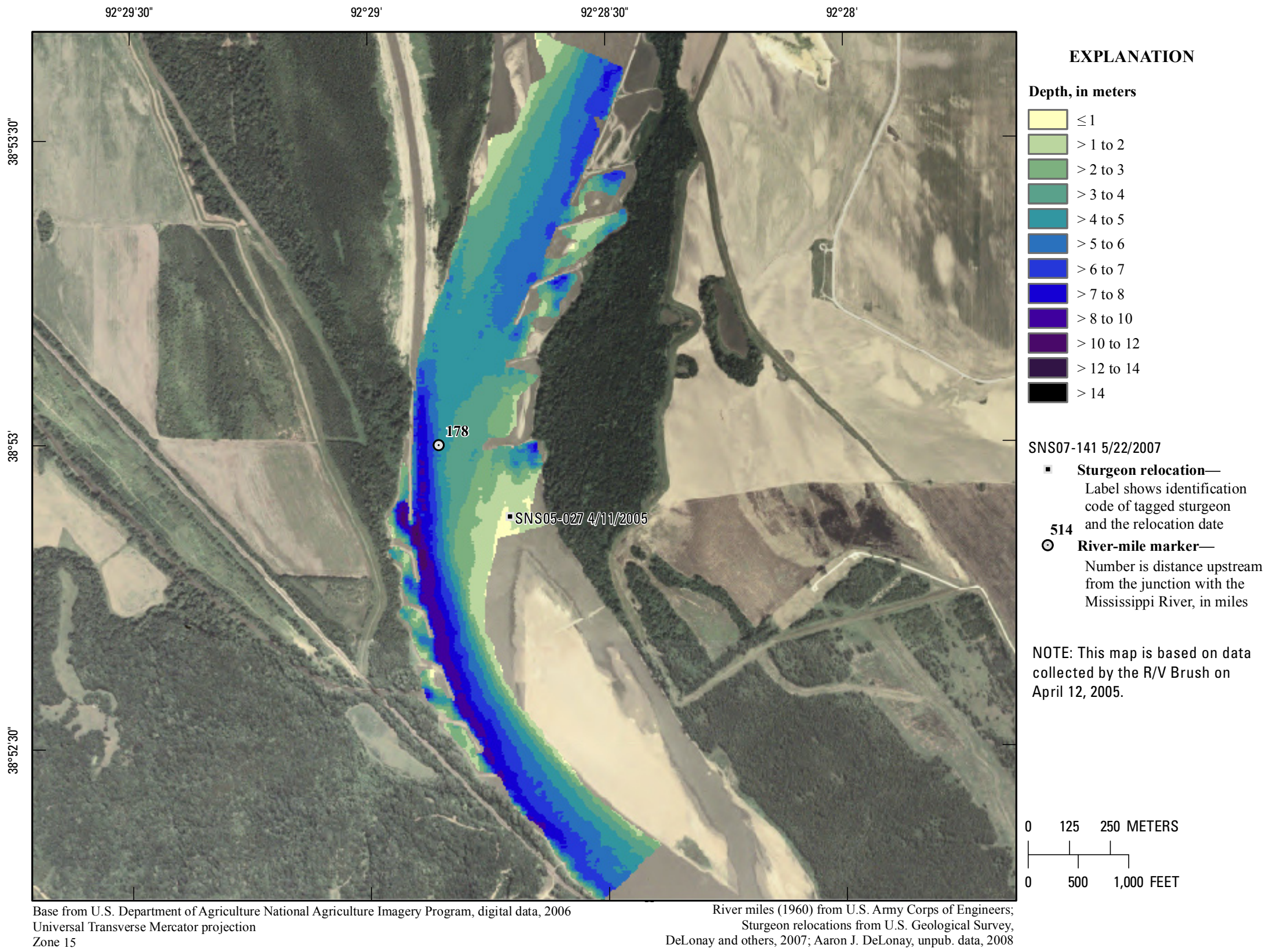

Figure 360. Map of depth based on data collected on April 12, 2005, in the vicinity of river mile 178. 


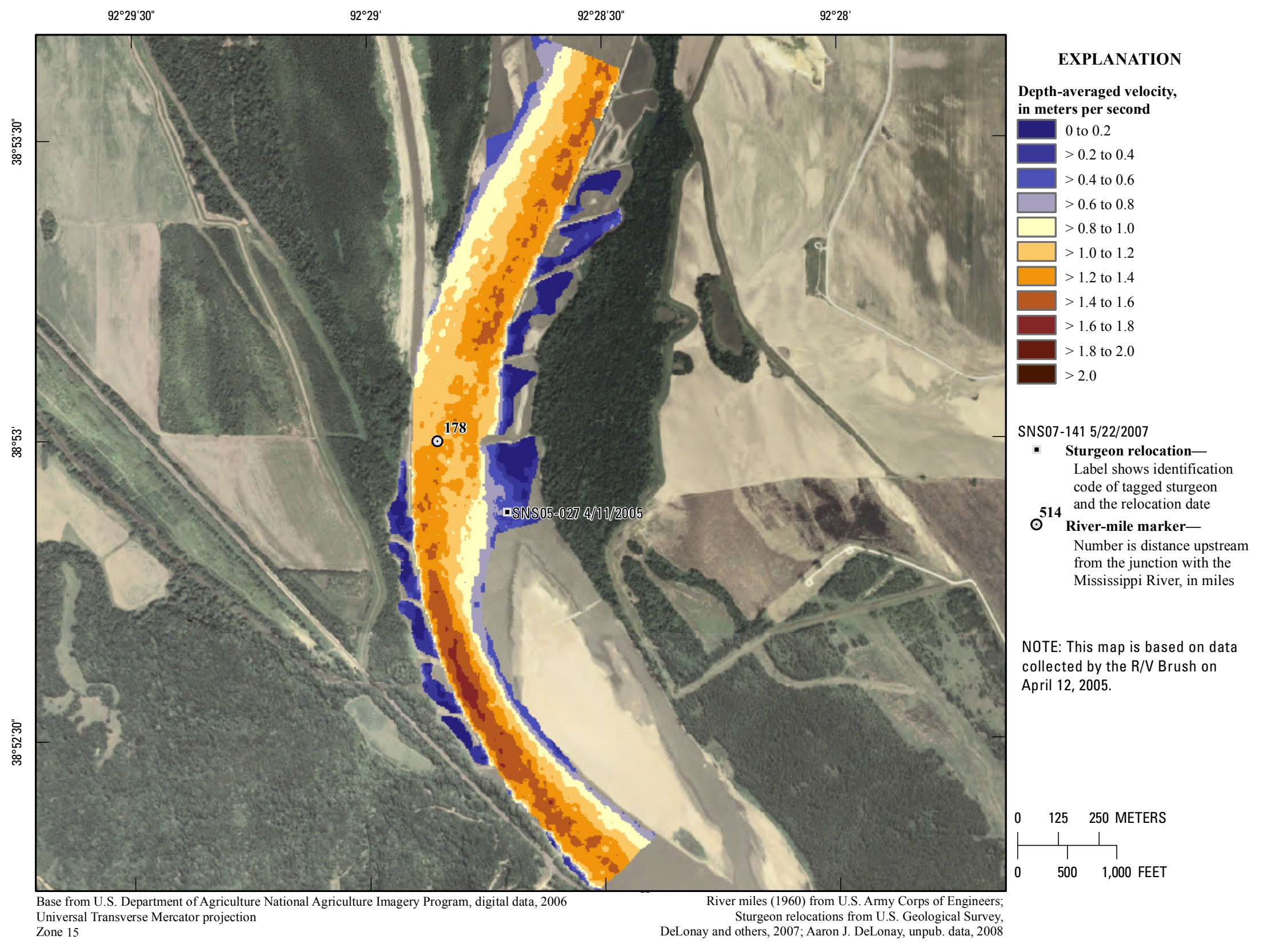

Figure 361. Map of depth-averaged velocity based on data collected on April 12, 2005, in the vicinity of river mile 178. 


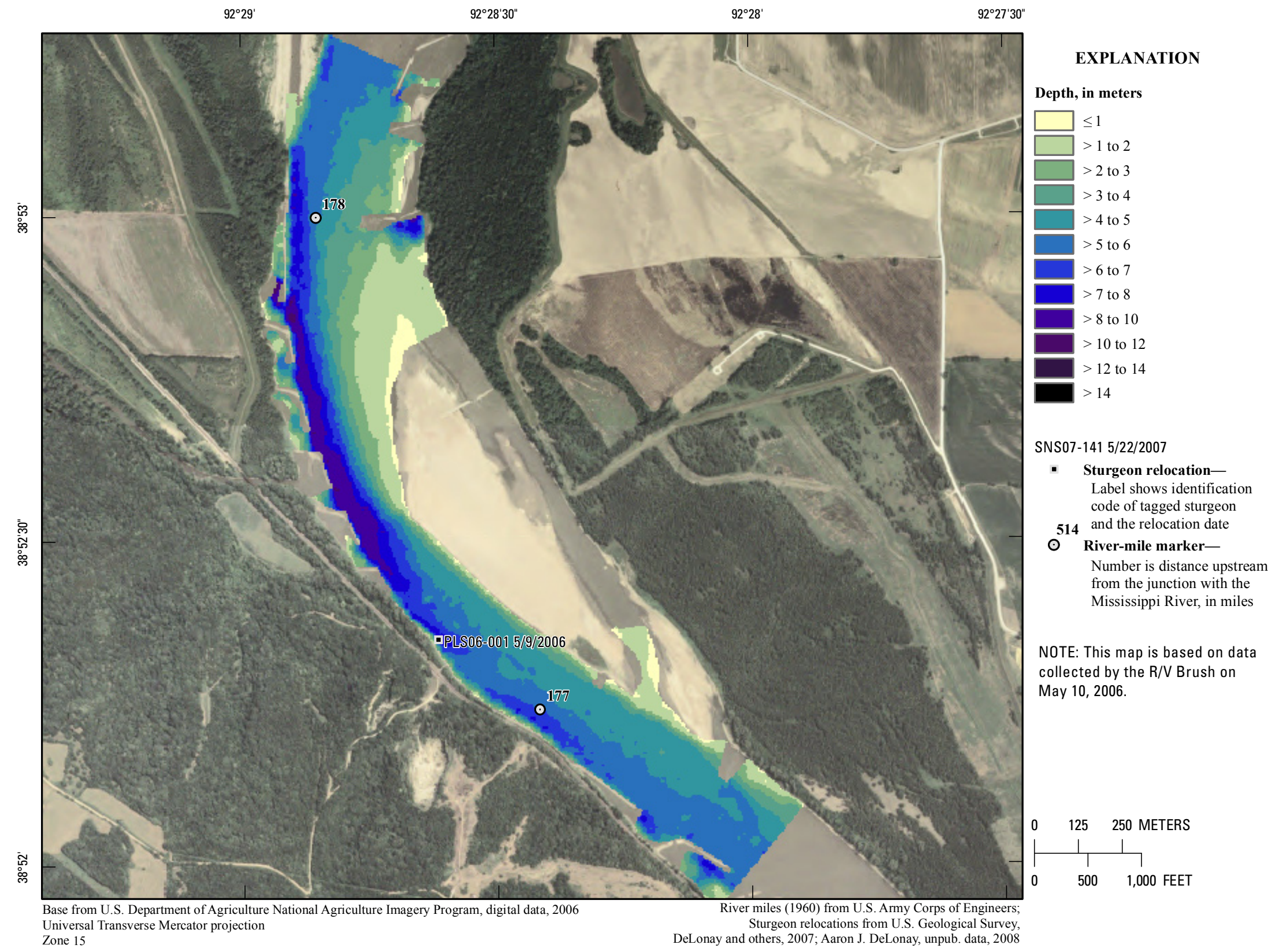

Figure 362. Map of depth based on data collected on May 10, 2006, in the vicinity of river mile 177. 


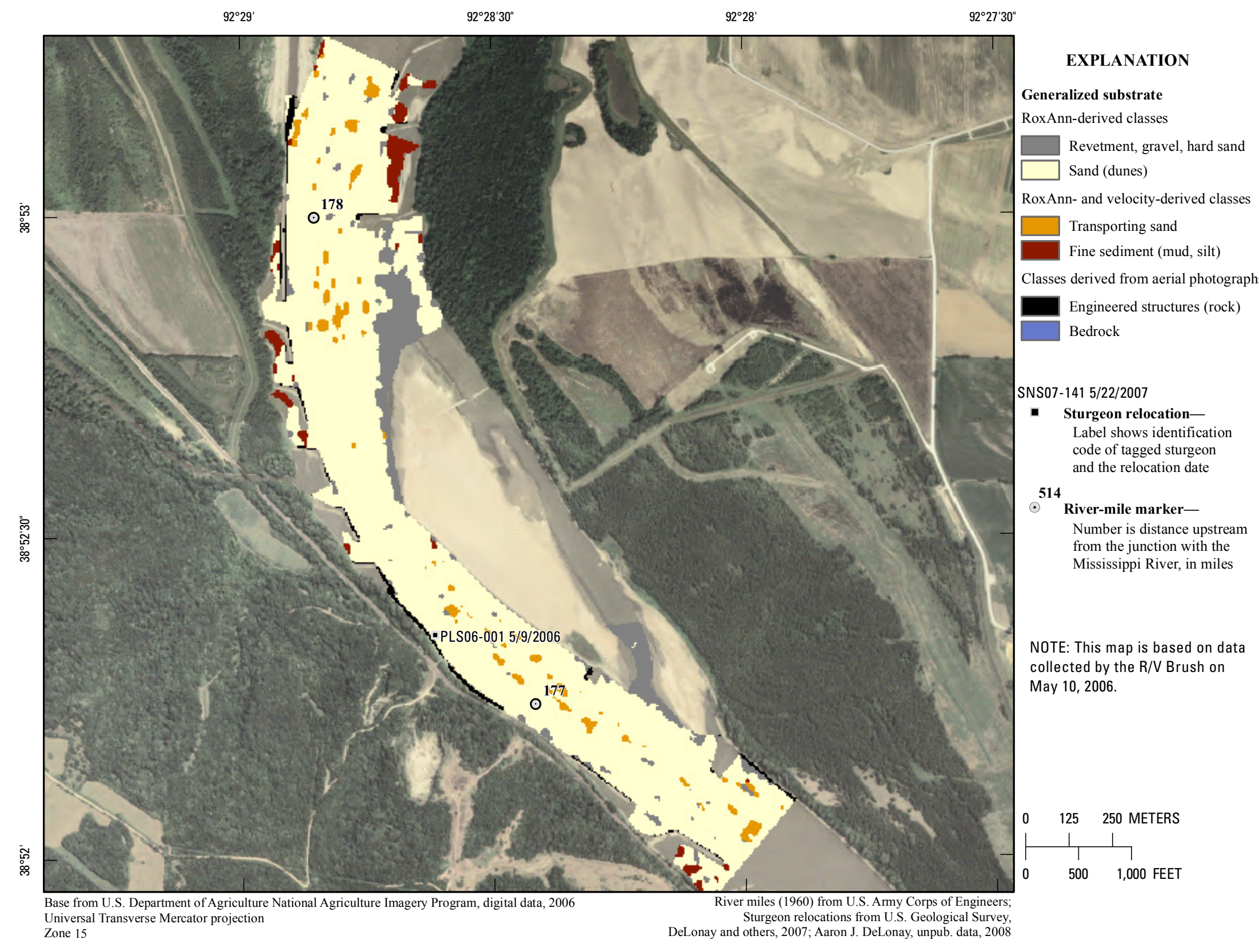

Figure 363. Map of generalized substrate based on data collected on May 10, 2006, in the vicinity of river mile 177 . 


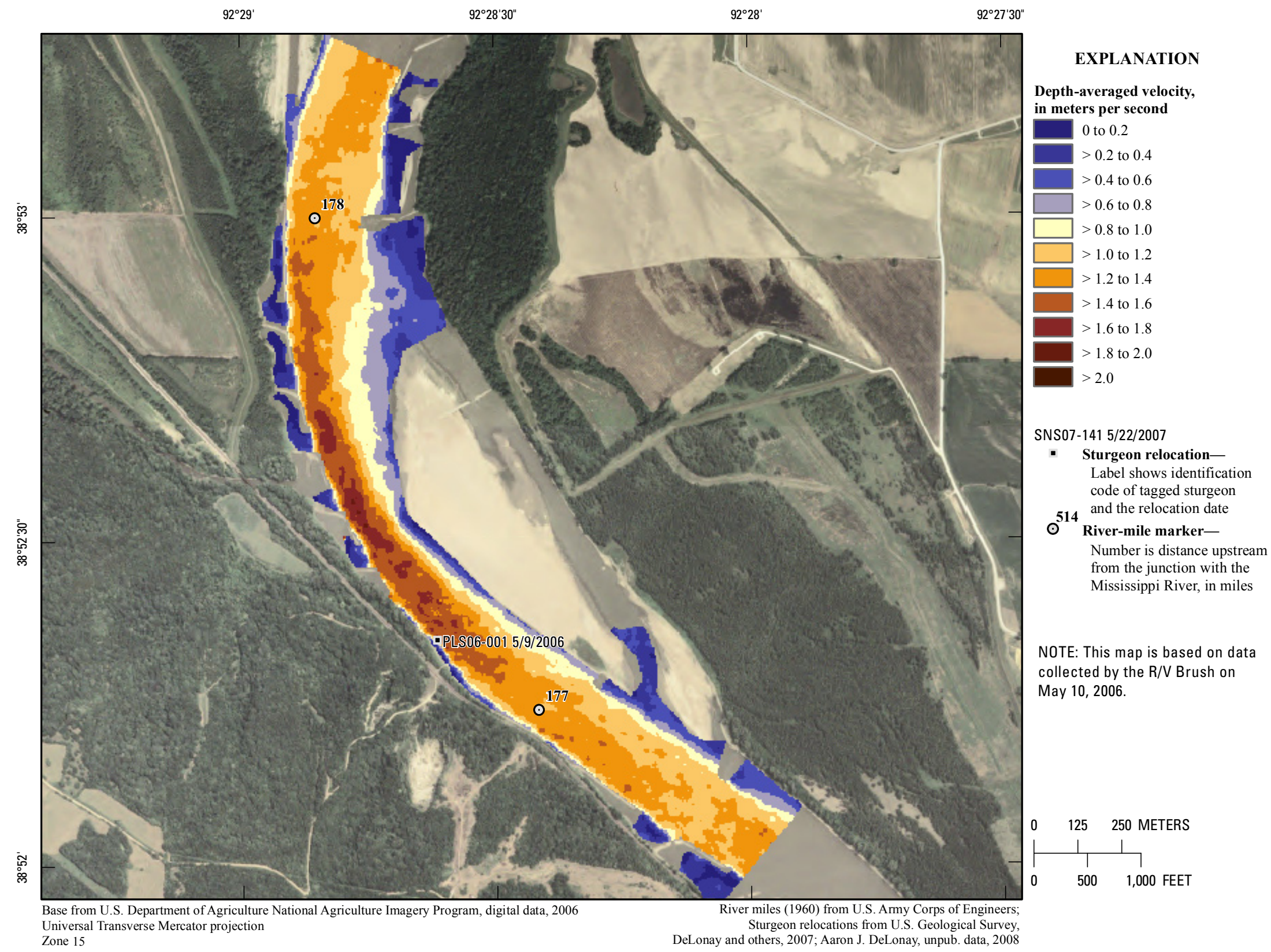

Figure 364. Map of depth-averaged velocity based on data collected on May 10, 2006, in the vicinity of river mile 177 . 


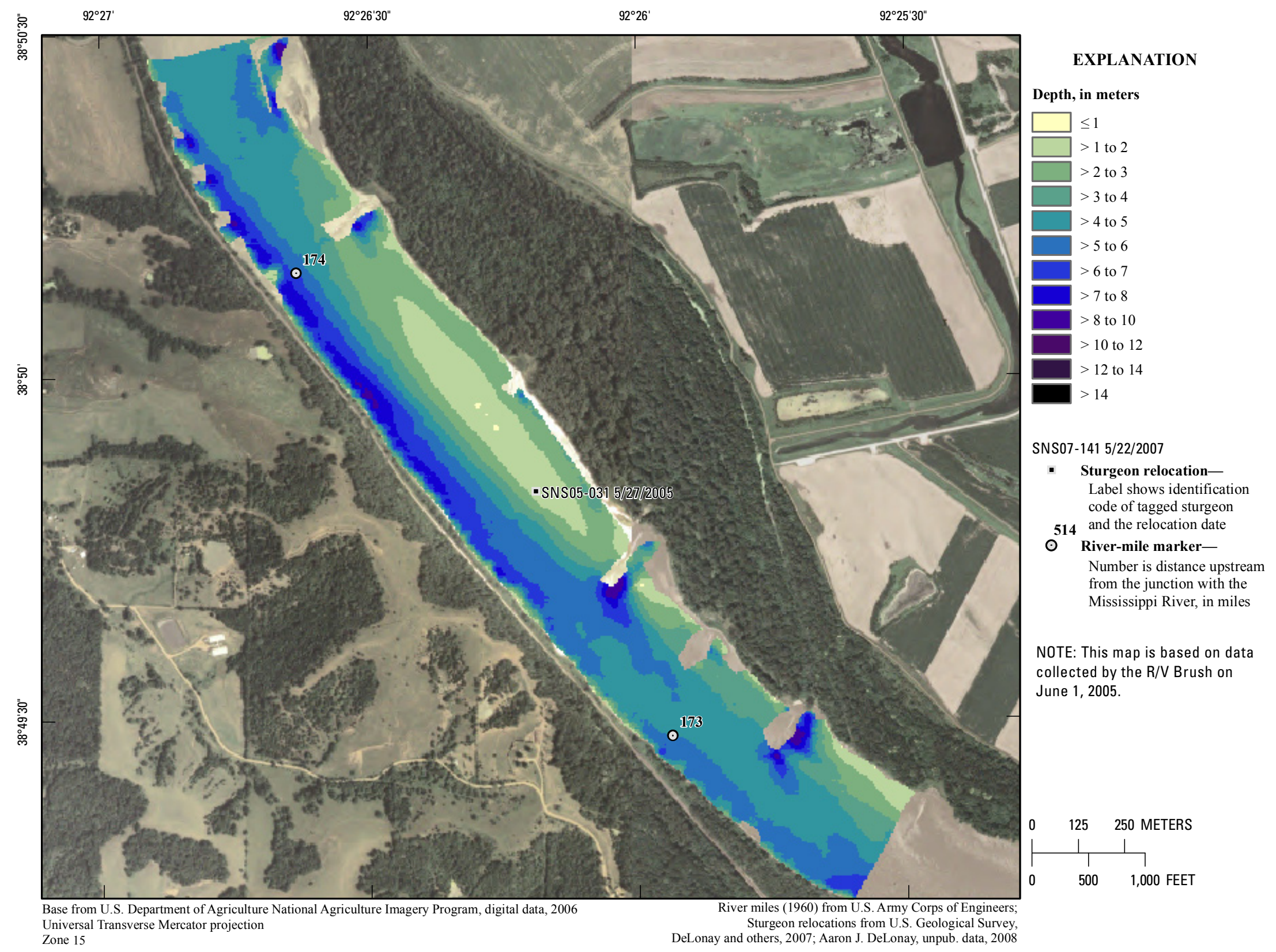

Figure 365. Map of depth based on data collected on June 1, 2005, in the vicinity of river mile 174 . 


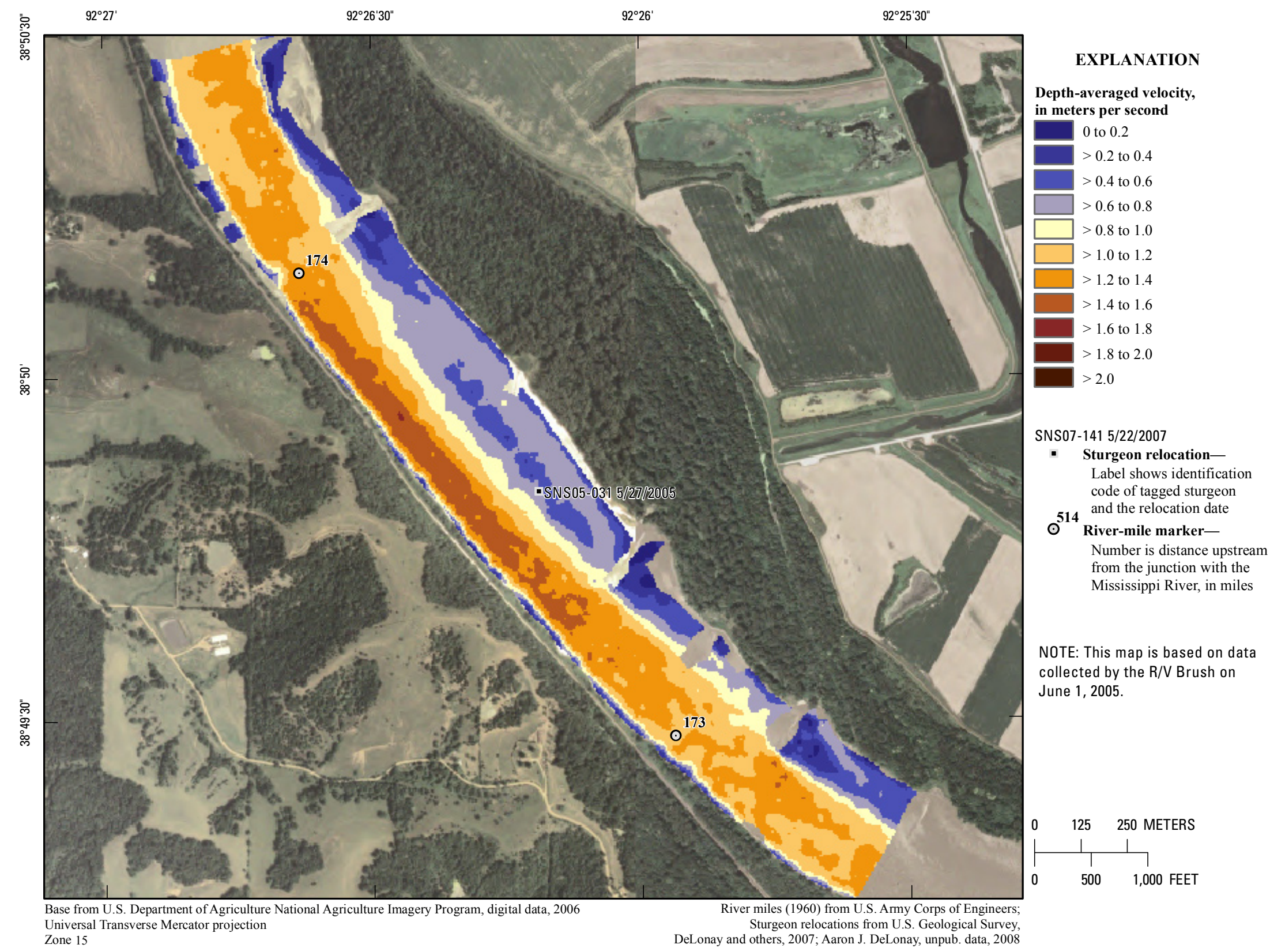

Figure 366. Map of depth-averaged velocity based on data collected on June 1, 2005, in the vicinity of river mile 174 . 


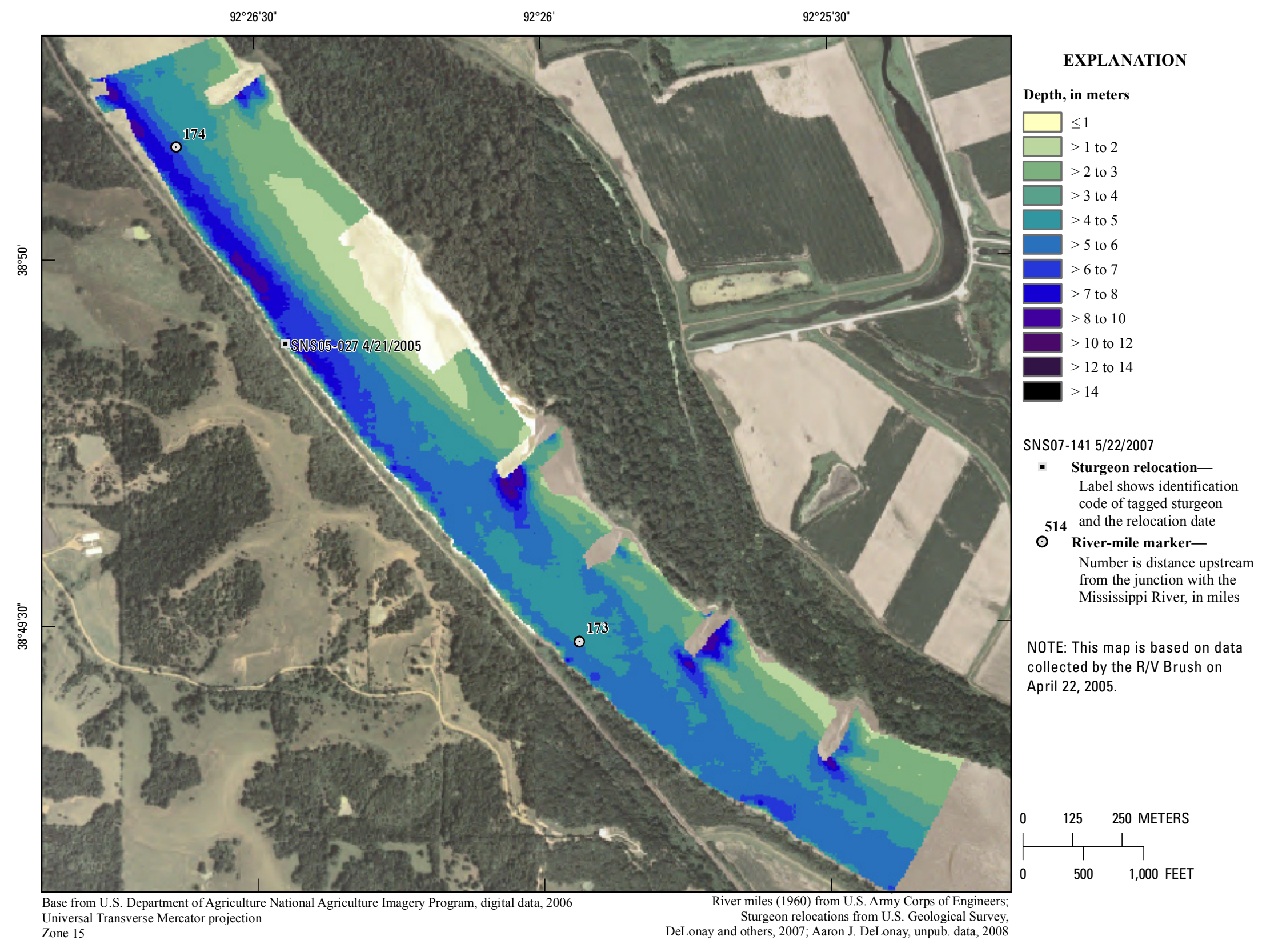

Figure 367. Map of depth based on data collected on April 22, 2005, in the vicinity of river mile 173. 


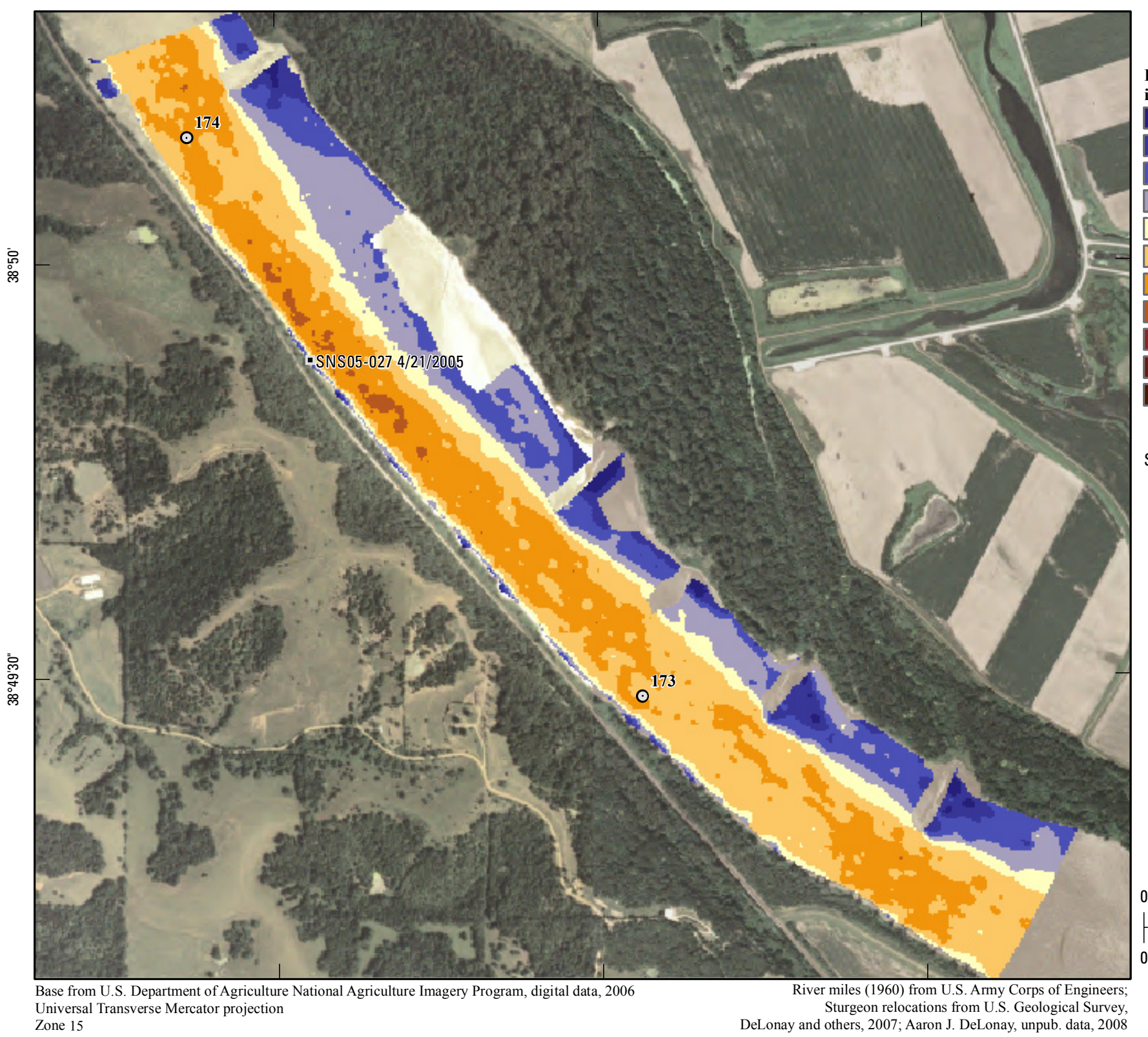

EXPLANATION

Depth-averaged velocity,

in meters per second

0 to 0.2

$>0.2$ to 0.4

$>0.4$ to 0.6

$>0.6$ to 0.8

$>0.8$ to 1.0

$>1.0$ to 1.2

$>1.2$ to 1.4

$>1.4$ to 1.6

$>1.6$ to 1.8

$>1.8$ to 2.0

$>2.0$

\section{SNS07-141 5/22/2007}

Sturgeon relocationLabel shows identification code of tagged sturgeo

$\mathbf{5 1 4}$ and the relocation date

Number is distance upstream

from the junction with the Mississippi River, in miles

NOTE: This map is based on data

collected by the $\mathrm{R} / \mathrm{V}$ Brush on Ap ril 22, 2005.

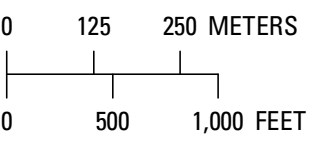




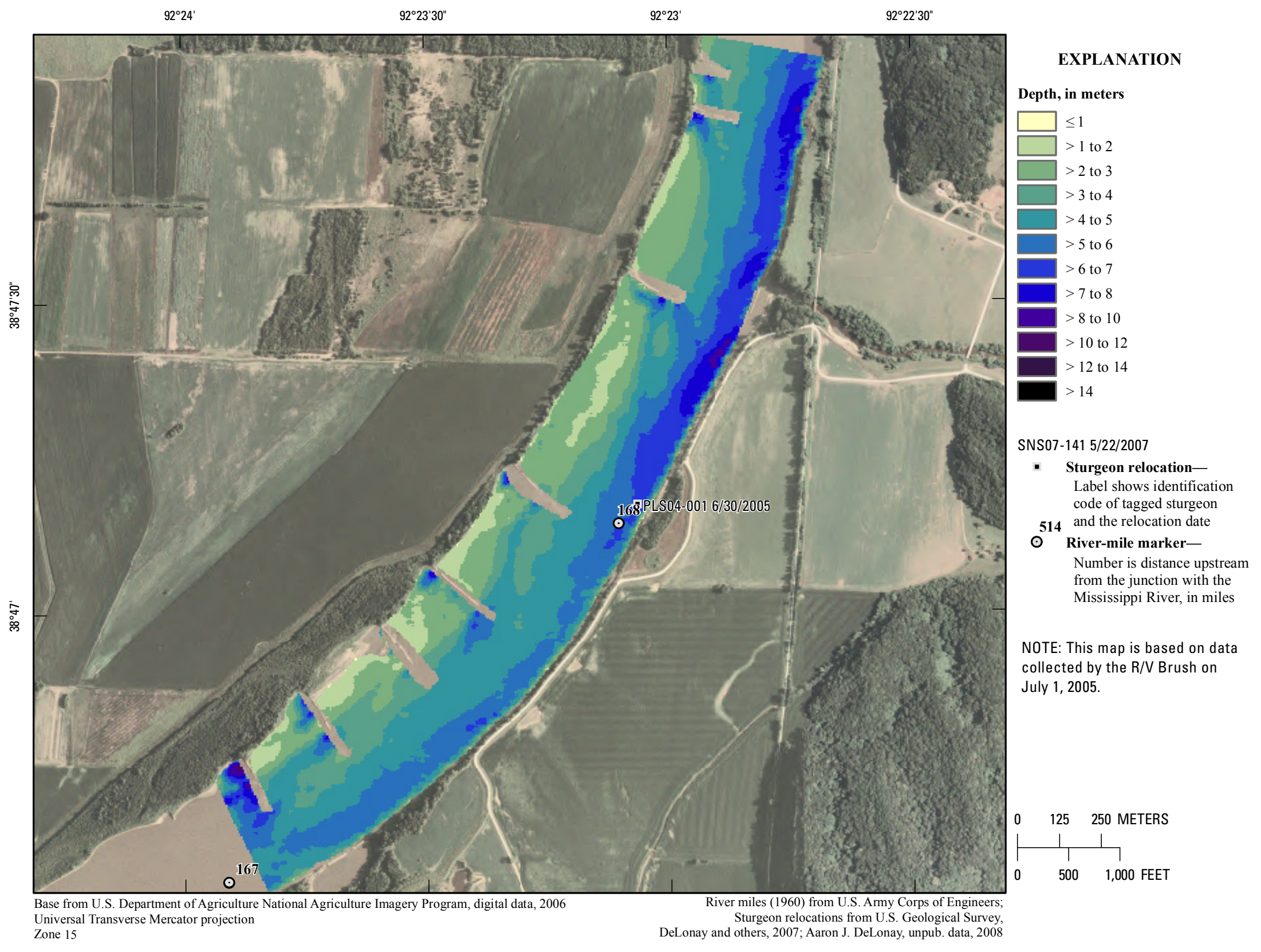

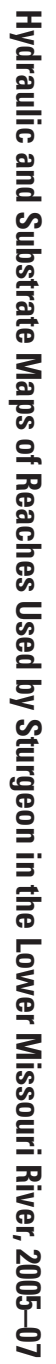

Figure 369. Map of depth based on data collected on July 1, 2005, in the vicinity of river mile 168 . 


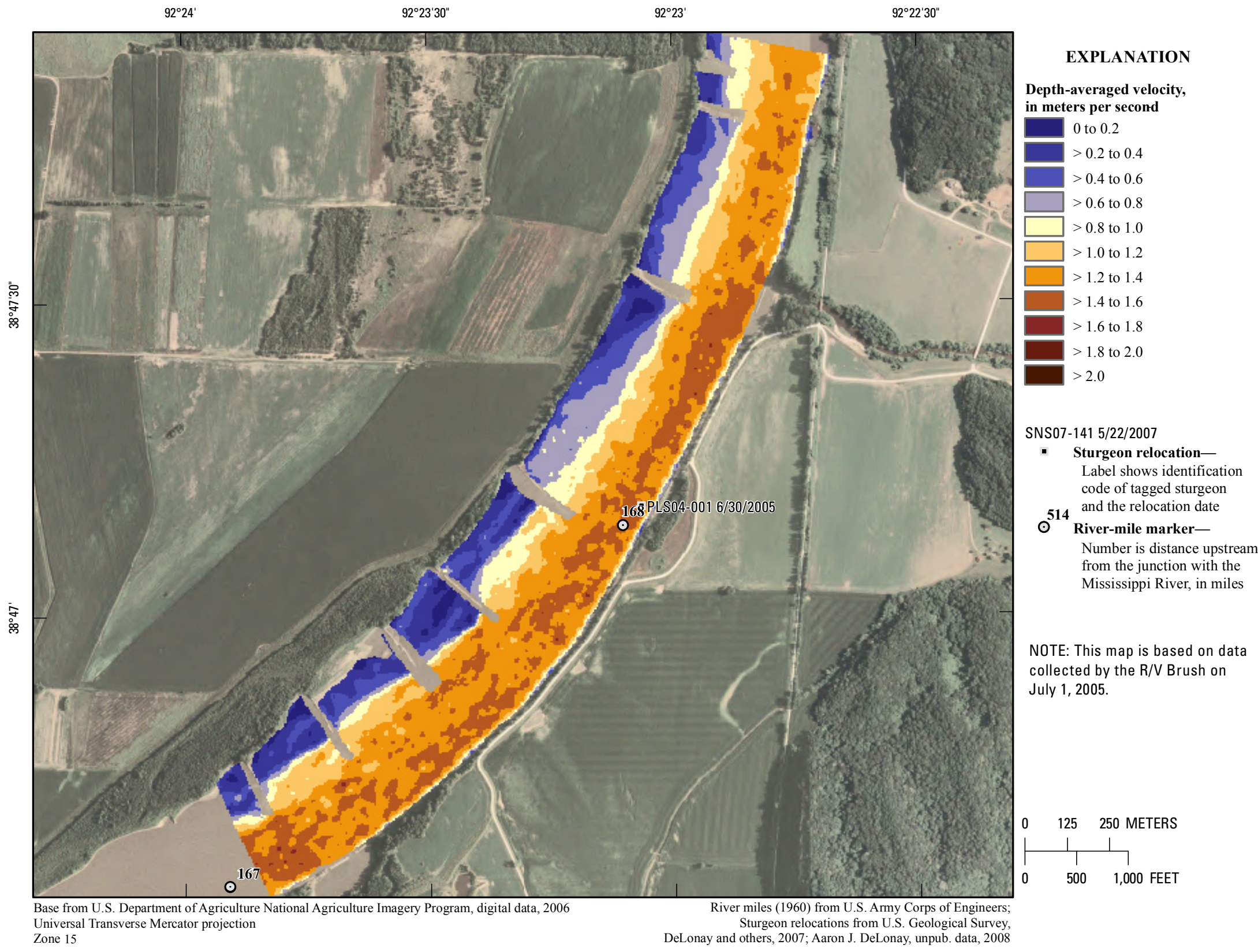

Figure 370. Map of depth-averaged velocity based on data collected on July 1, 2005, in the vicinity of river mile 168. 


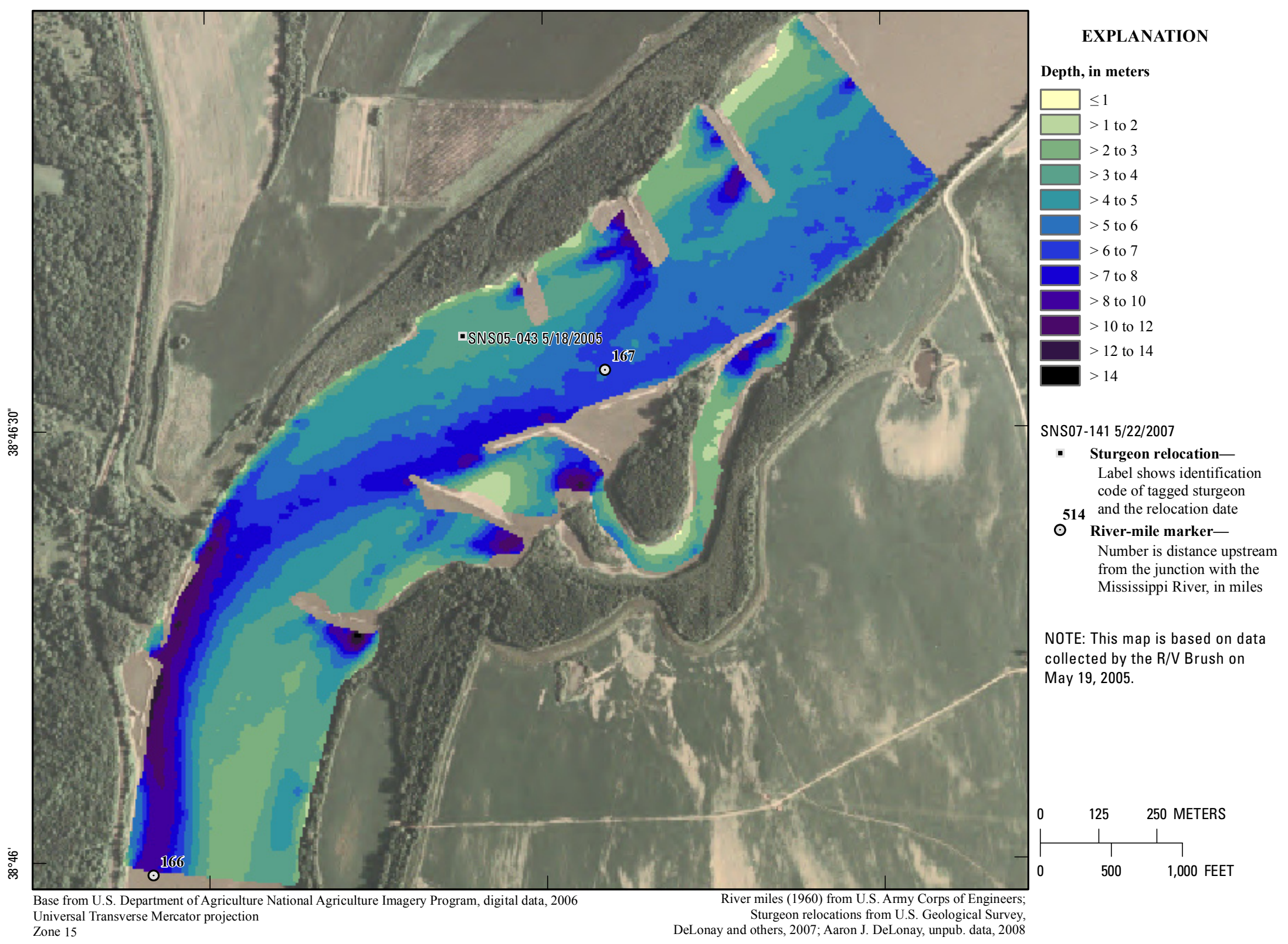

Figure 371. Map of depth based on data collected on May 19, 2005, in the vicinity of river mile 167. 


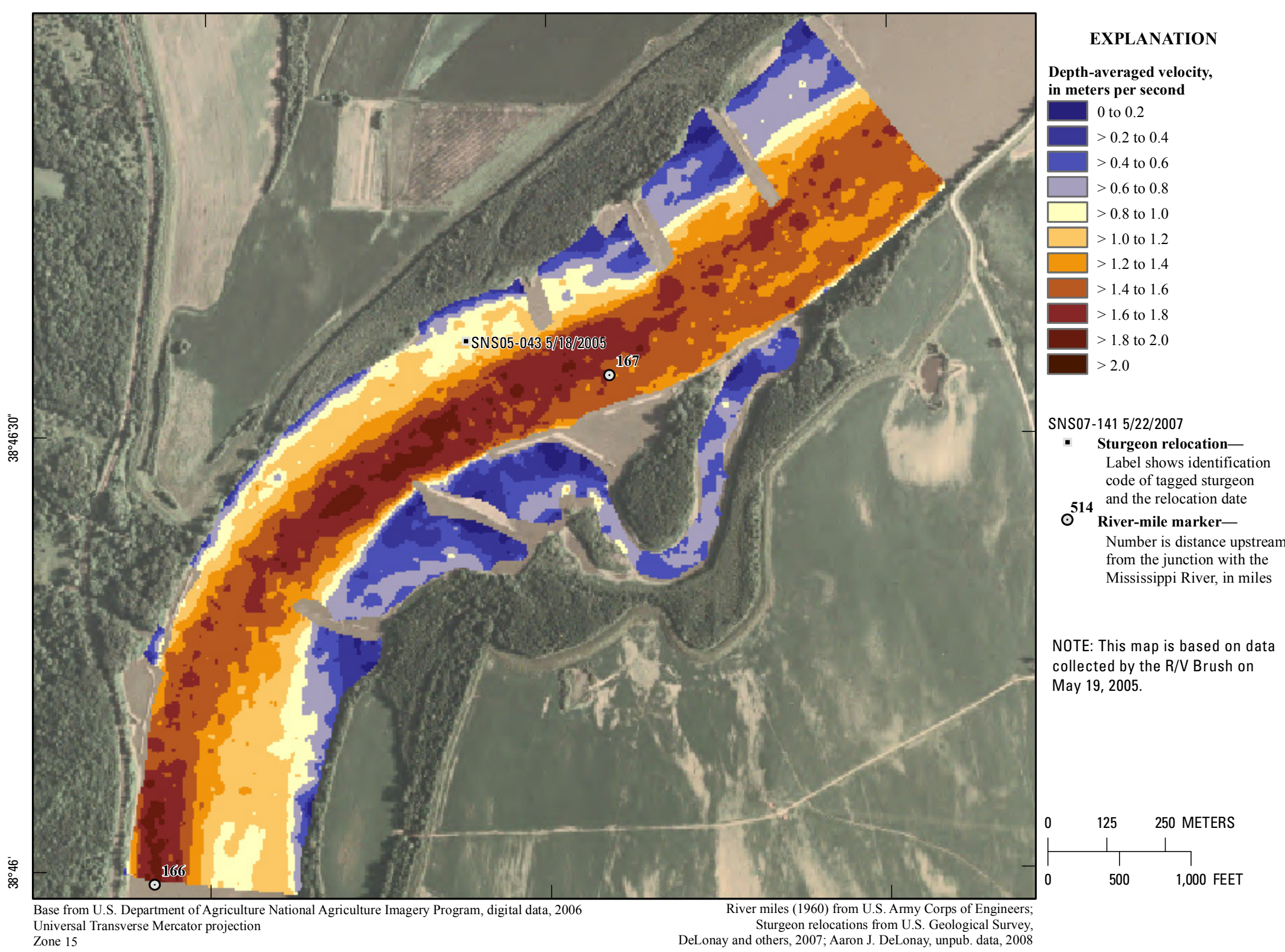




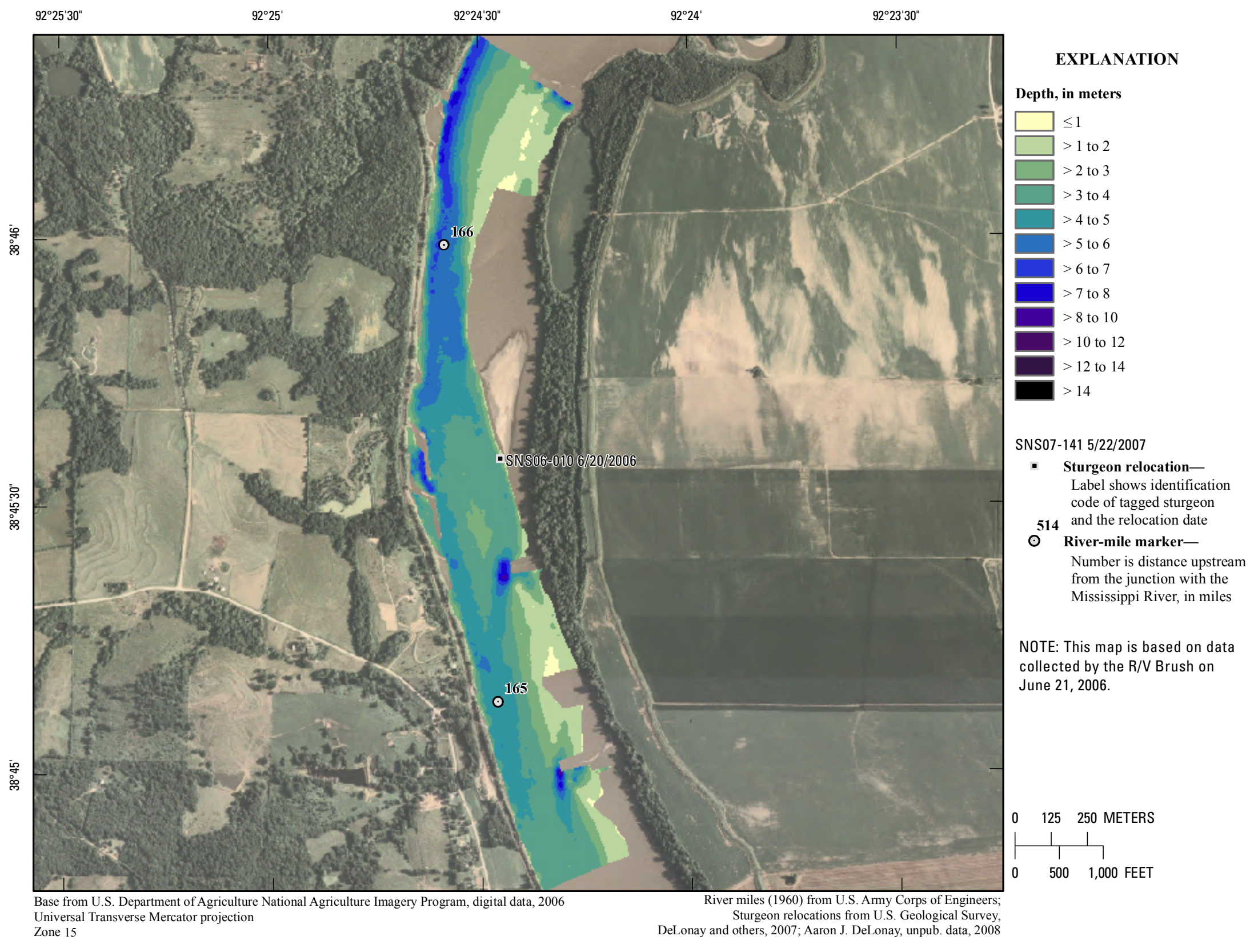

Figure 373. Map of depth based on data collected on June 21, 2006, in the vicinity of river mile 166 . 


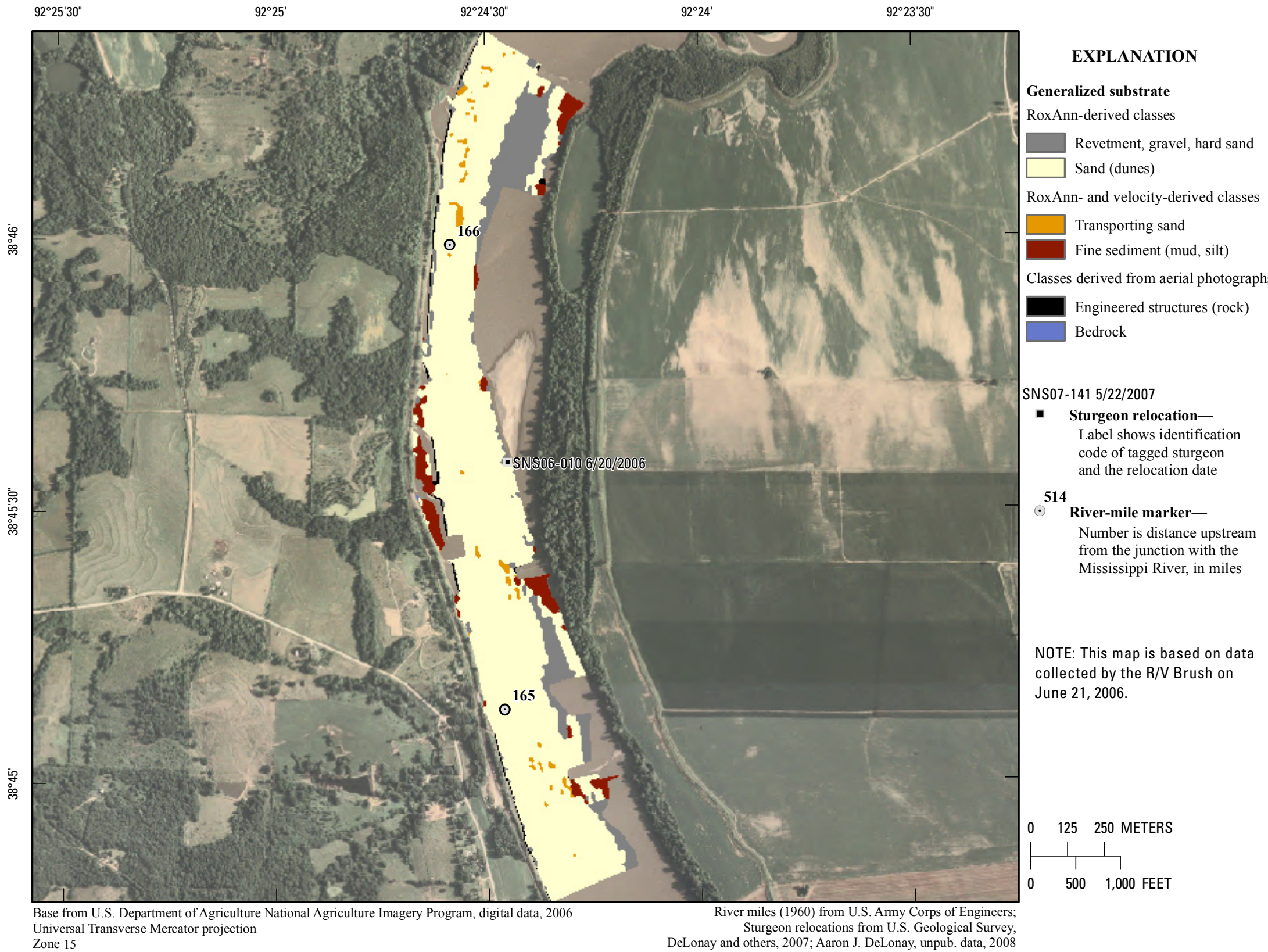

Figure 374. Map of generalized substrate based on data collected on June 21, 2006, in the vicinity of river mile 166 . 


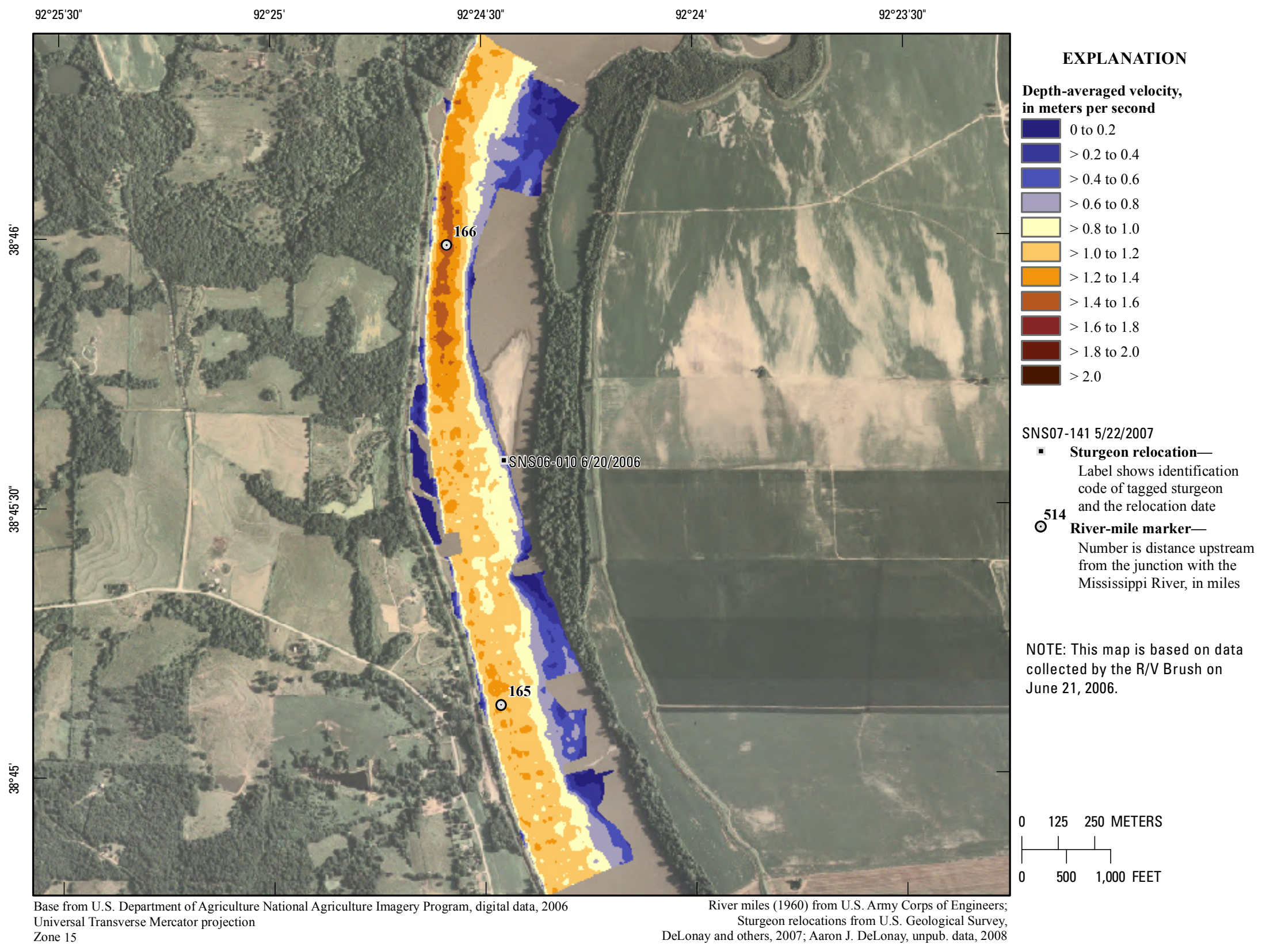

Figure 375. Map of depth-averaged velocity based on data collected on June 21, 2006, in the vicinity of river mile 166. 


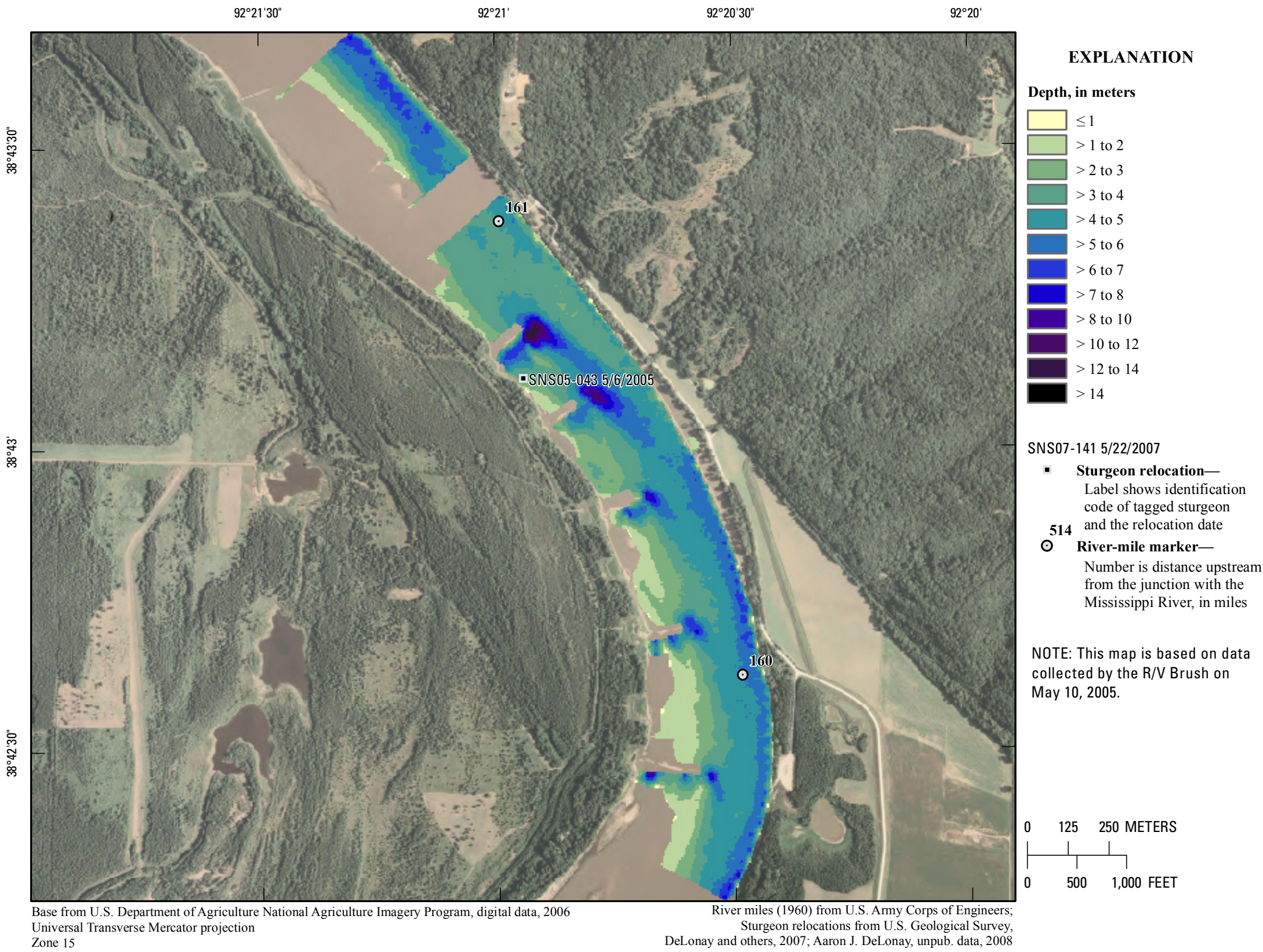

Figure 376. Map of depth based on data collected on May 10, 2005, in the vicinity of river mile 161. 


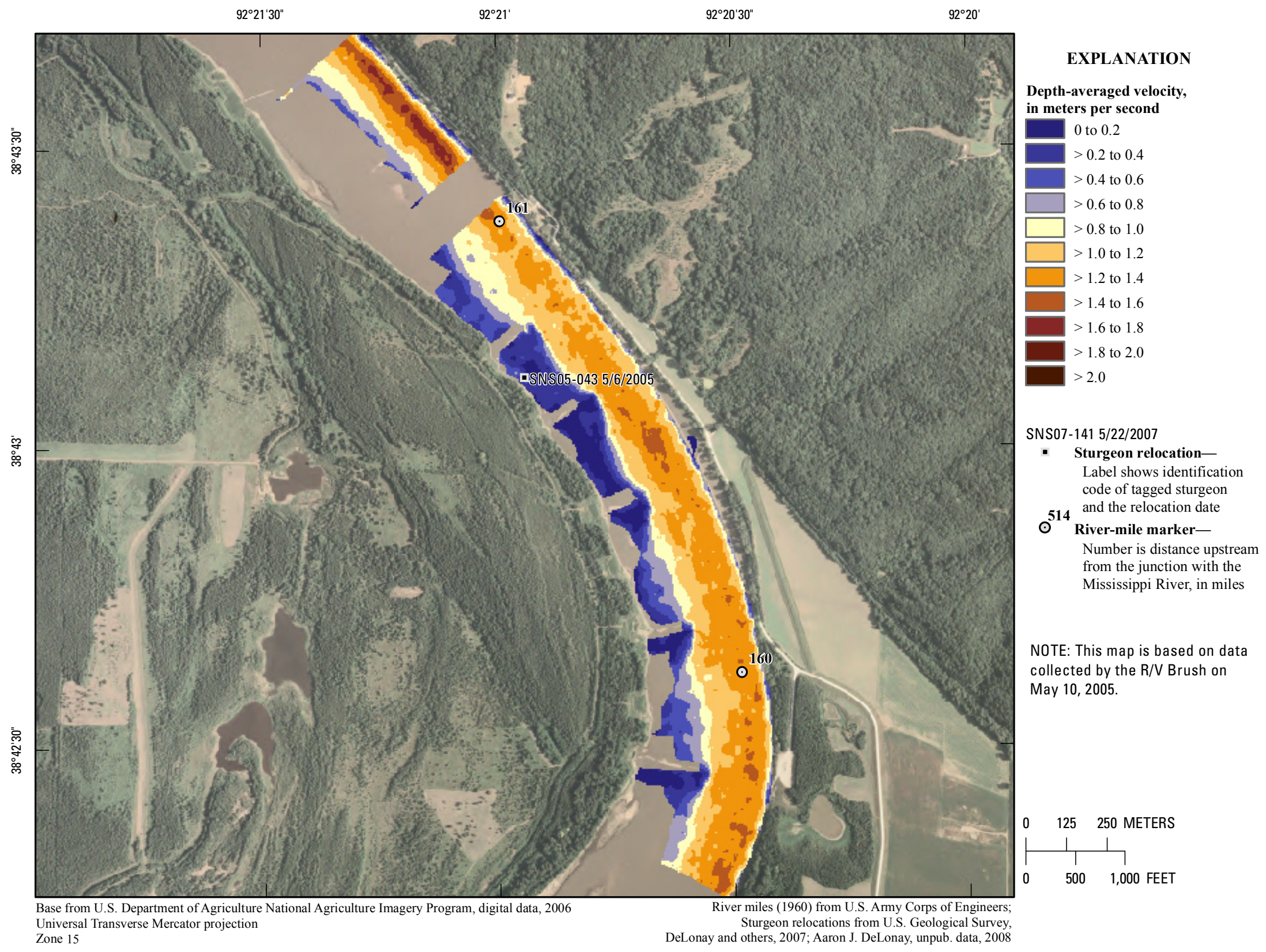

Figure 377. Map of depth-averaged velocity based on data collected on May 10, 2005, in the vicinity of river mile 161 . 


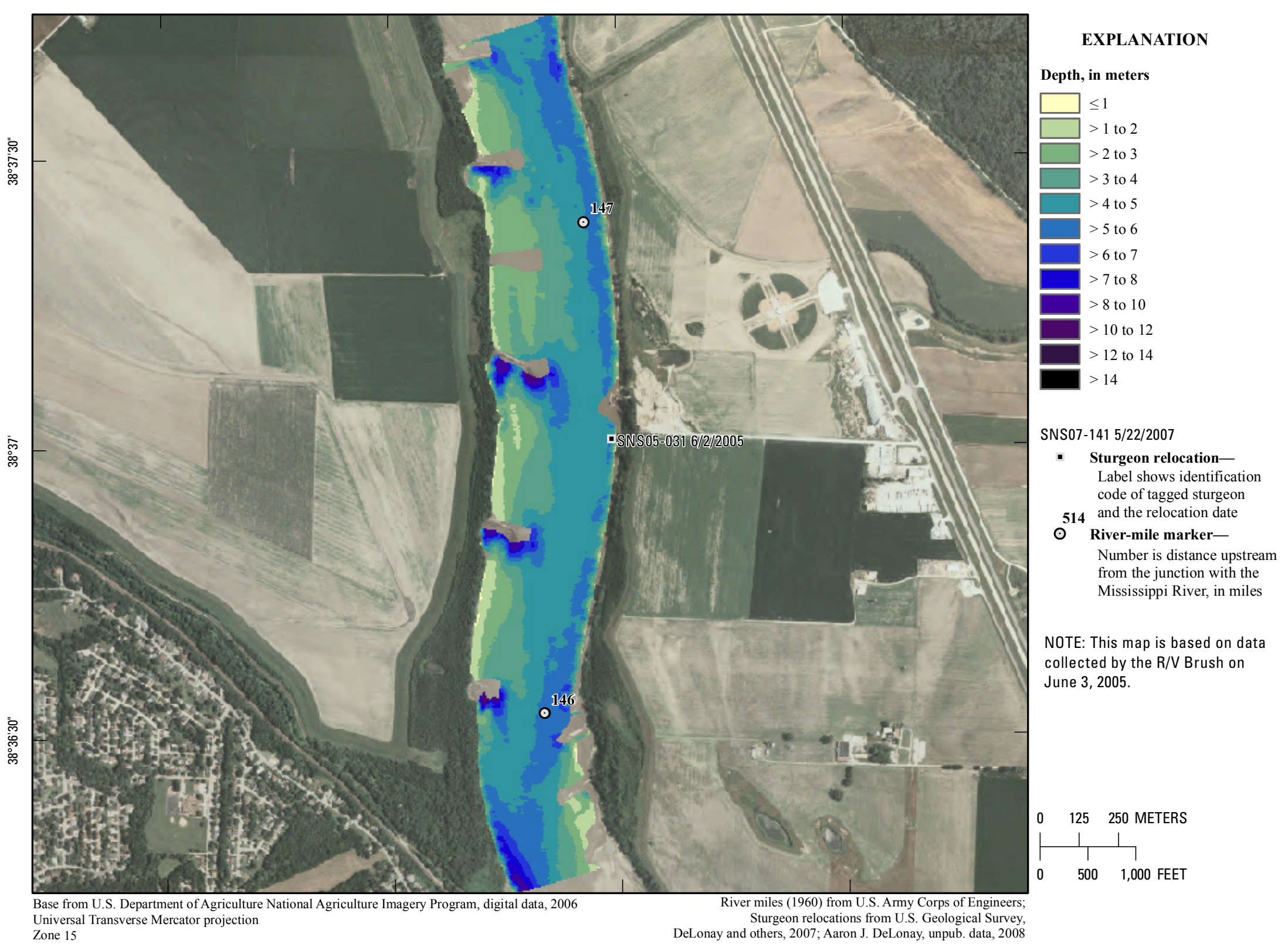




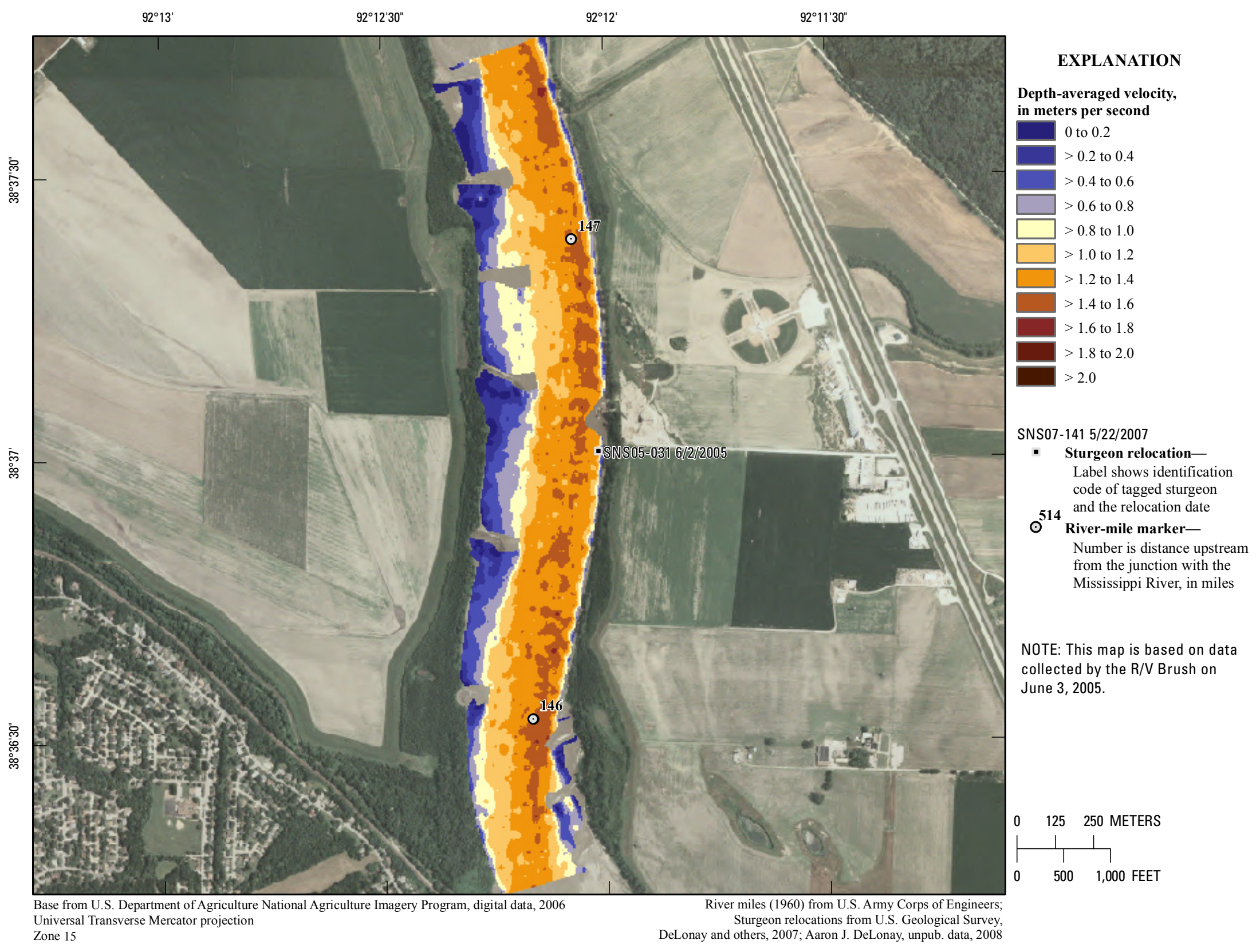

Figure 379. Map of depth-averaged velocity based on data collected on June 3, 2005, in the vicinity of river mile 147 . 


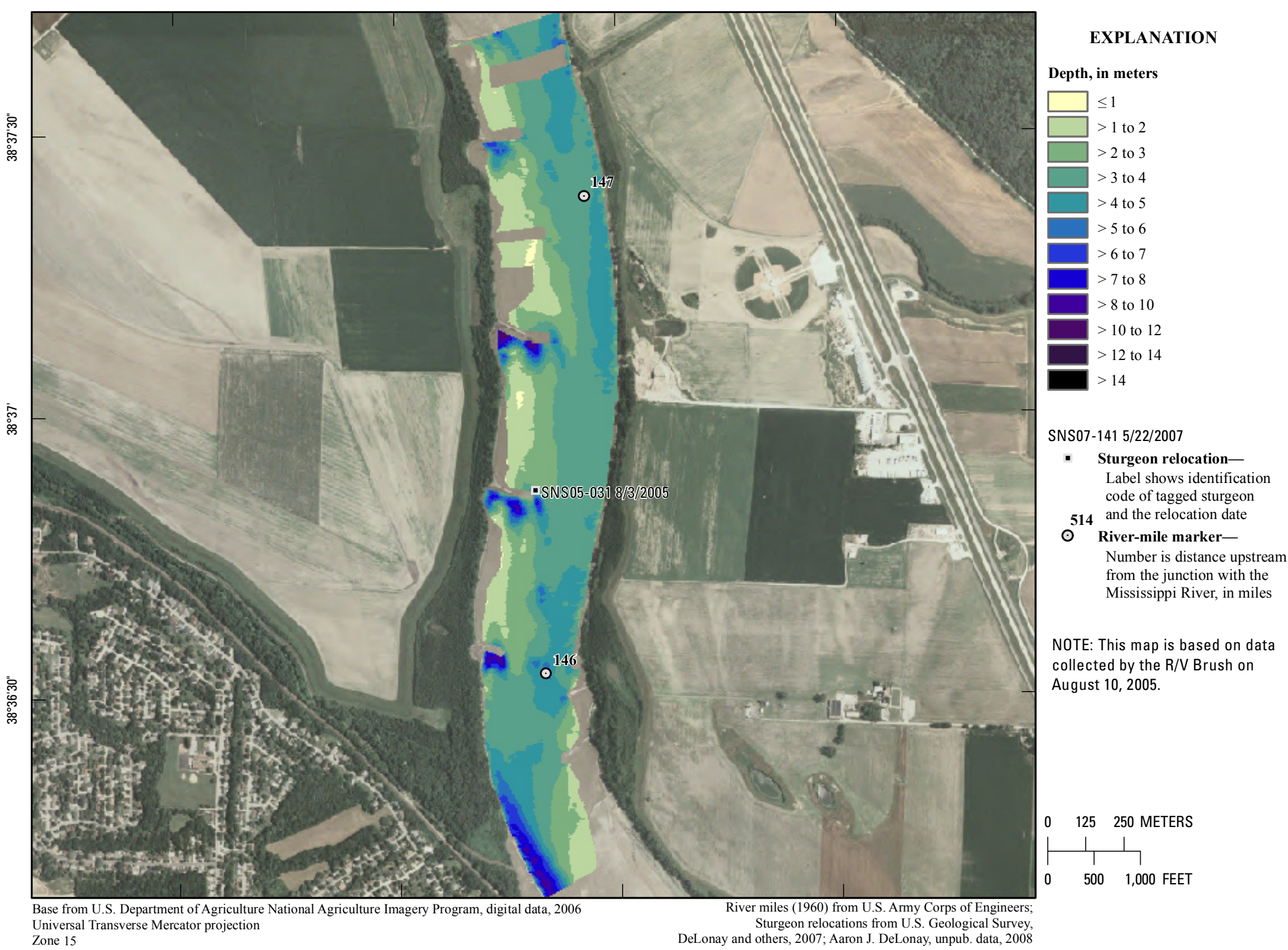
Universal Transverse Mercator projection Sturgeon relocations from U.S. Geological Survey,
DeLonay and others, 2007; Aaron J. DeLonay, unpub. data, 2008 


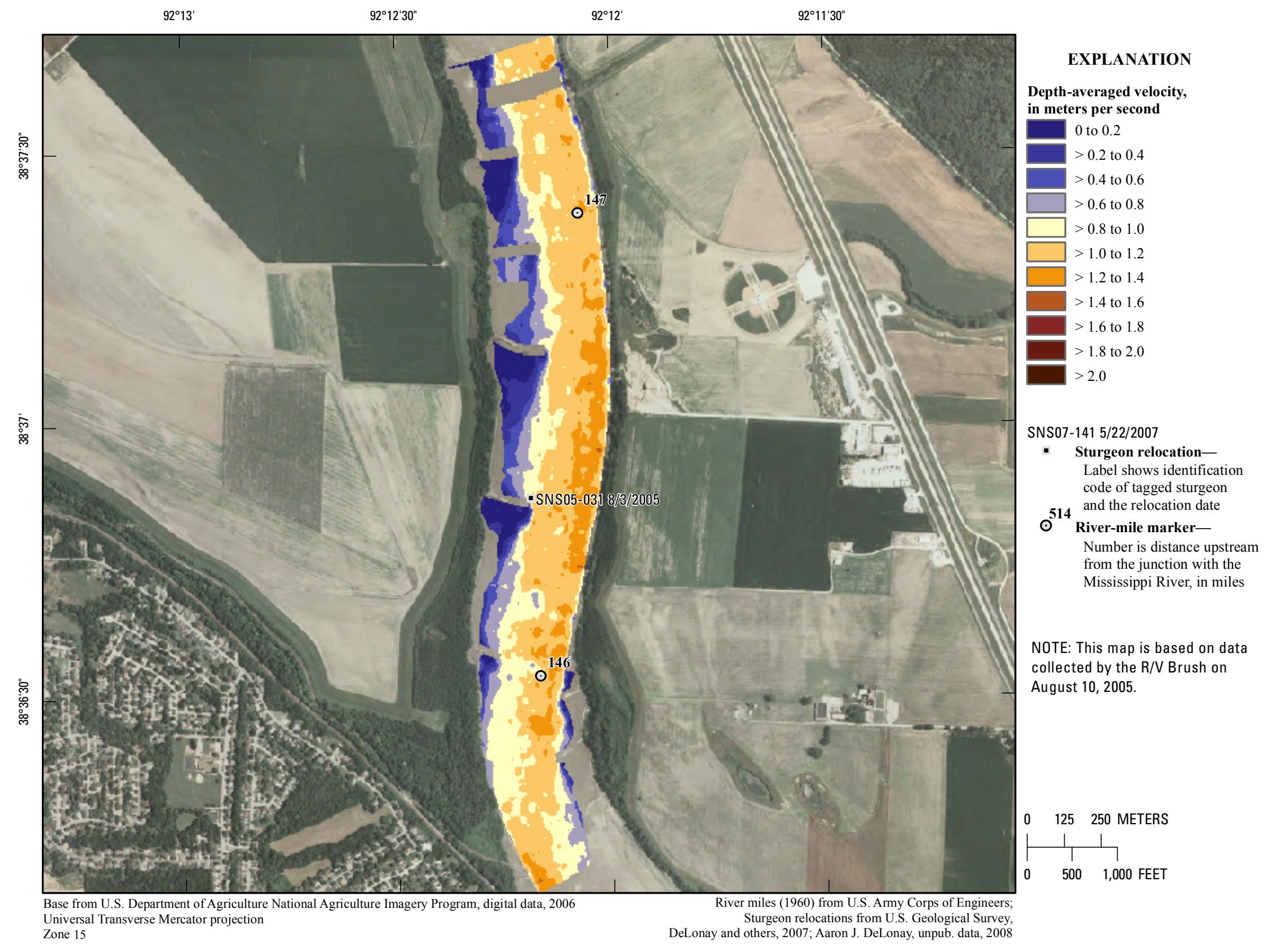

Figure 381. Map of depth-averaged velocity based on data collected on August 10, 2005, in the vicinity of river mile 147. 


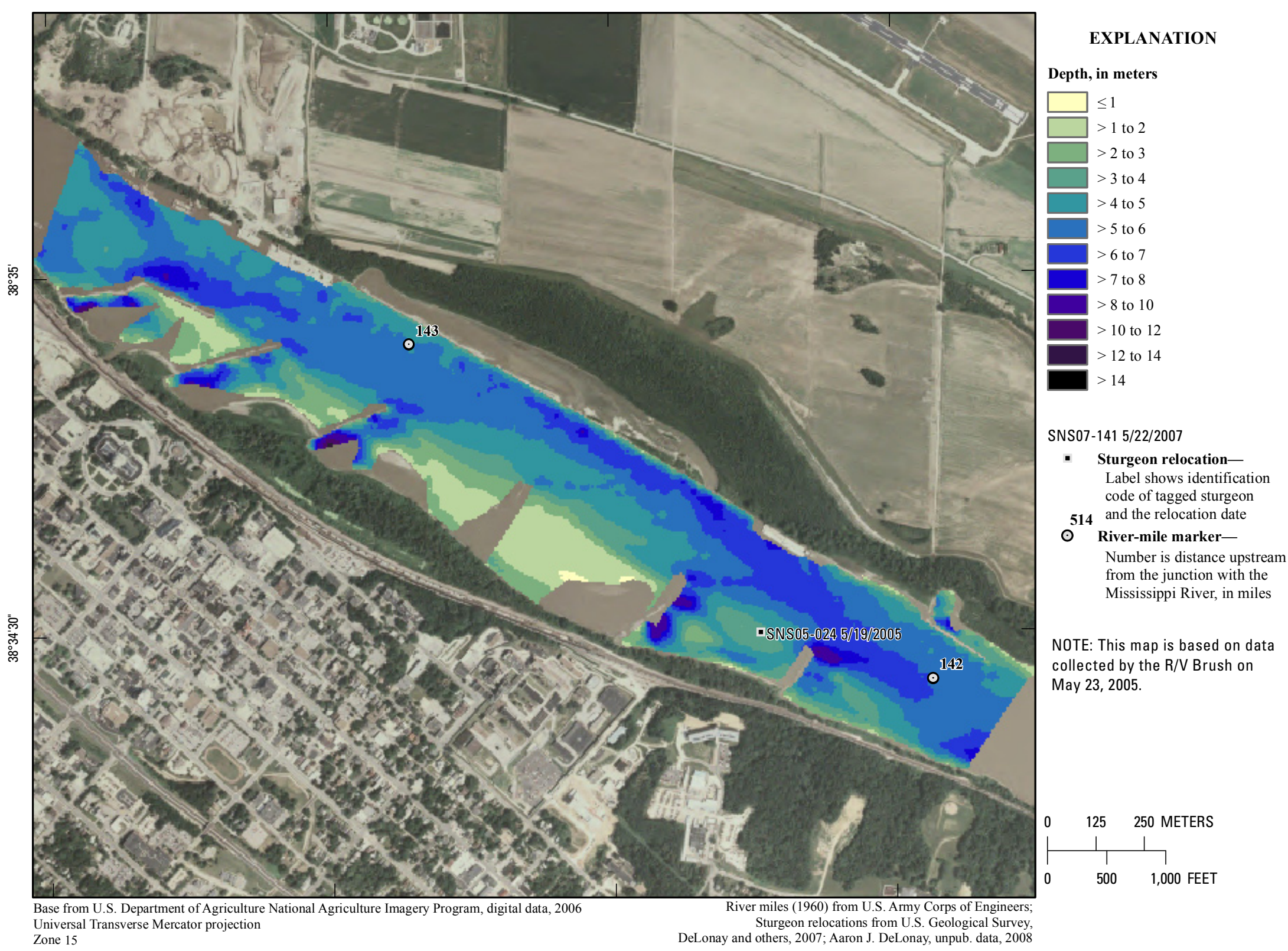
Universal Transverse Mercator projection

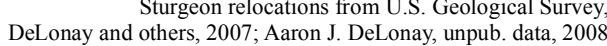




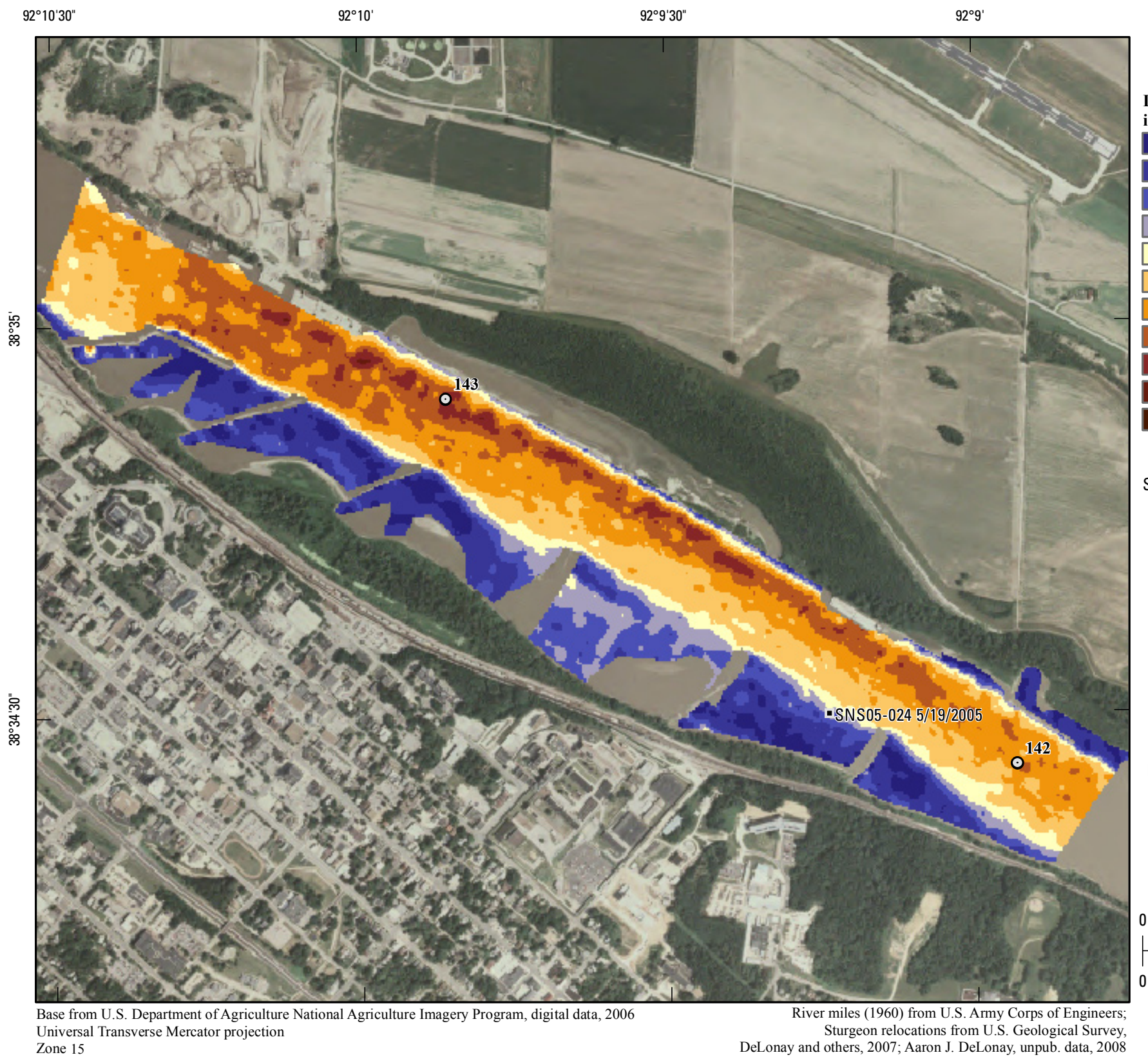

EXPLANATION

Depth-averaged velocity,

in meters per second

0 to 0.2

$>0.2$ to 0.4

$>0.4$ to 0.6

$>0.6$ to 0.8

$>0.8$ to 1.0

$>1.0$ to 1.2

$>1.2$ to 1.4

$>1.4$ to 1.6

$>1.6$ to 1.8

$>1.8$ to 2.0

$>2.0$

SNS07-141 5/22/2007

- Sturgeon relocationLabel shows identification code of tagged sturgeon 514 and the relocation date

River-mile marker-

Number is distance upstream from the junction with the Mississippi River, in miles

NOTE: This map is based on data

collected by the R/V Brush on

May 23, 2005.

Figure 383. Map of depth-averaged velocity based on data collected on May 23, 2005, in the vicinity of river mile 143. 


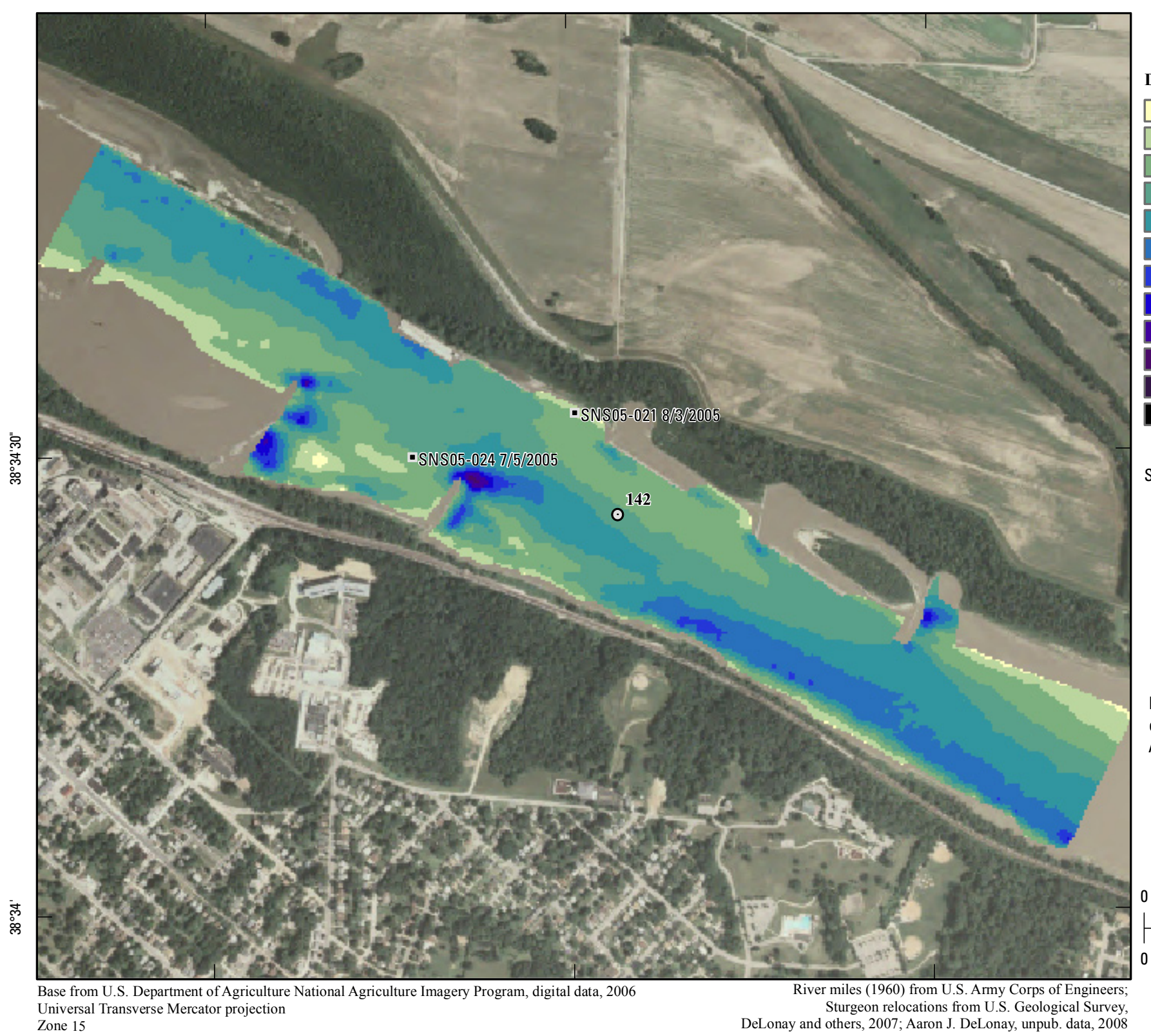

EXPLANATION

Depth, in meters

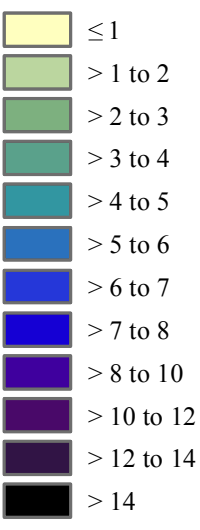

SNS07-141 5/22/2007

- Sturgeon relocationLabel shows identification code of tagged sturgeon 514
0

Number is distance upstream Number is distance upstrea
from the junction with the Mississippi River, in miles

NOTE: This map is based on data collected by the R/V Brush on August 9, 2005

\begin{tabular}{|c|c|}
\hline 125 & 250 METERS \\
\hline$\perp$ & \\
\hline 500 & 1,000 FEET \\
\hline
\end{tabular}
Universal Transverse Mercator projection

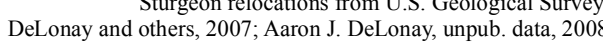




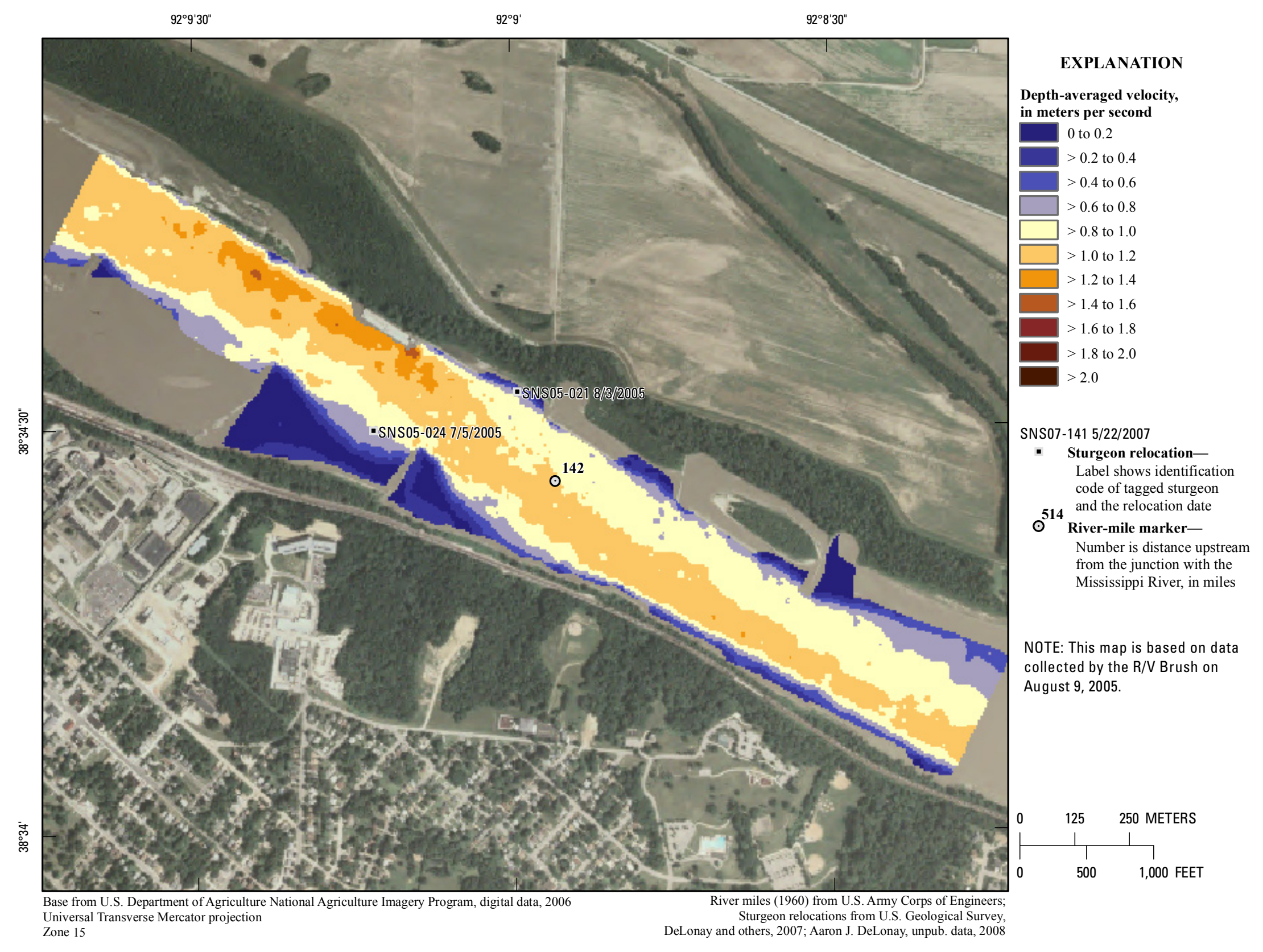

Figure 385. Map of depth-averaged velocity based on data collected on August 9, 2005, in the vicinity of river mile 142 . 


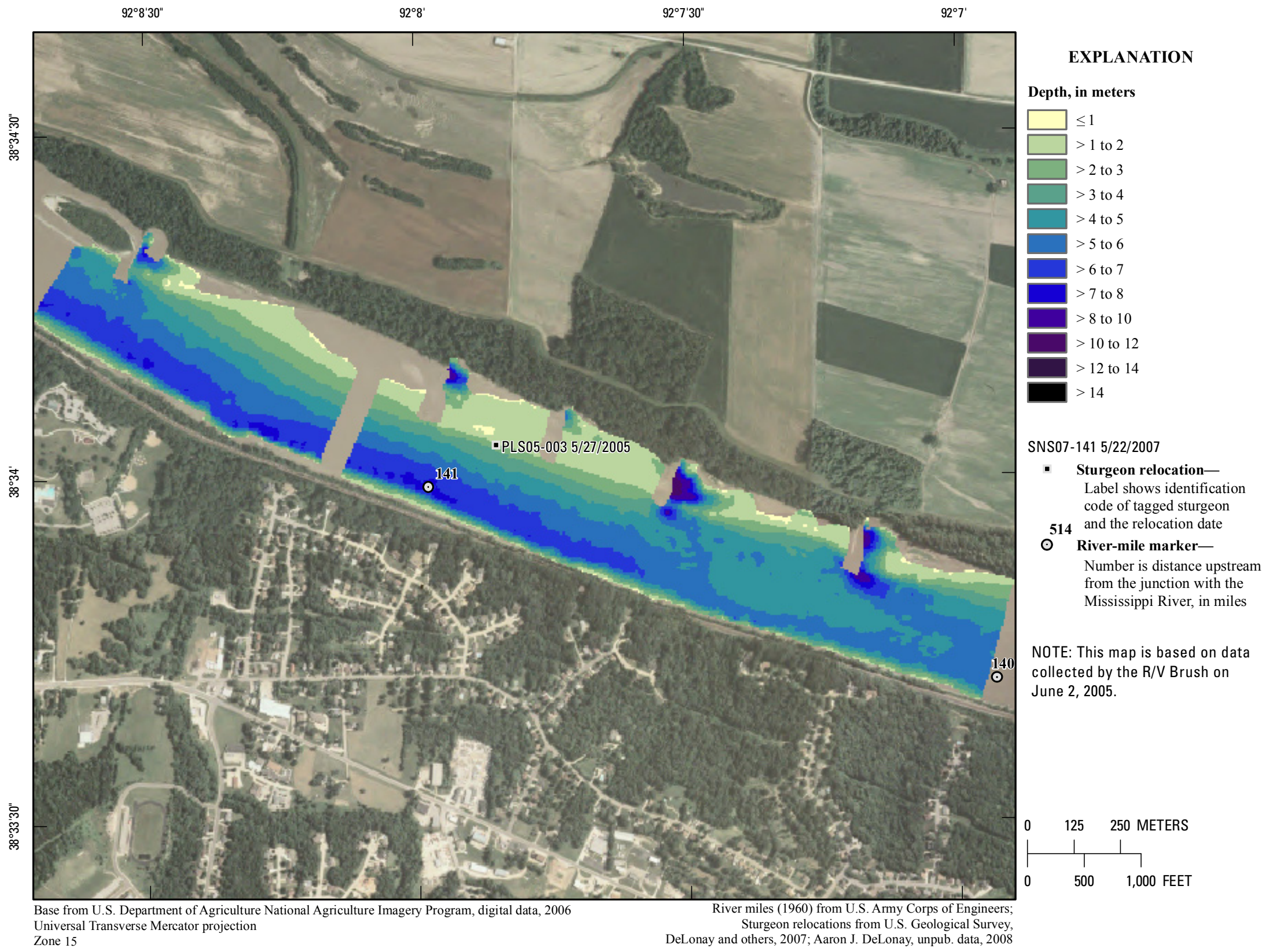

Figure 386. Map of depth based on data collected on June 2, 2005, in the vicinity of river mile 141 . 


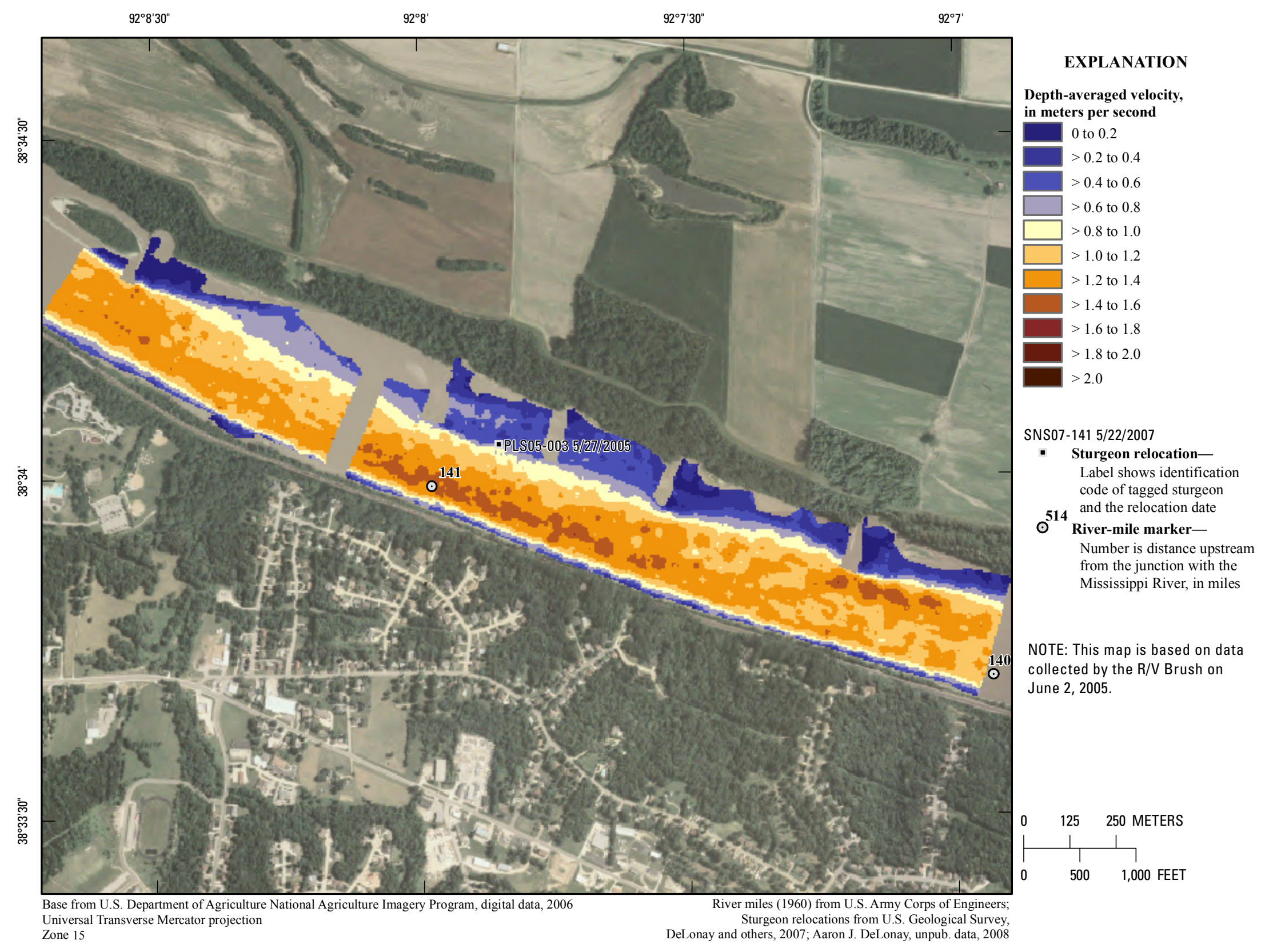

Figure 387. Map of depth-averaged velocity based on data collected on June 2, 2005, in the vicinity of river mile 141 . 


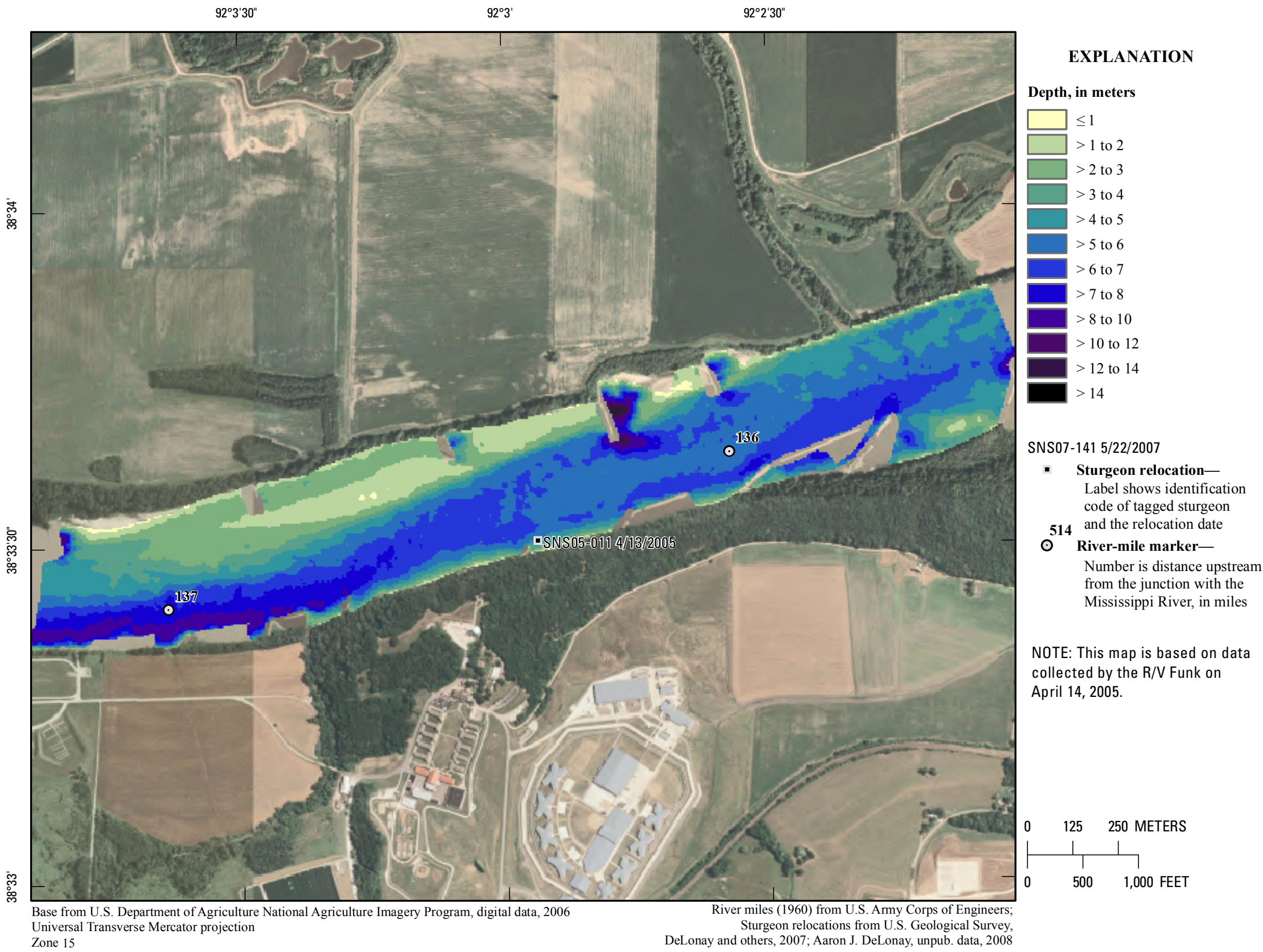

Figure 388. Map of depth based on data collected on April 14, 2005, in the vicinity of river mile 136. 


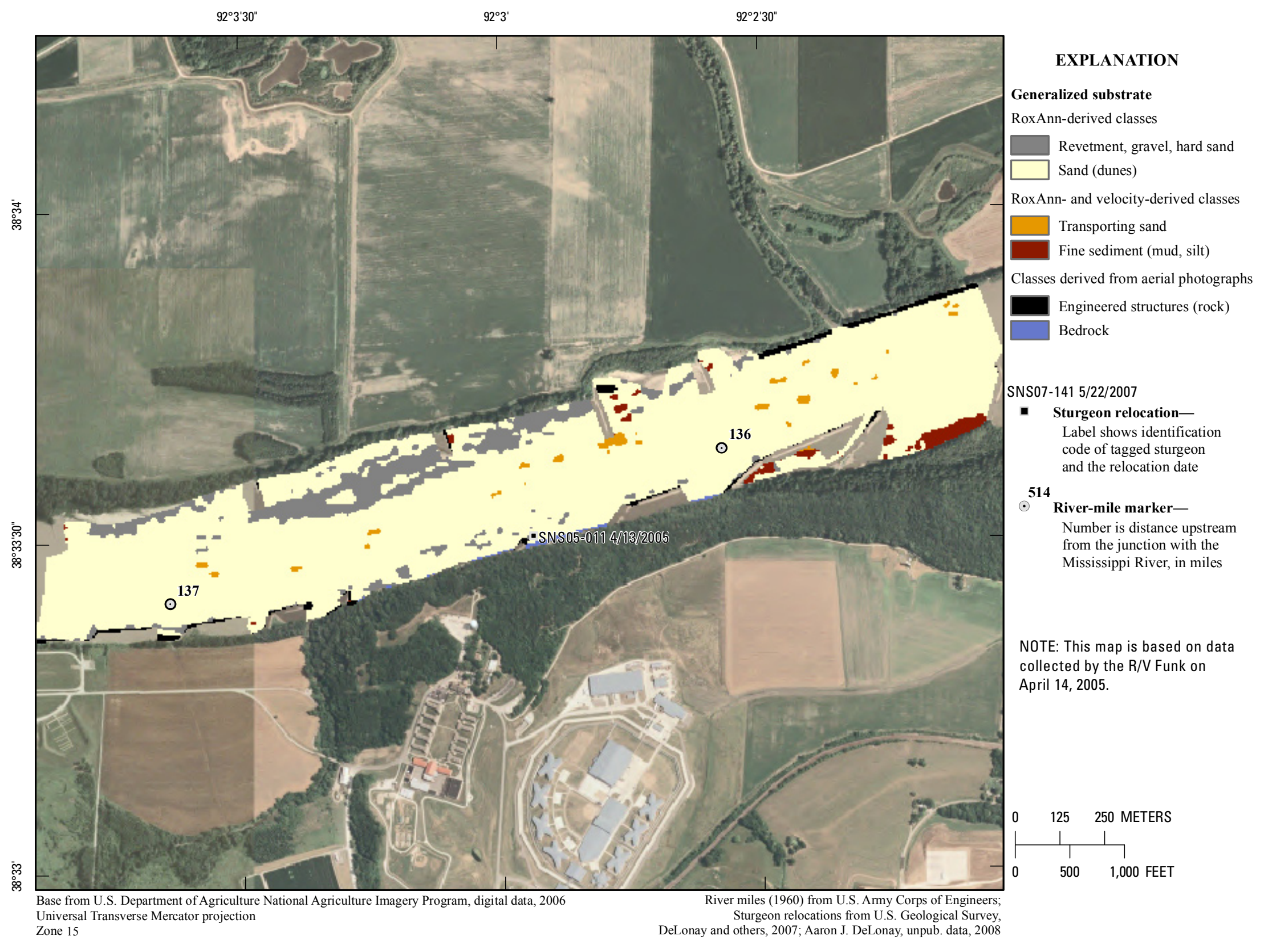

Figure 389. Map of generalized substrate based on data collected on April 14, 2005, in the vicinity of river mile 136 . 


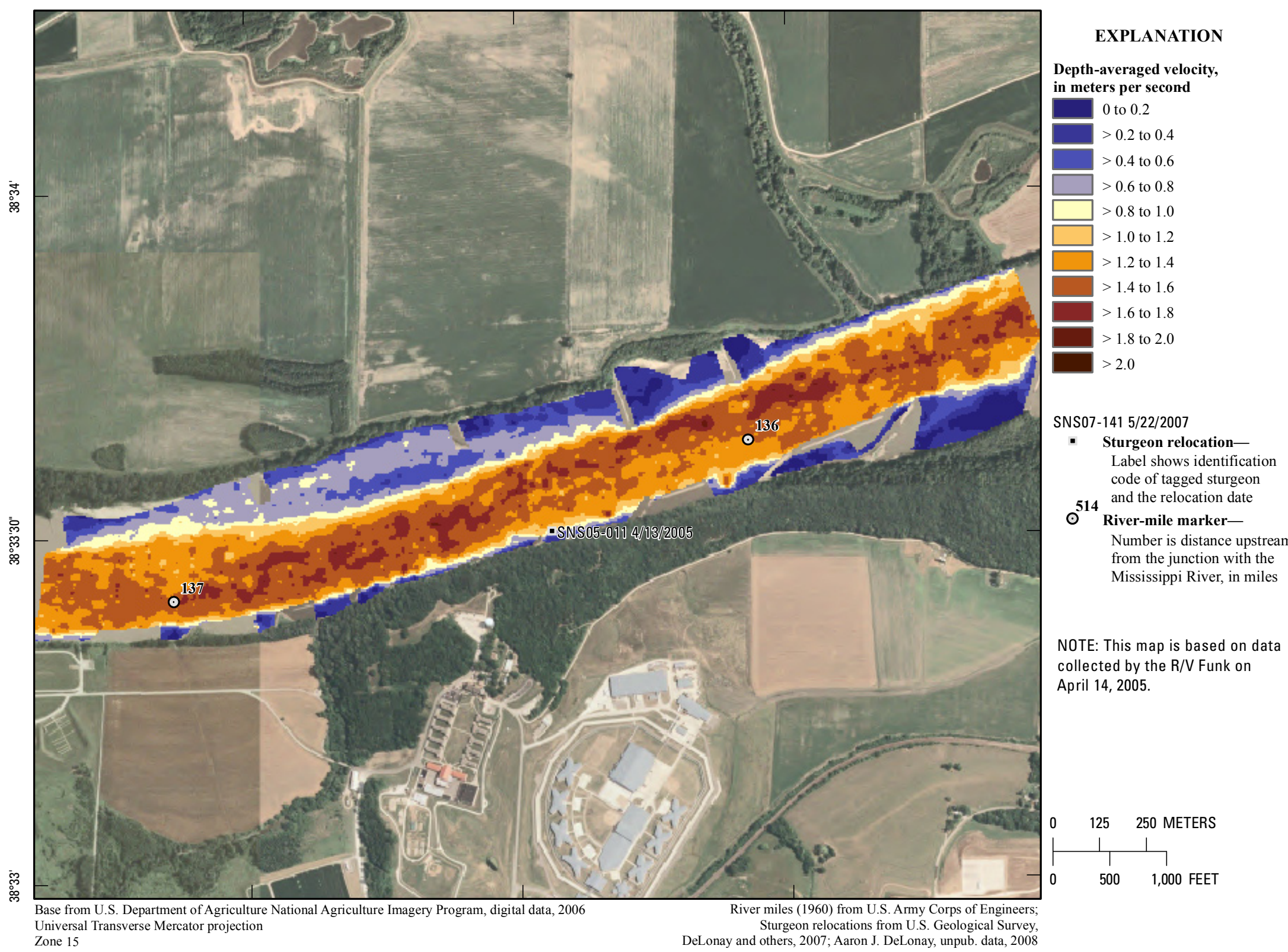
Universal Transverse Mercator projection Sturgeon relocations from U.S. Geological Survey,
DeLonay and others, 2007; Aaron J. DeLonay, unpub. data, 2008 


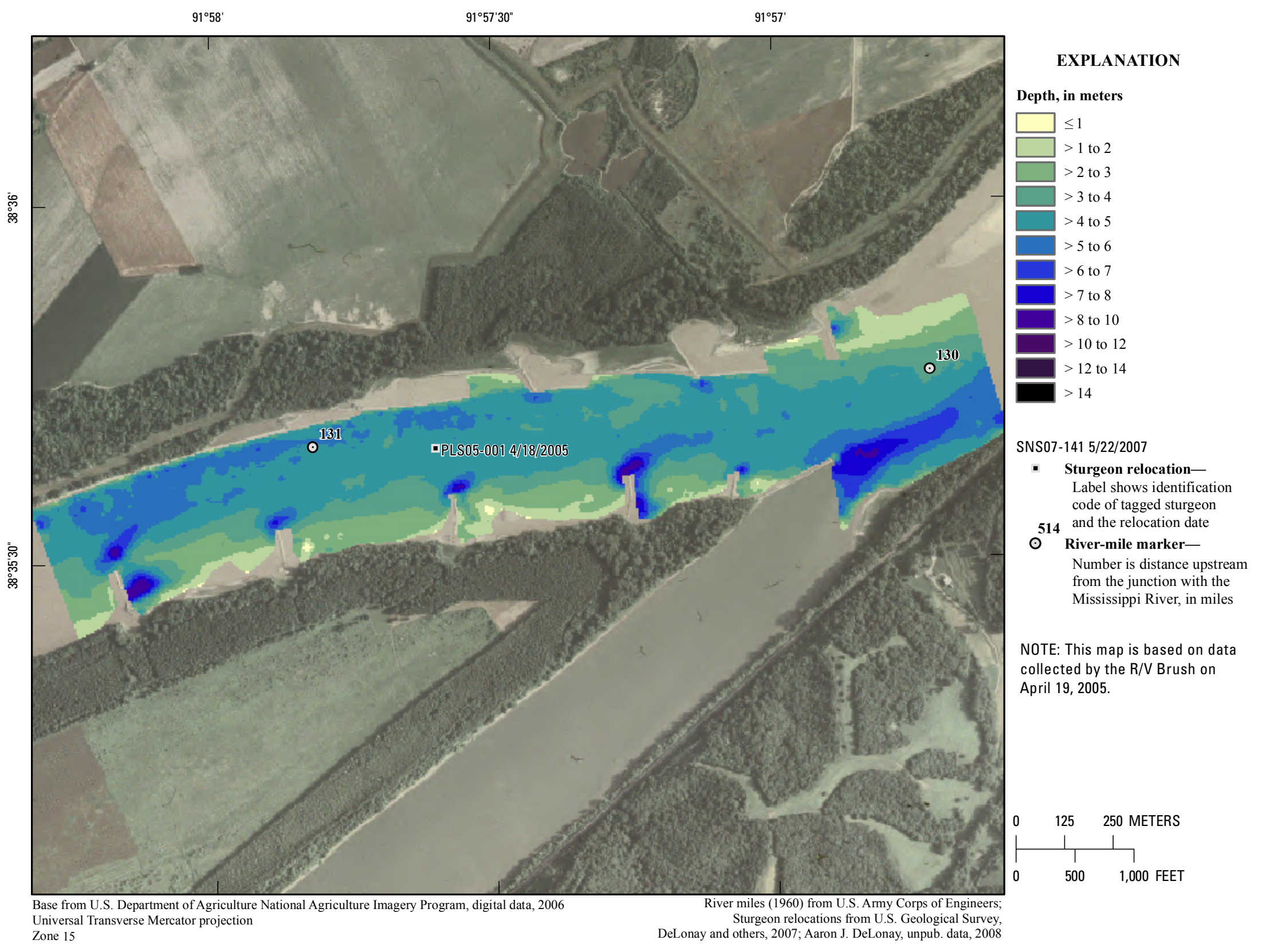

Figure 391. Map of depth based on data collected on April 19, 2005, in the vicinity of river mile 131. 


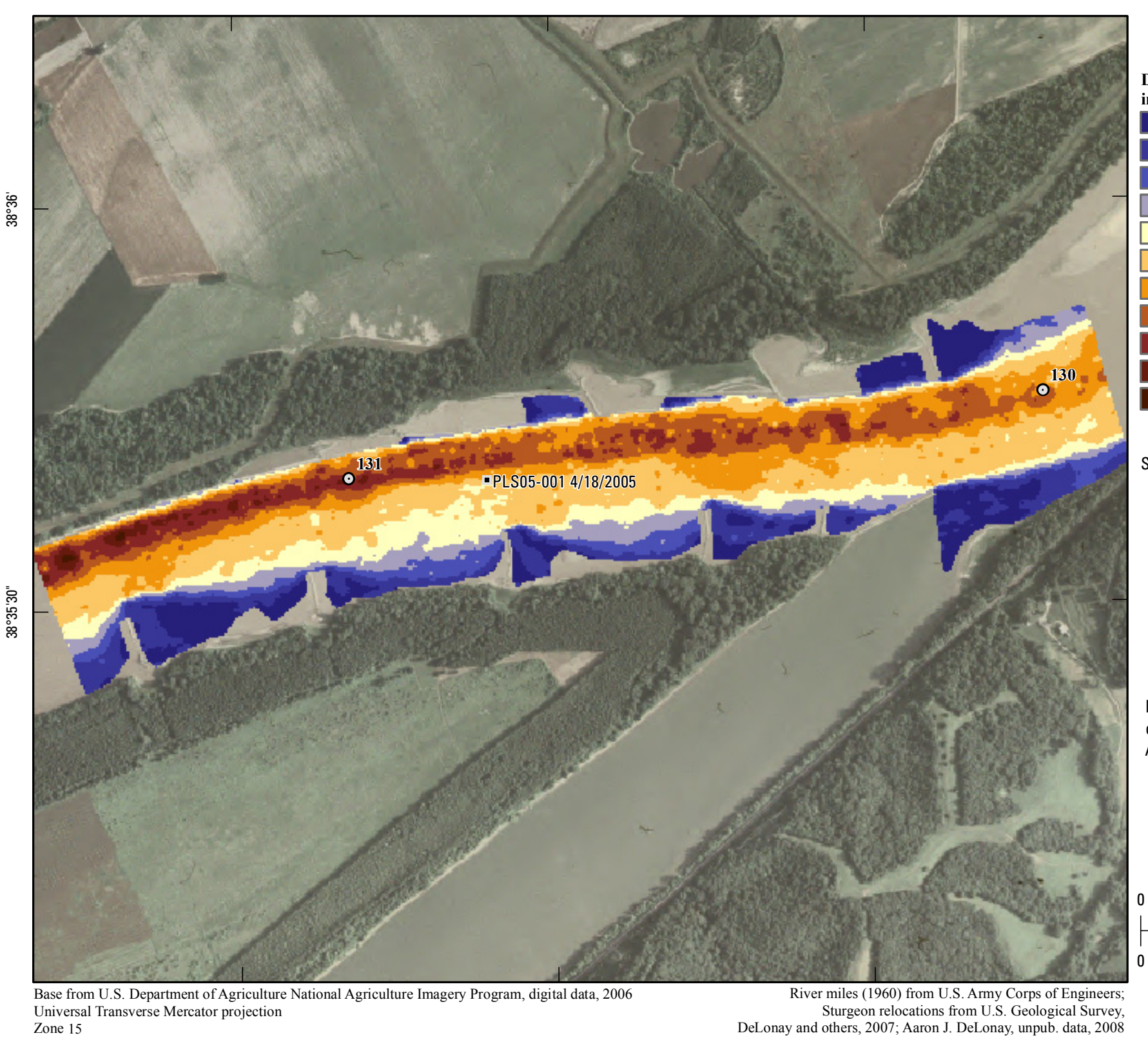

EXPLANATION

Depth-averaged velocity,

in meters per second

0 to 0.2

$>0.2$ to 0.4

$>0.4$ to 0.6

$>0.6$ to 0.8

$>0.8$ to 1.0

$>1.0$ to 1.2

$>1.2$ to 1.4

$>1.4$ to 1.6

$>1.6$ to 1.8

$>1.8$ to 2.0

$>2.0$

\section{SNS07-141 5/22/2007}

Sturgeon relocation-

Label shows identification

code of tagged sturgeo

514 and the relocation date

$\odot$ River-mile marker-

Number is distance upstream

from the junction with the Mississippi River, in miles

NOTE: This map is based on data

collected by the R/V Brush on

April 19, 2005.

\section{$125 \quad 250$ METERS

1 \\ $500 \quad 1,000$ FEET}

Figure 392. Map of depth-averaged velocity based on data collected on April 19, 2005, in the vicinity of river mile 131 . 


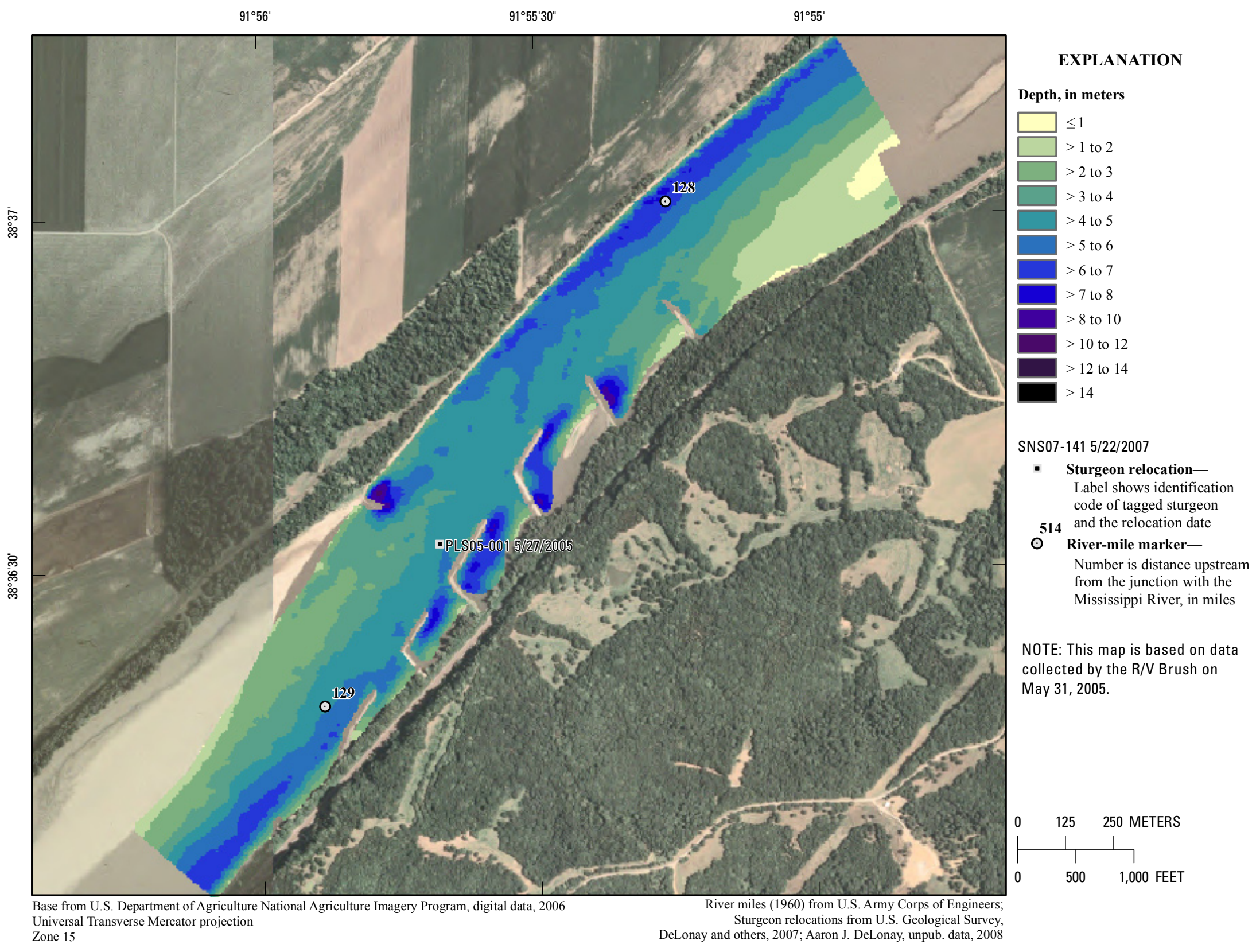

Figure 393. Map of depth based on data collected on May 31, 2005, in the vicinity of river mile 129. 


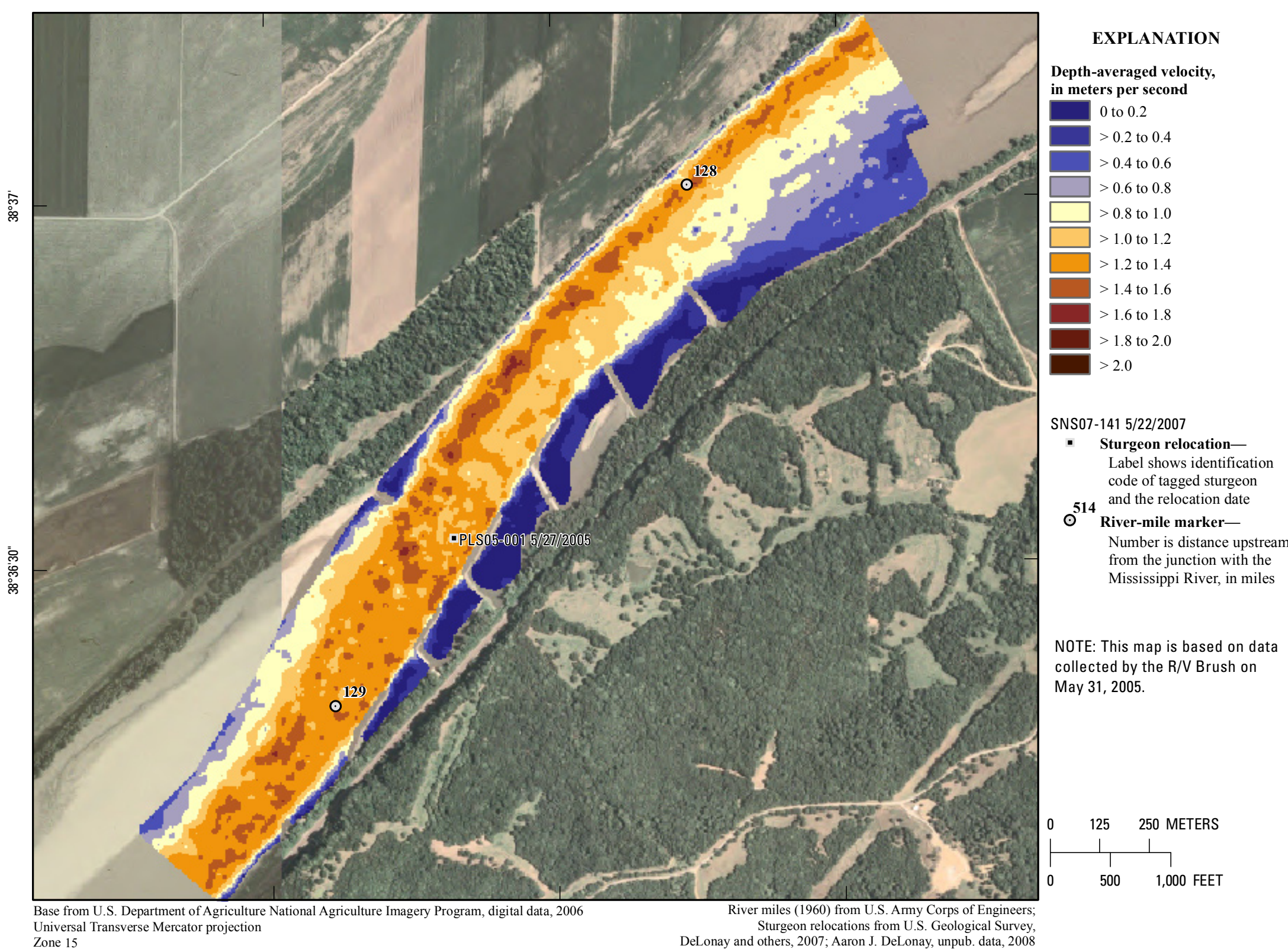




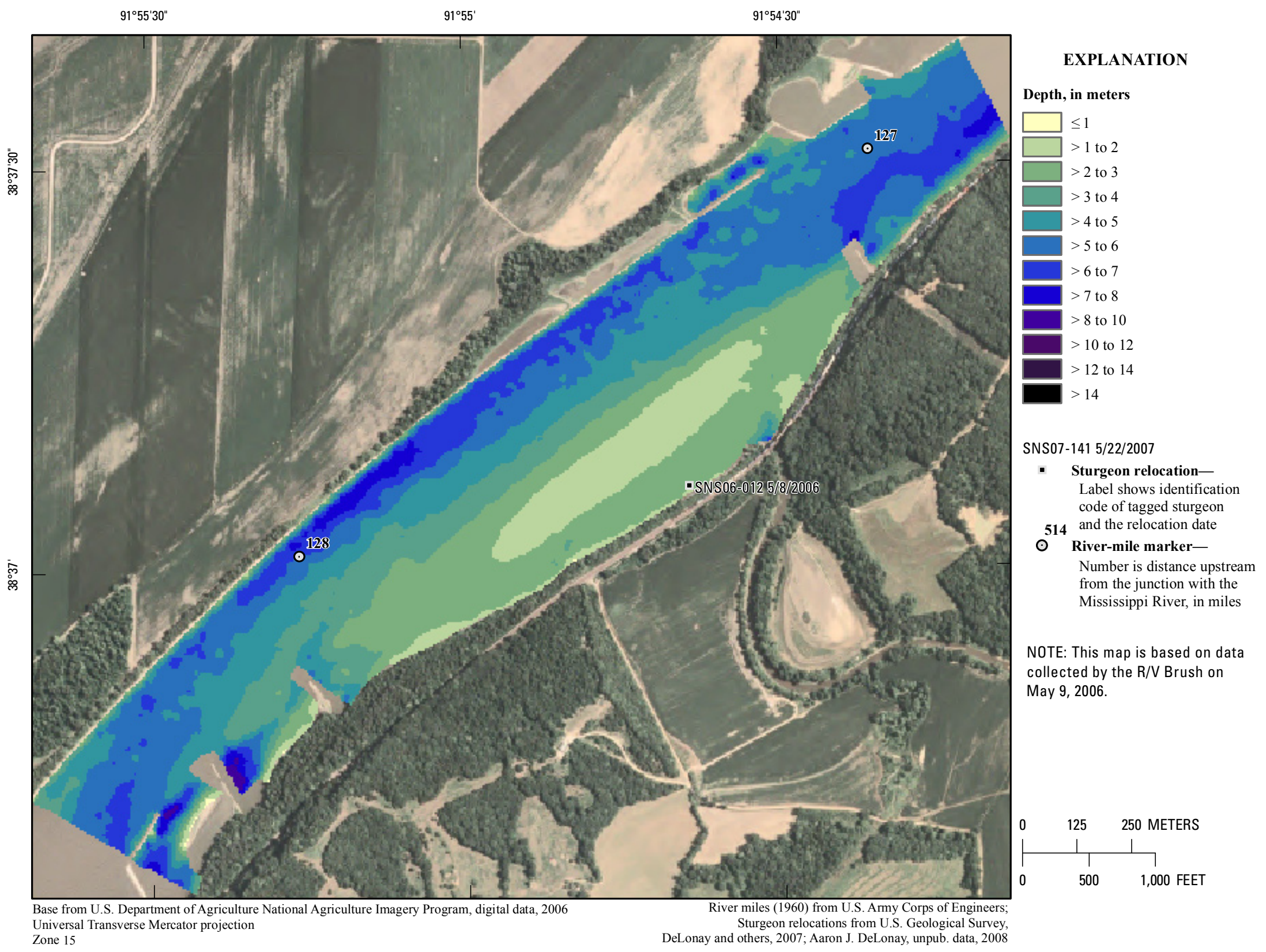

Figure 395. Map of depth based on data collected on May 9, 2006, in the vicinity of river mile 128. 


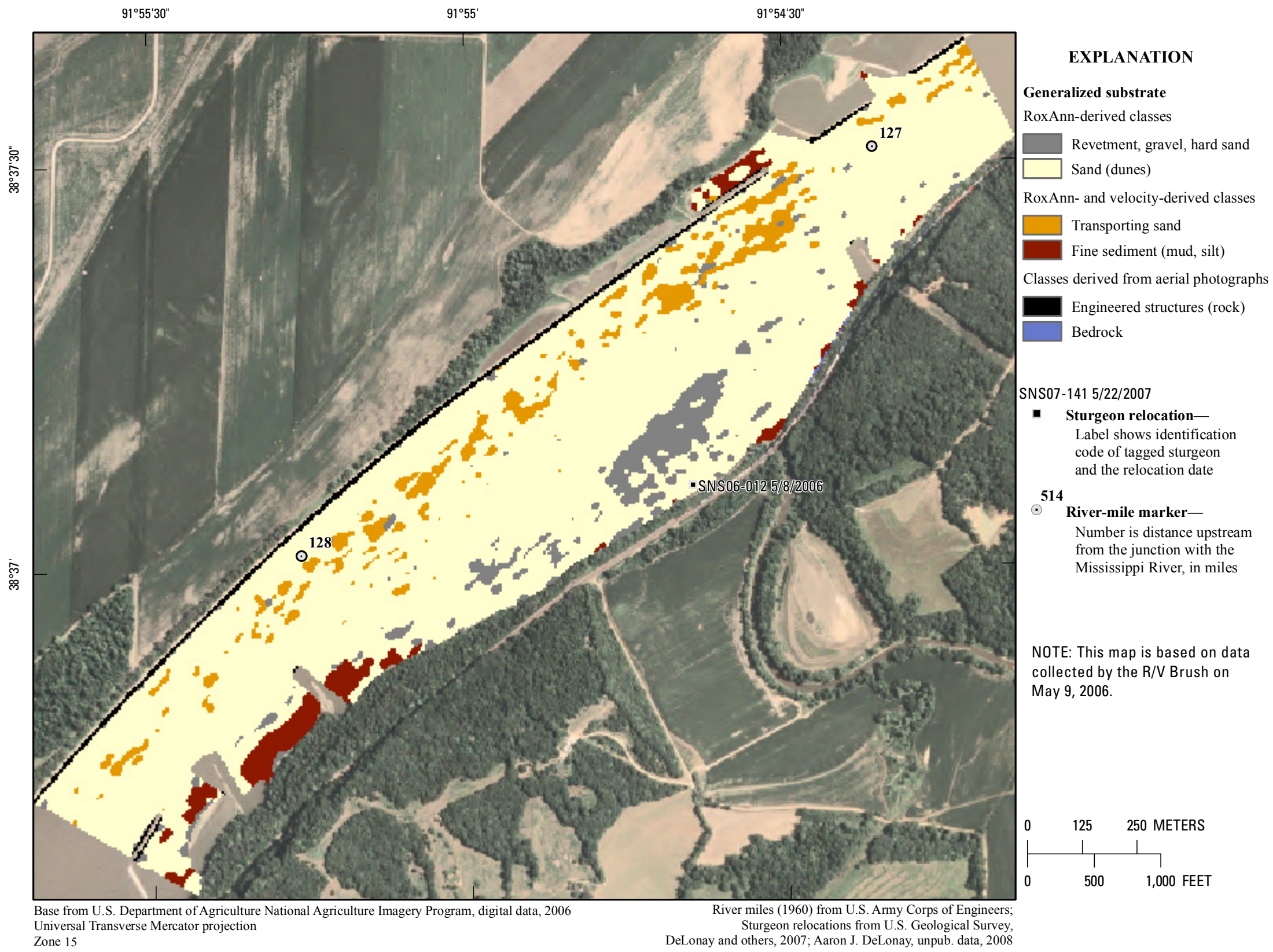




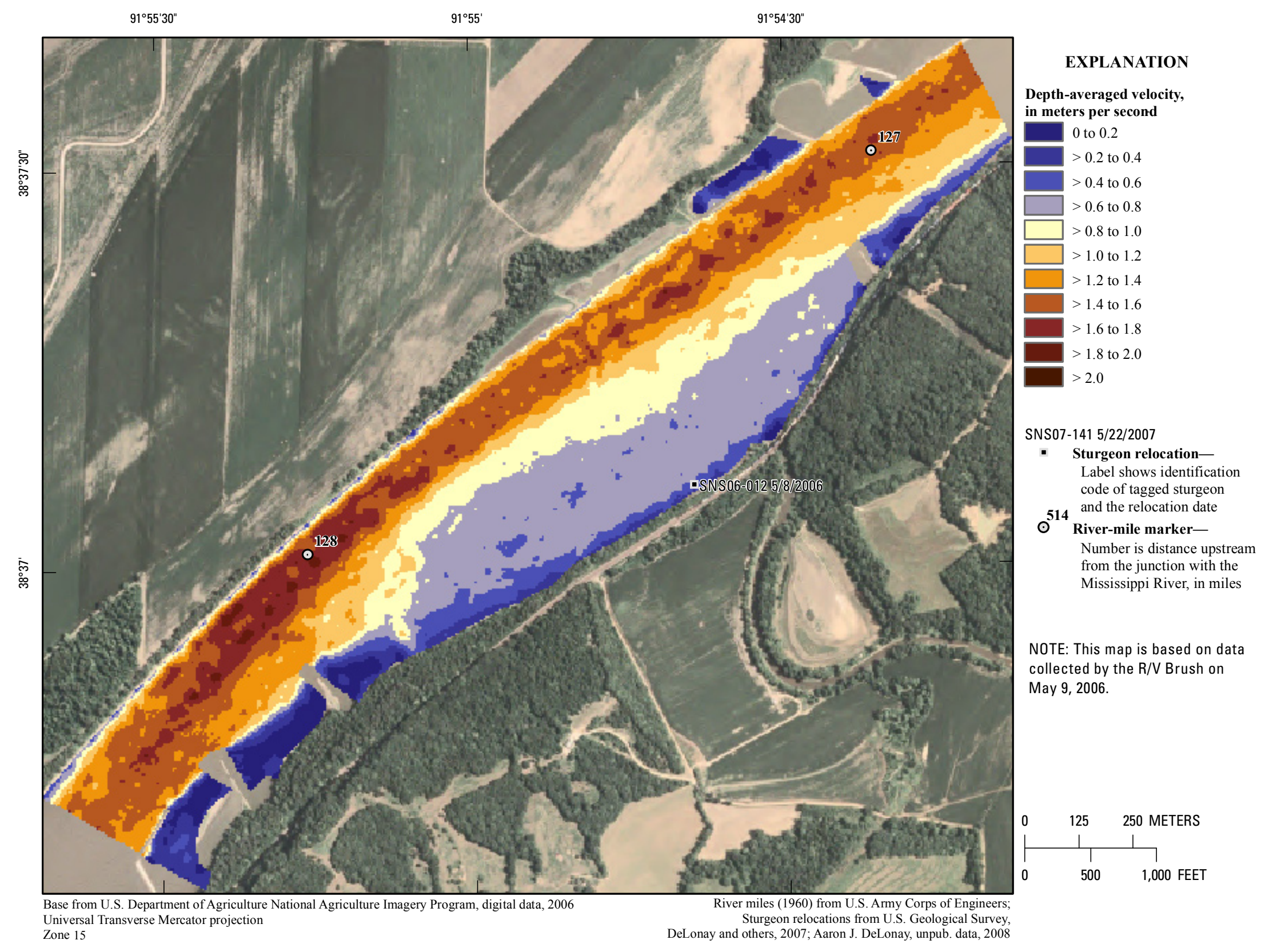

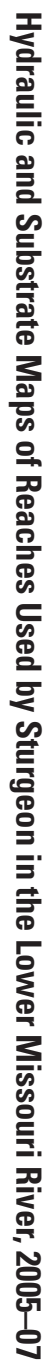

Figure 397. Map of depth-averaged velocity based on data collected on May 9, 2006, in the vicinity of river mile 128 . 


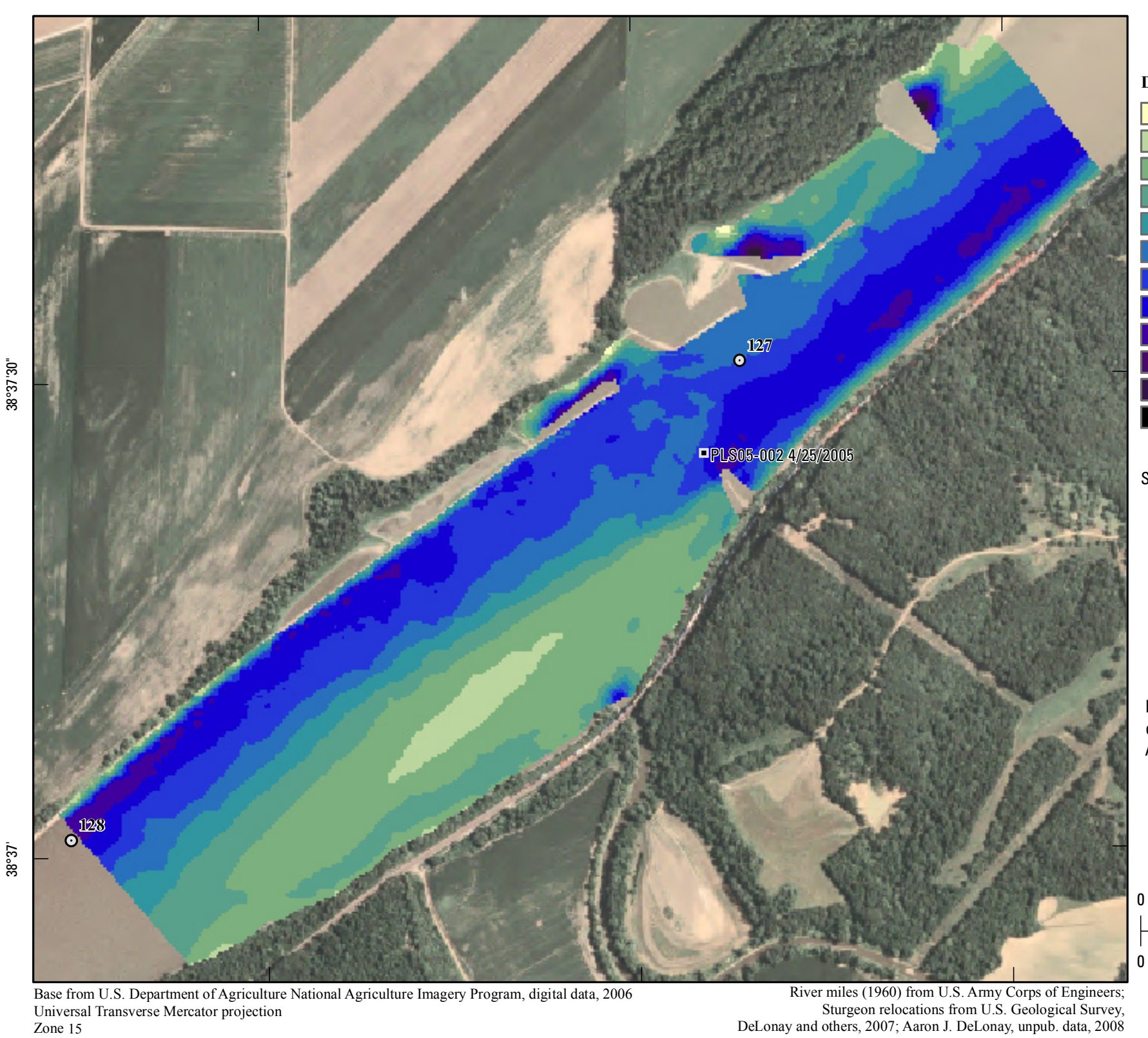

EXPLANATION

Depth, in meters

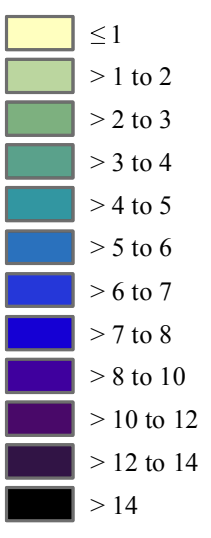

SNS07-141 5/22/2007

- Sturgeon relocationLabel shows identification code of tagged sturgeon 514 and the relocation date

Number is distance upstream from the junction with the Mississippi River, in miles

NOTE: This map is based on data collected by the R/V Brush on April 26, 2005. 


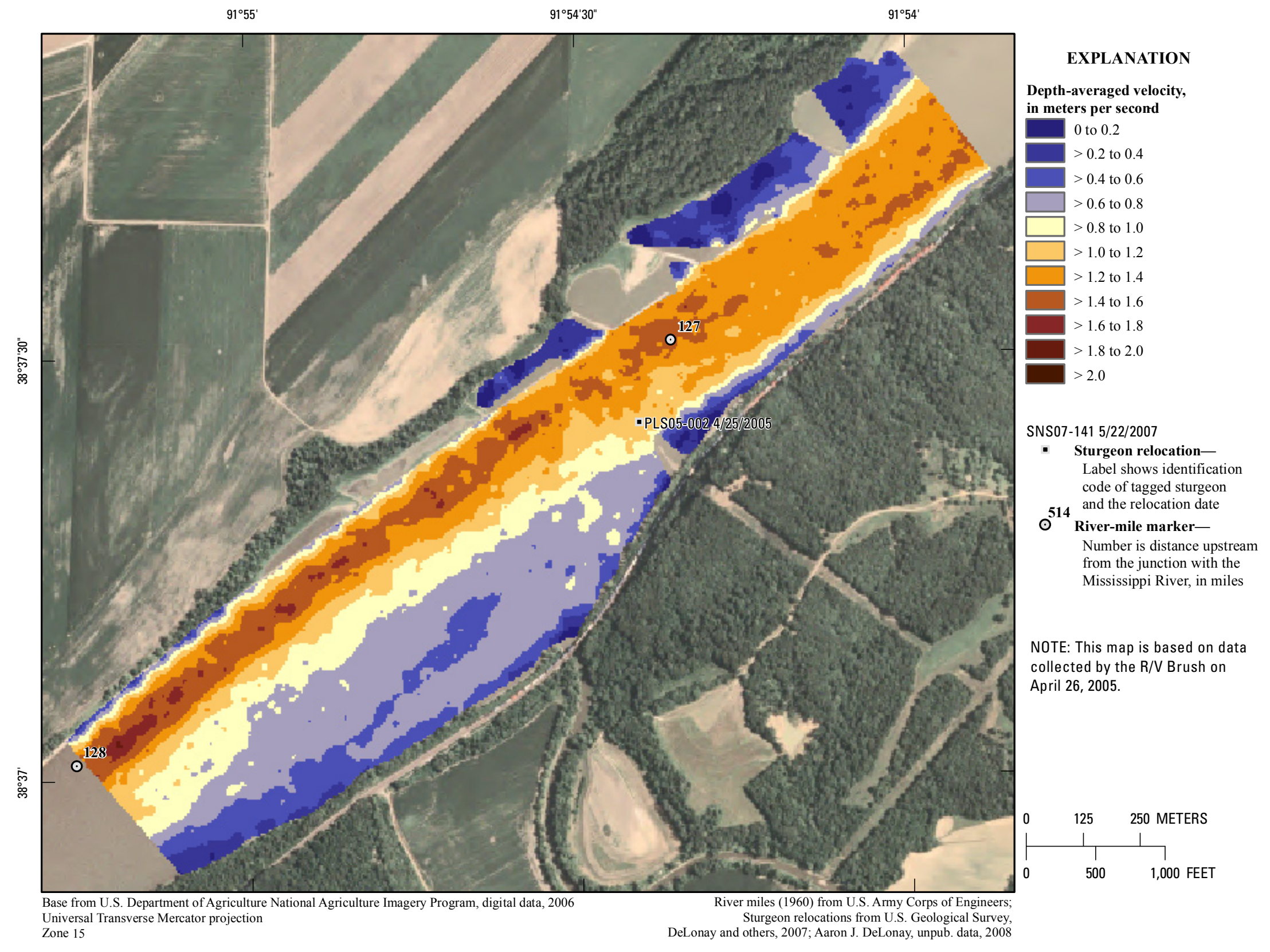

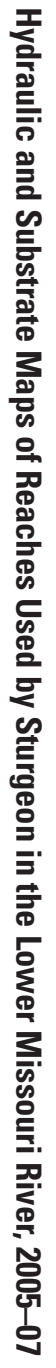

Figure 399. Map of depth-averaged velocity based on data collected on April 26, 2005, in the vicinity of river mile 127. 


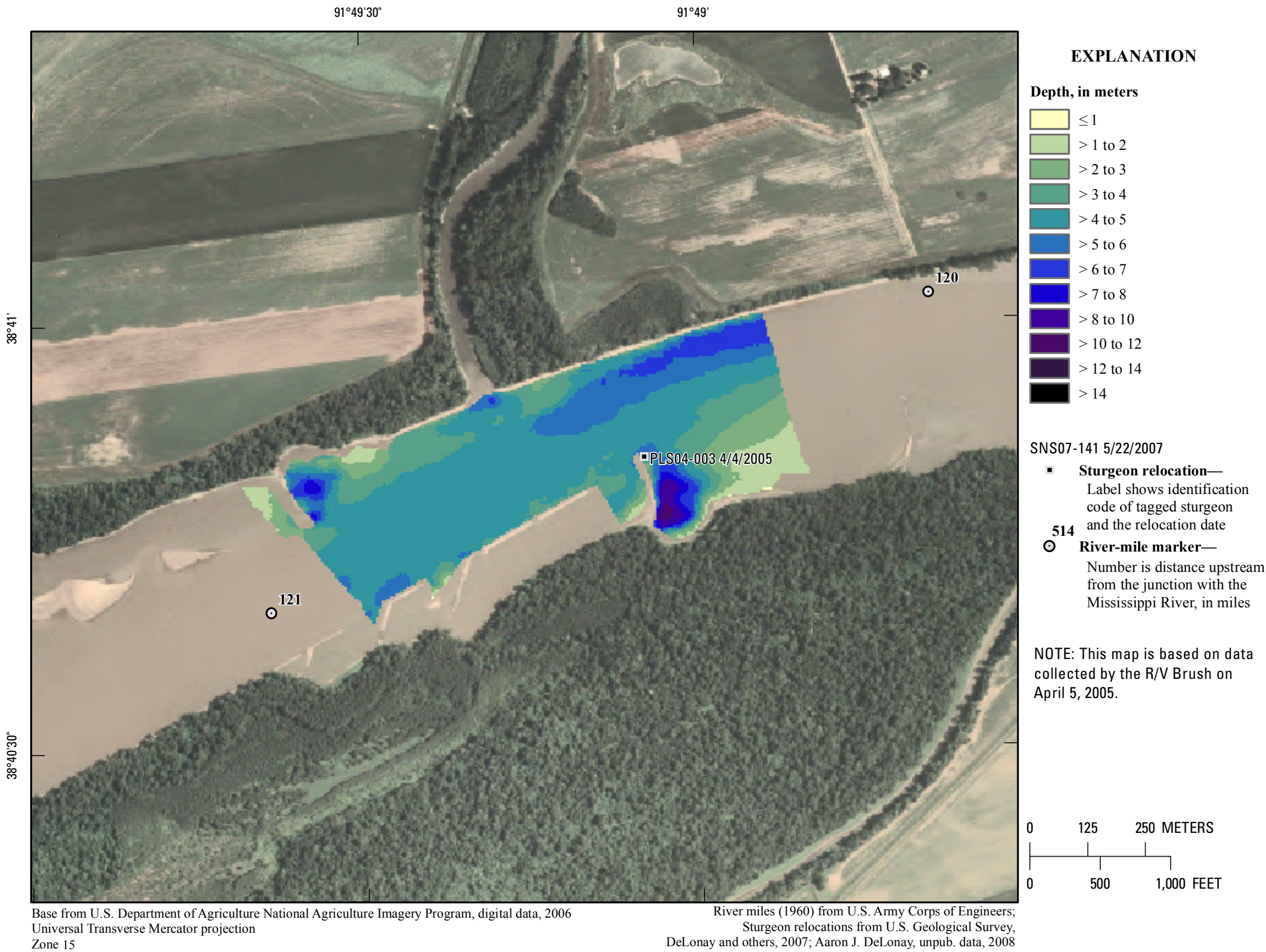

Figure 400. Map of depth based on data collected on April 5, 2005, in the vicinity of river mile 121. 


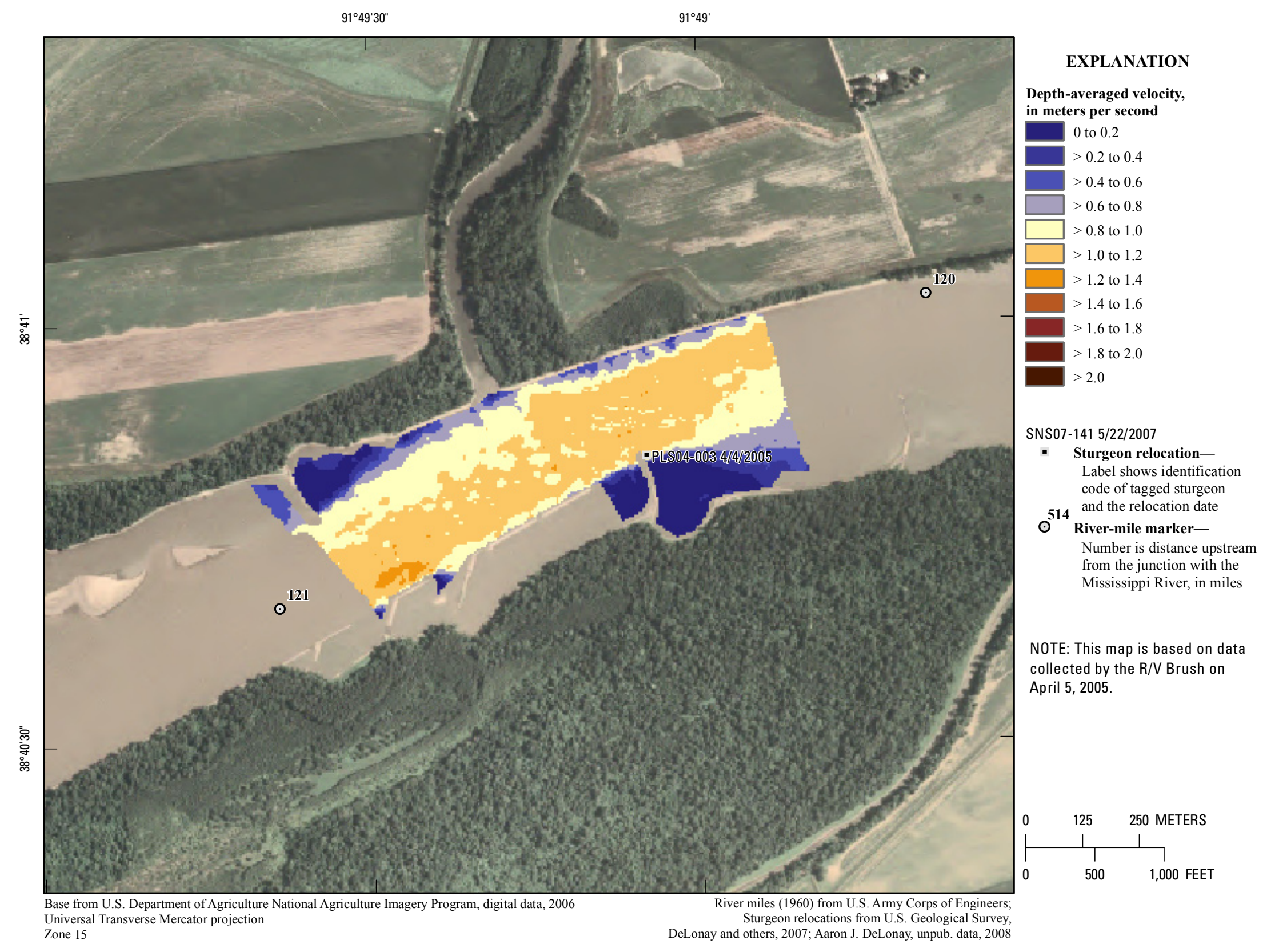

Figure 401. Map of depth-averaged velocity based on data collected on April 5, 2005, in the vicinity of river mile 121 . 


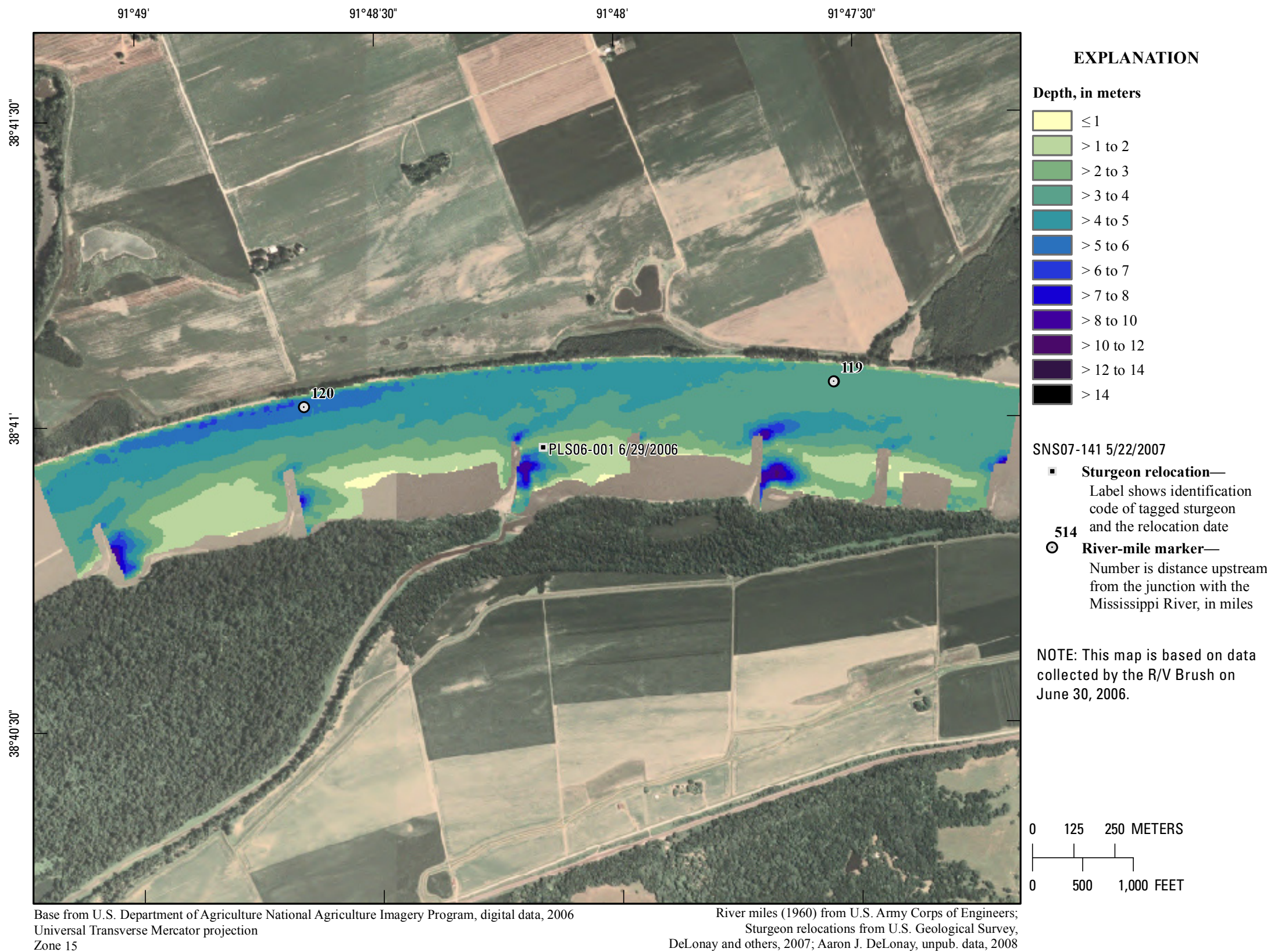

Figure 402. Map of depth based on data collected on June 30, 2006, in the vicinity of river mile 120 . 


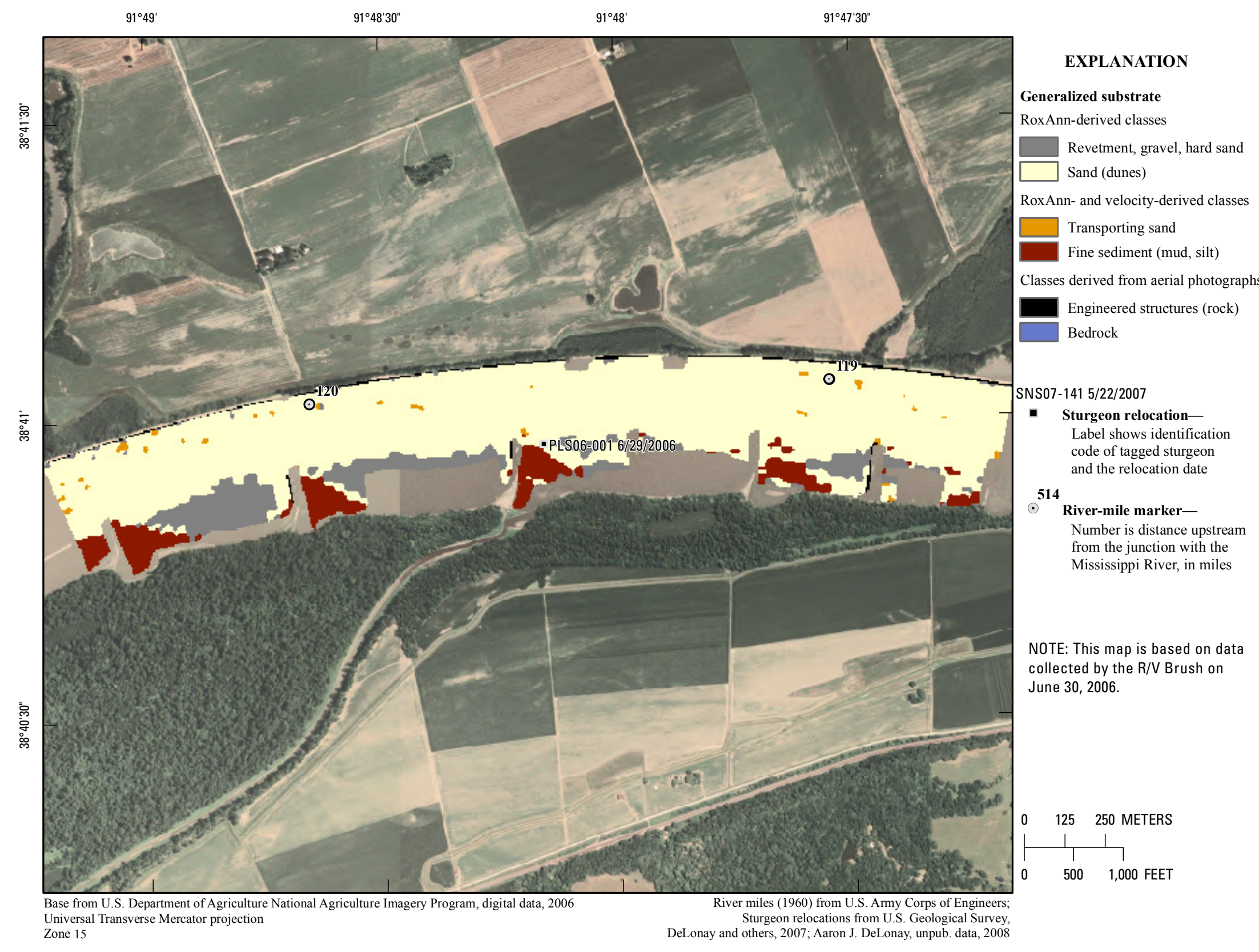

Figure 403. Map of generalized substrate based on data collected on June 30, 2006, in the vicinity of river mile 120 . 


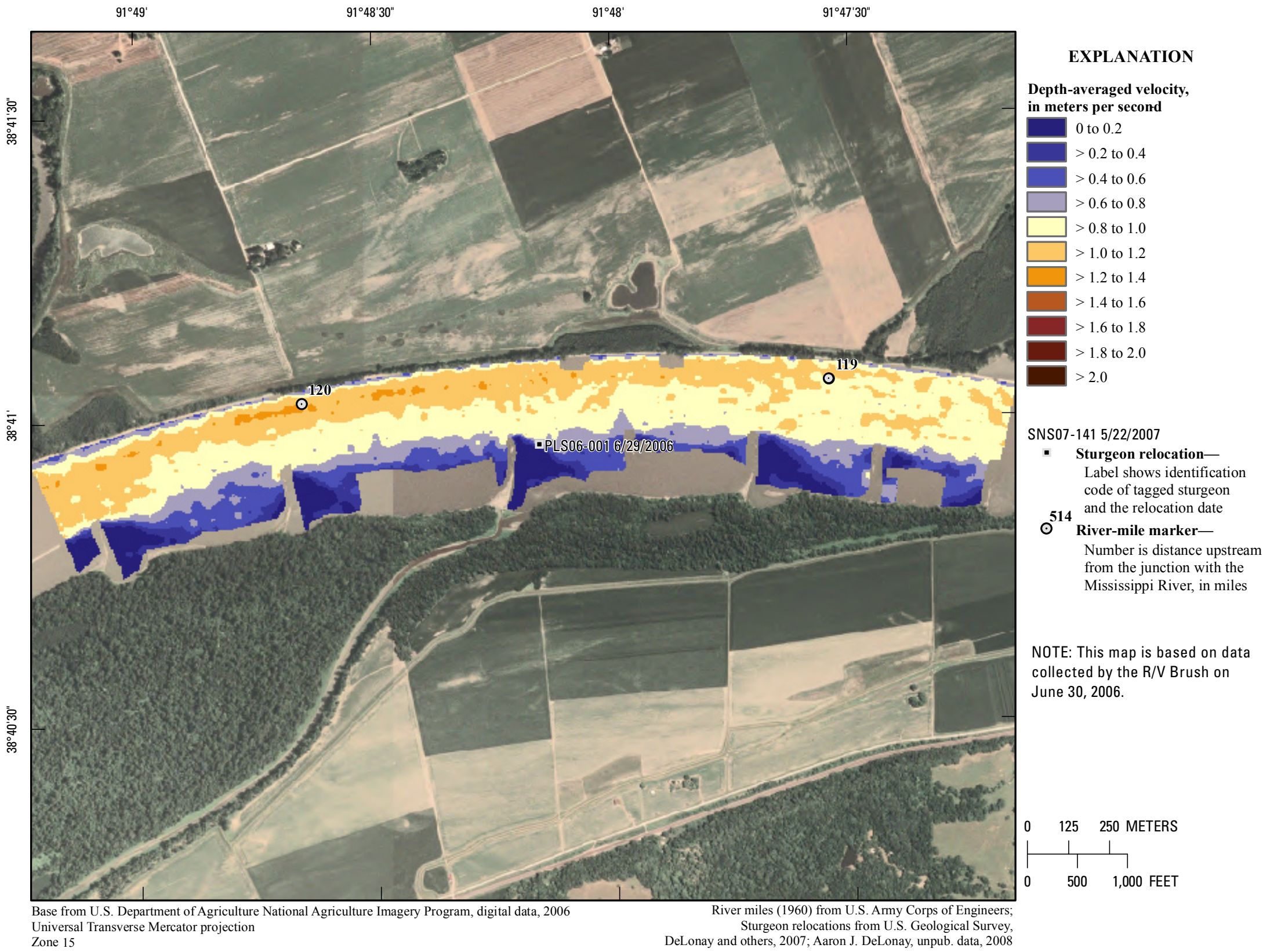

Figure 404. Map of depth-averaged velocity based on data collected on June 30, 2006, in the vicinity of river mile 120. 


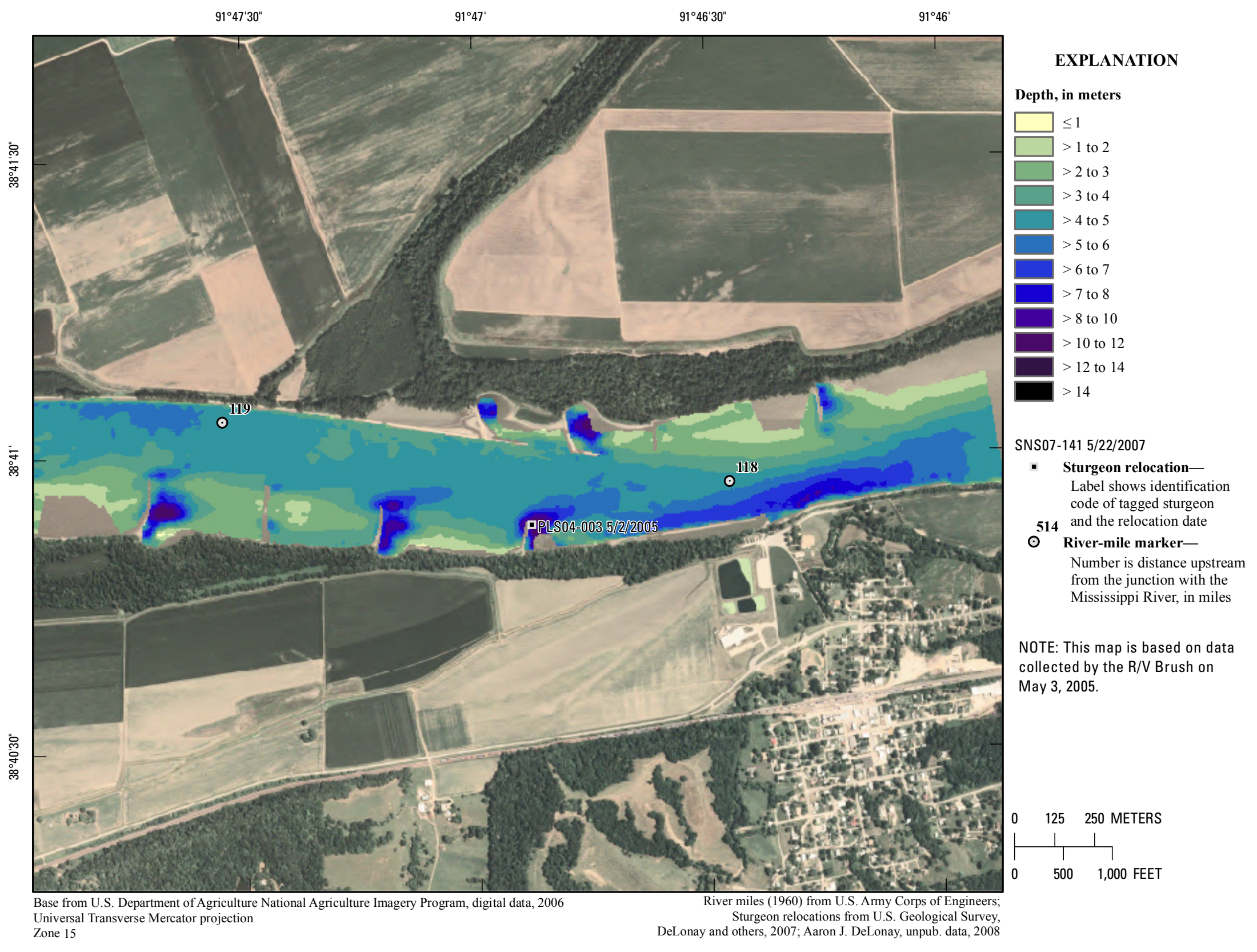

Figure 405. Map of depth based on data collected on May 3, 2005, in the vicinity of river mile 119. 


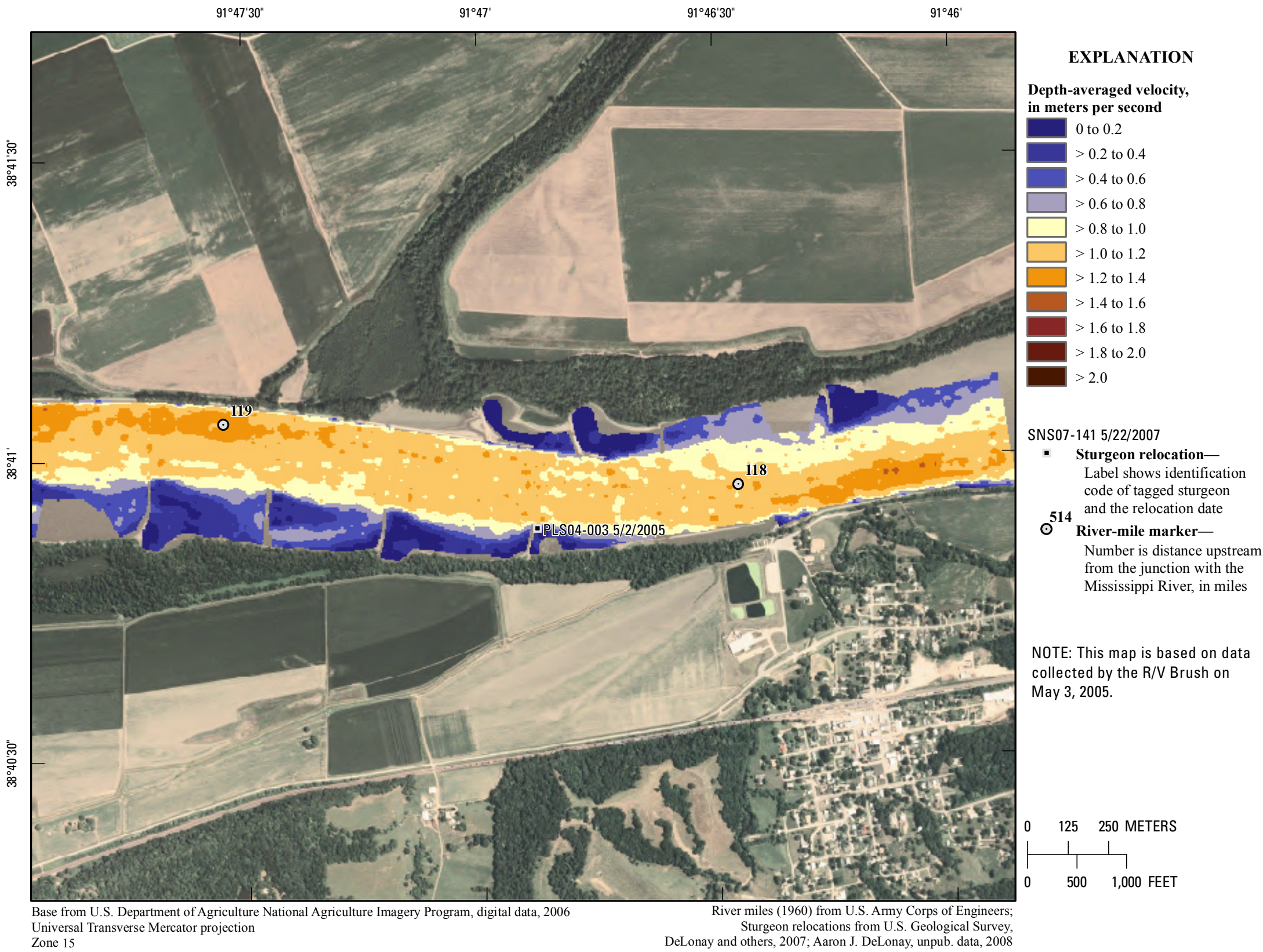

Figure 406. Map of depth-averaged velocity based on data collected on May 3, 2005, in the vicinity of river mile 119. 


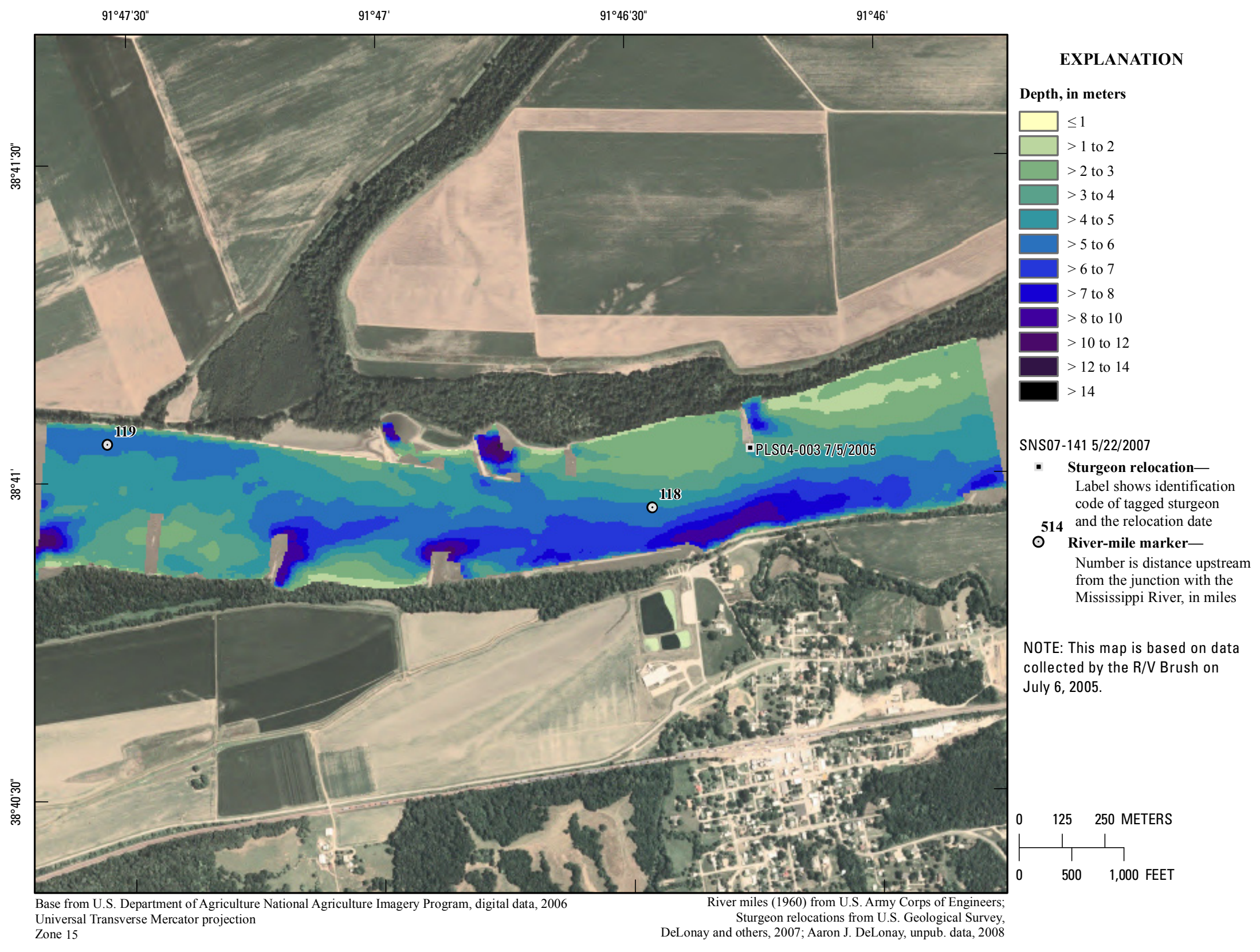

Figure 407. Map of depth based on data collected on July 6, 2005, in the vicinity of river mile 118 . 


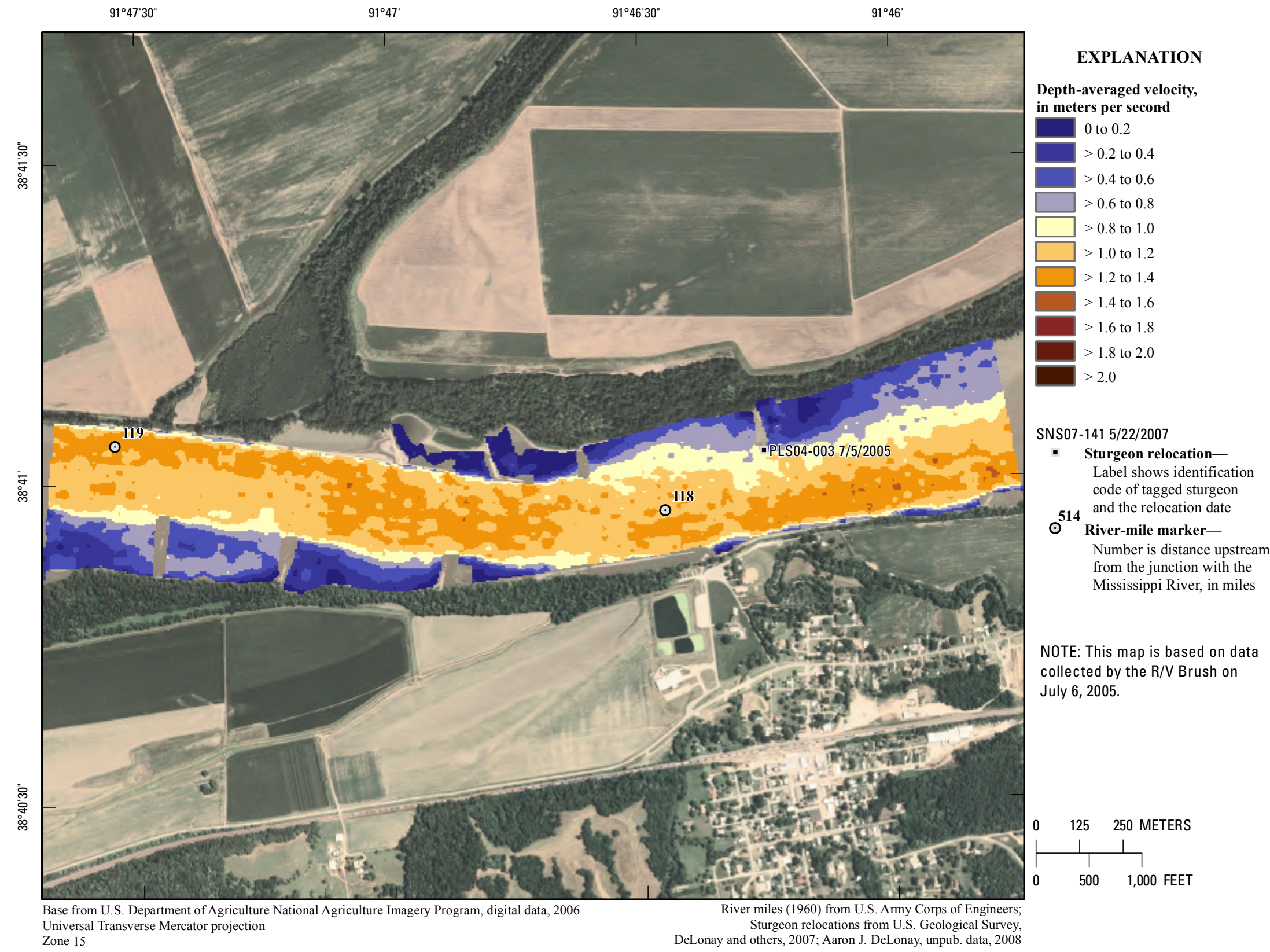

Figure 408. Map of depth-averaged velocity based on data collected on July 6, 2005, in the vicinity of river mile 118 . 


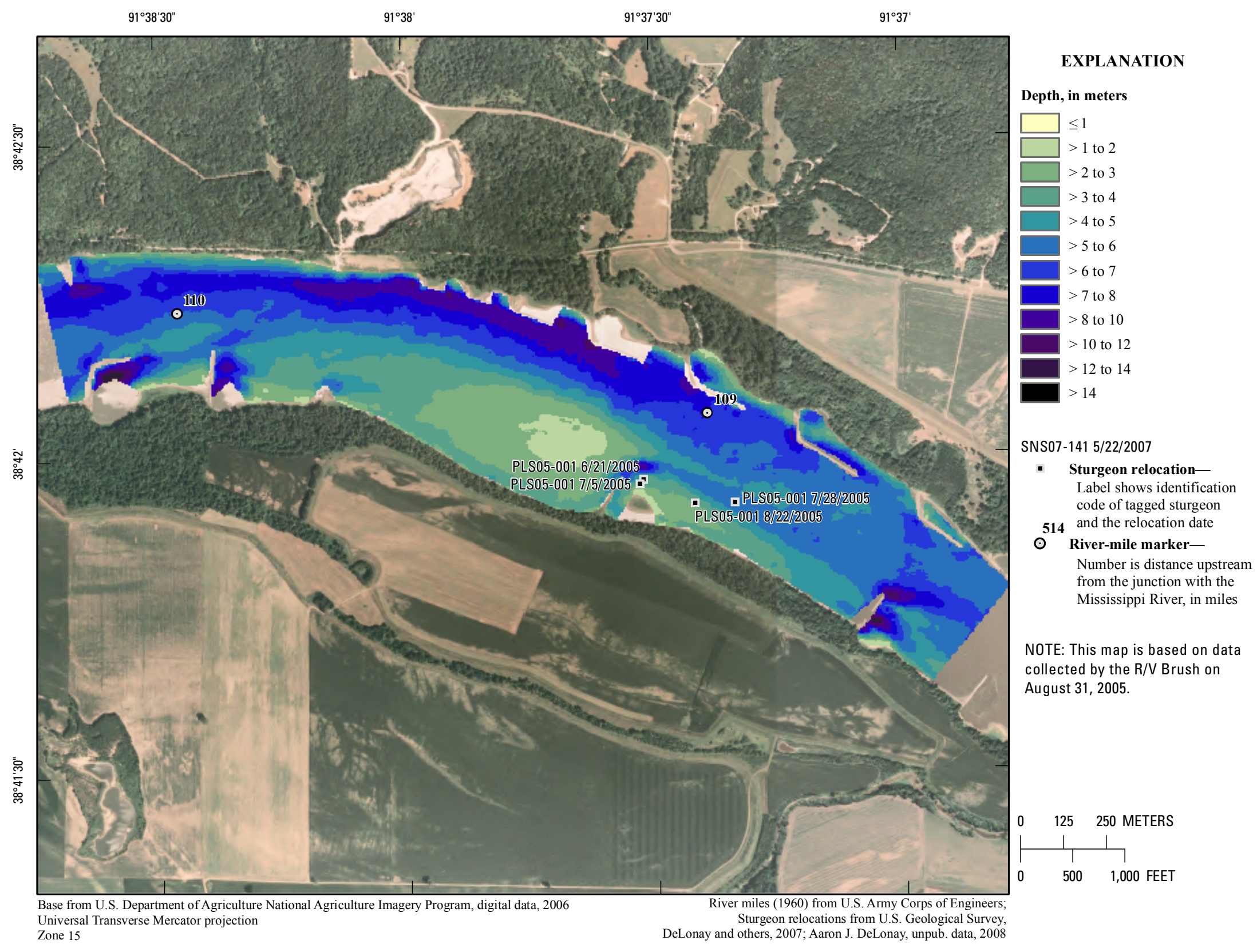

Figure 409. Map of depth based on data collected on August 31, 2005, in the vicinity of river mile 109. 


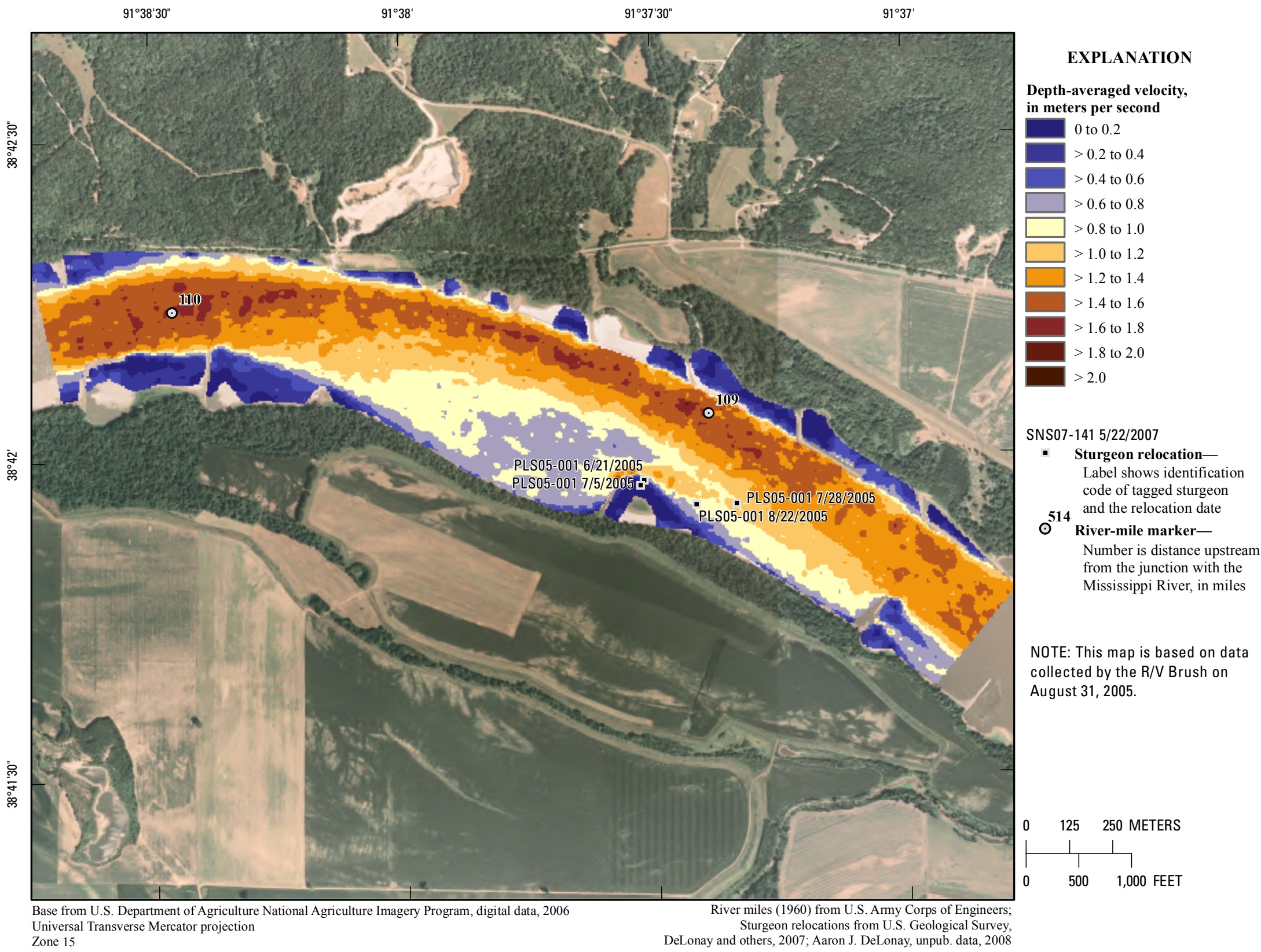

Figure 410. Map of depth-averaged velocity based on data collected on August 31, 2005, in the vicinity of river mile 109. 


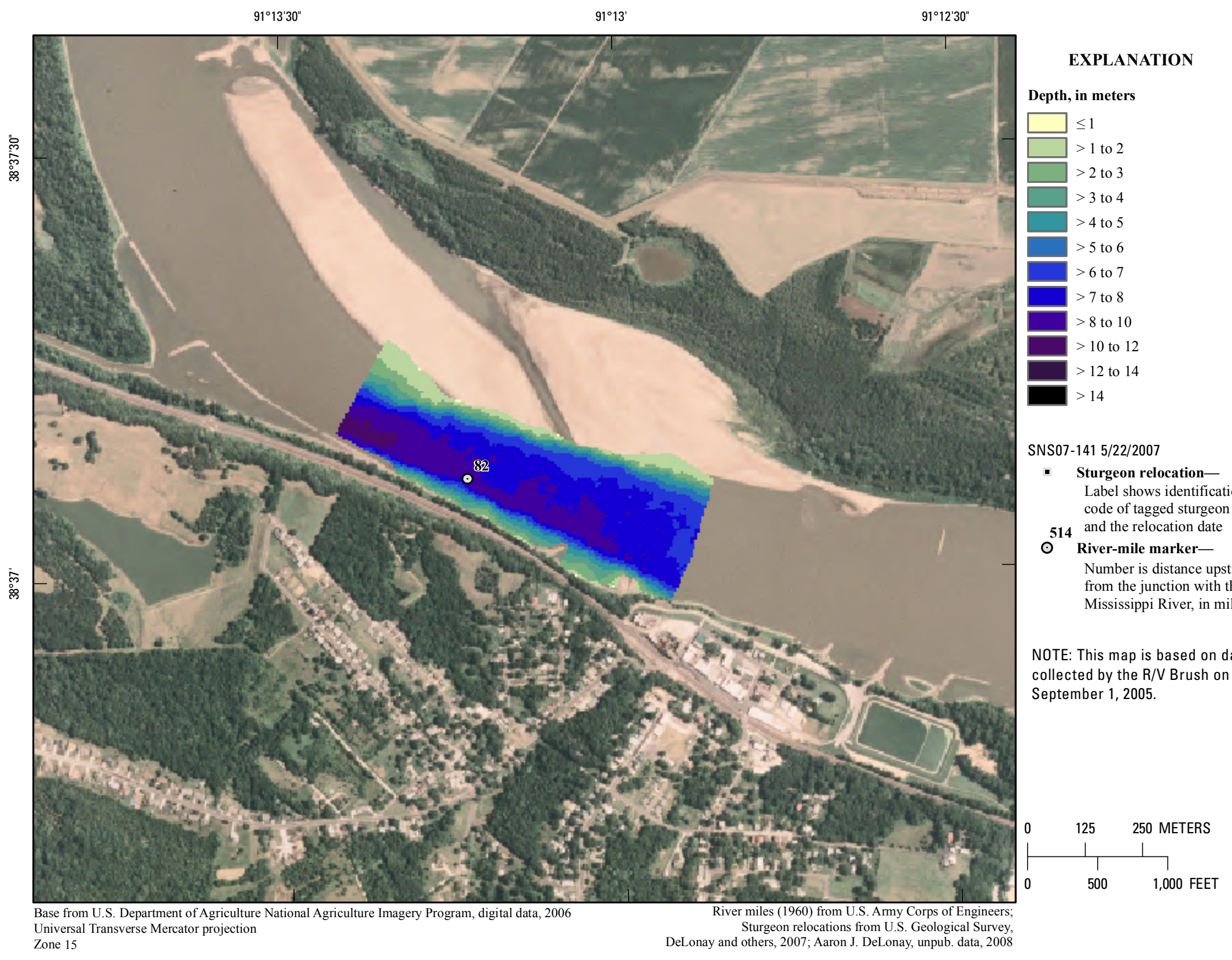

Figure 411. Map of depth based on data collected on September 1, 2005, in the vicinity of river mile 82. 


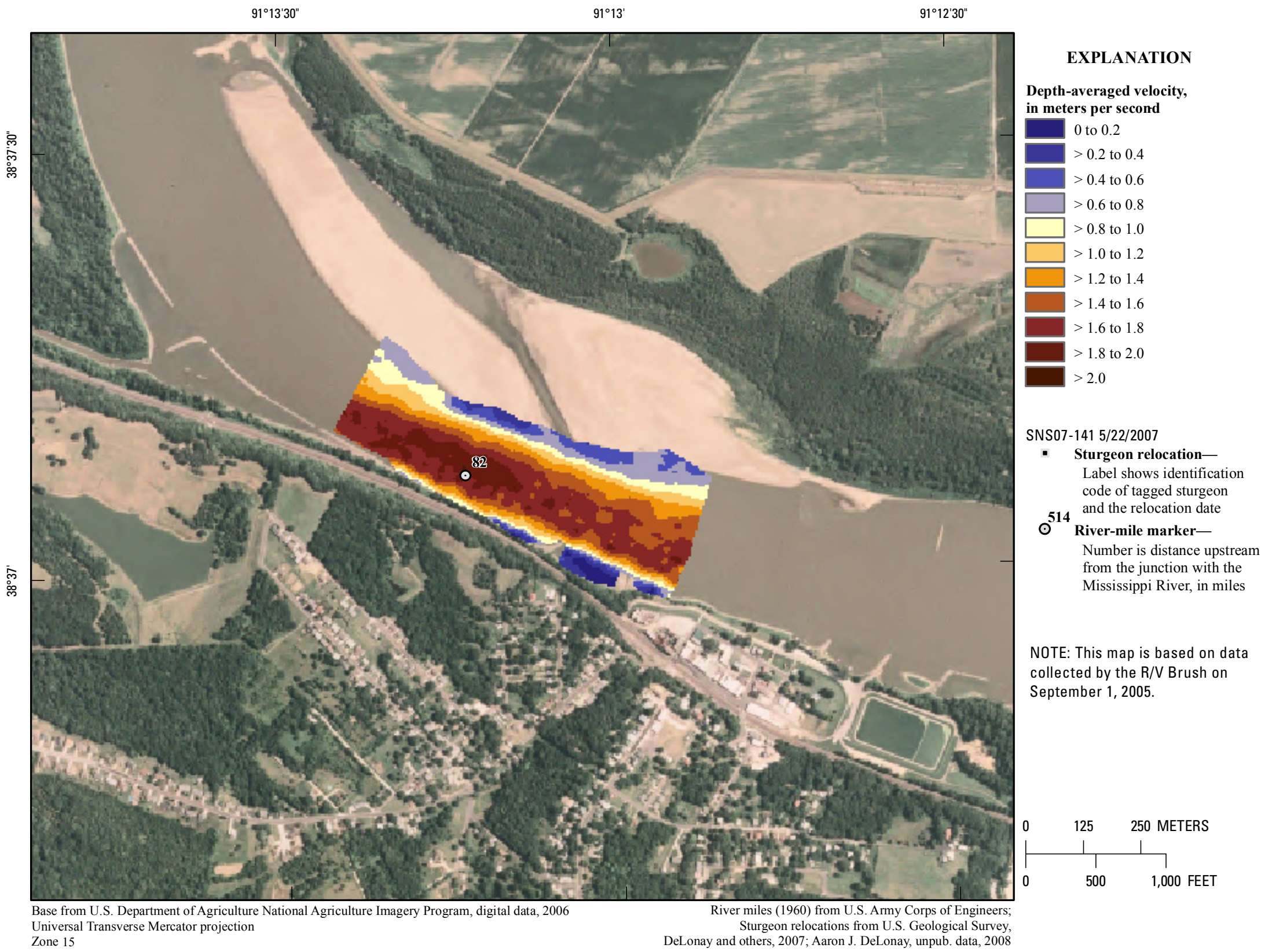

Figure 412. Map of depth-averaged velocity based on data collected on September 1, 2005, in the vicinity of river mile 82 . 


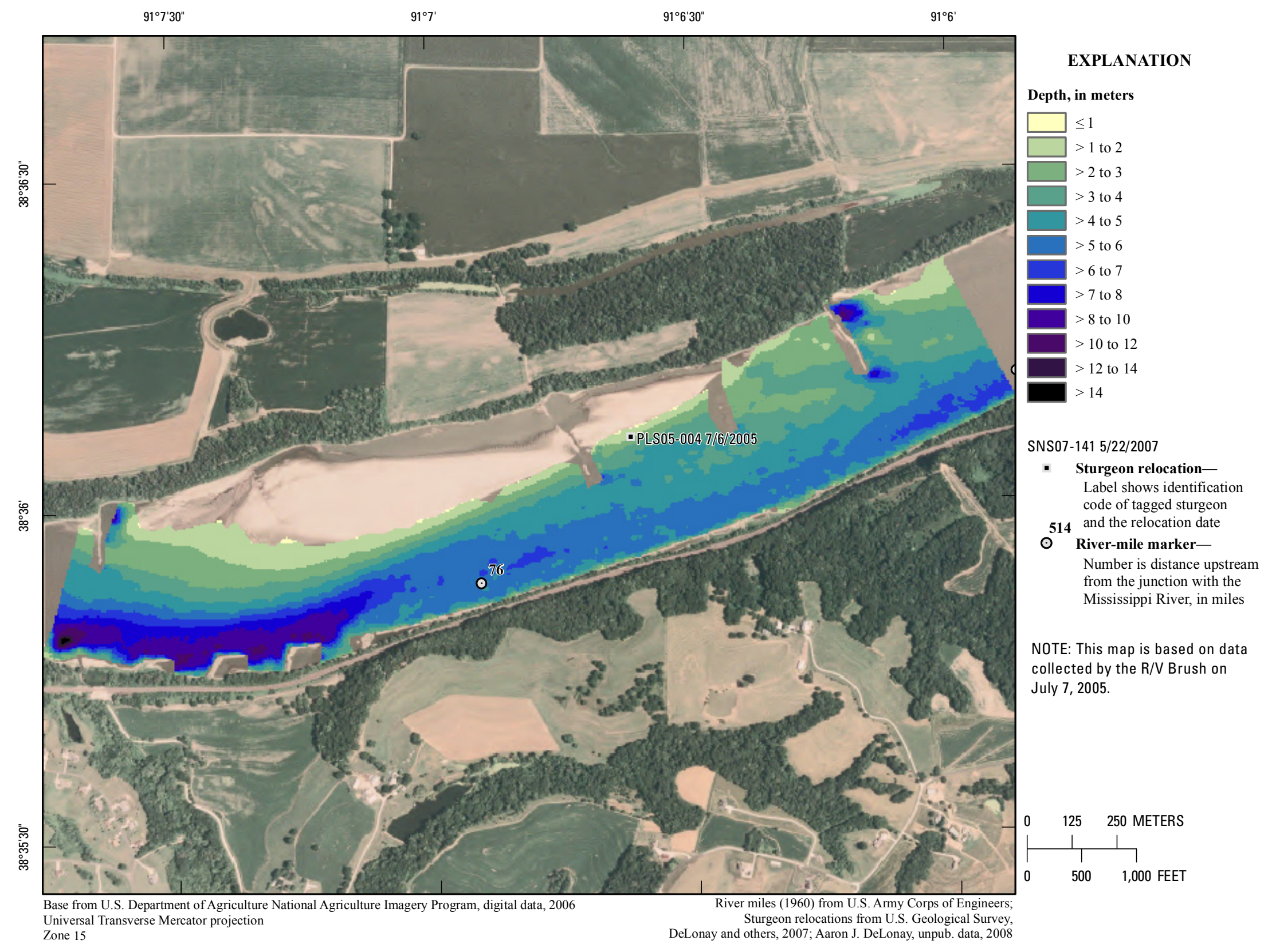

Figure 413. Map of depth based on data collected on July 7, 2005, in the vicinity of river mile 76 . 


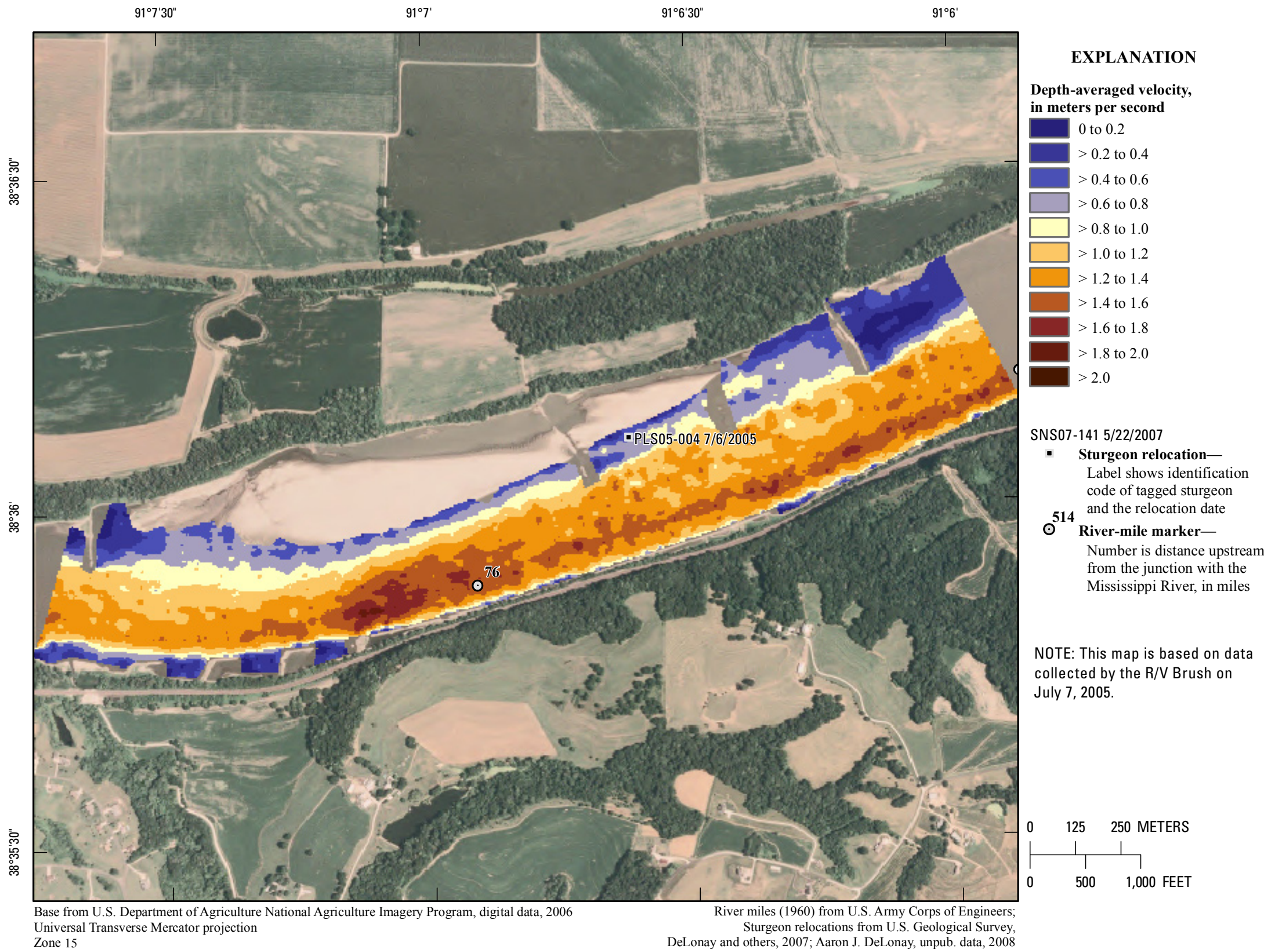

Figure 414. Map of depth-averaged velocity based on data collected on July 7, 2005, in the vicinity of river mile 76 . 


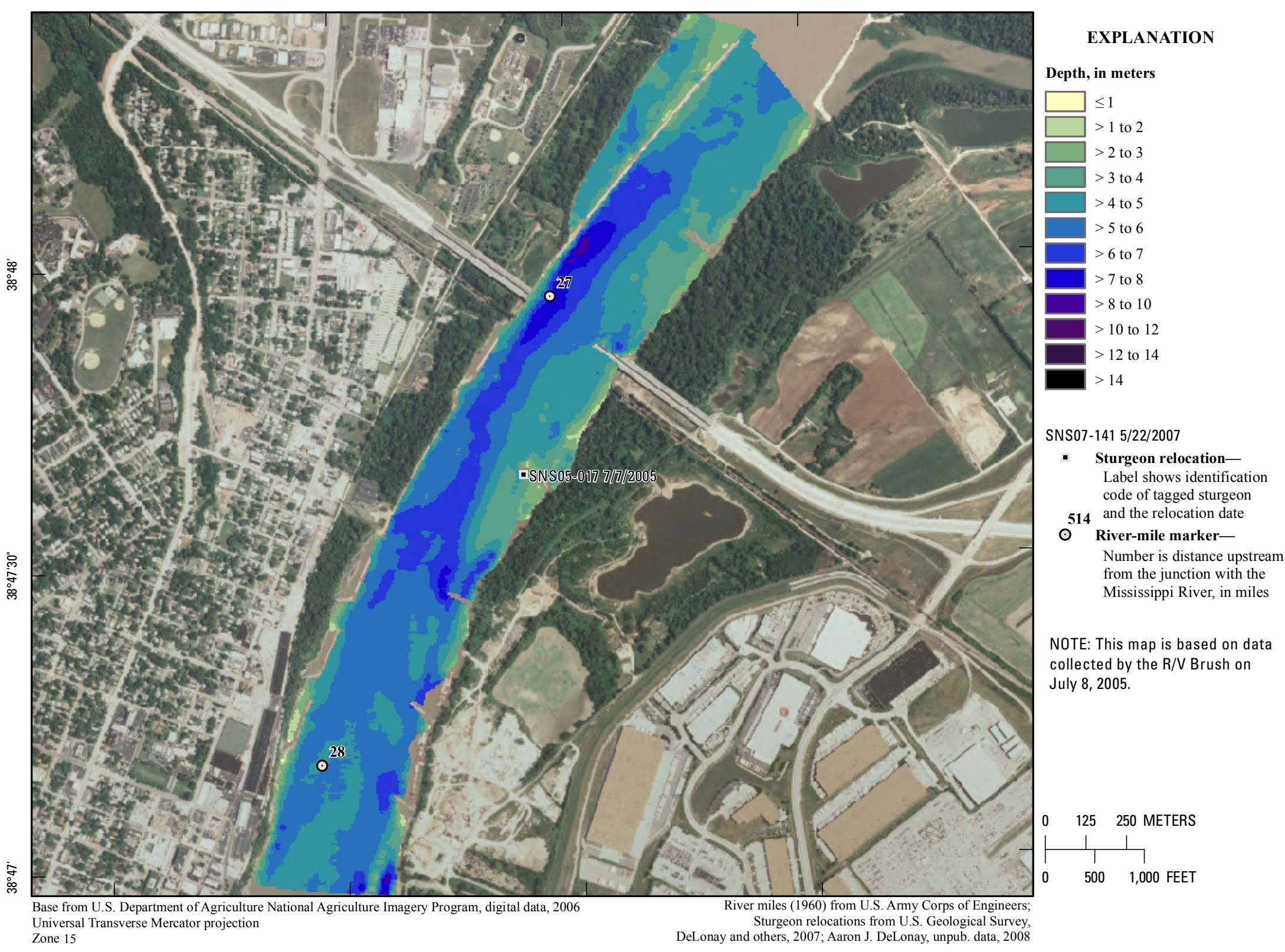

Figure 415. Map of depth based on data collected on July 8, 2005, in the vicinity of river mile 27 . 


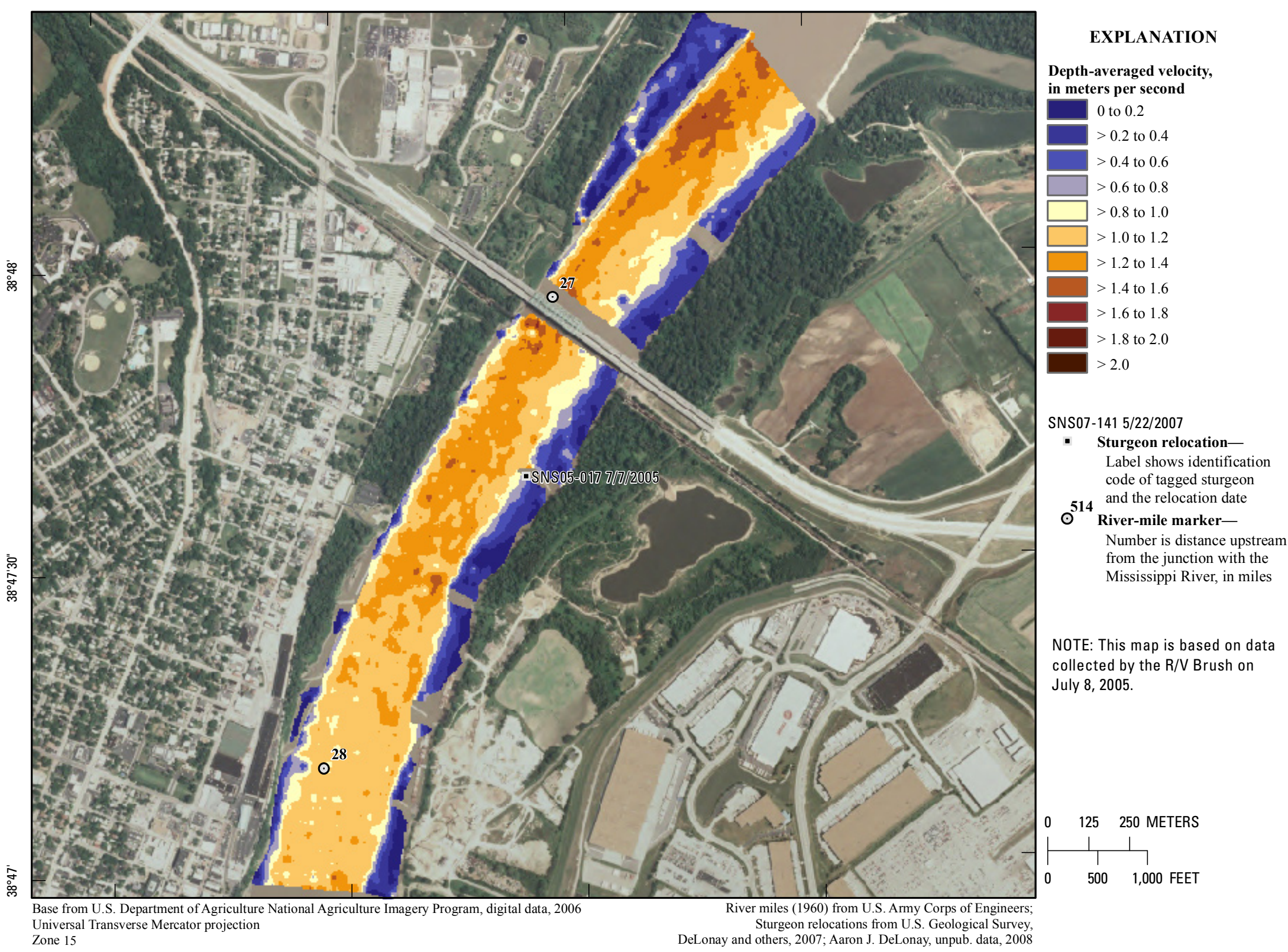
Universal Transverse Mercator projection
Zone 15 Sturgeon relocations from U.S. Geological Survey,
DeLonay and others, 2007; Aaron J. DeLonay, unpub. data, 2008 
Publishing support provided by:

Denver Publishing Service Center

For more information concerning this publication, contact:

Director

U.S. Geological Survey

Columbia Environmental Research Center

4200 New Haven Road

Columbia, M0 65201

(573) 875-5399

Or visit the Columbia Environmental Research Center website at:

http://www.cerc.usgs.gov 
